\title{
MRS/MRSI Aquisition
}

Metabolite cycled density-weighted concentric rings k-space trajectory (DW-CRT) enables $1 \mathrm{H}$ magnetic resonance spectroscopic imaging at 3 Tesla in a clinically feasible timeframe

Adam Steel ${ }^{1,2}$, Mark Chiew ${ }^{3}$, Peter Jezzard ${ }^{4}$, Natalie Voets ${ }^{4}$, Puneet Plaha ${ }^{4}$, M. Albert Thomas ${ }^{5}$, Charlotte J Stagg ${ }^{4}$, and Uzay E Emir ${ }^{6}$

${ }^{1}$ Nuffield Department of Medicine, University of Oxford, Headington, United Kingdom, ${ }^{2}$ National Institute of Mental Health, National Institutes of Health, Bethesda, DC, United States, ${ }^{3}$ Nuffield Department of Clinical Neuroscience, University of Oxford, Headington, United Kingdom, ${ }^{4}$ Nuffield Department of Clinical Neurosciences, University of Oxford, Headington, United Kingdom, ${ }^{5}$ Department of Radiology, University of California Los Angelas, Los Angeles, CA, United States, ${ }^{6}$ School of Health Sciences, Purdue University, West Lafayette, IN, United States

In this study, we demonstrate that a metabolite-cycled semi-LASER pulse localization with density-weighted concentric rings trajectory (DW-CRT) enables high-resolution MRSI to be acquired at 3 Tesla within a clinically feasible acquisition time. High-resolution $(5 \times 5 \times 10 \mathrm{~mm} 3)$ DW-CRT feasibility at $3 T$ was assessed in 6 healthy volunteers. Subsequently, the clinical utility of this approach was demonstrated by mapping the presence of 2-HG in a patient with a grade III oligodendroglioma tumor.

Standardized Parameterization of Echo-Planar Compressed Sensing MRSI Acquisition and Reconstruction

Jason C. Crane ${ }^{1}$, Marram P Olson ${ }^{1}$, Yan Li ${ }^{1}$, Maryam Vareth ${ }^{1}$, Hsin-Yu Chen ${ }^{1}$, Zihan Zhu ${ }^{1}$, Sukumar Subramaniam ${ }^{1}$, Peder E. Z. Larson ${ }^{1}$, Duan Xu ${ }^{1}$, Daniel B. Vigneron ${ }^{1}$, and Sarah J. Nelson ${ }^{1}$

Advanced MRSI acquisition strategies can be complex to implement and require customized reconstruction software, typically designed for a specific raw file format and that relies upon a priori knowledge of the specific implementation of the pulse sequence being applied. The ISMRMRD format ${ }^{1}$ has begun to address standardization in describing data acquisition parameters for different types of imaging data, but further development is needed. Here we build on this strategy by demonstrating XML encoding of parameters that describe flyback echo-planar, compressed sensing MRSI acquisitions being implemented on scanners from multiple vendors at UCSF that can be supported with generalized reconstruction software.

SNR and PSF Simulations for k-t Trajectories in MRSI: CSI, EPSI, Rosettes, and Concentric Rings

Amir Seginer ${ }^{1}$ and Assaf Tal

${ }^{1}$ Weizmann Institute of Science, Rehovot, Israel

We compare, using numeric simulations, the point spread functions (PSF) and the SNR of different trajectories in k-t space for magnetic resonance spectral imaging (MRSI). This is a first step towards evaluating the trajectory of choice while balancing SNR efficiency, scan time, and localization of signal (resolution vs. bleed).

JSASSI: A B1 Insensitive Technique for J-Resolved 2D Magnetic Resonance Spectroscopy at 7T

Judy Alper ${ }^{1,2}$, Rebecca E Feldman ${ }^{1}$, Francesco Padormo ${ }^{1}$, Priti Balchandani ${ }^{1}$, and Gaurav Verma ${ }^{1}$

${ }^{1}$ Radiology, Icahn School of Medicine At Mount Sinai, New York, NY, United States, ${ }^{2}$ Biomedical Engineering, City College of New York, New York, NY, United States

Magnetic resonance spectroscopy (MRS) can be used to investigate metabolite concentration changes correlated to neurological and psychiatric diseases. Improved spectral resolution and metabolite quantification in these disorders would add to our understanding of neurodegenerative diseases. JSASSI is a novel technique for localized two-dimensional (2D) MRS, based in part on the JPRESS spectroscopic sequence while implementing pulses from the SASSI sequence. An incrementing $\triangle \mathrm{t}_{1}$ time delay is introduced for resolving J-coupled metabolites from overlapping resonances. JSASSI was applied in phantoms and in vivo. Metabolite peaks for NAA, GIx, Cr and others were clearly identified using JSASSI. Unambiguous detection and resolution of J-coupled metabolites could facilitate reliable quantification of metabolites such as GABA, with potential applications in characterization and treatment monitoring in psychiatric disorders. 
${ }^{1}$ High Feld MR Centre, Medical University of Vienna, Vienna, Austria, ${ }^{2}$ Christian Doppler Laboratory for Clinical Molecular MR Imaging, Vienna, Austria, ${ }^{3}$ Athinoula A. Martinos Center for Biomedical Imaging, Department of Radiology, Massachusetts General Hospital, Harvard Medical School, Boston, MA, United States, ${ }^{4}$ Russell H. Morgan Department of Radiology and Radiological Science, The Johns Hopkins University School of Medicine, Baltimore, MD, United States, ${ }^{5}$ F. M. Kirby Research Center for Functional Brain Imaging, Kennedy Krieger Institute, Baltimore, MD, United States

Recently, ultra-high resolution (UHR-) MRSI of the brain at $7 \mathrm{~T}$ was successfully demonstrated, allowing metabolic mapping at near-anatomical resolution. With this work, we propose further optimised sequences, one for shorter measurement times of under $5 \mathrm{~min}$ and one for even higher in-plane resolutions down to $12 \mu \mathrm{L}$, which will allow a more flexible application of UHR_MRSI, and show their possibilities and limitations. Furthermore, the effects of slice thickness for UHR-MRSI were investigated with a second set of measurements.

Cross-vendor standardization of a 3 T MRS protocol with semi-LASER

Adam Berrington ${ }^{1,2}$, Dinesh K Deelchand ${ }^{3}$, James Joers ${ }^{3}$, Michal Považan ${ }^{1,2}$, Michael Schär ${ }^{1}$, Joseph Gillen ${ }^{1,2}$, Peter B Barker ${ }^{1,2}$, and Gülin Öz ${ }^{3}$

${ }^{1}$ Russell H. Morgan Department of Radiology, Johns Hopkins University School of Medicine, Baltimore, MD, United States, ${ }^{2}$ F. M. Kirby Center for Functional Brain Imaging, Kennedy Krieger Institute, Baltimore, MD, United States, ${ }^{3}$ Center for Magnetic Resonance Reseach, University of Minnesota, Minneapolis, MN, United States

Acceptance of ${ }^{1} \mathrm{H}-\mathrm{MRS}$ for clinical use is hindered by variability in methodology across platforms. Cross-vendor standardization is thus desirable for large-scale studies to be conducted. Here, we standardize a semi-LASER scheme $(T E=30 \mathrm{~ms})$ with identical pulses, inter-pulse durations and acquisition protocol in phantom and healthy volunteers on Philips and Siemens $3 \mathrm{~T}$ systems. The implemented method resulted in high quality spectra with matched SNR, linewidth and spectral patterns in phantom and similar estimated metabolite concentrations in vivo: between-subject CVs for NAA were (2.6-11.0)\% and (3.3-10.2)\% for Philips and Siemens, respectively. This method highlights the potential for pooling data across multiple sites.

Intrinsic inversion recovery-based macromolecular nulling in MEGA-PRESS 1H-MR brain spectra

Alexander Gussew ${ }^{1}$, Andreas Masek ${ }^{1}$, Martin Krämer $^{1}$, and Jürgen R. Reichenbach ${ }^{1}$

${ }^{1}$ Medical Physics Group, Institute of Diagnostic and Interventional Radiology, Jena University Hospital - Friedrich Schiller University Jena, Jena, Germany

The reliability of ${ }^{1} \mathrm{H}$-MRS MEGA-PRESS measurements of inhibitory neurotransmitter GABA in the human brain typically suffers from macromolecular (MM) contaminations of GABA resonances. In this work, we present a novel MM suppression approach, which relies on adiabatic inversion of the longitudinal magnetization of both metabolites and MMs prior to playing out the MEGA-PRESS editing scheme, which is applied after an inversion time delay ( $\mathrm{TI})$ corresponding to the zero-crossing of MM magnetization. As demonstrated in healthy subjects, this new approach ensures appropriate MM suppression and provides additional GABA signal gain compared to the commonly applied approach with symmetrical MM editing.

What is the optimal ROI size for single voxel MRS in global brain pathology?

Maike Hoefemann ${ }^{1}$, Victor Adalid ${ }^{1}$, and Roland Kreis ${ }^{1}$

${ }^{1}$ Depts. Radiology and Biomedical Research, University of Bern, Bern, Switzerland

The purpose of this study was to investigate optimal voxel size (VS) as a compromise between increasing SNR and decreasing linewidth under the side-constraint of minimal artifact levels and to investigate potential benefits from considering signals from single coil elements separately. Eight different VS were evaluated; hinting at optimal VS of $60 \mathrm{~cm}^{3}$ and indicating that lineshape information from unsuppressed water should be included in the fitting process. Differences in single coil elements show substantial impacts on spectral quality, indicating that individual processing and exclusion of certain channels is superior to the standard procedure of an indiscriminate weighted sum.

ISIS based Relaxation Enhanced MR spectroscopy (iRE-MRS) for downfield spectroscopy at short echo times

Sonia I. Goncalves ${ }^{1}$ and Noam Shemesh ${ }^{1}$

${ }^{1}$ Neuroplasticity and Neural Activity Lab, Champalimaud Foundation, Lisbon, Portugal

MRS is a versatile technique that allows for the non-invasive in-vivo exploration of tissue metabolism. In most MRS pulse sequences based on broadband excitation, the acquisition is preceded by water saturation pulses that suppress the water bulk signal and implicitly also exchangeable protons downfield of water. We introduce a new method for short-TE downfield MRS and show that it detects multiple peaks in-vivo that extend beyond $9 \mathrm{ppm}$. 
${ }^{1}$ Philips, Stockholm, Sweden, ${ }^{2}$ Department of Medical Physics, Uppsala University Hospital, Uppsala, Sweden, ${ }^{3}$ Philips, Best, Netherlands, ${ }^{4}$ Department of Psychology, Uppsala University, Uppsala, Sweden, ${ }^{5}$ Department of Clinical Neuroscience, Karolinska Institutet, Stockholm, Sweden, ${ }^{6}$ Department of Psychology, Stockholm University, Stockholm, Sweden, ${ }^{7}$ Centre for Neuroscience, Central European Institute of Technology, Masaryk University, Brno, Czech Republic, ${ }^{8}$ Department of Neuroscience, Psychiatry, Uppsala University, Uppsala, Sweden

Proton MRS of the anterior cingulate cortex (ACC) is an attractive biomarker as it provides non-invasive methods to quantify GABA levels that are linked with several psychiatric disorders. This study validates a MEGA-PRESS sequence that combines phase cycling with real time frequency drift correction to measure GABA spectra in phantom and human brain. The GABA levels of the ACC were repeatable and reproducible at two different scanning sites. Consequently, the technique is appropriate for future longitudinal psychiatric studies.

Comparison of adiabatic and non-adiabatic inversion pulses for lipid suppression in human calf muscle

Andreas Masek ${ }^{1}$, Alexander Gussew ${ }^{1}$, Martin $\mathrm{Krämer}^{1}$, and Jürgen R. Reichenbach ${ }^{1,2,3,4}$

${ }^{1}$ Medical Physics Group, Institute of Diagnostic and Interventional Radiology, Jena University Hospital - Friedrich Schiller University Jena, Jena, Germany, ${ }^{2}$ Michael Stifel Center for Data-driven and Simulation Science Jena, Friedrich Schiller University Jena, Jena, Germany, ${ }^{3}$ Abbe School of Photonics, Friedrich Schiller University Jena, Jena, Germany, ${ }^{4}$ Center of Medical Optics and Photonics, Friedrich Schiller University Jena, Jena, Germany

Overlapping signal contributions originating from different metabolites with similar molecular structure is a common problem of in vivo ${ }^{1} \mathrm{H}-\mathrm{MR}$ spectroscopy with magnetic field strengths of $\leq 3 \mathrm{~T}$. One prominent example is the "contamination" of the resonances of lactate with fat signals in ${ }^{1} \mathrm{H}-\mathrm{MR}$ muscle spectra. The goal of this work was to implement a MRS sequence with inversion recovery based adiabatic/ nonadiabatic lipid suppression and to test this approach in vivo in two different human calf muscles.

Finger tapping induces lactate increase in the human motor cortex detected by J-edited $1 \mathrm{H}-\mathrm{MRS}$ at $4 \mathrm{~T}$

Yury Koush ${ }^{1}$, Robin A. de Graaf ${ }^{1}$, Lihong Jiang ${ }^{1}$, Douglas L. Rothman ${ }^{1}$, and Fahmeed Hyder ${ }^{1}$

${ }^{1}$ MRRC, Yale University, New Haven, CT, United States

While functional MRI (fMRI) localizes regions of activation, functional MRS (fMRS) provides metabolic response to activation. fMRS, using short echo-time (TE) non-edited ${ }^{1} \mathrm{H}-\mathrm{MRS}$ protocols, has been shown to be capable of detecting a lactate increase in sensory-induced activations. Because short TE non-edited lactate spectra are susceptible to functional hyperemia and contamination from lipids/macromolecules, we posited if long TE J-edited ${ }^{1} \mathrm{H}-\mathrm{MRS}$ detection of lactate can reliably detect metabolic changes in the motor cortex (MC) during the standard finger-tapping paradigm. Our fMRS results at 4T showed significant physiological modulation of the MC lactate level.

Glycine quantification via S-PRESS difference editing of myo-inositol

Thomas Lange ${ }^{1}$ and Michael Dacko

$1283{ }^{1}$ Dept. of Radiology, Medical Physics, Medical Center - University of Freiburg, Faculty of Medicine, Freiburg, Germany

The quantification of glycine (Gly) with in vivo MRS is challenging due to the strong spectral overlap with myo-inositol ( $\mathrm{ml}$ ) so that only the concentration sum ml+Gly can be accurately measured with standard MRS methods at clinical field strengths. In this work, the distinction and quantification of ml and Gly is demonstrated with S-PRESS difference editing, which enables unequivocal detection of the strongly coupled $\mathrm{ml}$ resonances through suppression of the overlapping uncoupled Gly resonance.

High resolution localized 1D homonuclear decoupled in phase MR spectroscopy via z-filtered 2D J-spectroscopy

Lin Yanqin ${ }^{1}$, Bo Duan ${ }^{1}$, Dan Tian ${ }^{1}$, Qing Zeng ${ }^{1}$, and Zhong Chen ${ }^{1}$

${ }^{1}$ Department of Electronic Science, Xiamen University, Xiamen, China

Proton 1D MR spectroscopy is an important tool in the study of a number of diseases. However, due to multiplet structure and narrow proton chemical shift range, 1D spectra become complicated for direct assignment and quantification. Homonuclear broadband decoupled spectra can be obtained by separating the chemical shift and $\mathrm{J}$ coupling information into orthogonal axes in the conventional JPRESS spectra. However, they suffer low resolution because of phase-twisted lineshape. Here, a J-resolved alike experiment with z-filtered module is introduced for the selection of in phase magnetization, and thus high resolution phase sensitive localized 1D spectra can be obtained. 
Co-editing of macromolecule(MM) resonances is a major problem in J-difference based editing (e.g. MEGA-PRESS) at 3T and lower field strengths. Symmetrical pulsing centered at the $1.7 \mathrm{ppm}$ MM resonance alleviates this problem but results in loss of desired GABA signal, in addition to loss of unwanted MM signal, due to high bandwidth of frequency-selective editing pulses. Larger separation of editing pulses at 7T reduces the problem, but large chemical shift displacement errors, especially at low B1, make MEGA-PRESS non-viable at 7T. Using a low-power MEGA-LASER sequence, we measured macromolecule minimized GABA at 7T with editing pulses having bandwidths available in most scanners.

Simultaneous MRSI of GABA and glutathione using HERMES spectral editing at 3T

Kimberly Chan ${ }^{1,2,3}$, Richard Edden ${ }^{2,3}$, Georg Oeltzschner ${ }^{2,3}$, Muhammad Saleh ${ }^{2,3}$, and Peter Barker ${ }^{2,3}$

${ }^{1}$ Biomedical Engineering, Johns Hopkins School of Medicine, Baltimore, MD, United States, ${ }^{2}$ Russell H. Morgan Department of Radiology and Radiological Science, Johns Hopkins School of Medicine, Baltimore, MD, United States, ${ }^{3}$ F. M. Kirby Research Center for Functional Brain Imaging, Kennedy Krieger Institute, Baltimore, MD, United States

HERMES with single-voxel PRESS localization has been used to simultaneously edit multiple compounds. It's often desirable to measure spectra from multiple brain regions, using MR spectroscopic imaging (MRSI). This study examined the feasibility of HERMES editing of GABA and GSH with a PRESS-localized MRSI sequence at $3 T$, and compared it to conventional MEGA-edited MRSI acquisitions. It's found that adding symmetrical lipid suppression pulses to HERMES allows the sequence to be used in vivo and has an editing efficiency equivalent to that of separate acquisitions of GABA and GSH using MEGA-PRESS MRSI without an increase in measurement variability relative to MEGA-PRESS.

High resolution mapping of GABA+ and Glx using motion-corrected, spiral-accelerated, edited 1D-semiLASER MRSI in the human brain at 7T

Philipp Moser ${ }^{1,2}$, Bernhard Strasser ${ }^{3}$, Lukas Hingerl ${ }^{1}$, Michal Považan ${ }^{4,5}$, Gilbert Hangel ${ }^{1}$, Eva Heckova ${ }^{1}$, Borjan Gagoski ${ }^{6}$, Andre van der Kouwe ${ }^{7}$, Ovidiu C. Andronesi ${ }^{7}$, Siegfried Trattnig $^{1,2}$, and Wolfgang Bogner ${ }^{1}$

${ }^{1}$ High Field MR Centre, Department of Biomedical Imaging and Image-guided Therapy, Medical University of Vienna, Vienna, Austria, ${ }^{2}$ Christian Doppler Laboratory for Clinical Molecular MR Imaging, Vienna, Austria, ${ }^{3}$ Athinoula A. Martinos Center for Biomedical Imaging, Department of Radiology, Massachusetts General Hospital, Harvard Medical School, Boston, MA, United States, ${ }^{4}$ Russell H. Morgan Department of Radiology and Radiological Science, The Johns Hopkins University School of Medicine, Baltimore, MD, United States, ${ }^{5} \mathrm{~F}$. M. Kirby Research Center for Functional Brain Imaging, Kennedy Krieger Institute, Baltimore, MD, United States, ${ }^{6}$ Fetal-Neonatal Neuroimaging; Developmental Science Center, Boston Children's Hospital, Harvard Medical School, Boston, MA, United States, ${ }^{7}$ Athinoula A. Martinos Center for Biomedical Imaging, Department of Radiology, Massachusetts General Hospital, Harvard Medical School, Charlestown, MA, United States

In vivo detection of gamma-aminobutyric acid (GABA) and glutamate (Glu), both major neurotransmitters in the human brain, benefits from the higher sensitivity and SNR at ultra-high field $(7 \mathrm{~T})$ compared to lower field strengths. However, strong $\mathrm{B}_{1}{ }^{+}$inhomogeneities and chemical shift displacement errors, as well as subject motion and carrier frequency drifts can significantly impair the experiment. We preliminarily propose the first high resolution full-slice in vivo mapping of GABA ${ }^{+}$at $7 \mathrm{~T}$. Combining spatial-spectral spiral encoding for MRS acceleration with B1-insensitive adiabatic pulses and real-time motion correction allows unprecedented high resolution J-difference editing at $7 \mathrm{~T}$ in comparably short scan time.

Optimized Crusher Design for Magnetic Resonance Spectroscopy

Karl Landheer ${ }^{1}$ and Christoph Juchem ${ }^{1,2}$

${ }^{1}$ Biomedical Engineering, Columbia University, New York, NY, United States, ${ }^{2}$ Radiology, Columbia University, New York, NY, United States

Modern magnetic resonance spectroscopic (MRS) pulse sequences frequently overlook the issue of unwanted coherence pathways. A novel and robust algorithm which only requires input of the desired coherence(s) was developed to optimally crush all unwanted coherence pathways for any MRS pulse sequence. Experiments were performed on the GE BRAINO phantom comparing crusher schemes obtained from the literature with those obtained from the developed optimization algorithm for sLASER and MEGA-sLASER. The results demonstrate that the effects of unwanted coherences can be drastically reduced through the implementation of an optimized crusher scheme, without the need for additional or stronger crushers.

Improving time resolution in the imaging of metabolic dynamics using Compressed Sensing from 2D Heteronuclear Multiple Quantum Coherence

Utako Yamamoto ${ }^{1}$, Hirohiko Imai ${ }^{1}$, Kei Sano ${ }^{1}$, Masayuki Ohzeki ${ }^{2}$, Tetsuya Matsuda ${ }^{1}$, and Toshiyuki Tanaka ${ }^{1}$

${ }^{1}$ Department of Systems Science, Kyoto University, Kyoto, Japan, ${ }^{2}$ Department of Applied Information Sciences, Tohoku University, Sendai, Japan

We propose a compressed sensing reconstruction method with high time resolution for imaging fast metabolic dynamics from sequential data measured using $2 \mathrm{D}{ }^{1} \mathrm{H}-{ }^{13} \mathrm{C}$ heteronuclear multiple quantum coherence (HMQC) MRSI. Optimization using the alternating direction method of multipliers (ADMM) is employed to incorporate prior knowledge about the substance distribution.

The 2D-HMQC MRSI with pseudo-random undersampling is applied to tumor-bearing mice after the injection of [U- $\left.{ }^{13} \mathrm{C}\right]$ glucose. From the resulting data, we successfully reconstruct time-series of the in vivo density of three substances (glucose, lactate, and fat) at a high time resolution of $2.25 \mathrm{~min}$. 
Fast In Vivo Metabolite T2 Quantification by RF-Driven Steady State

Ningzhi Li ${ }^{1}$, Linqing $\mathrm{Li}^{1}$, Yan Zhang ${ }^{1}$, and Jun Shen ${ }^{1}$

${ }^{1}$ National Institute of Mental Health, Bethesda, MD, United States

MARzss method is a novel method for brain metabolite $T_{2}$ quantification without varying echo time. This study evaluates the feasibility of shortening the scan time of the MARzs method by more than $80 \%$ using minimum TR and two-FA measurements. Phantom and preliminary in vivo studies show that metabolite $T_{2}$ quantifications using two-FA measurements agree well with $\mathrm{T}_{2}$ values obtained by the originally proposed seven-FA measurements. In addition, Monte Carlo simulations indicate that under the same total scan time, the two-FA measurements can significantly improve the precision of $T_{2}$ quantification.

Test-retest reliability of real-time frequency and motion corrected Hadamard encoded spectral editing (CHASE)

Anna Lind ${ }^{1}$, Vincent O. Boer ${ }^{1}$, Mads Andersen ${ }^{2}$, Esben T. Petersen ${ }^{1,3}$, and Anouk Marsman ${ }^{1}$

${ }^{1}$ Danish Research Centre for Magnetic Resonance, Centre for Functional and Diagnostic Imaging and Research, Copenhagen University Hospital Hvidovre, Hvidovre, Denmark, ${ }^{2}$ Philips, Copenhagen, Denmark, ${ }^{3}$ Center for Magnetic Resonance, Dept. Electrical Engineering, Technical University of Denmark, Lyngby, Denmark

Inhibitory neurotransmitter GABA and antioxidant GSH are suggested to be implicated in psychiatric and neurological disorders. Because of their relatively weak signals, spectral editing is necessary to assess GABA and GSH in the human brain. Hadamard encoding can be applied simultaneously for spectral editing of GABA and GSH. As both small metabolite signals and Hadamard encoding are highly susceptible to frequency drift and motion, real-time frequency and motion correction significantly improves spectral quality. The data obtained in this study so far suggest good test-retest reliability of real-time frequency and motion corrected Hadamard encoded spectral editing (CHASE) for GABA and GSH.

Flip Angle Corrected Multi-TR, Multi-TE 1H MR Spectroscopy

Gavin Hamilton ${ }^{1}$, Alexandra N Schlein ${ }^{1}$, and Claude B Sirlin ${ }^{1}$

${ }^{1}$ Liver Imaging Group, Department of Radiology, University of California, San Diego, La Jolla, CA, United States

Multi-TR, multi-TE ${ }^{1} \mathrm{H}$ MRS estimates T1 and T2 of fat and water and liver proton density fat fraction in a single breath-hold. This approach uses a steady state solution, which assumes a perfect $90^{\circ}$ pulse is generated which is not guaranteed in vivo, possibly introducing T1 errors. We introduce a flip angle corrected multi-TR, multi-TE ${ }^{1} \mathrm{H}$ MRS sequence based on a non-steady state approach and demonstrate, in phantoms, that while the multi-TR, multi-TE MRS sequence estimates T1 dependent on the flip angle, the flip angle corrected multi-TR, multi-TE MRS sequence estimates T1 independent of flip angle.

Accuracy and Reproducibility of NAD+, NADH and Redox Ratio Measurement in Human Brain by LCModel

Lijing Xin ${ }^{1}$, Ozlem Ipek ${ }^{1}$, Bernard Cuenoud ${ }^{2}$, Maurice Beaumont ${ }^{2}$, Maya Shevlyakova ${ }^{2}$, and Rolf Gruetter 3,4

${ }^{1}$ Centre d'Imagerie BioMédicale (CIBM), Ecole Polytechnique Fédérale de Lausanne (EPFL), Lausanne, Switzerland, ${ }^{2}$ Nestec Ltd, Avenue Nestlé 55 , Vevey, Switzerland, ${ }^{3}$ Departments of Radiology, University of Lausanne, Lausanne, Switzerland, ${ }^{4}$ Laboratory of Functional and Metabolic Imaging, Ecole Polytechnique Fédérale de Lausanne, Lausanne, Switzerland

The aims of this study were to test the feasibility of $\mathrm{NAD}^{+}, \mathrm{NADH}$ and redox ratio([NAD+/NADH]) measurement in vivo in the human brain at $7 \mathrm{~T}$ using $\mathrm{LCModel}$ and to further evaluate the measurement accuracy and reproducibility. High ${ }^{31} \mathrm{P}$ spectral quality was achieved and LCModel provides excellent fitting quality. Monte-Carlo simulations and test-retest experiments demonstrated good measurement accuracy and reproducibility with sufficient SNR achieved. The values are in agreement with those previously published. Therefore, LCModel can be used as an alternative tool to achieve automated and objective measurement of $\mathrm{NAD}^{+}$, NADH and redox ratio in human brain in vivo.

Accelerated Correlated Spectroscopic Imaging in Two Spectral-Three Spatial Dimensions with Slice-selective Adiabatic Refocusing Pulses in Human Calf Muscles

Manoj K Sarma ${ }^{1}$, Andres Saucedo ${ }^{1}$, Christine H Darwin ${ }^{2}$, Neil Wilson ${ }^{1}$, Zohaib lqbal ${ }^{1}$, Cathy C Lee ${ }^{2,3}$, Catherine Carpenter ${ }^{4}$, Theodore Hahn ${ }^{2,3}$, and M. Albert Thomas ${ }^{1}$

${ }^{1}$ Radiological Sciences, David Geffen School of Medicine at UCLA, Los Angeles, CA, United States, ${ }^{2}$ Medicine, David Geffen School of Medicine at UCLA, Los Angeles, CA, United States, ${ }^{3}$ Greater Los Angeles Veterans Affairs Medical Center, Los Angeles, CA, United States, ${ }^{4}$ School of Nursing, David Geffen School of Medicine at UCLA, Los Angeles, CA, United States

An optimized version of the five-dimensional (5D) echo-planar correlated spectroscopic sequence using an adiabatic full passage (AFP) RF pulse pair has been implemented on a $3 T$ MRI/MRS scanner equipped with a 15-channel transmit/receive coil. The sequence was initially tested using a corn oil phantom. The calf muscle of twelve healthy subjects (age $27.5 \pm 3.1$ years) and six diabetic type 2 subjects was studied (age $62.3 \pm 9.8$ years). The AFP pulse pair enabled a sharper profile and minimal chemical shift misregistration. The localization of the volume of interest showed differential distribution of metabolites and lipids in human calf muscle and tibial marrow. 
Uncovering Long Range J-coupled Lipid Resonances in Human Calf In-Vivo: Pilot Findings Using Localized Two Dimensional Total Correlated Spectroscopy

Manoj K Sarma ${ }^{1}$, Andres Saucedo ${ }^{1}$, Christine H Darwin² ${ }^{2}$ Cathy C Lee ${ }^{2,3}$, Ravinder R. Regatte ${ }^{4}$, and M. Albert Thomas ${ }^{1}$

${ }^{1}$ Radiological Sciences, David Geffen School of Medicine at UCLA, Los Angeles, CA, United States, ${ }^{2}$ Medicine, David Geffen School of Medicine at UCLA, Los Angeles, CA, United States, ${ }^{3}$ Greater Los Angeles Veterans Affairs Medical Center, Los Angeles, CA, United States, ${ }^{4}$ Center for Biomedical Imaging, New York University School of Medicine, New York, NY,

United States

Based on the same principle of localized correlated spectroscopy (L-COSY) of coherence transfer during mixing period, total correlated spectroscopy (TOCSY) is a powerful technique that can provide correlations for both direct and long range coupled spins via relayed coherence transfer. Due to the SAR issue, the potential of TOCSY has not been fully exploited invivo and only few versions of TOCSY have been evaluated in brain. Here we have implemented a novel version of localized TOCSY technique for implementation in human calf muscle in-vivo, and compared results from three mixing strategies. Results are presented from a corn oil phantom, and in-vivo 2D spectra from 4 healthy volunteers and 1 diabetic patient obtained on $3 \mathrm{~T}$ clinical platforms. We demonstrated that TOCSY can uncover the hidden relayed peaks, particularly that of IMCL/EMCL in calf muscle which can play an important role in better estimation of degree of unsaturation.

In vivo detection of NAD+ in human calf muscle at $7 \mathrm{~T}$ using 28-channel knee volume coil

Puneet Bagga ${ }^{1}$, Neil Wilson ${ }^{1}$, Catherine DeBrosse ${ }^{1}$, Hari Hariharan ${ }^{1}$, and Ravinder Reddy ${ }^{1}$

Nicotinamide adenine dinucleotide $\left(\mathrm{NAD}^{+}\right)$is a ubiquitous molecule present in all cells and tissues of the body with an important role in the redox reactions and metabolism. Smal changes in NAD ${ }^{+}$levels may lead to oxidative stress and may be a cause for various disorders. NAD is usually be detected in vivo by ${ }^{31} P$ NMR spectroscopy. Recently, NAD measurement with ${ }^{1} \mathrm{H}$ MRS in the human brain was demonstrated. In the present study, we show for the first time, in vivo single voxel localized ${ }^{1} \mathrm{H}$ MRS detection of NAD+ from the human calf muscle at $7 \mathrm{~T}$.

Profiling lipid composition in whole breast tumours using two dimensional (2D) double quantum filtered (DQF) correlation spectroscopy (COSY) and multiple quantum coherence (MQC) magnetic resonance spectroscopy (MRS)

Sai Man Cheung ${ }^{1}$, Ehab Husain ${ }^{2,3}$, Yazan Masannat ${ }^{3,4}$, Vasiliki Mallikourti ${ }^{1}$, Steven D Heys ${ }^{3,4}$, and Jiabao He ${ }^{1}$

${ }^{1}$ Aberdeen Biomedical Imaging Centre, University of Aberdeen, Aberdeen, United Kingdom, ${ }^{2}$ Pathology Department, Aberdeen Royal Infirmary, Aberdeen, United Kingdom, ${ }^{3}$ School of Medicine, University of Aberdeen, Aberdeen, United Kingdom, ${ }^{4}$ Breast Unit, Aberdeen Royal Infirmary, Aberdeen, United Kingdom

Changes in lipid composition, such as polyunsaturated fatty acids (PUFA), have found to be potential biomarker of breast cancer. It has been shown that PUFA has a role in breast cancer initiation. The relationship in human between lipid composition and breast tumour grading warrants urgent investigation, as a pathway towards improved treatment. Conventional MRS suffers from overlap of nearby lipid and water peaks, and is insufficient for lipid composition measurement. We conducted double quantum filtered (DQF) correlation spectroscopy (COSY) to resolve lipid composition from the whole breast tumour, and multiple quantum coherence (MQC) MRS for further close investigation of PUFA.

High Quality Magnetic Resonance Spectroscopy Reconstruction with Vandermonde Factorization on Low Rank Hankel Matrix

Xiaobo Qu ${ }^{1}$, Jiaxi Ying ${ }^{1}$, Di Guo ${ }^{2}$, Jian-Feng Cai $^{3}$, Gongguo Tang ${ }^{4}$, and Zhong Chen ${ }^{1}$

${ }^{1}$ Department of Electronic Science, Xiamen University, Xiamen, China, ${ }^{2}$ School of Computer and Information Engineering, Xiamen University of Technology, Xiamen, China, ${ }^{3}$ Department of Mathematics, Hong Kong University of Science and Technology, Hong Kong, China, ${ }^{4}$ Department of Electrical Engineering \& Computer Science, Colorado School of Mines, Golden, CO, United States

Magnetic resonance spectroscopy (MRS) is commonly converted from its free induction decay (FID) data with Fourier transform. How to reconstruct high quality spectra is one of the fundamental problems for MRS. In this work, a reconstruction method is proposed to explore the general exponential property of FID. Each exponential function of FID is explicitly enforced with the Hankel matrix Vandermonde Factorization (HVaF). This model is then applied to spectrum reconstruction of sparsely sampled FID in fast MRS. Results on synthetic and realistic MRS show that the new approach requires fewer data to allow successful reconstruction and provides better reconstruction on low-intensity signals than the state-of-the-art low rank Hankel matrix method. Thus, the new approach would be useful for faster data acquisition or recovery of weak spectral peaks in MRS applications.

Indirect Detection and Spin Amplification of Non-Proton MRS and MRI by Solvent Proton Signals

Zhao Li ${ }^{1}$ and Yung-Ya Lin ${ }^{1}$ 
A general indirect-detection and spin-amplification scheme has been developed to enhance the sensitivity of heteronuclear MRS and MRI based on dynamic instability of the solvent proton magnetization under collective feedback fields of radiation damping and the distant dipolar field. The heteronuclear solute spins are first detected by the solvent proton spins through various magnetization transfer mechanisms and serve as small "input" signals to perturb the solvent proton magnetization, which is prepared in an unstable state. The weakly detected signal is then amplified through subsequent nonlinear evolution of the solvent proton magnetization to achieve $10 x$ SNR improvement for $13 \mathrm{C}$ MRS and MRI.

Reproducibility of the measurement of hepatic lipid composition with $1 \mathrm{H}$ MRS at 3T

Pandichelvam Veeraiah ${ }^{1,2}$, Kay H.M Roumans ${ }^{2}$, Joachim E Wildberger ${ }^{1}$, Patrick Schrauwen ${ }^{2}$, Vera B Schrauwen-Hinderling ${ }^{1,2}$, and Lucas Lindeboom ${ }^{1,2}$

${ }^{1}$ Department of Radiology, NUTRIM School for Nutrition and Translational Research in Metabolism, Maastricht University Medical Center, Maastricht, Netherlands, ${ }^{2}$ Department of Human Biology and Movement Sciences, NUTRIM School for Nutrition and Translational Research in Metabolism, Maastricht University Medical Center, Maastricht, Netherlands

The total intrahepatic lipid (IHL) content can reliably be determined with ${ }^{1} \mathrm{H}$-MRS, but measuring lipid composition (saturated, mono- and poly-unsaturated fatty acids) is very challenging At $3 \mathrm{~T}$ the allylic peak is contaminated with the alpha-carbonyl methylene resonance, which hampers accurate measure of lipid composition. Recently, we developed a new approach to determine the lipid composition using prior knowledge to correct the signal intensity for alpha carbonyl group using methyl resonance. Here, we determined the in vivo reproducibility of our approach and robust quantification of lipid composition in a group of subjects with a wide range of total liver fat content.

Preliminary study of proton magnetic resonance spectroscopy with multi-echo-time for simultaneous quantification and T2 measurement of glutamate.

Chi-Hyeon Yoo ${ }^{1,2}$, Kyu-Ho Song ${ }^{1}$, Song-I Lim ${ }^{1,2}$, Dong-Choel Woo ${ }^{2}$, and Bo-Young Choe ${ }^{1}$

${ }^{1}$ Department of Biomedical Engineering, Research Institute of Biomedical Engineering, College of Medicine, The Catholic University of Korea, Seoul, Republic of Korea, ${ }^{2}$ Asan Institute for Life Sciences, Asan Medical Center, Seoul, Republic of Korea

This study presents our preliminary concept of multi-echo-time (TE) in vivo proton magnetic resonance spectroscopy $\left({ }^{1} \mathrm{H}\right.$ MRS) for the simultaneous quantification and T2 measurement of the brain metabolites, particularly glutamate. The feasibility of the proposed method was verified by comparing metabolite concentrations to that of conventional short-TE, and 2 relaxation times to that of conventional T2 measurement. Although TE points must be further optimized, the multi-TE in vivo ${ }^{1} \mathrm{H}$ MRS could be used to simultaneously investigate the changes of brain metabolism and microenvironments in a scan time comparable to that of the conventional method.

Feasibility of Echo Time Optimization for Glutamate and Myoinositol Detection using TE-Averaged PRESS Spectral Editing Technique in Human Brain at 3T.

Gokce Hale Hatay ${ }^{1}$ and Esin Ozturk Isik ${ }^{1}$

${ }^{1}$ Biomedical Engineering Institute, Bogazici University, Istanbul, Turkey

This study aims to investigate the feasibility of echo time (TE) optimization for TE-averaged PRESS for faster detection of glutamate (Glu) and myoinositol ( $\mathrm{ml}$ ) in human brain at $3 \mathrm{~T}$ Proton MR spectroscopic imaging (1H-MRSI) data of a brain phantom and a healthy volunteer were acquired at $3 \mathrm{~T}$ using 10 different TEs, which were selected based on prior Monte Carlo simulation results. TE-averaged PRESS spectra were created with best TE combinations, and metabolites were quantified in MATLAB. Our results indicated that TE-averaged PRESS with upto 5 TE's could reliably detect separate Glu and $\mathrm{ml}$ metabolites.

$1 \mathrm{H}$-localised 13C DEPT measurement of glutamate and glutamine turnover in human frontal lobe using [1-13C]glucose infusion at $7 \mathrm{~T}$

Bernard Lanz ${ }^{1}$, Chen Chen ${ }^{1}$, Carolina Campanha Fernandes ${ }^{1}$, Liz Simpson ${ }^{2}$, Adriana Anton ${ }^{3}$, Mohammad Katshu ${ }^{4}$, Mohan Rathnaiah ${ }^{4}$, Andrew Peters ${ }^{1}$, lan Macdonald ${ }^{2}$, Stephen Williams ${ }^{5}$, Bill Deakin ${ }^{3}$, Peter Liddle ${ }^{4}$, and Peter Gordon Morris ${ }^{1}$

${ }^{1}$ Sir Peter Mansfield Imaging Centre, The University of Nottingham, Nottingham, United Kingdom, ${ }^{2}$ School of Life Sciences, University of Nottingham Medical School, Queen's Medical Centre, Nottingham, United Kingdom, ${ }^{3}$ Neuroscience and Psychiatry Unit, Division of Neuroscience and Experimental Psychology, University of Manchester, Manchester, United Kingdom, ${ }^{4}$ Institute of Mental Health, The University of Nottingham, Nottingham, United Kingdom, ${ }^{5}$ Centre for Imaging Science, University of Manchester, Manchester, United Kingdom

Human ${ }^{13} \mathrm{C}$ MRS has recently shown its further potential in understanding neurological disorders. In the field of schizophrenia, ${ }^{1} \mathrm{H}$ MRS has been applied with findings of abnormal

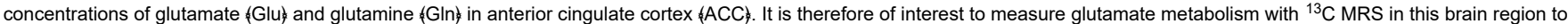
get deeper understanding of these changes. In the present study, we applied localized ${ }^{13} \mathrm{C}$ MRS at $7 \mathrm{~T}$ upon $\left[1-{ }^{13} \mathrm{C}\right]$ glucose infusion, using a ${ }^{13} \mathrm{C} /{ }^{1} \mathrm{H}$ volume coil and polarisation transfer (DEPT) to test the feasibility of measuring glutamate turnover in ACC. 
${ }^{1}$ Institute of Radiology, University Hospital Erlangen, Friedrich-Alexander-Universität Erlangen-Nürnberg (FAU), Erlangen, Germany, ${ }^{2}$ High Field MR Center, Department of Biomedical Imaging and Image-guided Therapy, Medical University of Vienna, Vienna, Austria, ${ }^{3}$ Research Unit of Medical Imaging, Physics and Technology, University of Oulu, Oulu, Finland, ${ }^{4}$ Center for Medical Physics and Engineering, Friedrich-Alexander-Universität Erlangen-Nürnberg (FAU), Erlangen, Germany, ${ }^{5}$ Division of Medical Physics in Radiology, German Cancer Research Centre (DKFZ), Heidelberg, Germany

An iterative reconstruction algorithm for sodium magnetic resonance imaging $\left({ }^{23} \mathrm{Na} \mathrm{MRI}\right)$ with multi-channel receiver coils is implemented and compared to a conventional gridding reconstruction. Based on compressed sensing $(\mathrm{CS})$ it utilizes a total variation $\left(\mathrm{TV}^{(2)}\right)$, combined with anatomical weighting factors $\left(\right.$ AnaWeTV $\left.{ }^{(2)}\right)$ to preserve known tissue boundaries Simulated and measured ${ }^{23} \mathrm{Na}$ multi-channel data sets of the female breast were reconstructed. The TV ${ }^{(2)}$ and in particular the AnaWeTV ${ }^{(2)}$ lead to an improved image quality, due to effective noise reduction and the highlighting of structure. The presented CS reconstruction is beneficial especially for high undersampling factors.

Traditional Poster

\section{MRS/MRSI Reconstruction \& Quantification}

Evaluation of different postprocessing-based B0 inhomogeneity correction methods for application in 7T FID-MRS

Stanislav Motyka ${ }^{1}$, Philipp Moser ${ }^{1,2}$, Bernhard Strasser ${ }^{3}$, Lukas Hingerl ${ }^{1}$, Michal Považan ${ }^{4,5}$, Gilbert Hangel ${ }^{1}$, Eva Heckova ${ }^{1}$, Stephan Gruber ${ }^{1}$, Siegfried Trattnig ${ }^{1,2}$, and Wolfgang Bogner $^{1}$

${ }^{1}$ High Field MR Centre, Department of Biomedical Imaging and Image-guided Therapy, Medical University of Vienna, Vienna, Austria, ${ }^{2}$ Christian Doppler Laboratory for Clinical Molecular MR Imaging, Vienna, Austria, ${ }^{3}$ Athinoula A. Martinos Center for Biomedical Imaging, Department of Radiology, Massachusetts General Hospital, Harvard Medical School, Boston, MA, United States, ${ }^{4}$ Russell H. Morgan Department of Radiology and Radiological Science, The Johns Hopkins University School of Medicine, Baltimore, MD, United States, ${ }^{5} \mathrm{M}$. Kirby Research Center for Functional Brain Imaging, Kennedy Krieger Institute, Baltimore, MD, United States

The information from B0 maps can be used to improve the spectral quality in MRSI. Two post-processing methods, SPREAD and odMRSI, were implemented and evaluated on: i) simulation model, ii) phantom data, and iii) high-resolution in vivo data acquired by 2D FID-MRSI with CAIPIRINHA acceleration at 7T. Both methods were capable to improve the spectral quality, however, the SPREAD only in high SNR situations which are not present in clinical reality. The spectral quality improvement brought by odMRSI was equivalent to the averaging of 6 averages but this improvement could not be directly translated into the same metabolic map quality.

MOSAIC - a generalized multi-channel coil combination for $1 \mathrm{H}-\mathrm{MRSI}$ via interleaved calibration scans

Philipp Moser ${ }^{1,2}$, Bernhard Strasser $^{3}$, Lukas Hingerl ${ }^{1}$, Michal Považan ${ }^{4,5}$, Gilbert Hangel $^{1}$, Eva Heckova ${ }^{1}$, Stephan Gruber ${ }^{1}$, Siegfried Trattnig ${ }^{1,2}$, and Wolfgang Bogner ${ }^{1}$

${ }^{1}$ High Field MR Centre, Department of Biomedical Imaging and Image-guided Therapy, Medical University of Vienna, Vienna, Austria, ${ }^{2}$ Christian Doppler Laboratory for Clinical Molecular MR Imaging, Vienna, Austria, ${ }^{3}$ Athinoula A. Martinos Center for Biomedical Imaging, Department of Radiology, Massachusetts General Hospital, Harvard Medical School, Boston, MA, United States, ${ }^{4}$ Russell H. Morgan Department of Radiology and Radiological Science, The Johns Hopkins University School of Medicine, Baltimore, MD, United States, ${ }^{5}$ F. M. Kirby Research Center for Functional Brain Imaging, Kennedy Krieger Institute, Baltimore, MD, United States

The optimal combination of signals from all receive elements is a prerequisite in MRSI especially at high field ( $\geq 7 \mathrm{~T})$, not only for SNR-efficient acquisition, but also for good parallel imaging reconstruction [1,2]. Phantom and in vivo experiments showed superior performance of MOSAIC including higher SNR, smaller FWHM and anatomically detailed metabolic maps compared to Brown and WSDV coil combination. MOSAIC is a flexible and robust approach for efficient MRSI coil combination under challenging conditions (B0 $\geq 7 \mathrm{~T}$, many coil elements, no reference coil, low SNR, possible spectral artifacts, motion/instability related artifacts, 1st-order phase error), especially with an outlook on parallel-imaging non-Cartesian MRSI.

3D EPSI Hadamard spectral editing of GABA and GSH at 7T

Vincent Oltman Boer ${ }^{1}$, Nam Gyun Lee ${ }^{1}$, Anouk Marsman ${ }^{1}$, and Esben Thade Petersen ${ }^{1,2}$

${ }^{1}$ Danish Research Centre for Magnetic Resonance, Centre for Functional and Diagnostic Imaging and Research, Copenhagen University Hospital Hvidovre, Hvidovre, Denmark, ${ }^{2}$ Center for Magnetic Resonance, Department of Electrical Engineering, Technical University of Denmark, Lyngby, Denmark

A 3D MRSI sequence was developed for simultaneous editing of GABA and GSH using a Hadamard editing scheme at 7T. 3D MRSI was performed using a 1D echo planar spectroscopic readout (EPSI). Volume selection was perfomed using a sLASER volume selection box using adiabatic refocusing pulses. 
${ }^{1}$ Institute of Radiology, University Hospital Erlangen, Friedrich-Alexander-Universität Erlangen-Nürnberg (FAU), Erlangen, Germany, ${ }^{2}$ Pattern Recognition Lab, Friedrich-AlexanderUniversität Erlangen-Nürnberg (FAU), Erlangen, Germany, ${ }^{3}$ Division of Medical Physics in Radiology, German Cancer Research Center (DKFZ), Heidelberg, Germany, ${ }^{4}$ Erlangen Graduate School in Advanced Optical Technologies, Erlangen, Germany

Sodium magnetic resonance imaging requires dedicated acquisition techniques and reconstruction approaches due to the low in-vivo signal and ultra-short relaxation times. For this purpose a compressed sensing reconstruction technique using dictionary learning is applied to raw data acquired with an anisotropic 3D density-adapted radial acquisition sequence. The anisotropic acquisition allows an adjustment of projections in different directions to increase the in-plane resolution. In the following evaluation the possible benefits of the compressed sensing reconstruction using the increased in-plane resolution are shown for in-vivo sodium magnetic resonance imaging and quantification of ${ }^{23} \mathrm{Na}$.

Accelerated in vivo Phosphorus Magnetic Resonance Spectroscopic Imaging combining flyback-EPSI and Compressed Sensing

Alejandro Santos Diaz ${ }^{1}$ and Michael Noseworthy ${ }^{1,2}$

${ }^{1}$ School of Biomedical Engineering, McMaster University, Hamilton, ON, Canada, ${ }^{2}$ Electrical and Computer Engineering, McMaster University, Hamilton, ON, Canada

Long acquisition time is still a major limitation in performing clinical 31P MRSI studies. To overcome this limitation we implemented and tested a pulse sequence that combines flyback EPSI readout and compressed sensing (CS). Our results, in human skeletal muscle, show the feasibility of performing ${ }^{31} \mathrm{P}$ MRSI using this combined approach.

Optimization of Radial Echo Planar Spectroscopic Image Reconstruction for Hyperpolarized [1-13C]-Pyruvate Imaging

Joshua Niedzielski ${ }^{1}$, Chang-yu Sun ${ }^{1}$, Keith Michel $^{1}$, Christopher Walker $^{1}$, Samuel Einstein ${ }^{1}$, and James Bankson ${ }^{1}$

${ }^{1}$ Imaging Physics, Univ. of Texas-MD Anderson Cancer Center, Houston, TX, United States

Radial echo planar spectroscopic imaging (EPSI) is an efficient method for imaging hyperpolarized (HP) substrates. However, symmetric data sampling between even/odd echo components can lead to ghost artifacts that can interfere with spectral undersampling strategies that enhance SNR. The purpose of this study was to optimize the acquisition and reconstruction of a symmetric radial EPSI sequence for dynamic HP $\left[1{ }^{13} \mathrm{C}\right]$-pyruvate imaging. In this work, we show that the generalized Fourier transform technique preserves spectral bandwidth, reduces ghost and aliasing artifacts, and improves SNR compared to alternative strategies that separately consider even and odd echo subsets.

In vivo validation of OVS-localized navigator for prospective frequency correction in MRSI

Chu-Yu Lee ${ }^{1}$, In-Young Choi ${ }^{1,2,3}$, and Phil Lee ${ }^{1,3}$

${ }^{1}$ Hoglund Brain Imaging Center, University of Kansas Medical Center, Kansas City, KS, United States, ${ }^{2}$ Department of Neurology, University of Kansas Medical Center, Kansas City, KS, United States, ${ }^{3}$ Department of Molecular \& Integrative Physiology, University of Kansas Medical Center, Kansas City, KS, United States

Data acquisition for MRS and MRSI requires a stable scanner frequency during the relatively long scan time. However, gradient heating and subject motion during the scan result in drifts of the scanner frequency. The effects of frequency drifts include reduced SNR, broad linewidth, and errors in spatial encoding and metabolite quantification. We had recently proposed a new navigator approach: outer volume suppression (OVS)-localized navigator, to prospectively correct frequency drifts without introducing SNR losses, overcoming the shortcomings of previous PRESS-localized navigator. The purpose of this study is to validate the OVS-localized navigator approach through the comparison with non-localized navigator and the quantitative evaluations of spectral quality and metabolite concentrations in 10 healthy subjects.

Reconstruction of motion affected prostate MRSI data using navigators and compressed sensing

Rashmi Reddy ${ }^{1}$, Ryan Kalmoe ${ }^{2}$, Greg Metzger ${ }^{2}$, and Sairam Geethanath ${ }^{1,3}$

${ }^{1}$ Dayananda Sagar Institutions, Bangalore, Karnataka, India, ${ }^{2}$ Center for Magnetic Resonance Research and Department of Radiology, University of Minnesota, Minneapolis, MN, United States, ${ }^{3}$ Department of Radiology, Columbia University Medical Centre, New York, NY, United States

This work focuses on reconstruction of 2D prostate in vitro and in vivo MRSI data. Motion affected phase encodes are tracked using a free induction decay navigator. The proposed work utilizes Compressed Sensing (CS) reconstruction technique to compensate for the loss of motion affected information. Comparison between data without motion considered as ground truth (GT) is performed with data with motion and CS reconstructed data. Qualitative and quantitative performance measures indicate improvement in spectral quality with the application of the navigator led CS MRSI reconstruction. Current and future work involves the application of this method on an increased sample size. 
The purpose of this study was to quantitatively evaluate biases caused by the use of ideal PRESS simulations. Metabolite basis spectra were simulated for an ideal PRESS sequence as well as with real shaped RF-pulses. Theoretical ground truth spectra were constructed for different TE and shim settings. They were fitted using both basis sets. It is shown that the fitting accuracy decreases when using ideal simulations and they depend on TE and metabolite. Therefore, simulation of basis sets should include the effects of the real pulse shapes even for the presented case of short TE and fairly large B1 amplitude.

Toward Absolute Quantification Using External Reference Standards at 3T and 9.4T

Andrew Martin Wright ${ }^{1,2}$, Sahar Nassirpour ${ }^{1,2}$, Paul Chang ${ }^{1,2}$, and Anke Henning ${ }^{1,3}$

${ }^{1}$ Biological Cybernetics, Max Planck Institute, Tübingen, Germany, ${ }^{2}$ IMPRS for Cognitive and Systems Neuroscience, Eberhard-Karls University of Tübingen, Tübingen, Germany,

${ }^{3}$ Institute of Physics, Ernst-Moritz-Arndt University Greifswald, Greifswald, Germany

Absolute quantification is a challenge with many paths to reach the final goal of quantifying metabolites in absolute units (e.g. Molarity and molality). Utilizing an external reference standard (ERF) is an attractive method for quantifying in vivo metabolites due to the ability for direct comparison between a known concentration of a metabolite and the in vivo data. A major concern in utilization of an ERF is the differences in coil loading between in vivo and in vitro measurements. To that end, this work describes a method to calibrate and adjust the transmitter voltage in order to maximize signal detection independently of coil load.

On the exploitation of slow macromolecular diffusion for baseline estimation in MR spectroscopy using 2D simultaneous fitting

André Döring ${ }^{1}$, Victor Adalid ${ }^{1}$, Chris Boesch ${ }^{1}$, and Roland Kreis

$1315 \quad{ }^{1}$ Depts. Radiology and Biomedical Research, University of Bern, Bern, Switzerland

The slow diffusivity of macromolecules was exploited in 2D signal modeling with FiTAID to estimate the macromolecular baseline in MRS of human brain. Two approaches were used for baseline modeling: (i) a predefined model derived from high-field and $\mathrm{T}_{1}$-based baseline determination and (ii) a model-free description by equally spaced Voigt resonances. Inspection of fit residues and comparison with literature reveals that the second model is more appropriate.

Simultaneous modeling of sum and difference spectra improves quantitative outcomes for edited MRS

Daniel Luc Rimbault ${ }^{1}$, Georg Oeltzschner ${ }^{2,3}$, Ali Alhamud ${ }^{1,4}$, Ernesta Meintjes ${ }^{1,4}$, and Richard A. E. Edden ${ }^{2,3}$

${ }^{1}$ Division of Biomedical Engineering, Department of Human Biology, University of Cape Town, Cape Town, South Africa, ${ }^{2}$ Russell H. Morgan Department of Radiology and Radiological Science, The Johns Hopkins University School of Medicine, Baltimore, MD, United States, ${ }^{3}$ F. M. Kirby Research Center for Functional Brain Imaging, Kennedy Krieger Institute, Baltimore, MD, United States, ${ }^{4}$ Cape Universities Body Imaging Centre (CUBIC-UCT), Cape Town, South Africa

J-difference-edited MR spectroscopy allows for the detection of several low-concentration compounds at 3T, but suffers from long acquisition times. Multiplexed editing experiments provide simultaneous detection of two or three metabolites by differentially modulating the spin systems of interest, and separating edited signals into distinct sum or difference spectra. For a novel multiplexed experiment (HERCULES), with simulated metabolite basis functions we demonstrate that simultaneously modeling the sum and difference spectra results in comparable metabolite levels with lower coefficients of variation, compared to separate modeling of the sum and difference spectra.

The Effect of B0 and B1+ Inhomogeneities on Spinal Cord MRS

Nicholas Maurice Simard ${ }^{1}$, Aimee J Nelson ${ }^{2}$, and Michael D. Noseworthy ${ }^{1,3}$

${ }^{1}$ School of Biomedical Engineering, McMaster University, Hamilton, ON, Canada, ${ }^{2}$ Department of Kinesiology, McMaster University, Hamilton, ON, Canada, ${ }^{3}$ Department of Electrical and Computer Engineering, McMaster University, Hamilton, ON, Canada

Spinal cord ${ }^{1} \mathrm{H}$ MR Spectroscopy $\left({ }^{1} \mathrm{H}-\mathrm{MRS}\right)$ is a promising method for musculoskeletal research. However, due to the spine's anatomical location there is a significant degradation of signal quality due to magnetic field inhomogeneities, rendering most MRS approaches inaccurate. Although there has been measurement of $\Delta \mathrm{B}_{0}$ in spinal cord MRS, there are no comprehensive assessments of temporal changes in $\mathrm{B}_{0}$ and $\mathrm{B}_{1}{ }^{+}$relating physiological disturbances with MRS accuracy. Thus our goal was to continually measure temporal changes in $\mathrm{B}_{0}$ and $\mathrm{B}_{1}{ }^{+}$during the length of a typical MEGA-PRESS scan (10min). 
Glutamate and glutamine play an important role in CNS. Both are quantifiable via $1 \mathrm{H}$ in-vivo MRS, although a correct, separate quantification of both metabolites is often very

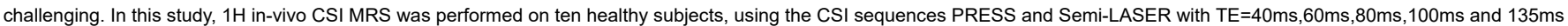
at 3T. The inner 64 spectra of each CSI matrix at each TE were averaged to a single spectrum. Averaged spectra were analysed using LCModel. The quantification of glutamate and glutamine, using this method, which is also a popular approach in MRS research, was shown to be inconsistent.

Novel methodology for processing, quality assessment, and artifact mitigation of raw 2D Correlation Spectroscopy data

Laura J Mariano ${ }^{1}$, Marcia Sahaya Louis ${ }^{2}$, Benjamin Rowland ${ }^{3}$, Huijun Liao ${ }^{4}$, Kristin Heaton ${ }^{5}$, John Irvine ${ }^{1}$, and Alexander P Lin ${ }^{3,6}$

${ }^{1}$ The Charles Stark Draper Laboratory, Inc., Cambridge, MA, United States, ${ }^{2}$ Electrical and Computer Engineering, Boston University, Boston, MA, United States, ${ }^{3}$ Center for Clinical Spectroscopy, Brigham and Women's Hospital, Boston, MA, United States, ${ }^{4}$ Psychiatric Neuroimaging Laboratory, Brigham and Women's Hospital, Boston, MA, United States, ${ }^{5}$ US Army Institute of Environmental Medicine, Natick, MA, United States, ${ }^{6}$ Harvard Medical Institute, Boston, MA, United States

2D Correlation Spectroscopy (COSY) can be used to identify and study coupled resonances that cannot be observed or distinguished in 1D NMR spectra. However, resources and literature on best practices for processing raw 2D COSY data are limited. In this work, we describe a novel pipeline of signal processing algorithms and visualizations for quality assessment and artifact mitigation designed specifically for raw 2D COSY data, including detection of residual $\mathrm{H} 2 \mathrm{O}$ and lipid contamination, correction for drift across averages, and peak location correction to enable more accurate comparisons of metabolites across subjects.

NMRScopeB - an open-source simulator for metabolite quantitation and pulse sequence development

Zenon Starčuk ${ }^{1}$ and Jana Starčuková ${ }^{1}$

${ }^{1}$ Magnetic Resonance and Cryogenics, Institute of Scientific Instruments of the CAS, Brno, Czech Republic

The architecture and function of the release version of a spectroscopic simulator NMRScopeB is described. It includes the jMRUI-related GUI and an open-source calculation server communicating with the kernel via sockets. While standard metabolite set simulations needed for quantitation by jMRUI or LCModel can be prepared in a few steps, more complex research task can be handled as well. The operation is described by control and data flow charts. After a period of beta-testing, the simulator is released as part of the recent jMRUI package.

Implications of magnetic susceptibility difference between grey and white matter for spectroscopy quantification at $7 \mathrm{~T}$.

Donghyun Hong ${ }^{1}$, Jack JA van Asten ${ }^{2}$, Seyedmorteza Rohani Rankouhi ${ }^{1}$, Jan-Willem Thielen ${ }^{1}$, and David G. Norris ${ }^{1,3}$

${ }^{1}$ Erwin L. Hahn Institute for MRI, University of Duisburg-Essen, Essen, Germany, ${ }^{2}$ Department of Radiology and Nuclear Medicine, Radboud University Medical Center, Nijmegen, Netherlands, ${ }^{3}$ Donders Institute for Brain, Cognition and Behavior, Radboud University, Nijmegen, Netherlands

Magnetic susceptibility differences between grey matter (GM) and white matter (WM) can potentially affect lineshapes and chemical shifts in single voxel spectroscopy. Hitherto, analytical techniques such as LCModel assumed a single lineshape per voxel. Separated GM and WM signals using multi-echo GRE image sequence in combination with literature values for the metabolite distribution between GM and WM enable to construct a realistic basis set for LCModel. With this information we can test how magnetic susceptibility induced lineshape modification affects metabolic quantification, which uses spectral prior knowledge.

Spectral denoising for MR Spectroscopy using orthogonal polynomials

Mathieu Naudin ${ }^{1,2,3}$, Benoit Tremblais ${ }^{1}$, Carole Guillevin ${ }^{2}$, Rémy Guillevin ${ }^{2}$, and Christine Fernandez-Maloigne ${ }^{1}$

${ }^{1}$ Univ. Poitiers, XLIM, CNRS UMR 7252, Poitiers, France, ${ }^{2}$ Univ. Poitiers, LMA, CHU Poitiers, CNRS UMR 7348, Poitiers, France, ${ }^{3}$ Siemens Healthineers, Saint-Denis, France

We propose a new methodology to denoise MRS spectrum with a focus on the acquisition time diminution. Using a discrete orthogonal polynomials, we detect two types of areas : homogenous and non-homogenous (metabolite peaks). Once these areas detected, we compute the Noise Level Function (NLF). Then, using the NLF, we use orthogonal polynomials to reconstruct a signal with a strategy for each type of area. As results, a denoising method is provided and it helps to correct the noise due to the acquisition time diminution with a good metabolite peaks conservation.

Ryan J Larsen ${ }^{1}$, Joseph L. Holtrop ${ }^{1,2}$, and Brad P. Sutton ${ }^{1,2}$ 
${ }^{1}$ Beckman Institute, University of Illinois at Urbana-Champaign, Urbana, IL, United States, ${ }^{2}$ Department of Bioengineering, University of Illinois at Urbana-Champaign, Urbana, IL, United States

Quantitation of MRSI data using water-scaling requires correction of the water signal for relaxation and CSF partial volume effects. We demonstrate the use of a rapid MRF sequence to characterize the water signal used to quantify MRS data, which we call WAter-scaling Quantification using MRF (WAQ-MRF) scan. WAQ-MRF provides subject-specific corrections of partial volume and relaxation effects for water-scaled data. By adding a one minute scan to a standard MRSI acquisition it is possible to eliminate the need for assuming literature values of relaxation and proton density to correct the water signal.

Spectral Quantification for Multiple-TE Spectroscopy Using Spectral Priors and Measured Lineshape Distortion Function

Fan Lam ${ }^{1}$, Yudu Li ${ }^{1,2}$, and Zhi-Pei Liang ${ }^{1,2}$

${ }^{1}$ Beckman Institute for Advanced Science and Technology, University of Illinois at Urbana-Champaign, Urbana, IL, United States, ${ }^{2}$ Department of Electrical and Computer Engineering, University of Illinois at Urbana-Champaign, Urbana, IL, United States

This work presents a new method for quantifying multiple-TE/two-dimensional spectroscopy data, characterized by the use of spectral priors obtained by quantum mechanical simulations and an experimentally measured lineshape distortion function derived from a set of multi-TE water spectroscopic data. Results from in vivo J-resolved spectroscopy data demonstrated the excellent fitting produced by the proposed method, and improved robustness over a standard parametric-model-based method. With further developments, such as extensions to different sequences and Cramer-Rao bound analysis, the proposed method should prove useful for a range of 2D spectroscopy experiments.

Classification of brain tumors by $1 \mathrm{H}$ MRSI and MRI using convolutional neural networks

Jacopo Acquarelli ${ }^{1,2}$, Arend Heerschap ${ }^{3}$, Geert J. Postma ${ }^{2}$, Twan van Laarhoven ${ }^{1}$, Jeroen J. Jansen ${ }^{2}$, Elena Marchiori ${ }^{1}$, and Lutgarde M.C. Buydens ${ }^{2}$

${ }^{1}$ Data Science, Radboud University Nijmegen, Nijmegen, Netherlands, ${ }^{2}$ Analytical Chemistry, Radboud University Nijmegen, Nijmegen, Netherlands, ${ }^{3}$ Radiology and Nuclear Medicine, Radboud University Nijmegen, Nijmegen, Netherlands

Several machine learning approaches have been used to classify brain tumors using MR images and spectra. Here we explore the specific properties of convolutional neural networks (CNN) for this task. We designed a CNN that could be trained on combined MR image and spectroscopic image data by exploiting their specific properties (spatial and spectral locality). Using a 'leave-one-out' validation, we demonstrate that our method outperforms state-of-the-art classification methods to distinguish tumor grades. These results demonstrate that CNNs are a powerful approach for tumor classification using MRSI data.

Highly Accelerated Simulation of Model Spectra for TE-Averaged Spectral Fitting

Yan Zhang ${ }^{1}$ and Jun Shen ${ }^{1}$

${ }^{1}$ National Institute of Mental Health, Bethesda, MD, United States

One-dimensional projection method was applied to the simulation of spatially localized J-resolved magnetic resonance spectroscopy with real RF pulses. As a comparison, the same pulse sequence was simulated using non-localized ideal RF pulses. The resultant TE-averaged spectra of glutamate were compared with phantom experiment at $3 T$. Conspicuous differences between ideal pulse simulated spectrum and phantom spectrum were found. For vivo comparisons, metabolite quantification was performed with real RF pulse basis set and ideal pulse basis set, respectively. Real RF pulse generated basis set significantly improved the reproducibility of glutamate quantification in vivo.

How does inclusion of different macromolecular baseline models affect reproducibility of $1 \mathrm{H}-\mathrm{FID}$ MRSI in the brain at 7T?

Eva Heckova ${ }^{1}$, Ursel Antpusat ${ }^{1,2}$, Michal Považan ${ }^{3,4}$, Bernhard Strasser ${ }^{5}$, Gilbert Hangel ${ }^{1}$, Lukas Hingerl ${ }^{1}$, Philipp Moser ${ }^{1}$, Stephan Gruber ${ }^{1}$, Siegfried Trattnig ${ }^{1,6}$, and Wolfgang Bogner ${ }^{1,6}$

${ }^{1}$ High Field MR Centre, Department of Biomedical Imaging and Image-guided Therapy, Medical University of Vienna, Vienna, Austria, ${ }^{2}$ Hamm-Lippstadt University of Applied Sciences, Hamm, Germany, ${ }^{3}$ Russell H. Morgan Department of Radiology and Radiological Science, The Johns Hopkins University School of Medicine, Baltimore, MD, United States, ${ }^{4}$ F. M. Kirby Research Center for Functional Brain Imaging, Kennedy Krieger Institute, Baltimore, MD, United States, ${ }^{5}$ Athinoula A. Martinos Center for Biomedical Imaging, Department of Radiology, Massachusetts General Hospital, Harvard Medical School, Boston, MA, United States, ${ }^{6}$ Christian Doppler Laboratory for Clinical Molecular Molecular MR Imaging, Vienna, Austria

The goal was to investigate how the use of different macromolecular baseline models affects both the accuracy and test-retest reproducibility of metabolite quantification for clinically attractive FID-MRSI scan with in-plane resolution of $3.4 \times 3.4 \mathrm{~mm}^{2}$ and acquisition time of $5 \mathrm{~min}$. We confirmed that our $1 \mathrm{H}$-FID-MRSI sequence provides information about abundance and spatial distribution of several neurometabolites with high accuracy. Including the information about the macromolecular background into the quantification process does not decrease its reproducibility. 
Highly Accelerated (R=14) Water Reference Acquisition for High Resolution 1H MRSI using Compressed Sensing

Paul Chang ${ }^{1,2}$, Sahar Nassirpour ${ }^{1,2}$, and Anke Henning ${ }^{1,3}$

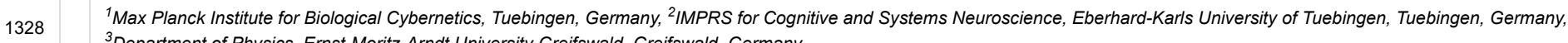

${ }^{3}$ Department of Physics, Ernst-Moritz-Arndt University Greifswald, Greifswald, Germany

In this study, the acquisition of a high resolution (64x64) water reference MRSI data is accelerated by a factor of R=14 using compressed sensing. The results show that this highly accelerated water reference can reliably be used for eddy current and phase correction purposes, as well as internal referencing and quantification. This enables the acquisition of the high resolution water reference MRSI data in 80 seconds at $9.4 \mathrm{~T}$.

MRF in Single Voxel Spectroscopy: Signal to Noise Ratio or Dictionary Length - Which is more important?

Alexey Kulpanovich ${ }^{1}$ and Assaf Tal ${ }^{1}$

${ }^{1}$ Weizmann Institute of Science, Rehovot, Israel

We use MR spectroscopic fingerprinting (MRSF) to quantify T1,T2 and concentration addressing the tradeoff between fingerprint lengths and averaging. Methods. MRSF using 25,50 and 100 fingerprint lengths were compered to inversion recovery (IR) and multi-TE using Monte-Carlo simulations and in-vivo experiments. Bias and variance were estimated for NAA, Creatine and Choline. Results. Simulations of all MRSF sequences show better accuracy and bias over IR. In-vivo experiments show improved T1 and concentration estimation. Conclusion. The low SNR emphasizes the tradeoff between fingerprint length and averaging. The In-vivo results show clear advantage using shorter fingerprint and increasing the SNR.

Estimation of T2 Relaxation Times of Downfield Peaks in Human Brain at $9.4 \mathrm{~T}$

Saipavitra V. Murali Manohar ${ }^{1}$, Tamas Borbath ${ }^{1}$, Nicole Fichtner ${ }^{2,3}$, Ioannis Angelos Giapitzakis ${ }^{1}$, Daniel Zaldivar ${ }^{1}$, Roland Kreis ${ }^{3}$, and Anke Henning ${ }^{1,4}$

$1330 \quad{ }^{1}$ Max Planck Institute for Biological Cybernetics, Tuebingen, Germany, ${ }^{2}$ Institute for Biomedical Engineering, UZH and ETH Zurich, Zurich, Switzerland, ${ }^{3}$ Depts. Radiology and Biomedical Research, University of Bern, Bern, Switzerland, ${ }^{4}$ Institute of Physics, Ernst-Moritz Arndt University Greifswald, Greifswald, Germany

$\mathrm{T}_{2}$ relaxations times for the downfield metabolites in human brain ${ }^{1} \mathrm{H}$ MR spectra were estimated at $9.4 \mathrm{~T}$. A possible new peak at 8.35 ppm with rapid $\mathrm{T}_{2}$ decay is reported. Due to the use of a non-water suppressed MRS method, the $T_{2}$ of slowly exchanging peaks could be assessed. The shorter $T_{2}$ relaxation times in the downfield compared to the upfield spectral areas leads us to suspect a macromolecular contribution, while also exchange effects may contribute to the short apparent $\mathrm{T}_{2} \mathrm{~s}$.

Multivariate Analysis of Developmental-Dependent Differences in Metabolites in White and Gray Matter: An Ultra-Short TE ${ }^{1} \mathrm{H}$ MRS Study at $3 \mathrm{~T}$

Jack Knight-Scott ${ }^{1}$

${ }^{1}$ Radiology, Children's Healthcare of Atlanta, Atlanta, GA, United States

Application of multivariate analysis of variance (MANOVA) to a developmental data set of ${ }^{1} \mathrm{H}$ spectra from white and gray matter brain tissue shows not only significant tissue differences but also significant gender and age differences. By specifically controlling for metabolite correlations, MANOVA results show higher sensitivity and power than individual ANOVAs.

A comparison of reference-based methods for removing artifacts in non-water-suppressed $1 \mathrm{H}$ MRSI data

Zhengchao Dong ${ }^{1,2}$, Feng $\mathrm{Liu}^{1,2}, \mathrm{Min} \mathrm{Li}^{2,3}$, Matthew Milak${ }^{2}$, and Sachin Jambawalikar ${ }^{4}$

${ }^{1}$ New York State Psychiatric Institute, New York, NY, United States, ${ }^{2}$ Psychiatry, Columbia University, New York, NY, United States, ${ }^{3}$ Collage of Internet of Things, Hohai University, Changzhou, China, ${ }^{4}$ Radiology, Columbia University, New York, NY, United States

Sideband artifacts is the major obstacle to $1 \mathrm{H}$ MRSI without water suppression. To remove the sideband artefacts, several reference-based methods have been proposed, in which the reference signals are acquired from a water phantom with identical experimental parameters as those of in vivo scan are acquired. The reference-based methods do not suffer scan time penalty and they are compatible with any accelerated sequences such as SENSE-SI. The aim of the present work is to improve and compare the performance of two kinds of referencebased methods, namely, the phase compensation method and the artifact subtraction method. 
Materials such as biological tissue are often characterized by considerable heterogeneity. This can manifest itself in significant variability of certain physicochemical parameter values across the measured volume. If the chemical shift of a particular MR resonance varies systematically with such a parameter, the resulting lineshape can be used to quantitatively characterize the heterogeneity with respect to this parameter. This is achieved by transforming the MRS lineshape into a curve representing the statistical distribution of the parameter values in question, followed by the derivation of a histogram. We study here two important conditions for the statistical evaluation of such spectrum-derived histograms.

Effects of non-linearity correction on statistical descriptors of $\mathrm{pH}$ heterogeneity, obtained from 3-APP and inorganic phosphate resonances of tumor 31P MR spectra

Norbert W Lutz ${ }^{1}$ and Monique Bernard

We recently presented a method for extracting statistical descriptors of $\mathrm{pH}$ heterogeneity from lineshapes of $\mathrm{pH}$-sensitive ${ }^{31} \mathrm{P}$ MRS resonances. The first step in this analysis is the conversion of the resonance in question into the corresponding $\mathrm{pH}$ profile. The latter is then corrected for non-linearity between chemical shift and $\mathrm{pH}$. However, this procedure is insufficient since the unequal spacing of the digital points making up such pH profiles needs to be compensated for by appropriate weighting. Exact statistical descriptor values are of importance in quantification of tissue $\mathrm{pH}$ heterogeneity, an issue that has received major attention in recent cancer research.

Restoration of truncated FID by machine learning

Hyochul Lee ${ }^{1}$ and Hyeonjin Kim ${ }^{1,2}$

The potential applicability of a recurrent neural network (RNN) in the reconstruction of spectra from truncated FIDs was explored. A RNN was trained on a set of simulated full FIDs with varying metabolite concentrations. Then, the performance of the trained RNN was tested on severely truncated FIDs ( 95\% truncation). Our preliminary study suggests that RNNs may be used in the restoration of truncated FIDs and thus reconstruction of spectra including tiny multiplets. A well trained RNN may be applicable to the situations where data sampling is highly limited such as in cardiac MRS and spectroscopic magnetic resonance fingerprinting (sMRF).

Traditional Poster

\section{Spectroscopy: NMR \& Other}

Time-domain EPR imaging with slice selection

Ayano Enomoto ${ }^{1}$, Ken-ichiro Matsumoto ${ }^{2}$, Shun Kishimoto ${ }^{1}$, Shingo Matsumoto ${ }^{3}$, Murali C Krishna ${ }^{1}$, and Nallathamby Devasahayam ${ }^{1}$

${ }^{1}$ National Cancer Institute, National Institutes of Health, Bethesda, MD, United States, ${ }^{2}$ Department of Basic Medical Sciences for Radiation Damages, National Institute of Radiological Sciences, Chiba, Japan, ${ }^{3}$ Graduate school of Information Sicence and technology, Hokkaido University, Sapporo, Japan

The slice selection imaging has advantages of reducing imaging time and obtaining optimum dynamic range in image for EPR imaging as well as for MRI. However, the slice selection using a selective pulse, which is used in MRI, is difficult to implement in EPR imaging because of ultra-fast relaxation time compared to gradient settling time. Therefore, we used a modulated gradient field to achieve slice selection in pulsed EPR imaging in this study. We demonstrated the slice selection imaging with tubes and a living mouse to show the effect of slice selection in pulsed EPR imaging.

Metabolic characteristics of oncogenically transformed mouse neural progenitor cells using one dimensional 1H NMR

Magretta Adiamah ${ }^{1}$, Liam Mistry $^{2}$, Andrew Houlton ${ }^{2}$, Elizabeth Stoll ${ }^{3}$, and Ross Maxwell ${ }^{4}$

${ }^{1}$ Northern Institute for Cancer Research, Newcastle University, Newcastle, United Kingdom, ${ }^{2}$ School of Natural and Environmental sciences, Newcastle University, Newcastle, United Kingdom, ${ }^{3}$ Institute of Neuroscience, Newcastle University, Newcastle, United Kingdom, ${ }^{4}$ Northern Institute for cancer research, Newcastle University, Newcastle, United Kingdom

Metabolic profiles of oncogenically transformed neural progenitor cells (NPCs) derived from 3 and 12 month old mice were evaluated using one dimensional $1 \mathrm{H}$ NMR spectroscopy. Principal component analysis revealed two distinct clusters which corresponded to the differently-aged NPCs. Metabolites identified in these cell lines were similar but differed in their relative abundance. The 3 month NPCs were characterised by high lipid CH2, creatine and choline. The metabolic signature of 12 month NPCs featured high levels of taurine, myoinositol and branched-chain amino acids. This data suggests alterations in metabolic phenotype of aged NPCs which may arise from differences in enzymatic capacity. 
Gene Expression Profiling to Understand the 1H MRS Characterization of the VEGF Metabolic Secretome from a Triple Negative Human Breast Cancer Xenograft

Santosh Kumar Bharti ${ }^{1}$, Balaji Kirshnamachary ${ }^{1}$, Louis Dore-Savard ${ }^{2}$, Brett Stark ${ }^{1}$, Aleksander S. Popel ${ }^{3}$, and Zaver M Bhujwalla ${ }^{1,4}$

${ }^{1}$ Division of Cancer Imaging Research, Department of Radiology, Johns Hopkins University, School of Medicine, Baltimore, MD, United States, ${ }^{2}$ McGill University Health Centre and RIMUHC, Montreal, QC, Canada, ${ }^{3}$ Systems Biology Laboratory, Department of Biomedical Engineering, Johns Hopkins University, School of Medicine, Baltimore, MD, United States, ${ }^{4}$ Department of Oncology, Johns Hopkins University, School of Medicine, Baltimore, MD, United States

Vascular endothelial growth factor (VEGF A) is a potent regulator of angiogenesis, invasion, and metastasis, especially in breast cancer. Secreted VEGF that forms a part of the interstitial milieu along with other metabolites shapes the microenvironment. Here, using $1 \mathrm{H}$ MR spectroscopy and microarray, we have characterized the metabolic and gene signature of the tumor tissue derived from MDA-MB-231 cells that stably overexpressed VEGF gene. Metabolic changes supported by gene array data provide new insight into the role played by VEGF in breast cancer progression

1H MRS Reveals Major Changes in Brain Metabolites Induced by Human Pancreatic Cancer Xenografts

Santosh Kumar Bharti ${ }^{1}$, Paul T Winnard Jr. ${ }^{1}$, Yelena Mironchik ${ }^{1}$, Marie-France Penet ${ }^{1}$, Anirban Maitra ${ }^{2}$, and Zaver M Bhujwalla ${ }^{1,3}$

${ }^{1}$ Division of Cancer Imaging Research, Department of Radiology, Johns Hopkins University, School of Medicine, Baltimore, MD, United States, ${ }^{2}$ Department of Pathology, The University of Texas MD Anderson Cancer Center, Houston, TX, United States, ${ }^{3}$ Department of Oncology, Johns Hopkins University, School of Medicine, Baltimore, MD, United States

Our ongoing efforts are focused on understanding systemic metabolic changes that occur during cancer-induced cachexia using human pancreatic ductal adenocarcinoma (PDAC) xenografts, since the syndrome occurs with the highest frequency and severity in PDAC. We used $1 \mathrm{H}$ MRS to analyze brain metabolite levels in mice with and without cachexia inducing human PDAC xenografts. Spectra revealed depletion of several metabolites, including neurotransmitters, in cachectic mice. These findings provide new insights into disruption of brain metabolism that may compromise central nervous system (CNS) function. Identifying alterations of brain metabolism may provide novel interventions to prevent or reduce CNS injury and cachexia.

Effect of sampling method on HR-MAS NMR spectra of caprine brain biopsies

Annakatrin Häni ${ }^{1}$, Gaelle Diserens ${ }^{2}$, Anna Oevermann ${ }^{3}$, Peter Vermathen ${ }^{2}$, and Christina Precht ${ }^{1}$

${ }^{1}$ Department of Clinical Veterinary Medicine, University of Bern, Bern, Switzerland, ${ }^{2} D B M R$, University of Bern, Bern, Switzerland, ${ }^{3} D C R-V P H$, University of Bern, Bern, Switzerland

Metabolic profiling of tissue biopsies using HR-MAS NMR has potential diagnostic and prognostic value, but alterations in the biochemical profile due to factors such as sampling method may lead to misinterpretation. Therefore we investigated the effect of two different sampling methods in normal caprine brain tissue, in vivo sampling by stereotactic biopsy and direct post mortem surgical sampling. We found significant differences between the two biopsy types with elevated lactate and creatine, and altered choline-containing compounds. We conclude that metabolite alterations depend on sampling methods and suggest the use of in vivo biopsy in animal models.

13C-NMR to study cancer cell metabolic plasticity following PDK inhibition. Influence of dichloroacetate and long-term exposure to acidic environment on glucose and glutamine metabolic pathways.

Céline Schoonjans ${ }^{1}$, Nicolas Joudiou ${ }^{1}$, Cyril Corbet $^{2}$, Olivier Feron ${ }^{2}$, and Bernard Gallez ${ }^{1}$

${ }^{1}$ Biomedical Magnetic Resonance Group (REMA), Louvain Drug Research Institute, Catholic university of Louvain, Bruxelles, Belgium, ${ }^{2}$ Pharmacotherapy Group (FATH), Institute of Experimental and Clinical Research, Catholic university of Louvain, Bruxelles, Belgium

Many cancer cells present an exacerbated glycolytic flux that provides advantage for growth and leads to extracellular acidosis. Dichloroacetate (DCA), a PDK inhibitor, shifts metabolism from glycolysis to glucose oxidation and decrease various cancer cells lines proliferation. However, as tumor cells are presenting metabolic plasticity, PDK inhibition may lack efficacy. To measure metabolic adaptations of cancer cells to acidic environment and in response to DCA, we studied metabolic fluxes using ${ }^{13} \mathrm{C}-\mathrm{NMR}$ spectroscopy. With this technology, we measured differences in metabolic profiles between parental cancer cells line and acidic clones and we quantified specific changes in metabolism following DCA treatment.

Non-invasive mapping of glutathione levels in mouse brains by electron paramagnetic resonance (EPR) imaging

Miho C Emoto ${ }^{1}$, Hirotada G Fujii ${ }^{1}$, and Hideo Sato-Akaba²

${ }^{1}$ Sapporo Medical University, Sapporo, Japan, ${ }^{2}$ Osaka University, Toyonaka, Japan 
Glutathione (GSH) is an important antioxidant that can protect cells under oxidative stress. Thus, a non-invasive method to measure GSH levels in live animals is needed. To map the levels of GSH in mouse brains, a new method using electron paramagnetic resonance (EPR) imaging with nitroxide imaging probes was developed. By analyzing the relationship between reduction rates for nitroxides in brains measured by EPR and brain GSH levels measured by biochemical assay, pixel-based mapping of brain GSH levels was successfully obtained. The newly developed method was applied to a kindling mouse model of epilepsy to clarify the role of GSH.

Comparing the Reproducibility of Commonly Used Magnetic Resonance Spectroscopy Techniques to Quantify Cerebral Glutathione at $3 \mathrm{~T}$

Andrea Wijtenburg ${ }^{1}$, Jamie Near ${ }^{2}$, Stephanie Korenic ${ }^{1}$, Frank Gaston ${ }^{1}$, Hongji Chen ${ }^{1}$, Mark Mikkelsen ${ }^{3,4}$, Robert McMahon ${ }^{1}$, Peter Kochunov ${ }^{1}$, Elliot Hong ${ }^{1}$, and Laura Rowland ${ }^{1}, 5$

${ }^{1}$ Psychiatry, University of Maryland School of Medicine, Baltimore, MD, United States, ${ }^{2}$ Centre d'Imagerie Cérébrale, Douglas Mental Health Institute, Montreal, QC, Canada, ${ }^{3}$ Russell H. Morgan Department of Radiology and Radiological Science, Johns Hopkins University School of Medicine, Baltimore, MD, United States, ${ }^{4}$ F. M. Kirby Research Center for Functional Brain Imaging, Kennedy Krieger Institute, Baltimore, MD, United States, ${ }^{5}$ Russell H. Morgan Department of Radiology and Radiological Sciences, Johns Hopkins University School of Medicine, Baltimore, MD, United States

Cerebral glutathione (GSH), a marker of oxidative stress processes, has been quantified in neurodegenerative diseases and psychiatric disorders using proton magnetic resonance spectroscopy. However, no studies to date have compared the reproducibility of the most commonly used magnetic resonance spectroscopy techniques for GSH quantification. Here, we scanned ten healthy adults twice and acquired spectroscopic data using PRESS, PR-STEAM, SPECIAL, and MEGA-PRESS at 3 Tesla. We assess reproducibility via mean coefficients of variation (CV) and mean absolute difference (AD).

On spectrally selective measurements of irreversible and reversible transverse relaxation rates from single voxel, single echo time PRESS acquisitions

Robert Mulkern ${ }^{1}$ and Mukund Balasubramanian ${ }^{1}$

${ }^{1}$ Radiology, Children's Hospital, Boston, Boston, MA, United States

We developed a methodology to measure the reversible and irreversible transverse relaxation rates $R_{2}{ }^{\prime}$ and $R_{2}$, respectively, of multiple spectral peaks from spectroscopic sampling of both sides of a single spin echo. The methodology was applied to resonances in muscle and brain and the irreversible relaxation rates $R_{2}$ were compared with conventional measurements made from right side only spectra acquired at multiple PRESS echo times.

Aberrant Glutamatergic Neurotransmission in the Left Dorsolateral Prefrontal Cortex in Patients with Mild Cognitive Impairment: Preliminary Evidence from Task-Based Proton Magnetic Resonance Spectroscopy

Anupa A Vijayakumari ${ }^{1}$, Bejoy Thomas ${ }^{1}$, Ramshekhar N Menon ${ }^{2}$, and Chandrasekharan Kesavadas ${ }^{1}$

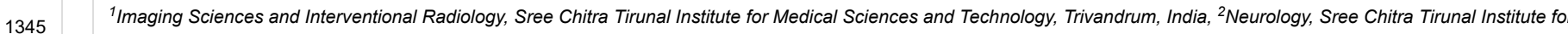
Medical Sciences and Technology, Trivandrum, India

Much less is known about the changes in glutamate during working memory (WM) in patients with mild cognitive impairment (MCl). In this study, we aimed to understand the glutamatergic response to functional activation in patients with $\mathrm{MCl}$ and healthy subjects (HS) during WM. The changes in glutamate were examined before, during, and after the WM task in both groups using point resolved spectroscopic sequence. We observed increased glutamate in $\mathrm{HS}$ during the task which was absent in $\mathrm{MCl}$. This suggests the disruption in the glutamatergic neurotransmission, which may be a part of the underlying pathophysiology in $\mathrm{MCl}$.

Traditional Poster

\section{MRS Human Applications}

Tracking changes in glutamate using dynamic MRS in response to an acutely painful stimulus.

Jessica Archibald ${ }^{1,2}$, Erin L Macmillan ${ }^{3,4,5}$, Carina Graf ${ }^{2,6}$, Cornelia Laule ${ }^{2,6,7}$, and John L.K Kramer ${ }^{1,2}$

${ }^{1}$ Kinesiology, University of British Colombia, Vancouver, BC, Canada, ${ }^{2}$ International Collaboration on Repair Discoveries (ICORD), Vancouver, BC, Canada, ${ }^{3}$ Radiology, University of British Colombia, Vancouver, BC, Canada, ${ }^{4}$ ImageTech Lab, SFU, Simon Fraser University, Vancouver, BC, Canada, ${ }^{5}$ Philips Healthcare Canada, Philips, Vancouver, BC, Canada, ${ }^{6}$ Physics and Astronomy, University of British Colombia, Vancouver, BC, Canada, ${ }^{7}$ Pathology and Laboratory Medicine, University of British Colombia, Vancouver, BC, Canada

Current treatment and diagnosis of pain conditions are dependent on self-reported measures. The objective of this study was to establish the feasibility of determining changes in excitatory neurotransmitter concentrations (glutamate) in the anterior cingulate cortex (ACC) as an objective measure of pain using dynamic single voxel magnetic resonance spectroscopy (MRS). Glutamate levels can accurately be detected with this paradigm, although a general trend in relation to pain was not observed across subjects. This is the first study to report dynamic levels of glutamate in the ACC in relation to pain in healthy individuals using optimized MRS acquisition and processing methods. 
Hippocampal metabolite changes in response to chronic corticosterone exposure: in vivo magnetic resonance spectroscopy at $9.4 \mathrm{~T}$

Song-I Lim ${ }^{1,2,3}$, Kyu-Ho Song ${ }^{1}$, Chi-Hyeon Yoo ${ }^{1}$, Hyeon-Man Baek ${ }^{3}$, and Bo-Young Choe ${ }^{1}$

${ }^{1}$ The Catholic University of Korea College of Medicine, Seoul, Republic of Korea, ${ }^{2}$ Asan Institute for Life Sciences, Asan Medical Center, Seoul, Republic of Korea, ${ }^{3}$ Lee Gil Ya Cancer \& Diabetes Institute, Gachon University School of Medicine, Incheon, Republic of Korea

The purpose of the study is to investigate neurochemical changes in a mouse model using proton magnetic resonance spectroscopy. Animals received $1 \%$ of ethanol drinking water solution or $100 \mu \mathrm{g} / \mathrm{mL}$ of corticosterone dissolved in $1 \%$ of ethanol drinking water for 4 weeks. MRS spectra were acquired at the end of the experiment. Mice that ingested corticosterone show elevated glutamate, glycerophosphocholine and taurine levels in the hippocampus compared with those shown by the control group. Increased corticosterone levels are considered a sign of stress or metabolic disturbance. Therefore we suggest that chronic corticosterone exposure can affect the hypothalamic-pituitary-adrenal dysregulation and neurochemical alteration.

[Asp], [Glu] and [NAA] changes following traumatic brain injury revealed by J-edited $1 \mathrm{H}$ MRS

Petr Menshchikov ${ }^{1,2}$, Natalia Semenova $^{1,2,3}$, Andrei Manzhurtsev $^{2,3}$, Maxim Ublinskii $^{2,3}$, llya Melnikov ${ }^{2}$, and Tolib Akhadov $^{2}$

${ }^{1}$ Semenov Institute of Chemical Physics, Russian Academy of Sciences, Moscow, Russian Federation, ${ }^{2}$ Clinical and Research Institute of Emergency Pediatric Surgery and Trauma, Moscow, Russian Federation, ${ }^{3}$ Emanuel Institute of Biochemical Physics, Russian Academy of Sciences, Moscow, Russian Federation

For the first time new method based on MEGA-PRESS pulse sequence for simultaneous aspartate (Asp), glutamate (Glu) and N acetyl aspartate (NAA) cerebral in vivo concentrations quantification were used for monitoring important metabolic changes after severe traumatic brain injury. Revealed Glutamate and Aspartate decrease is associated with excititoxicity (rapidly release of Glu and Asp from vesicles). In addition, Asp reduction might result from reduced availability of Glu.[NAA], marker of neuronal activity, reduction may be associated with synthesis disruption due to reduction of major NAA precussor (Asp)

Magnetization transfer among non-aqueous species and between them and water in spinal cord

Uzi Eliav ${ }^{1}$, Peter J. Basser ${ }^{2}$, and Gil Navon

${ }^{1}$ School of Chemistry, Tel Aviv University, Tel Aviv, Israel, ${ }^{2}$ SQITS/NICHD, NIH, Bethesda, MD, United States

Previous publications demonstrated that the intensity of white matter (WM) images of spinal cord stem from aqueous and non-aqueous protons (having a peak at 3.5ppm). The peak of the non-aqueous protons was analyzed to be a superimposition of signals with a distribution of $\mathrm{T}_{2}^{*}(10-1000 \mu \mathrm{s})$. Questions unanswered by these studies are whether the peaks with short and long $\mathrm{T}_{2}^{*}$ exchange magnetization among themselves, and whether they transfer magnetization (MT) to water. In the present publication these questions are addressed by combining double quantum filtering with magnetization transfer. The results demonstrate exchange between non-aqueous species and between them and water.

Multi-channel signal combination algorithms for polyunsaturated fatty acids (PUFA) using multiple quantum coherence (MQC) MRS in breast cancer

Vasiliki Mallikourti ${ }^{1}$, Sai Man Cheung ${ }^{1}$, Yazan Masannat ${ }^{2,3}$, Ehab Husain ${ }^{3,4}$, Steven D Heys ${ }^{2,3}$, and Jiabao He ${ }^{1}$

${ }^{1}$ University of Aberdeen, Aberdeen, United Kingdom, ${ }^{2}$ Breast Unit, Aberdeen Royal Infirmary, Aberdeen, United Kingdom, ${ }^{3}$ School of Medicine, University of Aberdeen, Aberdeen, United Kingdom, ${ }^{4}$ Pathology Department, Aberdeen Royal Infirmary, Aberdeen, United Kingdom

Polyunsaturated fatty acid (PUFA) is associated with malignant transformation of breast cancer and can be extracted from overwhelming background signals using multiple quantum coherence (MQC) MRS. Since MQC loses half of the signal, SNR enhancement through effective combination of signals acquired from multi channel coils holds significant potential. Investigations so far focused on conventional brain MRS, with drastically different metabolites and cluttered appearance compared to MQC MRS in breast. We therefore acquired PUFA spectra from 17 fresh breast tumour specimens and a patient on a clinical $3 T$ scanner, and current algorithms of adaptively optimised combination (AOC), S/N ${ }^{2}, \mathrm{~S} / \mathrm{N}$, Signal evaluated.

Detection of acute changes in glutamate with MR Spectroscopy using an $\mathrm{N}$-acetylcysteine challenge

Ruth Tuura ${ }^{1}$, Geoffrey Warnock ${ }^{2}$, Alfred Buck ${ }^{2}$, Valerie Treyer $^{2}$, Ralph Noeske ${ }^{3}$, and Michael Sommerauer ${ }^{2}$

${ }^{1}$ University Children's Hospital, Zurich, Switzerland, ${ }^{2}$ University Hospital, Zurich, Switzerland, ${ }^{3}$ GE Healthcare, Potsdam, Germany

We examined acute changes in MRS-visible glutamate and glutamine after stimulation with N-acetylcysteine (NAC), since NAC reportedly decreases synaptic glutamate via activation of inhibitory metabotropic glutamate receptors. In 10 healthy adults, NAC significantly reduced GIx in the basal ganglia and prefrontal cortex. In the basal ganglia, the changes in Glx were driven by changes in GIn, suggesting that GIn might represent a proxy marker for synaptic glutamate. In the frontal lobe, the MEGAPRESS edited spectra showed greater sensitivity to changes in GIx than short TE PRESS or the edit OFF subspectra. Acute compartmental shifts in glutamate are detectable with MRS. 
Characterizing altered glucose and glutamine metabolism in castration-resistant prostate cancer using high-resolution NMR

Jinny Sun ${ }^{1}$, Renuka Sriram ${ }^{2}$, Robert Bok ${ }^{2}$, Romelyn Delos Santos ${ }^{2}$, Mark Van Criekinge ${ }^{2}$, Daniel Vigneron ${ }^{2}$, and John Kurhanewicz ${ }^{2}$

${ }^{1}$ UC Berkeley - UCSF Graduate Program in Bioengineering, University of California, San Francisco, San Francisco, CA, United States, ${ }^{2}$ Department of Radiology and Biomedical Imaging, University of California, San Fracisco, San Francisco, CA, United States

This study demonstrates significant increases in flux through aerobic glycolysis, oxidative phosphorylation, and glutaminolysis with development of therapeutic resistance to androgen deprivation therapy using patient-derived cell lines and a transgenic murine model. Based on these metabolic differences between androgen-sensitive and insensitive prostate cancer, a combination of hyperpolarized $\left[1-{ }^{13} \mathrm{C}\right]$ pyruvate, $\left[2-{ }^{13} \mathrm{C}\right]$ pyruvate and $\left[5-{ }^{13} \mathrm{C}\right]$ glutamine can be used to noninvasively predict therapeutic resistance in future patient studies using $\mathrm{HP}{ }^{13} \mathrm{C}$ MRI.

Increase in Glutamate concentration during motor activation measured using functional Magnetic Resonance Spectroscopy (fMRS) at 3T.

Osnat Volovyk ${ }^{1}$ and Assaf Tal ${ }^{1}$

$1353 \quad{ }^{1}$ Chemical Physics, Weizmann Institute of Science, Rehovot, Israel

In the presented study we've demonstrated that small changes in Glutamate concentration associated with performing simple motor task can be reliably detected with 3T system using functional ${ }^{1} \mathrm{H}$ MR spectroscopy. Comparison between two differently timed paradigms for motor activation revealed a clear preference for longer-block designs. This suggests that motor activity-induced changes in Glutamate concentration are of minutes-long time-scale.

A 1H/31P MRS study of ATP and GABA modulation induced by anodal transcranial direct current stimulation in primary motor cortex of healthy subjects

Harshal Jayeshkumar Patel ${ }^{1}$, Chang-Hoon $\mathrm{Choi}^{2}$, N. Jon Shah ${ }^{2,3}$, and Ferdinand Binkofski ${ }^{1,2}$

${ }^{1}$ Division of Clinical Cognitive Sciences, Department of Neurology, RWTH Aachen University Hospital, Aachen, Germany, ${ }^{2}$ Institute of Neuroscience and Medicine - 4, Forschungszentrum Jülich GmbH, 52425, Juelich, Germany, ${ }^{3}$ Faculty of Medicine, Department of Neurology, RWTH Aachen University, JARA, Aachen, Germany

Transcranial direct current stimulation (tDCS) modulates cerebral energy and cortical inhibition. In this study we investigated long-term effects of anodal stimulation on inhibitory neurotransmitter and energy phosphate concentration using proton and phosphorous magnetic resonance spectroscopy. Our results indicate immediate GABA reduction following anodal tDCS and further maintaining the decreased state until the end of the experiment. ATP/Pi and PCr/Pi show initial reduction following anodal tDCS and further sign of recovery by the end of the experiment.

7T Magnetic Resonance Spectroscopy in the Hippocampus of MRI Normal Temporal Lobe Epilepsy Patients

John Adams ${ }^{1,2}$, Simona Nikolova ${ }^{3,4,5}$, Suzan Brown ${ }^{6}$, Robert Bartha ${ }^{1,2}$, and Jorge Burneo ${ }^{6,7}$

${ }^{1}$ Department of Medical BioPhysics, University of Western Ontario, London, ON, Canada, ${ }^{2}$ Centre for Functional and Metabolic Mapping, Robarts Research Institute, University of Western Ontario, London, ON, Canada, ${ }^{3}$ Department of Physics and Astronomy, University of California, Irvine, CA, United States, ${ }^{4}$ Department of Neurobiology and Behavior, University of California, Irvine, CA, United States, ${ }^{5}$ Center for the Neurobiology of Learning and Memory, University of California, Irvine, CA, United States, ${ }^{6}$ Epilepsy Program, London Health Sciences Centre, London, ON, Canada, ${ }^{7}$ Department of Clinical Neurological Studies, University of Western Ontario, London, ON, Canada

The utility of magnetic resonance spectroscopy for studying temporal lobe epilepsy (TLE) has been limited by magnetic field inhomogeneities. Using a 7T head-only MR system, we have successfully measured a number of metabolites which are challenging to measure in the hippocampus, including glutamate and glutathione, and we have observed a trend suggesting a decrease in creatine between contralateral and ipsilateral hippocampi in patients with unilateral, 1.5T MRI normal TLE.

Exploring metabolite profiling of patients with secondary progressive multiple sclerosis

Anita Monteverdi ${ }^{1}$, Bhavana Shantilal Solanky ${ }^{2}$, Floriana De Angelis ${ }^{2}$, Domenico Plantone ${ }^{2}$, Jonathan Stutters ${ }^{2}$, Nevin John ${ }^{2}$, Letizia Casiraghi ${ }^{1,3}$, lan Marshall ${ }^{4}$, Sue Pavitt ${ }^{5}$, Gavin Giovannoni $^{6}$, Christopher Weir ${ }^{7}$, Nigel Stallard ${ }^{8}$, Clive Hawkins ${ }^{9}$, Basil Sharrack ${ }^{10}$, Siddharthan Chandran ${ }^{4}$, Jeremy Chataway ${ }^{2}$, and Claudia Angela Gandini Wheeler-Kingshott ${ }^{1,2,11}$

${ }^{1}$ Department of Brain and Behavioural Sciences, University of Pavia, Pavia, Italy, ${ }^{2}$ Queen Square MS Centre, UCL Institute of Neurology, Faculty of Brain Sciences, University College London, London, United Kingdom, ${ }^{3}$ Brain Connectivity Center, C.Mondino National Neurological Institute, Pavia, Italy, ${ }^{4}$ Centre for Clinical Brain Sciences, University of Edinburgh, Edinburgh, United Kingdom, ${ }^{5}$ Dental Translational and Clinical Research Unit, School of Dentistry, Faculty of Medicine and Health, University of Leeds, Leeds, United Kingdom, ${ }^{6}$ Department of Neurology, Barts and the London NHS Trust, London, United Kingdom, ${ }^{7}$ Edinburgh Clinical Trials Unit, Usher Institute of Population Health Sciences and Informatics, University of Edinburgh, Edinburgh, United Kingdom, ${ }^{8}$ Division of Health Sciences, University of Warwick, Coventry, United Kingdom, ${ }^{9}$ Institute for Science and Technology in Medicine, Keele University, Keele, United Kingdom, ${ }^{10}$ Academic Department of Neuroscience, Royal Hallamshire Hospital, Sheffield, United Kingdom, ${ }^{11}$ Brain MRI $3 T$ Research Centre, C. Mondino National Neurological Institute, Pavia, Italy 
Proton magnetic resonance spectroscopic imaging (MRSI) quantifies brain metabolism in vivo and has the potential of uncovering the mechanism of action of therapeutic drugs. In this study, we assessed the baseline metabolic profile of 161 patients with secondary progressive multiple sclerosis (SPMS) against a control population by applying a short TE PRESS MRSI protocol at $3 \mathrm{~T}$. Based on the results the SPMS population could be divided into different groups (normal/biochemically abnormal) suggesting biochemical heterogeneity within SPMS patients.

Anterior cingulate cortex glutathione decreases with age - faster in women than in men?

Adriana Anton ${ }^{1}$, Catherine Gregory ${ }^{1}$, Richard Smallman ${ }^{1}$, Silke Conen ${ }^{1}$, Faezeh Sanaei-nezhad $^{2}$, Bill Deakin ${ }^{1}$, and Steve Williams ${ }^{2}$

${ }^{1}$ Neuroscience and Psychiatry Unit, Division of Neuroscience and Experimental Psychology, University of Manchester, Manchester, United Kingdom, ${ }^{2}$ Division of Informatics, Imaging and Data Sciences, University of Manchester, Manchester, United Kingdom

The anti-oxidant glutathione (GSH) may protect against ageing. Significantly lower GSH in the occipital cortex has been reported in elderly compared to young healthy volunteers. Here we show that $\mathrm{GSH}$ is also decreased in middle-aged ( $\mathrm{N}=8,39-54 \mathrm{y})$ compared to young $(\mathrm{N}=8,22-32 \mathrm{y})$ healthy subjects in the anterior cingulate but not the occipital cortex using $\mathrm{GSH}$ edited MEGA-PRESS at 3T. This significant difference is driven by the women in the middle-age sub-group (significantly lower GSH than in men). This suggests that age-related oxidative stress begins earlier in women compared to men and sex composition of a studied group could influence results.

Higher apparent diffusion coefficients in the older human brain

Dinesh K Deelchand ${ }^{1}$, J. Riley McCarten ${ }^{1,2}$, Laura S Hemmy ${ }^{1,2}$, Edward J Auerbach ${ }^{1}$, and Małgorzata Marjańska ${ }^{1}$

${ }^{1}$ University of Minnesota, Minneapolis, MN, United States, ${ }^{2}$ Veterans Affairs Health Care System, Minneapolis, MN, United States

The goal of this study was to compare the apparent diffusion coefficients (ADC) of the five major metabolites between young and older adults. Three brain regions were studied at $3 \mathrm{~T}$ using STEAM: prefrontal, posterior cingulate and occipital cortices. This study shows that the diffusivities of total $\mathrm{N}$-acetyl aspartate, glutamate and $m y o$-inositol are higher ( $7 \%$ on average) in the posterior cingulate cortex in older adults while no significant differences in ADC for the five major metabolites are observed in the other two brain regions studied. The ADCs of water are also higher in older adults in all three brain regions.

Contribution of Intramyocellular Lipids to the Decrease in Muscle Density with Age

Nicholas A. Brennan ${ }^{1}$, Kenneth W. Fishbein ${ }^{1}$, David A. Reiter ${ }^{2}$, Richard G. Spencer ${ }^{1}$, and Luigi Ferrucci ${ }^{3}$

${ }^{1}$ Laboratory of Clinical Investigation, National Institute on Aging, National Institutes of Health, Baltimore, MD, United States, ${ }^{2}$ Department of Radiology and Imaging Sciences, Emory University School of Medicine, Atlanta, GA, United States, ${ }^{3}$ Longitudinal Studies Section, National Institute on Aging, National Institutes of Health, Baltimore, MD, United States

Muscle density has been shown to decrease with age. However, the basis for this decrease remains unclear. We hypothesize that this decrease is associated with increased IMCL, and evaluated this relationship using localized $1 \mathrm{H}$ MRS of the vastus medialis muscle. We find that increased IMCL and decreased muscle density are strongly correlated across a large age range, even after controlling for multiple potential confounding variables.

${ }^{13} \mathrm{C}$ Magnetic Resonance Spectroscopy: Study of sperm metabolism under a hypoxic atmosphere.

Nurul Fadhlina Ismail ${ }^{1,2}$, Steven Reynolds ${ }^{1}$, Sarah Calvert ${ }^{3}$, Martyn Paley ${ }^{1}$, and Allan Pacey ${ }^{3}$

${ }^{1}$ Academic Unit of Radiology, University of Sheffield, Sheffield, United Kingdom, ${ }^{2}$ Faculty of Health Science, Universiti Sultan Zainal Abidin, Terengganu, Malaysia, ${ }^{3}$ Academic Unit of Reproductive \& Developmental Medicine, University of Sheffield, Sheffield, United Kingdom

Studying energy metabolism in sperm may be helpful in understanding the relationship between motility and infertility. To understand sperm metabolism, we acquired ${ }^{13} \mathrm{C}$ MR spectra during incubation with ${ }^{13} \mathrm{C}$-glucose in a normal and hypoxic atmosphere. Studies suggested that glycolysis is the main pathway for energy metabolism in sperm but whether glycolysis or oxidative phosphorylation(OXPHOS) dominates varies among species. This study examined the effect of hypoxia on sperm energy metabolism, with a secondary aim to observe Krebs cycle intermediates in the MR spectrum. Lactate signal in the hypoxia group was significantly higher than in the normoxia group. No Krebs cycle intermediate was detected.

Traditional Poster

\section{MRS Animal Studies}


Nathalie Just ${ }^{1}$ and Cornelius Faber ${ }^{1}$

${ }^{1}$ AG Experimentelle Magnetische Kernresonanz Translational Research Imaging Center (TRIC) Institut für, University Hospital Münster, Germany, Münster, Germany

For a better understanding of metabolic processes underlying neurovascular mechanisms, fMRS represents a suitable technique. The combination of fMRS and optogenetics (O-fMRS) should allow targeting the metabolism of specific cell populations during their activation. Our study aims at developing O-fMRS methodology in rat to provide further insight into brain energetics during activation. Here we establish a comparison between O-fMRS and sensory-fMRS in the rat forepaw cortex to investigate whether energetic demands are similar.

Comparison of in vivo MRS and ex vivo HR-MAS MRS for assessment of metabolite content in the GOT1 small intestine neuroendocrine tumour model

Mikael Montelius ${ }^{1}$, Johan Spetz ${ }^{2}$, Diana Bernin ${ }^{3}$, Oscar Jalnefjord ${ }^{1,4}$, Maria Ljungberg ${ }^{1,4}$, and Eva Forssell-Aronsson ${ }^{1,4}$

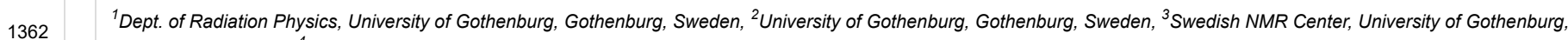
Gothenburg, Sweden, ${ }^{4}$ Dept. of medical physics and biomedical engineering, Sahlgrenska University hospital, Gothenburg, Sweden

In vivo characterisation of tumour metabolism using MRS would facilitate tumour therapy response assessment, but in vivo conditions may obscure the metabolic information acquired. In this study we investigate the information contained in in vivo MRS spectra of a neuroendocrine tumour model by correlating it to ex vivo HR-MAS MRS on excised tumour samples. Effects of post-mortem tissue degradation and tumour sample site on in vivo-ex vivo correlations are evaluated, and interpretation of in vivo data is discussed.

A neuroimaging study of the effects of early vs. late anti-inflammatory treatment in a rodent model of Alzheimer's disease

Caitlin Fowler ${ }^{1}$, Dan Madularu ${ }^{2}$, John Breitner ${ }^{3}$, and Jamie Near ${ }^{3}$

${ }^{1}$ Engineering, McGill University, Montreal, QC, Canada, ${ }^{2}$ McGill University, Montreal, QC, Canada, ${ }^{3}$ Douglas Mental Health University Institute and Department of Psychiatry, McGill University, Montreal, QC, Canada

Alzheimer's disease (AD) is a progressive neurodegenerative disorder with no effective treatments or known biomarkers for definitive diagnosis, substantiating the need for early detection of $A D$ and early intervention. This project employs Magnetic Resonance Spectroscopy (MRS) to measure changes in neurometabolites as compared to behavioural measures of cognitive function, in a transgenic rat model of $A D$ under treatment conditions. Preliminary results suggest that changes in metabolite levels are present before the onset of cognitive impairment, and between treatment and control groups, with some of these changes being sexually dimorphic.

Longitudinal follow-up of brain metabolism in rat models of progressive Parkinson's disease using Magnetic Resonance Spectroscopy Imaging

Carine Chassain ${ }^{1}$, Christophe Melon ${ }^{2}$, Guilhem Pages ${ }^{3}$, Yann Le Fur ${ }^{4}$, Pascal Salin ${ }^{2}$, Lydia Kerkerian-Le Goff ${ }^{2}$, and Franck Durif 5,6

${ }^{1}$ MRI department, CHU Clermont-Ferrand, Clermont-Ferrand, France, ${ }^{2}$ IBDM, UMR 7288 CNRS / Aix-Marseille Université, Marseille, France, ${ }^{3}$ AgroResonance-UR370 QuaPA, Saint Genes Champanelle, France, ${ }^{4}$ Centre de Résonance Magnétique Biologique et Médicale UMR 7339 CNRS / Aix-Marseille Université, Marseille, France, ${ }^{5}$ Neurology department, CHU Clermont-Ferrand, Clermont-Ferrand, France, ${ }^{6} 6$ Université Clermont Auvergne (UCA), EA7280 NPSY-Sydo, Clermont-Ferrand, France

The development of animal models that reproduce the selective and progressive loss of nigral dopamine neurons characterizing Parkinson's disease has opened new possibilities to study the disease evolution. Here magnetic resonance spectroscopy imaging was used to follow up the distributions of metabolites in key basal ganglia components in two rat models of progressive parkinsonism at three time points over a period of 120 days following injury. First results on overtime changes in NAA and glutamate repartition will be presented. Completion of this project may provide novel insights onto the pathological alterations associated with the progression of the neurodegenerative process.

Metabolic Consequences in the Heart and Skeletal Muscle of Human Pancreatic Cancer Xenograft Growth

Santosh Kumar Bharti ${ }^{1}$, Paul T Winnard $\mathrm{Jr}^{1}{ }^{1}$, Yelena Mironchik ${ }^{1}$, Marie-France Penet ${ }^{1}$, and Zaver M Bhujwalla ${ }^{1,2}$

${ }^{1}$ Division of Cancer Imaging Research, Department of Radiology, Johns Hopkins University, School of Medicine, Baltimore, MD, United States, ${ }^{2}$ Department of Oncology, Johns Hopkins University, School of Medicine, Baltimore, MD, United States

To understand the metabolic events that occur during cancer-induced cachexia, here we analyzed the effects of human pancreatic cancer xenografts on heart and skeletal muscle metabolites using $1 \mathrm{H}$ MRS. Studies were performed with cachexia-inducing Pa04C and non-cachexia inducing Panc1 human pancreatic cancer xenografts, since cachexia occurs most frequently in pancreatic cancer. 1H MR spectra identified differences in heart and skeletal muscle metabolites of cachectic and non-cachectic mice, as well as between normal mice and cachectic as well as non-cachectic mice. Our data highlight the systemic metabolic changes that occur with tumor growth and provide new insights in cancer-induced cachexia. 
Tatsuya Kawai ${ }^{1}$, Jeffery Brender ${ }^{2}$, Kevin Camphausen ${ }^{1}$, and Murali C Krishna ${ }^{2}$

${ }^{1}$ Radiation Oncology Branch, National Cancer Institute, NIH, Bethesda, MD, United States, ${ }^{2}$ Radiation Biology Branch, National Cancer Institute, NIH, Bethesda, MD, United States

Dynamic nuclear polarization-MRI along with hyperpolarized [1-13C] pyruvate was conducted to evaluate the difference in glycolytic profile between a glioblastoma cell line and cancer stem-like cells using the orthotopic xenograft mouse model.

Does maternal swimming during gestation protects the neonatal brain from hypoxic-ischemic injury?

Yohan van de Looij ${ }^{1,2,3}$, Eduardo Sanchez ${ }^{1}$, Petra S Hüppi ${ }^{1}$, and Stéphane V Sizonenko ${ }^{1}$

${ }^{1}$ Service développement et croissance, Université de Genève, Geneva, Switzerland, ${ }^{2}$ Laboratoire d'imagerie fonctionnelle et métabolique, Ecole polytechnique fédérale de Lausanne, Lausanne, Switzerland, ${ }^{3}$ Institut translationnel d'imagerie moléculaire, Université de Genève, Geneva, Switzerland

There are growing evidences that swimming during gestation has a neuroprotective effect on offspring perinatal brain injuries. The aim of this work was to assess this neuroprotective effect on P3 hypoxic-ischemic model by ${ }^{1} \mathrm{H}-\mathrm{MRS}$ and diffusion MRI (DTI and NODDI) at $9.4 \mathrm{~T}$. A moderate, but real effect of swimming during gestation on the neurochemical profile $24 \mathrm{~h}$ after $\mathrm{HI}$ was observed. Difference in neurochemical profile between sedentary and swimming rats may lead to a different response to the injury. At long-term, diffusion MRI derived parameters changes following $\mathrm{HI}$ were restored in the swimming $\mathrm{HI}$ group, providing evidence of a neuroprotective effect.

Differences between neurochemical profiles of male and female C57BL/6 mice

Sarah N Larson ${ }^{1}$ and Ivan Tkac ${ }^{1}$

1368 'Center for Magnetic Resonance Research, University of Minnesota, Minneapolis, MN, United States

The purpose of this study was to demonstrate whether neurochemical profiles of male and female C57BL/6 mice were affected in a sex-related manner. In vivo $1 \mathrm{H}$ MRS data were acquired from four different groups of mice, each group consisting of 10 male and 10 female mice. Highly significant differences between male and female groups were consistently observed in each group. These results have serious implications for appropriate quantification referencing (water vs. creatine, male or females in treated vs. control group) for avoiding bias in data interpretation.

Traditional Poster

\section{Cartilage}

Ability of MRI to Predict the Severity and Location of Chondral and Labral Pathology at Arthroscopy

Alissa J. Burge ${ }^{1}$, Stephen Lyman ${ }^{1}$, Matthew F. Koff ${ }^{1}$, Hollis G. Potter ${ }^{1}$, Sydney Kersten ${ }^{1}$, Bin Lin ${ }^{1}$, Kara Fields ${ }^{1}$, and Bryan Kelly ${ }^{1}$

${ }^{1}$ Hospital for Special Surgery, New York, NY, United States

Preoperative MRI and intraoperative arthroscopic images were independently reviewed in a cohort of 24 hips with femoroacetabular impingement with respect to severity and location of chondral, labral, and osseous pathology. Initial calculation of agreement between MRI and arthroscopic findings demonstrated fair to near perfect agreement for the severity of pathology; however, agreement for the location of pathology was highly variable. MR images were subsequently re-scored utilizing the indirect head of the rectus femoris as an anatomic landmark, in accordance with the system used by the operating surgeon, resulting in overall increased agreement across position-dependent variables.

Correlation time mapping is associated with permeability of articular cartilage

Mikko T. Nissinen ${ }^{1,2}$, Nina Hänninen ${ }^{3}$, Petri Tanska ${ }^{1}$, Olli Nykänen ${ }^{1}$, Mithilesh Prakash ${ }^{1}$, Matti Hanni ${ }^{2,3,4}$, Juha Töyräs ${ }^{1,5}$, Rami K. Korhonen $^{1}$, Mikko J. Nissi $^{1}$, and Miika T. Nieminen ${ }^{2,3,4}$

${ }^{1}$ Applied Physics, University of Eastern Finland, Kuopio, Finland, ${ }^{2}$ Medical Research Center, University of Oulu and Oulu University Hospital, Oulu, Finland, ${ }^{3}$ Research Unit of Medical Imaging, University of Oulu, Oulu, Finland, ${ }^{4}$ Department of Diagnostic Radiology, Oulu University Hospital, Oulu, Finland, ${ }^{5}$ Diagnostic Imaging Center, Kuopio University Hospital, Kuopio, Finland

Correlation time $\mathrm{T}_{\mathrm{C}}$ is a parameter that describes the relaxation properties of soft tissues. In this study, articular cartilage from human cadaver patellae was studied using MR imaging and biomechanical testing and modeling. The statistical analysis revealed an association between the permeability, as revealed by mechanical modeling, and the correlation time measured for articular cartilage. 
T2* Enhancement for Multi-Echo Data Image Combination -- Using least squares for echo prediction

Zhang Qiong ${ }^{1}$, Chen Shi ${ }^{1}$, Wei Binyan ${ }^{2}$, and Kang Yuanyuan ${ }^{1}$

This work provides a virtual echo prediction method for Multi Echo Data Image Combination (Medic) based on least square estimation. The strong dependences between multi-echoes in Medic sequences are used to predict virtual echoes with assumed echo times, and then such predictions are combined with real acquired echoes for heavier T2* contrast enhancement.

Comparison of Conventional and Synthetic MRI for Quantitative Cartilage T2 Mapping of the Patella

Le Roy Chong ${ }^{1}$, Gideon $\mathrm{Ooi}^{1}$, Jia Hui $\mathrm{Ng}^{1}$, and Hafiz Bin Abu Hassan ${ }^{1}$

Synthetic MRI has been shown to be of comparable performance to conventional MRI in the assessment of intracranial abnormalities. This study compares synthetic MRI with conventional T2 mapping for quantitative assessment of cartilage T2 relaxation times. T2 values acquired via synthetic MRI are highly correlated with but not equivalent to conventional T2 mapping. Synthetic MRI could be a potential alternative in the quantitative assessment of chondral abnormalities, without the need for prolonged scan times and providing the benefit of dynamic tissue contrasts from a single acquisition.

Associations between Osteoarthritis Molecular Biomarkers and MR-based cartilage composition and Knee Joint Morphology: Data from the Osteoarthritis Initiative

Gabby B Joseph ${ }^{1}$, Michael C Nevitt ${ }^{2}$, Charles E McCulloch², Jan Neumann ${ }^{1}$, John A Lynch² ${ }^{2}$ Ursula Heilmeier ${ }^{1}$, Nancy E Lane ${ }^{3}$, and Thomas M Link ${ }^{1}$

${ }^{1}$ Department of Radiology and Biomedical Imaging, University of California, San Francisco, San Francisco, CA, United States, ${ }^{2}$ Department of Epidemiology and Biostatistics, University of California, San Francisco, San Francisco, CA, United States, ${ }^{3}$ Department of Rheumatology, University of California, Davis, Davis, CA, United States

This study assessed the relationships of serum/urine biomarkers for osteoarthritis with MR imaging measures of joint structure and composition, using data from the Osteoarthritis Initiative (OAI). Significant positive correlations between the serum/urine biomarkers (sHA, sMMP3) and MRI cartilage T2 relaxation time measurements, compositional markers of early cartilage degeneration were observed. However, no significant associations were found with cartilage morphology or Kellgren-Lawrence (KL) grade. Therefore, serum biomarkers and cartilage $\mathrm{T} 2$ composition may reflect similar features of the pathophysiology of cartilage matrix degenerative disease.

Detailed T2-mapping analysis reveal disc characteristics that may be of significance for low back pain patients

Christian Waldenberg ${ }^{1}$, Hanna Hebelka $^{2}$, Helena Brisby ${ }^{3}$, and Kerstin Magdalena Lagerstrand ${ }^{1}$

${ }^{1}$ Dept. of Medical Physics and Techniques, Sahlgrenska University Hospital, Gothenburg, Sweden, Institute of Clinical Sciences, Sahlgrenska Academy, University of Gothenburg, Gothenburg, Sweden, Gothenburg, Sweden, ${ }^{2}$ Dept. of Radiology, Sahlgrenska University Hospital, Gothenburg, Sweden, Institute of Clinical Sciences, Sahlgrenska Academy, University of Gothenburg, Gothenburg, Sweden, Gothenburg, Sweden, ${ }^{3}$ Dept. of Orthopaedics, Sahlgrenska University Hospital, Gothenburg, Sweden., Institute of Clinical Sciences, Sahlgrenska Academy, University of Gothenburg, Gothenburg, Sweden, Gothenburg, Sweden

In this study, we address the lack of studies comparing intervertebral disc characteristics between symptomatic and asymptomatic individuals. Based on quantitative T2-mapping, small but relevant differences between low back pain patients and a control cohort were found on a global and regional level.

Magnetization Transfer Ratio (MTRNOE) as a Biomarker of Hip Osteoarthritis

Hatef Mehrabian ${ }^{1}$, Jasmine Rossi-Devries ${ }^{1}$, Alan L Zhang ${ }^{2}$, Richard B Souza ${ }^{3}$, and Sharmila Majumdar ${ }^{1}$

${ }^{1}$ Radiology \& Biomedical Imaging, University of California, San Francisco, San Francisco, CA, United States, ${ }^{2}$ Orthopaedic Surgery, University of California, San Francisco, San Francisco, CA, United States, ${ }^{3}$ Physical Therapy, University of California, San Francisco, San Francisco, CA, United States

Loss of cartilage collagen, proteoglycans (PG), glycosaminoglycans (GAG) are responsible for osteoarthritis (OA). MRI biomarkers $T_{2}$ (sensitive to collagen), magnetization transfer (MT) and $T_{1 p}$, (sensitive to $P G$ ), and GAG $_{\text {CEST }}$ (sensitive to GAG) can detect OA at early stages. Similar to GAG $_{\text {CEST, }}$, CEST signal of Nuclear Overhauser Effect (NOE changes with OA. However, unlike $\mathrm{GAG}_{\mathrm{CEST}}$, this $\mathrm{NOE}_{\mathrm{CEST}}$ is measurable at $3 \mathrm{~T}$ which is suitable for hip. MT ratio at this $-1.6 \mathrm{ppm}$ (MTR $\mathrm{ROE}_{\mathrm{N}}$ ) represents the combination of MT, $\mathrm{T}_{2}$, $\mathrm{NOE}_{\mathrm{CEST}}$ effects. OA-related changes in these three parameters result in decreased MTR $\mathrm{NOE}_{\mathrm{N}}$ making it a reliable biomarker for $O A$. 
T2 and T1rho mapping of ankle cartilage of female and male ballet dancers

Saya Horiuchi ${ }^{1}$, Hon J. Yu ${ }^{1}$, Alex Luk ${ }^{1}$, Adam Rudd ${ }^{1}$, Jimmy Ton ${ }^{1}$, Edward Kuoy ${ }^{1}$, Jeff Russell ${ }^{2}$, Kelli Sharp ${ }^{3}$, and Hiroshi Yoshioka ${ }^{1}$

${ }^{1}$ Radiological Sciences, University of California, Irvine, Irvine, CA, United States, ${ }^{2}$ Science and Health in Artistic Performance, Ohio University, Athens, OH, United States, ${ }^{3}$ Department of Dance, The Claire Trevor School of the Arts, University of California, Irvine, Irvine, CA, United States

This study demonstrated T2 and T1rho profiles of talar dome and tibial plafond cartilage from male and female ballet dancers using angular-segmentation methodology for quantitative assessment of cartilage in vivo. The results in this study showed both $\mathrm{T} 2$ and T1rho relaxation time indicated the lowest value over the central weight-bearing portion, while they indicated relatively higher values in the anterior and posterior portion. These findings can be due to the combination of the magic angle effect which has higher influence on $\mathrm{T} 2$ value and early cartilage degenerative changes which are more sharply detected by T1rho value.

Analysis of the Local Associations between Morphology and Biochemical Composition of the Articular Cartilage after Anterior Cruciate Ligament Injury and Reconstructive Surgery using Voxel-Based Relaxometry

Onyekachi Ezinna Nnabue ${ }^{1,2}$, Hatef Mehrabian¹, Valentina Pedoia ${ }^{1}$, Berk Norman ${ }^{1}$, Benjamin C. Ma², and Sharmila Majumdar ${ }^{1}$

${ }^{1}$ Department of Radiology and Biomedical Imaging, University of California, San Francisco, San Francisco, CA, United States, ${ }^{2}$ Department of Orthopaedic Surgery, University of California, San Francisco, San Francisco, CA, United States

This study uncovered new insights on the local associations between cartilage thickness and $\mathrm{T}_{1 \rho}$ relaxation time (a marker of cartilage proteoglycan content). Using Voxel-based relaxometry, this study quantified the longitudinal and cross-sectional thickness changes that occur in both the ACL-injured knee and the healthy contralateral in the lateral femoral condyle, medial femoral condyle, trochlea, medial tibia, lateral tibia, and patella and examined compartment-specific associations with relaxometry at various time points.

CS+SENSE for Fast UTE Knee Imaging: Technical Feasibility

Yongxian Qian ${ }^{1}$, Li Feng ${ }^{1}$, Tiejun Zhao², Richardo Otazo ${ }^{1}$, and Fernando E. Boada ${ }^{1}$

${ }^{1}$ Radiology, New York University, New York, NY, United States, ${ }^{2}$ Siemens Healthineers USA, New York, NY, United States

Ultrashort echo time (UTE<1ms) imaging has advantages over traditional long TE (>10ms) imaging to detect asymptomatic (subclinical) cartilage damages in the knee joint, such as fissuring, fracturing and collagen fiber breakdown. To advance UTE imaging toward clinical use, its long scan time needs to be reduced to meet clinical requirement of short protocols. Compressed sensing (CS) and sensitivity encoding (SENSE) parallel imaging have the potential to do so. However, individual use of them has limitations. A combined use of both techniques has been shown in dynamic imaging to be able to achieve higher acceleration factor without SNR loss. This study explores the technical feasibility to extend CE+SENSE to static UTE imaging.

Quantitative evaluation of knee cartilage after anterior cruciate ligament reconstruction using UTE-T2* mapping in a rabbit model

Yiwen $\mathrm{Hu}^{1}$ and Jianxun $\mathrm{Qu}^{2}$

${ }^{1}$ Fudan University affiliated Huashan Hospital, Shanghai, China, ${ }^{2} \mathrm{GE}$ Healthcare, CHINA, Beijing, China

Our study is a prospective longitudinal study conducted to find outcome of anterior cruciate ligament reconstruction in rabbit model. We evaluated degenerative changes of cartilage by UTE-T2* mapping. ACLR knees shows cartilage matrix degeneration at early stage of "ligamentization", though rabbit tibiofemoral cartilage is definitely thin.

Comparison of T2 Relaxation Times in Knee Cartilage Between Breaststroke and Nonbreaststroke Swimmers

James Yoder ${ }^{1}$, Feliks Kogan ${ }^{1}$, and Garry E. Gold ${ }^{1,2,3}$

${ }^{1}$ Radiology, Stanford University, Stanford, CA, United States, ${ }^{2}$ Bioengineering, Stanford University, Stanford, CA, United States, ${ }^{3}$ Orthopaedic Surgery, Stanford University, Stanford, CA, United States

While MRI has been widely used to examine the effects of translational forces on cartilage matrix structure, studies looking at rotational forces are limited. Breaststroke swimmers are a population of interest since the repeated use of the breaststroke kick has been cited as a source of knee pain. However, the cartilage of breaststrokers has not been quantitatively measured to investigate possible differences and the potential increased risk of cartilage degeneration and osteoarthritis development. This study compares the T2 relaxation times of various compartments for patellar, femoral, and tibial cartilage at the superficial, deep, and aggregate levels between breaststrokers and nonbreaststrokers. 
Grey-Level Co-Occurrence Matrix Texture Analysis of T2, Adiabatic T1 $\rho$, Adiabatic T2 $\rho$ and Dual-Echo Steady-State Magnetic Resonance Imaging Contrasts in Osteoarthritic Knee Articular Cartilage

Ines Barros ${ }^{1,2}$, Arttu Peuna ${ }^{2}$, Victor Casula1,3, Marianne Haapea ${ }^{1,2}$, Eveliina Lammentausta ${ }^{2}$, and Miika T. Nieminen ${ }^{1,2,3}$

${ }^{1}$ Research Unit of Medical Imaging, Physics and Technology, University of Oulu, Oulu, Finland, ${ }^{2}$ Department of Diagnostic Radiology, Oulu University Hospital, Oulu, Finland, ${ }^{3}$ Medical Research Center, University of Oulu and Oulu University Hospital, Oulu, Finland

Grey-level co-occurrence matrix (GLCM) based texture analysis is a sensitive image processing tool for the evaluation of cartilage in knee osteoarthritis (OA). Texture analysis of $\mathrm{T}_{2}$, Adiabatic $\mathrm{T}_{1 \rho}\left(\mathrm{AdT}_{1 \rho}\right)$, Adiabatic $\mathrm{T}_{2 \rho}\left(\mathrm{AdT}_{2 \rho}\right)$ relaxation time maps as well as Dual-Echo Steady-State (DESS) images showed the ability to distinguish OA patients and asymptomatic volunteers. Moreover, texture analysis turned out to be more sensitive to cartilage degeneration than mean relaxation time values. Texture analysis can therefore supplement existing quantitative MRI techniques of articular cartilage.

Simulated $1 \mathrm{H}-1 \mathrm{H}$ residual dipolar couplings of collagen-associated water

Jouni Karjalainen ${ }^{1}$, Mikko J. Nissi², Miika T. Nieminen ${ }^{1,3,4}$, and Matti Hanni ${ }^{1,3,4}$

${ }^{1}$ Research Unit of Medical Imaging, Physics and Technology, University of Oulu, Oulu, Finland, ${ }^{2}$ Department of Applied Physics, University of Eastern Finland, Kuopio, Finland, ${ }^{3}$ Medical Research Center, University of Oulu and Oulu University Hospital, Oulu, Finland, ${ }^{4}$ Department of Diagnostic Radiology, Oulu University Hospital, Oulu, Finland

Residual dipolar couplings have been suggested as the cause of the orientational dependence of relaxation times in anisotropic tissues, such as articular cartilage. We use molecular dynamics simulations to compute the residual dipolar couplings of water protons associated with a model collagen molecule. The results suggest that significant residual dipolar couplings appear without strong binding between the water and the collagen.

Quantitative GagCEST MRI in Juvenile Bovine Articular Cartilage Exhibit Correlations between 3T and 7T

Lauren Watkins ${ }^{1}$, Feliks Kogan ${ }^{2}$, Marianne Black ${ }^{3}$, Marc Levenston ${ }^{1,2,3}$, and Garry Gold ${ }^{1,2}$

${ }^{1}$ Bioengineering, Stanford University, Stanford, CA, United States, ${ }^{2}$ Radiology, Stanford University, Stanford, CA, United States, ${ }^{3}$ Mechanical Engineering, Stanford University, Stanford, $C A$, United States

GagCEST is a quantitative MR technique that shows promise at $7 \mathrm{~T}$ to specifically detect cartilage glycosaminoglycan content; however, its potential at $3 \mathrm{~T}$ is still uncertain. This study utilizes a new optimized 3D GagCEST sequence to maximize SNR and GagCEST contrast at 3T. Comparison of GagCEST asymmetry maps obtained at 3T and 7T suggest that GagCEST can be used to distinguish zonal differences in cartilage composition at both 3T and 7T. This work demonstrates potential for whole joint GagCEST knee imaging at 3T with improved dynamic range.

Automated segmentation of the cartilage from high-resolution isotropic T1rho MRI

Henry Rusinek ${ }^{1}$, Rahman Baboli ${ }^{2}$, Artem Mikheev², Azadeh Sharafi ${ }^{2}$, and Ravinder R Regatte ${ }^{2}$

${ }^{1}$ Radiology, New York University School of Medicine, New York, NY, United States, ${ }^{2}$ Radiology, New York Unversity School of Medicine, New York, NY, United States

We analyze the accuracy of atlas-based cartilage segmentation from isotropic T1 $\rho$ MRI and compare it to semi-automated "seed and blanket" method and manual segmentation (ground truth). Reference 3D cartilage masks were taken as the consensus of two human experts. For patella, our implementation of template matching yielded the root mean square volume measurement error RMSE of $0.66 \mathrm{~cm}^{3}$, with interclass correlation coefficient (ICC) $=0.765$ and sufficient precision to detect the gender effect. Over two-fold improvement in accuracy, RMSE $=0.25 \mathrm{~cm}^{3}$ and ICC $=0.960$ was achieved with a fast, semi-automated algorithm. Similar results hold for the accuracy of the average thickness of segmented masks.

The novel and quantitative MRI technique: Q-space imaging for evaluating intervertebral disc degeneration: basic and clinical study.

Daisuke Nakashima ${ }^{1}$, Nobuyuki Fujita ${ }^{2}$, Junichi Hata ${ }^{3,4}$, Takeo Nagura $^{2}$, Kanehiro Fujiyoshi $^{5}$, Hideyuki Okano ${ }^{3}$, Masahiro Jinzaki ${ }^{2}$, Morio Matsumoto ${ }^{2}$, and Masaya Nakamura ${ }^{2}$

${ }^{1}$ Department of Orthopaedic Surgery, Keio University School of Medicine, Tokyo, Japan, ${ }^{2}$ Keio University School of Medicine, Tokyo, Japan, ${ }^{3}$ Central Institute for Experimental Animals, Kawasaki, Japan, ${ }^{4}$ Department of Physiology, Keio University School of Medicine, Tokyo, Japan, ${ }^{5}$ Murayama Medical Center, Tokyo, Japan

The conventional qualitative classification of intervertebral disc (IVD) degeneration: Pfirrmann classification on T2 weighted imaging does not have the enough sensitivity for the evaluation of IVD degeneration. In the present study, probability at zero displacement obtained from Q-space imaging (QSI) has a high sensitivity of IVD degeneration in both basic and clinical study compared with the conventional method: T2 mapping. In particular, probability at zero displacement made it possible to observe the effect of the regenerative drug: N-Acetyl Cystaine on IVD degeneration which could not be observed by using T2 mapping. Probability at zero displacement obtained from QSI has the possibility to be a novel biomarker of IVD degeneration. 
Effect of Fat-contamination and Fat-suppression on T2 Quantitation of Knee Articular Cartilage In Vivo

Petri Paakkari ${ }^{1}$, Stefan Zbyn ${ }^{1,2}$, Mikko J Nissi $^{3}$, Eveliina Lammentausta ${ }^{4}$, Miika T Nieminen ${ }^{1,2,4}$, and Victor Casula ${ }^{1,2}$

${ }^{1}$ Research Unit of Medical Imaging, Physics and Technology, University of Oulu, Oulu, Finland, ${ }^{2}$ Medical Research Center, University of Oulu and Oulu University Hospital, Oulu, Finland, ${ }^{3}$ Department of Applied Physics, University of Eastern Finland, Kuopio, Finland, ${ }^{4}$ Department of Diagnostic Radiology, Oulu University Hospital, Oulu, Finland

This study aims to investigate the effect of fat contamination and fat suppression (FS) on in vivo T2 mapping of knee cartilage. Four volunteers were imaged on a 3T MRI scanner and T2 values were calculated in several regions of tibiofemoral cartilage using a MSME sequence with and without FS. The use of FS improved repeatability of cartilage segmentation in several regions and reduced the chemical shift artifacts. However, the regional heterogeneity in FS sequence introduced further uncertainties in T2 measurements.

T1 Relaxation Time Mapping of Articular Cartilage for Femoroacetabular Impingement (FAI) - A Clinical Pilot Study

Jutta Ellermann ${ }^{1}$, Douglas Martin², Casey P Johnson ${ }^{3}$, Robert Gao ${ }^{4}$, Luning Wang ${ }^{1}$, and Patrick Morgan ${ }^{5}$

${ }^{1}$ Radiology, CMRR, University of Minnesota, Minneapolis, MN, United States, ${ }^{2}$ Radiology, Stanford University, Palo Alto, CA, United States, ${ }^{3}$ Radiology, University of Minnesota, Minneapolis, MN, United States, ${ }^{4}$ University of Minnesota, Minneapolis, MN, United States, ${ }^{5}$ Orthopaedics, University of Minnesota, Minneapolis, MN, United States

In this pilot study we demonstrate the clinical utility of quantitative T1 relaxation time mapping to assess acetabular cartilage damage in patients with Femoroacetabular Impingement (FAI).

Analysis of Knee Cartilage using Magnetization Transfer and Multi-exponential T2* Fitting

Sooyeon $\mathrm{Ji}^{1}$, Se-Hong Oh${ }^{2}$, Young-Han Lee ${ }^{3}$, Dongmyung Shin ${ }^{1}$, Doohee Lee ${ }^{1}$, Taehyun Hwang ${ }^{1}$, Woojin Jung ${ }^{1}$, Hyeong-Geol Shin ${ }^{1}$, and Jongho Lee ${ }^{1}$

${ }^{1}$ Department of Electrical and Computer Engineering, Seoul National University, Seoul, Republic of Korea, ${ }^{2}$ Department of Biomedical Engineering, Hankuk University of Foreign Studies, Seoul, Republic of Korea, ${ }^{3}$ Department of Radiology, Research Institute of Radiological Science, Yonsei University College of Medicine, Seoul, Republic of Korea

In this study, we explored the combined use of magnetization transfer (MT) weighting and bi-exponential $\mathrm{T}_{2}{ }^{*}$ fitting as a potential tool to analyze the composition and microscopic geometry of the knee cartilage. The analysis results of deep cartilage areas showed that the MT ratio of the short $\mathrm{T}_{2}{ }^{*}$ component had significantly larger values than that of the long $\mathrm{T}_{2}{ }^{*}$ component. This observation may be explained by the geometry of collagen fibrils and proteoglycans.

T1-T2 correlation of site-specific changes and zone-dependent anisotropy of osteoarthritic cartilage using multi-resolution MRI

Farid Badar ${ }^{1}$ and Yang Xia ${ }^{1}$

${ }^{1}$ Physics, Oakland University, Rochester Hills, MI, United States

Topographical and zonal based studies of healthy and OA canine tibial cartilage are shown to be essential for the early detection of osteoarthritis. A high-resolution T1-T2 correlation with the low-resolution imaging of depth-dependent $\mathrm{T} 2$ profiles shows a more detailed and sensitive method of measuring the early sign of cartilage degradation, beneficial to human OA MRI.

Effect of spin-lock field direction on chemical exchange spin-lock (CESL) and evaluate its feasibility of glycosaminoglycan (GAG) detection at 3.0T

Baiyan Jiang ${ }^{1}$ and Weitian Chen ${ }^{1}$

${ }^{1}$ Imaging and Interventional Radiology, The Chinese University of Hong Kong, Shatin, Hong Kong

Chemical exchange spin-lock (CESL) is sensitive to fast exchange metabolites. CESL is performed across a range of resonance frequency offsets. At any frequency offset, either antiparallel or parallel spin-lock directions can be used. However, different directions can affect the z-spectrum and the magnetization transfer ratio asymmetry analysis. We used simulations and in vivo experiments to demonstrate this effect and provided theoretical analysis. We also presented preliminary results of CESL for imaging of chemical exchange associated with glycosaminoglycan (GAG) in human knee at 3.0T. 
${ }^{1}$ Radiology, University of California, San Diego, San Diego, CA, United States, ${ }^{2}$ GE Healthcare, San Diego, CA, United States, ${ }^{3}$ Radiology Service, VA San Diego Healthcare System, San Diego, CA, United States

Ultrashort echo time (UTE) MRI is able to assess long T2 tissues such as articular cartilage (AC) and short T2 tissues such as meniscus. Early stage of osteoarthritis is hypothesized to affect the mechanical properties of AC, sooner and quicker than its morphology. This study focused on the application of UTE imaging, including UTE magnetization transfer (UTE-MT) modelling, adiabatic T1r, T1 and T2* measurements in cadaveric human knee joints subject to sequential mechanical loading. Compression load application resulted in significant increases in macromolecular fraction estimated in AC and meniscus, obtained by two-pool MT modeling. T1, T1 $\rho$ and T2* biomarkers did not show consistent trends.

Quantitative DCE-MRI perfusion imaging of the subchondral bone in knee osteoarthritis

Bas A. de Vries ${ }^{1}$, Joost Verschueren ${ }^{1}$, Dirk H.J. Poot ${ }^{2}$, Gabriel P. Krestin ${ }^{1}$, and Edwin H.G. Oei ${ }^{1}$

${ }^{1}$ Radiology \& Nuclear Medicine, Erasmus MC, Rotterdam, Netherlands, ${ }^{2}$ Medical Informatics, Erasmus MC, Rotterdam, Netherlands

Changes in subchondral bone in knee osteoarthritis could be a marker of altered fluid dynamics. Perfusion can be visualized and quantified with MRI using dynamic contrast enhanced MRI (DCE-MRI). Using quantitative analysis of DCE-MRI, we compared perfusion in the affected compartment with the non-affected compartment in patients with unicompartmental knee osteoarthritis. We also evaluated the perfusion in subchondral bone marrow lesions (BMLs). Perfusion of the subchondral bone measured with DCE-MRI is not significantly different between the affected and non-affected compartment. Subchondral BMLs are significantly associated with increased perfusion parameters compared to subchondral bone regions without BMLs.

Low-field MRI of osteoarthritis in humans: correlations between load-dependent cartilage properties and relaxation parameters

Erik Roessler ${ }^{1}$, Carlos Mattea ${ }^{1}$, Miika Nieminen ${ }^{2}$, Sakari Karhula ${ }^{2}$, Simo Saarakkala $^{2}$, and Siegfried Stapf ${ }^{1}$

${ }^{1}$ IImenau University of Technology, IImenau, Germany, ${ }^{2}$ University of Oulu, Oulu, Finland

At low magnetic fields, $T_{1}$ variation within cartilage is a robust parameter that is employed to quantify the layered structure in the tissue and is sensitive to factors such as enzymatic degradation, external load, and diseases such as osteoarthritis. Variable-field relaxometry provides access to the content and local order of glycosaminoglycans and collagen via protonnitrogen quadrupolar dips. In this study on 20 human cartilage samples, load-dependent low-field and variable-field techniques were combined for the first time to correlate NMR parameters with the severity of osteoarthritis.

Traditional Poster

\section{Muscle}

Exhibition Hall 1394-1411

Monday 8:15 - 10:15

Impact of Rate of Cuff Inflation on the Post-Ischemia Hyperemic Response

Rajiv S Deshpande ${ }^{1}$, Erin K Englund ${ }^{2}$, and Felix W Wehrli

${ }^{1}$ Department of Bioengineering, University of Pennsylvania, Philadelphia, PA, United States, ${ }^{2}$ Department of Radiology, University of Pennsylvania, Philadelphia, PA, United States

The ischemia-reperfusion paradigm can be used to evaluate skeletal muscle and peripheral vascular function. To induce ischemia, a cuff is inflated to a suprasystolic pressure, which leads to occlusion of the blood vessels, and reactive hyperemia results upon cuff deflation. This study was done to determine whether the rate at which the cuff inflates affects the hyperemic response. MRI data were acquired using the ischemia-reperfusion paradigm under slow and fast cuff inflation rates with PIVOT and projection velocity mapping in eight healthy subjects. The results suggest that there were no significant differences between hyperemic responses from slow and fast inflations.

Simultaneous magnetic resonance elastography of the supraspinatus and the trapezius muscles

Daiki Ito ${ }^{1,2,3}$, Tomokazu Numano ${ }^{1,3}$, Koichi Takamoto $^{4}$, Kazuyuki Mizuhara ${ }^{3,5}$, and Hisao Nishijo ${ }^{6}$

${ }^{1}$ Department of Radiological Sciences, Graduate School of Human Health Sciences, Tokyo Metropolitan University, Tokyo, Japan, ${ }^{2}$ Office of Radiation Technology, Keio University Hospital, Tokyo, Japan, ${ }^{3}$ Health Research Institute, National Institute of Advanced Industrial Science and Technology, Tsukuba, Japan, ${ }^{4}$ Department of Judo Neurophysiotherapy, Graduate School of Medicine and Pharmaceutical Sciences, University of Toyama, Toyama, Japan, ${ }^{5}$ Department of Mechanical Engineering, Tokyo Denki University, Tokyo, Japan, ${ }^{6}$ Department of System Emotional Science, Graduate School of Medicine and Pharmaceutical Sciences, University of Toyama, Toyama, Japan 
Palpation is difficult to distinguish stiffness of the supraspinatus and trapezius muscles. Magnetic resonance elastography (MRE) can measure stiffness of tissues quantitatively only if vibrations reach the tissues. We developed simultaneous MRE of the supraspinatus and trapezius muscles by adjusting the shape of a wave transducer and vibration frequency. MREs were performed using self-made wave transducer at $50-150 \mathrm{~Hz}$, with a $25 \mathrm{~Hz}$ step. Both wave images of the supraspinatus and trapezius muscles showed clear wave propagation at 50 and $75 \mathrm{~Hz}$. The results demonstrated that our techniques allow simultaneous MRE of the supraspinatus and trapezius muscles at $75 \mathrm{~Hz}$.

Multi-centric evaluation of stability of quantitative outcome measures in healthy calf muscles

Lara Schlaffke ${ }^{1,2,3}$, Alberto De Luca ${ }^{4}$, Louise Otto ${ }^{5}$, Robert Rehmann ${ }^{1}$, Marlena Rohm ${ }^{1}$, Jedrzej Burakiewicz ${ }^{3}$, Celine Baligand ${ }^{3}$, Jithsa Monte ${ }^{6}$, Chiel den Harder $^{6}$, Aart Nederveen $^{6}$, Hermien $\mathrm{Kan}^{3}$, and Martijn Froeling ${ }^{2}$

${ }^{1}$ Neurology, BG UK Bergmannsheil gGmbH, Bochum, Germany, ${ }^{2}$ Radiology, Universitiy Medical Center Utrecht, Utrecht, Netherlands, ${ }^{3} \mathrm{C} . J$. Gorter Center for High Field MRI, Leiden University Medical Center, Leiden, Netherlands, ${ }^{4}$ Image Science Institute, Universitiy Medical Center Utrecht, Utrecht, Netherlands, ${ }^{5}$ Brain Centre Rudolf Magnus, Neurology, Universitiy Medical Center Utrecht, Utrecht, Netherlands, ${ }^{6}$ Radiology, Academic Medical Center Amsterdam, Amsterdam, Netherlands

Clinical feasible, comparable muscle MR-techniques are crucial for monitoring disease progression and therapy in patients with neuromuscular diseases. We developed and evaluated a multi-modal quantitative MR protocol at 3T. Diffusion parameters, water T2 relaxation time and fat-fraction were measured and tested for temporal stability, multicenter reproducibility and covariate influence. Diffusion parameters stabilized after 15 minutes and were comparable between centers. Water T2 decreased $1 \mathrm{~ms}$ within 1 hour. In dorsal muscles fat-fraction increased slightly, due to a decrease in muscle size. Temporal stability of quantitative parameters was shown and showed that T2 decrease needs to be considered when planning protocols.

Exploring the Textural Differences between Diseased and Normal Muscle on T1 Weighted MRIs of the Mid-calf and Mid-thigh

Chang Tung Harold Yip ${ }^{1}$, Phua Hwee Tang ${ }^{2}$, and Kein Meng Wendy Liew ${ }^{3}$

${ }^{1}$ Duke-NUS Medical School, Singapore, Singapore, ${ }^{2}$ Department of Diagnoistic and Interventional Imaging, KK Women's And Children's Hospital, Singapore, Singapore, ${ }^{3}$ Paediatric Neurology, KK Women's and Children's Hospital, Singapore, Singapore

Textural analysis is a non-invasive objective method to characterize MRIs of subjects with muscular disorders. It has the potential to characterize muscle abnormalities that are not visible to the human eye. This allows the detection of muscle abnormalities earlier hence aiding early diagnosis and prognostication. This also allows textural analysis to be a potential quantitative outcome measure for clinical trials of drug treatments for muscular disorders. This study shows that the textural parameter entropy remains stable as age increases and can distinguish between diseased and normal muscle tissue.

Fully automatic segmentation of all lower body muscles from high resolution MRI using a two-step DCNN model

Anudeep Konda ${ }^{1}$, Katherine Crump ${ }^{1}$, Daniel Podlisny ${ }^{1}$, Craig H Meyer ${ }^{1}$, Silvia S Blemker ${ }^{1}$, Joe Hart ${ }^{1}$, and Xue Feng ${ }^{1}$

${ }^{1}$ Springbok, Inc., Charlottesville, VA, United States

Lower limb skeletal muscles play an essential role in athletic performance as wellas muscular health in patients with dystrophies. Quantitative mapping of all 35 lower body muscles from high resolution MRI has the potential to improve power and agility in athletes and assist the diagnosis and follow-up for certain musculardystrophies in medical applications. However, due to the weak contrast and insufficient boundary information, the accurate segmentation of each individual muscle is challenging. In this study we developed a fully automatic segmentation framework using a two-step DCNN model and showed accurate segmentation for all muscles.

Robust multi-atlas MRI segmentation with corrective learning for quantification of local quadriceps muscles inflammation changes during a longitudinal study in athletes

Hoai-Thu Nguyen ${ }^{1}$, Pierre Croisille ${ }^{1,2}$, Magalie Viallon ${ }^{1,2}$, Charles de Bourguignon $^{2}$, Rémi Grange $^{2}$, Sylvain Grange ${ }^{1,2}$, and Thomas Grenier ${ }^{3}$

${ }^{1}$ Univ Lyon, UJM-Saint-Etienne, INSA-Lyon, Université Claude Bernard Lyon 1, CNRS, Inserm, CREATIS UMR 5220, U1206, F-42023, Saint-Etienne, France, ${ }^{2}$ Department of Radiology, Centre Hospitalier Universitaire de Saint-Etienne, Université Jean-Monnet, Saint-Etienne, France, ${ }^{3}$ Univ Lyon, INSA-Lyon, Université Claude Bernard Lyon 1, UJM-Saint Etienne, CNRS, Inserm, CREATIS UMR 5220, U1206, F-69621, Villeurbanne, France

This study propose an improved automatic segmentation of longitudinal MRI dataset of mountain ultra-marathon runners' upper thighs acquired during the Tor des Géants 2014 by using a multi-atlas segmentation strategy with corrective learning with a small number of training set. Our highly accurate and robust segmentations allow us to locally study the inflammation of each quadriceps head induced by the extreme conditions of the race, a method that is of high interest to monitor the impact of eccentric efforts during the race, identify local physiopathology changes in patients, and benefits of eventual therapy or intervention. 
With the popularity of magnetic resonance technology in recent years, the detection rate of soft tissue tumors has been greatly improved. The soft tissue tumors in MR images show various signal intensity distribution in different modalities. This work investigated and evaluated the role of texture analysis on T2WI, DWI and delayed T1-enhanced images to characterize the soft tissue tumors, and then evaluate the textures by support vector machine classifiers (SVM) to differentiate benign and malignant soft tissue tumors. Results showed that the application of texture analysis in T2WI, DWI and T1-enhanced imaging is helpful to distinguish benign and malignant soft tissue tumors by SVM.

Measurement of skeletal muscle extraceullar volume $(\mathrm{ECV})$ in the healthy thigh: determination of the time to contrast equilibrium

Alex F Goodall ${ }^{1}$, Dr David A Broadbent ${ }^{1}$, Dr Raluca B Dumitru ${ }^{2,3}$, Prof David L Buckley ${ }^{4}$, Prof Maya Buch ${ }^{2,3}$, Dr Ai Lyn Tan ${ }^{2,3}$, and Dr John D Biglands ${ }^{1,2}$

${ }^{1}$ Department of Medical Physics \& Engineering, Leeds Teaching Hospitals NHS Trust, Leeds, United Kingdom, ${ }^{2}$ NIHR Leeds Biomedical Research Centre, Leeds, United Kingdom, ${ }^{3}$ Leeds Institute of Rheumatic and Musculoskeletal Medicine, University of Leeds, Leeds, United Kingdom, ${ }^{4}$ Department of Biomedical Imaging Science, University of Leeds, Leeds, United Kingdom

Five healthy volunteers were scanned at $3 \mathrm{~T}$ to determine the time to contrast equilibrium in skeletal leg muscle to establish whether extracellular volume (ECV) mapping is clinically feasible for skeletal muscle (as it has proved to be for myocardium). Time to contrast equilibrium was 13 minutes, and native T1 values were validated against the literature. It was also found that the difference in measurement of ECV using the aorta compared to the femoral artery was small. It is hoped that advancements in this technique could aid in the diagnosis and treatment of scleroderma patients with muscle involvement.

Multi-parametric MRI-based classification for generating muscle percentage index in muscular dystrophy

Aydin Eresen ${ }^{1}$, Noor E. Hafsa ${ }^{2}$, Lejla Alic ${ }^{2}$, Sharla M. Birch ${ }^{1}$, Jay F. Griffin ${ }^{1}$, Joe N. Kornegay ${ }^{1}$, and Jim X. Ji ${ }^{1,2}$

${ }^{1}$ Texas A\&M University, College Station, TX, United States, ${ }^{2}$ Texas A\&M University at Qatar, Doha, Qatar

Imaging biomarker for muscular dystrophies, such as muscle percentage index (MPI), successfully differentiates between healthy and dystrophic muscles. However, the current methods to generate this biomarker are not well defined and therefore lack robustness and reproducibility. This study imaged ten Golden Retriever Muscular Dystrophy (GRMD) pectineus-muscle samples at a 4.7T MRI scanner. To facilitate estimation of MPI and to validate the results, we use trichrome-stained histology images. These images were registered accurately to multiparametric quantitative MRI (qMRI). We use local gradient and texture information to classify qMRI into muscle and non-muscle with respective accuracies of 0.86 and 0.71 .

MRI characterization of skeletal muscles of two dystrophic mouse models

Ravneet Singh Vohra ${ }^{1}$, Joshua Park ${ }^{1}$, Philip Kramer ${ }^{1}$, David Marcinek ${ }^{1}$, Jeffrey Chamberlain ${ }^{2,3}$, and Donghoon Lee

${ }^{1}$ Department of Radiology, University of Washington, Seattle, WA, United States, ${ }^{2}$ Department of Neurology, University of Washington, Seattle, WA, United States, ${ }^{3}$ Senator Paul D. Wellstone Muscular Dystrophy Cooperative Research Center, University of Washington, Seattle, WA, United States

The $m d x$ mouse model is one of the most commonly used animal model for Duchenne muscular dystrophy (DMD). However, it has a milder phenotype compared to patients with DMD. Evidence has demonstrated the presence of genetic modifiers that lead to phenotypic variability even with an identical gene mutation in both human and animal models of muscular dystrophy. We performed multi-parametric, high resolution MRI to demonstrate severity of disease progression in dystrophic mouse models on two different genetic backgrounds.

Application of MR Elastography to Transvertebral Psoas Major Muscle

Tomokazu Numano $^{1,2}$, Daiki Ito ${ }^{1,2,3}$, Koichi Takamoto $^{4}$, Kazuyuki Mizuhara $^{5}$, and Hisao Nishijo ${ }^{6}$

${ }^{1}$ Department of Radiological Sciences, Graduate School of Human Health Sciences, Tokyo Metropolitan University, Tokyo, Japan, ${ }^{2}$ Health Research Institute, National Institute of Advanced Industrial Science and Technology, Tsukuba, Japan, ${ }^{3}$ Office of Radiation Technology, Keio University Hospital, Tokyo, Japan, ${ }^{4}$ Department of Judo Neurophysiotherapy, Graduate School of Medicine and Pharmaceutical Sciences, University of Toyama, Toyama, Japan, ${ }^{5}$ Mechanical Engineering, Tokyo Denki University, Tokyo, Japan, ${ }^{6}$ Department of System Emotional Science, Graduate School of Medicine and Pharmaceutical Sciences, University of Toyama, Toyama, Japan

The aim of the present work was to develop the vibration techniques for the psoas major muscle (PM) MR elastography (MRE). The results indicated that the PM well vibrated, due to transmission of vibration from the lumbar spine. These findings suggest that placement of a narrow vibration pad under the supine body, along the lumbar spine, would allow PM MRE. The present techniques for the PM MRE provide a quantitative diagnostic tool for LBP-associated changes in the muscles, since increased stiffness of the muscle due to continuous contraction is suggested to be an important cause of LBP. 
Martin Schwartz ${ }^{1,2}$, Günter Steidle ${ }^{1}$, Petros Martirosian ${ }^{1}$, Michael Erb $^{3}$, Bin Yang ${ }^{2}$, Klaus Scheffler $^{3,4}$, and Fritz Schick ${ }^{1}$

${ }^{1}$ Section on Experimental Radiology, University Hospital of Tübingen, Tübingen, Germany, ${ }^{2}$ Institute of Signal Processing and System Theory, University of Stuttgart, Stuttgart, Germany, ${ }^{3}$ Biomedical Magnetic Resonance, University Hospital of Tübingen, Tübingen, Germany, ${ }^{4}$ High-Field Magnetic Resonance Center, Max Planck Institute for Biological Cybernetics, Tübingen, Germany

Reliable assessment and analysis of spontaneous mechanical activities in musculature (SMAM) visible in repetitive DWI is a relatively new technique for non-invasive characterization of skeletal musculature. To correct for data corrupted by intentional contractions, a surface electromyography-based contraction state analysis was investigated to reject undesired DWI data. It is demonstrated that the presented method enables a more reliable quantification of SMAMs and improved spontaneous activity maps.

Validation of an Osirix Plugin for automatic fat infiltration measurements in Paraspinal muscles using T2 weighted images

Cristobal Arrieta ${ }^{1}$, Julio Urrutia ${ }^{2}$, Pablo Besa ${ }^{2}$, Ignacio Osorio ${ }^{1}$, Cristian Montalba ${ }^{1}$, Daniel Hasson ${ }^{3}$, Marcelo E Andia ${ }^{4}$, and Sergio Uribe ${ }^{4}$

${ }^{1}$ Biomedical Imaging Center, Pontificia Universidad Catolica de Chile, Santiago, Chile, ${ }^{2}$ Department of Orthopaedic Surgery, School of Medicine, Pontificia Universidad Catolica de Chile, Santiago, Chile, ${ }^{3}$ Department of Radiology, Universidad del Desarrollo, Santiago, Chile, ${ }^{4}$ Department of Radiology, School of Medicine, Pontificia Universidad Catolica de Chile, Santiago, Chile

Paraspinal muscle fat infiltration has been related with low back pain. This measurements are typically evaluated using T2w images, however, the accuracy of this method needs a proper validation, since inhomogeneities may produce severe signal changes. In this work, we developed and validated an OsiriX plugin which allows to segment infiltrated fat in T2w images. This tool also allowed us for validating the use of T2w images, considering Dixon fat images as gold-standard. To validate our plugin, we evaluated 5 cross sectional areas (L1S1) of 4 paraspinal muscle groups for T2w images of 37 patients. To validate T2w images, we analyzed 10 healthy volunteers and 10 patients. We found that T2w segmentation with our OsiriX plugin is a reliable and an accurate method to evaluate the fat infiltration in paraspinal muscles.

Ex vivo MRS evaluation of severe burn injury in mice shows metabolic changes in skeletal muscle

Leo L. Cheng ${ }^{1}$, Bailing Li ${ }^{1,2}$, Lindsey. A. Vandergrift ${ }^{1}$, Jiake Chai ${ }^{3}$, and Zhongcong $\mathrm{Xie}^{4}$

${ }^{1}$ Pathology, Massachusetts General Hospital, Boston, MA, United States, ${ }^{2}$ Burns and Plastic Surgery, First Affiliated Hospital of General Hospital of PLA, Beijing, China, ${ }^{3}$ Burn and Plastic surgery, First Affiliated Hospital of PLA General Hospital, Beijing, China, ${ }^{4}$ Anesthesia, Critical Care and Pain Medicine, Massachusetts General Hospital, Boston, MA, United States

Patients of severe burn injury often suffer from sepsis, which results in multiple organ failure and prolonged metabolic derangement, leading to higher mortality. Accurate measurements of burn injury-associated metabolic changes may provide the burn clinic with quantitative tools to assess patient status. We tested the efficacy of High-Resolution Magic Angle Spinning (HRMAS) magnetic resonance spectroscopy (MRS) in evaluation of tissue metabolic changes with mouse skeletal diaphragm and gastrocnemius muscles after burn injury. HRMAS measurements indicated that IMTG and plasma FFA levels were increased after severe burn injury, with more pronounced differences detected in diaphragm muscle than in gastrocnemius muscle.

Sensitivity of Quantitative Texture Metrics to Variations in Image Acquisition Parameters

Bruce Damon ${ }^{1}$, Yuan $\mathrm{Xie}^{2}, \mathrm{Ke} \mathrm{Li}^{1}$, Susan $\mathrm{Kroop}^{1}$, and Jane Park ${ }^{1}$

${ }^{1}$ Vanderbilt University School of Medicine, Nashville, TN, United States, ${ }^{2}$ Vanderbilt University, Nashville, TN, United States

The purpose of this study was to examine the dependence of a quantitative texture metric, the high gray level run length emphasis (HGRE) in T2-weighted images, on common variations in image acquisition parameters. We studied 13 muscle disease patients with quantitative fat/water MRI and contrast-based images. The ability of the HGRE was unaffected by image matrix size. We also measured the dependence of the regression parameters on TR and TE. The results support the use of quantitative texture analysis to study clinically acquired MR images in muscle disease patients.

Assessment of perfusion-metabolism matching in exercising muscle from dynamic contrast-enhanced MRI and T2 mapping

Gwenael Layec ${ }^{1}$, christopher Conlin², Jiawei Dong ${ }^{2}$, Stephen Decker ${ }^{3}$, Corey R Hart ${ }^{3}$, Nan $\mathrm{Hu}^{2}$, Mariya A Chadovich², Michelle A Mueller², Lillian Khor ${ }^{3}$, Christopher Hanrahan $^{2}$, Vivian $S L^{2}{ }^{2}$, and Jeff L Zhang ${ }^{2}$

${ }^{1}$ VA Medical Center GRECC 182, 1D23A 500 Foothill Drive, University of Utah, Salt Lake City, UT, United States, ${ }^{2}$ Radiology, University of Utah, Salt Lake City, UT, United States,

${ }^{3}$ University of Utah, Salt Lake City, UT, United States

Using an MR approach combining DCE-MRI and T2 mapping, this study revealed that unlike PAD patients, muscle tissue perfusion was tightly correlated to exercise-induced changes in R2 in the lower leg muscles of healthy individuals. These findings suggest Q/Met mismatch following exercise in the skeletal muscle of PAD patients. The combination of DCE-MRI and T2 mapping opens a new avenue of research to investigate perfusion-metabolism heterogeneity in normal physiological conditions and muscle-related pathologies. 
Effects of PDE5A inhibition on skeletal muscle $1 \mathrm{H} 2 \mathrm{O}$ T2 following an acute bout of downhill running and endurance training in dystrophic mice

Abhinandan Batra ${ }^{1}$, Ravneet Vohra ${ }^{2}$, Steve Chrzanowski ${ }^{1}$, Donovan J Lott ${ }^{1}$, Glenn A Walter ${ }^{1}$, Krista Vandenborne $^{1}$, and Sean C Forbes ${ }^{1}$

${ }^{1}$ University of Florida, Gainesville, FL, United States, ${ }^{2}$ University of Washington, Seattle, WA, United States

This study examined the effects of phosphodiesterase $5 \mathrm{~A}$ inhibition with sildenafil citrate on skeletal muscle ${ }^{1} \mathrm{H}_{2} \mathrm{O} \mathrm{T}_{2}$ in dystrophic mice ( $m d x$ ) following downhill running and during four weeks of low-intensity treadmill training. Skeletal muscle ${ }^{1} \mathrm{H}_{2} \mathrm{O} \mathrm{T}_{2}$ was measured from spectra acquired with a single voxel ${ }^{1} \mathrm{H}$-MRS STEAM sequence. Our findings showed less altered $\mathrm{T}_{2}$ after downhill running with sildenafil citrate treatment indicating less muscle damage and improved running performance during endurance training. Collectively, the results support the use of sildenafil citrate when combined with acute and chronic bouts of exercise as a potential therapeutic intervention in muscular dystrophies.

Multi-Parametric MRI characterization for damaged dystrophic muscle

Joshua Park ${ }^{1}$, Ravneet Vohra ${ }^{1}$, Jeffrey S Chamberlain ${ }^{2,3}$, and Donghoon Lee ${ }^{1}$

${ }^{1}$ Radiology, University of Washington, Seattle, WA, United States, ${ }^{2}$ Neurology, University of Washington, Seattle, WA, United States, ${ }^{3}$ Senator Paul D. Wellstone Muscular Dystrophy Cooperative Research Center, Seattle, WA, United States

Muscular dystrophy is a family of inherited diseases characterized by progressive muscle weakness that leads to muscle damage and wasting. Clinical measures of muscular dystrophy rely on surgical biopsy, which is invasive and limited. Magnetic resonance imaging (MRI) can provide valuable information pertaining to tissue characteristics of this disease noninvasively. We performed multi-parametric MRI to assess the changes due to muscle damage and subsequent recovery over 3 weeks starting at 12 weeks of age in disease affected mice. The differences observed through MRI measurements demonstrate MRI can be used effectively to track disease progression and responses to future therapy.

Traditional Poster

\section{MSK: Other}

A Prospective, Longitudinal Assessment of Adverse Local Tissue Reactions in Resurfacing Hip Arthroplasty Versus Primary Total Hip Arthroplasty

Jacqui C. Zhu ${ }^{1}$, Matthew F. Koff ${ }^{1}$, Bin Lin ${ }^{1}$, Kara Fields ${ }^{1}$, Danyal G. Nawabi ${ }^{1}$, Edwin Su ${ }^{1}$, Douglass Padgett ${ }^{1}$, and Hollis G. Potter ${ }^{1}$

${ }^{1}$ Hospital for Special Surgery, New York, NY, United States

The purpose of this prospective study was to compare the prevalence of magnetic resonance imaging detected adverse local tissue reactions (ALTRs) in metal-on-metal hip resurfacing arthroplasty (HRA) and ceramic-on-poly (COP) total hip arthroplasty subjects. Images acquired at 4 time points with a 1-year interval showed a higher prevalence of ALTRs in the HRA than COP subjects. The self-assessed symptomatology scores did not significantly differ between the two groups at follow-up, indicating that ATLRs can be clinically silent. This study will permit better understanding of the natural history and follow up of ALTRs complicating hip arthroplasty.

Dynamic contrast enhanced MR imaging in early stage knee osteoarthritis: A test-retest repeatability study

Faezeh Sanaei Nezhad ${ }^{1,2}$, James MacKay ${ }^{3}$, Josh Kaggie ${ }^{3}$, Martin Graves ${ }^{3}$, Fiona Gilbert ${ }^{3}$, Andrew McCaskie ${ }^{4}$, Rob Janiczek ${ }^{5}$, Geoff JM Parker ${ }^{1,2}$, Alexandra R Morgan $^{5}$, and Jose Ulloa $^{1,2}$

${ }^{1}$ Bioxydyn, Manchester, United Kingdom, ${ }^{2}$ Centre for Imaging Sciences, University of Manchester, Manchester, United Kingdom, ${ }^{3}$ Department of Radiology, University of Cambridge, Cambridge, United Kingdom, ${ }^{4}$ Department of Trauma \& Orthopaedics, University of Cambridge, Cambridge, United Kingdom, ${ }^{5} \mathrm{GSK}$, Stevenage, United Kingdom

Dynamic contrast-enhanced MRI (DCE-MRI) has proven to be an effective method for qualitative and quantitative measurement of synovitis in the knee. Here we evaluate the test-retest repeatability of DCE-MRI measurements in the knee at $3 \mathrm{~T}$. Eight patients with mild/moderate knee osteoarthritis (OA) were scanned twice, 4 weeks apart. DCE biomarkers from the extended Tofts model were measured. This is the first demonstration of the repeatability of DCE-MRI in knee OA. This evaluation provides data to enable sample size calculations for further longitudinal and interventional studies using DCE-MRI as a biomarker of inflammation in OA. 
${ }^{1}$ Oxford Centre for Clinical Magnetic Resonance Research (OCMR), Division of Cardiovascular Medicine, Radcliffe Department of Medicine, University of Oxford, Oxford, United Kingdom

Tendon exhibits changes in $\mathrm{T}_{2}, \mathrm{~T}_{2}{ }^{*}$, and resonant frequency as a function of its orientation with respect to $\mathrm{B}_{0}$. An ultrashort echo time (UTE) sequence was employed to characterize the frequency of fresh bovine digital flexor tendon at angles of $0^{\circ}$ to $90^{\circ}$ relative to $B_{0}$, causing a maximal frequency shift of $1.0 \mathrm{ppm}$. Factors that could influence the frequency of tendon were evaluated. It was found that the frequency of tendon was affected by the enclosing container, but not the geometry of the tendon.

Cartilage and Meniscus T2 Relaxation Time in Subjects With and Without Meniscus Tears

Richard Kijowski ${ }^{1}$, Shivhumar Kambhampati ${ }^{1}$, Joshua Bunting ${ }^{1}$, Benjamin Beduhn ${ }^{1}$, Kaitlin Woo ${ }^{1}$, and Fang Liu ${ }^{1}$

${ }^{1}$ Department of Radiology, University of Wisconsin-Madison, Madison, WI, United States

This study was performed to compare cartilage T2 between subjects with and without meniscus tears. T2 mapping was performed on the knees of 30 control subjects without meniscus tears and 93 subjects with meniscus tears. Medial and lateral compartment cartilage T2 was measured. Radiographic osteoarthritis severity was assessed using the Kellgren-Lawrence $(\mathrm{KL})$ grading scale. The $30 \mathrm{KL}-0$ control subjects without meniscus tears had significantly lower $(\mathrm{p}<0.001)$ medial compartment cartilage $T 2$ than $\mathrm{KL}-0$ ( $n=46$ ), $\mathrm{KL} 1$ ( $n=27$ ), and $\mathrm{KL}-2$ $(n=20)$ subjects with meniscus tears and significantly lower $(p<0.01)$ lateral compartment cartilage $T 2$ than $K L-1$ and $K L-2$ subjects with meniscus tears.

Accuracy of MRI-based measurements of aponeurosis dimensions

Lachlan Bird ${ }^{1,2}$, Arkiev D'Souza $^{1,3}$, lain Ball ${ }^{4}$, Caroline Rae ${ }^{1,3}$, Robert Herbert $^{1,3}$, and Bart Bolsterlee ${ }^{1,3}$

${ }^{1}$ Neuroscience Research Australia, Randwick, Australia, ${ }^{2}$ Sydney University, Camperdown, Australia, ${ }^{3}$ University of New South Wales, Kensington, Australia, ${ }^{4}$ Philips Electronics Australia, Sydney, Australia

Aponeuroses are the thin, sheet-like tendons that cover substantial parts of muscles. We validated measurements of the dimensions of aponeuroses from T1, mDixon and ultrashort echo time (UTE) scans by comparing to direct measurements from dissection and digitisation. We used sequences that are feasible for human studies. Aponeurosis widths and lengths, measured on 20 lamb muscles, were substantially underestimated from mDixon scans. More accurate measurements were obtained from T1 and UTE scans, which had root mean square errors of $8-10 \%$ and $5-13 \%$ of the aponeurosis width and length, respectively, and did not systematically underestimate or overestimate aponeurosis width or length.

Elevated conversion of hyperpolarized [1-13C]pyruvate to [1-13C]lactate is not associated with tissue acidosis, as measured with hyperpolarized [13C]bicarbonate, in a murine model of rheumatoid arthritis.

Alan J. Wright ${ }^{1}$, Zoé M. A. Husson ${ }^{2}$, De-en $\mathrm{Hu}^{1}$, Gerard Callejo ${ }^{2}$, Kevin M. Brindle ${ }^{1,3}$, and Ewan St. John Smith ${ }^{2}$

${ }^{1}$ CRUK Cambridge Institute, University of Cambridge, Cambridge, United Kingdom, ${ }^{2}$ Department of Pharmacology, University of Cambridge, Cambridge, United Kingdom, ${ }^{3}$ Department of Biochemistry, University of Cambridge, Cambridge, United Kingdom

Measurements of synovial fluid $\mathrm{pH}$ in patients with rheumatoid arthritis suggest acidosis can occur at inflamed joints. A widely used model of rheumatoid arthritis is produced by injecting complete Freund's adjuvant into the hind paw of a mouse. We have investigated whether inflammation is associated with acidosis in this model using Magnetic Resonance Spectroscopic Imaging of injected hyperpolarised [1-13C]pyruvate, to detect the metabolic changes associated with inflammation, and hyperpolarised [13C]bicarbonate to measure extracellular $\mathrm{pH}$. A significant increase in the [1-13C]lactate/[1-13C]pyruvate was observed throughout the inflamed tissue, but there is no apparent acidosis

Is the anterolateral ligament affected by the rupture of anterior cruciate ligament? A tentative investigation based on magnetic resonance imaging

qian wang ${ }^{1}$, Cuiping Ren ${ }^{1}$, Jingliang Cheng ${ }^{1}$, and Zhizheng Zhuo ${ }^{2}$

${ }^{1}$ The First Affiliated Hospital of Zhengzhou University, Zhengzhou, China, ${ }^{2}$ Clinical Science, Philips Healthcare, Beijing, China

This study aimed to demonstrate the incidence of injured of ALL following ACL rupture, as well as observe the characteristics of thus injury based on MRI. In the study, we used the high resolution 3D TSE-based sequences including the optimized T1W-VISTA and T1W-VISTA-SPAIR to evaluate the 43 knees of patients who have ligament ruptured through clinical test. Chi-square test was performed to analyze the categorical variables. Binary logistic regression was performed to investigate the main cause. It indicated that $\mathrm{ACL}$ injuries has closer association with $A C L$ injuries but less association with LM injuries, and the femoral portions of ALL were easily ruptered 
${ }^{1}$ The First Affiliated Hospital of Zhengzhou University, Zhengzhou, China, ${ }^{2}$ Clinical Science, Philips Healthcare, Beijing, China

This study aimed to demonstrate the feasibility of optimized 3D high resolution MR imaging for scanning anterolaterl ligament, as well as provide more accurate imaging technique for patient with ACL and ALL injured. In the study, we used the high resolution 3D TSE-based sequences including the optimized T1W-VISTA, PDW-VISTA, and T1W-VISTA-SPAIR to evaluate the 60 knees of thirty healthy volunteers. There was significant difference between the three techniques for both the radiologists, and there was high consistency between the scores of two radiologists. 3D T1W-VISTA imaging technique has a high superiority in the three techniques, which may provide more information for clinical diagnosis.

A machine learning method for tissue characterisation in the human thigh

Terence Jones ${ }^{1,2}$, Sarah Wayte ${ }^{3}$, Abhir Bhalerao ${ }^{4}$, Nicola Gullick ${ }^{5}$, and Charles Edward Hutchinson ${ }^{1,2}$

${ }^{1}$ Medical School, University of Warwick, Coventry, United Kingdom, ${ }^{2}$ Radiology, University Hospitals Coventry \& Warwickshire NHS Trust, Coventry, United Kingdom, ${ }^{3}$ Medical Physics, University Hospitals Coventry \& Warwickshire NHS Trust, Coventry, United Kingdom, ${ }^{4}$ Computer Science, University of Warwick, Coventry, United Kingdom, ${ }^{5}$ Department of Rheumatology, University Hospitals Coventry \& Warwickshire NHS Trust, Coventry, United Kingdom

Inflammatory idiopathic myositis is a debilitating inflammatory muscle condition. Diagnosis relies on a battery of tests, but monitoring of disease severity can be challenging. We present a novel machine learning approach to classifying tissues using multi-parametric analysis of routine MRI sequences. A logistic regression model was trained to predict tissue type based on T1 and STIR signal intensity and 10-fold cross-validated. The system attained $93.8 \%$ sensitivity and $96.9 \%$ specificity overall (ROC area 0.991 ). Testing of this model showed a low level of ostensible muscle inflammation in 9/11 asymptomatic controls - likely due to misclassification of vessels.

Usefulness of PETRA imaging for frozen shoulder patients

Ryuji Nojiri ${ }^{1}$, Yasuaki Tsurushima ${ }^{1}$, Hiroko Fukushima ${ }^{1}$, Masaaki Hori ${ }^{2}$, Murata Katsutoshi ${ }^{3}$, Nobuhisa Shinozaki ${ }^{4}$, Yasui Kenji ${ }^{5}$, Kazuhiro Maeda ${ }^{5}$, and Ken Okazaki ${ }^{5}$

${ }^{1}$ Radiology, Tokyo medical clinic, Tokyo, Japan, ${ }^{2}$ Radiology, Jyuntendou University Hospital, Tokyo, Japan, ${ }^{3}$ SIEMENS Healthcare Co., Tokyo, Japan, ${ }^{4}$ Orthopedics, Tokyo-kita medical center, Tokyo, Japan, ${ }^{5}$ Orthopedics, Tokyo Women's Medical University, Tokyo, Japan

Pointwise encoding time reduction with radial acquisition (PETRA) has made it possible to visualize those tissues which have a short T2* value such as ligaments and tendons as high signal images by using ultra-short echo time (TE). In this study, we evaluated the significant difference of the thickness of the joint capsule in the axillary pouch, depending on the stage or the symptom of patients with frozen shoulder.

MRI Cytography: a biomarker of microstructural myofiber damage in Amyotrophic Lateral Sclerosis

Natenael B Semmineh ${ }^{1}$, Alberto Fuentes ${ }^{1}$, David Medina ${ }^{1}$, Rachael Sirianni ${ }^{1}$, and C Chad Quarles ${ }^{1}$

${ }^{1}$ Barrow Neurological Institute, Phoenix, AZ, United States

For patients diagnosed with Amyotrophic Lateral Sclerosis (ALS),

the clinical heterogeneity of disease presentation and progression continues to confound the identification of robust outcome measures and biomarkers that can be used as surrogates of $p$ making during clinical trials. To overcome this limitation we developed a non-invasive imaging strategy, termed MRI Cytography (MRC) that is uniquely sensitive to abnormal muscle cytoarı able to reliably differentiate between normal and degenerated muscle microstructure.

Preliminary study of BOLD fMRI for the differentiation of musculoskeletal benign and malignant tumors

Nan Sun ${ }^{1}$, Cuiping Ren ${ }^{1}$, Ying $\mathrm{Li}^{1}$, Jingliang Cheng ${ }^{1}$, and Zhizheng Zhuo ${ }^{2}$

${ }^{1}$ Dept. of MRI, The First Affiliated Hospital of Zhengzhou University, Zhengzhou, China, ${ }^{2}$ Philips Healthcare, Beijing, China

This work investigated and evaluated the role of Blood Oxygenation Level-Dependent (BOLD) based functional MRI in characterizing the musculoskeletal tumors, and furtherly evaluate the ability of the power calculated from the fMRI time series to differentiate benign and malignant tumors, which might be helpful for clinical diagnosis and studies. 
${ }^{1}$ Imaging and Interventional Radiology, The Chinese University of Hong Kong, Hong Kong, Hong Kong, ${ }^{2}$ Jockey Club Centre for Osteoporosis Care and Control, The Chinese University of Hong Kong, Hong Kong, Hong Kong, ${ }^{3}$ Medicine and Therapeutics, The Chinese University of Hong Kong, Hong Kong, Hong Kong

This study investigated the correlation between MRI parameters and clinical assessment in 106 treatment naïve patients presenting with early rheumatoid arthritis (ERA) i.e. symptoms < 24 months. The degree of synovial and tenosynovial proliferation, bone marrow oedema and bone erosions were semi-quantitatively and quantitatively measured on MR imaging Quantitative MRI parameters showed better correlation with clinical assessment than semi-quantitative methods. Only quantitative MRI methods showed significant change after treatment for one year.

MR based changes in normal ACL hamstring graft over two years following reconstruction

Fan Xiao ${ }^{1}$, Jacky Ka Long Ko ${ }^{1}$, Alex Wing Hung $\mathrm{Ng}^{1}$, Jason Chi Shun Leung ${ }^{2}$, David Ka Wai Yeung ${ }^{1}$, Patrick, Shu Hang Yung ${ }^{3}$, and James Griffith ${ }^{1}$

${ }^{1}$ Imaging and Interventional Radiology, The Chinese University of Hong Kong, Hong Kong, Hong Kong, ${ }^{2}$ Jockey Club Centre for Osteoporosis Care and Control, The Chinese University of Hong Kong, Hong Kong, Hong Kong, ${ }^{3}$ Orthopaedics and Traumatology, The Chinese University of Hong Kong, Hong Kong, Hong Kong

This study investigates that normal changes seen on MRI of the ACL graft over first two years after reconstruction. The graft and perigraft tissues were assessed on serial MRI examinations addressing features such as graft size, signal intensity and perfusion. MR changes were compatible with the histological process known as changes in the ACL graft, usually called 'ligamentization of the graft' seems to have stabilized by 24 months.

Anisotropic analysis and decay characteristics of T2* relaxation of the human Achilles tendon studied with $7 \mathrm{~T}$ MR-microscopy

Benedikt Hager ${ }^{1,2}$, Vladimir Juras ${ }^{1,2,3}$, Martin Zalaudek ${ }^{1,2}$, Joachim Friske $^{1,2}$, Xeni Deligianni $^{4}$, Oliver Bieri ${ }^{4}$, Lena Hirtler ${ }^{5}$, Andreas Berg ${ }^{6}$, Markus Schreiner $^{1,7}$, Sonja Walzer $^{7}$, and Siegfried Trattnig ${ }^{1,2}$

${ }^{1}$ High-Field MR Centre, Department of Biomedical Imaging and Image-guided Therapy, Medical University of Vienna, Vienna, Austria, ${ }^{2}$ Christian Doppler Laboratory for Clinical Molecular MR Imaging, Vienna, Austria, ${ }^{3}$ Department of Imaging Methods, Institute of Measurement Science, Bratislava, Slovakia, ${ }^{4}$ Division of Radiological Physics, Department of Radiology, University of Basel Hospital, Basel, Switzerland, ${ }^{5}$ Center for Anatomy and Cell Biology, Department for Systematic Anatomy, Medical University of Vienna, Vienna, Austria, ${ }^{6}$ Center for Medical Physics and Biomedical Engineering, Medical University of Vienna, Vienna, Austria, ${ }^{7}$ Department of Orthopaedic Surgery, Medical University of Vienna, Vienna, Austria

The fiber-to-field angle dependence and the $\mathrm{T}_{2}{ }^{*}$ characteristics of a human Achilles tendon were investigated. The results show an increase of approx. factor 20 in $\mathrm{T}_{2}{ }^{*}$ values when the long axis of the tendon is change from $0^{\circ}$ to $55^{\circ}$, which is much higher than previously reported. Moreover, in contrast to previous findings we found no homogenous biexponential decay behavior for the tendon on a small sized voxel basis. The results reported here are to our knowledge the first MR-microscopy evaluations of the orientational dependence of $\mathrm{T}_{2}{ }^{*}$ relaxation in the Achilles tendon.

MRI Methods for Exercise-based Perfusion Assessment of Diabetic Feet with Ulcers

Masoud A Edalati ${ }^{1}$, Mary K Hastings ${ }^{1}$, Zayed Mohamed ${ }^{1}$, David Muccigrosso ${ }^{1}$, Ran Li $^{1}$, Michael J Mueller ${ }^{1}$, and Jie Zheng

${ }^{1}$ Washington Univesity in St Louis, Saint Louis, MO, United States

The purpose of this study was to develop MRI methods for comprehensive evaluation of foot muscle perfusion and perfusion reserve in patients with diabetes and foot ulcers. Healthy controls and patients with diabetic foot ulcers were scanned with a non-contrast MRI protocol at rest and during a standardized foot flexion exercise. Ischemic regions around foot ulcers were clearly identified with quantitative perfusion data during the exercise.

T1p, T2, and RAFF are Sensitive to Acute Ischemic Injury to the Femoral Head in a Piglet Model of Legg-Calvé-Perthes Disease

Casey P. Johnson ${ }^{1,2}$, Cathy S. Carlson ${ }^{3}$, Ferenc Toth ${ }^{3}$, Harry K. W. Kim ${ }^{4,5}$, and Jutta M. Ellermann ${ }^{1,2}$

${ }^{1}$ Center for Magnetic Resonance Research, University of Minnesota, Minneapolis, MN, United States, ${ }^{2}$ Radiology, University of Minnesota, Minneapolis, MN, United States, ${ }^{3}$ Veterinary Population Medicine, University of Minnesota, Saint Paul, MN, United States, ${ }^{4}$ Texas Scottish Rite Hospital for Children, Dallas, TX, United States, ${ }^{5}$ Orthopaedic Surgery, UT Southwestern Medical Center, Dallas, TX, United States

We demonstrate that quantitative $\mathrm{T} 1 \rho, \mathrm{T} 2$, and RAFF relaxation time maps are highly sensitive to bone/marrow and cartilage changes within 48 hours following ischemic injury to the growing femoral head. This work has important implications for the diagnosis and treatment of diseases associated with avascular necrosis of bone and cartilage. 
Darryl B Sneag ${ }^{1}$, Jacqui C Zhu ${ }^{1}$, Susan Lee ${ }^{1}$, Tina Jeon ${ }^{1}$, Bin Lin ${ }^{1}$, and Maggie M Fung ${ }^{2}$

${ }^{1}$ Radiology, Hospital of Special Surgery, New York, NY, United States, ${ }^{2}$ Applications \& Workflow, GE Healthcare, New York, NY, United States

This study's purpose was to compare non-respiratory and respiratory- triggered proton density and T2-weighted DIXON fat suppression sequences for high-resolution brachial plexus MRI. In a cohort of 5 volunteers and 20 patients, we were able to demonstrate that respiratory triggering substantially reduced ghosting artifact and improved delineation of nerve fascicular architecture with acceptable increased scan time.

Advanced Knee Imaging Study in NCAA Division 1 Basketball: Protocol Development and Preliminary Results

Katherine A Young ${ }^{1}$, Feliks Kogan ${ }^{1}$, Robert D Peters ${ }^{2}$, Matthew F. Koff ${ }^{3}$, Valentina Pedoia ${ }^{4}$, Marc Safran ${ }^{5}$, Ben Ma ${ }^{4}$, Riley Williams ${ }^{3}$, Tom Wickiewicz ${ }^{3}$, Marianne S Black ${ }^{1}$, John M Sabol ${ }^{2}$, Kimberly K. Amrami ${ }^{6}$, Hollis Potter ${ }^{3}$, Sharmila Majumdar ${ }^{4}$, and Garry Gold ${ }^{1}$

${ }^{1}$ Radiology, Stanford, Stanford, CA, United States, ${ }^{2}$ GE Healthcare, Waukesha, WI, United States, ${ }^{3}$ Hospital of Special Surgeries, New York City, NY, United States, ${ }^{4}$ University of California San Francisco, San Francisco, CA, United States, ${ }^{5}$ Stanford, Redwood City, CA, United States, ${ }^{6}$ Radiology, Mayo Clinic, Rochester, MN, United States

Chronic knee injuries are especially common in jumping athletes, and in particular high-level basketball players. In this work, we developed an advanced quantitative MRI protocol to longitudinally study early degenerative changes in high-level basketball players across multiple sites. Studying these changes, between high and low impact athletes, within one season as well as over three seasons for a cumulative effect, will help provide better insight into these changes. In developing this protocol for a multi-center study, we use a common phantom to assess biases in quantitative measurements across study scanners.

Ultra-short echo-time (UTE) imaging of the knee with curved surface reconstruction-based extraction of the patellar tendon

Martin Krämer ${ }^{1}$, Marta B Maggioni ${ }^{1}$, Christoph von Tycowicz ${ }^{2}$, Nick Brisson ${ }^{3}$, Stefan Zachow ${ }^{2}$, Georg N Duda ${ }^{3}$, and Jürgen R Reichenbach ${ }^{1,4,5,6}$

${ }^{1}$ Medical Physics Group, Institute of Diagnostic and Interventional Radiology, Jena University Hospital - Friedrich Schiller University Jena, Jena, Germany, ${ }^{2}$ Zuse Institute Berlin, Berlin, Germany, ${ }^{3}$ Julius Wolff Institute and Center for Musculoskeletal Surgery, Charité - Universitätsmedizin Berlin, Berlin, Germany, ${ }^{4}$ Michael Stifel Center for Data-driven and Simulation Science Jena, Friedrich Schiller University Jena, Jena, Germany, ${ }^{5}$ Abbe School of Photonics, Friedrich Schiller University Jena, Jena, Germany, ${ }^{6}$ Center of Medical Optics and Photonics, Friedrich Schiller University Jena, Jena, Germany

Due to very short $T_{2}$ relaxation times, imaging of tendons is typically performed using ultra-short echo-time (UTE) acquisition techniques. In this work, we combined an echo-train shifted multi-echo 3D UTE imaging sequence with a 3D curved surface reconstruction to virtually extract the patellar tendon from an acquired 3D UTE dataset. Based on the analysis of the acquired multi-echo data, a $\mathrm{T}_{2}{ }^{*}$ relaxation time parameter map was calculated and interpolated to the curved surface of the patellar tendon.

Analysis of collagen fibrillogenesis of a caprine patella tendon with magic angle imaging

Karyn Elizabeth Chappell ${ }^{1}$, Catherine Van Der Straeten ${ }^{1}$, Donald McRobbie ${ }^{2}$, Wladyslaw Gedroyc ${ }^{1}$, Mihailo Ristic ${ }^{3}$, and Djordje Brujic ${ }^{3}$

${ }^{1}$ Medicine, Surgery and Cancer, Imperial College London, London, United Kingdom, ${ }^{2}$ University of Adelaide, Adelaide, Australia, ${ }^{3}$ Mechanical Engineering, Imperial College London, London, United Kingdom

It is known that our collagen fiber alignment changes as we develop, reach maturity and then age: the crosslinking of collagen is considered one of the best biomarkers of aging. This study used magic angle imaging to visualise the collagen fiber changes between development and skeletal maturity in caprine knees. Immature tendons are less aligned during development, becoming more aligned as skeletal maturity is reached. This method has great potential to non-invasively improve our understanding of the development and degeneration of collagen rich structures.

Feasibility of monosodium urate assessment using multi-echo gradient echo based quantitative imaging

Seung hee Han $^{1}$, Yoonho Nam ${ }^{1}$, Joon-Yong Jung ${ }^{1}$, and Won-Hee Jees ${ }^{1}$

1433 'Seoul St.Mary's Hospital, College of Medicine, The Catholic University of Korea, Seoul, Republic of Korea

Gout is a common disease caused by monosodium urate (MSU) accumulation in joints. Although conventional MR imaging well describes generic features of inflammation, sensitivity of MSU is relatively low compared to dual energy CT. Because MSU has diamagnetic susceptibility, high sensitivity can be expected in magnetic susceptibility related contrast imaging. However, calcium is another diamagnetic material existing in joints. Therefore, distinguishing MSU and calcium is an essential step for imaging MSU. In this context, we investigate the feasibility of multi-echo gradient echo based quantitative imaging for MSU assessment. 
Akshaykumar Nana Kamble ${ }^{1}$ and Gaurav Gangavani ${ }^{2}$

${ }^{1}$ Radio-diagnosis, Institute of Nuclear Medicine and Allied Sciences (INMAS), Delhi, India, ${ }^{2}$ Sir Ganga Ram Hospital, New Delhi, India

SWI has been used for detection of calcification and hemosiderin deposits in diagnosis of the neurological disorders, hemorrhagic disorders and neuroinfectious conditions. Our study tries to answer the question that whether the susceptibility weighted MR imaging can provide alternative to the CT scan and thus decreasing our dependency on the modality which has significant drawback of having radiation dose especially to our young patients. We compared SWi and CT for the characterization of lesion calcification and hemorrhage and we found there was no significant difference in detection rate of these characteristics between two modalities, thus proving SWI as equally sensitive.

T1 and T2 Mapping of Delayed Gadolinium Enhancement in Osteoarthritis with MR Fingerprinting

Joshua D Kaggie ${ }^{1,2}$, James MacKay ${ }^{1,2}$, Guido Buonincontri ${ }^{3}$, Fiona J Gilbert ${ }^{1,2}$, Rolf F Schulte ${ }^{4}$, Alexandra R Morgan ${ }^{5}$, Robert L Janiczek ${ }^{5}$, Michela Tosetti ${ }^{3}$, Andrew McCaskie ${ }^{2,6}$, and Martin J Graves ${ }^{1,2}$

${ }^{1}$ Radiology, University of Cambridge, Cambridge, United Kingdom, ${ }^{2}$ Addenbrooke's Hospital, Cambridge University Hospitals NHS Foundation Trust, Cambridge, United Kingdom, ${ }^{3}$ IRCCS Stella Maris and IMAGO7 Foundation, Pisa, United Kingdom, ${ }^{4}$ GE Healthcare, Munich, Germany, ${ }^{5}$ Experimental Medicine Imaging, GlaxoSmithKline, London, United Kingdom, ${ }^{6}$ Division of Trauma and Orthopaedic Surgery, University of Cambridge, Cambridge, United Kingdom

Mapping of quantitative MRI relaxation values is promising for improving the assessment of MSK disease. Magnetic Resonance Fingerprinting (MRF) is a new method that enables fast quantitative MRI by exploiting the transient signals caused by the variation of pseudorandom sequence parameters.

This proof-of-concept work demonstrates the utility of MR Fingerprinting in the knee. Seven participants, four of which had Kellgren-Lawrence (KL) grade 2 or 3 , were imaged eighty minutes after gadolinium injection with MRF on a 3.0T MRI. The mean T1 relaxation times were shorter in cartilage by $5-20 \%$ in $\mathrm{KL}=2,3$ subjects when compared to normal subjects.

Significant Metabolic Differences Between Benign Lipomatous Lesion and Liposarcoma Identified by High-Resolution 1H and 31P MRS: A Pilot Study

Santosh Kumar Bharti ${ }^{1}$, Brett Shannon ${ }^{2}$, Adam Levin $^{2}$, Carol D Morris ${ }^{2}$, Laura Fayad ${ }^{3}$, and Zaver M Bhujwalla $a^{1,4}$

${ }^{1}$ Division of Cancer Imaging Research, Department of Radiology, Johns Hopkins University, School of Medicine, Baltimore, MD, United States, ${ }^{2}$ Department of Orthopaedic Surgery, Johns Hopkins University, School of Medicine, Baltimore, MD, United States, ${ }^{3}$ Musculoskeletal Radiology, Department of Radiology, Johns Hopkins University, School of Medicine, Baltimore, MD, United States, ${ }^{4}$ Department of Oncology, Johns Hopkins University, School of Medicine, Baltimore, MD, United States

Adipocytic tumors present a spectrum of neoplastic disease including benign lipomas and their variants, atypical lipomatous tumors, and malignant liposarcomas. Distinguishing areas of malignant dedifferentiation from benign and atypical lipomatous tumors is a diagnostic challenge due to overlapping magnetic resonance imaging characteristics, and pre-operative diagnostic accuracy is poor. Here we have identified dramatic differences in the metabolic profile of water-soluble and lipid extracts of adipocytic tumors, suggesting that magnetic resonsance spectroscopy may have the potential to improve diagnostic accuracy. Our data may also lead to potential metabolic targets for treatment.

Automated Seed Points Selection Based Radial-Search Segmentation Method For Sagittal and Coronal View Knee MRI Imaging

Sandeep Panwar Jogi ${ }^{1,2}$, Rafeek T. ${ }^{1}$, Sriram Rajan ${ }^{3}$, Krithika Rangarajan $^{3}$, Anup Singh ${ }^{1}$, and Amit Mehndiratta ${ }^{1}$

${ }^{1}$ Centre of Biomedical Engineering, Indian Institute of Technology Delhi, New Delhi, India, ${ }^{2}$ BME, ASET, Amity University Haryana, Gurgaon, India, ${ }^{3}$ Mahajan Imaging Centre, New Delhi, India

Knee disorders are generally marked in tibio-femoral bone junction. Most of available segmentation techniques use time consuming semi-automatic approach as radial search method, in sagittal view only. However, coronal view MRI Knee images are clinically equal important. Proposed approach automates seed points selection process for the radial search method, which work equally good on both sagittal and coronal view for identification of tibio-femoral junction.

Traditional Poster

\section{Bone}


We investigated whether chemical shift imaging (CSI) is useful for differentiating multiple myeloma infiltration from hematopoietic bone marrow and for quantitatively assessing disease severity. For those myeloma patients with relatively high cellularity in the bone marrow, a lower signal drop on oppose phase images indicated a higher tumor burden. For bone marrow with relatively low cellularity, disease severity was not reflected on CSI. CSI did not prove useful for differentiating myeloma infiltration from hematopoietic bone marrow, which implies that differentiation between regrowth of hematopoietic bone marrow and minimal residual disease or relapse after chemotherapy might be difficult with CSI.

Towards Whole-Skeleton Fat Fraction Mapping: The Impact of Parallel Imaging

Vruti Dattani ${ }^{1}$, Tim Bray², Alan Bainbridge ${ }^{3}$, and Margaret A Hall-Craggs ${ }^{2}$

${ }^{1}$ Royal Free Hospital, London, United Kingdom, ${ }^{2}$ Centre for Medical Imaging, University College London, London, United Kingdom, ${ }^{3}$ Department of Medical Physics, University College London Hospitals, London, United Kingdom

Whole body MRI (WB-MRI) is increasingly used to image the skeleton in haematological diseases such as multiple myeloma (MM) and inflammatory disorders such as spondyloarthritis. WB-MRI can be used to acquire fat fraction (FF) maps, which can assess disease severity and treatment response. However, patients with bone pain find it difficult to lie in the scanner for long periods, necessitating the use of parallel imaging to accelerate the acquisition. The aim of this study was to determine the extent to which parallel imaging causes noise artifacts and fat-water swaps in FF maps, and to assess their impact on FF measurements.

Fat Fraction Thresholds for Defining Bone Marrow Edema and Fat Metaplasia in Spondyloarthritis: More Objective than 'A Tiny Bit of White'

Timothy J P Bray ${ }^{1,2}$, Alan Bainbridge ${ }^{3}$, Corinne Fisher ${ }^{2}$, Debajit Sen ${ }^{2}$, and Margaret A Hall-Craggs ${ }^{1,2}$

${ }^{1}$ Centre for Medical Imaging, University College London, London, United Kingdom, ${ }^{2}$ Arthritis Research UK Centre for Adolescent Rheumatology, University College London, London, United Kingdom, ${ }^{3}$ Department of Medical Physics, University College London Hospitals, London, United Kingdom

MRI is now widely used to diagnose spondyloarthritis, but existing methods for image analysis rely on qualitative visual analysis by radiologists, and suffer from poor reproducibility between observers. Here, we show that proton density fat fraction (PDFF) measurements can be used as an objective, quantitative alternative to visual analysis. Using receiver operating characteristic (ROC) analysis, we find that PDFF measurements enable accurate separation of bone marrow edema (active inflammation) and fat metaplasia (structural damage) from normal marrow. The described approach is more objective than looking for 'a tiny bit of white' on fat-suppressed images, which is the current clinical standard.

Measure for Measure: Machine Learning Models for Osteoporosis MRI data

Uran Ferizi ${ }^{1}$, Harrison Besser ${ }^{1}$, Chamith S Rajapakse ${ }^{2}$, Punam K Saha ${ }^{3}$, Stephen Honig ${ }^{1}$, and Gregory Chang ${ }^{1}$

${ }^{1}$ New York University School of Medicine, New York, NY, United States, ${ }^{2}$ University of Pennsylvania School of Medicine, Philadelphia, PA, United States, ${ }^{3}$ University of lowa College of Medicine, lowa City, IA, United States

We examine how Machine Learning can be used to identify novel risk factors of osteoporotic bone fracture. Using measurements from patient MRI scans at five anatomical sites, we sought to find which specific regions are best for stratifying the risk of osteoporotic fracture. Further studies on these models and other data will help improve clinicians' ability to accurately diagnose Osteoporosis, so that patients at risk for bone fracture may be caught and treated earlier.

Performance of different classifiers in the diagnosis of benign and malignant bone tumors based on MR diffusion kurtosis imaging

Zhizheng Zhuo ${ }^{1}$, Ying $\mathrm{Li}^{2}$, Cuiping Ren², and Jingliang Cheng ${ }^{2}$

${ }^{1}$ Clincial Science, Philips Healthcare, Beijing, China, ${ }^{2}$ Radiology Department of First Affiliated Hospital of Zhengzhou University, Zhengzhou, China

Recently, the Al (Artificial Intelligence) is popular in the clinical diagnosis based on medical imaging. The major target is to identify or classify the disease condition through the features extracted from the clinical images. Different algorithms (or classifiers) can be applied to classify the disease and the performance might be different for a specific clinical issue. In this work, we tried to investigate the performance of different classifiers in the diagnosis of benign and malignant bone tumors based on MRI diffusion kurtosis imaging.

Xing Lu ${ }^{1,2}$, Saeed Jerban ${ }^{1}$, Michael Carl ${ }^{3}$, Yajun Ma ${ }^{1}$, Annette von Drygalski ${ }^{4}$, Eric Y Chang ${ }^{5}$, and Jiang Du ${ }^{1}$

${ }^{1}$ Department of Radiology, University of California, San Diego, San Diego, CA, United States, ${ }^{2}$ Institute of Electrical Engineering, Chinese Academy of Science, Beijing, China, ${ }^{3}$ GE Healthcare, San Diego, CA, United States, ${ }^{4}$ Department of Medicine, Division of Hematology/Oncology, University of California, San Diego, San Diego, CA, United States, ${ }^{5}$ Radiology Service, VA San Diego Healthcare System, San Diego, CA, United States 
Bone mineral density (BMD) evaluation is crucial for the diagnosis of osteoporosis and related fractures. The purpose of this pilot study was to use a chemical-shift QSM method based on a 3D UTE-Cones sequence to assess the susceptibility values of human cortical bone specimens with consideration of gender and donor age, ranging over 5 decades. Significant differences between QSM values were observed for the different genders. A decaying trend between the minus QSM value and advancing age exists, which suggests a relationship between QSM values and BMD.

Study of mono-exponential and intravoxel incoherent motion models in differentiation of metastasis from myeloma

Xiaoying Xing ${ }^{1}$, Ning Lang ${ }^{1}$, and Huishu Yuan ${ }^{1}$

${ }^{1}$ Peking University 3rd Hospital, Beijing, China

This study aimed to evaluate the diagnostic performance of diffusion weighted imaging (DWI) to differentiate metastasis from myeloma using the apparent diffusion coefficient (ADC) and parameters derived from the intravoxel incoherent motion (IVIM) theory. 40 patients with metastasis and 12 with myeloma underwent diffusion-weighted magnetic resonance (MR) imaging and dynamic contrast enhanced MRI (DCE-MRI). ADC, diffusion coefficient(D), pseudodiffusion coefficient( $\left.D^{*}\right)$, and perfusion fraction ( $\left.f\right)$ were calculated.Through our study it is feasible to $d$ ifferentiate metastasis from myeloma by mono-exponential and IVIM models. IVIM-derived $D$ and $D^{*}$ values showed significantly better diagnostic performance than ADC values in differentiating metastasis from myeloma.

Clinical value of semi-quantitative and quantitative MR perfusion imaging in distinguishing malignant from benign bone tumors

Ying $\mathrm{Li}^{1}$, Cuiping Ren ${ }^{1}$, Jingliang Cheng ${ }^{1}$, and Zhizheng Zhuo ${ }^{2}$

${ }^{1}$ The First Affiliated Hospital of Zhengzhou University, Zhengzhou, China, ${ }^{2}$ Philips Healthcare, Beijing, China

The dynamic contrast-enhanced magnetic resonance imaging(DCE-MRI) is a common scanning technology which contains semi-quantitative and quantitative perfusion information. This work investigated and evaluated the ability of semi-quantitative and quantitative perfusion information in characterizing the bone tumors, and furtherly evaluate the ability of semiquantitative and quantitative parameters to differentiate benign and malignant tumors.

Proton Density Zero Echo Time(ZTE) Imaging for Evaluating the Bone Involvement in the Femoral Tumor

Xin $\mathrm{Lou}^{1}$, Jinfeng $\mathrm{Li}^{1}$, Lin $\mathrm{Xu}^{1}$, Xigang Zhao ${ }^{2}$, Jianxun $\mathrm{Qu}^{2}$, and $\mathrm{Lin} \mathrm{Ma}^{1}$

${ }^{1}$ Radiology and Imaging, China Army General Hospital, Beijing, China, ${ }^{2}$ General Electric Healthcare, Beijing, China

MRI can display the compositions of different tissues and adjacent involvements. In the patients of bone tumors, the integrity of cortical bone needed to be assessed for the preoperative planning. This study used proton density ZTE to display the bone involvement in patients of femoral tumors. Substantial agreement was found between $C T$ and $Z T E$ ( $r=0.98-0.99$ ) and there was not statics significance between the measured diameters from CT and ZTE MRI ( $p=0.34-0.99)$. further development of ZTE may obviate the need of $C T$ in evaluating the bone involvement of femoral tumors.

Preliminary study of T1rho imaging technique in assessment of early intervertebral disc degeneration in asymptomatic pilots at 3.0T magnetic resonance

XiuLan Zhang ${ }^{1}$, Yongmin $\mathrm{Bi}^{2}$, and Lizhi Xie ${ }^{3}$

${ }^{1}$ Radiology Department, The First Affiliated Hospital of Yangtze University, JingZhou, China, ${ }^{2}$ Department of CT\&MRI, Air Force General Hospital, Beijing, China, ${ }^{3}$ GE Healthcare, China, Beijing, China

T1rho MRI in the lumbar spine may provide a tool for the diagnosis of early degenerative changes in the disc. In this study, the mean T1rho value of pilots was significantly lower than that of the control group. The degenerative grades of pilots mainly were grade III and IV, but control group were grade I and II. There were significant differences in T1rho values at each age group between pilots and control group. And overload on spine column of pilots may be the important reason in degeneration and accelerate the degeneration process.

Utility of ZTE for the Characterization of Acute Ankle Fractures

Alissa J. Burge ${ }^{1}$, Ryan E Breighner ${ }^{1}$, Megan Sahr ${ }^{1}$, Matthew F. Koff ${ }^{1}$, Ogonna K Nwawka ${ }^{1}$, Darryl B. Sneag ${ }^{1}$, Gabrielle Konin ${ }^{1}$, Bin Lin², David Helfet ${ }^{1}$, and Hollis G. Potter ${ }^{1}$

${ }^{1}$ Hospital for Special Surgery, New York, NY, United States, ${ }^{2}$ Department of Radiology and Imaging - MRI, Hospital for Special Surgery, New York, NY, United States 
ZTE MRI provides CT-like tissue contrast, facilitating evaluation of mineralized bone. The utility of ZTE for evaluation of acute ankle fractures was evaluated in a series of 14 patients who underwent preoperative clinical MRI with an additional ZTE sequence, and subsequently underwent surgical fracture fixation. Fractures were characterized in a blinded fashion utilizing ZTE and CT, with subsequent operative confirmation. ZTE provided accurate characterization of fractures relative to both CT and surgery, with excellent inter- and intra-observer reliability.

Analysis of the relationship between mandibular joint motion trajectory and masticatory muscle properties (volume, shape, T1\&T2 value) with MR dynamic imaging

Ryusuke Nakai ${ }^{1,2}$, Takashi Azuma ${ }^{3}$, Toshihiro Togaya ${ }^{4}$, and Hiroo Iwata ${ }^{2}$

${ }^{1}$ Kokoro Research Center, Kyoto University, Kyoto, Japan, ${ }^{2}$ Institute for Frontier Life and Medical Sciences, Kyoto University, Kyoto, Japan, ${ }^{3}$ The Graduate School of Engineering, Kyoto University, Kyoto, Japan, ${ }^{4}$ Osaka Dental University, Osaka, Japan

For the diagnosis of temporomandibular joint disease, it is important to analyze with complete accuracy the range of mandibular motion and the tissue properties of the masticatory muscle in individual patients. In this study, we explored the parameters for accurate imaging of the mandibular motion trajectory using MR dynamic imaging, and then analyzed the relationship between the range of mandibular motion and the tissue properties of the masticatory muscle. As a result, we successfully identified the optimal imaging parameters and clarified that the range of side-to-side motion of the mandibular joint correlated with the tissue properties of the masticatory muscle.

Macromolecular and water pools distribution maps in bovine cortical bone using ultrashort echo time (UTE) MRI combined with magnetization transfer (MT) modeling

Saeed Jerban ${ }^{1}$, Yajun Ma ${ }^{1}$, Wei Zhao ${ }^{1}$, Xing Lu ${ }^{1}$, Michael Carl' ${ }^{2}$ Eric Y Chang ${ }^{1,3}$, and Jiang Du ${ }^{1}$

${ }^{1}$ Radiology, University of California, San Diego, San Diego, CA, United States, ${ }^{2}$ GE Healthcare, San Diego, CA, United States, ${ }^{3}$ Radiology Service, VA San Diego Healthcare System, San Diego, CA, United States

Collagenous matrix, bound and pore water pools are main responsible components for viscoelastic properties of the cortical bone. Quantitative ultrashort echo time MR imaging (UTEMRI) has been shown to be able to assess bound and pore water components as indexes for bone microstructure. UTE magnetization transfer (UTE-MT) modelling can evaluate the macromolecular (MM) components of the bone (collagen). Pixel mapping of MR properties of collagen and water components in cortical bone helps to localize pathologic or traumatic bone defects. This study focused on deriving the pixel maps of MR properties of these key bone components on seven bovine bone specimens.

Traditional Poster

MR Safety

Exhibition Hall 1451-1475

Monday 13:45 - 15:45

Assessment of Peripheral Nerve Stimulation due to MR gradient induced Electric Field around Implantable Device

Xiyao Xin ${ }^{1}$, Xi Lin Chen ${ }^{1}$, Xin Huang $^{1}$, and Shiloh Sison ${ }^{1}$

Time varying magnetic gradient fields can induce electric field (E-field) in the human body and may cause peripheral nerve stimulation (PNS) during MR scan. As metallic implant may cause local E-field enhancement, there is speculation that it may increase risk of PNS. In this study, gradient coil modeling is used to investigate induced E-fields around implantable devices. The maximum E-field in the proximity of implants is compared to the whole body maximum E-field of the human body without implant. The result shows that the local enhanced E-field near implants does not exceed the whole body maximum E-field in human body.

Impacts of 3.0 Tesla magnetic resonance imaging noise on hearing function in neonates with hearing protection

Huifang Zhao ${ }^{1}$, Chao $\mathrm{Jin}^{1}$, Xinyu Li ${ }^{1}$, Heng Liu ${ }^{1}$, Xiaoyu Wang ${ }^{1}$, Xingxing Tao ${ }^{1}$, Yannan Cheng ${ }^{1}$, and Jian Yang ${ }^{1}$

${ }^{1}$ Department of Diagnostic Radiology, the First Affiliated Hospital of Xi'an Jiaotong University, Xian', China

Loud acoustic noise generated from magnetic resonance (MR) imaging remains the great concern for neonatal exams. This study therefore aims to clarify whether this noise would cause the hearing loss to neonates who underwent MRI exam by auditory brainstem response (ABR). Results indicated that there was no significant difference in all the six ABR indices (waves I, III, V amplitudes and wave I-III, III-V, I-V intervals) between before and after the MRI examinations. Our findings may suggest the rarely temporary impact of MRI noise on ABR in neonates who underwent a $3.0 T$ MRI. 
The transfer function for implanted wires when a second wire is near

Peter R.S. Stijnman ${ }^{1}$, Janot P. Tokaya ${ }^{1}$, Cornelis A.T. van den Berg ${ }^{1}$, and Alexander J.E. Raaijmakers ${ }^{1}$

Lead wires of medical implants can pose a severe safety risk due to RF-induced heating. Risk assessment typically involves determination of the transfer function. This study shows that the transfer function may drastically change if a second wire is located close to the lead wire. An explorative simulation study has been performed investigating the impact of inter-wire spacing and wire length on the alteration of the transfer function by the second wire. Results reveal that in particular insulated wires may show very strong enhancements $(>100 \%)$ in induced currents if a second wire is present.

Analysis and Design of Lead Wires with Metallic Shielding for Reduction of RF Heating during MRI for Active Implants

Krishna Singhal ${ }^{1}$ and John A. Nyenhuis ${ }^{1}$

${ }^{1}$ Purdue University, West Lafayette, IN, United States

The purpose of this work is to provide a quantitative understanding of how a conducting metallic shield over a lead will reduce RF heating at the electrode during MRI scans. A physical model and equations for reduction of RF heating by a shielded lead are presented. Temperature rise were calculated for different lengths of shielded and unshielded leads. Confirming measurements were made for a quarter wavelength coaxial cable model of the lead. Measured temperature rise and transfer function depended on terminations conditions, with the open lead exhibiting a temperature rise approximately 10 times greater than the shorted lead.

MRI compatible neural electrodes for simultaneous deep brain stimulation and fMRI mapping

Siyuan Zhao ${ }^{1,2,3}$, Gen Li ${ }^{1}$, Wenjing Chen ${ }^{4}$, Zhifeng Liang ${ }^{4}$, and Xiaojie Duan ${ }^{1,2,3}$

${ }^{1}$ Department of Biomedical Engineering, College of Engineering, Peking University, Beijing, China, ${ }^{2}$ Academy for Advanced Interdisciplinary Studies, Peking University, Beijing, China, ${ }^{3}$ Center for Nanochemistry, Beijing Science and Engineering Center for Nanocarbons, Peking University, Beijing, China, ${ }^{4}$ Institute of Neuroscience, Chinese Academy of Sciences, Shanghai, China

Functional magnetic resonance imaging (fMRI) under deep brain stimulation (DBS) provides important insights into understanding the connection of the neural networks. However, such research has been limited by incompatibility of common electrode in the MR environment. To address such issue, we fabricated a novel graphene based neural microelectrode, which exhibited excellent charge storage capacity and MRI compatibility. Using such microelectrode, we successfully demonstrate deep brain stimulation of subthalamic nucleus (STN) evoked robust BOLD activation in cortex and basal ganglia nucleus of the Parkinsonian rats with minimal image artifact. Therefore, MR-compatible graphene microelectrode could provide unique opportunity for simultaneous DBS-fMRI studies.

RF-induced heating of a conducting wire entering into a dielectric medium along right-left axis on the presence of another wire during MRI

Pallab K Bhattacharyya ${ }^{1,2}$, Tanvir Baig ${ }^{3}$, Bhumi Bhusal ${ }^{3}$, Mark J Lowe ${ }^{1}$, Michael Martens ${ }^{3}$, and Stephen Jones ${ }^{1}$

${ }^{1}$ Imaging Institute, Cleveland Clnic, Cleveland, $\mathrm{OH}$, United States, ${ }^{2}$ Radiology, Cleveland Clinic Lerner College of Medicine, Cleveland, OH, United States, ${ }^{3}$ Physics, Case Western Reserve University, Cleveland, $\mathrm{OH}$, United States

RF-induced heating of stereo encephalography (SEEG) electrodes during MRI scans could be of concern. The change in heating pattern of an electrode in the presence of another electrode was investigated by measuring the heating at the tip of a conducting and insulated (bare at tip) wire parallel to B0-field and entering a poly-acrylic gel phantom along left-right axis in the presence of another wire. While the resonance length for maximum heating of the wires did not depend on the number of wires, the temperature rise at the wire tips depended on the relative lengths (resonance / anti-resonance) of the wires.

Safety of MRI scans of partially implanted entirely insulated conducting wire with spine matrix coil at 3T

Pallab K Bhattacharyya ${ }^{1,2}$, Bhumi Bhusal ${ }^{3}$, Anna Crawford ${ }^{1}$, Thomas Masaryk ${ }^{1}$, and Mark J Lowe ${ }^{1}$

${ }^{1}$ Imaging Institute, Cleveland Clnic, Cleveland, $\mathrm{OH}$, United States, ${ }^{2}$ Radiology, Cleveland Clinic Lerner College of Medicine, Cleveland, OH, United States, ${ }^{3} \mathrm{Phy}$ sics, Case Western Reserve University, Cleveland, $\mathrm{OH}$, United States

RF-induced heating of an entirely insulated partially implanted conducting wire in a gel phantom was measured at two different 3 tesla systems with a receive-only spine matrix coil. Presence of inner spiral-wound stainless steel helix in Arrow AK-05502 intrathecal catheters raises concern about possible RF-induced heating during MRI. Temperature of the catheter was measured by using fiber optic sensors with fluoroptic monitoring with the catheter inserted into an ASTM gel phantom. Different configurations representing in vivo settings were tested at different E-fields in the phantom. No significant heating was observed in any of the configurations. 
Transmit Coil impedance measurements to estimate radiofrequency induced currents on wires in MRI

Brandon J Coles ${ }^{1}$, Kevan J Anderson ${ }^{2}$, Greig C Scott ${ }^{3}$, Christopher W Ellenor ${ }^{3}$, and Graham A Wright ${ }^{1,2}$

${ }^{1}$ Medical Biophysics, University of Toronto, Toronto, ON, Canada, ${ }^{2}$ Sunnybrook Research Institute, Toronto, ON, Canada, ${ }^{3}$ Electrical Engineering Department, Stanford University, Stanford, CA, United States

$\mathrm{MRI}$ introduces a safety risk when performing imaged guided interventions caused by induced currents on interventional devices that potentially lead to dangerous temperature increases near their tip. This safety issue can be reduced using parallel RF transmission approaches, although it is difficult to ensure safety when device motion is involved. In this work, impedance changes of a transmit coil are used to estimate the coil's induced current on a device, and this is extended to a two coil array to determine individual transmit signals needed to reduce the total induced current on a device with simple device geometry.

The feasibility study about the protection circuit for unplugged local transceiver coil in MRI bore

Seunghoon $\mathrm{Ha}^{1}$, Adam Morris ${ }^{1}$, Jay Berres ${ }^{1}$, and Jonathan Nass ${ }^{1}$

${ }^{1}$ Philips Healthcare, Pewaukee, WI, United States

The local transceiver coil such as a birdcage coil has still been equipped for local extremity or brain MRI in clinical study. By accident, the local transceiver coil is disconnected from an MRI system and inadvertently leaves linked to strong MRI RF fields during imaging procedures using other RF coils. It makes the local transceiver coil damaged such as components burnt as well as worse plastic housing melt and even causes patients' skin to burn during clinical scanning. To prevent from these damages, we propose a new protection circuit to prevent the unplugged local transceiver coil in MR bore from RF power radiated by the whole body transmitter coil.

The effect of fetal dielectric properties, position and blood-flow in maternal tissues on fetal temperature for fetal MRI at 3T

Shaihan J Malik ${ }^{1}$, Jeffrey W Hand ${ }^{1}$, and Joseph V Hajnal ${ }^{1}$

${ }^{1}$ School of Biomedical Engineering and Imaging Sciences, King's College London, London, United Kingdom

Effects of age adjusted dielectric properties for fetal tissues compared to adult values, fetal position, and blood-flow in maternal tissues on fetal temperature in a model of a 7 month pregnant woman within a $3 T$ birdcage coil were investigated numerically. Age adjusted properties resulted in small increases in peak and mean fetal temperatures and reduced time to reach a peak fetal temperature of $39^{\circ} \mathrm{C}$. Changes in fetal position produced a greater effect on peak and mean fetal temperatures. Temperature dependent blood-flow in maternal superficial tissues had little effect on fetal temperature.

T2 Relaxation in Evaluating Gd deposition: comparison between MultiHance and Magnevist

Ning HUA ${ }^{1}$, Pedro V. Staziaki² ${ }^{2}$ Mohamad Assayuri² ${ }^{2}$ Vanesa Carlota Andreu Arasa ${ }^{2}$, Hernan Jara ${ }^{1}$, and Osamu Sakai ${ }^{2}$

${ }^{1}$ Boston University, Boston, MA, United States, ${ }^{2}$ Boston Medical Center, Boston, MA, United States

Purpose: To evaluation quantitative T2 mapping in exploring the effects of prior Gd exposure. Methods: Dual-echo MRI was performed in three groups of subjects; 1) without prior Gd exposure history, 2) only with prior exposure to MultiHance $₫$, and 3 ) only with prior exposure to Magnevist $₫$. T2 relaxation times were measured in pons, dentate nuclei, globus pallidi and thalami. Results: T2 relaxation time decrease was observed for both contrast agents in dentate nuclei and globus pallidi. Conclusion: Quantitative T2 mapping is a valuable tool in the investigation of $\mathrm{Gd}$ deposition in the brain.

Preliminary Experience in Off-Label Use of Ferumoxytol Contrast-enhanced Magnetic Resonance Angiography in Pregnancy

Lindsay M Griffin ${ }^{1}$, Kim-Lien Nguyen², Thomas M Grist ${ }^{1}$, Christopher J Francois ${ }^{1}$, Scott B Reeder ${ }^{3}$, J Paul Finn ${ }^{2}$, and Mark L Schiebler ${ }^{1}$

${ }^{1}$ Radiology, University of Wisconsin, Madison, WI, United States, ${ }^{2}$ Radiology and Medicine, University of California Los Angeles, Los Angeles, CA, United States, ${ }^{3}$ Radiology, Medical Physics, Biomedical Engineering, Medicine, and Emergency Medicine, University of Wisconsin, Madison, WI, United States

Recent debate about potential long-term safety of gadolinium-based contrast agents has amplified concerns about their use in pregnancy, greatly limiting options for advanced imaging in this critical patient group. We report our experience on the use of ferumoxytol contrast-enhanced magnetic resonance angiography (MRA) during pregnancy. We identified eight pregnant subjects, at two institutions, with contrast-enhanced MRI/MRA using ferumoxytol. There was one mild possible adverse event during contrast administration. There were no premature deliveries ( $<35$ weeks) or birth defects in five babies with available postpartum data. While preliminary, ferumoxytol holds promise as a versatile MR contrast agent in pregnancy. 
Jillian Katrina Chan ${ }^{1}$, Anthony Traboulsee ${ }^{2}$, Emanuel Kanal ${ }^{3}$, Kenneth Maravilla ${ }^{4}$, Lori Saslow ${ }^{5}$, Laura Barlow ${ }^{2}$, Bruce Cohen ${ }^{6}$, Kathleen Costello ${ }^{7}$, June Halper ${ }^{8}$, Colleen Harris ${ }^{9}$, David Jones $^{10}$, Flavia Nelson ${ }^{11}$, Scott Newsome ${ }^{12}$, Jiwon Oh $^{13}$, Daniel Pelletier ${ }^{14}$, Kottil Rammonhan ${ }^{15}$, Daniel Reich ${ }^{16}$, Alex Rovira ${ }^{17}$, Lael Stone ${ }^{18}$, Kevin Terashima ${ }^{16}$, Jerry Wolinsky ${ }^{11}$, and David $\mathrm{Li}^{2}$

${ }^{1}$ Neurology, University of British Columbia, Vancouver, BC, Canada, ${ }^{2}$ University of British Columbia, Vancouver, BC, Canada, ${ }^{3}$ University of Pittsburgh Medical Center, Pittsburgh, PA, United States, ${ }^{4}$ University of Washington, Seattle, WA, United States, ${ }^{5}$ LS Science and Medical Communications, LLC, Great Neck, NY, United States, ${ }^{6}$ Northwestern University Medical SChool, Chicago, IL, United States, ${ }^{7}$ Nathional MS Society, Maryland, MD, United States, ${ }^{8}$ Consortium of MS Centers, Hackensack, NJ, United States, ${ }^{9}$ University of Calgary, Calgary, AB, Canada, ${ }^{10}$ University of Virginia, Charlottesville, VA, United States, ${ }^{11}$ UT Health McGovern Medical School, Houston, TX, United States, ${ }^{12}$ Johns Hopkins Hospital, Baltimore, MD, United States, ${ }^{13}$ University of Toronto, Toronto, ON, Canada, ${ }^{14} \mathrm{Keck}$ School of Medicine of USC, Los Angeles, CA, United States, ${ }^{15}$ University of Miami Multiple Sclerosis Center, Miami, FL, United States, ${ }^{16}$ Translational Neuroradiology Unit, NINDS, Bethesda, MD, United States, ${ }^{17}$ Section of Neuroradiology, Hospital Vall d'Hebron, Barcelona, Spain, ${ }^{18}$ Mellen Center for MS Treatment and Research, Cleveland, $\mathrm{OH}$, United States

Clinical guidelines for the diagnosis and follow-up of multiple sclerosis recommends brain MR imaging with gadolinium based contrast agents. Our aim was to address concerns about the use of gadolinium, the risk of accumulation in the brain and propose changes to clinical guidelines published in 2016. Group consensus is that GBCA remain essential in the diagnostic evaluation of a patient suspected of having MS to demonstrate active inflammatory lesions. GBCA should be used judiciously, minimizing gadolinium exposure and dose when possible.

The impact of altering MRI equipment and scanning parameters on phantom signal intensity ratio measurements - possible implications for interpreting Gadolinium signal changes within the brain

Laura Kate Young ${ }^{1}$, Shona Matthew ${ }^{1}$, Stephen Gandy ${ }^{2}$, Lukasz Priba ${ }^{2}$, and John Graeme Houston ${ }^{1,3}$

${ }^{1}$ Division of Molecular and Clinical Medicine, University of Dundee, Ninewells Hospital and Medical School, Dundee, United Kingdom, ${ }^{2}$ Medical Physics, NHS Tayside, Ninewells Hospital and Medical School, Dundee, United Kingdom, ${ }^{3}$ Clinical Radiology, NHS Tayside, Ninewells Hospital and Medical School, Dundee, United Kingdom

Signal hyper-intensities within brain regions have been attributed to the deposition of gadolinium following repeat administrations of MR contrast agents. These have been mainly investigated retrospectively, but acquisition parameters may have varied. We investigated the impact of altering imaging parameters when measuring phantom signal intensity ratios (SIR). By changing parameters from a baseline, it was established that the application of filters, number of coil receiver channels, and changes to TR and TE resulted in percentage signal fluctuations of similar magnitude to hyper-intensities. It is recommended that imaging parameters are standardised where possible when interpreting SIR data in longitudinal brain studies.

Estimated Measurement Uncertainty (EMU) in Calorimetrically-Determined Whole Body SAR Values for Medical Device Evaluation Using Benchtop Radiofrequency Exposure Systems

Krzysztof Wawrzyn ${ }^{1}$, Jack Hendriks ${ }^{1}$, William B. Handler ${ }^{1}$, and Blaine A. Chronik ${ }^{1}$

${ }^{1}$ The xMR Labs, Department of Physics and Astronomy, Western University, London, ON, Canada

The in vitro assessment of true radiofrequency whole body averaged specific absorption rate (WB-SAR) is described in the technical specification standard of ASTM F2182-11a, by direct measure of RF-induced heating within a standardized phantom centered inside the RF birdcage coil. F2182-11a does not address uncertainty assessment of the heating experiment. In this study, we present our measured values for short-term measurement repeatability and long-term measurement reproducibility. These measurements support the conclusion that RF-induced WB-SAR measurements made with bench-top RF exposure systems can be made with a total estimated measurement uncertainty of approximately $7 \%$ $(k=1)$.

Impact of tissue image segmentation errors on SAR

Asha Singanamalli ${ }^{1}$, Matthew Tarasek ${ }^{1}$, Qin $\mathrm{Liu}^{2}$, Desmond Yeo ${ }^{1}$, and Thomas Foo

${ }^{1}$ GE Global Research, Niskayuna, NY, United States, ${ }^{2}$ GE Healthcare, Waukesha, WI, United States

In this study, we evaluate the sensitivity of peak and global SAR to false positive (FP) and false negative (FN) errors in segmentation for three major brain tissue types: Gray Matter (GM), White Matter (WM) and Cerebrospinal Fluid (CSF). Voxel probability maps of GM, WM and CSF are thresholded at various intervals to generate multiple anatomical head models from a simulated T1W MRI dataset. FP and FN errors in segmentation are evaluated for each anatomical model with respect to the ground truth. Electromagnetic simulations are performed to relate these errors to peak and global SAR values at $3 \mathrm{~T}$.

Safety and EEG Data Quality of Concurrent High-Density EEG and High-Speed fMRI at 3 Tesla

Mette Thrane Foged ${ }^{1,2}$, Ulrich Lindberg ${ }^{3}$, Kishore Vakamudi ${ }^{4,5}$, Henrik BW Larsson ${ }^{2,3}$, Lars Pinborg $^{1,2}$, Troels W Kjær ${ }^{2,6}$, Martin Fabricius $^{6}$, Claus Svarer $^{1}$, Brice Ozenne ${ }^{7}$, Carsten Thomsen 2,8 , Sándor Beniczky $6,9,10$, Olaf Bjarne Paulson ${ }^{1,2}$, and Stefan Posse ${ }^{4,5,11}$ 
${ }^{1}$ Neurobiology Research Unit, Department of Neurology, Rigshospitalet, Copenhagen, Denmark, ${ }^{2}$ Dept. of Clinical Medicine, University of Copenhagen, Copenhagen, Denmark, ${ }^{3}$ Functional Imaging Unit, Dept. of Clinical Physiology, Nuclear Medicine and PET, Rigshospitalet, Copenhagen, Denmark, ${ }^{4}$ Department of Neurology, University of New Mexico, Albuquerque, NM, United States, ${ }^{5}$ Department of Physics and Astronomy, University of New Mexico, Albuquerque, NM, United States, ${ }^{6}$ Dept. of Clinical Neurophysiology, Rigshospitalet, Copenhagen, Denmark, ${ }^{7}$ Department of Biostatistics, University of Copenhagen, Copenhagen, Denmark, ${ }^{8}$ Dept. of Radiology, Rigshospitalet, Copenhagen, Denmark, ${ }^{9}$ Danish Epilepsy Centre, Dianalund, Denmark, ${ }^{10}$ Aarhus University, Aarhus, Denmark, ${ }^{11}$ Department of Electrical and Computer Engineering, University of New Mexico, Albuquerque, NM, United States

Using concurrent high-density EEG and different high-speed fMRI methods, we investigate safety of RF heating, effect on image SNR and assess EEG data quality. RF related electrode heating during a 30 -minute scan did not exceed $1.0^{\circ} \mathrm{C}$ with any of the pulse sequences. No significant differences in the EEG data quality were found between high-speed fMRI and conventional EPI $(p=0.78)$. Residual ballistocardiographic artifacts resulted in $58 \%$ of EEG data being rated as poor quality. This study demonstrates that high-density EEG can be safely implemented in conjunction with high-speed fMRI and that high-speed fMRI does not adversely affect EEG data quality.

Active Implantable Medical Device - Can its Radio Frequency Radiation be a Potential Source of MR Image Artifact?

Xi Lin Chen ${ }^{1}$, Perry Li ${ }^{1}$, and Shiloh Sison

${ }^{1}$ Abbott Laboratories, Sylmar, CA, United States

To assess if an active implantable medical device (AIMD) may unintentionally generate radio frequency signals near the receiver band of an MRI RF coil and cause image artifact, a method is proposed in this study to quantify the maximum AIMD radiated signal strength near the MR Lamor frequencies at $1.5 \mathrm{~T}$ and $3 \mathrm{~T}$. Three commercially available AIMDs were investigated and the maximum radiated signal level was found to be around $-120 \mathrm{dBm}$ at the 64 and $128 \mathrm{MHz}$ range. Such information can be utilized in conjunction with MR RF receiver specifications to determine the potential impact on image artifacts

Implantable Lead MRI RF Heating in-vivo Transfer Function Modeling to Determine Suitable Test Medium

Xi Lin Chen ${ }^{1}$, Shi Feng ${ }^{1}$, Xiyao Xin ${ }^{1}$, Xin Huang ${ }^{1}$, Ruoli Jiang ${ }^{1}$, and Shiloh Sison ${ }^{1}$

${ }^{1}$ Abbott Laboratories, Sylmar, CA, United States

This abstract presents a novel technique to determine the suitable tissue simulating medium (TSM) conductivity for MRI lead electrode RF heating transfer function (TF) determination. The proposed method utilizes validated numerical lead model in conjunction with tissue models extracted along lead trajectories in anatomical models to produce in-vivo transfer function models. When combined with in-vivo incident electric fields, the power deposition or temperature rise predicted by the in-vitro and in-vivo TFs can be compared to assess the suitability and conservativeness of the selected TSM conductivity.

Comparison of RF Induced Device Heating at $0.35 T$ and $1.5 T$

Jessica A. Martinez ${ }^{1,2}$, Kévin Moulin ${ }^{1}$, Yu Gao ${ }^{1}$, Peng Huํㄹ , and Daniel B. Ennis ${ }^{1,2}$

${ }^{1}$ Radiological Sciences, UCLA, Los Angeles, CA, United States, ${ }^{2}$ Bioengineering, UCLA, Los Angeles, CA, United States

RF induced heating is a safety concern for patients with implanted electronic devices (IEDs). At lower field strengths (0.35T) heating is expected to be lower than at higher field strengths (1.5T). However, little experimental data has been acquired at field strengths below $1.5 \mathrm{~T}$. The purpose of this work is to compare the effects of field strength on RF induced heating by applying the same RF power in a metallic rod at $0.35 \mathrm{~T}$ and $1.5 \mathrm{~T}$. We found that heating was substantially lower at $0.35 \mathrm{~T}$ than $1.5 \mathrm{~T}$, which may be substantially beneficial for patients with IEDs.

Resonant heating study of a partially immersed implant in ASTM phantom and Human Mode

Bhumi Bhusal $^{1}$, Tanvir Baig ${ }^{1}$, Pallab Bhattacharyya ${ }^{2}$, Stephen Jones ${ }^{2}$, and Michael Martens ${ }^{1}$

14711 Physics, Case Western Reserve University, Cleveland, $\mathrm{OH}$, United States, ${ }^{2}$ Imaging Institute, Cleveland Clinic, Cleveland, $\mathrm{OH}$, United States

The RF heating of partially immersed implants in homogenous phantoms is reported to be highest for conductors at the resonant length. When addressing RF safety concerns, it is important to understand if these results apply to the heterogeneous structure within the human head. In this study, numerical simulations of RF heating of a partially immersed wire in an ASTM phantom are compared to an IT'IS virtual human model (Duke) for a head-only RF transmit coil in a 3 T MRI. We find that the resonant lengths are the same in both cases but the peak SAR changes slightly. 
${ }^{1}$ IHU Liryc, Electrophysiology and Heart Modeling Institute, Fondation Bordeaux Université, Bordeaux, France, ${ }^{2}$ Univ. Bordeaux, Centre de recherche Cardio-Thoracique de Bordeaux, U1045, Bordeaux, France, ${ }^{3}$ INSERM, Centre de recherche Cardio-Thoracique de Bordeaux, U1045, Bordeaux, France, ${ }^{4}$ Siemens Healthineers, Saint-Denis, France, 5 Image Guided Therapy SA, Bordeaux, France

This study presents a fast MR-thermometry sequence interleaved with a tunable SAR deposition module to simulate energy deposition of any clinically relevant MR-acquisition sequence. Validation of the method was performed on a 1.5T scanner using an MR-compatible catheter inserted into an agar-agar gel. Maximal temperature increase measured during equivalent SAR of a cardiac cine sequence was $41.8^{\circ} \mathrm{C}$ for a $90^{\circ}$ flip angle. This sequence may help quantifying the maximal acceptable SAR for any patient wearing implanted device and/or for volumetric imaging of local heating in multi-transmit technology at high field.

MRI RF Safety of Active Implantable Medical Devices (AIMDs): Experimental Study of the Effect of Conductivity of Tissue Simulating Media

Jingshen Liu ${ }^{1}$, Krishna Kurpad ${ }^{2}$, Paul Stadnik² ${ }^{2}$ Jeffrey VonArx², Larry Stotts ${ }^{2}$, Wolfgang Kainz ${ }^{3}$, and Ji Chen ${ }^{1}$

Experimental study of the effect of conductivity of tissue simulating media is performed for MRI RF safety of active implantable medical devices. The influence of medium surrounding the implantable lead tip, and the influence of medium surrounding implantable pulse generator are analyzed.

Electro-Optic E-field Mapping of Medical Implants with High Spatial Resolution: Resonant Excitation of Metallic Stents

Simon Reiss ${ }^{1}$, Thomas Lottner $^{1}$, Ali Caglar Özen ${ }^{1}$, Michael Bock $^{1}$, and Andreas Bitzer ${ }^{1,2}$

Electrically conducting implants with small and complex geometrical structures such as stents require electric field measurements with high spatial resolution to assess local MRI safety. So far, E-fields have been measured with dipole antennae that are limited in spatial resolution to several millimeters. In this study, we present an optical setup for $2 \mathrm{D}$ spatially resolved $\mathrm{E}$ field measurements of medical implants with high spatial resolution. Resonant excitation of metallic NiTi stents with varying lengths is assessed and the sub-millimeter spatial resolution of the setup is demonstrated.

Development and evaluation of a single-phase alloy with magnetic susceptibility equivalent to that of mammalian tissue for coil embolization of a cerebral aneurysm

Ryusuke Nakai ${ }^{1,2}$, Takashi Azuma ${ }^{3}$, Mitsuaki Toda ${ }^{2}$, Tomonobu Kodata ${ }^{4}$, and Hiroo Iwata ${ }^{2}$

${ }^{1}$ Kokoro Research Center, Kyoto University, Kyoto, Japan, ${ }^{2}$ Institute for Frontier and Medical Life Sciences, Kyoto University, Kyoto, Japan, ${ }^{3}$ Graduate School of Engineering, Kyoto University, Kyoto, Japan, ${ }^{4}$ Department of Neurosurgery, Jikei University School of Medicine, Tokyo, Japan

Relatively less invasive MRI has recently been increasingly used for examination after coil embolization of a cerebral aneurysm, but there is a risk of misdiagnosis due to magnetic susceptibility artifacts. In this study, we developed a device composed of a highly biocompatible alloy with magnetic susceptibility equivalent to that of mammalian tissue, and evaluated it using both an in vitro model and rabbits. We found that this alloy markedly reduced magnetic susceptibility artifacts and can be used as a device in the body. We are planning to develop various implantable medical devices using this alloy.

Traditional Poster

\section{MR-Guided Interventions}

Valéry Ozenne ${ }^{1,2,3}$, Pierre Bour ${ }^{1,2,3,4}$, Mathieu Santin ${ }^{5,6}$, Romain Valabrègue $^{5,6}$, Charlotte Constans ${ }^{7}$, Aurélien Trotier $^{8}$, Sylvain Miraux ${ }^{8}$, Jean-Francois Aubry ${ }^{7}$, and Bruno Quesson ${ }^{1,2,3}$

${ }^{1}$ IHU Liryc, Electrophysiology and Heart Modeling Institute, Fondation Bordeaux Université, Bordeaux, France, ${ }^{2}$ Univ. Bordeaux, Centre de recherche Cardio-Thoracique de Bordeaux, U1045, Bordeaux, France, ${ }^{3}$ INSERM, Centre de recherche Cardio-Thoracique de Bordeaux, U1045, Bordeaux, France, ${ }^{4}$ Image Guided Therapy SA, Bordeaux, France, ${ }^{5}$ CENIR, Centre de Neurolmagerie de Recherche, Paris, France, ${ }^{6}$ ICM, Inserm U 1127, CNRS UMR 7225, Sorbonne Universités, UPMC Université Paris 06 UMR S 1127 , Institut du Cerveau et de la Moelle épinière, Paris, France, ${ }^{7}$ Institut Langevin Ondes et Images, ESPCI ParisTech, CNRS 7587, UMRS 979 INSERM, Paris, France, ${ }^{8}$ Centre de Résonance Magnétique des Systèmes Biologiques, UMR5536, CNRS, Univ. Bordeaux, Bordeaux, France 
MR-guided HIFU in the brain currently lacks from insufficient spatial and temporal monitoring of the effect of ultrasound. In this study, we combine simultaneous multi-slice (SMS) echo planar imaging (EPI) technique with in-plane parallel imaging to achieve high spatial resolution with large volume coverage and/or short acquisition time during temperature mapping at 3T. The sequence was tested in vivo in a human brain with different multiband (MB) factors. SMS reconstruction and temperature mapping were computed using the Gadgetron framework. Then, validation was performed on an ex vivo chicken muscle during HIFU sonication to validate the method.

Accelerated imaging for visualizing interventional devices using parallel acquisition and compressed sensing reconstruction

Samira Vafay Eslahi ${ }^{1}$, Caiyun $\mathrm{Shi}^{2}$, Haifeng Wang ${ }^{2}$, Yifeng $\mathrm{Ye}^{3}$, Hanwei Chen ${ }^{3}$, Guoxi Xie ${ }^{4}$, and Jim $\mathrm{Ji}^{1}$

${ }^{1}$ Electrical and Computer Engineering, Texas A\&M university, College Station, TX, United States, ${ }^{2}$ Shenzhen Institutes of Advanced Technology, Lauterbur Research Center for Biomedical Imaging, Shenzhen, China, ${ }^{3}$ Department of Radiology, Panyu Central Hospital, Guangzhou, China, ${ }^{4}$ Department of Biomedical Engineering, Guangzhou Medical University, Qingyuan, China

Visualizing implanted and/or surgical devices is crucial for interventional radiology. Conventional MRI shows the devices as dark voids or with metal artifacts. Recent methods based on susceptibility mapping using fast spin-echo sequences can offer positive contrast visualizations, but they are relatively slow. In this work, parallel acquisition and compressed sensing reconstruction are integrated to accelerate the phase-sensitive acquisition and reconstruction. Applications in brachytherapy, biopsy and stent placement are demonstrated with simulations from real data. The proposed method can increase the acquisition speed by four while preserving the images quality.

Proton resonance frequency based MR thermometry using shifted-echo bSSFP

Seohee So ${ }^{1}$, Jaejin $\mathrm{Cho}^{1}$, Kinam Kwon ${ }^{1}$, Byungjai Kim ${ }^{1}$, Wonil Lee ${ }^{1}$, and Hyunwook Park ${ }^{1}$

Magnetic resonance thermometry provides noninvasive temperature measurements for thermal therapy. In this abstract, we exploit linear phase relation generated by echo shifting in the bSSFP acquisition to measure PRF change. Echo-shifting from TE=TR/2 in bSSFP provides a linear relation between phase of transverse magnetization and phase evolution in TR This linearity enables frequency prediction from the phase information, which makes temperature measurement with PRF shift possible. The performed simulations show shifted-echo bSSFP of TE=TR/4 well estimates frequency change.

Dependence of Focused-Ultrasound Induced Blood-Brain Barrier Opening Effect with Exposure Time: Evaluation via Dynamic Contrast-Enhanced Magnetic-Resonance Imaging Wen-Yen Chai ${ }^{1,2}$, Po-Chun Chun ${ }^{3}$, Sheng-Kai Wu ${ }^{4}$, Chih-Hung Tsai ${ }^{2}$, Hsin-Yi Lai ${ }^{5}$, and Hao-Li Liu ${ }^{2}$

${ }^{1}$ Department of Diagnostic Radiology and Intervention, Chang-Gung Memorial Hospital, Taoyuan, Taiwan, ${ }^{2}$ Department of Electrical Engineering, Chang-Gung University, Taoyuan, Taiwan, ${ }^{3}$ Department of Research and Development, NaviFUS corp., Taipei, Taiwan, ${ }^{4}$ Institute of Biomedical Engineering, National Taiwan University, Taipei, Taiwan, ${ }^{5}$ Interdisciplinary Institute of Neuroscience and Technology, Zhejiang University, Hangzhou, China

FUS exposure with presence of microbubbles can transiently open the BBB at targeted brain tissues. The study purpose is to investigate the dependency of the BBB opening effect with ultrasound exposure time by DCE-MRI. Our result showed extending exposure time can effectively increase FUS-induced BBB opening degree without causing tissue damage. We also proposed a strategy by adjusting exposure time during the multiple exposures to overcome the effects that microbubbles concentration dynamic changed after IV bolus injection. This approach of control FUS exposure time may bring technology advances of FUS-induced BBB opening to deliver drug for CNS disease treatment.

Correction of Motion-Induced Artifacts in PRFS MR Thermometry During Mild Hyperthermia in the Pelvis

Mingming Wu ${ }^{1}$, Paul Baron ${ }^{2}$, Hendrik T. Mulder ${ }^{2}$, Eduardo Coello ${ }^{1,3}$, Marion I. Menzel ${ }^{3}$, Gerard C. Van Rhoon ${ }^{2}$, and Axel Haase ${ }^{1}$

${ }^{1}$ Munich School of Bioengineering, Garching bei München, Germany, ${ }^{2}$ Erasmus Medical Center, Rotterdam, Netherlands, ${ }^{3}$ GE Research Center, Garching bei München, Germany

Digestive motion including gas is the predominant source of artifacts for PRFS MR Thermometry monitored RF hyperthermia inside the pelvis. Gastrointestinal motion of gas introduces large field variations inside the pelvis, thus significantly hampers PRFS based MR temperature reading. The estimation of these dipolar field disturbances from a changing susceptibility distribution is very exact in case we know the mask of $\Delta \mathrm{X}$, as shown with a phantom experiment. But using the PDF method, which allows a heterogeneous distribution of $\Delta \mathrm{X}$-values in the background, the temperature error could be reduced to noise level for in-vivo data in presence of susceptibility artefacts as well. 
Many medical applications such as brain surgery or stimulation require the clinician to identify an internal target location. Mixed reality see-through displays that enable a holographic visualization of brain MRI superimposed on a subject's head can help clinicians identify internal target locations but require tracking methods that keep the holographic brain MRI aligned with the subject's head as they move. We present a method for marker-less tracking of a subject's using a depth-sensing camera, which tracks facial features and sends location and rotation information to a see-through display to update the location in space of the MRI holograms.

Inertial Cavitation Induced Magnetic Resonance Signal Changes in a Rat Model

Cheng-Tao Ho ${ }^{1}$, Chen-Hua Wu ${ }^{1}$, Po-Hung Hsu ${ }^{2}$, Hao-Li Liu ${ }^{3}$, Chih-Kuang Yeh ${ }^{1}$, Ching-Hsiang Fan ${ }^{1}$, Wen-Shiang Chen ${ }^{4,5}$, and Hsu-Hsia Peng ${ }^{1}$

${ }^{1}$ Department Of Biomedical Engineering And Environmental Sciences, National Tsing Hua University, Hsinchu City, Taiwan, ${ }^{2}$ Center for Advanced Molecular Imaging and Translation, Chang Gung Memorial Hospital, Taoyuan city, Taiwan, ${ }^{3}$ Department Of Electrical Engineering, Chang-gung University, Taoyuan city, Taiwan, ${ }^{4}$ Department Of Physical Medicine And Rehabilitation, National Taiwan University Hospital, Taipei city, Taiwan, ${ }^{5}$ Division Of Medical Engineering Research, National Health Research Institutes, Miaoli city, Taiwan

We aim to real-time monitor the inertial cavitation (IC)-induced signal intensity (SI) changes in the presence of microbubbles and explore the correlation between the extent of IC-induced $\mathrm{SI}$ changes and the location of blood-brain barrier opening in a rat model. The computed |slopel map illustrated the territory of tissue with substantial SI changes and was consistent with the difference map (calculated from T1WI with and without Gd) and Evens Blue dyed region. In conclusion, we verified the feasibility of using FLASH sequence to distinguish the location of BBB-opening through the computed |slope| map in a rat model.

MR imaging simulator and optimized multi-echo z-shimmed sequence for temperature mapping near metallic ablation probes

Megan E Poorman ${ }^{1,2}$, Yue Chen ${ }^{3}$, Robert J Webster III,4, Eric J Barth ${ }^{3}$, and William A Grissom ${ }^{1,2}$

${ }^{1}$ Department of Biomedical Engineering, Vanderbilt University, Nashville, TN, United States, ${ }^{2}$ Vanderbilt University Institute of Imaging Science, Nashville, TN, United States,

${ }^{3}$ Department of Mechanical Engineering, Vanderbilt University, Nashville, TN, United States, ${ }^{4}$ Department of Neurological Surgery, Vanderbilt University, Nashville, TN, United States

Signal loss near metallic ablation probes can prevent quality MR thermometry guidance of treatment. Previously we proposed an orientation-independent multi-echo Z-shimmed sequence that could recover the lost signal and improve temperature precision near the probe. However, this method was not feasible for online implementation due to the need to acquire high resolution off-resonance maps around the ablator followed by a computationally-intensive optimization. Here we present an MR imaging simulator that calculates images near metallic ablation probes and successfully use it for offline optimization of the multi-echo Z-shimmed pulse sequence.

Development of a Tissue Mimicking Phantom for Focal Laser Ablation of the Prostate

Rory Geoghegan ${ }^{1,2}$, Alan Priester ${ }^{2,3}$, Alvaro Santamaria $^{3}$, Le Zhang ${ }^{4}$, Samantha Mikaiel ${ }^{4}$, Holden Wu ${ }^{4}$, Warren Grundfest ${ }^{1,2}$, Leonard Marks ${ }^{3}$, and Shyam Natarajan ${ }^{1,2,3}$

${ }^{1}$ Department of Bioengineering, University of California, Los Angeles, Los Angeles, CA, United States, ${ }^{2}$ Center for Advanced Surgical \& Interventional Technology, University of California, Los Angeles, Los Angeles, CA, United States, ${ }^{3}$ Department of Urology, University of California, Los Angeles, Los Angeles, CA, United States, ${ }^{4}$ Department of Radiology, University of California, Los Angeles, Los Angeles, CA, United States

There is a need to further develop real-time feedback systems for monitoring focal laser ablation (FLA). Here we have developed a tissue mimicking phantom to facilitate research on the use of magnetic resonance thermometry (MRT) and interstitial thermal probes as feedback systems. The tissue mimicking phantom was designed to match the optical and thermal properties of prostatic tissue at $980 \mathrm{~nm}$. The thermal response of the phantom to FLA was then compared to previously acquired clinical data and found to be qualitatively and quantitively similar to prostatic tissue. MRT and real-time quantification of damage zone progression are also demonstrated.

Monitoring and Guidance on High-Intensity Focused Ultrasound Treatment by Multiple Fast Field Echo at 3.0 T MRI: Ex-Vivo Studies with Multiparametric Mapping

Jong-Min Kim ${ }^{1,2}$, Chulhyun Lee ${ }^{3}$, Young-Seung Jo ${ }^{1}$, Han-Jae Chung ${ }^{1,2}$, Seong-Dae Hong ${ }^{1,2}$, You-Jin Jeong ${ }^{1,2}$, Jeong-Hee Kim ${ }^{4}$, and Chang-Hyun Oh ${ }^{1,2}$

${ }^{1}$ Department of Electronics and Information Engineering, Korea University, Seoul, Republic of Korea, ${ }^{2}$ ICT convergence technology for Health\&Safety, Korea University, Sejong,

Because the multiple Fast Field Echo (mFFE) is rich in contrast manipulation, such as, in water-fat, susceptibility, conductivity, and temperature imaging, it is well suited to guide the thermal treatment. In this study, we sought to investigate the feasibility of the mFFE for monitoring and guidance of HIFU treatment in ex-vivo swine tissue. To demonstrate this study, we present the conductivity, temperature, and susceptibility mapping results. We have shown that the mFFE is very useful for guidance and monitoring of the HIFU treatment. Simultaneous temperature, conductivity, and susceptibility mapping has been tried using the mFFE sequence and its utility has been shown in this paper. 
${ }^{1}$ Department of Biomedical Engineering, Tsinghua University, Beijing, China, ${ }^{2}$ Department of Engineering Physics, Tsinghua University, Beijing, China, ${ }^{3}$ Department of Orthopedics, First Affiliated Hospital of PLA General Hospital, Beijing, China, ${ }^{4}$ Key Laboratory of Particle and Radiation Imaging, Ministry of Education, Medical Physics and Engineering Institute, Tsinghua University, Beijing, China

Microwave ablation requires high temperature measurement accuracy to monitor the curative effect of the lesions. PRFS-based MR thermometry is the most commonly used temperature monitoring technique. However, PRFS is hampered by temperature-dependent magnetic susceptibility changes. It has been proved in the Quantitative Susceptibility Mapping(QSM) that susceptibility can be measured from the phase changes ,which is derived from Maxwell's Equation. In this work, we proposed a practical method to calculate the errors caused by temperature-induced susceptibility changes based on the method in QSM. Both Simulation studies and microwave heating experiments validated the accuracy of the method.

The effect of transducer position on signal-to-noise ratio in magnetic resonance guided focused ultrasound

Emilee S. Minalga ${ }^{1}$, Robb Merrill ${ }^{1}$, Dennis L Parker ${ }^{1}$, Allison H Payne ${ }^{1}$, and J. Rock Hadley ${ }^{1}$

${ }^{1}$ Utah Center for Advanced Imaging Research, University of Utah, Salt Lake City, UT, United States

Hardware requirements can be a roadblock to implementing procedure-specific coils in magnetic resonance guided focused ultrasound. In order to more effectively implement coils in the system, the effects of the focused ultrasound transducer's position on SNR needs to be considered. This work characterizes the SNR and noise correlation variability of the RF coils by evaluating the SNR tradeoffs and noise correlation as a function of device orientation and transducer position and report such variances. Understanding the SNR tradeoffs of system placement during treatment can aid in increased SNR within the treatment volume and can be a factor to consider in treatment planning

A hardware and algorithm framework for focal spot and slice positioning in MRgFUS treatments

Robb P Merrill ${ }^{1}$, J Rock Hadley ${ }^{1}$, Katelynn R Stroth ${ }^{1}$, Dylan E Palomino ${ }^{1}$, Dennis L Parker ${ }^{1}$, and Allison H Payne ${ }^{1}$

${ }^{1}$ Utah Center for Advanced Imaging Research (UCAIR), University of Utah, Salt Lake City, UT, United States

MRgFUS systems can be designed with a high degree of transducer positioning variability for precise focal point placement during tissue ablation procedures. This study evaluates hardware design and complementary algorithmic adaptations that predict the focal spot location and MRI slice orientation as a function of transducer adjustment settings. These design features were evaluated by comparing the physical focus of a mock transducer to the computed focus location from the prediction algorithm. The mean error between the measured and predicted point position was found to be $2.9 \pm 1.8 \mathrm{~mm}(\mathrm{~N}=20)$. Predicted slice orientation parameters also showed good agreement with hardware adjustment measurements.

Self-adaptive Bio-heat Transfer Model Modified Hybrid for Monitoring Temperature in Microwave Therapies

Jinchao Wu ${ }^{1}$, Shihan $\mathrm{Qiu}^{2}$, Bingyao $\mathrm{Chen}^{3}$, Jiafei Yang ${ }^{3}$, Xing Wei ${ }^{3}$, and Kui Ying ${ }^{1,4}$

${ }^{1}$ Department of Engineering Physics, Tsinghua University, Beijing, China, ${ }^{2}$ Department of Biomedical Engineering, Tsinghua University, Beijing, China, ${ }^{3}$ Department of Orthopedics, First Affiliated Hospital of PLA General Hospital, Beijing, China, ${ }^{4}$ Key Laboratory of Particle and Radiation Imaging, Ministry of Education, Medical Physics and Engineering Institute,

Tsinghua, Beijing, China

A BHT model was introduced to modify the penalty term of hybrid method for monitoring microwave ablation. Simulation results demonstrate that the proposed method is robust with the BHT model and can reconstruct more accurate temperature maps with different regularization parameters. Ex vivo experiment shows that the proposed method can achieve improved performance for rapid background shifting.

Detection of Acoustic Radiation Force-Induced Aggregated Bubbles by Velocity and Vorticity Maps

Che-Wei Wu ${ }^{1}$, Po-Hung Hsu ${ }^{2}$, Hao-Li Liu ${ }^{3}$, Chen-Hua Wu ${ }^{1}$, Ching-Hsiang Fan ${ }^{1}$, Chih-Kuang Yeh ${ }^{1}$, Wen-Shiang Chen ${ }^{4,5}$, and Hsu-Hsia Peng

${ }^{1}$ Biomedical Engineering and Environmental Sciences, National Tsing Hua University, Hsinchu, Taiwan, ${ }^{2}$ Center for Advanced Molecular Imaging and Translation, Chang Gung Memorial Hospital, Taoyuan, Taiwan, ${ }^{3}$ Electrical Engineering, Chang-gung University, Taoyuan, Taiwan, ${ }^{4}$ Physical Medicine and Rehabilitation, National Taiwan University Hospital, Taipei, Taiwan, ${ }^{5}$ Division of Medical Engineering Research, National Health Research Institutes, Miaoli, Taiwan

The aim of this study was to real-time localize the occurrence of secondary ARF and the aggregated bubbles by velocity and vorticity maps. During FUS transmission, the flow velocity and vorticity downstream to the FUS focus increased substantially. By observing the pixel-wise flow behavior in a scatter plot with information of velocity and vorticity, the position of aggregated bubbles could be localized in the regions with decreased velocity and vorticity. In conclusion, we verified the feasibility of using phase-contrast MRI to real-time detect secondary ARF and aggregated bubbles by combining pixel-wise velocity and vorticity information. 
Che-Wei Wu ${ }^{1}$, Po-Hung Hsu ${ }^{2}$, Hao-Li Liu ${ }^{3}$, Chen-Hua Wu ${ }^{1}$, Ching-Hsiang Fan ${ }^{1}$, Chih-Kuang Yeh ${ }^{1}$, Wen-Shiang Chen ${ }^{4,5}$, and Hsu-Hsia Peng ${ }^{1}$

${ }^{1}$ Biomedical Engineering and Environmental Sciences, National Tsing Hua University, Hsinchu, Taiwan, ${ }^{2}$ Center for Advanced Molecular Imaging and Translation, Chang Gung Memorial Hospital, Taoyuan, Taiwan, ${ }^{3}$ Electrical Engineering, Chang-gung University, Taoyuan, Taiwan, ${ }^{4}$ Physical Medicine and Rehabilitation, National Taiwan University Hospital, Taipei, Taiwan, ${ }^{5}$ Division of Medical Engineering Research, National Health Research Institutes, Miaoli, Taiwan

We hypothesized that the aggregated bubbles could be seen as a barrier, which might alter the flow pattern by shifting the high velocity core of flowing fluid. The aim was to assess the secondary acoustic radiation force and the size of aggregated bubbles, and thereby to estimate the amount of delivered drug in the targeting tissue. We found that larger displacement generally occurred with higher acoustic pressure, higher microbubble concentration, and slower flow velocity. In conclusion, we verified the feasibility of using phase-contrast MRI to evaluate the displacement of high velocity core in a phantom with flow microbubbles.

Volumetric and rapid MR-acoustic radiation force imaging using simultaneous multi-slice imaging

Pierre Bour ${ }^{1,2,3,4}$, Valéry Ozenne ${ }^{1,2,3}$, Stanislas Rapacchi ${ }^{5}$, Marylène Delcey ${ }^{1,2,3,6}$, Rainer Schneider ${ }^{7}$, Wadie Ben Hassen ${ }^{6}$, and Bruno Quesson ${ }^{1,2,3}$

${ }^{1}$ IHU-LIRYC, PESSAC, France, ${ }^{2}$ Univ. Bordeaux, Centre de recherche Cardio-Thoracique de Bordeaux, Bordeaux, France, ${ }^{3}$ INSERM U1045, Bordeaux, France, ${ }^{4}$ Image Guided Therapy, Pessac, France, ${ }^{5}$ Center for Magnetic Resonance in Biology and Medicine - UMR 7339, Marseille, France, ${ }^{6}$ Siemens Healthcare, Saint-Denis, France, ${ }^{7}$ Siemens Healthcare, Erlangen, Germany

The local tissue displacement induced by acoustic radiation force impulses (ARFI) during MR guided HIFU can be used to localize the focal spot position before thermal ablation and to monitor qualitative changes in tissue elasticity during ablation. However current MR-sequence implementations lack of spatial coverage, for a temporal resolution in the order of the timescale $(<1 \mathrm{~Hz})$ of displacement changes during sonication. To address this limitation, we developed a simultaneous multislice MR-ARFI sequence with a slice acceleration factor up to

3. Displacement estimations measured with accelerated sequences are compared to reference values using a non-accelerated sequence.

Application of hybrid MR-ultrasound imaging to multi-baseline thermometry

Pei-Hsin Wu ${ }^{1}$, Cheng-Chieh Cheng ${ }^{1}$, Frank Preiswerk ${ }^{1}$, and Bruno Madore ${ }^{1}$

1493 Radiology, Brigham and Women's Hospital, Harvard Medical School, Boston, MA, United States

MR thermometry, and more specifically the proton resonance frequency (PRF) shift method, has been widely employed for monitoring temperature change. However, breathing motion tends to corrupt the image phase that PRF relies upon. An existing free-breathing method called 'multi-baseline thermometry' was improved here by including a small ultrasound-based sensor fixed to the abdomen of the volunteer, to further help monitor and handle breathing motion. Utilizing both morphology (as in multi-baseline thermometry) and sensor information, better estimates of temperature changes could be achieved during breathing.

Hybrid Proton Resonance Frequency Shift and Variable Flip Angle T1 Temperature Mapping using a Golden-Angle 3D Stack-of-Radial Technique

Le Zhang ${ }^{1}$, Tess Armstrong ${ }^{1,2}$, Samantha Mikaiel ${ }^{1,2}$, Alan Priester ${ }^{3}$, Rory Geoghegan ${ }^{4}$, Shyam Natarajan ${ }^{3,4}$, and Holden Wu $\mathrm{H}^{1,2,4}$

${ }^{1}$ Radiological Sciences, University of California Los Angeles, Los Angeles, CA, United States, ${ }^{2}$ Physics and Biology in Medicine, University of California Los Angeles, Los Angeles, CA, United States, ${ }^{3}$ Urology, University of California Los Angeles, Los Angeles, CA, United States, ${ }^{4}$ Bioengineering, University of California Los Angeles, Los Angeles, CA, United States

Proton resonance frequency shift (PRF) is widely used for MR temperature mapping, but is not applicable in adipose tissues. $T_{1}$ measurement is an alternative MR temperature mapping method that can be applied in adipose tissues. Combined PRF- $T_{1}$ mapping has been evaluated for Cartesian MRI, but there is a lack of research for non-Cartesian techniques. In this work, we propose a new multi-echo 3D stack-of-radial technique that combines PRF and variable-flip-angle $\mathrm{T}_{1}$ measurement for MR temperature mapping. Preliminary results from laser ablation in phantoms demonstrate good agreement between temperature derived from both PRF and $\mathrm{T}_{1}$ compared to readings of temperature probes.

Detecting T1-based signal reduction in focused ultrasound heating of bone at 1.5T using a 3D spiral ultra-short echo time sequence

Helen Sporkin ${ }^{1}$, Yekaterina K Gilbo ${ }^{1}$, Sam W Fielden ${ }^{2}$, John P Mugler ${ }^{3}$, G. Wilson Miller ${ }^{3}$, Josef Pfeuffer ${ }^{4}$, Berthold Kiefer ${ }^{4}$, and Craig H Meyer ${ }^{1,3}$

${ }^{1}$ Biomedical Engineering, University of Virginia, Charlottesville, VA, United States, ${ }^{2}$ Autism and Developmental Medicine Institute, Geisinger Health System, Danville, PA, United States, ${ }^{3}$ Radiology and Medical Imaging, University of Virginia, Charlottesville, VA, United States, ${ }^{4}$ Application Development, Siemens Healthcare, Erlangen, Germany

MR-guided Focused Ultrasound (MRgFUS) is used transcranially to ablate brain tissue for the treatment of essential tremor and Parkinson's disease symptoms. Proton resonance frequency shift MR thermometry detects changes in temperature in tissues with sufficiently long T2, but fails to detect heating in the cortical bone of the skull. T1-based MR thermometry uses T1 mapping to observe a linear increase in T1 with temperature but requires long acquisitions. We demonstrate a thermometry method using the linear relationship between signal magnitude from a T1-weighted 3D Spiral Ultra-short Echo Time sequence and temperature in focused ultrasound heated bone with improved temporal resolution. 
Radiofrequency applicator concepts for RF hyperthermia treatment and MR imaging of glioblastoma multiforme at 7.0 T (298 MHz)

Eva Oberacker ${ }^{1}$, Andre Kuehne ${ }^{2}$, Helmar Waiczies ${ }^{2}$, Jacek Nadobny $^{3}$, Mirko Weihrauch ${ }^{3}$, Sebastian Zschaeck ${ }^{3}$, Pirus Ghadjar ${ }^{3}$, Peter Wust $^{3}$, Thoralf Niendorf $^{1,2,4}$, and Lukas Winter ${ }^{1}$

${ }^{1}$ Berlin Ultrahigh Field Facility (B.U.F.F.), Max Delbrueck Center for Molecular Medicine in the Helmholtz Association, Berlin, Germany, ${ }^{2}$ MRI.TOOLS GmbH, Berlin, Germany, ${ }^{3}$ Clinic for Radiation Oncology, Charite University Medicine, Berlin, Germany, ${ }^{4}$ Experimental and Clinical Research Center (ECRC), a joint cooperation between the Charite Medical Faculty and the Max Delbrueck Center for Molecular Medicine in the Helmholtz Association, Berlin, Germany

Glioblastoma multiforme is the most frequent and most aggressive malignant brain tumor with de facto no prognosis of long-term survival by the use of current multimodal therapeutic approaches. RF heating at ultrahigh fields $\left(B_{0}=7.0 T, f=298 M H z\right)$ has the potential of delivering sufficiently large thermal dosage for hyperthermia of relatively large tumor areas. This work focuses on EMF simulations and compares RF applicator designs tailored for simultaneous RF heating and MRI. Our results suggest that RF power can be focused to small tumor areas and to large clinical target volumes derived from segmented patient data.

Bio Heat Transfer Model Based Temporally Constrained Reconstruction for Accelerated MR Temperature Imaging

Shihan Qiu ${ }^{1}$, Jinchao Wu², Bingyao Chen ${ }^{3}$, Jiafei Yang ${ }^{3}$, Xing Wei ${ }^{3}$, and Kui Ying ${ }^{2,4}$

${ }^{1}$ Department of Biomedical Engineering, Tsinghua University, Beijing, China, ${ }^{2}$ Department of Engineering Physics, Tsinghua University, Beijing, China, ${ }^{3}$ Department of Orthopedics, First Affiliated Hospital of PLA General Hospital, Beijing, China, ${ }^{4}$ Key Laboratory of Particle and Radiation Imaging, Ministry of Education, Medical Physics and Engineering Institute, Tsinghua University, Beijing, China

Thermal therapies require accurate and real-time temperature monitoring to guide the treatment. To achieve higher temporal resolution in MR temperature imaging, we introduced bio heat transfer model to predict temperature maps, which are combined with previous image to act as constraints in the reconstruction of under-sampled data. An inverse optimization is also included to make the BHT model self-adaptive. Through robustness verifying experiment and heating simulation, the ability of the proposed method to provide accurate reconstruction at a high reduction factor is demonstrated in this study.

Accelerated MR-Thermometry Using Gradient Echo Keyhole for Focused Ultrasound

Radhika Tibrewala ${ }^{1}$, Viola Rieke ${ }^{1}$, and Eugene Ozhinsky ${ }^{1}$

${ }^{1}$ Radiology and Biomedical Imaging, University of California San Francisco, San Francisco, CA, United States

MRgFUS treatments require rapid imaging to visualize the temperature and accurately determine thermal dose. We propose accelerated gradient echo keyhole trajectories for MRthermometry, which acquire the middle of k-space densely (keyhole) while interleaving the outer k-space data. The trajectory acquisitions were synchronized to the ultrasound pulse to increase temperature accuracy. Different combinations of the keyhole size and number of interleaves were created and their accuracy was tested in a MATLAB simulation that uses the Bioheat Transfer Equation as a gold standard for temperature. The trajectories were implemented in RTHawk and results validated in a phantom experiment during focused ultrasound.

Passive Marker Tracking with Phase-Only Cross Correlation (POCC) in Highly Undersampled Radial Images: Improvements by Point-Spread-Function Considerations Andreas Reichert ${ }^{1}$, Michael Bock ${ }^{1}$, and Axel Joachim Krafft ${ }^{1}$

${ }^{1}$ Dept. of Radiology, Medical Physics, Medical Center University of Freiburg, Faculty of Medicine, University of Freiburg, Freiburg, Germany

Passive tracking with the phase-only cross correlation (POCC) algorithm can be used to accurately detect the position of MR-markers for needle procedures. The POCC tracking sequence continuously visualizes the planned needle trajectory during movement, however, image acquisition is interleaved with the measurement of two tracking images which degrades the temporal resolution. Here, it is shown that highly undersampled radial imaging together with the incorporation of the point-spread-function into the POCC algorithm can track the marker at substantially shorter acquisition times. This is an important step to improve the overall temporal resolution and might help to reduce durations of percutaneous procedures.

Cheng-Tao Ho ${ }^{1}$, Chen-Hua Wu ${ }^{1}$, Po-Hung Hsu², Hao-Li Liư ${ }^{3}$, Chih-Kuang Yeh ${ }^{1}$, Ching-Hsiang Fan ${ }^{1}$, Wen-Shiang Chen ${ }^{4,5}$, and Hsu-Hsia Peng ${ }^{1}$

${ }^{1}$ Department Of Biomedical Engineering And Environmental Sciences, National Tsing Hua University, Hsinchu City, Taiwan, ${ }^{2}$ Center for Advanced Molecular Imaging and Translation, Chang Gung Memorial Hospital, Taoyuan city, Taiwan, ${ }^{3}$ Department Of Electrical Engineering, Chang-gung University, Taoyuan city, Taiwan, ${ }^{4}$ Department Of Physical Medicine And Rehabilitation, National Taiwan University Hospital, Taipei city, Taiwan, ${ }^{5}$ Division Of Medical Engineering Research, National Health Research Institutes, Miaoli city, Taiwan 
The purpose of this study was to comprehend the mechanism of stable cavitation (SC)-induced signal intensity (SI) changes by fast spin-echo images in a phantom with flowing MBs. We postulated that the different patterns of SI changes might be related to transmitting FUS pulses at different timing of k-line acquisitions. The SC-induced microstreaming and shear force could generate hypo- and hyper-SI changes, respectively. In conclusion, the illustration of the mechanism could be helpful for designing experiments in monitoring SC-induced SI changes.

Simultaneous displacement and T2 mapping of High-intensity focused ultrasound therapy

Yangzi Qiao ${ }^{1}$, Chao Zou ${ }^{1}$, Zongwei Xu ${ }^{1,2}$, Chuanli Cheng ${ }^{1,3}$, Qian Wan ${ }^{1}$, Changjun Tie ${ }^{1}$, Xin Liu $^{1}$, and Hairong Zheng ${ }^{1}$

${ }^{1}$ Shenzhen Institutes of Advanced Technology, Chinese Academy of Sciences, Shenzhen, China, ${ }^{2}$ Zhengzhou University, Zhengzhou, China, ${ }^{3}$ University of Chinese Academy of Sciences, Beijing, China

In this work, a hybrid ARFI sequence based on segmented SE-EPI is proposed to simultaneously monitor the displacement and T2 change of tissue during HIFU therapy. The reliability of this sequence was validated first. The quantified displacement and T2 show good consistence with the reference ARFI and SE results. The hybrid sequence was then applied before and after HIFU therapy to evaluate the treatment effects. With the occurrence of ablative lesion, T2 relaxation time decreased in the lesion center and increased in the boundary. While the displaced region (region with obvious displacement) and the maximal displacement at focus both enlarged. In general, this hybrid ARFI is a potentially useful HIFU monitoring method in clinical application.

Monitoring of Acute Thermal Coagulation in Muscle Using PSIF Sequence in MRI-Guided High-Intensity Focused Ultrasound Therapy

Changjun Tie ${ }^{1}$, Chao Zou ${ }^{1}$, Qian Wan ${ }^{1,2}$, Yangzi Qiao ${ }^{1}$, Chuanli Cheng ${ }^{1,2}$, Xin Liu ${ }^{1}$, and Hairong Zheng ${ }^{1}$

${ }^{1}$ Shenzhen Institutes of Advanced Technology, Chinese Academy of Sciences, Shenzhen, GuangDong, China, ${ }^{2}$ University of Chinese Academy of Sciences, Beijing, China

MR-guided high intensity focused ultrasound (MRgHIFU) is a new noninvasive approach for thermal ablation of focal lesions with clinical applications in uterus, bone, prostate, brain, breast, and liver.Traditionally, the volume of tissue coagulation is evaluated through contrast enhanced T1-weighted images (CET1). However, there are several limitations for CET1 used for thermal lesion detection.

In this study, acute thermal damage following HIFU ablation in muscle was assessed using a PSIF images. this preclinical study demonstrates that PSIF sequence offers a good T2 contrast for visualizing acute thermal damage in muscle tissue during HIFU treatment, and has an obvious advantage in acquisition time, making PSIF a suitable sequence for real-time monitoring tissue changes during thermotherapy at high field system.

Feasibility Study for Off-Center Targets using ExAblate transcranial MR Guided Focused Ultrasound (tMRgFUS) System

Sijia Guo ${ }^{1}$, Jiachen Zhuo $^{1}$, and Rao P. Gullapalli ${ }^{1}$

${ }^{1}$ Department of Diagnostic Radiology and Nuclear Medicine, University of Maryland Medical Center, Baltimore, MD, United States

Recent approval by the FDA for treating essential tremors has created increased interest in targeting other critical regions within the brain. Relatively low frequency $220 \mathrm{kHz}$ tMRgFUS system has the potential to reach off-center targets compared to the $670 \mathrm{kHz}$ system used for essential tremor treatment. In this work, we assess the feasibility of the $220 \mathrm{kHz}$ reaching targets such as the central lateral thalamus $(\mathrm{CL})$ due to its role in neuropathic pain and the more laterally located temporal lope for the role it plays in temporal lobe epilepsy. Results suggest that temporal lobe interventions are possible but may require a careful optimization.

MR-HIFU setup for preclinical treatment of a mouse model of pancreatic ductal adenocarcinoma

Joshua Park ${ }^{1}$, Ravneet Vohra ${ }^{1}$, Mark Mathis ${ }^{1}$, Ari Partanen ${ }^{2}$, Cecil Hayes ${ }^{1}$, Yak-Nam Wang ${ }^{3}$, Stella Whang ${ }^{4}$, Joo Ha Hwang ${ }^{4}$, and Donghoon Lee

${ }^{1}$ Radiology, University of Washington, Seattle, WA, United States, ${ }^{2}$ Clinical Science MR Therapy, Philips, Andover, MA, United States, ${ }^{3}$ Applied Physics Laboratory, University of Washington, Seattle, WA, United States, ${ }^{4}$ Gastroenterology, University of Washington, Seattle, WA, United States

Preclinical studies using animal disease models on clinical MR-HIFU systems are important for human clinical translations but are often very challenging. We developed and tested a set of hardware components to treat a transgenic mouse model of pancreatic ductal adenocarcinoma on our clinical MR-HIFU system. The hardware components include an optimized RF coil, filter, RF switches and coil/animal holder. A gel phantom and a fixed mouse body were sonicated using the developed devices and a mild hyperthermia protocol on a $3 T$ MR-HIFU system. Pulse sequences for multi-parametric MRI were also tested to acquire optimum signal-to-noise ratio on the samples. 
During brain surgery, location of lesions can change in real-time due to leakage of cerebrospinal fluid. Therefore, navigating an MR-Endoscope probe with real-time intraoperative MRI is important in clinical application. However, conventional tracking system attached to the endoscope probe induces severe artifacts and is expensive and bulky. In this study, we propose a technique for navigating the endoscope probe without additional tracking system through segmentation of signals from tunable lens filled with gadolinium contrast agents. We also demonstrated tunable liquid-filled lens endoscope for intraoperative MRI. The proposed system/approach would be a good alternative as a tracking system for intraoperative MRI.

Improved MR thermometry for laser-induced thermal therapy - tradeoffs between imaging approaches

Henrik Odéen ${ }^{1}$ and Dennis L Parker

${ }^{1}$ Radiology and Imaging Sciences, University of Utah, Salt Lake City, UT, United States

MR thermometry is often used to monitor thermal therapies such as focused ultrasound and laser induced thermal therapies (LITT). As in MRI in general, there is an inherent tradeoff between measurement accuracy, precision, and spatial and temporal resolution in MR thermometry. In this work we present improved acquisition protocols for 2D and 3D MR thermometry for LITT applications. We investigate and compare image quality and temperature precision for 8 different 2D and 3D GRE, and 3D segmented EPI protocols. Experiments are performed in a healthy volunteer (non-heating) and tissue-mimicking gel (with heating).

MRI biomarkers for focused-ultrasound treatment of pancreatic ductal adenocarcinoma

Ezekiel Maloney ${ }^{1}$, Ravneet Vohra ${ }^{1}$, Yak-Nam Wang ${ }^{1}$, Tatiana Khokhlova ${ }^{1}$, Stella Whang ${ }^{1}$, Kayla Gravelle $^{1}$, Joshua Park ${ }^{1}$, JooHa Hwang $^{1}$, and Donghoon Lee ${ }^{1}$

${ }^{1}$ University of Washington, Seattle, WA, United States

Pancreatic cancer is a devastating disease with poor prognosis. Pancreatic tumor therapy has been ineffective in part because pancreatic tumors have high interstitial fluid pressure (IFP), driven by high hyaluronan concentration and a dense desmoplastic stroma that inhibit penetration of drugs into the tumor. We performed multi-parametric MRI at high resolution to non-invasively assess tumor response in a KPC mouse model to pulsed focused ultrasound treatments. T1 and T2 relaxation as well as diffusion, magnetization transfer, and chemical exchange saturation transfer methods were used to characterize the tumors before and after focused ultrasound treatment.

Performance evaluation of a B0-shim multi-coil system for small animal temperature mapping at 3T

Qiaoyan Chen ${ }^{1,2}$, Jo Lee ${ }^{1,2}$, Jianghong Wen ${ }^{1,2}$, Chao Zou ${ }^{1,2}$, Xiaoliang Zhang ${ }^{3,4}$, Xin Liu ${ }^{1,2}$, and Ye Li ${ }^{1,2}$

${ }^{1}$ Lauterbur Imaging Research Center, Shenzhen Institutes of Advanced Technology, Chinese Academy of Sciences, Shenzhen, China, ${ }^{2}$ Shenzhen Key Laboratory for MRI, Shenzhen, China, ${ }^{3}$ Department of Radiology and Biomedical Imaging, University of California San Francisco, San Francisco, CA, United States, ${ }^{4}$ UCSF/UC Berkeley Joint Graduate Group in Bioengineering, San Francisco, CA, United States

The magnetic field variation is a critical factor affecting the accuracy of temperature measurement in MRT. In this study, a 5-channel B0-shim coil was constructed for small animal temperature mapping in the MRI guided high intensity focused ultrasound (HIFU) at 3T. Firstly, the shimming ability was evaluated by the phantom study with a result that the standard deviation (STD) value of the offset magnetic field has reduced to $69 \%$ after currents optimized. Secondly, the relationship between T2* and SNR improvement has been studied. The results demonstrate that the temperature measurement accuracy is improved by $8 \%$ with the local multiple B0-shim coils.

Traditional Poster

\section{Cancer Imaging} Robinson ${ }^{1}$

${ }^{1}$ Division of Radiotherapy and Imaging, The Institute of Cancer Research, London, United Kingdom, ${ }^{2}$ Developmental Biology and Cancer Research Programme, Birth Defects Research Centre, Great Ormond Street Institute of Child Health, University College London, London, United Kingdom, ${ }^{3}$ Division of Clinical Studies, The Institute of Cancer Research, London, United Kingdom 


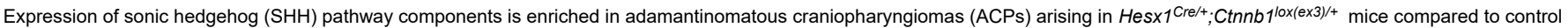
pituitaries. An MRI-embedded trial of smoothened inhibitor vismodegib in this genetically-engineered mouse model was undertaken to assess SHH pathway inhibition in ACP. Longitudinal MRI identified accelerated solid tumour growth in response to 28 days vismodegib treatment, which was associated with increased tumour cell proliferation, and resulted in shorter survival. 7 days of treatment induced early tumoural lesions in $\mathrm{Hes}{ }^{\mathrm{Cre} /{ }^{+}} ;{ }$Ctnnb $1^{\text {lox }(\mathrm{ex} 3) /+}$ pituitaries, resulted in a more undifferentiated and proliferative phenotype, and was associated with an elevated number of cells with clonogenic potential.

MRI-based radiomic to assess lipomatous soft tissue tumors malignancy: a pilot study

Benjamin Leporq ${ }^{1}$, Amine Bouhamama ${ }^{2}$, Fabrice Lame ${ }^{2}$, Catherine Bihane ${ }^{2}$, Michaël Sdika ${ }^{1}$, Jean-Yves Blay ${ }^{3}$, Olivier Beuf ${ }^{1}$, and Frank Pilleul ${ }^{1,2}$

${ }^{1}$ Laboratoire CREATIS (CNRS UMR 5220, Inserm U1206, INSA-Lyon, UCBL Lyon 1), Université de Lyon, Lyon, France, ${ }^{2}$ Department of radiology, Centre de lutte contre le cancer Léon Berard, Lyon, France, ${ }^{3}$ Department of oncology, Centre de lutte contre le cancer Léon Berard, Lyon, France

Aim of this study was to develop a MRI-based radiomic method to assess lipomatous soft tissue tumors malignancy. 105 subjects with lipomatous soft tissue tumors whose histology was known and with fat-suppressed T1w contrast enhanced MR images available were retrospectively enrolled to constitute a database. Based on histology, three groups were constituted according to malignancy from lipomas to high grade liposarcomas. A decisional algorithm based on 2 multivariate radiomic models was built to distinguish between these groups. Results demonstrate that the evaluation of lipomatous tumor malignancy is feasible using a routinely used MRI acquisition in clinical practice.

Magnetic resonance fingerprinting on a 1.5T MRI-Linac for tumor response monitoring

Tom Bruijnen ${ }^{1}$, Bjorn Stemkens ${ }^{1}$, Jan J W Lagendijk ${ }^{1}$, Cornelis A T van den Berg ${ }^{1}$, and Rob H N Tijssen ${ }^{1}$

${ }^{1}$ Radiotherapy, University Medical Center Utrecht, Utrecht, Netherlands

Magnetic resonance fingerprinting (MRF) is the ideal tool for rapid daily tumor response monitoring on a MRI-Linac (MRL). The 1.5T MRL used in our institution has a modified gradient coil and magnet coil design that potentially complicates the parameter quantification in MRF. In this work we are the first to demonstrate the feasibility of 2D MRF in phantoms and in-vivo on a 1.5T MRL. Moreover, we investigate the accuracy and precision of the parametric maps.

MRI-compatible intravital imaging window for longitudinal imaging of orthotopic mouse ovarian and pancreatic tumor stroma

Filip Bochner ${ }^{1}$, Vishnu Mohan ${ }^{1}$, Inbal Biton ${ }^{2}$, and Michal Neeman ${ }^{1}$

${ }^{1}$ Biological Regulation, Weizmann Institute of Science, Rehovot, Israel, ${ }^{2}$ Veterinary Resources, Weizmann Institute of Science, Rehovot, Israel

Longitudinal multi-modal imaging of abdominal organs remains a challenge. In cancer research, where data acquired at multiple spatial and temporal scales is especially valuable, combination of powerful microscopic methods with MRI can yield complementary information about ECM and vascular components of the tumor stroma, both constituting a hallmark of pancreatic and ovarian tumors. Here we present the MRI compatible optical imaging window for longitudinal imaging of ovary and pancreas.

Exploring the use of MR Elastography to probe immune cell-stromal interaction in tumour microenvironment

Ralph Sinkus ${ }^{1}$, Rachel Evans ${ }^{2}$, Fabian Flores-Borja ${ }^{3}$, and Tony $\mathrm{Ng}^{2}$

${ }^{1}$ Department of Radiological Imaging, King's College London, London, United Kingdom, ${ }^{2}$ School of Cancer and Pharmaceutical Sciences, King's College London, London, United Kingdom, ${ }^{3}$ School of Cancer and Pharmaceutical Sciences King's College London, London, United Kingdom

There is great, unmet need in understanding and monitoring non-invasively the immune cell changes within the tumour stromal microenvironment during cancer treatment. Howeve there is as yet no reliable non-invasive method of identifying at very early time points patients who are most likely to benefit from this relatively expensive class of treatments which generally are only associated with a clinical response in $25-30 \%$ of patients ${ }^{1}$. We show here in a mouse model that changes 11 days after implantation in the liquid-to-solid ratio (phase angle y) of the tumour biomechanics are indicative for successful immune cell - stromal cell interactions.

DKI can early detect radio-insensitive human nasopharyngeal carcinoma xenograft in nude mice

Xiang Zheng ${ }^{1}$, Yunbin Chen ${ }^{1}$, Youping Xiao ${ }^{1}$, and Dechun Zheng ${ }^{1}$

${ }^{1}$ Fujian Provincial Cancer Hospital, Fuzhou, China 
In order to evaluate feasibility of DKI sequence in early differentiating radio-insensitivity of nasopharyngeal carcinoma xenografts, Seventy-two nude mice were implanted with CNE1 (low radiosensitivity) and CNE-2(high radiosensitivity) and the xenografts were obtained. MRI scanning was performed after fractional irradiation. There are differences of the changes of DKI parameters (both D and K) between CNE-1 and CNE-2 before tumor volumes changed. Therefore, Both $\mathrm{D}$ and K can early (before volumes changed) distinguish radio-insensitive NPC xenografts from others.

Adult eye segmentation in MRI using active shape model: towards a personalized eye model for radiation treatment of uveal melanoma

Huu-Giao Nguyen, $\mathrm{PhD}^{1,2,3}$, Raphael Sznitman, Prof. ${ }^{2}$, Marta Peroni, $\mathrm{PhD}^{1}$, Jan Hrbacek, $\mathrm{PhD}^{1}$, Damien C. Weber, Prof. MD ${ }^{1}$, Alessia Pica, MD ${ }^{1}$, and Meritxell Bach Cuadra, PhD ${ }^{3,4}$

${ }^{1}$ Proton therapy Center, Paul Scherrer Institut (PSI), ETH Domain, Villigen, Switzerland, ${ }^{2}$ Ophthalmic Technology Laboratory, ARTORG Center of the University of Bern, Bern,

We aim to construct a 3-dimensional patient-specific eye model from MRI data in order to later be integrated into proton radiation treatment planning. Our major challenge is the presence of motion, as subjects are awake and physiologically blink eyes. Additionally, fixing a point during acquisition might be challenging for some patients with ocular tumors. As such, in this study we evaluated an Active Shape Model (ASM) segmentation on a data set of 31 subjects, including 3 uveal melanoma (UM) patients. Quantitative evaluation in comparison with manual delineations shows good accuracy, even for images with the presence of UM and tantalum clips.

Automatic classification between high grade gliomas and brain metastasis using Bag-Of-Features in comparison to statistical and morphologic features

Moran $\operatorname{Artzi}^{1,2}$, Gilad Liberman ${ }^{1,3}$, and Dafna Ben Bashat ${ }^{1,2,4}$

${ }^{1}$ Functional Brain Center, Tel Aviv Sourasky Medical Center, Tel Aviv, Israel, ${ }^{2}$ Sackler Faculty of Medicine, Tel Aviv University, Tel Aviv, Israel, ${ }^{3}$ Department of Chemical Physics, Weizmann Institute, Rehovot, Israel, ${ }^{4}$ Sagol School of Neuroscience, Tel Aviv University, Tel Aviv, Israel

This study suggests a clinical decision-support tool for automatic classification of brain tumors. Classification was performed on $179 \mathrm{MRI}$ patients: 81 patients with high grade-gliomas (HGG) and 98 patients with brain metastases (MET, 55 breast, 43 lung, cancer origin). The input data were Bag-Of-Features (BoF) and statistical-\&-morphologic features extracted from T1Wl+Gd. Classification was performed using five ensemble classifiers and results were evaluated using five-fold cross-validation. Best classification results produced accuracy $=83 \%$, sensitivity $=87 \%$, and specificity $=81 \%$ for discriminating between HGG and MET using Statistical-\&-morphologic features, and accuracy $=79 \%$, sensitivity $=76 \%$, and specificity $=80 \%$ for discriminating between breast and lung MET using BoF + Statistical-\&-morphologic features.

Dedicated 1.5T 16 channel array for MR-guided radiation treatment planning of head and neck tumors

Stefan Weick ${ }^{1}$, Kathrin Breuer ${ }^{1}$, Titus Lanz ${ }^{2}$, Michael Sauer ${ }^{2}$, Victor Lewitzki ${ }^{1}$, Bülent Polat ${ }^{1}$, Thorsten Bley ${ }^{3}$, and Michael Flentje

${ }^{1}$ Department of Radiation Oncology, University of Würzburg, Würzburg, Germany, ${ }^{2}$ Rapid Biomedical GmbH, Rimpar, Germany, ${ }^{3}$ Department of Radiology, University of Würzburg, Würzburg, Germany

Precise target delineation and safety margin definitions are mandatory in radiation treatment of head and neck tumors. In this context, magnetic resonance imaging (MRI) is increasingly used in addition to computed tomography (CT) in the treatment planning system because of its superior soft tissue contrast. In this work, a novel 16 channel head and neck array coil is presented, which is adapted to the special requirements of radiotherapy planning. It allows for MR imaging of patients with brain and head and neck tumors in treatment planning position in individual immobilization masks.

Investigating the effect of macromolecular cross-linking and increasing fiber density on the diffusion and viscoelastic properties of extracellular matrix materials using multiparametric MR

Hannah Macdonald ${ }^{1,2}$, Jeffrey Bamber ${ }^{1}$, David Collins ${ }^{1}$, Mihaela Rata ${ }^{1}$, Maxim Ryadnov $^{2}$, and Nandita deSouza ${ }^{1}$

${ }^{1}$ Institute of Cancer Research, London, United Kingdom, ${ }^{2}$ National Physical Laboratory, London, United Kingdom

Synthetic polymer polyvinylpyrrolidone and fibrous protein collagen were used to investigate the effect of macromolecular cross-linking and increasing fiber density on the physicochemical properties of extracellular matrix models using clinical MRI parameters and torsional rheometry. T1 and T2 decreased with increasing viscoelastic moduli of both materials. Covalent cross-linking of macromolecules by irradiation affected stiffness, but had a smaller effect than polymer concentration on T1, T2 and ADC. Collagen at increasing concentrations sufficient to substantially affect tissue stiffness (reflecting increasing fiber density) affected the structure of water within tissue, (changes in T1 and T2), but did not hinder water diffusion. 
${ }^{1}$ Department of Radiological Sciences, University of California, Los Angeles, Los Angeles, CA, United States, ${ }^{2}$ Physics and Biology in Medicine IDP, University of California, Los Angeles, Los Angeles, CA, United States, ${ }^{3}$ Ming Hsieh Department of Electrical Engineering, University of Southern California, Los Angeles, CA, United States

$\mathrm{B}_{1}{ }^{+}$correction is essential for quantitative prostate DCE-MRI. A simplified approximated analytical $\mathrm{B}_{1}{ }^{+}$correction method was proposed previously, and we assess this method on a digital reference object (DRO) with various SNR levels and on 110 in-vivo cases from two 3.0 T systems. We find that the approximated analytical $\mathrm{B}_{1}{ }^{+}$correction method achieves comparable performance to conventional correction method with substantially reduced computation. The approximated analytical correction method is simple and practical for application in the clinic.

Characterization of endometrioid adenocarcinoma microcirculation using distributed parameter model in DCE MRI

Zhi Jun Ye ${ }^{1}$, Gang Ning ${ }^{1}$, Hui Zhu Chen ${ }^{1}$, and Yan Song ${ }^{1}$

${ }^{1}$ West China Second University Hospital, Chengdu, China

Objective: To clarify the features of vascular proliferation and permeability in endometrioid adenocarcinoma. Methods: The DCE-MRI was applied to 55 women who confirmed as endometrioid adenocarcinoma with postoperative pathology. The receiver operating characteristic (ROC) analysis was employed using parameters derived with the DP model to differentiate tumor and normal myometrium and assess the diagnostic efficiency of these parameters. Results: $E$ and $P S$ in tumor was lower. $F$ in tumor was faster. Vp and Ve in tumor were lower. Areas under ROC curve (AUCs) for $E$ and PS attained values of 0.906 and 0.844 . AUCs for $F$ attained value of 0.548 . Vp and Ve in tumor with AUC values of 0.796 and 0.871 . Conclusion: The permeability of vascular wall was significantly lower in endometrioid adenocarcinoma, and the vascularity was moderately lower, suggestive of very different cell growth environment in endometrioid adenocarcinoma in comparison with most solid tumours.

Repeatability of intravoxel incoherent motion diffusion-weighted MRI during chemoradiation therapy in head and neck cancers

Ramesh Paudyal ${ }^{1}$, Nadeem Riaz ${ }^{2}$, Vaios Hatzoglou ${ }^{3}$, Xie Peng ${ }^{2,4}$, Jonathan Leeman ${ }^{2}$, David Aramburu Nunez ${ }^{1}$, Yonggang Lu ${ }^{5}$, Joseph O. Deasy ${ }^{1}$, Nancy Lee ${ }^{2}$, and Amita ShuklaDave $^{1,3}$

${ }^{1}$ Medical Physcis, Memorial Sloan Kettering Cancer Center, New York, NY, United States, ${ }^{2}$ Radiation Oncology, Memorial Sloan Kettering Cancer Center, New York, NY, United States, ${ }^{3}$ Radiology, Memorial Sloan Kettering Cancer Center, New York, NY, United States, ${ }^{4}$ Radiation Oncology, Shandong Cancer Hospital \& Institute, Jinan, China, ${ }^{5}$ Radiology, Medical College of Wisconsin, Milwaukee, WI, United States

The aim of this study is to determine the repeatability of pre- treatment (TX) and intra- TX week 1 imaging metrics derived from intravoxel incoherent motion diffusion weighted imaging (IVIM-DWI) in head and neck (HN) cancer patients during chemoradiation therapy. ADC, D, and $D^{*}$ imaging metrics showed better repeatability measurement than $f$ in the metastatic node of $\mathrm{HN}$ cancer patients.

Brain metastases developing pseudoprogression have poor vascular function and supply

Ingrid Digernes ${ }^{1}$, Endre Grøvik ${ }^{1}$, Line B. Nilsen ${ }^{1}$, Cathrine Saxhaug ${ }^{2}$, Oliver Geier ${ }^{1}$, Edmund Reitan ${ }^{2}$, Dag Ottar Sætre ${ }^{3}$, Birger Breivik ${ }^{4}$, Kari Dolven Jacobsen ${ }^{5}$, Åslaug Helland ${ }^{5}$, and Kyrre Eeg Emblem ${ }^{1}$

${ }^{1}$ Department of Diagnostic Physics, Oslo University Hospital, Oslo, Norway, ${ }^{2}$ Department of Radiology and Nuclear Medicine, Oslo University Hospital, Oslo, Norway, ${ }^{3}$ Department of Radiology, Østfold Hospital Trust, Kalnes, Norway, ${ }^{4}$ Deptartment of Radiology, Hospital of Southern Norway, Kristiansand, Norway, ${ }^{5}$ Department of Oncology, Oslo University Hospital, Oslo, Norway

Stereotactic radiosurgery of brain metastases can cause pseudoprogression. In this study, we use Vessel Architectural Imaging, based on dual echo DSC, to investigate the course of vascular function of brain metastases, both prior to and after pseudoprogression have occurred. Our results show that pseudoprogressing metastases were characterized by underperfused and oxygen-deprived tissue, and micro- and macrovessel pruning in the peritumoral regions. This was in contrast to peritumoral regions of responding metastases as well as normal-appearing brain tissue.

Grading of gliomas using Neurite orientation dispersion and density imaging (NODDI) on a clinical scanner

Arush Honnedevasthana Arun ${ }^{1}$, Aarthi Deepesh ${ }^{2}$, Dhritiman Chakrabarti², and Jitender Saini ${ }^{2}$

${ }^{1}$ Dayananda Sagar Institutions, Bangalore, India, ${ }^{2}$ National Institute of Mental Health and Neuroscience, Bangalore, India

Diffusion tensor imaging is sensitive to movement of water molecules but not specific as a biomarker in evaluating the highly complex microstructural environment of gliomas. Neurite orientation dispersion and density imaging (NODDI) uses different strengths of diffusion gradients to provide more specific indices of tissue microstructure than DTI. Patients with grade IV gliomas exhibited significant increase in both neurite density and orientation dispersion index as compared to grade III and II glioma cases. This study demonstrates clinical feasibility of using NODDI as a biomarker to grade tumors. 
Convolution-Difference Method for Feature Segmentation of Low-Resolution Images

Andrew A Maudsley

${ }^{1}$ Radiology, University of Miami, Miami, FL, United States

Automated lesion segmentation of clinical imaging studies is of potential value for treatment monitoring and radiation treatment planning. With low spatial resolution imaging systems, such as MR Spectroscopic Imaging, segmentation based on image intensity variations must take into consideration the broad spatial response function. In addition, the relative lesion-tobackground intensity variation and the object size must be considered. In this report a new automated image segmentation method is presented that accounts for these factors, which is based on a subtraction of a smoothed version of the MRSI maps from the original data.

A comparison of pseudo continuous arterial spin labeling perfusion MRI ( $\mathrm{PCASL}$ ) and permeability imaging with dynamic contrast-enhanced MRI (DCE-MRI) in human rectal cancer Yuichi Kumagae $^{1}$, Yoshihiko Fukukurra ${ }^{1}$, Koji Takumi $^{1}$, Hiroto Hakamada ${ }^{1}$, Tomoyuki Okuaki $^{2}$, and Takashi Yoshiura ${ }^{1}$

${ }^{1}$ Department of Radiology, Kagoshima University Graduate School of Medical and Dental Sciences, Kagoshima, Japan, ${ }^{2}$ Philips Electronics, Tokyo, Japan

Our purpose was to investigate potential correlations between the blood flow (BF) measured by pCASL and dynamic contrast-enhanced (DCE) MRI-derived pharmacokinetic parameters in rectal cancer. There were significant positive correlations between BF and $K^{\text {trans }}(p=0.006, r=0.579)$ or $K^{\text {ep }}(p=0.002, r=0.644)$. These results suggested that $p C A S L$ may have the potential to be a noninvasive alternative to DCE MRI

Surveillance in Germline TP53 Mutation Carriers Utilizing Whole-Body Magnetic Resonance Imaging

Kate Moodie ${ }^{1}$, Nick Ferris ${ }^{2}$, David Thomas ${ }^{3}$, Mandy Ballinger ${ }^{3}$, Emma Galligan ${ }^{1}$, Marion Harris ${ }^{4}$, Paul James ${ }^{1}$, Gillian Mitchell ${ }^{1}$, Eveline Niedermayr ${ }^{1}$, Bimal Parameswaran ${ }^{5}$, Deborah Schofield ${ }^{3}$, Sue Shanley ${ }^{6}$, Alison Trainer ${ }^{1}$, and Mary-Anne Young ${ }^{1}$

${ }^{1}$ Peter MacCallum Cancer Centre, Melbourne, Australia, ${ }^{2}$ Monash Imaging, Monash Health, Melbourne, Australia, ${ }^{3}$ Garvan Institute of Medical Research, Sydney, Australia, ${ }^{4}$ Monash Health, Melbourne, Australia, ${ }^{5}$ Eastern Health, Melbourne, Australia, ${ }^{6}$ Peter MacCallum Cancer Institute, Melbourne, Australia

Germline TP53 mutations are associated with Li-Fraumeni syndrome (LFS). Mutation carriers ascertained on family history have an extremely high lifetime risk of cancers arising from one or more of many possible sites. There is no established screening strategy for early detection and treatment of these cancers. Herein, we report preliminary data from a prospective study of a whole-body screening program that includes whole-body. Five new malignancies ( 3 de novo, 2 recurrent) have been identified in five of the first 30 participants, suggesting potentially significant benefits from screening in this population.

Assessment of micronecrotic tumor tissue using dynamic contrast-enhanced magnetic resonance imaging

Olga Schimpf ${ }^{1}$, Stefan Hindel ${ }^{1}$, and Lutz Lüdemann ${ }^{1}$

1527 'Strahlenklinik, Med. Physik, Universitätsklinikum Essen, Essen, Germany

Compartmental models for evaluation of dynamic contrast-enhanced magnetic resonance imaging (DCE-MRI) datasets assume a homogeneous interstitital volume distribution and homogeneous contrast agent (CA) distribution within each compartment, neglecting effects of CA diffusion within the compartments. When necrotic or micronecrotic tumor tissue is present, these assumptions may no longer be valid. Therefore, the present study investigates the validity of three compartmental models in assessing tumors with necrotic components.

Early biomarkers of response to neoadjuvant chemotherapy in lung cancer: preliminary data from a multicenter international study

Dominic Carlin ${ }^{1,2}$, Alexander Weller ${ }^{1,2}$, Joost Kuijer ${ }^{3}$, Gerbrand M Kramer $^{3}$, Arturo Chiti ${ }^{4}$, Mary E. R. O'Brien ${ }^{5}$, Sanjay Popat ${ }^{5}$, Yan Liu ${ }^{6}$, and Nandita M deSouza ${ }^{1,2}$

${ }^{1}$ CRUK Imaging Centre, Institute of Cancer Research, London, United Kingdom, ${ }^{2}$ MRI Unit, The Royal Marsden NHS Foundation Trust, Sutton, United Kingdom, ${ }^{3}$ VU University Medical Center, Amsterdam, Netherlands, ${ }^{4}$ Humanitas University, Milan, Italy, ${ }^{5}$ The Lung Unit, The Royal Marsden NHS Foundation Trust, Sutton, United Kingdom, ${ }^{6}$ European Organisation for Research and Treatment of Cancer Headquarters, Brussels, Belgium

Whole tumor ADC histogram parameters were assessed as early response biomarkers to platinum-based neo-adjuvant chemotherapy in 14 patients with non small cell lung cancer. On completion of treatment, 3 of 11 patients with DW-MRI at baseline and day 14 were classed responders by RECIST criteria. At Day 14 of treatment, there was a significant reduction in $\mathrm{ADC}$ metrics in responders ( 2 of 3 beyond limits of agreement) compared to non-responders ( 2 of 11 beyond limits of agreement). An increase in ADC 75 th centile (indicating more voxels with higher $A D C$ values), was consistent with necrosis; non-responders did not show this change. 
Developing a Halbach Array for Brain Tumor Targeting

Areej Alghamdi ${ }^{1}$, Munitta Munitta Muthana ${ }^{1}$, and Martyn Paley ${ }^{2}$

${ }^{1}$ Oncology and Metabolism, University of Sheffield, Sheffield, United Kingdom, ${ }^{2}$ Academic Radiology, University of Sheffield, Sheffield, United Kingdom

Steering magnetic nanoparticles (MNPs) in a desired trajectory has been proposed for guiding magnetically labelled drugs to clinical targets1. In order to steer MNPs to a desired location, a strong magnetic field and field gradient is necessary and the deeper the location, the stronger the magnetic force required. External permanent magnets can provide a strong magnetic field and gradient. We hypothesise that external magnetic field/field gradient arrays of 1.1T can be designed to capture MNPs into tumors. Brain tumors are one of the most difficult cancers to treat due to the complex anatomy of the brain. Therefore, we are developing a 3D printed brain tumor model to investigate trapping of MNPs into a tumor using Halbach arrays.

Lentiviral shRNA-mediated targeting of GDPD5 and GDPD6 in Orthotopic Human Breast Cancer Xenograft Models: A Metabolomics Study

Kanchan Sonkar ${ }^{1}$, Marina Stukova ${ }^{2}$, Caitlin M. Tressler ${ }^{1}$, Balaji Krishnamachary ${ }^{1}$, Zaver M. Bhujwalla ${ }^{1,3}$, and Kristine Glunde ${ }^{1,3}$

${ }^{1}$ The Russell H. Morgan Department of Radiology and Radiological Science, Johns Hopkins University School of Medicine, Baltimore, MD, United States, ${ }^{2}$ San Juan Bautista School of Medicine, Caguas, PR, United States, ${ }^{3}$ The Sidney Kimmel Comprehensive Cancer Center, The Johns Hopkins University School of Medicine, Baltimore, MD, United States

Activated choline phospholipid metabolism is a hallmark of cancer. Aggressive breast cancers are characterized by high tumoral phosphocholine and glycerophosphocholine. In our ongoing efforts of evaluating the glycerophosphodiesterases GDPD5 and GDPD6 as cancer treatment targets, we have systemically injected mice growing orthotopic triple-negative MDA-MB-231 breast tumors with lentiviral vectors that silence the GDPD5 or GDPD6 genes as compared to mice injected with control viruses. We have analyzed extracted tumor tissue by means of high-resolution ${ }^{1} \mathrm{H}$ MRS-based metabolomics. Differences in tumor growth and metabolic profiles were observed following silencing of GDPD5 and GDPD6 genes when compared to control mice.

Development of a 3D radial MP2RAGE sequence for free-breathing T1 mapping of the mouse abdomen

Thibaut L Faller ${ }^{1}$, Aurélien J Trotier ${ }^{1}$, Sylvain Miraux ${ }^{1}$, and Emeline J Ribot ${ }^{1}$

${ }^{1}$ CRMSB UMR5536, CNRS-Univ.Bordeaux, Bordeaux, France

T1 mapping could be useful to quantify the evolution of metastases over time and evaluate therapy efficiencies. The MP2RAGE sequence enables to obtain 3D T1 maps in reasonable scan time. Nevertheless, the standard sequence is too sensitive to respiratory motion, preventing its use at the abdominal level. Consequently, a 3D radial MP2RAGE sequence has been developed. The accuracy of the T1 measurements was evaluated in vitro and on the mouse brain. Then, abdominal 3D T1 maps were obtained without motion artifact while free breathing. Finally, the radial MP2RAGE sequence was used for the early detection and characterization of hepatic metastases.

Co-registration of MRI and histological habitats in pre-clinical tumor models

Bruna Victorasso Jardim-Perassi ${ }^{1}$, Suning Huang ${ }^{1}$, William Dominguez-Viqueira ${ }^{1}$, Epifanio Ruiz ${ }^{1}$, Mikalai Budzevich ${ }^{1}$, Jan Poleszczuk ${ }^{2}$, Marilyn Bui ${ }^{3}$, Robert Gillies ${ }^{1}$, and Gary Martinez ${ }^{1}$

${ }^{1}$ Cancer Imaging and Metabolism, Moffitt Cancer Center, Tampa, FL, United States, ${ }^{2}$ Nalecz Institute of Biocybernetics and Biomedical Engineering, Warsaw, Poland, ${ }^{3}$ Pathology Anatomic, Moffitt Cancer Center, Tampa, FL, United States

Tumor heterogeneity, may give insight into natural selection through detection of tumor sub-regions, referred as imaging habitats. We used statistical clustering of multiple pixels based on multiple MRI parameter maps to identify tumor habitats in pre-clinical models of sarcoma and breast cancer using T2, T2* ${ }^{*}$ ADC and three model free parameter maps determined from dynamic contrast enhanced images. MRI-derived habitat maps were determined by clustering multidimensional voxels using a Gaussian mixture model. 3D-printed tumor molds were used to successfully co-register MR imaging slices with their histological habitat-counterparts. Four distinct tumor habitats were detected by MRI and biologically corroborated by histology.

The Immune Checkpoint PD-L1 and Choline Kinase- $\alpha$ are inversely related in triple negative human breast cancer cells

Jesús Pacheco-Torres ${ }^{1}$, Marie-France Penet ${ }^{1,2}$, Yelena Mironchik ${ }^{1}$, Balaji Krishnamachary ${ }^{1}$, and Zaver M Bhujwalla ${ }^{1,2}$

${ }^{1}$ Division of Cancer Imaging Research, The Russell H. Morgan Department of Radiology and Radiological Science, The Johns Hopkins University School of Medicine, Baltimore, MD, United States, ${ }^{2}$ Sidney Kimmel Comprehensive Cancer Center, The Johns Hopkins University School of Medicine, Baltimore, MD, United States 
Immune checkpoint inhibition to activate the immune system has emerged as an exciting treatment option for several cancers. Programmed death-ligand 1 (PD-L1) plays a major role in immune suppression. We investigated the relationship between the aberrant choline metabolism observed in most cancers and PD-L1 expression in triple negative human MDA-MB-231 breast cancer cells. Using siRNA to downregulate choline kinase- $\alpha$ (Chk- $\alpha$ ) or PD-L1 or both, we identified a close inverse interdependence between Chk- $\alpha$, PD-L1 and phosphocholine. These results have significant implications for treatments that decrease Chk- $\alpha$ expression as these may drive up PD-L1 expression allowing escape of cancer cells from immune surveillance.

The relationship of $\mathrm{R} 1$ rho to aqueous $\mathrm{pH}$ and macromolecular density

Petros Fessas ${ }^{1}$, Syed Omar Ali ${ }^{1}$, Joshua Kaggie ${ }^{1}$, Martin Graves ${ }^{1}$, Scott Reid ${ }^{1}$, Gavin Houston ${ }^{1}$, and Ferdia Gallagher

${ }^{1}$ University of Cambridge, Cambridge, United Kingdom

We investigated the sensitivity of R1rho MRI to $\mathrm{pH}$ and macromolecular density in in vitro phantoms and in brains of volunteers to assess its suitability as an imaging modality for detecting and assessing the response of brain tumours. We find the dependence of $\mathrm{R} 1$ rho signal on $\mathrm{pH}$ in the presence of macromolecules, but a lack of $\mathrm{pH}$ dependence in their absence. We confirm R1rho sensitivity to macromolecular density at constant $\mathrm{pH}$.

Multiparametric MR approach for monitoring the pathological response of breast cancer patients to neoadjuvant chemotherapy

Naranamangalam R Jagannathan ${ }^{1}$, Uma Sharma ${ }^{1}$, Khusbhu Agarwal ${ }^{1}$, Rani G Sah ${ }^{1}$, Sandeep Mathur ${ }^{2}$, Vurthaluru Seenu ${ }^{3}$, Siddhartha D Gupta ${ }^{2}$, and Rajinder Parshad ${ }^{3}$

${ }^{1}$ Department of NMR and MRI Facility, All India Institute of Medical Sciences, New Delhi, India, ${ }^{2}$ Department of Pathology, All India Institute of Medical Sciences, New Delhi, India,

${ }^{3}$ Department of Surgery, All India Institute of Medical Sciences, New Delhi, India

A multiparametric MR approach using total choline (tCho), apparent diffusion coefficient (ADC) and tumor volume was undertaken for prediction of pathological response in 42 locally advanced breast cancer (LABC) patients undergoing neoadjuvant chemotherapy (NACT). 24 were pathologically responders (complete and partial) while 18 were non-responders. Percentage change in tCho, ADC and volume was higher in pathological responders than in non-responders after III NACT. Individually, all three parameters showed equal sensitivity $(66.7 \%)$ with specificity in the range $64.7 \%$ to $70.6 \%$ for pathological response prediction. Combination of all three MR parameters yielded $66.7 \%$ sensitivity and a specificity of $64.7 \%$.

Functional MRI at ultra-high field strength $(11.7 \mathrm{~T})$ for evaluation of rectal cancer stromal heterogeneity ex vivo: correlation with histopathology

Trang Thanh Pham ${ }^{1,2,3,4,5}$, Timothy Stait-Gardner ${ }^{6}$, C. Soon Lee Le, $^{3,4,7}$, Michael B. Barton ${ }^{1,3,4}$, Gary Liney ${ }^{1,3,4}$, Karen Wong ${ }^{1,3,4}$, and William S. Price ${ }^{5,6}$

${ }^{1}$ Radiation Oncology, Liverpool Cancer Therapy Centre, Liverpool Hospital, Sydney, Australia, ${ }^{2}$ Sydney West Radiation Oncology Network, Westmead, Blacktown and Nepean Hospitals, Sydney, Australia, ${ }^{3}$ Faculty of Medicine, University of New South Wales, Sydney, Australia, ${ }^{4}$ Ingham Institute for Applied Medical Research, Sydney, Australia, ${ }^{5}$ School of Medicine, Western Sydney University, Sydney, Australia, ${ }^{6}$ Nanoscale Organisation and Dynamics Group, Western Sydney University and National Imaging Facility, Sydney, Australia, ${ }^{7}$ Anatomical Pathology, Liverpool Hospital, Sydney, Australia

Diffusion Tensor Imaging (DTI) MRI at ultra-high field (11.7 T) was used to examine the stromal ultrastructure of malignant and normal rectal tissue ex vivo, and findings were correlated with histopathology. DTI was able to distinguish tumour from desmoplasia: tumour was found to have isotropic diffusion, whereas desmoplastic reaction or fibrous tissue had moderately anisotropic diffusion. DTI was useful in assessing depth of tumour infiltration into rectal wall: tumour was able to be distinguished from muscularis propria which was highly organised and anisotropic. This study showed that DTI-MRI can assist in more accurately defining tumour extent in rectal cancer.

Assessment of treatment response of lymphoma in an animal model with in vivo MR elastography

Jing Guo ${ }^{1}$, Animesh Bhattacharya ${ }^{2}$, Gergely Bertalan ${ }^{1}$, Jürgen Braun ${ }^{3}$, Clemens A. Schmitt²,4, , and Ingolf Sack ${ }^{1}$

${ }^{1}$ Radiology, Charité - Universitätsmedizin Berlin, Berlin, Germany, ${ }^{2}$ Medica Department of Hematology, Oncology, and Tumor Immunology, and Molecular Cancer Research Center (MKFZ), Charité - Universitätsmedizin Berlin, Berlin, Germany, ${ }^{3}$ Medical Informatics, Charité - Universitätsmedizin Berlin, Berlin, Germany, ${ }^{4}$ Berlin Institute of Health (BIH), Berlin, Germany, ${ }^{5}$ Max-Delbrück-Center for Molecular Medicine (MDC), Berlin, Germany

In this feasibility study, we have characterized the mechanical properties of lymphoma directly in the cervical lymph nodes with in vivo multifrequency MRE for the first time. Both MRE and diffusion weighted imaging were used to investigate the tumor's response to chemotherapy. We found that lymphomas stiffened 24 hours after chemotherapy which was accompanied by increased apparent diffusion coefficient (ADC) and reduced tumor volume. Wave speed obtained from MRE is sensitive in detecting the mechanical response of lymphoma to chemotherapy. Observed tumor stiffening post treatment needs to be validated by larger group size and should be explained by histological analysis. 
${ }^{1}$ Department of Radiology, Zhujiang Hospital, Southern Medical University, Guangzhou, China, ${ }^{2}$ Philips Healthcare, Guangzhou, China

There is attractive focus in developing non-invasive methods that assess tumor hypoxia. We applied tissue oxygen level dependent (TOLD) MRI to explore tumor oxygenation using VX2 liver tumor xenografts in a rabbit model. In this study, we demonstrated alteration in tumor oxygen inhalation and correlation in different hypoxia levels.

Dual-modality molecular imaging of choline kinase expression in lung cancer

Sofya A Osharovich ${ }^{1}$, Anatoliy V Popov ${ }^{1}$, David Holt ${ }^{2}$, Sunil Singhal ${ }^{3}$, and E. Jim Delikatny ${ }^{1}$

${ }^{1}$ Radiology, University of Pennsylvania, Philadelphia, PA, United States, ${ }^{2}$ School of Veterinary Medicine, University of Pennsylvania, Philadelphia, PA, United States, ${ }^{3}$ Surgery, University of Pennsylvania, Philadelphia, PA, United States

MR spectroscopy of tumors show elevated tCho resonances, reflecting increased levels of phosphocholine. This arises from overexpression of choline kinase (ChoK), which can be detected in breast tumor models using targeted near-infrared (NIR) probes and fluorescence optical imaging. This study translates these findings into lung cancer models, measuring elevated ChoK expression and activity in murine and human lung cancer cells and elevated ChoK levels in spontaneous canine adenocarcinomas. Dual modality molecular imaging could be employed using MRI and MRS for tumor staging, followed by NIR imaging for intraoperative surgical guidance, margin detection, and residual tumor removal, increasing patient survival.

MP2RAGE-Compressed Sensing for fast metastasis detection and characterization in mice

Aurélien Trotier ${ }^{1}$, Stanislas Rapacchi ${ }^{2}$, Thibaut Faller ${ }^{1}$, Sylvain Miraux ${ }^{1}$, and Emeline Ribot ${ }^{1}$

${ }^{1}$ CRMSB UMR5536, CNRS-Univ.Bordeaux, Bordeaux, France, ${ }^{2}$ CRMBM UMR7339, CNRS/Aix-Marseille Univ., Marseille, France

In order to detect and characterize metastases in preclinical studies, 3D T1 maps can be obtained with the MP2RAGE sequence. As high spatial resolution is required, the acquisition duration becomes prohibitive for the monitoring of metastases. Thus, acceleration via Compressed Sensing technique was achieved, necessitating a new undersampling scheme. T1 maps of the mouse whole brain were obtained in $<1 \mathrm{~min}$. The $\mathrm{T} 1$ of brain metastases was not affected by CS acceleration. Then, ultra-high spatially resolved maps $(130 \times 125 \times 141 \mu \mathrm{m})$ were acquired without lengthening scan time, to detect early-growing metastases and accurately measure their volumes.

Tumor Metabolism, Diffusion, and Perfusion in Head and Neck Cancer: Pretreatment Multimodality Imaging with DCE-MRI, IVIM DW-MRI, 18F-FMISO PET/CT, and 18F-FDG PET/CT David Aramburu Nunez ${ }^{1}$, Milan Grkovski ${ }^{1}$, Nancy Lee $^{2}$, Vaios Hatzoglou ${ }^{3}$, Heiko Schoder $^{3}$, Ramesh Paudyal ${ }^{1}$, Nadeem Riaz ${ }^{2}$, Joseph O Deasy ${ }^{1}$, John Humm $^{1}$, and Amita Shukla-Dave ${ }^{4}$

${ }^{1}$ Medical Physics, Memorial Sloan-Kettering Cancer Center, New York City, NY, United States, ${ }^{2}$ Radiation Oncology, Memorial Sloan-Kettering Cancer Center, New York City, NY, United States, ${ }^{3}$ Radiology, Memorial Sloan-Kettering Cancer Center, New York City, NY, United States, ${ }^{4}$ Medical Physics and Radiology, Memorial Sloan-Kettering Cancer Center, New York City, NY, United States

The aim of this study is to understand the correlation of pretreatment quantitative imaging metrics obtained from multimodality imaging (MMI) techniques, such as DCE-MRI, IVIM DWMRI, 18F-FMISO PET/CT, and 18F-FDG PET/CT giving us a comprehensive characterization of the tumor in head and neck cancer (HNC) patients. The results show complementary, rather than competitive, information about tumor metabolism, diffusion, and perfusion.

MRI exploration of the subventricular region of the third ventricle and its association with neurofibromatosis type-1 and white matter integrity in children with optic pathway glioma Natalie R Boonzaier ${ }^{1}$, Patrick W Hales ${ }^{1}$, Felice D'Arco ${ }^{2}$, Kshitij Mankad ${ }^{2}$, Darren Hargrave ${ }^{3}$, and Christopher Clark $^{1}$

${ }^{1}$ Developmental Imaging and Biophysics Section, Developmental Neurosciences, University College London Great Ormond Street Institute of Child Health, London, United Kingdom, ${ }^{2}$ Radiology Department, Great Ormond Street Children's Hospital, London, United Kingdom, ${ }^{3}$ Haematology and Oncology Department, Great Ormond Street Children's Hospital, London, United Kingdom

The lateral subventricular zone has been explored in association with high-grade gliomas, both in-vivo and with MRI. The third ventricle subventricular zone (TVZ) has been explored invivo, using immunohistochemistry and microarray analysis, with regard to neurofibromatosis type-1-associated low-grade optic pathway gliomas. This remains unexplored with MRI. This study examined diffusion MRI features of the TVZ and its association with NF1-status and peri-tumour white matter integrity. TVZ features correlated with NF1-status, and peri-tumour white matter integrity. These results suggest that the state of the TVZ environment can potentially indicate whether a sporadic tumour might behave like its less disruptive NF1associated counterpart 
Noa Urman ${ }^{1}$, Shay Levi ${ }^{1}$, Avital Frenkel ${ }^{1}$, Ariel Naveh ${ }^{1}$, Doron Manzur ${ }^{1}$, Gitit Lavy-Shahaf ${ }^{1}$, Hadas Sara Hershkovich ${ }^{1}$, Cornelia Wenger ${ }^{2}$, Ofir Yesharim ${ }^{1}$, Eilon Kirson ${ }^{1}$, and Ze'ev Bomzon $^{1}$

${ }^{1}$ Novocure, Haifa, Israel, ${ }^{2}$ Novocure GbmH, Root, Switzerland

Tumor Treating Fields (TTFields) are electric fields at an intermediate frequency approved for treatment of Glioblastoma Multiforme. Understanding how TTFields distribution in the brain influences disease progression can be studied using numerical simulations. Creation of computational patient models involves accurate segmentation of patient MRIs, a task that cannot be performed automatically, and is therefore time-consuming. We present a method for rapidly creating patient head models using a healthy head model as a deformable template. The method is robust even when MRI data quality is low. It is enabling a study correlating the spatial distribution of TTFields and patient outcome.

Dose reduction in myxoid liposarcomas: Initial descriptive results in the evaluation of response using multiparametric MRI.

Evanthia Kousi ${ }^{1}$, Maria A Schmidt ${ }^{1}$, Shane Zaidi ${ }^{2}$, Khin Thway ${ }^{3}$, Cyril Fisher ${ }^{3}$, Myles Smith ${ }^{4}$, Dirk Strauss ${ }^{4}$, Andrew Hayes ${ }^{4}$, Eleanor Moskovic ${ }^{5}$, Nicos Fotiadis $^{5}$, Elizabeth Barquin ${ }^{2}$, Komal Amin ${ }^{6}$, Rick Haas ${ }^{7}$, Christina Messiou ${ }^{5}$, and Aisha Miah ${ }^{2}$

${ }^{1}$ CR-UK and EPSRC Cancer Imaging Centre, Royal Marsden NHS Foundation Trust \& Institute of Cancer Research, Sutton, United Kingdom, ${ }^{2}$ Sarcoma Unit, Royal Marsden NHS Foundation Trust, London, United Kingdom, ${ }^{3}$ Pathology, Royal Marsden NHS Foundation Trust, London, United Kingdom, ${ }^{4}$ Academic Surgery, Royal Marsden NHS Foundation Trust, London, United Kingdom, ${ }^{5}$ Radiology, Royal Marsden NHS Foundation Trust, London, United Kingdom, ${ }^{6}$ Radiotherapy, Royal Marsden NHS Foundation Trust, London, United Kingdom,

${ }^{7}$ Radiotherapy, Neetherlands Cancer Institute, Amsterdam, Netherlands

Compared to other soft tissue sarcomas (STSs), myxoid liposarcomas (MLSs) are exquisitely radiosensitive. The clinicopathological response following pre-operative radiotherapy at 50 Gy/25\# in MLS might be due to radiation induction vascular damage. Here we report initial results in using multiparametric MRI (diffusion-weighted imaging, pharmacokinetic modelling and T2* measurements) to evaluate MLS response during and after preoperative RT. Dynamic contrast-enhanced examinations demonstrated both heterogeneous and homogeneous enhancement patterns. The tissue enhancement curve was monotonically-increasing in all cases, suggesting a distinct vascular pattern. Permeability and perfusion decreases from baseline in responders show Ktrans and IAUGC60 can potentially predict response.

Superpixels-based Segmentation and Automated Identification of Active Tumour and Necrotic regions in Bone Tumor using T1 and Diffusion Weighted Imaging

Amit Mehndiratta ${ }^{1}$, Esha Baidya Kayal ${ }^{1}$, Sneha Patil Kulkarni ${ }^{1}$, Raju Sharma ${ }^{2}$, Devasenathipathy Kandasamy ${ }^{2}$, and Sameer Bakhshi ${ }^{3}$

${ }^{1}$ Centre for Biomedical Engineering, Indian Institute of Technology Delhi, New Delhi, India, ${ }^{2}$ Department of Radiology, All India Institute of Medical Sciences, New Delhi, India,

${ }^{3}$ Department of Medical Oncology, IRCH, All India Institute of Medical Sciences, New Delhi, India

Proper Delineation of the tumour boundary and assessment of tumour size can take crucial part in treatment planning and monitoring treatment response. We investigate a fully automated Simple linear iterative clustering (SLIC) superpixel-based method for detection and segmentation of pathological tissues like oedema, tumour and necrosis associated with Osteosarcoma. Experimental results provide a close match to expert delineation and was able to estimate areas of active tumor and necrosis with good accuracy.

Prostate MR Elastography: a comparison of image acquisitions strategies in healthy volunteers

Kay Pepin ${ }^{1}$, Kevin Glaser ${ }^{1}$, Yi Sui ${ }^{1}$, Roger Grimm ${ }^{1}$, Arvin Arani ${ }^{1}$, Phillip Rossman ${ }^{1}$, Richard Ehman ${ }^{1}$, Michael Herman ${ }^{1}$, and Lance Mynderse ${ }^{1}$

$1546 \quad{ }^{1}$ Mayo Clinic, Rochester, MN, United States

The purpose of this study was to compare image acquisition strategies for prostate MRE using external drivers. Additionally, to assess the normal heterogeneity of prostate mechanical properties in an age-matched cohort to the prostate cancer population. Improved resolution using higher MRE vibration frequencies, larger acquisition matrices, and distortion-reduction techniques, may help advance the clinical application of prostate MRE.

Liver metabolomic investigation of lentiviral targeting of GDPD5 and GDPD6 for breast cancer treatment in a preclinical model

Kanchan Sonkar ${ }^{1}$, Marina Stukova ${ }^{2}$, Caitlin M. Tressler ${ }^{1}$, Balaji Krishnamachary ${ }^{1}$, Zaver M. Bhujwalla ${ }^{1,3}$, and Kristine Glunde ${ }^{1,3}$

${ }^{1}$ The Russell H. Morgan Department of Radiology and Radiological Science, Johns Hopkins University School of Medicine, Baltimore, MD, United States, ${ }^{2}$ San Juan Bautista School of Medicine, Caguas, PR, United States, ${ }^{3}$ The Sidney Kimmel Comprehensive Cancer Center, The Johns Hopkins University School of Medicine, Baltimore, MD, United States

High-resolution ${ }^{1} \mathrm{H}$ MRS is a powerful technique for metabolomics studies of tissues, cells, and body fluids. Here we have used this technique to explore metabolomic changes in the livers of mice that have been treated with lentiviral particles that silence either of the two glycerophosphodiesterase GDPD5 (GDPD5-shRNA) or GDPD6 (GDPD6-shRNA). We systemically administered lentiviral shRNA in mice with orthotopic breast tumor xenografts. We identified distinct increases in leucine, valine, glutathione, creatine, glucose, tyrosine, and histidine in the GDPD5-shRNA treated group, whereas cholesterol, isoleucine, beta-hydroxy butyrate, alanine, glutamate, glutamine, aspartate, fumerate, phenylalanine, and formate were elevated in the GDPD6-shRNA treated group. 
Vascular-induced spin dephasing in real vascular networks reveals useful decay characteristics to differentiate glioblastoma from healthy brain tissue

Artur Hahn¹, Thomas Kruewel ${ }^{2}$, Julia Bode ${ }^{2}$, Lukas Reinhold Buschle ${ }^{1,3}$, Björn Tews ${ }^{2}$, Sabine Heiland ${ }^{1}$, Martin Bendszus ${ }^{1}$, Christian Herbert Ziener ${ }^{1,3}$, and Felix Tobias Kurz ${ }^{1,3}$

${ }^{1}$ Neuroradiology, Heidelberg University Hospital, Heidelberg, Germany, ${ }^{2}$ Molecular Mechanisms of Tumor Invasion (V077), German Cancer Research Center (DKFZ), Heidelberg, Germany, ${ }^{3}$ E010 Radiology, German Cancer Research Center (DKFZ), Heidelberg, Germany

The transverse relaxation attributed to spin dephasing, caused by microscopic field inhomogeneities throughout a single imaging voxel, induced by the BOLD-mechanism, is studied using realistic three-dimensional microvascular structures, attained with fluorescence ultramicroscopy from mouse brains, and custom-written simulations to uncover differences between glioblastoma and healthy brain tissue. The signal attenuation is weaker and more heterogeneous in tumor tissue. Relaxation rates scale differently with varying field strengths or blood properties and the relaxation processes exhibit strong deviations from Lorentzian decay. The results are important for the development of signal processing methods for tumor diagnosis without contrast agents.

Effect of corrections for image distortion and gradient nonlinearity on longitudinal DTI tumor measurements in breast patients receiving neoadjuvant chemotherapy

Lisa J Wilmes ${ }^{1}$, Ek-Tsoon Tan ${ }^{2}$, Evelyn Proctor $^{1}$, Wen Li ${ }^{1}$, Jessica Gibbs ${ }^{1}$, Nola Hylton ${ }^{1}$, and David C Newitt ${ }^{1}$

${ }^{1}$ University of California San Francisco, San Francisco, CA, United States, ${ }^{2}$ GE Global Research, Niskayuna, NY, United States

Diffusion weighted imaging has shown promise for assessing tumor response to treatment, but suffers from gradient nonlinearity and image distortion that may adversely affect quantitative accuracy. This work evaluates corrections for image distortion (susceptibility-induced and eddy current) and bias from gradient non-linearity (GN) on breast tumor DTI metrics prior to treatment (TO) and at an early-treatment time point (T1), in six breast cancer patients undergoing neaoadjuvnt chemotherapy. Both GN and distortion correction had significant effects on tumor ADC and FA values at T0 and T1. The addition of distortion correction also improved the alignment of DTI and DCE-MRI tumor ROIs.

18F-FDG PET/MRI in Children with Oncologic Diseases: Initial Experience

Hansel Javier Otero ${ }^{1}$, Carolina L Maya ${ }^{1}$, Sabah E Servaes ${ }^{2}$, Jeffrey P Schmall ${ }^{1}$, and Lisa J States ${ }^{1}$

${ }^{1}$ Radiology, Children's Hospital of Philadelphia, Philadelphia, PA, United States, ${ }^{2}$ Raidology, Children's Hospital of Philadelphia, Philadelphia, PA, United States

We describe our initial experience with integrated whole-body Fluor-18-Fluordesoxyglucose-PET/MR imaging in children in a retrospective study of all 18F-FDG-PET/MR at our institution. 51 studies were carried out in 41 children ( 34 girls, 17 boys) with a mean age of 10.16 years (10 months-24 years). Primary diagnosis included rhabdomyosarcoma ( $\mathrm{n}=18$ ) and Osteosarcoma ( $n=5)$. The majority of studies $(n=29,57.9 \%)$ were performed for treatment response/restaging. All studies were diagnostic (technical success rate $100 \%)$. The mean effective dose was $5.25 \mathrm{mSv}$ (2.1-11.5 mSv). Mean total imaging time was 80 minutes (42-138 minutes). Thirty-eight (74.5\%) cases had an average of 2.2 additional MR sequences. $18 F-F D G$ PET/MR is technically feasible for the evaluation of oncologic processes in children at a fraction of the radiation dose.

Integrating Magnetic Resonance Imaging with Live Lung Intravital Microscopy: A Novel Platform to Evaluate the Effect of Radiation on Lung Tumors

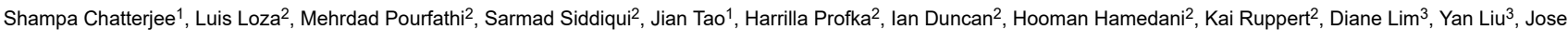
Conejo-Garcia ${ }^{4}$, Mary Spencer ${ }^{2}$, Tahmina Achekzai², Stephen Kadlecek², and Rahim R. Rizi

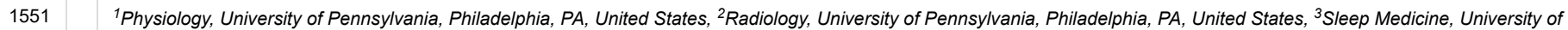
Pennsylvania, Philadelphia, PA, United States, ${ }^{4}$ Moffitt Cancer Center, Tampa, FL, United States

We propose that, when used in combination with MRI imaging, live lung intravital fluorescence microscopy can be a powerful tool for detecting the effects of radiotherapy on lung tumors. In this study, we monitored pulmonary nodules pre- and post-radiation in a novel murine model (Kras(G12D)/p53/f/l/myr-p110) with tumor regulation by Cre-recombinase. Using the reporter gene EGFP fluorescence, a significant loss of the tumor was observed post-radiation, which correlated with reduced fluorescent signal from the same region of the lung.

Effect of Stereotactic Body Radiotherapy on Perfusion and Diffusion in Prostate Tumor and Benign Tissue

Kristen Zakian ${ }^{1}$, Hebert Vargas Alvarez ${ }^{1,2}$, Andreas Wibmer ${ }^{2}$, Aditi lyer $^{2}$, Neelam Tyagi $^{2}$, Aditya Apte ${ }^{2}$, Marissa Kollmeier ${ }^{2}$, Boris Mychalczak ${ }^{2}$, Karen Borofsky $^{2}$, Oren Cahlon $^{2}$, Yousef $^{2}$ Mazaheri Tehrani ${ }^{2}$, Margie Hunt ${ }^{2}$, and Michael Zelefsky ${ }^{2}$

Multimodality MRI including DCE-MRI and DW-MRI were performed in patients prior to and following hypofractionated stereotactic body radiotherapy (SBRT). Diffusion and perfusion related parameters in both tumor and non-tumor benign tissue were calculated at 3,6 , and 12 months after SBRT. Radiation-induced changes were observed in perfusion and diffusion related parameters in tumors. In the non-tumor transition zone, SBRT induced changes in perfusion-related parameters. Multimodality MRI has potential for treatment effect monitoring in the prostate after SBRT. 
An integrated, semi-automated 3D printed Breast DCE-MRI phantom solution to generate diverse pharmacokinetic curves

Nithin N Vajuvalli ${ }^{1}$, Amaresha Shridhar Konar ${ }^{1}$, Shivaprasad Ashok Chikop ${ }^{1,2}$, Ramesh Venkatesan ${ }^{2}$, and Sairam Geethanath ${ }^{1,3}$

${ }^{1}$ Medical Imaging Research Centre, Dayananda Sagar Institution, Bangalore, India, ${ }^{2}$ Wipro GE healthcare, Bangalore, India, ${ }^{3}$ Department of Radiology, Columbia University Medical Centre, New York, NY, United States

In vitro phantoms play a critical role in the assessment of novel Dynamic Contrast Enhanced MRI (DCE-MRI) methods related to acquisition and reconstruction, among other advantages such as repeatability and reproducibility. In this work, we demonstrate a 3D printed breast DCE-MRI phantom that is capable of producing diverse kinetic curves as those seen in human patients. The wash-in and wash-out characteristics were controlled through user controlled $\mathrm{K}^{\text {trans }}$ values and the geometry of the phantom respectively. The phantom demonstrated in this work is 3D printed, cost effective, user interface controlled, and integrated with a peristaltic pump to obtain different kinetic curves.

Traditional Poster

\section{Fiber Orientation \& Fiber Tracking}

Damped Richardson-Lucy deconvolution for multi-shell diffusion MRI

Fenghua Guo ${ }^{1}$, Alexander Leemans ${ }^{1}$, Max Viergever ${ }^{1}$, Flavio Dell'acqua ${ }^{2}$, and Alberto De Luca ${ }^{1}$

${ }^{1}$ Image Sciences Institute, University Medical Center Utrecht, Utrecht, Netherlands, ${ }^{2}$ NATBRAINLAB, Department of Neuroimaging and NIHR Biomedical Research Centre, Institute of Psychiatry, King's College London, London, United Kingdom

The damped Richardson-Lucy (DRL) algorithm is a popular spherical deconvolution technique to quantify fiber orientation distributions from single-shell brain diffusion MRI (dMRI) data. Thanks to the progress of acquisition hardware, it is becoming increasingly common to acquire multi-shell dMRI data, which has the potential, to deliver additional information on the microstructure of tissues. In this work we extended the DRL framework to accommodate multi-shell data while accounting for multiple tissue types in the brain, to reduce partial volume contamination on the main FODs. The approach was tested on two dataset and proved to be stable over different acquisition schemes.

Bundle-specific tractography using voxel-wise orientation priors

Francois Rheault ${ }^{1}$, Etienne St-Onge ${ }^{2}$, Quentin Chenot $^{3}$, Laurent Petit ${ }^{3}$, and Maxime Descoteaux ${ }^{2}$

${ }^{1}$ Computer Science, Université de Sherbrooke, Lac-Etchemin, QC, Canada, ${ }^{2}$ Computer Science, Université de Sherbrooke, Sherbrooke, QC, Canada, ${ }^{3}$ Groupe d'Imagerie Neurofonctionnelles, Institut des Maladies Neurodégénératives (GIN-IMN) - UMR 5293, CNRS, CEA, Université de Bordeaux, Bordeaux, France

Diffusion tractography allows the investigation of white matter (WM) pathways of interest. However, to cover the full spatial extent of the desired bundles, tractography requires a large amount of streamlines (millions) to be generated. In this work, we developed a bundle-specific tractography algorithm using voxel-wise orientation priors. Our method aims to be more efficient than a classical whole brain tractography and increase the quality of virtual WM dissection.

Exploring Local Geometric Structure of Fiber Tracts Using Tract-Based Director Field Analysis

Jian Cheng ${ }^{1,2}$, Tao Liu ${ }^{3}$, Feng Shi ${ }^{4}$, Ruiliang Bai ${ }^{5}$, Jicong Zhang ${ }^{3}$, Haogang Zhu ${ }^{3}$, Dacheng Tao ${ }^{2}$, and Peter J. Basser ${ }^{1}$

${ }^{1}$ National Institutes of Health, Bethesda, MD, United States, ${ }^{2}$ University of Sydney, Sydney, Australia, ${ }^{3}$ Beihang University, Beijing, China, ${ }^{4}$ Cedars Sinai Medical Center, Los Angeles, CA, United States, ${ }^{5}$ Zhejiang University, Hangzhou, China

Inspired by distortion analysis of liquid crystals [1], we propose a novel mathematical framework, called tract-based director field analysis (TDFA), to explore the local geometric structure of fiber tracts after tractography. TDFA provides 6 scalar indices along tracts to quantify local orientational dispersion and orientational distortion (splay, bend, and twist) of fiber tracts. To our knowledge, this is the first work to quantify "splay", "bend" and "twist" of fiber tracts, although the three terms have been widely used to qualitatively describe the complexity of fiber tracts for about 20 years [2]. Synthetic and real data experiments demonstrate the effectiveness of the proposed scalar indices.

Divya Varadarajan ${ }^{1}$ and Justin P. Haldar ${ }^{1}$

${ }^{1}$ University of Southern California, Los Angeles, CA, United States 
Hiqh-quality diffusion tractography depends on the accurate estimation of orientation distribution functions (ODFs). Existing estimation methods often use modeling assumptions that are violated by real data, lack theoretical characterization, and/or are only applicable to a narrow class of q-space sampling patterns. As a result, existing approaches may be suboptimal. This work proposes a novel ODF estimation approach that learns a linear ODF estimator from training data. The approach can be applied to arbitrary q-space sampling schemes, has strong theoretical justification, and it can be shown that the trained estimators will generalize to new settings they weren't trained for.

Investigating the streamline count required for reproducible structural connectome construction across a range of brain parcellation resolutions

Chun-Hung Yeh ${ }^{1}$, Robert Elton Smith ${ }^{1}$, Xiaoyun Liang ${ }^{1}$, Fernando Calamante ${ }^{1,2}$, and Alan Connelly ${ }^{1,2}$

${ }^{1}$ The Florey Institute of Neuroscience and Mental Health, Melbourne, Australia, ${ }^{2}$ The Florey Department of Neuroscience and Mental Health, University of Melbourne, Melbourne, Australia

This study systematically investigates a fundamental question for tractogram-based connectomics research: for a given resolution of brain parcellation, how many streamlines are required for reproducible connectome construction? We incorporate state-of-the-art tractography techniques with surface parcellation schemes of multiple granularities to investigate the influence of streamline count on the connectome variability. Our results suggest that selecting an appropriate number of streamlines is crucial for global and per-edge variability of the connectome, revealing important implications for subsequent network analysis and inferences. Methods that investigate structural connectivity with different brain parcellation resolutions should benefit from the experimental workflow and outcomes of this study.

Spherical deconvolution of diffusion MRI data with tensor-valued encodings

Ben Jeurissen ${ }^{1}$ and Filip Szczepankiewicz ${ }^{2,3}$

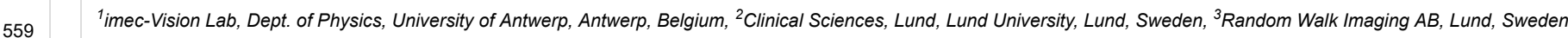

Multi-tissue constrained spherical deconvolution (MT-CSD) exploits the characteristic b-value dependency of each tissue type to estimate both the apparent tissue densities and the full white matter (WM) fiber orientation distribution function from diffusion MRI data. In this work, we extend the MT-CSD approach to account for data acquired with nonlinear and multiple btensor shapes and show that multiple b-tensor shapes can provide a new means of contrast between tissue types, in particular between gray matter and WM. Our approach provides high-quality apparent tissue density maps and high-quality fiber tracking from data with multiple b-tensor shapes, even with sparse q-space samplings.

Free Water Elimination Improves Tractography Through Multiple Sclerosis Lesions

Brittany Gilchrist ${ }^{1,2}$, Sidong Liu ${ }^{1,3,4}$, Chenyu Wang ${ }^{3,4}$, Ofer Pasternak ${ }^{5}$, Yuyi You ${ }^{1,2}$, and Alexander Klistorner ${ }^{1,2,3}$

${ }^{1}$ Save Sight Institute, Sydney Medical School, University of Sydney, Sydney, Australia, ${ }^{2}$ Faculty of Medicine and Health Sciences, Macquarie University, Sydney, Australia, ${ }^{3}$ Brain and Mind Centre, University of Sydney, Sydney, Australia, ${ }^{4}$ Sydney Neuroimaging Analysis Centre, Sydney, Australia, ${ }^{5}$ Brigham and Women's Hospital, Harvard Medical School, Boston, MA, United States

Axonal loss within chronic MS lesions is typically accompanied by increase of extra-cellular space. Reduction of anisotropy caused by this excessive extra-cellular water may limit the ability of tractography techniques to accurately detect fibre bundles. The aim of this study was to examine if application of free water elimination (FWE) algorithm may improve deterministic tractography through MS lesions. We show that elimination of free water markedly increases detection of lesional fibre bundles. While this effect was observed in the majority of lesions, it was more apparent in lesions with small initial number of fibres and in lesions categorised as severely damaged.

Is removal of weak connections necessary for dense weighted structural connectomes?

Oren Civier ${ }^{1}$, Robert Elton Smith ${ }^{1,2}$, Chun-Hung Yeh $^{1}$, Alan Connelly ${ }^{1,2}$, and Fernando Calamante ${ }^{1,2}$

${ }^{1}$ Florey Institute of Neuroscience and Mental Health, Melbourne, Australia, ${ }^{2}$ Florey Department of Neuroscience and Mental Health, University of Melbourne, Melbourne, Australia

Recent advances in tractography enable the generation of weighted structural connectomes where connection strengths are biologically meaningful. However, use of probabilistic tracking algorithms leads to dense graphs with many low-strength connections, many of which may be considered erroneous. Historically, the existence of such false positives necessitated thresholding of weak connections; this was especially relevant when constructing binary connectomes. Here we show that in dense weighted structural connectomes, the contribution of weak connections to network metrics is negligible and, thus, their removal is not necessary; indeed, the confounds introduced by an arbitrary cut-off value may in fact render this process undesirable.

Luka Liebrand ${ }^{1,2}$, Guido van Wingen ${ }^{1,2}$, Damiaan Denys ${ }^{1,2,3}$, and Matthan Caan ${ }^{2,4,5}$ 
${ }^{1}$ Dept. of Psychiatry, Academic Medical Center - University of Amsterdam, Amsterdam, Netherlands, ${ }^{2}$ Amsterdam Neuroscience, Amsterdam, Netherlands, ${ }^{3}$ Netherlands Institute for Neuroscience, Amsterdam, Netherlands, ${ }^{4}$ Dept. of Radiology, Academic Medical Center - University of Amsterdam, Amsterdam, Netherlands, ${ }^{5}$ Spinoza Centre for Neuroimaging, Amsterdam, Netherlands

Deep brain stimulation of the ventral part of the anterior limb of the internal capsule (vALIC) could potentially benefit from tractography-guided targeting, since it contains two major fiber bundles. In order to develop a diffusion-weighted sequence that has the greatest bundle specificity within the vALIC, we compared tractography results from a single-shell $3 \mathrm{~T}$ sequence with multi-shell $3 \mathrm{~T}$ and high-resolution 7T sequences. Although the multi-shell sequence showed superior SNR, it did not allow increased bundle discernibility in the vALIC. The highresolution sequence showed more anatomical detail, with more radially constrained tractography, and proved superior for separating the two bundles.

A preliminary application of the diffusion tensor imaging in estimating the functional and structural recovery of the visual pathway in Dysthyroid Optic Neuropathy patients after intravenous methylprednisolone pulse therapy.

ping liu ${ }^{1}$ and jing zhang ${ }^{1}$

${ }^{1}$ department of radiology, Tongji Hospital, Tongji Medical College, Huazhong University of Science and Technology, wu han, China

The management of DON (dysfunction optic neuropathy) is complex, an effective method to reflect the response of treatment is indispensable. We use the MRI-DTI combine d with DtiStudio software to assess the visual pathway changes in DON patients pre and post intravenous methylprednisolone pulse therapy. The results did demonstrate the improvement of visual pathway. The DTI can be regarded as a reliable tool to assess and follow up DON patients during therapy.

A multi-shell self-calibrating Richardson-Lucy deconvolution approach for the simultaneous quantification of ODF and tissue properties of different diffusion domains in the kidneys. Alberto De Luca ${ }^{1}$, Martijn Froeling ${ }^{2}$, and Alexander Leemans ${ }^{1}$

${ }^{1}$ Image Sciences Institute, UMC Utrecht and University Utrecht, Utrecht, Netherlands, ${ }^{2}$ Department of Radiology, UMC Utrecht, Utrecht, Netherlands

The advent of multi-shell diffusion MRI (dMRI) offers a viable substrate to apply deconvolution profiles in tissues characterized by partial volume of multiple diffusion domains, as the kidneys. In this work we present a modified damped Richardson-Lucy (mdRL) algorithm to perform spherical deconvolution over multiple diffusion domains. This method does not need to define a prior response function, which is dynamically estimated for each voxel, and allows to compute a fiber orientation distribution as well as relevant scalar metrics, as mean diffusivity and fractional anisotropy, for each diffusion domain. Applicability on two sample datasets is demonstrated as proof of concept.

Automatic reconstruction of cortico-striato-thalamo-cortical loops with application to obsessive-compulsive disorder

Dogu Baran Aydogan ${ }^{1}$, David Sean Thylur ${ }^{2}$, Junyan Wang ${ }^{1}$, Yuchun Tang $^{3}$, Janet Sobell ${ }^{1}$, James Knowles ${ }^{4}$, and Yonggang Shi ${ }^{1}$

${ }^{1}$ Keck School of Medicine of USC, Los Angeles, CA, United States, ${ }^{2}$ Emory University, Atlanta, GA, United States, ${ }^{3}$ Shandong University Cheeloo College of Medicine, Shandong, China, ${ }^{4}$ SUNY Downstate Medical Center, Brooklyn, NY, United States

Cortico-striato-thalamo-cortical (CSTC) loops are thought to play critical roles in the pathophysiology of several brain disorders. Despite the widespread evidence of CSTC circuits' crucial roles in brain disorders, a systematic approach to map their fiber pathways is missing. In order to advance our understanding on these critical circuits and how they are related to brain disorders, we propose a fully automatic approach for the in-vivo reconstruction based on diffusion MRI tractography. To demonstrate our approach, we studied MRI data from 19 patients with obsessive-compulsive disorder and 15 controls. Our approach enables in-dept analysis of the individual connections and also the full CSTC networks of the motor and lateral orbitofrontal loops.

Predictive Value of Two-tensor Unscented Kalman Filter Tractography in the Reconstruction of the Arcuate Fasciculus (AF) in Patients with Gliomas Involving Eloquent Language Areas Jing Yan ${ }^{1}$, Jingliang Cheng ${ }^{1}$, Shaoyu Wang ${ }^{2}$, and Xianzhi Liu ${ }^{3}$

${ }^{1}$ Department of MRI, the First Affiliated Hospital of Zhengzhou University, Zhengzhou, China, ${ }^{2}$ Siemens Healthcare, Scientific marketing, Beijing, China, ${ }^{3}$ Department of Neurosurgery, the First Affiliated Hospital of Zhengzhou University, Zhengzhou, China

This study aimed to preliminarily investigate the postoperative changes of AF in glioma patients detected by two-tensor UKF tractography from the perspective of the usefulness as a reference for postoperative recovery of language functions. The postoperative changes of AF were evaluated chronologically in relation to postoperative changes in language functions after surgery. Our study preliminarily shows that postoperative changes in the long segment of the left AF detected by two-tensor UKF tractography may be a predicting factor for postoperative language functional outcomes. Postoperative changes in the long and posterior segment of the left AF may be related with the language comprehending and repeating ability in glioma patients. 
Alexis Sánchez ${ }^{1}$, Cecilia Hernández ${ }^{1}$, Cyril Poupon² ${ }^{2}$ Jean-François Mangin² ${ }^{2}$, and Pamela Guevara ${ }^{1}$

${ }^{1}$ Faculty of Engineering, University of Concepción, Concepción, Chile, ${ }^{2}$ Neurospin, I2BM, CEA, Gif-sur-Yvette, France

We propose a fiber clustering algorithm composed by several steps, with the objective of representing the whole dataset by a small set of cluster centroids. First, a clustering is performed separately for a subset of points within the streamlines. The obtained point clusters are then used to regroup the fibers having common point clusters. Next, fiber clusters are filtered out by size and finally regroup using a quick merge based on a maximum Euclidean distance. A reduced set of regular and thin clusters is finally obtained. In contrast to previous works, the proposed method is only based on streamline structure.

Mitigating the effects of imperfect fixel correspondence in Fixel-Based Analysis

Robert Elton Smith ${ }^{1,2}$ and Alan Connelly $y^{1,2}$

${ }^{1}$ The Florey Institute of Neuroscience and Mental Health, Melbourne, Australia, ${ }^{2}$ Florey Department of Neuroscience and Mental Health, The University of Melbourne, Melbourne, Australia

A requisite step in performing a Fixel-Based Analysis (FBA) is the determination of "fixel correspondence", which defines how discrete fibre elements (fixe/s) for a particular subject map to the fixels defined in each voxel in template space. The method used thus far for this purpose - simply selecting the subject fixel that best aligns with the template fixel - fails to take into consideration the possibility for substantial variations in fixel segmentation across subjects. We propose a more sophisticated algorithm for determining fixel correspondence, which better accounts for differences in fixel segmentation, and demonstrate how this reduces the variance observed in fixel data across healthy controls.

Accuracy of response function estimation algorithms for 3-tissue spherical deconvolution of diverse quality diffusion MRI data

Thijs Dhollander ${ }^{1}$, David Raffelt ${ }^{1}$, and Alan Connelly ${ }^{1,2}$

${ }^{1}$ The Florey Institute of Neuroscience and Mental Health, Melbourne, Australia, ${ }^{2}$ The Florey Department of Neuroscience, University of Melbourne, Melbourne, Australia

Multi-shell multi-tissue constrained spherical deconvolution (MSMT-CSD) and single-shell 3-tissue CSD (SS3T-CSD) resolve white matter (WM) fibre orientation distributions and grey matter (GM) and CSF tissue compartments by deconvolving WM, GM and CSF response functions from the diffusion MRI data. To estimate these response functions from the data itself, a T1-based method was originally proposed. Recently, an unsupervised DWI-based method that doesn't rely on a co-registered T1-weighted image was also introduced. We evaluated the performance of both methods on high-quality HCP-data and clinical-quality single-shell data of an elderly patient with extensive lesions. The DWI-based method was more accurate in both scenarios.

Tissue-Encoded Colour Fluid-Attenuated Inversion Recovery (TEC-FLAIR) map: contrast fusion designed for improved characterisation of white matter lesion heterogeneity

Thijs Dhollander ${ }^{1}$, Remika Mito ${ }^{1,2}$, and Alan Connelly ${ }^{1,2}$

${ }^{1}$ The Florey Institute of Neuroscience and Mental Health, Melbourne, Australia, ${ }^{2}$ The Florey Department of Neuroscience, University of Melbourne, Melbourne, Australia

FLAIR MR images feature striking contrast, allowing easy identification of white matter hyperintense lesions. While such lesions have been explained by a range of microstructural characteristics, FLAIR itself doesn't provide specificity to distinguish these heterogeneous origins. 3-tissue CSD techniques resolve white matter (WM), grey matter (GM) and CSF compartments. In lesions, GM-like and CSF-like diffusion-weighted signals have been hypothesised to be related to certain origins, e.g. gliosis or increased interstitial fluid. We propose a fusion of 3-tissue encoded colours and FLAIR via panchromatic sharpening techniques, designed for improved characterisation of white matter lesion heterogeneity.

Linking neurocognitive measures with whole brain structure using Diffusion ODFs in the HCP dataset

Steven H. Baete ${ }^{1,2}$, Ying-Chia Lin ${ }^{1,2}$, Jingyun Chen $^{1,2,3}$, Ricardo Otazo ${ }^{1,2}$, and Fernando E. Boada ${ }^{1,2}$

${ }^{1}$ Center for Advanced Imaging Innovation and Research (CAIR), NYU School Of Medicine, New York, NY, United States, ${ }^{2}$ Center for Biomedical Imaging, Dept of Radiology, NYU School Of Medicine, New York, NY, United States, ${ }^{3}$ Dept of Psychiatry, NYU School Of Medicine, New York, NY, United States

Higher dimensional diffusion protocols are now routinely acquired in large-scale studies. While these diffusion data sets contain a wealth of information about white matter architecture, this information is not fully exploited when their dimensionality is reduced to simplify statistical correlations with neurocognitive markers over the whole brain. To overcome this limitation, we analyze the full Orientation Distribution Function (ODF) at each voxel using a Low-Rank plus Sparse decomposition to identify key ODF features. We use this approach to link neurocognitive measures to brain structure in a cohort of healthy Human Connectome Project volunteers. 
Suheyla Cetin Karayumak ${ }^{1,2}$, Marek Kubicki ${ }^{1,2}$, and Yogesh Rathi ${ }^{1,2}$

${ }^{1}$ Harvard Medical School, Boston, MA, United States, ${ }^{2}$ Brigham and Women's Hospital, Boston, MA, United States

Diffusion MRI (dMRI) data obtained from a 7T scanner has novel and improved microstructural tissue information missing from data acquired on 3T scanners. In this work, we propose to use deep Convolutional Neural Networks (CNN) that use rotation invariant spherical harmonic (RISH) features to map the dMRI data (the raw signal) between scanners without changing the fiber orientation. We validate our algorithm on 40 Human Connectome Project (HCP) subjects with scans on both $3 T$ and $7 \mathrm{~T}$ (10 training +30 test). Our preliminary results on 30 test subjects shows that CNN can indeed reliably obtain $7 \mathrm{~T}$ dMRI data quality from $3 \mathrm{~T}$ scans.

Pipeline for post-processing peripheral nerve DTI

Tina Jeon ${ }^{1}$, Jerome J Maller ${ }^{2}$, Maggie M.K. Fung ${ }^{3}$, and Darryl B Sneag ${ }^{1}$

${ }^{1}$ Radiology and Imaging, Hospital for Special Surgery, New York, NY, United States, ${ }^{2}$ General Electric Healthcare, Melbourne, Australia, ${ }^{3}$ General Electric Healthcare, New York, NY, United States

The purpose of the study is to evaluate and formalize a post-processing pipeline for DTI of the peripheral nerves using existing open source software suites. Our method integrates image registration, nerve segmentation, and DTI fiber tracking using the FMRIB software library (FSL) and MRtrix3, two popular software suites primarily used in the brain. 6 normal volunteers/patients and 9 nerves were analyzed and image quality was assessed. Using this protocol, image quality significantly improved in addition reducing processing time to 10 minutes using a semi-automated method.

Traditional Poster

\section{Diffusion MRI: Signal Reconstruction \& Representation}

Investigating noise distribution changes after motion correction and its effects on subsequent diffusion MRI processing

Samuel St-Jean ${ }^{1}$, Alberto De Luca ${ }^{1}$, Max A. Viergever ${ }^{1}$, and Alexander Leemans ${ }^{1}$

The quantification of diffusion MRI assumes the absence of motion and anatomical correspondence between diffusion sensitizing factors. To investigate the impact of processing order between motion correction and two denoising methods, we evaluated DKI and NODDI derived maps. Using repeated scans acquired with and without voluntary motion, three processing orders were compared. Results show that processing order moderately influences NODDI maps. However, two of the three denoising strategies can reduce outliers in mean kurtosis between $28 \%$ and $59 \%$ when compared to motion correction only.

Optimal b-value selection for IVIM-DWI: identification of pancreastic lesions based on entire-tumor

Jiali $\mathrm{Li}^{1}$, Daoyu $\mathrm{Hu}^{1}$, and $\mathrm{Zhen} \mathrm{Li}^{1}$

${ }^{1}$ Tongji Hospital, Huazhong University of Science and Technology, Wuhan, China

The purpose of this paper is to explore the successful b-value combination of IVIM-DWI that maximizes the diagnostic efficiency of parameters in differenting pancreatic cancer and normal tissues. IVIM parameters were measured by different $b$ value combinations, and then the diagnostic performance of each significant parameter in identificating tumors and normal tissues was calculated and compared between different combinations. The results show that in different $b$ value combinations, the diagnostic efficiency of the parameters are also different. The final conclusion is that $b$ value combination of 0-1700 may be the best selection in clinical practice. 
A voxel-wise multivariate analysis based on the Mahalanobis distance is presented. Upon implementation on simulated DTI data, the method demonstrates the ability to detect regions of pathology at an individual level with respect to a reference healthy control group. This multivariate approach could enhance the clinical value of diffusion weighted MRI in the assessment of individual patients with highly spatially heterogeneous brain conditions such as traumatic brain injury or autism spectrum disorder.

Anatomical atlas of MAP MRI-derived 3D diffusion propagators and microstructural parameters

Alexandru V Avram ${ }^{1}$, Adam S Bernstein², M. Okan Irfanoglu ${ }^{1}$, Amber Simmons $^{2}$, Martin Cota ${ }^{3}$, Neville Gai ${ }^{4}$, Neekita Jikaria ${ }^{3}$, Anita Moses ${ }^{3}$, Christine L Turtzo ${ }^{3}$, Lawrence Latour ${ }^{3}$, Dzung Pham ${ }^{4}$, John A Butman ${ }^{4}$, and Peter J Basser ${ }^{5}$

${ }^{1} \mathrm{NIBIB}$, National Institutes of Health, Bethesda, MD, United States, ${ }^{2} \mathrm{NICHD}$, National Institutes of Health, Bethesda, MD, United States, ${ }^{3}$ NINDS, National Institutes of Health, Bethesda, MD, United States, ${ }^{4}$ Diagnostic Radiology, National Institutes of Health, Bethesda, MD, United States, ${ }^{5}$ National Institutes of Health, Bethesda, MD, United States

We describe the construction of an anatomical template of 3D probability distributions water molecule displacements in tissues (i.e., diffusion propagators) measured with MAP MRI in a population of healthy volunteers. From the template of $3 \mathrm{D}$ diffusion propagators, we compute normative values of MAP MRI microstructural parameters and visualize the orientational characteristics of water net displacement profiles using orientation distribution functions (ODFs). This atlas could provide a reference for protocol development in longitudinal and multicenter studies, and for clinical studies seeking to detect and characterize subtle microstructural changes, such as those occurring in mild traumatic brain injury (mTBI), or metastatic cancer.

Spatial normalization of individual fractional anisotropy (FA) maps to widely used population templates for analysis can increase variability and create spurious differences in the measured FA values

Amritha Nayak ${ }^{1,2}$, Elisabeth Wilde ${ }^{3}$, Brian Taylor ${ }^{3}$, CENC Neuroimaging Core Investigators ${ }^{4}$, Laura Reyes ${ }^{1,2}$, and Carlo Pierpaoli ${ }^{1}$

${ }^{1}$ Quantitative Medical Imaging Section, NIBIB, NIH, Bethesda, MD, United States, ${ }^{2}$ The Henry M. Jackson Foundation for the Advancement of Military Medicine Inc, Bethesda, MD, United States, ${ }^{3}$ Michael E.DeBakey VA Medical Center and Baylor College of Medicine, Houston, TX, United States, ${ }^{4}$ Chronic Effects of Neurotrauma Consortium, Richmond, VA, United States

In this study we evaluate the effects of spatial normalization of individual fractional anisotropy (FA) maps to widely used population templates for analysis and its introduction of variability, creating spurious differences in the measured FA values.

Clinical assessment of simultaneous diffusion tensor imaging and T2 relaxometry of lumbar nerve roots in patients with low back pain

Takayuki Sakai ${ }^{1,2}$, Masami Yoneyama ${ }^{3}$, Tosiaki Miyati ${ }^{4}$, Atsuya Watanabe ${ }^{5,6}$, Eunju $\mathrm{Kim}^{7}$, and Noriyuki Yanagawa

${ }^{1}$ Eastern Chiba Medical Center, Tougane, Japan, ${ }^{2}$ Division of Health Sciences, Graduate School of Medical Sciences, Kanazawa University, Kanazawa, Japan, ${ }^{3}$ Philips Japan, Tokyo, Japan, ${ }^{4}$ Faculty of Health Sciences, Institute of Medical, Pharmaceutical and Health Sciences, Kanazawa University, Kanazawa, Japan, ${ }^{5}$ Orthopaedic Surgery, Eastern Chiba Medical Center, Tougane, Japan, ${ }^{6}$ Chiba University Graduate School of Medicine, Chiba, Japan, ${ }^{7}$ Philips Healthcare Korea, Seoul, Korea, Democratic People's Republic of

We developed a single-shot dual-echo EPI-DTI sequence (Diffusion-Relaxation Matrix: DRM) that can simultaneously provide the diffusion tensor parameters and T2 values. The purpose of this study was to investigate the clinical feasibility of DRM for the lumbar nerve roots in patients with low back pain. FA values were negatively correlated with each quantitative value. Prolongation of T2 values were observed in case of abnormally enlarged nerve roots. Therefore,simultaneous acquisition of diffusion tensor imaging and T2 map by using DRM technique might be able to evaluate the extent of nerve disorders more accurately.

q-Space Deep Learning for Alzheimer's Disease Diagnosis: Global Prediction and Weakly-Supervised Localization

Vladimir Golkov ${ }^{1}$, Phillip Swazinna ${ }^{1}$, Marcel M. Schmitt ${ }^{1}$, Qadeer A. Khan ${ }^{1}$, Chantal M.W. Tax ${ }^{2}$, Marat Serahlazau ${ }^{1}$, Francesco Pasa ${ }^{1,3}$, Franz Pfeiffer ${ }^{3}$, Geert Jan Biessels ${ }^{4}$, Alexander Leemans $^{5}$, and Daniel Cremers ${ }^{1}$

${ }^{1}$ Department of Informatics, Technical University of Munich, Munich, Germany, ${ }^{2}$ CUBRIC, Cardiff University, Cardiff, United Kingdom, ${ }^{3}$ Physics Department, Technical University of Munich, Munich, Germany, ${ }^{4}$ Department of Neurology, University Medical Center Utrecht, Utrecht, Netherlands, ${ }^{5}$ mage Sciences Institute, University Medical Center Utrecht, Utrecht, Netherlands

Most diffusion MRI approaches rely on comparably long scan time and a suboptimal processing pipeline with handcrafted physical/mathematical representations. They can be outperformed by recent handcrafted-representation-free methods. For instance, q-space deep learning (q-DL) allows unprecedentedly short scan times and optimized voxel-wise tissue characterization. We reformulate q-DL such that it estimates global (i.e. scan-wise rather than voxel-wise) information. We use this formulation to distinguish Alzheimer's disease (AD) patients from healthy controls based solely on raw q-space data without handcrafted representations such as DTI. Classification quality is very promising. Weakly-supervised localization techniques indicate that the neural network attends to AD-relevant brain areas. 
${ }^{1}$ Graduate School of Health Sciences, Fujita Health University, Toyoake, Japan, ${ }^{2}$ Department of Radiology, Fujita Health University Hospital, Toyoake, Japan, ${ }^{3}$ School of Medicine, Fujita Health University, Toyoake, Japan, ${ }^{4}$ Department of Radiology, Tokyo Metropolitan Health and Medical Treatment Corporation Ebara Hospital, Tokyo, Japan

IVIM analysis can provide the perfusion fraction $f$ and the pseudodiffusion coefficient $D^{*}$ or $D_{p}$ in addition to the diffusion parameters. The product of $f$ and $D^{*}$ is known to relate to cerebral blood flow. Recently, a higher diagnostic performance of $f D^{*}$ than $f$ and $D^{*}$ has been reported. We propose a method to estimate $f D_{p}$ without estimating $f$ and $D_{p}$ using $D K \mathrm{~K}$ analysis. The DKI based IVIM analysis can be implemented easily and provides $f D_{p}$ values with a high degree of precision.

Histogram Analysis of Diffusion Weighted Image for Body Tumors

Manabu Arai ${ }^{1}$, Koichi Oshio ${ }^{1}$, Shigeo Okuda ${ }^{1}$, and Masahiro Jinzaki ${ }^{1}$

${ }^{1}$ Department of Radiology, Keio Univerisity School of Medicine, Tokyo, Japan

Weighted diffusion subtraction (WDS) is a new imaging tool which may be useful for estimating the tissue characteristics within a voxel. In this study, DWI histogram (low b vs. high b) was generated and referred to WDS. On the histogram, the data distribution represents the tissue composition with blurring caused by partial volume. DWI histogram can visualize the relationship between T2WI (low b value DWI) and WDS.

Regularized nonnegative least-square fitting for intravoxel incoherent motion data processing: a simulation study

André Monteiro Paschoal ${ }^{1}$, Renata Ferranti Leoni ${ }^{1}$, and Fernando Fernandes Paiva ${ }^{2}$

$1583{ }^{1}$ InBrain Lab - FFCLRP, University of Sao Paulo, Ribeirao Preto, Brazil, ${ }^{2}$ Physics Institute of Sao Carlos, University of Sao Paulo, Sao Carlos, Brazil

Fitting model plays a crucial role in the analysis of intravoxel incoherent motion (IVIM) data due to limited number of points and to typical noisy data. Also, injured tissues can change the diffusion coefficient $(D)$ value so that the number of $D$ that contributes to total signal might be unknown. A possible solution for this problem is the nonnegative least-square (NNLS) fitting. This study aimed to evaluate the impact of the parameters used in the fitting and its applicability to simulated IVIM signal data processing.

New analysis and visualization tools AFNI-FATCAT (and implementing other software)

Paul Taylor ${ }^{1}$, Justin Rajendra ${ }^{1}$, Amritha Nayak $^{2,3}$, M. Okan Irfanoglu ${ }^{2}$, Daniel R Glen ${ }^{1}$, and Richard C Reynolds ${ }^{1}$

${ }^{1} \mathrm{NIMH}, \mathrm{NIH}$, Bethesda, MD, United States, ${ }^{2}$ NIBIB, NIH, Bethesda, MD, United States, ${ }^{3}$ Henry M. Jackson Foundation for the Advancement of Military Medicine, Bethesda, MD, United States

The typical size of MRI data sets being processed for a study is rapidly increasing, particularly with the growth of publicly available data sets and "big data" strategies for approaching problems. This produces a dual need in analysis: having scriptable and reproducible pipelines for analysis, as well as having a method for visualizing data both during intermediate steps and for final results presentation. Here, we describe new AFNI-FATCAT tools that provides a succinct set of processing steps for a full DTI analysis pipeline, from DICOM conversion to tractography and statistical anlyses; these tools create QC images and quantitative checks at each step for pipeline evaluation.

A review of the oscillating-gradient spin-echo signal model: Does a finite gradient duration alter

Jeff Kershaw ${ }^{1}$ and Takayuki Obata ${ }^{1}$

${ }^{1}$ Applied MRI Research, National Institute of Radiological Sciences, QST, Chiba, Japan

The oscillating gradient spin-echo (OGSE) sequence has emerged as a promising diffusion-weighted imaging (DWI) technique for probing in vivo tissue microstructure. However, due to the finite duration of the diffusion gradients, there are some aspects of the signal model that should be considered in more detail. This work re-examines the derivation of the OGSE method to better understand how the properties of the selected MPG are reflected in the signal equation. 
${ }^{1}$ InBrain Lab, Department of Physics, Faculty of Philosophy, Sciences and Letters of Ribeirão Preto, University of São Paulo, Ribeirão Preto, São Paulo, Brazil, ${ }^{2}$ Department of Internal Medicine, Ribeirão Preto Medical School, University of São Paulo, Ribeirão Preto, São Paulo, Brazil

The aim of this work is to compare FA and AFD as integrity parameter of white matter between groups of different ages to evaluate which areas of the white matter are affected in its fiber composition in the healthy aging process, and to evaluate if it happens in a global or specific manner. The results show that the largest decreases in FA and AFD occur in the brain of the elderly (over 60 years) due to more advanced axonal degeneration. AFD seems to show complementary information for understanding the white matter integrity alterations throughout the lifespan.

Diffusion exchange spectroscopic imaging of the spinal cord

Dan Benjamini ${ }^{1}$, Michal E Komlosh ${ }^{1,2}$, and Peter J Basser ${ }^{1}$

${ }^{1}$ Section on Quantitative Imaging and Tissue Sciences, National Institutes of Health, Bethesda, MD, United States, ${ }^{2}$ Center for Neuroscience and Regenerative Medicine, The Henry M. Jackson Foundation for the Advancement of Military Medicine, Bethesda, MD, United States

Diffusion exchange spectroscopy (DEXSY) is successfully used in conjunction with imaging on the spinal cord, and with excellent prospects for preclinical and clinical applications. DESXY is a model-free approach to measure water migration between and among distinct microenvironments. The time dependency of water migration from the intra- and extracellular microdomains indicates that different regions within gray or white matter exhibit different exchange kinetics, and points to the importance of the spatial scale of this heterogeneity.

A Novel Strategy For Morphologically Faithful Registration and Template Creation for Diffusion MRI Data

M. Okan Irfanoglu ${ }^{1}$, Neda Sadeghi ${ }^{1}$, Carlo Pierpaoli ${ }^{1}$, and Moebius Syndrome Research Consortium ${ }^{2}$

${ }^{1}$ QMI/NIBIB, National Institutes of Health, Bethesda, MD, United States, ${ }^{2}$ National Institutes of Health, Bethesda, MD, United States

Spatial alignment of diffusion tensor MRI (DTI) data is of fundamental importance for voxelwise statistical analysis and creation of population specific atlases of diffusion MRI metrics. Most available DTI-based spatial normalization algorithms emphasize alignment of anisotropic structures and disregard the quality of alignment for gray matter and CSF-filled regions. Additionally, standard atlas creation strategies using these registration tools do not generate templates that are morphologically representative of average features of the population. In this work, we propose a new DTI-based registration and atlas creation method that aims to overcome these challenges.

Reproducibility of Diffusion Tensor Imaging Data between Morning and Evening Scans

Domitille Dempuré 1,2 , Jia Fan ${ }^{2,3}$, André J.W. van der Kouwe ${ }^{4}$, Ernesta M. Meintjes $2,3,5$, and A. A. Alhamud $2,3,5$

${ }^{1}$ Higher Institute of Bioscience of Paris (ISBS), Paris, France, ${ }^{2}$ MRC/UCT Medical Imaging Research Unit, Division of Biomedical Engineering, University of Cape Town, Cape Town, South Africa, ${ }^{3}$ Department of Human Biology, University of Cape Town, Cape Town, South Africa, ${ }^{4}$ Massachusetts General Hospital, Boston, MA, United States, ${ }^{5}$ Cape Universities Body Imaging Centre (CUBIC-UCT), Cape Town, South Africa

Diffusion Tensor Imaging (DTI) is widely used to study brain white matter integrity. However, instability of the MRI scanner including heating of the iron plates in the shim trays or physiological changes during the day may influence DTI indices. The aim of this work was to evaluate DTI parameters through scans performed at two different times of the day, early morning and late afternoon, and repeated over six days. The results showed that DTI data acquired at different times of day differed, as mean diffusivity was higher in the morning than the evening.

IVIM D and f - Optimal estimation technique and their potential for tissue differentiation

Oscar Jalnefjord ${ }^{1,2}$, Mats Andersson ${ }^{3}$, Mikael Montelius ${ }^{1}$, Anna-Karin Elf $^{4}$, Viktor Johanson ${ }^{4}$, Johanna Svensson $^{5}$, Göran Starck $^{1,2}$, and Maria Ljungberg $^{1,2}$

${ }^{1}$ Department of Radiation Physics, University of Gothenburg, Gothenburg, Sweden, ${ }^{2}$ Department of Medical Physics and Biomedical Engineering, Sahlgrenska University Hospital, Gothenburg, Sweden, ${ }^{3}$ Department of Radiology, University of Gothenburg, Gothenburg, Sweden, ${ }^{4}$ Department of Surgery, University of Gothenburg, Gothenburg, Sweden,

${ }^{5}$ Department of Oncology, University of Gothenburg, Gothenburg, Sweden

IVIM parameter estimation restricted to $D$ and $f$ (avoiding $D^{*}$ ) has gained increased popularity. In this study we show that the commonly used segmented fitting approach is preferable. We also show that differentiation between tumor and healthy liver tissue is substantially enhanced by the combined use of $D$ and $f$. 
${ }^{1}$ Image Sciences Institute, University Medical Center Utrecht, Utrecht, Netherlands, ${ }^{2}$ Cardiff University Brain Research Imaging Centre, Cardiff, United Kingdom

Gradient non-linearities affects diffusion weighted imaging (DWI) as it can result in geometric distortions and spatially varying b-values and gradient directions. The effect is more severe at high gradient strengths. Spherical deconvolution, in particular, relies on a spherical sampling of q-space, which might be affected due to gradient nonlinearities. In this work, we explored the sensitivity of two widely used spherical deconvolution approaches to the gradient non-linearity effect by investigating FOD peak orientation deviations, and evaluate a modified version of DRL that can take into account spatially varying diffusion gradients and weighting. Monte-Carlo simulations and two datasets from the HCP project were used for evaluation.

Value of Whole-Tumor Histogram Analysis of Diffusion Tensor Imaging in Differentiating Intrahepatic Mass-forming Cholangiocarcinoma and Solitary Hypovascular Hepatic Metastases

Ying Zhao ${ }^{1}$, Ailian Liu ${ }^{1}$, Lihua Chen ${ }^{1}$, Lizhi Xie ${ }^{2}$, and Ye $\mathrm{Li}^{1}$

${ }^{1}$ Department of Radiology, The First Affiliated Hospital of Dalian Medical University, Dalian, China, ${ }^{2}$ MR Research, GE Healthcare, Beijing, China

Diffusion tensor imaging (DTI) is an imaging modality that detects the microstructural and pathological changes of organisms according to the diffusive characteristics of water molecules in the tissues. MR histogram analysis reflects the tumor heterogeneity. In the current study, histogram analysis of DTI was demonstrated to be capable to differentiate mass-forming cholangiocarcinoma and solitary hypovascular hepatic metastases, which can provide quantitative information for further clinical diagnosis.

Characterization and Correction of Abnormally Low Mean Kurtosis Values

Fan Zhang ${ }^{1}$, Lipeng Ning ${ }^{1}$, Lauren J. O'Donnell ${ }^{1}$, and Ofer Pasternak ${ }^{1}$

${ }^{1}$ Harvard Medical School, Boston, MA, United States

Diffusion kurtosis imaging (DKI) often yields abnormally low mean kurtosis (MK) values that are physically and/or biologically implausible. We aim to characterize the relationship between abnormally low MK and baseline (b0) values. We show that too low b0 signals explain abnormally low MK values. We propose an automatic and threshold free approach for the identification of low MK voxels, along with a correction strategy based on adaptive smoothing. Our results suggest that modifying the b0 is sufficient to resolve the vast majority of low MK values, and is preferred over two other popular correction methods.

A novel method for the detection of the number of compartments in diffusion MRI data

Emma Metcalfe-Smith ${ }^{1,2,3}$, Niloufar Zarinabad ${ }^{2,3}$, Jan Novak $^{2,3}$, Hamid Dehghani $^{1,4}$, and Andrew Peet ${ }^{2,3}$

${ }^{1}$ Physical Sciences for Health Doctoral Training Centre, University of Birmingham, Birmingham, United Kingdom, ${ }^{2}$ Institute of Cancer and Genomic Sciences, University of Birmingham, Birmingham, United Kingdom, ${ }^{3}$ Department of Oncology, Birmingham Children's Hospital, Birmingham, United Kingdom, ${ }^{4}$ School of Computer Science, University of Birmingham, Birmingham, United Kingdom

There is a need for a method that can detect the number of components within multi b-value diffusion-weighted imaging. In particular, this would aid in the identification and correction of partial volume effects (PVE) within the brain. A PVE model was simulated to contain varying ratios of cerebrospinal fluid and white matter. Multi-exponential fitting methods were applied and found to be unsuccessful in identifying the number of components within the model. A novel fitting method, the Autoregressive Discrete Acquisition Points Transformation, was applied to simulations. Following manipulation through the discrete Z-domain, the number of components were correctly identified.

Multicompartment modelling of diffusion-weighted MRI data with no prior assumptions

Emma Metcalfe-Smith ${ }^{1,2,3}$, Niloufar Zarinabad ${ }^{2,3}$, Jan Novak $^{2,3}$, Hamid Dehghani $^{1,4}$, and Andrew Peet ${ }^{2,3}$

${ }^{1}$ Physical Sciences for Health Doctoral Training Centre, University of Birmingham, Birmingham, United Kingdom, ${ }^{2}$ Institute of Cancer and Genomic Sciences, University of Birmingham, Birmingham, United Kingdom, ${ }^{3}$ Department of Oncology, Birmingham Children's Hospital, Birmingham, United Kingdom, ${ }^{4}$ School of Computer Science, Birmingham, United Kingdom

Multi-compartment modelling of Diffusion-Weighted MRI data can provide additional diffusion related parameters. However, to ensure meaningful parameters are attained, multicompartment models have to make several assumptions prior to fitting, including initial parameter values and multi-step fitting procedures. The novel Autoregressive Discrete Acquisition Points Transformation (ADAPT) method was applied to in vivo data. ADAPT demonstrated that it could infer the number of compartments within the data. When 1-and 2-compartment ADAPT models were investigated, the ADAPT coefficients were found to correlate with the parameters attained by the Apparent Diffusion Coefficient (ADC) and the Intravoxel Incoherent Motion (IVIM) models. 
${ }^{1}$ Institute of Medical Device and Imaging, National Taiwan University College of Medicine, Taipei, Taiwan, ${ }^{2}$ Molecular Imaging Center, National Taiwan University, Taipei, Taiwan, ${ }^{3}$ Graduate Institute of Brain and Mind Sciences, National Taiwan University College of Medicine, Taipei, Taiwan, ${ }^{4}$ Department of Medical Imaging, National Taiwan University Hospital, Taipei, Taiwan

In the study, we proposed a regularization method for MAP-MRI estimation, called ReMAP. This method includes a regularization term in the cost functional in order to penalize the coefficients. The penalty is a simple diagonal matrix with entries determined only by the order of the Hermite functions, where higher order functions take more penalization, therefore, this method is easy to implement. In addition, ReMAP outperforms MAP-MRI in both estimation efficiency and accuracy, revealing that the regularization term is crucial for a robust estimation. Therefore, ReMAP is an improved version of MAP-MRI and would be beneficial for clinical studies.

Are Intravoxel Incoherent Motion and Dynamic Contrast-Enhanced Perfusion Parameters Related in Glioblastomas?

Nicholas Majtenyi ${ }^{1}$, Thanh B. Nguyen ${ }^{2,3}$, Gerd Melkus ${ }^{2}$, Ryan Gotfrit ${ }^{4}$, Gregory O. Cron ${ }^{2,3,5}$, and lan G. Cameron ${ }^{1,2,3}$

${ }^{1}$ Department of Physics, Carleton University, Ottawa, ON, Canada, ${ }^{2}$ Medical Imaging, The Ottawa Hospital, Ottawa, ON, Canada, ${ }^{3}$ Radiology, University of Ottawa, Ottawa, ON,

Intravoxel incoherent motion (IVIM) is an MR-based diffusion-weighted imaging technique that can measure both diffusion and perfusion. Currently, no link has been established between the perfusion parameters obtained from IVIM to those from dynamic contrast-enhanced (DCE)-MRI, particularly in the human brain. This study determined that no correlation exists between these two perfusion measurement techniques in patients with glioblastomas. This indicates that these two imaging techniques measure two separate effects; however, IVIM may be able to provide complementary, additional perfusion information that can potentially aid clinical diagnoses when used in conjunction with DCE-MRI parameters.

A non-Gaussian bi-exponential diffusion model with CUSP74 sampling for improved myocardial helix angle quantification and segmentation.

Cyril Tous ${ }^{1}$, Alistair Young ${ }^{1}$, and Beau Pontre

${ }^{1}$ Anatomy and Medical Imaging, The University of Auckland, Auckland, New Zealand

The non-Gaussianity of diffusion at high b-value, leads to poor estimates of fast diffusion components when using diffusion models that assume Gaussian diffusion distributions. Including the diffusion kurtosis in a bi-exponential model allows better quantification of the partial volume effects when large b-values are used. This study investigates how this improved model can provide a better estimate of the helix angle in fixed heart specimens.

Where's my water? Untangling the diffusion signal using the phasor representation

Michael J van Rijssel ${ }^{1}$, Martijn Froeling ${ }^{1}$, and Josien P W Pluim ${ }^{1,2}$

${ }^{1}$ Center for Image Sciences, UMC Utrecht, Utrecht, Netherlands, ${ }^{2}$ Department of Biomedical Engineering, Eindhoven University of Technology, Eindhoven, Netherlands

The recently proposed phasor representation and associated unmixing method allow separation of multi-exponentially decaying signals. This method has achieved promising results on diffusion MRI data and boasts sub-second analysis of full datasets on regular desktop PCs. This work investigates the noise propagation properties of this method and the influence of misplacing the vertex of a component in phasor space when performing unmixing. Results indicate that the phasor method is feasible and that the influence of component misplacement is systematic, but smaller than the errors due to noise at regular diffusion MR signal-to-noise-ratio levels.

Intra- and inter-subject variability of diffusivity by DTI and DKI: An small animal study on $7 \mathrm{~T}$

Hung-Yu Fu' ${ }^{1}$, Wei-Cheng Lee ${ }^{1}$, Sheng-Min Huang ${ }^{1}$, Shin-Lei Peng ${ }^{2}$, Kung-Chu Ho ${ }^{3}$, and Fu-Nien Wang ${ }^{1}$

${ }^{1}$ Biomedical Engineering and Environmental Science, National Tsing Hua University, Hsinchu, Taiwan, ${ }^{2}$ Department of Biomedical Imaging and Radiological Science, China Medical University, Taichung, Taiwan, ${ }^{3}$ Linkou Chang Gung Memorial Hospital, Taoyuan, Taiwan

Diffusivity can be acquired by both DTI and DKI model on the same set of images. To investigate intra- and inter-subject variability of DKI and DTI derived diffusivities, five SpragueDawley rats were scanned on a 7T small animal scanner. In intra-subject variability test, lower coefficients of variation are found on DKI derived parameters. In inter-subject analysis, higher values were estimated by DKI in mean diffusivity, axial diffusivity, and radial diffusivity. The CNR between white matter and gray matter of these parameters are also better with DKI. However, the CNR of FA is higher with DTI than with DKI 
${ }^{1}$ Department of Biomedical Engineering, Linköping University, Linköping, Sweden, ${ }^{2}$ Department of Mathematics, Linköping University, Linköping, Sweden, ${ }^{3}$ Department of Radiology, Brigham and Women's Hospital, Harvard Medical School, Boston, MA, United States

The compartmentalized structure of biological tissues demands a representation of individual compartments and a description of diffusion within them. We identified a quadratic potential energy profile, recently studied in-depth by Yolcu et al. (Phys Rev E, 93, 052602, 2016), as the effective energy landscape for restricted diffusion as far as gradient waveforms featuring long pulses are concerned. Our simulations suggest that the stochastic effective force on the center-of-mass position is approximately linear, thus providing further support for the Hookean effective force model.

The diagnostic values of DTI and DKI techniques in degeneration of corpus callosum of chronic alcoholism

Ke-ning Xu', ${ }^{1,2}$ Guo-shi $\mathrm{LYU}^{1}$, and Lizhi $\mathrm{Xie}^{3}$

${ }^{1}$ Imaging Center, the 251st Hospital of PLA, Zhangjiakou, China, ${ }^{2}$ The Graduate School of HeBei North University, Zhangjiakou, China, ${ }^{3}$ GE Healthcare, China, Beijing, China

Chronic alcoholism is a common disease, and many patients are often associated with corpus callosal degeneration. In this study, the values of fractional anisotropy (FA) and apparent diffusion coefficient (ADC), diffusion tensor imaging (DTI) and the mean kurtosis (MK) values in diffusion kurtosis imaging (DKI) were used to analyze chronic alcoholism with corpus callus (MBD) patients, to explore the diagnostic value of these three parameters in MBD patients. Receiver operating characteristic curve (ROC)analysis of the parameters of the diagnosis of the disease. The results showed that FA is better than ADC and MK, and the sensitivity and specificity are better.

Comparison of intravoxel incoherent motion DWI, diffusion kurtosis imaging, and conventional DWI in predicting the chemotherapeutic response of colorectal liver metastases: preliminary experience

Huan Zhang ${ }^{1}$, Wenhua $\mathrm{Li}^{2}$, Robert Grimm ${ }^{3}$, Caixia $\mathrm{Fu}^{4}, \mathrm{Xu} \mathrm{Yan}^{5}$, and Tong Tong ${ }^{1}$

${ }^{1}$ Department of Radiology, Fudan University Shanghai Cancer Center; Department of Oncology, Shanghai Medical College, Fudan University, Shanghai, China, ${ }^{2}$ Department of Medical Oncology, Fudan University Shanghai Cancer Center; Department of Oncology, Shanghai Medical College, Fudan University, Shanghai, China, ${ }^{3}$ MR Application Predevelopment, Siemens Healthcare, Erlangen, Germany, ${ }^{4}$ APPL, Siemens Shenzhen Magnetic Resonance Ltd., Shenzhen, China, ${ }^{5}$ MR Collaboration NE Asia, Siemens Healthcare, Shanghai, China

The aim of this study was to compare the performance of pre-treatment intravoxel incoherent motion DWI (IVIM-DWI), diffusion kurtosis imaging (DKI), and conventional DWI for predicting the chemotherapeutic response in patients with colorectal liver metastases (CRLMs). The results indicates that they are all potentially useful for predicting the chemotherapeutic response of CRLMs, with mean diffusion derived from DKI having the best performance.

Quantitative Comparison of Multiple High Angular Resolution Diffusion Imaging Techniques for Myocardium

Sifangyuan Wang ${ }^{1}$, Lihui Wang ${ }^{1}$, Jian Zhang ${ }^{1}$, Rongpin Wang ${ }^{2}$, Xinfeng Liu², and Yuemin Zhu ${ }^{3}$

${ }^{1}$ Key Laboratory of Intelligent Medical Image Analysis and Precise Diagnosis of Guizhou Province, School of Compute Science and Technology, Guizhou University, Guiyang, China, ${ }^{2}$ Department of Radiology, Guizhou Provincial People's Hospital, Guiyang, China, ${ }^{3}$ Univ.Lyon, INSA-Lyon, Université Claude Bernard Lyon 1, UJM-Saint Etienne, CNRS, Inserm, CREATIS UMR 5220, U1206, F-69621, Lyon, France

We compared quantitatively three commonly used HARDI schemes for describing the myocardium structure in a unified frame-work. One pig heart was firstly scanned with 256 diffusion directions, and then the diffusion ODFs of q-ball imaging (QBI), diffusion spectrum imaging (DSI) and generalized q-space imaging (GQI) were reconstructed respectively, from which the myocardiac fiber orientations and the diffusion metrics were finally extracted and compared. The results show that the cardiac fiber crossing locations, crossing numbers, and the generalized fractional anisotropy detected by three schemes are totally different.

Diffusion gradient performance optimization for B-tensor encoded q-space trajectory imaging of the human brain

Jan Martin ${ }^{1}$, Andreas Wetscherek ${ }^{2}$, Lars Müller ${ }^{3,4}$, Tristan Anselm Kuder ${ }^{3}$, Michael Uder ${ }^{1}$, Bernhard Hensel ${ }^{5}$, and Frederik Bernd Laun ${ }^{1}$

${ }^{1}$ Institute of Radiology, University Hospital Erlangen, Friedrich-Alexander-Universität Erlangen-Nürnberg (FAU), Erlangen, Germany, ${ }^{2}$ Joint Department of Physics, The Institute of Cancer Research and The Royal Marsden NHS Foundation Trust, London, United Kingdom, ${ }^{3}$ Department Medical Physics in Radiology, German Cancer Research Center (DKFZ), Heidelberg, Germany, ${ }^{4}$ CUBRIC, School of Psychology, University of Cardiff, Cardiff, United Kingdom, ${ }^{5}$ Center for Medical Physics and Engineering, Friedrich-Alexander-Universität Erlangen-Nürnberg (FAU), Erlangen, Germany

q-Space trajectory imaging is a recently introduced approach for determining microscopic diffusion tensor properties like $\mu \mathrm{FA}$ and orientation coherence. To create the necessary higher order B-tensors special gradient trajectories are needed. The initial implementation of q-space trajectory imaging was based on magic-angle-spinning of the q-vector, and required echo times of $160 \mathrm{~ms}$ for b-values of $2000 \mathrm{~s} / \mathrm{mm}^{2}$. In the current abstract, numerically optimized gradient trajectories were implemented, which reduced the required echo time to $115 \mathrm{~ms}$. The resulting parameter maps benefited from the increase in signal-to-noise ratio. 
Application value of DKI in grading of pancreatic cancer

Meiying Yan $^{1}$, Xiaoqi Wang ${ }^{2}$, and Rengen $\mathrm{Xu}^{1}$

${ }^{1}$ Department of Radiology, Jiangxi Cancer Hospital, Nanchang, China, ${ }^{2}$ Philips Healthcare, Beijing, Beijing, China

Tumor cells and the complex micro-environment would lead to restricted the water molecules diffusion, in the form of non-Gaussian distribution at space, and diffusion kurtosis imaging $(\mathrm{DKI})^{(1)}$ describes the degree of non-Gaussian distribution, and it has shown to reflect more sensitive diffusion information comparing with regular diffusion weighted image(DWI) ${ }^{(2)}$. It was reported that DKI helped to classify tumors like astrocytomas ${ }^{(3)}$. However, there is challenges on the DKI application mostly due to low SNR in pancreas diffusion images and motion artifacts. pancreatic cancer is a malignant pancreatic tumor,and the recent prognosis of patients with pancreatic cancer is determined by the histopathological grade of tumor. Herein, we reported the investigation on applying DKI to differentiate the histological grade of pancreatic cancer.,by assessing DKI parameters.

Return-to-the-origin probability calculation in single shell acquisitions

Santiago Aja-Fernandez ${ }^{1}$, Antonio Tristan-Vega ${ }^{1}$, Malwina Molendowska ${ }^{2}$, Tomasz Pieciak ${ }^{2}$, and Rodrigo de Luis-Garcia ${ }^{1}$

${ }^{1}$ Universidad de Valladolid, Valladolid, Spain, ${ }^{2}$ AGH University of Science and Technology, Kraków, Poland

One of the problems of estimating q-space scalar measures is the need of a high number of samples in the q-space in order to properly reconstruct the diffusion signal without aliasing. In this work we propose an alternative method to estimate the return-to-origin probability (RTOP) from a single shell acquisition using a prior assumption over the diffusion signal. The method provides significant structural information even for single shell acquisitions with moderate b-values.

Comparison between readout segmented diffusion weighted imaging and single shot echo planar imaging for differential diagnosis of prostate cancer

Chuangbo YANG ${ }^{1}$, Qi YANG ${ }^{1}$, Nan YU ${ }^{1}$, Hui TAN ${ }^{1}$, Wei WEI ${ }^{1}$, Guangming MA ${ }^{1}$, Shaoyu WANG ${ }^{1}$, and Shenglin $\mathrm{LI}^{2}$

${ }^{1}$ Departments of Diagnostic Radiology, Affiliated Hospital of Shaanxi University of Chinese Medicine, Xianyang, China, China, ${ }^{2}$ Shaanxi University of Chinese Medicine, Xianyang, China, China

Readout segmented diffusion weighted imaging (Rs-EPI) with ultra-high b value (1000、2000、3000s/mm²) have high sensitivity , specificity, PPV and NPV in the differential diagnosis of prostate cancer than single shot echo planar imaging (SS-EPI) does.

Comparison of three diffusion models: monoexponential vs. intravoxel incoherent vs. stretched model

Jeong Hee Yoon ${ }^{1}$, Eunju Kim², and Jeong Min Lee ${ }^{1}$

${ }^{1}$ Seoul National University Hospital, Seoul, Republic of Korea, ${ }^{2}$ Philips Healthcare Korea, Seoul, Republic of Korea

A diffusion heterogeneity index $(\alpha)$ derived from a stretched exponential model may serve as a more sensitive parameter for hepatic fibrosis compared with paramters from mono-or biexponential diffusion weighted imaging (DWI).

Multi-platform reproducibility of advanced diffusion weighted MRI parameters in phantoms and healthy volunteers

Shah Islam ${ }^{1}$, Matthew Grech-Sollars ${ }^{2}$, Matthew Orton ${ }^{3}$, Lesley Honeyfield ${ }^{4}$, Eric Aboagye ${ }^{2}$, and Adam Waldman 1,5

${ }^{1}$ Brain Sciences, Imperial College London, London, United Kingdom, ${ }^{2}$ Surgery and Cancer, Imperial College London, London, United Kingdom, ${ }^{3}$ CRUK and EPSRC Cancer Imaging Centre, Institute of Cancer Research, London, United Kingdom, ${ }^{4}$ Department of Imaging, Imperial Healthcare NHS Trust, London, United Kingdom, ${ }^{5}$ Centre for Clinical Brain Sciences, University of Edinburgh, Edinburgh, United Kingdom

Quantitative diffusion imaging has an evolving role in tumour characterisation and disease monitoring. Most clinical DWI sequences use ADC derived from two b-values. Multiple b-value acquisition allows further biologically-relevant diffusion components to be interrogated using bi-, multi- and stretched exponential models; these require validation for application in multicentre trials. This study compared the reproducibility of ADC, IVIM and stretched exponential parameters across MRI platforms in two phantoms and healthy volunteers. Our initial results suggest highly reproducibility of all measured parameters in phantoms, and of ADC and IVIM in healthy brains. Stretched exponential data appear less reproducible in vivo. 
DWI body phantom development and signal to noise calculation based on RSNA QIBA protocol guidelines for identically configured MRI machines shows machine variability and resultant $\mathrm{ADC}$ calculation error propagation.

Non-Gaussian diffusion restriction effects in intravoxel incoherent motion imaging acquired at b-values below $1000 \$ \$ \$ 1 t t \mathrm{~s} / \mathrm{mm} \wedge\{2\} \$ \$ \$$

Hajime Tamura ${ }^{1}$, Hideki Ota ${ }^{2}$, Tatsuo Nagasaka² ${ }^{2}$ Naoko Mori ${ }^{2}$, and Shunji Mugikura ${ }^{2}$

${ }^{1}$ Tohoku University School of Medicine, Sendai, Japan, ${ }^{2}$ Tohoku University Hospital, Sendai, Japan

To know how much the intravoxel incoherent motion (IVIM) parameters deduced by a bi-exponential model are affected by neglecting non-Gaussian diffusion restriction effects, we performed Monte-Carlo simulations: fitting the bi-exponential model to simulated data containing the diffusion restriction effects. The results showed that non-Gaussian diffusion restriction effects may considerably affect estimation of IVIM parameters even when data acquired with low $b$-values $\left(b \leq 1000 \mathrm{~s} / \mathrm{mm}^{2}\right)$ are used. This should be taken into account when interpreting the results of IVIM analyses based on the bi-exponential model.

Traditional Poster

\section{Diffusion MRI: Acquisition \& Reconstruction}

A comparison of multi-ADC and DTI fit metrics of diffusion MRI data acquired with Stejskal-Tanner and asymmetric bipolar gradients at identical echo time.

Alberto De Luca ${ }^{1}$, Alexander Leemans ${ }^{1}$, and Martijn Froeling ${ }^{2}$

${ }^{1}$ Image Sciences Institute, UMC Utrecht and University Utrecht, Utrecht, Netherlands, ${ }^{2}$ Department of Radiology, UMC Utrecht, Utrecht, Netherlands

Asymmetric-Bipolar (AS) gradients have been proposed in diffusion MRI (dMRI) experiments as alternative to Stejskal-Tanner (ST) gradients to achieve flow and motion-compensation. However, it remains unclear whether the gradient shape affects commonly derived metrics. Data at multiple diffusion-weightings was acquired on 4 subjects with ST and flowcompensated gradients, then fit with a multi-ADC model and DTI. Results showed that some metrics, as free water signal fraction and fractional anisotropy were comparable between AS and ST, whereas diffusion coefficients and perfusion fraction were remarkably different. Great care is suggested when comparing studies using different waveforms despite other identical acquisition parameters.

Motion Compensated, Optimized Diffusion Encoding (MODE) Gradient Waveforms

Waqas Majeed ${ }^{1}$, Prateek Kalra ${ }^{1}$, and Arunark Kolipaka ${ }^{1}$

${ }^{1}$ Radiology, The Ohio State University Wexner Medical Center, Columbus, $\mathrm{OH}$, United States

We present a framework to obtain motion compensated diffusion encoding waveforms that are shorter than all diffusion encoding waveforms available to date. These waveforms can be obtained analytically. We successfully demonstrate the use of these waveforms for cardiac DWI.

Optimal Diffusion-weighting Gradient Waveform Design (ODGD): Formulation and Experimental Validation

Óscar Peña-Nogales ${ }^{1}$, Yuxin Zhang ${ }^{2,3}$, Rodrigo de Luis-Garcia ${ }^{1}$, Santiago Aja-Fernandez ${ }^{1}$, James H. Holmes ${ }^{2}$, and Diego Hernando ${ }^{2,3}$

${ }^{1}$ Laboratorio de Procesado de Imagen, Universidad de Valladolid, Valladolid, Spain, ${ }^{2}$ Radiology, University of Wisconsin-Madison, Madison, WI, United States, ${ }^{3}$ Medical Physics, University of Wisconsin-Madison, Madison, WI, United States

Diffusion-Weighted MRI often suffers from signal attenuation due to long TE, sensitivity to physiological motion, and dephasing due to concomitant gradients (CGs). These challenges complicate image interpretation and may introduce bias in quantitative diffusion measurements. Motion moment-nulled diffusion-weighting gradients have been proposed to compensate motion, however, they frequently result in high TE and suffer from CG effects. In this work, the Optimal Diffusion-weighting Gradient waveform Design method that overcomes limitations of state-of-the-art waveforms is revisited and validated in phantom and in-vivo experiments. These diffusion-weighting gradient waveforms reduce the TE and increase the SNR of stateof-the-art waveforms without and with CG-nulling. 
Spatio-Temporal dMRI Acquisition Design: Reducing the Number of Samples

Patryk Filipiak $^{1}$, Rutger Fick ${ }^{1}$, Alexandra Petiet ${ }^{2}$, Mathieu Santin ${ }^{2}$, Anne-Charlotte Philippe ${ }^{2}$, Stephane Lehericy ${ }^{2}$, Rachid Deriche ${ }^{1}$, and Demian Wassermann ${ }^{1,3}$

${ }^{1}$ Université Côte d'Azur - Inria Sophia Antipolis-Méditerranée, Valbonne, France, ${ }^{2}$ CENIR - Center for Neurolmaging Research, ICM - Brain and Spine Institute, Paris, France, ${ }^{3}$ Inria, CEA, Université Paris-Saclay, Paris, France

Acquisition time is a major limitation in recovering brain white matter microstructure with diffusion magnetic resonance imaging. Finding a sampling scheme that maximizes signal quality and satisfies given time constraints is NP-hard. Therefore, we propose a heuristic method based on genetic algorithm that finds sub-optimal solutions in reasonable time. Our diffusion model is defined in the $\$ \$ \$$ qltau $\$ \$ \$$-space, so that it captures both spacial and temporal phenomena. The experiments on synthetic data and in-vivo diffusion images of the C57BI6 wildtype mouse corpus callosum reveal superiority of our approach over random sampling and even distribution in the $\$ \$ \$$ q $\mid$ tau $\$ \$ \$$-space.

High resolution in vivo diffusion weighted imaging of the human occipital cortex: enabled by $300 \mathrm{mT} / \mathrm{m}$ gradients and flexible radio-frequency surface coils.

Evgeniya Kirilina ${ }^{1,2}$, Fakhereh Movahedian Attar ${ }^{1}$, Luke J. Edwards ${ }^{1}$, Kerrin J. Pine ${ }^{1}$, and Nikolaus Weiskopf ${ }^{1}$

${ }^{1}$ Department of Neurophysics, Max Planck Institute for Human Cognitive and Brain Sciences, Leipzig, Germany, ${ }^{2}$ Center for Cognitive Neuroscience Berlin, Free University Berlin, Berlin, Germany

Information about intracortical fibers and connectivity can potentially be obtained using diffusion weighted imaging (DWI). However, in vivo intracortical DWI requires extraordinarily high spatial resolution. We demonstrate in vivo DWI imaging in the human occipital cortex with an isotropic resolution of $800 \mu \mathrm{m}$ enabled by a high-performance $300 \mathrm{mT} / \mathrm{m}$ gradient system and flexible high-sensitivity RF receive coil optimized for cortical imaging. Robust detection of intracortical features was achieved in a reasonable scanning time. The described setup opens the exciting possibility to study intracortical connectomics in humans in vivo.

In-vivo line-scan diffusion MR at 250 micron inline resolution within human cerebral cortex at $7 \mathrm{~T}$

Mukund Balasubramanian ${ }^{1,2}$, Robert V. Mulkern ${ }^{1,2}$, Jeffrey J. Neil ${ }^{1,3}$, Stephan E. Maier ${ }^{1,4,5}$, and Jonathan R. Polimeni ${ }^{1,6,7}$

${ }^{1}$ Harvard Medical School, Boston, MA, United States, ${ }^{2}$ Department of Radiology, Boston Children's Hospital, Boston, MA, United States, ${ }^{3}$ Department of Neurology, Boston Children's Hospital, Boston, MA, United States, ${ }^{4}$ Department of Radiology, Brigham and Women's Hospital, Boston, MA, United States, ${ }^{5}$ Department of Radiology, Sahlgrenska University Hospital, Gothenburg, Sweden, ${ }^{6}$ Athinoula A. Martinos Center for Biomedical Imaging, Department of Radiology, Massachusetts General Hospital, Charlestown, MA, United States, ${ }^{7}$ Harvard-MIT Division of Health Sciences and Technology, Massachusetts Institute of Technology, Cambridge, MA, United States

We used the line-scan technique to measure in-vivo diffusion at $7 \mathrm{~T}$ within human primary somatosensory cortex (S1) and primary motor cortex (M1), achieving voxel sizes as low as 0.25 $\mathrm{mm}$ in the radial direction (i.e., orthogonal to the cortical surface). Our results are consistent with recent reports of predominantly tangential diffusion in $\mathrm{S} 1$ and, to a lesser extent, radial diffusion in M1; however, the smaller voxel sizes used in our study alleviate concerns regarding partial-volume effects and, perhaps more importantly, enable the study of fine-scale variations in diffusion structure across cortical layers.

Evaluation of Monopolar Diffusion-Prepared TSE for Diffusion Imaging

Jialu Zhang ${ }^{1,2,3}$, Xiufeng $\mathrm{Li}^{3}$, Kamil Ugurbil ${ }^{3}$, Anna Wang Roe ${ }^{1,2}$, Xiaotong Zhang ${ }^{1,2,4}$, and Dingxin Wang ${ }^{3}$

${ }^{1}$ Interdisciplinary Institute of Neuroscience and Technology, Qiushi Academy for Advanced Studies, Zhejiang University, Hangzhou, China, ${ }^{2}$ College of Biomedical Engineering \& Instrument Science, Zhejiang University, Hangzhou, China, ${ }^{3}$ Center for Magnetic Resonance Research, School of Medicine, University of Minnesota, Minneapolis, MN, United States, ${ }^{4}$ Key Laboratory for Biomedical Engineering of Ministry of Education, Zhejiang University, Hangzhou, China

EPI-based diffusion imaging methods are dominantly used, but suffer from susceptibility associated distortion and signal loss, making it challenging to achieve high-quality highresolution diffusion imaging results. To overcome these challenges, we implemented monopolar diffusion preparation module for TSE sequence (DP-TSE) and evaluated the performance in comparison to readout segmented multi-shot echo planner (RESOLVE) sequence for diffusion weighted imaging (DWI). Our study results suggest that Diffusion-Prepared TSE is a promising alternative for distortion-free, high-resolution diffusion imaging with superior diffusion SNR.

Meizhen $\operatorname{Han}^{1}$ and Jia-Hong Gao ${ }^{1}$

${ }^{1}$ Center for MRI Research, Peking University, Beijing, China 
High angular resolution diffusion MRI (HARDI), the most widely used method in in-vivo brain imaging experiments to delineate white matter pathways, has been found sufficient for resolving 2-way fiber crossings but unstable for detecting 3-way fiber crossings. Therefore, if more sensitive and accurate tractography is wanted, researchers need to use high b-value with multi-shell q-ball models, which can be time-consuming. In this study, we compared 3 diffusion MRI acquisition protocols by tracking callosal connections between motor areas with both probabilistic and deterministic fiber tracking algorithms and provided a new scheme for the future diffusion MRI experiment

Optimization of $\mathrm{b}$ values and reproducibility of perfusion and diffusion parameters using IntraVoxel Incoherent Motion (IVIM) with peripheral pulse triggering

Yu Ueda ${ }^{1}$, Minoru Hayashida ${ }^{2}$, Koji Yoshida ${ }^{2}$, Tomoyuki Okuaki ${ }^{1}$, Katsuyoshi Ito ${ }^{3}$, Makoto Obara ${ }^{1}$, and Marc Van Cauteren ${ }^{4}$

${ }^{1}$ Philips Japan, Tokyo, Japan, ${ }^{2}$ Kawasaki Medical School, Kurashiki, Japan, ${ }^{3}$ Yamaguchi University Graduate School of Medicine, Ube, Japan, ${ }^{4}$ Philips HealthTech, Tokyo, Japan

To investigate the reproducibility of IVIM-derived parameters with peripheral pulse unit (PPU) triggering and optimized b values combination to decrease scan time, we assessed the reproducibility by calculating coefficient of variation $(\mathrm{CV})$ for each parameter. Moreover, $D^{*}$ and $F$ calculated with some $b$ value patterns were compared to those with all $b$ values using the Pearson correlation. Our results suggest that cardiac gating does not improve reproducibility of perfusion and diffusion parameter. $F$ with only $4 \mathrm{~b}$ values (e.g. $\mathrm{b}=0-200-500-1000)$ can provide robust information on perfusion noninvasively with significantly shortened scan time.

Impact of slew rates on the performance of a novel high-gradient breast diffusion probe

Theresa Palm ${ }^{1}$, Jan Martin ${ }^{1}$, Bernhard Hensel ${ }^{2}$, Feng Jia ${ }^{3}$, Maxim Zaitsev ${ }^{3}$, Tristan A. Kuder ${ }^{4}$, Mark E. Ladd ${ }^{4}$, Michael Uder ${ }^{1}$, and Frederik B. Laun ${ }^{1}$

${ }^{1}$ Institute of Radiology, University Hospital Erlangen, Friedrich-Alexander-Universität Erlangen-Nürnberg (FAU), Erlangen, Germany, ${ }^{2}$ Center for Medical Physics and Engineering, Friedrich-Alexander-Universität Erlangen-Nürnberg (FAU), Erlangen, Germany, ${ }^{3}$ Department of Diagnostic Radiology, Medical Physics, University Hospital Freiburg, Freiburg, Germany, ${ }^{4}$ Medical Physics in Radiology, German Cancer Research Center, Heidelberg, Germany

Recent advances in gradient technology, in particular based on the use of local gradient coils, have increased the available gradient strength by almost an order of magnitude. In this context, the question arises what slew rates are required to translate the higher gradient amplitudes into the improved assessment of shorter diffusion times given a certain b-value. This work shows that slew rates are important in high-gradient diffusion experiments ( $\mathrm{G} \geq 300 \mathrm{mT} / \mathrm{m})$, in particular in low $b$-value applications $\left(b \leq 1000 \mathrm{~s} / \mathrm{mm}^{2}\right)$.

Low b-values and limited diffusion directions introduce bias in FA and MD that increases with decreasing voxel volumes.

Ofentse Noko ${ }^{1}$, Stephen Jermy ${ }^{1,2}$, Ali Alhamud $^{1,2}$, and Ernesta Meintjes ${ }^{1,2}$

${ }^{1}$ Department of Human Biology, University of Cape Town, Cape Town, South Africa, ${ }^{2}$ Cape Universities Body Imaging Centre (CUBIC), University of Cape Town, Cape Town, South Africa

Due to ECG triggering and breath hold techniques required to compensate for motion of the beating heart and respiration, acquisition times for cardiac diffusion tensor imaging (DTI) are limited. As such, lower b-values and fewer diffusion directions are typically used, together with larger slice thicknesses. This study aims to assess the impact of these changes on fractional anisotropy (FA) and mean diffusivity (MD) in a pineapple phantom. Smaller voxels were found to be more sensitive to changes in b-values and number of diffusion directions.

Progress in the use of SQUASHER for Diffusion weighted imaging

Steen Moeller ${ }^{1}$, Sudhir Ramanna ${ }^{1}$, Essa Yacoub ${ }^{1}$, and Mehmet Akcakaya ${ }^{1,2}$

${ }^{1}$ Center for Magnetic Resonance Research, University of Minnesota, Minneapolis, MN, United States, ${ }^{2}$ Department of Electrical and Computer Engineering, University of Minnesota, Minneapolis, MN, United States

The applicability of SQUASHER to EPI, along with a kz-dependent reconstruction approach for highly-accelerated 3D segmented EPI in dMRI

Caspar Florin ${ }^{1}$ and Jürgen Finsterbusch ${ }^{1}$

${ }^{1}$ Systems Neuroscience, University Medical Center Hamburg-Eppendorf, Hamburg, Germany 
Inner field-of-view EPI is widely used for diffusion-weighted acquisitions of the human spinal cord. However, due to the high in-plane resolution required acquisition times to achieve a reasonable signal-to-noise ratio are usually rather long. In this study, inner-field-of-view EPI based on 2D-selectove RF excitations is accelerated with multiband excitations. Two different approaches are considered that differ with respect to the orientation of the 2DRF trajectory and whether side excitations must be suppressed or can be used to cover the bands excited and acquired simultaneously. Results obtained in the human brains stem and cervical spinal cord in vivo are presented.

Anisotropic Diffusion Filter for Simultaneous Combination and Denoising of Multiple Acquisitions in DWI of the Spinal Cord

Sevgi Gokce Kafali ${ }^{1,2}$, Cagri Aydinkarahaliloglu ${ }^{1}$, Tolga Çukur ${ }^{1,2}$, and Emine Ulku Saritas ${ }^{1,2}$

${ }^{1}$ Electrical and Electronics Engineering, Bilkent University, Ankara, Turkey, ${ }^{2}$ National Magnetic Resonance Research Center (UMRAM), Ankara, Turkey

In diffusion weighted imaging (DWI), multiple acquisitions are acquired and averaged to attain a reasonable SNR level, especially for high spatial resolution or high b-value imaging. However, bulk or involuntary physiological motion during diffusion-sensitizing gradients alters the k-space, creating unpredictable global and/or local phases across multiple acquisitions. Therefore, direct complex averaging of these multiple acquisitions is prohibited. Here, we propose a reconstruction scheme based on modified anisotropic diffusion filtering, which starts with complex-valued acquisitions and corrects the phase issues while improving the SNR. The proposed reconstruction is demonstrated with in vivo DWI of the cervical spinal cord at $1.5 \mathrm{~T}$.

Improvement of diffusion-weighted image quality by iShim toward realization of cervical spinal cord region QSI

Yoshifumi Sone ${ }^{1}$, Zhouchen $\mathrm{Lu}^{1}$, Junichi Hata ${ }^{2}$, Daisuke Nakashima ${ }^{3}$, Katsuya Maruyama ${ }^{4}$, Alto Stemme ${ }^{5}$, Takeo Nagura ${ }^{3}$, Morio Matsumoto ${ }^{3}$, and Masaya Nakamura ${ }^{3}$

${ }^{1}$ Medical Scanning Tokyo, Tokyo, Japan, ${ }^{2}$ Central Institute for Experimental Animals, Japan, Tokyo, Japan, ${ }^{3}$ Department of Orthopaedic Surgery, Keio University School of Medicine, Tokyo, Japan, ${ }^{4}$ MR Research \& Collaboration Dpt., Diagnostic Imaging Business Area, Siemens Healthcare K.K., Tokyo, Japan, ${ }^{5}$ Siemens Healthcare GmbH, Erlangen, Germany, Erlangen, Germany

Herein, we adopted diffusion-weighted imaging (DWI) with a high fat suppression effect and high signal-to-noise ratio (SNR) in the cervical region, where magnetic field inhomogeneity may occur, using integrated slice-by-slice shimming (iShim), which improves static magnetic field (BO) shimming accuracy. We examined spinal cord SNR and standard deviation in healthy volunteers and performed cervical DWI with the conventional B0 shimming method and iShim, respectively. Furthermore, to verify whether short TI inversion recovery (STIR) or water excitation (WE) was appropriate as a fat suppression method, we used DWI with a high SNR at the cervical region by combining iShim with WE.

DTI-based free-water elimination with T2-weighting using dedicated anisotropic diffusion fibre phantoms

Ezequiel Farrher ${ }^{1}$, Kuan-Hung Cho ${ }^{2}$, Richard Buschbeck ${ }^{1}$, Husan-Han Chiang ${ }^{2}$, Ming-Jye Chen ${ }^{2}$, Farida Grinberg ${ }^{1,3}$, N. Jon Shah ${ }^{1,3,4,5,6}$, Chang-Hoon Choi ${ }^{1}$, and Li-Wei Kuo ${ }^{2,7}$

${ }^{1}$ Institute of Neuroscience and Medicine 4, Forschungszentrum Jülich, Jülich, Germany, ${ }^{2}$ Institute of Biomedical Engineering and Nanomedicine, National Health Research Institutes, Miaoli, Taiwan, ${ }^{3}$ Department of Neurology, Faculty of Medicine, RWTH Aachen University, Aachen, Germany, ${ }^{4}$ JARA - BRAIN - Translational Medicine, RWTH Aachen University, Aachen, Germany, ${ }^{5}$ Institute of Neuroscience and Medicine 11, Forschungszentrum Jülich, Jülich, Germany, ${ }^{6}$ Biomedical Imaging, School of Psychological Sciences, Monash University, Melbourne, Australia, ${ }^{7}$ Institute of Medical Device and Imaging, National Taiwan University College of Medicine, Taipei, Taiwan

In this work we demonstrate the use of two dedicated anisotropic diffusion fibre phantoms for the study of free-water elimination DTI. In particular, we make use of the recently proposed approach in which an extra dimension to the diffusion weighting, namely transverse relaxation weighting, is added to the model.

In vivo DTI-based free-water elimination with T2-weighting

Ezequiel Farrher ${ }^{1}$, Richard Buschbeck ${ }^{1}$, Chang-Hoon Choi $^{1}$, Li-Wei Kuo ${ }^{2,3}$, Seong-Dae Yun ${ }^{1}$, Farida Grinberg ${ }^{1,4}$, and N. Jon Shah ${ }^{1,4,5,6,7}$

${ }^{1}$ Institute of Neuroscience and Medicine 4, Forschungszentrum Jülich, Jülich, Germany, ${ }^{2}$ Institute of Biomedical Engineering and Nanomedicine, National Health Research Institutes, Miaoli, Taiwan, ${ }^{3}$ Institute of Medical Device and Imaging, National Taiwan University College of Medicine, Taipei, Taiwan, ${ }^{4}$ Department of Neurology, Faculty of Medicine, RWTH Aachen University, Aachen, Germany, ${ }^{5}$ JARA - BRAIN - Translational Medicine, RWTH Aachen University, Aachen, Germany, 6 Institute of Neuroscience and Medicine 11, Forschungszentrum Jülich, Jülich, Germany, ${ }^{7}$ Biomedical Imaging, School of Psychological Sciences, Monash University, Melbourne, Australia

Free-water elimination allows one to reduce the bias in DTI metrics induced by partial-volume effects. Unfortunately the fitting problem for this model is ill-conditioned. However, it has been recently demonstrated that the introduction of a second dimension determined by the echo-time, leads to a well-conditioned fitting problem. In this work we investigate the experimental design and data analysis pipeline of such experiments in vivo. 
Free water elimination (FWE) paradigms provide information about underlying pathology-induced tissue changes, based on a multi-compartment fit to the dMRI acquisition. Non-uniform intensity in MR signal, either due to coil or acquisition sequence, produces inhomogeneous tissue intensity profiles. This negatively affects FWE paradigms, producing artifactual multicompartment fits. In this work, through extensive application on varied datasets, we demonstrate the effect of using bias field correction, an optimized non-uniform intensity normalization, on reducing artifacts in FWE and producing physiologically relevant maps. This suggests that bias correction should be maintained as an essential step in dMRI preprocessing for FWE.

Navigated Multi-shot Diffusion-Weighted Imaging with Multiplexed Sensitivity Encoding

Valentina Taviani ${ }^{1}$, Ann Shimakawa ${ }^{1}$, Lloyd Estkowski ${ }^{1}$, Arnaud Guidon ${ }^{2}$, Ersin Bayram ${ }^{3}$, and Robert Peters ${ }^{4}$

${ }^{1}$ Global MR Applications and Workflow, GE Healthcare, Menlo Park, CA, United States, ${ }^{2}$ Global MR Applications and Workflow, GE Healthcare, Boston, MA, United States, ${ }^{3}$ Global MR Applications and Workflow, GE Healthcare, Houston, TX, United States, ${ }^{4}$ Global MR Applications and Workflow, GE Healthcare, Waukesha, WI, United States

MUltiplexed Sensitivity Encoding (MUSE) has been successfully used to correct for motion-induced phase errors in multi-shot diffusion-weighted imaging. However, this technique relies heavily on parallel imaging (PI) and can result in residual aliasing and excessive noise amplification when the number of shots is similar to the number of receiver coil elements. We propose a navigated multi-shot approach with multiplexed sensitivity encoding to handle cases where the coil geometry would otherwise limit the maximum number of interleaves. We show that both PI and 2D-selective excitation pulses can be used to reduce the scan duration, while maintaining similar levels of distortion.

Automatic and Spatially Varying Phase Correction for Diffusion Weighted Images

Marco Pizzolato ${ }^{1}$ and Rachid Deriche ${ }^{2}$

${ }^{1} E P F L$, Lausanne, Switzerland, ${ }^{2}$ Athena, Inria, Sophia Antipolis, France

Phase Correction is a post-processing procedure exploiting the phase of magnetic resonance images in order to obtain real-valued images containing tissue contrast with additive Gaussian noise, as opposed to magnitude images which are typically affected by a bias due to the Rician distribution of noise. This bias is particularly relevant in Diffusion Weighted Images where the signal-to-noise ratio is intrinsically low. We propose a method for automatically assessing the optimal amount of required correction based on properties of the noise affecting the images: its variance and positional non-stationarity. We present results for diffusion metrics such as FA, AD, and MD.

Image-based Multi-Scale Distortion Correction: Application to Diffusion Imaging

Lars Bielak ${ }^{1}$, Hatice Bunea ${ }^{2}$, Nicole Wiedenmann ${ }^{2}$, Anca-Ligia Grosu² ${ }^{2}$ and Michael Bock ${ }^{1}$

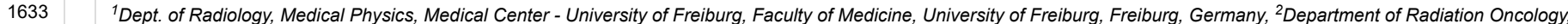
Medical Center - University of Freiburg, Faculty of Medicine, University of Freiburg, German Cancer Consortium (DKTK), Partner Site Freiburg, Freiburg, Germany

This work presents an algorithm that calculates a distortion field to correct a geometrically distorted image using an anatomically precise reference image. The algorithm employs mutual information based rigid image registration with a pyramidal architecture. Validation was performed on simulated distortion fields and in vivo comparison to a measured $\mathrm{B}_{0}$-fieldmap.

High Resolution Reconstruction of Diffusion Weighted Imaging Using EPI-Corrected Snapshots Acquired with Rotated K-spaces

Hengameh Mirzaalian ${ }^{1}$, Benoit Scherrer ${ }^{1}$, Onur Afacan ${ }^{1}$, Ali Gholipour ${ }^{1}$, and Simon K. Warfield ${ }^{1}$

${ }^{1}$ Harvard Medical School, Boston, MA, United States

We propose a non-Cartesian high resolution reconstruction of diffusion-weighted magnetic resonance imaging (DW-MRI) using multi-snapshots acquired with rotated K-spaces. Our technique boosts the signal level by reducing the echo time and by increasing voxel size for each snapshot. The final high resolution image is reconstructed by fusion of the snapshots, which were corrected for Echo-Planar-Imaging (EPI) distortions. We applied and evaluated different EPI correction methods. Through qualitative and quantitative evaluations based on in-vivo experiments, we showed that our protocol and image reconstruction technique leads to high spatial resolution and high signal-to-noise ratio DW-MRI. 
${ }^{1}$ Quantitative Medical Imaging Section, NIBIB, NIH, Bethesda, MD, United States, ${ }^{2}$ The Henry M. Jackson Foundation for the Advancement of Military Medicine Inc, Bethesda, MD, United States, ${ }^{3}$ Baylor College of Medicine, Houston, TX, United States, ${ }^{4}$ Chronic Effects of Neurotrauma Consortium, Richmond, VA, United States

In this study we evaluate the effect of echo planar imaging (EPI) distortion artifact as a contributing factor in inter-site variability. With living phantom data acquired with opposite phase encoding direction protocol (blipup-blipdown), we show the effectiveness of a robust EPI distortion correction method in reducing inter-site variability.

High-resolution off-resonance maps improve conformity between distortion-corrected EPI acquisitions and distortion-free references

Michael J van Rijssel ${ }^{1}$, Frank Zijlstra ${ }^{1}$, Peter R Seevinck ${ }^{1}$, Peter R Luijten ${ }^{1}$, Dennis W J Klomp ${ }^{1}$, and Josien P W Pluim ${ }^{1,2}$

${ }^{1}$ Center for Image Sciences, UMC Utrecht, Utrecht, Netherlands, ${ }^{2}$ Department of Biomedical Engineering, Eindhoven University of Technology, Eindhoven, Netherlands

The majority of diffusion acquisitions is affected by geometrical distortions due to susceptibility induced off-resonance effects in the EPI readout. This hampers the use and effectiveness of these images in multiparametric cancer protocols, especially in lipid-rich environments such as the human breast where tissue interfaces cause large but local discontinuities. Preliminary results show that improvements upon existing correction techniques can be made by using high-resolution off-resonance information in distortion correction algorithms.

Effects of phase error on image reconstruction for simultaneous multi-slice readout-segmented diffusion MRI

SeyyedKazem HashemizadehKolowri ${ }^{1}$, Rong-Rong Chen ${ }^{1}$, Edward V. R. DiBella ${ }^{1,2,3}$, Edward W. Hsu ${ }^{3}$, Leslie Ying ${ }^{4}$, and Ganesh Adluru ${ }^{2}$

${ }^{1} E C E$, University of Utah, SALT LAKE CITY, UT, United States, ${ }^{2}$ Radiology and Imaging Sciences, University of Utah, SALT LAKE CITY, UT, United States, ${ }^{3}$ Bioengineering, University of Utah, SALT LAKE CITY, UT, United States, ${ }^{4}$ Biomedical Engineering, The State University of New York (SUNY) at Buffalo, Buffalo, NY, United States

In this work, we study the effect of phase errors on the quality of image reconstructions for simultaneous multi-slice (SMS) readout-segmented echo planar imaging (RS-EPI) acquisitions. We propose an iterative split slice-GRAPPA (I-SSG) algorithm to train improved kernels using estimated diffusion weighted images (DWIs) rather than baseline images. Results from stroke patients show that the proposed I-SSG algorithm produces consistently better reconstructions than the SSG algorithm in the presence of baseline phase errors.

Distortion Correction using Reverse Polarity Gradient Method: Algorithm Optimization for Prostate Imaging using a Hybrid Weighting Metric

Maggie M Fung ${ }^{1}$, Pauline Worters ${ }^{2}$, Ek Tsoon Tan ${ }^{3}$, Arnaud Guidon ${ }^{4}$, and Ersin Bayram ${ }^{5}$

${ }^{1}$ Applications \& Workflow, GE Healthcare, New York, NY, United States, ${ }^{2}$ Applications \& Workflow, GE Healthcare, Menlo Park, CA, United States, ${ }^{3}$ Global Research Center, GE, Niskayuna, NY, United States, ${ }^{4}$ Applications \& Workflow, GE Healthcare, Boston, MA, United States, ${ }^{5}$ Applications \& Workflow, GE Healthcare, Houston, TX, United States

Prostate Diffusion Weighted Echo Planar imaging (DW-EPI) routinely suffers from nonlinear geometric distortion due to B0 inhomogeneity. Although reverse phase-encoding polaritybased distortion correction method works well in the brain, the same technique causes artifacts in prostate DWI due to the low SNR nature of body DWI scans, and the inconsistency of image content between the reverse and forward polarity images. In this study, we showed that a hybrid weighting metric method could improve the distortion correction performance in prostate DWI.

An integrated model-based framework for the correction of signal pile-up and translational offsets in prostate diffusion MRI

Muhammad Usman ${ }^{1}$, Lebina Kakkar ${ }^{2}$, Karin Shmueli ${ }^{3}$, Simon Arridge ${ }^{1}$, and David Atkinson ${ }^{2}$

${ }^{1}$ Centre for Medical Image Computing, Department of Computer Science, University College London, London, United Kingdom, ${ }^{2}$ Centre for Medical Imaging, Division of Medicine, University College London, London, United Kingdom, London, United Kingdom, ${ }^{3}$ Medical Physics and Biomedical Engineering, University College London, London, United Kingdom, London, United Kingdom

Prostate diffusion EPI scans suffer from geometric distortions, signal pile-up and signal drop-out due to differences in susceptibility values at interface between prostate and rectal-air. In this work, an integrated model based framework is proposed that can correct for signal pile-up in regions of severe distortions and can compensate for any translational offsets that may exist between different scans. In-vivo validation of the proposed method is done in patients. 
${ }^{1}$ Department of Biomedical Engineering, The University of North Carolina at Chapel Hill, Chapel Hill, NC, United States, ${ }^{2}$ Biomedical Research Imaging Center (BRIC), The University of North Carolina at Chapel Hill, Chapel Hill, NC, United States, ${ }^{3}$ Department of Radiology, The University of North Carolina at Chapel Hill, Chapel Hill, NC, United States

The magnetic field in a MR scanner varies slightly in strength over time and causes the signal to drift. This drift can vary from voxel to voxel both in extent and direction. In this abstract, we show using diffusion MRI data that signal drift can be corrected more accurately when done locally than globally over the whole image volume ${ }^{1}$. For this purpose, we employ a nonparametric correction method using non-diffusion-weighted scans interspersed in the diffusion-weighted image series.

Local Optimization of Diffusion Encoding Gradients Using a Z-Gradient Array for Echo Time Reduction in DWI

Koray Ertan ${ }^{1,2}$, Soheil Taraghinia ${ }^{1,2}$, Emine Ulku Saritas ${ }^{1,2}$, and Ergin Atalar ${ }^{1,2}$

${ }^{1}$ National Magnetic Resonance Resarch Center (UMRAM), Bilkent University, ANKARA, Turkey, ${ }^{2}$ Department of Electrical and Electronics Engineering, Bilkent University, ANKARA, Turkey

Spatial dependency of the gradient fields can be dynamically optimized using a gradient array coils driven by independent gradient amplifiers. Such dynamic optimization allows to maximize gradient strengths inside a target volume such as slice rather than the entire VOI. Gradient linearity error constraints can also be relaxed to obtain higher gradient strengths. Higher gradient strength can be utilized as diffusion gradients for shorter diffusion durations and TEs for fixed b-value, which increases the SNR of the DWI. Nine channel z-gradient array is used to create optimized gradient fields, which lead to $50 \%$ reduction of TE in phantom experiments.

2D Single-Shot Radial Diffusion-Weighted Imaging free of geometric distortion and optimization of SNR using Variable Flip-Angle and Random View-Ordering

Kyle Jeong ${ }^{1,2}$ and Eun-Kee Jeong ${ }^{1,3}$

${ }^{1}$ Utah Center for Advanced Imaging and Research, University of Utah, Salt Lake City, UT, United States, ${ }^{2}$ Department of Bioengineering, University of Utah, Salt Lake City, UT, United States, ${ }^{3}$ Radiology and Imaging Sciences, University of Utah, Salt Lake City, UT, United States

The 2D ss-DWEPI is routinely used for in-vivo DW imaging, because of its immunity to motion-induced artifact, but prone to susceptibility-induced geometric distortion. We present a novel DWI technique using single-shot radial imaging, which produces DW images with minimal geometric distortion, no motion artifact, and with optimized SNR and reduced effect of undersampling radial streak artifact. Variable-flip angle (VFA) and random-view ordering (RVO) were implemented to improve the SNR and reduce the geometric distortion, respectively.

Single-scan Mapping of Mean Diffusivity Using the Incomplete Initial Nutation Diffusion Imaging (INDI) framework

Andrada lanus ${ }^{1,2}$ and Noam Shemesh

${ }^{1}$ Champalimaud Neuroscience Programme, Champalimaud Centre for the Unknown, Lisbon, Portugal, ${ }^{2}$ Centre for Medical Image Computing, Department of Computer Science, University College London, London, United Kingdom

Diffusion MRI techniques require at least two different acquisitions separated by a repetition time in order to map mean diffusivity. Thus, dynamic imaging techniques, such as diffusion functional MRI, which aim to measure rapid diffusivity changes, might provide results confounded by T2 changes over the repetition time. This study introduces and validates the INDI (incomplete initial nutation diffusion imaging) framework, which can be used to accelerate diffusion acquisition so that the reference and diffusion weighted images are acquired within a few tens of milliseconds of each other.

Removal or correction of volumes affected by bulk motion: impact on DTI and NODDI metrics

Kerstin Pannek ${ }^{1}$, John Welsh ${ }^{2}$, Jurgen Fripp ${ }^{1}$, Joanne George ${ }^{3}$, Paul Colditz $^{3}$, Roslyn Boyd ${ }^{3}$, and Stephen Rose ${ }^{1}$

${ }^{1}$ CSIRO, Brisbane, Australia, ${ }^{2}$ University of Newcastle, Newcastle, Australia, ${ }^{3}$ The University of Queensland, Brisbane, Australia

In difficult patient populations, the interleaved acquisition of diffusion weighted volumes often leads to images that are not self-consistent due to movement. Here, we investigated the effect of removing or correcting volumes with movement artefacts on the DTI measures FA and MD, as well as on NODDI measures. While removal of affected volumes is typically used, we found that a simple correction strategy leads to markedly lower bias and variability in all diffusion measures. Data that may need to be rejected entirely if volume removal is used, may be salvaged if correction is used.

Quantifying deviations from gradient design in multi-platform longitudinal DWI QC for on-scanner correction of diffusion weighting bias

Dariya I Malyarenko ${ }^{1}$, Yuxi Pang ${ }^{1}$, Lisa J Wilmes ${ }^{2}$, Ek T Tan ${ }^{3}$, Johan Tondeur ${ }^{4}$, Ajit Devaraj ${ }^{5}$, Julien Sénégas ${ }^{6}$, Johannes Peeters ${ }^{7}$, John E Kirsch ${ }^{8}$, Michael A Jacobs ${ }^{9}$, David C Newitt $^{2}$ and Thomas L Chenevert ${ }^{1}$ 
${ }^{1}$ Radiology, University of Michigan, Ann Arbor, MI, United States, ${ }^{2}$ Radiology and Biomedical Imaging, University of California San Francisco, San Francisco, CA, United States, ${ }^{3}$ GE Global Research, Niskayuna, NY, United States, ${ }^{4}$ Siemens Medical Solutions, Cary, NC, United States, ${ }^{5}$ Philips Research Laboratories, Cambridge, MA, United States, ${ }^{6}$ Philips Research Laboratories, Hamburg, Germany, ${ }^{7}$ Philips MR Clinical Science, Best, Netherlands, ${ }^{8}$ Radiology, Massachusetts General Hospital, Boston, MA, United States, ${ }^{9}$ Radiology and Radiological Science, John Hopkins University School of Medicine, Baltimore, MD, United States

The most practical correction of nonuniform diffusion weighting due to gradient nonlinearity would use scanner-specific gradient design information similar to current mitigation of geometric image distortions. To check the feasibility of this approach in a multi-center, multi-scanner setting, longitudinal DWI quality control studies using a quantitative diffusion phantom were performed on representative MRI platforms in collaboration with three vendors. Here we report preliminary results for proposed descriptive metrics that adequately reflect the amount and source of deviations from system gradient design to guide implementation of comprehensive bias correction for quantitative DWI applications.

Intravoxel Incoherent Motion (IVIM) Fingerprinting

Qiuting Wen ${ }^{1}$, Li Feng ${ }^{2}$, Kun Zhou ${ }^{3}$, and Yu-Chien $\mathrm{Wu}^{1}$

${ }^{1}$ Center for Neuroimaging, Department of Radiology and Imaging Sciences, Indiana University, School of Medicine, Indianapolis, IN, United States, ${ }^{2}$ Center for Advanced Imaging Innovation and Research (CAI2R), New York University, School of Medicine, New York, NY, United States, ${ }^{3}$ Siemens Shenzhen Magnetic Resonance Ltd., Shenzhen, China

Intravoxel incoherent motion (IVIM) imaging employs a bi-exponential diffusion model to estimate capillary contributions to the diffusion-weighted signal. Major challenges of IVIM are long acquisition time, long processing time, and image distortion associated with EPI acquisition. In this work, we proposed a novel framework for rapid and distortion-free IVIM imaging called IVIM-Fingerprinting. It employs a single-shot acquisition scheme and an advanced image reconstruction scheme in combination with the recently proposed concept of MR Fingerprinting. Its performance was demonstrated both for simulation and for in-vivo studies.

Investigating the effect of gradient nonlinearities on Diffusional Kurtosis Imaging parameters: Results from the Human Connectome Project

Hamed Y. Mesri ${ }^{1}$, Szabolcs David ${ }^{1}$, Max A. Viergever ${ }^{1}$, and Alexander A. Leemans ${ }^{1}$

${ }^{1}$ Image Sciences Institute, University Medical Center Utrecht and Utrecht University, Utrecht, Netherlands

Gradient field nonlinearities in diffusion-weighted MRI may lead to systematic errors in the diffusion metrics. Despite previous works highlighting the adverse impact of gradient field nonuniformities on diffusion-weighted MRI, these effects are usually neglected and left uncorrected. In this work we use simulations and real data from the Human Connectome Project to investigate the effect of gradient field nonlinearities on the measures from Diffusional Kurtosis Imaging. Our results demonstrated that in general, the effect for the diffusion tensor metrics is larger than the effect for diffusional kurtosis metrics. However, the effect of the gradient nonlinearities on the kurtosis metrics should not be neglected.

Error estimation and evaluation of spatial smoothing processing for diffusion kurtosis imaging

Suguru Yokosawa ${ }^{1}$, Yoshitaka Bito ${ }^{2}$, and Hisaaki Ochi ${ }^{1}$

${ }^{1}$ Research \& Development Group, Hitachi, Ltd., Tokyo, Japan, ${ }^{2}$ Healthcare Business Unit, Hitachi, Ltd., Tokyo, Japan

DKI often suffers from error estimation such as unphysical negative kurtosis values which result in black voxels on mean kurtosis (MK) map. In this study, causes of the estimation error are investigated by using simulation. In addition, effect of smoothing processing is quantitatively evaluated in terms of reduction in estimation error and image sharpness. Our findings will be useful for clinical diagnosis using DKI.

Improved diffusion propagator reconstruction using Hermite functions and compressed sensing

Gabriel Varela-Mattatall ${ }^{1,2}$, Carlos Castillo-Passi ${ }^{1,2}$, Joaquin Mura $^{1}$, and Pablo Irarrazaval ${ }^{1,2,3}$

${ }^{1}$ Biomedical Imaging Center, Pontificia Universidad Católica de Chile, Santiago, Chile, ${ }^{2}$ Department of Electrical Engineering, Pontificia Universidad Católica de Chile, Santiago, Chile, ${ }^{3}$ Institute for Biological and Medical Engineering, Pontificia Universidad Católica de Chile, Santiago, Chile

Mean apparent propagator (MAP) reconstructs the diffusion pdf using a dictionary based on Hermite functions. The first element corresponds to a tensor approximation; and the following elements add non-gaussian components. To improve non-gaussian accuracy, one needs to increase the size of the dictionary, but it also increases the number of q-space samples for a robust optimisation. We propose the use of compressed sensing to efficiently increase the number of atoms in the dictionary by exploiting its sparsity for a better reconstruction. 
${ }^{1}$ School of Computer Science and Technology, Beijing Institute of Technology, Beijing, China, ${ }^{2}$ Center for Biomedical Imaging Research, Department of Biomedical Engineering, Tsinghua University, Beijing, China, ${ }^{3}$ Department of Biomedical Engineering, School of Medicine, Tsinghua University, Beijing, China

The estimation of voxel anisotropic properties from diffusion tensor imaging is critical for fiber tracking. Here machine learning was used to estimate the voxel anisotropic properties from undersampled data that were reconstructed by dictionary learning.

Deep learning with synthetic data for free water elimination in diffusion MRI

Miguel Molina-Romero ${ }^{1,2}$, Pedro A. Gómez ${ }^{1,2}$, Shadi Albarqouni ${ }^{1}$, Jonathan I. Sperl ${ }^{2}$, Marion I. Menzel ${ }^{2}$, and Bjoern H. Menze ${ }^{1}$

${ }^{1}$ Technical University of Munich, Munich, Germany, ${ }^{2}$ GE Global Research Europe, Munich, Germany

Diffusion metrics are typically biased by Cerebrospinal fluid (CSF) contamination. In this work, we present a deep learning based solution to remove the CSF contribution. First, we train an artificial neural network (ANN) with synthetic data to estimate the tissue volume fraction. Second, we use the resulting network to predict estimates of the tissue volume fraction for real data, and use them to correct for CSF contamination. Results show corrected CSF contribution which, in turn, indicates that the tissue volume fraction can be estimated using this joint data generation and deep learning approach.

A supervised learning approach for diffusion MRI quality control with minimal training data

Mark S Graham¹, Ivana Drobnjak ${ }^{1}$, and Hui Zhang ${ }^{1}$

${ }^{1}$ Centre for Medical Image Computing \& Department of Computer Science, University College London, London, United Kingdom

Quality control (QC) in diffusion-weighted MRI (DW-MRI) involves identifying problematic volumes in datasets. The current gold standard involves time-consuming manual inspection of data, and even supervised learning techniques that aim to replace the gold standard require manually labelled datasets for training. In this work we show the need for manual labelling can be greatly reduced by training a supervised classifier on realistic simulated data, and using a small amount of labelled data for a final calibration step. Such an approach may have applications in other image analysis tasks where labelled datasets are expensive or difficult to acquire.

Efficient Reconstruction of Diffusion Kurtosis Imaging Based on a Hierarchical Convolutional Neural Network

Ting Gong ${ }^{1}$, Hongjian $\mathrm{He}^{1}$, Zhiwei Li ${ }^{2}$, Zhichao Lin², Qiqi Tong ${ }^{1}$, Chen $\mathrm{Li}^{1}$, Yi Sun ${ }^{3}$, Feng Yu ${ }^{2}$, and Jianhui Zhong ${ }^{1,4}$

${ }^{1}$ Center for Brain Imaging Science and Technology, Key Laboratory for Biomedical Engineering of Ministry of Education, College of Biomedical Engineering and Instrumental Science, Zhejiang University, Hangzhou, China, ${ }^{2}$ Department of Instrument Science \& Technology, Zhejiang University, Hangzhou, China, ${ }^{3}$ MR Collaboration NE Asia, Siemens Healthcare, Shanghai, China, ${ }^{4}$ Department of Imaging Sciences, University of Rochester, Rochester, NY, United States

Diffusion kurtosis imaging (DKI) captures more complex microstructural properties than the widely used diffusion tensor imaging (DTI) but requires a longer acquisition time. To accelerate its acquisition, and thus facilitate its practical clinical use, a hierarchical convolution neural network $\left(\mathrm{H}_{-} \mathrm{CNN}\right)$ reconstruction method was proposed. The results showed that the $\mathrm{H}_{-} \mathrm{CNN}$ method provides efficient reconstruction of all eight DTI and DKI measures using as few as nine DWIs, with improved robustness against noise and the retention of fine structures, compared to artificial neural network-based methods. The H_CNN method potentially enables DKI clinical applications with an acquisition time of one minute.

Principal component analysis for model-free denoising of multi b-value diffusion-weighted images

Oliver J Gurney-Champion ${ }^{1}$, David J Collins ${ }^{2}$, Mihaela Rata ${ }^{2}$, Andreas Wetscherek ${ }^{1}$, Uwe Oelfke ${ }^{1}$, Kevin J Harrington ${ }^{3}$, and Matthew R Orton ${ }^{2}$

${ }^{1}$ Joint department of physics, Institute of Cancer Research and The Royal Marsden NHS Foundation Trust, London, United Kingdom, ${ }^{2}$ CRUK Cancer Imaging Centre, Institute of Cancer Research and The Royal Marsden NHS Foundation Trust, London, United Kingdom, ${ }^{3}$ Division of Radiotherapy \& Imaging, Institute of Cancer Research and The Royal Marsden NHS Foundation Trust, London, United Kingdom

We introduce principal component analyses (PCA) as a denoising technique for diffusion-weighted MRI (DWI) that is independent of the diffusion attenuation model. PCA denoises DWI data using only informative components while removing noisy ones. We show that it outperforms model-based denoising in simulations as well as in vivo. In simulations, PCA-denoising resulted in smaller systematic errors, while random errors were similar. In vivo, PCA-denoising rendered less noisy images and when motion was present, PCA recovered certain structures that were obscured by motion in model-based denoising. In conclusion, PCA-denoising is a powerful modelfree tool for denoising DWI data. 
Rafael Neto Henriques ${ }^{1,2}$ and Marta Morgado Correia ${ }^{2}$

${ }^{1}$ Champalimaud Research, Champalimaud Centre for the Unknown, Lisbon, Portugal, ${ }^{2}$ Cognition and Brain Sciences Unit, MRC, Cambridge, United Kingdom

Recent studies showed that PCA denoising algorithms using random matrix theory provide an optimal compromise between noise suppression and loss of anatomical information for standard diffusion measures and tractography approaches. In this study, we show that this algorithm seems also to optimally preserve the non-Gaussian diffusion properties. Several factors that influence the performance of the PCA denoising algorithm are also assessed, such as the spatial heterogeneity of diffusion parameters across neighbour voxels and different scanning protocols. Moreover, the compatibility of PCA denoising with Gibbs artefact suppression and noise bias correction is evaluated.

Traditional Poster

\section{Diffusion MRI: Applications}

Exhibition Hall 1656-1675

Tuesday 8:15 - 10:15

Diffusion MRI as a descriptive imaging marker of the pathogenesis of treatment-resistant depression.

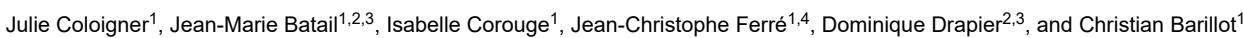

${ }^{1}$ Univ Rennes, INRIA, CNRS, Inserm, IRISA UMR 6074, VISAGES ERL U-1228, F-35000, Rennes, France, ${ }^{2}$ Academic Psychiatry Department, Centre Hospitalier Guillaume Régnier, Rennes, France, ${ }^{3} E A 4712$ Behavior and Basal Ganglia, CHU Rennes, University of Rennes 1, Rennes, France, ${ }^{4}$ Department of Neuroradiology, University Hospital of Rennes, Rennes, France

Despite the extensive therapy options available for depression, treatment-resistant depression (TRD) occurs in $20-30 \%$ of depressed patients. . Consequently, identification of neural changes in TRD could support to better understand the mechanism of resistance and to improve the treatment of individual depressed patients. We aimed to investigate the white-matter microstructure in a sample of depressed patients in which response to treatment was subsequently evaluated 6 months after. Our findings suggest the abnormalities of the white-matter integrity in multiple white matter tracts, such as anterior limb of internal capsule and genu of corpus may play a role in the pathogenesis of treatment-resistant depression.

Diffusion tensor MR imaging of optic radiation in advanced bilateral glaucoma patients in comparison to normal control subjects

Chanon Ngamsombat ${ }^{1}$, Thanakorn Chareankarunyuta ${ }^{1}$, Prapaporn Pornwuthi ${ }^{1}$, Panida Charnchaowanish ${ }^{1}$, Yudthaphon Vichianin ${ }^{2}$, Ngamkae Ruangvaravate ${ }^{3}$, Shuo Zhang ${ }^{4}$, and Orasa Chawalparit ${ }^{1}$

${ }^{1}$ Department of Radiology, Faculty of Medicine, Siriraj Hospital, Mahidol University, Bangkok, Thailand, ${ }^{2}$ Department of Radiological Technology, Faculty of Medical Technology, Mahidol University, Bangkok, Thailand, ${ }^{3}$ Department of Ophthalmology, Faculty of Medicine, Siriraj Hospital, Mahidol University, Bangkok, Thailand, ${ }^{4}$ Philips Healthcare, Singapore, Singapore

Glaucoma is a worldwide leading cause of irreversible vision loss characterized by degeneration of retinal ganglion cells. The damage can be found in visual pathway beyond retina and optic disc to visual cortex. Diffusion tensor MR imaging (DTI) is widely used for evaluation of early microstructural change in the brain parenchyma. Here we reported abnormal change of the optic radiation in advanced bilateral glaucoma patients using DTI as compared to the age-matched normal control subjects. The obtained DTI parameters may serve as potential quantitative imaging biomarkers to provide complementary indication of the disease condition in glaucoma.

Altered white matter tracts in schizophrenia with persistent negative symptoms

Jing-Ying Huang ${ }^{1,2}$, Chih-Min Liü ${ }^{3,4}$, Tzung-Jeng Hwang ${ }^{3,4}$, Yung-Chin Hsu' ${ }^{1}$, Hai-Gwo Hwu ${ }^{3,4}$, and Wen-Yih Isaac Tseng ${ }^{1,4,5,6}$

${ }^{1}$ Institute of Medical Device and Imaging, National Taiwan University College of Medicine, Taipei, Taiwan, ${ }^{2}$ Department of Radiology, Wei Gong Memorial Hospital, Miaoli, Taiwan, ${ }^{3}$ Department of Psychiatry, National Taiwan University Hospital, Taipei, Taiwan, ${ }^{4}$ Graduate Institute of Brain and Mind Sciences, National Taiwan University College of Medicine, Taipei, Taiwan, ${ }^{5}$ Department of Medical Imaging, National Taiwan University Hospital, Taipei, Taiwan, ${ }^{6}$ Molecular Imaging Center, National Taiwan University, Taipei, Taiwan

This article aimed to investigate the alteration of white matter tracts in schizophrenia with persistent negative symptoms (PNS) in an attempt to identify white matter tracts that are characteristic of PNS. We performed diffusion spectrum imaging (DSI) and whole brain tract-based automatic analysis (TBAA) to compare the tract integrity among healthy controls, PNS and non-PNS groups. Our results showed that the right uncinate fasciculus and bilateral thalamic radiations of the ventral lateral prefrontal cortex are tract correlates of PNS.

Functional organisation of the hyperdirect pathway by in vivo structural connectivity imaging in healthy humans at 3T

Gizem Temiz ${ }^{1,2}$, Chantal François ${ }^{1}$, Carine Karachi ${ }^{1,3}$, Sonia Pujol ${ }^{4}$, Eric Bardinet ${ }^{1,2}$, and Sophie Bernadette Sébille ${ }^{1,2}$ 
${ }^{1}$ Brain and Spine Institute, CNRS UMR 7225 - INSERM U 1127 - UPMC-P6 UMR S 1127, Paris, France, ${ }^{2}$ Center of Neurolmaging Research - CENIR, Paris, France, ${ }^{3}$ AP-HP, Hôpital de la Pitié-Salpêtrière, Department of Neurosurgery, Paris, France, ${ }^{4}$ Surgical Planning Laboratory, Department of Radiology, Brigham and Women's Hospital, Harvard Medical School, Boston, MA, United States

The goal of this study is to investigate the anatomo-functional organization of the hyperdirect pathway between the subthalamic nucleus (STN) and the cortex in humans. We identified motor, limbic and associative areas of the whole cortex. We used DWI from 30 healthy subjects and probabilistic tractography between the STN and 39 cortical areas. The motor part of the hyperdirect pathway was found predominant compare to the limbic and above all the associative parts.

Utility of Advanced Diffusion Models in Assessing Abscess Structure

Robert Wujek ${ }^{1}$, Mona Al-Gizawiy ${ }^{1}$, Kathleen Schmainda ${ }^{1}$, and Rodney Willoughby ${ }^{2}$

${ }^{1}$ Radiology, Medical College of Wisconsin, Milwaukee, WI, United States, ${ }^{2}$ Pediatrics, Medical College of Wisconsin, Milwaukee, WI, United States

MR imaging is commonly used in the diagnosis and monitoring of cerebral abscess, especially diffusion weighted imaging. However, the use of advanced diffusion models has yet to be seen with respect to this type of brain mass. The stretched-exponential, intra-voxel incoherent, and kurtosis diffusion models not only generate diffusivity coefficients, but also other parameters that may prove valuable in properly understanding the structure and progression of such lesions.

Optic radiation tractography in pediatric brain tumor and epilepsy surgery: a test-retest reliability assessment of the tractography method

Joseph Yuan-Mou Yang ${ }^{1,2,3}$, Richard Beare ${ }^{1,4}$, Michelle Hao Wu ${ }^{5}$, Sarah M. Barton ${ }^{1,6,7}$, Charles B. Malpas ${ }^{1,8}$, Vicki Anderson ${ }^{6,8,9,10}$, Wirginia J Maixner ${ }^{2,3}$, and Marc L Seal ${ }^{1,6}$

${ }^{1}$ Developmental Imaging, Murdoch Children's Research Institute, Melbourne, Australia, ${ }^{2}$ Neuroscience Research, Murdoch Children's Research Institute, Melbourne, Australia, ${ }^{3}$ Neurosurgery, The Royal Children's Hospital, Melbourne, Australia, ${ }^{4}$ Medicine, Monash University, Melbourne, Australia, ${ }^{5}$ Medical Imaging, The Royal Children's Hospital, Melbourne, Australia, ${ }^{6}$ Paediatrics, University of Melbourne, Melbourne, Australia, ${ }^{7}$ Neurology, the Royal Children's Hospital, Melbourne, Australia, ${ }^{8}$ Melbourne School of Psychological Sciences, University of Melbourne, Melbourne, Australia, ${ }^{9}$ Clinical Sciences, Murdoch Children's Research Institute, Melbourne, Australia, ${ }^{10}$ Psychology, the Royal Children's Hospital, Melbourne, Australia

Existing optic radiation (OR) tractography methods lack pediatric and surgical focus. We proposed a clinically feasible tractography framework and examined its test-retest reliability using both the preoperative and intraoperative MRI from eight pediatric epilepsy and brain tumor patients. Good to excellent intra- and inter-rater reproducibility was demonstrated in the assessments of all diffusion and morphological track metrics. The reconstructions closely resembled classic anatomy. All OR images were used to assist surgical planning and resection. Postoperatively, no patient had new visual field deficits. Our tractography method generates reproducible OR images that can be safely implemented in routine, non-emergency pediatric neurosurgical settings.

Impaired executive and visual network integrity in patients with Parkinson's disease and psychosis: A structural connectome based study

Abhishek Lenka ${ }^{1}$, Apurva Shah ${ }^{2}$, Jitender Saini ${ }^{3}$, Pramod Kumar Pal ${ }^{1}$, and Madhura Ingalhalikar ${ }^{2}$

${ }^{1}$ Neurology, NIMHANS, Bengaluru, India, ${ }^{2}$ Department of Electronics, Symbiosis Institute of Technology, Symbiosis International University, Pune, India, ${ }^{3}$ Radiology, NIMHANS,

Bengaluru, India

Psychosis manifested as formed visual hallucinations is one of the debilitating non-motor symptoms of Parkinson's disease (PD), the patho-physiology of which remains unclear. To gain insights into the neural correlates of psychosis in PD this study analyzed the structural connectomic sub-networks of visual, executive and memory circuits between patients with PD and psychosis (PD-P), PD without psychosis (PD-NP) and controls (HC). When PD-P and HCs were compared, a global connectivity deficit was observed in the visual and executive circuits and multiple connections within the visual network demonstrated significantly lower connectivity in PD-P. Such changes were not observed in PD-NP vs. HCs.

A comparison of different brain connectivity markers for classifying Gulf-war illness

Bang-Bon $\mathrm{Koo}^{1}$ and Kimberly Sullivan ${ }^{2}$

${ }^{1}$ Anatomy and Neurobiology, Boston University, Boston, MA, United States, ${ }^{2}$ Boston University School of Public Health, Boston, MA, United States

Gulf War Illness (GWI) represents a cluster of multi-system chronic symptoms experienced by a third of veterans who served in the Gulf War. The exact cause of GWI remains unknown and efforts directed towards developing treatments have been hampered by the lack of meaningful objective biomarkers of the illness. Combining machine learning technology to brain connectivity imaging may allow for better understanding of the complex pathobiology of GWI. Choosing optimal imaging index should be a first step to maximize its classification performance. 
Yuan Tian ${ }^{1}$, Lin Ma ${ }^{1}$, Gang Liu ${ }^{1}$, Mengyu Liu ${ }^{1}$, and Mingge $\mathrm{Li}^{1}$

${ }^{1}$ radiology department, General Hospital of the People's Liberation Army, Beijing, China

In the current study, we evaluated the performance of the field-of-view optimized and constrained undistorted single shot (FOCUS) DWI in assessing the optic nerve and chiasma abnormalities of acute optic neuritis. Visual assessment was obtained for the FOCUS-DWI and the conventional-DWI (c-DWI). We found that FOCUS-DWI provided better visual assessments of the optic nerve and chiasma abnormalities in acute optic neuritis (AON), with much reduced blurring effects and geometric deformations. It might indicated that the FOCUS-DWI would improve the diagnostic accuracy and prognosis evaluation in AON.

A Method to Quantitatively Assess and Compare Diffusion MRI Protocols between MR Systems

Samuel Anthony Hurley ${ }^{1,2}$ and Alan B McMillan

${ }^{1}$ Radiology, University of Wisconsin, Madison, WI, United States, ${ }^{2}$ Neuroscience, University of Wisconsin, Madison, WI, United States

MRI systems and protocols capable of achieving diffusion measurements with comparable imaging parameters and equal or better performance to the Human Connect Project (NCP) acquisitions will enable studies in additional populations or patient groups to leverage existing HCP data as control data, decreasing costs and increasing statistical power of findings. To evaluate new MRI systems and potential protocols, we present an automated and quantitative method for evaluation of diffusion imaging performance from in-vivo data, use this method to evaluate the performance of a dMRI protocol acquired in a prototype wide bore $3 \mathrm{~T}$ MRI system.

Tractography based parcellation of the frontal lobe: reproducibility \& functional significance.

Michel Thiebaut de Schotten ${ }^{1}$, Marika Urbanski ${ }^{1}$, Leonardo Cerliani ${ }^{1}$, and Emmanuelle Volle ${ }^{1}$

${ }^{1}$ BCBlab, Institut du Cerveau et de la Moelle, Paris, France

Dividing the brain based on structural connectivity is a challenge that we circumvented using the principal component analysis framework. By doing so, we reliably divided the frontal lobe into 12 areas across datasets and participants. Additionally, these areas showed neat functional specificity as defined by functional magnetic resonance imaging.

Application of DTI on hyroid-associated ophthalmopathy (TAO) with Dysthyroid Optic Neuropathy (DON) or diplopia patient after intravenous methylprednisolone strategy.

ping liu ${ }^{1}$ and jing zhang ${ }^{1}$

${ }^{1}$ department of radiology, Tongji Hospital, Tongji Medical College, Huazhong University of Science and Technology, wu han, China

The pathogenesis of DON and diplopia is totally different. This study use the MRI-DTI on DON and diplopia patients with good therapeutic efficacy, the multiple DTI parameters of optic nerve were calculated and assessed. The final results furtherly confirmed this difference. And the statistical difference of DTI parameter changes in DON patients validate the DTI can exactly, objectively and reliably detect the microstructure and functional repair of optic nerve after iv MP therapy.

Automated fibre quantification predicts early Wallerian degeneration of the CST after acute ischemic stroke

Min TANG ${ }^{1}$, Wei $\mathrm{DI}^{2}$, Xin ZHANG ${ }^{1}$, Jie GAO ${ }^{1}$, Xiaoling ZHANG ${ }^{1}$, Zhizheng ZHUO ${ }^{3}$, Xia ZHE ${ }^{1}$, Dongsheng ZHANG ${ }^{1}$, and Xuejiao YAN ${ }^{1}$

${ }^{1}$ Shaanxi provincial people's hospital, Xi'an, China, ${ }^{2}$ Department of neurology, Shaanxi provincial people 's hospital, Xi'an, China, ${ }^{3}$ Philips Healthcare, Beijing, China

This study aimed to observe the microstructural alterations in corticospinal tract (CST) after motor pathway infarction and predict early Wallerian degeneration based on automated fiber quantification (AFQ). 53 patients with first-onset stroke in motor pathway and 29 health age-matched controls were enrolled. FA, MD, AD and RD values were significantly reduced on lesions of the affected side, while DKI values (MK, AK and RK) exhibited significant increase. AFQ was performed to identify differences on the whole CST pathway in the affected side between control and patient group. AD and MD values in CST of the affect side were significantly higher than them in healthy control. The findings of $A D$ and MD have the same pathological changes on the affected CST pathway no matter the primary stroke lesions located in any regions (brainstem, posterior limb of internal capsule or above centrum semiovale). Our findings suggest that AFQ has the potential to detect the early Wallerian degeneration in the central nervous system in vivo after the first 24 hours in stroke. 
${ }^{1}$ Radiology, Center for Magnetic Resonance Research, University of Minnesota, Minneapolis, MN, United States, ${ }^{2}$ Department of Pediatrics, University of Minnesota, Minneapolis, MN, United States, ${ }^{3}$ Department of Medicine, University of Minnesota, Minneapolis, MN, United States

Cystic fibrosis (CF) is the most common fatal autosomal recessive disorder in Caucasians. As the effects of CF on the brain structure remain unexplored, we piloted initial MRI investigations of brain structure by diffusion weighted imaging in CF and cystic fibrosis related diabetes (CFRD), a common complication in CF patients. Diffusion metrics were obtained in selected white and gray matter regions of 5 healthy controls $(\mathrm{HC})$ and $5 \mathrm{CF}$ patients with CFRD. Diffusion metrics of deep gray matter structures appeared to differ between patients with $\mathrm{CF}$ and $\mathrm{HC}$, possibly related to increased iron deposition, warranting more comprehensive MRI investigations in larger cohorts of patients.

DW-MRI in assessment of 3D Cell Culture

Jui-Heng Lin ${ }^{1}$, Hao-Chun Peng ${ }^{1}$, Shao-Chieh Lin ${ }^{2}$, Yi-Jui Liu², Ruey-Hwang Chou ${ }^{3}$, Ke-Sin Yan ${ }^{3}$, Tan-Wei Liao ${ }^{3}$, Chia-Wei Lin ${ }^{4}$, Chao-Chun Lin ${ }^{4}$, Wei-Ching Lin ${ }^{4}$, and Wu-Chung Shen ${ }^{4}$

${ }^{1}$ Master's Program of Biomedical Informatics and Biomedical Engineering, Feng Chia University, Taichung, Taiwan, ${ }^{2}$ Department of Automatic Control Engineering, Feng Chia University, Taichung, Taiwan, ${ }^{3}$ Center for Molecular Medicine, China Medical University Hospital, Taichung, Taiwan, ${ }^{4}$ Department of Radiology, China Medical University Hospital, Taichung, Taiwan

In extracellular and intracellular space, the Brownian motion of water is restricted by organelles, cellmembranes, and extracellular fibers. DWI is sensitivity to microscopic motion, which is due to Brownian motion of water molecules. In this study, 3D cell culture with hydrogels ECM was used to investigate whether DWI may provide information on these microenvironmental parameters and the microenvironment-associated metastatic propensity of tumors. Our results demonstrated DW-MRI may provide the potential biomarkers on the change of microenvironment in the application of 3D cell culture experiment.

Structural and functional brain connectivity highlights in neurosensorial profound deafness

Pedro Henrique Rodrigues da Silva ${ }^{1}$, Antonio Carlos Santos Senra Filho ${ }^{2}$, Karol Dell Ducas Senra ${ }^{3}$, Renata Ferranti Leoni ${ }^{1}$, Luiz Otavio Murta Junior ${ }^{2}$, and Antonio Carlos dos Santos ${ }^{3}$

${ }^{1}$ Department of Physics, FFCLRP, University of São Paulo, Ribeirão Preto, Brazil, ${ }^{2}$ Department of Computing and Mathematics, FFCLRP, University of São Paulo, Ribeirão Preto, Brazil, ${ }^{3}$ Department of Medical Clinics, FMRP, University of São Paulo, Ribeirão Preto, Brazil

The absence of auditory stimuli for a long period leads to modifications in brain structural and functional connectivity. However, the relationship between the brain changes and neurosensorial hearing loss is not fully clarified. In this study we considered a group of subjects with pre-lingual congenital deafness and analyzed their structural and functional connectivity. Our results suggest that auditory input deprivation not only alters the activity of sensory areas but also reshape the structural and functional organization of cognitive-related networks. These findings can be instructive to clinical practice.

Novel Multi-band accelerated, Reference-less, Multifaceted Icosahedral and Multishell Diffusion MRI Protocol for human whole brain clinical applications

Khader M Hasan ${ }^{1}$, Refaat E Gabr ${ }^{1}$, John A Lincoln² ${ }^{2}$ and Ponnada A Narayana ${ }^{1}$

${ }^{1}$ Diagnostic and Interventional Radiology, UThealth, Houston, TX, United States, ${ }^{2}$ Neurology, UThealth, Houston, TX, United States

We describe a comprehensive multishell and multifaceted icosahedral diffusion MRI protocol that enables whole brain coverage in less than 10 minutes using multiband (MB) technology at $3 \mathrm{~T}$. We show the protocol utility in providing estimates of blood fraction, extent of CSF-contamination, diffusion tensor and kurtosis derived measures including fractional, axonal water fraction and extracellular tortuosity. The diffusion gradient encoding is based the Icosa 6 and Icosa15 sets forming the Icosa21 for additional quality assurance. In this report we describe the protocol, show feasibility and utility for mapping a host of useful quantitative measures in the same session without repeated scans.

Role of intravoxel incoherent motion diffusion-weighted imaging in the assessment of invasiveness for bladder cancer

Fang Wang ${ }^{1}$, Guangyu $\mathrm{Wu}^{1}$, and Weibo $\mathrm{Chen}^{2}$

${ }^{1}$ Ren Ji Hospital, School of Medicine, Shanghai Jiao Tong University, Shanghai, China, ${ }^{2}$ Philips Healthcare, Shanghai, China

The degree of bladder wall invasion by bladder cancer determines the clinical management, for muscle invasive bladder cancer (MIBC, Stage T2 or more) recommended neoadjuvant chemotherapy before radical cystectomy and non-muscle invasive bladder cancer (NMIBC, Stage T1 or lower) treated with transurethral resection (TUR). Thus, differentiating NMIBC from MIBC using preoperative imaging plays a crucial role in clinical practice. 
${ }^{1}$ West China Hospital, Sichuan University, Chengdu, China, ${ }^{2}$ Department of Psychiatry and Behavioral Neuroscience, University of Cincinnati, Cincinnati, OH, United States

To our knowledge, this is the first time to study the association between plasm IL10 level and WM disruption in stable medicated schizophrenia using diffusion tensor imaging (DTI). The present study provided empirical evidence that dysregulation of inflammation contributes to anatomical dysconnectivity in schizophrenia.

Prediction of histological grade of hepatocellular carcinoma using quantitative diffusion-weighted magnetic resonance imaging: a retrospective multi-vendor study

Yoshio Kitazume $^{1}$, Yusuke Ogihara ${ }^{1,2}$, and Ukihide Tateishi

${ }^{1}$ Tokyo Medical and Dental University, Tokyo, Japan, ${ }^{2}$ JA Toride Medical Center, Ibaraki, Japan

Eighty-three patients with 100 histologically diagnosed hepatocellular carcinomas (HCCs) who preoperatively underwent diffusion-weighted (DW) imaging at any of 6 institutes were retrospectively studied. Receiver-operating characteristic analysis revealed that quantitative measurements such as the relative contrast ratio (RCR) and the contrast-to-noise ratio (CNR) between lesion and liver parenchyma on DW images were superior to the apparent diffusion coefficient (ADC) in predicting poorly differentiated HCCs, and intraclass correlation coefficients for the RCR tended to be greater than for the CNR and the ADC.

Traditional Poster

\section{Diffusion MRI: Microstructure}

Diffusion Weighted Imaging with uniform fat suppression using a Modified Dixon based Single Shot Turbo Spin Echo

Xinzeng Wang ${ }^{1}$, Holger Eggers ${ }^{2}$, Marco C. Pinho ${ }^{1,3}$, Ivan Pedrosa ${ }^{1,3,4}$, Robert E. Lenkinski ${ }^{1,3}$, and Ananth J. Madhuranthakam ${ }^{1,3}$

${ }^{1}$ Radiology, UT Southwestern Medical Center, Dallas, TX, United States, ${ }^{2}$ Philips Research, Hamburg, Germany, ${ }^{3}$ Advanced Imaging Research Center, UT Southwestern Medical Center, Dallas, TX, United States, ${ }^{4}$ Kidney Cancer Program, Simmons Comprehensive Cancer Center, UT Southwestern Medical Center, Dallas, TX, United States

Diffusion weighted imaging using single-shot turbo spin echo (DW-SShTSE) with Dixon showed uniform fat suppression without geometric distortions, compared to DW-EPI and DWSShTSE with spectrally selective fat suppression (SPIR). However, the phase insensitive preparation used in DW-SShTSE reduces the SNR by half, impeding the robustness of Dixon reconstruction. In this work, we developed a hybrid DW-SShTSE, where the $b=0 \mathrm{~s} / \mathrm{mm}^{2}$ image was acquired without the phase insensitive preparation for improved SNR. This combined with modified acquisition order improved the robustness of fat/water separation and generated diffusion-weighted images of the cervical spine with improved spatial resolution.

Diagnostic value of diffusion tensor imaging and positron emission tomography in early stages of frontotemporal dementia

Julia Krämer ${ }^{1}$, Gero Lueg ${ }^{2}$, Jan-Gerd Tenberge ${ }^{1}$, Patrick Schiffler ${ }^{1}$, Alexis Vrachimis ${ }^{3}$, Matthias Weckesser ${ }^{3}$, Christian Wenning ${ }^{3}$, Andreas Johnen ${ }^{1}$, Matthias Pawlowski ${ }^{1}$, Sven G. Meuth ${ }^{1}$, and Thomas Duning ${ }^{1}$

${ }^{1}$ Department of Neurology, Westfälische Wilhelms University, Münster, Germany, ${ }^{2}$ Department of geriatric medicine and early rehabilitation, Marien Hospital Herne, Herne, Germany, ${ }^{3}$ Department of Nuclear Medicine, Westfälische Wilhelms University, Münster, Germany

The study intended to investigate the sensitivity of DTI and FDG-PET in 30 patients with early behavioral variant frontotemporal dementia (bvFTD) despite inconspicuous conventional MRI. Based on individual FDG-PET data analysis, 20 patients were rated as bvFTD "typical" with bifrontal/ bitemporal hypometabolism (bvFTD/PET+) and 10 patients as "not typical/normal" (bvFTD/PET-). DTI voxel-based group analyses revealed bifrontal/ bitemporal microstructural degeneration in all patients. However, individual DTI data analysis revealed alterations in only $14 \%$. Neuropsychological symptoms were associated to DTI and FDG-PET identifiable cerebral changes. Summarising improvement of individual DTI analysis tools is necessary to make this technique applicable for clinical routine.

Monte Carlo simulations of diffusion in myelin spirals: Impact on diffusional water exchange

Lorenza Brusini ${ }^{1}$, Gloria Menegaz ${ }^{1}$, and Markus Nilsson ${ }^{2}$

1678 'University of Verona, Verona, Italy, ${ }^{2}$ Lund University, Lund, Sweden

How does the myelin structure impact water diffusion? The answer is still not clarified but is important for interpreting diffusion MRI in conditions with altered myelin structure such as neurological disorders or developing brain. Myelin is sometimes modelled as permeable to explain exchange between compartments. This work investigates the impact of the spiralling nature of myelin on water exchange, until now only indirectly explored in one case. Findings emphasized that small axons and low number of myelin wraps lead to exchange times shorter than a second, which can be assessed at clinical scanners. 
Measuring water exchange using cumulant expansions

Lipeng Ning ${ }^{1,2}$, Markus Nilsson ${ }^{3}$, Carl-Fredrik Westin ${ }^{1,2}$, and Yogesh Rathi ${ }^{1,2}$

${ }^{1}$ Harvard Medical School, Boston, MA, United States, ${ }^{2}$ Brigham and Women's Hospital, Boston, MA, United States, ${ }^{3}$ Lund University, Lund, Sweden

Diffusion MRI (dMRI) can provide important information about water exchange between different tissue compartments. In this abstract, we introduce a generalized model to measure the exchange rate using arbitrary gradient sequences. We present a unified theory that incorporates water diffusion and exchange as a stochastic diffusion-exchange process. Our work for the first time allows to compare different diffusion sequences and allows to determine the optimal experimental configurations to measure the exchange rate. In the most common situation with single- or double-diffusion encoding (SDE, DDE) sequences, our theory shows that DDE is more sensitive to water exchange at short time scale. We validate our theory using Monte-Carlo simulations.

Using GPUs to accelerate computational diffusion MRI: From microstructure estimation to tractography and connectomes

Moises Hernandez-Fernandez ${ }^{1,2}$, Istvan Reguly ${ }^{3,4}$, Saad Jbabdi $^{1}$, Mike Giles ${ }^{3}$, Stephen Smith ${ }^{1}$, and Stamatios N. Sotiropoulos ${ }^{1,5}$

${ }^{1}$ Oxford Centre for Functional MRI of the Brain (FMRIB), University of Oxford, Oxford, United Kingdom, ${ }^{2}$ Section for Biomedical Image Analysis (SBIA), University of Pennsylvania, Philadelphia, PA, United States, ${ }^{3}$ Oxford e-Research Centre, University of Oxford, Oxford, United Kingdom, ${ }^{4}$ Faculty of Information Technology and Bionics, Pazmany Peter Catholic University, Budapest, Hungary, ${ }^{5}$ Sir Peter Mansfield Imaging Centre, School of Medicine, University of Nottingham, Nottingham, United Kingdom

The great potential of computational diffusion MRI (dMRI) relies on indirect inference of tissue microstructure and brain connections, as modelling and tractography frameworks map diffusion measurements to neuroanatomical features. This mapping however can be computationally expensive, particularly given the trend of increasing dataset sizes and/or the increased complexity in biophysical modelling. We present here a number of frameworks for accelerating dMRI computations using Graphics Processing Units (GPUs), for both microstructure estimation and tractography/connectome generation. We show that despite differences in challenges for parallelising these problems, GPU-based designs can offer accelerations of more than two orders of magnitude.

On the estimation of the apparent bundle-wise diffusivity profiles for axon damage detection

Ricardo Coronado-Leija ${ }^{1}$, Alonso Ramirez-Manzanares ${ }^{1}$, Jose Luis Marroquin ${ }^{1}$, Luis Concha², Gilberto Rojas-Vite ${ }^{2}$, and Ramsés Noguez-Imm²

${ }^{1}$ Computer Science, Centro de Investigacion en Matematicas, Guanajuato, Mexico, ${ }^{2}$ Institute of Neurobiology, Universidad Nacional Autonoma de Mexico, Queretaro, Mexico

To estimate the physical features of intra-voxel axon bundles in the detection of axon damage it is important to compute bundle-wise apparent diffusivities. There is a first family of methods that factors-out the effects of the orientation-dispersion under a convolution model (e.g. Spherical Mean), and a second family that associates the diffusivity properties with specific orientations (e.g. Gaussian-Mixture-Models). Here we demonstrate that only the second family provides bundle-wise apparent diffusivities, and thus it provides the useful information for clinical applications. This is demonstrated on a broad synthetic validation as well as on ad-hoc rat ex-vivo phantom with a damaged bundle.

Diffusion-Weighted MR Imaging of the Parotid glands in healthy volunteers before and after a gustatory stimulation to quantify relative function

Matthew George Birkbeck ${ }^{1}$, Fiona Elizabeth Smith ${ }^{1}$, and Andrew Matthew Blamire ${ }^{1}$

${ }^{1}$ Newcastle Magnetic Resonance Centre, Newcastle University, Newcastle upon Tyne, United Kingdom

Diffusion Weighted MR Imaging has been used to quantify the function of parotid glands. Clinically gland function is measured using Scintigraphy, but MR offers a non-invasive, nonionising alternative to this method. A DWI sequence for investigating parotid gland function is presented and tested in five healthy volunteers scanned on two occasions. We used four parameters to represent gland function: perfusion fraction $\left(f_{v}\right)$, apparent perfusion coefficient $\left(A D C_{\text {perfusion }}\right)$, diffusion fraction $\left(f_{d}\right)$ and apparent diffusion coefficient $\left(A D C_{\text {diffusion }}\right)$. Statistically significant changes were observed in $\mathrm{f}_{v}, \mathrm{f}_{d}$ and $A D C_{\text {diffusion }}$ in volunteers. Results indicate a normal range for these parameters.

DWI virtual MR elastography of the upper abdominal organs in healthy volunteers

Min Wang ${ }^{1}$, Yu Shi ${ }^{1}$, Xiaoqi Wang ${ }^{2}$, Yanqing Liu ${ }^{1}$, Ruoyun $\mathrm{Ji}^{1}$, Lizhuo Cang ${ }^{1}$, and Qiyong Guo ${ }^{1}$

Le et al ${ }^{1}$ recently found that apparent diffusion coefficient (ADC) calculated from 2 key b values ("shifted ADC", or sADC) can be directly and quantitatively represent healthy liver stiffness and be compared with results obtained by standard MR elastography (MRE). In this study, we found that there is a strong linear relationship between sADC and stiffness in both liver and pancreas, and a weak relationship in spleen, but no coherence in kidney in healthy volunteers. 
Investigation of diffusion, susceptibility, and vessel morphology effects on R2 in characterizing normal and tumorous vasculature using simulations

Mohammed Salman Shazeeb ${ }^{1,2}$, Jayashree Kalpathy-Cramer², and Bashar Issa ${ }^{1}$

${ }^{1}$ UAE University, Al-Ain, Abu Dhabi, United Arab Emirates, ${ }^{2}$ Radiology, MGH \& Harvard Medical School, Boston, MA, United States

Brain vasculature is conventionally represented as straight cylinders when simulating BOLD contrast effects in fMRI. In reality, the vasculature is more complicated with branching and coiling especially in tumors. We applied a cylinder fork model to reflect the bifurcation, rotations, and size of vessels and performed simulations to study the effect of the rotation angle ( $\phi$ ) on R2 at different bifurcation angles, vessel diameters, diffusion rates, and susceptibility values. This model clearly showed an R2 dependence on $\phi$, which could potentially be used, in addition to R2*, as a tool to differentiate between normal and tumor vessels.

Obtaining the barrier distribution in the micro-structure from diffusion spectra

Carlos Castillo-Passi ${ }^{1,2}$, Gabriel Varela-Mattatall ${ }^{1,2}$, Claudia Prieto ${ }^{1,2,3}$, Carlos Sing-Long ${ }^{2,4,5}$, and Pablo Irarrazaval ${ }^{1,2,5}$

${ }^{1}$ Department of Electrical Engineering, Pontificia Universidad Católica de Chile, Santiago, Chile, ${ }^{2}$ Biomedical Imaging Center, Pontificia Universidad Católica de Chile, Santiago, Chile, ${ }^{3}$ Division of Imaging Sciences and Biomedical Engineering, King's College London, London, United Kingdom, ${ }^{4}$ Institute for Computational and Mathematical Engineering, Stanford University, Stanford, CA, United States, ${ }^{5}$ Institute for Biological and Medical Engineering, Pontificia Universidad Católica de Chile, Santiago, Chile

Inspired in the solution of the diffusion equation in the restricted case, we propose to express the diffusion $\$ \$ \$ q \$ \$$-space information in a restricted basis. This representation allows to obtain the distribution of barriers separations, thus providing useful information about the micro-structure. Previous methods used multiple Diffusion Spectrum Imaging (DSI) images with different diffusion times, which is impractical to characterize barriers in multiple directions. Our method proposes to obtain the barrier distribution with only a single DSI image.

Furthermore, the model does not use a strong assumption for the geometry of the barriers (or axons) nor for the probability distribution of the barrier separation.

Increasing Mixing Time in STEAM-DTI Enhances Inter-Muscle Heterogeneity Patterns in the Lower Leg of Healthy Subjects

Celine Baligand ${ }^{1}$, Thom TJ Veeger ${ }^{1}$, Jedrek Burakiewicz ${ }^{1}$, Melissa T Hooijmans ${ }^{1}$, Jan JGM Verschuuren², Erik H Niks ${ }^{2}$, and Hermien E Kan ${ }^{1}$

${ }^{1}$ Radiology, Leiden University Medical Center, C.J. Gorter Center for High-field MRI, Leiden, Netherlands, ${ }^{2}$ Neurology, Leiden University Medical Center, Leiden, Netherlands

Hereditary muscular disorders are characterized by progressive skeletal muscle wasting and weakness. Although these diseases are caused by ubiquitous genetic mutations, the symptoms appear at different rates in different muscles. We investigated the differences in microstructural properties of different muscles of the lower leg in healthy subject using STEAM-DTI with varying diffusion times at 3 T. We identified a characteristic pattern of differences in fractional anisotropy and diffusivity in healthy muscles than can serve as a knowledge base for future studies on disease progression in muscular disorders.

Residual analysis reveals variation of the intrinsic diffusivity throughout the brain in neurite orientation dispersion and density imaging (NODDI)

Jose M Guerrero ${ }^{1}$, Nagesh Adluru², Steven Kecskemeti ${ }^{2}$, Richard Davidson ${ }^{3}$, Hui Zhang ${ }^{4}$, and Andrew L Alexander

${ }^{1}$ Medical Physics, University of Wisconsin - Madison, Madison, WI, United States, ${ }^{2}$ Waisman Center, University of Wisconsin - Madison, Madison, WI, United States, ${ }^{3}$ Psychology and Psychiatry, University of Wisconsin - Madison, Madison, WI, United States, ${ }^{4}$ Department of Computer Science, University College London, London, United Kingdom, ${ }^{5}$ Medical Physics, Psychiatry, University of Wisconsin - Madison, Madison, WI, United States

NOODI and its widely used estimation toolbox assume the intrinsic diffusivity to a fixed value suitable for healthy adult brains. For broader applicability of the model in neurological diseases it is important to understand the validity of assumed fixed intrinsic diffusivity. Using multi-shell diffusion data we investigated the variability of estimated NODDI indices as well as the model residuals with respect to variations in intrinsic diffusivity. The results suggest significant differences between optimum intrinsic diffusivity for white and gray matter regions as derived from intrinsic diffusivity values that generate smallest model residuals. The variability analysis indicates appreciable differences in the estimated parameters in the range of probable diffusivities predicted by the residual analysis.

Fitting MAP-MRI in 2 shell DWI Datasets using Model-based Extrapolation

maryam afzali ${ }^{1}$, Sharlene Newman ${ }^{1}$, Eleftherios Garyfallidis ${ }^{2}$, and Hu Cheng ${ }^{1}$

${ }^{1}$ Department of Psychological and Brain Sciences, Indiana University, Bloomington, IN, United States, ${ }^{2}$ Department of Intelligent Systems Engineering, Indiana University, Bloomington, IN, United States

We showed that three shells are sufficient to result in good approximations of MAP-MRI indices from numerical simulation. We used multiple compartment microstructure models to fit the two shell data and extrapolate the third shell with a higher b-value. We compared the performance of two models, NODDI and NODDI with fiber crossing (NODDIx), on the Human Connectome Project (HCP) DWI data. NODDIx showed improvement in the white matter with extrapolation but NODDI did not. Both NODDI and NODDIx failed to improve the results in the gray matter. Our approach also provides a new mechanism in validating or comparing microstructure models. 
Traditional Poster

\section{RF Coils \& Electronics}

Construction of an open PXIe based scalable MRI console

Andrew Ang ${ }^{1}$, Sergei Obruchkov², and Robin Dykstra ${ }^{1}$

${ }^{1}$ School of Engineering and Computer Science, Victoria University of Wellington, Wellington, New Zealand, ${ }^{2}$ Robinson Research Institute, Victoria University of Wellington, Wellington, New Zealand

We have developed an open source PXle platform tailored for MRI console development. The example design has a multichannel RF transceiver, and signal generation for gradient drive.

Software defined radio-based platform for parallel transmission MRI research

Fred Tam ${ }^{1}$, Benson Yang ${ }^{1}$, and Simon J Graham ${ }^{1,2}$

${ }^{1}$ Physical Sciences, Sunnybrook Research Institute, Toronto, ON, Canada, ${ }^{2}$ Department of Medical Biophysics, University of Toronto, Toronto, ON, Canada

Parallel transmission (PTx) research platforms are challenging to implement and to integrate with commercial MRI systems. A prototype PTx research platform was demonstrated that leverages off-the-shelf software-defined radio (SDR) for flexibility and scalability, with easy integration and moderate cost. The SDR system was evaluated on the bench and connected to a commercial 3-T MRI system for an initial RF shimming demonstration. Substantial latency was found, likely due to the preliminary software implementation, but overall measurements and images were promising. Scaling to 32 transmit channels and applications other than RF shimming are expected to be practical.

A Gate Modulated Digitally Controlled Modified Class-E Amplifier for On-Coil Applications in 1.5 T MRI

Bismillah Nasir Ashfaq ${ }^{1,2}$, Fatima Tu Zahra ${ }^{1,2}$, Berk Silemek ${ }^{2}$, Uğur Yılmaz ${ }^{2}$, and Ergin Atalar ${ }^{1,2}$

${ }^{1}$ Department of Electrical and Electronics Engineering, Bilkent University, Ankara, Turkey, ${ }^{2}$ National Magnetic Resonance Research Center (UMRAM), Ankara, Turkey

A novel technique of modulating both the amplitude and frequency of the desired MR Radiofrequency pulse in a class-E amplifier topology, without utilizing supply-modulation, is presented. Amplifier's MATLAB model is developed and the carrier frequency bitstream is intelligently controlled to achieve both the amplitude and phase modulation of the output waveform. Benchtop experiments are performed showing accurate translation of software predictions on hardware, however requiring some additional optimization steps. MR experiments are performed to demonstrate the slice-selective capability of the generated RF pulse. Images are acquired at input powers of up to $80 \mathrm{~W}$ with $89 \%$ peak drain efficiency.

Accurate Noise Figure Measurements for Highly Mismatched Preamplifiers

Daniel Højrup Johansen ${ }^{1}$, Juan D. Sanchez-Heredia ${ }^{1}$, Vitaliy Zhurbenko ${ }^{1}$, and Jan H. Ardenkjær-Larsen ${ }^{1,2}$

${ }^{1}$ Department of Electrical Engineering, Technical University of Denmark, Kgs. Lyngby, Denmark, ${ }^{2}$ GE Healthcare, Brøndby, Denmark

A method reducing the uncertainty of noise figure measurements of highly mismatched preamplifiers is presented. In many cases when measuring the noise figure of preamplifiers for MRI receive arrays the uncertainty is approximately $\pm 0.4 \mathrm{~dB}$. Since the noise figure of the preamplifier is also in this range, a more accurate method is needed. Here we show an increase of $59 \%$ in noise figure accuracy by adding an attenuator between the noise source and preamplifier.

Xiaoyu Yang ${ }^{1}$, Haoqin Zhu ${ }^{1}$, Tsinghua Zheng ${ }^{1}$, and Yong $\mathrm{Wu}^{1}$

${ }^{1}$ Quality Electrodynamics, LLC, Mayfield Village, $\mathrm{OH}$, United States 
Typical Tx/Rx coils require a separate local transmitter and complicated T/R switches to make a local transmitter. They are expensive and may degrade receiver coil performance. We propose a novel Tx/Rx coil concept using the same receiver array coils. All receiver coils are allowed to inductively couple to the WBC in Tx mode. The combined induced amplified Tx field from the array coils is uniform and can be used as local Tx $B_{1}$ field. This new concept simplifies Tx/Rx coil design and enables highly parallel array coil design with local Tx capability.

A Low Cost Prototype Pre-Gate Amplifier to Study Radiofrequency Power Amplification for Parallel Transmission MRI at 3 T

Benson Yang ${ }^{1}$ and Simon J Graham ${ }^{1,2}$

There is a growing interest to increase the channel count on parallel transmit systems. With system cost always a major consideration, substantial savings may be possible as the channel count becomes high (ie. $\geq 32$ ). Typically, radiofrequency power amplifier (RFPA) designs involve multiple amplification stages to achieve a target output power. Three stages are identified in the design approach of the present work: (1) a low noise pre-amplifier; (2) a driver amplifier; and (3) a power gain amplifier. The present goal is introduce and characterize system architecture for a prototype "pre-gate" amplifier (stage 1 and 2) to explore power amplification technology for stage 3 of the RFPA.

A Prototype Four-Channel Parallel Transmission System to Investigate MRI Safety at $3 \mathrm{~T}$

Benson Yang ${ }^{1}$, Fred Tam ${ }^{1}$, Pei-Shan Wei ${ }^{1}$, Clare E McElcheran ${ }^{2}$, and Simon J Graham ${ }^{1,3}$

Interest in parallel transmission (pTx) continues to grow with many research groups investigating methods to increase channel count and applications on commercial MRI systems. It can be challenging, however, to integrate pTx hardware onto existing systems without disrupting normal operation. The present work successfully interposes a four-channel pTx system on an existing 3 T Siemens Prisma system and performs validation to demonstrate: (1) four-channel radiofrequency (RF) shimming; and (2) reduced RF heating in an electrically conductive implant.

A meander slot element with microstrip line match and tune

Dheyaa Alkandari ${ }^{1}$, Chung-Huan Huang ${ }^{1}$, and Steven M Wright

${ }^{1}$ Texas A\&M University, College station, TX, United States

lot antennas have been widely used in communications because of their obvious low-profile nature. In MRI applications, the ability to 'hide' ancillary electronic components behind a shield containing a slot antenna could lead to interesting and very "clean" transmit antenna designs. Using the meander slot as elements for multi-channel coils allows for shield containing a slot antenna could lead to interesting and very "clean" transmit antenna designs. Using the meander slot as elements for multi-channel coils allows for
more compact multi-channel transmit coil designs with a shielded "clean" imaging area. This shielded imaging area provides a desirable environment for placing a more compact multi-channel transmit coil designs with a shielded "clean" imaging area. This shielded imaging area provides a desirable environment for placing a
receiver coil. More importantly, using meander slot coil elements can potentially allow for the design of multi-channel coils without the need of using matching and tuning networks or decoupling circuits. We believe this may significantly simplify the design of multi-channel transmit coils.

A 32-Channel Array Coil for Bilateral Breast Imaging and Spectroscopy at $7 \mathrm{~T}$

Romina Del Bosque ${ }^{1}$, Matthew Wilcox ${ }^{1}$, Jiaming Cui $^{2}$, Sergey Cheshkov ${ }^{3,4}$, Ivan Dimitrov ${ }^{4,5}$, Craig Malloy ${ }^{3,4,6}$, Steve Wright ${ }^{1,2}$, and Mary McDougall ${ }^{1,2}$

${ }^{1}$ Biomedical Engineering, Texas A\&M University, College Station, TX, United States, ${ }^{2}$ Electrical and Computer Engineering, Texas A\&M University, College Station, TX, United States, ${ }^{3}$ Radiology, UT Southwestern Medical Center, Dallas, TX, United States, ${ }^{4}$ Advanced Imaging Research Center, UT Southwestern Medical Center, Dallas, TX, United States, ${ }^{5}$ Philips Medical Systems, Cleveland, $\mathrm{OH}$, United States, ${ }^{6}$ Internal Medicine, UT Southwestern Medical Center, Dallas, TX, United States

This work describes the design, construction, and performance of a 32-channel array coil for bilateral breast imaging at $7 \mathrm{~T}$. Imaging indicated an increase in average SNR over a T/R volume coil of 5.5 times, with a three times increase in the center and up to 20 times along the periphery. Channel noise correlations indicated well decoupled elements and highly unilaterally isolated sets of 16 elements. In combination with high field strength benefits, this array will enable high resolution accelerated breast imaging

Fabian J. Kratzer ${ }^{1}$, Reiner Umathum ${ }^{1}$, Sebastian Flassbeck ${ }^{1}$, Thomas M. Fiedler ${ }^{1}$, Andreas K. Bitz ${ }^{1,2}$, Mark E. Ladd ${ }^{1,3}$, Gregor Adriany ${ }^{4}$, and Sebastian Schmitter ${ }^{1,5}$

${ }^{1}$ Medical Physics in Radiology, German Cancer Research Center (DKFZ), Heidelberg, Germany, ${ }^{2}$ Faculty of Electrical Engineering and Information Technology, FH Aachen - University of Applied Sciences, Aachen, Germany, ${ }^{3}$ Erwin L. Hahn Institute for MRI, University Duisburg-Essen, Essen, Germany, ${ }^{4}$ Center for Magnetic Resonance Research, University of Minnesota Medical School, Minneapolis, MN, United States, ${ }^{5}$ Medical Physics and Metrological Information Technology, Physikalisch-Technische Bundesanstalt (PTB), Berlin, Germany 
Stroke is one of the most common causes of death, often caused by accumulation of plaques in the carotid arteries. This motivates investigating the anatomy and blood hemodynamics in the carotid bifurcation with high resolution. For early diagnostics, this work presents a new, saddle-shaped neck-adapted 4-channel parallel transceive coil for imaging at 7T. Coil design and optimization were performed using numerical simulations, and a safety assessment was performed with an anatomical body model. A head-shoulder phantom was built and used to validate measurements. High-resolution anatomical images and flow measurements were acquired in the common carotid artery.

\section{A Fast MOSFET RF Switch for TRASE MRI at Low Magnetic Field}

Pierre-Jean Nacher ${ }^{1}$, Sashika Kumaragamage ${ }^{2}$, Geneviève Tastevin ${ }^{1}$, and Christopher P Bidinosti ${ }^{3}$

${ }^{1}$ Laboratoire Kastler Brossel, ENS-PSL Research University, CNRS, UPMC-Sorbonne Université, Collège de France, Paris, France, ${ }^{2}$ Rady Faculty of Health Sciences, College of Medicine, University of Manitoba, Winnipeg, MB, Canada, ${ }^{3}$ Department of Physics, University of Winnipeg, Winnipeg, MB, Canada

TRansmit Array Spatial Encoding (TRASE) MRI uses trains of $\mathrm{B}_{1}$ pulses alternatively produced by distinct transmit coils. Commonly used coil switching involving PIN diodes is too slow for low-field MRI and would introduce wait times between pulses typically as long as each individual pulse (hence, significant diffusion-induced resolution loss in TRASE MRI of gas samples). A MOSFET-based RF switch is described and characterised. Up to $200 \mathrm{kHz}$, it allows for sub- $\mu$ s switching of RF currents from a single amplifier to several coils with sufficient isolation ratio and no delay between pulses.

A 22-Channel RF coil array for fetus MR imaging at 3T

Chao Luo ${ }^{1,2}$, Guoxi Xie ${ }^{3}$, Jo Lee ${ }^{1,2}$, Xing Yang ${ }^{4}$, Xiaoliang Zhang ${ }^{5,6}$, Xin Liu ${ }^{1,2}$, and Ye Li ${ }^{1,2}$

${ }^{1}$ Lauterbur Research Center for Biomedical Imaging, Shenzhen Institutes of Advanced Technology, Chinese Academy of Sciences, Shenzhen, China, ${ }^{2}$ Shenzhen Key Laboratory for MRI, Shenzhen, China, ${ }^{3}$ School of Basic Science, Guangzhou Medical University, Guangzhou, China, ${ }^{4}$ High-Field Magnetic Resonance Brain Imaging Key Laboratory of Sichuan Province, Chengdu, China, ${ }^{5}$ Department of Radiology and Biomedical Imaging, University of California San Francisco, San Francisco, CA, United States, ${ }^{6}$ UCSF/UC Berkeley Joint Graduate Group in Bioengineering, San Francisco, CA, United States

Due to lack of dedicated fetal imaging RF coils, the system body coil is often used to acquire fetal images. This setup is not optimized and offers limited sensitivity and image quality. In this work, we designed and manufactured a 22-channel flexible coil array for fetal examinations. Compared with Siemens 6-channel body coil, the proposed fetal coil array achieves significant improvements in imaging coverage, image SNR and parallel acceleration capability.

Magnetically coupled RF coil for optimizing noise correlation

Yosuke Otake $^{1}$, Kohjiro Iwasawa ${ }^{1}$, Hisaaki Ochi ${ }^{1}$, Masayoshi Dohata ${ }^{2}$, and Yoshihisa Soutome ${ }^{1}$

${ }^{1}$ Research \& Development Group, Hitachi, Ltd., Tokyo, Japan, ${ }^{2}$ Healthcare Business Unit, Hitachi, Ltd., Tokyo, Japan

A magnetically coupled radiofrequency (RF) coil (MC coil) for optimizing noise correlation has been developed. The electric fields of each RF coil, which determine noise correlation, were controlled by a small magnetic coupling between a pair of RF coils. The MC coil was implemented as a two-channel loop coil in $1.5 \mathrm{~T}$ magnetic resonance imaging (MRI). The experimental results show that noise correlation can be controlled by using a small magnetic coupling without signal-to-noise ratio (SNR) loss. MC coils that can optimize noise correlation give a new degree of freedom to coil design.

Small self-decoupled RF coils

Xinqiang Yan ${ }^{1,2}$, John C. Gore ${ }^{1,2,3}$, and William A. Grissom ${ }^{1,2,3}$

${ }^{1}$ Institute of Imaging Science, Vanderbilt University, Nashville, TN, United States, ${ }^{2}$ Department of Radiology and Radiological Sciences, Vanderbilt University, Nashville, TN, United States, ${ }^{3}$ Department of Biomedical Engineering, Vanderbilt University, Nashville, TN, United States

The self-decoupled coil that is intrinsically decoupled proves to be a simple way to solve coupling issues in RF arrays. Small mode capacitances are needed to balance the dipole- and loop-mode coupling in self-decoupled coils, which then requires the addition of inductors to maintain the resonant frequency. But inductors may lead to loss and thus decrease transmit efficiency. In this work, we investigated the performance of small self-decoupled coils at $7 \mathrm{~T}$ and compared it to ideal conventional coils. It was found that the coil performance of selfdecoupled array could be well preserved so long as the sample loss is dominated. Based on these simulation and experimental results, the self-decoupled coil is a good candidate for dense coil arrays at ultrahigh fields. 
PET insert for the existing MRI systems can be a potential affordable alternative of body PET/MRI system. To avoid mutual interference between PET front-end electronics and the MRI system, PET front-end (F/E) electronics are enclosed in RF shielded Faraday cage that is connected to the RF ground for shielding purpose. On the other hand, strip transmission line RF coil requires a grounded plane in parallel with a strip conductor as coil that are connected by shunt capacitors. In this study, we proposed a strip transmission line coil that replaced the ground one layer conductor with the shielded PET detector module. The combined system shows promise for a compact PET/RF coil modality as insert for simultaneous PET/MR imaging with existing MRI systems, suitable even at ultrahigh field MRI.

Fixed-phase prostate imaging with a 8-channel transmit/receive dipole antenna array on a conventional 3T system

Aidin Ali Haghnejad ${ }^{1}$, Mark Gosselink ${ }^{1}$, Ingmar Voogt ${ }^{1}$, Dennis Klomp ${ }^{1}$, Peter Luijten ${ }^{1}$, and Alexander Raaijmakers ${ }^{1,2}$

${ }^{1}$ Radiology, UMC Utrecht, Netherlands, Utrecht, Netherlands, ${ }^{2}$ Eindhoven University of Technology, Biomedical Image Analysis, Eindhoven, Netherlands

Local multi-transmit arrays at 3T provide reduced power requirements and reduced local SAR. However, it requires $3 T$ scanners with multi-transmit functionality which are rare. This work presents add-on hardware that enables the use of local transmit/receive arrays. An exploration on prostate imaging with fixed phase settings using a 8-channel dipole array has been performed on four subjects. $\mathrm{B}_{1}{ }^{+}$levels range from 5 to $8.5 \mathrm{uT}$ for $8 \times 215-300 \mathrm{~W}$ input power. T2 $\mathrm{w}$ images have been acquired successfully for each subject. The modest intersubject variation in $\mathrm{B}_{1}{ }^{+}$demonstrates the feasibility of this approach.

Large FOV 16-channel receive array with a volume transmit coil for human forearm/wrist/hand imaging at $7 \mathrm{~T}$

Özlem Ipek ${ }^{1}$, Jérémie Clément ${ }^{2}$, and Maria Isabel Vargas ${ }^{3}$

${ }^{1}$ CIBM-AIT, Ecole Polytechnique Federale de Lausanne, Lausanne, Switzerland, ${ }^{2}$ LIFMET, Ecole Polytechnique Federale de Lausanne, Lausanne, Switzerland, ${ }^{3}$ Neuroradiology division, Geneva University Hospitals, Geneva, Switzerland

A large-field-of-view 16-channel circular loop receive array with a volume transmit coil for the human forearm, wrist and hand imaging at 7 Tesla was constructed. While the volume transmit coil yields homogeneous transmit field distribution along the $350-\mathrm{mm}$ in length, the 16-channel receiver array enables two times faster imaging with a similar MR image quality. In conclusion, the use of this large field-of-view RF coil configuration for a total MR protocol of 15 minutes is feasible, and it enables visualization of different anatomical structures on the human forearm and hand at 7 Tesla.

A Flexible Transceiver Array for Cardiac MRI at 7 T: Performance Evaluation on a Torso Phantom

Sajad Hosseinnezhadian ${ }^{1,2}$, Roberta Frass-Kriegl ${ }^{2}$, Sigrun Goluch² ${ }^{2}$ Michael Pichler ${ }^{2}$, Jürgen Sieg ${ }^{2}$, Marie Poirier-Quinot ${ }^{1}$, Luc Darrasse ${ }^{1}$, Ewald Moser ${ }^{2}$, Jean-Christophe Ginefri $^{1}$, and Elmar Laistler ${ }^{2}$

${ }^{1}$ IR4M (Imagerie par Résonance Magnétique Médicale et Multi-Modalités), Univ. Paris-Sud, CNRS, Université Paris-Saclay, Orsay, France, ${ }^{2}$ Division MR Physics - Center for Medical Physics and Biomedical Engineering, Vienna, Austria

A flexible 12-channel transceiver transmission line resonator (TLR) array for $7 \mathrm{~T}$ cardiac ${ }^{1} \mathrm{H}$ MRI compatible with parallel transmission systems was developed. The size of the array is $38 \mathrm{~cm} \times 28.5 \mathrm{~cm}$ with individual TLRs of $84 \mathrm{~mm}$ diameter. A decoupling ring-based inter-element decoupling technique was used where the basic TLR geometry is surrounded by a conducting ring. Its efficiency was demonstrated with the array bent on a torso phantom and a human torso $\left(\mathrm{S}_{\mathrm{ij}}<-16 \mathrm{~dB}\right)$. Acceleration factors up to 3 in bent configuration can be employed without significant SNR degradation ( $g$-factor $<1.6$ ).

Sensitivity Improvement of Quadrature Surface Coil using Isotropic Metamaterial Flat Lens

Tejkiran A. Patil ${ }^{1}$, A. Sidhique ${ }^{1}$, Pulkit Sharma ${ }^{1}$, Rajesh Harsh $^{1}$, and P. H. Rao ${ }^{2}$

${ }^{1}$ Indigenous Magnetic Resonance Imaging Laboratory, SAMEER, Mumbai, India, ${ }^{2}$ SAMEER-CEM, Chennai, India

Metamaterial lens has previously been used to improve the sensitivity of phased array coils and the improvement is specifically seen at the epicenters of the loops and a sharper notch is formed at the critical overlapping region because of high resolving capability of the lens and it is not desirable for larger field of view (FOV). This work proposed a novel concept of nearly constant improvement in receiver sensitivity over the FOV using a combination of both metamaterial flat lens and quadrature surface coil. 
${ }^{1}$ Electrical and Electronics Engineering, Bilkent University, Ankara, Turkey, ${ }^{2}$ National Magnetic Resonance Research Center (UMRAM), Bilkent University, Ankara, Turkey, ${ }^{3}$ Aselsan, REHIS Power Amplifier Technologies, Ankara, Turkey

In this work, we designed and manufacture a 12-channel body birdcage degenerate transmit array coil. After determining the size of the coil, the trace thickness for each of the conductors and the location of the capacitors, an EM solver is used to find the equivalent circuit model of the coil. The capacitor values are tuned by solving the circuit model and recalculating the EM model iteratively. After reaching the minimum total reflection of $14 \%$, we constructed the 12 -channel body degenerate birdcage transmit array coil. The strongest coupling was observed between adjacent channels measuring as $-15.7 \mathrm{~dB}$.

A double resonant $(1 \mathrm{H} / 23 \mathrm{Na})$ whole-body RF system for MRI at $3 \mathrm{~T}$

Matthias Malzacher ${ }^{1}$, Nadia Paschke ${ }^{1}$, Jorge Chacon-Caldera ${ }^{1}$, and Lothar R. Schad ${ }^{1}$

${ }^{1}$ Computer Assisted Clinical Medicine,Medical Faculty Mannheim, Heidelberg University, Mannheim, Germany

${ }^{23} \mathrm{Na}$ MRI keeps increasingly demonstrating diagnostic value in a multitude of studies and clinical applications due to its capability to provide information on tissue viability. In order to coregister ${ }^{23} \mathrm{Na}$ and ${ }^{1} \mathrm{H}$ MR images, a double resonant ${ }^{23} \mathrm{Na} /{ }^{1} \mathrm{H}$ RF system is the optimal solution. In this work we present a clinical double-resonant RF system consisting of a shielded ${ }^{23} \mathrm{Na} \mathrm{BC}$ coil, a 16 channel ${ }^{23} \mathrm{Na} \mathrm{Rx}$ array and a local ${ }^{1} \mathrm{H}$ Helmholtz coil inside the shielded ${ }^{23} \mathrm{Na} \mathrm{BC}$ coil. The complete system is demonstrated in EM simulations and initial feasibility measurements are performed.

300 W Modified Class-E RF Amplifiers for $64 \mathrm{MHz}$ Transmit Array System

Fatima tu Zahra ${ }^{1,2}$, Bismillah Nasir Ashfaq ${ }^{1,2}$, Berk Silemek $^{2}$, Ugur Yilmaz $^{2}$, Redi Poni $^{3}$, and Ergin Atalar ${ }^{1,2}$

${ }^{1}$ Department of Electrical and Electronics Engineering, Bilkent University, Ankara, Turkey, ${ }^{2}$ National Magnetic Resonance Research Center (UMRAM), Ankara, Turkey,

${ }^{3}$ ValoTec, Engineering Consultant, Paris, France

In this work, highly efficient $300 \mathrm{~W}$ digitally controlled supply-modulated Class-E amplifiers for two-channel RF transmit array are presented. Load pull analysis is performed for load optimization purposes. Coupling between the transmit coils is measured to be $8 \%$ when $12 \mathrm{~cm}$ diameter coils are placed with a distance of $7 \mathrm{~cm}$. The performance of amplifiers while working simultaneously at same frequency and at different frequencies is evaluated. MR experiments are conducted and it is observed that MR images show no artifact in the presence of amplifier near transmit coil inside the scanner.

Ideal Coil Decoupling in Receive Arrays using Negative Resistance Preamplifiers

Daniel Højrup Johansen ${ }^{1}$, Juan D. Sanchez-Heredia ${ }^{1}$, Vitaliy Zhurbenko ${ }^{1}$, and Jan H. Ardenkjær-Larsen ${ }^{1,2}$

${ }^{1}$ Department of Electrical Engineering, Technical University of Denmark, Kgs. Lyngby, Denmark, ${ }^{2}$ GE Healthcare, Brøndby, Denmark

This work presents the method of achieving ideal decoupling between elements in a receive coil array. Generally, preamplifier decoupling is limited by nonidealities of the implemented components. It is shown analytically and numerically, that for the ideal (lossless) matching circuits the input resistance of the preamplifier should be zero, while for the realistic lossy case a small negative resistance can be used to achieve ideal decoupling. Here we use a negative input resistance preamplifier (NIRP) to compensate for the loss of the circuit. The analysis is verified experimentally showing a decoupling of $-62 \mathrm{~dB}$ when a NIRP with an input resistance of $-0.023 \Omega$ is used.

Using Noise Waves for Simulation and Measurement of Array SNR Penalty due to Passive Impedance Match

Arne Reykowski ${ }^{1}$, Christian Findeklee ${ }^{2}$, Paul Redder ${ }^{1}$, Tracy Wynn ${ }^{1}$, Tim Ortiz $^{1}$, Randy Duensing ${ }^{2}$, and Scott B King ${ }^{1}$

${ }^{1}$ Invivo Corporation, Gainesville, FL, United States, ${ }^{2}$ Philips Research, Hamburg, Germany

Active impedance matching versus passive impedance matching of array coils is a concept well understood when designing transmit arrays. Lesser known however is that this concept also applies to receive arrays. Even though it appears that preamplifiers are noise matched to the passive port impedance (usually 50 Ohms), preamplifier noise coupling creates active noise match impedances which are mode dependent. In this context, a mode is defined by a signal vector and the corresponding weighting factors for optimum combined SNR. We use coupled noise waves to explain by simple concepts how the weighted and combined coupled noise changes the active noise match impedance. 
${ }^{1}$ McMaster University, Hamilton, ON, Canada, ${ }^{2}$ Imaging Research Centre, St. Joseph's Healthcare, Hamilton, ON, Canada, ${ }^{3}$ Electrical and Computer Engineering, McMaster University, Hamilton, ON, Canada

We compared a fractal patterned micro-strip surface coil with a simple circular micro-strip surface coil for hyperpolarized ${ }^{129}$ Xe lung imaging applications. Both patterns were simulated using a finite element solver and electric and magnetic fields were calculated in the surface coil and adjacent air volume. The fractal-patterned coil showed relatively higher magnetic field compared to the circular coil in both the micro-strip surface and the air volume. Although, further simulations are required, fractal-patterned designs of MRI coils could offer specific improvement in signal penetration and magnetic field homogeneity.

A Dual-Tuned $70 \mathrm{~cm}$ Whole-Body Resonator for $13 \mathrm{C}$ and Proton MRI/MRS at $3 \mathrm{~T}$

Ed Boskamp ${ }^{1}$, Zhentian $\mathrm{Xie}^{2}$, Victor Taracila ${ }^{1}$, Amy Stephen ${ }^{2}$, Mike Edwards $^{2}$, Tim Skloss ${ }^{2}$, Ralph Hurd ${ }^{3}$, Fraser Robb ${ }^{1}$, and Joe Murphy-Boesch ${ }^{4}$

${ }^{1}$ G. E. Healthcare Technologies, Aurora, OH, United States, ${ }^{2}$ G. E. Healthcare Technologies, Waukesha, WI, United States, ${ }^{3}$ Radiological sciences lab, Stanford University, Palo Alto, CA, United States, ${ }^{4}$ NINDS-NIH, Bethesda, MD, United States

Hyperpolarized $13 \mathrm{C}$ enhances the SNR of signals from $13 \mathrm{C}$ metabolites. Separate transmit and receive coils are inserted into the magnet bore to image $13 \mathrm{C}$, limiting patient space. Here, a dual tuned $13 \mathrm{C} / 1 \mathrm{H}$ body coil is developed that is capable of imaging both proton and $13 \mathrm{C}$ in one exam. The coil has the same $70 \mathrm{~cm}$ inner diameter as the standard body coil and can be used stand-alone as the Tx/Rx coil, or as the transmit coil for proton and $13 \mathrm{C}$ receive arrays. The efficiency for proton excitation is comparable to that of the standard proton only body coil.

High precision MR-TEM cell for in-situ calibration of RF field probes in clinical MR systems

Frank Seifert ${ }^{1}$ and Bernd Ittermann ${ }^{1}$

${ }^{1}$ Physikalisch-Technische Bundesanstalt (PTB), Braunschweig und Berlin, Germany

An MR-TEM cell is a transverse electromagnetic (TEM) cell operated as a Tx/Rx coil directly inside an MR scanner. From a precise flip angle measurement in a tiny sphere of water the $\mathrm{RF}$ electric field inside the cell can be determined using the TEM condition $|\mathrm{E}|=2 \mathrm{c}_{0}\left|\mathrm{~B}_{1}{ }^{+}\right|$. Thus, an MR-TEM cell can be utilized for the calibration of RF E- and $\mathrm{H}$-field probes as well as for the determination of the RF voltages and RF currents at its ports which is important e.g. for experimental validation of simulation results in RF safety research. We report here on the high precision flip angle calibration of an MR-TEM cell with $0.1 \%$ uncertainty

Dual-resonant helmet coil for $1 \mathrm{H} / 31 \mathrm{P}$ at $3 \mathrm{~T}$ MRI

Suk-Min Hong ${ }^{1}$, Chang-Hoon Choi ${ }^{1}$, Jörg Felder ${ }^{1}$, and N. Jon Shah ${ }^{1,2}$

${ }^{1}$ Institute of Neuroscience and Medicine - 4, Forschungszentrum Jülich, Jülich, Germany, ${ }^{2}$ Department of Neurology, Faculty of Medicine, RWTH Aachen University, JARA, Aachen, Germany

The partial volume helmet coil is the intermediate coil type between surface coil and volume coil in terms of SNR and $\mathrm{B}_{1}$ uniformity. The helmet coil was introduced to increase the filling factor leading to increasing SNR. In this study, we modified the helmet coil geometry by inserting additional ring to achieve a dual resonance, which is tuned for ${ }^{1} \mathrm{H} /{ }^{31} \mathrm{P}$. The feasibility of dual-tuned helmet coil was evaluated by simulation and MR measurement, and the results were compared with those acquired by commercial single- and dual-tuned birdcage coils.

A genuine design for a dual-tuned $\$ \$ \$^{\wedge}\{1\} \mathrm{H} / \wedge\{31\} \mathrm{P} \$ \$ \$$ coil with no lumped elements operating at $4.7 \mathrm{~T}$

Anna Hurshkainen ${ }^{1}$, Anton Nikulin ${ }^{1}$, Stanislav Glybovski ${ }^{1}$, Christophe Vilmen $^{2}$, Marc Dubois $^{3}$, Djamel Berrahou ${ }^{3}$, Stefan Enoch ${ }^{3}$, Irina Melchakova $^{1}$, Pavel Belov $^{1}$, Redha Abdeddaim $^{3}$, and David Bendahan ${ }^{2}$

${ }^{1}$ Department of Nanophotonics and Metamaterials, ITMO University, Saint-Petersburg, Russian Federation, ${ }^{2} \mathrm{CNRS} / \mathrm{CRMBM}$, Aix-Marseille University, Marseille, France, ${ }^{3} \mathrm{CNRS} / \mathrm{Institute}$ Fresnel, Aix-Marseille University, Marseille, France

For a wide range of MRI and MRS applications dual-tuned MR coils are used capable of multi-nuclear studies. Conventional ultra-high-field preclinical dual-tuned coils are either surface loops having high SNR over a limited FOV or volumetric coils with ultimate coverage compromised by low SNR while used in Tx and Rx regimes. In this contribution we propose an alternative design of the dual-tuned ${ }^{1} \mathrm{H} /{ }^{31} \mathrm{P}$ coil based on an open self-resonant periodic structure, which doesn't require variable lumped capacitors for tuning and matching. It has been shown that the proposed coil is suitable for studying energetics in human forearm muscles at $4.7 \mathrm{~T}$.

Decoupling strategies for Double Tuned Radio Frequency coils at $7 \mathrm{~T}$

F. Maggiorelli' ${ }^{1}$ E. B. Boskamp ${ }^{2}$, A. Retico ${ }^{1}$, G. Tiberi ${ }^{3}$, J. D. Kaggie ${ }^{4}$, F. Robb ${ }^{5}$, and M. Tosetti ${ }^{3}$ 
${ }^{1}$ Pisa Division, National Institute of Nuclear Physics, Pisa, Italy, ${ }^{2}$ GE Healthcare, San Diego, CA, United States, ${ }^{3}$ IRCCS Stella Maris, Imago7 Foundation, Calambrone,Pisa, Italy,

${ }^{4}$ University of Cambridge, Cambridge, United Kingdom, ${ }^{5} \mathrm{GE}$ Healthcare, Aurora, $\mathrm{OH}$, United States

Magnetic Resonance Imaging (MRI) and Spectroscopy (MRS) with nuclei different from protons, often require the acquisition of proton signal for shimming and coregistration procedures. For this purpose Double Tuned Radio Frequency (DT-RF) coils are needed. The drawback of DT-RF coils is basically the coupling between the two resonant structures, which reduces SNR and increases focal heating. The aim of this study is to compare active and passive decoupling strategies in terms of $Q$ factor and $\mathrm{S}_{21}$ parameter. Workbench measurements show that PIN Diode active decoupling is an interesting alternative for DT-RF coils.

Optimization study of a double-tuned nested birdcage RF coil for $1 \mathrm{H} / 23 \mathrm{Na}$ MRI

Angelo Galante ${ }^{1,2,3}$, Marco Fantasia ${ }^{1,2,3}$, and Marcello Alecci ${ }^{1,2,3}$

${ }^{1}$ Life, Health and Environmental Science, L'Aquila University, L'Aquila, Italy, ${ }^{2}$ Laboratori Nazionali del Gran Sasso, Istituto Nazionale di Fisica Nucleare, L'Aquila, Italy, ${ }^{3}$ Istituto SPINCNR, CNR, L'Aquila, Italy

The nested birdcage design is useful to develop dual tuned volume transceiver RF coils. Despite its apparently simple design, coupling among coils affects the resonance frequencies, making its practical realization cumbersome. FEM simulations were first validated by workbench measurements for a specific nested double-birdcage suitable for $1 \mathrm{H} / 23 \mathrm{Na} \mathrm{MRI}$ at $2.35 \mathrm{~T}$. Then were used to study the isolation and RF efficiency for a set of different geometrical parameters. We demonstrate that an optimized nested design is obtained if the disposition of the birdcages rugs, lengths and shield diameter are carefully taken into account.

The Design of A Short Solenoid with Homogeneous B1 for A Low-field Portable MRI Scanner Using Genetic Algorithm

Zhi Hua Ren ${ }^{1}$ and Shao Ying Huang ${ }^{1}$

${ }^{1}$ Engineering Product Development, Singapore University of Technology and Design, Singapore, Singapore

A short solenoid that provides field homogeneity with relatively low inductance and low length-to-radius ratio was successfully designed and validated to work in a Halbach array based portable MRI scanner. The optimization is done by applying genetic algorithm and by using Bio-Savart Law as a forward calculation model. The optimized design shows advantages of much higher homogeneity with a practically small length-to-radius ratio compared with a constant-pitch solenoid.

Remote tuning and matching of a non-resonant wire loop

J. Rock Hadley ${ }^{1}$, Laura Slusser ${ }^{1}$, Robb Merrill ${ }^{1}$, and Dennis L. Parker ${ }^{1}$

${ }^{1}$ Department of Radiology and Imaging Sciences, University of Utah, Salt Lake City, UT, United States

There are several situations, such as some interventional applications or intracavitary placement, where it would be desirable to remotely tune and match a local RF coil. Although remote lumped element placement will result in decreased SNR, it is likely that net loss in SNR may be a function of the designs used. This study investigated the SNR trade-off of different methods of remote tuning by comparing the SNR that could be achieved with the placement of lumped elements at the coil. A large variation in SNR based on method was observed.

Endoluminal coil-sensitivity degradation with the coil-orientation effect with respect to B0 field: preliminary results

HAMZA RAKI ${ }^{1,2}$, SIMON A. LAMBERT ${ }^{1}$, KEVIN TSE VE KOON $^{1}$, HENRI SOUCHAY $^{2}$, FRASER ROBB $^{3}$, ISABELLE SANIOUR ${ }^{1}$, and OLIVIER BEUF ${ }^{1}$

${ }^{1}$ Univ. Lyon, INSA-Lyon, Université Claude Bernard Lyon 1, UJM-Saint Etienne, CNRS, Inserm, CREATIS UMR 5220, U1206, F-69000, LYON, France, ²General Electric Healthcare, Buc, France, ${ }^{3}$ General Electric Healthcare, Aurora, $\mathrm{OH}$, United States

Single-loop endoluminal RF-coils are a possible solution for the SNR limitations of external coils. However, they suffer from signal variations due to the coil sensitivity dependence with the coil orientations with respect to the $\mathrm{B}_{0}$ field. We simulated (electromagnetic simulations with Feko) an RF-coil along the $\mathrm{Ox}$ axis $\left(0^{\circ}\right)$ taken to be that of the $\mathrm{B} 0$ field and for specific coil orientations $\left(30,45,60\right.$ and $\left.90^{\circ}\right)$ around and in oblique position with respect to the Ox axis $\left(\mathrm{B}_{0}\right)$. We then evaluated the signal distribution ( $\mathrm{H}$-field $2 \mathrm{D}$ map) variation with the coil orientations to can propose an adequate architecture. 
${ }^{1}$ Department of Biomedical Engineering, Vanderbilt University, Nashville, TN, United States, ${ }^{2}$ Vanderbilt University of Imaging Science, Vanderbilt University, Nashville, TN, United States, ${ }^{3}$ Department of Radiology, Vanderbilt University, Nashville, TN, United States, ${ }^{4}$ Department of Electrical Engineering, Vanderbilt University, Nashville, TN, United States

Array-compressed parallel transmission was recently proposed as a way to reduce the number of RF power amplifiers required for many-coil parallel transmission [1]. This is achieved by connecting a large number of coils to a small number of amplifiers via an array compression network that implements optimized coil-to-channel combinations using ratio-adjustable power splitter (RAPS) circuits [2,3] and phase shifters. Currently, the RAPS circuit ratios are determined by tuning coaxial cable lengths within the RAPS circuit (Figure 2), but this prevents dynamic switching of the compression weights via remote tuning. Remotely tunable RAPS circuits and phase shifters would also be useful for dynamic mode switching in MR fingerprinting $[4,5]$. To achieve this, here we describe the design and validation of a quad hybrid-based phase shifter that can be tuned by varying terminating capacitors, and integrate it into a RAPS circuit. Bench tests and 7T imaging and B1+ mapping experiments were performed to validate the phase shifters and new RAPS circuit design.

A low cost Internet of Things solution for real time magnetic field measurement for MRI polarization coils using a computer numeric control machine

Priyanka Harish ${ }^{1}$, Likith $P S^{1}$, Mamatha $M R^{1}$, Meghana $S^{1}$, Vikas Vasisht $A^{1}$, and Sairam Geethanath ${ }^{1,2}$

${ }^{1}$ Medical Imaging Research Center, Dayananda Sagar Institutions, Bengaluru, India, ${ }^{2}$ Department of Radiology, Columbia University Medical Center, New York, NY, United States

An Internet of Things solution for real time Magnetic Field Measurement of polarization coils using Computer Numeric Control (CNC) machine was developed in order to automatically map the static magnetic field at a low cost (\$722). The results were transformed into a visualization report of the magnetic field and uploaded on the cloud server. This report can be accessed by any authorized user with an internet connection from any device, to conduct further analysis. The magnetic field measuring $\mathrm{CNC}$ is a multipurpose 3 -axis robotic system which can be equipped with other field probes to serve as a multi-parametric measurement device.

Optoelectronical-based multiplexed transmission of analog signals in a magnetic environment.

Christophe Vilmen ${ }^{1}$, Louis Bortoli ${ }^{1,2}$, Evan Gallouin ${ }^{1,3}$, Maxime Guye $^{1,4}$, Monique Bernard $^{1}$, David Bendahan ${ }^{1}$, and Alexandre Fouré ${ }^{1}$

${ }^{1}$ Aix-Marseille Univ, CNRS, CRMBM, Marseille, France, ${ }^{2}$ Aix-Marseille Univ, Polytech ${ }^{\circ}$ Marseille, Ecole d'ingénieurs, Marseille, France, ${ }^{3}$ ESTIA Ecole supérieure des technologies industrielles avancées, Bidart, France, ${ }^{4}$ APHM, Hôpital Universitaire Timone, CEMEREM, Marseille, France

This study describes the methodological developments to both convert and transmit several mechanical signals in a magnetic environment (3T Verio Siemens) as optical signals. Multiple sensors were connected to a MR-compatible ergometer used to assess dynamic knee extensions kinetics. The corresponding signals were analog to digital converted and transmitted as optical signals through a single optical fiber. The quality of mechanical and ${ }^{31} \mathrm{P}$ MR spectroscopy $\left({ }^{31} \mathrm{P}-\mathrm{MRS}\right)$ signals remained high and disclosed no adverse interference from the transducers ensuring both conversion and transmission. The multiplexed signals transmission allowed an accurate assessment of human movement kinetics in a magnetic environment.

Pulseq-GPI Compatible console for 9.5mT MRI system

Syed Saad Siddiq ${ }^{1}$, Sneha Potdar $^{1}$, and Sairam Geethanath ${ }^{1,2}$

${ }^{1}$ MIRC, Dayananda Sagar Institutions, Bangalore, India, ${ }^{2}$ Department of Radiology, Columbia University Medical Center, New York, NY, United States

A cost effective console, compatible with Pulseq-GPI has been designed for $9.5 \mathrm{mT}$ using general purpose microcontroller boards. Data from Pulseq-GPI was extracted in a text file and uploaded on the microcontroller to play the gradient waveforms ( $\mathrm{Gx}, \mathrm{Gy}$ ) and radio frequency (RF) pulses, with a dwell time of 5 us. Current work involves integration of Analog to Digital Convertor $(A D C)$ for Gradient Recalled Echo (GRE) sequence and reducing the time required to upload the waveforms for the entire sequence. Future work involves interfacing the console with coil driver apparatus to integrate with $9.5 \mathrm{mT}$ lab MRI systems.

$10 \mu \mathrm{m}$ isotropic voxels acquired with a CMOS-based planar microcoil at $14.1 \mathrm{~T}$ : Preliminary results

Marlon Arturo Pérez Rodas ${ }^{1,2}$, Jonas Handwerker ${ }^{3,4}$, Hellmut Merkle $^{1}$, Rolf Pohmann ${ }^{1}$, Jens Anders ${ }^{3,4}$, and Klaus Scheffler ${ }^{1,5}$

${ }^{1}$ High-Field MR Center, Max Planck Institute for Biological Cybernetics, Tübingen, Germany, ${ }^{2}$ Graduate Training Centre of Neuroscience, IMPRS for Cognitive and Systems Neuroscience, University of Tübingen, Tübingen, Germany, ${ }^{3}$ Institute of Microelectronics, University of Ulm, Ulm, Germany, ${ }^{4}$ Institute of Theory of Electrical Engineering, University of Stuttgart, Stuttgart, Germany, ${ }^{5}$ Department for Biomedical Magnetic Resonance, University of Tübingen, Tübingen, Germany

The quest for high resolution MR have push the technology to miniaturization. Thus, microcoils have been used for imaging with very high resolution. Here, we have designed and constructed a fully integrated CMOS NMR transceiver containing an on-chip microcoil, integrated amplifiers and demodulator for the high-frequency MR signal. In the present work, the initial microimaging results of this fully-integrated NMR transceiver in a $14.1 \mathrm{~T}$ animal scanner are presented. The on-chip microcoil allows imaging with a spatial resolution down to 10 $\mu \mathrm{m}$ with an SNR of 64 and with an improvement in SNR/volume ratio of 150 compared to a $10 \mathrm{~mm}$ surface coil. 
High Definition Sodium (23Na) In Vivo MRI of the Human Eye at 7.0 Tesla: Need for Substantially Enhanced Spatial Resolution than Commonly Used in Brain MRI

Daniel Wenz ${ }^{1}$, Andre Kuehne ${ }^{2}$, Till Huelnhagen ${ }^{1}$, Armin M. Nagel $^{3,4}$, Helmar Waiczies $^{2}$, Oliver Stachs ${ }^{5}$, Erdmann Seeliger ${ }^{6}$, Bert Flemming ${ }^{6}$, and Thoralf Niendorf ${ }^{1,2}$

${ }^{1}$ Berlin Ultrahigh Field Facility, Max Delbrueck Centrum, Berlin, Germany, ${ }^{2}$ MRI.TOOLS GmbH, Berlin, Germany, ${ }^{3}$ Institute of Radiology, Unviersity Hospital Erlangen, Erlangen, Germany, ${ }^{4}$ Division of Medical Physics in Radiology, German Research Centre (DKFZ), Heidelberg, Germany, ${ }^{5}$ Department of Ophthalmology, University of Rostock, Rostock, Germany, ${ }^{6}$ Institute of Physiology, Charite University Medicine, Berlin, Germany

Sodium ions are crucial in the physiology of human eye and its compartments like vitreous humor, aqueous humor, lens and retina. In this work we used a six-channel transceiver array dedicated for ocular ${ }^{23} \mathrm{Na} \mathrm{MRI}$ and obtained in vivo images of the eye of exceptional quality with enhanced spatial resolution like $(1.0 \times 1.0 \times 1.0) \mathrm{mm}{ }^{3}$ and demonstrated why spatial resolutions currently used for sodium MRI of the human brain are not sufficient in the context of ${ }^{23} \mathrm{Na}$ in vivo MRI of the human eye. Enhancing spatial resolution is essential to investigate changes of sodium concentration in subtle eye compartments (aqueous humor, lens).

A 3D printed lung phantom for exploration of the limits of ${ }^{19} \mathrm{~F}-\mathrm{C}_{3} \mathrm{~F}_{8}$ ventilation imaging resolution and SNR

Adam Maunder ${ }^{1}$, Fraser Robb ${ }^{1,2}$, Madhwesha Rao ${ }^{1}$, and Jim Wild ${ }^{1}$

${ }^{1}$ POLARIS, Academic Radiology, University of Sheffield, Sheffield, United Kingdom, ${ }^{2}$ GE Healthcare Inc., Aurora, OH, United States

Fluorinated gas imaging is a complementary method to hyperpolarized gas ventilation imaging, but suffers from lower SNR by virtue of low spin density and thermal polarisation. We present a 3D printed lung phantom based on a gold standard lung ventilation scan acquired from ${ }^{3} \mathrm{He}$ MRI used to explore the limits of fluorinated gas MR in terms of spatial resolution and SNR. Images acquired with unrealistically long imaging times for in-vivo exams were compared to lower resolution images. The results demonstrate that resolutions obtainable with in-vivo fluorinated gas imaging miss potentially important spatial variation information.

Clinical Improvement of 19F Image Sensitivity using the Inductive Coupling at 7.0T Animal MRI

Bu S Park ${ }^{1}$, Sunder S Rajan ${ }^{2}$, and Brenton McCright ${ }^{1}$

${ }_{1}^{1}$ Division of Cellular and Gene Therapies, FDA/CBER/ Office of Tissues and Advanced Therapies, Silver Spring, MD, United States, ${ }^{2}$ Biomedical Physics, FDA/CDRH/Office of Science and Engineering Laboratories, Silver Spring, MD, United States

We present numerical simulations and experimental validation data testing the feasibility to improve $19 \mathrm{~F}$ image sensitivity of perfluorocarbon labeled cells using the secondary resonator tuned at $287 \mathrm{MHz}$ to make an enhancing induced RF magnetic field (B1) at 7.0T 19F/1H MRI. The numerical simulation results of $|\mathrm{B} 1+|$ and corresponding experimental $19 \mathrm{~F}$ images without and with the secondary resonator tuned at $287 \mathrm{MHz}$ show the improvement of $|\mathrm{B} 1+|$ and $19 \mathrm{~F}$ image uniformity. To model a potential clinical application, we used inductive coupling MR to image 19F perfluorocarbon labeled cells encapsulated in polyethylene glycol (PEG) after their transplantation into mice.

A Tool For Rapid Power Analysis for Arbitrary Circular Surface Coil Near Arbitrary Spherical Sample at Any Frequency

Giuseppe Carluccio $^{1,2}$, Karthik Lakshmanan ${ }^{1,2}$, and Christopher Michael Collins ${ }^{1,2}$

${ }^{1}$ Radiology, Center for Advanced Imaging Innovation and Research (CAI2R), New York, NY, United States, ${ }^{2}$ Radiology, Bernard and Irene Schwartz Center for Biomedical Imaging, New York, NY, United States

We present a tool to quickly estimate the noise induced by the resistance of a surface coil and the noise induced by the coil in a sphere. The tool relies on two analytical solutions, and results depend on many parameters. We show plots of the dissipated power in the sample and the coil as function of some of these parameters such as the diameter of the coil, the distance of the coil from the sphere and the wire diameter of the coil. The tool can be useful in the design process of coils, especially dense receive arrays.

Nested Birdcage Receive Array for Simultaneous Multislice EPI

Kenneth M Bradshaw ${ }^{1}$, Daniel Sheltraw ${ }^{2}$, Greyson Tarbox ${ }^{3}$, and Ben Inglis ${ }^{4}$

${ }^{1}$ ECEn Department, Brigham Young University, Provo, UT, United States, ${ }^{2}$ University of California - Berkeley, Berkeley, CA, United States, ${ }^{3}$ ECEn, Brigham Young University, Provo, UT, United States, ${ }^{4}$ UC BerkeleyUniversity of California - Berkeley, Berkeley, CA, United States

We present a novel RF coil design that is capable of simultaneous multi-slice (SMS) echo planar imaging (EPI) for functional MRI along the $z$ axis at 3 T, while maintaining high in-plane $(x-y)$ homogeneity to minimize the effects of receive field contrast on subject motion and motion correction. The coil is symmetric and is open front and rear, making it compatible with fMRI stimulus devices including transcranial magnetic stimulation (TMS) coils. 
A new dual-mode RF-coil array element for 7T MRI based on dipole antennas

Georgiy Solomakha ${ }^{1}$, Stanislav Glybovski ${ }^{2}$, Alexander J.E. Raaijmakers ${ }^{3}$, Constantin Simovski ${ }^{4}$, Alexander Popugaev ${ }^{5}$, Irina Melchakova ${ }^{2}$, Pavel Belov $^{2}$, and Redha Abdeddaim ${ }^{6}$

${ }^{1}$ Depatment of Nanophotonics and metamaterials, ITMO University, Saint-Petersburg, Russian Federation, ${ }^{2}$ Nanophotonics and metamaterials, ITMO University, Saint-Petersburg, Russian Federation, ${ }^{3}$ Department of Radiology, UMC Utrecht, Utrecht, Netherlands, ${ }^{4}$ Electronics and Nanoengineering, Aalto University, School of Electrical Engineering, Helsinki, Finland, ${ }^{5} \mathrm{RF}$ and SatCom Systems, Fraunhofer Institute for Integrated Circuits IIS, Erlangen, Germany, ${ }^{6} \mathrm{CNRS}$, Institut Fresnel, Aix-Marseille Universite, Marsel, France

In this work, we demonstrate a new RF-coil for 7 Tesla ultrahigh field MRI with two orthogonal channels to achieve better SAR and SNR of images. The first phase of the work involves numerical study of different multimode structures consisting of coupled electrical dipoles to form a radiofrequency coil that may operate both as a surface loop [1] or a single radiative electrical dipole [2] depending on the driven channel.

Design of an electromagnetic actuator for magnetic resonance elastography

Yuan Feng ${ }^{1}$, Xuefeng Zhao ${ }^{1}$, Suhao $\mathrm{Qiu}^{1}$, Mo Zhu ${ }^{2}$, Ping Shen ${ }^{2}$, Shengyuan $\mathrm{Ma}^{1}$, Chun-hong $\mathrm{Hu}^{2}$, and Liang Guo ${ }^{2}$

${ }^{1}$ Soochow University, Suzhou, China, ${ }^{2}$ the First Affiliated Hospital of Soochow University, Suzhou, China

We introduced a novel design of electromagnetic actuator for magnetic resonance elastography. The actuator consists of a vibration control module and an actuation module. The actuation frequency and magnitude were manually tuned in a control panel of the control module. The actuation module could be easily converted to imaging phantom, organs of the abdomen region and the brain. Results showed a steady elastic wave propagation at gel phantom, liver, and brain tissues.

MR-Compatible, Organic Light-Emitting Diode (OLED) display for functional MRI

YunKyoung Ko ${ }^{1}$, Seond Dae Yun ${ }^{1}$, Jörg Felder ${ }^{1}$, Chang-Hoon Choi ${ }^{1}$, and N.Jon Shah ${ }^{1}$

Functional MRI (fMRI) frequently relies on visual stimulation. In this study, we designed and implemented a MR compatible display unit based on organic light-emitting diodes (OLED) and evaluated its performance on a 3T clinical MRI scanner by carrying out a visual block-paradigm fMRI experiment using the OLED display. The OLED display was successfully operated during the MR measurements. And an fMRI examination was successfully demonstrated with a visual functional study using the OLED display.

Ultra-low power transmitter for encoding non-MR signals in Magnetic Resonance (MR) recordings

Jan Raagaard Petersen ${ }^{1}$, Jan Ole Pedersen ${ }^{1,2}$, Vitaliy Zhurbenko ${ }^{1}$, Jan Henrik Ardenkjær-Larsen ${ }^{1}$, and Lars G. Hanson ${ }^{1,2}$

${ }^{1}$ Department of Electrical Engineering, Technical University of Denmark, Kgs. Lyngby, Denmark, ${ }^{2}$ Research Centre for Magnetic Resonance, Dept 714 Centre for Functional and Diagnostic Imaging and Research, Copenhagen University Hospital Hvidovre, Hvidovre, Denmark

Advancing Magnetic Resonance Imaging (MRI) technology requires integration of the MRI scanners with sensors and systems for monitoring various non-MRI signals. In this paper, we present design and integration of a low power AM radio transmitter into a 3T MRI scanner, which can be used for efficient collection of data from non-MRI sensors. The transmitter consumes only $1.3 \mathrm{~mW}$ while transmitting $2.7 \mu \mathrm{W}$ at $120 \mathrm{MHz}$ with high frequency stability. The presented design is useful in low power applications requiring high frequency stability and is intended for wireless transmission of non-MR signal recordings during MRI scanning.

Traditional Poster

\section{PET \& Hybrid Systems}

Development of a radiolucent 64-channel on-body receive array to enhance image quality of the MR-linac

Stefan E. Zijlema ${ }^{1}$, Luca van Dijk ${ }^{1}$, Sara L. Hackett ${ }^{1}$, Jan J.W. Lagendijk ${ }^{1}$, Rob H.N. Tijssen ${ }^{1}$, and Cornelis A.T. van den Berg ${ }^{1}$

${ }^{1}$ Radiotherapy, UMC Utrecht, Utrecht, Netherlands 
To improve the spatiotemporal resolution of 3D imaging on the MR-linac, we are developing a new radiolucent 64-channel receive array, which can be placed directly onto the patient during treatments. Coil prototypes caused no significant dosimetric changes. Measurements with 4-channel prototypes showed that overlapping coil loops lead to the highest potential imaging performance. Imaging comparisons with the current MR-linac array showed that the signal-to-noise ratio is improved.

Concentric PET shields and wide-bore 1.5 T MR birdcage for optimal MR and PET signal

Deb Rivera ${ }^{1,2}$, Erik R Huijing ${ }^{3}$, Cezar Alborahal ${ }^{2,4}$, Flavio Meliado ${ }^{3,5}$, Bart Steensma $^{3}$, Thomas Dey ${ }^{6}$, Volkmar Schulz ${ }^{7}$, Björn Weißler ${ }^{7}$, E Versteeg ${ }^{3}$, Hugo de Jong $^{3}$, Martino Borgo ${ }^{8}$, Michel Italiaander ${ }^{2}$, and Dennis Klomp ${ }^{2,3}$

${ }^{1}$ Academic Medical Center, Amsterdam (AMC), Amsterdam, Netherlands, ${ }^{2}$ MR Coils BV, Zaltbommel, Netherlands, ${ }^{3}$ University Medical Center Utrecht (UMCU), Utrecht, Netherlands, ${ }^{4}$ MR Focus BV, Zaltbommel, Netherlands, ${ }^{5}$ MR Code BV, Zaltbommel, Netherlands, ${ }^{6}$ Rheinisch-Westfaelische Technische Hochschule Aachen, Aachen, Netherlands, ${ }^{7}$ RheinischWestfaelische Technische Hochschule Aachen, Aachen, Germany, ${ }^{8}$ Furtura, Heerhugowaard, Netherlands

Prioritizing signal fidelity for PET and MR, we simulated, built, and tested a wide-bore 1.5T body coil with a concentric ring of novel PET shields. With such an approach, the inherent reduced transmit efficiency can be compensated for by applying more power. Through B1+ measurements in phantoms and in the head, we validate that dual RF power amplifiers meet the power requirements.

Design and evaluation of RF coils for hybrid MR-PET imaging of the prostate

Chang-Hoon Choi $^{1}$, Karl Ziemons ${ }^{2}$, Tim Felder ${ }^{1,2}$, Hans-Peter Wegener ${ }^{2}$, and N. Jon Shah ${ }^{1,3}$

${ }^{1}$ Forschungszentrum Juelich, Juelich, Germany, ${ }^{2}$ Faculty of Medical Engineering and Technomathematics, FH Aachen University of Applied Sciences, Juelich, Germany, ${ }^{3}$ Faculty of Medicine, Department of Neurology, RWTH Aachen University, JARA, Aachen, Germany

Prostate cancer is one of the most common diseases in men, and using multimodality, hybrid systems, such as MR-PET provides valuable data for early diagnosis. A human prostate is quite flexible and can move into different positions under external conditions so it is important to localise the critical region-of-interest using both MRI and PET under the same circumstances. In this study, we focused on various MRI RF coil designs suitable for use in MR-PET prostate imaging, and investigated their performance by evaluating SNRs and penetration depths as a function of coil tilting angle against $B_{0}$

A comprehensive study on electrically floating PET insert for efficient RF penetrability at 3 T MRI system

Md Shahadat Hossain Akram¹ ${ }^{1}$, Craig S. Levin², Takayuki Obata ${ }^{1}$, Genki Hirumi ${ }^{1}$, and Taiga Yamaya

${ }^{1}$ National Institute of Radiological Sciences, Japan, Chiba, Japan, ${ }^{2}$ Stanford University, Stanford, CA, United States

A comprehensive experimental study has been conducted on the geometrical aspects of electrically floating radio frequency (RF) penetrable PET inserts to improve the RF penetration efficiency for acceptable MR imaging performance. Several one ring and two ring PET insert prototypes were used to do experiments in a 700-mm bore diameter 3 T clinical MRI system with a homogeneous cylindrical phantom. Study results provide guidance for optimized PET ring design for efficient RF field penetration inside the shielded ring

MR Compatibility of MADPET4: A Small Animal PET Insert for a 7T MRI System

Geoffrey Topping ${ }^{1}$, Negar Omidvari ${ }^{1}$, Jorge Cabello ${ }^{1}$, Stephan Paul ${ }^{2}$, Markus Schwaiger ${ }^{1}$, and Sibylle Ziegler ${ }^{1,3}$

The impacts of operating a small animal PET insert in a 7T MRI system were studied. The MRI's performance was compared with and without the insert by measuring the static field, flip angle distribution, RF noise, and several imaging sequences with two RF volume coils. With the insert inside a large ${ }^{1} \mathrm{H}$ volume coil, the MR was limited to T1-weighted anatomical tested MRI sequences were functional.

Low cost Earth Field NMR Spectrometer with improved Shimming (LESS)

Chennagiri Rajarao Padma ${ }^{1}$, ThejasVishnu Ramesh ${ }^{1}$, Syed Saad Siddiq ${ }^{1}$, Darshan Shivaramu Keelara ${ }^{1}$, and Sairam Geethanath ${ }^{1,2}$ 
A simple, portable and low cost Earth's Field NMR (EFNMR) spectrometer with improved shimming has been demonstrated. Basic NMR principles such as signal transmission, signal detection, and the pulse sequence for MR signal formation have also been demonstrated. The EFNMR spectrometer has been benchmarked with the commercially available Terranova system. The spectrometer was designed with inexpensive and readily available electronic components, costing less than $\$ 130$. The current work focuses on improving the signal-tonoise ratio of the system using conventional shimming methods, which is a challenge in ultra-low field systems. Future work involves incorporation of gradients and time-shared pulse sequence design.

A New Yokeless Permanent Magnet Array with High Field Strength and High Field Homogeneity for Low-field Portable MRI System

Zhi Hua Ren ${ }^{1}$, Wen Chuan $\mathrm{Mu}^{1}$, and Shao Ying Huang ${ }^{1}$

Permanent magnet array is a good candidate for providing the main magnetic field for a low-field portable MRI system. In this abstract, we present the design of a new yokeless permanent magnet array that generates a longitudinal magnetic field with a significant increase in field strength and in homogeneity compared to a traditional two-ring structure. It is compatible with existing RF coils thus the advancement in coil designs can be applied. The optimization was done based on genetic algorithm and a current model which shows much higher calculation efficiency than finite element method. The effectiveness of the optimization is validated by realistic simulations using COMSOL.

Traditional Poster

\section{Pre-Clinical}

3-Fold SNR Enhancement of Small Animal $\$ \$ \$\left\{^{\wedge} 1\right\}\left\{{ }^{\wedge} 3\right\} \$ \$ \$ C$ MRI using a Cryogenically Cooled (88 K) RF Coil

Juan Diego Sánchez-Heredia ${ }^{1}$, Daniel Højrup Johansen ${ }^{1}$, Rafael A. Baron ${ }^{1}$, Matthias Schneider ${ }^{2}$, Gabriele Spörl ${ }^{2}$, Jarek Wosik ${ }^{3}$, Vitaliy Zhurbenko ${ }^{1}$, and Jan H. Ardenkjær-Larsen ${ }^{1}$

${ }^{1}$ Department of Electrical Engineering, Technical University of Denmark, Kgs. Lyngby, Denmark, ${ }^{2}$ Institut für Luft- und Kältetechnik gemeinnützige GmbH, Dresden, Germany, ${ }^{3}$ Electrical and Computer Engineering Department, University of Houston, Houston, TX, United States

SNR in hyperpolarized ${ }^{13} \mathrm{C}$ MRI is often limited by the low sensitivity of the receive RF chain at the low Larmor frequency of ${ }^{13} \mathrm{C}$. In this study we present an RF transparent (nonmetallic) cryostat designed for small animal imaging, which allows a coil temperature of $88 \mathrm{~K}$, with a coil-to-sample distance below $3 \mathrm{~mm}$. Performance of the cryostat equipped with a 30 $\times 40 \mathrm{~mm}^{2}{ }^{13} \mathrm{C}$ surface coil ( $3 \mathrm{~T}, 32 \mathrm{MHz}$ ) was tested and 3-fold SNR gain over room temperature coil was achieved.

A coil-noise-dominated flexible array inside a whole-head coil to improve temporal SNR in non-human primate imaging

Kyle M Gilbert ${ }^{1}$, Peter Zeman ${ }^{1}$, Jorn Diedrichsen ${ }^{2}$, Julio C Martinez-Trujilloc ${ }^{3}$, J Andrew Pruszynski ${ }^{3}$, and Ravi S Menon ${ }^{1}$

${ }^{1}$ Centre for Functional and Metabolic Mapping, The University of Western Ontario, London, ON, Canada, ${ }^{2}$ Department of Computer Science, The University of Western Ontario, London, ON, Canada, ${ }^{3}$ Department of Physiology and Pharmacology, The University of Western Ontario, London, ON, Canada

Typically, coil elements or arrays are dispersed on a two-dimensional surface to ensure their sensitivity profiles do not overlap, since correlated noise mitigates an SNR improvement when overlapping coils are operating in the sample-noise-dominated regime. In this study, we show that a small flexible array, operating in the coil-noise-dominated regime, can locally improve temporal SNR when placed inside a whole-head array. The two concentric arrays are inductively decoupled using preamplifier decoupling, and the contribution of coil noise to the overall noise reduces the noise correlation. Up to a two-fold increase in temporal SNR is achieved in the motor cortex.

Feasibility test of magnetron surface coil for preclinical MRI at $11.7 \mathrm{~T}$

Sergio E Solis-Najera ${ }^{1}$, Fabian Vazquez ${ }^{1}$, Rodrigo Martin ${ }^{1}$, Oscar Marrufo ${ }^{2}$, and Alfredo Odon Rodriguez ${ }^{3}$

${ }^{1}$ Department of Physics, Faculty of Sciences, UNAM, Mexico City, Mexico, ${ }^{2}$ Department of Neuroimage, INNN MVS, Mexico City, Mexico, ${ }^{3}$ Electrical Engineering Department, UAM Iztapalapa, Mexico City, Mexico

A magnetron surface coil was developed for rodent MRI at 11.7 T. The prototype performance was $Q_{l} 6.5=Q_{u}$, and, the noise figure was 1.6. Phantom images were acquired with the magnetron coil to prove its feasibility. A circular coil was also used to acquire phantom images for comparison purposes. A SNR roll-off comparison was computed and showed an improvement of the magnetron coil over the circular one. Image SNR values were also calculated showing a $28.14 \%$ improvement of our coil over the circular coil. These results demonstrate the versatility and feasibility of the magnetron design to be used at UHF MRI. 
An 8 Channel Dipole Transmit Array and 8 Channel Loop Receive Array for Head Imaging of Non-Human Primates at 10.5T

Russell Luke Lagore ${ }^{1}$, Lance DelaBarre ${ }^{1}$, Jerahmie Radder ${ }^{1}$, Noam Harel ${ }^{1}$, Essa Yacoub ${ }^{1}$, Edward J Auerbach ${ }^{1}$, Kamil Ugurbil ${ }^{1}$, and Gregor Adriany ${ }^{1}$

Described herein is the design, construction, and testing of a head coil for imaging non-human primates at 10.5T. The coil is composed of an 8-channel decoupled dipole array for transmit and an 8-channel loop array for receive. We present preliminary transmit efficiency, SNR, noise correlation, and g-factor results for a phantom with immediate plans to acquire in vivo images. This coil is a proof of concept for higher channel count receive arrays of 16 or 24 loops for head imaging of non-human primates at $10.5 \mathrm{~T}$.

Investigating the Coverage of Receive Coil Arrays Through the SNR and Parallel Imaging Performance: A Simulation Study on A Realistic Monkey Head Model at 7 T

Yang Gao ${ }^{1,2}$ and Xiaotong Zhang ${ }^{1,2,3}$

${ }^{1}$ Interdisciplinary Institute of Neuroscience and Technology, Qiushi Academy for Advanced Studies, Zhejiang University, Hangzhou, China, ${ }^{2}$ College of Biomedical Engineering \&

The coverage of receive coil array is an important concern in coil design especially for monkey head coil. The simulation of receive coil array is helpful in decision-making. For macaque brain imaging at $7 \mathrm{~T}$, five coil array configurations with different coil coverage under realistic considerations were systematically evaluated through quantifying their spatial SNR profiles and parallel imaging acceleration performance. Extending the traditional helmet coverage design for monkey head to whole-head coverage demonstrated substantial improvement in acceleration performance in deep brain region, but less pronounced enhancement can be observed in spatial SNR profiles in brain area.

Development of an integrated RF coil and restraint system for awake rat scanning at $7 \mathrm{~T}$

Dan Madularu ${ }^{1}$, Chathura Kumaragamage ${ }^{1}$, Axel Mathieu ${ }^{1}$, Sricharana Rajagopal ${ }^{1}$, and Jamie Near ${ }^{1}$

${ }^{1}$ McGill University/Douglas Hospital, Montreal, QC, Canada

Research utilizing awake rodents has been conducted for the past 10-15 years, however limitations still exist surrounding this technique. Our goal is to build a restraining/RF coil system that circumvents some of the shortcomings present in existing systems, while allowing for the delivery of various stimuli during preclinical neuroimaging. The proposed design (i.e. TriCoil) has integrated access ports for binocular visual stimulation, gustatory and olfactory stimuli presentation, as well as intranasal delivery. SNR obtained with the TriCoil was superior to a volumetric RF coil for awake rat imaging, while a $\mathrm{CO} 2$ challenge yielded significant brain-wide BOLD changes.

Traditional Poster

\section{Gradients \& Other Effects on B0}

An actively-shielded planar gradient coil design scheme in limited coil-layer-placing space

Yaohui Wang ${ }^{1}$, Xuegang $\mathrm{Xin}^{1}$, Lei Guo ${ }^{2}$, Zhifeng Chen ${ }^{1}$, and Feng Liu ${ }^{2}$

${ }^{1}$ South China University of Technology, Guangzhou, China, ${ }^{2}$ The University of Queensland, Brisbane, Australia

A novel gradient coil design scheme was proposed for use in planar MRI systems. Unlike conventional scheme in a limited magnet pole-pole space which usually applies unshielded design, the novel strategy integrated a set of actively-shielded gradient coils in only four layers in the pole-pole space with the utilization of the system peripheral sections. The design largely improved the shielding effect of the gradient coils and meanwhile left adequate space for the patients and installation of cooling device. The design scheme did not significantly increase the system manufacturing complexity either.

High-performance of Multi-axes DWI sequences using Advanced Charge of Gradient power supply

Sho Kawajiri ${ }^{1}$, Yuki Takai $^{1}$, Motohiro Miura ${ }^{1}$, and Masashi Hori ${ }^{1}$

${ }^{1}$ MRI Systems Development Department, Toshiba Medical Systems Corporation, Tochigi, Japan 
Optimizing the energy distribution to the 3 axes output sections of the gradient power supply allows attaining high-performance Multi-axes DWI sequences. In this study, we propose an 'Advanced Charge' method for preferential energy supply with one axis emitting the largest fraction of output energy of all 3 axes. To realize it, the energy consumption simulation model for gradient power supply and gradient coil was updated accounting for the energy distribution to each individual axis. The new simulation model was implemented in the Advanced Charge control and the feasibility of high-performance of Multi-axes DWI sequences was then confirmed.

Calibration of Siemens MAGNETOM(TM) Terra 7T Shim System and Analysis of Static 3rd-order B0-Shimming of the Heart Using B0DETOX

Michael Hock ${ }^{1}$, Maxim Terekhov ${ }^{1}$, David Lohr ${ }^{1}$, Maria Roxana Stefanescu ${ }^{1}$, Anja Schröder ${ }^{2}$, Heike Walles $^{2}$, Christoph Juchem ${ }^{3}$, and Laura Maria Schreiber ${ }^{1}$

${ }^{1}$ Chair of Cellular and Molecular Imaging, Comprehensive Heart Failure Center (CHFC), University Hospital, Wuerzburg, Germany, ${ }^{2}$ Translational Center Regenerative Therapies (TLCRT), Fraunhofer Institute for Silicate Research (ISC), Wuerzburg, Germany, ${ }^{3}$ Departments of Biomedical Engineering and Radiology, Columbia University, New York, NY, United States

Susceptibility-induced field inhomogeneities in both space and time make $\mathrm{B}_{0}$-shimming a prerequisite for cardiac MRI at ultra-high field. All individual terms of the static $3^{\text {rd }}$-order spherical harmonics shim system were calibrated. Field mapping and calculation of shim currents are performed in customized B0DETOX software. Analysis of $B_{0}$-inhomogeneities is later tested both in measurement of an ex-vivo pig heart and in-vivo in humans. The adjustment of the shim volume to the three measured slices in a healthy volunteer reduced the standard deviation of the field map by $4 \%, 19 \%$ and $18 \%$ compared to shimming of the global heart.

Interferences of local B0-shim coils and RF coils on a 3T MRI scanner

Qiaoyan Chen ${ }^{1,2}$, Jo Lee ${ }^{1,2}$, Jianghong Wen ${ }^{1,2}$, Chao Zou ${ }^{1,2}$, Xiaoliang Zhang ${ }^{3,4}$, Xin Liu ${ }^{1,2}$, and Ye Li ${ }^{1,2}$

${ }^{1}$ Lauterbur Imaging Research Center, Shenzhen Institutes of Advanced Technology, Chinese Academy of Sciences, Shenzhen, China, ${ }^{2}$ Shenzhen Key Laboratory for MRI, Shenzhen, China, ${ }^{3}$ Department of Radiology and Biomedical Imaging, University of California San Francisco, San Francisco, CA, United States, ${ }^{4}$ UCSF/UC Berkeley Joint Graduate Group in Bioengineering, San Francisco, CA, United States

In this work, we quantitatively studied the impact of the local shim coil to RF coil in a combined B0 shim coil and RF coil system in terms of SNR, transmit B1+ and receive B1. By using the results as a design guideline, a 5-channel shim coil was constructed, of which interferences on RF coils were minimized with the appropriate shim coil diameters, number of turns and distances between the shim coil and the sample.

Comparison of patient bore tube supporting structures for a high-performance gradient whole-body MRI system to reduce acoustic noise

Hiromitsu Takamori ${ }^{1}$, Kaoru Ikeda $^{1}$, Shoji Ishizaki ${ }^{1}$, Kazuya Okamoto ${ }^{1}$, Hitoshi Kanazawa ${ }^{1}$, and Kazuto Nakabayashi ${ }^{1}$

${ }^{1}$ Yokohama Development Center, Toshiba Medical Systems, Yokohama, Japan

A whole-body MRI scanner with high-performance gradient system produces loud acoustic noise during scan. In the present study we have evaluated the acoustic noise performance for a new gantry structure aimed at noise reduction with a vacuum chamber insert between the gradient coil cylinder and the patient bore tube cylinder. Two different supporting structures for the bore tube were compared. The method supporting the bore tube by means of a beam structure mounted on the feet of magnet scored better performance than the alternative method supporting it by short brackets mounted at the edges of magnet bore opening.

A feasibility study of ultra-high-strength gradient system on 3T: demonstration using DTI on anisotropic diffusion fibre phantoms

Ming-Jye Chen ${ }^{1}$, Kuan-Hung Cho ${ }^{1}$, Chang-Hoon Choi ${ }^{2}$, Ezequiel Farrher ${ }^{2}$, Richard Buschbeck ${ }^{2}$, Hsuan-Han Chiang ${ }^{1}$, N. Jon Shah ${ }^{2,3}$, Hsu Chang ${ }^{1}$, and Li-Wei Kuo ${ }^{1}$

${ }^{1}$ Institute of Biomedical Engineering and Nanomedicine, National Health Research Institutes, Miaoli, Taiwan, ${ }^{2}$ Institute of Neuroscience and Medicine - 4, Forschungszentrum Juelich, Juelich, Germany, Juelich, Germany, ${ }^{3}$ Department of Neurology, Faculty of Medicine, RWTH Aachen University, Aachen, Germany

In this study, we aimed to integrate an ultra-high-strength gradient system (15 gauss/cm) on a 3T scanner and to demonstrate its feasibility by employing diffusion tensor imaging (DTI) on dedicated anisotropic diffusion fibre phantoms. Two DTI experiments were performed to explore the feasibility of this gradient system, i.e. comparisons of gradient strengths and number of averages. Our results demonstrate reasonable SNR and diffusion contrast acquired on this system using pulsed gradient spin echo diffusion weighted scans could provide useful information. Consistently, it also suggests higher gradient strength could be beneficial to improve the quality of diffusion MRI experiments and its ability to resolve fibre orientations, especially when higher $b$-values are used. 
${ }^{1}$ National Magnetic Resonance Resarch Center (UMRAM), Bilkent University, ANKARA, Turkey, ${ }^{2}$ Department of Electrical and Electronics Engineering, Bilkent University, ANKARA, Turkey

Gradient array systems recently have gained attention due to their various flexibilities and capabilities in different applications. Reducing the mutual-coupling between the coil elements is one of the constraints during the process of the coil design. However, by determining any existing coupling value between the array elements, required decoupling can be achieved. For a typical trapezoidal gradient current waveform, desired voltage values during rise/fall times, are recalculated considering all mutual-couplings between the array elements. This method is evaluated experimentally for different trapezoidal current combinations and can be used in any gradient array system with mutually coupled elements.

Design of breast gradient coil with the control of field nonlinearity

Feng Jia ${ }^{1}$, Sebastian Littin ${ }^{1}$, stefan kroboth ${ }^{1}$, Huijun $\mathrm{Yu}^{1}$, Theresa Palm² ${ }^{2}$ Frederik B. Laun ${ }^{2}$, Mark E. Ladd ${ }^{3}$, and Maxim Zaitsev

${ }^{1}$ Dept. of Radiology, Medical Physics, Medical Center University of Freiburg, Faculty of Medicine, University of Freiburg, Freiburg, Germany, ${ }^{2}$ Institute of Radiology, University Hospital Erlangen, Friedrich-Alexander-Universität Erlangen-Nürnberg (FAU), Erlangen, Germany, ${ }^{3}$ Medical Physics in Radiology, German Cancer Research Center, Heidelberg, Germany

High performance gradient coils are required to assess the tissue microstructure in human breast in vivo with diffusion-weighted imaging. A deisgn methodology of nonlinear breast gradient coil is proposed to increase resultant gradient strength with the control of field nonlinearity. The method is tested by designing a unilateral breast gradient coil for diffusion weighting. The results are analysis to reveal new insights of coil designs.

A Bo Tapestry: MRI Magnet Technology, 1977-2017

Gregory Hurst ${ }^{1}$, Ewald Moser ${ }^{2}$, Martyn Paley ${ }^{3}$, and Franz Schmitt

${ }^{1}$ Upstate Medical University, Syracuse, NY, United States, ${ }^{2}$ Medical University of Vienna, Vienna, Austria, ${ }^{3}$ University of Sheffield, Sheffield, United Kingdom, ${ }^{4}$ Lakeside Imaging-e, Erlangen, Germany

This is a preliminary report from a project to gather and organize an objective historical record of human MRI scanner technology. This report spans magnet technology from 1977 to present (2017), covering about 100 magnets and scanners, and invites additional information.

Magnetic gradient mapping of a 3T MRI scanner using a modular array of novel three-axis Hall sensors

Joris Pascal ${ }^{1}$, Nicolas Weber ${ }^{2,3}$, Jacques Felblinger ${ }^{2,3}$, and Julien Oster ${ }^{2,3}$

${ }^{1}$ FHNW, University of Applied Sciences and Arts Northwestern Switzerland, Muttenz, Switzerland, ${ }^{2}$ U947, Inserm, Nancy, France, ${ }^{3}$ IADI, Université de Lorraine, Nancy, France

This paper presents a multi-point and modular magnetic field sensor system compatible with a 3T-MRI environment. The system features a three-axis magnetometer on a chip. This monolithic sensor is to our knowledge the only integrated sensor commercially available that provides full field vector information as well as sufficient dynamic range and acquisition rate for MRI-applications. We have validated experimentally our demonstrator through the measurement of static magnetic field and magnetic field gradients simultaneously acquired at nine locations within a MRI bore (Prisma, Siemens, Erlangen, Germany).

Switched Gradient Impulse Response Measurement with Uniform Excitation of Eigenmodes

Magdoom Kulam ${ }^{1}$, Malisa Sarntinoranont ${ }^{1}$, William W Brey², and Mareci H Thomas ${ }^{1}$

${ }^{1}$ University of Florida, Gainesville, FL, United States, ${ }^{2}$ National High Magnetic Field Laboratory, Tallahassee, FL, United States

For pulsed field gradient experiments, it is important to characterize gradient switching to correct for errors in measured diffusivity and velocity resulting from imbalances in the gradient time integrals. Accurate characterization of the system requires the time derivative of the test gradient pulse mimic that of an impulse function which excite all the gradient eigenmodes uniformly. We introduce a new test pulse, called the Fresnel pulse whose derivative is a chirp function, which has a uniform spectrum like the impulse function. We also introduce a MR imaging based method to measure the spatiotemporal magnetic fields generated after the test pulse. 
${ }^{1}$ Medical Imaging Research Center, Dayananda Sagar Institution, Bangalore, India, ${ }^{2}$ Dept.of Radiology,Medical Physics, University of Freiburg, Medical Center, Freiburg, Germany,

${ }^{3}$ Dept.of Radiology, Columbia University Medical Center, NewYork, NY, United States

Gradient coils are traditionally designed using variations of the target field method. For asymmetric coils it may however be advantages to allow for a flexible field offset and specify the field gradient as a target instead. In this work we evaluate the performance of the target gradient method for generating head gradient inserts with a window in a lower face region.

Optimization of a traversable wire path of a gradient coil for a magnetic resonance microscope

Takahiro Nishigaki $^{1}$, Shin-ichi Urayama ${ }^{2}$, Naozo Sugimoto ${ }^{1}$, and Tomohiro Ueno ${ }^{1}$

${ }^{1}$ Human Health Sciences, Graduate School of Medicine, Kyoto University, Kyoto, Japan, ${ }^{2}$ Center for the Promotion of Interdisciplinary Education and Resarch, Kyoto University, Kyoto, Japan

We designed $1 \mathrm{~T} / \mathrm{m}$ gradient coils for a $14.1 \mathrm{~T}$ magnetic resonance microscope. The calculated contour wire pattern, however, should be transformed to a traversable wire path for actual construction. In this study, we optimized a connecting method by comparing three loop connecting patterns with the inside and outside return paths as a function of the transition size. We found that larger transition size in smooth parts of the loop reduced more the root mean square of deviations from the center gradient value. This optimization is applicable to gradient coils of larger size.

Biplanar PCB based Micro-Gradient-System-Insert for a Small Animal MRI

Thomas Hüfken ${ }^{1}$

${ }^{1}$ UIm University, Ulm, Germany

MR microscopy demands dedicated gradient systems for providing sufficient spatial resolution, which can normally not be met on conventional small animal or whole-body systems. In this contribution a dedicated gradient insert based on a rather simple biplanar design realized with PCB technique is presented. The gradient shows excellent linearity and provides 1.2 $\mathrm{T} / \mathrm{m}$ amplitude in continuous mode.

Gradient system characterization of a 1.5T MRI-Linac with application to UTE imaging

Tom Bruijnen ${ }^{1}$, Bjorn Stemkens ${ }^{1}$, Jan J W Lagendijk ${ }^{1}$, Cornelis A T van den Berg ${ }^{1}$, and Rob H N Tijssen ${ }^{1}$

${ }^{1}$ Radiotherapy, University Medical Center Utrecht, Utrecht, Netherlands

We characterize the gradient system of a hybrid 1.5T MRI-Linac, which has been developed as the ideal platform for MRI-guided radiotherapy. The system is equipped with a split gradient coil that potentially complicates reconstruction of non-Cartesian sequences such as ultra short echo time (UTE) imaging, which is a promising sequence for pseudo-CT generation and lung imaging. Here, we determine the zeroth and first spatial order gradient impulse responses. These are used to show that UTE imaging is feasible and image quality can be increased significantly using the gradient impulse response.

Traditional Poster

\section{Neonatal \& Pediatric Neuroimaging}


High-Resolution Radial Diffusivity Images Provide Insights of Fetal Brain Development

Akiko Uematsu ${ }^{1,2,3}$, Keigo Hikishima ${ }^{4}$, Junichi Hata ${ }^{1,3}$, and Hideyuki Okano ${ }^{2,3}$

${ }^{1}$ Central Institute for Experimental Animals, Kanagawa, Japan, ${ }^{2}$ RIKEN Brain Science Institute, Saitama, Japan, ${ }^{3}$ Keio University School of Medicine, Tokyo, Japan, ${ }^{4}$ Okinawa Institute of Science and Technology, Okinawa, Japan

Investigating prenatal neural development provide depth knowledge of brain ontogeny. DTI-derived radial diffusivity (RD) imaging has advantage to provide information of microstructural tissue organization information without damaging the tissues. In this study, we investigate the changes of the radial diffusivity (RD) values during fetal development in non-human primate. The RD image contrast was enough to clearly depict the emergence of each brain regions as well as major white matter bundles during prenatal period. In addition, its whole brain intensity distribution histogram provided the information of critical period for the growth of myelination.

Preeclampsia related to delayed development of white matter and cortical infolding.

Ting Liu ${ }^{1}$, Miaomiao Wang ${ }^{1}$, Chao $\mathrm{Jin}^{1}$, Xianjun $\mathrm{Li}^{1}$, and Jian Yang ${ }^{1}$

${ }^{1}$ Department of Diagnostic Radiology, the first Affiliated Hospital of Xi'an Jiaotong University, Xi'an, China

Offspring born from preeclampsia exhibit deficits in cognitive impairment. But the pathogenesis is not clear. We assessed brain maturation and white matter development in neonatal period using total maturation score and tract-based spatial statistics. TMS showed the scores of TMS, B and C scores were lower in preeclampsia group. TBSS results displayed FA values decreased, while $\mathrm{AD}$ and $\mathrm{RD}$ values increased on anterior \& posterior limb of internal capsule, external capsule, splenium of corpus callosum, optic radiation and centrum semiovale in preeclampsia group. The results indicated preeclampsia is associated with delayed development of white matter and cortical infolding.

Is cortical microstructure related to folding during development? A longitudinal MRI study in preterms

Alexandra Hertz ${ }^{1}$, Antonietta Pepe ${ }^{2}$, Julien Lefevre ${ }^{2}$, Marie Zomeno ${ }^{1}$, Francois Leroy ${ }^{1}$, Jessica Lebenberg ${ }^{1,3}$, Linda de Vries ${ }^{4}$, Floris Groenendaal ${ }^{4}$, David Germanaud ${ }^{5}$, Manon Benders $^{4}$, and Jessica Dubois ${ }^{1}$

${ }^{1}$ INSERM, Gif-sur-Yvette, France, ${ }^{2}$ Aix-Marseille University, CNRS, Marseille, France, ${ }^{3}$ CEA, Gif-sur-Yvette, France, ${ }^{4}$ Wilhelmina Children's Hospital, University Medical Center, Utrecht, Netherlands, ${ }^{5}$ APHP, INSERM, Paris, France

The human brain cortex develops dramatically during the preterm period, in terms of both morphology, intra-cortical maturation and dendritic arborization. Here we aimed to investigate whether different stages of microstructural maturation are observed in cortical regions that fold successively. We studied preterm infants longitudinally at around 30 and 40 weeks of post-menstrual age, and combined measures from diffusion tensor imaging (DTI) and spectral analysis of gyrification (SPANGY). We highlighted that proxies of primary folds have an advanced microstructural maturation early on, and that the progression until term age is more intense in proxies of secundary folds than in gyri.

Changes in neonatal regional brain volume associated with preterm birth and perinatal factors

Bonnie Alexander ${ }^{1}$, Claire E Kelly ${ }^{1}$, Chris Adamson ${ }^{1}$, Richard Beare ${ }^{1,2}$, Diana Zannino ${ }^{1}$, Jian Chen ${ }^{1,2}$, Andrea Murray ${ }^{1}$, Wai Yen Loh ${ }^{1,3,4}$, Lillian G Matthews ${ }^{5}$, Simon K Warfield ${ }^{6}$, Peter $J$ Anderson $^{1,7,8}$, Lex W Doyle ${ }^{1,8,9,10}$, Marc Seal ${ }^{1,8}$, Alicia Spittle ${ }^{1,9,11}$, Jeanie Cheong ${ }^{1,9,10}$, and Deanne K Thompson ${ }^{1,3,8}$

${ }^{1}$ Murdoch Children's Research Institute, Melbourne, Australia, ${ }^{2}$ Dept of Medicine, Monash University, Melbourne, Australia, ${ }^{3}$ Florey Institute of Neuroscience and Mental Health, Melbourne, Australia, ${ }^{4}$ The Florey Department of Neuroscience and Mental Health, The University of Melbourne, Melbourne, Australia, ${ }^{5}$ Dept of Newborn Medicine, Harvard Medical School, Boston, MA, United States, ${ }^{6}$ Dept of Radiology, Harvard Medical School, Boston, MA, United States, ${ }^{7}$ Monash Institute of Cognitive and Clinical Neurosciences, Monash University, Melbourne, Australia, ${ }^{8}$ Dept of Paediatrics, The University of Melbourne, Melbourne, Australia, ${ }^{9}$ Neonatal services, Royal Women's Hospital, Melbourne, Australia, ${ }^{10}$ Dept of Obstetrics and Gynaecology, The University of Melbourne, Melbourne, Australia, ${ }^{11}$ Dept of Physiotherapy, The University of Melbourne, Melbourne, Australia

In a cohort of 285 preterm and term infants at term equivalent age, associations were investigated between gestational age (GA) at birth, perinatal factors, and volumes of 100 regions of the M-CRIB neonatal brain atlas. Volumes increased with increasing GA in some regions, and decreased with increasing GA in other regions including primary visual, motor and somatosensory cortices. Robust increases in many regional volumes were found for birthweight standard deviation score, and male sex. These results provide increased insight into the complex array of correlates of preterm birth. 
T2 relaxometry brain MRI could be of prognostic value in preterm infants. The maturation patterns of periventricular white matter differed according to neurodevelopmental outcomes. T2 relaxation values over mid-body periventricular white matter at $>1$ month old of corrected age could predict CP. T2 relaxometry brain MRI provides neuroimaging-outcome correlation among preterm infants, especially when interpreted with age-specific and area-selective considerations.

Automatic Brain Segmentation in a Neonatal Population Using a Multi-Delay Multi-Echo Sequence

Maarten Naeyaert ${ }^{1}$, Tim Vanderhasselt ${ }^{1}$, Marcel Warntjes ${ }^{2}$, and Hubert Raeymaekers ${ }^{1}$

Synthetic MRI using a multi-delay multi-echo sequence was applied to a pre-term neonatal and full term neonatal population. The brain was segmented into different tissue types using the relaxometric data and using an improved algorithm which suppresses CSF partial volume fractions in grey matter. The volumes and volume fractions were calculated. The relation between volumetric quantities and either gestational age (preterm patients only), or corrected age (whole population) was investigated. The Brain Parenchymal and grey matter fraction were found to be dependent on gestational age at birth, while grey matter, CSF, intracranial and brain parenchymal volume are dependent on age.

Longitudinal Mapping of Local Relationship of Surface Area, Cortical Thickness and Cortical Folding in Infants

Dingna Duan ${ }^{1,2}$, Shunren $\mathrm{Xia}^{2}$, Zhengwang Wu${ }^{1}$, Fan Wang ${ }^{1}$, Weili Lin ${ }^{1}$, John H Gilmore ${ }^{3}$, Dinggang Shen ${ }^{1}$, and Gang Li ${ }^{1}$

${ }^{1}$ Department of Radiology and BRIC, University of North Carolina at Chapel Hill, Chapel Hill, NC, United States, ${ }^{2}$ Key Laboratory of Biomedical Engineering of Ministry of Education, Zhejiang University, Hangzhou, China, ${ }^{3}$ Department of Psychiatry, University of North Carolina at Chapel Hill, Chapel Hill, NC, United States

A simple physical law on the global relationship of surface area, cortical thickness, and cortical folding is found across a full range of mammalian species' brains, including adult human brains $^{1,2}$. However, little is known about the local relationship of these cortical properties, especially in infant brains with rapid development in the first two years of life. To fill this knowledge gap, we explored the local relationship of surface area, cortical thickness and cortical folding on 73 normal infants, each of which was longitudinally scanned at 0 , 1 , and 2 years of age. We reveal that the relationship of these three cortical properties is age-specific and region-specific.

Evaluation of cortical thickness estimation methods in neonates.

Martina Lucignani ${ }^{1}$, Andrea Pittella ${ }^{2}$, Maria Camilla Rossi Espagnet ${ }^{3}$, Daniela Longo ${ }^{3}$, Giulia Lucignani ${ }^{3}$, Maurizio Schmid ${ }^{2}$, and Antonio Napolitano ${ }^{1}$

${ }^{1}$ Medical Physics Department, IRCCS Bambino Gesù Children's Hospital, Rome, Italy, ${ }^{2}$ Enginerring Department, Roma Tre University, Rome, Italy, ${ }^{3}$ Imaging Department, IRCCS Bambino Gesù Children's Hospital, Rome, Italy

Cortical thickness (CT) is a sensitive indicator of normal brain structural and functional development, aging, as well as a variety of neuropsychiatric disorders. The state of the art for cortical thickness estimation in children in not as good as the one for adults. We then compared two different algorithms and assess the agreement between these methods and their local variability.

Asynchrony of the cortical maturation in the infant brain studied with MRI

Jessica Lebenberg ${ }^{1,2}$, Jean-François Mangin ${ }^{1,3}$, Cyril Poupon ${ }^{4}$, Lucie Hertz-Pannier ${ }^{5}$, François Leroy², Parvaneh Adibpour ${ }^{2}$, Claire Kabdebon ${ }^{2}$, Ghislaine Dehaene-Lambertz $^{2}$, and Jessica Dubois ${ }^{2}$

${ }^{1}$ UNATI, CEA DRF/Institut Joliot, Université Paris-Sud, Université Paris-Saclay, NeuroSpin center, Gif-sur-Yvette, France, ${ }^{2}$ Cognitive Neuroimaging Unit U992, INSERM, CEA DRF/Institut Joliot, Université Paris-Sud, Université Paris-Saclay, NeuroSpin center, Gif-sur-Yvette, France, ${ }^{3}$ Multicenter Neuroimaging Platform, CATI, cati-neuroimaging.com, France, ${ }^{4}$ UNIRS, CEA DRF/Institut Joliot, Université Paris-Sud, Université Paris-Saclay, NeuroSpin center, Gif-sur-Yvette, France, ${ }^{5}$ UNIACT, CEA DRF/Institut Joliot, INSERM U1129, Université Paris-Sud, Université Paris-Saclay, Université Paris-Descartes, NeuroSpin center, Gif-sur-Yvette, France

Intense changes in cortical microstructure occur during early infancy. Here, we aimed to study cortical maturation over this largely unexplored developmental period using quantitative MRI in 17 infants from 1 to 5 post-natal months. By taking benefit of robust intra- and inter-individual registrations of anatomical images and parametric maps, we measured T1, T2 relaxation times, and DTI longitudinal diffusivity over cortical surfaces and regions of interest. Results showed that each parameter relevantly but differently reflects the progressive maturation. This suggests that multi-parametric approaches might provide interpretable measures of the developing microstructure by accounting for the parameters complementarity. 
Quantitative MRI promises to allow objective and reproducible tissue metrics which are of special interest in newborn brain maturation characterization. However, such methods require acquisition times above 20 minutes which hinders their clinical applicability. With an increasing trend towards examination without sedation during natural sleep, subject motion is an important issue for neonatal applications. With this in mind, this work builds on the previously described Joint System Relaxometry framework and presents a neonatal specific protocol which allows $1.25 \mathrm{~mm}$ isotropic 3D maps of Proton Density, T1 and T2 relaxation times in a total of 10minutes examination time.

Anatomo-functional correlates of auditory development in infancy

Parvaneh Adibpour ${ }^{1}$, Jessica Lebenberg ${ }^{1,2}$, Claire Kabdebon ${ }^{1}$, Francois Leroy ${ }^{1}$, Ghislaine Dehaene-Lambertz ${ }^{1}$, and Jessica Dubois ${ }^{1}$

${ }^{1}$ Cognitive Neuroimaging Unit, INSERM, UMR992; CEA, NeuroSpin Center, Gif-sur-Yvette, France, ${ }^{2}$ UNATI, CEA DRF Institut Joliot, Gif-sur-Yvette, France

Early infancy is a period of intense behavioral acquisitions and brain development. Nevertheless, how functional and structural maturations are inter-related has been little explored so far. Following studies of visual domain, we aimed to address this question for the auditory modality in 1 to 5 -month-old infants, by combining EEG and quantitative MRI measures supposed to reflect fiber myelination and intra-cortical development of dendritic arborization. We investigated the relationships between the functional maturation of auditory-evoked responses in terms of latency and speed, and the maturation of microstructural properties for both white matter tracts and cortical regions of the auditory network.

Optimization of phase-contrast MRI for cerebral blood flow quantification in neonates

Peiying Liu ${ }^{1}$, Charlamaine Parkinson ${ }^{1}$, Dengrong Jiang ${ }^{1}$, Jill B De Vis ${ }^{1}$, Li Pan ${ }^{2}$, Himanshu Bhat ${ }^{2}$, Andrea Poretti ${ }^{1}$, Frances Northington ${ }^{1}$, Aylin Tekes ${ }^{1}$, Thierry Huisman ${ }^{1}$, and W Christopher Golden ${ }^{1}$

Knowledge of $\mathrm{CBF}$ in neonates may provide valuable information in many pathological conditions. When applied to very young children, $\mathrm{CBF}$ mapping using arterial-spin-labeling (ASL) MRI suffers from low signal-to-noise ratio and poor quantification, whereas phase-contrast (PC) MRI may provide reliable estimation of global CBF. This study aimed to optimize the PCMRI protocol for future applications in neonates. By comparing the cardiac-gated and non-gated implementations, we found non-gated PC-MRI could provide accurate CBF measurement with shorter scan time. We also found lower imaging resolution would over-estimate $\mathrm{CBF}$, and therefore recommend the use of $0.3 \mathrm{~mm}$ resolution with 6 averages in neonates.

Clinical application of 4D ASL-MRA in neonatal Vein of Galen malformation

Magdalena Sokolska ${ }^{1}$, Subhabrata Mitra ${ }^{2}$, Yuriko Suzuki ${ }^{3}$, Matthias van Osch ${ }^{3}$, H Rolf Jäger $^{4}$, Adam Rennie ${ }^{4,5}$, Fergus Robertson ${ }^{5}$, Giles Kendall ${ }^{2}$, and Alan Bainbridge ${ }^{1}$

${ }^{1}$ Medical Physics and Biomedical Engineering, University College Hospital, London, United Kingdom, ${ }^{2}$ University College Hospital, London, United Kingdom, ${ }^{3}$ Leiden University Medical Center, Leiden, Netherlands, ${ }^{4}$ UCL National Hospital for Neurology \& Neurosurgery, London, United Kingdom, ${ }^{5}$ Great Ormond Street Hospital for Children, London, United Kingdom

This work investigates the feasibility of using time-resolved magnetic resonance angiography, based on arterial-spin-labelling (ASL), to investigate neonatal vein of Galen malformation for the purpose of aiding diagnosis and surgical treatment planning.

Intraoperative Volatile Anesthetic Exposure Predicts Reduced Frontal Lobe Connectivity Compared to Dexmedetomidine in Infants with Congenital Heart Disease

Vincent Kyu Lee ${ }^{1}$, Phillip Adams ${ }^{2}$, Benjamin Meyers ${ }^{1}$, Lauren Dennis ${ }^{3}$, Nancy Beluk ${ }^{1}$, Tracy Baust ${ }^{4}$, Lucas Saenz ${ }^{4}$, Yulia Domnina ${ }^{4,5}$, Joan $_{\text {Sanchez de Toledo }}^{4,5}$, Vincent J Schmithorst ${ }^{1}$, and Ashok Panigrahy ${ }^{1,6}$

${ }^{1}$ Radiology, University of Pittsburgh, Pittsburgh, PA, United States, ${ }^{2}$ Anesthesiology, Children's Hospital of Pittsburgh UPMC, Pittsburgh, PA, United States, ${ }^{3}$ Science Technology and Mathematics, Regent University, Pittsburgh, PA, United States, ${ }^{4}$ Critical Care Medicine, University of Pittsburgh, Pittsburgh, PA, United States, ${ }^{5}$ Critical Care Medicine, Children's Hospital of Pittsburgh of UPMC, Pittsburgh, PA, United States, ${ }^{6}$ Radiology, Children's Hospital of Pittsburgh of UPMC, Pittsburgh, PA, United States

Anesthetic neurotoxicity in infants with repetitive exposure is a risk factors for adverse neurodevelopmental outcomes. Dexmedetomidine exposure is thought to have neuroprotective effects. We tested the hypothesis that intraoperative volatile anesthetic exposure is predictive of aberrant brain connectivity in the post-operative period in CHD infants, relative to dexmedetomidine exposure using DTI and BOLD imaging. Using both hypothesis driven and data driven approaches, as well as graph analysis we showed that Increased volatile anesthetic exposure in the intraoperative period is associated with reduced post-operative frontal brain connectivity in CHD infants, while DEX exposure was associated with metrics of improved brain connectivity. 
Jenna M Schabdach ${ }^{1}$, Rafael Ceschin ${ }^{1,2}$, Vince Lee $^{2}$, Vincent Schmithorst ${ }^{2}$, and Ashok Panigrahy ${ }^{1,2}$

${ }^{1}$ Department of Biomedical Informatics, University of Pittsburgh, Pittsburgh, PA, United States, ${ }^{2}$ Department of Radiology, Children's Hospital of Pittsburgh of UPMC, Pittsburgh, PA, United States

Functional connectivity studies commonly use resting-state BOLD MR images to study the neurodevelopment of healthy and at-risk neonates. BOLD images are highly sensitive to motion; post-acquisition motion correction techniques can be applied to BOLD data to compensate for motion. We compare the corrective performance of two motion correction techniques on a cohort of 17 healthy neonates: the traditional correction to the first volume technique and a novel, HMM-based motion correction technique. We evaluate the corrected images in terms of the Power et al. thresholds and show the HMM-based technique can be used to recover neonatal BOLD data corrupted by motion.

Anisotropic similarity, a constrained affine transformation: application to brain development analysis

Antoine Legouhy ${ }^{1}$, Olivier Commowick ${ }^{1}$, François Rousseau ${ }^{2}$, and Christian Barillot ${ }^{1}$

${ }^{1}$ Univ Rennes, INRIA, CNRS, INSERM, IRISA UMR 6074, VISAGES ERL U-1228, F-35000, Rennes, France, ${ }^{2}$ IMT Atlantique, LaTIM U1101 INSERM, UBL, Brest, France

The study of brain development provides insights in the normal trend of brain evolution and enables early detection of abnormalities. We propose a method to quantify brain growth in three arbitrary orthogonal directions of the brain through linear registration. We introduce a 9 degrees of freedom transformation that gives the opportunity to extract scaling factors describing brain growth along those directions by registering a database of subjects in a common basis. We apply this framework to create a longitudinal curve of scaling ratios along fixed orthogonal directions from 0 to 16 years highlighting anisotropic brain development.

New microstructural asymmetries in the brain

Junyu Guo ${ }^{1}$, Yuanyuan $\mathrm{Han}^{2}$, Yimei $\mathrm{Li}^{2}$, and Wilburn E. Reddick ${ }^{1}$

${ }^{1}$ Diagnostic Imaging, St Jude Children's Research Hospital, Memphis, TN, United States, ${ }^{2}$ Biostatistics, St Jude Children's Research Hospital, Memphis, TN, United States

Brain microstructural asymmetry can provide more direct causal explanations of functional lateralization than can macrostructural asymmetry. In this study, we discovered two new types of microstructural asymmetry that help to bridge the gap between macrostructural asymmetry and functional lateralization. Myelin-related asymmetry was prominent in the back brain, and axon-related asymmetry occurred in both the front brain and the back brain. These asymmetries early in development indicate that white matter is more mature and more myelinated in the left back brain, providing an explanation for the leftward lateralization of language and visual functions. The asymmetries continue to increase throughout childhood and adolescence.

Comparison of Thalamus Segmentation Using Publicly Available Segmentation Methods in a Pediatric Population

Salem Hannoun ${ }^{1}$, Rayyan Tutunji ${ }^{2}$, Maria El Homsi² ${ }^{2}$ and Roula Hourany ${ }^{2}$

${ }^{1}$ Nehme and Therese Tohme Multiple Sclerosis Center, American University of Beirut Medical Center, Beirut, Lebanon, ${ }^{2}$ Radiology Department, American University of Beirut Medical Center, Beirut, Lebanon

107 subjects were recruited between the ages of one month and 18 years. The study aimed to investigate the differences in the accuracy of five publicly available segmentation techniques on T1-enhanced and non-enhanced images compared to manual segmentation of the thalamus in a pediatric population. volBrain had the best outcomes in enhanced and non-enhanced images. Image segmentation using volBrain is the ideal methodology for thalamus segmentation. Gadolinium-enhancement negatively affects the outcomes of all the tested automated segmentation.

Magnetization transfer ratio in cortical gray matter: a longitudinal study.

Yash P. Patel ${ }^{1,2}$, Jean Shin ${ }^{2,3}$, Penny A. Gowland ${ }^{4}$, and Tomas Paus ${ }^{2,5,6,7}$

${ }^{1}$ Institute of Medical Sciences, University of Toronto, Toronto, ON, Canada, ${ }^{2}$ Rotman Research Institute, University of Toronto, Toronto, ON, Canada, ${ }^{3}$ The Hospital for Sick Children, University of Toronto, Toronto, ON, Canada, ${ }^{4}$ Sir Peter Mansfield Imaging Centre, School of Physics and Astronomy, University of Nottingham, Nottingham, United Kingdom, ${ }^{5}$ Center for Developing Brain, Child Mind Institute, New York, NY, United States, ${ }^{6}$ Department of Psychiatry, University of Toronto, Toronto, ON, Canada, ${ }^{7}$ Department of Psychology, University of Toronto, Toronto, ON, Canada

To assess the change in magnetization transfer ratio (MTR) in the human cerebral cortex during adolescence(14 to 19 years of age). We observe an age-related increase in average MTR in both sexes. Inter-regional profiles of MTR measured at a single time-point correlate with gene-expression profiles of CA1 pyramidal cells (membranes of dendritic arbor) but not of oligodendrocytes (myelin). On the other hand, profiles of the MTR change (from 14 to 19 years) correlate with gene-expression profiles of oligodendrocytes, suggesting that the change may be sensitive to intra-cortical myelination. 
Paediatric brain tissue properties measured with magnetic resonance elastography

Jade Yeung ${ }^{1}$, Lauriane Jugé ${ }^{1,2}$, and Lynne E. Bilston ${ }^{1,3}$

${ }^{1}$ Neuroscience Research Australia, Randwick, NSW, Australia, ${ }^{2}$ University of New South Wales, School of Medical Sciences, Kensington, NSW, Australia, ${ }^{3}$ University of New South Wales, Prince of Wales Clinical School, Kensington, NSW, Australia

Magnetic resonance (MR) elastography is a technique to noninvasively measure the mechanical properties of soft tissues. While adult brain data obtained with MR elastography is readily available, there is little data for healthy paediatric brains throughout development. MR elastography was performed on 25 healthy paediatric subjects aged between $7-18$ years at three frequencies, and the shear moduli of white and grey matter were calculated and compared to data obtained from 10 healthy adults. The shear modulus of paediatric brains was not found to be age dependent, with no significant differences between adult and paediatric brains.

Clinical Equivalence Assessment of T2 Synthetic Pediatric Brain MRI

Basile Kerleroux ${ }^{1}$, Tobias Kober ${ }^{2}$, Tom Hilbert ${ }^{2}$, Mohamed El Ouali ${ }^{3}$, Dominique Sirinelli ${ }^{3}$, and Baptiste Morel ${ }^{4}$

${ }^{1}$ Pediatric Radiology, CHRU de Tours, Tours, France, ${ }^{2}$ Advanced Clinical Imaging Technology, Siemens Healthcare AG, Lausanne, Switzerland, ${ }^{3}$ Pediatric Radiology, CHRU de Tours, Tours, Switzerland, ${ }^{4}$ Pediatric Radiology, CHRU Tours, Tours, France

In a prospective randomized study, we compared the image quality of a synthetized T2 with conventional turbo spin echo T2 during pediatric brain MRI. According to several assessment criteria, synthetic T2 seemed to be an overall equivalent to standard TSE T2, with the advantage of new available T2 quantitative data with a similar acquisition time.

Motor connectivity of the midbrain in healthy children defined using connectivity based parcellation

Sonja Soskic ${ }^{1}$, Hannah Cooper $^{2}$, Alexandra Bonthrone ${ }^{3}$, and Chris A. Clark ${ }^{1}$

${ }^{1}$ Developmental Imaging and Biophysics Section, UCL GOS Institute of Child Health, University College London, London, United Kingdom, ${ }^{2}$ UCL Ear Institute, University College London, London, United Kingdom, ${ }^{3}$ Cognitive Neuroscience and Neuropsychiatry Section, UCL GOS Institute of Child Health, University College London, London, United Kingdom

Delineation of midbrain regions connected with the motor cortex may be useful in evaluating disruptions of motor pathways in paediatric patients. We used the established winner-takesit-all method to parcellate the midbrain according to cortical connectivity in healthy children aged 6-12 years. The percentage of ipsilateral midbrain occupied by motor parcels was negatively associated with age on the right side only, producing an association between age and interhemispheric asymmetry. Our findings indicate that age and interhemispheric differences need to be taken into account if this method is to be utilised for quantitative comparisons of midbrain-motor connectivity in children.

Assessing white matter development in peri-pubertal children using longitudinal fixel-based analysis

Sila Genc ${ }^{1,2}$, Robert E Smith ${ }^{3}$, Charles B Malpas ${ }^{2}$, Vicki A Anderson ${ }^{4,5}$, Jan M Nicholson ${ }^{6}$, Daryl Efron ${ }^{5}$, Timothy J Silk ${ }^{1,2}$, and Marc L Seal ${ }^{1,2}$

${ }^{1}$ Department of Paediatrics, The University of Melbourne, Parkville, Australia, ${ }^{2}$ Developmental Imaging, Murdoch Childrens Research Institute, Parkville, Australia, ${ }^{3}$ The Florey Institute of Neuroscience and Mental Health, Heidelberg, Australia, ${ }^{4}$ Clinical Sciences, Murdoch Childrens Research Institute, Parkville, Australia, ${ }^{5}$ The Royal Children's Hospital, Parkville, Australia, ${ }^{6}$ Judith Lumley Centre, La Trobe University, Melbourne, Australia

Recent evidence suggests that the pubertal period corresponds with changes to white matter microstructure above and beyond age-related development. This study uses a longitudinal fixel-based analysis to investigate which regions of the brain correspond to changes in white matter fibre density and cross-section during pubertal development. We show that, over a 16-month follow-up period, increases in fibre density and cross-section are predominantly in the posterior white matter. These results add to evidence that white matter develops in a posterior-anterior fashion, and signifies the dynamic nature of brain development during puberty.

Longitudinal myelin development in children born very preterm compared with typically developing peers

Deanne Thompson ${ }^{1,2,3,4}$, Joseph Yang ${ }^{2,5,6}$, Jian Chen ${ }^{2}$, Claire Kelly ${ }^{1,2}$, Bonnie Alexander ${ }^{1,2}$, Lillian Matthews ${ }^{7}$, Katherine Lee L $^{1,3,8}$, Rod Hunt ${ }^{1,3,9}$, Jeanie Cheong $^{1,10,11}$, Megan SpencerSmith $^{1,12}$, Marc Seal ${ }^{2,3}$, Jeffrey Neil ${ }^{7}$, Terrie Inder ${ }^{1,7}$, Lex Doyle ${ }^{1,3,10,11}$, and Peter Anderson ${ }^{1,3,12}$

${ }^{1}$ Victorian Infant Brain Studies (VIBeS), Murdoch Children's Research Institute, Melbourne, Australia, ${ }^{2}$ Developmental Imaging, Murdoch Children's Research Institute, Melbourne, Australia, ${ }^{3}$ Department of Paediatrics, The University of Melbourne, Melbourne, Australia, ${ }^{4}$ Florey Institute of Neuroscience and Mental Health, Melbourne, Australia, ${ }^{5}$ Neuroscience Research, Murdoch Children's Research Institute, Melbourne, Australia, ${ }^{6}$ Department of Neurosurgery, The Royal Children's Hospital, Melbourne, Australia, ${ }^{7}$ Department of Pediatric Newborn Medicine, Brigham and Women's Hospital, Harvard Medical School, Boston, MA, United States, ${ }^{8}$ Clinical Epidemiology \& Biostatistics Unit, Murdoch Children's Research Institute, Melbourne, Australia, ${ }^{9}$ Neonatal Medicine, The Royal Children's Hospital, Melbourne, Australia, ${ }^{10}$ Department of Obstetrics and Gynaecology, The University of Melbourne, Melbourne, Australia, ${ }^{11}$ The Royal Women's Hospital, Melbourne, Australia, ${ }^{12}$ Monash Institute of Cognitive and Clinical Neurosciences, Monash University, Melbourne, Australia 
Myelin development over time in preterm children remains unclear. This study compared $T_{1} / T_{2}$ myelin maps for 81 very preterm (VP) and 29 full-term children between 7 and 13 years of age. On average, VP children had higher $T_{1} / T_{2}$ ratios than full-term children in most white matter tracts and deep gray matter structures at both time points. This may reflect compensation or developmental catch-up. $T_{1} / T_{2}$ ratios increased from childhood to adolescence in both VP and full-term children, shedding light on typical and atypical myelin maturation.

Regional Brain Myelin Changes in Patients with Single Ventricle Heart Disease

Sadhana Singh ${ }^{1}$, Bhaswati Roy ${ }^{2}$, Xiaopeng Song ${ }^{1}$, Nancy Halnon ${ }^{3}$, Alan Lewis ${ }^{4}$, Mary Woo ${ }^{2}$, Nancy Pike ${ }^{2}$, and Rajesh Kumar ${ }^{1,5,6,7}$

${ }^{1}$ Department of Anesthesiology, University of California Los Angeles, Los Angeles, CA, United States, ${ }^{2}$ UCLA School of Nursing, University of California Los Angeles, Los Angeles, CA, United States, ${ }^{3}$ Division of Pediatric Cardiology, University of California Los Angeles, Los Angeles, CA, United States, ${ }^{4}$ Division of Pediatric Cardiology, Children's Hospital Los Angeles, Los Angeles, CA, United States, ${ }^{5}$ Department of Radiological Sciences, University of California Los Angeles, Los Angeles, CA, United States, ${ }^{6}$ Department of Bioengineering, University of California Los Angeles, Los Angeles, CA, United States, ${ }^{7}$ Brain Research Institute, University of California Los Angeles, Los Angeles, CA, United States

Single ventricle heart disease (SVHD) subjects show brain injury in multiple gray and white matter based on MRI procedures. However, the extent of regional myelin integrity in SVHD is unclear. We examined the regional brain myelin integrity in SVHD adolescents using the ratio of T1-weighted and T2-weighted MRI signal intensity, and found decreased values in critical autonomic, mood, and cognitive control sites, functions that are deficient in the condition, likely resulting from hypoxic/ischemic processes.

Regional CBF differences underlie neurocognitive outcomes in older children with congenital heart disease: a voxelwise mediation analysis

Vincent Jerome Schmithorst ${ }^{1}$ and Ashok Panigrahy ${ }^{2}$

1791 'Radiology, Children's Hospital of Pittsburgh, Pittsburgh, PA, United States, ${ }^{2}$ Children's Hospital of Pittsburgh, Pittsburgh, PA, United States

We investigate in more detail the relationship between congenital heart disease (CHD), CBF, and neurocognitive outcome in older children by employing a novel voxelwise mediation analysis with $\mathrm{CHD}$ status the independent variable, $\mathrm{NIH}$ Toolbox scores the dependent variable, and voxelwise $\mathrm{CBF}$ the mediating variable. $\mathrm{CHD}$ patients display reduced $\mathrm{CBF}$ in the salience network (insula, medial prefrontal, caudate) which mediates lower performance on tests of memory and language function. However, the reduced CBF in the salience network mediates improved performance of executive function (flanker inhibitory control) likely due to less filtering out of presumed irrelevant but actually relevant information.

Relationships between brain structure and behavior in children with specific learning disabilities revealed by diffusion spectrum imaging

Yi-Chun Liu ${ }^{1}$, Hsiao-Lan Sharon Wang ${ }^{2}$, Shan-Chih Lee ${ }^{1}$, and Jun-Cheng Weng ${ }^{3,4}$

${ }^{1}$ Department of Medical Imaging and Radiological Sciences, Chung Shan Medical University, Taichung, Taiwan, ${ }^{2}$ Department of Special Education, National Taiwan Normal University, Taipei, Taiwan, ${ }^{3}$ Department of Medical Imaging and Radiological Sciences, Chang Gung University, Taoyuan, Taiwan, ${ }^{4}$ Department of Psychiatry, Chang Gung Memorial Hospital, Chiayi, Taiwan

We used diffusion spectrum imaging (DSI) to investigate the relationships between brain structure and behavior in children with specific learning disabilities (SLD). The correlation between reading comprehension scores and the DSI indices was found in corpus callosum. The correlation between Chinese character recognition and the DSI indices was found in cingulate and corpus callosum. The correlation between tone awareness scores and the DSI indices was found in cingulate, superior frontal gyrus and corpus callosum. In summary, SLD not only had difficulty reading and spelling individual words but also more likely to have poorer phonological awareness.

Altered regional brain activities and functional connectivities in children with nonsyndromic cleft and/or lip palate: a resting-state functional MRI study.

Hua CHENG ${ }^{1}$, BO RAO $^{2}$, YANG FAN ${ }^{3}$, YingZi Gao ${ }^{1}$, WenJing Zhang ${ }^{4}$, and Yun Peng ${ }^{1}$

${ }^{1}$ Imaging Center, Beijing Children's Hospital affiliated to Capital Medical University, Beijing, China, ${ }^{2}$ Capital Medical University, Beijing, China, ${ }^{3}$ GE Healthcare, MR Research China, Beijing, China, ${ }^{4}$ Beijing Stomatological Hospital, Capital Medical University, Beijing, China

Rs-fMRI has been widely used as an effective method to evaluate the brain functional changes in physiological and pathological process. Altered both regional brain activities and functional connectivities, especially in verbal and cognitive areas, were found in children with nonsyndromic $\mathrm{CL} / \mathrm{P}$ using resting-state fMRI. It helps to understand the abnormality of functional architecture of CL/P which implies different structures and cognitive patterns in CL/P compared with normal development children. 
${ }^{1}$ Faculty of Mathematical and Physical Sciences, BUAP, Puebla, Mexico, ${ }^{2}$ Imaging Department, Hospital Infantil de México, Federico Gómez, Mexico City, Mexico, ${ }^{3}$ UAM Iztapalapa, Mexico City, Mexico, ${ }^{4}$ Neurology Department, Hospital Infantil de México, Federico Gómez, Mexico City, Mexico

Impulsivity is a multi-dimensional construct of behaviors. Here we compared two cohorts of impulsive and control children. Both groups underwent a functional magnetic resonance imaging experiment which food related odor cues. Activations were larger for the impulsive group in: temporal lobe, cerebellum, supplementary motor area, frontal cortex, medial cingulate cortex, insula, precuneus, precentral, para-hippocampal \& clacarine. Connectivity results showed that emotional reward based on the smell and processed in temporal lobes was the main cue driving impulsive children. This was followed by a focused attention and sensations of comfort and happiness modulated by precuneus and cingulum.

Investigation of sickle cell related changes in the basal ganglia of pediatric subjects using QSM and R2*

Richard A Jones ${ }^{1}$, Binjian Sun ${ }^{1}$, Deqiang Qiu², Susan Palasis ${ }^{1}$, Thomas G Burns ${ }^{1}$, and Clark Brown ${ }^{3}$

${ }^{1}$ Radiology, CHOA, Atlanta, GA, United States, ${ }^{2}$ Radiology, Emory University, Atlanta, GA, United States, ${ }^{3}$ Pediatrics, Emory University, Atlanta, GA, United States

In previous work on susceptibility differences between controls and subjects with sickle cell disease (SCD) receiving chronic transfusions we found no significant differences in the basal ganglia (BG). In this abstract we added a group of non-transfused SCD subjects and included an analysis of the R2* in order to better understand the nature of any observed changes. Significant differences between the groups were observed in the BG for both susceptibility and R2*, but the pattern of the changes was inconsistent, probably due to the multifactorial nature of $2^{*}$ in tissues where iron is not the dominant contrast mechanism.

Quantitative subcortical morphometry in mTOR/AKT/PI3K pathway disorders: A novel clinical biomarker

Matthew J Barkovich ${ }^{1}$, Ryan M Nillo ${ }^{1}$, Chin Hong Tan ${ }^{1}$, Leo Sugrue ${ }^{1}$, Anthony James Barkovich ${ }^{1}$, and Rahul S Desikan ${ }^{1}$

${ }^{1}$ Radiology and Biomedical Imaging, University of California, San Francisco, San Francisco, CA, United States

Subcortical volumes were quantitatively evaluated on clinical MRI exams of neurofibromatosis type 1 (NF1) and tuberous sclerosis complex (TSC) patients. Robustly larger volumes of several subcortical structures, including the thalamus, hippocampus and ventral diencephalon, were found in NF1; characteristic NF1 imaging abnormalities are found in these areas. In TSC, we found smaller cerebellar volumes; findings that have been associated with autistic phenotypes. Cluster analysis reveals three distinct clustering patterns, each corresponding to a patient class. These results show the feasibility of obtaining automatic quantitative measurements of anatomic structures from clinical MRI exams.

\section{ROTATING FRAME MRI CONTRASTS FOR ASSESSMENT OF WHITE MATTER ALTERATION IN MUCOPOLYSACCHARIDOSIS TYPE I}

Alena Svatkova ${ }^{1}$, Bryon A. Mueller ${ }^{2}$, Petr Bednařik ${ }^{3}$, Carol Nguyen ${ }^{1}$, Lubomír Vojtišek ${ }^{4}$, Silvia Mangia ${ }^{3}$, Mikko Nissi ${ }^{5}$, Shalom Michaeli ${ }^{3}$, and Igor Nestrasil ${ }^{1}$

${ }^{1}$ Department of Pediatrics, University of Minnesota, Minneapolis, MN, United States, ${ }^{2}$ Department of Psychiatry, University of Minnesota, Minneapolis, MN, United States, ${ }^{3}$ Radiology, CMRR, University of Minnesota, Minneapolis, MN, United States, ${ }^{4}$ Central European Institute of Technology, Masaryk University, Brno, Czech Republic, ${ }^{5}$ Department of Applied Physics, University of Eastern Finland, Kuopio, Finland

Mucopolysaccharidosis type I (MPSI) is an inherited metabolic disease with severe and attenuated disease subtypes. While both MPSI subtypes manifest pronounced morphological brain changes, little has been discovered about alterations of white matter (WM) microstructure. Here, we utilized rotating frame MRI contrasts along with DTI to detect WM alterations between in 11 severe and 9 attenuated MPSI patients at 3T. T1 $\rho$ and RAFF4 detected WM differences between MPS subtypes that were not depicted by DTI. Outcomes demonstrate an exceptional sensitivity of rotating frame methods to probe WM microstructure in MPSI.

REDUCED INTRACRANIAL VOLUME IN FABRY DISEASE: A VOLUMETRIC MRI STUDY

Giuseppe Pontillo ${ }^{1}$, Sirio Cocozza ${ }^{1}$, Arturo Brunetti ${ }^{1}$, Vincenzo Brescia Morra ${ }^{2}$, Eleonora Riccio ${ }^{2}$, Camilla Russo ${ }^{1}$, Francesco Saccà2 ${ }^{2}$ Enrico Tedeschi ${ }^{1}$, Antonio Pisani ${ }^{2}$, and Mario Quarantelli ${ }^{3}$

${ }^{1}$ Department of Advanced Medical Sciences, University of Naples Federico II, Naples, Italy, ${ }^{2}$ University of Naples Federico II, Naples, Italy, ${ }^{3}$ nstitute of Biostructure and Bioimaging, National Research Council, Naples, Italy

To investigate the possibility that in Fabry Disease (FD), similarly to other LSD, an abnormal brain development could occur, we performed a volumetric MRI analysis on 42 FD patients and 38 healthy controls (HC). MRI data were processed using SPM12 to obtain ICV values, as well as brain parenchymal (BPF) and gray matter (GMF) fractions. Mean ICV of FD patients was $8.1 \%$ smaller compared to $\mathrm{HC}(\mathrm{p}<5 \cdot 10-5)$, without significant differences in terms of BPF or GMF, thus suggesting a harmonious volumetric reduction of intracranial structures, as a reflection of a possible abnormal brain development in this condition. 
Aashim Bhatia ${ }^{1}$, Bryson Reynolds², Samantha By², Bhavesh Ramkorun², Quinn Weinberg ${ }^{2}$, Mark Adams ${ }^{3}$, John Clifton Wellons III ${ }^{4}$, and Seth Smith²

${ }^{1}$ Radiology, Vanderbilt Childrens's Hospital, Nashville, TN, United States, ${ }^{2}$ Vanderbilt University Institute of Imaging Science, Nashville, TN, United States, ${ }^{3}$ Urology, Vanderbilt Childrens's Hospital, Nashville, TN, United States, ${ }^{4}$ Neurosurgery, Vanderbilt Childrens's Hospital, Nashville, TN, United States

The goal of the study was to apply optimized Diffusion Tensor Imaging (DTI) in the pediatric spinal cord and quantified to determine normative DTI-derived indices based on age. DTI was acquired in 35 patients, 22 being normal and $A D, F A, M D$, and $R D$ were calculated.

DTI of the spinal cord in the pediatric population can be performed in the clinical setting to produce reliable DTI values. AD and MD demonstrated statistically significant changes based on age in both normal patients and the complete patient population.

Tag-Based CSF Imaging Performance in Pediatric Patients and Adult Volunteers

Jieun Kwak ${ }^{1,2}$, Tai-Wei Wu${ }^{1}$, Skorn Ponrartana ${ }^{3,4,5}$, Benita Tamrazi ${ }^{3,5}$, Wende Gibbs ${ }^{5}$, Thomas Chavez ${ }^{1}$, William Bradley ${ }^{6}$, Marvin D Nelson ${ }^{3,5}$, J. Gordon McComb ${ }^{7,8}$, Stefan Blüml ${ }^{3,4}$, and Matthew Borzage $1,3,4$

${ }^{1}$ Center for Fetal and Neonatal Medicine, Division of Neonatology | Children's Hospital Los Angeles, Department of Pediatrics, Keck School of Medicine, University of Southern California, Los Angeles, CA, Los Angeles, CA, United States, ${ }^{2}$ USC/LAC+USC Neonatal-Perinatal Medicine Fellowship Program, Division of Neonatology LAC+USC Medical Center \& Children's Hospital Los Angeles, Keck School of Medicine of USC, Los Angeles, CA, Los Angeles, CA, United States, ${ }^{3}$ Department of Radiology, Children's Hospital Los Angeles, Los Angeles, CA, Los Angeles, CA, United States, ${ }^{4}$ Rudi Schulte Research Institute, Santa Barbara, CA, Santa Barbara, CA, United States, ${ }^{5}$ Department of Radiology, Keck School of Medicine, University of Southern California, Los Angeles, CA, Los Angeles, CA, United States, ${ }^{6}$ Department of Radiology, University of California San Diego, La Jolla, CA, La Jolla, CA, United States, ${ }^{7}$ Division of Neurosurgery, Children's Hospital Los Angeles, Los Angeles, CA, Los Angeles, CA, United States, ${ }^{8}$ Department of Neurological Surgery, Keck School of Medicine, University of Southern California, Los Angeles CA, Los Angeles, CA, United States

We compared tag-based CSF imaging techniques (TimeSLIP and TimeSTAMP) in 10 healthy adults and 19 pediatric patients with cerebrospinal fluid (CSF) abnormalities. In adults, TimeSLIP and TimeSTAMP contrasts were quantitatively compared. TimeSTAMP sequences showed higher contrasts with decreased contrast variability versus TimeSLIP sequences. In pediatric patients, TimeSTAMP sequences were acquired to observe clinical utility and had similar contrast to the healthy adults. TimeSTAMP may be a superior imaging technique with clinical implications in adults and pediatric patients.

Factor analysis to determine white matter injury patterns following pediatric traumatic brain injury.

Brenda Bartnik Olson ${ }^{1}$, Nirmalya Ghosh ${ }^{2}$, Udo Oyoyo ${ }^{1}$, Barbara Holshouser ${ }^{1}$, Joy Nichols ${ }^{2}$, Jamie Pivonka-Jones ${ }^{2}$, Karen Tong ${ }^{1}$, and Stephen Ashwal ${ }^{2}$

Several studies have shown regional disruptions in white matter integrity following TBI although conventional methods don't account for the relationship between regions. In this study we used factor analysis, a data reduction technique, to identify patterns of WM injury that are associated with neurocognitive outcome in pediatric TBI patients. Our findings identified 3 dominant patterns of WM injury in pediatric TBI patients, describing regional changes in: 1) subcortical + cortical diffusivity, 2) subcortical diffusivity, and 3) subcortical + cortical anisotropy. Factor analysis provides a unique statistical approach to analyze DTI data and potentially could be used to combine different data streams (DTI, MR spectroscopy, SWI) representing different elements of injury.

Structural MRI derived connectivity in Paediatric Mild Traumatic Brain Injury: Acute Neuroimaging and its relationship with executive function outcomes

Daniel J King ${ }^{1}$, Stefano Seri ${ }^{1}$, Vicki Anderson ${ }^{2}$, Cathy Catroppa ${ }^{2}$, Miriam H Beauchamp ${ }^{3}$, and Amanda G Wood ${ }^{1,4}$

${ }^{1}$ Aston Brain Center \& School of Life and Health Sciences, Aston University, Birmingham, United Kingdom, ${ }^{2}$ Clinical Sciences, Murdoch Childrens Research Institute, Melbourne,

The aim of the current study was to identify acute differences in the topology of the structural covariance network of children after a mild traumatic brain injury (TBI). This was to assess the potential utility of this connectivity analysis applied to T1-weighted MR images, novel in the TBI literature. The main findings of this study were i) both patients and controls exhibited typical frequency distribution of few, highly connected nodes, ii) at a group level, patients exhibited connections between nodes a greater distance apart, iii) these differences were not associated with differences in executive function outcome. Future work will have to move to individual-level SCNS to allow for more complex analyses and to enable investigation of more subtle individual differences in structural covariance.

Traditional Poster

\section{Psychoradiology}


Morphological interrelationships in mid-line white-matter structures are altered in individuals carrying rare neuropsychiatric copy number variants.

Mark Drakesmith ${ }^{1,2}$, Greg D Parker ${ }^{1}$, Jacqueline Smith ${ }^{2}$, Elliot Rees ${ }^{2}$, Michael Owen $^{2}$, Derek K Jones ${ }^{1,2}$, and David E Linden ${ }^{2}$

${ }^{1}$ CUBRIC, Cardiff University, Cardiff, United Kingdom, ${ }^{2}$ Neuroscience and Mental Health Research Institute, Cardiff University, Cardiff, United Kingdom

Neuropsychiatric copy number variants (CNVs) provide unique insights into the genetic basis of neuropsychiatric disorders. This study utilised a novel approach for characterising morphology of white-matter fibres and combines them with more traditional volumetric and microstructural indices of white-matter to study their relation to penetrance for psychopathology in a CNV cohort. Results show cingulum morphology is significantly affected by the presence of CNVs with high-penetrance for schizophrenia and developmental disorders. Additionally, volumetric interrelationships across several white-matter structures are also altered. In particular, the ratios of tract volumes across segments of the corpus callosum are altered. It is likely that both these effects stem from a single neurodevelopmental trajectory characteristic of neuropsychiatric CNVs.

Quantitative magnetization transfer imaging in schizophrenia: a closer look at myelin dysfunction

Yu Sui ${ }^{1}$, Pippa Storey ${ }^{1}$, Hilary Bertisch ${ }^{2}$, Matthew Lustberg ${ }^{1}$, Taylor Coats ${ }^{1}$, Donald Goff ${ }^{3}$, Alexey Samsonov ${ }^{4}$, and Mariana Lazar ${ }^{1}$

${ }^{1}$ Bernard and Irene Schwartz Center for Biomedical Imaging, Department of Radiology, New York University School of Medicine, New York, NY, United States, ${ }^{2}$ Department of Rehabilitation Medicine, New York University School of Medicine, New York, NY, United States, ${ }^{3}$ Department of Psychiatry, New York University School of Medicine, New York, NY, United States, ${ }^{4}$ Department of Radiology, University of Wisconsin at Madison, Madison, WI, United States

Myelin dysfunction has frequently been identified as one of the neural abnormalities in schizophrenia, yet systematic in vivo examination of myelin content in patients is lacking. The current study compared the degree of myelination in schizophrenia patients and comparison healthy controls. Myelin content was estimated by constructing quantitative whole-brain maps of macromolecular proton fraction, which is believed to be one of the biomarkers for myelination in neural tissues. Statistical analysis revealed that SZ patients were associated with a significant reduction in myelin content throughout white matter, as well as in several grey matter regions including cingulate cortex and hippocampus.

Acutely treated antipsychotics haloperidol enhances BOLD responses to the somatosensory stimulation in anesthetized rats.

Yunbok Kim ${ }^{1}$, Jeong Pyo Son ${ }^{1}$, SoHyun Han ${ }^{1}$, and Seong-Gi Kim ${ }^{1,2}$

${ }^{1}$ Center for Neuroscience Imaging Research (CNIR), Institute for Basic Science (IBS), Suwon, Republic of Korea, ${ }^{2}$ Department of Biomedical Engineering, Sungkyunkwan University, Suwon, Republic of Korea

The use of BOLD fMRI is rapidly increasing for probing the effects of antipsychotics in schizophrenia. Since fMRI BOLD is an indirect measurement of neural activities, it is critical to examine the effect of antipsychotics on neurovascular coupling to prevent misinterpretation of MR data. Acutely treated haloperidol (0.2mg/kg, i.v.) increased BOLD fMRI to the somatosensory stimulation in the $1.5 \%$ isoflurane-anesthetized rats $(n=5)$. In parallel with the BOLD results, evoked CBF and LFP by somatosensory stimuli were increased after haloperidol administration $(n=8)$. Our results indicate that acutely treated haloperidol could influence somatosensory responses and the increased BOLD signal is coupled with enhanced neural activities.

Convolutional Neural Networks on Functional Connectivity Derived From r-fMRI: Explore the Effects of Thresholds

Xingjuan $\mathrm{Li}^{1}, \mathrm{Yu} \mathrm{Li}^{1}$, and Xue $\mathrm{Li}^{1}$

In this study, we propose a novel CNN to predict autism from functional brain networks. Experimental results demonstrate that the predictive ability of CNN outperforms a logistic regression method by $8 \%$ and a five-layer fully-connected network (FCN) by approximately $7 \%$. Network thresholding is often used to control false connections arising in the process of constructing functional brain networks. We also compare the influence of different thresholds on the performance of proposed CNN. Experimental results show that CNN is robust to false connections. Our study will contribute to predict reliable clinical outcomes in autism using deep learning on brain networks.

Hippocampus and parietal lobe glutamate changes as a function of age in schizophrenia

Frank E. Gaston ${ }^{1}$, S. Andrea Wijtenburg ${ }^{1}$, Stephanie A. Korenic ${ }^{1}$, Hongji Chen ${ }^{1}$, and Laura M. Rowland ${ }^{1}$

${ }^{1}$ Psychiatry, University of Maryland School of Medicine, Baltimore, MD, United States

MRS was used to examine the aging effects of glutamate in participants with schizophrenia versus healthy controls. The parietal lobe and hippocampus, regions associated with general aging and the pathophysiology of schizophrenia, were assessed. Results revealed that hippocampal glutamate was lower in older adults with schizophrenia versus older controls. In contrast, parietal glutamate was lower in schizophrenia versus controls, irrespective of age group. These results suggest that the hippocampus may be particularly vulnerable to aging in schizophrenia. Interventions that halt hippocampal glutamate decline may be beneficial for patients with schizophrenia. 
Amygdala dysfunction during negative emotional situation in Obsessive-Compulsive Disorder

Hyunsil Cha ${ }^{1}$, Sang Won Lee ${ }^{2}$, Kyung Eun Jang ${ }^{1}$, Hyejeong Choi ${ }^{1}$, Eunji Kim ${ }^{1}$, Moojin Yang ${ }^{1}$, Jiung Yang ${ }^{1}$, Moon Jung Hwang ${ }^{3}$, Huijin Song ${ }^{4}$, Seung Jae Lee ${ }^{2}$, and Yongmin Chang ${ }^{1,5}$

${ }^{1}$ Department of Medical \& Biological Engineering, Kyungpook national university, Daegu, Republic of Korea, ${ }^{2}$ Department of Psychiatry, Kyungpook national university hospital, Daegu, Republic of Korea, ${ }^{3}$ GE Healthcare, Seoul, Republic of Korea, ${ }^{4}$ Biomedical Engineering Research, Kyungpook national university, Daegu, Republic of Korea, ${ }^{5}$ Department of Radiology and Molecular Medicine, Kyungpook national university, Daegu, Republic of Korea

We investigated brain activation in obsessive-compulsive disorder (OCD) patient using thought-action fusion (TAF) task to assess the influence of OCD symptom on amygdala response to the task. Within and between group analysis of close and neutral condition showed decreased amygdala activation in patients with OCD compared to healthy control.

Assessment of brain volume and shape abnormalities in the major depressive disorders with and without suicidal ideation

Hui-Ming Tseng ${ }^{1}$, Vincent Chin-Hung Chen ${ }^{2,3}$, Yuan-Hsiung Tsai ${ }^{4}$, and Jun-Cheng Weng ${ }^{3,5}$

${ }^{1}$ Department of Medical Imaging and Radiological Sciences, Chung Shan Medical University, Taichung, Taiwan, ${ }^{2}$ School of Medicine, Chang Gung University, Taoyuan, Taiwan, ${ }^{3}$ Department of Psychiatry/ Health Information and Epidemiology Laboratory, Chang Gung Memorial Hospital, Chiayi, Taiwan, ${ }^{4}$ Department of Diagnostic Radiology, Chang Gung Memorial Hospital, Chiayi, Taiwan, ${ }^{5}$ Department of Medical Imaging and Radiological Sciences, Chang Gung University, Taoyuan, Taiwan

There is very strong connection between patients with major depressive disorders (MDD) and suicide. We used voxel-based morphometry (VBM) and vertex-wise shape analyses to observe the difference between the MDD patients with and without suicidal ideation in their brain volume of gray and white matter as well as shape. We found the negative correlation between the brain volume of limbic system in MDD patients. We also found the significant difference in brain volume and shape of limbic system between suicidal ideation and nonsuicidal ideation.

Atypical associations between language comprehension network and attention pathways in autism spectrum disorders

Yu-Chun Lo ${ }^{1,2}$, Susan Shur-Fen Gau ${ }^{3}$, Yu-Jen Chen ${ }^{1}$, Yung-Chin Hsu ${ }^{1}$, and Wen-Yih Isaac Tseng ${ }^{1}$

${ }^{1}$ Institute of Medical Device and Imaging, National Taiwan University College of Medicine, Taipei, Taiwan, ${ }^{2}$ The Ph.D. Program for Neural Regenerative Medicine, Taipei Medical University, Taipei, Taiwan, ${ }^{3}$ Department of Psychiatry, National Taiwan University Hospital and College of Medicine, Taipei, Taiwan

Impaired language comprehension has been consistently found in autism spectrum disorder (ASD). Development of language comprehension highly corresponds to joint attention and impulsivity. We used diffusion spectrum imaging to measure white matter integrity of the language comprehension network and the attention pathways in 60 ASD and 55 typically developing (TD) boys. ASD showed partially reduced white matter integrity in the targeted tracts as compared to TD. The tract covariance between the language comprehension network and the attention pathways showed different patterns in both groups which may shed light in the relationships of language and attention in ASD.

Connectome analysis of brain functional network alterations in depressed patients with and without self-harm

Yu-Syuan Chou ${ }^{1}$, Vincent Chin-Hung Chen ${ }^{2,3}$, Yuan-Hsiung Tsai ${ }^{4}$, Shan-Chih Lee ${ }^{1}$, and Jun-Cheng Weng ${ }^{3,5}$

${ }^{1}$ Department of Medical Imaging and Radiological Sciences, Chung Shan Medical University, Taichung, Taiwan, ${ }^{2}$ School of Medicine, Chang Gung University, Taoyuan, Taiwan, ${ }^{3}$ Department of Psychiatry/ Health Information and Epidemiology Laboratory, Chang Gung Memorial Hospital, Chiayi, Taiwan, ${ }^{4}$ Department of Diagnostic Radiology, Chang Gung Memorial Hospital, Chiayi, Taiwan, ${ }^{5}$ Department of Medical Imaging and Radiological Sciences, Chang Gung University, Taoyuan, Taiwan

We aimed to use resting-state fMRI (rs-fMRI) to investigate the functional connectivity difference between depressed patients with and without self-harm history as well as healthy participants. The graph theoretical analysis (GTA) and network-based statistic (NBS) analysis were also used to find the network difference between each group. In GTA and NBS analyses revealed different topological organization and poor global integration of the brain network in depressed participants compared with healthy participants. We suggested that depressed patients with or without self-harm history may affect their brain functional connectivity

Measurements of rat hippocampus Glu, GIn and GABA using NMR, MRS and HPLC in animal models of autism

Pawel Senator ${ }^{1}$, Elzbieta Zieminska² ${ }^{2}$ Wojciech Hilgier $^{2}$, Jaroslaw Orzel ${ }^{2}$, and Beata Toczylowska ${ }^{1,3}$

${ }^{1}$ Nalecz Institute of Biocybernetics and Biomedical Engineering Polish Academy of Sciences, Warsaw, Poland, ${ }^{2}$ Mossakowski Medical Research Center Polish Academy of Sciences, Warsaw, Poland, ${ }^{3}$ Institute of Biochemistry and Biophysics Polish Academy of Sciences, Warsaw, Poland

The goal of our studies was to compare different measuring methods of glutamine, glutamate and GABA of rat hippocampus used for study of pathogenesis of autism. The methods under consideration were: in vivo MRS and two in vitro ones, NMR and HPLC. Univariate statistical analysis of ratios of tested amino acids with respect to glutamate concentration was performed using General Linear Model. This demonstrated statistically significant differences between the results from three methods for both, glutamine and GABA ratios. OPLS-DA analysis allowed build models for differentiation of two animal models of disease and control group in NMR and HPLC. 
Resting-state brain functional alteration in dorsal attention network associated with post-chemotherapy breast cancer

Chao-Yu Shen ${ }^{1,2,3}$, Vincent Chin-Hung Chen ${ }^{4,5}$, Xuan-Ru Zhang ${ }^{2}$, Meng-Syuan Lin ${ }^{2}$, Dah-Cherng Yeh ${ }^{6}$, Yeu-Sheng Tyan ${ }^{2,3}$, Ming-Chih Chou ${ }^{1,7}$, and Jun-Cheng Weng ${ }^{5,8}$

${ }^{1}$ Institute of Medicine, Chung Shan Medical University, Taichung, Taiwan, ${ }^{2}$ Department of Medical Imaging and Radiological Sciences, Chung Shan Medical University, Taichung, Taiwan, ${ }^{3}$ Department of Medical Imaging, Chung Shan Medical University Hospital, Taichung, Taiwan, ${ }^{4}$ School of Medicine, Chang Gung University, Taoyuan, Taiwan, ${ }^{5}$ Department of Psychiatry/ Health Information and Epidemiology Laboratory, Chang Gung Memorial Hospital, Chiayi, Taiwan, ${ }^{6}$ Breast Center, Taichung Tzu Chi Hospital, Taichung, Taiwan, ${ }^{7}$ Division of Thoracic Surgery, Department of Surgery, Chung Shan Medical University Hospital, Taichung, Taiwan, ${ }^{8}$ Department of Medical Imaging and Radiological Sciences, Chang Gung University, Taoyuan, Taiwan

The current study was to investigate post-chemotherapy breast cancer with rs-fMRI using mfALFF analysis and correlated with clinical cognitive testing. The results showed altered brain activity in the dorsal attention network in breast cancer patients compared to healthy controls and the affected areas were associated with MMSE, CAMS-R and IES-R scores.

Principal Component Analysis of Schizophrenia Reveals Link Between Auditory Hallucination Severity and Fractional Anisotropy in the Corpus Callosum

Meighen M Roes ${ }^{1}$, Alexander Mark Weber ${ }^{2}$, and Todd S Woodward ${ }^{1}$

${ }^{1}$ Psychiatry, University of British Columbia, Vancouver, BC, Canada, ${ }^{2}$ Pediatrics, University of British Columbia, Vancouver, BC, Canada

A PCA analysis of fractional anistropy (FA) was conducted from a sample of schizophrenia patients $(n=42)$ and healthy controls ( $n=40)$ resulted in three major components: "corpus callosum", "internal capsule/temporal/brainstem", and "corona radiata". Average component scores did not differ as a function of group, but a correlation of PSYRATS scores and principal components revealed the frequency, amount of distress associated with voices, and disruption associated with voices correlated significantly with the corpus callosum component. Our findings suggest that reduced interhemispheric connectivity of the prefrontal cortex is related to hallucination severity in schizophrenia, perhaps mediated through topdown processes such as source monitoring

Diffusion kurtosis imaging and white matter model analysis of the brains of patients with major depressive disorder

Kouhei Kamiya ${ }^{1,2}$, Naohiro Okada ${ }^{3}$, Kingo Sawada ${ }^{3}$, Yusuke Watanabe ${ }^{1}$, Ryusuke Irie ${ }^{1,2}$, Yuichi Suzuki ${ }^{4}$, Shohei Hanaoka ${ }^{1}$, Takeyuki Watadani ${ }^{1}$, Shinsuke Koike ${ }^{3}$, Harushi Mori ${ }^{1}$, Akira Kunimatsu ${ }^{1,5}$, Masaaki Hori ${ }^{2}$, Shigeki Aoki ${ }^{2}$, Kiyoto Kasai $^{3}$, and Osamu Abe ${ }^{1}$

${ }^{1}$ Department of Radiology, the University of Tokyo, Tokyo, Japan, ${ }^{2}$ Department of Radiology, Juntendo University, Tokyo, Japan, ${ }^{3}$ Department of Neuropsychiatry, the University of Tokyo, Tokyo, Japan, ${ }^{4}$ Department of Radiology, the University of Tokyo Hospital, Tokyo, Japan, ${ }^{5}$ Department of Radiology, the Institute of Medical Science, the University of Tokyo, Tokyo, Japan

We investigated the brain microstructural changes in major depressive disorder (MDD) using DKI and biophysical modelling. Twenty-six patients with MDD and 42 healthy control subjects were enrolled. TBSS whole brain analyses showed decrease of MK and RK in the patients as compared to the controls, predominantly in the frontal lobe, but widely distributed in the cerebral white matter. Model analysis revealed smaller intra-axonal volume fraction in the corpus callosum. The present results indicate the ability of DKI to demonstrate MDD pathology that are not fully depicted by DTI, and possibly to provide a new insights into the pathophysiology of MDD.

Upregulation of hippocampal glutamatergic neurotransmission during acute episodes of major depression: Excitotoxic effects might be related to reduced hippocampal volumes

Jochen Bauer ${ }^{1}$, Patricia Ohrmann ${ }^{2}$, Bendix Labeit ${ }^{2}$, Elke Scherbiski ${ }^{2}$, and Harald Kugel ${ }^{1}$

${ }^{1}$ Department of Clinical Radiology, University of Muenster, Muenster, Germany, ${ }^{2}$ Department of Psychiatry, University of Muenster, Muenster, Germany

Investigation of the glutamatergic metabolism with ${ }^{1} \mathrm{H}$-spectroscopy revealed a significant higher glutamate level in the hippocampus in patients with major depression. The excitotoxicity of increased glutamate levels on neural brain structures might be causally related to reduced volumes of hippocampi as found in patients with recurrend episodes. $\mathrm{Li}^{2,4,5}$, and Martin Walter ${ }^{1,2,4,5,7}$

${ }^{1}$ Department of Psychiatry and Psychotherapy, University of Tübingen, Tübingen, Germany, ${ }^{2}$ High-Field Magnetic Resonance, Max Planck Institute for Biological Cybernetics, Tübingen, Germany, ${ }^{3}$ Otto von Guericke University, Magdeburg, Germany, ${ }^{4}$ Clinical Affective Neuroimaging Laboratory (CANLAB), Magdeburg, Germany, ${ }^{5}$ Leibniz Institute for Neurobiology, Magdeburg, Germany, ${ }^{6}$ School of Psychology and Cognitive Science, East China Normal University, Shanghai, China, ${ }^{7}$ Department of Psychiatry and Psychotherapy, Otto von Guericke University, Magdeburg, Germany, ${ }^{8}$ Biomagnetical Resonance, Otto von Guericke University, Magdeburg, Germany 
Increasing evidence suggests a hypoglutamatergic state in major depressive disorder (MDD), however spatial- and metabolite specific abnormalities have not been fully characterized. Using short TE/TM STEAM MRS, we evaluated Glu, Gln, GIn/Glu and GABA metabolism in two histoarchitectonically distinct subdivisions of the anterior cingulate cortex (ACC). The pregenual ACC, involved in emotion processing, showed altered glutamine-glutamine cycling but not altered GABAergic metabolism in MDD, whereas no differences between patients and controls were found in the anteromedial ACC. Increased GIn/Glu in MDD in pgACC but not aMCC confirms a regionally specific role of altered glutamatergic metabolism and neuronal-glial interaction.

MR Spectroscopic evaluation of brain white matter metabolite abnormalities in Psychotic Spectrum Disorders

Ines Blockx ${ }^{1}$, Matthew Lustberg ${ }^{1}$, Taylor C Coats ${ }^{1}$, Hillary C Bertisch ${ }^{2}$, Oded Gonen ${ }^{1,3}$, Donald C Goff ${ }^{4}$, and Mariana Lazar ${ }^{1}$

${ }^{1}$ Department of Radiology, Bernard and Irene Schwartz Center for Biomedical Imaging, New York University School of Medicine, New York, NY, United States, ${ }^{2}$ Department of Rehabilitation Medicine, New York University School of Medicine, New York, NY, United States, ${ }^{3}$ Department of Radiology, Center for Advanced Imaging Innovation and Research (CAI2R), New York University School of Medicine, New York, NY, United States, ${ }^{4}$ Department of Psychiatry, New York University School of Medicine, New York, NY, United States

${ }^{1} \mathrm{H}-\mathrm{MRS}$ has been widely applied in studies with Psychotic Spectrum Disorders, however, findings are mixed and the exact cause of these disorders remains to be elucidated.The preliminary results of the present study show increased $\mathrm{Gln} / \mathrm{Cr}$ levels in schizophrenia and schizoaffective patients in central WM reaching statistical significance in the bipolar group. The increase in $\mathrm{Gln} / \mathrm{Cr}$ levels has been proposed to occur in the early stages of the disorder which is consistent with the population included here. The current study brings WM as a relevant area susceptible to damage into focus, which is likely to be involved in the early stages of PSD.

Auditory system altered in auditory verbal hallucination studied using diffusion spectrum imaging, T1-weighted image and fMRI

Kayako Matsuo ${ }^{1}$

${ }^{1}$ Department of Biological Psychiatry and Neuroscience, Dokkyo Medical University, Tochigi, Japan

To understand the pathology of auditory verbal hallucination (AVH), we investigated $3 \mathrm{MRI}$ indices: generalized fractional anisotropy (GFA) using diffusion spectrum imaging in the auditory radiation, gray matter volume (GMV) using T1-weighted images in Heschl's gyrus (i.e., auditory cortex) and BOLD contrast estimates using task-fMRI in the auditory cortex. The BOLD relative to the GFA was significantly greater in controls than in patients with schizophrenia who had AVH. The GMV relative to the GFA also tended to show greater values in controls than in patients. An unregulated auditory sensation attributed to a dysfunction in the cortex might eventually encompass AVH.

Grey abnormalities associate with suicide related behaviour in first episode non-affective psychosis patients

Manuel Canal-Rivero ${ }^{1,2}$, Rosa Ayesa-Arriola ${ }^{2,3}$, Esther Setien-Suero ${ }^{2,3}$, Manuel Delgado-Alvarado ${ }^{1}$, Benedicto Crespo-Facorro ${ }^{2,3}$, and Diana Tordesillas-Gutierrez ${ }^{1,2}$

${ }^{1}$ Neuroimaging Unit, Technological Facilities, IDIVAL, Santander, Spain, ${ }^{2}$ CIBERSAM, Santander, Spain, ${ }^{3}$ University Hospital Marqués de Valdecilla, School of Medicine, University of Cantabria, IDIVAL, Santander, Spain

Little is known about brain abnormalities associated with suicide-related behaviours in first episode psychosis patients and controversial results have been reported. The main aim of the present study was to examine brain abnormalities related with suicidal behaviours in a large sample of first episode psychosis (FEP) patients. In particular, we found reduction grey matter volume in frontal area, middle temporal gyrus as well as posterior cingulate gyrus and precuneus. These areas appear to be associated with some of the greatest features related to suicidal behaviour such as impulsivity, emotional processing information, responses to pain and aggressiveness.

The Differences of Amplitude of Low Frequency Fluctuation between Methamphetamine and Heroin use disorder: a resting-state functional magnetic resonance imaging study

Yan Liu' ${ }^{1,2}$, Wei Wang ${ }^{1}$, Wei Li ${ }^{1}$, Qiang Li ${ }^{1}$, Yongbin Li ${ }^{1}$, Jiajie Chen ${ }^{1}$, Jing Chen ${ }^{1}$, and Shan Dang ${ }^{1}$

1821

${ }^{1}$ Department of Radiology, Tangdu Hospital, the Air Force Medical University, XI AN, China, ${ }^{2}$ Department of Radiology, Changqing Xinglongyuan Hospital, Affiliated Hospital of Changqing Oilfield, XI AN, China

These findings indicated different brain regions between MA users and heroin users in resting-state, as well as it's function correlation with emotion.

Myelin content and axonal size/density is reduced in early-course schizophrenia: Evidence from multi-echo T2 imaging study

Shivali R. Patel ${ }^{1}$, Jennifer Losiowski ${ }^{2}$, Muzamil Arshad ${ }^{3}$, Naftali Raz $^{4,5}$, Vaibhav A. Diwadkar ${ }^{2}$, and Jeffrey A. Stanley ${ }^{2}$

${ }^{1}$ MD Program, Wayne State University School of Medicine, Detroit, MI, United States, ${ }^{2}$ Psychiatry and Behavioral Neurosciences, Wayne State University School of Medicine, Detroit, MI, United States, ${ }^{3}$ MD/PhD Program, Wayne State University School of Medicine, Detroit, MI, United States, ${ }^{4}$ Psychology, Wayne State University, Detroit, MI, United States, ${ }^{5}$ Institute of Gerontology, Wayne State University, Detroit, MI, United States 
White matter aberrations have been well documented in schizophrenia using diffusion tensor or weighted imaging, but the differences in myelin macrostructure morphology have not been extensively explored. Here we used multi-echo $\mathrm{T}_{2}\left(\mathrm{ME}-\mathrm{T}_{2}\right)$ imaging to examine myelin content and axonal size and packing density in schizophrenia in white matter regions, specifically association, commissural, and projection fiber tracts. We demonstrate reduced myelin content as well as increased axonal packing density in association and projection tracts, which may contribute to neural dysconnectivity mechanisms underlying the neuropathology of schizophrenia.

Resting-state Network Evaluation of First-episode Schizophrenia Patients by fMRI

Kangkang Xue ${ }^{1}$, Dandan Zheng ${ }^{2}$, and Jingliang Cheng ${ }^{1}$

${ }^{1}$ Medical Imaging and Nuclear Medicine, The First Affiliated Hospital of Zhengzhou University, Zhengzhou, China, ${ }^{2}$ GE Healthcare, China, Beijing, China

Schizophrenia is a chronic mental illness whose symptoms are thought to have a strong neurobiological basis. This work is to study the resting state networks changes in first-episode schizophrenia patients by resting-state functional magnetic resonance imaging. The current study explored that there were RSNs damages or multiple brain regions functional connectivity abnormalities in first-episode schizophrenia patients compared with healthy controls, which behave functional connectivity increase and decrease.

A voxel-based diffusion kurtosis imaging study of whole-brain in chronic alcohol dependent patients

Hong-yan $\mathrm{Nie}^{1}$, Jun Chen ${ }^{1}$, Ya-qi Wang ${ }^{1}$, and Yang Fan ${ }^{2}$

${ }^{1}$ Department of Radiology, Renmin Hospital of Wuhan University, Wuhan, China, ${ }^{2} \mathrm{GE}$ Healthcare China, Beijing, China

In the present study, diffusion kurtosis imaging (DKI), which is based on the method of voxel-based analysis(VBA), was used to investigate the alterations of microstructure of white matter and gray matter in chronic alcohol dependent patients. Thirty patients with chronic alcohol dependence and twenty healthy volunteers were scanned with DKI. Compared with the healthy control group, the brain regions associated with visual information processing, memory, movement coordination and emotional control capacity have been found to be abnormal in different degrees.

Structural correlates of trait anxiety: Volume reduction in hypothalamus

SHILPI MODI ${ }^{1}$, DIVESH THAPLOO ${ }^{1}$, PAWAN KUMAR $^{1}$, and SUBASH KHUSHU ${ }^{1}$

$825 \quad{ }^{1}$ NMR Research Centre, Institute of Nuclear Medicine and Allied Sciences (INMAS), Delhi, India

Trait anxiety affects brain functioning and cognition as suggested by various neuroimaging and behavioural studies. It is also a a prone phenotype for the development of psychiatric disorders. Therefore, in order to identify individuals that are at risk for the development of clinical anxiety disorders and depression, identifying hallmarks of trait anxiety becomes important, to fascilitate timely preventive interventions. We investigated the structural correlates of trait anxiety in healthy participants using high resolution structural MRI. Results suggest that a reduction in the gray matter volumes of the hypothalamus may be putative imaging marker for trait anxiety.

Increased functional connectivity between medial prefrontal cortex and nucleus accumbens in morphine craving rats

Hannes Michel Wiesner ${ }^{1}$, Shinho Cho ${ }^{1}$, Yi Zhang ${ }^{1}$, Erin Larson ${ }^{2}$, Mark J. Thomas ${ }^{3}$, Xiao-Hong Zhu ${ }^{1}$, and Wei Chen ${ }^{1}$

${ }^{1}$ CMRR, Department of Radiology, University of Minnesota Medical School, Minneapolis, MN, United States, ${ }^{2}$ MnDRIVE Optogenetics and Neuromodulation Core, Neuroscience Department, University of Minnesota Medical School, Minneapolis, MN, United States, ${ }^{3}$ Departments of Neuroscience and Psychology, University of Minnesota Medical School, Minneapolis, MN, United States

Morphine is a potent analgesic with a high addictive potential. In this study we have shown a difference in brain connectivity related to drug-seeking behavior involving key neural decision and reward systems using rs-fMRI. The finding contributes to a better understanding of the neural underpinnings of opioid addiction and could help in a better assessment of relapse risk in individuals.

Alterations in amplitude of low frequency fluctuation in drug-free major depressive disorder

Hu Xiaoxiao ${ }^{1}$, Hu Xinyu ${ }^{1}$, Li Hailong ${ }^{1}$, Zhang Lianqing ${ }^{1}$, Lu Lu ${ }^{1}$, Bu Xuan ${ }^{1}$, Tang Shi ${ }^{1}$, Gong Qiyong ${ }^{1}$, and Huang Xiaoqi ${ }^{1}$ 
The objective of this study was 1) to confirm whether the intrinsic brain activities (as evaluated by ALFF) in the anterior cingulate cortex (ACC) is associated with antidepressant treatment in a relative large sample of drug-free major depressive disorder (MDD) patients and 2) to determine whether the pretreatment ALFF activities predict the effect of the follow-up antidepressant treatment in MDD. Our findings demonstrate that intrinsic brain activities in the ACC was influenced by disease itself rather than antidepressant treatment and threw light on predictive value of the right thalamus as a marker of short term antidepressant treatment outcome in MDD.

A pilot study of cerebral blood flow changes in patients undergoing electroconvulsive therapy

Karl D Spuhler ${ }^{1}$, Laura Kunkel ${ }^{2}$, Adeeb Yacoub ${ }^{2}$, Kenneth Wengler ${ }^{1}$, Xiang $\mathrm{He}^{3}$, and Chuan Huang ${ }^{1,2,3}$

${ }^{1}$ Biomedical Engineering, Stony Brook University, Stony Brook, NY, United States, ${ }^{2}$ Psychiatry, Stony Brook Medicine, Stony Brook, NY, United States, ${ }^{3}$ Radiology, Stony Brook Medicine, Stony Brook, NY, United States

Electroconvulsive therapy (ECT) is an effective choice for patients with untreatable depression. Although it is very effective, the mechanisms through which ECT works are poorly understood. We have previously collected PET/MRI data in patients receiving ECT which suggest that this treatment strongly affects the hippocampus. Herein, we supplement these preexisting data with arterial spin labeling data showing significantly reduced blood flow to the hippocampus following ECT in three responders.

In search for a neuroimaging marker for neuroinflammation in neuropsychiatric systemic lupus erythematosus

Marjolein Bulk ${ }^{1}$, Ece Ercan² ${ }^{2}$ Cesar Magro-Checa ${ }^{3}$, Louise van der Weerd ${ }^{1}$, and Itamar Ronen ${ }^{1}$

${ }^{1}$ Radiology, LUMC, Leiden, Netherlands, ${ }^{2}$ Radiology, UT Southwestern Medical Center, Dallas, TX, United States, ${ }^{3}$ Rheumatology, LUMC, Leiden, Netherlands

We explored the link between neuroinflammation and related changes in tissue susceptibility by using quantitative susceptibility mapping (QSM) in a clinically well characterized cohort including inflammatory NP-SLE, ischemic NP-SLE and SLE patients. No significant differences were found after stratifying all patients for antibodies, SLE activity, cumulative SLE damage or complement components in subcortical structures. Subanalysis of inflammatory NP-SLE patients showed a residual correlation between QSM values in the globus palidus and low C1q levels, which need further investigation. Current work is underway to analyse QSM in a bigger sample size to further investigate its potential in identifying NP-SLE patients.

Trait anxiety associated metabolic alterations in thalamus: An MRS study

SHILPI MODI ${ }^{1}$, DIVESH THAPLOO ${ }^{1}$, PRABHJOT KAUR ${ }^{1}$, and SUBASH KHUSHU ${ }^{1}$

${ }^{1}$ NMR Research Centre, Institute of Nuclear Medicine and Allied Sciences (INMAS), Delhi, India

Trait anxiety is a prone phenotype for the development of anxiety disorders and depression. Therefore, in order to identify the individuals 'at risk', identifying the hallmarks of trait anxiety becomes important. Ones identified, timely preventive interventions may be given to such individuals. This study is an attempt to study the trait anxiety associated metabolic/ neurochemical alterations in the brain using proton magnetic resonance spectroscopy. We obtained an increase in the concentrations of Choline compounds in the thalamus as a function of trait anxiety of the subjects suggesting an altered cell membrane metabolism.

Hippocampus Glutamate Concentrations in Schizophrenia and Bipolar Disorder

Nicolas R. Bolo ${ }^{1,2}$, Olivia J. Lutz ${ }^{1}$, Gautami Shashidhar ${ }^{1}$, Li Yao ${ }^{1}$, Yungxiang Tang ${ }^{1}$, Brett A. Clementz ${ }^{3}$, Godfrey Pearlson ${ }^{4}$, Elliot Gershon ${ }^{5}$, John A. Sweeney ${ }^{6}$, Carol A. Tamminga ${ }^{6}$, and Matcheri S. Keshavan ${ }^{1,2}$

${ }^{1}$ Psychiatry, Beth Israel Deaconess Medical Center, Boston, MA, United States, ${ }^{2}$ Psychiatry, Harvard Medical School, Boston, MA, United States, ${ }^{3}$ Psychology, University of Georgia, Athens, GA, United States, ${ }^{4}$ Psychiatry, Yale University, Hartford, CT, United States, ${ }^{5}$ Psychiatry, University of Chicago, Chicago, IL, United States, ${ }^{6}$ Psychiatry, UT Southwestern Medical Center, Dallas, TX, United States

Deficient hippocampus glutamatergic function could underlie cognitive deficits and positive-negative symptoms in schizophrenia (SZ) and bipolar disorder (BP). Using ${ }^{1} \mathrm{H}$ MRS, we found that the glutamate concentration of left anterior hippocampus was significantly lower in $\mathrm{SZ}(6.3 \pm 1.8 \mathrm{mM})$ vs. healthy controls $(\mathrm{HC}, 7.8 \pm 1.2 \mathrm{mM}, \mathrm{p}=0.021)$ and $\mathrm{BP}(8.5 \pm 1.3 \mathrm{mM}$, $p=0.001)$ and trended higher in BP vs. $\mathrm{HC}(\mathrm{p}=0.179)$. Decreased glutamate is consistent with deficient excitatory neurotransmission in the hippocampus of patients with $\mathrm{SZ}$, which could alter synaptic plasticity underlying memory and cognition. Our findings are consistent with the glutamate hypothesis of SZ.

Change of cortical thickness and hippocampal volume in adolescents with autism spectrum disorder

I-Ting Su' ${ }^{1}$ Tzu-chao Chuang ${ }^{1}$, Ming-Ting Wu ${ }^{2,3}$, and Pinchen Yang ${ }^{4}$

${ }^{1}$ Electrical Engineering, National Sun Yat-Sen University, Kaohsiung, Taiwan, Taiwan, ${ }^{2}$ School of Medicine, National Yang-Ming University, Taipei, Taiwan, Taiwan, ${ }^{3}$ Radiology, Kaohsiung Veterans General Hospital, Kaohsiung, Taiwan, Taiwan, ${ }^{4}$ Psychiatry, Kaohsiung Medical University and Kaohsiung Medical University Hospital, Kaohsiung, Taiwan, Taiwan 
By using a surface-based method (Freesurfer), the cortical thickness, hippocampal volume, and amygdala volume measurement were performed on adolescents with autism spectrum disorder $(n=17)$ and age-matched typically developing controls $(n=10)$. ASD patients showed a thicker cortex in temporal and occipital regions, a thinner cortex in frontal regions, and larger right hippocampal volume compared to the controls.

A meta-analysis of altered resting-state functional activity in medication-naive patients with first-episode major depression versus healthy controls

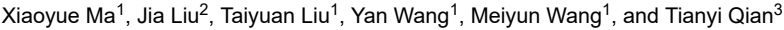

${ }^{1}$ Zhengzhou University People's Hospital \& Henan Provincial People's Hospital\& Henan Key Laboratory for Medical Imaging of Neurological Diseases, Zhengzhou, China, ${ }^{2}$ Union Hospital, Tongji Medical College, Huazhong University of Science and Technology, Wuhan, China, ${ }^{3}$ Siemens Healthcare, MR Collaboration NE Asia, Beijing, China

This study aimed to use the voxel-based meta-analytic technique called anisotropic effect size-signed differential mapping (AES-SDM) to determine consistent regional brain activity alterations in medication-naive patients with first-episode unipolar major depression disorder (MDD) versus healthy controls (HCs). The pooled and subgroup meta-analyses found that MDD patients showed resting-state brain decreased activity in the left anterior lobe of the cerebellum and increased activity in the left amygdala and left hippocampus which have hitherto been neglected in previous studies and provide new implications for the pathophysiology of cognitive and emotional impairment in MDD patients.

Neurometabolic alterations in patients with major depression measured with short echo-time whole-brain MR spectroscopic imaging

Xiao-Qi Ding ${ }^{1}$, Sirin Atalay ${ }^{2}$, Andrew A Maudsley ${ }^{3}$, Sulaiman Sheriff ${ }^{3}$, Anna Cummings ${ }^{2}$, Birte Schmitz ${ }^{1}$, Heinrich Lanfermann ${ }^{1}$, and Kai G Kahl ${ }^{2}$

${ }^{1}$ Institute of Diagnostic and Interventional Neuroradiology, Hannover Medical School, Hannover, Germany, ${ }^{2}$ Department of Psychiatry, Social Psychiatry and Psychotherapy, Hannover Medical School, Hannover, Germany, ${ }^{3}$ Department of Radiology, University of Miami School of Medicine, Miami, FL, United States

Major depressive disorder (MDD) is a common mental disorder with unclear pathophysiology. Metabolite concentrations over brain lobes or cerebellum in patients with MDD were studied. The results revealed that brain metabolic alterations associated with MDD were related to brain region and metabolite, and were particularly present in right and left frontal lobes. The findings indicate neuronal dysfunction and altered glutamatergic neuronal activity in patients.

Longitudinal structural white matter alterations in adolescents at risk for psychopathology: a Randomised Controlled Trial.

Stijn Michielse ${ }^{1}$, Jindra Bakker ${ }^{1}$, Iris Lange ${ }^{1}$, Liesbet Goossens ${ }^{1}$, Koen Schruers ${ }^{1,2}$, Ritsaert Lieverse ${ }^{1}$, Therese van Amelsvoort ${ }^{1}$, Marieke Wichers $^{3}$, Jim van Os ${ }^{1,4,5}$, and Machteld Marcelis ${ }^{1,6}$

${ }^{1}$ Psychiatry \& Neuropsychology, Maastricht University, Maastricht, Netherlands, ${ }^{2}$ Faculty of Psychology, University of Leuven, Leuven, Belgium, ${ }^{3}$ Department of Psychiatry, University Medical Center Groningen, Groningen, Netherlands, ${ }^{4}$ Department of Psychosis Studies, Institute of Psychiatry, King's College London, London, United Kingdom, ${ }^{5}$. Department of Psychiatry, Brain Center Rudolf Magnus, University Medical Center Utrecht, Utrecht, Netherlands, ${ }^{6}$ Institute for Mental Health Care, Eindhoven, Netherlands

This project is an RCT in 51 individuals with mild psychopathology randomly assigned to Acceptance and Commitment Therapy (ACT) or topic discussion group conditions. Participants underwent Diffusion Weighted Imaging (DWI), Experience Sampling Method (ESM) and a Community Assessment of Psychic Experiences (CAPE) questionnaire before and after intervention. Results show no differences between conditions after the intervention in the white matter (DWI) or the amount of psychotic experiences (CAPE). The suspicious mood ESM item showed was significantly changed due to ACT-intervention. Therefore white matter changes do not seem to occur, while mood changes as a result after 12 week intervention.

Investigation of resting-state fMRI and cognitive function changes in patients with late-onset depression after one year follow-up

Hongmin $\mathrm{Xu}^{1}$, Hongmei $\mathrm{Fu}^{2}$, Naying $\mathrm{He}^{3}$, and Fuhua $\mathrm{Yan}^{3}$

${ }^{1}$ Radiology, Ruijin Hospital,Shanghai Jiao Tong University School of Medicine, Shanghai, China, ${ }^{2}$ Pudong new area mental health center, Shanghai, China, ${ }^{3}$ Ruijin Hospital, Shanghai Jiao Tong University School of Medicine, Shanghai, China

Late-onset depression is a common psychiatric disorder, depressed elderly often exhibit cognitive impairment that are substantial, prevalent, and disabling. The LOD patients with cognitive impairment has increased risk of conversion to dementia. The amplitude low-frequency fluctuation analysis based on resting state fMRI can directly reflect the intensity of spontaneous activity of neurons and provide information of local neurons in brain areas. In this study, we observed the changes of cognitive function and local brain functional activity in patients with LOD after one year follow-up, investigated the correlation between cognitive function and brain activity. And possibly provide an objective imaging basis for the early intervention in LOD patients with cognitive impairment before deteriorate into dementia.

Structural magnetic resonance imaging study on schizophrenic patients with violence risk

Yingna Li ${ }^{1}$, Fengmei Fan ${ }^{2}$, Zhiyuan Feng ${ }^{1}$, Shuping $\operatorname{Tan}^{3}$, and Fude Yang ${ }^{3}$ 
${ }^{1}$ Radiology department, Beijing Huilongguan Hospital, Beijing, China, ${ }^{2}$ Psychiatric research center, Beijing huilongguan Hospital, Beijing, China, ${ }^{3} P$ sychiatric research center, Beijing Huilongguan Hospital, Beijing, China

To explore the brain structual imaging differences between schizophrenic patients with or without violence risk. By structual MRI and Freesurfer software,the study founds that schizophrenic patients with violence risk show the brain cortex thickness and volum reduction and cortical meancurvature increase , especially the reduction of the cortex thickness in the postdorsal cingulate gyrus .

\section{SUBCORTICAL VOLUMETRIC CHANGES IN PATIENTS WITH MAJOR DEPRESSIVE DISORDER: ROLE OF MRI}

Mariia Viktorovna Rezakova ${ }^{1}$, Elena Andreevna Filimonova ${ }^{1}$, Khurshed J. Ibrogimov ${ }^{2}$, Olga Anatolevna Subbotina ${ }^{1}$, and Alexandr Vladimirovich Shevchenko

${ }^{1}$ Stare Scientific-Research Institute of Physiology and Basic Medicine, Novosibirsk, Russian Federation, ${ }^{2}$ Novosibirsk State University, Novosibirsk, Russian Federation

We analyzed subcortical structures in patients with MDD $(\mathrm{N}=15)$ and control $(\mathrm{N}=15)$ using FreeSurfer. Patients with MDD had significantly lower left thalamus $(\mathrm{p}<0,01)$, left putamen $(p<0,05)$, left hippocampus $(p<0,05)$ and some hippocampal subfields volumes, relative to control. We found correlations $(p<0,05)$ between patient's age and putamen volume $(r=-0,56)$, number of depressive episodes and molecular layer volume $(r=-0,52)$. We didn't reveal correlation between segmentation data and MDD severity.

Voxel-based morphometry using silent T1-weighted sequence elucidates the brain volume difference between autism spectrum disorder and children with typical development

Yoshiyuki Watanabe ${ }^{1}$, Masahiro Fujiwara ${ }^{1}$, Takuya Fujiwara ${ }^{1}$, Hiroto Takahashi ${ }^{1}$, Hisashi Tanaka ${ }^{1}$, Kuriko Shimono ${ }^{2}$, Mariko Nakanishi ${ }^{2}$, Ryuzo Hanaie $^{2}$, Ikuko Mohri ${ }^{2}$, and Noriyuki Tomiyama $^{1}$

${ }^{1}$ Radiology, Osaka University, Suita, Japan, ${ }^{2}$ United Graduate School of Child Development, Osaka University, Suita, Japan

Silent MR sequences are expected to be useful and promising in the evaluation of hyperacusia patients, especially autism spectrum disorder (ASD). The aim of this research was to apply silent T1W to evaluate the brain volume changes between ASD and children with typical development (TD). Results showed that the brain volume of ASD was significantly increased at the left inferior temporal lobe and the right cerebellar tonsils and decreased at the right insular cortex and the right medial frontal lobe compared to that of TD. Silent T1W sequence can detect brain volume difference between ASD and TD.

White Matter Abnormalities in Never-Treated Patients with Long Term Schizophrenia

Yuan Xiao ${ }^{1}$, Huaiqiang Sun ${ }^{1}$, Bo Tao ${ }^{1}$, Youjin Zhao ${ }^{1}$, Wenjing Zhang ${ }^{1}$, Qiyong Gong ${ }^{1}$, John Adrian Sweeney ${ }^{2}$, and Su Lui ${ }^{1}$

${ }^{1}$ Dept. of Radiology, West China Hospital of Sichuan University, Chengdu, China, ${ }^{2}$ Dept. of Psychiatry and Behavioral Neuroscience, University of Cincinnati, Cincinnati, OH, United States

Do white matter abnormalities increase over the long-term course of schizophrenia, and is their trajectory influenced by antipsychotic treatment? In this cross-sectional study, more alteration of white matter microstructure were found in long-term but never-treated schizophrenia patients than duration-matched chronically treated patients. In the genu of the corpus callosum, there was an accelerated age-related reduction of fiber tract integrity in the never-treated patients. The more attenuated white matter changes in the treated patient group suggests that long-term antipsychotic treatment may have a neuroprotective effect on white matter tracts.

Gray Matter Network Organization in Psychotic Disorders

Wenjing Zhang ${ }^{1}$, Du Lei ${ }^{1}$, Brett Clementz ${ }^{2}$, Carol Tamminga ${ }^{3}$, Matcheri Keshavan ${ }^{4}$, Sarah Keedy ${ }^{5}$, Godfrey Pearlson ${ }^{6}$, Elliot Gershon ${ }^{5}$, Jeffrey Bishop ${ }^{7}$, Jieke Liu ${ }^{1}$, Qiyong Gong ${ }^{1}$, John Sweeney ${ }^{8}$, and Su Lui ${ }^{1}$

${ }^{1}$ Huaxi MR Research Center (HMRRC), Department of Radiology, West China Hospital of Sichuan University, Chengdu, China, ${ }^{2}$ Department of Psychology, University of Georgia, Athens, GA, United States, ${ }^{3}$ Department of Psychiatry, University of Texas Southwestern Medical Center, Dallas, TX, United States, ${ }^{4}$ Department of Psychiatry, Beth Israel Deaconess Medical Center, Harvard Medical School, Boston, MA, United States, ${ }^{5}$ Department of Psychiatry and Behavioral Neuroscience, University of Chicago, Chicago, IL, United States, ${ }^{6}$ Department of Psychiatry, School of Medicine, Yale University, New Haven, CT, United States, ${ }^{7}$ Department of Experimental and Clinical Pharmacology, College of Pharmacy, University of Minnesota, Minneapolis, MN, United States, ${ }^{8}$ Department of Psychiatry, University of Cincinnati, Cincinnati, OH, United States

Recently, new approaches have been developed using graph theory to identify deficits in gray matter networks at individual level. In the current study, by investigating single-subject graphs based on gray matter morphology to define neuroanatomic networks in a large group of individuals across psychotic disorders ( $n=330$ ), we observed disrupted network organizations associated with superior temporal and prefrontal regions within the gray matter networks in patients, which were also negatively associated with severity of psychotic symptoms. These findings showed the utility of graph theory based measures of neuroanatomic network organization to extend our understanding of the neurobiology underlying psychotic disorders. 
Peripheral oxytocin and vasopressin modulates regional brain activity differently in men and women with schizophrenia

Siyi Li ${ }^{1}$, Leah Rubin² ${ }^{2} \mathrm{Li} \mathrm{YaO}^{1}$, and Su Lui ${ }^{1}$

${ }^{1}$ Radiology, West China Hospital, Sichuan University, Chengdu, China, ${ }^{2}$ Psychiatry, Women's Mental Health Research Program, University of Illinois at Chicago, Chicago, IL, United States

Oxytocin (OT) and arginine vasopressin (AVP) exert sexually dimorphic effects on cognition and emotion processing in healthy individuals, and abnormalities in these neuroendocrine systems are observed in schizophrenia with a sex-dependent manner. Here we examined sex-dependent hormone associations with resting brain activity by applying resting-fMRI and their clinical associations in schizophrenia patients relative to healthy controls. We found that hormones differentially associate with brain networks, the sex-dependent alternation of hormone and brain activity are important for cognition and emotion processing in men and women with schizophrenia.

Higher variability of individual functional brain networks in young children with autism

Chenying Zhao ${ }^{1}$, Qinmu Peng ${ }^{2,3}$, Minhui Ouyang ${ }^{2}$, Hua Cheng ${ }^{4}$, Yun Peng ${ }^{4}$, Bo Hong ${ }^{5}$, and Hao Huang 2,3

${ }^{1}$ Department of Bioengineering, School of Engineering and Applied Science, University of Pennsylvania, Philadelphia, PA, United States, ${ }^{2}$ Department of Radiology, Children's Hospital of Philadelphia, Philadelphia, PA, United States, ${ }^{3}$ Department of Radiology, Perelman School of Medicine, University of Pennsylvania, Philadelphia, PA, United States, ${ }^{4}$ Beijing Children's Hospital, Capital Medical University, Beijing, China, ${ }^{5}$ Department of Biomedical Engineering, School of Medicine, Tsinghua University, Beijing, China

Individual's functional brain networks are sensitive indicators of behaviors. Atypical functional connectivity have been observed in children with autistic spectrum disorder (ASD), manifesting characteristic and distinctive behavior at ages of 2- to 7-years. However, little is known about individual variability of the functional brain networks in children with ASD. In this study, using resting-state fMRI and variability analysis, we quantified distinguished variability pattern in children with ASD from typically developing (TD) children from 2- to 7-years of age, especially in higher-order functional networks. The higher inter-subject variability in children with ASD may be associated with their impaired behaviors.

Brain Gray Matter Abnormalities in First-Episode, Treatment-Naïve Patients with Obsessive-Compulsive Disorder

Junhong Liu' ${ }^{1}$, Dandan Zheng ${ }^{2}$, and Jingliang Cheng ${ }^{3}$

${ }^{1}$ The First Affiliated Hospital of Zhengzhou University, Zhengzhou City, China, ${ }^{2}$ GE Healthcare, MR Research China, Zhengzhou, China, ${ }^{3}$ The First Affiliated Hospital of Zhengzhou University, Zhengzhou, China

Examinations of 36 first-episode, treatment-naive pediatric OCD patients without any comorbidities and 37 matched healthy controls (HCs) were performed with $3.0 \mathrm{~T}$ magnetic resonance imaging (MRI). Voxel-based morphometry (VBM) following Diffeomorphic Anatomical Registration using Exponentiated Lie algebra (DARTEL) was used to conduct voxel-wise tests for group differences in regional gray matter volume (GMV). Compared to $\mathrm{HCs}$, the patient group exhibited significantly different GMV in bilateral anterior cingulate cortex (ACC), left fusiform gyrus and the left postcentral gyrus. It is believed that this noninvasive method might be useful for exploring the pathophysiology of OCD.

Recuperative white matter integrity in long-term abstinent heroin addicts

wei $\mathrm{Li}^{1}$, qiang $\mathrm{Li}^{1}$, yan $\mathrm{Liu}^{1}$, jing Chen ${ }^{1}$, shan Dang ${ }^{1}$, and wei Wang ${ }^{1}$

${ }^{1}$ Radiology, Tangdu Hospital, The Fourth Military Medical University, Xi'an, China

Heroin-induced white matter integrity disruption and the restorability during long-term abstinence have been reported. However, the characteristic of these recover during different stage of abstinence has not been well understood. Use the voxel-wised diffusion tensor method, we compared the white matter difference within 17 long-term abstinence heroin addicts (LA), 22 short-term abstainers (SA) and 20 healthy controls $(\mathrm{HC})$. We found significantly decreased white matter integrity in SA and the time-dependent recover of white matter integrity, especially the restoration of myelin sheath, in LA,. These structural recover may contributed to the improvement of function in the duration of long-term abstinence.

Traditional Poster

\section{Myelin Imaging: From Mice to People}


${ }^{1}$ Department of Radiology, University of Pennsylvania, Philadelphia, PA, United States, ${ }^{2}$ Translational and Molecular Imaging Institute, Icahn School of Medicine at Mount Sinai, New York, NY, United States, ${ }^{3}$ Department of Chemistry, Temple University, Philadelphia, PA, United States, ${ }^{4}$ SAIF Core Facility, Children's Hospital of Philadelphia, Philadelphia, PA, United States

Direct detection of myelin using solid-state imaging methods is challenging due to the extremely short lifetime of the myelin matrix ${ }^{1} \mathrm{H}$ MR signal, which significantly limits its observability. In this work, the fraction of total myelin matrix ${ }^{1} \mathrm{H}$ MR signal that is observable by an inversion-recovery (IR)-prepared zero echo-time (ZTE) imaging with pointwise encoding time reduction with radial acquisition (PETRA) sequence using various acquisition parameters is estimated by Bloch equation simulations. Only approximately $5 \%$ of total magnetization is observable under realistic experimental conditions. The adiabatic inversion-recovery pulse is mostly responsible for this low fractional observability.

Magnetic Resonance Imaging (MRI) Assessment of Dimethyl Fumarate in Protecting Myelin in a Cuprizone Mouse Model

Peter Cheng-te Chou ${ }^{1}$, Benxiu Ji ${ }^{2}$, Jon Archbold ${ }^{1}$, Ankur Thomas ${ }^{2}$, Davide Gianni ${ }^{2}$, Daniel Bradley ${ }^{1}$, Haiying Liu ${ }^{1}$, and Brian Wipke ${ }^{2}$

${ }^{1}$ Research and Early Development Biomarker, Biogen, Cambridge, MA, United States, ${ }^{2}$ Neuroimmunology and Acute Neurology Research Unit, Biogen, Cambridge, MA, United States

Multiple sclerosis (MS) is a debilitating disease that affects the central nervous system. Immune system destroys the myelin that protects the axon which leads to physical, neurocognitive, and psychiatric disorders. Symptoms may improve, but permanent neurological problems often remain. There is no known cure for MS but current treatments can improve symptoms and prevent relapse. MRI has a role in MS diagnosis and management. We demonstrated that advances in MRI techniques such as Magnetization Transfer Ratio Imaging and Diffusion Tensor Imaging can detect the protective effects of dimethyl fumarate, clinically approved MS treatment, in the corpus callosum of mice.

Relevance of microglia receptor TREM2 for remyelination as revealed by multimodal MRI in the cuprizone mouse model

Anna E. Mechling ${ }^{1}$, Eva Mracsko ${ }^{1}$, Andreas Bruns ${ }^{1}$, Thomas Mueggler ${ }^{1}$, Irene Knuesel ${ }^{1}$, and Basil Künnecke

${ }^{1}$ NORD Discovery \& Translational Area, Pharmaceutical research and Early Development, Roche Innovation Center Basel, F. Hoffmann-La Roche Ltd, Basel, Switzerland

Demyelination and ensuing axonal damage are hallmarks of numerous neurodegenerative disorders. Novel treatment strategies seek to enhance remyelination and axonal recovery through acceleration of myelin debris clearance by phagocytic microglia. TREM2 is a receptor expressed by microglia that has been implicated in the regulation of phagocytosis, migration and anti-inflammatory activity. Here, we further elucidated the role of TREM2 in de- and remyelination processes by means of multiparametric in vivo MRI. We combined a TREM2 loss-of-function mouse model with cuprizone feeding as an accepted model for demyelination. Deficiency of TREM2 leads to progressive structural disintegration and absence of proper remyelination.

Three-Dimensional Inversion Recovery Ultrashort Echo Time (3D IR-UTE) Magnetic Resonance Imaging of Myelin in Rats and Mice Subject to Cuprizone Treatment

Yajun $\mathrm{Ma}^{1}$, Adam Searleman ${ }^{1}$, Robert Bussell ${ }^{1}$, Eric Y Chang ${ }^{1,2}$, Srihari Sampath ${ }^{3}$, Srinath Sampath ${ }^{3}$, Lisa Deaton ${ }^{3}$, Andrew Shumacher ${ }^{3}$, and Jiang Du ${ }^{1}$

${ }^{1}$ University of California, San Diego, San Diego, CA, United States, ${ }^{2}$ VA San Diego Healthcare System, San Diego, CA, United States, ${ }^{3}$ Genomics Institute of the Novartis Research Foundation (GNF), San Diego, CA, United States

Ultrashort echo time (UTE) MRI is capable of directly imaging myelin protons. We present the first application of a UTE sequence to study an animal model of demyelination, using inversion recovery (IR) and 3D radial sampling. Mice treated with $0.2 \%$ cuprizone for 5 weeks show loss of the $3 D$ IR-UTE signal in the lateral corpus callosum, which is expected to be maximally demyelinated at this time point. Future studies of histologically validated demyelination and remyelination in this model will further confirm the capability of 3D IR-UTE to selectively image myelin.

Measurement of T1 and T2* Relaxation Times of Purified Animal Myelin by 3D UTE Cones Sequences at 3T

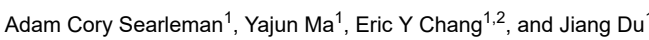

${ }^{1}$ Radiology, University of California San Diego, La Jolla, CA, United States, ${ }^{2}$ Radiology, VA San Diego Healthcare System, San Diego, CA, United States

Determination of accurate $\mathrm{T} 1$ and $\mathrm{T}^{*}$ values of myelin protons is challenging because it is comprised of multiple lipid and protein components with an ultrashort T2*, but would be important for ultrashort echo time (UTE) sequence development. In this study, we present the first T1 and T2* measurements of intact myelin directly purified from white matter, with T1 measured using a 3D UTE Cones adaptation of actual flip-angle imaging (UTE-AFI) with variable TRs, and T2* measured using 3D UTE acquisitions with variable TEs. We find that myelin has a T1 of $367 \mathrm{~ms}$ and T2* of $225 \mathrm{~ms}$ at 3T. 
${ }^{1}$ Radiology, University of California San Diego, La Jolla, CA, United States, ${ }^{2}$ Radiology, University of California, San Francisco, San Francisco, CA, United States, ${ }^{3}$ Radiology, VA San Diego Healthcare System, San Diego, CA, United States

Quantification of myelin has the potential to be used as a specific biomarker for demyelinating diseases of the nervous system such as multiple sclerosis. Ultrashort echo time (UTE) MRI has been shown to be able to directly detect signal from myelin protons, but the dynamic sensitivity of the 3D UTE Cones sequence remains unclear. This study examined the correlation between 3D UTE Cones signal intensities and different concentrations of myelin extract in $\mathrm{D}_{2} \mathrm{O}$, and found a strong linear correlation up to a myelin concentration of $24 \%$ (w/v).

Effect of aldehyde fixation on the myelin water fraction measurements in rat cervical spinal cord

Henry Szu-Meng Chen ${ }^{1}$, Jie Liu ${ }^{2}$, Alex L. MacKay ${ }^{1,3,4}$, Wolfram Tetzlaff ${ }^{2,5}$, and Piotr Kozlowski ${ }^{1,4}$

${ }^{1}$ Radiology, University of British Columbia, Vancouver, BC, Canada, ${ }^{2}$ ICORD, Vancouver, BC, Canada, ${ }^{3}$ Physics and Astronomy, University of British Columbia, Vancouver, BC, Canada, ${ }^{4}$ UBC MRI Research Centre, Vancouver, BC, Canada, ${ }^{5}$ Zoology, University of British Columbia, Vancouver, BC, Canada

This study investigated the effect of tissue fixation on myelin water fraction (MWF), an MR derived measurement of myelin content. MWF was found to increase during aldehyde fixation due to an increase in myelin water. Differences in MWF between immersion fixation and perfusion fixation with immersion post-fixation were quantified. This study demonstrated that the measured MWF is sensitive to the changes induced by chemical fixation. The results bridge the interpretation of MWF in the in vivo situation to that of the ex vivo situation and provide a guideline for designing MWF studies with histological validation.

Sequential Changes of Diffusion Anisotropy and Mean Kurtosis in Cuprizone-Induced Demyelination: A Rat Model

Ping-Huei Tsai ${ }^{1,2,3}$, Hua-Shan Liu ${ }^{4}$, Fei-Ting Hsu ${ }^{1,2}$, Yu-Chieh Kao ${ }^{1,3}$, Chia-Feng Lu ${ }^{3,5}$, Hsiao-Wen Chung ${ }^{6}$, and Cheng-Yu Chen ${ }^{1,2,3}$

${ }^{1}$ Department of Radiology, School of Medicine, College of Medicine, Taipei Medical University, Taipei, Taiwan, ${ }^{2}$ Department of Medical Imaging, Taipei Medical University Hospital, Taipei Medical University, Taipei, Taiwan, ${ }^{3}$ Research Center of Translational Imaging, College of Medicine, Taipei Medical University, Taipei, Taiwan, ${ }^{4}$ School of Biomedical Engineering, College of Biomedical Engineering, Taipei Medical University, Taipei, Taiwan, ${ }^{5}$ Department of Anatomy and Cell Biology, School of Medicine, College of Medicine, Taipei Medical University, Taipei, Taiwan, ${ }^{6}$ Graduate Institute of Biomedical Electrics and Bioinformatics, National Taiwan University, Taipei, Taiwan

The verification of cuprizone-induced demyelination in a rat model remains controversial. This study aims to develop a reliable cuprizone-induced demyelination rat model and to test the ability of DKI to monitor the sequential changes during brain demyelination. Our findings demonstrated that DKI could provide complementary information, associated with pathophysiological processes after demyelination in rat brain, which may have potential to detect microstructural changes at both acute and chronic stages and contribute to evaluations of further therapeutic strategies.

Multicomponent relaxation analysis of myelin in the brains of rare progressive solitary sclerosis, compared to multiple sclerosis and healthy control subjects in vivo Lisa Eunyoung Lee ${ }^{1}$, Jillian Chan ${ }^{1}$, Irene Vavasour ${ }^{2}$, Roger Tam ${ }^{2}$, Anthony Traboulsee ${ }^{1}$, Robert Carruthers ${ }^{1}$, and Shannon Kolind ${ }^{1,2}$

${ }^{1}$ Department of Medicine (Neurology), University of British Columbia, Vancouver, BC, Canada, ${ }^{2}$ Department of Radiology, University of British Columbia, Vancouver, BC, Canada

Progressive solitary sclerosis (PSS) presents with an isolated demyelinating lesion along the corticospinal tract that results in progressive motor deficits. We used mcDESPOT-derived parameters to better understand the pathology in the normal-appearing white matter tracts (WMT) of PSS compared to relapsing-remitting multiple sclerosis (RRMS) and healthy control (HC) subjects. Overall, we found a trend of lower MWF (myelin content) and higher qT (inflammation/edema) in WMT in PSS, compared to RRMS and HC subjects. This suggested that there might be more extensive myelin damage in the normal-appearing brain, beyond the lesional site, that may be driving disease progression in PSS

A new rapid and high-resolution multi-slice inhomogeneous Magnetization Transfer protocol to evaluate diffuse and regional cervical cord myelination at $3 T$ Henitsoa Rasoanandrianina ${ }^{1,2,3}$, Guillaume Duhamel ${ }^{1,2}$, Aurélien Massire ${ }^{1,2,3}$, Olivier Girard ${ }^{1,2}$, Maxime Guye ${ }^{1,2}$, Jean Pelletier ${ }^{1,2,4}$, Bertrand Audoin $^{1,2,4}$, and Virginie Callot $^{1,2,3}$

${ }^{1}$ Aix-Marseille Univ, CNRS, CRMBM, Marseille, France, ${ }^{2}$ APHM, Hôpital Universitaire Timone, CEMEREM, Marseille, France, ${ }^{3}$ iLab-Spine International Associated Laboratory, Montreal, Marseille, France, ${ }^{4}$ Department of Neurology, CHU Timone, AP-HM, Marseille, France

The inhomogeneous Magnetization Transfer (ihMT) technique has recently been proposed as a new method to probe the cervical spinal cord (CSC) myelin-content. Studies reported so far were limited to single-slice acquisition, hence precluding investigation of the whole CSC within a short acquisition time. To overcome this limitation, a $2 \mathrm{D}$ multi-slice single-shot SpinEcho-Echo-Planar Imaging (SE-EPI) read-out approach was implemented at 3T along with strategies to correct for inherent susceptibility-induced image-distortions and post-saturation relaxation effect for each slice. Validated on phantom and applied to healthy subjects and a patient with multiple sclerosis, this preliminary study shows the promising value of SE-EPI inMT in the clinical context. 
Alessio Fracasso ${ }^{1}$, Carlien A Roelofzen ${ }^{2}$, Giorgio L Porro ${ }^{3}$, Douwe P Bergsma ${ }^{4}$, Mies van Genderen ${ }^{5}$, Serge O Dumoulin ${ }^{6}$, and Natalia Petridou ${ }^{3}$

${ }^{1}$ Spinoza Center for Neuroimaging, Amsterdam, Netherlands, ${ }^{2}$ Utrecht University, Utrecht, Netherlands, ${ }^{3}$ University Medical Centre Utrecht, Utrecht, Netherlands, ${ }^{4}$ Donders Institute, Njmegen, Netherlands, ${ }^{5}$ Bartimeus Institute for the Visually Impaired, Zeist, Netherlands, ${ }^{6}$ Spinoza Centre for Neuroimaging, Amsterdam, Netherlands

High resolution 7T MRI allows to investigate the functional and structural organization of human cerebral cortex at an unprecedented level of detail, visualizing myelination patterns over the cortical surface and identifying a large number of cortical areas. In this study we hypothesize that myelin content co-varies with loss of visual input. We used a modified T1-W MPRAGE to enhance myelin visualization within gray matter and acquired data from patients with hemianopsia, a visual field defect consisting of an absolute scotoma limited to a single hemifield, and evaluate whether the clinical symptoms are reflected in gray matter myelination in the occipital cortex.

Myelin-Water Quantification: Orthogonal Matching Pursuit versus Non-Negative Least Squares

Gerhard Drenthen ${ }^{1,2}$, Walter Backes $^{1,2}$, Albert Aldenkamp $^{3}$, and Jacobus Jansen ${ }^{1,2}$

${ }^{1}$ Department of Radiology \& Nuclear Medicine, Maastricht University Medical Center, Maastricht, Netherlands, ${ }^{2}$ School for Mental Health and Neuroscience, Maastricht University Medical Center, Maastricht, Netherlands, ${ }^{3}$ Department of Behavioral Sciences, Epilepsy Center Kempenheaghe, Heeze, Netherlands

Myelin-water quantification relies on modeling of multi-exponential T2-relaxation time decay. For this, we explore the greedy Orthogonal Matching Pursuit (OMP) method and compare it to the most commonly applied non-negative least squares (NNLS) method. The two methods are evaluated by means of simulations, phantom measurements and in vivo image data.

Reproducibility of Myelin Water Fraction for GRASE sequences with a varying SENSE factor

Gerhard Drenthen ${ }^{1,2}$, Walter Backes ${ }^{1,2}$, Albert Aldenkamp $^{3}$, and Jacobus Jansen ${ }^{1,2}$

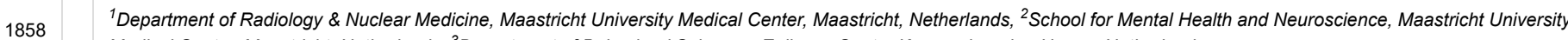
Medical Center, Maastricht, Netherlands, ${ }^{3}$ Department of Behavioral Sciences, Epilepsy Center Kempenheaghe, Heeze, Netherlands

For myelin-water quantification to become a feasible method in a clinical setting a rapid whole brain coverage acquisition is required, as well as reproducible results. Therefore, this study aims to measure the reproducibility of the Gradient-Spin Echo (GRASE) sequence with and without utilizing parallel imaging with sensitivity coding (SENSE) to investigate the impact of the acceleration (e.g. increased SENSE factor).

Training induced myelin and iron changes in healthy subjects using novel quantitative MRI techniques

Michela Azzarito ${ }^{1}$, Eveline Huber ${ }^{1}$, Maryam Seif ${ }^{1}$, Gabriel Ziegler ${ }^{2,3}$, and Patrick Freund ${ }^{1,4,5,6}$

${ }^{1}$ Spinal Cord Injury Center Balgrist, University Hospital Zurich, University of Zurich, Zurich, Switzerland, zürich, Switzerland, ${ }^{2}$ German Center for Neurodegenerative Diseases (DZNE), Magdeburg, Germany, magdeburg, Germany, ${ }^{3}$ Institute of Cognitive Neurology and Dementia Research, Magdeburg, Germany, Magdeburg, Germany, ${ }^{4}$ Department of Neurophysics, Max Planck Institute for Human Cognitive and Brain Sciences, Leipzig, Germany, leipzig, Germany, ${ }^{5}$ Department of Brain Repair and Rehabilitation, UCL Institute of Neurology, University College London, London, UK, Iondon, United Kingdom, ${ }^{6}$ Wellcome Trust Centre for Neuroimaging, UCL Institute of Neurology, University College London, London, UK, london, United Kingdom

Activity-dependent plasticity has significant implications for healthy development, learning, memory, and recovery from brain damage. However, the exact time course and the neural mechanisms behind brain plasticity are still not completely understood. In this study, longitudinal quantitative MRI protocols were used to assess training associated microstructural changes using markers sensitive to myelin and iron. We show that training improvements during a sensorimotor task performed over 4 weeks induces linear and non-linear increases in myelin and iron content in the primary motor cortex and cerebellum. This study provides new tools to assess training effects in healthy controls.

Decreased myelin water fraction in the corpus callosum at 6 months post mild traumatic brain injury

Bretta Russell-Schulz ${ }^{1}$, Ivan J Torres ${ }^{2}$, Manraj K.S. Heran ${ }^{3}$, Alex MacKay ${ }^{1,4}$, and William Panenka ${ }^{5}$

${ }^{1}$ Radiology, UBC MRI Research Centre, Vancouver, BC, Canada, ${ }^{2}$ Psychiatry, University of British Columbia, Vancouver, BC, Canada, ${ }^{3}$ Radiology, Diagnostic \& Therapeutic Neuroradiology, Vancouver, BC, Canada, ${ }^{4}$ Physics and Astronomy, University of British Columbia, Vancouver, BC, Canada, ${ }^{5}$ Psychiatry, British Columbia Provincial Neuropsychiatry Program, Vancouver, BC, Canada

Monitoring mild traumatic brain injury (TBI) presents challenges for conventional MRI and underlying myelin changes are not well understood. Myelin water fraction (MWF) presents an opportunity to examine myelin changes post injury. At 6 months post injury, corpus callosum genu and body MWF decreased from baseline (acute) in 8 out of 9 subjects. Splenium MWF decreased in 7 out of 9 subjects. When averaged across subjects, the average decrease in MWF was $2 \%$ for the genu and $5 \%$ for the splenium, not significantly different from baseline; the lack of significance was due to large MWF increases in one of the participants. 
Spinal Cord (C1 to T12) Demyelination Measured by Magnetization Transfer Imaging: Characteristics of Acute, Sub-Acute and Chronic Disease Phases

Sze Nok Tam ${ }^{1}$, Katie E Silva ${ }^{1}$, David Zurakowski ${ }^{1}$, Leslie Benson ${ }^{1}$, Mark Gorman ${ }^{1}$, David Borsook ${ }^{1}$, and Nadia Barakat ${ }^{1}$

The diagnostic utility of Magnetization Transfer Imaging (MTI) was tested in a large cohort of patients with transverse myelitis - a demyelinating myelopathy affecting the spinal cord. We measured the reproducibility of MTI in a pediatric clinical model, at different disease stages. Our results showed that obtaining repeatable measures in the entire spinal cord (C1 to T12) is feasible. Our findings also showed significant differences in MTR values between patients and healthy controls, and between three sub-groups of patients (acute, sub-acute and chronic disease phases).

mcDESPOT-derived measurements are sensitive to differences in myelin content and thickness in the corpus callosum of neuromyelitis optica patients and healthy controls Shawna Abel ${ }^{1}$, Irene Vavasour ${ }^{2}$, Lisa Lee ${ }^{1}$, Roger Tam ${ }^{2}$, Cornelia Laule ${ }^{2}$, Robert Carruthers ${ }^{1}$, Anthony Traboulsee ${ }^{1}$, Anna Combes ${ }^{3}$, and Shannon Kolind ${ }^{1}$

${ }^{1}$ Neurology, University of British Columbia, Vancouver, BC, Canada, ${ }^{2}$ Radiology, University of British Columbia, Vancouver, BC, Canada, ${ }^{3}$ Neuroimaging, King's College London, London, United Kingdom

Histological studies suggest that white matter microstructure varies across different subregions of the corpus callosum (CC). We used mcDESPOT-derived measures to examine myelin content and thickness in vivo in 3 subregions of the $\mathrm{CC}$ in healthy controls $(\mathrm{HC})$ and individuals with neuromyelitis optica spectrum disorder (NMOSD). Differences in both myelin content and thickness were observed in different subregions of the CC in HC. Myelin content was decreased in posterior CC in NMOSD relative to HC. mcDESPOT-derived myelin measurements are sensitive to differences in white matter microstructure and can be used to investigate the underlying pathology contributing to demyelinating diseases.

Quantitative MRI of diffusely abnormal white matter in multiple sclerosis at 3T

Irene M Vavasour ${ }^{1}$, Roger Tam ${ }^{1,2}$, Shannon H Kolind ${ }^{1,2,3,4,5}$, Robert L Carruthers ${ }^{3}$, Anthony Traboulsee ${ }^{2,3}$, David KB Li $i^{1,2,3}$, and Cornelia Laule ${ }^{1,4,5,6}$

${ }^{1}$ Radiology, University of British Columbia, Vancouver, BC, Canada, ${ }^{2}$ MS/MRI Research Group, University of British Columbia, Vancouver, BC, Canada, ${ }^{3}$ Medicine (Neurology), University of British Columbia, Vancouver, BC, Canada, ${ }^{4}$ Physics and Astronomy, University of British Columbia, Vancouver, BC, Canada, ${ }^{5}$ International Collaboration on Repair Discoveries, University of British Columbia, Vancouver, BC, Canada, ${ }^{6}$ Pathology \& Laboratory Medicine, University of British Columbia, Vancouver, BC, Canada

Diffusely abnormal white matter (DAWM) is found in the brain of some multiple sclerosis (MS) and clinically isolated syndrome (CIS) subjects. DAWM has poorly defined boundaries, with signal intensity higher than normal appearing white matter (NAWM) but not as high as lesions on FLAIR, proton density and $\mathrm{T}_{2}$-weighted MRI. We compared results from myelin water imaging, $T_{1}$ and diffusion basis spectrum imaging in areas of DAWM and corresponding areas of NAWM in 20 MS/CIS participants. No significant differences in measures sensitive to myelin, axons, oedema and inflammation were found, although trends for increased $\mathrm{T}_{1}$ and reduced fibre fraction were observed.

Rapid estimation of myelin for diagnostic imaging (REMyDI): A clinical and histopathological validation in multiple sclerosis

Russell Ouellette ${ }^{1,2,3,4}$, Marcel Warntjes ${ }^{5,6}$, Yngve Forslin ${ }^{1,2}$, Michael Plattén ${ }^{1,2}$, Martin Uppman ${ }^{1}$, Åsa Bergendal ${ }^{1,7}$, Fredrik Piehl ${ }^{1,8}$, Sten Fredrikson ${ }^{1,8}$, Maria Kristoffersen-Wiberg $^{1,2}$, Caterina Mainero ${ }^{3,4}$, and Tobias Granberg ${ }^{1,2,3,4}$

${ }^{1}$ Clinical Neuroscience, Karolinska Institutet, Stockholm, Sweden, ${ }^{2}$ Department of Radiology, Division of Neuroradiology, Karolinska University Hospital, Stockholm, Sweden, ${ }^{3}$ Martinos Center for Biomedical Imaging, Department of Radiology, Massachusetts General Hospital, Boston, MA, United States, ${ }^{4}$ Harvard Medical School, Boston, MA, United States, ${ }^{5}$ Synthetic MR, Stockholm, Sweden, ${ }^{6}$ Center for Medical Image Science and Visualization, Linköping University, Linköping, Sweden, ${ }^{7}$ Department of Medical Psychology, Karolinska University Hospital, Stockholm, Sweden, ${ }^{8}$ Department of Neurology, Karolinska University Hospital, Stockholm, Sweden

Multiple sclerosis is a chronic inflammatory and neurodegenerative disease characterized by demyelination. To follow patients longitudinally and monitor treatment response, there is a need for robust and tissue-specific imaging biomarkers reflective of the heterogeneous disease course. Here, we aimed to validate REMyDI as an MRI-based measure of myelin ex vivo and in vivo. Histopathologically, REMyDI correlates well with all three of the studied myelin staining methods. In vivo, REMyDI revealed a strong sensitivity in differentiating white matter as compared to normal appearing white matter with associations to both cognitive (information processing speed) and physical disability (Expanded Disability Status Scale).

Christoph Birkl ${ }^{1,2}$, Vanessa Wiggermann ${ }^{1,3,4}$, Verena Endmayr ${ }^{5}$, Enedino Hernandez-Torres ${ }^{1,4}$, Gregor Kasprian $^{6}$, Romana Hoeftberger ${ }^{7}$, Stefan Ropele ${ }^{2}$, Simon Hametner $^{5,8}$, and Alexander Rauscher ${ }^{1,3,4,9}$

${ }^{1}$ UBC MRI Research Centre, University of British Columbia, Vancouver, BC, Canada, ${ }^{2}$ Department of Neurology, Medical University of Graz, Graz, Austria, ${ }^{3}$ Department of Physics and Astronomy, University of British Columbia, Vancouver, BC, Canada, ${ }^{4}$ Department of Pediatrics (Devision of Neurology), University of British Columbia, Vancouver, BC, Canada, ${ }^{5}$ Center for Brain Research, Medical University of Vienna, Vienna, Austria, ${ }^{6}$ Department of Biomedical Imaging and Image-Guided Therapy, Medical University of Vienna, Vienna, Austria, ${ }^{7}$ Institute of Neurology, Medical University of Vienna, Vienna, Austria, ${ }^{8}$ Institute of Neuropahtology, University Medical Center Goettingen, Goettingen, Germany, ${ }^{9}$ Child and Family Research Institute, University of British Columbia, Vancouver, BC, Canada 
Magnetic-susceptibility sensitive MRI as measure for tissue damage in multiple sclerosis (MS) lesions has been controversial, since the relationship between the MR signal and the underlying pathology is not fully understood. Here we assessed $R_{2}{ }^{*}$ of different white matter MS lesion types and normal appearing white matter (NAWM) in relation to the underlying iron and myelin densities. We observed lower $\mathrm{R}_{2}{ }^{*}$ in all MS lesion types compared to NAWM, driven by lower iron and myelin densities. Shadow plaques showed significant higher $\mathrm{R}_{2}{ }^{*}$ values than other MS lesions, in line with the hypothesis of remyelination and supported by myelin histology.

A Comparison of R1 and Magnetization Transfer Saturation for Mapping Intracortical Myelin

Cecil Chern-Chyi Yen ${ }^{1}$, Kimberly Lara Desmond ${ }^{2}$, Afonso C. Silva ${ }^{1}$, and Nicholas Adam Bock ${ }^{2}$

${ }^{1}$ National Institute of Neurological Disorders and Stroke, National Institutes of Health, Bethesda, MD, United States, ${ }^{2}$ Psychology, Neuroscience and Behaviour, McMaster University, Hamilton, ON, Canada

Contrasts based on T1 and R1 (1/T1), including T1/T2-weighted hybrid contrast, have been proposed to map intracortical myelin in the mammalian brain. However, iron in the cortex may obscure changes in myelin investigated by T1-based contrast since T1 is also sensitive to myelin. Here we explore magnetization transfer contrast for mapping ICM, as it may be more specific for myelin. We compare R1 maps measured by MP2RAGE with MTsat measured by MT-FLASH in two marmosets, a species of small non-human primate. Although MTsat shows a similar pattern as R1 in some regions of the cortex, MTsat suffers from signal inhomogeneity issues and care is needed to correct these in future measurement protocols to shows a similar pattern as R1 in some regions
properly compare R1 and MTsat contrasts.

Comparison between quantitative magnetization transfer imaging and ratio of $\mathrm{T} 1 \mathrm{w} / \mathrm{T} 2 \mathrm{w}$ approach in myelin mapping

Yu Sui ${ }^{1}$, Pippa Storey ${ }^{1}$, Alexey Samsonov ${ }^{2}$, and Mariana Lazar $^{1}$

${ }^{1}$ Bernard and Irene Schwartz Center for Biomedical Imaging, Department of Radiology, New York University School of Medicine, New York, NY, United States, ${ }^{2}$ Department of Radiology, University of Wisconsin at Madison, Madison, WI, United States

Myelination is one of the essential indicators of brain maturation, and various abnormalities in myelin content have been found for different psychiatric disorders. However, reliable imaging techniques for human in vivo myelin measurement are still under intensive research, thus the degree and significance of myelin deficits for specific pathology remain indeterminate. The current study compared myelin mapping proposed as part of the Human Connectome Protocol using the ratio of T1 and T2 weighted image intensity to quantitative magnetization transfer mapping (qMT). The relationship between myelin content estimated by these two methodologies in various brain regions is discussed.

Traditional Poster

\section{Neurovascular Imaging Methods}

Magnetic resonance angiography and venography was not useful for correcting underestimated susceptibility measurements of sub-voxel objects on quantitative susceptibility maps Natalie M Wiseman ${ }^{1}$, Sagar Buch², Yongsheng $\mathrm{Chen}^{3}$, E Mark Haacke ${ }^{3,4}$, and Zhifeng Kou ${ }^{3,4}$

${ }^{1}$ Department of Psychiatry and Behavioral Neurosciences, Wayne State University School of Medicine, Detroit, MI, United States, ${ }^{2}$ Center for Functional and Metabolic Mapping, Robarts' Research Institute, Western University, London, ON, Canada, ${ }^{3}$ Department of Radiology, Wayne State University, Detroit, MI, United States, ${ }^{4}$ Department of Biomedical Engineering, Wayne State University, Detroit, MI, United States

We investigated two magnetic resonance angiography and venography (MRAV) methods for use in correcting quantitative susceptibility mapping (QSM) estimates in sub-voxel veins. An MRAV generated from an interleaved rephased/dephased gradient echo sequence (without contrast agent) suffered from low SNR in veins, whereas the contrast-enhanced T1-MRAV caused the vessels to appear larger than those in the pre-contrast images. Neither method offered a reliable correction of partial-volumed susceptibility measurements.

Increased cerebral oxygen extraction fraction measured in the ischemic stroke using an asymmetric spin echo EPI approach

Yong Zhang ${ }^{1}$, Qiao $\mathrm{Li}^{2}$, Jun Zhang ${ }^{2}$, and Bing $\mathrm{Wu}^{3}$

${ }^{1}$ GE Healthcare, Shanghai, China, ${ }^{2}$ Radiology, Huashan Hospital, Shanghai, China, ${ }^{3}$ GE Healthcare, Beijing, China

The oxygen consumption by brain tissue can be measured with oxygen extraction fraction (OEF), a potential indicator of the occurrence of stroke. A single shot asymmetric spin echo (ASE) EPI sequence was implemented for OEF measurement in stroke patients. Increased OEF corresponded with the decrease blood flow in the ischemic brain region, as reported in the previous literature. ASE EPI showed the potential to provide quantitative OEF maps with good brain coverage and without the need of gas challenges. The measurement of OEF may provide a better assessment of viable brain stroke after a stroke attack for potential treatment 
Vector Field Perfusion Imaging: A Validation Study by Using Multiphysics Model

Liangdong Zhou ${ }^{1}$, Pascal Spincemaille ${ }^{1}$, Qihao Zhang ${ }^{1}$, Thanh Nguyen ${ }^{1}$, Vincent Doyeux ${ }^{2}$, Sylvie Lorthois ${ }^{2}$, and Yi Wang ${ }^{1,3}$

${ }^{1}$ Department of Radiology, Weill Cornell Medical College, New York, NY, United States, ${ }^{2}$ Institut de Mécanique des Fluides de Toulouse, Toulouse, France, ${ }^{3}$ Department of Biomedical Engineering, Cornell University, Ithaca, NY, United States

A multiphysics model based on Navier-Stokes equation and continuity equation is built to simulate the arterial spin labeled (ASL) blood flow in the blood vessels. Blood velocity distribution is reconstructed by measuring the 4D time-resolved labeled blood concentration and doing inversion data fitting processing. The conventional lumped-element Kety's equation provides a quantitative measurement of whole brain cerebral blood flow (CBF) suffering from the inaccurate estimation of arterial input function (AIF). The multiphysics model validates that the blood velocity involved vector field perfusion (VFP) with multiple post label delays does not rely on the AIF.

A rapid scan for simultaneous MRAV, MRA, tSWI, and QSM on 1.5T

Wei Xu${ }^{1}$, Yu Wang ${ }^{1,2}$, Feng Huang ${ }^{1}$, Tie cheng $\mathrm{Li}^{1}$, Hongyu Guo ${ }^{1}$, Yongsheng Chen ${ }^{3,4}$, and Ewart Mark Haacke ${ }^{2,3,4}$

${ }^{1}$ Neusoft Medical System, Shanghai, China, ${ }^{2}$ Shanghai Key Laboratory of Magnetic Resonance, East China Normal University, Shanghai, China, ${ }^{3}$ Department of Radiology, School of Medicine, Wayne State University, Detroit, FL, United States, ${ }^{4}$ The MRI Institute for Biomedical Research, Detroit, FL, United States

Numerous diseases such as stroke, arteriovenous malformation (AVM), traumatic brain injury (TBI) and tumor evaluation require detailed vascular information for the best diagnostic interpretation ${ }^{1-4}$. Being able to collect both MR angiography and venography with sufficient SNR, CNR and co-registration in short time is critical for these diseases, especially for emergency patients. In this work, we developed a rapid 3D interleaved GRE sequence to acquire these vascular images simultaneously. Co-registered MRAV, MRA, QSM and tSWI for imaging arteries, veins and basal ganglia in 4 minutes and 24 seconds on a NMS 1.5 T system covering the whole brain with $0.67 \times 1.33 \times 2.7 \mathrm{~mm}^{3}$ resolution

Brain Cloud of Carbogen-based Cerebrovascular Reserve : territorial and cortical specificity

Tzu-chen Yeh ${ }^{1,2}$, Chou-ming Cheng ${ }^{3}$, and Chi-che $\mathrm{Chou}^{3}$

${ }^{1}$ Department of Radiology, Taipei Veterans General Hospital, Taipei, Taiwan, ${ }^{2}$ Institute of Brain Science, National Yang-ming University, Taipei, Taiwan, ${ }^{3}$ Integrated Brain Research Unit, Department of Medical Research, Taipei Veterans General Hospital, Taipei, Taiwan

To explore the spatial characters of carbogen-based cerebrovascular reserve (CO2-CVR), grouped analyses of CO2-CVR was obtained using BOLD-based fMRI for ninety normal subjects with the fully automatic delivery system of carbogens and parametric inhalation of 1-5\% CO2. Distal territories of ACA, segment 3, showed the highest of CO2-CVR at v23ab (ventral portion of Brodmann area 23) as verified by territorial and cortical parcellation. Our findings supported the biological adapation of CVR for resting activity, e.g. default mode network.

Simultaneous acquisition of T1- and T2-weighted images using Volumetric Isotropic Turbo spin echo Acquisition (VISTA): A feasibility study towards cerebral venous thrombus imaging

Yunduo Li ${ }^{1}$, Shuo Chen ${ }^{1}$, Zechen Zhou ${ }^{2}$, Rui Li ${ }^{1}$, and Chun Yuan ${ }^{1,3}$

${ }^{1}$ Center for Biomedical Imaging Research, Department of Biomedical Engineering, Tsinghua University, Beijing, China, ${ }^{2}$ Philips Research North America, Cambridge, MA, United States, ${ }^{3}$ Department of Radiology, University of Washington, Seattle, WA, United States

This study demonstrated the feasibility of simultaneously acquiring T1 and T2-weighted images using dual-echo VISTA sequence. Phantom experiments showed that dual-echo VISTA can provide T1- and T2-weighted images as conventional T1/2 imaging sequences, and the performance of proposed sequence was further validated by in-vivo scan. By assembling flow-suppression, T1 and T2 contrast in one sequence, dual-echo VISTA has its potential to differentiate stages of thrombus more accurately.

A comparative study of arterial spin labeling and CT perfusion on evaluation of cerebral perfusion changes after carotid endarterectomy

Ying Liu ${ }^{1}$, Huimin $\mathrm{Xu}^{1}$, Zheng Wang ${ }^{1}$, and Huishu Yuan ${ }^{1}$ 
Justine DEBATISSE ${ }^{1,2}$, Nikolaos MAKRIS ${ }^{3}$, Nicolas COSTES ${ }^{4}$, Michael VERSET ${ }^{5}$, Océane WATEAU ${ }^{5}$, Karine PORTIER ${ }^{1}$, Mohamed AGGOUR ${ }^{1}$, Jean-Baptiste LANGLOIS ${ }^{4}$, Christian TOURVIEILLE ${ }^{4}$, Didier LE BARS ${ }^{4}$, Thomas TROALEN ${ }^{2}$, Hugues CONTAMIN 5 , Tae-Hee CHO ${ }^{3,6}$, and Emmanuelle CANET-SOULAS ${ }^{1}$

${ }^{1}$ Univ Lyon, CarMeN Laboratory, INSERM, INRA, INSA Lyon, Université Claude Bernard Lyon 1, Lyon, France, ${ }^{2}$ Siemens Healthcare SAS, Saint-Denis, France, ${ }^{3}$ CREATIS, CNRS UMR 5220, INSERM U1206, Université Lyon 1, INSA Lyon, Université Jean Monnet Saint-Etienne, Lyon, France, ${ }^{4}$ CERMEP - Imagerie du vivant, Lyon, France, ${ }^{5}$ Cynbiose SAS, MarcyL'Etoile, France, ${ }^{6}$ Department of Neurology, Hospices Civils de Lyon, Lyon, France

Reliable estimation of cerebral blood flow (CBF) is crucial for a precise diagnosis of acute ischemia. PET using $\left.{ }^{15} \mathrm{O}\right] \mathrm{H}_{2} \mathrm{O}$ remains the reference method to assess $\mathrm{CBF}$ but it can also be assessed using MRI. Several post-processing algorithms of perfusion MRI can be used to derive MRI-CBF values. CBF was simultaneously assessed with PET and MRI in a Macaca fascicularis model of stroke using a Siemens PET-MRI hybrid scanner. Four MRI post processing algorithms (sSVD, cSVD, oSVD and Bayesian) were compared against PET estimation of CBF. Bayesian algorithm seems to derive the most reliable estimation of CBF.

Standard and Look-Locker FAIR-TrueFISP for arterial spin labelling on mouse at $9.4 \mathrm{~T}$

Michael Gottschalk ${ }^{1}$

${ }^{1}$ Lund University Bioimaging Center, Lund University, Lund, Sweden

The study investigates TrueFISP readout for FAIR either as standard inversion recovery (IR) or as Look-Locker (LL) inversion recovery. These two methods are compared to EPI readout as implemented by Bruker. The aim was to show the improved image quality using TrueFISP and to evaluate the alternatives standard IR and LL. For FAIR-TrueFISP an in-house written method was created. The method was tested on a group of C57BL/6 mice at the field strength of $9.4 \mathrm{~T}$. The results show cerebral blood flow maps with less distortion than EPI and the values found are in agreement with the literature.

QSM in stroke: Veins, Tissue and Cerebral Microbleeds

Parisa Badihi Najafabadi ${ }^{1}$, Ana Klahr ${ }^{2}$, Hongfu Sun ${ }^{1}$, Ahmed Elkady ${ }^{1}$, Derek J Emery ${ }^{3}$, Kenneth S Butcher ${ }^{2}$, and Alan H Wilman ${ }^{1}$

${ }^{1}$ Biomedical Engineering, University of Alberta, Edmonton, AB, Canada, ${ }^{2}$ Medicine, University of Alberta, Edmonton, AB, Canada, ${ }^{3}$ Radiology \& Diagnostic Imaging, University of

We assessed microbleed burden and cerebral oxygenation in veins and tissue in stroke patients by means of Quantitative Susceptibility Mapping (QSM). Results showed significant susceptibility difference for ipsi- and contralateral veins, and smaller area measurements for cerebral microbleeds compared to SWI and magnitude images due to elimination of blooming effects. Strong susceptibility difference of microbleeds compared to other brain tissue suggest the possibility of quantifying microbleeds by thresholding the images. QSM may be employed in stroke studies to study cerebral oxygenation in veins and microbleed assessment.

Ultra-High resolution SWI at 3T

Harshan Ravi ${ }^{1}$, Wen-Tung Wang ${ }^{1}$, Andrew Knutsen ${ }^{1}$, Dzung L Pham ${ }^{1}$, and John A Butman ${ }^{1,2}$

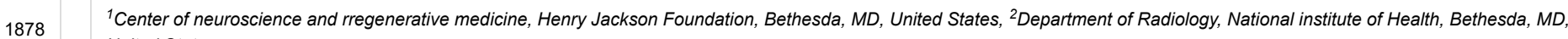
United States

Susceptibility weighted imaging (SWI) uses phase and magnitude data to increase the conspicuity of sources such as blood vessels and hemorrhages. Typical resolution used in clinical SWI are approximately 0.5-1 mm in-plane and 1-2 mm through plane. Higher resolution has been achieved using 7.0 T MRI, but such units have limited availability. In this work, we generated ultra-high resolution ( $400 \mu \mathrm{m}$ isotropic) SWI at $3.0 \mathrm{~T}$ using registration and averaging.

Vascular Change Assessed by Calibrated Multi-delay Arterial Spin Labeling Under Oxygen and Carbogen Gas Challenge

Michael L Rohan ${ }^{1}$, Clara Wellons ${ }^{2}$, Megan Shevenell ${ }^{3}$, Nicolette Schwarz ${ }^{4}$, Xingfeng Shao ${ }^{5}$, Daniel JJ Wang ${ }^{5}$, and Blaise Frederick ${ }^{3}$

${ }^{1}$ Imaging, McLean Hospital, Belmont, MA, United States, ${ }^{2}$ imaging, McLean Hospital, Belmont, MA, United States, ${ }^{3}$ McLean Hospital, Belmont, MA, United States, ${ }^{4}$ Mc:ean Hospital,

Arterial Spin Labeling (ASL) measurements are employed here in a suite of hemodynamic assessments in our study of cerebrovascular reactivity. In this project we test a 3D Gradient and Spin Echo (GRASE) Multiple delay Pseudo-Continuous Arterial Spin Labeling (MPCASL) Magnetic Resonance (MR) acquisition in order to measure the change in these measures under gas challenge. Subjects were scanned with 3D GRASE MPCASL while breathing medical air, oxygen, or Carbogen (5\% CO2 +95\% O2) under controlled conditions. Changes in blood flow, volume, and arrival time that were observed will be used to calibrate novel delay assessment methods. 
Template maps of vascular function and structure in the healthy brain

Endre Grøvik ${ }^{1}$, Kyrre Eeg Emblem ${ }^{1}$, Ingrid Digernes ${ }^{1}$, Line Brennhaug Nilsen ${ }^{1}$, Cornelius Eichner ${ }^{2}$, Kourosh Jafari $^{2}$, Thomas Witzel ${ }^{2}$, Behroze Vachha ${ }^{2}$, Elizabeth Gerstner ${ }^{2}$, Jayashree Kalpathy-Cramer ${ }^{2}$, Kawin Setsompop ${ }^{2}$, and Steven Stufflebeam ${ }^{2}$

${ }^{1}$ Department for Diagnostic Physics, Oslo University Hospital, Oslo, Norway, ${ }^{2}$ Athinoula A. Martinos Center for Biomedical Imaging, Harvard Medical School, Boston, MA, United States

In recent years, Vessel Architectural Imaging (VAl) has emerged as a promising tool in tumor diagnosis to reveal unique MRI-based information on vessel architecture, hemodynamic efficacy and metabolic activity. Healthy control data may further advance our knowledge on the VAI method and its underlying mechanisms, as well as serve as study controls. Here we propose a set of healthy-tissue template maps of all VAI derived parameters which may act as a toolbox to identify anomalies of various vascular brain diseases and ultimately help improve diagnostic and outcome assessment in clinical settings.

Simultaneous measures of brain oxygenation and perfusion using a 9.4T MRI in rats

Kevin Lee ${ }^{1}$, Matthew Bouchard ${ }^{1}$, Sara Bohnert ${ }^{2}$, and Jeff F Dunn ${ }^{1}$

${ }^{1}$ Radiology, University of Calgary, Calgary, AB, Canada, ${ }^{2}$ Casualty Management Section, Defence Research and Development Canada- Suffield Research Centre, Suffield, AB, Canada

We developed a novel method to simultaneously measure tissue oxygenation and cerebral blood flow. This technique combines chronically implanted fiber-optic oxygen sensors and continuous arterial spin labeling MRI. An added benefit is that one can measure oxygen while the animals are awake and freely moving.

One minute Brain MR venography with Compressed SENSE at 3T.

Kayoko Abe ${ }^{1}$, Kazufumi Suzuki ${ }^{1}$, and Shuji Sakai ${ }^{1}$

${ }^{1}$ Department of Diagnostic Imaging and Nuclear Medicine, Tokyo Women's Medical University, Tokyo, Japan

Brain MR venograpy based on phase-contrast technique (MRV) contributes in the diagnosis of venous sinus thrombosis and helps to clarify venous anatomy before brain operations. However, MRV is not commonly taken in routine brain MRI examinations because it requires a longer acquisition time. Recently, Compressed SENSE, which is a combination of compressed sensing and parallel imaging technique: SENSE, has been developed, and can shorten acquisition times with minimum image quality deterioration. Therefore, we investigated the optimization of 1 minute MRV, which the acquisition time was 1 minute, using Compressed SENSE at 3T.

Microstructural Characterization of Post-Stroke Lesions in the Posterior Limb of the Internal Capsule in Subacute Patients using DTI and NODDI

Alfonso Mastropietro ${ }^{1}$, Lucia Fontana ${ }^{2}$, Maria Luisa Malosio ${ }^{3,4}$, Laura Straffi $^{5}$, Simona Marcheselli ${ }^{5}$, Marco Grimaldi ${ }^{2}$, and Giovanna Rizzo ${ }^{1}$

${ }^{1}$ Institute of Bioimaging and Molecular Physiology, Consiglio Nazionale delle Ricerche, Segrate, Italy, ${ }^{2}$ Neuroradiology Unit \& Neuro Center, Humanitas Clinical and Research Center, Rozzano, Italy, ${ }^{3}$ Institute of Neuroscience, Consiglio Nazionale delle Ricerche, Milano, Italy, ${ }^{4}$ Laboratory of Brain Pathology and Pharmacology, Humanitas Clinical and Research Center, Rozzano, Italy, ${ }^{5}$ Stroke Unit \& Neuro Center, Humanitas Clinical and Research Center, Rozzano, Italy

The purpose of his work was to characterize the Posterior Limb of the Internal Capsule (PLIC) of subacute stroke patients using both DTI and NODDI approaches to investigate microstructural changes occurring in the lesioned with respect to the unlesioned hemisphere. Six patients having a brain damage involving the Corticospinal Tract (CST) were enrolled. MRI was carried out on a 3T scanner about 14 days after stroke occurrence. DTI and NODDI analysis showed CST alterations in subacute stroke patients. FA and ODI were the only parameters that underwent significant modifications in PLIC regions.

Clinical application of QSM sequence in cerebral microbleeds of patients with essential hypertension

Sainuchral Borjigin ${ }^{1}$, Guang-ming Niu ${ }^{1}$, and Lizhi $\mathrm{Xie}^{2}$

${ }^{1}$ Department of Radiology, Affiliated Hospital of Inner Mongolia Medical University, Hohhot, China, ${ }^{2}$ GE Healthcare, China, Beijing, China

QSM sequence can quantitativeaccess tissue magnetic susceptibility, and applied to understand the distribution of iron content in the cerebral microbleeds. In the study, 3.0T MRI was applied to investigate intracerebral micro-hemorrhage in 33 patients with essential hypertension. QSM sequence was also adminstrated to quantitatively analyze the magnetic susceptibility of CMBs in hypertensive patients. The resultes indicated a significant difference between the magnetic susceptibility of the lesions in the basal ganglia and that of the lesions in the subcortical and infratentorial regions, respectively. Moreover, there was a positive correlation observed between the lesion area and the susceptibility value in each region. 
${ }^{1}$ Cardiovascular Research and Development, Fraunhofer MEVIS, Berlin, Germany, ${ }^{2}$ Institute for Computational and Imaging Science in Cardiovascular Medicine, Charité Universitaetsmedizin Berlin, Berlin, Germany, ${ }^{3}$ Dept. of Radiology Medical Physics, University Medical Center Freiburg, Freiburg, Germany, ${ }^{4}$ Neurology and Clinical Neurophysiology, University Medical Center Freiburg, Freiburg, Germany, ${ }^{5}$ Cardiovascular Research and Development, Fraunhofer MEVIS, Bremen, Germany

The quantitative analysis of vessel wall thickness in the carotid bifurcation region based on blackblood MR imaging is a difficult problem because of partial volume effects, strong variations in surrounding tissue contrast, and flow artifacts, which frequently appear in the carotid bulbus. The abstract presents an automatic vessel wall thickness quantification approach based on a segmentation that integrates the information from a TOF-MRA sequence and a MSD-T2-weighted variable flip angle 3D MRI sequence without changing the image data.

The method is validated using a crossection-wise comparison with contours corrected by 6 different observers on 60 vessel crossections.

The results show a good overall agreement. Major deviations between observers and automatic segmentation occur in regions with strong artefact.

Evaluation of cerebral perfusion changes using arterial spin labeling after carotid endarterectomy

Huimin $\mathrm{Xu}^{1}$, Ying Liu ${ }^{1}$, and Huishu Yuan ${ }^{1}$

${ }^{1}$ Peking University Third Hospital, Peking, China

Carotid endarterectomy (CEA) is a common surgical method for patients with significant carotid stenosis. The evaluation of perioperative cerebral perfusion is extremely important to evaluate the efficacy of CEA. It can provide information of both the etiology of stroke due to carotid stenosis and cerebral hemodynamic changes after CEA.[1] Arterial spin labeling (ASL) is a magnetic resonance imaging (MRI) technique that uses the protons of arterial blood water molecules as endogenous tracers to evaluate cerebral blood flow (CBF) noninvasively and repeatedly.[2] Territorial ASL (t-ASL), a modified ASL technique, allows independently labeling a single brain-feeding artery to visualize its cerebral blood perfusion territory. [3] Therefore, we supposed that ASL techniques have ability to provide more information for the evaluation of cerebral perfusion changes pre- and post- CEA.

Feasibility of cerebral blood volume mapping by using velocity selective arterial spin labeling with 3D radial gradient echo acquisition

Mulan Jen ${ }^{1}$, James $\mathrm{H} \mathrm{Holmes}^{2}$, Patrick A Turski², and Kevin M Johnson ${ }^{1,2}$

Theoretically, velocity selective arterial spin labeling (VS-ASL) can be utilized for obtaining cerebral blood volume (CBV). However, challenges such as large vessel contamination and readout dependent artifacts make it difficult to obtain quantitative values. This work investigates the feasibility of VS-ASL CBV mapping by using three-dimensional gradient echo radial acquisition. Measured CBV's were found to be comparable to previous literature, however results highlight the potential resolution dependence of low SNR ASL based CBV mapping

Quantitative assessment of USPIO uptake in cerebral small vessel disease

Michael Jonathan Thrippleton ${ }^{1}$, Gordon Blair ${ }^{1}$, Maria Valdes-Hernandez ${ }^{1}$, Andreas Glatz ${ }^{1}$, Iona Hamilton ${ }^{1}$, Fergus Doubal ${ }^{1}$, lan Marshall ${ }^{1}$, Scott I K Semple ${ }^{2}$, David E Newby ${ }^{2}$, Alex Vesey $^{2}$, and Joanna M Wardlaw ${ }^{1}$

${ }^{1}$ Centre for Clinical Brain Sciences, University of Edinburgh, Edinburgh, United Kingdom, ${ }^{2}$ Centre for Cardiovascular Science, University of Edinburgh, Edinburgh, United Kingdom

A method for assessing cerebral blood volume and inflammation in small vessel disease was piloted, employing $T_{1}$ relaxometry and USPIO contrast agent. 12 stable patients with a history of minor stroke were recruited and scanned pre- and post-contrast, and at 24-30 hours. $R_{1}$ increased following USPIO administration and remained elevated at $24-30$ hours; apparent cerebral blood volume did not change significantly in any tissue at 24-30 hours versus post-contrast $(\mathrm{p}>0.20)$. Our work demonstrates the feasibility of $T_{1}$ relaxometry for quantitative assessment of USPIO distribution but larger studies are required to determine whether detectable inflammatory uptake occurs.

Simultaneous depiction of arterial and venous vasculature at high spatial resolution with $3 \mathrm{D}$ spoiled gradient multi-echo acquisition at $7 \mathrm{~T}$

Hana Hlavata ${ }^{1}$, Mauro Costagli ${ }^{2}$, Janine M Lupo $^{3}$, Emiliano Perticaroli ${ }^{4}$, Michela Tosetti ${ }^{2}$, and Mirco Cosottini ${ }^{5}$

${ }^{1}$ IRCCS Stella Maris, Pisa, Italy, ${ }^{2}$ Imago 7 Research Center, IRCCS Stella Maris, Pisa, Italy, ${ }^{3}$ University of California San Francisco, San Francisco, CA, United States, ${ }^{4}$ Azienda Ospedaliero-Universitaria Pisana, Pisa, Italy, ${ }^{5}$ University of Pisa, Pisa, Italy

The simultaneous depiction of both arterial and venous vasculature has recently been demonstrated by using multi-echo sequences. We quantitatively and qualitatively assessed the simultaneous representation of intracranial arteries and veins at a higher resolution than previously reported using a customized 3D spoiled gradient multi-echo sequence at $7 \mathrm{~T}$. Such custom sequence had an overall better capability of depicting the arterial vasculature compared to conventional time-of-flight (TOF) arteriography. On the contrary, veins were in general better depicted by conventional susceptibility-weighted venography, however the custom multi-echo sequence provided superior quality images of the superficial veins. 
Quantitative Inhomogeneous Magnetization Transfer (ihMT) in Acute Stroke: A Preliminary Study

Chien-Yuan Eddy Lin ${ }^{1}$, Xiaocheng Wei ${ }^{2}$, Bing Wu ${ }^{2}$, Yen-Chien Wu ${ }^{3}$, and Chi-Jen Chen $^{3}$

Inhomogeneous magnetization transfer (ihMT) has been recent developed and has shown promise for myelin-specific imaging. The abnormal lipid pattern in the myelin of the white matter has been observed and could play an important role on ischemic lesion after stroke. The aim of this study was to investigate the myelin change within ischemic lesions using inMT. In our presentative case, the abnormal area on DWI appears larger than that on inMT. The difference may result from heterogeneous tissue characteristic in acute ischemic brain, which might evolve with the time after symptom onset and indicate a different clinical outcome.

Banding free DANTE prepared vessel wall imaging incorporating multiple acquisition and phase cycling

Jianxun $\mathrm{Qu}^{1}$, Tianye $\mathrm{Lin}^{2}$, Xiaocheng $\mathrm{Wei}^{1}$, Bing $\mathrm{Wu}^{1}$, and Feng Feng ${ }^{2}$

1891

${ }^{1}$ GE Healthcare, Shanghai, China, ${ }^{2}$ Radiology, Peking Union Medical College Hospital, Beijing, China

Phase cycling was used to address the banding artefact in DANTE prepared black blood imaging. Simulation, phantom and in-vivo experiment were performed to illustrate and validate the effectiveness

Intravascular Signal Suppression and Micro-Vascular Signal Mapping obtained from ASL Perfusion Imaging with DANTE Pulse

Yasuhiro Fujiwara ${ }^{1}$, Hirohiko Kimura ${ }^{2}$, Shota Ishida ${ }^{3}$, Masayuki Kanamoto ${ }^{3}$, Naoyuki Takei ${ }^{4}$, Tsuyoshi Matsuda $^{5}$, R Marc Lebel ${ }^{6}$, and Toshiki Adachi ${ }^{3}$

${ }^{1}$ Department of Medical Imaging, Faculty of Life Sciences, Kumamoto University, Kumamoto, Japan, ${ }^{2}$ Radiology, University of Fukui, Fukui, Japan, ${ }^{3}$ Radiological Center, University of Fukui Hospital, Fukui, Japan, ${ }^{4}$ Global MR Applications and Workflow, GE Healthcare Japan, Tokyo, Japan, ${ }^{5}$ Division of Ultrahigh Field MRI, Institute for Biomedical Science, Iwate Medical University, Iwate, Japan, ${ }^{6}$ GE Healthcare, Calgary, Canada

In ASL perfusion imaging, the signal from the label that is still present in larger arteries at the time of imaging causes vascular artifact, which reduces the accuracy of quantification of cerebral blood flow. The purpose of this study is to eliminate the vascular artifacts in larger vessels using the delays alternating with nutation for tailored excitation (DANTE) pulse as vascular crushing gradients and to evaluate the efficiency of the DANTE pulse. The optimized DANTE pulse makes it possible to suppress the vascular signal depending on the flow velocity, which decreased the ASL signal of the arterial region. The relative vascular signal mapping may be helpful to reveal altered hemodynamic state, since the amount of suppressed signal directly associate with flow velocity.

Visualizing the Lenticulostriate Arteries at 3T with a Dual-Echo White-Blood and Black-Blood Imaging Technique

M Louis Lauzon ${ }^{1,2}$

${ }^{1}$ Radiology and Clinical Neurosciences, Hotchkiss Brain Institute, University of Calgary, Calgary, AB, Canada, ${ }^{2}$ Seaman Family MR Research Centre, Foothills Medical Centre, Calgary, $A B$, Canada

A dual-echo white-blood (WB) and black-blood (BB) imaging technique was developed to visualize the lenticulostriate arteries at 3T. The WB echo, effectively a flow-compensated timeof-flight image, and the flow-sensitized BB echo complement each other such that using these two inherently co-registered echoes in unison helps to better depict and delineate the vessels.

Mirja Wolf ${ }^{1}$, Stephan Kaczmarz ${ }^{2,3}$, Jens Göttler ${ }^{2,3}$, Claus Zimmer $^{3}$, Christian Schwarzbauer ${ }^{1}$, and Christine Preibisch ${ }^{3,4}$

${ }^{1}$ Applied Sciences and Mechatronics, University of Applied Sciences Munich, Munich, Germany, ${ }^{2}$ Yale University, New Haven, CT, United States, ${ }^{3}$ Neuroradiology, Technical University of Munich, Munich, Germany, ${ }^{4}$ Clinic for Neurology, Technical University of Munich, Munich, Germany 
High-grade internal carotid artery stenosis is a widespread cause of ischemic stroke. A recent study proposed an iterative correlation-based image analysis method allowing quick identification of regions with perfusion deficits in dynamic susceptibility contrast magnetic resonance imaging. Here, we evaluate whether correlation-based methods can successfully detect perfusion delay in brain tissue in patients with asymptomatic carotid artery stenosis. In addition, we employed a subtraction method to segment regions of delayed perfusion. Volumes segmented by the subtraction method showed good spatial correspondence with dynamic susceptibility contrast-based time-to-peak maps.

A phantom set-up to evaluate slow flow artefacts in vessel wall MRI of intracranial aneurysms

Eva L. Leemans ${ }^{1,2}$, Bart M.W. Cornelissen ${ }^{1,2,3}$, Rebecca J.I. Bot ${ }^{1,4}$, Gerben A. te Rieg o/g Scholten ${ }^{3}$, Charles B.L.M. Majoie ${ }^{2}$, Bram F. Coolen ${ }^{1}$, Henk A. Marquering $^{1,2}$, and Cees H. Slump ${ }^{3}$

${ }^{1}$ Biomedical engineering and physics, AMC, Amsterdam, Netherlands, ${ }^{2}$ Radiology and Nuclear Medicine, AMC, Amsterdam, Netherlands, ${ }^{3}$ MIRA Institute for Biomedical Engineering and Technical Medicine, University of Twente, Enschede, Netherlands, ${ }^{4}$ Biomedical Sciences, VU university, Amsterdam, Netherlands

To reliably assess the vessel wall, adequate blood suppression is of high importance. Most black-blood vessel wall MRI sequences rely on flow sensitive signal attenuation. Intraaneurysmal flow is often chaotic with slower flows near the aneurysm wall. Therefore, certain regions within the aneurysm might be more difficult to suppress. In this study we developed a phantom set-up to evaluate slow flow artefacts in vessel wall MRI of intracranial aneurysms. This setup allows to study the sensitivity of different vessel wall MRI sequences (e.g. DANTE, MSDE, 3D TSE) in relation to specific aneurysm geometries and contrast agent concentrations.

Mean Transit Time as a Marker of Vascular Change in Asymptomatic White Matter Disease

Blake E. Dewey ${ }^{1,2}$, Xiang Xü2,3, Linda Knutsson ${ }^{3,4}$, Amod Jog $^{5}$, Jerry L. Prince ${ }^{1,3}$, Peter B. Barker ${ }^{2,3}$, Peter C. M. van Zij $\left.\right|^{2,3}$, and Paul Nyquist ${ }^{6}$

${ }^{1}$ Department of Electrical and Computer Engineering, Johns Hopkins University, Baltimore, MD, United States, ${ }^{2}$ Kirby Center for Functional Brain Imaging, Kennedy Krieger Institute, Baltimore, MD, United States, ${ }^{3}$ Department of Radiology and Radiological Sciences, Johns Hopkins University, Baltimore, MD, United States, ${ }^{4}$ Department of Medical Radiation Physics, Lund University, Lund, Sweden, ${ }^{5}$ Athinoula A. Martinos Center for Biomedical Imaging, Harvard Medical School, Boston, MA, United States, ${ }^{6}$ Department of Neurology, Johns Hopkins University, Baltimore, $M D$, United States

White matter hyperintensity (WMH) has been associated with cognitive and motor decline. The condition is of presumed vascular origin and may involve decreased blood brain barrier (BBB) integrity. A double contrast injection scheme was used to access both dynamic contrast enhanced (DCE) and dynamic susceptibility contrast (DSC) perfusion-related parameters in an asymptomatic population with high prevalence of WMH. The mean transit time (MTT) was found to be significantly prolonged (5.87, $\mathrm{p}=0.002)$ in $\mathrm{WMH}$ when compared to normal appearing white matter and that there was no significant change in $\mathrm{K}^{\text {trans }}(0.018, \mathrm{p}=0.351)$ between the lesions and the white/gray matter

3D multi-shot(ms) Spin-Stimulated Echo(STE) EPI sequence Technique for accurate T1 quantification of contrast uptake within vulnerable large artery plaque

Seong-Eun Kim ${ }^{1}$, J Scott Scott McNalley ${ }^{1}$, Adam de Havenon ${ }^{2}$, Dennis L Parker ${ }^{1}$, and Gerald S Treiman ${ }^{3}$

${ }^{1}$ UCAIR, Department of Radiology and Imaging Sciences, University of Utah, Salt Lake City, UT, United States, ${ }^{2}$ Department of Neurology, University of Utah, Salt Lake City, UT, United States, ${ }^{3}$ Department of Veterans Affairs, VASLCHCS, Salt Lake City, UT, United States

Large artery atherosclerotic disease is one of the most common causes of ischemic stroke. Post-contrast plaque enhancement (PPE), which may result from endothelial dysfunction or be secondary to intraplaque inflammation, is a vulnerable plaque feature that correlates with increased stroke risk independent of stenosis. Although PPE can be detected with vessel wall MRI better quantitative methods to measure PPE are needed. This work presents a new $3 \mathrm{D}$ high resolution $\mathrm{T}_{1}$ mapping technique for accurate $\mathrm{T}_{1}$ quantification of contrast uptake within vulnerable large artery plaque.

Cross-vendor comparison of cerebrovascular reactivity MRI using hypercapnia challenge

Peiying Liu ${ }^{1}$, Dengrong Jiang ${ }^{1}$, Yang Li ${ }^{1}$, Xirui Hou ${ }^{1}$, Jay J Pillai ${ }^{1}$, and Hanzhang Lu ${ }^{1}$

${ }^{1}$ Johns Hopkins University School of Medicine, Baltimore, MD, United States

Cerebrovascular reactivity (CVR) is an important marker of the brain's vascular health. BOLD MRI with hypercapnia challenge has been shown to be a promising method to measure CVR in various cerebrovascular conditions. To prepare this method for larger-scale multi-site studies, a cross-vendor comparison was performed to evaluate the variability of this CVR mapping method across different scanner platforms. CVR, bolus arrival time and functional connectivity networks were found to be measured reliably from both Philips and Siemens $3 T$ scanners using this method. Although CVR was highly correlated between the two scanners, there was slight difference in CVR values between them. 
Different parameters of Dynamic contrast-enhanced magnetic resonance imaging (DCE-MRI) has been provided for noninvasive evluating gliomas pathology status. But the diagnostic performance of those parameters were variant among the recent reports during different type of gliomas. This study included 17 DCE-MRI studies regarding to differentiating different types of gliomas. The meta-analysis results demonstrated that Ve parameter of DCE-MRI has higher AUC in distinguishing HGGs from LGGs, gradeII from grade III and grade III from gradeIV,respectively, Ktrans has higher AUC in distinguishing gradeIIfrom grade IV; Among all the pamameters from DCE, Ktrans,Ve,Vp showed higher diagnostic performance in distinguishing different grade of gliomas.

Remote effects of hemodynamic impairment on network efficiency in chronic steno-occlusive disease of the anterior circulation: A resting-state functional MRI study

Junjie $\mathrm{Wu}^{1}$, Seena Dehkharghani ${ }^{2}$, Fadi Nahab ${ }^{3}$, Jason W. Allen ${ }^{1}$, Ranliang $\mathrm{Hu}^{1}$, and Deqiang Qiu ${ }^{1}$

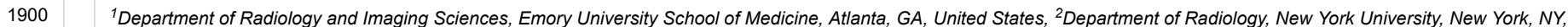
United States, ${ }^{3}$ Department of Neurology, Emory University School of Medicine, Atlanta, GA, United States

In this abstract we explored remote effects of cerebrovascular hemodynamic impairment on the efficiency of functional connectivity in patients with chronic, anterior circulation stenoocclusive disease. We further evaluated the correlation between network efficiency and cerebrovascular reactivity (CVR), a measure of cerebral hemodynamics.

Visualizing Wall Enhancement Over Time in Unruptured Intracranial Aneurysms Using 3D Vessel Wall Imaging

Bing Tian ${ }^{1}$, Shahed Toossi ${ }^{1}$, Laura Eisenmenger ${ }^{1}$, Christopher Hess ${ }^{1}$, and David Saloner ${ }^{1}$

$1901 \quad{ }^{1}$ UCSF, San Francisco, CA, United States

Advances in vessel wall imaging techniques using high-resolution MR sequences now allow for improved visualization of the walls of intracranial vessels. In this study, we present results obtained with a 3D SPACE to visualize the walls of intracranial aneurysms and to grade the extent of aneurysm wall enhancement in subjects whose aneurysms were monitored over time. Our studies showed that visualization of the aneurysm wall is significantly better on post-contrast images than on pre-contrast images, and the majority of unruptured aneurysms show wall enhancement. Furthermore, we found the wall enhancement scores to remain essentially unchanged on follow up studies.

Comparison of Cerebral Blood Flow in a Rat Model of Hypertension and Age-Matched Controls

Abinand C. Rejimon ${ }^{1}$, Diana Y. Lee ${ }^{1}$, Rebecca L. McPherson ${ }^{2}$, Mustapha Bouhrara ${ }^{1}$, Akshay Naraine ${ }^{2}$, Kenneth W. Fishbein ${ }^{1}$, Olga V. Fedorova ${ }^{2}$, and Richard G. Spencer

${ }^{1}$ Laboratory of Clinical Investigation, Magnetic Resonance Imaging and Spectroscopy Section, National Institute on Aging, Baltimore, MD, United States, ${ }^{2}$ Laboratory of Cardiovascular Sciences, National Institute on Aging, Baltimore, MD, United States

Continuous arterial spin labeling (CASL) was used to quantify and compare cerebral blood flow (CBF) in Dahl salt-sensitive (DSS) and Sprague-Dawley (SD) rats. CBF quantification was greatly facilitated through use of the recently-introduced NESMA non-local noise reduction filter. A blunted response to hypercapnia was observed in the DSS rats. These results demonstrate the dysregulation of cerebral vasodilatory responses in hypertension, and may have important implications in the understanding of the vascular basis for cognitive impairment in humans.

Traditional Poster

\section{Neurovascular Clinical Studies}

Quantitative Susceptibility Mapping analysis of cerebral microbleeds in hypertensive patients

Jinyu Song ${ }^{1}$, Shengzhang $\mathrm{Ji}^{1}$, Junjie Ren ${ }^{1}$, Ling $\mathrm{Li}^{1}$, and Zhizheng ZHUO ${ }^{2}$

${ }^{1}$ The 4 th center hospital of TianJin, China, TianJin, China, ${ }^{2}$ Philips Healthcare Beijing China, Beijing, China

Cerebral microbleeds (CMBs) was often found in hypertensive patients.Quantitative susceptibility mapping (QSM) could detect iron-containing lesions with high sensitivity and spatial accuracy in the presence of potentially confounding tissue abnormalities. The results of retrospective study showed that there was significant difference in CMBs between the hypertensive group and the control group.So the conclussion is MR quantitative susceptibility could directly explicate the evolution law of CMBs in hypertensive patients, timely intervention of hypertension could reduce the occurrence of CMBs. 
Coupling of the regional cerebral blood flow and resting state functional connectivity in stroke patients with unilateral middle cerebral artery infarction

jiaxin zeng ${ }^{1}$, yuan $x^{1 a o^{1}}{ }^{1}$, biqiu tang ${ }^{1}$, lu liu ${ }^{1}$, wenjing zhang ${ }^{1}$, jieke liu ${ }^{1}$, and su lui ${ }^{1}$

${ }^{1}$ Radiology, West China Hospital, Chengdu, China

Coupling of rCBF and FC in stroke patients with unilateral middle cerebral artery infarction reveals positive correlation between rCBF and FC, especially in the ipsilateral hemisphere, which indicates improving the CBF in ipsilateral hemisphere in stroke patients.

Language reorganization in pre-and post-operative drug refractory extra temporal lobe epilepsy patients: An fMRI based study

Kapil Chaudhary ${ }^{1}$, Senthil Kumaran ${ }^{2}$, Sarat $\mathrm{P}$ Chandra $^{3}$, Ashima Nehra Wadhawan ${ }^{4}$, and Manjari Tripathi ${ }^{1}$

${ }^{1}$ Department of Neurology, All India Institute of Medical Sciences, New Delhi, India, ${ }^{2}$ Department of NMR and MRI Facility, All India Institute of Medical Sciences, New Delhi, India, ${ }^{3}$ Department of Neuro-Surgery, All India Institute of Medical Sciences, New Delhi, India, ${ }^{4}$ Department of Clinical Neuropsychology, All India Institute of Medical Sciences, New Delhi, India

Drug refractory epilepsy (DRE) patients have atypical language lateralization with ipsilateral and contra lateral hemispheric lesions and pathological abnormalities. Such kind of patients may have different language recovery after surgery. In this study, we have used a standardized Hindi-language paradigm using semantic, syntactic, judgement and comprehension components for testing in the North-Indian population. We observed greater improvement in language skills in ETLE-patients with correspondingly greater recruitment of the bilateral hemisphere.

The value of diffusion tensor imaging (DTI) and tractography (DTT) in lumbar nerve roots display and lumbar disc herniation assessment

Qingwei Song ${ }^{1}$, Shaowei Zheng ${ }^{1}$, Yu Song ${ }^{1}$, Qiang Wei ${ }^{1}$, Bin Xu ${ }^{1}$, and Lizhi Xie ${ }^{2}$

${ }^{1}$ The first affiliated hospital of Dalian medical university, Dalian, China, ${ }^{2}$ GE Healthcare, Beijing, China

This is a prospective study on lumbar disc herniation patient and healthy control with diffusion tensor imaging (DTI) and tractography (DTT). We obtained a high success rate (>90\%) of achieving the DTI with tractography of lumbar nerve roots was in this study, and revealed that DTI and DTT technique can both display intensity and morphology changes in the compressed areas of lumbar nerve roots. DTI with tractography provides an abundant diagnostic information with specificity on both qualitative- and quantitative-wise, which is great helpful to assess the disorders with lumbar nerve root compression.

The effect of small vessel disease lesions on structural brain network

Xiaopei Xu1 ${ }^{1}$, Kui Kai Lau ${ }^{2}$, Yuen Kwun Wong ${ }^{2}$, Henry KF Mak ${ }^{1,3}$, Queenie Chan ${ }^{4}$, and Edward S Hui ${ }^{1,3}$

${ }^{1}$ Department of Diagnostic Radiology, The University of Hong Kong, HKSAR, China, ${ }^{2}$ Department of Medicine, The University of Hong Kong, HKSAR, China, ${ }^{3}$ The State Key Laboratory of Brain and Cognitive Sciences, The University of Hong Kong, HKSAR, China, ${ }^{4}$ Philips Healthcare, HKSAR, China

We aim to explore the influences of cerebral small vessel disease (SVD) lesion on the structural brain network of patients with transient ischemic attack or acute stroke. Our results demonstrated that the efficiency of both global and regional network of patients with SVD were lower compared to those without, and that higher total SVD burden was significantly associated with decreased network efficiency. These results suggested that both presence and severity of SVD related lesion load is associated with disrupted network organization, and brain network analysis is a sensitive method to monitor and assess SVD.

7T TOF-MRA showed a decreased contrast-to-noise ratio of the lenticulostriate arteries in hemispheres with unilateral lacunar stroke

Qingle Kong ${ }^{1,2,3}$, Haiqiang Qin $^{4}$, Jing $\mathrm{An}^{5}$, Yan Zhuo ${ }^{1,3}$, and Zihao Zhang ${ }^{1,3}$

${ }^{1}$ State Key Laboratory of Brain and Cognitive Science, Institute of Biophysics, Chinese Academy of Sciences, Beijing, China, ${ }^{2}$ University of Chinese Academy of Sciences, Beijing, China, ${ }^{3}$ The Innovation Center of Excellence on Brain Science, Chinese Academy of Sciences, Beijing, China, ${ }^{4}$ Department of Neurology, Beijing Tiantan Hospital, Capital Medical University, Beijing, China, ${ }^{5}$ Siemens Shenzhen Magnetic Resonance Ltd., Shenzhen, China

7T TOF-MRA has demonstrated an exquisite capacity for imaging the lenticulostriate artery (LSA) due to its high spatial resolution and in-flow effect. However, due to the morphological variability of the LSA, a clinical application is needed to identify abnormalities of this vessel. In this study, we analyzed the LSA using 7T TOF-MRA on patients with unilateral lacunar stroke. Comparing the results with other morphological parameters, we found that a reduced contrast-to-noise ratio (CNR) was a more sensitive parameter for reflecting impairment of the LSA on the ipsilateral side of the lacunae. 
The usefulness of thick slice-basal ganglia rapid pCASL for acute ischemic stroke.

Daisuke Oura ${ }^{1}$, Yoshimasa Niiya² ${ }^{2}$ Masahito Kawabori ${ }^{3}$, Shinpei Sato ${ }^{1}$, Kadoya Tomoka $^{1}$, and Takumi Yokohama ${ }^{1}$

$1909 \quad{ }^{1}$ Department of Radiology, Otaru General Hospital, Otaru, Japan, ${ }^{2}$ Department of Neurosurgery, Otaru General Hospital, Otaru, Japan, ${ }^{3}$ Department of Neurosurgery, Hokkaido University Graduate School of Medicine, Sapporo, Japan

In this study, we demonstrated the efficacy of the thick slice-basal ganglia pCASL (TB-pCASL) for acute ischemic stroke. The limited scan range and selection of thick slice retain signal noise to ratio (SNR) even in approximately $1 \mathrm{~min}$ scan. TB-pCASL can rapidly estimate an ischemic region corresponding occlusion-stenosis region, and to combine with DWI can depict penumbra within $2 \mathrm{~min}$. TB-pCASL is reliable and useful tool for diagnosis of acute ischemic stroke in the emergency medical field.

Imaging Patterns and Implications of Time-of-Flight Magnetic Resonance Angiography in Intracranial Atherosclerotic Stenosis

Jinhao Lyu ${ }^{1}$, Ning $\mathrm{Ma}^{2}$, Xiaoxiao Ma ${ }^{1}$, Lin Ma ${ }^{1}$, and Xin Lou ${ }^{1}$

${ }^{1}$ Department of Radiology, Chinese PLA General Hospital, Beijing, China, ${ }^{2}$ Department of Interventional Radiology, Beijing Tiantan Hospital, Beijing, China

The imaging pattern and implication of intracranial atherosclerotic stenosis on TOF MRA had not been fully understood. In patients with middle cerebral artery stenosis, we had used high-resolution vessel wall imaging to evaluate plaque morphology and conventional angiography to evaluate cerebral hemodynamics in groups with different TOF MRA pattern. We had found that the TOF MRA pattern was associated with stenosis percentage, the middle cerebral artery branch signal intensity distal to the site of stenosis on TOF MRA was associated with hemodynamic impairments and was determined by the status of antegrade flow.

Visualization of lenticulostriate arteries by high-resolution vessel wall imaging on a 3T MRI system: a comparison study between subjects with and without lacunar infarction in the basal ganglia region

Weiwei Xie ${ }^{1,2}$, Tianyi Qian ${ }^{3}$, Jinxia Zhu³ ${ }^{3}$ Wen Shen ${ }^{2}$, and Shuang $\mathrm{Xia}^{2}$

${ }^{1}$ First Central Clinical College of TianJin Medical University, Tianjin, China, ${ }^{2}$ Department of Radiology, Tianjin First Central Hospital, Tianjin, China, ${ }^{3}$ MR Collaboration NEA, Siemens Healthcare, Beijing, China

The lenticulostriate artery may be associated with lacunar infarction. We aimed to visualize the lenticulostriate artery and explore the correlation between the number and length of lenticulostriate arteries and the number and volume of lacunar infarctions using High-Resolution Vessel Wall Imaging (HR-VWI) on a 3T MR scanner. The results indicated that the length of the lenticulostriate artery was associated with the number of lacunar infarctions. The lenticulostriate artery can be well visualized with HR-VWI, and the length of the artery may be associated with lacunar infarction.

Ferumoxytol vascular imaging of the central nervous system in pediatric patients compared to noncontrast MRA: a single center's initial experience

Josephine Ndolo ${ }^{1}$ and Aashim Bhatia ${ }^{1}$

1912

${ }^{1}$ Vanderbilt Children's Hospital, Nashville, TN, United States

Ferumoxytol-enhanced MRA allows for improved visualization and characterization of vascular pathologies in the brain compared to noncontrast MRA.

Evaluation of Treatment Effect for Saccular Aneurysm by DANTE T1-SPACE

Yasutaka Fushimi ${ }^{1}$, Hidehisa Nishi ${ }^{2}$, Akira Ishii $^{2}$, Tomohisa Okada ${ }^{3}$, Akira Yamamoto ${ }^{1}$, Tsutomu Okada ${ }^{1}$, Takuya Hinoda ${ }^{1}$, Takayuki Yamamoto ${ }^{1}$, Hikaru Fukutomi ${ }^{1}$, Yusuke Yokota ${ }^{1}$, Sonoko Oshima ${ }^{1}$, John Grinstead ${ }^{4}$, Sinyeob Ahn ${ }^{5}$, and Kaori Togashi ${ }^{1}$

${ }^{1}$ Department of Diagnostic Imaging and Nuclear Medicine, Kyoto University Graduate School of Medicine, Kyoto, Japan, ${ }^{2}$ Department of Neurosurgery, Kyoto University Graduate School of Medicine, Kyoto, Japan, ${ }^{3}$ Human Brain Research Center, Kyoto University Graduate School of Medicine, Kyoto, Japan, ${ }^{4}$ Siemens Healthineers, Portland, OR, United States, ${ }^{5}$ Siemens Healthineers, San Francisco, CA, United States

The purpose of this study is to evaluate the therapeutic effect by FD stent on DANTE T1-SPACE imaging by comparing contrast enhanced 3D T1-weighted imaging. Patients underwent MR imaging for evaluation of pre-, post FD stent placement, and follow-up at 3T MR scanners were included. DANTE T1-SPACE of aneurysm showed dark intensity in pre-treatment study, and higher intensity in follow-up study, then darker intensity later. Enhancement ratio showed high value in pre-treatment study, and low value in follow-up study. DANTE T1SPACE of aneurysm and enhancement ratio were negatively associated in all patients and exams. 
Comparison of Image Reconstruction Algorithms of "Flexible PET/MRI" with and without Non-Local Mean Regularization.

Yasutaka Fushimi ${ }^{1}$, Tomohisa Okada ${ }^{2}$, Mizue Suzuki ${ }^{1}$, Takuya Hinoda $^{1}$, Ryusuke Nakamoto ${ }^{1}$, Yuji Nakamoto $^{1}$, and Kaori Togashi ${ }^{1}$

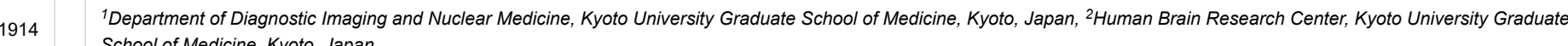
School of Medicine, Kyoto, Japan

Flexible PET (fxPET) is a prototype of MR-compatible mobile PET system. We have compared two different image reconstruction algorithms called as dynamic row-action maximumlikelihood algorithm (DRAMA) and DRAMA with non-local mean (DRAMA-NLM) by evaluating image quality and SUV. NLM filter can reduce artifacts and noise with keeping contrast. The image quality was almost similar between two algorithms and DRAMA-NLM shows significantly higher SUV than DRAMA.

Noninvasive measurements of human brain temperature in patients with arteriovenous malformations using magnetic resonance spectroscopy

Takashi Inoue $^{1}$, Tomohisa Ishida ${ }^{1}$, Shunsuke Omodaka ${ }^{2}$, Miki Fujimura ${ }^{2}$, Masayuki Ezura $^{1}$, Hiroshi Uenohara $^{1}$, and Teiji Tominaga ${ }^{3}$

${ }^{1}$ Neurosurgery, Sendai Medical Center, Sendai, Japan, ${ }^{2}$ Neurosurgery, Kohnan Hospital, Sendai, Japan, ${ }^{3}$ Neurosurgery, Tohoku University, Sendai, Japan

The present study investigated whether brain temperature measured by proton magnetic resonance (MR) spectroscopy can detect cerebral hemodynamic impairment in patients with arteriovenous malformations (AVMs) as shown by single photon emission computed tomography (SPECT). Brain temperature, cerebral blood flow, and cerebrovascular reactivity were measured using proton MR spectroscopy and SPECT in five healthy volunteers and six patients with AVMs. A significant correlation was observed between brain temperature difference (affected side - contralateral side) and cerebrovascular reactivity ratio (affected side/contralateral side) $(r=0.82, p=0.0480)$. Brain temperature measured by proton MR spectroscopy can detect cerebral hemodynamic impairment in patients with AVMs.

Cerebral blood flow in different severity degree moyamoya disease before and after artery bypass surgery

Chuanying Shi ${ }^{1}$, Weidong Liu ${ }^{1}$, Jianxun $\mathrm{Qu}^{2}$, Jipeng Wang ${ }^{1}$, and Chuanchen Zhang ${ }^{1}$

1916 ' 1 Liaocheng People's Hospital, Liaocheng, China, ${ }^{2}$ GE Healthcare, MR Research China, Beijing, China

The goal of the present study was to assess the improvement of CBF after STA-MCA bypass surgery in the mid, moderate, and severe regions based on Tmax value in Moyamoya disease patients. For this purpose, 13 Moyamoya patients were scanned using 3D pc-ASL, and the different perfusion territories were separated based on ASPECT scoring system. The results indicate that mid regions did not get obvious CBF improvement after the surgery and the mid patients did not need to get the bypass surgery.

The ischemic penumbra assessment using 3D ASL at different post labeling delays in patients with unilateral middle cerebral artery severe stenosis or occlusion

Du Hui ${ }^{1}$ and Miao yan wei ${ }^{2}$

${ }^{1}$ Radiology, The First Affiliated Hospital of Dalian Medical University, Dalian, Dalian, China, ${ }^{2}$ Radiology, The First Affiliated Hospital of Dalian Medical University, Dalian, China

It is necessary to consider the different PLDs to assess IP by 3D pCASL in ischemic cerebrovascular disease.

Pseudo Continuous ASL for Quantification of Regional Cerebral Hypoperfusion in Chronic Fatigue

Deirdre M McGrath ${ }^{1}$, Katija Khan ${ }^{2,3}$, Annalena Venneri², and lain D Wilkinson ${ }^{4}$

${ }^{1}$ Electronic and Electrical Engineering, University of Sheffield, Sheffield, United Kingdom, ${ }^{2}$ Department of Neuroscience, University of Sheffield, Sheffield, United Kingdom, ${ }^{3}$ Department of Clinical Medical Sciences, University of the West Indies, St Augustine, Trinidad and Tobago, ${ }^{4}$ Academic Radiology, University of Sheffield, Sheffield, United Kingdom

In this study pseudo continuous arterial spin labelling (pCASL) was employed to measure cerebral blood flow (CBF) in chronic fatigue syndrome (CFS) patients and healthy volunteers, to determine if CBF was reduced in CFS and in post-exertional malaise. Normalised regional CBF was found to be reduced in CFS for 11 brain regions, predominantly in the left hemisphere, including 8 previously identified regions, along with the left paracentral lobe, and the left and right posterior cingulate. Patients were asked to return for a second scan during post-exertional malaise, in which rCBF was found to be reduced in the left temporal pole. 
Hyperperfusion syndrome (HPS) is a rare but potentially fatal postoperative complication deriving from carotid artery stenting (CAS) and endarterectomy (CEA), while the pattern of postoperation cerebral blood flow (CBF) changes relating to HPS remained unclear. We had used pseudo continuous arterial spin labeling (pCASL) to monitore 4 consecutive time points at $24 \mathrm{~h}, 48 \mathrm{~h}, 72 \mathrm{~h}$, and $96 \mathrm{~h}$ after CEA and CAS in patients with internal carotid artery (ICA) stenosis. We had found that attention should be focused on 72 hours after CAS and 48 hours after CEA to control blood pressure and prevent potential HPS.

Territory Arterial Spin Labeling technique in evaluation of Superficial Temporal Artery to Middle Cerebral Artery Bypass Surgery in Moyamoya Disease

JING YUAN ${ }^{1}$, JIANXUN QU ${ }^{2}$, and PEIYI GAO ${ }^{1}$

The purpose of this study was to evaluate cerebral blood flow and territory through superficial temporal artery (STA) to middle cerebral artery (MCA) bypass in patients with Moyamoya disease after direct revascularization surgery using territory arterial spin labeling (tASL) technique. ASL and tASL scan were performed before and after bypassing surgery. our study demonstrated some bypasses can effectively supply blood flow into the brain and others cannot. tASL technique can selectively demonstrate perfusion territory through STA to MCA bypass. Thus, provide information about patency of STA to MCA bypass.

Silent Susceptibility Weighted MR Angiography; Clinical and Phantom Study

Takuya Fujiwara ${ }^{1}$, Yoshiyuki Watanabe ${ }^{1}$, Hisashi Tanaka ${ }^{1}$, Hiroto Takahashi ${ }^{1}$, Atsuko Arisawa ${ }^{1}$, Chisato Matsuo ${ }^{1}$, Masahiro Fujiwara ${ }^{1}$, Tetsuya Wakayama ${ }^{2}$, Pauline Worters ${ }^{2}$, Christopher $\mathrm{J} \mathrm{Hardy}^{3}$, and Noriyuki Tomiyama ${ }^{1}$

${ }^{1}$ Diagnostic and Interventional Radiology, Osaka University Graduate School of Medicine, Suita, Japan, ${ }^{2}$ GE Healthcare, MR Collaboration and Development, Tokyo, Japan, ${ }^{3}$ GE Global Research, Niskayuna, NY, United States

We compared silent susceptibility-weighted angiography (SWAN) with conventional SWAN (cSWAN) in the depiction of hemorrhagic lesions. We measured acoustic noise and performed phantom and clinical study using silent SWAN, cSWAN, and T2*-weighted images (T2*-WI). Acoustic noise of silent SWAN was significantly lower compared to cSWAN. In clinical and phantom study, the contrast-noise ratio (CNR) for silent SWAN and cSWAN were similar. The CNR for T2*-WI was lower than them. In clinical study, imaging quality was almost the same. T2*-WI had more artifact. Conventional SWAN may be replaced with silent SWAN which yields comparable imaging quality and lower acoustic noise.

Decreased Cerebral Blood Volume among those with Chronic Brain Insult in HIV

Karen $\mathrm{Chu}^{1}$, Ke Wei ${ }^{1}$, Thao Tran ${ }^{1}$, Timothy Yao ${ }^{1}$, Kim Shriner $^{2}$, and Kevin King ${ }^{1}$

${ }^{1}$ Huntington Medical Research Institutes, Pasadena, CA, United States, ${ }^{2}$ Phil Simon Clinic, Pasadena, CA, United States

Despite advances in medications and modern practices of immediate antiretroviral therapy, chronic HIV infection remains associated with brain insults, cognitive decline, and related neurological disorders. Reduced $\mathrm{N}$-acetyl-aspartete (NAA), a metabolic marker of neuronal injury, was associated with advanced age and lower CD4 nadir count in a chronic, asymptomatic HIV cohort. Using a novel, BOLD MR protocol incorporating hypercapnic and hyperoxic stimuli, NAA showed no relation to cerebrovascular reactivity (CVR) but was significantly correlated to cerebral blood volume (CBV). Our results may indicate future use of NAA and CBV as complementary non-invasive metrics to track brain health in HIV.

Cerebral blood flow in a resuscitated septic shock population: an ASL study

Marie Anne Richard ${ }^{1,2}$, Marie-Hélène Masse ${ }^{1,2}$, Frédérick D'Aragon ${ }^{1,2}$, Charles St-Arnaud $^{1}$, Michael Mayette ${ }^{1}$, Steven Palanchuck ${ }^{1}$, Etienne Croteau ${ }^{1,2}$, Neil Adhikari $^{3}$, William Fraser ${ }^{1,2}$ André Carpentier ${ }^{1,2}$, David Gauthier ${ }^{1}$, Luc Lanthier ${ }^{1}$, Matthieu Touchette ${ }^{1}$, Albert Lamontagne ${ }^{1}$, Jean Chénard ${ }^{1}$, Sangeeta Mehta ${ }^{4}$, Yanick Sansoucy ${ }^{1}$, François Lamontagne ${ }^{1,2}$, and Martin Lepage ${ }^{1,2}$

${ }^{1}$ Université de Sherbrooke, Sherbrooke, QC, Canada, ${ }^{2}$ Centre de recherche du CHUS, Sherbrooke, QC, Canada, ${ }^{3}$ Sunnybrook Health Sciences Centre, Toronto, ON, Canada, ${ }^{4}$ Mount Sinai Hospital, Toronto, ON, Canada

Reduced cerebral blood flow (CBF) is often blamed for sepsis-associated encephalopathy. The present study compares the $\mathrm{CBF}_{\text {and }}$ blood oxygen consumption ( $\mathrm{CMRO} \mathrm{O}_{2}$ ) of healthy subjects and resuscitated septic patients under vasopressor (norepinephrine) treatment. Methods used are pseudo-continuous arterial spin labeling (PCASL) and T2-relaxation-underspin-tagging (TRUST). We find that septic patients have elevated global and regional CBF, whereas $\mathrm{CMRO}_{2}$ seems reduced. Further studies are needed to elucidate the underlying mechanisms of this apparent uncoupling 


\section{Parkinson's Disease}

Determination of White Matter Tracts Implicated in Postural Gait Instability Disorder through Tract-Based Automated Analysis

Leon Qi Rong Ooi ${ }^{1}$, Chu Ning Ann ${ }^{1}$, Yun-Chin Hsu ${ }^{2}$, Chen-Hsiang Weng ${ }^{2}$, Ming-Ching Wen ${ }^{1}$, HuiHua Li ${ }^{3}$, Helmut Rumpel ${ }^{4,5}$, Eng King Tan ${ }^{1,5,6}$, Wen-Yih Isaac Teng ${ }^{2,7}$, and Ling Ling $\operatorname{Chan}^{4,5}$

${ }^{1}$ Department of Research, National Neuroscience Institute, Singapore, Singapore, ${ }^{2}$ Institute of Medical Device and Imaging, National Taiwan University College of Medicine, Taipei, Taiwan, ${ }^{3}$ Health Services Research Unit, Singapore General Hospital, Singapore, Singapore, ${ }^{4}$ Department of Diagnostic Radiology, Singapore General Hospital, Singapore, Singapore, ${ }^{5}$ Duke-NUS Medical School, Singapore, Singapore, ${ }^{6}$ Department of Neurology, Singapore General Hospital, Singapore, Singapore, ${ }^{7}$ Molecular Imaging Center, National Taiwan University, Taipei, Taiwan

Tract-Based Automated Analysis (TBAA) in Diffusion Tensor Imaging allows for the study of microstructural properties along the tracts in white matter. Diffusivity measures extracted from TBAA for various tracts of the brain were correlated to Tinetti Balance Scale scores in Parkinson's Disease and Postural Gait Instability Disorder patients, allowing identification of tracts of interest in the pathological study of the diseases.

The longitudinal changes in white matter of patients with Parkinson's disease as detected by using Fixel-Based Analysis

Shi-Ming Wang ${ }^{1}$, Sung-han Lin ${ }^{1}$, Chin-Song Lu ${ }^{2}$, Yi-Hsin Weng ${ }^{2}$, Yao-Liang Chen ${ }^{3}$, Shu-Hang Ng ${ }^{4}$, Yi-Ming Wu ${ }^{4}$, Chih-Chien Tsai ${ }^{1}$, Jacques-Donald Tournier ${ }^{5}$, and Jiun-Jie Wang ${ }^{1}$

${ }^{1}$ Medical Imaging and Radiological Sciences, Chang Gung University, Taoyuan, Taiwan, ${ }^{2}$ Neurology, Chang Gung Memorial Hospital, Taoyuan, Taiwan, ${ }^{3}$ Diagnostic Radiology, Keelung Chang Gung Memorial Hospital, Keelung, Taiwan, ${ }^{4}$ Diagnostic Radiology, Chang Gung Memorial Hospital, Taoyuan, Taiwan, ${ }^{5}$ Division of Imaging Sciences \& Biomedical Engineering, King's College London, London, United Kingdom

Parkinson's disease (PD) is a neurodegenerative disease as the result from the loss of cell in basal ganglia. Fixel-Based Analysis can qualified the fiber density and fibre-bundle crosssection in the white matter. The fiber density and fibre-bundle cross-section is feasible to interpret the microstructure changes in the brain of patients with PD. Therefore, the current study aimed to investigate the long-term white matter changes in Parkinson's disease by using Fixel-Based Analysis.

Quantifying nigral degeneration indicates rapid eye movement sleep behavior disorder being a predictor of Parkinson's disease

hiroto takahashi ${ }^{1}$, Yoshiyuki Watanabe ${ }^{2}$, Masahito Mihara ${ }^{3}$, Hideki Mochizuki ${ }^{3}$, Hiroyoshi Adachi ${ }^{4}$, Tian Liu ${ }^{5}$, Yi Wang ${ }^{5}$, and Noriyuki Tomiyama ${ }^{2}$

${ }^{1}$ Department of Radiology, Osaka University Graduate School of Medicine, Suita, Japan, ${ }^{2}$ Department of Diagnostic and Interventional Radiology, Osaka University Graduate School of Medicine, Suita, Japan, ${ }^{3}$ Department of neurology, Osaka University Graduate School of Medicine, Suita, Japan, ${ }^{4}$ Department of Psychiatry, Osaka University Graduate School of Medicine, Suita, Japan, ${ }^{5}$ Departments of Biomedical Engineering and Radiology, Cornell University, New York, NY, United States

RBD is thought to be prodromal Parkinson's disease (PD), so we aimed to assess the utility of rapid eye movement sleep behavior disorder (RBD) as a predictor of PD using neuromelanin imaging and quantitative susceptibility mapping (QSM). Our results indicated that RBD-related dopamine cell loss and iron deposition in the substantia nigra pars compacta occur in the developmental process of PD. Thus, we conclude that RBD is prodromal PD and quantifying nigral degeneration in RBD is useful in predicting PD.

Characterizing Neuronal Loss To Differentiate Parkinsonian Subtypes Using Automated Deep Grey Nuclear Volumetry

Chu-Ning Ann ${ }^{* 1}$, Bénédicte Maréchal ${ }^{\star 2,3,4}$, Eric Fang ${ }^{5}$, Jie-Xie Lim ${ }^{6}$, Celeste Chen ${ }^{1}$, Julian Gan ${ }^{7}$, Eng-King Tan ${ }^{1,8}$, and Ling-Ling Chan ${ }^{5,8}$

${ }^{1}$ National Neuroscience Institute, Singapore, Singapore, ${ }^{2}$ Advanced Clinical Imaging Technology, Siemens Healthcare AG, Lausanne, Switzerland, ${ }^{3}$ Department of Radiology, CHUV, Lausanne, Switzerland, ${ }^{4}$ LTS5, EPFL, Lausanne, Switzerland, ${ }^{5}$ Singapore General Hospital, Singapore, Singapore, ${ }^{6}$ Nanyang Technological University, Singapore, Singapore, ${ }^{7}$ Siemens Healthcare, Singapore, Singapore, ${ }^{8}$ Duke-NUS Medical School, Singapore, Singapore

Postural Instability Gait Disorder (PIGD), a Parkinson's Disease (PD) motor subtype, progresses rapidly with a higher prevalence of neurobehavioural changes. Using automated deep grey nuclear tissue classification combined with atlas-based segmentation, we investigated the performance of resulting estimated lesion load to aid differential diagnosis. Caudate lesion load in PIGD and idiopathic PD subtypes correlated with clinical balance and gait assessment. Combining caudate with abnormal white matter volumetric characterization further improved the discriminative power and could potentially support differential diagnosis of PD.

Ming ming Huang ${ }^{1}$ and Hui $\mathrm{Yu}^{1}$ 
Previous morphometric studies of Parkinson disease (PD) were mainly conducted by measuring gray matter volume and cortical thickness, and little attention has been paid to whether structure MRI improves PD diagnosis or helps differentiating between phenotypes, such as postural instability gait difficulty (PIGD) and tremor dominant (TD). From this study, compared with the control group, PIGD patients had significantly thinning cortical thickness in multiple brain regions, such as bilateral inferiorparietal, paracentral, postiocingulate, superiorfrontal, precuneus, caudalmiddlefrontal, superfrontal and right parsorbitals. TD patients had significantly thinning cortical thickness in left posteriocingulate, inferioparietal and right superiofrontal, superiortemporal, postcentral, precuneus, fusiform and parahippacampal . In addition, subcortical volume atrophy was identified in the bilateral hippocampus and bilateral amygdala of the patients with PIGD, only little bilateral hippocampus changes was found in the TD group.

Brain morphological changes in early-stage Parkinson's disease

Lanbo Wang ${ }^{1,2}$, Xishan $Y^{1}{ }^{1}$, Thyagarajan Subramanian ${ }^{3,4}$, Qing X Yang ${ }^{1,5}$, and Jianli Wang ${ }^{1}$

${ }^{1}$ Radiology, Penn State University College of Medicine, Hershey, PA, United States, ${ }^{2}$ Radiology, Shengjing Hospital of China Medical University, Shenyang, Liaoning, China, ${ }^{3}$ Neurology, Penn State University College of Medicine, Hershey, PA, United States, ${ }^{4}$ Neural \& Behavioral Sciences, Penn State University College of Medicine, Hershey, PA, United States, ${ }^{5}$ Neurosurgery, Penn State University College of Medicine, Hershey, PA, United States

At disease onset clinically, the motor symptoms and signs are usually asymmetric or unilateral in majority of Parkinson's disease (PD) patients. When disease progresses to a later stage, the asymmetry becomes less significant. The cause of this asymmetry, and the relationship between functional deficits and the structural changes in the brain are not clear. In this study, we investigated the morphological changes in the brain hemispheres corresponding to the early-onset and late-onset body sides through a longitudinal study on 24 early-stage PD patients. Significant atrophy was observed in the motor cortex and basal ganglia nuclei.

Disrupted Functional Connectivity and Network Topology in Early Parkinson's Disease

Karthik R Sreenivasan ${ }^{1}$, Virendra Mishra ${ }^{1}$, Zhengshi Yang ${ }^{1}$, Christopher Bird ${ }^{1}$, Xiaowei Zhuang ${ }^{1}$, Dietmar Cordes ${ }^{1,2}$, and Ryan R Walsh ${ }^{3}$

${ }^{1}$ Cleveland Clinic Lou Ruvo Center for Brain Health, Las Vegas, NV, United States, ${ }^{2}$ University of Colorado Boulder, Boulder, CO, United States, ${ }^{3}$ Muhammad Ali Parkinson Center at Barrow Neurological Institute, Phoenix, AZ, United States

Imaging biomarkers that reliably capture the impact of the spreading pathology of Parkinson's disease (PD), including its impact on both white and graymatter, remain elusive. In this study, we applied graph-theoretical techniques to multi-site resting-state fMRI data from a cohort of unmedicated early PD-subjects in Parkinson's Progressive Markers Initiative (PPMI) database. Altered functional connectivity and disrupted topological brain organization was seen in early PD-subjects. Our study opens new avenues to understanding disease progression and severity of PD from graph-theoretical approach.

Functional brain connectome architecture in a large cohort of Parkinson's disease patients

Silvia Basaia ${ }^{1}$, Federica Agosta ${ }^{1}$, Homa Zahedmanesh ${ }^{1,2}$, Tanja Stojkovic ${ }^{3}$, Vladana Markovic ${ }^{3}$, Iva Stankovic ${ }^{3}$, Igor Petrovic ${ }^{3}$, Elka Stefanova ${ }^{3}$, Vladimir Kostic $^{3}$, and Massimo Filippi ${ }^{1,4}$

${ }^{1}$ Neuroimaging Research Unit, INSPE, Division of Neuroscience, San Raffaele Scientific Institute, Vita-Salute San Raffaele University, Milan, Italy, ${ }^{2}$ Department of Electronics, Information, and Bioengineering (DEIB), Politecnico di Milano, Milano, Italy, ${ }^{3}$ Clinic of Neurology, Faculty of Medicine, University of Belgrade, Belgrade, Yugoslavia, ${ }^{4}$ Department of Neurology, San Raffaele Scientific Institute, Vita-Salute San Raffaele University, Milan, Italy

In this study, we investigated functional neural pathway organization in patients with Parkinson's disease (PD) using advanced network-based techniques. At the regional network level, compared to controls, PD groups showed decreased functional connectivity within basal ganglia/sensorimotor network and parietal regions. Compared to early PD cases, mild-to-severe PD patients were characterized by a greater involvement of basal ganglia/sensorimotor networks. This study suggests that graph analysis and connectomics might represent a powerful approach to understand the pathophysiological process across different stages of the disease.

Sensorimotor resting-state functional connectivity at 7T: contrasting Huntington's and Parkinson's disease.

Sirius Boessenkool ${ }^{1}$, Stefania Evangelisti ${ }^{1}$, Patrick Pflanz ${ }^{1}$, Stuart Clare ${ }^{1}$, Campbell Le Heron ${ }^{2}$, Johannes Klein ${ }^{1}$, Richard Armstrong ${ }^{2}$, Kinan Muhammed ${ }^{2}$, Andrea Nemeth $^{2}$, Michele $^{2}$ $\mathrm{Hu}^{2}$, and Gwenaelle Douaud ${ }^{1}$

This preliminary study aims to explore high-resolution functional sensorimotor connectivity using resting-state fMRI in healthy controls (HC), Parkinson's (PD) and Huntington's (HD) disease patients. This $7 \mathrm{~T}$ study therefore includes subjects showing all three states of the basal ganglia inhibitory function. Group ICA and dual regression analyses identified 2 sensorimotor networks: one in which PD and HD showed the same lower cortical connectivity pattern compared with HC in M1 (face area), but opposite pattern in the subthalamic nucleus; and another in which PD and HD showed opposite pattern in M1 and S1 (hand area). This demonstrates the capacity of 7T rs-fMRI to identify with remarkable detail meaningful differences between these two movement disorders. 
Virendra R Mishra ${ }^{1}$, Zhengshi Yang ${ }^{1}$, Karthik Sreenivasan $^{1}$, Xiaowei Zhuang $^{1}$, and Dietmar Cordes ${ }^{1}$

${ }^{1}$ Imaging, Cleveland Clinic Lou Ruvo Center for Brain Health, Las Vegas, NV, United States

In this study, we utilized the diffusion MRI (dMRI) data of early Parkinson's disease (PD) patients and healthy controls (HC) from the Parkinson's Progressive Markers Initiative (PPMI) database and performed a plethora of multivariate and univariate statistical tests ranging from voxelwise measures, skeleton-wise measures from both TBSS and DTI-TK, and region of interest (ROI) analysis of major white matter tracts from JHU atlas at various smoothing levels. Our study revealed only voxelwise measures could classify $\mathrm{HC}$ from PD patients if a minimum smoothing level has reached, and skeleton-wise and ROI analysis (both univariate and multivariate) were associated with the disease.

Baseline Symptoms and Basal Forebrain Volume Predict Future Psychosis in Early Parkinson Disease

Jamie Blair ${ }^{1}$, Matthew Barrett ${ }^{2}$, Scott Sperling ${ }^{2}$, Mark Smolkin ${ }^{3}$, and T. Jason Druzgal ${ }^{1}$

${ }^{1}$ Radiology and Medical Imaging, University of Virginia, Charlottesville, VA, United States, ${ }^{2}$ Neurology, University of Virginia, Charlottesville, VA, United States, ${ }^{3}$ Public Health Services, University of Virginia, Charlottesville, VA, United States

Psychosis is a common neuropsychiatric symptom of Parkinson's disease, and can serve as a clinical marker of advanced disease. Our study aimed to investigate the characteristics of psychosis in a longitudinal PD cohort, to verify baseline clinical risk factors for future psychotic symptoms in de novo PD patients, and to evaluate the relationship between baseline gray matter density in the nucleus basalis of Meynert and future psychotic symptoms in PD. We found lower NBM density at baseline to be associated with increased psychotic symptom burden compared to controls, suggesting utility for the NBM as a neuroimaging biomarker for advanced PD.

Studying the neural correlates of motor fatiguability in controls and people with Parkinson's Disease

Yue Lily Xing ${ }^{1,2}$, Saadnah Naidu ${ }^{1,2}$, Nin Bajaj $^{3}$, and Dorothee Auer ${ }^{1,2,4}$

${ }^{1}$ Radiological Sciences, Division of clinical neuroscience, University of Nottingham, Nottingham, United Kingdom, ${ }^{2}$ Sir Peter Mansfield Imaging Centre, University of Nottingham, Nottingham, United Kingdom, ${ }^{3}$ Division of Neurology, University of Nottingham, Nottingham, United Kingdom, ${ }^{4}$ Nottingham NIHR Biomedical Research Centre, University of Nottingham, Nottingham, United Kingdom

Fatiguability, an objective decline in the amplitude of movements during sustained or fast repetitive motor tasks, is one of the primary clinical features demonstrated in Parkinson's disease (PD). However, our understanding of its underlying pathophysiology is still limited. Here, we propose a fMRI protocol to study the neuronal correlates of fatiguability and present preliminary data in PD and control subjects while performing sustained finger tapping. There was significant reduction of tapping-related activation in the primary motor cortex, somatosensory cortex, premotor cortex and middle frontal gyrus in the fatiguing vs. no-or-less fatiguing subgroups, suggesting that those regions were involved in fatigue.

Brain Motor Asymmetry in PD using Positron Emission Tomography and Diffusion Tensor Imaging

Dan Stein ${ }^{1}$, Natalia Goldberg ${ }^{1}$, Liran Domachevsky ${ }^{1}$, Hanna Bernstine ${ }^{1,2}$, Meital Nidam ${ }^{1}$, David Groshar ${ }^{1}$, Mordechai Lorberboym ${ }^{1,3}$, Simon Israeli-Korn ${ }^{4}$, Moshe Gomori ${ }^{5}$, Yaniv Assaf ${ }^{6}$, and Sharon Hassin-Baer ${ }^{2,7}$

${ }^{1}$ Assuta Medical Center, Tel-Aviv, Israel, ${ }^{2}$ Sackler Faculty of Medicine, Tel Aviv University, Tel-Aviv, Israel, ${ }^{3}$ Tel-Aviv University, Tel-Aviv, Israel, ${ }^{4}$ Movement Disorders Institute, Sagol Neuroscience Center and Department of Neurology, Chaim Sheba Medical Center, Tel Hashomer, Tel-Aviv, Israel, ${ }^{5}$ Department of Radiology, Hadassah-Hebrew University Medical Center, Jerusalem, Israel., Jerusalem, Israel, ${ }^{6}$ Sagol School of Neuroscience, Tel Aviv University, Tel-Aviv, Israel, ${ }^{7}$ Movement Disorders Institute, Sagol Neuroscience Center and Department of Neurology, Chaim Sheba Medical Center, Tel Hashomer, Israel, Tel-Aviv, Israel

The accuracy of clinical diagnosis of Parkinson disease is currently not satisfying, particularly in early Parkinson disease where clinical signs are not yet fully present. Imaging nigral structures has been proposed as a biomarker for PD but fails to provide effective differential diagnosis. In this study we compared motor brain regions between hemispheres in patients with asymmetrical motor symptoms using voxel based analysis and network analysis and have found significant regional differences between the more and less affected hemispheres as well as connectivity differences in frontal and cerebral regions as the main hubs.

The fronto-parietal connectivity in freezing of gait: a left/right imbalance?

Céline Tard ${ }^{1}$, Caroline Moreau ${ }^{2}$, Romain Viard ${ }^{3}$, Christine Delmaire ${ }^{2}$, David Devos ${ }^{2}$, Pierre Lenfant ${ }^{2}$, Kathy Dujardin $^{2}$, Luc Defebvre ${ }^{2}$, Arnaud Delval $^{2}$, and Renaud Lopes ${ }^{2}$

${ }^{1}$ Neurology Department, Lille University Hospital Center, Lille, France, ${ }^{2}$ Lille University Hospital Center, Lille, France, ${ }^{3}$ Radiology Department, Lille University Hospital Center, Lille, France

The multimodal MRI assessment is here used to better understand the previous known parietoprefrontal networks' abnormalities in parkinsonian patients with freezing of gait. Anatomic disconnection was observed in the right prefrontal cortex in those patients and functional disconnection was major from the left one. The imbalance between left and right networks is discussed heyard the pathophysiology of freezing. 
${ }^{1}$ Bioengineering Department / Institute for Systems and Robotics, Instituto Superior Técnico, University of Lisbon, Lisbon, Portugal, ${ }^{2}$ Neurological Imaging Department, Hospital de Santa Maria, Centro Hospitalar Lisboa Norte, Lisbon, Portugal, ${ }^{3}$ Clinical Pharmacology Unit, Instituto de Medicina Molecular, Faculdade de Medicina, University of Lisbon, Lisbon, Portugal, ${ }^{4}$ Neurology Department, Hospital de Santa Maria, Centro Hospitalar Lisboa Norte, Lisbon, Portugal

Neuromelanin(NM)-sensitive MRI is a promising technique for enlightening pathological changes in NM-containing structures. Fast-Spin-Echo (FSE) based NM-MRI sequences have been applied at $3 \mathrm{~T}$ for improved resolution and signal-to-noise ratio but scanner availability and safety concerns may prevent imaging at this field strength. A $1.5 \mathrm{~T}$ NM-MRI FSE sequence was developed and compared to the standard 3T NM-MRI sequence. Semi-automatic segmentation of the Substantia Nigra (SN) was performed with good reliability at both fields. The Bland-Altman method was used to compare SN areas between field strengths showing good agreement, supporting the possibility for using NM-MRI at $1.5 \mathrm{~T}$, widening its scope of applicability.

A Cycling Exercise Study of Parkinson's Disease: The Effect of Exercises on Motor Cortex Functional Connectivity Revealed by Resting State FMRI

Jian Lin ${ }^{1}$, Katherine A Koenig ${ }^{1}$, Erik Beall ${ }^{2}$, Mark J Lowe ${ }^{1}$, Amy E Jansen $^{3}$, Amanda L Penko ${ }^{3}$, and Jay Alberts ${ }^{3}$

${ }^{1}$ Radiology, Cleveland Clinic, Cleveland, OH, United States, ${ }^{2}$ Hema Imaging LLC, Minneapolis, MN, United States, ${ }^{3}$ Biomedical Engineering, Cleveland Clinic, Cleveland, OH, United States

Parkinson's disease (PD) is a progressive neurological disorder which produces a general poverty of movement. Lower extremity forced exercise (FE) has been shown to provide therapeutic benefits for PD motor symptoms similar to that of antiparkinson medication ${ }^{1}$. In the current study, both voluntary exercise (VE) and FE were evaluated. Our results suggest that both modes of aerobic exercise have effects on motor functional connectivity similar to changes associated with antiparkinson medication.

BOLD responses to light stimulus frequency in the rat visual pathway reveal profound effects of Parkinson's disease in the Superior Colliculus

Emmanuelle Bellot ${ }^{1}$, Arnaud Pautrat ${ }^{1}$, Yassamine Rahmani Bouzina ${ }^{1}$, Nora Collomb $^{2}$, Olivier Montigon ${ }^{2}$, Véronique Coizet ${ }^{1}$, and Michel Dojat ${ }^{1}$

Sensory disorders are associated with Parkinson Disease (PD) at an early stage. We explored with fMRI the visual pathway response to light stimulus frequency in PD rat models. Activation of the Superior Colliculus (SC) was exacerbated at low frequency (1-3\%) and rapidly saturated compared to controls. These results confirm the possible role of SC as an early biomarker of the disease.

QSM versus R2* to study iron deposition in the substantia nigra and subthalamic nucleus in Parkinson's disease and REM sleep behavior disorders

Mathieu David Santin ${ }^{1,2}$, Nadya Pyatigorskaya ${ }^{1,2}$, Romain Valabregue ${ }^{1,2}$, Rahul Gaurav ${ }^{1,2}$, Lydia Yahia Cherif ${ }^{1,2}$, Sara Fernandez-Vidal ${ }^{1,2}$, Eric Bardinet ${ }^{1,2}$, Graziella Mangone ${ }^{2}$, Isabelle Arnulf $^{2}$, Marie Vidailhet ${ }^{2}$, Jean-Christophe Corvol $^{2}$, and Stéphane Lehéricy ${ }^{1,2}$

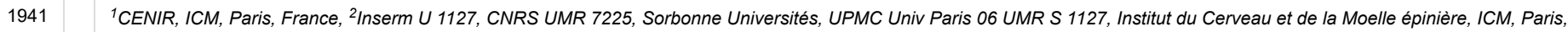
France

Here, we compared R2* relaxation rate and QSM to study iron deposition in the substantia nigra (SN) and subthalamic nucleus (STN) in patients with early PD and idiopathic REM sleep behavior disorders with two different segmentation methods. PD patients showed increased iron deposition in the SN and STN as compared with healthy controls with QSM and R2*. iRBD only showed an increase tendency of QSM values compared to healthy controls. Obtained p-values were more systematically lower in QSM than in R2*.

Iron Deposition Quantification in Patients with Parkinson's Disease by Quantitative Susceptibility Mapping

Zhangxuan $\mathrm{Hu}^{1}$, Yuhui Xiong ${ }^{1}$, Xuesong $\mathrm{Li}^{2}$, Rongsong Zhou ${ }^{3}$, Suhua $\mathrm{Miao}^{3}$, Le He${ }^{1}$, Yu Ma ${ }^{3}$, and Hua Guo

${ }^{1}$ Biomedical Engineering, Tsinghua University, Beijing, China, ${ }^{2}$ School of Computer Science and Technology, Beijing Insitute of Technology, Beijing, China, ${ }^{3}$ Tsinghua University Yuquan Hospital, Beijing, China

Parkinson's disease $(\mathrm{PD})$ is one of the most common neurodegenerative disorders worldwide. This study explores the relationships between iron accumulation in different nucleus, including red nucleus (RN), caudate nucleus (CN), global pallidus (GP), putamen (PUT), and the severity of PD, which is characterized by the Unified Parkinson's Disease Rating Scale (UPDRS)-III. Significant bilateral difference was found in RN only. Significant correlations were found in bilateral GPe, PUT, RN, and contralateral GPi, which can serves as an evidence that iron deposition can be an important biomarker for the severity of PD. 
Use of Functional MRI to assess the differences of STN and GPI Deep Brain Stimulation in Parkinson Disease

Marisa DiMarzio ${ }^{1}$, lleana Hancu² ${ }^{2}$ Eric Fiveland ${ }^{2}$, Julia Prusik ${ }^{3}$, Radhika Madhavan ${ }^{4}$, Suresh Joel ${ }^{4}$, Michael Gillogly ${ }^{3}$, Jeffery Ashe ${ }^{2}$, Tanweer Rashid $^{1}$, Jennifer Durphy $^{5}$, Roy Hwang $^{3}$, and Julie Pilitsis 1,3

${ }^{1}$ Neuroscience and Experimental Therapeutics, Albany Medical College, Albany, NY, United States, ${ }^{2}$ GE Global Research Center, Niskayuna, NY, United States, ${ }^{3}$ Neurosurgery, Albany Medical College, Albany, NY, United States, ${ }^{4}$ GE Global Research Center, Bangalore, India, ${ }^{5}$ Neurology, Albany Medical College, Albany, NY, United States

Deep brain stimulation (DBS) of both the subthalamic nucleus (STN) and globus pallidus interna (GPi) are well-recognized effective treatments for Parkinson's disease (PD). The mechanism of DBS and network responses produced by stimulation of these targets remains unknown. Conditional labeling of DBS now allows fMRI to be performed in the ON state. We examine whether GPI DBS and STN DBS affect blood oxygen level dependent (BOLD) brain activation/deactivation patterns similarly. Results show that both types of DBS activate the thalamus and deactivate the primary motor cortex; while the STN cohort showed activation in the cerebellum, an opposite effect was apparent in the GPi cohort.

Altered marginal division connectivity in Parkinson disease with mild cognitive impairment revealed by resting-state fMRI

Li mingge ${ }^{1,2}$, Chen yuanyuan ${ }^{3}$, Feng jie ${ }^{1}$, Zhang shiyu ${ }^{1}$, Lou xin ${ }^{1}$, and Ma lin ${ }^{1}$

1944

${ }^{1}$ Chinese PLA general hospital, beijing, China, ${ }^{2}$ Nankai University, tianjin, China, ${ }^{3}$ Tianjin University, tianjin, China

The marginal division $(\mathrm{MrD})$ functional connectivity is disrupted during mild cognitive impairment in Parkinson's disease.

Microstructural Changes in Brain Gray Matter Nuclei of Patients with Parkinson's Disease:A Study Based on MR Diffusion Kurtosis Imaging

Qiyuan Sun ${ }^{1}$, Heng Meng ${ }^{1}$, and Zhizheng Zhuo

$1945 \quad{ }^{1}$ Affiliated Hospital Of BeiHua University, Jilin, China, ${ }^{2}$ Philips Healthcare, Beijing, China

Parkinson's disease is the most common extrapyramidal disease in the elderly people, and the overall prevalence rate is increasing year by year. Diffusion kurtosis imaging (DKI) which was an based on the extension of diffusion tensor imaging (DTI) to reflect the diffusion motion of water molecules in the non-Gaussian distribution between tissues have been proved reliable for the brain microstructural changes. Previous studies have shown that DKI could facilitate the detection of subtle structural changes in the gray matter nuclei of patients with $\mathrm{PD}$, which may be related to the reduction of dopaminergic neurons, iron deposition and gliosis.

Iron Quantification in Brain Gray Matter Nuclei of Patients with Parkinson's Disease: A Study Based on MR Quantitative Susceptibility Mapping

Qiyuan Sun ${ }^{1}$, Heng Meng ${ }^{1}$, and Zhizheng Zhuo ${ }^{2}$

${ }^{1}$ Affiliated Hospital Of BeiHua University, Jilin, China, ${ }^{2}$ Philips Healthcare, Beijing, China

Parkinson's disease (PD) is the most common extrapyramidal disease in the elderly people, and the overall prevalence rate is increasing year by year. Quantitative susceptibility mapping (QSM) is based on the basis of susceptibility weighted imaging (SWI), and has more advantages in quantitative detection of brain iron content and display of microstructure. In this study, we tried to use QSM to analyze brain iron variations and microstructural changes in brain gray matter nuclei of patients with PD.

\section{ROLE OF SUSCEPTIBILITY-WEIGHTED ANGIOGRAPHY (SWAN) QUANTITATIVE MAPPING IN PARKINSON DISEASE DIAGNOSIS}

Mariia Viktorovna Rezakova ${ }^{1}$, Khurshed J. Ibrogimov ${ }^{2}$, Elena Andreevna Filimonova ${ }^{1}$, Olga Anatolevna Subbotina ${ }^{1}$, and Alexandr Vladimirovich Shevchenko ${ }^{1}$

\section{Epilepsy}


Resting state activity is depressed in regions of MRSI identified dysfunction in epilepsy

Jing Huei Lee ${ }^{1}$, Arun Antony ${ }^{2}$, Victor Yushmanov ${ }^{2}$, R. Mark Richardson ${ }^{2}$, and Jullie W Pan ${ }^{2}$

${ }^{1}$ University of Cincinati, Cincinati, $\mathrm{OH}$, United States, ${ }^{2}$ University of Pittsburgh, pittsburgh, PA, United States

This study describes co-registered rsfMRI and MRSI data in poorly localized epilepsy patients with the goal of identifying the aberrant epilepsy network. We used $3 \mathrm{~T}$ rosette encoded spectroscopic image covering the fronto-parietal-temporal brain regions in conjunction with resting fMRI data. The MRSI defined masks of metabolic dysfunction which was then forward warped using Bo maps to define the equivalent regions in the rsfMRI data. The rsfMRI data was analyzed with a model-free evaluation of local connectivity (regional homogeneity). Regions identified by MRSI as metabolically abnormal exhibited lower local rsfMRI coherence in comparison to gray matter or temporal regions.

Resting-state functional connectivity of hippocampus in patients with drug-resistant idiopathic generalized epilepsy

Zhengge Wang ${ }^{1}$, Lipei Cao ${ }^{1}$, Bing Zhang ${ }^{1}$, and Bin Zhu ${ }^{1}$

${ }^{1}$ Department of Radiology, The Affiliated Drum Tower Hospital of Nanjing University Medical School, Nanjing, China

Previous studies have found altered resting-state functional connectivity in default mode network in drug-resistant patients with idiopathic generalized epilepsy (IGE). Recent studies showed that the volume of the hippocampus is decreased in IGE patients. Hippocampus abnormalities are often related to drug-resistant epilepsy. We investigated the alteration of resting-state functional connectivity of hippocampus in drug-resistant IGE patients by using seed-based functional connectivity and found divergent changes in drug-resistant and drugsensitive IGE patients. Our findings indicate that the hippocampus and the related network may play an important role in drug-resistant IGE patients.

Functional connectivity changes during epileptogenesis: a longitudinal rs-fMRI study

Emma Christiaen ${ }^{1}$, Marie-Gabrielle Goossens ${ }^{2}$, Benedicte Descamps ${ }^{1}$, Paul Boon $^{2}$, Robrecht Raedt ${ }^{2}$, and Christian Vanhove ${ }^{1}$

${ }^{1}$ MEDISIP, Department of Electronics and Information Systems, Ghent University - IMEC, Ghent, Belgium, ${ }^{2}$ Laboratory for Clinical and Experimental Neurophysiology, Neurobiology and Neuropsychology (LCEN3), Department of Neurology, Ghent University, Ghent, Belgium

Abnormal functional brain networks could be involved in the development of temporal lobe epilepsy (TLE). In this longitudinal resting-state fMRI study, changes in functional networks during epileptogenesis in the intraperitoneal kainic acid (IPKA) rat model for TLE were mapped. Therefore, resting-state fMRI was acquired at several time points during epileptogenesis to identify functional networks that were analysed and compared with graph theory. Our results suggest that network connections in the functional brain network progressively become weaker during epileptogenesis. We also find a decreased segregation and integration of the network.

Whole-Brain connectomics reveals network differences in patients with Non-Lesional Frontal Lobe Epilepsy

Maria Eugenia Caligiuri ${ }^{1}$, Andrea Cherubini ${ }^{1}$, Antonio Gambardella ${ }^{2}$, and Angelo Labate ${ }^{2}$

${ }^{1}$ Institute of Molecular Bioimaging and Physiology (IBFM-CNR), Catanzaro, Italy, ${ }^{2}$ Institute of Neurology, University Magna Graecia, Catanzaro, Italy

In frontal lobe epilepsy (FLE) seizure onset is usually caused by the presence of lesions or cortical dysplasias of different location and size, challenging the identification of homogeneous samples for neuroimaging studies. However, there are patients in which, even if seizures start in the frontal lobe, no clearly identifiable abnormality can be seen on magnetic resonance imaging (MRI). Thus, it has been hypothesized that non-lesional FLE is indeed a network syndrome, rather the result of focal pathology. In the light of this probabilistic tractography and graph analysis seem the ideal methodology to investigate the presence and extent of network alterations.

MRI and CT derived 3D-printed patient specific brain model for localizing depth elecrodes for epilepsy surgery planning

Sarah L Hurrell ${ }^{1}$, Sean M Lew ${ }^{2}$, Wade Mueller ${ }^{3}$, and Peter S LaViolette ${ }^{1}$

${ }^{1}$ Radiology, The Medical College of Wisconsin, Milwaukee, WI, United States, ${ }^{2}$ Pediatric Neurosurgery, The Medical College of Wisconsin, Milwaukee, WI, United States, ${ }^{3}$ Neurosurgery, The Medical College of Wisconsin, Milwaukee, WI, United States

We present a method for creating a patient specific, 3D printed model of depth electrode location in an epilepsy patient. We utilized a pre-surgery structural MRI scan and a postelectrode placement CT, which were aligned, and combined to visualize a cortical anatomy and electrode position. 3D models were then generated, edited, and 3D-printed to provide a visual and physical aid for surgical planning 


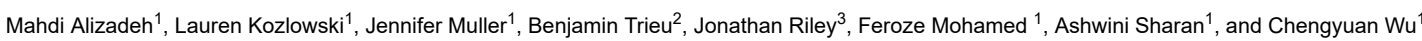

${ }^{1}$ Thomas Jefferson University, Philadelphia, PA, United States, ${ }^{2}$ Temple University, Philadelphia, PA, United States, ${ }^{3}$ University at Buffalo, Buffalo, NY, United States

Diffusion tensor imaging and diffusion tensor tractography help to better understand the pathological alterations in white matter structures, and in tracing axonal pathways involved in patients with temporal lobe epilepsy.

Automated Hippocampal Subfield Segmentation using Ultrahigh Field MRI in Patients with Epilepsy

Judy Alper ${ }^{1,2}$, Rebecca E Feldman ${ }^{1}$, Long Xie ${ }^{3}$, Alexandru L Rus ${ }^{4}$, Lara V Marcuse ${ }^{5}$, Madeline C Fields ${ }^{5}$, Bradley N Delman $^{6}$, Hung-Mo Lin ${ }^{7}$, Patrick Hof $^{8}$, and Priti Balchandani ${ }^{1}$

${ }^{1}$ Radiology, Icahn School of Medicine At Mount Sinai, New York, NY, United States, ${ }^{2}$ Biomedical Engineering, City College of New York, New York, NY, United States, ${ }^{3}$ Biomedical Engineering, University of Pennsylvania, Philadelphia, PA, United States, ${ }^{4}$ Icahn School of Medicine At Mount Sinai, New York, NY, United States, ${ }^{5}$ Neurology, Mount Sinai Medical Center, New York, NY, United States, ${ }^{6}$ Radiology, Mount Sinai Medical Center, New York, NY, United States, ${ }^{7}$ Population Health Science and Policy Department, Icahn School of Medicine At Mount Sinai, New York, NY, United States, ${ }^{8}$ Neuroscience, Icahn School of Medicine At Mount Sinai, New York, NY, United States

Epilepsy is a widely prevalent, disabling condition, whose anatomical source is not clearly identifiable on clinical MRI scans. Identifying hippocampal subfields associated with epilepsy may elucidate mechanisms of epileptigenesis and assist treatment planning. We performed high-resolution 7T-MRI, enabling precise subfield measurements in thirty patients and matched controls. Greater CA1 and DG asymmetries were found in patients compared to controls. In a subset of mesial-temporal lobe epilepsy patients, we found reduced CA2 on the ipsilateral side in patients compared to controls. Identifying hippocampal subfield biomarkers in epilepsy can result in better treatment planning and monitoring in epilepsy.

Comparison between two different post-processing techniques in the presurgical evaluation of Focal Cortical Displasya in a paediatric population.

Elena Bassanelli ${ }^{1}$, Maria Camilla Rossi Espagnet ${ }^{2}$, Nicola Pietrafusa ${ }^{3}$, Luca De Palma ${ }^{3}$, Nicola Specchio ${ }^{3}$, Daniela Longo ${ }^{2}$, and Antonio Napolitano ${ }^{1}$

${ }^{1}$ Medical Physics Department, IRCCS Bambino Gesù Children's Hospital, Rome, Italy, ${ }^{2}$ Imaging Department, IRCCS Bambino Gesù Children's Hospital, Rome, Italy, ${ }^{3}$ Department of Neurosciences, IRCCS Bambino Gesù Children's Hospital, Rome, Italy

The purpose of this study is to compare two different techniques for cortical dysplasia detection: Opti-MAP and the SUPR-FLAIR. The Opti-MAP is a children-optimized version of the Morphological analysis program (MAP), which is able to detect the "blurred-junction", peculiar characteristics of focal cortical dysplasia in children, thanks to a voxel-based morphological analysis in which neuroanatomical differences are detected by comparison with a normal template. The SUPR-FLAIR analysis, instead, is a technique able to highlight hyperintensities in FLAIR images. These methods have been applied on paediatric subjects affected by pharmaco-resistant epilepsy.

Imaging and involvement of visual pathways in children undergoing epilepsy surgery

Luis Miguel Lacerda ${ }^{1}$, Martin Tisdall ${ }^{2}$, Gavin Winston ${ }^{3}$, Sian Handley ${ }^{4}$, Alki Liasis ${ }^{4}$, and Chris A Clark ${ }^{1}$

${ }^{1}$ Developmental Imaging and Biophysics Section, UCL Great Ormond Street Institute of Child Health, London, United Kingdom, ${ }^{2}$ Neurosurgery, UCL Great Ormond Street Institute of Child Health, London, United Kingdom, ${ }^{3}$ Department of Clinical and Experimental Epilepsy, UCL Institute of Neurology, London, United Kingdom, ${ }^{4}$ Ophthalmology, UCL Great Ormond Street Institute of Child Health, London, United Kingdom

Surgery is a key approach for achieving seizure control in children with epilepsy but it can affect or be in the vicinity of the optic radiations. Whilst tractography has shown that damage to optic radiations leads to postoperative visual field defects in adults it has not yet been properly explored in children. In this study we successfully performed tractography reconstructions in a paediatric cohort undergoing surgery. Furthermore, we showed that in cases with pre- and post-surgical visual function assessment, involvement of optic radiations corresponded to visual function disturbances. This highlights the importance of tractography to aid pre-surgical evaluation in children.

Case Study: Evaluation of White Matter Disorganization in Temporal Lobe Epilepsy

Laura Barlow ${ }^{1}$, Irene Vavasour ${ }^{1,2}$, David Li $^{1,2,3}$, Martin Parent $^{4}$, and Doris Doudet ${ }^{3}$

${ }^{1}$ UBC MRI Research Centre, University of British Columbia, Vancouver, BC, Canada, ${ }^{2}$ Radiology, University of British Columbia, Vancouver, BC, Canada, ${ }^{3}$ Neurology, University of British Columbia, Vancouver, BC, Canada, ${ }^{4}$ Laval University, Quebec City, QC, Canada

Temporal lobe epilepsy (TLE) assessment on MRI is limited to qualitative analysis in the clinical environment. Diffusion Tensor Imaging has been used to interrogate white matter changes in TLE while Myelin Water Fraction has not. With this case study we compare diffusion tensor imaging with myelin water imaging in a non-human primate (NHP) with TLE and a healthy control to assess if the two methods are complementary in evaluating white matter disorganization. 
Paul Summers ${ }^{1}$, Fulvia Palesi ${ }^{2}$, Francesco Padelli ${ }^{3}$, Ileana Zucca $^{3}$, Marcella Malagoli $^{4}$, Carmelo Maccagnano ${ }^{3}$, Stefano Meletti ${ }^{4,5}$, Giuseppe Didato $^{3}$, Claudia Wheeler-Kingshott ${ }^{1,2,6}$, and Paolo Vitali ${ }^{1}$

${ }^{1}$ C. Mondino National Neurological Institute, Pavia, Italy, ${ }^{2}$ University of Pavia, Pavia, Italy, ${ }^{3} I R C C S$ Foundation, C. Besta Neurological Institute, Milan, Italy, ${ }^{4}$ Civile Aziende OspedalieraUniversitaria, Modena, Italy, ${ }^{5}$ University of Modena and Reggio Emilia, Modena, Italy, ${ }^{6}$ University College London, London, United Kingdom

Quantitative characterization of MT, R1, R2*, and PD may aid in providing more consistent readings of alterations in temporal lobe epilepsy. As part of a multi-centric study we have set up a hMRI protocol for use at $3 \mathrm{~T}$ across two manufactures of MR scanners. Because of differences in MT pulses and SAR calculations, near matching was achieved only through use of commercial or research options. Initial results from one scanner show excellent reproducibility within and between subjects for MT and R1. A cross-scanner evaluation is in course.

Comprehensive assessment of white matter microstructural integrity and its change across lifespan in patients with tuberous sclerosis complex

Tei-Wei Kao ${ }^{1}$, Pi-Chuan Fan² ${ }^{2}$ Yung-Chin Hsu' ${ }^{1}$, and Wen-Yih Isaac Tseng ${ }^{1,3}$

${ }^{1}$ Institute of Medical Device and Imaging, National Taiwan University College of Medicine, Taipei, Taiwan, ${ }^{2}$ Department of Pediatrics, National Taiwan University Hospital and National Taiwan University College of Medicine, Taipei, Taiwan, ${ }^{3}$ Molecular Imaging Center, National Taiwan University, Taipei, Taiwan

In previous studies, white matter microstructural integrity and its lifetime change in patients with tuberous sclerosis complex (TSC) were not clearly identified. Therefore, we performed diffusion spectrum imaging using whole-brain tract-specific analysis to measure the generalized fractional anisotropy (GFA), and built an age-GFA quadratic linear model to investigate 76 major white matter tract bundles between TSC and healthy control groups. Twenty tract bundles showed a group effect with substantially lower GFA in childhood and older adulthood in patients with TSC. Our results suggest that TSC might pose detrimental effects on microstructural integrity in the developmental and aging periods of life.

Progressive white matter changes in the pilocarpine-induced temporal lobe epilepsy with focal seizure rat model: A diffusion tensor imaging study

Yao-Chia Shih ${ }^{1,2}$, Chih-Hsien Tseng ${ }^{1,2}$, Fang-Chia Chang ${ }^{3}$, Horng-Huei Liou ${ }^{4,5}$, and Wen-Yih Issac Tseng ${ }^{2,5,6}$

${ }^{1}$ Institute of Biomedical Engineering, National Taiwan University, Taipei, Taiwan, ${ }^{2}$ Institute of Medical Device and Imaging, National Taiwan University College of Medicine, Taipei, Taiwan, ${ }^{3}$ Department of Veterinary Medicine, School of Veterinary Medicine, National Taiwan University, Taipei, Taiwan, ${ }^{4}$ Department of Neurology, National Taiwan University Hospital and College of Medicine, Taipei, Taiwan, ${ }^{5}$ Graduate Institute of Brain and Mind Sciences, College of Medicine, National Taiwan University, Taipei, Taiwan, ${ }^{6}$ Molecular Imaging Center, National Taiwan University, Taipei, Taiwan

A more suitable pilocarpine rat model with microinjection into the left central nucleus of the amygdala and in-vivo diffusion tensor imaging acquisitions were used to investigate progressive changes in the white matter fibers at three different time points during epileptogenesis in temporal lobe epilepsy (TLE) with focal seizure. We found transient fractional anisotropy (FA) changes in the left fimbria of the hippocampus after status epilepticus and subsequent FA changes in the left cingulum after the presence of spontaneous recurrent seizure. The results demonstrate potential imaging markers for monitoring the progression and development of TLE with focal seizure.

Traditional Poster

\section{Head \& Neck}

Compressed-Sensing Accelerated 3-Dimensional Magnetic Resonance Imaging of Inner Ear: A Feasibility Study of Volunteer

Yuan Jiang ${ }^{1}$, Lina Zhu ${ }^{1}$, Jing Liư ${ }^{1}$, Xiaodong Zhang ${ }^{1}$, Shuai Ma ${ }^{1}$, Yi Liu ${ }^{1}$, Zhiyong Lin ${ }^{1}$, Ke Wang ${ }^{1}$, Zhizheng Zhuo ${ }^{2}$, and Xiaoying Wang ${ }^{1}$

Compressed-Sensing (CS) accelerated 3-dimensional magnetic resonance imaging (MRI) does not reduce image quality even with higher image quality scores compared to conventional MRI of inner ear, while significantly shortening the imaging time. It is a feasible protocol in inner ear imaging. 
The purposes of this study were to optimize the protocol of brachial plexus MRN for brachial plexus injuries, and to study the diagnostic performance of the protocol, using clinical contexts as the reference standard. Twenty-one patients with brachial plexus injury were performed brachial plexus MRN (T2-weighted image-high resolution, mDIXON and diffusion weighted image) before conventional myelography. The diagnostic yield of T2-weighted image-high resolution was comparable to conventional myelography. The combination of T2weighted image-high resolution and $\mathrm{mDIXON}$ had the highest diagnostic yield and recommended for the evaluation of brachial plexus injuries.

MRI Assessment of SPION Contrast in the Inner Ear

Wendy Oakden ${ }^{1}$, Maya Kuroiwa Rivero ${ }^{2,3}$, Lola Awofala ${ }^{3}$, Greg J Stanisz ${ }^{1,4,5}$, and Trung N Le ${ }^{2,3}$

${ }^{1}$ Physical Sciences Platform, Sunnybrook Research Institute, Toronto, ON, Canada, ${ }^{2}$ Department of Otolaryngology Head \& Neck Surgery, University of Toronto, Toronto, ON, Canada, ${ }^{3}$ Biological Sciences Platform, Sunnybrook Research Institute, Toronto, ON, Canada, ${ }^{4}$ Medical Biophysics, University of Toronto, Toronto, ON, Canada, ${ }^{5}$ Neurosurgery and Pediatric Neurosurgery, Medical University of Lublin, Lublin, Poland

A novel approach to diagnostic imaging and treatment of the inner ear disorders is magnetic targeting of therapy using superparamagnetic iron oxide nanoparticles (SPIONs). SPIONs were deposited onto the round window niche using a surgical approach, and then magnetic targeting was used, in the treatment group, to "pull" the SPIONs further into the inner ear. High resolution T2 weighted imaging was used to assess the treatment. Signal loss was observed in the vestibule and cochlea in both groups, while increased signal loss was observed at the apex of the cochlea in treated animals relative to the control group.

The feasibility of ultrashort echo time imaging for visualization of sinonasal and skull base bony structures: preliminary study

Miran $\mathrm{HAN}^{1}$, Jin Wook $\mathrm{Choi}^{1}$, and Sungmin $\mathrm{Gho}^{2}$

${ }^{1}$ Ajou Univeristy Medical Center, Suwon, Republic of Korea, ${ }^{2} G E$ healthcare, Seoul, Republic of Korea

We evaluate the feasibility of ultrashort echo time (UTE) imaging in the visualization of sinonasal and skull base bony structures. MRI with UTE imaging are feasible to assess not only the normal bony structures but also diverse anatomic variations of sinonasal cavity and skull base without radiation exposure. This technique may lead to a new application of diagnostic MRI in head and neck imaging and could be expected to prevent additional CT imaging and consequently reduce radiation exposure.

Differentiating Neuromyelitis Optica (NMO)-related and Multiple Sclerosis-related Acute Optic Neuritis using Conventional Magnetic Resonance Imaging Combined with Readoutsegmented Echo-planar Diffusion-weighted Imaging

Ping Lu ${ }^{1}$, Yan Sha ${ }^{1}$, Guohong Tian ${ }^{1}$, Xilan Liu ${ }^{1}$, Feng Wang ${ }^{1}$, Zhongshuai Zhang ${ }^{2}$, and Yi Sun ${ }^{2}$

$1965 \quad{ }^{1}$ Eye \& ENT hospital of Fudan University, Shanghai, China, ${ }^{2}$ Siemens Ltd, Shanghai, China

In clinical practice, acute optic neuritis (ON) associated with the development of neuromyelitis optica (NMO) after the first attack is often indistinguishable from that associated with multiple sclerosis (MS)1-3; and different therapeutic strategies are required for the two diseases because of their immunopathogenic differences4. Therefore, we aimed to determine the optimal combination of features derived from conventional magnetic resonance imaging (MRI) and diffusion-weighted imaging using readout-segmented echo-planar imaging (RESOLVE-DWI) for the differentiation of the two types of acute ON.

Diffusion-prepared magnetic resonance neurography for the visualization of the Facial nerve

Paula Bos ${ }^{1,2}$, Bas M.S. Jasperse ${ }^{1}$, Alfons J.M. Balm²,3, Leon C. ter Beek ${ }^{1}$, Fijs W.B. van Leeuwen ${ }^{2,4}$, Michiel W.M. van den Brekel ${ }^{2,3}$, Regina G.H. Beets-Tan ${ }^{1}$, and Tessa Buckle

${ }^{1}$ Radiology, The Netherlands Cancer Institute - Antoni van Leeuwenhoek Hospital, Amsterdam, Netherlands, ${ }^{2}$ Head and Neck Oncology and Surgery, The Netherlands Cancer Institute Antoni van Leeuwenhoek Hospital, Amsterdam, Netherlands, ${ }^{3}$ Oral and Maxillofacial Surgery, Academic Medical Center, Amsterdam, Netherlands, ${ }^{4}$ Radiology, Leiden University Medical Center, Leiden, Netherlands

The aim of the study was to investigate the feasibility of Diffusion-prepared MRI (D-prep MRI) to visualize the Facial nerve in head and neck cancer patients. Twenty-four patients (12 male, $60 \pm 11$ year) received a D-prep MRI, where the main trunk and branches of the Facial nerve is reviewed by one neuro/head and neck radiologists. The main trunk was visible in fifteen patients and in four, six, six and one patients for the posterior auricular, zygomaticofacial, cervicofacial and temporal branches respectively. D-prep MRI is able to visualize the Facial nerve in most cases, but further improvement is required. 
This work assessed the feasibility of quantitative parameters derived from quantitative dynamic contrast enhancement MR imaging (DCE-MRI) parameters in the prediction and evaluation of the response to chemotherapy in patients with malignant sinonasal tumors.

One-step high-resolution diffusion weighted imaging in ocular masses and optic nerve using a dedicated surface coil

Qinghua Chen ${ }^{1}$, Zongrui Zhang ${ }^{1}$, Xiaoqi Wang ${ }^{2}$, Fei Yan ${ }^{1}$, and Junfang Xian ${ }^{1}$

${ }^{1}$ Radiology Department, Beijing Tongren Hospital, Capital Medical University, Beijing, China, ${ }^{2}$ Philips Healthcare China, Beijing, China

It is challenging for the routine clinical ocular MRI protocol to use a large FOV covering the whole orbits and sellar region with high spatial resolution relatively. The aim of this study was to evaluate custom-made ocular surface coil in diagnosing images for ocular masses and the optic nerve by comparing TSE DWI images. The dedicated ocular coil obtained large FOV and high spatial resolution images with higher SNR in TSE DW images as examples. The custom-made surface coil can demonstrate ocular masses and the optic nerve more clearly, and provide more details with high SNR in one-step.

Three-dimensional fast spin echo with extended echo train acquisition (3D-FSE-Cube) integrate with two point water-fat separation Dixon methods (Flex): comparison with threedimensional fast spin echo Cube in lachrymal drainage system imaging

ping $\operatorname{liu}^{1}$ and jing zhang ${ }^{1}$

${ }^{1}$ department of radiology, Tongji Hospital, Tongji Medical College, Huazhong University of Science and Technology, wu han, China

The normal membranous lacrimal passage and tear fluid play a very essential role in protecting and lubricating the ocular surface. An ideal lacrimal imaging is very for clinical therapy stratage. The MRI combine fluid is noninvasive and efficient. This study compared the image quality on 3D-FSE-Cube MRD and 3D-FSE-Cube-Flex MRD. The results demonstrated both of the technique has its own advantage. The mutual complementation of each other can fulfill the thorough application of MRI for qualitative images.

MAGNETIC RESONANCE IMAGING TEXTURE ANALYSIS (MRTA) OF NASOPHARYNGEAL CARCINOMA IN T2W AND CE-T1W IMAGES

NAFIR ABDUL JALEEL ${ }^{1}$, LI JUN WANG ${ }^{1}$, and YAN WEI MIAO ${ }^{1}$

1970

${ }^{1}$ RADIOLOGY, THE FIRST AFFLIATED HOSPITAL OF DALIAN MEDICAL UNIVERSITY, DALIAN, China

Nasopharyngeal carcinoma is a common malignant tumour in Asian countries with nearly $80 \%$ of them being squamous cell carcinoma. The aim is to investigate the potential of MRI (T2W \& CE-T1W) texture analysis to predict response in patients with advanced Nasopharyngeal carcinoma(squamous cell carcinoma). The patients were grouped into Residual/NonResponders and Non-Residual/Responders based on the post-treatment MR images. Texture analysis was used to find significant parameters. On T2WI, significance were recorded with 2 parameters which showed potential to predict the response to treatment and can be further used in the future studies to predict and alter the treatment course and cycles

Regional cerebral blood flow alterations in patients with comitant exotropia: a pilot 3D-pCASL MRI study

Zhi Wen ${ }^{1}$, Xuefang Lư ${ }^{1}$, Xin Huang ${ }^{2}$, Yang Fan ${ }^{3}$, Yunfei Zha ${ }^{1}$, and Baojun Xie ${ }^{1}$

1971

${ }^{1}$ Dept. Radiology, Renmin Hospital of Wuhan University, Wuhan, China, ${ }^{2}$ Dept. Ophthalmology, Renmin Hospital of Wuhan University, Wuhan, China, ${ }^{3}$ GE Healthcare, Beijing, China

Strabismus is a common eye disease characterized by abnormal eye position and ocular motor disorder. In this study, we compared the cerebral blood flow (CBF) in patients with comitant extropia (CE) relative to healthy controls using 3D-pCASL MRI. We found that CE patients had significantly increased CBF in the right parahippocampal region, bilateral medial FG/ACC, bilateral IFG, left SFG, bilateral MCC, right MFG (BA8), and right paracentral lobule. This study demonstrates the hypothesis that CE involves the dysfunction of visual pathway. Interestingly, the most significant $C B F$ increase in the right parahippocampal region, suggests potential cognitive and mood compensation in $C E$.

Traditional Poster

\section{A Potpourri of Multiple Sclerosis}

Exhibition Hall 1972-1993

Tuesday 16:15 - 18:15

Periventricular innate immune cell activation drives tissue damage and clinical progression in multiple sclerosis

Emilie Poirion ${ }^{1}$, Benedetta Bodini ${ }^{1}$, Charline Benoit ${ }^{1}$, Matteo Tonietto ${ }^{1}$, Geraldine Bera ${ }^{1}$, and Bruno Stankoff ${ }^{1,2}$ 
${ }^{1}$ Institut du Cerveau et de la Moelle épinière (ICM), Inserm U 1127, CNRS UMR 7225, Sorbonne Universités, UPMC Univ Paris 06 UMR S 1127, Paris, France, ${ }^{2}$ Neurology Department, St Antoine Hospital, APHP, Paris, France, Paris, France

The objective of this study was to investigate the role of activated microglia in the periventricular damage of patients with MS, combining positron emission tomography with [18F]DPA714 and magnetisation transfert ratio (MTR). Using two-mm thick rings from the ventricular CSF surface to periventricular WM and thalamus, we describe the presence of a gradient of activated microglia together with a gradient of MTR, which correlate with the clinical worsening of patients. These results suggest that an increase of activated microglia and tissue damage might be triggered by the presence of CSF-derived factors, and could mediate the subsequent development of neuro-axonal irreversible damage in MS.

Microglia activation in multiple sclerosis lesions drives structural changes over time and correlates with clinical progression

Matteo Tonietto ${ }^{1}$, Charline Benoit ${ }^{1}$, Emilie Poirion ${ }^{1}$, Geraldine Bera ${ }^{1}$, Mattia Veronese ${ }^{2}$, Federico E. Turkheimer ${ }^{2}$, Marco Battaglini ${ }^{3}$, Benedetta Bodini ${ }^{1}$, and Bruno Stankoff ${ }^{1}$

${ }^{1}$ Brain and Spine Institute - ICM, Paris, France, ${ }^{2}$ King's College London, London, United Kingdom, ${ }^{3}$ University of Siena, Siena, Italy

In this study we develop a new method to generate individual maps of activated microglia from ${ }^{18} \mathrm{~F}$-DPA-714 positron emission tomography images and we use it to reproduce in-vivo the histopathological classification of multiple sclerosis white matter lesions. This method allowed us to identify chronically active lesions which are not detectable with standard MRI. These lesions were found to be the most structurally dynamic over time, having a higher chance of enlarging or shrinking after one year. Furthermore, a higher number of active lesions was associated with a more severe clinical progression.

Microglial activation is accompanied by diffuse axonal loss in multiple sclerosis: in vivo evidence by multimodal 11C-PBR28 MR-PET and multi-shell diffusion imaging

Elena Herranz ${ }^{1,2}$, Silvia De Santis ${ }^{3}$, Constantina Andrada Treaba ${ }^{1,2}$, Tobias Granberg ${ }^{1,2,4,5}$, Russell Ouellette ${ }^{1}$, Jacob Sloane ${ }^{2,6}$, Eric Klawiter ${ }^{1,2,7}$, Nicola Toschi ${ }^{8}$, and Caterina Mainero ${ }^{1,2}$

${ }^{1}$ Radiology, Athinoula A. Martinos Center for Biomedical Imaging, Massachusetts General Hospital, Boston, MA, United States, ${ }^{2}$ Harvard Medical School, Boston, MA, United States, ${ }^{3} \mathrm{CSIC}-U \mathrm{MH}$, Instituto de Neurociencias de Alicante, Alicante, Spain, ${ }^{4}$ Department of Clinical Science, Intervention and Technology, Karolinska Institutet, Stockholm, Sweden, ${ }^{5}$ Department of Radiology, Karolinska University Hospital, Stockholm, Sweden, ${ }^{6}$ Neurology, Beth Israel Deaconess Medical Center, Boston, MA, United States, ${ }^{7}$ Neurology, Massachusetts General Hospital, Boston, MA, United States, ${ }^{8}$ Department of Biomedicine and Prevention, University of Rome Tor Vergata, Rome, Italy

Neuropathological studies of multiple sclerosis (MS) established that diffuse microglia activation with axonal loss in the normal appearing white matter (NAWM) is a main determinant of disease progression. The in vivo study of neuroinflammation and axonal integrity is still challenging. We combined 11C-PBR28 MR-PET with multi-shell diffusion imaging to investigate neuroinflammation and microstructural abnormalities in the NAWM of MS subjects. Results showed evidence of diffuse neuroinflammation accompanied by microstructural diffusion abnormalities with decreased axonal density. The axonal density estimate from the Composite Hindered and Restricted Model of Diffusion was more sensitive than diffusion tensor imaging measures in disclosing axonal damage.

Cortical metabolic changes and glial cell activation in multiple sclerosis: An in vivo 11C-PBR28 MR-PET and magnetic resonance spectroscopy study.

Elena Herranz ${ }^{1,2}$, Constantina Andrada Treaba ${ }^{1,2}$, Eva Ratai ${ }^{1,2}$, Valeria Barletta ${ }^{1,2}$, Russell Ouellette ${ }^{1}$, Marco Loggia ${ }^{1,2}$, Jacob Sloane ${ }^{2,3}$, Eric Klawiter ${ }^{1,2,4}$, and Caterina Mainero ${ }^{1,2}$

${ }^{1}$ Radiology, Athinoula A. Martinos Center for Biomedical Imaging, Massachusetts General Hospital, Boston, MA, United States, ${ }^{2}$ Harvard Medical School, Boston, MA, United States,

${ }^{3}$ Neurology, Beth Israel Deaconess Medical Center, Boston, MA, United States, ${ }^{4}$ Neurology, Massachusetts General Hospital, Boston, MA, United States

We combined 11C-PBR28 imaging on a high resolution, integrated human MR-PET system with magnetic resonance spectroscopy to investigate brain metabolites abnormalities and microglia activation in the motor cortex of multiple sclerosis subjects relative to healthy controls. Our study provided increase of microglia activation and decrease of $\mathrm{N}$-acetylaspartate, the latter indicating neuronal injury and/or loss, in multiple sclerosis compared to controls. None of the other metabolites (choline, myoinositol, glutamine, glutamate, phosphocholine) showed significant differences between the two groups. Also, we did not find a correlation between 11C-PBR28 binding and the metabolites concentration, suggesting that the two measures reflect distinct pathological aspects.

19F MR characterization of teriflunomide, a fluorinated drug indicated in Multiple Sclerosis

Christian Prinz ${ }^{1}$, Jason M. Millward ${ }^{1}$, João dos Santos Periquito ${ }^{1}$, Ludger Starke ${ }^{1}$, Paula Ramos Delgado ${ }^{1}$, Stefanie Muenchberg ${ }^{1}$, Andreas Pohlmann ${ }^{1}$, Thoralf Niendorf ${ }^{1,2}$, and Sonia Waiczies ${ }^{1}$

${ }^{1}$ Berlin Ultrahigh Field Facility, Max Delbrueck Center for Molecular Medicine in the Helmholtz Association, Berlin, Germany, ${ }^{2}$ Experimental and Clinical Research Center, a joint cooperation between the Charite Medical Faculty and the Max Delbrueck Center for Molecular Medicine in the Helmholtz Association, Berlin, Germany

Teriflunomide is an anti-inflammatory drug indicated for the treatment of Multiple Sclerosis (MS). This disease presents with a wide spectrum of symptoms and available drugs have different effects, thereby posing a major treatment challenge. Due to its three fluorine atoms, teriflunomide can be detected non-invasively by fluorine-19 $\left({ }^{19} \mathrm{~F}\right)$ magnetic resonance. The objective of this work is to characterize the ${ }^{19} \mathrm{~F}$ MR properties of teriflunomide in order to adapt MR sequences for in vivo measurements. Here, we studied the relaxation times of teriflunomide and their modifications as a result of concentration, $\mathrm{pH}$ and temperature changes. 
Comparison of Two Methods for the Measurement of T1 Hyperintensity in Multiple Sclerosis Patients with Repeated Exposure to Gadolinium-Based Contrast Agents

Megan Hii ${ }^{1}$, Heejun Kang ${ }^{1,2}$, Megan Le ${ }^{1}$, Andrew Riddehough ${ }^{1}$, Anthony Traboulsee ${ }^{1}$, Shannon Kolind ${ }^{1}$, David Li ${ }^{1,2}$, and Roger Tam ${ }^{1,2}$

Exposure to gadolinium-based contrast agents is associated with long-term increase in $T_{1}$ signal intensity in deep grey brain structures, but the measurement methodologies have not been well investigated. We propose marking regions of interest (ROIs) on registered serial $\mathrm{T}_{2} \mathrm{w}$ images, and compared two methods for measuring the signal changes in the corresponding $T_{1} w$ images: 1) Align the $T_{1} w$ to the $T_{2} w$ images ( $T_{2}$-space), and 2) Map the ROls marked on the $T_{2} w$ images to the $T_{1} w$ images ( $T_{1}$-space). Applying these methods to frequent and infrequent scanning cohorts, we found signal increase to be associated with GBCA exposure, and $T_{1}$-space is more sensitive.

Gadolinium retention in the brain - an MRI relaxometry study comparing linear and macrocyclic types of gadolinium based contrast agents

Yngve Forslin $^{1,2}$, Juha Martola ${ }^{1}$, Sara Shams ${ }^{1}$, Åsa Bergendal ${ }^{1}$, Maria Kristoffersen-Wiberg ${ }^{1}$, Sten Fredrikson ${ }^{1}$, and Tobias Granberg ${ }^{1}$

${ }^{1}$ Department of Clinical Neuroscience, Karolinska Institutet, Stockholm, Sweden, ${ }^{2}$ Department of Radiology, Karolinska University Hospital, Stockholm, Sweden

Gadolinium contrast agents (GBCAs) have been shown to be retained in the brain after multiple linear GBCA administrations. We aimed to quantitatively investigate T1 in relation to linear and macrocyclic GBCA-administrations in DN and GP by relaxometry. $80 \mathrm{MS}$ patients who had received different types of GBCAs, were consecutively recruited. This study, in line with previous studies using semi-quantitatively methods, showed that exposure of GBCA leads to shorter T1 relaxation using linear GBCA in comparison to patients who had received macrocyclic types of GBCA, as well as patients without GBCA exposure and healthy controls.

An individual radiomics nomogram for differential diagnosis between multiple sclerosis and neuromyelitis optica spectrum disorder

Yaou Liu ${ }^{1}$, Di Dong ${ }^{2}$, Liwen Zhang ${ }^{2}$, Yunyun Duan ${ }^{1}$, Jie Tian² ${ }^{2}$ and Kuncheng Li ${ }^{3}$

${ }^{1}$ Department of Radiology, Beijing Tiantan Hospital, Capital Medical University, Beijing, China, ${ }^{2}$ CAS Key Laboratory of Molecular Imaging, Institute of Automation, Chinese Academy of Sciences, Beijing, China, ${ }^{3}$ Department of Radiology, Xuanwu Hospital, Capital Medical University, Beijing, China

Clinically distinguishing the multiple sclerosis (MS) and neuromyelitis optica spectrum disorder (NMOSD) is critical, since the prognosis and treatment of these disorders differ. We extracted nine radiomics features from 485 radiomics features combining with clinical measurements to build the model for differentiating MS and NMOSD. The area under receiver operating characteristic curve (AUC) of the model was 0.8808 and 0.7115 in the primary and validation cohort. The model demonstrated good calibration. The current study revealed the different radiomics features between MS and NMOSD, and developed and validated an individual model to differentiate the two diseases.

Sufficient Gradient Sampling for Diffusion Tensor Imaging in Clinical Trials

Ken Sakaie ${ }^{1}$, Jian Lin ${ }^{1}$, Josef Debbins ${ }^{2}$, Mark Lowe ${ }^{1}$, and Robert Fox ${ }^{3}$

${ }^{1}$ Imaging Institute, The Cleveland Clinic, Cleveland, OH, United States, ${ }^{2}$ Keller Center for Imaging Innovation, Barrow Neurological Institute, Phoenix, AZ, United States, ${ }^{3}$ Neurological Institute, The Cleveland Clinic, Cleveland, $\mathrm{OH}$, United States

Although many diffusion-weighting gradients are desirable for diffusion MRI, implementation may be difficult in a multicenter trial for practical reasons. This study retrospectively examines the adequacy of using as few as 6 directions, the minimum required for calculating the diffusion tensor, for tissue microstructure measurements.

Biophysically meaningful MRI features for accurate classification of multiple sclerosis phenotypes

Antonio Ricciardi ${ }^{1,2,3}$, Francesco Grussu ${ }^{1,3}$, Wallace Brownlee ${ }^{1}$, Baris Kanber ${ }^{1,4}$, Ferran Prados ${ }^{1,4}$, Sara Collorone ${ }^{1}$, Enrico Kaden ${ }^{3}$, Ahmed Toosy $^{1,5}$, Sebastien Ourselin $^{4}$, Olga $^{2}$ Ciccarelli ${ }^{1,5}$, Daniel C Alexander ${ }^{3}$, and Claudia Angela Gandini Wheeler-Kingshott ${ }^{1,6,7}$

${ }^{1}$ Queen Square MS Centre, Department of Neuroinflammation, UCL Institute of Neurology, Faculty of Brain Sciences, University College London, London, United Kingdom, ${ }^{2}$ Department of Medical Physics and Biomedical Engineering, University College London, London, United Kingdom, ${ }^{3}$ Centre for Medical Image Computing, Department of Computer Science, University College London, London, United Kingdom, ${ }^{4}$ Translational Imaging Group, Centre for Medical Image Computing, Medical Physics and Biomedical Engineering, University College London, London, United Kingdom, ${ }^{5}$ National Hospital of Neurology and Neurosurgery, London, United Kingdom, ${ }^{6}$ Department of Brain and Behavioral Sciences, University of Pavia, Pavia, Italy, ${ }^{7}$ Brain MRI $3 T$ Research Centre, C. Mondino National Neurological Institute, Pavia, Italy 
Quantitative MRI can provide maps of biophysically meaningful features (BMFs) that can be exploited using machine learning techniques to better correlate MR alterations with multiple sclerosis (MS) severity, and improve our understanding of the disease. In this study, a random forest classifier was trained over a rich multi-modal quantitative MRI dataset of healthy controls and MS patients with different phenotypes, to find the BMFs that best characterise disease course. Inflammation and atrophy were the most significant BMFs in distinguishing between controls and patients, with microstructural alterations arising particularly when comparing subjects who only experienced a clinically isolated syndrome with patients and controls.

Evolution of functional and structural connectivity of motor network during 2 years of fingolimod therapy for multiple sclerosis

Pallab K Bhattacharyya ${ }^{1,2}$, Robert Fox ${ }^{3}$, Hong Li ${ }^{4}$, Jian Lin ${ }^{1}$, Ken E Sakaie ${ }^{1}$, and Mark J Lowe ${ }^{1}$

${ }^{1}$ Imaging Institute, Cleveland Clnic, Cleveland, $\mathrm{OH}$, United States, ${ }^{2}$ Radiology, Cleveland Clinic Lerner College of Medicine, Cleveland, OH, United States, ${ }^{3} \mathrm{Neurological}$ Institute, Cleveland Clnic, Cleveland, $\mathrm{OH}$, United States, ${ }^{4}$ Quantitative Health Sciences, Cleveland Clnic, Cleveland, OH, United States

Evolution of resting state functional connectivity (fcMRI) between right and left primary motor cortices, and structural connectivity along corticospinal tract (CST) during 2 years of Filgolimod therapy of patients with multiple sclerosis were investigated. MS patients were scanned at baseline (just prior to start of treatment), $6,12,18$ and 24 months after the start of treatment. Using echoplanar imaging for fCMRI and high angular resolution diffusion imaging for assessing normal appearing white matter integrity along CST, it was found that both functional and structural connectivity damage of motor network stabilized after one year of fingolimod treatment.

Integration of Probabilistic Atlas and Graph Cuts for Automated Segmentation of Multiple Sclerosis lesions

Francesca Galassi ${ }^{1}$, Olivier Commowick ${ }^{1}$, and Christian Barillot

${ }^{1}$ Univ Rennes, Inria, CNRS, IRISA UMR 6074, VISAGES ERL U-1228, F-35000, Rennes, France

We propose a framework for automated segmentation of Multiple Sclerosis (MS) lesions from MR brain images. It integrates a priori tissues and MS lesions information into a GraphCuts algorithm for improved segmentation results. We formulate the energy terms to include a priori information as well as the information derived directly from the MR images. We validate our method on a dataset of $37 \mathrm{MS}$ subjects with a broad range of lesion loads. Results indicate that integrating a priori information with the information derived from the images can improve the segmentation outcome.

Reproducibility Study of a Longitudinal Pipeline for Brain Volumetry based on Partial Volume Estimation

Ricardo A. Corredor-Jerez $z^{1,2,3}$, Mário João Fartaria ${ }^{1,2,3}$, Adrian Tsang ${ }^{4}$, Robert Bermel ${ }^{5}$, Stephen E. Jones ${ }^{5}$, Izlem Izbudak ${ }^{6}$, Ellen M Mowry ${ }^{6}$, Yvonne W. Lui $^{7}$, Lauren Krupp ${ }^{7}$, Elizabeth Fisher $^{4}$, Tobias Kober ${ }^{1,2,3}$, and Bénédicte Maréchal ${ }^{1,2,3}$

${ }^{1}$ Advanced Clinical Imaging Technology, Siemens Healthcare AG, Lausanne, Switzerland, ${ }^{2}$ Department of Radiology, Centre Hospitalier Universitaire Vaudois (CHUV), Lausanne, Switzerland, ${ }^{3}$ Signal Processing Laboratory (LTS 5), École Polytechnique Fédérale de Lausanne (EPFL), Lausanne, Switzerland, ${ }^{4}$ Biogen, Cambridge, MA, United States, ${ }^{5}$ Cleveland Clinic, Cleveland, $\mathrm{OH}$, United States, ${ }^{6}$ Johns Hopkins University, Baltimore, MD, United States, ${ }^{7}$ New York University, New York, NY, United States

A reliable and accurate quantification of brain tissue loss is important to measure progressive atrophy caused by neurological diseases such as multiple sclerosis. However, accuracy and reproducibility of current methods are often limited by partial volume effects, especially at tissue interfaces where subtle atrophy patterns are likely to occur. We propose a longitudinal pipeline for brain tissue segmentation incorporating partial volume estimation to increase longitudinal robustness. Results show an increase in reproducibility of $44 \%$ compared to methods not including partial volume effects in volume estimation, suggesting that these effects should be taken into account for longitudinal atrophy measurements.

Diagnostic Accuracy of Semiautomatic T2 Subtraction plus Quantitative Susceptibility Mapping in the Detection of New Multiple Sclerosis Lesions

Shun Zhang ${ }^{1,2}$, Thanh D. Nguyen ${ }^{2}$, Yize Zhao ${ }^{3}$, Susan A. Gauthier ${ }^{4}$, Yi Wang $^{2,5}$, and Ajay Gupta ${ }^{2}$

${ }^{1}$ Radiology, Tongji Hospital, Tongji Medical College, Huazhong University of Science and Technology, Wuhan, China, ${ }^{2}$ Radiolgy, Weill Cornell Medical College, NewYork, NY, United States, ${ }^{3}$ Healthcare Policy and Research, Weill Cornell Medical College, NewYork, NY, United States, ${ }^{4}$ Neurology, Weill Cornell Medical College, NewYork, NY, United States, ${ }^{5}$ Biomedical Engineerring, Cornell University, Ithaca, NY, United States

The ability to identify new MRI lesions in patients with multiple sclerosis (MS) on follow-up imaging is of great importance in monitoring disease activity and informing therapeutic decision-making. Gadolinium (Gd)-enhancing lesions tend to be isointense or slightly hyperintense on QSM images whereas non-enhancing tend to be hyperintense. However, characterization of QSM signal of MS lesions in isolation can be difficult without coregistered T2-weighted imaging. For this reason, we developed an algorithm of T2-subtraction based on two time points of FLAIR images, as well as an automatic lesion mask to help detect new MS lesions with the overall goal of combining this technique with QSM to predict the enhancement status of MS lesions. We found that T2 subtraction+QSM has a sensitivity of $90.9 \%$ to predict new enhancing lesions that had been previously identified by experienced neuroradiologists on $\mathrm{T} 1 \mathrm{w}+\mathrm{Gd}$ imaging. In discriminating between new enhancing versus new but nonenhancing lesions, our T2 subtraction+QSM protocol had a sensitivity of $87.5 \%$, and specificity of $89.7 \%$. Receiver operating characteristic (ROC) curve analysis using region-of-interest of susceptibility values on QSM showed an optimal cutoff susceptibility value of -4.92 $\mathrm{ppb}$ (referenced to CSF) in distinguishing new enhancing lesions from new but nonenhancing lesions (sensitivity $88.9 \%$, specificity $80.0 \%$ ). Our results suggest that T2 subtraction plus QSM no Gd protocol can be a useful tool in detecting the new enhancing MS lesions in clinical practice without Gd injection. 
Automated Detection of Central Vessel Sign in Multiple Sclerosis using a 3D Deep Convolutional Neural Network

Richard Watts ${ }^{1}$

A 3D deep convolutional neural network ( $\mathrm{CCNN}$ ) was trained to differentiate MS from non-MS lesions based on the orientation and location of a central vein ('central vein sign') relative to the lesion. Excellent performance was achieved using simulated FLAIR and $\mathrm{T}_{2}{ }^{*}$-weighted imaging, with realistic noise levels. The dCNN may be capable of identifying other discriminatory features from multimodal human imaging data.

The corticospinal tract in relapsing-remitting multiple sclerosis: a preliminary tractography and fixel-based MRI analysis at ultra high-field

Myrte Strik ${ }^{1}$, Camille Shanahan ${ }^{1}$, Stacey Telianidis ${ }^{1}$, Anneke Van der Walt ${ }^{2,3}$, Rebecca Glarin ${ }^{1}$, Roger Ordidge ${ }^{1}$, Bradford Moffat ${ }^{1}$, Fary Khan $^{3}$, Andisheh Bastani $^{3}$, Eduardo Cofré Lizama $^{3}$, Mary Galea ${ }^{3}$, Trevor Kilpatrick ${ }^{1,2}$, Jon Cleary ${ }^{1}$, and Scott Kolbe ${ }^{1,4}$

${ }^{1}$ Anatomy and Neuroscience, University of Melbourne, Melbourne, Australia, ${ }^{2}$ Neurology, Royal Melbourne Hospital, Melbourne, Australia, ${ }^{3}$ Medicine, University of Melbourne, Melbourne, Australia, ${ }^{4}$ Florey Institute of Neuroscience and Mental Health, Melbourne, Australia

Lower limb disability in multiple sclerosis (MS) is likely related to axonal damage in the corticospinal tract (CST), the main motor pathway. This study aimed to compare the degree of CST degeneration to clinical motor disability using high field (7T) diffusion weighted MRI and subsequent analyses methods like tractography and fixel-based analysis. Eleven minimally impaired relapsing-remitting MS patients ( $1 \mathrm{~m} / 10 \mathrm{f}, 42 \pm 12.4 \mathrm{yrs})$ were tested. Results show loss of fiber density (FD) in the subcortical white matter of the CST was associated with increased pyramidal dysfunction ( $p_{\text {uncorrected }}<0.05$ ). FD could provide a useful marker of disease progression leading to loss of mobility.

Diffusivity and the neurocognitive domains of premorbid intelligence and visuospatial memory in Pediatric Multiple Sclerosis

Sindhuja T. Govindarajan ${ }^{1}$, M. Andrea Parra ${ }^{2}$, Tao Wang ${ }^{1}$, Kenneth Wengler ${ }^{1}$, Chuan Huang ${ }^{2}$, Xiang He ${ }^{2}$, Leigh Charvet $^{3}$, Lauren Krupp ${ }^{3}$, and Tim Q Duong ${ }^{2}$

${ }^{1}$ Stony Brook University, Stony Brook, NY, United States, ${ }^{2}$ Stony Brook University School of Medicine, Stony Brook, NY, United States, ${ }^{3}$ New York University Langone Medical Center, New York, NY, United States

DTI has been commonly used to study multiple sclerosis (MS) patients ${ }^{1-3}$ and many studies have correlate DTI parameters with neurocognitive functions. However, only a handful of studies ${ }^{4,5}$ have characterized such relationships in pediatric MS patients. The goal of this study was to investigate DTI characteristics in pediatric onset MS patients and to correlate them with neurocognitive functions (intelligence and visuospatial memory).

Multi-shell diffusion imaging is a sensitive marker for longitudinal axonal degeneration in multiple sclerosis

Nicola Toschi ${ }^{1,2}$, Silvia De Santis ${ }^{3,4}$, Tobias Granberg $^{2,5,6}$, Russel Ouellette IV ${ }^{2,5}$, Constantina Andrada Treaba ${ }^{2}$, Elena Herranz ${ }^{2}$, Qiuyun Fan $^{2}$, and Caterina Mainero ${ }^{2}$

${ }^{1}$ Biomedicine and Prevention, University of Rome Tor Vergata, Rome, Italy, ${ }^{2}$ Athinoula A. Martinos Center for Biomedical Imaging and Harvard Medical School, Boston, MA, United States, ${ }^{3} \mathrm{CSIC}-U M H$, Instituto de Neurociencias de Alicante, Alicante, Spain, ${ }^{4}$ Brain Research Imaging Centre (CUBRIC), Cardiff University, Cardiff, United Kingdom, ${ }^{5}$ Department of Clinical Neuroscience, Karolinska Institutet, Solna, Sweden, ${ }^{6}$ Department of Radiology, Karolinska University Hospital, Stockholm, Sweden

Axonal loss, a crucial pathological process in multiple sclerosis (MS), can be disentangled non-invasively by the CHARMED diffusion model. 8 early MS subjects were scanned at baseline and after 1 year follow-up. At follow-up, TBSS analysis showed statistically significant changes (decrease in FR/FA, increase in MD) compared to baseline in widespread brain regions. The most extensive change was evident in FR, which also showed the greatest sensitivity, especially in areas of fiber-crossing. FR was the only index which detected longitudinal change in axonal density in lesions and therefore holds promise as a biomarker for early diagnosis and disease-monitoring purposes.

A new texture-based method for assessing high angular diffusion MRI from patients with multiple sclerosis

Zahra Hosseinpour ${ }^{1}$, Olayinka Oladosu², Wei-qiao Liu², Bruce G Pike ${ }^{2}$, Luanne M Metz² , and Yunyan Zhang ${ }^{2}$ 
Multiple Sclerosis Gray Matter Shows Greater Abnormalities in Phosphate Metabolites than White Matter

Manoj K Sammi ${ }^{1}$, Yosef Berlow ${ }^{2}$, Randy West ${ }^{1,3}$, Katherine Powers ${ }^{1}$, Vijayshree Yadav ${ }^{3,4}$, Dennis Bourdette ${ }^{3}$, Rebecca Spain ${ }^{3}$, and William D Rooney ${ }^{1,3}$

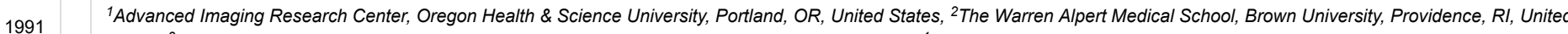
States, ${ }^{3}$ Department of Neurology, Oregon Health \& Science University, Portland, OR, United States, ${ }^{4}$ MS Center of Excellence West, VA Portland Health Care System, Portland, OR, United States

Phosphate metabolite distribution in gray matter and white matter in human brain is compared between healthy control $(\mathrm{HC})$ and subjects with Multiple Sclerosis (MS) using ${ }^{31} \mathrm{P}$ Magnetic Resonance Spectroscopic Imaging (MRSI) at 7T. Phosphate metabolites are decreased in GM in MS compared to healthy controls.

Gray Matter Atrophy and Microstructural White Matter Abnormalities Underlying Cognitive Impairment in Benign MS

Elisabetta Pagani ${ }^{1}$, Gianna Carla Riccitelli ${ }^{1}$, Marta Radaelli ${ }^{2}$, Paolo Preziosa ${ }^{1,2}$, Giancarlo Comi ${ }^{2}$, Andrea Falini ${ }^{3}$, Massimo Filippi ${ }^{1,2}$, and Maria A. Rocca ${ }^{1,2}$

${ }^{1}$ Neuroimaging Research Unit, INSPE, Division of Neuroscience, San Raffaele Scientific Institute, Vita-Salute San Raffaele University, Milan, Italy, ${ }^{2}$ Department of Neurology, San Raffaele Scientific Institute, Vita-Salute San Raffaele University, Milan, Italy, ${ }^{3}$ Department of Neuroradiology, San Raffaele Scientific Institute, Vita-Salute San Raffaele University, Milan, Italy

The definition of benign multiple sclerosis (BMS) is based on long disease duration and low level of disability, without considering cognitive deficits. Aim of the study was to apply voxelwise methods to investigate whether cognitive dysfunction in BMS patients is associated with specific patterns of regional damage in the brain gray matter (GM) and white matter (WM). High-resolution T1-weighted and diffusion tensor MRI scans were acquired from 50 healthy controls and 38 BMS patients, $42 \%$ of which were classified as cognitively impaired. Distinct regional patterns of abnormalities, functionally relevant for cognitive processing, were associated with cognitive impairment in BMS patients.

Clustering of Multiple Sclerosis Patient's Clinical Courses with White Matter Fiber-Bundle Profiles

Gabriel Kocevar ${ }^{1}$, Claudio Stamile ${ }^{1}$, François Cotton ${ }^{1,2}$, Françoise Durand-Dubief ${ }^{1,3}$, and Dominique Sappey-Marinier ${ }^{1,4}$

${ }^{1}$ CREATIS Laboratory, Université Claude Bernard - Lyon 1, Lyon, France, ${ }^{2}$ Centre Hospitalier Lyon-Sud - Service de Radiologie, Hospices Civils de Lyon, Lyon, France, ${ }^{3}$ Hôpital Neurologique - Service de Neurologie A, Hospices Civils de Lyon, Lyon, France, ${ }^{4}$ CERMEP - Imagerie du Vivant, Université de Lyon, Lyon, France

Predicting the individual multiple sclerosis (MS) patients evolution, based on markers available from disease onset, may help the neurologist in the patient care. However, such a prediction remains a challenge. In this study, we merged spatial information of fiber tracking with diffusivity metrics, measured in 68 patients presenting the three forms of MS, in order to classify patients using a white matter fiber-bundle profile analysis. The good performances of the clustering, reached with fractional anisotropy and mean diffusivity together, make our method a potential tool to better predict the disease evolution, especially the conversion of RR-MS to SP-MS.

Traditional Poster

\section{Alzheimer's Disease \& Other Dementias}

Test-retest reproducibility of quantitative susceptibility mapping in a multi-site study on Alzheimer disease: effect of reference region of interest choice

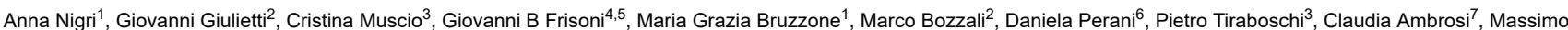
Caulo $^{8,9}$, Pietro Chiarini ${ }^{10}$, Elena Chipi ${ }^{11}$, Stefano Chiti ${ }^{12}$, Enrico Fainardi ${ }^{13}$, Stefania Ferraro ${ }^{1}$, Cristina Festari ${ }^{4}$, Roberto Gasparotti ${ }^{7}$, Ruben Gianeri $^{1}$, Andrea Ginestroni ${ }^{13}$, Andrea Ginestroni ${ }^{13}$, Lorella Mascaro ${ }^{14}$, Riccardo Navarra ${ }^{8}$, Lucilla Parnetti ${ }^{11}$, Alberto Redolfi ${ }^{4}$, Laura Serra ${ }^{2}$, Roberto Tarducci ${ }^{15}$, Fabrizio Tagliavini ${ }^{3,16}$, and Jorge Jovicich ${ }^{17}$

${ }^{1}$ Neuroradiology Department, Neurological Institute "Carlo Besta", IRCCS Foundation, Milan, Italy, ${ }^{2}$ Neuroimaging laboratory, IRCCS Santa Lucia Foundation, Rome, Italy, ${ }^{3}$ Division of Neurology V/Neuropathology, Neurological Institute "Carlo Besta", IRCCS Foundation, Milan, Italy, ${ }^{4}$ Laboratory of Alzheimer's Neuroimaging and Epidemiology, IRCCS Centro San Giovanni di Dio-FBF, Brescia, Italy, ${ }^{5}$ Memory Clinic and LANVIE-Laboratory of Neuroimaging of Aging, University Hospitals and University of Geneva, Geneva, Switzerland, ${ }^{6}$ Nuclear Medicine Unit, IRCCS San Raffaele Hospital, Milan, Italy, ${ }^{7}$ Department of Diagnostic Imaging, Neuroradiology Unit, University of Brescia, Brescia, Italy, ${ }^{8}$ Department of Neuroscience, Imaging and Clinical Sciences, University "G. d'Annunzio" of Chieti, Chieti, Italy, ${ }^{9}$ Institute for Advanced Biomedical Technologies (ITAB), University "G. d'Annunzio" of Chieti, Chieti, Italy, ${ }^{10}$ Neuroradiology Unit, Department of Diagnostic Imaging, R. Silvestrini Hospital, Perugia, Italy, ${ }^{11}$ Centre for Memory Disturbances, Lab of Clinical Neurochemistry, University of Perugia, Perugia, Italy, ${ }^{12}$ Department Health Professions - U.O.c Research and Development, Careggi University Hospital Florence, Florence, Italy, ${ }^{13}$ Department of Neuroradiology, Careggi University Hospital Florence, Florence, Italy, ${ }^{14}$ Medical Physics Unit, Spedali Civili di Brescia, Brescia, Italy, ${ }^{15}$ Section of Neurology, Center for Memory Disturbances, University of Perugia, Perugia, Italy, ${ }^{16}$ Scientific Direction, Neurological Institute "Carlo Besta", IRCCS Foundation, Milan, Italy, ${ }^{17}$ Center for Mind/Brain Sciences, University of Trento, Mattarello, Italy

Quantitative susceptibility mapping (QSM) is a neuroimaging marker of iron of interest as biomarker of Alzheimer disease (AD) progression. The purpose of this work was to assess the test-retest reproducibility of QSM in the Italian AD-NET project, a multi-site study on AD. We evaluated how QSM reproducibility is affected by acquisition (different clinical $3 T$ MRI sites using vendor provided sequences) and analyses factors (choice of the reference region of interest used to compute within-subject relative QSM). The goal is to minimize reproducibility errors and thus maximize the sensitivity to detect longitudinal QSM changes related to the progression of AD. 
Quantitative T1 and T2 mapping with standard and MR fingerprinting techniques to assess frontotemporal dementia: A pilot study

Stilyana Peteva Bakoeva ${ }^{1}$, Vera Catharina Keil ${ }^{1}$, Alina Jurcoane ${ }^{1}$, Mariya Doneva² ${ }^{2}$ Thomas Amthor ${ }^{2}$, Peter Koken², Burkhard Mädler ${ }^{3}$, Wolfgang Block ${ }^{1}$, Klaus Fließbach ${ }^{4}$, and Elke Hattingen ${ }^{1}$

${ }^{1}$ Department of Radiology, University Hospital Bonn, Bonn, Germany, ${ }^{2}$ Philips Research, Hamburg, Germany, ${ }^{3}$ Philips Healthcare, Bonn, Germany, ${ }^{4}$ Department of Psychiatry, University Hospital Bonn, Bonn, Germany

This pilot study explored the utility of MR fingerprinting (MRF) and standard T1 and T2 relaxometry to discover focal anomalies in patients with the primary progressive aphasic form of frontotemporal dementia (FTD). MRF and standard techniques revealed longer T1 and T2 relaxation times of cortex and deep white matter as well as the hippocampus (left>right) in FTD compared to healthy controls. Relaxation times between MRF and standard differed and not all techniques revealed all structures as altered in relaxation time. In conclusion, MRF and standard relaxometry have the potential to quantify brain anomalies in FTD, which may be used for diagnosis and monitoring, but are not interchangeable.

Detection of Alzheimer's Disease Patients Based on Single Brain Region by Convolution Neural Networks

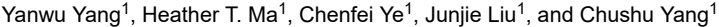

${ }^{1}$ Harbin Institute of Technology (Shenzhen), Shezhen, China

Diagnosis plays an important role in preventing progress and treating the Alzheimer's disease (AD). This paper proposed to predict the AD with a convolutional neural network (CNN), which can learn generic features capturing AD biomarkers. In particular, we extract some specific brain regions from structural MRI and apply MR features from the brain regions to detect $A D$ patients in $\mathrm{CNN}$ framework, achieving accuracy up to $99 \%$ and outperforming some other classifiers from other studies.

Structural and Functional Networks of Emotion Regulation Are Altered in Subjective Cognitive Decline

Pin-Yu Chen ${ }^{1}$, Yung-Chin Hsu ${ }^{1}$, Yu-Chen Wei ${ }^{1}$, Yu-Ling Chang ${ }^{2}$, Ming-Jang Chiu ${ }^{2,3,4,5}$, and Wen-Yih Tseng ${ }^{1,4,5}$

${ }^{1}$ Institute of Medical Device and Imaging, National Taiwan University College of Medicine, Taipei, Taiwan, ${ }^{2}$ Department of Psychology, National Taiwan University, Taipei, Taiwan, ${ }^{3}$ Department of Neurology, National Taiwan University Hospital, College of Medicine, National Taiwan University, Taipei, Taiwan, ${ }^{4}$ Molecular Imaging Center, National Taiwan University, Taipei, Taiwan, ${ }^{5}$ Graduate Institute of Brain and Mind Sciences, College of Medicine, National Taiwan University, Taipei, Taiwan

Previous research suggested that subjective cognitive decline (SCD) may also present with other psychiatric diseases, personal traits, physical conditions and medication use. We hypothesized the depressive trait was the early factor and its neural correlates of function or structure changes may reflect such mental feature. We investigated the functional and structural connectivity of the emotion regulation network in SCD. We found that left amygdala to left IFG especially displayed both functional and structure changes. Our study suggests that the altered patterns of the emotion regulation network could serve as the neural basis of the emotion regulation function and display the depressive trait is the critical risk factor for SCD progression to memory disease.

Riluzole improved Energy Metabolism in AßPP-PS1 Mouse Model of Alzheimer's Disease

Anant Bahadur Patel ${ }^{1}$ and Kamal Saba ${ }^{1}$

${ }^{1}$ NMR Microimaging and Spectroscopy, Centre for Cellular and Molecular Biology, Hyderabad, India

Alzheimer's disease $(A D)$ is a neurodegenerative disorder, characterized by degeneration of neurons leading to memory loss, deterioration in cognitive function and behavior. Despite intensive research of several decades treatment of $A D$ is still a major challenge. Riluzole is known to be neuro-protector and regulates the function of glutamatergic neurons by reducing glutamate release and helping astroglial uptake. In this study, we have evaluated the impacts of Riluzole on the neuronal activity in the $A \beta P P-P S 1$ mouse model of the $A D$ by ${ }^{1} \mathrm{H}-\left[{ }^{13} \mathrm{C}\right]-$ NMR spectroscopy together with infusion of $\left[1,6-{ }^{13} C_{2}\right]$ glucose. The finding of improved neurometabolism in $A D$ mice suggests riluzole improved cognitive function in Alzheimer's disease.

Influence of Different Anesthesia Protocols on Cerebrovascular Reactivity and Cerebral Blood Flow measured by Pseudo-continuous Arterial Spin Labeling

Marc Derieppe $^{1}$, Leon Munting ${ }^{1}$, Ernst Suidgeest ${ }^{1}$, and Louise van der Weerd ${ }^{1}$

Anesthesia protocols in animal studies greatly influence cerebral hemodynamics, so it is critical to devise standardized protocols in order to provide reproducible and comparable Cerebral Blood Flow (CBF) and Cerebral Vascular Reactivity (CVR) in different mouse strains or models. We compared strain-dependent sensitivity towards different anesthesia protocols for vascular reactivity experiments (high-dose isoflurane, medetomidine, low-induction dose isoflurane and high-dose isoflurane in intubated and mechanically ventilated mice), using pseudo-Continuous ASL ( $\mathrm{pCASL}$ ) and discuss the relative performance of these protocols. 
Quantification of Perfusion Asymmetries in MCl Subjects using Arterial Spin Labeling MR imaging

Li Liang ${ }^{1}$, Heather T. Ma ${ }^{1}$, ChenFei Ye ${ }^{1}$, and Susumu Mori ${ }^{2}$

In this study, we recruited seven subjects with $\mathrm{MCl}$ and ten subjects as cognitive normal groups. All subjects underwent PASL and T1-weighted MR imaging. Multimodal images were upload to MRICloud for segmentation and quantification of regional CBF. T-test was used to detect significant changes in brain region volumes and perfusion asymmetries in contralateral regions. We observed tendencies of atrophy in right posterior cingulate cortex and dilation in bilateral fornix, significant perfusion asymmetries were found in regions of temporal lobe, basal ganglia and posterior cingulate cortex among $\mathrm{MCl}$ subjects.

Changes in hippocampal and whole brain stiffness in 14-month old female mice with Alzheimer's disease

Miklos Palotai ${ }^{1}$, Katharina Schregel ${ }^{1,2}$, Navid Nazari ${ }^{1,3}$, Julie P. Merchant ${ }^{4}$, Walter M. Taylor ${ }^{4}$, Charles R.G. Guttmann ${ }^{1}$, Ralph Sinkus ${ }^{5}$, Tracy L. Young-Pearse ${ }^{4}$, and Samuel Patz ${ }^{1}$

${ }^{1}$ Department of Radiology, Brigham and Women's Hospital, Harvard Medical School, Boston, MA, United States, ${ }^{2}$ Institute of Neuroradiology, University Medical Center Goettingen, Goettingen, Germany, ${ }^{3}$ Department of Biomedical Engineering, Boston University, Boston, MA, United States, ${ }^{4}$ Ann Romney Center for Neurologic Diseases, Brigham and Women's Hospital, Harvard Medical School, Boston, MA, United States, ${ }^{5}$ Department of Radiological Imaging, Imaging Sciences \& Biomedical Engineering Division, King's College London, London, United Kingdom

Alzheimer's disease (AD) has been associated with human brain softening, but the underlying biomechanical mechanism is not fully elucidated. We used magnetic resonance elastography to investigate the effect of amyloid-beta accumulation on hippocampal and whole brain (WB) stiffness in transgenic AD and wild-type (WT) mice at 11 and 14 months of age. The only differences observed between AD and WT mice were that the longitudinal change in the loss modulus between 11 and 14 months for female AD mice was significantly different than that of either the WT or male AD mice.

A combined dual-tracer PET/diffusion tractometry analysis of the posterior cingulum in a mild cognitive impairment ketogenic intervention

Maggie Roy ${ }^{1}$, Stephen Cunnane ${ }^{1}$, Étienne Croteau ${ }^{1}$, Alexandre Castellano ${ }^{1}$, Mélanie Fortier ${ }^{1}$, Félix C Morency ${ }^{2}$, Jean-Christophe Houde ${ }^{1}$, and Maxime Descoteaux ${ }^{1}$

2002 1Université de Sherbrooke, Sherbrooke, QC, Canada, ${ }^{2}$ Imeka Solutions Inc., Sherbrooke, QC, Canada

In mild cognitive impairment $(\mathrm{MCl})$, posterior cingulate cortex glucose hypometabolism may results from posterior cingulum (PCg) alterations. We suggest that raising ketone availability to the brain may overcome the brain energy deficit. We developed a dual-tracer PET/dMRI tractometry method to assess whether a ketogenic supplement has impact on fuel uptake in the $\mathrm{PCg}$ of $\mathrm{MCl}$ participants. Mean fuel uptake in the PCg was unchanged post-supplementation, but tract-profiling enabled the identification of sections with lower glucose uptake. Energy supply in white matter fascicles is crucial to sustain adequate axonal function and may be linked to the pathogenesis of $\mathrm{MCl}$.

Unbalanced large-scale brain networks during static and dynamic states in Alzheimer's Disease

Xiaoqing $\mathrm{Ji}^{1}$, Haiyang Geng ${ }^{2,3}$, Rui $\mathrm{Li}^{1}$, Le He${ }^{1}$, and Chun Yuan ${ }^{1,4}$

${ }^{1}$ Center for Biomedical Imaging Research, Tsinghua University, Beijing, China, ${ }^{2}$ Institute of Affective and Social Neuroscience, Shenzhen University, Shenzhen, China, ${ }^{3}$ Neuroimaging Center,University Medical Center Groningen, University of Groningen, Groningen, Netherlands, ${ }^{4}$ Department of Radiology, Washington Univesity, Seattle, WA, United States

In this study, we applied Independent Component Analysis (ICA) and dynamic network approaches to explore the neural network mechanisms between Alzheimer's disease (AD) patients and normal aging healthy controls $(\mathrm{HC})$ from distinct brain states. We conducted rs-fMRI scanning on 12 ADs and $12 \mathrm{HCs}$. From ICA, we got three networks including DAN, VAN and DMN. From dynamic network analysis, we achieved three dynamic states. Two sample t-test results showed that, in AD, DAN had weaker connectivity, DMN had no difference both in static and dynamic states, VAN only had increased connectivity between IFG and other regions in static state.

Prediction of Cognitive Impairment and Amyloid Deposition through Metabolic and Vascular Deficits in ADNI Cohorts

David Ma ${ }^{1,2}$ and Ai-Ling Lin ${ }^{1}$

$2004{ }^{1}$ Sanders Brown Center on Aging, Lexington, KY, United States, ${ }^{2}$ Paul Laurence Dunbar High School, Lexington, KY, United States

Recent research has been focused on developing diagnostics based on amyloid- $\beta$ and tau. However, metabolic and vascular changes pre-date both by several decades. The aim of this study was to exploit the coupling between glucose uptake in aerobic glycolysis and cerebral blood flow to produce a biomarker for metabolic dysfunction and amyloid- $\beta$ deposition. Here we found that a decrease in glucose uptake in the presence of stable blood flow is spatially correlated with an increase in amyloid- $\beta$ deposition and that uncoupling between metabolic and vascular function could drive amyloid- $\beta$ deposition. 
MRI and PET alterations in Alzheimer's disease and cognitive normal HFEH63D polymorphism carriers

Carson J Purnell ${ }^{1}$, Qing X Yang ${ }^{2}$, James R Connor ${ }^{1}$, and Mark D Meadowcroft ${ }^{1,2}$

${ }^{1}$ Neurosurgery, The Pennsylvania State University - College of Medicine, Hershey, PA, United States, ${ }^{2}$ Radiology, The Pennsylvania State University - College of Medicine, Hershey, PA, United States

Anatomical MRI and PET data from the genetic cohort of the ADNI database was analyzed for MRI volumetric, FDG-PET, and AV-45 differences between HFEH63D polymorphism and HFEWT carriers. A decrease in the amount of AV-45 amyloid binding was observed in the AD HFEH63D carriers as well as an increase in FDG metabolism and a decrease in regional brain volume. HFEH63D appears to be preservative in $A D$ with respect to PET imaging biomarkers, but there was a negative interaction in the VBM analysis. This reinforces the hypothesis that HFEH63D has a preservative effect in AD.

Regional brain iron accumulation in an Alzheimer's mouse model fed lipophilic iron

Douglas G Peters ${ }^{1}$, Carson J Purnell ${ }^{1}$, Qing X Yang $^{2}$, James R Connor ${ }^{1}$, and Mark D Meadowcroft ${ }^{1,2}$

${ }^{1}$ Neurosurgery, The Pennsylvania State University - College of Medicine, Hershey, PA, United States, ${ }^{2}$ Radiology, The Pennsylvania State University - College of Medicine, Hershey, PA, United States

Alzheimer's disease (AD) is a progressive neurodegenerative disorder characterized pathologically by amyloid beta (A $\beta$ ) deposition, microgliosis, and iron dyshomeostasis. The goal of this work was to observe how brain iron levels temporally influence A $\beta$ plaque formation, plaque iron concentration, and microgliosis. Humanized APPNL-G-F knock-in and control mice were fed either lipophilic iron compound 3,5,5-trimethylhexanoyl ferrocene (TMHF), normal, or iron deficient diets for twelve months. Increased brain iron was observed in the olfactory, frontal and hippocampal regions and was associated with increased plaque-iron loading and microglial iron inclusions.

Sex Differences in Behavior, Brain Structure and Functional Connectivity in the APOE Epsilon 4 Knock-In Rat Model of Alzheimer's Disease: Are Females the Stronger Sex?

Praveen P Kulkarni ${ }^{1}$, Dan Madularu ${ }^{1,2}$, Thomas R Morrison ${ }^{1}$, and Craig F Ferris ${ }^{1}$

${ }^{1}$ Psychology, Northeastern University, Boston, MA, United States, ${ }^{2}$ Brain Imaging Center, Mc Gill University, Montreal, QC, Canada

APOE genotypes are a major focus for Alzheimer's disease (AD) research following the localization of ApoE on neurofibrillary tangles and amyloids of senile plaques AD patients' brain. The risk of developing $A D$ increases with the frequency of the $\varepsilon 4$ allele, with women outnumbering men. In this study we utilized multiple imaging modalities and behavioral assays to identify sex-specific anatomical biomarkers in a novel rat APOE- $\varepsilon 4$ knock-in model. $\varepsilon 4+$ males show greater variation in neural structure and function in terms of the proportion of brain areas affected; these results are reflected in sex-driven differences in behavior mirroring hippocampal function.

Investigation of cerebral perfusion differences between the 2xTg Alzheimer's disease mouse model and age-matched controls using FAIR ASL MRI

Diana Y. Lee ${ }^{1}$, Abinand C. Rejimon ${ }^{1}$, Rebecca L. McPherson ${ }^{2}$, Kenneth W. Fishbein ${ }^{1}$, Mustapha Bouhrara ${ }^{1}$, Simonetta Camandola ${ }^{3}$, Mark P. Mattson ${ }^{3}$, Edward G. Lakatta ${ }^{2}$, Olga V. Fedorova ${ }^{2}$, and Richard G. Spencer ${ }^{1}$

${ }^{1}$ Laboratory of Clinical Investigation, Magnetic Resonance Imaging and Spectroscopy Section, National Institute on Aging, The National Institutes of Health, Baltimore, MD, United States, 'Laboratory of Cardiovascular Science, National Institute on Aging, The National Institutes of Health, Baltimore, MD, United States, ${ }^{3}$ Laboratory of Neuroscience, National Institute on Aging, The National Institutes of Health, Baltimore, MD, United States

Cerebral blood flow (CBF) is an emerging biomarker of Alzheimer's disease (AD). To correlate CBF to other known measures of AD, such as cortical thinning and volume loss, we assessed perfusion differences between a $2 x T g-A D$ mouse model and age-matched wild-type mice using a FAIR RARE MRI sequence. Our results demonstrate greater systolic blood pressure (SBP) in AD mice as they age as well as hypoperfusion within the cerebral cortex at 12 months of age.

Quantitative Susceptibility Mapping to evaluate the Iron deposition and Venous Blood Oxygenation in the brain for the differentiation of Mild Cognitive Impairment and Alzheimer's disease: A pilot study

Sheelakumari $\mathrm{R}^{1}$, Bejoy Thomas ${ }^{1}$, Ramshekhar Menon ${ }^{2}$, Ramesh Venkatesan ${ }^{3}$, and Chandrasekharan Kesavadas ${ }^{1}$

${ }^{1}$ Department of Imaging Sciences and Interventional Radiology, Sree Chitra Tirunal Institute for Medical Sciences and Technology, Trivandrum, India, ${ }^{2}$ Department of Neurology, Sree Chitra Tirunal Institute for Medical Sciences and Technology, Trivandrum, India, ${ }^{3} \mathrm{GE}$ Healthcare, Banglore, India

Oxygen extraction fraction (OEF) in the brain can be obtained by Quantitative Susceptibility Mapping (QSM). QSM can also estimate iron which is implicated in the pathogenesis of Alzheimer's disease and its pre-symptomatic antecedents. The differences of OEF and iron values among the controls, Mild Cognitive Impairment and AD were investigated by ROI based comparisons using a one-way analysis of variance. These values in the posterior brain regions were found to have a trend towards increment in patient groups compared with controls, in this pilot study. Future studies are required to validate the usefulness of this technique as potential biomarker. 
APOE $\varepsilon 4$ Allele Effect on White Matter Perfusion and Diffusion in Cognitively Normal and MCl Groups

Youngkyoo Jung ${ }^{1}$, Jeongchul Kim¹, Megan E Johnston ${ }^{1}$, Christopher T Whitlow ${ }^{1}$, Laura D Baker ${ }^{2}$, and Suzanne Craft ${ }^{2}$

${ }^{1}$ Radiology, Wake Forest School of Medicine, Winston-Salem, NC, United States, ${ }^{2}$ Gerontology and Geriatric Medicine, Wake Forest School of Medicine, Winston-Salem, NC, United States

Hypo-perfusion was observed among APOE $\varepsilon 4$ carriers in both white and gray matter from the previous study in cognitively normal and mild cognitive impairment groups. Diffusion tensor imaging metrics in the white matter was further examined in the hypo-perfusion region and compared with perfusion metrics. Multiple statistical trends match with the observations from the perfusion metrics, which may suggest evidence of that a perfusion abnormality among APOE $\varepsilon 4$ carriers may precedes the disruption of white matter integrity in the group.

Effect of Antiepileptic Treatment on Hippocampal Activity in Alzheimer's Disease measured by ASL

Weiying Dai ${ }^{1}$, Song Chen ${ }^{1}$, Li Zhao ${ }^{2}$, David Alsop ${ }^{2}$, and Daniel Press ${ }^{3}$

${ }^{1}$ Computer Science, State University of New York at Binghamton, Binghamton, NY, United States, ${ }^{2}$ Department of Radiology, Beth Israel Deaconess Medical Center and Harvard Medical School, Boston, MA, United States, ${ }^{3}$ Department of Neurology, Beth Israel Deaconess Medical Center and Harvard Medical School, Boston, MA, United States

Increased hippocampal perfusion in early AD has been reported, but the underlying mechanism is still not clear. We hypothesized that epileptiform activity occurs in the hippocampus with $A D$ and causes increased perfusion. Here, we designed a placebo-controlled study using an antiepileptic drug, Levetiracetam to modulate epileptic activity of the hippocampus. Nine subjects with $\mathrm{AD}$ were scanned following drug or placebo. We observed decreased perfusion and increased perfusion fluctuation in entorhinal cortex with Levetiracetam. These findings support the potential epileptic activity effects of entorhinal cortex in AD. Due to neighboring locations of hippocampus and entorhinal cortex, further work will probe the effects of potential misregistration.

MR Spectroscopy in a Transgenic Rat Model of Alzheimer's Disease

Wendy Oakden ${ }^{1}$, Christina Beckett ${ }^{1}$, Bojana Stephanovic ${ }^{1,2}$, Greg J Stanisz $^{1,2,3}$, and JoAnne McLaurin ${ }^{4,5}$

${ }^{1}$ Physical Sciences Platform, Sunnybrook Research Institute, Toronto, ON, Canada, ${ }^{2}$ Medical Biophysics, University of Toronto, Toronto, ON, Canada, ${ }^{3}$ Neurosurgery and Pediatric Neurosurgery, Medical University of Lublin, Lublin, Poland, ${ }^{4}$ Biological Sciences Platform, Sunnybrook Research Institute, Toronto, ON, Canada, ${ }^{5}$ Department of Laboratory Medicine and Pathobiology, University of Toronto, Toronto, ON, Canada

The transgenic rat model of Alzheimer's Disease (AD), TgF344-AD rats, manifests a more complete spectrum of age-dependent $A D$ pathologies in conjunction with cognitive disturbance. Importantly, TgF344-AD rats exhibit amyloid and tau pathology as well as frank neuronal loss with aging. This study investigates brain metabolic changes, using magnetic resonance spectroscopy, in older TgF344-AD animals relative to younger and to non-transgenic littermate rats. Our data shows a statistically significant decrease in phosphocreatine, glutamate, and taurine, and a trend towards decreased NAA $(p=0.053)$ in comparison to the combined younger and non-transgenic littermate rats.

Understanding the Role of Gender in Progression and Severity of Alzheimer 's Disease: $\mathrm{A}{ }^{1} \mathrm{H}-\left[{ }^{13} \mathrm{C}\right]-\mathrm{NMR}$ investigation

Narayan Datt Soni ${ }^{1}$, Sreemnatula Arun Kumar ${ }^{1}$, Dipak Roy ${ }^{1}$, and Anant Bahadur Patel

${ }^{1}$ NMR Microimaging and Spectroscopy, CSIR-Centre for Cellular and Molecular Biology, Hyderabad, India

The epidemiological data suggested more prevalence of $A D$ in females than males. To understand the severity of $A D$ in females, we have performed behavioral and neurometabolic analysis in female and male $3 \times \mathrm{Tg}-\mathrm{AD}$ mice. Though, the learning and memory are impaired in both male and female AD mice, there is no neurometabolic impairment in female $3 \times \mathrm{Tg}-\mathrm{AD}$ mice. In contrary, neurometabolism was severely compromised in male AD mice. The data from the current study suggest more severe AD in males as compared to females till their reproductive age.

Quantitative vascular measurements in APOE- $\varepsilon 4$ knock-in female rats before the onset of AD

Codi Gharagouzloo ${ }^{1}$, Praveen Kulkarni ${ }^{2}$, Liam Timms ${ }^{3}$, Ju Qiao ${ }^{3}$, Srinivas Sridhar ${ }^{3}$, and Craig Ferris ${ }^{2}$

${ }^{1}$ Massachusetts General Hospital and Harvard Medical School, Boston, MA, United States, ${ }^{2}$ Center for Translational Neuroimaging (CTNI), Northeastern University, Boston, MA, United States, ${ }^{3}$ Nanomedicine Science and Technology Center, Northeastern University, Boston, MA, United States

There is an increasing body of evidence that suggests vascular dysfunction may play an important role in Alzheimer's Disease (AD)1. Hyperperfusion has been shown to be associated with mild cognitive impairment $(\mathrm{MCl})$ and hypoperfusion with the onset of $A D$, along with neurodegeneration2,3. In this study we utilized a novel imaging modality, QUTE-CE MRI4,5, to study the micro- and macro- vascular abnormalities in a APOE- $\varepsilon 4$ knock-in model, since the APOE- $\varepsilon 4$ allele is the single most important genetic risk factor for AD. While our $173-$ region characterization reveals both hyper- and hyop-vascularization, the changes in microvascularity are almost entirely hypervascular. 
Assessment of mild cognitive impairment detection in a community-dwelling population using quantitative, multiparametric MRI-based classification

Mark J.R.J. Bouts ${ }^{1,2,3}$, Jeroen van der Grond ${ }^{2}$, Meike W. Vernooij ${ }^{4,5}$, Tijn M. Schouten ${ }^{1,2,3}$, Frank de Vos ${ }^{1,2,3}$, Lotte G.M. Cremers ${ }^{4,5}$, Mark de Rooij ${ }^{1,3}$, Wiro J. Niessen ${ }^{4,6,7}$, M. Arfan $\mathrm{Ikram}^{4,5,8}$, and Serge A.R.B. Rombouts ${ }^{1,2,3}$

${ }^{1}$ Psychology, Leiden University, Leiden, Netherlands, ${ }^{2}$ Radiology, Leiden University Medical Center, Leiden, Netherlands, ${ }^{3}$ Leiden Institute for Brain and Cognition, Leiden University, Leiden, Netherlands, ${ }^{4}$ Radiology and Nuclear Medicine, Erasmus MC University Medical Center, Rotterdam, Netherlands, ${ }^{5}$ Epidemiology, Erasmus MC University Medical Center, Rotterdam, Netherlands, ${ }^{6}$ Medical Informatics, Erasmus MC University Medical Center, Rotterdam, Netherlands, ${ }^{7}$ Applied Sciences, Delft University of Technology, Delft, Netherlands, ${ }^{8}$ Neurology, Erasmus MC University Medical Center, Rotterdam, Netherlands

Multiparametric MRI-based classification algorithms improve classification of dementia over single measure classifications. Yet, how accurate these algorithms are in identifying subjects with mild cognitive impairment $(\mathrm{MCl})$ in a general population is unclear. We evaluated single and multiparametric algorithms that include structural and diffusion tensor MRI in their potential to accurately differentiate $\mathrm{MCl}$ from normal aging subjects in a community-dwelling population. While highest classification rates were observed for multiparametric algorithms, overall classification performance was low (AUC: $0.524-0.631$ ). Our results suggest that accurate MRI-based single subject detection of MCl within a population-based setting may be difficult to achieve using MR imaging alone.

Higher temporal lobe curvature in early Alzheimer's indicative of subsequent cognitive decline

Christopher Bird ${ }^{1}$, Sarah J Banks ${ }^{1}$, Dietmar Cordes ${ }^{1,2}$, Karthik Sreenivasan $^{1}$, Xiaowei Zhang ${ }^{1}$, Zhengshi Yang ${ }^{1}$, and Virendra Mishra ${ }^{1}$

${ }^{1}$ Cleveland Clinic, Las Vegas, NV, United States, ${ }^{2}$ University of Colorado Boulder, Boulder, CO, United States

We selected ADNI patients with an initial diagnosis of mild cognitive impairment (MCI) due to early Alzheimer's disease, a positive amyloid PET scan within 4 years, and comparable cognitive test scores during their initial visit. Patients were grouped according to their diagnosed outcome within 4 years of the initial visit, specifically, $\mathrm{MCl}$ subsequently diagnosed with dementia and stable $\mathrm{MCl}$. We found that curvature within the temporal lobe was greater among patients subsequently diagnosed with dementia. Established measurements of atrophy, including hippocampal volume and temporal lobe thickness, did not differ between these groups.

Functional and structural deficits in a novel transgenic rat model of Alzheimer's Disease.

Cynthia Anckaerts ${ }^{1}$, Ines Blockx ${ }^{1,2}$, Christina Kreutzer $^{3}$, Hervé Boutin ${ }^{4}$, Sébastien Couillard-Despres ${ }^{3}$, Marleen Verhoye ${ }^{1}$, and Annemie Van der Linden ${ }^{1}$

${ }^{1}$ Bio-Imaging Lab, University of Antwerp, Wilrijk, Antwerp, Belgium, ${ }^{2}$ Department of Radiology, NYU Langone Medical Center, Center for Biomedical Imaging, New York, NY, United States, ${ }^{3}$ Spinal Cord Injury and Tissue Regeneration Center Salzburg; Institute of Experimental Neuroregeneration; Paracelsus Medical University, Salzburg, Austria, ${ }^{4}$ Wolfson Molecular Imaging Centre, University of Manchester, Manchester, United Kingdom

As improving our understanding of the underlying mechanisms of Alzheimer's Disease (AD) pathology is of utmost importance, the development and characterization of innovative animal models is essential in AD-related research. Here, we further characterized a novel transgenic rat model of AD, the TgF344-AD rat, which manifests progressive AD pathology, much akin to human AD. Functional and structural deficits along the disease progression were assessed using resting state functional MRI (rsfMRI) and diffusion tensor imaging, respectively.

Follow up research of hippocampal subfield in patients with mild Alzheimer's disease

Ying Liu ${ }^{1}$ and Lizhi Xie ${ }^{2}$

2018 1 Radiology Department, Peking University Third Hospital, Beijing, China, ${ }^{2}$ GE Healthcare, China, Beijing, China

The aim of this study is to evaluate the atrophy pattern of hippocampal subfield and follow up the changes of hippocampal subfield by using automatic segmentation tool in patients with mild AD. The results indicate that volumes of hippocampal subfield decrease in patients with mild AD, and the declination are positive correlated with clinical scores. We conclude that substructures of hippocampal might serve as a good index to characterize subtle changes in AD patients.

Prediction of long-term evolution of cognitive impairment following stroke using resting-state functional connectivity.

Clément Bournonville ${ }^{1}$, Hilde Hénon ${ }^{1}$, Christine Delmaire ${ }^{1}$, Stéphanie Bombois ${ }^{1}$, Jean-Pierre Pruvo ${ }^{1}$, Xavier Leclerc ${ }^{1}$, Régis Bordet ${ }^{1}$, and Renaud lopes ${ }^{1}$

${ }^{1}$ Univ. Lille, INSERM, CHRU Lille, U1171 - Neurodegenerative and vascular disorders, Lille, France

The mechanisms of chronic post-stroke cognitive impairments are currently poor understood. However, the study of functional connectivity gives new opportunities to better elucidate the physiopathology. Here, using resting functional connectivity and a machine learning approach, we tried to predict the evolution of cognitive functions up to 36 months after stroke. The results showed that the prediction capacity depends on the studied cognitive domain, and that a particular focus should be done on frontal and temporal cortices. 
APPswe/PS1dE9 mice with cortical amyloid pathology show a reduced NAA/Cr ratio without apparent brain atrophy: A MRS and MRI study

Angela Kuhla ${ }^{1}$, Fatemah Sakr ${ }^{2}$, Claire Ruehlmann ${ }^{1}$, Tobias Lindner ${ }^{3}$, Stefan Polei ${ }^{3}$, Stefan Hadlich ${ }^{4}$, Bernd J Krause ${ }^{5}$, Brigitte Vollmar ${ }^{1}$, and Stefan Teipel ${ }^{2,6}$

${ }^{1}$ Institute for Experimental Surgery, Rostock University Medical Center, Rostock, Germany, ${ }^{2}$ Rostock University Medical Center, Rostock, Germany, ${ }^{3}$ Core Facility Multimodal Small Animal imaging, Rostock University Medical Center, Rostock University Medical Center, Rostock, Germany, ${ }^{4}$ Institute of Diagnostic Radiology \& Neuroradiology, University Medicine Greifswald, Gerifswald, Germany, ${ }^{5}$ Department of Nuclear Medicine, Rostock University Medical Center, Rostock, Germany, ${ }^{6}$ German Center for Neurodegenerative Diseases (DZNE),

Rostock, Germany

Amyloid- $\beta$ deposition is one of the hallmarks of Alzheimer's disease (AD) that starts to progress decades before the onset of cognitive impairment. With the rise of the new diagnostic criteria of $A D$ that considers the neuropathological changes as the main aspects for explaining the extent of the disease regardless the cognitive status of the patient \& further highlighted the importance of finding reliable in-vivo biological markers to identify those in the preclinical stage of AD. Through the use of the transgenic mice models, particularly APPswe/PS1dE9 we could study the different pathomechanics contributing to the development of AD. So, in this study, we assumed an approach combining morphometry based on high-resolution MRI as a measure for the brain atrophy \& proton magnetic resonance spectroscopy as a measure of neuronal functional viability. Then compare these data with a well known \& standardized method as the histopathological assessments of neuron \& amyloid plaques load. Using the quantitative neuroimaging allows us to translate these mechanistic findings in transgenic models to human phenotypes of brain morphology and function.

Increased Mode of Anisotropy in crossing-fibre areas predicts conversion from Mild Cognitive Impairment (MCI) to Alzheimer's disease (AD)

Matt C Gabel ${ }^{1}$, Meena Zaveri², Laura Serra ${ }^{3}$, Marco Bozzali ${ }^{1,3}$, and Mara Cercignani ${ }^{1}$

${ }^{1}$ Clinical Imaging Sciences Centre, Brighton and Sussex Medical School, Brighton, United Kingdom, ${ }^{2}$ School of Life Sciences, University of Sussex, Falmer, United Kingdom,

${ }^{3}$ Neuroimaging Laboratory, Santa Lucia Foundation IRCCS, Rome, Italy

Diffusion MRI was used to examine whether any change in the white matter tracts of patients with mild cognitive impairment (MCI) can predict conversion to Alzheimer's disease (AD) in a longitudinal study. Our data show increases in mode of anisotropy (MO) in a region of crossing fibres in the centrum semiovale for $\mathrm{MCl}$ patients who later converted to $\mathrm{AD}$.

Investigating Glumphatic Function During Early Tau Pathology Using Dynamic Contrast-Enhanced MRI

Ozama Ismail ${ }^{1}$, Ian F Harrison ${ }^{1}$, Jack A Wells ${ }^{1}$, Yolanda Ohene ${ }^{1}$, Payam Nahavandi ${ }^{1}$, Alexander V Gourine ${ }^{2}$, Zeshan Ahmed ${ }^{3}$, Alice Fisher ${ }^{3}$, Tracey K Murray ${ }^{3}$, Ross A Johnson ${ }^{4}$, Emily C Collins ${ }^{4}$, Michael J O'Neill ${ }^{3}$, and Mark F Lythgoe ${ }^{1}$

${ }^{1}$ UCL Centre for Advanced Biomedical Imaging, University College London, London, United Kingdom, ${ }^{2}$ Neuroscience, Physiology \& Pharmacology, University College London, London, United Kingdom, ${ }^{3}$ Eli Lilly \& Company, Surrey, United Kingdom, ${ }^{4}$ Eli Lilly \& Company, Indianapolis, IN, United States

Pathological accumulation of tau and amyloid in the brains of Alzheimer's disease (AD) patients leads to a continuum of irreversible biochemical and pathological changes and pronounced neurodegeneration. Impaired 'glymphatic' clearance may be one of the earliest biological changes in AD, occurring many years prior to neurodegeneration, and therefore presents a unique opportunity for strategic therapeutic intervention. Here, we have mapped the extent of glymphatic inflow of an MRI contrast agent from cerebrospinal fluid, into the brain parenchyma. Leading on from previous studies, we have demonstrated that glymphatic inflow is impaired during the onset of pathology in an AD animal model.

Correlation analysis between the gray matter volumes obtained with two different imaging sequences and the cognitive decline in Apolipoprotein E $\varepsilon 4$ carrier subjects

Na Young Choi ${ }^{1}$, Hak Young Rhee ${ }^{2}$, Soonchan Park ${ }^{1}$, Chang-Woo Ryu ${ }^{1}$, Geon-Ho Jahng ${ }^{1}$, Wook Jin ${ }^{1}$, and Dal Mo Yang ${ }^{1}$

${ }^{1}$ Radiology, Kyung Hee Univ. Hospital at Gangdong, Seoul, Republic of Korea, ${ }^{2}$ Neurology, Kyung Hee Univ. Hospital at Gangdong, Seoul, Republic of Korea

To evaluate the association between GMV loss and cognitive decline in the APOE e 4 carriers and to investigate alterations of GMV, MPRAGE and DIR images were acquired from 72 subjects ( 51 noncarriers, 21 carriers). Voxel- and ROI-based analyses were performed to evaluate the association between GMV loss and the MMSE score and to do the group differences of GMV for each sequence. GMV of carriers was positively correlated with the MMSE score for both sequences. DIR can be effective for identifying GMV loss in the carriers and may be useful to evaluate GMV changes in the early stage of dementia.

Zhizheng Zhuo ${ }^{1,2}$ and Haiyun $\mathrm{Li}^{2}$

${ }^{1}$ Clincial Science, Philips Healthcare, Beijing, China, ${ }^{2}$ Biomedical Engineering, Capital Medical University, Beijing, China 
Pearson's Correlation analysis has been applied to construct the connectivity network and describe the connectivity strength between different brain function areas. But the correlation coefficient was sensitive to the noise and just for stationary signals. In this study, a new functional connectivity network constructing method based on DCCA (Detrend Cross Correlation Analysis) for non-stationary signals was proposed and applied on $\mathrm{AD}$ and $\mathrm{MCl}$.

Diffusion Kurtosis Imaging Study on Brain Deep Grey Matter in Alzheimer' s Disease

Zhou Yujing ${ }^{1}$, Hu Rui ${ }^{1}$, and Miao Yanwei ${ }^{1}$

${ }^{1}$ Radiology, First Affiliated Hospital of Dalian Medical University, Dalian, China

We used Diffusion kurtosis imaging (DKI) to evaluate the microstructure changes of brain deep gray matter and to explore its relationship with cognitive function in $\mathrm{AD}$.

Changes in Functional and Structural Brain Connectome Along the Alzheimer's Disease Continuum

Federica Agosta ${ }^{1}$, Silvia Basaia ${ }^{1}$, Elisa Canu ${ }^{1}$, Francesca Imperiale ${ }^{1}$, Giuseppe Magnani ${ }^{2}$, Monica Falautano $^{2}$, Giancarlo Comi ${ }^{2}$, Andrea Falini $^{3}$, and Massimo Filippi ${ }^{1}, 2$

${ }^{1}$ Neuroimaging Research Unit, INSPE, Division of Neuroscience, San Raffaele Scientific Institute, Vita-Salute San Raffaele University, Milan, Italy, ${ }^{2}$ Department of Neurology, San Raffaele Scientific Institute, Vita-Salute San Raffaele University, Milan, Italy, ${ }^{3}$ Department of Neuroradiology, San Raffaele Scientific Institute, Vita-Salute San Raffaele University, Milan, Italy

We investigated structural and functional brain network architecture in patients with Alzheimer's disease (AD) and mild cognitive impairment (MCI); and assessed the relationship between healthy brain network functional connectivity and the topography of brain atrophy in patients along the AD continuum. Structural connectivity alterations distinguished $\mathrm{MCl}$ who converted to $\mathrm{AD}$ from those who did not. Brain regions most strongly connected with the disease-epicenter (left hippocampus) in the healthy functional connectome were also the most atrophic in both $\mathrm{AD}$ and converters $\mathrm{MCl}$. Graph theoretical analysis provides insight on how neurodegeneration propagates across the human brain in the early phase of AD.

Iron Deposition in Alzheimer's Dementia Hippocampus is Associated with Increased R2* Values

Grayson Tarbox ${ }^{1}$, Amin Nazaran ${ }^{1}$, Neal Bangerter ${ }^{1}$, and Jonathan J. Wisco ${ }^{2}$

${ }^{1}$ Electrical Engineering, Brigham Young University, Provo, UT, United States, ${ }^{2}$ Physiology and Developmental Biology, Brigham Young University, Provo, UT, United States

We describe the utilization of UTE-3D Cones to create T2* maps of iron deposition in the hippocampus of an Alzheimer's dementia subject, but not in a corticobasal degeneration subject. These results are consistent with histopathological studies involving post-mortem human brain tissue. UTE-3D Cones could be a promising imaging protocol for AD diagnostic imaging.

Characterizing Perfusion and Arterial Transit Time of the Choroid Plexus with Arterial Spin Labeling

LI Zhao ${ }^{1}$ and David C. Alsop ${ }^{1}$

${ }^{1}$ Radiology, Beth Israel Deaconess Medical Center \& Harvard Medical School, Boston, MA, United States

Choroid plexus signal is readily apparent on Arterial Spin Labeling images but its perfusion characteristics have not been systematically studied. Since the choroid plexus plays an important role in both cerebrospinal fluid production and composition, measuring its function may yield insights into cerebrospinal fluid physiology and disease. In this work, we report initial measurements of the choroid plexus blood flow with noninvasive arterial spin labeling methods using anatomically defined regional measurements.

Takashi Watanabe ${ }^{1}$, Ana Martinez-Hernandez ${ }^{2}$, Jens Frahm¹, and Thomas Michaelis ${ }^{1}$

${ }^{1}$ Biomedizinische NMR Forschungs GmbH, Max-Planck-Institut für biophysikalische Chemie, Göttingen, Germany, ${ }^{2}$ Abteilung Gene und Verhalten, Max-Planck-Institut für biophysikalische Chemie, Göttingen, Germany 
Cerebral MRS of APP/PS1/Ear2(-/-) mice in vivo reveals significant alterations of several metabolites suggesting (i) an impaired cellular respiration compensated for by accelerated anaerobic glycolysis (i.e., elevated lactate), (ii) a loss of neurons (reduced $\mathrm{N}$-acetylaspartate, glutamate, total creatine, and $\mathrm{Y}$-aminobutyric acid) possibly compensated for by osmoregulators (elevated myo-inositol and taurine), (iii) an accumulation of paramagnetic iron (shortened water proton $\mathrm{T}_{2}$ ) possibly associated with inflammation, and (iv) subsequent gliosis (elevated myo-inositol). More specifically, a $60-75 \%$ reduction of noradrenaline is shown to accelerate the reduction of $\mathrm{N}$-acetylaspartate and glutamate in the hippocampus as well as the $T_{2}$-shortening in the frontal cortex.

MRI Hippocampal subfield volume analysis: Comparison between Alzheimer's disease, mild cognitive impairment, and normal aging subjects in an amyloid PET project.

Natcha Wontaneeporn ${ }^{1}$, Chanon Ngamsombat ${ }^{1}$, Weerasak Muangpaisan ${ }^{2}$, Panida Charnchaowanish ${ }^{1}$, and Orasa Chawalparit ${ }^{1}$

${ }^{1}$ Department of Radiology, Faculty of Medicine Siriraj Hospital, Mahidol University, Bangkok, Thailand, ${ }^{2}$ Department of Geriatric Medicine, Faculty of Medicine Siriraj Hospital, Mahidol University, Bangkok, Thailand

Hippocampal atrophy evidenced by MRI is one of the most validated biomarkers of Alzheimer's disease (AD). The previous neuropathological data showed a differential vulnerability of hippocampal subfields to AD processes. This study aims to use an automated analysis technique for subfield hippocampal volume measurement in order to differentiate early detection of $\mathrm{AD}$. We demonstrated high diagnostic efficacy of using hippocampal subfield analysis for discriminating $\mathrm{AD}$ subjects from heathy control (HC) or mild cognitive impairment (MCI) than whole hippocampal volume and feasibility for discriminating $\mathrm{MCl}$ to $\mathrm{HC}$ as compared with amyloid PET result.

Nilvadipine slows progression of white matter hyperintensities in Alzheimer's disease

Anne Rijpma ${ }^{1}$, Brian Lawlor², and Jurgen Claassen ${ }^{1}$

${ }^{1}$ Radboudumc Alzheimer Center, Radboud university medical center, Donders Institute for Brain, Cognition and Behavior, Nijmegen, Netherlands, ${ }^{2}$ Trinity College Institute of Neuroscience, Trinity College Dublin, Dublin, Ireland

Cerebrovascular disease, such as presence of white matter hyperintensities (WMH), contributes to Alzheimer's disease (AD) pathology and progression. The antihypertensive nilvadipine may reduce WMH progression by reducing amyloid-induced vasoconstriction and improving cerebral perfusion. Here we show that in patients with mild to moderate $A D$, nilvadipine slows the increase of WMH after 6 months, but not after 18 months, when correcting for baseline WMH. This contradicts the view that reducing blood pressure in an elderly dementia population leads to progression of white matter damage and instead seems to have a beneficial effect on WMH.

Effects of perivascular progenitor cells in combination with Abeta clearance on neurovascular function following transient hypertension in a transgenic rat model of Alzheimer's Disease

Tina L Beckett ${ }^{1}$, Paolo Bazzigaluppi ${ }^{1}$, Margaret Koletar ${ }^{1}$, Conner Robert Adams ${ }^{2}$, Lynsie Thomason ${ }^{1}$, Adrienne Dorr ${ }^{1}$, Denis Gallagher ${ }^{3}$, Clifford Librach $^{1,3}$, JoAnne McLaurin ${ }^{1,4}$, and Bojana Stefanovic ${ }^{1,2}$

${ }^{1}$ Sunnybrook Research Institute, Toronto, ON, Canada, ${ }^{2}$ Medical Biophysics, University of Toronto, Toronto, ON, Canada, ${ }^{3} \mathrm{CReATe}$ Research Program, Toronto, ON, Canada, ${ }^{4}$ Laboratory Medicine and Pathobiology, University of Toronto, Toronto, ON, Canada

Examining the interplay between cerebrovascular compromise and $A D$ in the development of therapies is complicated by long prodromal phases of both conditions, necessitating preclinical studies. Four-month-old TgAD-F344 rats, which by six months of age present amyloid deposits and hyperphosphorylated tau, were treated with a nitric oxide synthase inhibitor L-NAME for one month to induce transient hypertension. Human umbilical cord perivascular cells were then given in combination with scyllo-inositol, an inhibitor of Abeta peptide oligomerization and fibrillization to elicit cerebrovascular repair and clear amyloid. Following L-NAME, non-transgenic rats showed transient cerebrovascular changes, whereas TgAD-F344 animals exhibited sustained increase in cerebrovascular reactivity. The latter effect was ameliorated by the treatment.

Diffusion MRI Changes in the Brain of the 3xTg Mouse Model of Alzheimer's Disease

Xingju Nie ${ }^{1,2}$, Maria Fatima Falangola ${ }^{1,2}$, Emilie T. McKinnon ${ }^{1,2,3}$, Joseph A. Helpern ${ }^{1,2,3}$, and Jens H. Jensen ${ }^{1,2}$

${ }^{1}$ Department of Neuroscience, Medical University of South Carolina, Charleston, SC, United States, ${ }^{2}$ Center for Biomedical Imaging, Medical University of South Carolina, Charleston, SC, United States, ${ }^{3}$ Department of Neurology, Medical University of South Carolina, Charleston, SC, United States

The triple transgenic mouse model (3xTg) of Alzheimer's disease (AD) exhibits both A $\beta$ and tau pathology. Although diffusion MRI (dMRI) is an established tool for tracking changes in brain microstructure for aging and $A D$ in humans, prior research using diffusion tensor imaging has called into question the sensitivity of dMRI for $3 \times T g$ mice. Here we investigated the sensitivity of an alternative dMRI method, diffusional kurtosis imaging, to detect brain changes associated with aging and disease progression in $3 \times T_{g}$ mice. Our results indicate that $\mathrm{dMRI}$ is able to capture age and/or pathology related alterations in brain tissue for this mouse model.

In vivo MR detection and automated quantification of amyloid plaques in a preclinical model of Alzheimer's disease

Steve J Sawiak ${ }^{1}$, Anne-Sophie Herard ${ }^{2}$, Mathieu D Santin ${ }^{3}$, Thierry Delzescaux ${ }^{2}$, and Marc Dhenain ${ }^{2}$ 
Amyloid plaque load is a key index of disease burden in Alzheimer's disease, but methods for its quantification are slow and operator dependent. Recent advances in the use of contrast agents allow the plaques to be visualized in vivo, but as yet no direct quantification methods are available. Here we present a new technique for automatic segmentation of amyloid plaques and to evaluate age-related or therapy related changes on a voxel-based basis with minimal user intervention. We report localized age-related changes of amyloid load across the whole brain of APP/PS1 mouse model of amyloidosis.

Traditional Poster

\section{Brain Imaging Methodology}

High Resolution Structural MRI of the of Eye: Initial Experience at Ultra High Field

Jon O Cleary ${ }^{1}$, Bao Nguyen ${ }^{2}$, Rebecca Glarin ${ }^{1}$, Scott C Kolbe ${ }^{1}$, Bradford A Moffat ${ }^{1}$, Rishma Vidyasagar ${ }^{1}$, Bang Bui ${ }^{2}$, Allison McKendrick ${ }^{2}$, and Roger J Ordidge ${ }^{1}$

While optical eye imaging techniques are available for examining anterior and retinal structures, they are limited in making 3 dimensional assessments of the whole eyeball. MRI is the preferred modality in these areas but fine eye structures are difficult to resolve on clinical systems. Ultra high field magnets offer increased signal-to-noise, providing higher resolution, but there have been only a limited number of studies so far. We performed an initial study to assess achievable resolution, the anatomy visible on differing image weightings and MR parameter measurements, in eyes of healthy subjects on a 7 Tesla system.

Investigating relevance of tumor shape features in overall survival prediction of glioblastoma multiforme patients using machine learning and multi-channel MR images

Parita Sanghani ${ }^{1}$, Ang Beng $\mathrm{Ti}^{2}$, Nicolas Kon Kam King ${ }^{2}$, and Hongliang Ren

${ }^{1}$ Department of Biomedical Engineering, National University of Singapore, Singapore, Singapore, ${ }^{2}$ Department of Neurosurgery, National Neuroscience Institute, Singapore, Singapore

In this work, we study the impact of combining shape features with texture and volumetric features derived from glioblastoma multiforme (GBM) tumors for overall survival (OS) prediction. A comprehensive set of features were obtained from multichannel MR images of 163 GBM patients. Support Vector Machine-Recursive Feature Elimination (SVM-RFE) was used for feature selection, followed by SVM regression for survival prediction. The shape features used in this study have not yet been used for OS prediction in GBM patients and were found to improve the prediction accuracy.

T1-weighted imaging of the orbitofrontal cortex in individuals with dental braces using 2D FLAIR

Sander Lamballais ${ }^{1,2}$, Piotr Wielopolski ${ }^{3}$, Aad van der Lugt ${ }^{3}$, Vincent Jaddoe ${ }^{1,2,4}$, Mohammad Arfan Ikram ${ }^{1,3,5}$, Tonya White ${ }^{3,6}$, and Juan Antonio Hernández Tamames ${ }^{3}$

${ }^{1}$ Department of Epidemiology, Erasmus MC University Medical Center Rotterdam, Rotterdam, Netherlands, ${ }^{2}$ The Generation R Study Group, Erasmus MC University Medical Center Rotterdam, Rotterdam, Netherlands, ${ }^{3}$ Department of Radiology, Erasmus MC University Medical Center Rotterdam, Rotterdam, Netherlands, ${ }^{4}$ Department of Pediatrics, Erasmus MC University Medical Center Rotterdam, Rotterdam, Netherlands, ${ }^{5}$ Department of Neurology, Erasmus MC University Medical Center Rotterdam, Rotterdam, Netherlands, ${ }^{6}$ Department of Child and Adolescent Psychiatry/Psychology, Erasmus MC University Medical Center Rotterdam, Rotterdam, Netherlands

Neuroimaging studies in (pre)adolescent populations have steadily increased in number over the last decade. However, dental braces are common amongst (pre)adolescents and introduce metal-related artifacts in the images. Excluding individuals with braces from epidemiological studies may lead to selection bias and missingness in repeated measures. To this end we configured a $T_{1}$-weighted 2D FLAIR sequence and compared it to a $T_{1}$-weighted 3D SPGR sequence. Compared to SPGR, the FLAIR sequence suffered less from metal artifacts and performed similarly in cortical reconstruction and volumetric segmentation. Thus, $T_{1}$-weighted $2 D$ FLAIR may be a useful alternative for neuroimaging in participants with dental braces.

Combined MRI and Ultrasound Measurements to Assess the Impact of Systemic Chemotherapy on the Developing Brain and Heart

Leigh Spencer Noakes ${ }^{1}$, Thomas Przybycien ${ }^{2}$, Amanda Forwell ${ }^{3}$, Yu-Qing Zhou ${ }^{1}$, Ellen van der Plas ${ }^{2}$, and Brian J. Nieman ${ }^{1,4}$

${ }^{1}$ The Mouse Imaging Centre, The Hospital for Sick Children, Toronto, ON, Canada, ${ }^{2}$ The Hospital for Sick Children, Toronto, ON, Canada, ${ }^{3}$ University of Waterloo, Waterloo, ON, Canada, ${ }^{4}$ Medical Biophysics, University of Toronto, Toronto, ON, Canada 
Combined multiple-mouse ex vivo MRI and high-frequency cardiac ultrasound were used to assess the impact of common chemotherapy agents on the developing brain and heart. Of the eight agents considered, vincristine had the most widespread impact on the brain. Doxorubicin, methotrexate, and L-asparaginase were also found to impact brain and/or heart development.

Variable flip angle RARE for High-Resolution Preclinical Brain and Spinal Cord Imaging

Matthew Budde ${ }^{1}$

${ }^{1}$ Neurosurgery, Medical College of Wisconsin, Milwaukee, WI, United States

Variable flip angle RARE imaging has seen widespread utility in clinical brain and body imaging, but it has not been available for similar gains in preclinical MRI. This work demonstrates implementation and applications of vfaRARE in a the rat brain and spinal cord.

Improving sensitivity of infiltrative glioma detection by multi-parametric magnetic resonance imaging

Georgia Kanli ${ }^{1,2}$, Anaïs Oudin ${ }^{1,2}$, Simone P. Niclou ${ }^{2,3}$, Rolf Bjerkvig ${ }^{2,3,4}$, and Olivier Keunen ${ }^{1,2}$

${ }^{1}$ In Vivo Imaging Facility, Department of Oncology, Luxembourg Institute of Health (LIH), Luxembourg, Luxembourg, ${ }^{2}$ NorLux Neuro-Oncology Laboratory, Department of Oncology, Luxembourg Institute of Health (LIH), Luxembourg, Luxembourg, ${ }^{3} \mathrm{KG}$ Jebsen Brain Tumour Research Center, Department of Biomedicine, University of Bergen, Bergen, Norway,

${ }^{4}$ NorLux Neuro-Oncology, Department of Biomedicine, University of Bergen, Bergen, Norway

Glioblastoma is characterized by poor prognosis and limited treatment efficacy. One main contributing factor is the presence of a large population of infiltrated tumor cells that are difficult to visualize and treat with resective surgery and radiochemotherapy. In the present study, we aim at establishing techniques that combine various contrast mechanisms available in MR and PET to improve the sensitivity of the detection of infiltrated tumour cells. Such techniques are likely to improve prognosis by early tumor detection, better delineation of the target for radiotherapy, and better assessment of the full extent of the tumor and its response to therapy.

Spiral TAPIR with Compressed Sensing for Fast Sub-Millimetre T1 Mapping of Rapidly Relaxing Compartments at 3 Tesla

Robert Claeser $^{1}$, Markus Zimmermann ${ }^{1}$, and Nadim Joni Shah ${ }^{1,2}$

${ }^{1}$ Institute of Neuroscience and Medicine - 4, Medical Imaging Physics, Forschungszentrum Juelich GmbH, Juelich, Germany, Juelich, Germany, ${ }^{2}$ Department of Neurology, Faculty of Medicine, JARA, RWTH Aachen University, Aachen, Germany

TAPIR is a highly accurate, precise and efficient method for T1 mapping of the brain. It combines an efficient slice-interleaving Look-Locker read-out to sample T1 relaxation by acquiring multiple k-space lines in one shot. However, mapping rapidly relaxing tissue requires the number of lines read in one shot to be small, thus increasing total measurement time. In this work we show how incorporating an interleaved spiral read-out into TAPIR enhances its T1 fitting abilities for rapidly relaxing tissue such as white matter myelin. Scanning time can be decreased by factors of up to 3.3 in comparison to classical Cartesian TAPIR.

Quantitative assessment of automatic cortical surface reconstructions from Wave-CAIPI MPRAGE: A validation study

Yulin V Chang ${ }^{1}$, Stephen F Cauley ${ }^{2,3}$, Wei Liu ${ }^{4}$, Daniel Polak ${ }^{2,5}$, Borjan Gagoski ${ }^{6}$, Berkin Bilgic ${ }^{2,3}$, Kawin Setsompop $^{2,3}$, and Jonathan R Polimeni ${ }^{2,3}$

${ }^{1}$ Siemens Medical Solutions USA, Boston, MA, United States, ${ }^{2}$ A. A. Martinos Center for Biomedical Imaging, Radiology, MGH, Charlestown, MA, United States, ${ }^{3}$ Harvard Medical School, Boston, MA, United States, ${ }^{4}$ Siemens Magnetic Resonance, Shenzhen, China, ${ }^{5}$ Medical Physics in Radiology, German Cancer Research Center, Heidelberg, Germany, ${ }^{6}$ Boston Children's Hospital, Boston, MA, United States

Structural imaging of the brain using conventional MPRAGE at high resolution is vulnerable to motion artifacts due to prolonged scan times. MPRAGE acquired with wave-CAIPIRINHA technique (waveMPRAGE) and a multi-channel receive coil can significantly improve imaging speed with minimal noise penalty. We show that head motion can be observed from multiple waveMPRAGE scans in a time span similar to a single conventional MPRAGE, and that registering and averaging multiple short (approx. 1 min) waveMPRAGE repetitions produces reliable and reproducible cortical surfaces reconstructed automatically using FreeSurfer

Muriel Marisa Katharina Bruchhage ${ }^{1,2}$, Stephen Correla $^{3}$, Paul Malloy ${ }^{4}$, Stephen Salloway $^{5}$, and Sean Deoni ${ }^{2,6}$

${ }^{1}$ Centre for Neuroimaging Sciences, King's College London, London, United Kingdom, ${ }^{2}$ Advanced Baby Imaging Lab, Memorial Hospital of Rhode Island, Providence, RI, United States, ${ }^{3}$ Veterans Affairs Medical Center, Providence, RI, United States, ${ }^{4}$ Neurology, Butler Hospital, Providence, RI, United States, ${ }^{5}$ Human Behavior and Psychiatry, Warren Alpert Medical School at Brown University, Providence, RI, United States, ${ }^{6}$ Warren Alpert Medical School at Brown University, Providence, RI, United States 
Alzheimer's disease $(A D)$ is one of the most common forms of dementia, marked by progressively degrading cognitive function. The cerebellum plays a role in $A D$ development, but its predictive contribution to early stages of AD remains unclear. We used MRI machine learning based classification within myelin and grey matter of the whole, anterior and posterior cerebellum and the whole brain, between individuals within the first two early stages of dementia and typically ageing controls. Our findings suggest myelin and grey matter loss in early stages of $A D$, with distinct patterns of anterior and posterior cerebellar atrophy for each tissue property.

Magnetic Resonance Electrical Impedance Tomography in Salamander Retina Neuronal Activity Imaging

Fanrui $\mathrm{Fu}^{1}$, Munish Chauhan ${ }^{1}$, and Rosalind Sadleir ${ }^{1}$

${ }^{1}$ Arizona State University, Tempe, AZ, United States

Magnetic resonance electrical impedance tomography (MREIT) is an innovate potential technique for imaging neural activity. To test its capability, we used salamander retina as a neuronal activity source. The neuronal activity activation was modulated by light, which enables us to compare the result between with and without neuronal activity. After obtained phase images, we expected that the standard deviation of phase in the region of interest (ROI) for the experiment group with neuronal activity is higher than that for the group without activity.

Comparison Study between Quantitative Susceptibility Mapping and CT

Sonoko Oshima ${ }^{1}$, Yasutaka Fushimi ${ }^{1}$, Tomohisa Okada ${ }^{2}$, Takuya Hinoda ${ }^{1}$, Takayuki Yamamoto ${ }^{1}$, Hikaru Fukutomi ${ }^{1}$, Yusuke Yokota ${ }^{1}$, Akira Yamamoto ${ }^{1}$, Tsutomu Okada ${ }^{1}$, and Kaori Togashi $^{1}$

${ }^{1}$ Department of Diagnostic Radiology and Nuclear Medicine, Graduate School of Medicine, Kyoto University, Kyoto, Japan, ${ }^{2}$ Human Brain Research Center, Graduate School of Medicine, Kyoto University, Kyoto, Japan

Quantitative susceptibility mapping (QSM) is a technique which can provide quantitative values of magnetic susceptibility. In this study, we compared magnetic susceptibility values with computed tomography (CT) numbers of brain structures and intracranial lesions in 30 subjects. QSM was able to differentiate between paramagnetic and diamagnetic substances. Susceptibility values showed positive correlations with CT numbers in globus pallidus and lesions with positive susceptibility, and negative correlations in choroid plexus and lesions with negative susceptibility.

MP2RAGE, enhanced T1 contrast and beyond

Yishi Wang ${ }^{1}$, Yajie Wang ${ }^{1}$, Jie Shi ${ }^{2}$, Wenjing Zhou ${ }^{2}$, Xuesong $\mathrm{Li}^{3}$, Chun Yuan ${ }^{1,4}$, and Hua Guo ${ }^{1}$

${ }^{1}$ Center for Biomedical Imaging Research, Department of Biomedical Engineering, School of Medicine, Tsinghua University, Beijing, China, ${ }^{2}$ Epilepsy Center, Tsinghua University Yuquan Hospital, Beijing, China, ${ }^{3}$ School of Computer Science and Technology,Beijing Institute of Technology, Beijing, China, ${ }^{4}$ Vascular Imaging Laboratory, Department of Radiology, University of Washington, Seattle, WA, United States

MP2RAGE has been modified to acquire two image volumes that can be used to suppress the signals from white matter and CSF respectively. In this study, we show that based on the two intrinsically co-registered volumes, enhanced T1 contrast images compared to traditional MPRAGE images as well as other contrasts such as gray matter image and angiogram can be generated using simple post-processing

Application of support vector machines to multi-modal hemo-metabolic data for classification of disease severity in patients with extreme arterial steno-occlusive diseases

Spencer L. Waddle ${ }^{1}$, Sarah K. Lants ${ }^{2}$, Larry T. Davis ${ }^{2}$, Meher R. Juttukonda ${ }^{2}$, Matthew R. Fusco ${ }^{3}$, Lori C. Jordan ${ }^{4}$, and Manus J. Donahue

${ }^{1}$ Chemical and Physical Biology Program, Vanderbilt, Nashville, TN, United States, ${ }^{2}$ Radiology and Radiological Sciences, Vanderbilt, Nashville, TN, United States, ${ }^{3}$ Neurosurgery, Vanderbilt, Nashville, TN, United States, ${ }^{4}$ Pediatrics - Division of Pediatric Neurology, Vanderbilt, Nashville, TN, United States

Traditional hemodynamic imaging approaches such as arterial spin labeling (ASL) and hypercapnic blood oxygenation level-dependent (BOLD) reactivity provide contrasts that are frequently difficult to interpret using conventional analyses in arterial steno-occlusive disease patients with extreme blood arrival and vascular reactivity delay times. We investigated applying a supervised learning procedure to exploit endovascular and vascular compliance artifacts as potential indicators of disease severity; results show that less-conventional variables which report on endovascular blood signal and delayed vascular compliance outperform conventional variables, such as mean ASL signal and BOLD signal change.

Mark Symms ${ }^{1}$, Florian Wiesinger ${ }^{2}$, Mauro Costagli $^{3}$, Doug Kelley ${ }^{4}$, Mirco Cosottini $^{3}$, and Michela Tosetti $^{3}$

${ }^{1}$ GE Healthcare, Pisa, Italy, ${ }^{2}$ GE Healthcare, Munich, Germany, ${ }^{3}$ Imago7, Pisa, Italy, ${ }^{4}$ GE Healthcare, Waukesha, WI, United States 
We applied the MP2RAGE formalism to a T1-weighted Zero Time Echo sequence. The complex ratio of ZTE images taken with and without inversion preparation showed a correction of the receive coil bias. Brain images of the head are presented showing improved contrast between grey and white matter.

A Simplified Method to Estimate Perfusion Characteristics of Gliomas based on Diffusion-weighted Imaging

Mengqiu Cao ${ }^{1}$, Shiteng Suo ${ }^{1}, \mathrm{Xu} \mathrm{Han}^{1}$, Yawen Sun ${ }^{1}$, Yao Wang ${ }^{1}$, Weina Ding ${ }^{1}, \mathrm{Ke} \mathrm{Jin}^{2}$, Xiaohua Zhang ${ }^{2}$, Jianxun Qu ${ }^{3}$, and Yan Zhou

${ }^{1}$ Department of Radiology, Renji Hospital, School of Medicine, Shanghai Jiao Tong University, Shanghai, P.R. China, Shanghai, China, ${ }^{2}$ Department of Neurosurgery, Renji Hospital, School of Medicine, Shanghai Jiao Tong University, Shanghai, P.R. China, Shanghai, China, ${ }^{3}$ GE Healthcare China, Shanghai, P.R. China, Shanghai, China

The purpose of the study was to evaluate the application of a simplified method to estimate the perfusion characteristics of glioma as an alternative less time-consuming approach. Fifty patients confirmed with glioma were assessed with multi-b-value DWI and DCE MR imaging. Results indicated that the simplified perfusion fraction (SPF) based on DWI acquired with three b-values showed strong correlation with IVIM-derived $f$ and $D^{*}$, and showed medium correlation with DCE MR imaging-derived Ktrans and vp. SPF achieved the highest accuracy for gliomas grading. SPF may serve as a valuable alternative to measure tumor perfusion in gliomas.

Quantitative comparison of image quality between averaged MPRAGE, averaged multi-echo MPRAGE, MP2RAGE and multi-echo MP2RAGE images at high field

Paolo Montagna ${ }^{1}$, Domenico Zacà ${ }^{1}$, and Jorge Jovicich ${ }^{1}$

${ }^{1}$ MRI Lab, Center for Mind/Brain Sciences, University of Trento, Trento, Italy

T1 structural neuroimaging is challenged by spatial inhomogeneities of $B_{1}$ and $B_{0}$, especially at high fields $(>=3 T$ ). Different strategies have been proposed, MP2RAGE (less sensitive to $\triangle \mathrm{B}_{1}$, giving also a T1 map) and multi-echo MPRAGE (MEMPRAGE, less sensitive to $\triangle \mathrm{B}_{0}$, giving also a T2* map). Here we evaluate the combination of both approaches: MEMP2RAGE. We compare gray-white matter contrast (tissue_CNR) and intensity non-uniformity (INU), between MEMP2RAGE and 3 sets of images under comparable acquisition time: MP2RAGE, two averages MPRAGE, two averages MEMPRAGE. Both MP2RAGE images provide higher tissue_CNR and INU correction than standard MPRAGE images.

Distortion-Free Imaging: A Double Encoding Method (DIADEM), High-Resolution Diffusion Imaging of Brain Tumors on a Compact 3T Scanner

Myung-Ho In ${ }^{1}$, Joshua D Trzasko ${ }^{1}$, Yunhong Shu ${ }^{1}$, Shengzhen Tao ${ }^{1}$, Erin M Gray ${ }^{1}$, Matt A Bernstein ${ }^{1}$, and John Huston ${ }^{1}$

${ }^{1}$ Department of Radiology, Mayo Clinic, Rochester, MN, United States

Recently, we developed a multi-shot method using spin-warp echo-planar encoding technique inspired by point-spread function mapping. Distortion-free imaging: a double encoding method (DIADEM) can achieve distortion-free, very high in-plane spatial resolution whole brain diffusion imaging in less than 10 minutes on a compact $3 T$ scanner with high performance gradients. A clinical feasibility study of brain tumor diffusion imaging was performed to explore the efficacy of this approach compared to standard single-shot, echo-planar imaging commonly used in clinical practice. The results demonstrate that the proposed method allows considerable improvements in characterizing brain tumors especially at regions of the brain typically degraded by high susceptibility artifacts.

An 8 channel Rhesus Head coil for Neuroimaging on 3T

Jo Lee ${ }^{1,2}$, Xing Yang ${ }^{3}$, Qiaoyan Chen ${ }^{1,2}$, Changjun Tie ${ }^{1,2}$, Xiaoliang Zhang ${ }^{4,5}$, Hairong Zheng ${ }^{1,2}$, and Ye Li ${ }^{1,2}$

${ }^{1}$ Lauterbur Imaging Research Center, Shenzhen Institutes of Advanced Technology, Chinese Academy of Sciences, Shenzhen, China, ${ }^{2}$ Shenzhen Key Laboratory for MRI, Shenzhen, China, ${ }^{3}$ High-Field Magnetic Resonance Brain Imaging Key Laboratory of Sichuan Province, School of Life Science and Technology, University of Electronic Science and Technology of China, Chengdu, China, ${ }^{4}$ Department of Radiology and Biomedical Imaging, University of California San Francisco, San Francisco, CA, United States, ${ }^{5}$ UCSF/UC Berkeley Joint Graduate Group in Bioengineering, San Francisco, CA, United States

In this study, a custom-designed 8-channel monkey coil was made to match the specific stereotaxic instrument and also better fit the shape of rhesus monkey brain. In comparison with a commercially available coil array, monkey brain images acquired using the dedicated monkey coil array at $3 T$ achieve better SNR, improved parallel imaging capability and higher spatial resolution.

Improved Identification of $\mathrm{MCl}$ Converters and Non-Converters using Voxel-Based Morphometry and Low-Rank Plus Sparse Matrix Decomposition

Xiuyuan Wang ${ }^{1,2}$, Steven H. Baete ${ }^{1,2}$, Ying-Chia Lin ${ }^{1,2}$, Ricardo Otazo ${ }^{1}$, and Fernando E. Boada ${ }^{1,2}$

${ }^{1}$ Center for Advanced Imaging Innovation and Research (CAI2R), NYU School of Medicine, New York, NY, United States, ${ }^{2}$ Center for Biomedical Imaging, Department of Radiology, NYU School of Medicine, New York, NY, United States 
Early identification of mild cognitive impairment $(\mathrm{MCl})$ patients presents significant challenges due to mild symptoms and low sensitivity of the algorithms proposed for $\mathrm{MCl}$ identification. In this study we employed low-rank plus sparse $(L+S)$ matrix decomposition for identifying gray matter volume differences in bilateral hippocampi between $M C l$ patients who converted to Alzheimer's disease within 18 months and $\mathrm{MCl}$ patients who did not. The $\mathrm{L}+\mathrm{S}$ decomposition identifies features that are common across subjects while minimizing the influence of individual variabilities and outliers. Sensitivity and accuracy are greatly improved and voxel-wise differences that couldn't be assessed by previous analyses are also identified.

Eight fold acceleration for isotropic T2w and T2FLAIR imaging using Multi-Contrast Second-Order Directional Total Generalized Variation (dTGV)

Youngwook Kee ${ }^{1}$, Junghun $\mathrm{Cho}^{2}$, Thanh Nguyen ${ }^{1}$, Pascal Spincemaille ${ }^{1}$, and Yi Wang ${ }^{1}$

${ }^{1}$ Weill Cornell Medical College, New York, NY, United States, ${ }^{2}$ Cornell University, Ithaca, NY, United States

We propose a second-order directional total generalized variation (dTGV) that makes use of directional edge information in T1w to reconstruct highly undersampled T2W and T2FLAIR data. This allows a further doubling of the acquisition speed over the standard four fold accelerated protocol. The proposed dTGV regularizer promotes structural similarity between contrasts.

Whole Tumor Histogram Analysis of T2-Weighted, Diffusion-weighted, and Postcontrast T1-Weighted Images in Medulloblatoma:Assessment Risk of Recurrence. QINGQING LV 1

${ }^{1}$ the First Affiliated Hospital of Zhengzhou University, zhengzhou, China

Retrospective analysis of 28 patients which were pathologically confirmed medulloblastoma.We find that MRI whole-tumor histogram analysis can be used as an important supplementary method to assess the risk of medulloblastoma recurrence.

Regional Brain Iron Mapping in Patients with Heart Failure

Bhaswati Roy ${ }^{1}$, Sadhana Singh ${ }^{2}$, Xiaopeng Song ${ }^{2}$, Ashish Sahib ${ }^{2}$, Cristina Cabrera-Mino ${ }^{1}$, Gregg C. Fonarow ${ }^{3}$, Mary Woo ${ }^{1}$, and Rajesh Kumar ${ }^{2,4,5,6}$

${ }^{1}$ UCLA School of Nursing, University of California at Los Angeles, Los Angeles, CA, United States, ${ }^{2}$ Department of Anesthesiology, University of California at Los Angeles, Los Angeles, CA, United States, ${ }^{3}$ Division of Cardiology, University of California at Los Angeles, Los Angeles, CA, United States, ${ }^{4}$ Department of Radiological Sciences, University of California at Los Angeles, Los Angeles, CA, United States, ${ }^{5}$ Department of Bioengineering, University of California at Los Angeles, Los Angeles, CA, United States, ${ }^{6}$ Brain Research Institute, University of California at Los Angeles, Los Angeles, CA, United States

$\mathrm{HF}$ subjects show brain injury in multiple areas, which may contribute to altered iron concentration in those sites. However, regional brain iron load in $\mathrm{HF}$ subjects is unclear. We examined regional iron deposition using R2*-relaxometry procedures and found altered R2*-values in the amygdala, brainstem, thalamus, globus pallidus, hippocampus, cerebellum, insula, and frontal and temporal white matter regions. The altered iron concentration in HF subjects may result from neural and white matter injury, including myelin and glial dysfunction, with iron potentially accelerating tissue degeneration. These data suggest that interfering with the iron action may reduce the exacerbation of injury in $\mathrm{HF}$

Identification of thalamic substructures in ultra-high b-value DWI

Nils Christoph Nuessle ${ }^{1}$, Benjamin Bender ${ }^{1}$, and Uwe Klose ${ }^{1}$

${ }^{1}$ Department for Neuroradiology, University Hospital of Tuebingen, Tuebingen, Germany

Precise implantation of deep brain stimulation devices in Parkinson, primary dystonia or epilepsy patients requires precise structural information about the thalamic region. Purpose of this study was to evaluate the capability of DWI in identifying thalamic substructures. Eight healthy volunteers underwent ultra-high b-value DWI (5000 s/mm $\left.{ }^{2}\right)$ at $3 \mathrm{~T}$. Images were denoised using total generalized variation and 7 substructures (Pulvinar and six nuclei) within the thalamus were drawn in and compared to histological atlases. In all volunteers, all seven structures could be identified due to signal intensities. High b-value diffusion weighted imaging therefore shows great potential in determining thalamic substructures.

Practical parameter setting for simultaneous measurement of CBF and ATT with Hadamard-encoded ASL: Special reference for clinical practice

Shota Ishida ${ }^{1,2}$, Hirohiko Kimura ${ }^{3}$, Naoyuki Takei ${ }^{4}$, Masayuki Kanamoto ${ }^{1}$, Yasuhiro Fujiwara ${ }^{5}$, Tsuyoshi Matsuda $^{6}$, R Marc Lebel $^{7}$, and Toshiki Adachi $^{1}$

${ }^{1}$ Radiological Center, University of Fukui Hospital, Yoshida-gun, Japan, ${ }^{2}$ Division of Health Sciences, Graduate School of Medical Sciences, Kanazawa Unversity, Kanazawa, Japan, ${ }^{3}$ Department of Radiology, Faculty of Medical Science, University of Fukui, Yoshida-gun, Japan, ${ }^{4}$ Global MR applications and Workflow, GE Healthcare Japan, Hino, Japan, ${ }^{5}$ Department of Medical Imaging, Faculty of Life Sciences, Kumamoto University, Kumamoto, Japan, ${ }^{6}$ Division of Ultrahigh Field MRI, Institute for Biomedical Sciences, Iwate Medical University, Shiwa-gun, Japan, ${ }^{7} G E$ Healthcare, Calgary, AB, Canada 
Hadamard-encoded ASL (H-ASL) is a time-efficient method for measuring arterial transit time (ATT). The larger encoding matrix extends the scan time, but the accuracy of the ATT with a different encoding matrix was not clarified. This study aimed to propose a practical parameter selection in H-ASL for clinical use. The ATT was not significantly different between 3 and 7 delay encodings. Cerebral blood flow (CBF) obtained with 3 delay encodings with a linear division block design was equivalent to that obtained without encoding. Three delay encodings with a linear division block design provides accurate ATT and CBF within 4 minutes.

Impact of coregistration approaches on the reliability of R2* and Quantitative Susceptibility Maps (QSM) at 7 T

Seongjin $\mathrm{Choi}^{1}, \mathrm{Xu} \mathrm{Li}^{2,3}$, and Daniel M Harrison ${ }^{1}$

${ }^{1}$ Department of Neurology, University of Maryland Baltimore, Baltimore, MD, United States, ${ }^{2}$ Department of Radiology and Radiological Science, Johns Hopkins University, Baltimore, MD, United States, ${ }^{3}$ F.M. Kirby Research Center for Functional Brain Imaging, Kennedy Krieger Institute, Baltimore, MD, United States

We assessed the unexplored reliabilities of QSM and R2* map acquired at $7 \mathrm{~T}$ by two different approaches that are 1) a direct coregistration of the quantitative maps from their native to a reference space and 2) processing the quantitative maps in a transformed space. R2* was reliable in both methods in the pixel value and the group-mean analyses. However, QSM was less reliable when it was processed in a transformed space in both analyses. Therefore, QSM is recommended to be calculated in its native space prior to any coregistration in a multi-modal study.

Prognostic value of phase images of 2D T2*-weighted GRE in cardiac arrest survivors: A pilot study

Jinhee Jang ${ }^{1}$, Sang Hoon $\mathrm{Oh}^{2}$, Yangsean $\mathrm{Choi}^{1}$, Yoonho Nam ${ }^{1}$, Kyu Nam Park ${ }^{2}$, and Kook-Jin Ahn ${ }^{1}$

${ }^{1}$ Radiology, Seoul St. Mary's hospital, College of Medicine, The Catholic University of Korea, Seoul, Republic of Korea, ${ }^{2}$ Emergency Medicine, Seoul St. Mary's hospital, College of Medicine, The Catholic University of Korea, Seoul, Republic of Korea

Because hypoxic ischemic injury of the brain occurs in cardiac arrest survivors, assessment of oxygen metabolism could be useful. In this work, we analyzed filtered phase images of $2 \mathrm{D}$ T2*-weighed gradient echo images in them. Three survivors with good neurologic outcome showed normal pattern of cortical and deep veins, as well as dural sinuses. However, patients with poor outcome showed two abnormal pattern of venous structures on filtered phase images; (1) attenuated contrast of venous structures and (2) strong and exaggerated venous contrast. Filtered phase images of 2D T2*-weighed gradient echo might useful to predict prognosis of cardiac arrest survivors.

Traditional Poster

\section{Brain Pathology \& Ageing Brain}

Revealing the three-dimensional intraparenchymal trajectory of the brainstem cranial nerve systems by diffusion MRI representation.

Elizabeth B Hutchinson ${ }^{1,2}$, Neda Sadeghi ${ }^{1}$, Martin Lizak ${ }^{3}$, Martha Quezado ${ }^{4}$, Irini Manoli ${ }^{5}$, and Carlo Pierpaoli ${ }^{1}$

${ }^{1}$ QMI/NIBIB, National Institutes of Health, Bethesda, MD, United States, ${ }^{2}$ Henry M. Jackson Foundation, Bethesda, MD, United States, ${ }^{3}$ NINDS, National Institutes of Health, Bethesda, $M D$, United States, ${ }^{4} \mathrm{NCI}$, National Institutes of Health, Bethesda, MD, United States, ${ }^{5} \mathrm{NHGRI}$, National Institutes of Health, Bethesda, MD, United States

The cranial nerve systems of the human brainstem are challenging to distinguish from their complex architectural surroundings, but anisotropy, orientation and tract-based diffusion MRI methods may address these challenges and enable mapping intraparenchymal trajectories of the cranial nerves. The objective of this study was to apply and evaluate DTI and tractography tools for segmentation and mapping of the cranial nerve systems at high spatial resolution in post-mortem human brainstems. Our findings demonstrate the salient features of scalar, directional and tract-based maps for distinguishing the cranial nerves and their nuclei with attention to their relative geometric complexity and architectural environment.

Linking neurotransmitter concentration and functional connectivity of the hippocampus after stress: an in-vivo MRI study

Ricardo Magalhães ${ }^{1,2}$, David Barriére ${ }^{3}$, Ashley Novais $^{1,2}$, Fernanda Marques $^{1,2}$, João Carlos Sousa $^{1,2}$, João Cerqueira ${ }^{1,2}$, Arnaud Cachia $^{3,4}$, Thérèse Jay $^{3}$, Nuno Sousa $^{1,2}$, Sébastien Mériaux ${ }^{5}$, and Fawzi Boumezbeur ${ }^{5}$

${ }^{1}$ Life and Health Sciences Institute, ICVS, School of Medicine, Universidade do Minho, Braga, Portugal, ${ }^{2}$ ICVS/3B's - PT Government Associate laboratory, Braga/Guimaraes, Portugal, ${ }^{3}$ Physiopathologie des Maladies Psychiatriques, UMR_S 894 Inserm, Paris, France, ${ }^{4}$ Univ. Paris Descartes, Paris, France, ${ }^{5}$ Neurospin, JOLIOT, CEA, Paris, France

Stress is a potent modulator of brain metabolism and function. Here we use a combined approach of blood corticosterone quantification, nuclear magnetic resonance spectroscopy and resting state functional magnetic resonance imaging to probe both metabolic and functional changes in the brain. We show correlations in the concentration of GABA/Glutamine and Glutamate/Glutamine in the hippocampus and how these two factors interact with the response to stress. Furthermore we explore how the changes in neurotransmitters correlate with functional networks, revealing several affected connections especially with the retrosplenial cortex, therefore suggesting a role of this relationship in the affected memory phenotype. 
White Matter Microvascular Changes in Healthy Aging

Ian J Tagge ${ }^{1}$, Valerie C Anderson ${ }^{1}$, James T Obayashi ${ }^{2}$, Xin Li ${ }^{1}$, Joseph F Quinn ${ }^{3}$, Jeffrey A Kaye ${ }^{3}$, Dennis N Bourdette ${ }^{3}$, Rebecca I Spain ${ }^{3}$, Manoj K Sammi ${ }^{1}$, and William D Rooney ${ }^{1}$

${ }^{1}$ Advanced Imaging Research Center, Oregon Health \& Science University, Portland, OR, United States, ${ }^{2}$ Neurological Surgery, Oregon Health \& Science University, Portland, OR, United States, ${ }^{3}$ Neurology, Oregon Health \& Science University, Portland, OR, United States

The extent to which changes in blood-brain-barrier permeability are associated with healthy aging is poorly understood. Pharmacokinetic modeling of dynamic-contrast-enhanced MRI yields quantitative estimates of BBB water permeability. DCE-MRI data were collected from 40 healthy controls (aged 34-80 yrs) at 7T. Declines in pharmacokinetic parameters were significant across the entire age range included in this study. Because changes in BBB permeability to water and other small molecules are likely to precede the leakage of CR and larger macromolecules, these estimates represent particularly important probes of the subtle BBB abnormalities that are likely to accompany healthy brain aging.

Retinal Vascular Fractal Dimension and Cerebral Blood Flow, a pilot study

Jeremy Nadal ${ }^{1,2}$, Jeremy Deverdun ${ }^{3}$, Nicolas Menjot de Champfleur ${ }^{3}$, Emmanuelle Le Bars ${ }^{3}$, and Vincent Daien ${ }^{4,5}$

${ }^{1}$ Department of Ophthalmology, Nîmes University Hospital, Nimes, France, ${ }^{2}$ Gui de Chauliac Hospital, I2FH, Institut d'Imagerie Fonctionnelle Humaine, Montpellier, France,

${ }^{3}$ Neuroradiology, I2FH - CHU Gui de Chauliac, Montpellier, France, ${ }^{4}$ U1061, INSERM, Montpellier, France, ${ }^{5}$ Department of Ophthalmology, Gui De Chauliac Hospital, Montpellier, France

The retinal vascular fractal dimension (FD) is a marker of retinal vascular complexity of the vascular tree. It has been associated with systemic disorders but also with neurodegenerative and cerebrovascular diseases. The purpose of this study was to explore the relationship between cerebral blood flow (CBF), retinal vascular FD and other retinal vascular markers. CBF was estimated in vascular territories using 2D PASL sequence. CBF was positively associated to venular FD (R2 $=0.32$, $p=0.03$ ). Non-invasive exploration of the retinal vasculature may be used as a proxy measure, with the condition of retinal vessels possibly reflecting the condition of the cerebral vasculature.

Relationships among cerebrovascular reactivity, grey matter volume and markers of successful aging

Brittany Intzandt ${ }^{1}$, Dalia Sabra ${ }^{2}$, Laurence Desjardins-Crepeau ${ }^{3,4}$, Said Mekary ${ }^{5}$, Louis Bherer ${ }^{3,4,6}$, Richard D Hoge ${ }^{7,8}$, Christopher J Steele ${ }^{9,10}$, and Claudine J Gauthier ${ }^{11,12}$

${ }^{1}$ Concordia University, Montreal, QC, Canada, ${ }^{2}$ Biomedical Sciences, Universite de Montreal, Montreal, QC, Canada, ${ }^{3}$ Montreal Heart Institute, Montreal, QC, Canada, ${ }^{4}$ Laboratoire d'Étude de la Santé Cognitive des Ainés, Centre de recherche de l'Institut universitaire de gériatrie de Montréal, Montreal, QC, Canada, ${ }^{5}$ Kinesiology, Acadia University, Wolfville, NS, Canada, ${ }^{6}$ Medicine, Universite de Montreal, Montreal, QC, Canada, ${ }^{7}$ Montreal Neurological Institute, Montreal, QC, Canada, ${ }^{8}$ Neurology and Neurosurgery, McGill University, Montreal, QC, Canada, ${ }^{9}$ Neurology, Max Planck Institute for Human Cognitive and Brain Sciences, Leipzig, Germany, ${ }^{10}$ Cerebral Imaging Center, Douglas Mental Health University InstituteMcGill University, Montreal, QC, Canada, ${ }^{11}$ Physics, Concordia University, Montreal, QC, Canada, ${ }^{12}$ PERFORM Centre, Montreal, QC, Canada

Aging causes decline in brain health, which has a complex relationship with fitness and cognition. Here, we aimed to disentangle the interactions between these outcomes in healthy older adults. MRI was used to acquire anatomical and cerebrovascular reactivity (CVR) in all participants. VO2max and cognitive outcomes were also tested. Results revealed that increased CVR was associated with decreased fitness and cognitive performance, whereas increased grey matter volume was associated with increased fitness. It is apparent that the relationship between brain health and fitness and cognitive outcomes is intricate and other parameters, such as cerebral blood flow, are necessary to gain further understanding.

Effect of autolysis, fixation, and storage in PBS on relaxation rates and macro-molecular tissue volume across fiber pathways of the human brain

Mohammad Ashtarayeh ${ }^{1}$, Tobias Streubel ${ }^{1}$, Klaus Püschel $^{2}$, and Siawoosh Mohammadi ${ }^{1}$

${ }^{1}$ Department of Systems Neuroscience, Medical Center Hamburg-Eppendorf, Hamburg, Germany, ${ }^{2}$ Center for Diagnostics, Institute of Legal Medicine, Medical Center HamburgEppendorf, Hamburg, Germany

We evaluated the effect of autolysis, brain tissue fixation, and embedding into PBS on three potential quantitative myelin MRI markers across different white matter fiber pathways: longitudinal (R1) and effective transverse (R2*) relaxation rates, and macro-molecular tissue volume (MTV) using the quantitative multi-parameter mapping (MPM) protocol. We found that the effect of autolysis was most apparent in R2* and MTV, R1 drastically changed its contrast after fixation, and R1 and R2* values increased after storage in Phosphate-Buffered Saline (PBS) solution.

Andrew Hall ${ }^{1}$, Laurentius Huber ${ }^{2}$, Daniel Handwerker ${ }^{2}$, Emily Finn ${ }^{2}$, and Peter Bandettini ${ }^{2}$

${ }^{1} \mathrm{NIH}$, Bethesda, MD, United States, ${ }^{2} \mathrm{NIH} / \mathrm{NIMH}$, Bethesda, MD, United States 
We investigated $166 \mathrm{~T} 1$-weighted datasets to identify neural biomarkers of sleep deprivation (3h sleep). We find that a linear classification algorithm is able to distinguish between sleep deprived and well-rested brains at $65 \%$ accuracy in T1-weighted images. The underlying hypothesis is that if glymphatic function is mediated by sleep, one should be able to tell the difference between sleepy and rested brains based on subtle shifts in T1 across brains.

Nonlinear pattern of the emergence of white matter hyperintensity in healthy Han Chinese: an adult lifespan study

Chu-Chung Huang ${ }^{1}$, Albert C. Yang ${ }^{2}$, Kun-Hsien Chou ${ }^{3}$, Mu-En Liư ${ }^{4}$, Shih-Jen Tsai ${ }^{4}$, and Ching-Po Lin ${ }^{5}$

${ }^{1}$ Aging and Health Research Center, National Yang-Ming University, Taipei, Taiwan, ${ }^{2}$ Division of Interdiscplinary Medicine and Biotechnology, Beth Israel Deaconess Medical Center, Harvard Medical School, Boston, MA, United States, ${ }^{3}$ Brain Research Center, National Yang-Ming University, Taipei, Taiwan, ${ }^{4}$ Department of Psychiatry, Taipei Veterans General Hospital, Taipei, Taiwan, 5 Insitute of Neuroscience, National Yang-Ming University, Taipei, Taiwan

WMH is one of the most obvious imaging traits in the aged brain. There is evidence that the WMH volume may have the potential to track with age and age-related cognitive decline. However, no study has investigated the trajectory of WMH progression and their impact on cognition during normal aging process. We show that increased age is nonlinearly correlated with increased PVWMH. In two-mediators mediation model, PVWMH is found to mediate the age-related decline of MMSE, but not DWMH. This study suggested that PVWMH could be a potential, and feasible biomarker in predicting age-related cognitive decline across the adult lifespan.

MR Spectroscopy Study: Neural Effects of Induced Hypothermia Treatment after Myocardial Infarction \& Anoxia

Lasya Sreepada ${ }^{1,2}$, Jong Woo Lee ${ }^{3}$, Huijun Liao ${ }^{1}$, Benjamin Rowland ${ }^{1,4}$, and Alexander P Lin ${ }^{1}$

${ }^{1}$ Center for Clinical Spectroscopy, Department of Radiology, Brigham and Women's Hospital, Boston, MA, United States, ${ }^{2}$ Yale University, New Haven, CT, United States, ${ }^{3}$ Neurology, Brigham and Women's Hospital, Boston, MA, United States, ${ }^{4}$ Cardiff University Brain Imaging Centre, Cardiff University, Cardiff, United Kingdom

Coma after cardiac arrest is a common and debilitating incidence. This study aims to determine the neurochemical changes that occur in comatose cardiac arrest patients who underwent targeted temperature management. Single Voxel MRS was acquired in the posterior cingulate gyrus (PCG) and parietal white matter (PWM) of patients and age-matched controls with no history of neurological disease. Patients showed decreases in NAA, as well as increases in total Choline and Lactate in both PCG and PWM. Patients also showed decreases in glutamate in the PCG. These neurometabolic changes reflect neuronal, axonal and glial loss that would result in reduced neurotransmission.

Cerebral Vascular Reactivity and Cognitive Decline in Healthy and in Early Stages of Pathological Aging.

Naila Boudiaf ${ }^{1}$, Jan Warnking ${ }^{2}$, Olivier Moreaud ${ }^{3}$, Johan Pietras ${ }^{4}$, Eric Condamine ${ }^{2}$, Nathalie Fournet ${ }^{5}$, Amandine Bossant $^{6}$, Monica Baciu ${ }^{7}$, and Alexandre Krainik ${ }^{1}$

${ }^{1}$ Neuroradiology, CHU Grenoble, Grenoble, France, ${ }^{2}$ Grenoble Institute of Neuroscience, Grenoble, France, ${ }^{3}$ Neurology, CMRR Grenoble, CHU Grenoble, Grenoble, France, ${ }^{4}$ IRMAGE, Grenoble, France, ${ }^{5} \mathrm{CNRS}$, LPNC, Chambéry, France, ${ }^{6} \mathrm{CHU}$ Grenoble, Grenoble, France, ${ }^{7} \mathrm{CNRS}$, LPNC, Grenoble, France

Cerebral blood flow (CBF) and cerebral vascular reactivity (CVR) decrease with age and might affect cognitive functions. In this study, we investigated their correlation with cognitive abilities during normal and pathological aging.

We performed neuropsychological assessments on thirty-four participants: 13 healthy-young, 10 healthy-old and 11 impaired-old. We measured CBF and CVR using hypercapnia and Arterial Spin Labeling imaging.

Regarding the impaired-old, we found specific decrease in executive functions, short-term and working memory. Significant correlations were found between CVR and cognitive scores. Global CBF correlated only with age.

Unlike CBF, CVR reduction was specifically associated with cognitive decline during aging.

Cingulum tractography in old subjects presenting low or high white matter lesion burden

Manon Edde ${ }^{1}$, Bixente Dilharreguy ${ }^{1}$, Catherine Helmer $^{2}$, Jean-François Dartigues ${ }^{2}$, Michèle Allard ${ }^{1}$, and Gwénaëlle Catheline ${ }^{1}$

${ }^{1}$ UMR5287, Aquitaine Institute for Cognitive and Integrative Neuroscience, Bordeaux, France, ${ }^{2}$ INSERM U897, Bordeaux Population Health (BPH Center), Bordeaux, France

Tractography frequently fails in aging brain because diffusion parameters dramatically decrease in regions of WM hyperintensities (WMH) which one are very common in this population. We developed here a pipeline taking into account this pitfall to truly investigate the microstructural properties of the cingulum bundles in presence and absence of WMH. 
${ }^{1}$ Neuroscience, Washington University School of Medicine, St. Louis, MO, United States, ${ }^{2}$ Radiology, Washington University School of Medicine, St. Louis, MO, United States,

${ }^{3}$ Neurosurgery, Washington University School of Medicine, St. Louis, MO, United States

This study is the first of its kind, and uses diffusion basis spectrum imaging (DBSI) to quantify, as well as differentiate the complex pathologies that underlies the white matter injury in post-hemorrhagic hydrocephalus $(\mathrm{PHH})$ in neonates, using a ferret model of $\mathrm{PHH}$.

Improving MRI assessment of whole-brain structural health in aging: an approach involving multiple sequences

Hui Guo ${ }^{1,2}$, Yunting Zhang ${ }^{1}$, Ryan C.N. D'Arcy ${ }^{2,3}$, and Xiaowei Song ${ }^{2,3}$

${ }^{1}$ Medical imaging department, Tianjin medical university general hospital, Tianjin, China, ${ }^{2}$ Health Sciences and Innovation, Fraser Health Authority \& SFU ImageTech Laboratory, Surrey Memorial Hospital, Surrey, BC, Canada, ${ }^{3}$ Schools of Engineering and Computing Sciences, Simon Fraser University, Burnaby, BC, Canada

The process of brain aging is characterized by the accumulation of multiple structural changes, several of which can be visualized using clinical MRI. Brain Atrophy and Lesion Index (BALI) has been validated to collectively assess MRI-based whole-brain structural changes. This study aims to improve the BALI assessment of whole-brain structural changes in aging using multiple routine clinical MRI sequences (T1WI, T2WI, T2-FLAIR and T2*GRE).

Nutritional intervention for developmental brain damage: neuroprotection with Lactoferrin following intrauterine growth restriction.

Yohan van de Looij ${ }^{1,2,3}$, Camille Larpin ${ }^{1}$, Petra S Hüppi ${ }^{1}$, and Stéphane V Sizonenko ${ }^{1}$

${ }^{1}$ Service développement et croissance, Université de Genève, Geneva, Switzerland, ${ }^{2}$ Laboratoire d'imagerie fonctionnelle et métabolique, Ecole polytechnique fédérale de Lausanne, Lausanne, Switzerland, ${ }^{3}$ Institut translationnel d'imagerie moléculaire, Université de Genève, Geneva, Switzerland

Lactoferrin (Lf) is an iron-binding glycoprotein secreted in milk known as antioxidant, antimicrobial and anti-inflammatory. Infants exposed to adverse prenatal conditions of intrauterine growth restriction (IUGR), are at high risk for neurological morbidities. The aim of this work was to assess neuroprotective effect of Lf on brain microstructure by using diffusion imaging and NODDI model at $9.4 \mathrm{~T}$ in a model of $50 \%$ gestational caloric restriction. Diffusion MRI derived parameters changes following IUGR were partially restored in the Lf supplemented group, providing evidence of a neuroprotective effect.

Differentiation of white matter hyperintensity severity using T2- and T1-weighted brain MRI.

Nina Linde Højland Reislev ${ }^{1,2}$, Henrik Lundell ${ }^{1}$, Hartwig Roman Siebner ${ }^{1,3}$, Christian Eriksen ${ }^{2,4}$, Michael Kjær $^{2,4}$, and Ellen Garde ${ }^{2,5}$

${ }^{1}$ Danish Research Centre for Magnetic Resonance, Centre for Functional and Diagnostic Imaging and Research, Copenhagen University Hospital Hvidovre, Hvidovre, Denmark, ${ }^{2}$ Center for Healthy Aging, Faculty of Health and Medical Sciences, University of Copenhagen, Copenhagen, Denmark, ${ }^{3}$ Department of Neurology, Copenhagen University Hospital Bispebjerg, Copenhagen, Denmark, ${ }^{4}$ Institute for Sports Medicine, Copenhagen University Hospital Bispebjerg, Copenhagen, Denmark, ${ }^{5}$ Department of Public Health, University of Copenhagen, Copenhagen, Denmark

This study presents a new method to differentiate brain white matter hyperintensity (WMH) severity using conventional T1-weighted and T2-weighted MRI. By combining normalized image intensity, heterogeneous tissue properties within lesions are revealed. Lesion severity is quantified through two distance measures of parallel and perpendicular deviation from normal appearing white matter. Correlations with diffusion imaging based measures suggest that multi-modal voxel-based lesion analysis provide comparable but high-resolution tissue information. Based on conventional MRI scans this method adds valuable insight into the differentiated impact of WMH lesions on brain structure and function.

The Aging Brain: Cerebrovascular responses to $\mathrm{CO} 2$.

Larissa McKetton ${ }^{1}$, Olivia Sobczyk ${ }^{2}$, Julien Poublanc ${ }^{1}$, Kevin Sam², ${ }^{2,3}$ Adrian P. Crawley ${ }^{1}$, Lakshmikumar Venkat Raghavan ${ }^{4}$, James Duffin ${ }^{4,5}$, Joseph A. Fisher $2,4,5$, and David J. Mikulis ${ }^{4,5}$

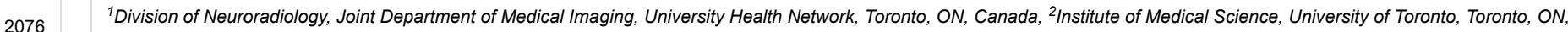
Canada, ${ }^{3}$ The Russell H. Morgan Department of Radiology \& Radiological Science, The John Hopkins University School of Medicine, Baltimore, MD, United States, ${ }^{4}$ Department of Anaesthesia and Pain Management, University Health Network, Toronto, ON, Canada, ${ }^{5}$ Department of Physiology, University of Toronto, Toronto, ON, Canada

Measures of cerebrovascular reactivity (CVR) are used to judge the health of the brain vasculature. We report the use of several different analyses of $\mathrm{BOLD}$ responses to $\mathrm{CO}_{2}$ to provide a number of metrics for various aspects of CVR. To assess possible differences in these metrics with age, we compiled atlases reflecting voxel-wise means and standard deviations for different age ranges and compared them. 
Petrice M Cogswell ${ }^{1}$, Sarah K Lants ${ }^{1}$, L Taylor Davis ${ }^{1}$, Spencer Waddle ${ }^{1}$, and Manus J Donahue ${ }^{1}$

${ }^{1}$ Vanderbilt University Medical Center, Nashville, TN, United States

Vessel wall imaging is becoming more widely applied, however normal, age-specific ranges for wall thickness have not been established. We applied a variable refocusing angle 3DTSE acquisition with and without a DANTE flow suppression module to healthy subjects (ages=8-79 years; $n=82$ ). Vessel wall measurements revealed no significant change in wall thickness with age for the supraclinoid internal carotid arteries and basilar artery. The outer wall diameter and wall thickness were measured to be less for the acquisition with versus without DANTE. These data suggest that unlike tissue volume, vessel wall thickness is relatively constant across the lifespan for healthy subjects.

Altered Intrinsic Brain Activity and Memory Function Improvement in Patients with End-Stage Renal Disease During A Single Dialysis Session

Peng $\mathrm{Li}^{1}$, Dun Ding ${ }^{2}$, Xueying Ma ${ }^{2}$, Huawen Zhang ${ }^{1}$, Jixin $\mathrm{Liu}^{3}$, and Ming Zhang ${ }^{2}$

${ }^{1}$ Department of Medical Imaging, NO.215 Hospital of Shaanxi Nuclear Industry, Xianyang, China, ${ }^{2}$ Department of Medical Imaging, First Affiliated Hospital of Xi'an Jiaotong University, Xi'an, China, ${ }^{3}$ Center for Brain Imaging, School of Life Science and Technology, Xidian University, Xi'an, China

The underlying neural mechanisms of the memory deficits in end-stage renal disease patients with dialysis treatment are poorly understood. Here we analyzed the resting-state brain activity changes and the related memory improvement by using mALFF and ReHo methods before dialysis ( $\mathrm{T} 1$ pre-dialysis) and after 24 hours ( $\mathrm{T} 2$ post-dialysis). The results indicated that regional spontaneous activity changes of the DLPFC were related with memory improvement after a single dialysis treatment, which may provide insight into the effect of hemodialysis on changes of brain function and cognitive impairments.

Development of Individual Evaluation System for White Matter Hyperintesity Recognition Using Deep Convolutional Neural Network

Kyung Mi Lee ${ }^{1}$, Hyug-Gi Kim² ${ }^{2}$ Jiwon Yoon ${ }^{2}$, Mi-hyun Kim ${ }^{3}$, Jang-Hoon Oh ${ }^{3}$, In Young Lee ${ }^{3}$, Soonchan Park ${ }^{4}$, Chang-Woo Ryu ${ }^{4}$, Eui Jong Kim ${ }^{1}$, Woo Suk Choi ${ }^{1}$, Na Rae Yang ${ }^{5}$, and Jihye Song ${ }^{6}$

${ }^{1}$ Kyung Hee University College of Medicine, Kyung Hee University Hospital, Seoul, Republic of Korea, ${ }^{2}$ Kyung Hee University Hospital, Seoul, Republic of Korea, ${ }^{3}$ Univeristy Industry Cooperation, Kyung Hee University, Seoul, Republic of Korea, ${ }^{4}$ Kyung Hee University Hospital at Gangdong, Seoul, Republic of Korea, ${ }^{5}$ Neurosurgery, Ewha Womans University School of Medicine, Mokdong Hospital, Seoul, Republic of Korea, ${ }^{6}$ College of Medicine, Konyang University Hospital, Konyang University Myunggok Medical Research Institute, Daejeon, Republic of Korea

White matter hyperintensity (WMH) is one of the important characteristics of cerebral small vessel disease (cSVD). The objective of this study to investigate the feasibility of WMH recognition using deep convolutional neural networks (CNN). Furthermore, individual evaluation system was proposed to classify WMH groups.

Integration and segregation of functional segmented anterior and posterior hippocampal networks in memory performance

Jingjing $\mathrm{Xu}^{1}$, Xiaojun Guan ${ }^{1}$, Xiaojun $\mathrm{Xu}^{1}$, and Minming Zhang ${ }^{1}$

${ }^{1}$ Radiology, the Second Affiliated Hospital of Zhejiang University, School of Medicine, Hangzhou, China

In this study, we used a novel functional segmentation method to subdivided the left and right hippocampus into anterior and posterior portions according to preferred functional connections with certain cortical regions. And we investigated the association between specific performance of verbal and visual memories and intra-hemispheric resting state FC across anterior and posterior hippocampal networks using resting functional MRI measures in healthy young volunteers. The present results demonstrated that, the anterior hippocampus was specifically involved in the visual memory processing, whereas the posterior hippocampus contributed to both the verbal and visual memories, which may have implications for a functionally synergetic and dissociable role of the hippocampus in different kinds of memory.

Presurgical planning: comparison between task activation and resting-state connectivity maps in the motor and language networks

Scott J. Peltier ${ }^{1,2}$ and Gaurang V. Shah ${ }^{3}$

${ }^{1}$ Functional MRI Laboratory, University of Michigan, Ann Arbor, MI, United States, ${ }^{2}$ Biomedical Engineering, University of Michigan, Ann Arbor, MI, United States, ${ }^{3}$ Radiology, University of Michigan, Ann Arbor, MI, United States

In this study, task and resting-state data from presurgical patients with brain tumors was analyzed. Task activation and data-driven resting-state connectivity maps for both motor and language networks were generated for each subject and compared for spatial overlap. 
Guangyu Chen ${ }^{1}$, Arun Singavi ${ }^{1}$, Nancy Wandersee ${ }^{2}$, Collin Hubler ${ }^{2}$, Amanda Brandow ${ }^{1}$, Simpson Pippa ${ }^{1,2}$, Shi-Jiang Li ${ }^{1}$, and Joshua Field ${ }^{1,2}$

${ }^{1}$ Medical College of Wisconsin, Milwaukee, WI, United States, ${ }^{2}$ BloodCenter of Wisconsin, Milwaukee, WI, United States

About half of Sickle Cell Disease (SCD) adults suffer from a chronic pain syndrome. What of the SCD brain contributes to the development and maintenance of the pain syndrome is unknown. We used resting state functional connectivity MRI (rfcMRI) technique, found significant differences between SCD and controls in areas known to contribute to the development and maintenance of a chronic pain syndrome, and the differences have significant associations with the pain phenotype measurements. The findings suggest that rfcMRI could be used as a biomarker to determine the efficacy of interventions targeted to chronic pain in SCD patients.

Impacts of Chronic Liver Injury on Brain Energy Metabolism: A ${ }^{1} \mathrm{H}-\left[{ }^{13} \mathrm{C}\right]-\mathrm{NMR}$ Study on Hepatic Encephalopathy

TK Sampath Kumar ${ }^{1}$, N Sairam² ${ }^{2}$ and Anant Bahadur Patel ${ }^{1}$

${ }^{1}$ NMR Microimaging and Spectroscopy, Centre for Cellular and Molecular Biology, Hyderabad, India, ${ }^{2}$ Animal House, Centre for Cellular and Molecular Biology, Hyderabad, India

It has been postulated that excess ammonia and neuroinflammation resulting from liver failure induces astrocytic swelling which can lead to increased BBB permeability and neuronal dysfunction. The impacts of high levels of blood ammonia on the brain energy metabolism is not clear. The objective of current study is to evaluate the neurotransmitter metabolism in $\mathrm{CCl}_{4}$ induced liver injury mouse model using using ${ }^{1} \mathrm{H}-\left[{ }^{13} \mathrm{C}\right]-\mathrm{NMR}$ spectroscopy together with $\left[1,6-{ }^{13} \mathrm{C}_{2}\right]$ glucose infusion. Our findings indicate reduction in the activity of glutamatergic and GABAergic neurons in the chronic liver damage condition.

Increased Glutamate in Anterior Cingulate Cortex in Crohn's Disease Patients with Abdominal Pain Revealed by Proton MR Spectroscopy

Kun Lv ${ }^{1}$, Wenwen Song ${ }^{2}$, Yihong Fan ${ }^{3}$, Yong Zhang ${ }^{4}$, Bin Lv³ , and Maosheng $\mathrm{Xu}^{2}$

${ }^{1}$ Zhejiang Chinese Medical University, Hangzhou, China, ${ }^{2}$ Radiology, The First Affiliated Hospital of Zhejiang Chinese Medical University, Hangzhou, China, ${ }^{3}$ Gastroenterology, The First Affiliated Hospital of Zhejiang Chinese Medical University, Hangzhou, China, ${ }^{4}$ MR research, GE Healthcare, Shanghai, China

Based on Brain-gut axis, the study used proton magnetic resonance (MR) spectroscopy, a noninvasive detection to reveal the alteration of metabolites in bilateral perigenual anterior cingulate cortex (pgACC) in patients with Crohn's disease (CD) with abdominal pain. Twenty nine CD patients (cases with/without abdominal pain, 16/13) and 20 healthy controls were recruited for comparison. The pain CD group showed increased Glutamate (Glu) levels in bilateral pgACC, which might provide new insight into the neural mechanism of the disease in abdominal pain processing.

Changes in Quantitative Free Water Content with Increasing BMI in Elderly Subjects

Melissa Schall ${ }^{1}$, Elene Iordanishvili ${ }^{1}$, Svenja Caspers ${ }^{2}$, N. Jon Shah ${ }^{1,3}$, and Ana-Maria Oros-Peusquens ${ }^{1}$

${ }^{1}$ Institute of Medical Imaging Physics INM-4, Research Centre Jülich, Jülich, Germany, ${ }^{2}$ Institute of Neuroscience and Medicine (INM-4), Research Centre Jülich, Jülich, Germany,

${ }^{3}$ Jülich Aachen Research Alliance (JARA), Jülich, Germany

A high body mass index is known to play a role in a variety of chronic diseases, which makes it an important biomarker. Using a 3D two-point quantitative mapping method, changes in several parameters including relaxation times, $\mathrm{H}_{2} \mathrm{O}$ and magnetisation transfer measures were investigated in lean and obese subjects. Preliminary results show a significant increase of $\mathrm{H}_{2} \mathrm{O}$ in corpus callosum $(p<0.05)$, thalamus $(p<0.005)$ and white matter of temporal lobe $(p<0.05)$ with increasing BMI. Changes in the other parameters did not reach significance. These findings suggest the existence of regional low-grade brain inflammation in obesity.

Traditional Poster

\section{Novel Neuroimaging Methods}

MRI based texture analysis on FLAIR and ADC to predict malignant transformation of Low Grade Gliomas

Shun Zhang ${ }^{1,2}$, Gloria Chia-Yi Chiang ${ }^{2}$, Yihao Yao ${ }^{1}$, Ramin Jafari $^{2}$, Rajiv S. Magge ${ }^{3}$, Howard Alan Fine ${ }^{3}$, Rohan Ramakrishna ${ }^{4}$, Yi Wang ${ }^{2,5}$, and Ilhami Kovanlikaya ${ }^{2}$

${ }^{1}$ Radiolgy, Tongji Hospital, Tongji Medical College, HUST, Wuhan, China, ${ }^{2}$ Radiolgy, Weill Cornell Medical College, NewYork, NY, United States, ${ }^{3}$ Neurology, Weill Cornell Medical College, NewYork, NY, United States, ${ }^{4}$ Neurological Surgery, Weill Cornell Medical College, NewYork, NY, United States, ${ }^{5}$ Biomedical Engineerring, Cornell University, Ithaca, NY, United States 
Low grade gliomas (LGG) may undergo malignant transformation into high-grade gliomas, which generally occur within 5 years in about $50 \%$ of patients. Hence assessing whether or not a LGG will convert to high grade is of great importance in treatment. In this study, we use texture and histogram analyses on preoperative MRI FLAIR and ADC images to predict malignant transformation from low grade to higher grade glioma, as well as to discriminate between astrocytoma and oligodendroglioma. Based on the receiver operating characteristic (ROC) curves from training data, texture analysis had a higher area under the curve (AUC) value than histogram parameters, and it also more accurately predicted whether LGGs would convert and discriminated between astrocytoma and oligodendroglioma in the testing data. Texture analysis on conventional preoperative FLAIR and ADC images can accurately predict malignant transformation of low grade gliomas, as well as discriminate between astrocytoma and oligodendroglioma.

Observing the Hyaluronan Component of the Extracellular Matrix in the Brain with Quantitative MRI

Riccardo Metere ${ }^{1}$, Carsten Jäger ${ }^{2,3}$, Markus Morawski ${ }^{3}$, and Harald E. Möller ${ }^{1}$

${ }^{1}$ NMR Unit, Max Planck Institute for Human Cognitive and Brain Sciences, Leipzig, Germany, ${ }^{2}$ Department of Neurophysics, Max Planck Institute for Human Cognitive and Brain Sciences, Leipzig, Germany, ${ }^{3}$ Paul Flechsig Institute for Brain Research, University of Leipzig, Leipzig, Germany

The tissue composition of the brain can be related to different contrast sources in quantitative MRI. Notably, $\mathrm{T}_{1}$ and $\mathrm{T}_{2}{ }^{*}$ strongly correlate with myelin and iron. However, other components may play a role in contrast generation. In particular, the hyaluronan component of the extracellular matrix has been recently proposed as a possible important contributor to MRI contrast. Here, we quantify the bulk contribution of hyaluronan to quantitative relaxation maps. This is obtained by characterizing the evolution of the relevant MRI parameters over time during the enzymatic digestion of the hyaluronan.

Noninvasive Analysis of Brain Shift Transformation in Closed Cranium using MR Images Acquired in Different Body Positions

Etsuko Kumamoto ${ }^{1,2}$, Shigeto Hayashi ${ }^{3}$, Kento Matsuda ${ }^{2}$, Katsusuke Kyotani ${ }^{4}$, Takashi Nishino ${ }^{5}$, Tomoaki Nakai ${ }^{6}$, and Eiji Kohmura ${ }^{6}$

${ }^{1}$ Information Science and Technology Center, Kobe University, Kobe, Japan, ${ }^{2}$ Graduate School of System Informatics, Kobe University, Kobe, Japan, ${ }^{3}$ Department of Neurosurgery, Hyogo Emergency Medical Center, Kobe Red Cross Hospital, Kobe, Japan, ${ }^{4}$ Department of Radiology, Kobe University, Kobe, Japan, ${ }^{5}$ Department of Chemical Science and Engineering Faculty of Engineering, Kobe University, Kobe, Japan, ${ }^{6}$ Department of Neurosurgery, Kobe University, Kobe, Japan

Although the transformation of brain tissue during craniotomy is a well-known phenomenon, there has been a lack of methodological analysis related to physiological brain shift in the closed cranium. In this study, we analyzed brain shift and transformation using MRI volume data acquired in different body positions. The volume data were divided into voxels. Each voxel of the prone or the right volume was registered using voxels of the supine or the left volume as templates, and movement and rotation of each voxel were recorded. Experimental result shows that the displacement in the depth of the brain tended to be conspicuous and rigid compared to the displacement of the brain surface.

Towards an optimized protocol for dynamic oxygen enhanced imaging of the brain.

William Lloyd ${ }^{1}$, Adam K Featherstone ${ }^{1}$, Alan Jackson ${ }^{1}$, and Geoff JM Parker ${ }^{1}$

${ }^{1}$ University of Manchester, Manchester, United Kingdom

$\mathrm{T}_{1}$ w dynamic oxygen-enhanced MRI (OE-MRI) has been shown to be a promising method for the assessment and quantification of tumour hypoxia. This work presents a comparison between two possible methods; FFE and IR-TFE based sequences. IR-TFE is shown to give greater contrast for oxygen induced signal change as well as increased SNR. Further sequence optimisation demonstrates the possibility of scanning at high resolution while maintaining contrast and SNR.

Differences in subcortical brain volumes between expert and novice chess players

Ethan $\mathrm{Li}^{1}$, David J Ouellette ${ }^{1}$, and Tim Q Duong

${ }^{1}$ Stony Brook University, Stony Brook, NY, United States

The goal of this study was to investigate the anatomical neural correlates underlying expertise acquisition between expert versus novice chess players using MRI. We found that the acquisition of expertise is accompanied by gray-matter volumetric changes in subcortical brain structures implicated in memory and reinforcement learning. By comparison, the anatomical circuits involved in acquired chess expertise differ from other expertise domains. Improving the understanding of the neural correlates underpinning expertise may prove useful in designing individualized training strategies. 
${ }^{1}$ Medical Sciences, University of Calgary, Calgary, AB, Canada, ${ }^{2}$ Radiology, University of Calgary, Calgary, AB, Canada, ${ }^{3} \mathrm{Psychiatry}$, University of Toronto, Toronto, ON, Canada, ${ }^{4}$ Medicine, Neurology, Sunnybrook Health Sciences Centre, Toronto, ON, Canada, ${ }^{5}$ Clinical Neurosciences, University of Calgary, Calgary, AB, Canada

The hippocampus atrophy rate (volumetric loss per year) might be a good biomarker for predicting disease progression. However, hippocampus atrophy rate assessment requires accurate delineation of the structure from longitudinal scans. In this work, we propose an automatic approach based on convolutional neural network (CNN) for robust and reliable hippocampus segmentation. Therefore, the CNN was pre-trained using weakly annotated T1-weighted MRI datasets and fine-tuned using fully-annotated datasets. Leave-one-out cross validation revealed that the proposed method leads to robust and reproducible segmentation results with an average Dice coefficient of 0.89 .

Concept of Gadolinium-Ferritin Interactions as Explanation of Signal Intensity Changes in Deep Brain Nuclei after Application of Gadolinium-Based Contrast Agents Josef Vymazal ${ }^{1}$, Jitka Neburkova ${ }^{2}$, Martin Dracinsky ${ }^{2}$, Mohan Pingle $^{3}$, Petr Cigler $^{4}$, and Aaron Michael Rulseh ${ }^{1}$

${ }^{1}$ Dept. of Radiology, Na Homolce Hospital, Prague, Czech Republic, ${ }^{2}$ Insittute of Organic Chemistry and Biochemistry, Prague, Czech Republic, ${ }^{3}$ Dept. of Radiology, Na Homolce Hospital, Praha, Czech Republic, ${ }^{4}$ Petr Cigler, Insittute of Organic Chemistry and Biochemistry, Prague, Czech Republic

Interaction between gadolinium-based contrast agents and metalloprotein ferritin may explain observed signal intensity changes in vivo due to T1 (and T2) shortening in the globus pallidus and dentate nucleus.

How does the weighting factor in a regularized quantitative BOLD approach affect the estimated oxygen extraction fraction?

Sebastian Thomas ${ }^{1}$, Simon Hubertus ${ }^{1}$, Sebastian Domsch ${ }^{1}$, and Lothar R. Schad ${ }^{1}$

${ }^{1}$ Computer Assisted Clinical Medicine, Medical Faculty Mannheim, Heidelberg University, Mannheim, Germany

Applying the quantitative blood-oxygenation-level-dependent (qBOLD) method for measuring the oxygen extraction fraction (OEF) often suffers from bad contrast due to the low SNR typical at clinical scan times. In order to improve the evaluation, the choice of the weighting factors in a proposed regularization approach was analyzed. Using the regularization approach, simulations showed increasing precision but decreasing accuracy for increasing weighting factors. For a range of weighting factors a good trade-off between noise suppression and data-fidelity was achieved, which resulted in optimal contrast.

Visualizing healthy aging: A comparative study of quantitative T1 and T2 relaxometry with standard and MR fingerprinting techniques

Vera Catharina Keil ${ }^{1}$, Stilyana Peteva Bakoeva ${ }^{1}$, Alina Jurcoane ${ }^{1}$, Thomas Amthor ${ }^{2}$, Mariya Doneva ${ }^{2}$, Peter Koken ${ }^{2}$, Burkhard Mädler ${ }^{3}$, Wolfgang Block ${ }^{1}$, and Elke Hattingen ${ }^{1}$

$2094 \quad{ }^{1}$ Department of Radiology, University Hospital Bonn, Bonn, Germany, ${ }^{2}$ Philips Research, Hamburg, Germany, ${ }^{3}$ Philips Healthcare, Bonn, Germany

Relaxometry aims at an absolute quantification of T1 and T2 times explained by physicochemical properties in the brain. MR Fingerprinting can be used for quantitative relaxometry, e.g. to explore the effect of age on brain structure. This study examined young and old age ( $n=26$ each) volunteers with standard and MRF mapping techniques. We found that MRF and standard technique multiecho-derived T1 and T2 maps do not identify the same brain structures as affected by age-related relaxometric changes and show in part contradictory relationships between age and especially T1 relaxation. This limited comparability has strong clinical implications for the interpretation of relaxometric studies beyond the topic "aging".

The hMRI analysis toolbox for quantitative MRI and in vivo histology using MRI (hMRI)

Evelyne Balteau ${ }^{1}$, Tobias Leutritz ${ }^{2}$, Antoine Lutti ${ }^{3,4}$, Martina F Callaghan ${ }^{5}$, Bogdan Draganski ${ }^{2,3}$, Christophe Phillips ${ }^{1}$, Enrico Reimer ${ }^{2}$, Lars Ruthotto ${ }^{6}$, Maryam Seif ${ }^{7}$, Nikolaus Weiskopf ${ }^{2}$, Gabriel Ziegler $^{8}$, Siawoosh Mohammadi ${ }^{9}$, and Karsten Tabelow ${ }^{10}$

${ }^{1}$ University of Liege, Liege, Belgium, ${ }^{2}$ Max Planck Institute for Human Cognitive and Brain Sciences, Leipzig, Germany, ${ }^{3}$ LREN, DNC - CHUV, University Lausanne, Lausanne, Switzerland, ${ }^{4}$ University of Lausanne, Lausanne, Switzerland, ${ }^{5}$ University College London, London, United Kingdom, ${ }^{6}$ Emory University, Atlanta, GA, United States, ${ }^{7}$ University of Zurich, Zurich, Switzerland, ${ }^{8}$ Otto-von-Guericke-University Magdeburg, Magdeburg, Germany, ${ }^{9}$ Medical Center Hamburg-Eppendorf, Hamburg, Germany, ${ }^{10}$ Weierstrass Institute for Applied Analysis and Stochastics, Berlin, Germany

Quantitative MRI finds increasing application in neuroscience and clinical research due to its greater specificity and its sensitivity to microstructural properties of brain tissue - myelin, iron and water concentration. We introduce the hMRI toolbox, an easy-to-use open-source tool for data handling and processing of quantitative MRI data. This toolbox, embedded in the SPM framework, allows the estimation of quantitative MRI maps (longitudinal and transverse relaxation rates $R_{1}$ and $R_{2}{ }^{*}$, proton density PD, and magnetization transfer MT), followed by spatial registration in common space for statistical analysis. It also offers flexibility for calculation of novel MRI biomarkers of tissue microstructure. 
${ }^{1}$ Department of Radiology and Radiation Oncology, Institute of Biomedical Sciences, Tokushima-shi, Japan, ${ }^{2}$ Institute of Biomedical Sciences, Tokushima University Graduate School, Tokushima-shi, Japan, ${ }^{3}$ Global MR Applications and Workflow, GE Healthcare Japan, Hino-shi, Japan

The purpose of this study is to investigate the relationship between CEST imaging and several neurodegenerative diseases to verify the feasibility of an estimation parameter derived from the CEST approach. For this study, patients with Parkinson's disease, progressive supranuclear palsy and multiple system atrophy as well as healthy volunteers were examined. Region-of-interest analysis was performed in the substantia nigra and red nucleus area. As the results, the CEST parameters were significantly different for each of the neurodegenerative diseases and healthy volunteers. CEST imaging might have the ability to obtain more detailed information concerning neurodegenerative disease.

Predicting the age from time of flight MR angiography using 3D convolutional neural network

Yoonho Nam ${ }^{1}$, Jaeho Lee ${ }^{2}$, Dong-Hyun Kim², Jinhee Jang ${ }^{1}$, Bumsoo Kim ${ }^{1}$, and Kook-Jin Ahn ${ }^{1}$

${ }^{1}$ Seoul St.Mary's Hospital, College of Medicine, The Catholic University of Korea, Seoul, Republic of Korea, ${ }^{2}$ Yonsei University, Seoul, Republic of Korea

The age-related changes involve the vasculatures of the brain because the brain has rich blood supply. Previous studies using time of flight (TOF) MR angiography suggested that the aging intracranial arteries were tortuous, irregular and heterogeneous in shape. However, the use of these hand-crafted features and qualitative visual assessments are limited in practical clinical use. Vascular aging could be used as an imaging biomarker for the brain if we could distinguish various age-related vascular changes automatically and quickly from MR angiography. In this study, we investigate the feasibility of deep learning based feature extraction as a tool for analysis of age-related change of brain vasculatures.

Simultaneous assessment of tDCS-induced neuronal responses with oxygen metabolic MRI

Yulin $\mathrm{Ge}^{1}$, Abhishek Datta ${ }^{2}$, Bryan Dobbs ${ }^{3}$, Michael Shaw ${ }^{3}$, Ashley Clayton ${ }^{3}$, Oded Gonen ${ }^{3}$, and Leigh Charvet $^{3}$

${ }^{1}$ Radiology, New York University School of Medicine, New York City, NY, United States, ${ }^{2}$ Soterix Medical, New York City, NY, United States, ${ }^{3}$ New York University School of Medicine, New York City, NY, United States

Although transcranial direct current stimulation (tDCS) offers a therapeutic solution in many neurological diseases, it is still poorly understood how tDCS works underlying neuronal activity in real time. This work was to investigate the real-time tDCS (during stimulation) neuronal response measured with oxygen metabolic MRI. We found cerebral metabolic rate of oxygen $\left(\mathrm{CMRO}_{2}\right)$ increased during tDCS as compared to sham and immediately reduced when tDCS was turned off but remained at slightly higher level than pre-tDCS. The results strongly support our hypothesis that electric current stimulation can induce neuronal activity and $\mathrm{CMRO}_{2}$ increase.

Visualizing the effects of ultrasound-based peripheral neuromodulation in the brain

Ileana Hancu ${ }^{1}$, Vickie Cotero ${ }^{1}$, Suresh Joel ${ }^{2}$, Chitresh Bhushan ${ }^{1}$, Jeanette Roberts ${ }^{1}$, Ying Fan ${ }^{1}$, Sireesha Kaanumalle ${ }^{1}$, Jeffrey Ashe ${ }^{1}$, and Chris Puleo $^{1}$

${ }^{1}$ GE Global Research Center, Niskayuna, NY, United States, ${ }^{2}$ GE Global Research Center, Bangalore, India

We have demonstrated visualization of functional brain changes caused by non-invasive, ultrasound-based stimulation of specific axonal projections within the liver. Following lipopolysaccharide (LPS) injections in a rat animal model, the site-specific liver ultrasound (US) stimulation affected blood glucose levels. The glucose concentration changes were accompanied by increases in the apparent diffusion coefficients (ADC's) in the paraventricular nucleus (PVN), a known center of afferent nerve pathway termination for integration of outgoing systemic signaling. The local sites of neuronal de-activation (as highlighted by diffusion fMRI) were confirmed by reduced hypothalamic cFOS staining (a marker of neuronal activation).

MR neuroimaging and proton spectroscopy in Wolfram syndrome

Stefania Evangelisti ${ }^{1,2}$, Chiara La Morgia ${ }^{1,3}$, Claudia Testa ${ }^{1,2}$, David Neil Manners ${ }^{1,2}$, Claudio Bianchini ${ }^{1,2}$, Michele Carbonelli $^{3}$, Giulia Amore ${ }^{1}$, Alessandra Maresca $^{1}$, Leonardo Caporali $^{1}$, Raffaele Lodi ${ }^{1,2}$, Valerio Carelli ${ }^{1,3}$, and Caterina Tonon ${ }^{1,2}$

${ }^{1}$ Department of Biomedical and NeuroMotor Sciences, University of Bologna, Bologna, Italy, ${ }^{2}$ Functional MR Unit, Policlinico S. Orsola - Malpighi, Bologna, Italy, ${ }^{3}$ IRCCS Institute of Neurological Sciences of Bologna, Bologna, Italy

We characterized neurodegeneration in Wolfram syndrome by combining MR neuroimaging and proton MRS, and evaluated pathological accumulation of brain lactate as a. mitochondrial oxidative impairment marker. Cerebellar white matter loss was widespread, while grey matter loss was stronger within sensorimotor and cognitive cerebellar lobules. Infratentorial neurodegeneration was confirmed by biochemical signs of neuro-axonal degeneration in cerebellum and pons. The lack of abnormal ventricle lactate suggests an absence of dysfunction of mitochondrial metabolism. These morphological, microstructural and biochemical alterations were in line with neuropathological findings of loss of myelinated axons in the visual system, smaller brainstem and cerebellar white matter loss. 
Yangyingqiu Liu ${ }^{1}$, Jin Shang ${ }^{1}$, and Yanwei Miao

${ }^{1}$ Radiology department, First Affiliated Hospital of Dalian Medical University, Dalian, China

The angiogenesis and microvascular permeability of atypical pituitary adenomas were quantitatively analyzed using DCE-MRI texture analysis based on whole tumor volume

Thin Slab Cerebral Quantitative Susceptibility Mapping

Chia-Chen Tsai ${ }^{1}$, Tzu-Cheng Chao ${ }^{1,2}$, Ming-Hong Ho${ }^{2}$, Yi-Jui Liu ${ }^{3}$, and Ming-Long Wu ${ }^{1,2}$

${ }^{1}$ Institute of Medical Informatics, National Cheng Kung University, Tainan, Taiwan, ${ }^{2}$ Department of Computer Science and Information Engineering, National Cheng Kung University, Tainan, Taiwan, ${ }^{3}$ Department of Automatic Control Engineering, Feng-Chia University, Taichung, Taiwan

Quantitative susceptibility mapping has been a useful tool to monitor magnetic properties of the tissues. Conventional QSM uses thick slab volumetric scan to ensure accurate deconvolution of the dipole kernel for susceptibility estimation. The requirement of large volume coverage and appropriate resolution lead to very long scan time, which has limited QSM's integration in a clinical protocol. After inspecting dipole kernel's property, the present work hypothesized QSM should still be performed with a thinner slab to reduce scan time. The results suggest that the reconstructed susceptibility from a whole brain and a thin-slab scan is highly correlated with conventional QSM and the scan time can be reduced up to 4 times.

Improving susceptibility mapping using multiple thresholding k-space division

Wen-Tung Wang ${ }^{1}$, Harshan Ravi ${ }^{1}$, Dzung Pham ${ }^{1}$, and John A Butman ${ }^{2}$

$21031{ }^{1} 9000$ Rockville Pike, CNRM, NIH/USU, Bethesda, MD, United States, ${ }^{2}$ National Institutes of Health, Bethesda, MD, United States

A major challenge in QSM is inverting the acquired phase measurement to estimate the underlying susceptibility. Thresholded K-space division (TKD) is a straightforward technique to calculate the magnetic susceptibility distribution from a single orientation phase images. In this work, we propose to obtain an optimal inverse dipole kernel by using multiple thresholding to minimize the RMSE of the resultant susceptibility map against a susceptibility map

Traditional Poster

\section{Neuroimaging: Animal Models}

Comparison of intravenous and intraperitoneal routes of Omniscan administration with respect to its retention in the rat brain

Serguei Liachenko ${ }^{1}$, Natalya Sadovova ${ }^{1}$, Sherry Ferguson ${ }^{1}$, Joseph Hanig $^{2}$, Merle G Paule ${ }^{1}$, Olayinka Dina ${ }^{3}$, Anthony Fotenos ${ }^{3}$, Adebayo Laniyonu ${ }^{3}$, and Ira Krefting ${ }^{3}$

Preclinical investigation into the brain retention of gadolinium contrast agents after repeated dosing requires extensive animal handling, particularly for intravenous injections. To decrease the potential stress caused by surgical implantation of intravenous catheters and constant maintenance of those catheters for repeated dosing, we proposed to administer Omniscan for such studies via the intraperitoneal route to laboratory rodents. After 20 dosed over 5 weeks, Omniscan retention was similar in both routes of administration.

Quantitative T2 mapping can reliably detect the retention of Omniscan in the rat brain

Serguei Liachenko ${ }^{1}$, Natalya Sadovova ${ }^{1}$, Sherry Ferguson ${ }^{1}$, Joseph Hanig $^{2}$, Zhen $\mathrm{He}^{1}$, Merle G Paule ${ }^{1}$, Olayinka Dina ${ }^{3}$, Anthony Fotenos ${ }^{3}$, Adebayo Laniyonu ${ }^{3}$, and Ira Krefting ${ }^{3}$

Current methods of investigating brain retention of gadolinium-based contrast agents use $T_{1}$-weighted MRI, and rarely $T_{1}$ quantitative mapping. The former does not provide easily quantifiable data and the latter require prolonged scanning time. We proposed the use of a simple 'off-the-shelf' $T_{2}$ mapping technique to reliably quantify relaxation changes in the rat brain due to gadolinium accumulation. The sensitivity of this method is much better compared to the commonly used $\mathrm{T}_{1}$-weighted MRI. 
Pharmacological MRI allows the visualization of brain pharmacological effects of drugs using fMRI. In order to clarify the relationship between fMRI signal and receptor occupancy or behavioral response, we performed $\left[{ }^{11} \mathrm{C}\right]$-raclopride PET, fMRI and the behavioral assessment with raclopride, dopamine D2 receptor antagonist. The positive fMRI response and cataleptic behavior were observed at the dose of raclopride showing $83 \%$ of D2 receptor occupancy, but not at the dose of raclopride showing $42 \%$ of D2 receptor occupancy. These results suggest that $\mathrm{fMRI}$ and behavioral response induced by raclopride will be needed the high $\mathrm{D} 2$ receptor occupancy.

Simian immunodeficiency virus infection transiently increases brain temperature in rhesus macaques as detected with magnetic resonance spectroscopy thermometry

Dionyssios Mintzopoulos ${ }^{1,2}$, Gilberto Gonzalez ${ }^{2,3}$, Eva-Maria Ratai ${ }^{2,3}$, and Marc J Kaufman ${ }^{1,2}$

${ }^{1}$ McLean Imaging Center, McLean Hospital, Belmont, MA, United States, ${ }^{2}$ Harvard Medical School, Boston, MA, United States, ${ }^{3}$ Martinos Center for Biomedical Imaging, Massachusetts General Hospital, Charlestown, MA, United States

Our prior proton Magnetic Resonance Spectroscopy (MRS) studies in Simian Immunodeficiency Virus (SIV)-infected macaques reported higher brain choline and myo-inositol levels at 2 weeks post-infection, suggestive of ongoing inflammation. As brain inflammation has been associated with brain hyperthermia, we used Magnetic Resonance Spectroscopy Thermometry retrospectively to determine whether SIV infection increases brain temperature. At 2 weeks post-infection, we detected increased brain temperature in the frontal and parietal cortex, basal ganglia, and in white matter, relative to pre-infection temperatures. Brain temperatures were strongly correlated with choline levels, suggesting that SIV transiently increases brain temperature by increasing brain inflammation.

Understanding the Impact of Anesthetics on Neuronal and Astroglial Metabolic Activity using ${ }^{1} \mathrm{H}-\left[{ }^{13} \mathrm{C}\right]-\mathrm{NMR}$ Spectroscopy

Anant Bahadur Patel ${ }^{1}$, Sreemantula Arun Kumar ${ }^{1}$, and Pooja Gautam ${ }^{1}$

${ }^{1}$ NMR Microimaging and Spectroscopy, Centre for Cellular and Molecular Biology, Hyderabad, India

Neurometabolic rate is coupled with neurotransmitter cycling, which is perturbed during various neurological disorders. Though, anesthetics are widely used in neurometabolic studies, their impacts on neural function is unclear. In the present study, effects of isoflurane and urethane on brain energy metabolism were investigated using ${ }^{1} \mathrm{H}-\left[^{13} \mathrm{C}\right]-\mathrm{NMR}$ spectroscopy in tissue extract during an infusion of $\left[1,6-{ }^{13} \mathrm{C}_{2}\right]$ glucose or $\left[2-{ }^{13} \mathrm{C}\right]$ acetate. The reduction in neuronal metabolic activity under isoflurane was higher than urethane in the cerebral cortex and striatum. The data from the study indicate that impacts of anesthetics on neuronal function is more compared to astroglia suggesting that astroglial function is less affected with increased brain activity.

Cerebral Reflections of Conditioned Pain Modulation in the Rat: An fMRI study

Silke Kreitz ${ }^{1,2}$, Tabea Klasfauseweh ${ }^{1}$, Sandra Strobelt ${ }^{1}$, Johannes Kaesser ${ }^{1}$, Isabel Wank ${ }^{1}$, Michael Uder ${ }^{2}$, and Andreas Hess ${ }^{1}$

${ }^{1 *}$ Institute for Pharmacology and Toxicology, Friedrich-Alexander-University Erlangen-Nuremberg, Erlangen, Germany, ${ }^{2}$ Department of Radiology, University Hospital of the FriedrichAlexander University Erlangen-Nuremberg, Erlangen, Germany

In this $\mathrm{fMRI}$ study we introduce an animal model to investigate the neural mechanisms of conditioned pain modulation (CPM) in rat brains. Here, a conditioning tonic cold ( $10^{\circ} \mathrm{C}$ water) stimulus at the right hindpaw was used to modulate nociceptive heat stimuli applied to the left hindpaw. Conditioned modulations in functional activation and related network connectivity could be observed in various brain structures involved in pain processing: brainstem and sensory input, lateral thalamus, sensorimotor cortex, frontal association cortex and limbic system. Additionally, over time decreasing resting state connectivity of brainstem and sensorimotor cortex due to cold water stimulation was found.

Vitamin B12 Deficiency Perturbed Energy Metabolism in Pre Frontol Cortex: ${ }^{1} \mathrm{H}-\left[{ }^{13} \mathrm{C}\right] \mathrm{NMR}$ Study

Anant Bahadur Patel ${ }^{1}$, Jitendra Kumar Sinha ${ }^{2}$, Shampa Ghosh ${ }^{2}$, TK Sampath Kumar ${ }^{1}$, and Manchala Raghunath ${ }^{2}$

${ }^{1}$ NMR Microimaging and Spectroscopy, Centre for Cellular and Molecular Biology, Hyderabad, India, ${ }^{2}$ National Institute of Nutrition, Hyderabad, India

The consequences of severe deficiencies in micronutrients especially vitamin B12 on the developing brain during infancy and early post-natal period is not very clear. The current study aim to understand the effects of B12 deficiency on cognitive function using ${ }^{1} \mathrm{H}-\left[{ }^{13} \mathrm{C}\right]-\mathrm{NMR}$ spectroscopy together with $\left[1,6-{ }^{13} \mathrm{C}_{2}\right]$ glucose infusion in vitamin $\mathrm{B} 12$ deficient mice. Our findings indicate reduction in the metabolic activity of glutamatergic and GABAergic neurons in the prefrontal cortex of mice maintained with moderate and severe vitamin B12 defcient diet. 
Notch1 signaling plays time-dependent roles in the sequential process of neurogenesis after stroke. In this study, we aim to detect the appropriate therapeutic time frame of DAPT treatment based on the Notch1 signaling activation and NSCs responses after stroke. Combing the in vivo monitor of comprehensive microstructure changes with diffusion MRI and the in vitro analysis of neurogenesis and remyelination with immunohistology, we ultimately demonstrate the neurorestorative effects of DAPT treatment at the subacute stage after stroke. Our results suggested the appropriate therapeutic time window of inhibiting Notch1 signaling to maximally promote endogenous neurogenesis and axonal reorganization, which could enhance the efficacy of Notch-1 signaling related therapy and promote its application to clinical trials.

Physical exercise enhances adult cortical plasticity in neonatal hypoxic ischemic injured rats: Evidence by BOLD-fMRI and LFP electrophysiological recording

Sun Young Chae ${ }^{1,2}$, Jun Ho Jang ${ }^{3}$, Geun Ho Im ${ }^{4,5}$, Moon-sun Jang ${ }^{4,5}$, Won-Beom Jung ${ }^{2,6}$, Seungsoo Chung ${ }^{7}$, and Jung Hee Lee ${ }^{1,2,4,6}$

${ }^{1}$ Department of Health Sciences and Technology, SAIHST, Sungkyunkwan university, Seoul, Republic of Korea, ${ }^{2}$ Center for Neuroscience Imaging Research, Institute for Basic Science (IBS), Suwon, Republic of Korea, ${ }^{3}$ BnH Research co.,Ltd, Goyang, Republic of Korea, ${ }^{4}$ Department of Radiology, Samsung Medical Center, Sungkyunkwan University School of Medicine, Seoul, Republic of Korea, ${ }^{5}$ Center for Molecular and Cellular Imaging, Samsung Biomedical Research Institute, Seoul, Republic of Korea, ${ }^{6}$ Department of Global Biomedical Engineering, Sungkyunkwan University, Suwon, Republic of Korea, ${ }^{7}$ Department of Physiology, Yonsei University Colleage of Medicine, Seoul, Republic of Korea

The developing brain has a powerful ability to modify its own structure and function for recovery from injury in efforts to compensate for loss of function ${ }^{1,2}$. In critical period, developing brain has maximal neuronal synaptic connections so it is most amenable to changes in response to external stimulus such as physical exercise ${ }^{3}$. However, after critical period, neuronal synaptic connections are reduced, and maintained at the reduced state ${ }^{3}$. Here, we demonstrate enhanced neuroplasticity with physical exercise performed beyond critical period for rats that are injured during critical period. We obtained the BOLD-fMRI response and the interneuron activity with LFP electrophysiological recording.

Differences in resting state functional networks during pregnancy in C57BI6 mice

Guadalupe Soria ${ }^{1,2}$, Raúl Tudela ${ }^{1,2}$, Emma Muñoz-Moreno $^{1}$, Xavier López-Gil ${ }^{1}$, Roberta Haddad-Tóvolli ${ }^{3}$, and Marc Claret ${ }^{3}$

${ }^{1}$ Experimental $7 T$ MRI Unit, IDIBAPS, Barcelona, Spain, ${ }^{2}$ CIBER de Bioingeniería, Biomateriales y Nanomedicina (CIBER-BBN) Group of Biomedical Imaging of the University of Barcelona, Barcelona, Spain, ${ }^{3}$ Neuronal Control of Metabolism (NeuCoMe) Laboratory, IDIBAPS, Barcelona, Spain

The purpose of this study was to investigate if resting state functional MRI is able to reveal brain network changes associated to pregnancy in C57BI6 mice. 12 mice were scanned before and 3 weeks after pregnancy using a classical resting state fMRI proptocol. Dual regression was performed using these 20 components to find the subject-specific time-series and spatial maps for each network. Significant differences were observed in the striatal, the insula-amygdala and the hippocampal-brainstem networks. Our results reveal that in pregnant C57BI6 female micethere is reorganization of brain connectivity in specific brain regions and networks.

Monitoring LPS-induced gray matter inflammation through endogenous contrasts: MT, CEST and NOE

Chenwang Jin ${ }^{1,2}$, Yanrong Chen ${ }^{1,3}$, Chenyan $\mathrm{Chu}^{1}$, Piotr walzcak ${ }^{1}$, and Xiaolei Song ${ }^{1}$

${ }^{1}$ Morgan Department of Radiology and Radiological Science, Cellular Imaging Section and Vascular Biology Program, Institute for Cell Engineering, The Johns Hopkins University School of Medicine, Baltimore, MD, United States, ${ }^{2}$ Medical Imaging, The First Affiliated Hospital of Xian Jiaotong University, Xian, China, ${ }^{3}$ School of information and technology, The Northwest University, Xian, China

Gray matter (GM) damage is a common phenomenon and clinically relevant in the onset and progression of many neuroinflammation diseases, including Multiple Sclerosis, Alzheimer's Disease and Depression. However, conventional MRI techniques are insensitive to the detection of GM damage. Chemical exchange saturation transfer (CEST) is an innovative molecular MRI technique that bridges the tissue microstructure and cellular metabolic function, possibly allowing sensing metabolic changes. Our preliminary results suggest that NOEMRI (Nuclear Overhauser Effect, NOE) may provide a novel biomarker in detection of slight inflammatory changes in cortex and deep GM and also potentially enable quantifing the diffusive GM damages. 
$\mathrm{N}$-methyl-Daspartate receptor (NMDAR) antagonists, such as phencyclidine (PCP), ketamine and dizocilpine (MK801), have been widely used for inducing schizophrenia animal models. As a noncompetitive selective NMDAR antagonist, MK801 has two stereoisomers, (+)MK801 and (-)MK801, which have been found to induce different behavioral phenotypes and histological changes in animals. In this study, we compared differential effects of (+)MK801 and (-)MK801 on brain structure and metabolism in adolescence rats with MRI/in vivo ${ }^{1} \mathrm{H}-$ MRS. The results showed that (+)MK801 induced more severe gray matter (GM) atrophy and more evident metabolic changes than (-)MK801, and the different effects were related to their potency at NMDA receptors.

Apparent Diffusion Coefficient Correlates with Histological Tumor Burden at the Infiltrating Margins of a Preclinical Glioblastoma Model

Gerard Thompson ${ }^{1}$, Antoine Vallatos ${ }^{1}$, Haitham Al-Mubarak ${ }^{2}$, Lesley Gilmour ${ }^{3}$, Joanna Birch ${ }^{3}$, Lindsay Gallagher ${ }^{2}$, James Mullin², Adam Waldman ${ }^{1}$, William M Holmes ${ }^{2}$, and Anthony J Chalmers ${ }^{3}$

${ }^{1}$ Centre for Clinical Brain Sciences, University of Edinburgh, Edinburgh, United Kingdom, ${ }^{2}$ Glasgow Experimental MRI Centre, University of Glasgow, Glasgow, United Kingdom, ${ }^{3}$ Institute of Cancer Sciences, University of Glasgow, Glasgow, United Kingdom

Assessing the imaging boundary of glioblastoma multiforme (GBM) has potential to characterize phenotypic invasiveness relevant to outcomes. Preoperative apparent diffusion coefficient (ADC) changes across this boundary predicts outcome in humans. The tissue specificity of this finding is unknown, hindering the interpretation, further development, and application of the technique. We selected and assessed a relevant preclinical murine infiltrating GBM orthotopic human xenograft model with a novel histological tissue tumor load assessment to investigate relationships between $A D C$ on imaging and cellular infiltration. A robust and strong inverse correlation between the histological tumor infiltration measure and $A D C$ transition is demonstrated, supporting the hypothesis that $A D C$ changes across GBM boundaries represent tumor infiltration and therefore relate to the previously-proposed invasive phenotype imaging biomarker.

Multiparametric magnetic resonance and phenotypic characterization of a mild depression rat model

Teresa Navarro-Hernanz ${ }^{1}$, David Alcázar ${ }^{1}$, Fátima Sanchis $^{1}$, and Pilar López-Larrubia ${ }^{1}$

${ }^{1}$ Instituto de Investigaciones Biomédicas "Alberto Sols", CSIC-UAM, Madrid, Spain

Depression is a common and serious medical illness with a direct impact both in the physical and mental health. It is a complex disorder of the mood with a high incidence in the world population and a tendency to continue increasing. The multifactorial and heterogeneous character of this disease hinders the understanding of the pathological mechanism. The use of an appropriate animal model of depression can contribute to improve the diagnosis and monitoring of the therapy outcome. In this work, we characterized with MRI and phenotyping studies a mild depression model developed in female rats.

Chronic Oral Methylene Blue Treatment in a Rat Ischemic Stroke Model

Lei Huang ${ }^{1,2}$, Yichu Liu ${ }^{1}$, Zhao Jiang ${ }^{1}$, and Timothy Q. Duong ${ }^{1}$

${ }^{1}$ Radiology and Preclinical Imaging Center, Stony Brook Medicine, Stony Brook, NY, United States, ${ }^{2}$ Loma Linda University, Loma Linda, CA, United States

Methylene blue (MB), an FDA-grandfathered drug, has been shown to reduce MRI-defined infarct volume in acute ischemic stroke. However, the efficacy of chronic MB treatment in stroke remains unknown. The goal of this study was to investigate the efficacy of chronic oral MB administration in ischemic stroke using MRI and behavioral tests. We found chronic MB treatment reduced MRI-defined total lesion volumes and improved functional behavioral outcomes, as well as reduced sub-acute hyperperfusion and white-matter damage. Our findings, for the first time, suggest that long-term MB oral administration is safe and has positive therapeutic effects in chronic stroke.

Protective effect of high creatine diet during chronic hepatic encephalopathy in young rats, an in vivo longitudinal 1H and 31P MRS study

Veronika Rackayova ${ }^{1}$, Olivier Braissant ${ }^{2}$, Dario Sessa ${ }^{3}$, Stefanita Mitrea ${ }^{4}$, Valerie McLin ${ }^{3}$, Rolf Gruetter ${ }^{4}$, and Cristina Cudalbu ${ }^{4}$

${ }^{1}$ Laboratory of Functional and Metabolic Imaging (LIFMET), Ecole Polytechnique Fédérale de Lausanne (EPFL), Lausanne, Switzerland, ${ }^{2}$ Neurometabolic Unit, Service of Clinical Chemistry, University Hospital of Lausanne, Lausanne, Switzerland, ${ }^{3}$ Swiss Center for Liver Disease in Children, University Hospitals Geneva, Geneva, Switzerland, ${ }^{4}$ Centre d'Imagerie Biomédicale (CIBM). Ecole Polytechnique Fédérale de Lausanne (EPFL), Lausanne, Switzerland

Chronic hepatic encephalopathy $(\mathrm{CHE})$ is a serious neuropsychiatric disease with altered neurological status and changes in brain metabolites (among others, decrease in brain tCr). If $\mathrm{CHE}$ is acquired in childhood these conditions might perturb normal brain development. Our aim was to test whether oral $\mathrm{Cr}$ supplementation dampens the neurometabolic changes observed in CHE in a longitudinal model of chronic liver disease in young rats. Using in vivo longitudinal brain $1 \mathrm{H}$ and 31P-MRS, we showed rescued tCr levels, enhanced energy metabolism (restoration of ATP), improved antioxidant capacity (increased Asc), positive effect on phospholipid metabolism and smaller increase in GIn (marker of CHE). 
${ }^{1}$ Pathology, University of New Mexico Health Sciences Center, Albuquerque, NM, United States, ${ }^{2}$ Zilkha Neurogenetic Institute, University of Southern California, Los Angeles, CA, United States

PTSD results from life-threatening fear. We use mouse as an experimental model to investigate acute and persistent fear responses, imaging brain activity by MEMRI, coupled with behavioral responses and histologic confirmation of activity with c-Fos staining. We imaged neural activity at multiple time points in mouse lacking the serotonin transporter, SERT, and with/without early life stress. This approach represents an unbiased comprehensive method to look at the dynamics of the brain's response to fear over time, not possible by other imaging methods. We find altered activity and circuits in mice after fear dependent on genotype and environment.

Diffusion tensor imaging reveals altered brain development of MECP2 overexpressing rat in cerebellar and limbic structures

Jian-kun Dai ${ }^{1}$, Yu-yan Chen ${ }^{1}$, and Zhifeng Liang ${ }^{1}$

${ }^{1}$ Institute of Neuroscience, Chinese Academy of Sciences, Shanghai, China

In this study, we used diffusion tensor imaging to investigate the effect of MECP2 overexpressing (MECP2-OE) on the rat brain development. Our results showed the MECP2-OE mainly affected the cerebellar fiber tracts and limbic structures. Behavior tests showed the MECP2-OE rats presented significant defects of social interaction than the wild type (WT) rats.

A novel transgenic rat model of evolving cerebral amyloid angiopathy (CAA)

Hedok Lee ${ }^{1}$, Xiaodan Liu ${ }^{1}$, Simon Sanggaard ${ }^{1}$, Sunil Koundal ${ }^{1}$, Feng Xu², William Van Nostrand ${ }^{2}$, and Helene Benveniste ${ }^{1}$

${ }^{1}$ Yale University, New Haven, CT, United States, ${ }^{2}$ University of Rhode Island, Kingston, RI, United States

Understanding the pathophysiology of cerebral amyloid angiopathy (CAA) has become increasingly important because there is evidence to suggest that vascular dysfunction plays an important role in early component in the development of Alzheimer's disease (AD). To study CAA, Tg-SwDI transgenic mouse model was recently extended to rat (Tg-DI) and here we report the first MRI studies to characterize CAA in Tg-DI in both in vivo as well as in vitro using 3D-GRE sequence. Conspicuous lesions were detected in thalamus in Tg-DI at very early stage, consisting of multiple pathological changes including micro-bleeds, extravasation of blood products and/or occluded vessels.

Comparison of BOLD and MION enhanced CBV fMRI to the noxious stimulus in anesthetized rhesus monkey

Eunha Baeg ${ }^{1}$, Boo-Hee Choi ${ }^{1}$, Chan-Ung Park ${ }^{1}$, Choong-Wan Woo ${ }^{1}$, and Seong-Gi Kim ${ }^{1}$

${ }^{1}$ Center for Neuroscience Imaging Research, Suwon, Republic of Korea

Using contrast agent in $\mathrm{FMRI}$ has the benefits of providing additional information of regional cerebral blood volume (CBV) and of enhancing sensitivity. Response comparisons of BOLD and MION enhanced CBV fMRI to the noxious stimulus in non-human primate showed signal increase for MION fMRI in the regions that are important in pain-processing network. Activities of some brain areas, including putamen, were captured with MION fMRI, not with BOLD. Capsaicin treatment augmented the responses of fMRI to the noxious stimulation for BOLD and MION fMRI. New insight can be obtained for the pain network through the comparison between BOLD and CBV fMRI.

Changes in corticospinal tract integrity in relation to recovery after cortical stroke as measured with DTI-based tractography in rat brain

Geralda AF van Tilborg ${ }^{1}$, Michel RT Sinke ${ }^{1}$, Anu E Meerwaldt ${ }^{1}$, Annette van der Toorn ${ }^{1}$, Caroline H van Heijningen ${ }^{1}$, Milou Straathof ${ }^{1}$, Mohamed Ali ${ }^{2}$, Khalid Al-Saad ${ }^{3}$, and Rick M Dijkhuizen ${ }^{1}$

${ }^{1}$ Biomedical MR Imaging and Spectroscopy Group, Center for Image Sciences, University Medical Center Utrecht, Utrecht University, Utrecht, Netherlands, ${ }^{2}$ Neurological Disorders Research Centre, Qatar Biomedical Research Institute (QBRI), Hamad Bin Khalifa University, Doha, Qatar, ${ }^{3}$ Department of Chemistry and Earth Sciences, College of Arts and Sciences, Qatar University, Doha, Qatar

Preserved or restored integrity of the corticospinal tract (CST) is critical for motor recovery after stroke. However, data on spatiotemporal alterations in CST integrity after stroke are largely lacking. Here we implemented diffusion tensor-based tractography to identify the CST in rat brain, which we applied to measure microstructural changes along the CST following experimental stroke to the sensorimotor cortex. Number of tractography streamlines, fractional anisotropy (FA) and axial diffusivity (AD) were reduced 1 week post-stroke, and recovered to control levels after 28 weeks. This temporal pattern, reflective of white matter remodeling, coincided with loss and recovery of sensorimotor function.

Jasmien Ellen Maria Jozef Orije ${ }^{1}$, Geert De Groof ${ }^{2}$, Sofie Van Massenhoven ${ }^{3}$, Elisabeth Jonckers² ${ }^{2}$ Veerle Darras ${ }^{4}$, and Annemie Van der Linden ${ }^{2}$ 
${ }^{1}$ Bio-Imaging Lab, University of Antwerp, Deurne, Belgium, ${ }^{2}$ Bio-Imaging Lab, University of Antwerp, Wilrijk, Belgium, ${ }^{3}$ University of Antwerp, Wilrijk, Belgium, ${ }^{4}$ Laboratory of Comparative Endocrinology, KU Leuven, Leuven, Belgium

The dynamic relationship between song performance and neuroplasticity induced by testosterone implantation was monitored longitudinally in a seasonal songbird (European starling) by using in vivo DTI. Voxel based analysis showed that the song bout length was positively correlated to the fractional anisotropy changes in different parts of the motor pathway. Meaning that the motor pathway strengthens as song performance advances under the influence of testosterone.

Dynamic Structural-Functional Relationship between Left and Right Somatosensory Cortex in Rats across the Lifespan

Michel R.T. Sinke ${ }^{1}$, Milou Straathof ${ }^{1}$, Paul L. Weerheim ${ }^{1}$, Willem M. Otte ${ }^{1,2}$, and Rick M. Dijkhuizen ${ }^{1}$

${ }^{1}$ Biomedical MR Imaging and Spectroscopy Group, Center for Image Sciences, University Medical Center Utrecht / Utrecht University, Utrecht, Netherlands, ${ }^{2}$ Department of Pediatric Neurology, Brain Center Rudolf Magnus, University Medical Center Utrecht / Utrecht University, Utrecht, Netherlands

The relationship between structural and functional brain connectivity across the mammalian lifespan is largely unknown. To elucidate the temporal characteristics of this relationship we longitudinally acquired high-field resting-state fMRI and diffusion-MRI in rats, from early infancy to old age. We specifically examined the interhemispheric connectivity between homologous primary somatosensory cortices, a major part of the sensorimotor system. The structure-function correlation increased from about 0 during infancy to 0.4 around adulthood followed by a further gradual increase towards old age. This reflects dynamic patterns of lifelong brain remodeling, which may underlie variations in brain disease etiology during development and ageing

Kinase-inactive Met mice show altered forebrain functional connectivity: A resting state functional MRI study

Shiyu Tang ${ }^{1}$, Elizabeth M Powell ${ }^{2}$, Reha S Erzurumlu², Wenjun Zhu ${ }^{1}$, Fu-Sun Lo², and Su Xu

${ }^{1}$ Diagnostic Radiology and Nuclear Medicine, University of Maryland School of Medicine, Baltimore, MD, United States, ${ }^{2}$ Anatomy and Neurobiology, University of Maryland School of Medicine, Baltimore, MD, United States

$M E T$, the gene encoding tyrosine kinase receptor for hepatocyte growth factor, is a susceptibility gene for autism spectrum disorder (ASD). Genetically altered mice with a kinaseinactive Met offer a potential model for understanding neural circuit organization changes in autism. We employed resting-state functional MRI to a kinase-inactive Met mouse model to test our hypothesis that aberrant functioning of the somatosensory-thalamocortical system is at the core of the conspicuous somatosensory behavioral phenotypes observed in autism. Results showed impaired organization of large-scale network and increased somatosensory-thalamocortical connectivity with a sex dependent manner and differences between heterozygous and homozygous Met-Emx1 mice.

Abnormal growth trajectories of white matter in spontaneously hypertensive rats when compared to non-hypertensive controls: Implications for small vessel disease progression Sunil Koundal ${ }^{1}$, Simon Sanggaard ${ }^{1,2}$, Kristian Mortensen $^{2}$, Helene Benveniste $^{1}$, Maiken Nedergaard ${ }^{2,3}$, and Hedok Lee ${ }^{1}$

${ }^{1}$ Yale University, New Haven, CT, United States, ${ }^{2}$ University of Copenhagen, Copenhagen, Denmark, ${ }^{3}$ University of Rochester, Rochester, NY, United States

The spontaneously hypertensive rat (SHR) is a clinically relevant animal model in studying small vessel disease. Whole brain morphological differences between SHR compare to normotensive Wistar-Kyoto (WKY) rats were evaluated in parallel with development of chronic hypertension. Voxel-wise deformation based morphometry indicated progressive enlargement of the cerebral ventricles in SHR compare to WKY, and a fraction of the body and splenium of corpus callosum in SHRs were significantly smaller in the middle-aged rats but not in young-aged rats.

In-Vivo Analysis of the Superficial White Matter in the Macaque Brain Using High-resolution Diffusion MRI: preliminary results

Yann Bihan-Poudec ${ }^{1}$, Slimane Tounekti ${ }^{1}$, Nathalie Richard ${ }^{1}$, Mathilda Froesel ${ }^{1}$, Franck Lamberton ${ }^{2}$, Thomas Troalen ${ }^{3}$, Suliann Ben Hamed ${ }^{1}$, Maxime Descoteaux ${ }^{4}$, and Bassem Hiba ${ }^{1}$

${ }^{1}$ CNRS, Bron, France, ${ }^{2}$ CERMEP, Bron, France, ${ }^{3}$ Siemens-Healthineers, Saint-Denis, France, ${ }^{4}$ Université de Sherbrooke, Sherbrooke, QC, Canada

This study was focused on the U-fibers located on the Superficial White Matter (SWM) of the Rhesus macaque brain.

A diffusion MRI (dMRI) pulse-sequence with a 3D multi-shot-EPI module was used to achieve a $0.5 \mathrm{~mm}$ isotropic dMRI data in 4 macaques.

The organization of white matter in the region of arcuate sulcus (AS) was analyzed using diffusion tensor and fiber orientation distribution data, the U-fiber over all the AS was tracked and its water diffusion metrics were quantitatively assessed.

The results, obtained using high-resolution dMRI, pave the way for quantitative analyses of SWM for clinical and neuroscientific applications 
No dynamic contrast-enhanced (DCE) study has been published so far in Experimental Allergic Encephalomyelitis (EAE), although DCE-MRI is used in human Multiple Sclerosis. This study reports a DCE protocol optimized for mouse brain imaging of subtle and delayed contrast agent accumulation and applies it to the study of EAE with moderate neurological signs. Two-fold signal increase with respect to the vascular volume fraction can be detected while even moderately enhancing lesions remain visually undetectable on pre and post-contrast T2w and T2* $w$ acquisitions. Ventricles, midbrain and ventral olfactory bulb are first to be affected in moderate EAE.

Advanced MR imaging characterization of a novel in vivo xenograft model mimicking recurrent glioblastoma

Mona M Al-Gizawiy ${ }^{1}$, Robert T Wujek ${ }^{1}$, Melissa A Prah ${ }^{1}$, Hisham S Alhajala², Ninh B Doan ${ }^{3}$, Jeffrey A Knipstein ${ }^{4,5}$, Jennifer M Connelly ${ }^{5,6}$, Shama P Mirza ${ }^{7}$, Christopher R Chitambar², and Kathleen M Schmainda ${ }^{1,8}$

${ }^{1}$ Radiology, Medical College of Wisconsin, Milwaukee, WI, United States, ${ }^{2}$ Medicine, Medical College of Wisconsin, Milwaukee, WI, United States, ${ }^{3}$ Neurosurgery, Medical College of Wisconsin, Milwaukee, WI, United States, ${ }^{4}$ Pediatrics, Medical College of Wisconsin, Milwaukee, WI, United States, ${ }^{5}$ Neuro-Oncology, Medical College of Wisconsin, Milwaukee, WI, United States, ${ }^{6}$ Neurology, Medical College of Wisconsin, Milwaukee, WI, United States, ${ }^{7}$ Chemistry \& Biochemistry, University of Wisconsin, Milwaukee, WI, United States, ${ }^{8}$ Biophysics, Medical College of Wisconsin, Milwaukee, WI, United States

We have developed a robust and reproducible rat xenograft model of recurrent GBM by irradiating adult and pediatric GBM cell lines in vitro prior to brain inoculation. Both advanced MR imaging and histological analyses highlight the amplified aggressiveness of the resultant tumor compared to the conventional U-87MG xenograft, as evidenced by profound vascularization and increased cell proliferation. Moreover, our recurrent GBM model exhibited invasive lesions with areas of infiltrating neutrophils and necrosis, all features that are not associated with conventional U-87MG xenograft tumors. Shortened survival of animals bearing irradiated U87-10Gy or SJGBM2-10Gy tumors further reinforces the aggressive nature of the model.

Traditional Poster

\section{Brain Tumours}

Quantifying tumour oxygenation using streamlined-qBOLD

Alan J Stone ${ }^{1}$, Esther AH Warnert ${ }^{2}$, Puneet Plaha ${ }^{3,4}$, Natalie L Voets ${ }^{1,3}$, and Nicholas P Blockley ${ }^{1}$

${ }^{1}$ Wellcome Centre for Integrative Neuroimaging, FMRIB, Nuffield Department of Clinical Neurosciences, University of Oxford, Oxford, United Kingdom, ${ }^{2}$ Department of Radiology \& Nuclear Medicine, Erasmus MC, Rotterdam, Netherlands, ${ }^{3}$ Department of Neurosurgery, John Radcliffe Hospital, Oxford University Hospitals NHS Foundation Trust, Oxford, United Kingdom, ${ }^{4}$ Nuffield Department of Clinical Neurosciences, University of Oxford, Oxford, United Kingdom

Hypoxia and high metabolic demand are important identifying features of high-grade gliomas. Imaging methods capable of mapping tissue oxygenation therefore have the potential to provide non-invasive information about the metabolic environment of tumour tissue and may produce useful markers for stage grading and monitoring treatment efficacy. Here we demonstrate the use of streamlined-qBOLD for mapping tumour oxygenation.

Volume-independent radiomic features from T2w-FLAIR MRI could reveal mutation of histones in diffuse intrinsic pontine glioma

Jessica Goya-Outi ${ }^{1}$, Fanny Orlhac ${ }^{1}$, Raphael Calmon ${ }^{2}$, Cathy Philippe ${ }^{3}$, Stéphanie Puget ${ }^{4}$, Nathalie Boddaert ${ }^{2}$, Irène Buvat ${ }^{1}$, Jacques Grill ${ }^{5}$, Vincent Frouin ${ }^{3}$, and Frédérique Frouin ${ }^{1}$

${ }^{1}$ IMIV, Inserm, CEA, CNRS, Université Paris-Sud, Université Paris-Saclay, Orsay, France, ${ }^{2}$ Pediatric Radiology, Hôpital Necker Enfants Malades, AP-HP, Paris, France, ${ }^{3}$ UNATI, Neurospin, CEA, Université Paris-Saclay, Gif-sur-Yvette, France, ${ }^{4}$ Pediatric Neurosurgery, Hôpital Necker Enfants Malades, AP-HP, Paris, France, ${ }^{5}$ Cancérologie de l'enfant et de l'adolescent, Gustave Roussy, CNRS UMR 8203, Université Paris-Saclay, Villejuif, France

In diffuse intrinsic pontine glioma, the mutations of histones (H3.1 versus $\mathrm{H} 3.3$ ) are correlated with patient survival. A new method to compute radiomic features free of tumor volume effect was applied to four structural MR modalities and patients were classified according to histone mutation. The tumor was scanned by a 5 mm radius sphere and textural indices were computed inside each position. A total of 37 features calculated from T2w-FLAIR yielded an area under the Receiver Operating Characteristics curve greater than 0.85 . T2w-FLAIR appears to be the most informative modality to predict mutation type. 
${ }^{1}$ Radiology and Imaging Sciences, Indiana University, Indianapolis, IN, United States, ${ }^{2}$ Health Sciences, Purdue University, West Lafayette, IN, United States, ${ }^{3}$ Neurosurgery, Dayton Children's Hospital, Dayton, $\mathrm{OH}$, United States

The purpose of this work was to evaluate the relative contributions of MR contrasts to tumor tissue classification. Seventeen (17) glioma patient datasets (WHO grade II-IV) containing T1, T1+gad, T2, FLAIR, and ADC were studied using multinomial logistic regression. T2 images had the highest individual classification accuracy (78.1\%). Classification accuracy improved with each additional contrast, leading to an overall accuracy of $84.1 \%$ for all 5 contrasts. The multinomial logistic regression showed that together the 5 contrasts had greater tumor tissue classification accuracy than individually, but that the improvement in accuracy was not linear and decreased as more MR data was included. Lower grade gliomas and GBM could be predicted by the percentage of voxels classified as suspicious by the regression model, but not by any other class. These results may aid in clinical protocol development and optimization for neuro-oncologic imaging, especially in situations where overall scan time is limited.

Multi-sequence and Habitat-based Radiomics Analysis to Predict MGMT Promoter Methylation Status in Grade II-IV Gliomas Using Magnetic Resonance Imaging

Jingwei Wei ${ }^{1}$, Jie Tian ${ }^{1}$, Dongsheng $\mathrm{Gu}^{1}$, Xiaohan $\mathrm{Hao}^{1}$, Guoqiang Yang ${ }^{2}$, and Yan $\mathrm{Tan}^{2}$

${ }^{1}$ Chinese Academy of Sciences, Beijing, China, ${ }^{2}$ Shanxi Medical University, Taiyuan, China

In this study, we performed multi-habitat and multi-sequence MRI radiomics to make preoperative prediction on MGMT promoter methylation in grade II-IV gliomas. Quantitative imaging features were extracted on each habitat from CE-T1WI, T2FLAIR and ADC maps to reveal the genetic heterogeneity of the tumor and describe the subtle textural characteristics of different molecular subtypes. The habitat-integrated radiomics signature behaved more stable and had better predictive efficacy than one-region based radiomics signature. The final constructed predictive model incorporating the proposed radiomics signature and traditional clinical predictors achieved the optimal performance on the MGMT status.

Comparative analysis of diffusion kurtosis imaging, diffusion tensor imaging and diffusion weighted imaging in grading and assessing cellular proliferation of meningioma

Lin LIN ${ }^{1,2}$, Yunjing Xue ${ }^{3}$, and Qing Duan ${ }^{3}$

${ }^{1}$ Radiology, Fujian medical university affiliated union hospital, Fuzhou, China, ${ }^{2}$ Fudan university affiliated huashan hospital, Shanghai, China, ${ }^{3}$ Fujian medical university affiliated union hospital, Fuzhou, China

An accurate evaluation of the WHO grade and cellular proliferation is particularly important in meningiomas, it may facilitate treatment decisions and improve clinical prognosis. But conventional magnetic resonance (MR) imaging were not sufficiently accurate in evaluating the meningioma grade and Ki-67 expression. This study prospectively evaluate and compare diffusion kurtosis imaging (DKI), diffusion tensor imaging(DTI) and diffusion weighted imaging (DWI) metrics in determining the grade and cellular proliferation of meningiomas. It was found that DKI is a better diffusion technique for assessing the grading and cellular proliferation of meningiomas compared to conventional diffusion imaging.

Growth patterns of non-enhancing glioma assessed on DTI-derived isotropic and anisotropic maps are not associated with IDH and 1p19q codeletion status

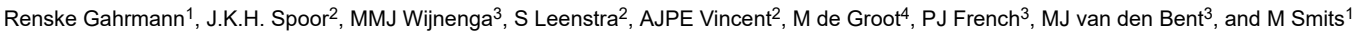

${ }^{1}$ Radiology and Nuclear Medicine, Erasmus MC, University Medical Center, Rotterdam, Netherlands, ${ }^{2}$ Neurosurgery, Erasmus MC, University Medical Center, Rotterdam, Netherlands,

${ }^{3}$ The Brain Tumor Center, Erasmus MC, University Medical Center, Rotterdam, Netherlands, ${ }^{4}$ Medical Informatics, Erasmus MC, University Medical Center, Rotterdam, Netherlands

Previous reports show that $I D H$-mutation status can be determined in glioblastoma using DTI-derived isotropic $(p)$ and anisotropic $(q)$ maps to measure infiltrative growth along white matter tracts by determining the extent or pattern of $p / q$ mismatch: abnormal $p$ overlaps normal $q$-areas by $>0.5 \mathrm{~cm}$. We use this method in presumed low-grade (i.e. non-enhancing) gliomas to see if infiltrative growth patterns correlate with $I D H$-mutation and $1 \mathrm{p} 19 \mathrm{q}$ codeletion status, which in turn are correlated with prognosis.

Gadolinium concentration map based on synthetic MRI and its application to brain metastases

Misaki Nakazawa ${ }^{1}$, Akifumi Hagiwara ${ }^{1,2}$, Christina Andica ${ }^{1}$, Masaaki Hori ${ }^{1}$, Moeko Horita ${ }^{1,3}$, Koji Kamagata $^{1}$, Haruyoshi Houshito ${ }^{1}$, and Shigeki Aoki ${ }^{1}$

${ }^{1}$ Department of Radiology, Juntendo University School of Medicine School of Medicine School of Medicine, Tokyo, Japan, ${ }^{2}$ Graduate School of Medicine, The University of Tokyo, Tokyo, Japan, ${ }^{3}$ Graduate School of Human Health Sciences, Tokyo Metropolitan University, Tokyo, Japan

Signal intensity measured on T1-weighted image is not proportional to the gadolinium concentration in vivo after administration of contrast agent. Thus, some calculations are required to estimate gadolinium concentration using quantitative values before and after gadolinium administration. We created gadolinium concentration maps that directly show the amount of contrast agent using quantitative maps calculated using synthetic MRI. The gadolinium concentration map we created using phantoms showed high accuracy and precision. The gadolinium concentration map could reliably measure the concentration of gadolinium in metastatic brain tumors. 
Angela Jakary ${ }^{1}$, Tracy Luks ${ }^{1}$, Susan Chang ${ }^{2}$, Jennifer Clarke ${ }^{2}$, Nicholas Butowski ${ }^{2}$, Nancy Ann Oberheim Bush ${ }^{2}$, and Jennie Taylor ${ }^{2}$

${ }^{1}$ Radiology and Biomedical Imaging, UCSF, San Francisco, CA, United States, ${ }^{2}$ Neurological Surgery, UCSF, San Francisco, CA, United States

Quality of life and neurocognitive function are important clinical outcome measures for patients with lower grade glioma. In this pilot study, we performed neurocognitive testing and quality of life assessments in radiologically and clinically stable grade II and III glioma patients who were not receiving active treatment. We found novel associations between standard clinical assessments and neuroimaging metrics at pre-surgical and follow-up timepoints. Further characterizing the longitudinal relationship between structural and functional neuroimaging, neurocognition and quality of life will better allow clinicians to proactively intervene to help patients in future.

Robust Quantification of Changes in Arterial Cerebral Vasculature Post Radiation Therapy in Pediatric Brain Tumor Survivors

Sivakami Avadiappan ${ }^{1}$, Sam Payabvash ${ }^{1}$, Angela Jakary ${ }^{1}$, Erin Felton ${ }^{2}$, Melanie Morrison ${ }^{1}$, Christopher P Hess ${ }^{1,2}$, Sabine Mueller $^{2,3}$, and Janine M Lupo ${ }^{1}$

${ }^{1}$ Department of Radiology and Biomedical Imaging, University of California, San Francisco, San Francisco, CA, United States, ${ }^{2}$ Department of Neurology, University of California, San Francisco, San Francisco, CA, United States, ${ }^{3}$ Department of Neurological Surgery, University of California, San Francisco, San Franciso, CA, United States

With the improved long-term survival of children with brain tumors, understanding the late effects of their therapy on small arterioles is of great importance. We developed a method for robust segmentation of arteries and quantification of their thickness using TOF-MRA at 7T and estimated the vessel radii distribution in irradiated patients compared to controls. Radiation-induced damage to the microvasculature resulted in a higher fraction of small vessels observed with time from radiation therapy, likely due to vessel thinning.

Importance of early spectral variations during 36 months of longitudinal follow MRI and MRS in 90 patients treated glioblastomas

J.-M. Constans ${ }^{1}$, A. Heintz ${ }^{1}$, O. Seloi ${ }^{1}$, J.P. Chombar ${ }^{1}$, N. Deleval ${ }^{1}$, R. Hanafi ${ }^{1}$, W. Dou ${ }^{2}$, S. Ruan ${ }^{3}$, J. Prades ${ }^{1}$, D. Le Gars ${ }^{1}$, O. Baledent ${ }^{1}$, H. Deramond ${ }^{1}$, A. Houessinon ${ }^{1}$, A. Fichten ${ }^{1}$, M. Lefranc ${ }^{1}$, A. Coutte ${ }^{1}$, P. Toussaint ${ }^{1}$, C. Desenclos ${ }^{1}$, B. Chauffert ${ }^{1}$, and M. Boone ${ }^{1}$

${ }^{1} \mathrm{CHU}$ et Université Amiens, Picardie, France, Amiens, France, Metropolitan, ${ }^{2}$ TsinghuaUniversity, Beijing, Chine, Beijing, China, ${ }^{3}$ Université de Rouen, France, Rouen, France, Metropolitan

MRS allows non-invasive follow-up of treated glioblastomas tumors. There is a large variability, but repetition and modelisation of spectroscopic measurements during longitudinal followup could allow us to diminish it and to improve prognostic evaluation especially in long survivors and patients with proliferation relapses. Studying the relationship between MRS measures, segmentation and perfusion parameters could lead to better understanding of tumoral processes and of therapeutic response, especially with regard to chemotherapy, radiotherapy and antiangiogenic molecules and in the future oxidative stress and hypoxia modulators.

A Large Scale Radiomics Profiling Strategy for Glioma Overall Survival Prediction

Pan Sun ${ }^{1}$, Defeng Wang ${ }^{2}$, Queenie Chan ${ }^{3}$, and Lin Shi ${ }^{1}$

${ }^{1}$ Medicine and Therapeutics, The Chinese University of Hong Kong, Hong Kong, China, ${ }^{2}$ Research Center for Medical Image Computing, The Chinese University of Hong Kong, Hong Kong, China, ${ }^{3}$ Philips Healthcare, Hong Kong, China

Glioma is the most common brain intracranial malignancy, which accounts for about $80 \%$ of malignant brain tumors in adults and its median survival rate is 12 months. In clinical, how to accurately predict the glioma overall survival (GOS) is a crucial work and it will be beneficial to monitor tumor progression, execute surgery as well as plan radiotherapy and follow-up studies. However, the glioma generally has highly heterogeneity degrees in the histological tumor sub-regions. we propose a comprehensive multi-modality MRI radiomics way of predicting the GOS. Different features are proposed committing to different image modalities. A feature selection strategy is applied for the optimal features and then random forest is contributed to the classification of short-survivors and long-survivors. With the performance evaluation criteria, our model showed promising classification ability for the brain tumor.

Diffusion kurtosis imaging (DKI) can help to differentiate low- and high-grade gliomas in pediatric patients: a prospective single centre experience with the simultaneous multislice (SMS) technique

Antonio Napolitano ${ }^{1}$, Ioan Paul Voicu ${ }^{2}$, Lorenzo Lattavo ${ }^{2}$, Maria Camilla Rossi Espagnet ${ }^{2}$, Chiara Carducci ${ }^{2}$, Angela Mastronuzzi ${ }^{3}$, Paolo Tomà ${ }^{2}$, and Giovanna Stefania Colafati ${ }^{2}$

${ }^{1}$ Medical Physics Department, IRCCS Bambino Gesù Children's Hospital, Rome, Italy, ${ }^{2}$ Imaging Department, IRCCS Bambino Gesù Children's Hospital, Rome, Italy, ${ }^{3}$ Department of Pediatric Onco-Hematology and Transfusion Medicine, IRCCS Bambino Gesù Children's Hospital, Rome, Italy

Pediatric brain glioma is a very devastating brain tumour and the most frequent solid tumour in children. Differentiating low-from high-grade glioma without the use of invasive biopsy is important to optimize patient management strategies yet difficult with imaging alone. Diffusion kurtosis imaging is then an emerging technique that has shown the ability of discriminating grades in adults. We make use of multislice approach to acquire and evaluate kurtosis metrics in brain gliomas and show how estimation of the heterogeneity of the tumour might be indicative of its grade. 
Quantitative proton density values compared to $1 \mathrm{H}$ MRSI in areas of contrast enhancement of glioma patients after surgical resection.

Felix Raschke ${ }^{1}$, Tim Wesemann ${ }^{2}$, Hannes Wahl ${ }^{2}$, Steffen Appold ${ }^{3}$, Mechthild Krause ${ }^{1,3,4,5,6}$, Jennifer Linn² ${ }^{2}$ and Esther G.C. Toost ${ }^{1,3,4,5,6}$

${ }^{1}$ National Center for Tumor Diseases, partner site Dresden, Germany, Dresden, Germany, ${ }^{2}$ Institute of Neuroradiology, University Hospital Carl Gustav Carus and Medical Faculty of Technische Universität, Dresden, Germany, ${ }^{3}$ Department of Radiotherapy and Radiooncology, University Hospital Carl Gustav Carus and Medical Faculty of Technische Universität, Dresden, Germany, ${ }^{4}$ OncoRay - National Center for Radiation Research in Oncology, Faculty of Medicine and University Hospital Carl Gustav Carus, Technische Universität Dresden, Helmholtz-Zentrum Dresden - Rossendorf, Dresden - Rossendorf, Germany, ${ }^{5}$ Institute of Radiooncology - OncoRay, Helmholtz-Zentrum Dresden-Rossendorf, Rossendorf, Germany, ${ }^{6}$ German Cancer Consortium (DKTK), partner site Dresden, Dresden, Germany

In this study we measured mean proton density (PD) values in MR spectroscopic imaging (MRSI) voxels showing contrast enhancement of glioma patients 30 days \pm 12 days after surgical resection. MRSI voxels with (partial) overlap with contrast enhancing areas were manually selected. Mean PD values showed a significant inverse correlation with NAA/Cho indicating that areas with higher PD are more likely to contain residual tumour tissue rather than surgery-related tissue damage. There was, however, no correlation of PD with $\mathrm{Cho/Cr}$, which suggests that quantitative PD values are unable to determine tumour aggressiveness.

Association between pharmacokinetic parameters from DCE-MRI and metabolic parameters from dynamic 18F-fluoromethylcholine PET in human brain glioma

Marianna Inglese ${ }^{1,2}$, Matthew Grech-Sollars ${ }^{1,3}$, Katherine Ordidge ${ }^{3}$, Vijaykumar Vaja ${ }^{4}$, Lesley Honeyfield ${ }^{3}$, Sameer Khan ${ }^{3}$, Tara Barwick ${ }^{1,3}$, Eric Aboagye ${ }^{1}$, and Adam D Waldman An $^{4,5}$

${ }^{1}$ Department of Surgery and Cancer, Imperial College London, London, United Kingdom, ${ }^{2}$ Department of Computer, Control and Management Engineering Antonio Ruberti, La Sapienza University of Rome, Rome, Italy, ${ }^{3}$ Department of Imaging, Imperial College Healthcare NHS Trust, London, United Kingdom, ${ }^{4}$ Department of Medicine, Imperial College London, London, United Kingdom, ${ }^{5}$ Centre for Clinical Brain Sciences, The University of Edinburgh, Edinburgh, United Kingdom

Magnetic resonance imaging (MRI) is the standard imaging technique in the diagnosis of primary brain lesions. However, novel PET imaging techniques such as choline-PET are currently being investigated in the clinic to characterize tumour metabolism. In this study, we compared pharmacokinetic parameters resulting from the modelling of dynamic contrast enhanced (DCE) MRI data, using the Tofts model (TM) and shutter speed model (SSM), with metabolic macroparameters derived from the application of the spectral analysis (SA) to dynamic PET data. We observe a correlation between some pharmacokinetic parameters and the parameters obtained through spectral analysis of the dynamic choline-PET data.

Quantitative susceptibility imaging for the assessment of early radiation-induced white matter injury in children with primary brain tumors

Junjie Wu ${ }^{1}$, Susan Palasis ${ }^{2}$, Natia Esiashvili ${ }^{3}$, Richard Jones ${ }^{2}$, Eduard Schreibmann ${ }^{3}$, and Deqiang Qiu ${ }^{1}$

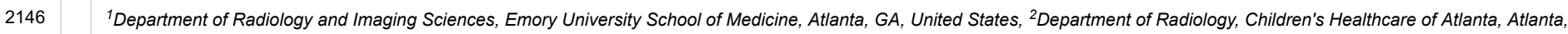
GA, United States, ${ }^{3}$ Department of Radiation Oncology, Emory University School of Medicine, Atlanta, GA, United States

We examined radiation-induced white matter injury using quantitative susceptibility mapping (QSM) in children with primary brain tumors. Following radiation therapy, susceptibility changed with time and dose. QSM may be a useful biomarker for irradiation damage.

Effects of Glioblastoma (GBM) on quantitative MRI of Contralateral Normal Appearing White Matter

Hatef Mehrabian ${ }^{1,2}$, Wilfred W Lam ${ }^{1}$, Sten Myrehaug ${ }^{3}$, Arjun Sahgal ${ }^{3}$, and Greg J Stanisz ${ }^{1}$

${ }^{1}$ Physical Sciences, Sunnybrook Research Institute, Toronto, ON, Canada, ${ }^{2}$ Radiology \& Biomedical Imaging, University of California, San Francisco, San Francisco, CA, United States, ${ }^{3}$ Sunnybrook Health Sciences Centre, Toronto, ON, Canada

Normal-appearing white matter on the contralateral hemisphere (cNAWM) of glioblastoma (GBM) has been shown with MRS and DTI to be abnormal which might be due to tumor cell infiltration into these distant normal appearing brain structures. Chemical exchange saturation transfer (CEST), quantitative magnetization transfer (qMT) and transverse relaxation time $\left(\mathrm{T}_{2}\right)$ are sensitive to changes in tissue microstructure and metabolism. CEST, qMT and $\mathrm{T}_{2}$-mapping were used to investigate abnormalities in cNAWM. Results demonstrated differences in white mater cellular density (measured with $\mathrm{T}_{2}$ and $\mathrm{qMT}$ ) as well as metabolism (measured with CEST) in cNAWM of GBM patients compared to healthy controls.

Relationship Between Tumor Cellularity and Metabolic Activity in IDH-Mutant Gliomas: A Correlative Study with 2-Hydroxyglutarate MRSI and a Novel Diffusion MRI Method

Ina Ly ${ }^{1}$, Ovidiu Andronesi ${ }^{1,2}$, Qiuyun Fan ${ }^{1,2}$, Barbara Wichtmann ${ }^{3}$, Aapo Nummenmaa ${ }^{1,2}$, Brian Nahed ${ }^{1}$, William Curry ${ }^{1}$, Daniel Cahill ${ }^{1}$, Tracy Batchelor ${ }^{1}$, Jayashree Kalpathy-Cramer ${ }^{1,2}$, Bruce Rosen ${ }^{1,2}$, and Elizabeth Gerstner ${ }^{1}$

${ }^{1}$ Massachusetts General Hospital, Boston, MA, United States, ${ }^{2}$ Athinoula A. Martinos Center for Biomedical Imaging, Charlestown, MA, United States, ${ }^{3}$ Computer Assisted Clinical Medicine, Medical Faculty Mannheim, Heidelberg University, Mannheim, Germany 
Anatomic T2/FLAIR sequences are the gold standard in the diagnostic and monitoring process of non-enhancing gliomas but do not provide accurate information about the underlying metabolic activity of the tumor. In this work, we investigated the combined use of 2-hydroxyglutarate (2HG) magnetic resonance spectroscopic imaging (MRSI) and a novel threecompartment diffusion MRI method (Linear Multi-Scale Model) to characterize isocitrate dehydrogenase-mutant gliomas, and found that high $2 \mathrm{HG}$ levels correlated with decreased restricted diffusion.

Differentiating glioma histologic grade using histogram analyses of Amide Proton Transfer MRI

Qihong Rui ${ }^{1}$, Yingjie Mei ${ }^{2}$, Hao Yu ${ }^{1}$, Xianlong Wang ${ }^{1}$, Shanshan Jiang ${ }^{3}$, Jinyuan Zhou ${ }^{3}$, and Zhibo Wen ${ }^{1}$

${ }^{1}$ Department of Radiology, Zhujiang Hospital of Southern Medical University, Guangzhou, China, ${ }^{2}$ Philips Healthcare, Guangzhou, China, ${ }^{3}$ Division of MR Research, Department of Radiology, Johns Hopkins University School of Medicine, Baltimore, MD, United States

A correct preoperatively grading of glioma is always the important issue in clinic. APT imaging is designed to assess glioma on the level of cell and molecule. In this study we used the APT MRI histogram analyses, to determine if it can help differentiate HGG from LGG.

Multiparametric metabolic and physiologic MR-Imaging models for differentiating tumor from treatment effects in patients suspected of recurrent glioblastoma

Julia Cluceru ${ }^{1}$, Sarah Nelson ${ }^{1}$, Annette Molinaro ${ }^{1}$, Joanna Phillips ${ }^{1}$, Marram Olson ${ }^{1}$, Marisa LaFontaine ${ }^{1}$, Angela Jakary ${ }^{1}$, Devika Nair ${ }^{1}$, Soonmee Cha $^{1}$, Susan Chang ${ }^{1}$, and Janine Lupo $^{1}$

Despite previous research on physiological and metabolic MR imaging techniques with standard clinical anatomical MRI of patients with recurrent glioma, there is still no one parameter that can differentiate recurrent glioblastoma (rGBM) from treatment-induced effects (TxE) with high enough accuracy to be used clinically. We assessed the value of incorporating anatomical, perfusion-weighted, diffusion-weighted, and spectroscopic imaging parameters to identify TxE in patients suspected of rGBM. nPH from DSC perfusion-weighted imaging and Choline-to-NAA Index from MR spectroscopic imaging were found to be the most related to pathological markers of tumor and TxE.

Comparison of R2* and quantitative susceptibility mapping in the characterizing tumor hypoxia in a mouse model of glioblastoma

Runze Yang ${ }^{1}$, A. Max Hamilton ${ }^{1}$, Hongfu Sun ${ }^{1}$, Susobhan Sarkar ${ }^{2}$, Reza Mirzaei², G. Bruce Pike ${ }^{1}$, V. Wee Yong ${ }^{2}$, and Jeff F. Dunn ${ }^{1}$

${ }^{1}$ Radiology, University of Calgary, Calgary, AB, Canada, ${ }^{2}$ Clinical Neuroscience, University of Calgary, Calgary, AB, Canada

Hypoxia (low levels of oxygen) is an important biomarker in many solid tumors, as it is responsible for promoting tumor angiogenesis and resistance to radiotherapy. Hypoxia can be indirectly monitored by measuring levels of deoxyhemoglobin with MRI, using either $\mathrm{R}_{2}{ }^{*}$ or quantitative susceptibility mapping (QSM). We compared the two methods for brain tumor and hypoxia imaging in a mouse model of glioblastoma. We found that both methods were sensitive at detecting a decrease in deoxyhemoglobin due to $100 \%$ oxygen. However, QSM provided better anatomical information and was better at detecting tumor heterogeneity. QSM is a promising tumor imaging method.

The diagnostic value of postcontrast susceptibility-weighted imaging in the assessment of intracranial brain neoplasm at 3T

Hyunkoo Kang ${ }^{1}$

${ }^{1}$ Department of Radiology, Seoul Veterans Hospital, Seoul, Republic of Korea

The aim of this study is to estimate the diagnostic value of postcontrast susceptibility-weighted imaging (CESWI) in the assessment of intracranial brain neoplasm at 3 T MRI. Our results showed that the SWI can be performed after gadolinium injection without information loss or signal change and the CESWI clearly visualized the characteristics and the architecture of brain neoplasm. The CESWI can be a match to the CET1 with regard to the visibility of tumor margin and internal architecture in intracranial tumors without information loss or signal change.

The diagnostic value of the distribution pattern of intratumoral susceptibility sign of intracranial tumors on susceptibility-weighted imaging

Hyunkoo Kang ${ }^{1}$ and Seongwon Jang ${ }^{1}$

${ }^{1}$ Department of Radiology, Seoul Veterans Hospital, Seoul, Republic of Korea 
The aim of this study is to determine whether the distribution pattern of intratumoral susceptibility sign (ITSS) derived from susceptibility-weighted imaging (SWI) could differentiate glioblastoma multiforme (GBM) and single brain metastasis. We compared the grade of the visibility of ITSS in the central portion of tumors (CITSS) and in the tumor capsular area (PITSS) on SWI. Our findings suggest that there were different characteristics of ITSS between GBM and brain metastasis on SWI due to the profound difference in histologic feature of capillary between the two tumor types.

Comparison of Dynamic Susceptibility Contrast and Arterial Spin Labeling at the Target Locations of Image guided Tissue Samples for Patients with Glioma

Marisa M Lafontaine ${ }^{1}$, Janine M Lupo ${ }^{1}$, Marram P Olson ${ }^{1}$, Joanna J Phillips ${ }^{2}$, Susan M Chang ${ }^{3}$, and Sarah J Nelson ${ }^{1}$

${ }^{1}$ Radiology, UCSF, San Francisco, CA, United States, ${ }^{2}$ Neuropathology, UCSF, San Francisco, CA, United States, ${ }^{3}$ Neurosurgery, UCSF, San Francisco, CA, United States

Arterial spin labeling and dynamic susceptibility contrast perfusion weighted imaging were both found to provide acceptable measures of blood vessel angiogenesis in brain tumors compared to pathological measures but dynamic susceptibility contrast may be better correlated with the underlying vascular morphology.

Differentiation of grade II/III and Grade IV glioma by combining 'T1 contrast enhanced brain perfusion imaging' and susceptibility weighted quantitative imaging

Jitender Saini ${ }^{1}$, Pradeep Kumar Gupta ${ }^{2}$, Prativa Sahoo ${ }^{3}$, Anup Singh ${ }^{4}$, Rana Patir ${ }^{5}$, Sunita Ahlawat ${ }^{6}$, Manish Beniwal ${ }^{7}$, K. Thennarasu ${ }^{8}$, Vani Santosh ${ }^{9}$, and Rakesh Kumar Gupta ${ }^{2}$

${ }^{1}$ Department of Neuroimaging \& Interventional Radiology, National Institute of Mental Health and Neurosciences, Bangalore, India, ${ }^{2}$ Department of Radiology and Imaging, Fortis Memorial Research Institute, Gurgaon, India, ${ }^{3}$ Beckman Research Institute, Mathematical Oncology, Duarte, CA, United States, ${ }^{4}$ Center for Biomedical Engineering, Indian Institute of Technology Delhi, Delhi, India, ${ }^{5}$ Department of Neurosurgery, Fortis Memorial Research Institute, Gurgaon, India, ${ }^{6}$ SRL Diagnostics, Fortis Memorial Research Institute, Gurgaon, India, ${ }^{7}$ Department of Neurosurgery, National Institute of Mental Health and Neurosciences, Bangalore, India, ${ }^{8}$ Department of Biostatistics, National Institute of Mental Health and Neurosciences, Bangalore, India, ${ }^{9}$ Department of Neuropathology, National Institute of Mental Health and Neurosciences, Bangalore, Heard Island And Mcdonald Islands

The purpose of this study is to evaluate the usefulness of T1-perfusion MRI and SWI in discriminating among grade II, III and IV gliomas. We found that combining T1-perfusion and SWI improves the diagnostic accuracy for discrimination of grade III from grade IV gliomas and T1-perfusion MRI derived rCBV alone appears to be an excellent measure for discriminating grade II from grade III glioma.

ADC-map-based classification of glioma-subtypes in diffusion-weighted MR-Imaging

Nils Christoph Nuessle ${ }^{1}$, Johann Martin Hempel ${ }^{1}$, Jens Schittenhelm² , and Uwe Klose

${ }^{1}$ Department for Neuroradiology, University Hospital of Tuebingen, Tuebingen, Germany, ${ }^{2}$ Institute of Neuropathology, Department of Pathology and Neuropathology, University Hospital of Tuebingen, Tuebingen, Germany

DWI showed great potential for estimation of histopathological and molecular profile of human glioma. 97 patients with suspected glioma underwent pre-operative MRI-scans, including high b-value DWI. ADC-maps from pairs of two b-values were calculated. Post-interventional histopathological tumor grading was realized on a molecular basis using the molecular markers IDH-mutation, 1p/19q- and ATRX-loss. Significant differences $(p<0.001)$ were found between oligodendroglioma, astrozytoma and GBM. Best discrimination was achieved when calculating the ADC-maps from b-values of 500 and $2500 \mathrm{~s} / \mathrm{mm}^{2}$. Therefore, ADC-map based evaluation of glioma in DWI provides great potential in accurate pre-interventional diagnosing of glioma subtypes.

Quantitative T1-difference maps and T1-weighted difference images: which modality is better at identifying tumor infiltration in high grade gliomas?

Ulrike Nöth ${ }^{1}$, Ralf Deichmann ${ }^{1}$, Oliver Bähr ${ }^{2}$, Julia Tichy ${ }^{2}$, Stephanie Lescher ${ }^{3}$, and Elke Hattingen ${ }^{4}$

${ }^{1}$ Brain Imaging Center (BIC), Goethe University, Frankfurt/Main, Germany, ${ }^{2}$ Dr Senckenberg Institute of Neurooncology, Goethe University, Frankfurt/Main, Germany, ${ }^{3}$ Institute of Neuroradiology, Goethe University, Frankfurt/Main, Germany, ${ }^{4}$ Funktionseinheit Neuroradiologie, Radiologische Klinik der UKB, Bonn, Germany

In glioblastoma patients, differences of quantitative T1 (qT1) maps acquired before and after contrast agent (CA) administration are visually compared to the respective differences of conventional T1-weighted (T1w) images. Quantitative T1-differences are determined in the following regions-of-interest (ROIs): (1) enhancing tumor, (2) edema, (3) $5 \mathrm{~mm}$-rim around (1)+ (2), (4) control tissue contralateral to the tumor. T1w- and qT1-differences clearly show the enhancing tumor, but only the qT1-difference maps show signal enhancement in the edema, which is in line with elevated qT1-difference values in this ROI. This indicates most likely CA leakage due to tumor infiltration.

Traditional Poster

\section{Perfusion Methods}


Comparing pCASL CBF measurements between 3D-GraSE and 2D-EPI on 1.5T and 3T systems

Koen P.A. Baas ${ }^{1,2}$, Henri J.M.M. Mutsaerts 2,3 , Jan Petr $^{3,4}$, Joost P.A. Kuijer ${ }^{2}$, and Kim C.C. van de Ven ${ }^{1}$

${ }^{1}$ BIU MR, Philips, Best, Netherlands, ${ }^{2}$ VUmc, Amsterdam, Netherlands, ${ }^{3}$ Kate Gleason College of Engineering, Rochester Institute of Technology (RIT), Rochester, NY, United States, ${ }^{4}$ PET Center, Institute of Radiopharmaceutical Cancer Research, Helmholtz-Zentrum Dresden-Rossendorf, Dresden, Germany

We have compared CBF value agreement in healthy subjects across two readouts, 3D-GraSE and 2D-EPI, and two field strengths, 1.5T and 3T, and investigated with which acquisition parameters we can reach the best agreement. Significantly higher GM CBF was observed with the 2D-EPI readout compared to the 3D-GraSE readout with equivalent acquisition voxel size $(p<0.005$ for $1.5 T$ and $p<0.05$ for 3T). Better agreement was observed between 3D-GraSE and 2D-EPI on 3T systems when the resolution of the 3D-GraSE readout was increased to match the effective resolution to the $2 \mathrm{D}-\mathrm{EPI}$ scan $(\mathrm{ICC}=0.772$ and ICC $=0.932$ respectively).

Reproducibility and repeatability of 3D-GraSE and 2D-EPI ASL on 1.5T and 3T systems in healthy elderly

Koen P.A. Baas ${ }^{1,2}$, Henri J.M.M. Mutsaerts ${ }^{2,3}$, Joost P.A. Kuijer ${ }^{2}$, and Kim C.C. van de Ven ${ }^{1}$

${ }^{1}$ BIU MR, Philips, Best, Netherlands, ${ }^{2}$ VUmc, Amsterdam, Netherlands, ${ }^{3}$ Kate Gleason College of Engineering, Rochester Institute of Technology (RIT), Rochester, NY, United States

We present the results of a reproducibility and repeatability study in 34 healthy elderly scanned on $1.5 \mathrm{~T}$ and 3T systems employing pCASL with a 3D-GraSE and 2D-EPI read-out. Best repeatability and reproducibility were achieved when using 3D-GraSE readout on $3 \mathrm{~T}$ systems leading to an average repeatability and reproducibility of GM CBF of $2.7 \% \pm 1.8 \%$ and $2.9 \% \pm 3.5 \%$ respectively. The repeatability and reproducibility of $2 \mathrm{D}$ read-out and of comparisons at $1.5 \mathrm{~T}$ and $1.5 \mathrm{~T}$ versus $3 \mathrm{~T}$ were slightly lower. These results imply that $3 \mathrm{D}-\mathrm{GraSE}$ pCASL at $3 \mathrm{~T}$ should be preferred in multi-center trials as well as for clinical imaging.

Background-suppression is more important for ASL at higher magnetic field strength

Lydiane Hirschler ${ }^{1}$, Suzanne L Franklin ${ }^{1,2}$, Sophie Schmid ${ }^{1}$, Wouter M Teeuwisse ${ }^{1}$, and Matthias JP van Osch ${ }^{1}$

$2160 \quad{ }^{1}$ Radiology, Leiden University Medical Center, Leiden, Netherlands, ${ }^{2}$ Imaging Division, University Medical Center Utrecht, Utrecht, Netherlands

Background suppression is a recommended and frequently employed strategy to improve the perfusion-temporal-SNR (tSNR) of ASL. Since physiological signal fluctuations are known to be a major source of data corruption in functional MRI at higher magnetic field-strengths, it might also be expected that the benefits of BGS are even stronger at higher field-strengths. In this study, we evaluated and compared the importance of the introduction of BGS-pulses at 3T and 7T and show that, at higher magnetic field, BGS is even more crucial.

A novel hybrid of time-encoded and sequential multi-PLD PCASL for improved cerebral blood flow estimation

Joseph G. Woods ${ }^{1}$, Michael A. Chappell ${ }^{2}$, and Thomas W. Okell ${ }^{1}$

$2161 \quad{ }^{1}$ Wellcome Centre for Integrative Neuroimaging, FMRIB, Nuffield Department of Clinical Neurosciences, University of Oxford, Oxford, United Kingdom, ${ }^{2}$ Institute of Biomedical 'Wellcome Centre for Integrative Neuroimaging, FMRIB, Nuffi
Engineering, University of Oxford, Oxford, United Kingdom

We present a novel hybrid combination of time-encoded and sequential multi-PLD pseudo-continuous ASL, which benefits from the advantages of both techniques, and demonstrate that the increased flexibility of this approach improves CBF precision compared to either method alone.

Comparison of optimized single-PLD, sequential multi-PLD and time-encoded PCASL for cerebral blood flow measurements

Joseph G. Woods ${ }^{1}$, Michael A. Chappell ${ }^{2}$, and Thomas W. Okell ${ }^{1}$

${ }^{1}$ Wellcome Centre for Integrative Neuroimaging, FMRIB, Nuffield Department of Clinical Neurosciences, University of Oxford, Oxford, United Kingdom, ${ }^{2}$ Institute of Biomedical Engineering, University of Oxford, Oxford, United Kingdom

In this work, we use an objective approach to optimize sequential and time-encoded multi-PLD protocols, and compare them to the recommended single-PLD protocol using simulations, with the aim of determining which method is capable of producing the most accurate CBF estimates across a range of ATTs. 
The tracer kinetic properties of velocity selective inversion pulses were characterized using a two compartment model. The properties of these pulses indicate that VSI pulses can produce large input functions and little or no transit time effects. These translate into speed and SNR gains for perfusion images of both grey and white matter without the use of contrast agents.

Patch based low rank and sparse decomposition for arterial spin labeling perfusion MRI signal denoising

Hancan Zhu' ${ }^{1}$, Jian Zhang ${ }^{2}$, and Ze Wang ${ }^{3}$

${ }^{1}$ Department of Mathematics, Shaoxing University, Shaoxing, China, ${ }^{2}$ Institutes of Psychological Science, Hangzhou Normal University, Hangzhou, China, ${ }^{3}$ Department of Radiology, Temple University, Philadelphia, PA, United States

Arterial spin labeling (ASL) perfusion fMRI has much less neurovascular effects than BOLD fMRI, but its application in time-series analysis is still depreciated due to the low signal-tonoise-ratio (SNR). In this study, we propose a patch based low rank and sparse decomposition method to denoise ASL MRI. Our results showed that the proposed method can markedly increase the sensitivity of ASL MRI-based task activation detection.

Blood-Brain Partition Coefficient Correction Improves Gray-White Matter Contrast in Blood Flow Measurement in Mice

Scott William Thalman ${ }^{1}$, David Powell ${ }^{1,2}$, and Ai-Ling Lin ${ }^{1,3}$

${ }^{1}$ Biomedical Engineering, University of Kentucky, Lexington, KY, United States, ${ }^{2}$ Magnetic Resonance Imaging and Spectroscopy Center, University of Kentucky, Lexington, KY, United States, ${ }^{3}$ Pharmacology and Nutritional Sciences, University of Kentucky, Lexington, KY, United States

The blood-brain partition coefficient (BBPC) is a tissue-specific parameter important in quantifying cerebral blood flow (CBF), but regional differences in BBPC are commonly ignored. Using an accelerated calibrated proton density imaging technique we measure BBPC directly, enabling a voxel-wise correction of CBF maps derived from arterial spin labeling acquisitions. We measure an elevated BBPC in the cortex $(0.99 \mathrm{~mL} / \mathrm{g})$ relative to the corpus callosum $(0.93 \mathrm{~mL} / \mathrm{g})$ and the hippocampus $(0.95 \mathrm{~mL} / \mathrm{g})$, and demonstrate that BBPCcorrection improves gray-white matter contrast in CBF maps by $15 \%$ in the cortex and $7 \%$ in the hippocampus.

Improved functional Arterial Spin Labeling by spatio-temporal ICTGV denoising

Stefan Manfred Spann ${ }^{1}$, Matthias Schloegl ${ }^{1}$, Christoph Stefan Aigner ${ }^{1}$, Karl Koschutnig ${ }^{2}$, Martin Holler ${ }^{3}$, Kristian Bredies ${ }^{3}$, and Rudolf Stollberger ${ }^{1,4}$

${ }^{1}$ Institute of Medical Engineering, Graz University of Technology, Graz, Austria, ${ }^{2}$ Institute of Psychology, University of Graz, Graz, Austria, ${ }^{3}$ Institute of Mathematics and Scientific Computing, University of Graz, Graz, Austria, ${ }^{4}$ BioTechMed-Graz, Graz, Austria

Functional Arterial Spin Labeling (fASL) provides important information of perfusion changes over time and is therefore suitable for detecting neuronal activation due to cognitive functions or motor tasks. However, the low signal to noise ratio of ASL images restrains its application in clinical and research areas. In this study we propose a method for denoising fASL data using infimal convolution of total generalized variations (ICTGV). Compared to standard Gaussian denoising ICTGV denoising incorporates spatial and temporal information of the perfusion weighted time series. This leads to a substantial improvement in noise-suppression for fASL data.

Measurement of Pulmonary Perfusion using PCASL True-FISP Imaging at 1.5 Tesla

Petros Martirosian ${ }^{1}$, Ferdinand Seith ${ }^{2}$, Rolf Pohmann ${ }^{3}$, Martin Schwartz ${ }^{1,4}$, Thomas Küstner ${ }^{1,4}$, Klaus Scheffler $^{3,5}$, Konstantin Nikolaou ${ }^{2}$, and Fritz Schick ${ }^{1}$

${ }^{1}$ Section on Experimental Radiology, University of Tübingen, Tübingen, Germany, ${ }^{2}$ Department of Diagnostic and Interventional Radiology, University of Tübingen, Tübingen, Germany, ${ }^{3}$ Max Planck Institute for Biological Cybernetics, Tübingen, Germany, Tübingen, Germany, ${ }^{4}$ Institute of Signal Processing and System Theory, University of Stuttgart, Stuttgart, Germany, ${ }^{5}$ Department of Biomedical Magnetic Resonance, University of Tübingen, Tübingen, Germany

Pseudo-continuous-arterial-spin-labeling (PCASL) has been successfully applied in the liver and kidney providing high signal-to-noise-ratio. The goal of this work is to assess the potential of PCASL technique to measure the pulmonary perfusion at $1.5 \mathrm{~T}$. Effective labeling of pulmonary blood flow was achieved by ECG triggering and an orientation of the labeling plane perpendicular to the pulmonary trunk. Fast True-FISP imaging with short TE of $0.9 \mathrm{~ms}$ was used to obtain high signal from lung parenchyma. The PCASL-True-FISP technique provides high quality perfusion images of the lung and allows quantitative measurements of pulmonary perfusion both in multiple breath-holds and under free breathing condition. 
${ }^{1}$ Electrical and Computer Engineering, Temple university, Philadelphia, PA, United States, ${ }^{2}$ Department of Radiology, Temple university, Philadelphia, PA, United States

In this study, we use Deep Learning-based (DL) method to denoising ASL CBF images. Convolutional neural networks with a "wide" structure, residual learning and batch normalization are utilized as the core of our denoising model. Comparing to non-DL-based methods, the proposed method showed a significant SNR increase as well as partial volume effects improvement. Also, the DL-based method requires less CBF input images, which significantly shorten the acquisition time and reduce the chance of head motion.

Introducing a fat-image guided registration technique for image-based retrospective motion compensation for free-breathing background suppressed renal pCASL

Isabell Katrin Bones ${ }^{1}$, Anita A Harteveld ${ }^{1}$, Suzanne L Franklin ${ }^{1,2}$, Matthias JP van Osch², Jeroen Hendrikse ${ }^{3}$, Chrit TW Moonen ${ }^{1}$, Clemens Bos ${ }^{1}$, and Marijn van Stralen ${ }^{1}$

${ }^{1}$ Center for Image Sciences, University Medical Center Utrecht, Utrecht, Netherlands, ${ }^{2}$ C.J.Gorter Center for High Field MRI, Leiden University Medical Center, Leiden, Netherlands, ${ }^{3}$ Radiology, University Medical Center Utrecht, Utrecht, Netherlands

Aiming for rapid and accurate perfusion measurement, background suppressed (BGS) ASL under free breathing is desired. Motion compensation on BGS ASL is challenging due to the lack of anatomical contrast. We investigated the benefit of BGS versus non-BGS ASL, guided by motion compensation based on the ASL-images themselves and additionally acquired fat-images. Registration effect on perfusion weighted signal (PWS) and temporal SNR (tSNR) was evaluated for ASL-image and fat-image based registration, proving increased tSNR and increased PWS robustness, without compromising signal intensity. We conclude that free-breathing BGS renal pCASL with image-based retrospective motion compensation yields better reproducibility than without BGS.

Simultaneous Acquisition of ASL, BOLD effect, Phase and QSM for Functional Multi-Parametric Brain Studies

Sagar Buch ${ }^{1}$, Hacene Serrai ${ }^{1}$, and Ravi S. Menon ${ }^{1,2}$

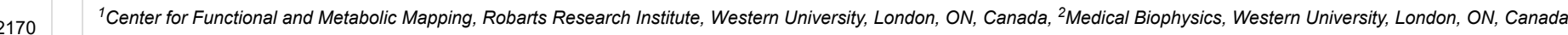

A 2D-GRE-EPI based sequence combined with the PICORE magnetization preparation technique was used to acquire functional Arterial Spin Labeling (ASL) perfusion data at high field (7T). BOLD and Cerebral Blood Flow (CBF) changes along with phase and susceptibility maps (QSM) are obtained and assessed from this scan. Using a pre-determined general linear model (GLM), a strong correlation between the change in these parameters in the activated region (visual cortex) has been found showing that this multi-parametric acquisition may help in resolving the multi-factorial BOLD signal for functional brain studies.

Reconstructing Pseudo-Continuous Arterial Spin Labeling Perfusion Signals through Modulation of Labeling RF Power and Fourier Analysis

Hyo-Im Heo ${ }^{1}$, Paul Kyu Han², Seung Hong $\mathrm{Choi}^{3}$, and Sung-Hong Park

${ }^{1}$ Department of Bio and Brain Engineering, Korea Advanced Institute of Science and Technology, Daejeon, Republic of Korea, ${ }^{2}$ Gordon Imaging Center, Massachusetts General Hospital, Harvard Medical School, Boston, MA, United States, ${ }^{3}$ Department of Radiology, Seoul National University College of Medicine, Seoul, Republic of Korea

The conventional pCASL is vulnerable to data corruption and has high specific absorption rate. In this study, we propose a new pCASL approach using modulation of labeling RF pulse power and Fourier analysis. The proposed approach enabled us to acquire perfusion images comparable to those of the conventional pCASL. Under data corruption, the proposed approach maintained the perfusion signals well with no observable effect, while the conventional method showed almost no perfusion signal. The proposed approach has relatively low average SAR and instantaneous SAR, potentially advantageous at high fields. These advantages of the proposed method warrant further investigation.

Evaluation of the Suitability of Hadamard Encoding Schemes for Pseudo-Continuous Arterial Spin Labelling

Jed Wingrove ${ }^{1}$, Marc Lebel ${ }^{2}$, and Fernando Zelaya ${ }^{1}$

${ }^{1}$ Department of Neuroimaging, King's College London, London, United Kingdom, ${ }^{2}$ GE Healthcare, Calgary, Canada

Multi delay Arterial Spin Labelling offers the advantage of measuring neurophysiological properties such as arterial transit delay which can be used to hep improve cerebral blood flow estimation. Hadamard encoding PCASL is a method with improved temporal resolution and SNR compared to sequential multi delay methods. This work presents the findings of an evaluation of three different Hadamard encoded schemes for perfusion and transit delay estimation. All schemes were comparable with regards to perfusion estimation however showed some interesting regional differences in TD estimation. 
${ }^{1}$ InBrain Lab - FFCLRP, University of Sao Paulo, Ribeirao Preto, Brazil, ${ }^{2}$ Physics Institute of Sao Carlos, University of Sao Paulo, Sao Carlos, Brazil

Arterial Spin Labeling (ASL) is a method designed to measure blood perfusion. In special, brain perfusion is measured as the cerebral blood flow (CBF), whose time-series fluctuations allow its use in functional analysis. This study aimed to run a dual-echo pseudo-continuous ASL acquisition and analyze its capacity to identify brain networks activated during a verbal fluency task and study the dynamic of brain areas during task and rest conditions. Results showed that it is possible to access language networks based on CBF-ASL, and reported differences in connectivity between both conditions analyzed.

Investigating Cerebrovascular Reactivity Using Pseudo-continuous ASL and Turbo QUASAR ASL at Varying Blood Flow Conditions

Moss Y Zhao ${ }^{1}$, Lena Vaclavu² ${ }^{2}$ Esben T Petersen ${ }^{3}$, Henk-Jan Mutsaerts ${ }^{2,4,5}$, Bart J Biemond ${ }^{6}$, Ed T van Bavel ${ }^{7}$, Charles B Majoie ${ }^{2}$, Aart J Nederveen², and Michael A Chappell ${ }^{1}$

${ }^{1}$ Institute of Biomedical Engineering, University of Oxford, Oxford, United Kingdom, ${ }^{2}$ Department of Radiology and Nuclear Medicine, Academic Medical Center, Amsterdam, Netherlands, ${ }^{3}$ Danish Research Centre for Magnetic Resonance, Copenhagen, Denmark, ${ }^{4}$ Department of Radiology, University Medical Center Utrecht, Utrecht, Netherlands, ${ }^{5}$ Rochester Institute of Technology, Rochester, NY, United States, ${ }^{6}$ Department of Haematology, Academic Medical Center, Amsterdam, Netherlands, ${ }^{7}$ Department of Biomedical Engineering and Physics, Academic Medical Center, Amsterdam, Netherlands

CVR has become an important biomarker to assess cerebrovascular health, and ASL is a non-invasive technique to quantify CVR. This work compared the CVR measurement from PCASL and Turbo QUASAR ASL at varying blood flow conditions induced by acetazolamide. Results showed that both ASL techniques were sensitive to CVR and that significant changes of ATT were detected by Turbo QUASAR ASL. The differences in CVR (higher in PCASL) may be due to the different sensitivity to ATT of the two ASL methods.

Pushing the Limits of ASL Imaging for the Lifespan Human Connectome Projects

Xiufeng $\mathrm{Li}^{1}$, Dingxin Wang ${ }^{1}$, Steen Moeller ${ }^{1}$, Danny JJ Wang ${ }^{2}$, Michael Chappell ${ }^{3}$, Essa Yacoub ${ }^{1}$, and Kamil Ugurbil ${ }^{1}$

${ }^{1}$ Center for Magnetic Resonance Research, School of Medicine, University of Minnesota, Minneapolis, MN, United States, ${ }^{2}$ Laboratory of FMRI Technology (LOFT), Mark \& Mary Stevens Neuroimaging and Informatics Institute, Keck School of Medicine, University of Southern California (USC), Los Angeles, CA, United States, ${ }^{3}$ Institute of Biomedical Engineering, University of Oxford, Headington, Oxford, United Kingdom

Arterial spin labeling (ASL) imaging is included in the Lifespan Human Connectome Projects (HCPs) in order to investigate the evolution of cerebral blood flow (CBF) in children and elderly populations. To push the limits of ASL imaging for the Lifespan HCPs, we optimized and evaluated high-resolution 2D slice accelerated protocols for multi-delay PCASL imaging. The results suggest that high quality arterial transit time (ATT) and CBF maps with a $2.5 \mathrm{~mm}$ resolution can be reliably achieved in about 5.5 minutes.

Regional Oxygen Extraction Fraction Measurements in the Middle Cerebral Artery Territory using Selective Localised T2-Relaxation-Under-Spin-Tagging (SL-TRUST)

Caitlin O'Brien ${ }^{1}$, Thomas Okell ${ }^{1}$, and Peter Jezzard ${ }^{1}$

${ }^{1}$ Wellcome Centre for Integrative Neuroimaging, FMRIB, University of Oxford, Oxford, United Kingdom

Regional measurements of brain tissue oxygen extraction fraction (OEF) are an important indicator of tissue physiology and disease. We present an improved Selective Localised T2relaxation-under-spin-tagging (SL-TRUST) sequence for regional venous blood $\mathrm{T}_{2}$ measurements, decoded in the superior sagittal sinus, from which cerebral tissue OEF can be calculated. A spatially selective WET saturation scheme is used to saturate signal outside the region of interest, enabling OEF measurements in a hemisphere and in the middle cerebral artery (MCA) territory. Using a multi-TI inversion recovery sequence we calculate subject specific blood hematocrit in the sagittal sinus and thus improve our OEF calibration.

Influence of background suppression and retrospective realignment on free-breathing renal perfusion imaging using ASL

Manuel Taso ${ }^{1}$, Arnaud Guidon ${ }^{2}$, and David C. Alsop ${ }^{1}$

${ }^{1}$ Division of MRI Research, department of Radiology, Beth Israel Deaconess Medical Center, Harvard Medical School, Boston, MA, United States, ${ }^{2}$ Global MR Applications and Workflow, GE Healthcare, Boston, MA, United States

While a consensus exists on the benefits of background suppression for brain ASL to reduce physiological noise, conflicting results have been presented for renal applications. Furthermore, bulk motion management remains a challenge for clinical applications. In the current work, we investigate the effects and interactions between background suppression and retrospective motion-correction when used for single-slice free-breathing renal ASL. We emphasize the influence of BS and motion-correction on thermal and physiological noise levels and show that BS is critical for renal ASL using PCASL while retrospective motion-compensation helps in increasing image sharpness. 
${ }^{1}$ Bioengineering, University of Texas at Dallas, Richardson, TX, United States, ${ }^{2}$ Radiology, UT Southwestern Medical Center, Dallas, TX, United States, ${ }^{3}$ Advanced Imaging Research Center, UT Southwestern Medical Center, Dallas, TX, United States

2D Flow Alternating Inversion Recovery (FAIR) has been applied to measure non-contrast pulmonary perfusion in research environments, but its lack of coverage limits its applicability for clinical perfusion evaluation, where whole-lung coverage is often necessary. In this study, we optimized the multi-slice FAIR technique, including background suppression for robust image quality and inflow saturation to minimize the blood volume contribution, to measure pulmonary perfusion across the whole-lung at $3 \mathrm{~T}$.

Automatic selection of local arterial input functions in perfusion MRI using cluster analysis and priority-flooding

Rami Tabbara ${ }^{1}$, Alan Connelly ${ }^{1,2}$, and Fernando Calamante ${ }^{1,2}$

${ }^{1}$ The Florey Institute of Neuroscience and Mental Health, Melbourne, Australia, ${ }^{2}$ Florey Department of Neuroscience and Mental Health, University of Melbourne, Australia

We present a robust, multi-stage automated local arterial input function (AIF) method to quantify perfusion using dynamic-susceptibility contrast (DSC)-MRI. We show how this approach reduces potential AIF misclassifications observed in existing automated solutions that can lead to quantification errors and artefacts. Examples of our new approach eliminating such artefacts from scans of subjects who exhibit various cerebrovascular abnormalities are provided, with generated perfusion maps further showing regions of higher cerebral blood flow (CBF) relative to established global AIF methods, consistent with a reduction in quantification errors associated with bolus dispersion.

Evaluation of dynamic DCE-MRI of the temporomandibular joint

Ondrej Macicek ${ }^{1}$, Erling Andersen ${ }^{2}$, Oskar Angenete ${ }^{3,4}$, Thomas Augdal ${ }^{5}$, Karen Rosendahl ${ }^{6,7}$, Radovan Jirik ${ }^{1}$, and Renate Grüner ${ }^{8,9}$

${ }^{1}$ The Czech Academy of Sciences, Institute of Scientific Instruments, Brno, Czech Republic, ${ }^{2}$ Department of Clinical Engineering, Haukeland University Hospital, Bergen, Norway, ${ }^{3}$ Department of Radiology and Nuclear Medicine, St Olav Hospital HF, Trondheim, Norway, ${ }^{4}$ Department of Circulation and Medical imaging, Norwegian University of Science and Technology, Faculty of Medicine, Trondheim, Norway, ${ }^{5}$ Department of Radiology, University Hospital of North Norway, Tromsø, Norway, ${ }^{6}$ Dept of Radiology, Haukeland University Hospital, Bergen, Norway, ${ }^{7}$ Dept of Clinical Sciences, University of Bergen, Bergen, Norway, ${ }^{8}$ Department of Radiology, Haukeland University Hospital, Bergen, Norway, ${ }^{9}$ Dept Physics and Technology, University of Bergen, Bergen, Norway

The feasibility of DCE-MRI as a tool to investigate perfusion of temporomandibular joints (TMJs) in case of Juvenile Idiopathic Arthritis (JIA) in children is investigated. The hypothesis in this current study is that inflammation is associated with increased vascularity and is the origin of experienced pain. Contrary to previous studies, high temporal resolution ( $4 \mathrm{~s})$ dynamic DCE-MRI using advanced pharmacokinetic models are for the first time applied when imaging the TMJ in JIA children aged 6-15. Results of deconvolution show that there is a difference in perfusion parameters between affected and unaffected patients, especially when permeability-surface area product (PS) and blood plasma flow (Fp) parameters are combined.

Quantitative Modeling of Sequence Parameter Choices to Support Standardization for Quantitative DCE-MRI

Jakob Meineke ${ }^{1}$ and Jochen Keupp ${ }^{1}$

${ }^{1}$ Philips Research Europe, Hamburg, Germany

Systematic and statistical errors in quantitative DCE-MRI measurements which adhere to standardization recommendations by the Quantitative Imaging Biomarker Alliance (QIBA) of the RSNA are assessed using EPG simulations. It is found that small sequence parameter changes, well within the bounds allowed by QIBA, can produce large changes in the estimated quantitative parameters.

Incorporating Bolus Arrival Time Offset into Fast Linear Analysis Could Shorten Acquisition Times for DCE-MRI

Sharon Peled ${ }^{1}$, Ron Kikinis ${ }^{1}$, Fiona Fennessy ${ }^{1}$, and Andrey Fedorov ${ }^{1}$

2182 'Brigham and Women's Hospital, Boston, MA, United States

Linearization of the Kety/Tofts model for DCE analysis drastically shortens computation time. We show that addition of bolus arrival time (BAT) compensation to the linearized analysis could also allow quicker acquisition times. With BAT inclusion, 3 minute sequences yield equivalent parameter estimation accuracy to 5 minute sequences without BAT compensation. The combination of shorter acquisition and real-time analysis would reduce the general time burden of DCE, which has potential implications for increased patient turnaround, and making DCE more acceptable as a tool, for example in evaluating response to therapy or in image guided therapy. 
${ }^{1}$ Electrical Engineering Department, University of Southern California, Los Angeles, CA, United States, ${ }^{2}$ Department of Radiological Sciences \& Physics and Biology in Medicine IDP, University of California, Los Angeles, Los Angeles, CA, United States, ${ }^{3}$ Imaging Physics, Sanford Health, Fargo, ND, United States

Spatial inhomogeneity in the transmitted RF introduces bias and increased variance in quantitative DCE-MRI metrics, which can dominate all other sources of error if uncorrected. The amount and pattern of inhomogeneity depends on the RF coil geometry, the driving circuits, and the vendor-specific pre-scan calibration. In this work, we (1) constructed human tissuemimicking torso and brain phantoms, (2) measured and compared the spatial RF transmit inhomogeneity across different scanners, vendors, and sites, and (3) evaluated vendorrecommended methods for RF transmit measurement.

Measuring transient $\mathrm{T}^{*}$ changes in vivo to validate Dynamic Distributed Spirals, a novel DSC-perfusion method

Dallas C Turley ${ }^{1}$ and James G Pipe ${ }^{2}$

${ }^{1}$ Department of Radiology, University of Chicago, Chicago, IL, United States, ${ }^{2}$ MR Technology Design Group, Barrow Neurological Institute, Phoenix, AZ, United States

Validating new contrast-enhances sequences is problematic, as risks associated with gadolinium contrast agents generally preclude testing in healthy volunteers. The Dynamic Distributed Spirals trajectory (DDS) is a promising new dynamic susceptibility-contrast (DSC)-perfusion method. In this work, we show that DDS is capable of measuring the transient $\mathrm{T}^{*}$ changes induced by breathholding which are much lower in magnitude than the susceptibility changes induced by contrast agent transit in conventional DSC-perfusion experiments.

Diffusion dependency of oxygenation measurements obtained with Vessel Architectural Imaging

Ingrid Digernes ${ }^{1}$, Atle Bjørnerud ${ }^{1,2}$, Grete Løvland ${ }^{1}$, Einar Vik-Mo ${ }^{3}$, Torstein Meling ${ }^{3,4}$, and Kyrre Eeg Emblem ${ }^{1}$

${ }^{1}$ Oslo University Hospital, Oslo, Norway, ${ }^{2}$ Department of Physics, University of Oslo, Oslo, Norway, ${ }^{3}$ Department of Neurosurgery, Oslo University Hospital, Oslo, Norway, ${ }^{4}$ Institute of Clinical Medicine, University of Oslo, Oslo, Norway

With the dual echo DSC-based technique Vessel Architectural Imaging (VAI), measurement of oxygenation level ( $\Delta$ SO2) can be obtained. However, how the $\Delta$ SO2-parameter is influenced by diffusion have previously not been investigated. Based on simulations, we show that the measured $\Delta \mathrm{SO} 2$ obtained from VAI have a diffusion dependency proportional to $1 /$ $\sqrt{ } \mathrm{D}$. ADC-maps from 10 glioblastoma patients were used to display the range correction factors in white matter and tumor regions. In conclusion, the diffusion dependency should be corrected for to obtain more accurate measurements of $\Delta \mathrm{SO}$, and may be especially relevant for brain diseases with aberrant diffusion characteristics.

Feasibility of measuring subtle Blood-Brain Barrier permeability change with reduced scan time using Dynamic Contrast-Enhanced Magnetic Resonance Imaging

Jonghyun Bae ${ }^{1,2,3}$, Jin Zhang ${ }^{2,3}$, Youssef Zaim Wadghiri ${ }^{2,3}$, Atul Singh Minhas ${ }^{4}$, Harish Poptani ${ }^{4}$, Yulin $^{2,3}$, and Sungheon Gene Kim²,3

${ }^{1}$ Sackler Institute of Graduate Biomedical Science, New York University School of Medicine, New York, NY, United States, ${ }^{2}$ Center for Biomedical Imaging, Radiology, New York University School of Medicine, New York, NY, United States, ${ }^{3}$ Center for Advanced Imaging Innovation and Research, Radiology, New York University School of Medicine, New York, NY, United States, ${ }^{4}$ Centre for Preclinical Imaging, Institute of Translational Medicine, University of Liverpool, liverpool, United Kingdom

The purpose of this study is to evaluate the feasibility of using a new contrast kinetic model to accurately measure changes in the low permeability of the blood-brain barrier due to the subtle vascular disruption in the development of neurodegenerative diseases. Our proposed kinetic model, named extended Patlak model (EPM), includes the plasma flow from the artery to capillary bed, which allows the accurate description of intake dynamics. We hypothesize that this extension allows EPM to estimate the permeability change more accurately than the conventional Patlak model (PM) with a reduced scan-time of around $10 \mathrm{~min}$

Cerebral Perfusion Imaging: The Vascular Territory of Middle Cerebral Artery is Optimal for Automatic Arterial-Input-Function Selection

Irene Klærke Mikkelsen ${ }^{1}$ and Simon Fristed Eskildsen

${ }^{1}$ Center for Functionally Integrative Neuroscience, Aarhus University, Aarhus, Denmark

A key issue in cerebral perfusion imaging is the selection of an arterial input function (AIF). AIF shape-properties have been used as criteria for automatic AIF selection. This study compares three brain regions for AIF target areas. The Middle Cerebral Artery (MCA) -M1 segment, the MCA-vascular territory and whole-brain. The prior displayed high noise levels, while the latter produced AIFs delayed compared to GM/WM tissue. The MCA-vascular territory is suggested as a region of interest for automatic AlF detection 
Brain tumor dynamic susceptibility contrast (DSC) MRI is adversely impacted by contrast agent leakage that results in confounding $T_{1}$ and $T_{2}{ }^{*}$ effects. While multi-echo acquisitions remove $T_{1}$ leakage effects, there is no consensus on the optimal set of acquisition parameters. Using a validated DSC-MRI digital reference object (DRO), we assessed the influence of preload dosing, pulse sequence parameters (number of echoes, TEs, TR, FA), and leakage correction method on cerebral blood volume (CBV) accuracy. This computational approach permits the systematic evaluation of a wide range of acquisition strategies to determine the optimal multi-echo DSC-MRI perfusion protocol.

Incremental modeling in DCE-MRI in gliomas

Magne Kleppestø ${ }^{1}$, Christopher Larsson ${ }^{1}$, and Atle Bjørnerud ${ }^{1}$

${ }^{1}$ Oslo University Hospital, Oslo, Norway

This work compares three kinetic models for evaluation of DCE-MRI in high-grade gliomas: the Tofts-Kermode (TK) model, the extended Tofts model (ETM) and the two-compartment exchange (TCE) model. 25 patients underwent a combined 238 examinations, and kinetic analysis was performed using the three models. In tumor regions where the data was better fitted using TK or TCE, median $K^{\text {trans }}$ estimates obtained from this model was compared to that from using ETM. It was found that in tumor regions in which TCE provides the best fit, median $K^{\text {trans }}$ was significantly underestimated when applying ETM.

Traditional Poster

\section{Quantitative Susceptibility Mapping}

COSMOS for Estimating Variation in Single Orientation Quantitative Susceptibility Mapping of the Brain: An Ultra High Field Study

Jon O Cleary ${ }^{1}$, Hongfu Sun ${ }^{2}$, Rebecca Glarin ${ }^{1}$, Peter Yoo ${ }^{1}$, Bradford A Moffat ${ }^{1}$, Roger J Ordidge ${ }^{1}$, and Scott C Kolbe ${ }^{1}$

${ }^{1}$ Melbourne Brain Centre Imaging Unit, Department of Anatomy and Neuroscience, University of Melbourne, Parkville, Australia, ${ }^{2}$ Department of Radiology, University of Calgary, Calgary, $A B$, Canada

The purpose of this study was to use high resolution Calculation of susceptibility through Multiple Orientation Sampling (COSMOS) reconstructed QSM (QSMC) as a gold standard to estimate the variation, distribution and magnitude of a single orientation QSM reconstruction pipeline. QSMc processing is an emerging technique for overcoming artefacts characteristic of single orientation QSM (QSMs). However it requires at least 4 fold increases in image acquisition times or reductions in resolution and SNR. We sought to produce high resolution QSMc reference datasets from healthy subjects to quantify the differences from QSMs values across a variety of cortical and subcortical brain regions.

Evaluating the Precision of Multi-Echo Combination Methods for Susceptibility Mapping by Analysing the Propagation of Single-Echo Phase Noise into Multi-Echo Field and Susceptibility Maps

Emma Biondetti ${ }^{1}$, Anita Karsa ${ }^{1}$, David L Thomas ${ }^{2,3}$, and Karin Shmueli ${ }^{1}$

${ }^{1}$ Medical Physics and Biomedical Engineering, University College London, London, United Kingdom, ${ }^{2}$ Academic Neuroradiological Unit, Department of Brain Repair and Rehabilitation, Institute of Neurology, University College London, London, United Kingdom, ${ }^{3}$ Leonard Wolfson Experimental Neurology Centre, Institute of Neurology, University College London, London, United Kingdom

In Susceptibility Mapping (SM) using multi-echo acquisitions, noise propagates from the single-echo phase images into the field map in a manner dependent on the method used fo multi-echo combination. Field noise then propagates into the susceptibility map, determining the precision of the measured susceptibility. Here, we characterised the propagation of single-echo phase noise into both the combined field and susceptibility maps using three methods for multi-echo combination: fitting, averaging and echo time-weighted averaging. We calculated susceptibility noise maps for both simulated and acquired data, showing that, when choosing a pipeline for multi-echo SM, it is important to consider its precision.

Ashmita De ${ }^{1}$, Hongfu Sun ${ }^{1}$, Ahmed Elkady ${ }^{1}$, Peter Seres ${ }^{1}$, and Alan H Wilman 
Typical Quantitative Susceptibility Mapping (QSM) sequences have a long acquisition time which may yield motion artifacts that alter magnitude, phase and susceptibility values in the brain. Simulations and motion experiments were conducted on patients suspected of stroke and healthy volunteers to calculate the variations of susceptibility, magnitude and local field in brain. Variations between susceptibility and magnitude images were compared. In general, magnitude images were found to be more affected by motion than QSM in the brain areas studied.

Fast Zoomed QSM of the Human Midbrain at 7T

Kyungmin Nam ${ }^{1,2,3,4}$, Namgyun Lee ${ }^{1}$, Anouk Marsman ${ }^{1}$, Vincent Oltman Boer ${ }^{1}$, Chulhyun Lee ${ }^{3,4}$, and Esben Thade Petersen ${ }^{1,2}$

${ }^{1}$ Danish Research Center for Magnetic Resonance, Centre for Functional and Diagnostic Imaging and Research, Copenhagen University Hospital Hvidovre, Hvidovre, Denmark, ${ }^{2}$ Center for Magnetic Resonance, Department of Electrical Engineering, Technical University of Denmark, Lyngby, Denmark, ${ }^{3}$ Bio-Imaging Research Team, Korea Basic Science Institute, Cheongju, Republic of Korea, ${ }^{4}$ Bio-Analysis Science, University of Science and Technology, Daejeon, Republic of Korea

In this work, zoomed quantitative susceptibility mapping (QSM) is proposed as an alternative way of accelerating high resolution QSM data acquisition at 7T. Inner volume excitation is realized with $2 \mathrm{D}$ spatially selective excitation, targeting the midbrain, which is the primary region of investigation for Parkinson's disease. The consequence of reducing the excited region on the reconstructed susceptibility maps was investigated via simulations, where the diameter of a brain mask was gradually decreased in the QSM processing pipeline. The susceptibility maps of a healthy volunteer at $7 \mathrm{~T}$ acquired with inner volume excitation are compared to those derived from a whole brain.

Phase Corrected Bipolar Acquisition for Simultaneous Water-Fat Separation and Quantitative Susceptibility Mapping of the Carotid Artery Wall

Pascal P R Ruetten ${ }^{1}$, Andrew N Priest ${ }^{1,2}$, Jianmin Yuan ${ }^{1}$, Ammara Usman $^{1}$, Jonathan H Gillard ${ }^{1}$, and Martin J Graves ${ }^{2}$

${ }^{1}$ Department of Radiology, University of Cambridge, Cambridge, United Kingdom, ${ }^{2}$ Department of Radiology, Cambridge University Hospitals NHS Foundation Trust, Cambridge, United Kingdom

In this work we investigated the feasibility and assessed the performance of a bipolar compared to a unipolar gradient echo readout for a combined method of water-fat separation and quantitative susceptibility mapping for application in the carotid artery wall.

Simultaneous quantification of fat fraction, susceptibility and R2* from a single GRE acquisition: flip-angle effects

Junmin Liu ${ }^{1}$, Spencer Christiansen ${ }^{1,2}$, and Maria Drangova ${ }^{1,2}$

${ }^{1}$ Imaging Research Laboratories, Robarts Research Institute, Schulich School of Medicine \& Dentistry, University of Western Ontario, London, ON, Canada, ${ }^{2}$ Medical Biophysics, Schulich School of Medicine \& Dentistry, University of Western Ontario, London, ON, Canada

We report on a systematic investigation of the flip-angle (FA) effects on the quantification of fat fraction (FF), susceptibility, and R2* simultaneously from a single multi-echo GRE (mGRE) acquisition. Using a phantom with a range of oil-water emulsions and aqueous Gadolinium solutions we tested five different FAs $\left(1^{\circ}, 3^{\circ}, 5^{\circ}, 8^{\circ}\right.$ and $\left.15^{\circ}\right)$ with a bipolar $\mathrm{mGRE}$ protocol and were able to successfully generate the FF, susceptibility and R2* maps for all cases. Our results demonstrate that a single mGRE scan with optimized TEs has the potential to accurately measure quantitative FF, susceptibility, and R2* with a FA of $8^{\circ}$.

Quantitative Susceptibility Mapping with Silent 3D Radial T2* Acquisition

Mauro Costagli ${ }^{1}$, Ana Beatriz Solana ${ }^{2}$, Guido Buonincontri ${ }^{1}$, Florian Wiesinger ${ }^{2}$, Michela Tosetti ${ }^{1}$, and Rolf F Schulte ${ }^{2}$

${ }^{1}$ Imago 7 Research Center, IRCCS Stella Maris, Pisa, Italy, ${ }^{2}$ ASL Europe, GE Healthcare, Munich, Germany

Recent implementations of radial Zero Echo Time (ZTE) techniques are capable of providing T2*-weighted signal. Quantitative Susceptibility Mapping (QSM) using such techniques might have several potential advantages, such as (i) robustness to head motion, flow artifacts and geometrical distortions, (ii) improved sampling efficiency, (iii) reduced acoustic noise, (iv) simultaneous acquisition of proton-density data. We assessed the QSMs obtained with two different silent radial techniques, and their accuracy was similar to that of QSM obtained with conventional scanning schemes, which encourages the development of ZTE-based techniques specifically tailored for efficient and silent QSM, to achieve important advantages in clinical applications.

Kevin Koch ${ }^{1}$, Tugan Muftuler ${ }^{2}$, Robin Karr ${ }^{1}$, and Andrew Nencka ${ }^{1}$

${ }^{1}$ Radiology, Medical College of Wisconsin, Wauwatosa, WI, United States, ${ }^{2}$ Medical College of Wisconsin, Wauwatosa, WI, United States 
One of the remaining translational challenges in QSM is the need for post-processing algorithms that are rapid, robust, and accurate. Here, we present an alternative formulation of the QSM inversion problem. The field-to-source inversion is divided into a multi-resolution decomposition, whereby each resolution stage is divided into small independent processing regions. The basic premise of this concept is the isolate local susceptibility fields and sources at varying levels of resolution. When the susceptibility problem is divided in this fashion, field-to-source inversions can occur in regions of very volumetric matrix sizes (with varying voxel sizes per inversion). After inverting each of the sub-volumes, a combination procedure is implemented to combine the volumes and the resolution layers. Due to the small size of the inversion volumes, the dimensionality of the problem lends itself to the use of convolutional neural network modeling and application.

DeepQSM - Solving the Quantitative Susceptibility Mapping Inverse Problem Using Deep Learning

Mads Kristensen ${ }^{1}$, Kasper Gade Bøtker Rasmussen ${ }^{1}$, Rasmus Guldhammer Blendal ${ }^{1}$, Lasse Riis Østergaard ${ }^{1}$, Maciej Plocharski ${ }^{1}$, Andrew Janke ${ }^{2}$, Christian Langkammer ${ }^{3}$, Kieran O'Brien ${ }^{2,4}$, Markus Barth ${ }^{2}$, and Steffen Bollmann ${ }^{2}$

${ }^{1}$ Department of Health Science and Technology, Aalborg University, Aalborg, Denmark, ${ }^{2}$ Centre for Advanced Imaging, University of Queensland, Brisbane, Australia, ${ }^{3}$ Department of Neurology, Medical University of Graz, Graz, Austria, ${ }^{4}$ Siemens Healthcare Pty Ltd, Brisbane, Australia

Quantitative susceptibility mapping (QSM) aims to extract the magnetic susceptibility of tissue by solving an ill-posed field-to-source-inversion. Current QSM algorithms require manual parameter choices to balance between smoothing, artifacts and quantitation accuracy. Deep neural networks have been shown to perform well on ill-posed problems and can find optimal parameter sets for a given problem based on real-world training data. We have developed a proof-of-concept fully convolutional deep network capable of solving QSM's ill-posed field-to-source inversion that preserves fine spatial structures and delivers accurate quantitation results.

Reconstruction of Quantitative Susceptibility Maps using Annihilating Filter-Based Low-Rank Hankel Matrix Approach

Hyun-Seo Ahn ${ }^{1}$, Sung-Hong Park ${ }^{1}$, and Jong Chul Ye ${ }^{1}$

${ }^{1}$ Department of Bio and Brain Engineering, Korea Advanced Institute of Science and Technology, Daejeon, Republic of Korea

In this study, we proposed a novel QSM image reconstruction algorithm using annihilating filter-based low-rank hankel matrix (ALOHA) approach. Unlike the conventional algorithm that requires additional anatomical information, the proposed method estimates susceptibility map using direct 3-D k-space domain interpolation in the Fourier domain. The proposed method showed superior performance over the conventional methods (SWIM, TSVD, TKD, MEDI, and TVSB) in a numerical phantom and in-vivo human brains.

Magnetic susceptibility source separation using multi-echo GRE data only

Taehyun Hwang ${ }^{1}$, Jingu Lee ${ }^{1}$, Hyeong-Geol Shin ${ }^{1}$, Doohee Lee ${ }^{1}$, Joon Yul Choi ${ }^{1}$, Hyunsung Eun ${ }^{1}$, Yoonho Nam ${ }^{2}$, and Jongho Lee ${ }^{1}$

${ }^{1}$ Electrical and Computer Engineering, Seoul National University, Seoul, Republic of Korea, ${ }^{2}$ Department of Radiology, Seoul St. Mary's Hospital, Colleg of Medicine, The Catholic University of Korea, Seoul, Republic of Korea

In this work, we explored an alternative approach of using nominal $\$ \$ \$ R \_2^{\wedge}\left\{1::^{\prime}\right\} \$ \$$ instead of measured $\$ \$ \$ R \quad 2^{\wedge}\{1: '\} \$ \$$ in separating the two susceptibility sources. The linear relationship between $\$ \$ \$ R \_2^{\wedge}\left\{{ }^{*}\right\} \$ \$$ and $\$ \$ \$ R \_2^{\wedge}\left\{1::^{\prime}\right\} \$ \$$ was investigated and used to obtain the nominal $\$ \$ \$ R \_2^{\wedge}\left\{1:{ }^{\prime}\right\} \$ \$$ values. The positive and negative magnetic susceptibility source maps using nominal $\$ \$ \$ R \_2^{\wedge}\left\{:^{\prime}\right\} \$ \$ \$$ showed similar susceptibility distribution to the map using measured $\$ \$ \$ R \_2^{\wedge}\{l: '\} \$ \$$.

Fast and accurate reconstruction for susceptibility source separation in QSM

Seyoon Ko ${ }^{1}$, Jingu Lee ${ }^{2}$, Joong-Ho Won ${ }^{1}$, and Jongho Lee ${ }^{2}$

${ }^{1}$ Department of Statistics, Seoul National University, Seoul, Republic of Korea, ${ }^{2}$ Laboratory for Imaging Science and Technology, Department of Electrical and Computer Engineering Seoul National University, Seoul, Republic of Korea

We investigate fast and accurate reconstruction methods for susceptibility source separation (S3) in quantitative susceptibility mapping (QSM). S3 separates positive and negative susceptibility sources within a voxel utilizing signal relaxation (R2') for dipole inversion. We propose new primal-dual (PD) methods for S3 and compare them with the alternating GaussNewton conjugate gradient (A-GNCG). A-GNCG alters the energy functional, and furthermore its convergence is not guaranteed. In contrast, the proposed PD methods are exact and have convergence guarantees. Validation on a simulated phantom and in-vivo data shows that the PD methods converge faster with better accuracies. 
${ }^{1}$ Department of Electrical Engineering, Pontificia Universidad Catolica de Chile, Santiago, Chile, ${ }^{2}$ Biomedical Imaging Center, Pontificia Universidad Catolica de Chile, Santiago, Chile, ${ }^{3}$ Martinos Center for Biomedical Imaging, Harvard Medical School, Charlestown, MA, United States, ${ }^{4}$ Department of Neurology, Medical University of Graz, Graz, Austria, ${ }^{5}$ Wellcome Centre for Human Neuroimaging, UCL Institute of Neurology, University College London, London, United Kingdom

In the context of QSM, the background pre-filtering step often leaves remnants in the local field, particularly in the vicinity of trustable-region boundary. Since such remnant fields must satisfy Laplace's equation, i.e. they must be harmonic functions within the ROI, we propose a new regularization term based on a weak-harmonics formulation (WH-QSM) to remove spurious non-local components during inversion. The WH extension resulted in more accurate and reproducible results than conventional total-variation (TV) regularized QSM.

Nonlinear projection onto dipole fields with preconditioning (nPDF)

Carlos Milovic ${ }^{1,2}$, Berkin Bilgic ${ }^{3}$, Bo Zhao ${ }^{3}$, Christian Langkammer ${ }^{4}$, Julio Acosta-Cabronero ${ }^{5}$, and Cristian Tejos ${ }^{1,2}$

${ }^{1}$ Department of Electrical Engineering, Pontificia Universidad Catolica de Chile, Santiago, Chile, ${ }^{2}$ Biomedical Imaging Center, Pontificia Universidad Catolica de Chile, Santiago, Chile, ${ }^{3}$ Martinos Center for Biomedical Imaging, Harvard Medical School, Charlestown, MA, United States, ${ }^{4}$ Department of Neurology, Medical University of Graz, Graz, Austria, ${ }^{5}$ Wellcome Centre for Human Neuroimaging, UCL Institute of Neurology, University College London, London, United Kingdom

QSM requires to remove fields originated outside a region of interest prior to inversion. This is prone to generating artifacts due to noise and error propagation from previous processing steps such as coil combination or phase unwrapping. To address this, we reformulated the widely used projection onto dipole fields (PDF) method as a nonlinear problem with preconditioning. This new formalism is wrap-insensitive, results in improved noise/error management, and might enable a more straightforward implementation of multi-coil/-echo combination and background removal steps into a single optimizer

Background Field Removal for Large Susceptibility Anatomical Structures in Human Brain with Orientation Variations

Jinsheng Fang ${ }^{1}$, Lijun Bao ${ }^{1}$, and Zhong Chen ${ }^{1}$

${ }^{1}$ Department of Electronic Science, Xiamen University, Xiamen, China

We propose a novel background field removal method for large susceptibility anatomical structures, e.g., tissues around paranasal sinuses and interfaces of the tissue and skull, under various scanning orientations. The proposed method employs the gradient and magnitude of the phase map, combined with a normalized wrap count. Experimental results were both validated on four-orientation numerical simulation and in vivo human brain, which demonstrated the proposed method suppressed the residual phase error better than the other methods.

Suitable image quality measures to evaluate quantitative susceptibility maps

Janis Stiegeler ${ }^{1}$ and Sina Straub ${ }^{1}$

${ }^{1}$ Medical Physics in Radiology, German Cancer Research Center (DKFZ), Heidelberg, Germany

The 2016 QSM Reconstruction Challenge urged the need for a suitable quality measure of susceptibility maps as classical image quality measures (root-mean-square error, highfrequency error-norm, structural similarity index) were no suitable indicators of the visual quality of susceptibility maps. Errors (noise, smoothing, streaking) were added to a reference susceptibility map and the sharpness-index-weighted structural similarity index was used to evaluate the degraded quantitative susceptibility maps and to compare the result with classical image quality measures. The sharpness-index-weighted structural similarity index was shown to be a suitable measure for QSM image quality with a strong devaluation of oversmoothed images.

An automatically referenced quantitative susceptibility mapping algorithm: QSMauto_ref

Jingu Lee ${ }^{1}$, Taehyun Hwang ${ }^{1}$, Yoonho $\mathrm{Nam}^{2}$, Se-Hong $\mathrm{Oh}^{3}$, and Jongho Lee ${ }^{4}$

${ }^{1}$ Department of Electrical and Computer Engineering, Seoul National University, Seoul, Republic of Korea, ${ }^{2}$ Department of Radiology, Seoul St. Mary's Hospital, College of Medicine, The Catholic University of Korea, Seoul, Republic of Korea, ${ }^{3}$ Division of Biomedical Engineering, Hankuk University of Foreign Studies, Yongin, Republic of Korea, ${ }^{4}$ Seoul National University, Seoul, Republic of Korea

We proposed a new QSM algorithm that automatically sets CSF as a susceptibility reference. The algorithm utilizes susceptibility effects on $\mathrm{R}_{2}{ }^{\prime}$ as a regularization term. The proposed algorithm does not require either segmentation of CSF or a well-refined brain mask and, therefore, can be used reliably.

Jingu Lee ${ }^{1}$, Taehyun Hwang ${ }^{1}$, Yoonho Nam² ${ }^{2}$ Jinhee Jang ${ }^{2}$, Woojun Kim ${ }^{3}$, Se-Hong $\mathrm{Oh}^{4}$, Masaki Fukunaga ${ }^{5}$, and Jongho Lee ${ }^{1}$ 
${ }^{1}$ Department of Electrical and Computer Engineering, Seoul National University, Seoul, Republic of Korea, ${ }^{2}$ Department of Radiology, Seoul St. Mary's Hospital, College of Medicine, The Catholic University of Korea, Seoul, Republic of Korea, ${ }^{3}$ Department of Neurology, Seoul St. Mary's Hospital, College of Medicine, The Catholic University of Korea, Seoul, Republic of Korea, ${ }^{4}$ Division of Biomedical Engineering, Hankuk University of Foreign Studies, Yongin, Republic of Korea, ${ }^{5}$ Division of Cerebral Integration, National Institute for Physiological Sciences, Okazaki, Japan

Magnetic susceptibility source separation is a recently proposed technique that generates positive and negative susceptibility maps corresponding to iron and myelin distributions in the brain. In this study, iron accumulation and myelin degradation in a few typical types of multiple sclerosis lesions were visualized using the magnetic susceptibility source separation method. Additionally, the well-known co-localization of iron and myelin in the Gennari line was demonstrated in an ex-vivo brain sample.

Structure tensor enhanced quantitative susceptibility mapping (ST-QSM)

Agnese Tamanti ${ }^{1}$, Kristian Bredies ${ }^{2}$, Marco Castellaro ${ }^{3}$, Stefan Ropele ${ }^{4}$, Berkin Bilgic ${ }^{5}$, and Christian Langkammer ${ }^{4}$

${ }^{1}$ University of Verona, Verona, Italy, ${ }^{2}$ Institute of Mathematics and Scientific Computing, University of Graz, Graz, Austria, ${ }^{3}$ Department of Information Engineering, University of Padova, Padova, Italy, ${ }^{4}$ Department of Neurology, Medical University of Graz, Graz, Austria, ${ }^{5}$ Athinoula A. Martinos Center for Biomedical Imaging, Department of Radiology, Harvard Medical School, MGH, Boston, MA, United States

Quantitative susceptibility mapping (QSM) is an MRI technique enabling the reconstruction of a basic physical property in vivo. However, retrieving susceptibility maps from the MRI phase data requires an ill-posed inverse problem to be solved, which is often achieved using regularization approaches. In this abstract, we extend an existing QSM algorithm by incorporating weights from the linear structure tensor (ST) of the magnitude images to stabilize the regularization. The new algorithm yields improvements regarding the visual appearance and the quantitative performance of the susceptibility maps obtained.

BuckyBall: Reproducible gradient-echo MRI measurements with variable magnetic field directions

Enrico Kaden ${ }^{1}$, Irina Y. Barskaya ${ }^{2}$, Nathaniel D. Kelm², Mark D. Does ${ }^{2}$, and Daniel C. Alexander ${ }^{1}$

${ }^{1}$ Centre for Medical Image Computing, Department of Computer Science, University College London, London, United Kingdom, ${ }^{2}$ Institute of Imaging Science, Department of Biomedical Engineering, Vanderbilt University, Nashville, TN, United States

The direction of the external magnetic field is typically fixed, although it is well-known that the signal of various MR modalities in brain white matter depends on the magnetic field direction. This work presents a general framework for analysing $B_{0}$-direction dependent contrast. Specifically, we have developed a holder device, called BuckyBall, that enables the uniform orientation of the scanned object in a reproducible manner. Its feasibility and practicality are demonstrated in a multi-echo gradient-echo experiment with 50 unique magnetic field directions using a monkey brain sample.

Measurement of Iron Concentration in Deep Gray Matter Nuclei over the Lifespan Using Quantitative Susceptibility Mapping

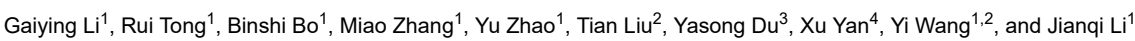

${ }^{1}$ Shanghai Key Laboratory of Magnetic Resonance and Department of Physics, East China Normal University, Shangha, China, ${ }^{2}$ Department of Radiology, Weill Medical College of Cornell University, New York, New York, NY, United States, ${ }^{3}$ Shanghai Mental Health Center, Shanghai Jiao Tong University School of Medicine, Shanghai, China, ${ }^{4}$ MR Collaboration NE Asia, Siemens Healthcare, Shanghai, China

Histological in vitro analysis has demonstrated that iron accumulation rates in various gray matter nuclei are different throughout an individual's lifetime. QSM provides excellent contrast of iron-rich deep nuclei to quantify iron in the brains. In this study, we investigated the linear and nonlinear correlation of magnetic susceptibility in the deep gray matter nuclei as a function of ageing using QSM. Compared with the published studies, the nonlinear analysis results showed the differential developmental trajectories of magnetic susceptibility in the deep gray matter nuclei over the lifespan.

Improved depiction of subthalamic nucleus and globus pallidus internus with optimized high-resolution quantitative susceptibility mapping at 7 Tesla

Fei Cong ${ }^{1,2}$, Yelong Shen ${ }^{3,4}$, Bo Wang ${ }^{1}$, Jing $\mathrm{An}^{5}$, Zihao Zhang ${ }^{1}$, Zhentao Zuo ${ }^{1}$, Yan Zhuo ${ }^{1}$, and Lirong Yan $^{3}$

${ }^{1}$ State Key Laboratory of Brain and Cognitive Science, Beijing MR Center for Brain Research, Institute of Biophysics, Chinese Academy of Sciences, Beijing, China, ${ }^{2}$ University of Chinese Academy of Sciences, Beijing, China, ${ }^{3}$ Laboratory of Functional MRI Technology (LOFT), Stevens Neuroimaging and Informatics Institute, Keck School of Medicine, University of Southern California, Los Angeles, CA, United States, ${ }^{4}$ Shandong Medical Imaging Research Institute, School of Medicine, Shandong University, Jinan, China, ${ }^{5}$ Siemens Shenzhen Magnetic Resonance Ltd., Shenzhen, China

Quantitative susceptibility mapping (QSM) shows a potential to image subthalamic nucleus (STN) and globus pallidus internus (GPi). However, the image quality of QSM is dependent on the selection of regularization parameter during reconstruction. Here we proposed an approach to determine the optimal regularization parameter for imaging the sub-cortical nuclei at different spatial resolution and field strengths. Optimized QSM images were further compared with the other susceptibility weighted images for visualization of STN and GPi at 3T and 7T. Our results suggest that optimized 7T QSM with spatial resolution of $0.35 \times 0.35 \times 1.0 \mathrm{~mm}^{3}$ provides better delineation of STN and GPi. 
High resolution MRI for functional and structural depiction of subthalamic nuclei in DBS pre-surgical mapping: a comparison between QSM and T2w

Alexey V. Dimov ${ }^{1,2}$, Ajay Gupta ${ }^{1}$, Brian H. Kopell ${ }^{3}$, and Yi Wang ${ }^{1,2}$

${ }^{1}$ Radiology, Weill Cornell Medical College, New York, NY, United States, ${ }^{2}$ Meinig School of Biomedical Engineering, Cornell University, Ithaca, NY, United States, ${ }^{3}$ Neurosurgery, Mount Sinai Health System, New York, NY, United States

In this work, we investigate the use of a sub-millimeter quantitative susceptibility mapping (QSM) protocol for preoperative imaging of the suthalamic nucleui (STN) for planning of deep brain stimulation (DBS). Image scoring revealed superior performance of QSM compared to the conventional T2 weighted (T2W) protocol. In contrast to T2W, image scores further increased for QSM when resolution was increased.

Magnetic susceptibility characterization of human habenula at 3T: comparison of QSM and R2*

Seulki Yoo ${ }^{1,2}$ and Seung-Kyun Lee ${ }^{1,2}$

${ }^{1}$ Department of Biomedical Engineering, Sungkyunkwan University, Suwon, Republic of Korea, ${ }^{2}$ IBS Center for Neuroscience Imaging Research, Suwon, Republic of Korea

To investigate the potential of magnetic susceptibility as a biomarker for habenula studies, we have obtained quantitative susceptibility maps (QSM) and R2* maps from 21 normal volunteers at high spatial resolution. Compared to R2* maps, QSM showed more conspicuous and localized contrast in habenula in about $75 \%$ of the volunteers. Measured susceptibility and $\mathrm{R} 2^{*}$ values exhibited clear positive correlation, indicating iron-dominance (as opposed to myelin) of the susceptibility contrast in habenula. Significant heterogeneity of the susceptibility contrast across the subjects and within the tissue appear to be a challenge for using QSM as a biomarker for human habenula research.

QSM susceptibility patterns and their clinical implications

Kelly Gillen ${ }^{1}$, Mayyan Mubarak ${ }^{2}$, Shun Zhang ${ }^{1}$, Somiah Dahlawi², Thanh D Nguyen ${ }^{1}$, David Pitt ${ }^{2}$, and Yi Wang ${ }^{1}$

Multiple sclerosis is an autoimmune disorder characterized by focal inflammatory demyelination. We combined quantitative susceptibility mapping (QSM) with histopathology on MS autopsy tissue to identify chronic activation of iron-positive macrophages/microglia. We demonstrate that the QSM susceptibility pattern gives insight into the lesion inflammatory state. Only rim positive lesions indicate smoldering inflammation in the presence of iron, and therefore are of particular relevance in the clinic.

Investigating the Effect of Prior Stroke on Regional Brain Iron Concentrations in Children with Sickle Cell Anaemia using MRI Susceptibility Mapping.

Russell Murdoch ${ }^{1}$, Jamie Kawadler ${ }^{2}$, Fenella Kirkham ${ }^{3}$, and Karin Shmueli ${ }^{1}$

${ }^{1}$ Medical Physics and Biomedical Engineering, University College London, London, United Kingdom, ${ }^{2}$ Imaging \& Biophysics Unit, UCL Institute of Child Health, London, United Kingdom, ${ }^{3}$ Neurosciences Unit, UCL Institute of Child Health, London, United Kingdom

Regional iron concentrations in the brains of children with Sickle Cell Anaemia (SCA) were examined using susceptibility mapping (SM), in the first study to apply SM to an African cohort in Tanzania. Mean susceptibility values in three deep-brain regions were compared to age, blood ferritin levels and history of clinical stroke. Mean susceptibility values increased linearly with age, but there was no significant correlation between susceptibility values and blood ferritin levels. SCA patients who had suffered stroke prior to MRI had significantly lower susceptibility values than stroke-free patients. This may suggest a role for iron deficiency in stroke in SCA.

Fast brain iron quantification using QSM with low spatial resolution

Xin Miao ${ }^{1}$, Krishna S Nayak ${ }^{1,2}$, and John C Wood ${ }^{1,3}$

${ }^{1}$ Biomedical Engineering, University of Southern California, Los Angeles, CA, United States, ${ }^{2}$ Ming Hsieh Department of Electrical Engineering, University of Southern California, Los Angeles, CA, United States, ${ }^{3}$ Cardiology, Children's Hospital Los Angeles, Los Angeles, CA, United States

This study investigates the impact of spatial resolution on QSM susceptibility mapping for brain iron quantification. We obtained 40 sub-millimeter resolution whole-brain QSM datasets, and simulated six levels of spatial resolution via k-space truncation. QSM-based iron quantification was performed at each spatial scale and compared against the reference. We found that estimation error was $\leq 5 \mathrm{ppb}$ in the basal ganglia when the voxel dimension along all three axes was $\leq 2.0 \mathrm{~mm}$. The finding suggests that scan time can be significantly shortened by reducing spatial resolution. 
QSM-MRI reveals increased brain iron deposition in anemia patients with blood transfusion

Xin Miao ${ }^{1}$, Soyoung Choi ${ }^{2}$, Krishna S Nayak ${ }^{1,3}$, and John C Wood ${ }^{1,4}$

${ }^{1}$ Biomedical Engineering, University of Southern California, Los Angeles, CA, United States, ${ }^{2}$ Neuroscience Graduate Program, University of Southern California, Los Angeles, CA, United States, ${ }^{3}$ Ming Hsieh Department of Electrical Engineering, University of Southern California, Los Angeles, CA, United States, ${ }^{4}$ Cardiology, Children's Hospital Los Angeles, Los Angeles, CA, United States

Sickle cell patients identified with high stroke risks and other genetically anemic patients are treated with chronic blood transfusions. Unfortunately, transfusions may cause iron overload. While transfusion-related iron overload has been shown in other major organs, less has been explored whether it impacts brain. This study compares brain iron content measured by quantitative susceptibility mapping (QSM) in 17 healthy controls and 33 patients with sickle cell or other types of anemia. We found significantly higher iron in the putamen of anemia patients receiving blood transfusion. The result of this study can provide insights on the neurological effects of blood transfusions.

Are all susceptibility maps created equal? - An investigation of the impact of the field-to-source inversion step on the study outcome in patient-control group studies.

Poonam Choudhary ${ }^{1,2}$, Niels Bergsland ${ }^{2,3}$, Akshay V Dhamankar ${ }^{2}$, Michael G. Dwyer ${ }^{2}$, Bianca Weinstock-Guttman ${ }^{4}$, Robert Zivadinov 2,5 , and Ferdinand Schweser 2,5

${ }^{1}$ Department of Medical Physics, University at Buffalo, The State University of New York, Buffalo, NY, United States, ${ }^{2}$ Buffalo Neuroimaging Analysis Center, Department of Neurology, Jacobs School of Medicine and Biomedical Sciences, University at Buffalo, The State University of New York, Buffalo, NY, United States, Buffalo, NY, United States, ${ }^{3}$ MR Research Laboratory, IRCCS, Don Gnocchi Foundation ONLUS, Milan, Italy, ${ }^{4}$ BairdMS Center, Department of Neurology, Jacobs School of Medicine and Biomedical Sciences, University at Buffalo, The State University of New York, Buffalo, NY, United States, ${ }^{5}$ Center for Biomedical Imaging, Clinical and Translational Science Institute, University at Buffalo, The State University of New York, Buffalo, NY, United States

Quantitative Susceptibility Mapping (QSM) is a relatively new post-processing technique for susceptibility-weighted gradient-recalled echo (GRE) phase images. The technique numerically solves an ill-posed inverse mathematical problem to reveal the tissue magnetic susceptibility distribution. Due to its uniquely high sensitivity on the tissue concentrations of myelin, calcium and iron, QSM is increasingly being applied in clinical studies of neurological diseases that are affected by demyelination and a disturbed iron homeostasis, such as multiple sclerosis (MS) and Parkinson's disease. In the present work, to better understand the comparability and reproducibility of QSM studies, we evaluated several widely-used inversion algorithms concerning their ability to detect differences in susceptibility between two different groups of subjects, a typical scenario in clinical research.

Reproducibility of Quantitative Susceptibility Mapping and R2* Mapping of the Human Brain at 7T: a Multi-Centre Pilot Study

Catarina Rua ${ }^{1}$, William T Clarke ${ }^{2}$, lan D Driver ${ }^{3}$, Olivier Mougin ${ }^{4}$, Stuart Clare ${ }^{2}$, Susan Francis ${ }^{4}$, Keith Muir ${ }^{5}$, Richard Wise ${ }^{3}$, Guy Williams ${ }^{1}$, Richard Bowtell ${ }^{4}$, and Adrian Carpenter ${ }^{1}$

${ }^{1}$ Department of Clinical Neurosciences, University of Cambridge, Wolfson Brain Imaging Centre, Cambridge, United Kingdom, ${ }^{2}$ University of Oxford, Welcome Centre for Integrative Neuroimaging (FMRIB), Oxford, United Kingdom, ${ }^{3}$ School of Psychology, Cardiff University, Cardiff University Brain Research Imaging Centre, Cardiff, United Kingdom, ${ }^{4}$ University of Nottingham, Sir Peter Mansfield Imaging Centre, Nottingham, United Kingdom, ${ }^{5}$ University of Glasgow, Institute of Neuroscience \& Psychology, Glasgow, United Kingdom

To perform cost-effective research with high-field imaging by increasing the size of the patient pool in investigations of brain diseases, it is important to guarantee cross-site reproducibility and consistency of the QSM and R2* results. This study is part of a pilot "travelling-heads" experiment from the UK7T network, in which we aim to develop harmonized approaches for T2*-weighted imaging in order to provide a framework for future multi-centre clinical studies at $7 \mathrm{~T}$.

Quantitative Susceptibility Mapping at high and ultra-high field: a reproducibility study

Marta Lancione ${ }^{1,2}$, Michela Tosetti ${ }^{2,3}$, Paolo Cecchi ${ }^{4}$, Graziella Donatelli ${ }^{5}$, Mirco Cosottini ${ }^{2,4,5}$, and Mauro Costagli ${ }^{2,3}$

${ }^{1}$ IMT School for Advanced Studies, Lucca, Italy, ${ }^{2}$ IMAGO7 Research Center, Pisa, Italy, ${ }^{3}$ IRCCS Stella Maris, Pisa, Italy, ${ }^{4}$ Unit of Neuroradiology, AOUP, Pisa, Italy, ${ }^{5}$ University of Pisa, Pisa, Italy

The aim of this study is to assess the reproducibility of Quantitative Susceptibility Mapping (QSM), which is crucial to enable the application of this technique in clinical follow-up and multi-center studies. Five healthy subjects underwent multiple QSM acquisition sessions using two MRI systems at different field strength (3T and 7T). Both voxel-wise and automated atlas-based ROI analyses proved the goodness of intra-scanner repeatability and inter-scanner reproducibility, the latter being slightly weaker than the former.

Ruiyang Zhao ${ }^{1,2}$, Valentina Taviani ${ }^{3}$, Shreyas Vasanawala ${ }^{4}$, Scott B. Reeder ${ }^{1,2,5,6,7}$, and Diego Hernando ${ }^{1,2}$

${ }^{1}$ Medical Physics, University of Wisconsin-Madison, Madison, WI, United States, ${ }^{2}$ Radiology, University of Wisconsin-Madison, Madison, WI, United States, ${ }^{3}$ Global MR Applications \& Workflow, GE Healthcare, Menlo Park, CA, United States, ${ }^{4}$ Radiology, Stanford University, Palo Alto, CA, United States, ${ }^{5}$ Biomedical Engineering, University of Wisconsin-Madison, Madison, WI, United States, ${ }^{6}$ Medicine, University of Wisconsin-Madison, Madison, WI, United States, ${ }^{7}$ Emergency Medicine, University of Wisconsin-Madison, Madison, WI, United States 
Accurate quantification of liver iron concentration (LIC) is needed for the assessment of iron overload. Quantification of magnetic susceptibility may enable accurate and reproducible estimation of LIC. SQUID-based biomagnetic liver susceptometry (BLS) is used clinically to measure magnetic susceptibility, but has very limited availability. MRI-based Quantitative Susceptibility Mapping (QSM) may enable liver susceptometry with much broader availability. However, the accuracy of QSM-BLS across field strengths remains unknown. In this abstract, we observed strong correlation $\left(r^{2}=0.90\right)$ between QSM-BLS (at both 1.5T and 3.0T) with SQUID-BLS in patients with known or suspected iron overload.

Traditional Poster

\section{CEST: Novel Methods \& Applications}

A novel normalization to correct APT-CEST in the presence of fat

Ferdinand Zimmermann ${ }^{1}$, Andreas Korzowski ${ }^{1}$, Patrick Schuenke ${ }^{1}$, Johannes Breitling ${ }^{1}$, Mark Ladd ${ }^{1}$, Peter Bachert ${ }^{1}$, and Steffen Goerke $^{1}$

${ }^{1}$ Medical Physics in Radiology, German Cancer Research Center, Heidelberg, Germany

Chemical Exchange Saturation Transfer (CEST) MRI in the human breast is affected by the fat content in the fibro glandular tissue. Although the spectral region of the amide proton transfer (APT) signal does not overlay with fat resonances, the fat signal leads to an incorrect normalization of the Z-spectrum and therefore to misleading CEST effects. We propose a novel method yielding a corrected normalization without the need for application of fat saturation schemes, thus enabling APT-CEST imaging corrected for fat signal contribution. Transfer of the gained insights to realize correct APT-CEST in the human breast at $7 \mathrm{~T}$ is currently under investigation.

Rapid and Quantitative Chemical Exchange Saturation Transfer (CEST) Imaging of In Vivo Rat Brain with Magnetic Resonance Fingerprinting (MRF)

Ouri Cohen ${ }^{1,2}$, Shuning Huang ${ }^{3}$, Michael T. McMahon ${ }^{4,5}$, Matthew S. Rosen ${ }^{1,2}$, and Christian T. Farrar ${ }^{1}$

${ }^{1}$ Martinos Center for Biomedical Imaging, Deprtment of Radiology, Massachusetts General Hospital and Harvard Medical School, Charlestown, MA, United States, ${ }^{2}$ Physics Department, Harvard University, Cambridge, MA, United States, ${ }^{3}$ Department of Biomedical Engineering, Texas A\&M University, College Station, TX, United States, ${ }^{4}$ Department of Radiology, Johns Hopkins University School of Medicine, Baltimore, MD, United States, ${ }^{5}$ F.M. Kirby Center for Functional Brain Imaging, Kennedy Krieger Institute, Baltimore, MD, United States

CEST MRI suffers from several limitations including long image acquisition times and the qualitative nature of the CEST contrast. Clinical translation of CEST MRI would benefit greatly from the development of quantitative and rapid CEST methods. Here we build on the recently developed Magnetic Resonance Fingerprinting (MRF) technique and report the use of a fast CEST fingerprinting method for generating quantitative exchange rate and exchangeable proton concentration maps of L-Arginine phantoms and in vivo rat brain tissue.

3D CEST MRI of human brain at $9.4 \mathrm{~T}$ reveals vessel correlation of the effect at $-1.7 \mathrm{ppm}$

Moritz Zaiss ${ }^{1}$, Jonas Bause ${ }^{1}$, Anagha Deshmane ${ }^{1}$, Kai Herz $^{1}$, and Klaus Scheffler ${ }^{1}$

${ }^{1}$ High-Field Magnetic Resonance, Max Planck Institute for Biological Cybernetics, Tuebingen, Germany

In vivo CEST imaging at $9.4 \mathrm{~T}$ reveals that the peak at $-1.7 \mathrm{ppm}$ which was recently associated with red blood cells is spatially localized to blood vessels. A $3 \mathrm{D}$ CEST sequence with high resolution and dense sampling of the Z-spectrum shows that only the $-1.7 \mathrm{ppm}$ contrast is vascularly localized.

Myocardial Creatine CEST in human heart using a segmented pseudo steady state acquisition over multiple short breathholds

Neil E Wilson ${ }^{1}$, Puneet Bagga ${ }^{1}$, Kevin D'Aquilla ${ }^{1}$, Hari Hariharan ${ }^{1}$, and Ravinder Reddy ${ }^{1}$

${ }^{1}$ Department of Radiology, University of Pennsylvania, Philadelphia, PA, United States

A technique to acquire creatine CEST of the myocardial muscle is presented here. The technique uses a pseudo steady state saturation, segmented readout, and multiple, short breathholds. 
${ }^{1}$ Howard University, Washington, DC, United States, ${ }^{2}$ National Institutes of Health, Bethesda, MD, United States

This study compared the endogenous glucoCEST contrast to the glucoCEST with exogenous glucose delivered as contrast agent in experimental TBI. By giving relatively low concentration $(0.3 \mathrm{~g} / \mathrm{kg})$ of $2 \mathrm{DG}$ solution, the post-contrast glucoCEST weighted images could magnify the contrast changes in the brains before and after TBI. Meanwhile, the endogenous glucoCEST weighted images also detected the same pattern of decreased contrast in the TBI brains and that was validated by 2DG autoradiography. Our findings substantiate that the glucoCEST technique has potential to detect the hypometabolic syndrome following TBI, even without using exogenous contrast agent.

Mapping elevated lactate levels after ischemic stroke using PROBE CEST/NOE: a feasibility study in patients at 3T

Tobias Lenich ${ }^{1}$, André Pampel ${ }^{1}$, Toralf Mildner ${ }^{1}$, Ralf Mekle ${ }^{2}$, Ramanan Ganeshan ${ }^{2}$, Jochen B. Fiebach ${ }^{2}$, and Harald E. Möller ${ }^{1}$

${ }^{1}$ Max Planck Institute for Human Cognitive and Brain Sciences, Leipzig, Germany, ${ }^{2}$ Center for Stroke Research Berlin (CSB), Charité - Universitätsmedizin Berlin, Berlin, Germany

In ischemic stroke, anaerobic glycolysis leads to a local increase in lactate concentration. Such elevated levels of lactate can be detected via CEST/NOE. In vivo, several broad tissue contributions as well as metabolites lead to a complex intermingled baseline in Z-spectra. With PROBE, such effects are compensated based on healthy tissue, and a flat baseline is achieved. Stroke affected areas can hence be identified in direct contrast to healthy tissue. Lactate contributions to the Z-spectrum became distinctively observable. The feasibility was demonstrated in-vivo for thalamic stroke in a clinical setting at 3T.

Dependence of rNOE-CEST signals on molecular weight

Steffen Goerke ${ }^{1}$, Johannes Breitling ${ }^{1}$, Karel D Klika ${ }^{2}$, Mark E Ladd ${ }^{1}$, and Peter Bachert ${ }^{1}$

${ }^{1}$ Division of Medical Physics in Radiology, German Cancer Research Center (DKFZ), Heidelberg, Germany, ${ }^{2}$ Molecular Structure Analysis, German Cancer Research Center (DKFZ), Heidelberg, Germany

In this study, rNOE-CEST signals of proteins have been demonstrated to depend on molecular weight. This finding can explain the observed intensity decrease of aliphatic rNOE-CEST signals in tumors in comparison to healthy tissue.

Optimization and acceleration of Selective Inversion Recovery imaging for practical whole-brain quantitative Magnetization Transfer measurements

Matthew J Cronin ${ }^{1}$, Junzhong Zhu ${ }^{1}$, Daniel Gochberg ${ }^{1}$, and Richard D Dortch ${ }^{1}$

${ }^{1}$ Institute of Imaging Science, Vanderbilt University Medical Center, Nashville, TN, United States

Selective inversion recovery quantitative magnetization transfer (SIR-qMT) imaging offers increased efficiency relative to conventional pulsed-saturation qMT due to its ability to quantify MT parameters without the need for independent estimates of $\mathrm{BO}, \mathrm{B}_{1}{ }^{+}$, and $\mathrm{T}_{1}$. Despite this, qMT acquisition at a reasonable resolution over a large field of view remains prohibitively time consuming. Here, we employ an optimised acquisition strategy and accelerated readouts to acquire whole-brain SIR-qMT data at $2 \times 2 \times 3$ mm ${ }^{3}$ resolution in $\sim 10$ minutes; opening the door to qMT imaging on a time scale practical for clinical application.

Multiple Interleaved Mode Saturation (MIMOSA) for B1+ inhomogeneity mitigation in chemical exchange saturation transfer.

Andrzej Liebert ${ }^{1}$, Moritz Zaiss ${ }^{2}$, Rene Gumbrecht ${ }^{3}$, Benjamin Schmitt ${ }^{4}$, Peter Linz ${ }^{1}$, Frederik B. Laun ${ }^{1}$, Michael Uder ${ }^{1}$, and Armin M. Nagel ${ }^{1}$

${ }^{1}$ Institute of Radiology, University Hospital Erlangen, Friedrich-Alexander-Universität Erlangen-Nürnberg (FAU), Erlangen, Germany, ${ }^{2}$ Max Planck Institute for Biological Cybernetics, Tuebingen, Germany, ${ }^{3}$ Siemens Healthcare GmbH, Erlangen, Germany, ${ }^{4}$ Siemens Healthcare Pty Ltd, Sydney, Australia

Due to high sensitivity to $\mathrm{B}_{1}{ }^{+}$-inhomogeneities, Chemical Exchange Saturation Transfer MRI requires a correction or mitigation of the $\mathrm{B}_{1}{ }^{+}$-inhomogeneity at ultra-high magnetic field strengths $\left(B_{0} \geq 7\right.$ Tesla). A novel approach for mitigation of $B_{1}{ }^{+}$-inhomogeneity effects that affects the saturation process is presented. The method employs two interleaved excitation modes during the saturation pulse train. Simulations show a decrease of the relative difference of the MTRRex metric caused by $B_{1}{ }^{+}$inhomogeneity. This "Multiple Interleaved Mode Saturation" scheme leads to improved homogeneity in both, phantom and in vivo measurements at 7 Tesla. 
${ }^{1}$ German Center for Neurodegenerative Diseases (DZNE), Bonn, Germany, ${ }^{2}$ Max-Planck Institute for Biological Cybernetics, Tübingen, Germany, ${ }^{3}$ Department of Physics and Astronomy, University of Bonn, Germany

We present a 3D CEST sequence that allows $2 \mathrm{~mm}$ isotropic whole-brain acquisition within $6 \mathrm{~s}$ per frequency offset. The $4.5 \mathrm{~s}$ CEST preparation is followed by a $1.5 \mathrm{~s}$ centric-reordered $3 \mathrm{D}$-EPI readout with water excitation. The single-shot readout improves robustness against physiological noise and provides complete freedom in the design of the saturation block. We acquired whole-brain CEST data at 7T and show metabolite maps obtained from a Lorentzian fit to the Z-spectra.

CEST Feasibility in Rectal Cancer Patients at 7T for Detection of Residual Tumor

Catalina S. Arteaga de Castro ${ }^{1}$, Quincy van Houtum ${ }^{1}$, Sieske Hoendervangers ${ }^{2}$, Alice M Couwenberg ${ }^{2}$, Martijn P.W. Intven², Helena M Verkooijen²,3, Dennis W.J. Klomp ${ }^{1}$, and Marielle E.P. Philippens ${ }^{2}$

${ }^{1}$ Imaging, University Medical Center Utrecht, Utrecht, Netherlands, ${ }^{2}$ Radiation Oncology, University Medical Center Utrecht, Utrecht, Netherlands, ${ }^{3}$ Utrecht University, Utrecht, Netherlands

Five patients were scanned at a 7T MR scanner, 9 weeks after chemoradiation treatment. Three patients showed extreme artifacts on the calculated CEST maps due to B0 artifacts from air contained in the rectum or poor B0 shimming. Two successful CEST measurements showed matching amide-CEST maps to the residual tumor observed in the MRI. CEST applied to rectum patients after chemoradiation might be the appropriate technique to avoid surgical resection in some patients without residual tumor after treatment.

Optimization of OH-CEST contrast at 3T for clinical application of glucoCEST MRI

Chirayu Gandhi ${ }^{1}$, Dario Longo ${ }^{2}$, Annasofia Anemone ${ }^{3}$, Kai Herz $^{1}$, Anagha Deshmane ${ }^{1}$, Tobias Lindig ${ }^{4}$, Benjamin Bender ${ }^{4}$, Silvio Aime ${ }^{3}$, Klaus Scheffler ${ }^{1}$, and Moritz Zaiss ${ }^{1}$

${ }^{1}$ High-Field Magnetic Resonance, Max Planck Institute for Biological Cybernetics, Tuebingen, Germany, ${ }^{2}$ Istituto di Biostrutture e Bioimmagini, Consiglio Nazionale delle Ricerche (CNR), Torino, Italy, ${ }^{3}$ Dipartimento di Biotecnologie Molecolari e Scienze per la Salute, Università degli Studi di Torino, Torino, Italy, ${ }^{4}$ Diagnostic \& Interventional Neuroradiology, University Hospital Tuebingen, Tuebingen, Germany

A 3D snapshot CEST sequence is optimized for contrast originating from hydroxyl groups of glucose molecules. Multi-B1-multi-pH measurements allow fitting of exchange rates of four glucose hydroxyl groups, which are then used to optimize pre-saturation parameters in simulation. The optimal protocol gave highly reproducible signals in 6 healthy volunteers, and showed no contrast when tested in a brain tumor patient. This protocol provides a robust baseline for glucose injection studies.

Concentration and relaxation rate independent clinical pH-weighted metabolic imaging at 3T using pulsed radiofrequency chemical exchange saturation transfer spin-and-gradient-echo echoplanar imaging (CEST-SAGE-EPI)

Jingwen $\mathrm{Yao}^{1,2}$ and Benjamin M. Ellingson ${ }^{1}$

${ }^{1}$ UCLA Brain Tumor Imaging Laboratory, Center for Computer Vision and Imaging Biomarkers, Department of Radiological Sciences, University of California, Los Angeles, Los Angeles, CA, United States, ${ }^{2}$ Department of Bioengineering, University of California, Los Angeles, Los Angeles, CA, United States

Noninvasive $\mathrm{pH}$ measurement with chemical exchange saturation transfer (CEST) MRI often suffers from various confounding factors. In this study, we investigate the feasibility of using a "ratiometric method" to obtain tissue relaxation rates and concentration independent pH-weighted MR image contrast at clinical field strengths using short RF saturation pulse trains and a multi-echo echoplanar readout. Results from numerical simulation and phantom experiments indicate that the new metric $R\left(\Delta \omega_{1}, \Delta \omega_{2}\right)$ has an approximately linear relationship with $\mathrm{pH}$, and is not sensitive to water relaxation rates or amino acid concentration. This approach will be highly valuable for investigating metabolic changes in many diseases.

Feasibility of ACIDOCEST using lodixanol in a Rat Glioma Model

Dushyant Kumar ${ }^{1}$, Ravi Nanga ${ }^{1}$, Puneet Bagga ${ }^{1}$, Kavindra Nath ${ }^{1}$, Ranjit Ittyerah ${ }^{1}$, Damodara Reddy ${ }^{1}$, Hari Hariharan ${ }^{1}$, and Ravinder Reddy

${ }^{1}$ Radiology, University of Pennsylvania, PHILADELPHIA, PA, United States

In vivo $\mathrm{pH}$ mapping within tumor and kidney have been successfully demonstrated in both preclinical and clinical settings using MRI based imaging modality, known as AcidoCEST. This method uses iodinated contrast agents (ICAs) as exogenous contrast agent which are normally used in CT scans. So far, these methods have mainly utilized CEST contrast from to the amide peaks $(\sim 4.2,5.6 \mathrm{ppm})$ ICAs. We demonstrate the feasibility of detecting the CEST contrast from both hydroxyl groups ( 0.8 ppm) and amide groups $(\sim 4.2 \mathrm{ppm})$ from lodixanol in the glioma model. 
The chemical exchange saturation transfer (CEST) MRI usually requires long RF irradiation before every data acquisition to achieve the steady-state CEST mechanism. To eliminate the repeatedly required RF irradiations and to increase the scan efficiency, a new CEST MRI technique that performs the RF irradiation in parallel with data acquisition is developed. The results of MR experiments demonstrate the feasibility of the proposed technique in amide proton CEST.

Ammonia-weighted imaging by chemical exchange saturation transfer - MRI at $3 \mathrm{~T}$

Helge Jörn Zöllner ${ }^{1,2}$, Markus Butz ${ }^{1}$, Gerald Kircheis ${ }^{3}$, Stefan Klinker ${ }^{4}$, Dieter Häussinger ${ }^{3}$, Benjamin Schmitt ${ }^{5}$, Alfons Schnitzler ${ }^{1}$, and Hans-Jörg Wittsack ${ }^{2}$

${ }^{1}$ Institute of Clincial Neuroscience and Medical Psychology, Heinrich Heine University, Düsseldorf, Germany, ${ }^{2}$ Department of Diagnostic and Interventional Radiology, Heinrich Heine University, Düsseldorf, Germany, ${ }^{3}$ Department of Gastroenterology, Hepatology and Infectiology, Heinrich Heine University, Düsseldorf, Germany, ${ }^{4}$ Institute of Physical Biology, Heinrich Heine University, Düsseldorf, Germany, ${ }^{5} 160$ Herring Road, Siemens Healthcare Pty Ltd., Macquarie Park NSW 2113, Australia

Chemical exchange saturation transfer (CEST) is an advanced MR contrast, which is sensitive to metabolic parameters as pH or protein content. The present study shows the ammoniasensitivity of amide proton CEST imaging at a fixed $\mathrm{pH}$ value. The in vivo applicability is tested in a population of patients suffering from hepatic encephalopathy (HE), which is linked to ammonia accumulation within the brain. In HE, the CEST signal is especially reduced within occipital and cerebral regions. This reduction may be related to increased ammonia levels in HE patients.

In vivo imaging of Nucleus of the solitary tract at ultra-high field: a preliminary study

Nikos Priovoulos ${ }^{1}$, Benedikt A Poser ${ }^{2}$, Roberta Sclocco ${ }^{3,4}$, Vitaly Napadow ${ }^{3,4,5}$, Frans Verhey ${ }^{1}$, and Heidi IL Jacobs ${ }^{1,2,6}$

${ }^{1}$ Alzheimer Center Limburg, Maastricht University, Maastricht, Netherlands, ${ }^{2}$ Department of Cognitive Neuroscience, Maastricht University, Maastricht, Netherlands, ${ }^{3}$ Department of Radiology, Martinos Center for Biomedical Imaging, Massachusetts General Hospital, Boston, MA, United States, ${ }^{4}$ Department of Anesthesiology, Perioperative and Pain Medicine, Brigham and Women's Hospital, Harvard Medical School, Boston, MA, United States, ${ }^{5}$ Department of Radiology, Logan University, Chesterfield, MT, United States, ${ }^{6}$ Department of Radiology, Division of Nuclear Medicine and Molecular Imaging, Harvard Medical School, Boston, MA, United States

The nucleus of the solitary tract consists of a set of nuclei in medulla oblongata involved in several homeostatic systems. No method has been proposed so far to image it in vivo, due to its low contrast with standard T1 and T2-weighted methods, its small size and its position deep in the medulla. In this study we present preliminary results that indicate that NTS may be sensitive to magnetization transfer effects.

How Valuable is T1 and T2 Information for Model-based Analysis of CEST MRI in Disease?

Paula L. Croal ${ }^{1}$, Kevin J. Ray ${ }^{2,3}$, James R. Larkin² ${ }^{2}$ Manon A. Simard ${ }^{2}$, Brad A. Sutherland ${ }^{4,5}$, James Kennedy ${ }^{4}$, Nicola Sibson ${ }^{2}$, and Michael Chappell

${ }^{1}$ Institute of Biomedical Engineering, University of Oxford, Oxford, United Kingdom, ${ }^{2}$ CRUK \& MRC Oxford Institute for Radiation Oncology, University of Oxford, Oxford, United Kingdom, ${ }^{3}$ Wellcome Centre for Integrative Neuroimaging, FMRIB, Nuffield Department of Clinical Neurosciences, University of Oxford, Oxford, United Kingdom, ${ }^{4}$ Acute Stroke Programme, Radcliffe Department of Medicine, University of Oxford, Oxford, United Kingdom, ${ }^{5}$ School of Medicine, Faculty of Health, University of Tasmania, Hobart, Australia

$T_{1}$ and $T_{2}$ are often altered by pathology, and while this may have significant impact on quantification of CEST MRI, acquisition of $T_{1}$ and $T_{2}$ maps may not be feasible within a clinical setting. However, Bayesian model-based analysis of CEST MRI can incorporate estimation of $T_{1}$ and $T_{2}$, with or without quantitative maps. Here we explore how valuable $T_{1}$ and $T_{2}$ knowledge is for the detection of pathological alterations in the CEST effect using APT MRI,in both ischaemic stroke and tumours, demonstrating acquisition and analysis of should in part be tailored to the pathology in question.

Steady-state CEST-MRI using a reduced saturation period

Johannes Breitling ${ }^{1}$, Steffen Goerke ${ }^{1}$, Jan-Eric Meissner ${ }^{1}$, Andreas Korzowski ${ }^{1}$, Patrick Schuenke ${ }^{1}$, Mark E. Ladd ${ }^{1}$, and Peter Bachert ${ }^{1}$

${ }^{1}$ Division of Medical Physics in Radiology, German Cancer Research Center (DKFZ), Heidelberg, Germany

In this study, we propose a novel approach to determine the steady-state of CEST experiments without the application of prolonged saturation periods. This is achieved by numerically calculating the steady-state from a measurement with a reduced saturation period (in the order of the water proton $\mathrm{T}_{1}$ ). This may allow quantitative CEST measurements, capable of providing information about $\mathrm{pH}$ and metabolite concentrations, in a reasonable and clinical relevant time frame. 
${ }^{1}$ Wellcome Centre for Integrative Neuroimaging, FMRIB, Nuffield Department of Clinical Neurosciences, University of Oxford, Oxford, United Kingdom, ${ }^{2}$ CRUK \& MRC Oxford Institute for Radiation Oncology, University of Oxford, Oxford, United Kingdom, ${ }^{3}$ Institute of Biomedical Engineering, University of Oxford, Oxford, United Kingdom

A Bayesian model-based approach to analysis of CEST MRI quantifies CEST effects more accurately than alternative approaches by fitting the Bloch-McConnell equations to measured $Z$-spectra and estimating the exchange rate and concentration of each labile proton population. However, estimates of exchange rate and concentration using this approach are correlated, making accurate estimation of either parameter in isolation difficult. In this study, we demonstrate using simulation and in vivo data that separation of this correlation may be possible by analysing data acquired with different $\mathrm{B}_{1}$ powers simultaneously.

Implications of tissue compartmentalisation on APT MRI

Kevin J Ray ${ }^{1,2}$, Michael A Chappell ${ }^{3}$, and Nicola R Sibson ${ }^{2}$

${ }^{1}$ Wellcome Centre for Integrative Neuroimaging, FMRIB, Nuffield Department of Clinical Neurosciences, University of Oxford, Oxford, United Kingdom, ${ }^{2}$ CRUK \& MRC Oxford Institute for Radiation Oncology, University of Oxford, Oxford, United Kingdom, ${ }^{3}$ Institute of Biomedical Engineering, University of Oxford, Oxford, United Kingdom

Amide proton transfer (APT) MRI is assumed to report on the intracellular environment. However, no attempt has been made to verify this assumption, or examine the extent to which tissue compartmentalisation contaminates measurements of biophysical parameters (e.g. protein concentration, pH) from APT MRI data. In this simulation study, we show that measurement of biophysical parameters by APT MRI is influenced by tissue compartmentalisation when the transcytolemmal exchange rate is slow (<2Hz). Since recent studies have reported transcytolemmal exchange to be in such a regime, it may be possible to separate intra- and extracellular APT signals.

A Phantom Investigation into the Biosynthesis Pathway of Serotonin Using CEST

Ryan T. Oglesby ${ }^{1,2}$, Wilfred W. Lam², and Greg J. Stanisz ${ }^{1,2}$

${ }^{1}$ Medical Biophysics, University of Toronto, Toronto, ON, Canada, ${ }^{2}$ Physical Sciences, Sunnybrook Research Institute, Toronto, ON, Canada

The four metabolites involved in the biosynthesis pathway of serotonin were scanned at 7T using CEST MRI in order to characterize the Z-spectrum of each. It was found that each metabolite was distinguishable from one another according to their peak location and amplitude at physiological temperature and pH within experimental uncertainty. Using a BlochMcConnell exchange model, each metabolite was fitted for $T_{1}, T_{2}$, peak location $\Delta_{0 c}$, exchange rate $R_{C}$, and pool size $M_{0}$. The in vitro CEST MRI data acquired during this investigation may increase the specificity of in vivo Z-spectrum interpretation during an investigation focused on detecting serotonin.

Presaturation power adjusted pulsed (PPAP)-CEST: A method to increase the independence of target CEST signals

Kazufumi Kikuchi ${ }^{1}$, Keisuke Ishimatsu ${ }^{1}$, Shanrong Zhang ${ }^{1}$, Ivan E. Dimitrov ${ }^{1,2}$, Hiroshi Honda ${ }^{3}$, A. Dean Sherry ${ }^{1}$, and Masaya Takahashi

${ }^{1}$ Advanced Imaging Research Center, The University of Texas Southwestern Medical Center, Dallas, TX, United States, ${ }^{2}$ Philips Healthcare, Gainesville, FL, United States, ${ }^{3}$ Kyushu University, Fukuoka, Japan

We previously demonstrated in the phantoms that the chemical exchange saturation transfer (CEST) peaks identified during 0 to 3.5 ppm are often quite broad and overlap with each other, which caused in obvious interference between the CEST signals. We attempted a presaturation power adjusted pulsed (PPAP)-CEST method which aimed to increase the independence of glutamate CEST signal by eliminating an interference from a neighboring CEST signal in the kidney in mice. The CEST signal of glutamate was less impacted by concentration changes in other exchanging species by subtracting CEST signals at two different power levels.

Investigating the Effect of Rapid Exchange Rate on the Accuracy of the Bayesian CEST Model at 7T

Alex K. Smith ${ }^{1}$ and Kevin Ray ${ }^{1}$

${ }^{1}$ Wellcome Centre for Integrative Neuroimaging, FMRIB, University of Oxford, Oxford, United Kingdom

The Bayesian model-based approach has shown a remarkable ability to accurately characterize the APT-CEST effect in vivo. However, no studies have been performed to ensure this performance is maintained when examining labile protons exchanging at faster exchange rates. Here, we examine how exchange rates in the intermediate-to-fast exchange regime affect the characterization of the CEST effect by a model-based approach, and compare it with the MTR asym measurement. The results suggest that the Bayesian model accurately characterizes the CEST effect in question at exchange rates up to $2000 \mathrm{~Hz}$, and outperforms the MTR asym when faced with multiple confounding pools 
${ }^{1}$ Biomedical Imaging and Image-guided Therapy, Medical University of Vienna, Vienna, Austria, ${ }^{2}$ Christian Doppler Laboratory for Clinical Molecular MR Imaging, Vienna, Austria,

${ }^{3}$ Department of Physics, University of Helsinki, Helsinki, Finland

Chemical Exchange Saturation Transfer is prone to inhomogeneities of the static magnetic field $\left(\mathrm{B}_{0}\right)$. Hence, accurate frequency correction is mandatory for reliable quantification. Currently established $B_{0}$ correction approaches assume $B_{0}$ inhomogeneities to be static during CEST experiments, but this is questionable in the presence of subject motion and scanner instabilities. Thus, we propose three different dynamic B0 correction methods for CEST that can compensate for $\mathrm{B}_{0}$ instability for each Z-spectral point separately and compare them to three established static $B_{0}$ correction approaches that apply the same frequency shift to all Z-spectral points in phantom and in vivo experiments.

gagCEST on patients with focal knee cartilage defects

Markus M. Schreiner ${ }^{1,2}$, Vladimir Mlynarik ${ }^{2,3}$, Stefan Zbyn $^{2,4}$, Vladimir Juras ${ }^{2}$, Pavol Szomolanyi ${ }^{2}$, Didier Laurent ${ }^{5}$, Celeste Scotti $^{5}$, Harry Haber ${ }^{5}$, and Siegfried Trattnig ${ }^{2,3}$

${ }^{1}$ Department of Orthopedics, Medical University of Vienna, Vienna, Austria, ${ }^{2}$ High-Field MR Centre, Department of Biomedical Imaging and Image-Guided Therapy, Medical University of Vienna, Vienna, Austria, ${ }^{3} \mathrm{CD}$ Laboratory for Clinical Molecular MR Imaging, Vienna, Austria, ${ }^{4}$ Research Unit of Medical Imaging, Physics and Technology, University of Oulu, Oulu,

Finland, ${ }^{5}$ Novartis Institutes for Biomedical Research, Basel, Switzerland

The gagCEST technique is a promising tool for determining concentration of glycosaminoglycans in articular cartilage. In this study, the performance of gagCEST in a group of patients with ICRS grade I-II knee cartilage defects was investigated. It was found that the method gives significantly different mean MTR asym values for cartilage defects, normal weight-bearing and normal non-weight-bearing femoral cartilage. The clinical use of the gagCEST technique is currently limited by its long measurement time and sensitivity to patient motion.

High-resolution total Creatine mapping of the mouse brain at $11.7 \mathrm{~T}$ using CEST

Lin Chen ${ }^{1,2,3}$, Zhiliang Wei ${ }^{1,2}$, Xiang Xu ${ }^{1,2}$, Yuguo Li ${ }^{1,2}$, Shuhui Cai ${ }^{3}$, Guanshu Liu ${ }^{1,2}$, Hanzhang Lu ${ }^{1,2}$, Peter B. Barker ${ }^{1,2}$, Robert G. Weiss ${ }^{1}$, Peter C.M. van Zijj ${ }^{1,2}$, and Jiadi Xu ${ }^{1,2}$

${ }^{1}$ Department of Radiology and Radiological Science, Johns Hopkins University, Baltimore, MD, United States, ${ }^{2}$ F.M. Kirby Research Center for Functional Brain Imaging, Kennedy Krieger Research Institute, Baltimore, MD, United States, ${ }^{3}$ Department of Electronic Science, Xiamen University, Xiamen, China

A combined polynomial and Lorentzian Fitting (PLOF) scheme was developed to map total creatine (tCr) signal using a CW-CEST sequence under short saturation time situation. At 11.7T, the guanidinium proton signals of $\mathrm{tCr}$ and tissue proteins are not coalesced with the water signal and the line-shape fitting procedure can correct the direct saturation and magnetization transfer contrast introduced spill-over effects, allowing the guanidinium CEST signal to be extracted and subsequently quantified. A series of Cr phantom and mouse brain studies with different saturation times and powers were carried out to determine the optimal parameters for protein-signal corrected creatine CEST quantification.

Low power Z-spectrum analysis for isolated NOE and amide CEST-MRI at 3T with comparison to 9.4T

Anagha Deshmane ${ }^{1}$, Moritz Zaiss ${ }^{1}$, Benjamin Bender ${ }^{2}$, Tobias Lindig ${ }^{2}$, Johannes Windschuh ${ }^{3}$, Kai Herz ${ }^{1}$, and Klaus Scheffler ${ }^{1}$

${ }^{1}$ High-Field Magnetic Resonance, Max Planck Institute for Biological Cybernetics, Tuebingen, Germany, ${ }^{2}$ Diagnostic \& Interventional Neuroradiology, University Hospital Tuebingen, Tuebingen, Germany, ${ }^{3}$ Center for Biomedical Imaging, New York University Langone Health, New York, NY, United States

A snapCEST sequence was optimized for imaging of protein CEST effects at 3T with low saturation power. Full Z-spectrum sampling allows Lorentzian fitting of amide, NOE, semisolid MT, and water pools. Validation against data acquired at 9.4T demonstrates effective labeling of selective amide and NOE CEST effects at 3 T. Data acquired in a brain tumor patients demonstrates clinical feasibility.

Traditional Poster

\section{Novel Contrast Mechanisms: Body}

Cheng Junying ${ }^{1,2}$, Mei Yingjie ${ }^{2,3}$, Chen Maodong ${ }^{2}$, Wang Changqing ${ }^{1,2}$, Liu Xiaoyun ${ }^{1}$, Chen Wufan ${ }^{1,2}$, and Feng Yanqiu ${ }^{2}$

${ }^{1}$ School of Automation Engineering, University of Electronic Science and Technology of China, Chengdu, China, ${ }^{2}$ Guangdong Provincial Key Laboratory of Medical Image Processing, School of Biomedical Engineering, Southern Medical University, Guangzhou, China, ${ }^{3}$ Philips Healthcare, Guangzhou, China 
Current phase-unwrapping algorithms are generally challenged by severe noise, rapid-varying phase or disconnected regions. We present a novel phase-unwrapping method by using phase jumps detection and local polynomial surface fitting. The proposed method first segments the whole phase map into blocks by exploiting the phase jumps that are automatically identified. Intra-block wrapping may still exist if the true phase difference between adjacent pixels exceeds $\pi$ inside a block. To address potential intra-block wraps, we further segment each block into subblocks using the phase partition method, and perform inter-subblock unwrapping using the block-growing method. Simulation and in vivo Dixon water-fat separation experiments were implemented to evaluate the performance of the proposed method, with comparisons to PRELUDE and CLOSE. This method has great potential in phase-related MRI applications in practice.

Quantitative estimation of sub-voxel fat and water fractions based on two T2-component fitting in calf muscle.

Jannette Nassar ${ }^{1}$, Dvir Radunsky ${ }^{1}$, Noam Omer ${ }^{1}$, Yann Le Fur ${ }^{2}$, David Bendahan ${ }^{2}$, and Noam Ben-Eliezer ${ }^{1,3,4}$

${ }^{1}$ Department of Biomedical Engineering, Tel Aviv University, Tel Aviv, Israel, ${ }^{2}$ Aix Marseille Univ, CNRS, CRMBM, Marseille, France, ${ }^{3}$ Center for Advanced Imaging Innovation and Research, New York University, New York, NY, United States, ${ }^{4}$ Sagol School of Neuroscience, Tel Aviv University, Tel Aviv, Israel

T2 relaxation is an effective biomarker for muscle pathology including inflammation, necrosis, or fatty infiltration. Accurate quantification of T2 values, however, is hampered due to the inherent bias of rapid multi-SE protocols by stimulated echoes. Recently, we introduced the echo modulation curve (EMC) algorithm, which successfully overcomes this problem and provide accurate T2 values that are stable across scanners and scan-settings. In this work, we present extension of the EMC algorithm for two component fitting, water and fat, allowing to quantify the sub-voxel infiltration of fat into the muscle, along with the corresponding T2 value of each component.

A dual-step iterative temperature estimation method for accurate and precise fat referenced PRFS temperature mapping

Chuanli Cheng ${ }^{1,2}$, Chao Zou ${ }^{1}$, Yangzi Qiao ${ }^{1}$, Changjun Tie ${ }^{1}$, Qian Wan ${ }^{1,2}$, Xin Liu $^{1}$, and Hairong Zheng ${ }^{1}$

Temperature imaging based on proton resonance frequency shift (PRFS) fails in fat containing tissues as the proton frequency of fat does not change with temperature. A dual-step iterative temperature estimation of fat referenced PRFS method is proposed to improve both the accuracy and precision of fat-referenced PRFS method. The method is evaluated with fat-water phantom and ex vivo BAT tissue excised from rats. Compared to the existing methods, the proposed method has least bias to the fluorescent optical fiber thermometer while maintaining the best noise performance.

Longitudinal Stability of a Quantitative Fat-Water Phantom

Benjamin A Ratliff ${ }^{1,2}$, Samir D Sharma ${ }^{2}$, Jean H Brittain ${ }^{2}$, Scott B Reeder ${ }^{1,2,3,4,5}$, and Diego Hernando ${ }^{2,3}$

${ }^{1}$ Biomedical Engineering, University of Wisconsin, Madison, Madison, WI, United States, ${ }^{2}$ Radiology, University of Wisconsin, Madison, Madison, WI, United States, ${ }^{3}$ Medical Physics, University of Wisconsin, Madison, Madison, WI, United States, ${ }^{4}$ Medicine, University of Wisconsin, Madison, Madison, WI, United States, ${ }^{5}$ Emergency Medicine, University of Wisconsin, Madison, Madison, WI, United States

The purpose of this work was to evaluate the long-term stability of a previously validated fat-water phantom under a range of environmental conditions. Two separate phantoms were constructed, each with a range of fat concentrations. The first phantom was subjected to three different temperature conditions over one year. The second fat phantom was kept at room temperature and studied over three years. Our results show that the fat-water phantom has excellent long-term stability at room temperature and is robust to different temperature conditions.

Evaluation of A Method for Simultaneous in vivo Measurements of Blood T1 and T2

Jialu Zhang ${ }^{1,2,3}$, Dingxin Wang ${ }^{3}$, Xiaotong Zhang ${ }^{1,2}$, Lynn E. Eberly ${ }^{4}$, Gregory J. Metzger ${ }^{3}$, Donald R. Dengel ${ }^{5}$, David Tupper ${ }^{6}$, Anne M. Murray ${ }^{7}$, and Xiufeng Li ${ }^{3}$

${ }^{1}$ Interdisciplinary Institute of Neuroscience and Technology, Qiushi Academy for Advanced Studies, Zhejiang University, Hangzhou, China, ${ }^{2}$ College of Biomedical Engineering \& Instrument Science, Zhejiang University, Hangzhou, China, ${ }^{3}$ Center for Magnetic Resonance Research, School of Medicine, University of Minnesota, Minneapolis, MN, United States, ${ }^{4}$ Division of Biostatistics, School of Public Health, University of Minnesota, Minneapolis, MN, United States, ${ }^{5}$ Laboratory of Integrative Human Physiology, School of Kinesiology, University of Minnesota, Minneapolis, MN, United States, ${ }^{6}$ Neuropsychology Section, Hennepin County Medical Center, Minneapolis, MN, United States, ${ }^{7}$ Berman Center for Clinical Research, Hennepin County Medical Center, Minneapolis, MN, United States

The longitudinal and transverse relaxation time constants of blood are important parameters for MRI methods and biomedical research studies. However, these parameters can vary largely across subjects, and change significantly across developmental stages, with physiological states, and due to specific diseases, which has motivated in vivo measurements of these parameters. We implemented a fast method for simultaneous in vivo measurements of blood $T_{1}$ and $T_{2}$. The study results suggest that the in vivo measurements of blood $T_{1}$ and $\mathrm{T}_{2}$ can be achieved in about $25 \mathrm{~s}$ using the implemented method. 
Silvin P. Knight ${ }^{1}$, James F. Meaney ${ }^{1}$, and Andrew J. Fagan ${ }^{1,2}$

${ }^{1}$ National Centre for Advanced Medical Imaging (CAMI), St James Hospital / School of Medicine, Trinity College University of Dublin, Dublin 8, Ireland, ${ }^{2}$ Department of Radiology, Mayo Clinic, Rochester, MN, United States

A novel anthropomorphic phantom test device was used to investigate the effects of temporal resolution $\left(\mathrm{T}_{\text {res }}\right), B_{1}{ }^{+}$-field non-uniformities, and pharmacokinetic (PK) model fitting methods on the absolute accuracy and precision of DCE-MRI measurements of the arterial input function (AIF), and resulting PK parameter estimates. Optimizing the $\mathrm{T}_{\text {res }}$ was found to reduce the maximum errors in PK parameter estimation from $\sim 47 \%$ to $\sim 20 \%$. By correcting for $B_{1}{ }^{+}$-field non-uniformities these errors were further reduced to $\sim 7 \%$. Using a linear rather than non-linear version of the standard Tofts model further increased the accuracy and precision of PK parameter estimations.

Baseline System Variability of Test-Retest and Cross-Platform Liver MR Elastography

Kevin Glaser ${ }^{1}$, Roger Grimm ${ }^{1}$, Brad Jr. Bolster ${ }^{2}$, Richard Ehman ${ }^{1}$, and Jun Chen ${ }^{1}$

1200 1st St Sw, Mayo Clinic, Rochester, MN, United States, ${ }^{2}$ Siemens Healthineers, Salt Lake City, UT, United States

Liver MR Elastography (MRE) has become an important noninvasive liver fibrosis imaging modality. It is essential to know the system variability of the stiffness measurements as only liver stiffness changes greater than the system variability are meaningful and reflect the true liver changes with $95 \%$ confidence. Our study was to perform a single-institution, singlereader study of fasting, normal subjects to assess the baseline (minimum possibly) MRE system variability within and between days, within and between platforms.

Robust chemical exchange spin-lock (CESL) using adiabatic pulses

Baiyan Jiang ${ }^{1}$, Jing Yuan², Queenie Chan ${ }^{3}$, Yi-Xiang Wang ${ }^{1}$, and Weitian Chen ${ }^{1}$

${ }^{1}$ Imaging and Interventional Radiology, The Chinese University of Hong Kong, Shatin, Hong Kong, ${ }^{2}$ Medical physics and research department, Hong Kong, Hong Kong, ${ }^{3}$ Philips Healthcare, Hong Kong, Hong Kong

Chemical exchange spin-lock (CESL) is a recently reported technology for probing metabolites which have intermediate to fast chemical exchange with bulk water. However, the conventional CESL is susceptible to B1 radiofrequency (RF) and B0 field inhomogeneity. The presence of these system imperfections leads to signal distortions and errors in contrast map. In this work, we report an approach to address this problem. We used simulation and in vivo experiments to demonstrate our proposed method.

Morphometric Adaptions of Rectus Femoris to Muscle Strain Revealed Through 'Dynamic Magnetic Resonance Elastography' (DMRE)

Michael Perrins ${ }^{1,2}$, Michiel Simons ${ }^{2,3}$, Andre Attard $^{4}$, Colin Brown ${ }^{5}$, Leela Biant ${ }^{6}$, Edwin J.R. van Beek ${ }^{2}$, and Neil Roberts ${ }^{2}$

${ }^{1}$ MRC Centre for Inflammation Research, University of Edinburgh, Edinburgh, United Kingdom, ${ }^{2}$ Edinburgh Imaging Facility, University of Edinburgh, Edinburgh, United Kingdom, ${ }^{3}$ Department of Clinical Surgery, University of Edinburgh, Edinburgh, United Kingdom, ${ }^{4}$ Department of Bioengineering, University of Strathclyde, Glasgow, United Kingdom, ${ }^{5}$ The Mentholatum Company Ltd., East Kilbride, Glasgow, United Kingdom, ${ }^{6}$ Department of Trauma \& Orthopeadic Surgery, University of Manchester, Manchester, United Kingdom

Magnetic Resonance Elastography (MRE) allows for the quantification of tissue stiffness. When MRE is applied in muscle it allows for the measurement of muscle strain, with strain having an impact on structure morphology. This research investigated whether the bi-articular design of the Rectus Femoris gives an anatomical advantage in adapting to muscle strain and avoiding injury, as this is a vital muscle for movement. It was found that the mono-articular Quadriceps muscles showed significant muscle strain from loading, whereas the Rectus Femoris showed significant changes in cylindrical shape, and as expected, adapted to increased loading.

Lipid nanocapsules for tissue oxygenation determination using MRI

Janske $\mathrm{Nel}^{1,2}$, Florence FRANCONI ${ }^{3}$, Nicolas JOUDIOU ${ }^{2}$, Bernard GALLEZ ${ }^{2}$, and Laurent LEMAIRE ${ }^{1}$

${ }^{1}$ Micro et Nanomedecines translationnelles, MINT, Université d'Angers, Angers, France, ${ }^{2}$ Biomedical Magnetic Resonance Unit (REMA), Université catholique de Louvain, WoluweSaint-Lambert, Belgium, ${ }^{3} P R I S M$, Université d'Angers, Angers, France To determine tissue $\mathrm{pO}_{2}$, lipid nanocapsules $(\mathrm{LNCs})$ were used in conjunction with the rapid mapping of changes in tissue oxygenation, based on the higher solubility of $\mathrm{O}_{2}$ in lipids than
in water, (MOBILE) MR sequence. LNCs were injected into the femoral muscle $(\mathrm{n}=5)$ of $\mathrm{C} 3 \mathrm{H}$ mice and $\mathrm{T}_{1}$ relaxation was measured whilst the animal was breathing air or carbogen (95 in water, (MOBILE) MR sequence. LNCs were injected into the femoral muscle $(n=5)$ of $C 3 \mathrm{H}$ mice and $\mathrm{T}_{1}$ relaxation was measured whilst the animal was breathing air or carbogen $(95$
$\left.\% \mathrm{O}_{2}, 5 \% \mathrm{CO}_{2}\right)$ gas. In all explored mice a shortening in $\mathrm{T}_{1}$ relaxation was observed following the carbogen challenge, and $\mathrm{T}_{1}$ relaxation maps were produced indicating a response of the LNCs to the tissue $\mathrm{O}_{2}$ environment. 
We demonstrate the feasibility of combining simultaneous multi-slice (SMS) excitation with in-plane acceleration to achieve highly accelerated MR elastography data acquisition. The proposed approach enables the acquisition of diagnostic liver MRE data in a single breath-hold, which was not possible using the previous approaches. Our results indicate excellent agreement between the data acquired with and without SMS

Tomoelastography of pancreatic tumors: Preliminary results

Stephan Rodrigo Marticorena Garcia ${ }^{1}$, Christian Burkhardt ${ }^{1}$, Rosa Schmuck ${ }^{2}$, Guo Jing ${ }^{1}$, Bernd Hamm ${ }^{1}$, Jürgen Braun ${ }^{3}$, and Ingolf Sack

${ }^{1}$ Radiology, Charité - Universitätsmedizin Berlin, Berlin, Germany, ${ }^{2}$ Experimental Surgery, Charité - Universitätsmedizin Berlin, Berlin, Germany, ${ }^{3}$ Medical Informatics, Charité Universitätsmedizin Berlin, Berlin, Germany

High-resolution stiffness maps of the pancreas were generated using multifrequency magnetic resonance elastography (MRE) and tomoelastography data processing in healthy controls (Ctr) and patients with pancreatic carcinoma ( $\mathrm{Pa}-\mathrm{Ca}$ ). Pa-Ca have higher stiffness than control tissue and non-tumorous pancreatic parenchyma in patients without overlap to normal values. Subregional analysis for pancreatic head, corpus and tail revealed no difference between these anatomical regions. Tomoelastogaphy is sensitive to pathological changes in viscoelastic properties of $\mathrm{Pa}-\mathrm{Ca}$ and offers a quantitative measure of stiffness of pancreatic tissue.

In vivo measurements of T1-dispersion maps in a kidney tumor mouse model using FFC-MRI around 1.5 T

Nicolas Chanet ${ }^{1}$, Geneviève Guillot ${ }^{1}$, Ingrid Leguerney ${ }^{2,3}$, Rose-Marie Dubuisson ${ }^{1}$, Catherine Sebrié ${ }^{1}$, Alexandre Ingels ${ }^{2,4}$, Noémie Assoun ${ }^{5}$, Estelle Daudigeos-Dubus ${ }^{5}$, Birgit Geoerger $^{5}$, Nathalie Lassau ${ }^{2,3}$, Lionel Broche ${ }^{6}$, and Ludovic de Rochefort ${ }^{7}$

${ }^{1}$ IR4M UMR8081 Univ Paris-Sud CNRS, Université Paris Saclay, Orsay, France, ${ }^{2}$ IR4M UMR8081 Univ Paris-Sud CNRS, Université Paris Saclay, Villejuif, France, ${ }^{3}$ Gustave Roussy, Villejuif, France, ${ }^{4}$ Département d'Urologie - Institut Mutualiste Montsouris, Paris, France, ${ }^{5}$ Gustave Roussy, Vectorology and Anticancer Therapies, UMR8203 CNRS Univ Paris-Sud, Université Paris-Saclay, Villejuif, France, ${ }^{6}$ Bio-Medical Physics, School of Medicine, Medical Sciences and Nutrition, University of Aberdeen, Aberdeen, United Kingdom, ${ }^{7}$ CRMBM UMR7339 CNRS Aix-Marseille Université, Marseille, France

Fast Field Cycling MRI offers the possibility to explore new contrasts generated from NMR dispersion (NMRD) profiles of tissue. Exploiting the dispersion properties of tissues may provide an additional biomarker of diseases through a deeper understanding of molecular mobility. Kidney tumors and healthy kidneys were analyzed among a cohort of twenty-seven mice to give insight into the potential of FFC-MRI for clinical applications. Here, we present $\mathrm{R}_{1}$-dispersion maps performed around $1.5 \mathrm{~T}$ to show that the intrinsic dispersion of tumors measured in vivo differs from the one of healthy kidneys.

MR Elastography of Kidneys Using SE-EPI: A Reproducibility Study and Comparison to ADC and FA Measurements

Deep Gandhi ${ }^{1}$, Prateek Kalra ${ }^{2}$, Huiming Dong ${ }^{1}$, Brian Raterman ${ }^{2}$, and Arunark Kolipaka ${ }^{2}$

${ }^{1}$ Biomedical Engineering, Ohio State University, Columbus, $\mathrm{OH}$, United States, ${ }^{2}$ Radiology, Ohio State University Wexner Medical Center, Columbus, OH, United States

Stiffness change has been associated with progress of disease. Magnetic resonance elastography(MRE) is an imaging-based alternative that can measure stiffness. Diffusion Tensor Imaging(DTI) provides apparent diffusion coefficient(ADC) and fractional anisotropy(FA) of the tissue. Previous studies have investigated stiffness and diffusion in kidneys individually. However, none of the studies have investigated the two together. Aim of this study is to show reproducibility of spin-echo echo-planar imaging(SE-EPI) MRE and correlate it with ADC and FA measurements obtained from DTI. Preliminary results showed good reproducibility in stiffness measurements and moderate correlation between MRE stiffness and ADC and FA values from DTI.

Changqing Wang ${ }^{1,2,3}$, Benjamin Andrew Ratliff ${ }^{3,4}$, Claude B. Sirlin ${ }^{5}$, Scott B. Reeder ${ }^{3,4,6,7,8}$, and Diego Hernando ${ }^{3,6}$

${ }^{1}$ School of Automation Engineering, University of Electronic Science and Technology of China, Chengdu, China, ${ }^{2}$ School of Biomedical Engineering and Guangdong Provincial Key Laboratory of Medical Image Processing, Southern Medical University, Guangzhou, China, ${ }^{3}$ Radiology, University of Wisconsin-Madison, Madison, WI, United States, ${ }^{4}$ Biomedical Engineering, University of Wisconsin-Madison, Madison, WI, United States, ${ }^{5}$ Radiology, University of California, San Diego, San Diego, CA, United States, ${ }^{6}$ Medical Physics, University of Wisconsin-Madison, Madison, WI, United States, ${ }^{7}$ Medicine, University of Wisconsin-Madison, Madison, WI, United States, ${ }^{8}$ Emergency Medicine, University of Wisconsin-Madison, Madison, WI, United States 
Recent studies using chemical shift-encoded MRI in patients with elevated liver fat content, but no iron overload, have shown a positive correlation between proton density fat fraction (PDFF) and R2*. In this work, we investigate the underlying biophysical mechanism of this observation using Monte Carlo simulations. Results from this Monte Carlo study show a positive correlation between PDFF and R2* consistent with previous in vivo observations. Based on the PDFF-R2* relationship, the Monte Carlo simulations may provide a new means to correct for the effect of fat on $\mathrm{R} 2^{*}$ quantification.

Monte Carlo Modeling of Multiple Spin Echo Signals in the Presence of Liver Iron Overload

Changqing Wang ${ }^{1,2,3}$, Scott B. Reeder ${ }^{3,4,5,6,7}$, and Diego Hernando $o^{3,4}$

${ }^{1}$ School of Automation Engineering, University of Electronic Science and Technology of China, Chengdu, China, ${ }^{2}$ School of Biomedical Engineering and Guangdong Provincial Key Laboratory of Medical Image Processing, Southern Medical University, Guangzhou, China, ${ }^{3}$ Radiology, University of Wisconsin-Madison, Madison, WI, United States, ${ }^{4}$ Medical Physics, University of Wisconsin-Madison, Madison, WI, United States, ${ }^{5}$ Biomedical Engineering, University of Wisconsin-Madison, Madison, WI, United States, ${ }^{6}$ Medicine, University of Wisconsin-Madison, Madison, WI, United States, ${ }^{7}$ Emergency Medicine, University of Wisconsin-Madison, Madison, WI, United States

Multiple spin echo (MSE) imaging may enable improved quantification and characterization of tissue iron deposition, with application for assessment of liver iron overload. However, iron deposition generally results in non-exponential signal decay in MSE imaging, and MSE-based R2 (1/T2) relaxometry can depend on the inter-echo time. Additionally, it is cumbersome and expensive to empirically calibrate the R2 relaxometry-iron concentration relationship. In this work, we investigate the effect of inter-echo time on MSE signal in the presence of liver iron overload using Monte Carlo simulations. This Monte Carlo approach may enable improved calibration of MSE-based measurements of iron concentration.

Traditional Poster

\section{Contrast Mechanisms}

Exhibition Hall 2266-2297

Wednesday 8:15 - 10:15

Quantification of T1 and T2 from Standard MR Images

Kelly C McPhee ${ }^{1}$ and Alan H Wilman ${ }^{1,2}$

$2266 \quad{ }^{1}$ Physics, University of Alberta, Edmonton, AB, Canada, ${ }^{2}$ Biomedical Engineering, University of Alberta, Edmonton, AB, Canada

Exact sequence modelling using the Bloch equations is employed to directly extract quantitative T1 and T2 relaxation maps from standard MRI sequences. The need for excess specialized sequences was eliminated by measuring relaxation directly from $\mathrm{T} 1, \mathrm{~T} 2$, and PD-weighted images, and a rapid B1 map. This approach enables wider use of quantitative MRI.

$\$ \$ \$ R \_2^{\wedge *} \$ \$ \$$ Correction for Gradient Echo with a Gaussian Excitation Pulse

Martin Soellradl ${ }^{1}$, Lukas Pirpamer ${ }^{1}$, Jan Sedlacik ${ }^{2}$, Franz Fazekas ${ }^{1}$, Stefan Ropele ${ }^{1}$, and Christian Langkammer ${ }^{1}$

${ }^{1}$ Department of Neurology, Medical University of Graz, Graz, Austria, ${ }^{2}$ Neuroradiology, University Medical Center Hamburg-Eppendorf, Hamburg, Germany

Macroscopic field inhomogeneities increase the effective transverse relaxation rate $R_{2}{ }^{*}$. In contrast to conventional models assuming ideal rectangular pulses, we developed an $R_{2}{ }^{*}$ correction model for Gaussian excitation pulses. After demonstrating the validity of the model in phantom measurements we measured 10 volunteers with $2 \mathrm{~mm}$ and $4 \mathrm{~mm}$ slice thickness, respectively. Uncorrected and corrected $R_{2}{ }^{*}$ values were assessed regionally and a significant effect of the correction was observed. An advantage of the proposed method is that it only requires two echoes, rendering it useful in clinical MRI.

Accuracy and precision of measured T1 in hepatic portal vein blood using a variety of Look- Locker and Modified Look-Locker Inversion Recovery sequences.

Svein Are Sirirud Vatnehol ${ }^{1,2,3}$, Atle Bjørnerud ${ }^{4}$, Camilla Haglerød ${ }^{2}$, Per Kristian Hol ${ }^{1,3}$, Mahmood Amiry-Moghaddam ${ }^{2,5}$, and Tryggve Holck Storås ${ }^{4}$

${ }^{1}$ The Intervention Center, Oslo University Hospital, Oslo, Norway, ${ }^{2}$ Oxy Solutions AS, Oslo, Norway, ${ }^{3}$ Faculty of Medicine, University of Oslo, Oslo, Norway, ${ }^{4}$ Dept. of Diagnostic Physics, Oslo University Hospital, Oslo, Norway, ${ }^{5}$ Institute of basic medical sciences, University of Oslo, Oslo, Norway

The longitudinal variation and averaged T1 measured in the hepatic portal vein (HPV) obtained with 12 variations of Look-Locker (LL) and Modified Look-Locker Inversion Recovery (MOLLI) sequences were compared to identify the sequence with least variation. Among the sequences studied, LL sequence with 5 beat readout and 450 flip angle and MOLLI with an acquisition scheme 10 beats readout, 5 beats recovery followed by 5 beat readout (10(5)5) were shown to be the most stable. Method of image analysis and the use of simulated versus real-time EKG did not significantly affect the stability of the T1-estimates. 
Assessing B1 map errors in vivo: measuring stability and absolute accuracy despite the lack of gold standard

Sofia Chavez ${ }^{1}$

${ }^{1} \mathrm{CAMH}$, Toronto, ON, Canada

B1+ field inhomogeneity is a major source of errors in quantitative mapping. The accuracy of B1 maps, depicting the effects of B1+ inhomogeneity on the flip angle, is thus critical. However, there is no gold standard B1 mapping method in vivo so absolute accuracy is difficult to determine. In this work, we propose steps that exploit known B1 effects in a small phantom to obtain absolute accuracy estimates in vivo. Two B1 mapping methods are required, but neither need be accurate. We demonstrate the proposed assessment by obtaining stability and absolute accuracy measurements of the Method of Slopes B1 maps.

Aqueous and Non-aqueous T1 relaxation in brain under six diverse initial conditions

Alan Manning ${ }^{1}$, Carl Michal ${ }^{1}$, and Alex MacKay ${ }^{1,2}$

${ }^{1}$ Physics, University of British Columbia, Vancouver, BC, Canada, ${ }^{2}$ Radiology, University of British Columbia, Vancouver, BC, Canada

A consistent view of $T_{1}$ relaxation in white matter remains elusive. We use an NMR spectrometer to observe white matter $T_{1}$ relaxation behavior in both aqueous and non-aqueous protons following six diverse initial magnetizations. The data is analyzed in the context of both an unrestricted and restricted four pool model. We show how the observed multicomponent $T_{1}$ relaxation behavior depends sensitively on the initial conditions of the different pools, suggesting that great care must be taken in interpreting $T_{1}$ relaxation measurements.

Towards Quantifying pO2 via 1H Longitudinal Relaxation of Water: Quantifying the Confounds

Kelsey Meinerz' ${ }^{1}$, Scott C. Beeman², James D. Quirk², Joel R. Garbow ${ }^{2,3}$, and Joseph J.H. Ackerman²,3,4,5

${ }^{1}$ Physics, Washington University in St Louis, St Louis, MO, United States, ${ }^{2}$ Radiology, Washington University in St Louis, Saint Louis, MO, United States, ${ }^{3}$ Siteman Cancer Center, Washington University in St Louis, Saint Louis, MO, United States, ${ }^{4}$ Internal Medicine, Washington University in St Louis, Saint Louis, MO, United States, ${ }^{5}$ Chemistry, Washington University in St Louis, Saint Louis, MO, United States

Crosslinked bovine serum albumin phantoms are used as tissue surrogates/mimics to investigate the physiologic confounds to $\mathrm{R}_{1}$-based tissue- $\mathrm{O}_{2}$ quantification under precise, laboratory controlled conditions. The relaxation-rate constants for both the rapidly relaxing and the slowly relaxing populations are affected by changes in temperature, $\mathrm{pH}$, and protein concentration.

Increased CEST specificity for amide and fast exchanging amine protons using exchange-dependent relaxation rate

Xiao-Yong Zhang ${ }^{1}$, Feng Wang ${ }^{2}$, Junzhong $\mathrm{Xu}^{2}$, Daniel Gochberg ${ }^{2}$, John Gore ${ }^{2}$, and Zhongliang Zu

${ }^{1}$ Institute of Science and Technology for Brain Inspired Intelligence, Fudan University, Shanghai, China, ${ }^{2}$ Vanderbilt University Institute of Imaging Science, Vanderbilt University, Nashville, TN, United States

It is challenging to remove overlapping chemical exchange saturation transfer (CEST) signals from nearby exchanging sites. Our previous study showed that the contributions of fast exchanging amines to CEST signals at 3 ppm induce a broad spectral region that overlaps with the amide proton transfer (APT) spectrum centered around 3.5 ppm. In this work, we apply an exchange-dependent relaxation rate $\left(R_{e x}\right)$ for quantifying CEST effects to increased CEST specificity for amide and fast exchanging amine protons. Our results demonstrate that $R_{\text {ex }}$ reduces the influences of overlapping CEST signals for APT imaging, and thus can significantly enhances the CEST detection specificity.

MR Elastography of the brain: Comparison between anisotropic and isotropic stiffness with age

Prateek Kalra ${ }^{1}$, Brian Raterman ${ }^{1}$, Xiaokui Mo $^{2}$, and Arunark Kolipaka ${ }^{1}$

${ }^{1}$ Radiology, Ohio State University Wexner Medical Center, Columbus, OH, United States, ${ }^{2}$ Center for Biostatistics, Ohio State University Wexner Medical Center, Columbus, OH, United States

Noninvasive measurement of mechanical properties of brain tissue using Magnetic Resonance Elastography has been a promising method for investigating neurological disorders such as multiple sclerosis, hydrocephalus and Alzheimer's. However, due to regional and directional dependency of brain stiffness, estimating anisotropic stiffness is important. Previous studies have investigated anisotropic and isotropic stiffness separately but none of them investigated the two together. Objective of this study is to investigate both isotropic and anisotropic stiffness together and independently compare with age and with each other. Results demonstrated a significant decrease in isotropic and anisotropic stiffness with age in some regions of the brain. 
Using healthy volunteers to optimize amide proton transfer CEST sequences.

Robert C. Brand ${ }^{1}$, Nicholas P. Blockley ${ }^{1}$, Michael A. Chappell ${ }^{2}$, and Peter Jezzard ${ }^{1}$

${ }^{1}$ Wellcome Centre for Integrative Neuroimaging, FMRIB, Nuffield Department of Clinical Neurosciences, University of Oxford, Oxford, United Kingdom, ${ }^{2} I B M E$, Department of Engineering, University of Oxford, Oxford, United Kingdom

Optimising CEST sequences for clinical use is difficult due to the lack of representative phantoms. Healthy volunteers do not show the variation in pH or concentration that these sequences seek to detect. However, in this work we show how the inherent $T_{1}$ sensitivity of CEST sequences [1] can be exploited to optimise them in healthy volunteers. We demonstrate that the sequence conditions that maximise the grey/white matter contrast in exchange maps are also the parameter conditions that maximise the exchange sensitivity. This method provides an effective way to optimise in vivo CEST sequences without the need for phantoms or simulations.

Chemical Exchange Saturation Transfer (CEST) MRI of glucosamine at 3T

Michal Rivlin ${ }^{1}$, Daniel Barazany ${ }^{2}$, and Gil Navon ${ }^{1}$

$2275 \quad{ }^{1}$ School of Chemistry, Tel Aviv University, Tel Aviv, Israel, ${ }^{2}$ Department of Neurobiology, Tel Aviv University, Tel Aviv, Israel

In our previous work using preclinical 7T MRI scanner we have shown that tumors in mice can be imaged using CEST-MRI of glucosamine. Moving toward clinical application, considering the excellent safety profile of glucosamine, we tested the CEST-MRI of glucosamine on a $3 T$ clinical scanner. Here we report significant CEST MRI signal up to $\sim 3.5$ ppm from the water signal corresponding to the exchangeable protons of the glucosamine hydroxyls and amine residues. Thus, CEST MRI using glucosamine has the potential to report on the activity of tumor metabolism, noninvasively by using MRI.

Live monitoring of red and yellow bone marrow in long bones of the mouse at $9.4 \mathrm{~T}$

Nicolas Kunz ${ }^{1}$, Josefine Tratwal ${ }^{2}$, and Olaia Naveiras ${ }^{2,3}$

When hematopoiesis is compromised, as after lethal irradiation, the red BM is rapidly infiltrated by fat, then slowly recovers hematopoietic function following BM transplantation. Monitoring this red-to-yellow-to-red BM transition non-invasively using a tree point Dixon technique would provide important information on the reconstitution of the hematopoietic system that precedes blood formation as measured by bleeding, and thus be extremely useful in experimental hematology.

In this preliminary study we investigate the feasibility to track differences in bone marrow adiposity in the C57B6 mouse femur and tibia post-irradiation by monitoring the fat content.

SafeNet: Artificial Neural Network for Real-Time T2 Mapping with Quality Assurance

Doohee Lee ${ }^{1}$, Woojin Jung ${ }^{1}$, Jingu Lee ${ }^{1}$, Jingyu Ko ${ }^{1}$, Hyeong-Geol Shin ${ }^{1}$, Hyunsung Eun ${ }^{1}$, Yoonho Nam², and Jongho Lee ${ }^{1}$

${ }^{1}$ Department of Electrical and Computer Engineering, Seoul National University, Seoul, Republic of Korea, ${ }^{2}$ Department of Radiology, Seoul St.Mary's Hospital, College of Medicine, The Catholic University of Korea, Seoul, Republic of Korea

Accurate $T_{2}$ mapping using multi-echo spin-echo data is a time-consuming process due to stimulated echo correction. In this study, we developed an artificial neural network for real-time $T_{2}$ mapping. The training dataset using both in-vivo data and model-based synthetic data demonstrated the best performance. The resulting $T_{2}$ map shows mean $T_{2}$ errors of less than $0.3 \mathrm{~ms}$ with minimal computation time (less than $1 \mathrm{sec}$ as opposed to 8.3 hours for conventional method). An additional algorithm was developed to ensure the fidelity of the $T_{2}$ map at the cost of slightly increased computation time.

Validation of intrinsic actuation MR Elastography through a $1 \mathrm{~Hz}$ experimental phantom system

Scott Gordon-Wylie ${ }^{1}$, Matthew McGarry ${ }^{1}$, Ligin Solamen ${ }^{1}$, Elijah Van Houten ${ }^{2}$, John Weaver ${ }^{1,3}$, and Keith Paulsen ${ }^{1,3}$

${ }^{1}$ Thayer School of Engineering, Dartmouth College, Hanover, NH, United States, ${ }^{2}$ Mechanical Engineering, Université de Sherbrooke, Sherbrooke, QC, Canada, ${ }^{3}$ Dartmouth Hitchcock Medical Center, Lebanon, NH, United States

A $1 \mathrm{~Hz}$ MR elastography (MRE) phantom system is presented to validate the spatial accuracy of mechanical property images in intrinsic actuation MRE. A custom hydraulically driven actuator generated $1 \mathrm{~Hz}$ shearing motions in gelatin phantoms with stiff inclusions which were measured using a retrospectively gated QFLOW sequence on a $3 T$ Philips Achieva MRI. Maps of the octahedral shear strain showed low strain in stiff inclusions, and high strains in areas of stress concentrations, as expected from theory. Shear modulus maps computed by a viscoelastic nonlinear inversion MRE algorithm were spatially accurate, and identified the correct stiffness contrast of phantom components. 
MR Elastography in a mouse model of Alzheimer's disease: 5XFAD Mice

Shreyan Majumdar ${ }^{1}$, Rachana Mishra ${ }^{2}$, Orly Lazarov ${ }^{2}$, and Dieter Klatt ${ }^{1}$

${ }^{1}$ Department of Bioengineering, University of Illinois at Chicago, Chicago, IL, United States, ${ }^{2}$ Department of Anatomy and Cell Biology, University of Illinois at Chicago, Chicago, IL, United States

In vivo magnetic resonance elastography (MRE) experiments on the 5XFAD Alzheimer's disease (AD) mouse model were conducted. The AD and Control mice were in the age group of $\sim 1$ month ( $n=2$ for both) and $3 \sim 4$ months ( $n=5$ for both). Median stiffness values were measured over different regions of the brain. The overall brain tissue was stiffer in the disease model when compared to the control, with results being significant at the 3 4-month time point. Further experiments are underway at the 1-month time-point for conclusive age-based comparisons.

Accuracy and precision of Synthetic MRI

Gabriel Mangeat ${ }^{1}$, Russell Ouellette ${ }^{2,3}$, Marcel Warntjes ${ }^{4,5}$, Michael Plattén ${ }^{2,3}$, Love Engström Nordin ${ }^{6,7}$, Nikola Stikov ${ }^{1,8}$, Tobias Granberg ${ }^{2,3}$, and Julien Cohen-Adad ${ }^{1,9}$

${ }^{1}$ NeuroPoly Lab, Institute of Biomedical Engineering, Polytechnique Montreal, Montreal, QC, Canada, ${ }^{2}$ Department of Clinical Neuroscience, Karolinska Institutet, Stockholm, Sweden, ${ }^{3}$ Division of Neuroradiology, Department of Radiology, Karolinska University Hospital, Stockholm, Sweden, ${ }^{4}$ Center for Medical Imaging Science and Visualization, CMIV, Linköping, Sweden, ${ }^{5}$ SyntheticMR, Linköping, Sweden, ${ }^{6}$ Department of Diagnostic Medical Physics, Karolinska University Hospital, Stockholm, Sweden, ${ }^{7}$ Division of Clinical Geriatrics, Department of Neurobiology, Care Sciences and Society, Karolinska Institutet, Stockholm, Sweden, ${ }^{8}$ Montreal Health Institute, Montreal, QC, Canada, ${ }^{9}$ Functional Neuroimaging Unit, CRIUGM, Université de Montréal, Montreal, QC, Canada

Synthetic MRI (SyMRI) provides quantitative PD, T1 and T2 maps via a rapid single-volume acquisition. Here, we aim to validate the precision and accuracy of SyMRI quantification by performing five scan re-scans, at two field strengths, of the quantitative NIST phantom and one control subject. Results show a good accuracy of T1 and T2 quantification at $3 \mathrm{~T}$, and a very good precision of all the phantom and subject measurements at $1.5 \mathrm{~T}$ and $3 \mathrm{~T}$ (95\% confidence intervals width are respectively lower than $6 \%$ and $4 \%$, of the measured value). This study brings confidence in comparing SyMRI quantitative measurements across subjects or time.

Dynamic Contrast Enhanced DWI in a split dynamic framework

Tryggve Holck Storås ${ }^{1}$, Endre Grøvik ${ }^{1}$, Kjell-Inge Gjesdal ${ }^{2,3}$, Sebastian Meltzer ${ }^{4,5}$, and Kathrine Røe Redalen ${ }^{4,6}$

${ }^{1}$ Diagnostic Physics, KRN, Oslo Universityhospital, Oslo, Norway, ${ }^{2}$ Department of Radiology, Akershus University Hospital, Lørenskog, Norway, ${ }^{3}$ Sunnmøre MR-klinikk, Alesund, Norway, ${ }^{4}$ Department of Oncology, Akershus University Hospital, Lørenskog, Norway, ${ }^{5}$ Faculty of Medicine, University of Oslo, Olso, Norway, ${ }^{6}$ Department of Physics, NTNU, Trondheim, Norway

Most MRI contrast agents distributes extracellular only. If the extracellular water signal is suppressed by diffusion weighting the short ranged T1-relaxation effect of a the contrast agent will be solely through water exchange through the cell membrane. In this paper we describe the implementation of a dynamic contrast enhanced diffusion weighted acquisition facilitating the study of diffusion weighting on the relaxivity of contrast agents. This is all done within a Split Dynamic framework allowing for this study to be performed without sacrificing assessment of the established pharmacokinetic parameters.

Low frequency excited MR elastography of the brain using displacement encoding with stimulated echoes and multi phase offset readouts

Johannes Strasser ${ }^{1}$, Franz Fazekas ${ }^{1}$, and Stefan Ropele ${ }^{1}$

${ }^{1}$ Medical University of Graz, Graz, Austria

In Magnetic Resonance Elastography (MRE), mechanical tissue parameters are assessed by sampling shear wave propagation via a set of motion encoded phase offset images. We here investigate a multiple phase offset image acquisition strategy based on displacement encoding with stimulated echoes (DENSE) for multi-slice human brain MRE together with a low frequency mechanical excitation and short echo times. Clear wave images could be acquired using the proposed imaging approach and estimates of the magnitudes of the complex shear modulus could be calculated from the derived wave image data set.

Can the slow compression wave in MRE data be inverted? An exploratory analysis

Eric Barnhill' ${ }^{1}$ Jürgen Braun ${ }^{2}$, and Ingolf Sack ${ }^{1}$

${ }^{1}$ Radiology, Charité, Berlin, Germany, ${ }^{2}$ Medical Informatics, Charité, Berlin, Germany 
Magnetic Resonance Elastography (MRE) data show high-amplitude, low frequency artifact which does not accord with the viscoelastic model in near-incompressible tissue. This exploratory study investigates whether the low frequency behavior is poroelastic, and if so whether slow compression wavelengths can be estimated. A cohort of abdominal MRE acquisitions at four frequencies were convolved with a fine-grained Gabor filter bank, and the frequency response of the acquisitions were pooled across subjects for liver and spleen regions. The pooled frequency responses for both liver and spleen showed a shifting peak in the response function mass that tracked with the increase in frequency, with wavespeeds in the shear regime. A second peak identified a lower frequency regime. This regime produced values similar to those observed in tissue poroelastic behaviors.

High Resolution Low Field MR Elastography

Muhammad Waqas ${ }^{1}$, Huihui $\mathrm{Xu}^{1}$, and Shadi F. Othman ${ }^{1}$

In this study, we extend MRE to a low field strength of $0.5 T$ that offers in-plane resolution of 150 micron $\times 150$ micron. To verify the method, shear wave images through gel phantoms were obtained at a mechanical excitation frequency of $370 \mathrm{~Hz}$. Preliminary studies on rat brains demonstrate the feasibility of the using low filed MRE in determining mechanical properties.

Wideband mechanical tests of the viscoelastic powerlaw behavior of phantom materials for Magnetic resonance elastography

Felix Schrank ${ }^{1}$, Heiko Tzschätzsch ${ }^{1}$, Angela Ariza de Schellenberger ${ }^{1}$, Paul Janmey ${ }^{2}$, Jürgen Braun ${ }^{3}$, and Ingolf Sack ${ }^{1}$

${ }^{1}$ Department of Radiology, Charité - Universitätsmedizin Berlin, Berlin, Germany, ${ }^{2}$ Institute for Medicine and Engineering, University of Pennsylvania, Philadelphia, PA, United States, ${ }^{3}$ Institute of Medical Informatics, Charité - Universitätsmedizin Berlin, Berlin, Germany

Shear rheometry was combined with magnetic resonance elastography (MRE) in a 1.5-T clinical system and a 0.5-T tabletop MRE system to investigate the viscoelastic powerlaw behavior of heparin and polyacrylamide (PAAm) over more than three orders of magnitude dynamic range. While heparin has softer properties than encountered in soft in-vivo tissues, crosslinked PAAm has similar stiffness as measured for in-vivo tissues, however, with lower dispersive properties. Overall both materials are good candidates for the use as standard phantom materials in MRE due to their well predictable springpot properties across the full frequency range relevant for MRE investigations.

Region-specific regularization of convection-reaction Magnetic Resonance Electrical Property Tomography (MREPT) for improving the accuracy and noise-tolerance of EP reconstruction Adan Jafet Garcia ${ }^{1}$, Shaoying Huang ${ }^{2}$, and Wenwei $\mathrm{Yu}^{1}$

${ }^{1}$ Center for Frontier Medical Engineering, Chiba University, Chiba, Japan, ${ }^{2}$ Bio-Medical Group, Engineering Product Development, Singapore University of Technology and Design, Singapore, Singapore

Magnetic resonance electrical property tomography (MREPT) is a technology for noninvasively reconstructing electrical properties (EPs) (permittivity, e, and electrical conductivity, s) of the human body from B1-map from MRI. Boundary inaccuracy and noise sensitivity are two problems of most MREPT methods. Previous studies showed that regularization can be one solution for both of the problems. However, there have been few reports on how to set up regularization terms. In this study, we show how region-specific regularization can achieve higher accuracy and noise tolerance.

Low Frequency Magnetic Resonance Conductivity Imaging By Means of Oscillating Gradient Fields

Hasan H. Eroglu ${ }^{1,2}$, Mehdi Sadighi ${ }^{1}$, and B. Murat Eyuboglu ${ }^{1}$

${ }^{1}$ Department of Electrical and Electronics Engineering, Middle East Technical University, Ankara, Turkey, ${ }^{2}$ Gaziler Physical Therapy and Rehabilitation Education and Research Hospital, Ankara, Turkey

Recently, low frequency (LF) magnetic resonance electrical conductivity imaging by means of oscillating gradient fields is reported to be infeasible. In these studies, LF phase measurements are modeled with radio frequency (RF) leakage due to geometric shifts in MR images. Although RF leakage is related with conductivity, we have not come across a conductivity image reconstructed using this model. In this study, LF conductivity imaging is evaluated for an MRI pulse sequence including multiple gradient pulses. Geometric shifts are evaluated by focusing on the MR magnitude. A procedure is proposed for the reconstruction of conductivity, based on LF phase measurements.

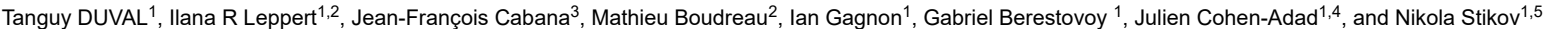

${ }^{1}$ NeuroPoly Lab, Polytechnique Montreal, Montreal, QC, Canada, ${ }^{2}$ Montreal Neurological Institute, McGill University, Montreal, QC, Canada, ${ }^{3}$ Centre Hospitalier de I'Université de Montréal, Montreal, QC, Canada, ${ }^{4}$ Functional Neuroimaging Unit, CRIUGM, Université de Montréal, Montreal, QC, Canada, ${ }^{5}$ Montreal Heart Institute, Montreal, QC, Canada 
Quantitative MR (qMR) methods exist for most MRI sequences (e.g. diffusion, magnetization transfer, inversion recovery). All these methods have a similar methodology: a biophysical model (i.e. an analytical equation), that relates the MRI contrast to some microstructural and physical features, is used to fit experimental data. Although open-source software packages are available online for certain qMR techniques, there does not exist a single stand-alone platform that can implement and compare a wide range of quantitative MRI methods. With qMRLab, we propose an open-source, MATLAB-based, object-oriented software with separate modules for each technique. We envision qMRLab as a standard platform with a growing list of contributors, where the qMR community can replicate and cross-validate a wide range of qMR methods. qMRLab includes a user-friendly graphical user interface (GUI), batch scripts examples, and qMR datasets. The software can be used to fit and check the quality of qMR data, to optimize protocols, compare fitting models, and simulate the effects of various model assumptions.

Implementation and validation of delta relaxation enhanced MRI at 3T: A system for quadrupole enhanced relaxation imaging

Markus Bödenler ${ }^{1}$, Martina Basini ${ }^{2}$, Maria Francesca Casula ${ }^{3}$, Evrim Umut ${ }^{4}$, Danuta Kruk $^{4}$, and Hermann Scharfetter $^{1}$

${ }^{1}$ Graz University of Technology, Graz, Austria, ${ }^{2}$ Università degli Studi di Milano, Milano, Italy, ${ }^{3}$ Università di Cagliari, Monserrato, Italy, ${ }^{4}$ University of Warmia and Mazury, Olsztyn, Poland

The frequency-selective nature of quadrupolar relaxation enhancement offers a high potential for designing smart molecular probes for the usage as novel MRI contrast agents by cycling the main magnetic field. Their validation and application requires a fast field-cycling MRI system. In this work, we present the first implementation and validation of such a system at the clinical field strength of 3 T. The complete FFC-MRI setup was successfully validated by $\mathrm{R}_{1}$ dispersion imaging with dispersive iron oxide magnetic nanoparticles, thus providing a ready-to-use hardware setup for the future investigation of new compounds.

Quadrupolar relaxation enhancement in selected Bismuth-Aryl compounds: Promising precursors for novel T1 MRI contrast agents Hermann Scharfetter ${ }^{1}$, Christian Gösweiner ${ }^{1}$, Evrim Umut ${ }^{2}$, Carina Sampl ${ }^{3}$, Roland Fischer ${ }^{3}$, Stefan Spirk ${ }^{4}$, Andreas Petrovic ${ }^{1}$, and Danuta Kruk ${ }^{2}$

${ }^{1}$ Institute of Medical Engineering, Graz University of Technology, Graz, Austria, ${ }^{2}$ Faculty of Mathematics and Computer Science, Universtiy of Warmia and Mazury, Olsztyn, Poland, ${ }^{3}$ Institute for Chemistry and Technology of Materials, Graz University of Technology, Graz, Austria, ${ }^{4}$ Institute of Paper, Pulp and Fibre Technology, Graz University of Technology, Graz, Austria

${ }^{209} \mathrm{Bi}$-aryl compounds have the potential for designing novel class of smart MRI $\mathrm{T}_{1}$ contrast agents which are sensitive to the chemical environment and the $\mathrm{B}_{0}$ field. We have confirmed quadrupolar relaxation enhancement (QRE) of protons as the underlying mechanism in two solid organobismuth-compounds in the $B_{0}$ range 0.5 - $3 T$. We also show first QRE peaks of solvent protons in a solution of Tris-(2-orthomethoxy-Phenyl)Bismuthane in tetrahydrofurane. This very important first step yields two promising candidates for the development of QREbased CAs and opens the way for the second step, i.e. grafting them onto water-soluble nanoparticles for optimizing the relaxivity.

MANGANESE ENHANCED MRI: A METHOD IN ORDER TO VALIDATE PHYSILOGICAL MARKERS OF TINNITUS IN RODENTS

Amandine Laboulais ${ }^{1,2}$, Maïda Cardoso ${ }^{1}$, Sergio Gonzalez ${ }^{2}$, Gaëlle Naert ${ }^{2}$, Yves Cazals² Arnaud J. Noreña $^{3}$, Sylvie Cosnier-Pucheu², Celia Belline ${ }^{2}$, and Christophe Goze-Bac ${ }^{1}$

${ }^{1}$ Charles Coulomb Laboratory, Montpellier, France, Metropolitan, ${ }^{2}$ CILCARE, Montferrier sur Lez, France, Metropolitan, ${ }^{3}$ Laboratoire de Neurosciences intégratives et Adaptatives, Marseille, France, Metropolitan

The present study is designed to show physiological markers of tinnitus in rodents. The tinnitus is an auditory phantom sensation experienced in absence of an external stimulus. The prevalence of tinnitus shows a worrying growth curve with the development of new lifestyles (exposure to noise, urbanization, ...). One promising tool is used, called Manganese Enhanced MRI (MEMRI). The use of manganese chloride as an MRI contrast agent enables to follow brain neuronal activity. T1-weighted MRI images are collected in order to investigate the specific areas activated in presence of tinnitus or not. Two analysis methods are used: Statistical analysis by Signal to Noise Ratio (SNR) and T2 rate cartography. Results enable to shown a complementary between the two analysis methods and allows us to discriminate between healthy and tinnitus rats.

Relative perfusion mapping using BOLD imaging with induced hypoxia

Chau Vu ${ }^{1}$, Julie Coloigner ${ }^{2}$, Soyoung $\mathrm{Choi}^{3,4}$, and John Wood $^{1,4}$

${ }^{1}$ Biomedical Engineering, University of Southern California, Los Angeles, CA, United States, ${ }^{2}$ Division of Radiology, Children's Hospital of Los Angeles, Los Angeles, CA, United States, ${ }^{3}$ Neuroscience Graduate Program, University of Southern California, Los Angeles, CA, United States, ${ }^{4}$ Division of Cardiology, Children's Hospital of Los Angeles, Los Angeles, CA, United States

DSC MRI is a popular perfusion technique that requires the use of an invasive exogenous contrast. This study proposes an alternative technique which uses BOLD imaging and $100 \%$ nitrogen inhalation to map relative perfusion values ( $\mathrm{rCBV}, \mathrm{rCBF}$ and $\mathrm{rMTT}$ ) without the use of contrast. We evaluated its performance on a cohort of healthy controls and sickle cell disease patients with a large range of global cerebral blood flow. 
Thomas Christen ${ }^{1}$, Jia Guo ${ }^{1}$, Wendi W. Ni ${ }^{1}$, Audrey P. Fan ${ }^{1}$, Michael M. Moseley ${ }^{1}$, and Greg Zaharchuk ${ }^{1}$

${ }^{1}$ Radiology, Stanford University, Stanford, CA, United States

A new MR approach has been proposed to obtain simultaneous measurements of blood oxygen saturation $\left(\mathrm{SO}_{2}\right)$ and hematocrit ( $\left.\mathrm{Hct}\right)$ by measuring and combining blood $\mathrm{MR}$ relaxation times. Although the first results were encouraging, the method has not been properly validated. In this study, we tested this approach in 10 volunteers subjected to gas challenges with the intent to modify $\mathrm{SO}_{2}$ while keeping $\mathrm{Hct}$ constant. The method was also tested in 10 Moyamoya patients and compared to photometric analysis. Results suggest that reliable MR estimates of both $\mathrm{SO}_{2}$ and $\mathrm{Hct}$ can be obtained in vivo.

Incidental magnetization transfer in qMRI: effects of multi-slice imaging with mixed-TSE.

Ning HUA ${ }^{1}$, Mitchell Horn ${ }^{1}$, Adam Aakil ${ }^{1}$, Stephan Anderson ${ }^{2}$, and Hernan Jara ${ }^{1}$

${ }^{1}$ Boston University, Boston, MA, United States, ${ }^{2}$ Boston Medical Center, Boston, MA, United States

Purpose: To evaluate the effect of inherent and incidental magnetization transfer (MT) on T1 and T2 measurements when using the mixed turbo spin echo sequence (mixed-TSE). Methods: mixed-TSE was applied to a phantom of 1-5\% agarose gel. The levels of the MT effects were induced and controlled by varying the number of slices per acquisition package. Results: T1 values were underestimated in multi-slice mixed-TSE. No obvious trend was observed for T2 measurements. Conclusion: mixed-TSE is powerful and efficient tool for qMRI, yet caution should be taken when interpreting the derived T1 values because of MT effects.

The Hematocrit Dependence of Blood T2 Relaxometry Parameters in the Weak Field Approximation

Avery JL Berman ${ }^{1,2}$, Jonathan R Polimeni ${ }^{1,3}$, and G Bruce Pike ${ }^{2,4}$

${ }^{1}$ Athinoula A. Martinos Center for Biomedical Imaging, Department of Radiology, Harvard Medical School, Massachusetts General Hospital, Charlestown, MA, United States,

${ }^{2}$ Department of Biomedical Engineering, McGill University, Montreal, QC, Canada, ${ }^{3}$ Division of Health Science and Technology, Massachusetts Institute of Technology, Cambridge, MA, United States, ${ }^{4}$ Department of Radiology and Hotchkiss Brain Institute, University of Calgary, Calgary, AB, Canada

The weak field approximation (WFA) is a theory that relates $T_{2}$ relaxation from tissue to the underlying tissue properties and is commonly applied to the analysis of relaxation from red blood cells (RBCs) in blood. This study examines the hematocrit-dependence of the different parameters of the WFA using simulated populations of RBCs and published experimental relaxometry results from two studies. Both the simulations and the experimental results show an unexpected result that the characteristic perturber size estimate is not constant with hematocrit but is negatively correlated with it. This has important implications for the implementation and interpretation of the WFA theory on blood relaxometry data.

Use of Entwined Magnitude and Phase-sensitive Inversion REcovery (EMPIRE) Pulse Sequences to Study the Brain and Knee

Yajun $\mathrm{Ma}^{1}$, Wei Zhao ${ }^{1}$, Adam Searleman ${ }^{1}$, Jiang Du${ }^{1}$, Nikolaus M Szeverenyi ${ }^{1}$, and Graeme M Bydder ${ }^{1}$

${ }^{1}$ Radiology, Univ of Cal, San Diego, LaJolla, CA, United States

The combination (addition/subtraction) of magnitude and phase-sensitive IR images (termed EMPIRE technique) when appropriate TIs were used was found to provide increased tissue contrast over specific ranges of tissue $T_{1}$. This behavior was explored numerically and summarized in signal intensity vs. $T_{1}$ plots. Clinically relevant applications were demonstrated in brain and knee cartilage using FSE and UTE data collections. In addition to increased contrast, this approach allowed detection of short $T_{2}$ tissue signals while suppressing unwanted signal from longer $\mathrm{T}_{1}$ tissue fluids.

Age-associated changes in skeletal muscle morphology assessed by intramuscular adipose and connective tissue

Bharath Ambale Venkatesh ${ }^{1}$, Yoko Kato ${ }^{2}$, Jaclyn Sesso ${ }^{2}$, Jason Ortman ${ }^{2}$, John Pitts ${ }^{3}$, Michio Ozaki², Yoshimori Kassai ${ }^{4}$, and Joao AC Lima ${ }^{2}$

${ }^{1}$ Radiology, Johns Hopkins University, Baltimore, MD, United States, ${ }^{2}$ Johns Hopkins University, Baltimore, MD, United States, ${ }^{3}$ Toshiba Medical Research Institute USA Inc, Mayfield Village, $\mathrm{OH}$, United States, ${ }^{4}$ Toshiba Medical Systems Corporation, Tochigi, Japan

In human skeletal muscles, the aging process causes a decrease of contractile function likely associated with an increase in intramuscular adipose and connective tissues. The accumulation of non-contractile tissues and loss of muscle tissue may contribute to sarcopenia and frailty observed at older age but their quantification is challenging1. The purpose of this study was to establish MR imaging-based methods to quantify the relative amounts of fat and connective tissue in healthy human subjects, and investigate their association with age. 
fMRI: Physiology

Cerebrovascular function in the middle cerebral artery measured using the cardiac-induced inflow effect on fast echo-planar imaging

Joseph Whittaker ${ }^{1}$, Patrick Liebig ${ }^{2}$, Fabrizio Fasano ${ }^{3}$, Marcello Venzi ${ }^{1}$, Robin Heidemann ${ }^{2}$, and Kevin Murphy ${ }^{1}$

${ }^{1}$ School of Physics and Astronomy, Cardiff University Brain Research Imgaging Centre (CUBRIC), Cardiff University, Cardiff, United Kingdom, ${ }^{2}$ Siemens Healthcare GmbH, Erlangen, Germany, ${ }^{3}$ Siemens Healthcare Ltd, Camberly, United Kingdom

We demonstrate that cardiac-induced pulsatile flow-related signal enhancement in fast EPI provides a dynamic assessment of cerebrovascular function in the brain's large feeding arteries. We show that cardiac pulsatile waveforms, derived from magnitude data taken at the site of the middle cerebral artery, are attenuated at longer TRs, suggesting they are related to pulsatile flow rather than volume changes. The same waveforms are modulated by a global flow-increasing hypercapnic challenge, showing that this endogenous signal contrast can be useful for exploring dynamic cerebrovascular function. We propose that a multi-shot segmented EPI approach will further increase this signal contrast.

Modelling the laminar GRE-BOLD signal: integrating anatomical, physiological and methodological determinants

Alberto Merola ${ }^{1}$ and Nikolaus Weiskopf ${ }^{1}$

${ }^{1}$ Max Planck Institute for Human Cognitive and Brain Sciences, Leipzig, Germany

An insight into the layered functional organization of grey matter can be offered by spatially accurate high resolution measurements of the laminar BOLD signal. However, their specificity is limited by anatomical, physiological and methodological features affecting the functional point spread function (fPSF). In order to examine these, an integrated model of the laminar GRE-BOLD signal has been formulated that combines a vascular geometric model of the cortex with a model describing the relationship between underlying physiological parameters and $\mathrm{R}^{*}$ changes. Using the new detailed model we are able to characterize the laminar GRE-BOLD signal dependency on physiological and partial volume effects.

Sex differences in resting-state cerebral activity alterations in Internet gaming disorder

Yawen Sun ${ }^{1}$, Yan Zhou ${ }^{1}$, Yao Wang ${ }^{1}$, Xu Han ${ }^{1}$, Weina Ding ${ }^{1}$, Yong Zhang ${ }^{2}$, Jianxun $\mathrm{Qu}^{2}$, and Jianrong Xu ${ }^{1}$

${ }^{1}$ Department of Radiology, Ren Ji Hospital, School of Medicine, Shanghai Jiao Tong University, Shanghai, China, ${ }^{2}$ Ge Applied Science Laboratory, GE Healthcare, Shanghai, China

The purpose of this study was to explore the sex-specific neuroimaging differences involved in IGD. Thirty IGDm, 23 IGDf, $30 \mathrm{HCm}$ and $22 \mathrm{HCf}$ underwent rs-fMRI. ALFF and seedbased FC maps were constructed. A two-factor ANCOVA model was specified using SPM8, with sex and diagnosis as the between-subject factors. When interaction effects occurred, post-hoc pair-wise comparisons were performed using two-sample t-tests within the interaction masks. IGDm and IGDf exhibited different regional and network-level functional changes. Lower ALFF values in the orbit part of SFG showed higher impulsivity in IGDm. The results may lead to improved sex-specific treatment and prevention strategies .

Human whole-brain sub-millimeter cerebral blood flow map using 7T ASL

Dimo Ivanov ${ }^{1}$, Sriranga Kashyap ${ }^{1}$, Roy AM Haast ${ }^{1}$, Shanice Janssens ${ }^{1}$, Laurentius Huber ${ }^{2}$, Benedikt A Poser ${ }^{1}$, and Kâmil Uludağ

$2301 \quad{ }^{1}$ Department of Cognitive Neuroscience, Maastricht University, Maastricht, Netherlands, ${ }^{2}$ SFIM, NIMH, Bethesda, MD, United States

Arterial spin labeling (ASL) offers non-invasive cerebral blood flow (CBF) measurements, but typically suffers from low signal-to-noise ratio, limiting the achievable spatial resolution. In this work, we employ 3D EPI ASL at 7T, partially-overlapping acquisition of multiple slabs and across-session averaging to achieve a high-quality whole-brain 0.7 mm ${ }^{3}$ isotropic resolution CBF map from a healthy volunteer. The dataset presents the highest spatial resolution CBF map in humans so far, and a unique opportunity to investigate the cortical distribution of baseline CBF across and within brain areas, including providing a physiological basis for the interpretation of laminar and columnar fMRI.

Comparison of neurovascular coupling and BOLD responses under medetomidine and isoflurane anesthesia in the rat somatosensory cortex.

Ryota Tokunaga ${ }^{1}$, Thierry Paquette ${ }^{2}$, Hugues Leblond ${ }^{2}$, Tomokazu Tsurugizawa ${ }^{3}$, and Mathieu Piché ${ }^{4}$

${ }^{1}$ Chiropractic, UQTR, Trois-Rivieres, QC, Canada, ${ }^{2}$ Anatomy, UQTR, Trois-Rivieres, QC, Canada, ${ }^{3}$ NeuroSpin, Commissariat à l'Energie Atomique-Saclay Center, Gif-sur-Yvette,

France, ${ }^{4}$ Chiropractic, UQTR, Trois-Rivières, QC, Canada 
In this study, we aimed at comparing the coupling between neuronal activity and hemodynamic changes evoked by hindpaw stimulation, under medetomidine and isoflurane anesthesia. Simultaneous recordings of local field potentials (LFP) and cerebral blood flow (CBF) were performed in the rat somatosensory cortex. In a separate experiment, hemodynamic changes evoked by hindpaw stimulation were measured using BOLD fMRI. The coupling between LFP amplitude and CBF changes was similar between isoflurane and medetomidine anesthesia. However, BOLD signal changes were smaller under isoflurane compared with medetomidine anesthesia. This suggests that isoflurane anesthesia may alter BOLD signal through alteration of $\mathrm{O} 2$-consumption or $\mathrm{O} 2$-saturation.

Brain activity and connectivity changes in response to glucose ingestion

Anna M van Opstal ${ }^{1}$, Anne Hafkemeijer ${ }^{1,2,3}$, Annette van den Berg-Huysmans ${ }^{1}$, Marco Hoeksma ${ }^{4}$, Cor Blonk ${ }^{4}$, Hanno Pijl ${ }^{5}$, Serge A.R.B Rombouts ${ }^{1,2,3}$, and Jeroen van der Grond ${ }^{1}$

${ }^{1}$ Department of Radiology, Leiden University Medical Center, Leiden, Netherlands, ${ }^{2}$ Department of Methodology and Statistics, Institute of Psychology, Leiden, Netherlands, ${ }^{3}$ Leiden Institute for Brain and Cognition (LIBC), Leiden, Netherlands, ${ }^{4}$ Unilever Research \& Development, Vlaardingen, Netherlands, ${ }^{5}$ Department of Internal Medicine, Leiden University Medical Center, Leiden, Netherlands

Understanding of functional brain responses yields insights into satiety signaling, nutrient sensing, energy seeking and feeding behavior. The current aim was to determine normal whole brain functional responses to the ingestion of glucose in healthy normal weight subjects using BOLD signal, network connectivity and Eigen vector centrality functional MRI analysis approaches. Our results show that ingestion of glucose in a fasted state leads to deactivation and decreased connectivity, which can be associated with satiation and reward effects in the brain and a decrease in energy seeking. In contrast, drinking plain water leads to activation and increased centrality and connectivity.

Anesthesia affects connectivity of default-mode sub-networks in the rat in a time-dependent and region-dependent manner

Punitkumar Makani ${ }^{1}$, Rolf Gruetter ${ }^{2}$, and lleana Ozana Jelescu ${ }^{2}$

${ }^{1}$ University of Tübingen, Tübingen, Germany, ${ }^{2}$ Centre d'Imagerie Biomédicale, Ecole Polytechnique Fédérale de Lausanne, Lausanne, Switzerland

Anesthetic agents affect brain connectivity and/or neurovascular coupling, with confounding effects on BOLD resting-state fMRI. To date, the most widespread anesthesia protocol for fMRI in rats consists in isoflurane induction followed by medetomidine sedation. We report that, using this protocol, connectivity of default-mode sub-networks is affected in a timedependent and region-dependent manner, with modules such as hippocampus becoming detectable as late as two hours into sedation. These spatio-temporal features have significant implications for the interpretation and comparison of resting-state studies in the rat, and of the default-mode network connectivity in particular.

Cortical propagation of slow oscillation-associated traveling waves resolved by fast line scanning in brain-state-informed BOLD fMRI

Andrea Kronfeld ${ }^{1}$, Felipe Aedo-Jury ${ }^{1}$, Lara Hamzehpour ${ }^{1}$, and Albrecht Stroh ${ }^{1}$

${ }^{1}$ Institute of Microanatomy and Neurobiology, University Medical Center of the Johannes Gutenberg University Mainz, Mainz, Germany

Cortical activity patterns - both spontaneous and stimulus-evoked - are significantly impacted by the respective functional brain state. Here, we explored activity patterns in two brain states: persistent state, maintained by sedation, and slow wave state, dominated by slow-oscillation-associated waves, maintained by rather deep anesthesia. Upon visual stimulation, we found localized activation of the visual cortex only in persistent state, whereas in slow wave state, large areas of the cortex are recruited. By applying fast line scanning methods, we could for the first time resolve a propagation of slow waves by fMRI, presumably evoked by visual stimulation.

Sex Differences in Stimulus Induced Blood Flow: The Importance of Sex Hormones

Samantha Cote $^{1}$, Russell Butler ${ }^{1}$, Jean-Francios Lepage ${ }^{1}$, Adrianna Mendrek $^{2}$, and Kevin Whittingstall ${ }^{1}$

${ }^{1}$ Université de Sherbrooke, Sherbrooke, QC, Canada, ${ }^{2}$ Bishop's University, Sherbrooke, QC, Canada

Sex differences in resting CBF has been reported, these differences may be explained through sex differences in sex hormones. There is currently no study that examines if this difference is maintained during stimulus-induced CBF. The current study evaluated men and naturally cycling women three times during their menstrual cycle at different sex hormone levels using a pCASL sequence. Preliminary results reveal sex differences in CBF response to the same stimulus, which is amplified when one considers sex hormones. These findings may reflect vascular effects of sex hormones, highlighting the importance of considering sex and hormone profiles when conducting fMR

Yameng Gu' and Xiao Liu

${ }^{1}$ Biomedical Engineering, Pennsylvania State University, State College, PA, United States 
Head motion has been shown to be associated with distinct changes in local and long-range rsfMRI connectivity, and the temporal scrubbing based on motion parameters has been proposed to remove such "motion-induced" artefacts. Here, we showed that scrubbing arousal-related time points resulted in a similar but stronger change on the rsfMRI connectivity than the motion-based scrubbing. Moreover, the effect of the motion-based scrubbing can be completely removed by retaining the part of scrubbed time points related to arousal changes. The findings suggest that arousal modulations may mediate the association between the motion and rsfMRI connectivity.

Robust arterial functional MRI (fMRI) data and its application

Jinxia Yao ${ }^{1}$, James Hao Wang ${ }^{2}$, Xin Shen ${ }^{2}$, and Yunjie Tong ${ }^{2}$

${ }^{1}$ Agricultural and Biological Engineering, Purdue University, West Lafayette, IN, United States, ${ }^{2}$ Weldon School of Biomedical Engineering, Purdue University, West Lafayette, IN, United States

Previous research conducted on Myconnectome project (90 resting scan sessions of one subjects) found that systemic low frequency oscillations (sLFOs) extracted from resting-state $\mathrm{fMRI}$ data of big arteries to be: 1) negatively correlated with; 2) temporally leading, the fMRI data from the veins. To generalize the finding, the resting state scans from 20 randomly selected subjects of Human Connectome Project (HCP) were analyzed. The findings were validated among around $80 \%$ of the data, which also showed that cerebral circulation time between females and males are significantly different.

BOLD-fMRI comparison of olfactory responses in the mouse whole brain, with different odors and anesthesia

Fuyu Hayashi ${ }^{1}$, Sosuke Yoshinaga ${ }^{1}$, Naoya Yuzuriha ${ }^{1}$, Mitsuhiro Takeda ${ }^{1}$, and Hiroaki Terasawa ${ }^{1}$

${ }^{1}$ Faculty of Life Sciences, Kumamoto University, Kumamoto, Japan

Mice have well-developed olfactory systems, and the odor response throughout the mouse whole brain is an important target of olfactory research. We previously applied independent component analysis (ICA), which identifies periodically activated regions, to detect BOLD responses from odor-stimulated mice. In this study, we successfully discriminated olfactory responses from different odors, isoamyl acetate and musocone, in the mouse whole brain, using the BOLD-ICA method. In addition, we investigated the effects of urethane and medetomidine anesthetics on the olfactory responses. This study demonstrated the utility of the BOLD-ICA method to trace the real-time activation of the mouse whole brain.

Vascular effect on cerebral blood flow in BOLD fMRI under fed-caffeinated effect

Ho-Ching Yang ${ }^{1}$, Xin Shen ${ }^{1}$, Matthew Robert Derdak ${ }^{1}$, Blaise deB Frederick ${ }^{2,3}$, and Yunjie Tong ${ }^{1}$

${ }^{1}$ Weldon School of Biomedical Engineering, Purdue University, West Lafayette, IN, United States, ${ }^{2}$ McLean Imaging Center, McLean Hospital, Belmont, MA, United States, ${ }^{3}$ Department of Psychiatry, Harvard Medical School, Boston, MA, United States

In this study, we explored the vascular effect of a fed-caffeinated condition versus a fasted-uncaffeinated condition in resting-state fMRI dataset from the Myconnectome project. We extracted the low frequency oscillation signal from the superior sagittal sinus (SSS) as a vascular seed to evaluate the propagation of these signals through the brain in these two conditions.

The footprint of physiology in ultra-fast RS-FMRI

Daniel P Gomez ${ }^{1}$, Zahra Fazal ${ }^{2}$, José P Marques ${ }^{2}$, and David G Norris ${ }^{2}$

${ }^{1}$ Radboud University, Nijmegen, Netherlands, ${ }^{2}$ Donders Centre for Cognitive Neuroimaging, Radboud University, Nijmegen, Netherlands

In the current contribution we study the footprint of physiology in ultra-fast RS-FMRI timeseries by examining RSNs obtained from full brain EPI data acquired with a sampling rate of $158 \mathrm{~ms}$.

BOLD-fMRI evaluation of different types of analgesic agents on allodynia-specific pain in a rat chronic pain model

Mikio Sameshima ${ }^{1}$, Naoya Yuzuriha ${ }^{1}$, Sosuke Yoshinaga ${ }^{1}$, Soichiro Ezaki ${ }^{1}$, Norihito Ishida ${ }^{1}$, Mitsuhiro Takeda ${ }^{1}$, Yuya Terashima ${ }^{2}$, Etsuko Toda ${ }^{2}$, Kouji Matsushima $^{2}$, and Hiroaki Terasawa $^{1}$

${ }^{1}$ Faculty of Life Sciences, Kumamoto University, Kumamoto, Japan, ${ }^{2}$ Graduate School of Medicine, The University of Tokyo, Tokyo, Japan 
The aims of this study are to reveal the underlying inhibitory mechanism of a compound against the chemokine signal, based on targeted protein structures, and to evaluate the analgesic effect on allodynia-specific responses in a rat chronic pain model, with our BOLD-fMRI-based pain evaluation system using a green laser. An NMR titration analysis demonstrated that the compound strongly binds to the chemokine receptor-binding protein. BOLD-fMRI revealed that the allodynia-specific responses were suppressed by the administration of the compound, in a similar manner to the existing analgesic, pregabalin, with a completely different mechanism of action from that of the compound.

Evidence of faster hemodynamic response function at weak sensory stimulus levels supports higher frequency intrinsic functional connectivity

Jingyuan Chen $^{1}$ and Gary Glover ${ }^{2}$

${ }^{1}$ MGH/HST Martinos Center for Biomedical Imaging, Charleston, MA, United States, ${ }^{2}$ Stanford University, Stanford, CA, United States

There has been emerging evidence that resting state spontaneous neural fluctuations can persist at frequencies not supported by the canonical hemodynamic response function (HRF). As RS may likely comprise varying levels of spontaneous stimuli, it is thus of interest to query whether BOLD fluctuations elicited by small-intensity stimuli occur at faster time scales than the canonical HRF and can account for certain high-frequency (HF) phenomena observed at rest. Here, we employ a vibrotactile stimulus with graded contrasts, and show that HRFs elicited by small intensity stimuli have faster time-to-peak and narrower dispersions than canonical HRFs, thus may promise elevated BOLD responses in higher frequency bands and explain part of the HF phenomena observed in recent RS studies.

Interactions between cardiac waves and resting-state BOLD signals exhibit high intra-subject consistency and high inter-subject variability

Jingyuan Chen ${ }^{1}$, Laura Lewis ${ }^{2}$, Marta Bianciardi ${ }^{3}$, and Jonathan Polimeni ${ }^{2}$

${ }^{1}$ MGH/HST Martinos Center for Biomedical Imaging, Charleston, MA, United States, ${ }^{2}$ Athinoula A. Martinos Center for Biomedical Imaging, Boston, MA, United States, ${ }^{3}$ Radiology, Athinoula A. Martinos Center for Biomedical Imaging, Boston, MA, United States

Low-frequency respiratory and systemic cardiovascular fluctuations can affect vascular oxygenation and manifest in the envelope of cardiac waves. Here, we examined the interaction between the envelope of cardiac waves collected by a piezoelectric (PO) sensor (POE) and fMRI signals, and found that POE may provide unique information about BOLD fluctuations that are not explained by changes of heart rate, respiratory volumes or end-tidal CO2 levels. We also observed that the interaction between fMRI, cardiac, and respiratory measures was relatively stable within individuals, but highly variable across individuals.

Functional connectivity and dynamic change of rat brain resting-state networks under morphine-induced condition

Wei Zhu ${ }^{1}$, Hannes M. Wiesner ${ }^{1}$, Xiao-Hong Zhu ${ }^{1}$, Yi Zhang ${ }^{1}$, Nanyin Zhang ${ }^{2}$, Yunchong Ma $^{2}$, and Wei Chen

${ }^{1}$ Center for Magnetic Resonance Research, Department of Radiology, University of Minnesota, MINNEAPOLIS, MN, United States, ${ }^{2}$ Department of Biomedical Engineering, Penn State University, State College, $P A$, United States

Resting-state fMRI (rs-fMRI) in animal is essential for studying neural networks and translational research. However, animal motion poses a major obstacle for performing rs-fMRI, and it is commonly requires anesthesia that could suppress and alter the resting-state networks (RSNs). In this work, we investigated the rat RSNs under morphine condition, and the differentiation and transition of RSNs when animal conditions were changing from isoflurane to morphine. We found that the number of RSNs was significantly increased from deep anesthesia to morphine-induced condition; the RSNs became highly specific to brain functions; and thus, RSN mapping became more reliable.

Force-related BOLD effects during naturalistic and symbolic effort observation

Letizia Casiraghi ${ }^{1,2}$, Adnan Alahmadi ${ }^{3,4}$, Anita Monteverdi $^{1}$, Fulvia Palesi ${ }^{2,5}$, Gloria Castellazzi ${ }^{2,6}$, Giovanni Savini ${ }^{2,7}$, Karl Friston ${ }^{8}$, Claudia Angela Gandini Wheeler-Kingshott ${ }^{1,4,9}$, and Egidio D'Angelo ${ }^{1,2}$

${ }^{1}$ Department of Brain and Behavioral Sciences, University of Pavia, Pavia, Italy, ${ }^{2}$ Brain Connectivity Center, C. Mondino National Neurological Institute, Pavia, Italy, ${ }^{3}$ Department of Diagnostic Radiology, Faculty of Applied Medical Science, King Abdulaziz University (KAU), Jeddah, Saudi Arabia, ${ }^{4}$ Queen Square MS Centre, UCL Institute of Neurology, Faculty of Brain Sciences, University College London (UCL), London, United Kingdom, ${ }^{5}$ Brain Connectivity Center, University of Pavia, Pavia, Italy, ${ }^{6}$ Department of Electrical, Computer and Biomedical Engineering, University of Pavia, Pavia, Italy, ${ }^{7}$ Department of Physics, University of Milan, Milan, Italy, ${ }^{8}$ University College London (UCL), London, United Kingdom, ${ }^{9}$ Brain MRI 3 T Research Centre, C. Mondino National Neurological Institute, Pavia, Italy

In this pilot study, we used a 3-condition fMRI squeezeball paradigm to study the non-linear BOLD response to varying grip force (GF) during action execution (AE, subjects performed the task), action observation ( $A O$, subjects watched a video of the task) and $A O$ with visual cue (AOvc). fMRI activity patterns in brain circuits controlling $A E, A O$ and $A O v c$ account for different GF applied to an object or perceived from others' action. AO and AOvc calls different processing depending on the presence or the absence of the visual cue indicating specific regions and BOLD-GF relations for the effort perception. 
Kenneth T Wengler ${ }^{1}$, Justina $\mathrm{Tam}^{2}$, Steven Weissbart ${ }^{2}$, and Xiang $\mathrm{He}^{3}$

${ }^{1}$ Biomedical Engineering, Stony Brook University, Stony Brook, NY, United States, ${ }^{2}$ Urology, Stony Brook University Hospital, Stony Brook, NY, United States, ${ }^{3}$ Radiology, Stony Brook University Hospital, Stony Brook, NY, United States

Overactive bladder affects a significant portion of women in the US $(\sim 15 \%)$. Women with this syndrome experience a frequent pathologic desire to urinate with a profound impact on their quality of life and productivity. It is unclear how cerebral perfusion changes as the bladder fills. In this study eight healthy female participants were imaged with a double-echo EPI sequence for simultaneous ASL and BOLD acquisition. Bladder filling by urethral catheter was used to assess the brain's response at filling volumes of 0,50,100, 200, 350 and $500 \mathrm{~mL}$. Increased blood flow was observed at low urgency compared to baseline while decreased blood flow was observed at high urgency compared to low urgency.

Multi-scale assessment of brain network response to sustained working memory task

Daniele Mascali ${ }^{1}$, Silvia Tommasin ${ }^{1,2}$, Marta Moraschi $^{1}$, Tommaso Gili ${ }^{1,3}$, Ibrahim Eid Assan ${ }^{4}$, Michela Fratini ${ }^{3}$, Richard G. Wise ${ }^{5}$, Silvia Mangia ${ }^{6}$, Emiliano Macaluso $^{7}$, and Federico Giove ${ }^{1,3}$

${ }^{1}$ Centro Fermi - Museo storico della fisica e Centro di studi e ricerche Enrico Fermi, Rome, Italy, ${ }^{2}$ Dipartimento di Neurologia e Psichiatria, Sapienza Università di Roma, Roma, Italy, ${ }^{3}$ Fondazione Santa Lucia IRCCS, Roma, Italy, ${ }^{4}$ Dipartimento di Fisica, Sapienza Università di Roma, Roma, Italy, ${ }^{5}$ Cardiff University Brain Research Imaging Centre (CUBRIC), Cardiff, United Kingdom, ${ }^{6}$ Center for Magnetic Resonance Research, University of Minnesota, Minneapolis, MN, United States, ${ }^{7}$ ImpAct Team, Lyon Neuroscience Research Center, Lyon, France

How low-frequency BOLD fluctuations (LFFs) are modulated when the brain is engaged in processing external stimuli is still poorly described. We exploited a non-conventional, longlasting, block-design paradigm to study LFF modulations during sustained performance of a working memory task. Task-associated modulations were characterized by increased synchronization between networks at the expense of reduced within-network coherence. Such pattern persisted at several spatial scales, indicating a scale-invariant feature of taskassociated modulations. Despite such clear-cut network behavior, no linear correlation between performance and connectivity changes was observed. Contrarily, high levels of connectivity at task and especially at rest were associated with greater performance.

Investigating the Functional Diffusion-Signal Response (DfMRI) in Living, CA1 Pyramidal Neurons Undergoing Chemical Activation with Kainate

Jeremy J. Flint ${ }^{1,2}$, Kannan Menon ${ }^{2,3,4}$, Brian Hansen ${ }^{5}$, John Forder ${ }^{2,3,4}$, and Stephen J. Blackband ${ }^{1,2,6}$

${ }^{1}$ Neuroscience, University of Florida, Gainesville, FL, United States, ${ }^{2}$ McKnight Brain Institute, University of Florida, Gainesville, FL, United States, ${ }^{3}$ Biomedical Engineering, University of Florida, Gainesville, FL, United States, ${ }^{4}$ Radiology, University of Florida, Gainesville, FL, United States, ${ }^{5}$ Center for Functionally Integrative Neuroscience, Aarhus University, Aarhus, Denmark, ${ }^{6}$ National High Magnetic Field Laboratory, Florida State University, Tallahassee, FL, United States

In the current study, we use MR microscopy and superfusion techniques to investigate changes in the diffusion properties of living, hippocampal neurons following activation with kainate. Acute hippocampal slice preparations $(n=6)$ were imaged at six different $b$ values $\left(b=0\right.$ to $\left.3200 \mathrm{~s} / \mathrm{mm}^{2}\right)$ both before and after exposure to kainate $(100 \mu M)$. Significant activation-based changes $(p=0.0043)$ in diffusion properties were detected in the stratum pyramidale: the tissue lamina comprised primarily of pyramidal neuron cell bodies. No changes were observed in either the strata oriens (apical dendrites) or radiatum (axons).

Characterization of the Central Analgesic Effects of Two Different Acupuncture Modalities in a Mouse Model of Nociception

Isabel Wank ${ }^{1,2}$, Jianliang Zhang ${ }^{3,4}$, Shuping Chen ${ }^{4}$, Vanja Nagy ${ }^{3,5}$, Liqun Zhang $^{6}$, Silke Kreitz ${ }^{1}$, Andreas Hess ${ }^{1}$, and Josef Penninger ${ }^{3}$

${ }^{1}$ Institute of Pharmacology and Toxicology, University of Erlangen-Nuremberg, Bayern - Erlangen, Germany, ${ }^{2}$ Department of Medicine 3 Rheumatology and Immunology, University Hospital Erlangen, Erlangen, Germany, ${ }^{3}$ Institute of Molecular Biotechnology, Austrian Academy of Sciences, Vienna, Austria, ${ }^{4}$ Institute of Acupuncture and Moxibustion, China Academy of Chinese Medical Sciences, Beijing, China, ${ }^{5}$ Ludwig Boltzmann Institute for Rare and Undiagnosed Diseases, Austrian Academy of Sciences, Vienna, Austria, ${ }^{6}$ Clinical Institute of Laboratory Medicine, Medical University of Vienna, Vienna, Austria

By using functional MRI, possible analgesic effects of two different acupuncture treatments (insertion of needles and electro-acupuncture) at Zusanli acupoint (ST36) were investigated. The brain's response in anaesthetized C57B//6J mice to noxious stimuli with and without acupuncture was analyzed by characterization of the classical stimulus-driven BOLD parameters but also the influence on stimulus- as well as non-stimulus-driven functional connectivity-based brain networks. Acupuncture was shown to modulate (pseudo-resting state) brain networks by enhancing functional connectivity within limbic structures and decreasing thalamic connectivity particular with electro-acupuncture. Thereby acupuncture exerts control over the processing of noxious stimuli by higher-order brain regions.

Approximation of $1 \mathrm{H}$ MRS glutamate from fMRI hemodynamic response function

Rangaprakash Deshpande ${ }^{1,2}$, Gopikrishna Deshpande ${ }^{2,3,4}$, Reza Tadayonnejad ${ }^{1}$, Joseph O'Neill ${ }^{1}$, and Jamie D. Feusner ${ }^{1}$ 
${ }^{1}$ Department of Psychiatry and Biobehavioral Sciences, University of California Los Angeles, Los Angeles, CA, United States, ${ }^{2}$ Department of Electrical and Computer Engineering, Auburn University, Auburn, AL, United States, ${ }^{3}$ Department of Psychology, Auburn University, Auburn, AL, United States, ${ }^{4}$ Alabama Advanced Imaging Consortium, Auburn University and University of Alabama Birmingham, Auburn, AL, United States

Functional MRI is a blood-based marker of neural activity. The transfer function relating the two is the hemodynamic response function (HRF), which varies across both brain regions and individuals. It is traditionally considered a confound in $\mathrm{fMRI}$ analysis; however, the underlying biophysics suggests that HRF might in part reflect local neurochemical substrates, specifically glutamate, GABA and serotonin. Here, we found evidence that HRF shape is associated with, and predictive of, ${ }^{1} \mathrm{H}$ MRS glutamate in thalamus. These results open the possibility of approximating neurochemical concentrations using resting-state fMRI. Future studies could validate this in an independent and larger sample.

Traditional Poster

\section{Task-Based fMRI: Acquisition \& Analysis}

A Weighted Square Averaging Method of Combining Primary and Temporal Derivative Parameter Estimates In General Linear Model Analysis of Functional MRI

Kwan-Jin Jung ${ }^{1}$ and Hae-Min Jung ${ }^{2}$

${ }^{1}$ Human Magnetic Resonance Center, Institute of Applied Life Sciences, University of Massachusetts Amherst, Amherst, MA, United States, ${ }^{2}$ Austen Riggs Center, Stockbridge, MA, United States

The temporal derivative has been considered as a mathematical solution for the latency variation of the hemodynamic response function (HRF) in the general linear model (GLM) analysis of the task-based functional MRI (fMRI). A method of combining the primary and derivate estimates was developed by Calhoun and its implementation was introduced. However, serious defects were revealed in the existing methods from a GLM analysis of an event-related fMRI. Here, the method is revised to provide a correct combined estimate using a weighted square average method. The proposed method was confirmed with event-related fMRI studies at various phases of the double Gamma HRF.

Energy-Period Characteristics of Brain Networks using Empirical Mode Decomposition

Dietmar Cordes ${ }^{1,2}$, Muhammad Kaleem ${ }^{3}$, Xiaowei Zhuang ${ }^{1}$, Karthik Sreenivasan ${ }^{1}$, Zhengshi Yang ${ }^{1}$, Tim Curran $^{2}$, and Virendra Mishra

${ }^{1}$ Cleveland Clinic Lou Ruvo Center for Brain Health, Las Vegas, NV, United States, ${ }^{2}$ University of Colorado, Boulder, CO, United States, ${ }^{3}$ University of Management \& Technology, Lahore, Pakistan

In this project, we have studied resting-state networks using Empirical Mode Decomposition (EMD) to obtain time-frequency-energy information. Intrinsic Mode Functions (IMFs) and associated spatial maps provide a data-driven decomposition of resting-state networks. We investigated the average energy-period relationship of IMFs of group independent components analysis (ICA) networks to better characterize temporal properties of networks and found that the IMFs of BOLD data provide inverted V-shaped energy-period signatures that allow a natural ranking of all resting-state networks when compared to signatures of pure noise.

Neurophysiological Basis of Multi-Scale Entropy Analysis of Brain Complexity and Its Relationship with Functional Connectivity

Danny JJ Wang ${ }^{1}$, Kay Jann ${ }^{1}$, Chang Fan ${ }^{1}$, Yang Qiao ${ }^{2}$, Yu-Feng Zang ${ }^{2}$, Hanbing Lu $^{3}$, and Yihong Yang ${ }^{3}$

${ }^{1}$ Laboratory of FMRI Technology, Stevens Neuroimaging and Informatics Institute, University of Southern California (USC), Los Angeles, CA, United States, ${ }^{2}$ Center for Cognition and Brain Disorders, Institutes of Psychological Sciences, Hangzhou Normal University, Hangzhou, China, ${ }^{3}$ Neuroimaging Research Branch, National Institute on Drug Abuse, Baltimore, MD, United States

Recently, non-linear statistical measures such as multi-scale entropy (MSE) have been introduced as indices of the complexity of BOLD fMRI time-series across multiple time scales. In this work, we investigated the neurophysiological underpinnings of complexity (MSE) of electrophysiology and fMRI signals and their relations to functional connectivity (FC). We include both simulation data using neural mess model based brain network model and animal models with concurrent recording of fMRI and electrophysiology in conjunction with pharmacological manipulations. Our results show that the complexity of regional electrophysiology and fMRI signals is positively correlated with network FC. 
Taking advantage of improvements to EPI imaging at 7T could allow for direct use of EPI volumes when registering multiple volumes to a common space. We proposed using an unsaturated EPI template in order to perform the spatial co-registration of multiple subjects. In this study, we compare the quality of a resulting group map resting state motor network connectivity in order to evaluate different registration pipelines, one utilizing an EPI template as well as one based on the commonly used T1 weighted image template.

Multivariate Second Level Analysis in fMRI with Canonical Correlation Analysis

Xiaowei Zhuang ${ }^{1}$, Zhengshi Yang ${ }^{1}$, Rajesh Nandy², Tim Curran ${ }^{3}$, and Dietmar Cordes ${ }^{1,3}$

${ }^{1}$ Cleveland Clinic Lou Ruvo Center for Brain Health, Las Vegas, NV, United States, ${ }^{2}$ University of North Taxes, Fort Worth, TX, United States, ${ }^{3}$ University of Colorado, Boulder, CO, United States

A multivariate CCA method is introduced for fMRI $2^{\text {nd }}$ level analysis to incorporate local neighboring information, and to improve the sensitivity in group activation and group difference detection in noisy fMRI data. Statistical thresholds for significance of the group-inferences in the multivariate method are computed non-parametrically. Results from both simulated data and real episodic memory data indicate that a higher detection sensitivity for a fixed specificity can be achieved in both $2^{\text {nd }}$ level activation and difference detection with the proposed method, as compared to the widely used univariate techniques.

Quantitative assessment of fMRI head motion metrics and motion correction methods using digital motion phantoms

James Voyvodic ${ }^{1}$ and Pamela Romero Cruz $^{2}$

${ }^{1}$ Radiology, Duke University, Durham, NC, United States, ${ }^{2}$ Duke University, Durham, NC, United States

Head motion remains a major obstacle in fMRI. We have used realistic human digital motion phantoms with empirically-derived head movements and known BOLD signals to address two unresolved questions: 1) how effective are motion correction algorithms? and 2) how much motion is too much when assessing scan quality? Our analysis evaluated different motion metrics and motion correction methods using both block-designed and event-related fMRI task data. The results show that head motion metrics need to distinguish between positional offsets versus active movement, that combining image realignment plus motion-censoring is most effective, and that residual motion after corrections determines acceptability thresholds.

3D Spatially-Adaptive Canonical Correlation Analysis for Episodic Memory Task fMRI Data: Local and Global Methods

Zhengshi Yang ${ }^{1}$, Xiaowei Zhuang ${ }^{1}$, Karthik Sreenivasan ${ }^{1}$, Virendra Mishra ${ }^{1}$, Tim Curran $^{2}$, Richard Byrd $^{2}$, Rajesh Nandy $^{3}$, and Dietmar Cordes ${ }^{1,2}$

${ }^{1}$ Cleveland Clinic Lou Ruvo Center for Brain Health, Las Vegas, NV, United States, ${ }^{2}$ University of Colorado, Boulder, CO, United States, ${ }^{3}$ University of North Texas, Fort Worth, TX, United States

Spatially adaptive multivariate methods based on local CCA have been used in fMRI data analysis to improve sensitivity of activation detection. To improve specificity, local CCA methods require spatial constraints. In the past, local CCA methods have been used exclusively in $2 \mathrm{D}$ applications because of limitations imposed by the computational time requirements for 3D neighborhoods. We have implemented an efficient algorithm to solve the 3D local constrained CCA problem and furthermore proposed a global kernel CCA method to analyze the time series of the whole brain simultaneously. Results show that global kernel CCA outperforms local CCA in detecting activations.

Human brain functional areas of unitary pooled activity identified with fMRI

Jie Huang

${ }^{1}$ Department of Radiology, Michigan State University, East Lansing, MI, United States

We define a functional area of unitary pooled activity (FAUPA) as an area in which the temporal variation of the activity is the same across the entire area, i.e., the pooled activity is a dynamically unitary activity. This unitary activity across the FAUPA implies a perfect temporal correlation for the activity-induced BOLD response, i.e., the Pearson correlation coefficient is 1 for the BOLD responses of any two locations within the FAUPA. A FAUPA may play the role of a functional unit at large-scale. We report the identification of FAUPAs for both restingstate and task fMRI.

Xuetao Hao ${ }^{1}$, Jan Petr ${ }^{2}$, Aart JJ Nederveen ${ }^{3}$, John Wood ${ }^{4}$, Danny JJ Wang ${ }^{1}$, Henk-Jan Mutsaerts ${ }^{3}$, and Kay Jann ${ }^{1}$

${ }^{1}$ USC Stevens Institute for Neuroimaging and Informatics, Keck School of Medicine at USC, University of Southern California, Los Angeles, CA, United States, ${ }^{2}$ Institute of Radiopharmaceutical cancer research, Helmholtz-Zentrum Dresden-Rossendorf, Dresden, Germany, ${ }^{3}$ Department of Radiology and Nuclear Medicine, Academic Medical Center, Amsterdam, Netherlands, ${ }^{4}$ Cardiology and Radiology, Children's Hospital of Los Angeles, Los Angeles, CA, United States 
We evaluate the use of Independent Component Analysis (ICA) to separate physiological and other noise from Arterial Spin Labeling (ASL) perfusion MRI as has been shown for BOLD fMRI. We show that this approach improves tSNR and cerebral blood flow (CBF) quantification in a cohort of healthy young controls and a group of children with sickle cell disease.

Functional MRI of the Letter Cancellation Test

Luke Chung $^{1}$, Nathan Churchill ${ }^{2}$, Megan Hird ${ }^{2}$, Tahira Tasneem², Fred Tam ${ }^{1}$, Simon Graham ${ }^{1,3}$, and Tom Schweizer ${ }^{2}$

${ }^{1}$ Physical Sciences, Sunnybrook Research Institute, Toronto, ON, Canada, ${ }^{2}$ St. Michael's Hospital, Toronto, ON, Canada, ${ }^{3}$ University of Toronto, Toronto, ON, Canada

Letter cancellation test (LCT) variants are widely used pen-and-paper assessment tools in clinical and experimental psychology, but brain regions that mediate LCT performance are not well understood. An fMRI study involving elderly healthy volunteers was conducted to establish the neural correlates of the LCT using a highly novel fMRI-compatible tablet system that enables investigation of drawing behavior. The resultant brain activation highlighted parietal and frontal regions, consistent with existing fMRI literature on visual attention. This is the first fMRI study of the LCT and the results have relevance for future clinically-oriented fMRI studies of this test.

Cardiac-Gated 4-Echo Spiral Sequence for ME-ICA Denoising of fMRI Data

Patricia Lan ${ }^{1}$, Christine Law ${ }^{2}$, and Gary Glover ${ }^{2}$

${ }^{1}$ Bioengineering, Stanford University, Stanford, CA, United States, ${ }^{2}$ Radiology, Stanford University, Stanford, CA, United States

One challenge in fMRI is separating neuronal from artifactual signal fluctuations. Recent developments in multi-echo fMRI (ME-fMRI) have enabled such classification by examining the TE-dependence of each component after ICA. However, ME-fMRI needs short readouts to fit multiple echoes before signal decay, requiring sparse sampling for EPI-sequences. Here we present our cardiac-gated 4-echo spiral sequence, which allows for short echo time and readout duration, which maximizes SNR and BOLD contrast. We were able to identify and remove $T_{1}$-artifacts resulting from cardiac-gating's variable-TR, an essential aspect for applications such as spinal cord fMRI, where cardiac-gating is required to remove CSF pulsation effects.

Exploiting the physiological properties of the global signal to correct for fluctuations in BOLD fMRI induced by heart rate and respiratory variations

Michalis Kassinopoulos ${ }^{1}$ and Georgios D. Mitsis ${ }^{2}$

${ }^{1}$ Graduate Program in Biological \& Biomedical Engineering, McGill University, Montreal, QC, Canada, ${ }^{2}$ Department of Bioengineering, McGill University, Montreal, QC, Canada

Functional connectivity (FC) in fMRI has generated major attention recently. Patterns of FC are consistently found in healthy subjects, whereas alterations of these patterns have been associated with many neuropsychiatric disorders. However, confounding factors arising from physiological processes have to be taken into consideration when analyzing and interpreting the results in order to ensure their validity. Even though physiological noise correction is commonly applied to fMRI, it is believed that the field would certainly benefit from more efficient techniques. In this study, we examine the relationship of the global BOLD signal with fluctuations in heart rate and respiration and propose a new method for removing the associated artifacts from whole-brain fMRI data.

Susceptibility distortion correction for fMRI

Gina Joue ${ }^{1}$, Tobias Sommer ${ }^{1}$, and Siawoosh Mohammadi ${ }^{1}$

${ }^{1}$ Department of Systems Neuroscience, University Medical Center Hamburg-Eppendorf, Hamburg, Germany

Multiband echo planar imaging (EPI) offers increased temporospatial resolution and statistical power for functional magnetic resonance imaging (fMRI) but the higher spatial resolution comes at the cost of higher susceptibility-related spatial distortions. In diffusion MRI (dMRI), studies have shown that distortion correction is better when using blip-reversed EPI data (known under the term blip-up/down images) as compared to the standard fieldmap approach. This has motivated fMRI studies to acquire their data with blip-up/down directions and to use these to reduce susceptibility distortion. Here, we qualitatively illustrate why this can lead to erroneous results and quantify this error across 10 subjects.

Comparison of MB and MBME in task fMR

Zahra Fazal ${ }^{1}$, Daniel Gomez ${ }^{1}$, José Marques ${ }^{1}$, Benedikt A Poser ${ }^{2}$, and David G Norris ${ }^{1,3}$

${ }^{1}$ Donders Center for Cognitive Neuroimaging, Radboud University, Nijmegen, Netherlands, ${ }^{2}$ Faculty of Psycology and Neuroscience, Masstricht Univeristy, Masstricht, Netherlands,

${ }^{3}$ Erwin L. Hahn Institute for Magnetic Resonance Imaging, Essen, Germany 
In this work a multiband protocol is compared to a Multiband Multi-echo (MBME) protocol in the context of task fMRI at 3T. Furthermore, we evaluate the use of FSL- FIX to clean both datasets and compare its impact on the acquisition protocols in terms of tSNR, sensitivity and statistical significance.

The case for 3D PRESTO fMRI: Improved temporal SNR via ghost suppression by temporal filtering

Jon-Fredrik Nielsen ${ }^{1}$, Tianrui Luo ${ }^{1}$, Scott J Peltier ${ }^{1}$, and Douglas C Noll ${ }^{1}$

We apply a recently-introduced method for more efficient RF-spoiling in dynamic imaging to PRESTO fMRI, and show that this improves temporal SNR significantly. For a whole-brain $\mathrm{fMRI}$ acquisition with high temporal resolution $\left(\mathrm{TR}_{\mathrm{vol}}=0.52 \mathrm{~s}\right)$ and $3.3 \mathrm{~mm}$ isotropic resolution, $\mathrm{tSNR}$ is maximized for a net gradient area of only about $1-1.5 \mathrm{cycles} / \mathrm{voxel}$ (applied to two gradient axes). We anticipate that the use of such low spoiler gradients will make PRESTO a much more viable alternative for 3D fMRI.

Impact of cerebral blood flow level on the fluctuations of resting-state BOLD fMRI in anesthetized rats

Sophie Achard ${ }^{1}$, Guillaume Becq ${ }^{1}$, Tarik Habet ${ }^{2}$, Nora Collomb² ${ }^{2}$, Margaux Faucher ${ }^{2}$, Chantal Delon-Martin², Véronique Coizet ${ }^{2}$, and Emmanuel L Barbier ${ }^{2}$

${ }^{1}$ Univ. Grenoble Alpes, CNRS, Grenoble INP, GIPSA-lab, Grenoble, France, ${ }^{2}$ Univ. Grenoble Alpes, INSERM, CHU Grenoble Alpes, GIN, Grenoble, France

The Blood Oxygen Level Dependent (BOLD) signal, used in resting-state functional Magnetic Resonance Imaging (fMRI), is tighly linked to Cerebral Blood Flow (CBF). This study evaluates the impact of the CBF on the low-frequencies BOLD fluctuations and four physiological parameters during restingstate in 5 groups of rats (Wistar and Long-Evans) anesthetized with Isoflurane, Medetomidine, Etomidate and Urethane. It is shown that the CBF is not related to physiological parameters, and there exists a range of CBF values where the BOLD fluctations are sufficiently high for being used in any other analysis.

Can measures for evaluating gambling strategies inform decisions about fMRI pipelines?

David Paul McAllindon ${ }^{1}$, Steve Patterson ${ }^{1}$, Chris Van Bowen ${ }^{1}$, Christopher O'Grady ${ }^{1}$, Jeff Kowalski ${ }^{1}$, and Steven Beyea ${ }^{1}$

In single-subject fMRI such as is used in presurgical mapping, processing decisions and choice of threshold can greatly affect the activation maps. In order to provide support for making these decisions, we propose a self-similarity approach that uses comparisons across randomly-created split-halves of the data and evaluating the maps using measures that come from a gambling model - the Bookmaker Informedness, Markedness and Matthews Correlation Coefficient - using an fMRI simulation. Early results indicate that features of Informedness and Matthews Correlation Coefficient data may be useful for making pipeline and threshold decisions.

Mapping drug resistant epilepsy with MREG signal coefficient of variance.

Janne Kananen ${ }^{1}$, Timo Tuovinen ${ }^{1}$, Hanna Ansakorpi ${ }^{2}$, Heta Helakari ${ }^{1}$, Niko Huotari ${ }^{1}$, Ville Raatikainen ${ }^{1}$, Aleksi Rasila ${ }^{1}$, Lauri Raitamaa ${ }^{1}$, Viola Borchardt ${ }^{1}$, Vesa Korhonen ${ }^{1}$, and Vesa Kiviniemi ${ }^{1}$

In the absence of detectable epileptiform activity, even combined EEG-fMRI scanning may fail to detect the epileptic foci. We utilize a novel measure of BOLD signal stability, the coefficient of variance (CV), with ultra-fast fMRI sequence MREG in drug resistant epilepsy (DRE). We detect a robust increase of MREG CV in patients with in white matter brainstem and cerebellum in DRE at group level. Importantly, thresholding the $\mathrm{CV}+3$ std above mean enables individual level mapping of epileptic abnormality in DRE patients.

Traditional Poster

\section{fMRI: Basic Neuroscience}


${ }^{1}$ Biomedical Engineering Graduate Program, University of Calgary, Calgary, AB, Canada, ${ }^{2}$ Department of Medicine, Snyder Institute for Chronic Diseases, University of Calgary, Calgary, $A B$, Canada, ${ }^{3}$ Radiology, Clinical Neurosciences, Psychiatry, The Hotchkiss Brain Institute, University of Calgary, Calgary, AB, Canada

Inflammatory bowel disease (IBD) is a chronic and painful inflammatory-mediated disease of the gastrointestinal system. Recent animal model evidence suggests that cognitive deficits and mood changes experienced by IBD patients are not merely emotional reactions, but result from structural and functional changes in the brain. We used dual-regression analysis of resting-state fMRI data to identify alterations in functional connectivity in IBD patients compared to controls. Connectivity was altered with auditory and pain perception networks, which may help explain behavioural symptoms (hearing loss, pain) commonly experienced by IBD patients.

Brain functional connectivity signatures of neuropathic pain-induced depression in a preclinical model

Meltem Karatas ${ }^{1,2,3,4}$, Muris Humo ${ }^{3}$, Laetitia Degiorgis ${ }^{1}$, Marion Sourty ${ }^{1}$, Thomas Bienert ${ }^{2}$, Céline Meillier ${ }^{1}$, Jean-Paul Armspach ${ }^{1}$, Dominik von Elverfeldt ${ }^{2}$, Ipek Yalcin ${ }^{3}$, and LauraAdela Harsan $1,2,5$

${ }^{1}$ ICube, University of Strasbourg, Strasbourg, France, ${ }^{2}$ Dept. of Radiology, Medical Physics, University Medical Center Freiburg, Freiburg im Breisgau, Germany, ${ }^{3}$ INCl, University of Strasbourg, Strasbourg, France, ${ }^{4}$ Faculty of Biology, University of Freiburg, Freiburg im Breisgau, Germany, ${ }^{5}$ Hautepierre Hospital, Department of Biophysics and Nuclear Medicine, Faculty of Medicine, University Hospital Strasbourg, Strasbourg, France

Chronic pain disorders are associated with high prevalence of depression which points to a link between two pathologies; although the underlying mechanisms remain elusive. As a translational approach, preclinical MR imaging offers a unique opportunity to reliably establish causal relations between the pathological conditions and brain function in vivo. In this study, we used a mouse model of neuropathic pain to investigate affective consequences of chronic pain. We performed behavioural assessments as well as resting-state fMRI and our results show a remodelling of functional connectivity in regions belonging to default-mode network and the reward system in mice with pain-induced depression

Research on the brain function of cervicogenic vertigo: A resting-state fMRI study

Kuang Cuili ${ }^{1}$ and Fan Yang ${ }^{2}$

${ }^{1}$ Radiological Department, Renmin Hospital of Wuhan University, Wuhan, China, ${ }^{2}$ GE Healthcare China, Beijing, China

People with cervicogenic vertigo(CV) due to vertebrobasilar insufficiency suffer lots of troubles. Through neuroimaging analysis method, this study finds significant difference in right cerebellum anterior lobe(RCAL) on mfALFF value and connectivities with other brain regions between CV and normal control(NC). Besides, the mfALFF and mReHo of RCAL are correlated to DHI(Dizziness Handicap Inventory) significant positively. These discoveries seem to indicate that a long-term vertebrobasilar insufficiency results in such alterations of these functional indexes and connectivities in RCAL of CV, then lead to the function degradation of maintain the basic balance of the body when occurrence of vertigo.

Hot-wiring of functional brain connectome in neurologically asymptomatic patients with primary insomnia

Xiaofen $\mathrm{Ma}^{1}$, Guihua Jiang ${ }^{1}$, Queenie Chan ${ }^{2}$, Zhizheng Zhuo ${ }^{3}$, Jin Fang ${ }^{1}$, Shishun $\mathrm{Fu}^{1}$, Guang Xu ${ }^{1}$, and Wenfeng Zhan ${ }^{1}$

${ }^{1}$ Department of Medical Imaging, Guangdong No. 2 Provincial People's Hospital, Guangzhou, China, ${ }^{2}$ Philips Healthcare, Hong Kong, China, ${ }^{3}$ Philips Healthcare, Beijing, China

Functional and structural neuroimaging studies have revealed abnormal of Primary Insomnia (PI) patient's brain, including decreased gray matter density, and increase of spontaneous brain activity and metabolism in hippocampus and fronto-parietal cortex, and so on. We use graph-based approaches to investigate the topological abnormalities of functional brain networks in PI patients and examine clinical correlates of the alterations. PI patients exhibited increased overall connectivity of functional brain networks and nodal efficiency in the default mode network (DMN) and emotional circuit. This abnormal organization of large-scale functional brain networks in PI, which could account for memory and emotion dysfunction in PI patients.

Preventive anti-NGF treatment suppresses alterations in functional connectivity imposed by cancer-induced bone pain in mice

David Buehlmann ${ }^{1,2}$, Giovanna Diletta lelacqua ${ }^{1}$, Jael Xandry ${ }^{3}$, and Markus Rudin ${ }^{1,2,3}$

${ }^{1}$ Inst. for Biomedical Engineering, ETH \& University of Zurich, Zürich, Switzerland, ${ }^{2}$ Neuroscience Center Zurich (ZNZ), Zürich, Switzerland, ${ }^{3}$ Inst. of Pharmacology \& Toxicology, University of Zurich, Zürich, Switzerland

The efficacy of an anti-nerve growth factor (NGF) antibody in preventing rearrangements of whole-brain functional connectivity elicited by nociceptive input following bone metastases was evaluated in a mouse model of cancer-induced bone pain using longitudinal resting-state fMRI. ROI-based network and seed-based connectivity analysis approaches revealed major hubs of ascending and descending pain pathways to be affected by the developing pain. Functional rearrangements within these regions could be prevented by prospective application of anti-NGF antibody mAb911 indicating the efficacy of anti-NGF treatment in preventing, or at least delaying, adaptations of the brain circuitry associated with development of a chronic pain state. 
Altered Voxel-based Functional Connectivity Density of Default Mode Network in Chronic Insomnia: A resting-state fMRI study

Zhonglin $\mathrm{Li}^{1,2}$, Enfeng Wang ${ }^{1,2}$, Tianyi Qian ${ }^{3}$, Zhi Zou ${ }^{1,2}$, Thomas Beck ${ }^{4}$, Yanrui Shen ${ }^{1,2}$, Xiaolin Wu ${ }^{1,2}$, Shewei Dou ${ }^{1,2}$, Dapeng Shi ${ }^{1,2}$, Meiyun Wang ${ }^{1,2}$, Hongju Zhang $^{5}$, and Yongli Li $i^{1,2,6}$

${ }^{1}$ Department of Radiology, People's Hospital of Zhengzhou University, Zhengzhou, China, ${ }^{2}$ Department of Functional Imaging, Henan Key Laboratory for Medical Imaging of Neurological Diseases, Zhengzhou, China, ${ }^{3}$ Siemens Healthcare, Beijing, China, ${ }^{4}$ Siemens Healthcare, Erlangen, Germany, ${ }^{5}$ Department of Neurology, People's Hospital of Zhengzhou University, Zhengzhou, China, ${ }^{6}$ Health Management Center, People's Hospital of Zhengzhou University, Zhengzhou, China

This study aimed to investigate the potentially altered functional connectivity (FC) of the default-mode network (DMN) in chronic insomnia disorder (CID) patients. A voxel-based functional connectivity density (FCD) analysis method was applied to identify abnormal FC among 44 CID patients and 31 healthy controls. A seed-based FC analysis and independent component analysis were also employed and compared. CID patients showed increased FCD in the right medial temporal gyrus (MTG), including long and short distance connections. Our results suggest that hyperarousal of the DMN may be related to increased FCD of the right MTG. Furthermore, the altered connectivity within or outside the DMN may further contribute to cognitive, emotional, and memory impairment.

Asymmetric Functional Connectivity in Major Depressive Disorder Revealed by Ultra-high Field Resting-state fMRI

Chan-A Park ${ }^{1}$, Enae Cheong ${ }^{1,2}$, Youngkyu Song ${ }^{1}$, Sungho Tak ${ }^{1},{\text { Gyunggoo } \text { Cho }^{1} \text {, Jin-Hun Sohn }}^{2}$, and Chaejoon Cheong ${ }^{1,3}$

${ }^{1}$ Bioimaging Research Team, Division of Bioconvergence Analysis, Korea Basic Science Institute, Ochang Center, Cheongju, Korea, Democratic People's Republic of, ${ }^{2}$ Department of Psychology, Brain Research Institute, Chungnam National University, Daejeon, Korea, Democratic People's Republic of, ${ }^{3}$ Department of Bio-Analytical Science, University of Science and Technology, Cheongju, Korea, Democratic People's Republic of

The purpose of the study is that the investigation of resting-state functional magnetic resonance imaging (MRI) with 7T MRI via seed-based correlation analysis is examined the significant difference of the whole-brain functional connectivity among major depressive disorder (MDD) patients and healthy subjects. The results showed that MDD had higher correlations compared with healthy group. Furthermore, MDD exhibited lateralization of connected regions, including the lateral occipital cortex, inferior temporal gyrus, angular gyrus, temporal fusiform cortex, occipital fusiform gyrus, and lingual gyrus, mainly located in the left hemisphere. These suggest that MDD is associated with disruptions in the asymmetric organization of brain

Changes in Brain Function induced by Chronic Neuropathic Pain in a Mouse Model of Chronic Nerve Constriction Injury

Katja Sauer ${ }^{1}$, Isabel Wank ${ }^{1}$, Silke Kreitz ${ }^{1}$, and Andreas Hess ${ }^{1}$

${ }^{1}$ University of Erlangen-Nuremberg, Institute of Pharmacology and Toxicology, Erlangen, Germany

The aim of this study was to investigate the effects of the well-established chronic constriction injury $(\mathrm{CCl})$ model on central nociceptive processing in mice over a period of 56 days. For this purpose two behavioral tests (Hargreaves and electronic pressure-meter test ["plantar test"]) and functional MRI were combined. The ligation of the sciatic nerve induced behavioral changes indicative of a neuropathic pain state. Graph theoretical analysis of functional connectivity revealed known effects of chronic pain for the first time also for the CCI model: modifications of the sensory as well as emotional system induced by thermal but also mechanical stimulation.

Resting-state fMRI predicts somatosensory-evoked BOLD fMRI in anesthetized mice

Hyun-Ji Shim ${ }^{1,2}$, Won Beom Jung ${ }^{1,3}$, Sangwoo Kim ${ }^{1}$, and Seong-Gi Kim ${ }^{1,2,3}$

${ }^{1}$ Center for Neuroscience Imaging Research (CNIR), Institute for Basic Science (IBS), Suwon, Republic of Korea, ${ }^{2}$ Department of Health Sciences and Technology (SAIHST), Sungkyunkwan University (SKKU), Seoul, Republic of Korea, ${ }^{3}$ Department of Biomedical Engineering (BME), Sungkyunkwan University (SKKU), Suwon, Republic of Korea

BOLD fMRI in rodents has been used to investigate brain functions in normal and diseased conditions. Until now, most animal fMRI have used anesthetics to reduce animal stress and minimize motions. Because anesthesia affects neurovascular coupling, maintaining the proper physiological condition under anesthesia is important. However, it is challenging in mice due to the limitation of physiological monitoring and high sensitivity to anesthetics used. Here we introduced ketamine and xylazine anesthesia in mice. Then, to examine the variability of fMRI response and indirectly measure the physiological condition, we use the resting state fMRI (RS-fMRI), which detects intrinsic brain state and connectivity.

Yuchuan Zhuang ${ }^{1}$, Lu Wang ${ }^{2}$, Madalina Tivarus ${ }^{3}$, Xing Qiu², Michael E. Yurcheshen ${ }^{4}$, Jianhui Zhong ${ }^{3}$, and Giovanni Schifitto ${ }^{5}$

${ }^{1}$ Electrical and Computer Engineering, University of Rochester, Rochester, NY, United States, ${ }^{2}$ Dept of Biostatistics and Computational Biology, University of Rochester, Rochester, NY, United States, ${ }^{3}$ Department of Imaging Sciences, University of Rochester, Rochester, NY, United States, ${ }^{4}$ Department of Medicine, University of Rochester, Rochester, NY, United States, ${ }^{5}$ Department of Neurology, University of Rochester, Rochester, NY, United States 
We investigated sleep quality in HIV infected individuals and its potential impact on cognitive performance and functional connectivity. Sleep quality was assessed using a self-report questionnaire, Pittsburgh Sleep Quality Index (PSQI). Cognitive performance was measured by a standard battery of neuropsychological tests assessing six cognitive domains, while functional connectivity was assessed by resting-state fMRI. We used a seed-based method to investigate the activation changes associated with the thalamus and frontoparietal network. We found a strong interaction between HIV infection and sleep quality, in the inferior temporal gyrus and the inferior parietal lobule but no deleterious effect on cognitive performance.

Alterations of neural activity patterns in pontine versus coronal radiata stroke

Chunxiang Jiang ${ }^{1,2}$, Siqi Cai ${ }^{1,2}$, Rui Meng ${ }^{1,2}$, Xiaojing Long ${ }^{1}$, Hang Zhang ${ }^{1}$, and Lijuan Zhang ${ }^{* 1}$

We compared the alteration of intrinsic neural functional networks in 15 patients with ischemic stroke (IS) in pontine (PS) and 21 patients with IS in coronal radiata (CRS) with 30 healthy controls (HCs). Degree centrality (DC) increased in posterior cingulate gyrus and ReHo decreased in sensorimotor cortex and default mode network in PS and CRS group relative to $\mathrm{HCs}$ group. DC increase was observed in cuneus in CRS group. These findings suggest that IS disrupts the functional integration of brain in an extensive scale, and the lesion location may substrate the functional outcomes for the IS patients.

Diaschisis of The Language Network in Resting State fMRI Functional Connectivity of Post-Stroke Chronic Aphasia

Kwan-Jin Jung ${ }^{1}$ and Jacquie Kurland ${ }^{2}$

${ }^{1}$ Human Magnetic Resonance Center, University of Massachusetts Amherst, Amherst, MA, United States, ${ }^{2}$ Department of Communication Disorders, University of Massachusetts Amherst, Amherst, MA, United States

Functional connectivity (FC) of intrinsic networks was compared between two groups: healthy controls and post-stroke aphasia, using resting state fMRI. While the FC of auditory, motor and default mode networks were preserved, FC of the language network was disrupted in the aphasia group. The aphasia group showed left ipsilateral frontal FC from the Broca area but not from the Wernicke area. Similarly, the aphasia group showed left ipsilateral temporo-parietal FC from the Wernicke area but not from the Broca area. Thus, a clear picture of diaschisis, not just structural disruption, was revealed in the FC of the aphasia group.

Ketamine-induced modulation of functional connectivity in male and female rats

Jaakko Paasonen ${ }^{1}$, Leena Penna², Tomi Rantamäki ${ }^{2}$, and Olli Gröhn ${ }^{1}$

${ }^{1}$ A.I.V. Institute for Molecular Sciences, University of Eastern Finland, Kuopio, Finland, ${ }^{2}$ Department of Biosciences, University of Helsinki, Helsinki, Finland

To get further insights into sustained and gender-dependent neurobiological effects of ketamine, an N-methyl-D-aspartate blocker carrying antidepressant and addictive properties, we investigated the resting-state functional connectivity (FC) in female and male rats 24 hours after a subanesthetic dose of ketamine. Ketamine tended to suppress FC between several brain regions such as hippocampus - medial prefrontal cortex and caudate putamen - medial prefrontal cortex. Significant interactions between treatment and gender were also observed. These observations shed light on the mechanisms underlying the complex neurobiological effects produced by ketamine.

Functional connectivity underlying attentional deficits in children born preterm

Trevor McComber Flynn ${ }^{1}$, Olga Tymofiyeva ${ }^{1}$, Dawn Gano ${ }^{2}$, Donna M Ferriero ${ }^{3}$, J James Barkovich $^{4}$, and Duan $\mathrm{Xu}^{1}$

${ }^{1}$ Radiology and Biomedical Imaging, University of California San Francisco, San Francisco, CA, United States, ${ }^{2}$ Neurology, University of California San Francisco, San Francisco, CA, United States, ${ }^{3}$ Pediatrics, University of California San Francisco, San Francisco, CA, United States, ${ }^{4}$ Radiology, University of California San Francisco, San Francisco, CA, United States

Children born very preterm are at a increased risk to develop attention deficits. Here we employ fMRI connectivity analysis to study the functional connectivity underlying these attention deficits in preterm children ages 10-14. Subjects were separated into normal and attention deficit groups and then group differences in voxelwise connectivity from 16 seed regions were delineated using AFNI. Significant clusters of hypoconnectivity in the attention deficit group were found in eight of the ROls, primarily from the middle frontal gyri and anterior cingulate cortices, as well as hyperconnectivity from the right anterior insula. 
${ }^{1}$ Department of Radiology, Centre Hospitalier Universitaire Vaudois (CHUV), and University of Lausanne, 1011 Lausanne, Switzerland, Unil, Lausanne, Switzerland, ${ }^{2}$ Neuropsychology and Neurorehabilitation Service, Centre Hospitalier Universitaire Vaudois (CHUV), and University of Lausanne, 1011 Lausanne, Switzerland, Unil, Lausanne, Switzerland, ${ }^{3}$ CIBM (Centre d'Imagerie Biomédicale), Dept. of Radiology, Centre Hospitalier Universitaire Vaudois (CHUV), and University of Lausanne, 1011 Lausanne, Switzerland, Unil, Lausanne, Switzerland

Prismatic adaptation (PA), a therapy for neglect after a stroke, showed conflicting results in the literature. The variability of brain lesions leading to neglect and their relation to structures affected by PA could explain those results. MRI studies found focal brain activations after PA. We aim to understand if there is an effect on functional networks after PA. To do so, we analyzed the resting state fMRI connectivity of healthy subjects before and after PA. We found that the ventral attention system (VAS) was less connected after PA, this provides new insights to select patients for this therapy.

Mirror Therapy Increases Resting-State Functional Connectivity in Stroke Recovery

Christine S Law ${ }^{1}$, Aarti Sharma ${ }^{2}$, and Iris Grunwald ${ }^{2}$

${ }^{1}$ Stanford University, Palo Alto, CA, United States, ${ }^{2}$ Southend Hospital, Southend-on-sea, United Kingdom

Mirror therapy increases resting-state functional connectivity when compared to conventional rehabilitation. In particular, connectivity increase is linearly proportional to increase in pinch strength. Our results strongly indicate neuronal changes resulting from mirror therapy, and that mirror therapy is of palpable benefit.

Comparison of Resting State Networks using EEG and Pseudo-continuous ASL

Wenna Duan ${ }^{1}$, Wen-Ming Luh², Mingzhao Chen ${ }^{1}$, and Weiying Dai ${ }^{1}$

${ }^{1}$ Computer Science, State University of New York at Binghamton, Vestal, NY, United States, ${ }^{2}$ Cornell MRI Facility, Uninversity of Cornell, Ithaca, NY, United States

Arterial spin labeling (ASL) and electroencephalography (EEG) data were acquired separately on young healthy subjects to verify whether EEG signal reflects the same brain networks corresponding to those extracted from ASL images. Four brain networks were derived from both resting state ASL images and resting state EEG recordings during both eye-open and eye-closed conditions.

Altered brain activation and connectivity during anticipation of uncertain threat in anxiety

Haiyang Geng ${ }^{1,2}$, Ruolei $\mathrm{Gu}^{3}$, and Xuebing $\mathrm{Li}^{1}$

${ }^{1}$ Key laboratory of Mental Health, Institute of Psychology, Chinese Academy of Sciences, Beijing, China, ${ }^{2}$ Shenzhen Key Laboratory of Affective and Social Cognitive Science, Shenzhen University, Shenzhen, China, ${ }^{3}$ Key laboratory of Behavioral Science, Institute of Psychology, Chinese Academy of Sciences, Beijing, China

In the present study, we used an emotional anticipation paradigm with functional magnetic resonance imaging (fMRI) to examine the anticipation processing of uncertain threat in anxious individuals by employing brain activation and general psychophysiological interactions (gPPI) analysis. Our findings show altered activations in dmPFC, precuneus, thalamus, and MTG; impaired connections of dmPFC-vmPFC, precuneus-FPN, precuneus-MTG, and precuneus-PHA during anticipation of uncertain threat in anxious individuals, which may be involved in estimation of, perception of, and emotional reactions to uncertain threat. All of these altered neural patterns may together contribute to pathology of anxiety. Our study also provides a new insight for neural and behavioral treatments focusing on the dmPFC-vmPFC circuit that underlies uncertainty estimation and emotion regulation in anxiety-related disorders.

BOLD responses in the posterior cerebellum differ when a motor task has a proprioception component

Wietske van der Zwaag ${ }^{1}$

The cerebellum receives proprioceptive information from the body, as well as tactile input. Here, we aimed to separate the proprioceptive BOLD responses from the motor/somatosensory clusters in the human cerebellum. Regions responding to a fingertapping task and a motion task requiring proprioceptive information were found to differ in the posterior cerebellum. Using high resolution 7T functional MRI, all proprioceptive clusters in lobule VIII of the cerebellum were found to be positioned medial to regions responding to the simple tapping task. 
${ }^{1}$ Queensland Brain Institute, The University of Queensland, Brisbane, Australia, ${ }^{2}$ Agency for Science Technology and Research, Singapore, Singapore, Singapore, ${ }^{3}$ Saw Swee Hock School of Public Health, National University of Singapore and National University Health System, Singapore, Singapore

Paradigm design in the functional MRI acquisition is of paramount importance to investigate brain activities non-invasively. This is the first time to introduce imagination tasks of walking on floors with different surface stiffness in studying the consequence of a disease associated with diabetic peripheral neuropathy. Our results from the study-specific paradigms show a strong involvement of central nervous system in diabetic peripheral neuropathy subjects as well as cognitive deficits in sensation as caused by the disease.

Spatial- and category-based attention have distinct functional organizations in human visual cortex

Pei Huang ${ }^{1}$, Marta M Correia ${ }^{1}$, Justin L Gardner ${ }^{2}$, and Johan D Carlin ${ }^{1}$

${ }^{1}$ MRC-Cognition and Brain Sciences Unit, University of Cambridge, Cambridge, United Kingdom, ${ }^{2}$ BIO-X, Stanford University, Stanford, CA, United States

Recent studies have challenged the two-streams division of primate visual cortex into a ventral object identity stream ('what') and a dorsal object location stream ('where'). We collected fMRI data while human participants performed a selective attention task. We used a multivariate discriminant method to separately decode the currently attended location and object category. We found a distinction between early visual regions coding the attended location and ventral/dorsal stream regions coding the attended category. Our study reveals a largescale functional organization for spatial- and category-based attention in visual cortex, but its principal axis is posterior-anterior rather than dorsal-ventral.

Title: Self-Regulation of vmPFC Activation Using Real-Time fMRI Neurofeedback

Ahmad Mayeli ${ }^{1,2}$, Vadim Zotev ${ }^{1}$, Raquel Phillips ${ }^{1}$, Hazem Refai ${ }^{2}$, Martin Paulus ${ }^{1}$, and Jerzy Bodurka ${ }^{1,3}$

${ }^{1}$ Laureate Institute for Brain Research, Tulsa, OK, United States, ${ }^{2}$ Electrical and Computer Engineering, University of Oklahoma, Tulsa, OK, United States, ${ }^{3}$ College of Engineering, Stephenson School of Biomedical Engineering, University of Oklahoma, Tulsa, OK, United States

In this study, we have examined the feasibility of training healthy human subjects to self-regulate the hemodynamic activity of the vmPFC using real-time fMRI neurofeedback (rtfMRI-nf). Eight healthy subjects took part in experimental group with real rtfMRI neurofeedback from vmPFC and four in control group with a sham feedback from HIPS region. The results show significant vmPFC BOLD activity differences between the groups, demonstrating the feasibility of targeted modulation of the vmPFC using the rtfMRI-nf.

Visual cortex and auditory cortex activation in early binocularly blind macaques: A BOLD-fMRI study using auditory stimuli

Lingjie Wu ${ }^{1}$, Rong Wang ${ }^{2}$, Zuohua Tang ${ }^{2}$, Xinghuai Sun ${ }^{3}$, Xiaoyuan Feng ${ }^{4}$, Weijun Tang ${ }^{4}$, Wen Qian ${ }^{2}$, Jie Wang ${ }^{1}$, Yufeng Zhong ${ }^{5}$, Zebin Xiao ${ }^{2}$, and Zhongshuai Zhang ${ }^{6}$

${ }^{1}$ Otolaryngology, Eye \& ENT Hospital of Fudan University, Shanghai, China, ${ }^{2}$ Radiology, Eye \& ENT Hospital of Fudan University, Shanghai, China, ${ }^{3}$ Eye, Eye \& ENT Hospital of Fudan University, Shanghai, China, ${ }^{4}$ Radiology, Huashan Hospital of Fudan University, Shanghai, China, ${ }^{5}$ Jinshan Hospital of Fudan University, Shanghai, China, ${ }^{6}$ Siemens Healthcare Ltd., Shanghai, China

We aimed to detect the changes in BOLD activity between the visual and auditory cortices of monocularly blind neonatal macaques by using pure tones as auditory stimuli. The changes in the BOLD response in the bilateral visual and auditory cortices were detected and further compared with the findings of the immunofluorescent staining. In monocularly bind macaques, we found a greater level of significant activation in the bilateral visual cortices while the number of activated volumes of the bilateral auditory cortices decreased. Therefore, cross-modal plasticity within the visual and auditory cortices was established in the monocularly blind macaques.

CMRO2 Changes During Sleep in Humans

Hyunyeol Lee ${ }^{1}$, Erin K Englund ${ }^{1}$, Ana E Rodriguez-Soto ${ }^{1}$, John A Detre ${ }^{2}$, Richard Schwab ${ }^{3}$, and Felix W Wehrli

${ }^{1}$ Radiology, University of Pennsylvania, Philadelphia, PA, United States, ${ }^{2}$ Neurology, University of Pennsylvania, Philadelphia, PA, United States, ${ }^{3}$ Division of Sleep Medicine, University of Pennsylvania, Philadelphia, PA, United States

Synaptic transmission is well known to be reduced during sleep, and yet very little is known about the extent to which the various stages of sleep affect neurometabolism. Here, we measured whole-brain $\mathrm{CMRO}_{2}$ in test subjects by means of the OxFlow technique while collecting data continuously for a period of 30 minutes, first during wakefulness and, in a second set of experiments, during sleep and subsequent arousal. During wakefulness $\mathrm{CMRO}_{2}$ was stable (average $\mathrm{CV} \sim 7 \%$ ). Following onset of sleep there was a rapid decrease in $\mathrm{CMRO} 2$ by up to $25 \%$, along with increased $\mathrm{SvO}_{2}$ but almost unaltered CBF. 
${ }^{1}$ Spinoza Centre for Neuroimaging, Amsterdam, Netherlands, ${ }^{2}$ Experimental Psychology, Utrecht University, Utrecht, Netherlands, ${ }^{3}$ Radiology, University Medical Centre Utrecht, Utrecht, Netherlands, ${ }^{4}$ Applied and Experimental Psychology, VU University, Amsterdam, Netherlands

Nearly all fMRI analysis methods assume a linear relationship between local neuronal activity and the BOLD signal. This assumption is supported for fMRI at conventional resolutions (>1 mm isotropic). We assess whether linearity of the BOLD signal holds at sub-millimetre resolution, over cortical depth. We acquired functional GE $3 D$-EPI data at $0.7 \mathrm{~mm}$ isotropic resolution (TR/TE $=57 / 28 \mathrm{~ms}$ ). Stimuli consisted of moving circular sine gratings at $80 \%, 20 \%$, and $5 \%$ contrast. Our results suggest that response profiles for one contrast are linearly scaled response profiles of any other contrast.

Information content carried by resting-state BOLD fMRI signals reduces differentially in sensory and memory compared with cognitive systems in MCS and UWS patients Xiaolin Liu ${ }^{1}$, Xinhuai Wu², Shanshan Chen ${ }^{3}$, Lubin Wang ${ }^{3}$, Bing Wü ${ }^{2}$, Yituo Wang ${ }^{3}$, Mingmei Ge ${ }^{2}$, Zhan Xu ${ }^{1}$, B. Douglas Ward ${ }^{1}$, Shi-Jiang Li ${ }^{1}$, and Zheng Yang ${ }^{3}$

${ }^{1}$ Medical College of Wisconsin, Milwaukee, WI, United States, ${ }^{2}$ Army General Hospital, Beijing, China, ${ }^{3}$ Beijing Institute of Basic Medical Sciences, Beijing, China

How brain injuries affect the information content carried by signals of brain imaging modalities in patients with consciousness disorders has received little attention. We proposed a novel principal-components-analysis-based approach to quantify regional information content in patients in a minimally conscious state (MCS) and with unresponsive wakefulness syndrome (UWS). We show a reduction of regional information content in both patient populations. Importantly, our analyses revealed differential patterns in the reduction of information content in the sensory and memory compared with high-order cognitive systems in MCS and UWS; such observations are consistent with the clinical symptoms in the two DOC patient populations.

Acute stress modulates cigarette cue-evoked neural activation: A neuropharmacological investigation among non-treatment-seeking cigarette smokers

Eric Woodcock ${ }^{1}$, Vaibhav Diwadkar ${ }^{2}$, Jeffrey Stanley ${ }^{2}$, Dalal Khatib ${ }^{2}$, and Mark Greenwald ${ }^{2}$

${ }^{1}$ Psychiatry and Radiology, Yale University School of Medicine, New Haven, CT, United States, ${ }^{2}$ Psychiatry and Behavioral Neurosciences, Wayne State University School of Medicine, Detroit, MI, United States

Separately, acute stress and cigarette cues are associated with cigarette smoking relapse, and each has been shown to increase nicotine seeking/self-administration. However, their potentially additive effects are understudied in humans. Using functional neuroimaging and a placebo-controlled double-blind design, we found acute stress suppressed cue-evoked activation in the medial orbitofrontal, parietal, and prefrontal cortices. Further, the effects of stress on nicotine withdrawal severity were inversely related to medial orbitofrontal and nucleus accumbens activation. Our findings illustrate acute stress exerts cooperative modulation of neural signals and subjective withdrawal severity, known to be important for long-term abstinence.

Brain fMRI responses during spinal cord stimulation in rats

Lauri Juhani Lehto ${ }^{1}$, Hanne Laakso ${ }^{1}$, Carlos Cuellar ${ }^{2}$, Igor Lavrov², Silvia Mangia ${ }^{1}$, and Shalom Michaeli ${ }^{1}$

${ }^{1}$ Center for Magnetic Resonance Research, University of Minnesota, Minneapolis, MN, United States, ${ }^{2}$ Neural Engineering Laboratory, Mayo Clinic, Rochester, MN, United States

Spinal cord stimulation (SCS) has had success in pain management and promising results were demonstrated in other pathologies. To our knowledge, no preclinical studies of SCS in combination with brain fMRI exist, which limit exploration of novel SCS strategies. Here, we show our first results of simultaneous SCS and brain fMRI in rats aiming to establish a framework for future SCS developments. Stimulating spinal cord segment L2 induced a BOLD activation in the primary somatosensory/motor cortex and the thalamus that was dependent on the stimulation frequency. These results demonstrate that monitoring modulation of brain activity due to SCS is feasible.

Piriform cortex involvement in odor imagery

Jérémy Weber ${ }^{1}$, Muriel Jacquot ${ }^{2,3}$, Faustine Noël ${ }^{2}$, Jacques Felblinger ${ }^{1,4}$, and Gabriela Hossu ${ }^{4}$

${ }^{1}$ IADI, U947, Université de Lorraine, INSERM, Nancy, France, ${ }^{2}$ Myrissi, Nancy, France, ${ }^{3}$ InnoCIM, ENSAIA, Université de Lorraine, Nancy, France, ${ }^{4}$ CIC1433, CHRU Nancy, INSERM, Université de Lorraine, Nancy, France

Current research relies on the piriform cortex as an indicator of successful odor imagery. Using colored arrangements as visual stimulation, we seek to show that the piriform cortex is mainly linked to odor perception. Our results support this hypothesis by showing a clear lack of activation in this area during odor imagery. Furthermore this study definitively highlights the use of colored arrangements in an odor imagery study compared to other visual stimulation and its benefits. 
${ }^{1}$ Department of Radiology, Tangdu Hospital, The Fourth Military Medical University, Xi'an, China, ${ }^{2}$ radiology department of Tangdu hospital, The Fourth Military Medical University, Xi'an, China, ${ }^{3}$ Radiology department of Tangdu hospital, The Fourth Military Medical University, Xi'an, China

Our research aimed to compare PA with MMT,to reveal which abstinence way is better to recover the brain function in heroin-dependent individuals.24 heroin-dependent patients under PA , 19 heroin-dependent patients under MMT and 20 healthy volunteers were recruited. The functional images were acquired by using a spin-echo EPI. In the last part of this study, we proved PA group is closer to healthy group. This study showed that PA is more advantageous than MMT to reduce heroin addiction in drug cue-reactivity.

BOLD activation pattern of dominant versus non-dominant hand wrist extension task in stroke patients and healthy subjects

Dixit Sharma ${ }^{1}$, Neha Singh ${ }^{1}$, Megha Saini ${ }^{2}$, Sneh Anand ${ }^{1}$, Nand Kumar ${ }^{3}$, MV Padma Srivastava ${ }^{4}$, S Senthil Kumaran ${ }^{5}$, and Amit Mehndiratta ${ }^{1}$

${ }^{1}$ Centre for Biomedical Engineering, Indian Institute of Technology Delhi, New Delhi, India, ${ }^{2}$ Department of Biomedical Engineering, All India Institute of Medical Sciences, NEw Delhi, India, ${ }^{3}$ Department of Psychiatry, All India Institute of Medical Sciences, NEw Delhi, India, ${ }^{4}$ Department of Neurology, All India Institute of Medical Sciences, NEw Delhi, India, ${ }^{5}$ Department of Nuclear Magnetic Resonance, All India Institute of Medical Sciences, NEw Delhi, India

Flexor-hypertonia being the most common symptom of stroke, overcoming it by attaining wrist-extension can be judged as key function of recovery of stroke. We compared activation pattern of dominant versus non-dominant hand movements of wrist-extension of 6 healthy-subjects with 6 dominant and 6 non-dominant stroke using fMRI. Results in healthy-subjects show differences in activation-pattern of dominant and non-dominant hand. Stroke patient's results shows ipsilesional activation-pattern with dominant-hemisphere stroke with activation in motor, sensory area and cerebellum as compared to no ipsilesional activation-pattern in non-dominant hemisphere stroke. These results might have further implication in structuring rehabilitation-protocol for different hemisphere stroke differently.

Effect of emotional enhancement of memory on recollection process in young adults: The influence factors and neural mechanisms

Xiaoshu Li ${ }^{1}$, Haibao Wang $^{1}$, Xiaohu Li ${ }^{1}$, Jiajia Zhu ${ }^{1}$, Yong Zhang ${ }^{2}$, and Yongqiang Yu ${ }^{1}$

${ }^{1}$ Department of Radiology, The First Affiliated Hospital of Anhui Medical University, Hefei, China, ${ }^{2}$ GE Healthcare China, Shanghai, China

This research explored how the inherent stimulus properties and amount of devoted attention influenced the emotional enhancement of memory (EEM) effect on recollection and evaluated the correlations between emotional memory/EEM and the spontaneous brain activity of hippocampus, perirhinal, and entorhinal cortex, and the correlations between emotional memory/EEM and the topological properties of three stipulated emotional memory processing networks in 59 young adults using resting-state fMRI. The EEM was elicited by incidental encoding, negative images, and positive high-arousal images. The hippocampus, perirhinal, and entorhinal cortex play distinct roles in the recollection and familiarity processes of emotional memory and the EEM effect.

Brain activity during the training period of the Hybrid Assistive Limb (HAL) for a subacute stroke: an fMRI case report

Kousaku Saotome ${ }^{1}$, Akira Matsushita $^{2}$, Aiki Marushima $^{3}$, Hiroaki Kawamoto $^{4}$, Hideo Tsurushima ${ }^{1}$, Masashi Yamazaki ${ }^{5}$, Akira Matsumura $^{3}$, and Yoshiyuki Sankai ${ }^{1}$

${ }^{1}$ Center for Cybernics Research, University of Tsukuba, Tsukuba, Japan, ${ }^{2}$ Department of Neurosurgery, Ibaraki Prefectural University of Health Sciences Hospital, Ami, Japan, ${ }^{3}$ Department of Neurosurgery, Faculty of Medicine, University of Tsukuba, Tsukuba, Japan, ${ }^{4}$ Faculty of Engineering, Information and Systems, University of Tsukuba, Tsukuba, Japan, ${ }^{5}$ Department of Orthopaedic Surgery, Faculty of Medicine, University of Tsukuba, Tsukuba, Japan

The effectiveness of hybrid assistive limb (HAL) training, which is the new rehabilitation robotic approach, for recovery of brain function after stroke remains to be clarified. This is the first report to show the brain activation alteration during the training period for HAL for subacute stroke by using motor task-based functional magnetic resonance imaging (fMRI) 4 times. Our major finding was that fMRI results demonstrated rearrangement of the cortical activation pattern in a form that induces cerebral lateralization in M1 toward the contralateral hemisphere.

Neural mechanism of reward circuit in exercise addiction : an fMRI study

Kyung Eun Jang ${ }^{1}$, Yang-Tae Kim² ${ }^{2}$ Jingu Kim ${ }^{3}$, Hyunsil Cha ${ }^{1}$, Heajung Choi ${ }^{1}$, Eunji Kim ${ }^{1}$, Moojin Yang ${ }^{1}$, Jiung Yang ${ }^{1}$, Huijin Song ${ }^{4}$, Moon Jung Hwang ${ }^{5}$, and Yongmin Chang ${ }^{1,6}$

${ }^{1}$ Medical \& Biological Engineering, Kyungpook National University, Daegu, Republic of Korea, ${ }^{2}$ Psychiatry, School of Medicine, Keimyung University, Daegu, Republic of Korea, ${ }^{3}$ Physical Education, Kyungpook National University, Daegu, Republic of Korea, ${ }^{4}$ Biomedical Engineering Research, Kyungpook National University, Daegu, Republic of Korea, ${ }^{5}$ GE Health Korea, Seoul, Republic of Korea, ${ }^{6}$ Radiology and Molecular Medicine, College of Medicine, Kyungpook National University, Daegu, Republic of Korea

We investigate neural activation of physical exercise related pictures in exercise addiction. Our results demonstrate that exercise addiction group showed lower activation in ventra striatum than the moderate exercise group, indicating that dopamine release of the ventral striatum in exercise addiction group may reduce because of withdrawal symptoms and negative prediction error. Moreover, we found that exercise addiction group showed less activation in the posterior orbitofrontal cortex than the other groups, suggesting that exercise addiction group may not deliberate fitness equipment and body shape of exerciser as reward value. 
Functional connectivity of intrinsic brain networks in chronic low back pain

Arman Tadjibaev ${ }^{1,2}$, William Cottam ${ }^{1,2,3}$, and Dorothee Auer ${ }^{1,2,3}$

${ }^{1}$ Arthritis UK Pain Centre, University of Nottingham, Nottingham, United Kingdom, ${ }^{2}$ Sir Peter Mansfield Imaging Centre, University of Nottingham, Nottingham, United Kingdom, ${ }^{3}$ NIHR Nottingham BRC, Nottingham, United Kingdom

Understanding pathological changes in intrinsic connectivity networks may advance our knowledge of chronic pain. We performed resting state seed-based functional connectivity analysis of main intrinsic brain networks in 34 chronic low back pain patients and 34 healthy controls. Results of present study are in accordance with studies that demonstrated weaker connectivity within the default mode network and reduced anticorrelation between the default mode and salience networks in chronic pain. In addition, we have identified abnormal sensorimotor network (SMN) connectivity and more profound medial prefrontal - hippocampal connectivity dysfunction in chronic low back pain.

Ventral intermediate nucleus involved in tremor and Postural instability and gait disability-related networks in Parkinson's disease

Qiaoling Zeng ${ }^{1}$, Xiaojun Guan², Tao Guo ${ }^{2}$, Jason C. F. Law Yan Lun ${ }^{2}$, and Minming Zhang ${ }^{1}$

${ }^{1}$ Department of Radiology, The 2nd Affiliated Hospital, Department of Radiology, School of Medicine, Zhejiang University, hangzhou, China, ${ }^{2}$ The 2nd Affiliated Hospital, Department of Radiology, School of Medicine, Zhejiang University, hangzhou, China

To investigate the core pathophysiology between Parkinson's disease $(\mathrm{PD})$ motor subtypes in subregions of thalamus and their different directory connectivity patterns, we collected multi-model magnetic resource imaging of 79 PD patients and 31 normal controls. We compared the grey matter volume and perfusion characteristics within the thalamus between PD phenotypes. Granger causality analysis was used to compare the effective connectivity between different subtypes. Our study revealed that core pathophysiology in tremor-dominant subtype may lie in the ventral intermediate nucleus, and a differential effective connectivity pattern existed in tremor and posture instability gait difficulty-related networks that related to behavioral heterogeneity in PD.

Perspective taking modulates inter-subject correlated hemodynamic brain responses in movie watching

Yu Ching Lam ${ }^{1}$, Kuan-yi Lu ${ }^{1}$, Shu-Yu Huang ${ }^{1}$, and Fa-hsuan Lin ${ }^{1}$

${ }^{1}$ National Taiwan University, Taipei, Taiwan

2376

We used function Magnetic Resonance Imaging (fMRI) to obtain the hemodynamic brain responses during perspective modulated naturalistic movie presentations to find the influence of perception to individual's cognitive and affective reactions. Inter-subject correlation (ISC) was used as the analysis tool and Interpersonal Reactivity Index (IRI) was used as the behavioral measurement tool. The study applied selected group ISC analysis to distinguish neural substrates related to physical, cognitive, and affective perspective-taking using naturalistic perspective modulated movie presentation. The finding helps understanding the neural mechanism of perspective taking and would be a useful for future social cognition studies.

Traditional Poster

\section{fMRI: Contrast Mechanisms}

Hypercapnic manipulation of baseline blood volume alters coupling between BOLD and CBV visual responses

Marcello Venzi ${ }^{1}$, Joseph Whittaker ${ }^{1}$, Jessica Steventon ${ }^{1}$, Laurentius Huber ${ }^{2}$, Harald Möller ${ }^{3}$, and Kevin Murphy ${ }^{1}$

${ }^{1}$ School of Physics and Astronomy, Cardiff University Brain Research Imaging Centre (CUBRIC), Cardiff University, Cardiff, United Kingdom, ${ }^{2}$ SFIM, NIMH, Bethesda, MD, United States, ${ }^{3}$ Max-Planck-Institut für Kognitions- und Neurowissenschaften, Leipzig, Germany

The utility of VASO to study disease-related alterations in CBV is demonstrated. Manipulation of baseline CBV with hypercapnia mimicking arteriolar disease states produces a change in dynamic coupling of BOLD and CBV visual evoked responses. Although no significant changes in signal amplitude were detected, the expected trend for amplitude reduction was observed in the VASO signals with increasing $\mathrm{CO} 2$ levels. The time-to-peak of BOLD responses lengthens but CBV peak times, being longer at baseline, remain the same. This study indicates that combining VASO and BOLD contrasts can be sensitive enough to investigate the consequences of patho-physiological changes in baseline CBV. 
${ }^{1}$ Center for Functional and Metabolic Mapping, Robarts Research Institute, Western University, London, ON, Canada, ${ }^{2}$ Medical Biophysics, Western University, London, ON, Canada

Phase imaging and QSM abet the magnitude $\mathrm{fMRI}$ by revealing and quantifying the draining veins of the activation areas. Consequently, QSM sheds light on calibrating the \% BOLD change and, when combined with CBF, has a potential to determine the basis of negative BOLD signal; in particular if it is due to increased oxygenation during rest periods or reduced oxygenation during the activation.

Simultaneous acquisition of $\$ \$ \$$ T_ $1 \$ \$$ Maps and BOLD fMRI Signal During Brain Activation Using Multi-Echo EPI

Xianglun $\mathrm{Mao}^{1}$, J. Andrew Derbyshire ${ }^{2}$, Vinai Roopchansingh ${ }^{2}$, Thomas M Talavage ${ }^{1,3}$, and Peter A Bandettini ${ }^{2}$

${ }^{1}$ School of Electrical and Computer Engineering, Purdue University, West Lafayette, IN, United States, ${ }^{2}$ Functional MRI facility, NIMH, National Institute of Health, Bethesda, MD, United States, ${ }^{3}$ Weldon School of Biomedical Engineering, Purdue University, West Lafayette, IN, United States

A quantitative $\$ \$ \$ T 1 \$ \$ \$$ map and blood oxygenation level-dependent (BOLD) signals are simultaneously measured during a flickering checkerboard using a multi-echo echo-planar imaging (ME-EPI) based fMRI sequence. The acquired EPI-based $\$ \$ \$ T$ 1 $\$ \$$ maps provide a means of tissue identification and allow direct comparison with BOLD activation maps on a voxel-wise basis, and thus offer an alternative of tissue segmentation and avoid the need for image registration between anatomical and functional imaging data

Comparing cortical layer activation using gradient echo with phase regression and spin echo in the human visual cortex

Olivia W Stanley ${ }^{1,2}$, Alan B Kuurstra ${ }^{2}$, L Martyn Klassen ${ }^{1,2}$, Ravi S Menon ${ }^{1,2}$, and Joseph S Gati ${ }^{1,2}$

High resolution fMRI sequence selection is often a compromise between specificity to tissue (SE-EPI) and sensitivity to the BOLD effect (GE-EPI). Our work compared the laminar activation profiles of SE-EPI and GE-EPI once phase regression based macrovascular filtering has been applied. We demonstrated that GE-EPI with macrovascular filtering produces a laminar profile more similar to SE-EPI than GE-EPI without filtering. This shows that GE-EPI could be used for high resolution imaging and achieve a more sensitive profile when phase regression is included.

Field strength dependent somatosensory-evoked mouse fMRI: $9.4 \mathrm{~T}$ vs. $15.2 \mathrm{~T}$

Won Beom Jung ${ }^{1,2}$, Hyun-Ji Shim ${ }^{1,3}$, Sangwoo Kim ${ }^{1}$, and Seong-Gi Kim ${ }^{1,2,3}$

${ }^{1}$ Cener for Neuroscience Imaging Research (CNIR), Institute for Basic Science (IBS), Suwon, Republic of Korea, ${ }^{2}$ Department of Biomedical Engineering, Sungkyunkwan University, Suwon, Republic of Korea, ${ }^{3}$ Department of Health Sciences and Technology (SAIHST), Sungkyunkwan University, Seoul, Republic of Korea

BOLD fMRI is highly sensitive to magnetic field strength. However, there is a notion that 7-9.4T is an optimal field strength for fMRI and higher fields do not increase BOLD signal changes. Here, we compared the BOLD response in the mouse somatosensory cortex at both magnetic fields of 9.4T and 15.2T to determine the BOLD dependence on magnetic field strength and found that the BOLD FMRI response is indeed increased as the field strength increases.

Mouse BOLD fMRI at 15.2 T: Detection of the entire somatosensory pathway including thalamic nuclei

Won Beom Jung ${ }^{1,2}$, Hyun-Ji Shim ${ }^{1,3}$, Sangwoo Kim¹ ${ }^{1}$, and Seong-Gi Kim¹,2,3

${ }^{1}$ Cener for Neuroscience Imaging Research (CNIR), Institute for Basic Science (IBS), Suwon, Republic of Korea, ${ }^{2}$ Department of Biomedical Engineering, Sungkyunkwan University, Suwon, Republic of Korea, ${ }^{3}$ Department of Health Sciences and Technology (SAIHST), Sungkyunkwan University, Seoul, Republic of Korea

BOLD-functional MRI is a useful tool to identify the brain function and to examine the functional effects on development, recovery, and reorganization. The most common stimulusparadigm is the electrical forepaw/hindpaw stimulation to generate the evoked BOLD response. Although specific brain areas are involved in the somatosensory system, most studies in the anesthetized rodents have focused on the somatosensory cortex as it is difficult to detect activation in the subcortical areas. BOLD sensitivity is dependent on the field strength and affected by physiological condition under the anesthesia. Here, we demonstrated the thalamo-cortical BOLD response in mice under the ketamine-xylazine at $15.2 \mathrm{~T}$. 
Passband bSSFP is an excellent alternative to gradient-echo EPI for BOLD fMRI at high field but properties of the BOLD signal have not been reported at ultra-high field (14T). Here, we show that the BOLD amplitude is similar for short and intermediate TR ( 6 and $12 \mathrm{~ms}$, respectively) which suggests that, in spite of the high field, BOLD contrast in passband bSSFP has limited $T_{2}{ }^{*}$ and off-band contributions, and dominant $T_{2}$ contributions for TR $\leq 12 \mathrm{~ms}$. A short TR can thus be used to increase temporal or spatial resolution, as well as coverage, with no penalty in intrinsic sensitivity.

Optimization of Serial Correlation Correction Methods Based on Autoregressive Model in Fast fMRI

Qingfei Luo ${ }^{1}$, Masaya Misaki ${ }^{1}$, Beni Mulyana ${ }^{1}$, Chung-Ki Wong ${ }^{1}$, and Jerzy Bodurka ${ }^{1,2}$

${ }^{1}$ Laureate Institute for Brain Research, Tulsa, OK, United States, ${ }^{2}$ Stephenson School for Biomedical Engineering, University of Oklahoma, Norman, OK, United States

Serial correlation (SC) of noise inflates T-statistics in simultaneous multi-slice excitation (SMS) fMRI studies with short repetition times (TR<2s). The SC can be corrected using noise pre-whitening methods based on the high-order autoregressive (AR) model. This study aims to determine the optimal order selection (OS) method of AR model to achieve the best SC correction accuracy. By evaluating the false positive characteristics in rest/null datasets, our study showed that the corrected Akaike information criterion (AICc) has the best performance among the OS criteria. We recommend use the AR model with AICc to correct the SC in SMS fMRI experiments.

Estimation of physiological sources of nonlinearity in BOLD signals

Daehun Kang ${ }^{1}$, Yul-Wan Sung ${ }^{1}$, and Satoshi Shioiri²

${ }^{1}$ Kansei Fukushi Research Institute, Tohoku Fukushi University, Sendai, Japan, ${ }^{2}$ Tohoku University, Sendai, Japan

The BOLD signals related to brain activation is often nonlinear with change in TE. In contrast to extravascular component, the nonlinearity is attributable to intravascular component due to chemical exchange between plasma and deoxy- $\mathrm{Hb}$. Recently, activity-evoked $\mathrm{pH}$ change on the brain has been demonstrated. Since the chemical exchange is often pH-dependent, the time for the chemical exchange would change. Thus, the two-compartment model that incorporates the change would be more accurate for estimation of parameters than the model with fixed exchange time. In this study, we measured the nonlinearity by multi-echo GRE-EPI and estimated parameters of the proposed model.

More than BOLD: dual spin populations create functional contrast

David Ress ${ }^{1}$, Elizabeth Halfen ${ }^{1}$, Vimal Singh ${ }^{2}$, and Amanda Taylor ${ }^{1}$

${ }^{1}$ Neuroscience, Baylor College of Medicine, Houston, TX, United States, ${ }^{2}$ Electrical Engineering, University of Texas at Austin, Austin, TX, United States

The "classical" description of functional contrast postulates a single spin population with transverse lifetime modulated by neurovascular coupling. A variety of studies have cast doubt on this description. To better understand such issues, novel methods were used to probe functional contrast in the gray matter of human visual cortex as a function of echo time and flip angle. We find evidence that two spin populations with disparate lifetimes contribute to functional contrast.

Exploring the Origin of the Low Frequency Oscillation Signal in dual-echo Arterial Spin Labeling MRI

Xin Shen ${ }^{1}$, Ho-Ching Yang ${ }^{1}$, Blaise deB. Frederick ${ }^{2,3}$, Danny JJ Wang ${ }^{4}$, and Yunjie Tong ${ }^{1}$

${ }^{1}$ Biomedical Engineering, Purdue University, West Lafayette, IN, United States, ${ }^{2}$ Brain Imaging Center, McLean Hospital, Belmont, MA, United States, ${ }^{3}$ Department of Psychiatry, Harvard University Medical School, Boston, MA, United States, ${ }^{4}$ Laboratory of FMRI Technology, University of Southern California, Los Angeles, CA, United States

Arterial spin labeling (ASL), which is a non-invasive technique providing perfusion values in the unit of $\mathrm{ml} / 100 \mathrm{~g} / \mathrm{min}$, has been limited by low signal-to-noise ratio (SNR). Although doing average of several repeating scans might be a solution, it is essential to identify the 'physiological noise', i.e. low frequency oscillations (LFOs). In a study of 9 healthy subjects, the similarity and amplitude of LFOs in ASL and in blood oxygenation level dependent (BOLD) were compared to explore the origin of LFOs as well as to discover a potential method for denoising and decreasing scanning time.

Reduced basal ganglia adaptability in patients with diabetic peripheral neuropathy

Yijia Zheng ${ }^{1}$, Ye Wang ${ }^{1,2}$, Geheng Yuan ${ }^{3}$, Xin Qi ${ }^{4}$, Rui Wang ${ }^{5}$, Zhanyang Ma ${ }^{3}$, Xiaohui Guo ${ }^{3}$, Xiaoying Wang ${ }^{1,5}$, Jue Zhang ${ }^{1,6}$, and Jing Fang ${ }^{1,6}$

${ }^{1}$ Academy for Advanced Interdisciplinary Studies, Peking University, Beijing, China, ${ }^{2}$ Neuroscience and Intelligent Media Institute, Communication University of China, Beijing, China, ${ }^{3}$ Department of Endocrinology, Peking University First Hospital, Beijing, China, ${ }^{4}$ Department of Plastic Surgery \& Burns, Peking University First Hospital, Beijing, China, ${ }^{5}$ Department of Radiology, Peking University First Hospital, Beijing, China, ${ }^{6}$ College of Engineering, Peking University, Beijing, China 
Diabetic peripheral neuropathy (DPN) is one of the most common complications of diabetes mellitus and the patients often have no symptoms in the early stage. Notably, basal ganglia is an important hub in the sensorimotor loop, we hypothesized that dysfunction of basal ganglia in diabetic patients with DPN. Based on this hypothesis, we assessed the function of basal ganglia in diabetic patients using resting-state functional magnetic resonance imaging (fMRI). And our results found the reduced basal ganglia adaptability in DPN patients, which is expected to providing a new perspective for the guidance of early clinical diagnosis and efficacy evaluation.

Why elder adults have a higher fall risk in dual-task daily life: A Preliminary fMRI Study

Yijia Zheng ${ }^{1}$, Ye Wang ${ }^{1,2}$, Yi Zhu ${ }^{1}$, Xiaoying Wang ${ }^{1,3}$, Jue Zhang ${ }^{1,4}$, and Jing Fang ${ }^{1,4}$

${ }^{1}$ Academy for Advanced Interdisciplinary Studies, Peking University, Beijing, China, ${ }^{2}$ Neuroscience and Intelligent Media Institute, Communication University of China, Beijing, China, ${ }^{3}$ Department of Radiology, Peking University First Hospital, Beijing, China, ${ }^{4}$ College of Engineering, Peking University, Beijing, China

Reduced plantar sensation can lead to weakened balance ability in elder adults and an addition of cognitive tasks will further weaken it. Thereby, we attempted to explore the brain activity pattern of the elder and of young adults under foot stimuli and in dual-task condition. The results revealed that elder adults have significantly stronger cortical excitability than young do, and that foot stimuli induced stronger cortical excitability compared with dual-task condition. In conclusion, these phenomena may be due to the elder adults' inadequate central reserve. Besides, added cognitive task can further reduce the brain's response through diminished sensory input.

Traditional Poster

\section{fMRI: Connectivity Methods}

Investigating Local and Global Connectivity to Inform Seizure Generation in Epilepsy: a Feasibility Study

Bianca De Blasi ${ }^{1}$, Ilaria Boscolo Galazzo ${ }^{2}$, Marian Galovic ${ }^{3}$, Enrico De Vita ${ }^{4}$, Ashley Groves ${ }^{5}$, Martin Tisdall ${ }^{6}$, Anna Barnes ${ }^{5}$, and Matthias Koepp ${ }^{3}$

${ }^{1}$ Medical Physics and Biomedical Engineering, University College London, London, United Kingdom, ${ }^{2}$ University of Verona, Verona, Italy, ${ }^{3}$ UCL Institute of Neurology, London, United Kingdom, ${ }^{4}$ King's College London, London, United Kingdom, ${ }^{5}$ UCLH Institute of Nuclear Medicine, London, United Kingdom, ${ }^{6}$ Great Ormond Street Hospital, London, United Kingdom

In this work, we combined local and global functional connectivity to provide a more complete picture of the epileptogenic brain in temporal lobe epilepsy (TLE). Local functional connectivity was assessed by computing regional homogeneity $(\mathrm{ReHo})$ maps which were compared between left $(n=9)$ or right $(n=10)$ TLE patients and controls $(n=20)$. Areas of increased ReHo were used in a seed-to-voxel analysis to investigate global functional connectivity changes. We report a different pattern of alteration between left and right TLE patients. Left TLE patients showed a more profound bilateral increased connectivity which might highlight compensatory mechanisms.

Oxycodone BOLD activation and connectivity signature by Mu opioid receptor in anaesthetized mice fMRI

Md Taufiq Nasseef ${ }^{1}$, Emmanuel Darcq ${ }^{1}$, Jai Puneet Singh ${ }^{1}$, Praveen Kulkarni ${ }^{2}$, and Brigitte L. Kieffer ${ }^{1}$

${ }^{1}$ Douglas Mental Health University Institute, Department of Psychiatry, Mcgill University, Montreal, QC, Canada, ${ }^{2}$ Center for Translational Neuro-Imaging, Northeastern University, Boston, MA, United States

Mu opioid receptors (MORs) mediate biological effects of oxycodone, including their analgesic and euphoric properties. To assess the effect of oxycodone on neuronal communication, we used non-invasive mouse fMRI and tested oxycodone effects in both wild-type and MOR-knockout mice in order to extract MOR-dependent effects. Analysis was performed 2 to 7 minutes after drug administration, a time where BOLD activation was minimal in knockout animals. Here, we show that oxycodone reduces functional activity of the Nucleus Accumbens seed with several brain regions, establishing a first receptor-mediated FC connectivity signature of a MOR agonist.

Using Social Network Analysis to enhance the understanding of Brain Connectivity

Claudio Tomazzoli ${ }^{1}$, Silvia Francesca Storti ${ }^{1}$, Ilaria Boscolo Galazzo ${ }^{1}$, Matteo Cristani ${ }^{1}$, and Gloria Menegaz ${ }^{1}$

${ }^{1}$ University of Verona, Verona, Italy

Graph-based network modelling is becoming increasingly pervasive touching at very different fields, ranging from social networks to brain connectivity. This works is a first attempt to borrow the concept of "transtopic messaging" from social network for its exploitation in the functional connectivity framework. Basically, different functional tasks are mapped to different "semantic topics", and the overall relevance (according to given metrics) of the nodes of the network graph in ruling the spread of the different "topics" is assessed. This rises the connectivity analysis of one level of abstraction allowing to assess the overall transtopical relevance of each node of the graph providing information on the higher-level structure of the network. 
High-Performance Correlation and Mapping Engine for Brain Connectivity Networks from High Resolution fMRI Data

John David Lusher II ${ }^{1}$, Jim Xiuquan $\mathrm{Ji}^{1}$, and Joseph Orr²

${ }^{1}$ Department of Electrical and Computer Engineering, Texas A\&M University, College Station, TX, United States, ${ }^{2}$ Department of Psychological and Brain Sciences, Texas A\&M Institute for Neuroscience, Texas A\&M University, College Station, TX, United States

Seed-based Correlation Analysis (SCA) of fMRI data has been used to create brain connectivity networks. With close to a million unique voxels in a fMRI dataset, the number of calculations involved in SCA becomes high. With the emergence of the dynamic functional connectivity analysis, and the studies relying on real-time neurological feedback, the need for rapid processing methods becomes even more critical. This work aims to develop a new approach which produces high-resolution brain connectivity maps rapidly. Preliminary results show that this process can improve processing by a factor of 27 or more over that of a conventional PC workstation.

Regression does not Eliminate the Effects of Nuisance terms in Dynamic Functional Connectivity Estimates

Alican Nalci ${ }^{1}$ and Thomas T. Liu ${ }^{1}$

${ }^{1}$ UCSD Center for Functional MRI, La Jolla, CA, United States

Nuisance regression is commonly used in dynamic functional connectivity (DFC) studies to reduce the influence of nuisance factors, such as head motion or physiological activity. Here, we show that DFC estimates before nuisance regression are significantly correlated with the norms of various nuisance terms. Furthermore, we find that nuisance regression does not eliminate the correlations between DFC estimates and the nuisance norms.

Malfunction of cerebellum functional connectivity in patients with mTBI. rsfMRI study.

Maxim Ublinskiy ${ }^{1,2}$, Natalia Semenova $^{1,2,3}$, Petr Menshchikov $^{1,3}$, Andrei Manzhurtsev $^{1,2}$, llya Melnikov $^{1}$, and Tolib Akhadov ${ }^{1}$

${ }^{1}$ Clinical and Research Institute of Emergency Pediatric Surgery and Trauma, Moscow, Russian Federation, ${ }^{2}$ Emanuel Institute of Biochemical Physics, Russian Academy of Sciences, Moscow, Russian Federation, ${ }^{3}$ Semenov Institute of Chemical Physics, Russian Academy of Sciences, Moscow, Russian Federation

Mild TBI appears to be a possible reason of connectivity malfunction in normal-appearing flocculus.

Using Temporal ICA to Selectively Remove Global Noise While Preserving Global Signal in Functional MRI Data

Matthew F. Glasser ${ }^{1,2}$, Timothy S. Coalson ${ }^{1}$, Janine D. Bijsterbosch ${ }^{3}$, Samuel J. Harrison ${ }^{3}$, Michael P. Harms ${ }^{1}$, Alan Anticevic ${ }^{4}$, David C. Van Essen ${ }^{1}$, and Stephen M. Smith ${ }^{3}$

${ }^{1}$ Washington University in St. Louis, Saint Louis, MO, United States, ${ }^{2}$ St. Luke's Hospital, Saint Louis, MO, United States, ${ }^{3}$ University of Oxford., Oxford, United Kingdom, ${ }^{4}$ Yale University, New Haven, CT, United States

A major unresolved methodological issue in fMRI is how to address the problem of spatially global noise, particularly in resting state functional connectivity data. Global signal regression is effective at removing global noise, which largely arises from physiological sources; however, it has the drawback of additionally removing global or semi-global neural signal as well. Here we present a method to selectively remove global noise while preserving global neural signal using temporal ICA. Thus, we remove a global positive bias in functional connectivity without inducing the network-specific negative bias that results from global signal regression.

Preserving Maximal Spatial Specificity in Resting State Group Analysis at 7 Tesla

Anna-Thekla Schmidt ${ }^{1,2}$, Julia M Huntenburg ${ }^{1}$, Christine L Tardif ${ }^{3,4}$, Claudine J Gauthier ${ }^{5}$, Arno Villringer ${ }^{1}$, Christopher J Steele ${ }^{1,6}$, and Pierre-Louis Bazin ${ }^{1,7,8}$

${ }^{1}$ Neurology, Max Planck Institute for Human Cognitive and Brain Sciences, Leipzig, Germany, ${ }^{2}$ MaxNetAging, Max Planck Institute for Demographic Research, Rostock, Germany, ${ }^{3}$ Montreal Neurological Institute and Hospital, Montreal, QC, Canada, ${ }^{4}$ McGill University, Montreal, QC, Canada, ${ }^{5}$ Physics, Concordia University / PERFORM Centre, Montreal, QC, Canada, ${ }^{6}$ Cerebral Imaging Center, Douglas Mental Health University Institute, McGill University, Montreal, QC, Canada, ${ }^{7}$ Netherlands Institute for Neuroscience, Amsterdam, Netherlands, ${ }^{8}$ Spinoza Centre for Neuroimaging, Amsterdam, Netherlands

Most studies use standard software pipelines for processing and analyzing fMRI data. These pipelines were designed to work with data from 3 Tesla scanners. With more widespread availability of ultra-high field MRI scanners, new processing techniques need to be applied to address the unique demands of high resolution data and to fully take advantage of the high spatial specificity. Here, we propose a novel approach for processing and analysing high resolution resting state fMRI data. 
The traditional resting-state fMRI studies are based on the blood oxygenation level dependent (BOLD) contrast. Compared with BOLD, the oxygen extraction fraction (OEF) can more directly reflect the neuronal activities. However, due to the poor temporal resolution of existing OEF techniques, there is no study detecting resting-state networks with OEF contrast. In this study, the OEF contrast based resting-state networks were investigated through a newly proposed technique. Both seed-based correlation and independent component analysis were used and the results suggested that OEF can be used as an effective contrast to study resting-state brain networks.

Estimating the time-lag of neuronal activity for the default mode network using multi-band EPI acquisitions in resting-state fMRI

Atsushi Tachibana ${ }^{1,2,3}$, Yoko Ikoma ${ }^{1}$, Yasuhiko Tachibana ${ }^{1}$, Jeff Kershaw ${ }^{1}$, Yoshiyuki Hirano ${ }^{4}$, Katsutoshi Murata ${ }^{5}$, Tatsuya Higashi ${ }^{1}$, and Takayuki Obata ${ }^{1,2}$

${ }^{1}$ Applied MRI Research, Department of Molecular Imaging and Theranostics, National Institute of Radiological Sciences, QST, Chiba, Japan, ${ }^{2}$ Department of Radiological Sciences, Graduate School of Human Health Sciences, Tokyo Metropolitan University, Tokyo, Japan, ${ }^{3}$ Department of Radiology, AIC Yaesu Clinic, Tokyo, Japan, ${ }^{4}$ Research Center for Child Mental Development, Chiba University, Chiba, Japan, ${ }^{5}$ Siemens Healthcare K.K., Tokyo, Japan

Conventional EPI requires a temporal resolution of 2-3 seconds to obtain whole-brain data for resting-state fMRI (rsfMRI). More recently, multi-band EPI (MB) acquisition can be used to improve temporal resolution and obtain whole-brain coverage in less than 1 second. Our hypothesis is that MB acquisition can be used to detect the time-lag of neuronal activity. In this study, we estimated the time-lag in the default mode network using conventional (TR $2000 \mathrm{~ms}$ ) and MB (TR 500 ms) rsfMRI. Significant time-lags between PCC and AG, and between mPFC and AG were detected only for the MB acquisition

The investigation of brain functional alterations of $\mathrm{MCl}$ patients by using two novel non-linear analysis techniques

Lijiang Wei ${ }^{1}$, Zhe Ma ${ }^{1}$, Zhizheng Zhuo ${ }^{1}$, Bin Jing ${ }^{1}$, Haiyun $\mathrm{Li}^{1}$, and Yingjie $\mathrm{Mei}^{2}$

${ }^{1}$ Capital Medical University, Beijing, China, ${ }^{2}$ Clincial Science, Philips Healthcare, Guangzhou, China

In this paper, we proposed two novel non-linear analysis methods including cross-sample entropy of ordinal pattern and inner composition alignment (IOTA) of ordinal pattern to construct brain network based on functional magnetic resonance imaging. Group-level statistical comparisons were performed to investigate the differences of brain networks. The results showed that the network related to hippocampus, amygdala and posterior cingulate cortexin in mild cognitive impairment (MCl) participants significantly differ from in normal controls. Our results suggest that both the non-linear methods can be applied to estimate the characteristics of brain network in $\mathrm{MCl}$.

Functional connectivity sensitivity to image acceleration and orientation in simultaneous PET/MRI

Alessandro Palombit ${ }^{1,2}$, Marco Castellaro ${ }^{1,2}$, Erica Silvestri ${ }^{1,2}$, Enrico De Vita $^{3}$, Diego Cecchin ${ }^{2,4}$, and Alessandra Bertoldo ${ }^{1,2}$

${ }^{1}$ Department of Information Engineering, University of Padova, Padova, Italy, ${ }^{2}$ Padova Neuroscience Center, University of Padova, Padova, Italy, ${ }^{3}$ Department of Biomedical Engineering, King's College London, London, United Kingdom, ${ }^{4}$ Department of Diagnostic Medical Sciences, University of Padova, Padova, Italy

Resting state fMRI (rs-fMRI) permits in-vivo characterization of brain's functional connectivity (FC). Multi-Band accelerated EPI allows to improve the temporal resolution of rs-fMRI data and, potentially, to achieve a better characterization of the brain network correlations. However, the impact of image acceleration and orientation on FC structure has not been quantified. In this work we investigated FC changes related to image acceleration effects in a test/retest rs-fMRI protocol. We found FC differences involving relevant networks, confirmed even by graph analysis of the FC maps. Our findings explore the lower bound of single-subject FC reliability and network-dependent acceleration sensitivity.

Multiband-enabled Resting State Functional Connectivity Mapping in Simultaneous PET/MRI

Alessandro Palombit ${ }^{1,2}$, Erica Silvestri ${ }^{1,2}$, Marco Castellaro ${ }^{1,2}$, Enrico De Vita ${ }^{3}$, Diego Cecchin 2,4 , and Alessandra Bertoldo ${ }^{1,2}$

${ }^{1}$ Department of Information Engineering, University of Padova, Padova, Italy, ${ }^{2}$ Padova Neuroscience Center, University of Padova, Padova, Italy, ${ }^{3}$ Department of Biomedical Engineering, King's College London, London, United Kingdom, ${ }^{4}$ Department of Diagnostic Medical Sciences, University of Padova, Padova, Italy

PET/MRI scanner is the ideal instrument to simultaneously study brain's metabolism and fMRI-based functional connectivity (FC). State-of-the-art fMRI multiband (MB) EPI sequences on those scanners can be limited by the PET-transparent head coil receiver capabilities as the longitudinal coil elements organisation along head-feet direction is theoretically unable to provide sensitivity variation along this axis. In this work we provided optimal sequence settings for FC studies encompassing available out-of-plane (MB) and in-plane (iPAT) accelerations with two slice orientations demonstrating MB-EPI reliability for FC studies and how non conventional slice orientations can enhance supported MB acceleration factors. 
Hemodynamic reorganization approach to estimate the functional connectivity in task based functional MRI study

Swati Agrawal ${ }^{1}$, Vijayakumar $C^{1}$, Ardaman Kaur ${ }^{1}$, Subash Khushu ${ }^{1}$, Rinku Sharma ${ }^{2}$, and Suresh Sharma ${ }^{2}$

Modulations in brain connectivity by task reveal more insights into complex interaction and neuronal communication occurs between various cortexes. However, assessment of these modulation is limited by dynamic hemodynamic (HRF) spread ( 3 to $6 \mathrm{sec}$ ) occurs at every brain regions by various task stimulus. This dynamic HRF limits methods of resting-state studies to be adopted directly in task-fMRI. Thus, in this study, a novel hemodynamic reorganization method is proposed to rearrange the dynamic HRF of every stimulus such that functional connectivity modulation caused by every stimulus and their mutual correlations in visual search based target detection task can be assessed.

Correcting for erroneous assessment of resting-state functional connectivity caused by prolonged arterial arrival time: a study in Moyamoya patients

Hesamoddin Jahanian ${ }^{1}$, Thomas Christen², Michael Moseley², and Greg Zaharchuk ${ }^{2}$

${ }^{1}$ Radiology, University of Washington, Seattle, WA, United States, ${ }^{2}$ Radiology, Stanford University, Palo Alto, CA, United States

We studied the default mode network in a group of Moyamoya patients using ICA method and observed erroneous assessments of functional connectivity in regions with prolonged arterial arrival time. We showed that these arterial delays could lead to erroneous elimination of affected brain regions from a functional connectivity network. We proposed a method called "temporal realignment" to mitigate this problem.

Functional Connectivity within the Cognitive Networks is associated with the Complexity of Network Node Dynamics

Kay Jann ${ }^{1}$ and Danny JJ Wang ${ }^{1}$

${ }^{1}$ USC Stevens Institute for Neuroimaging and Informatics, Keck School of Medicine at USC, University of Southern California, Los Angeles, CA, United States

While static and dynamic functional connectivity $(\mathrm{sFC} / \mathrm{dynFC}$ ) provide estimates of the integrity and information transfer between brain network nodes, the signal dynamics at each node represents the local information processing. Here we assessed the relation between static FC, dynFC and complexity of signal fluctuations wishing nodes within four networks. We found that more complex and thus less predictable signal in networks allows for a more dynamic functional connectivity and hence a richer repertoire of different FC states.

Alterations of Resting State fMRI Functional Connectivity in Hypercapnia

Yu-Chia Cheng ${ }^{1}$, Teng-Chieh Cheng ${ }^{2}$, Wen-Chau Wu ${ }^{3}$, Teng-Yi Huang ${ }^{4}$, Chao-Chun Lin ${ }^{5}$, Chia-Wei Lin ${ }^{5}$, Wu-Chung Shen ${ }^{5}$, and Yi-Jui Liu ${ }^{2}$

${ }^{1}$ Master 's Program of Biomedical Informatics and Biomedical Engineering of Feng Chia University, Taichung City 407, Taiwan, ${ }^{2}$ Department of Automatic Control Engineering, Feng Chia University, Taichung City 407, Taiwan, ${ }^{3}$ Graduate Institute of Oncology, National Taiwan University, Taipei, Taiwan, ${ }^{4}$ Department of Electrical Engineering, National Taiwan University of Science and Technology, Taipei, Taiwan, ${ }^{5}$ Department of Radiology, China Medical University Hospital, Taichung City 407, Taiwan

The purpose of this study is to explore the alterations of brain functional connectivity among different hypercapnia using resting-state functional magnetic resonance imaging (rs-fMRI). 10 healthy males were enrolled in this study. A high-resolution T1WI image and BOLD-EPI were performed by a 3 Tesla MR scanner. The CO2 gas mixture (air, $3 \%, 5 \%$ and $7 \%$ ) was given at the different hypercanpic for each experiment. Our results show that the brain functional connectivity in resting state is changed in hypercapnia. FC is gradual reduction as the increased $\mathrm{CO} 2$ fraction in the most primary functional networks, expect the executive control network.

Adaptive global signal regression for resting-state functional connectivity MRI

Narges Moradi ${ }^{1}$, Mehdy Dousty ${ }^{1}$, and Roberto C Sotero ${ }^{2}$

${ }^{1}$ Biomedical Engineering Graduate Program, University of Calgary, Calgary, AB, Canada, ${ }^{2}$ Department of Radiology, Hotchkiss Brain Institute, University of Calgary, Calgary, AB, Canada

One of the primary steps in exploring resting-state functional connectivity MRI is to identify and remove the global signal (GS). Plenty of methods have been proposed for this. However, the majority of them are based on an averaging approach known to produce spurious connectivity values. In this work, we used a nonlinear adaptive method to construct voxel-specific GS. The method is tested for task-positive, task-negative and reference ROIs by computing the Pearson correlation coefficient. Our results show a high level of precision for the proposed approach, while the conventional method could not provide an accurate brain functional mapping. 
${ }^{1}$ Department of Computer Science, University of Verona, Verona, Italy, ${ }^{2}$ Department of Neuroradiology, University Hospital Verona, Verona, Italy, ${ }^{3}$ King's College London, London, United Kingdom, ${ }^{4}$ Institute of Nuclear Medicine, University College London, London, United Kingdom

Nowadays, the assessment of brain functional connectivity (FC) patterns, ranging from resting-state networks to network modelling, can rely on Arterial Spin Labeling (ASL) MRI as an alternative to the gold-standard sequence represented by the blood-oxygenation-level-dependent contrast. We evaluated FC mapping from different perspectives (experimental protocols, populations and analysis methods), trying to overcome some of the present challenges related to the ASL applicability in this framework. The results demonstrate how FC patterns and changes can be reliably detected using ASL, with the added value of allowing the simultaneous quantification of brain perfusion, a direct marker of neuronal activity.

Improving the resting state fMRI detection in anesthetized monkeys using multiband MRI technique

Chun-Xia Li ${ }^{1}$, Doty Kempf ${ }^{1}$, Leonard Howell ${ }^{1,2}$, and Xiaodong Zhang ${ }^{1,2}$

${ }^{1}$ Yerkes Imaging Center, Yerkes National Primate Research Center, Emory University, Atlanta, GA, United States, ${ }^{2}$ Division of Neuropharmacology and Neurologic Diseases, Yerkes National Primate Research Center, Emory University, Atlanta, GA, United States

Neuroimaging studies of non-human primates are generally conducted with anesthesia using anesthetics like isoflurane which is known to suppress the neuronal activation of the brain substantially. The resting state functional MRI (rsfMRI) examination in anesthetized monkeys is hindered by limited choices of anesthetics compared to rodent studies. In the present study, multiband MRI technique was explored to improve the rsfMRI detection in anesthetized macaque monkeys. The preliminary results suggest the multiband MRI can be employed to dramatically improve the rsfMRI detection in examining the functional connectivity of default mode network in anesthetized monkeys using a clinical $3 \mathrm{~T}$ setting.

Effective connectivity of brain regions involved in word processing: an fMRI study of Chinese character and pinyin in reading

Guoyuan Yang ${ }^{1}$, Jianqiao $\mathrm{Ge}^{1}$, and Jia-Hong Gao ${ }^{1}$

${ }^{1}$ Peking University, Beijing, China

Reading words has been thought to be consist of three underlying constituents including orthographic, phonological, and semantic processing. The relationship between orthographic, phonological, and semantic processing in nonalphabetic language were still unclear. In the present study, we used functional magnetic resonance imaging (fMRI) to scan subjects when they were reading Chinese character and pinyin. Using dynamic causal modeling, we found that Chinese character reading processing was apparently involved ventral stream, and Chinese pinyin reading significantly involved dorsal stream. We conclude that nonalphabetic language with logographic system like Chinese character may needs less assembling phonology when word processing.

Investigation of Physiological Variability Effects on fMRI Dynamic Functional Connectivity using Independent Component Analysis

Foivia Nikolaou ${ }^{1,2}$, Christina Orphanidou ${ }^{2}$, Kevin Murphy $^{3}$, Richard G. Wise ${ }^{3}$, and Georgios D. Mitsis ${ }^{4}$

${ }^{1}$ Electrical and Computer Engineering, University of Cyprus, Nicosia, Cyprus, ${ }^{2} \mathrm{KIOS}$ Research and Innovation Center of Excellence, Nicosia, Cyprus, ${ }^{3}$ Cardiff University Brain Research Imaging Center (CUBRIC), School of Psychology, Cardiff University, Cardiff, United Kingdom, ${ }^{4}$ Department of Bioengineering, McGill University, Montreal, QC, Canada

The BOLD fMRI signal is influenced not only by neuronal activity but also by fluctuations in physiological signals. It has been shown that estimates of resting dynamic functional connectivity (DFC) may be confounded by the effects of physiological signal fluctuations. Here we examine the relation between DFC patterns for the DMN, visual and somatosensory networks and the time-varying properties of simultaneously recorded end-tidal CO2 and HR signals by using resting-state fMRI data and several variants of ICA. A modulatory effect, which was more pronounced in specific frequency bands, of the physiological signals on the resting DFC patterns is revealed.

Using graph theory measurements acquired from resting state fMRI data combine with machine learning methods to investigate abnormalities in temporal lobe epilepsy and classification.

Mohsen Mazrooyisebdani ${ }^{1}$, Veena A. Nair ${ }^{2}$, Bruce Hermann ${ }^{3}$, Beth Meyerand ${ }^{4}$, Vivek Prabhakaran ${ }^{2}$, and Raheel Ahmed ${ }^{3}$

${ }^{1}$ Electrical and engineering, University of Wisconsin Madison, Madison, WI, United States, ${ }^{2}$ Radiology, University of Wisconsin Madison, Madison, WI, United States, ${ }^{3}$ Neurology, University of Wisconsin Madison, Madison, WI, United States, ${ }^{4}$ Medical Physics, University of Wisconsin Madison, Madison, WI, United States

Many studies has shown structural damage in TLE caused by seizure propagation. We use graph theoretical approach to look at network differences in TLE's brain in order to find abnormalities that may cause seizure. We find out that subcortical regions such as thalamus and hippocampus are abnormally more connected together and with cerebellar regions and these regions are generally less involved in transferring information to other part of the brain from graph theoretical respect of view. In other word, any information pulse that generated in these regions, will circulate faster within these regions which might be the reason for seizure. 
Optimal Time-Dependent Window-Size Reveals a More Accurate Picture of Dynamic Functional Connectivity

Xiaowei Zhuang ${ }^{1}$, Zhengshi Yang ${ }^{1}$, Brent Bluett ${ }^{1}$, Sarah Banks $^{1}$, and Dietmar Cordes $^{1,2}$

${ }^{1}$ Cleveland Clinic Lou Ruvo Center for Brain Health, Las Vegas, NV, United States, ${ }^{2}$ University of Colorado, Boulder, CO, United States

We have introduced a new method to determine the optimal time-dependent window-size for calculating sliding-window correlations between two non-stationary time series. The timedependent window-size is calculated from the local information of intrinsic mode functions of each time series computed using empirical mode decomposition. Results from simulation demonstrate that the running-correlation computed with a time-dependent window-size is able to capture local transients without creating unstable fluctuations. By incorporating the optimal window-size in a whole-brain dynamic functional connectivity analysis, we are able to view differences in whole-brain temporal dynamics between normal control subjects and PD subjects more precisely.

A new analysis of resting state connectivity and graph theory reveals distinctive short-term modulations due to whisker stimulations in rats.

Silke Kreitz ${ }^{1,2}$, Benito de Celis Alonso ${ }^{3}$, Michael Uder ${ }^{2}$, and Andreas Hess ${ }^{1}$

${ }^{1}$ Institute for Pharmacology and Toxicology, Friedrich-Alexander-University Erlangen-Nuremberg, Erlangen, Germany, ${ }^{2}$ Department of Radiology, University Hospital of the FriedrichAlexander University Erlangen-Nuremberg, Erlangen, Germany, ${ }^{3}$ Faculty of Mathematical \& Physical Sciences, Benemerita Universidad Autonoma de Puebla, Puebla, Mexico

In this study we introduced a powerful new method to analyze resting state functional connectivity. The MSRA approach integrates classical seed based correlation and modern graphtheory. In comparison to two undirected graph-theoretical approaches, it resembles ICA components best and is characterized by its high specificity and reproducibility. In combination with an adaptation of the network based statistics to paired samples, it promises to be a powerful tool to investigate short term modulations of sensory stimuli related resting state connectivity and ultimately impact our understanding of basic brain functions like fear to higher functions such as plasticity, learning and memory.

Cross State Interference in Dynamic Functional Connectivity

Victor D. Vergara ${ }^{1}$ and Vince D. Calhoun ${ }^{1}$

$2415 \quad{ }^{1}$ The Mind Research Network, Albuquerque, NM, United States

Several parameters need to be set in a dynamic connectivity analysis. The window length (time interval used to estimate windowed correlation) gained recent attention after simulated data showed that a minimum length should be observed. This work presents evidence that large window lengths are not free of nuisances and proposes a method to find an appropriate window length. The proposed length is found to be half the average duration of a dynamic connectivity state. Longer window lengths produces cross-talk interference among states.

Resting State ASL : Toward an optimal sequence duration

Corentin Vallée ${ }^{1}$, Pierre Maurel ${ }^{1}$, Isabelle Corouge ${ }^{1}$, and Christian Barillot ${ }^{1}$

${ }^{1}$ Univ Rennes, Inria, CNRS, Inserm, IRISA UMR 6074, VISAGES ERL U-1228, F-35000, Rennes, France

Resting-state functional Arterial Spin Labeling (rs-fASL) in clinical daily practice and academic research stay discreet compared to resting-state BOLD. However, by giving direct access to cerebral blood flow maps, rs-fASL leads to significant clinical subject scaled application as CBF can be considered as a biomarker in common neuropathology. Our work here focuses on the link between overall quality of rs-fASL and duration of acquisition. To this end, we consider subject self-Default Mode Network (DMN), and assess DMN quality depletion compared to a gold standard DMN depending on the duration of acquisition.

Resting-state fMRI functional connectivity is confounded by the hemodynamic response function (HRF)

Rangaprakash Deshpande ${ }^{1,2}$, Guo-Rong Wü3 ${ }^{3,4}$, Daniele Marinazzo ${ }^{3}$, Xiaoping Hu ${ }^{5}$, and Gopikrishna Deshpande ${ }^{2,6,7}$

${ }^{1}$ Department of Psychiatry and Biobehavioral Sciences, University of California Los Angeles, Los Angeles, CA, United States, ${ }^{2}$ Department of Electrical and Computer Engineering, Auburn University, Auburn, AL, United States, ${ }^{3}$ Department of Data Analysis, University of Ghent, Ghent, Belgium, ${ }^{4}$ Key Laboratory of Cognition and Personality, Southwest University, Chongqing, China, ${ }^{5}$ Department of Bioengineering, University of California Riverside, Riverside, CA, United States, ${ }^{6}$ Department of Psychology, Auburn University, Auburn, AL, United States, ${ }^{7}$ Alabama Advanced Imaging Consortium, Auburn University and University of Alabama Birmingham, Auburn, AL, United States

Functional MRI is an indirect measure of neural activity, as it is the convolution of the hemodynamic-response function (HRF) and latent neural response. Recent studies show variability in HRF across brain regions and individuals, with the potential to confound resting-state functional connectivity (FC) if HRF variability were ignored. Using resting-state fMRI obtained at $7 \mathrm{~T}(\mathrm{~N}=47)$, we estimated HRF parameters using deconvolution, and tested the hypothesis that HRF variability confounds $\mathrm{FC}$. We found evidence, with simulations (up to $50 \%$ error in $\mathrm{FC}$ ) and experimental data (mean/median error $=30.5 / 11.5 \%$ in FC) quantifying the impact the HRF variability on FC. 


\section{Breast Imaging}

A MRI-based breast density measure which is directly comparable to mammographic density

Jie Ding ${ }^{1}$, Alison T Stopeck ${ }^{2,3}$, Yi Gao ${ }^{4,5,6}$, Marilyn T Marron ${ }^{7}$, Betsy C Wertheim ${ }^{7}$, Maria I Altbach ${ }^{7,8}$, Jean-Philippe Galons ${ }^{7,8}$, Denise J Roe ${ }^{7,9}$, Fang Wang ${ }^{2}$, Gertraud Maskarinec ${ }^{10}$, Cynthia A Thomson ${ }^{7}$, Patricia A Thompson ${ }^{2,11}$, and Chuan Huang ${ }^{1,12,13,14}$

${ }^{1}$ Biomedical Engineering, Stony Brook University, Stony Brook, NY, United States, ${ }^{2}$ Stony Brook University Cancer Center, Stony Brook, NY, United States, ${ }^{3}$ Hematology and Oncology, Stony Brook Medicine, Stony Brook, NY, United States, ${ }^{4}$ Biomedical Engineering, Shenzhen University, Shenzhen, China, ${ }^{5}$ Guangdong Key Laboratory for Biomedical Measurements and Ultrasound Imaging, Shenzhen, China, ${ }^{6}$ Applied Mathematics and Statistics, Stony Brook University, Stony Brook, NY, United States, ${ }^{7}$ University of Arizona Cancer Center, Tucson, AZ, United States, ${ }^{8}$ Medical Imaging, University of Arizona, Tucson, AZ, United States, ${ }^{9}$ Epidemiology and Biostatistics, University of Arizona, Tucson, AZ, United States, ${ }^{10}$ University of Hawaii Cancer Center, Honolulu, HI, United States, ${ }^{11}$ Pathology, Stony Brook Medicine, Stony Brook, NY, United States, ${ }^{12}$ Computer Science, Stony Brook University, Stony Brook, NY,

United States, ${ }^{13}$ Radiology, Stony Brook Medicine, Stony Brook, NY, United States, ${ }^{14}$ Psychiatry, Stony Brook Medicine, Stony Brook, NY, United States

High breast density is an independent risk factor for breast cancer. Mammography, the most widely used method for breast density determination, is limited by ionizing radiation exposure and its relatively low reliability for density assessment. We propose an automated, safe, and highly reproducible breast density measurement based on fat-water decomposition MRI. The technique yields a measure directly comparable to mammographic density which is easy for clinicians to use and for patients to understand.

Rapid and Simultaneous T1, T2 and Diffusion Quantification using MR Fingerprinting in the Breast

Yun Jiang ${ }^{1}$, Katherine L. Wright ${ }^{1}$, Jesse Hamilton ${ }^{2}$, Wei-Ching Lo ${ }^{2}$, Ananya Panda ${ }^{1}$, Gregor Körzdörfer ${ }^{3,4}$, Shota Hodono ${ }^{5}$, Michael A. Boss ${ }^{6}$, Nicole Seiberlich ${ }^{1,2}$, Vikas Gulani ${ }^{1,2}$, and Mark A. Griswold ${ }^{1,2}$

${ }^{1}$ Radiology, Case Western Reserve University, Cleveland, OH, United States, ${ }^{2}$ Biomedical Engineering, Case Western Reserve University, Cleveland, OH, United States, ${ }^{3}$ Siemens Healthcare GmbH, Erlangen, Germany, ${ }^{4}$ Friedrich-Alexander Universität Erlangen-Nürnberg, Erlangen, Germany, ${ }^{5}$ Department of Physics and Astronomy, Ohio Northern University, Ada, $\mathrm{OH}$, United States, ${ }^{6}$ National Institute of Standards and Technology, Boulder, CO, United States

High quality, distortion-free T1, T2 and diffusivity maps in breast imaging are simultaneously generated using MRF framework. A good agreement of T1, T2 and ADC between the proposed MRF method and the traditional spin echo methods is demonstrated in a phantom and in vivo in breast imaging. This method enables the simultaneous collection of T1, T2 and diffusion maps for tissue characterization without the need to co-register separately acquired maps as in conventional MRI.

Automatic Breast and Fibroglandular Tissue Segmentation Using Deep Learning by A Fully-Convolutional Residual Neural Network

Yang Zhang ${ }^{1}$, Vivian Youngjean Park ${ }^{2}$, Min Jung Kim ${ }^{2}$, Peter Chang ${ }^{3}$, Melissa Khy ${ }^{1}$, Daniel Chow ${ }^{1}$, Jeon-Hor Chen ${ }^{1}$, Alex Luk ${ }^{1}$, and Min-Ying Su ${ }^{1}$

${ }^{1}$ Department of Radiological Sciences, University of California, Irvine, CA, United States, ${ }^{2}$ Department of Radiology and Research Institute of Radiological Science, Severance Hospital, Yonsei University College of Medicine, Seoul, Republic of Korea, ${ }^{3}$ Department of Radiology, University of California, San Francisco, CA, United States

A deep learning method using the fully-convolutional residual neural network (FCR-NN) was applied to segment the whole breast and fibroglandular tissue in 289 patients. The Dice similarity coefficient (DSC) value and accuracy were calculated as evaluation metrics. For breast segmentation, the mean DSC was 0.85 with an accuracy of 0.93 ; for fibroglandular tissue segmentation, the mean DSC was 0.67 with an accuracy of 0.75 . The percent density calculated from ground truth and network segmentations were correlated, and showed a high coefficient of $r=0.9$. The initial results are promising, suggesting deep learning has a potential to provide an efficient and reliable breast density segmentation tool.

T2 star for breast invasive ductal carcinoma histopathological grade

Meiying Yan ${ }^{1}$, Xiaoqi Wang ${ }^{2}$, and Rengen $\mathrm{Xu}^{1}$

${ }^{1}$ Department of Radiology, Jiangxi Cancer Hospital, Nanchang, China, ${ }^{2}$ Philips Healthcare, Beijing, Beijing, China

Chemical shift encoded MRI (CSE-MRI) utilizes the water-fat signal model method, and its corresponding T2*mapping has less artifacts from water-fat shift. We extracted the fatinfluence-free $\mathrm{T} 2^{*}$ to investigate the correlation between T2 * mapping and histological grading of breast invasive ductal carcinoma, and found T2 * value for IDC-3 significantly higher than in IDC-2. This finds may provide more understanding of invasive ductal carcinoma microstructure and metabolism. 
${ }^{1}$ Radiology, The First affiliated hospital of Dalian Medical University, Dalian, China, ${ }^{2}$ Life science, GE Healthcare, Shenyang, China, ${ }^{3}$ GE healthcare, Beijing, China

The distinction between phyllodes tumor of breast (PTB) and fibroadenoma(FA) is clinically important, as approximately $20-30 \%$ of resected PTBs are malignant. Only limited information on the MRI characteristics of PTB is available. This study was performed to compare the MRI features (radiomics) of PTBs and FAs, which may resemble each other on conventional MRI.

Application of multiple b-value diffusion weighted imaging in diagnosing ductal carcinoma in situ

Lina Zhang ${ }^{1}$, Kai Zhang ${ }^{2}$, Qingwei Song ${ }^{1}$, Ailian Liu $^{1}$, and Lizhi Xie ${ }^{3}$

${ }^{1}$ Radiology, The First affiliated hospital of Dalian Medical University, Dalian, China, ${ }^{2}$ Radiology, The Second affiliated hospital of Dalian Medical University, Dalian, China, ${ }^{3}$ GE healthcare, China, Beijing, China

Multiple b-value diffusion weighted imaging (DWI) provides quantitative measurement of ADCslow for cellularity and $A D C_{\text {fast }}$ and $f_{\text {fast }}$ for vascularity. It is helpful for the differentiation between benign and malignant breast lesions. This study concerned perfusion as well as diffusion information in normal breast tissues and breast lesions from intravoxel incoherent motion (IVIM) imaging based on the biexponential analysis of multiple b-value DWI and then compared these parameters to ADC obtained with monoexponential analysis on the diagnosis of different grades of ductal carcinoma in situ (DCIS).

Proton MR Spectroscopy in Breast: Lipid Metabolite Concentrations as Valuable Quantitative Imaging Biomarkers for Cancer Diagnosis

Sunitha B Thakur ${ }^{1}$, Sandra Brennan², Ileana Hancu ${ }^{3}$, Blanca Bernard-Davila ${ }^{4}$, Michael Weber ${ }^{5}$, Elizabeth Manderski ${ }^{2}$, Elizabeth Morris ${ }^{2}$, and Katja Pinker ${ }^{2}$

${ }^{1}$ Medical Physics, Memorial Sloan Kettering Cancer Center, New York, NY, United States, ${ }^{2}$ Radiology, Memorial Sloan Kettering Cancer Center, New York, NY, United States, ${ }^{3}$ GE Global Research, Niskayuna, NY, United States, ${ }^{4}$ Memorial Sloan Kettering Cancer Center, New York, NY, United States, ${ }^{5}$ Medical University Vienna, Vienna, Austria

Differential expression of lipid metabolism-related proteins was recently reported in breast cancer patients. In this retrospective MR spectroscopy (MRS) study, the spectral lipid profile was assessed in breast cancer patients with malignant and benign lesions. Single-voxel MRS data from 176 breast lesions was analyzed to quantify multiple lipid metabolite concentrations using LCModel. Lipid peak analysis highlighted significant differences in lipid metabolite concentrations with significantly low concentrations in malignant compared to benign lesions and in luminal cancers compared to other molecular subtypes. MRS-based lipid metabolite profile may provide a valuable tool for breast cancer diagnosis.

Diffusion tensor and Intravoxel incoherent motion magnetic resonance imaging of the normal breast in young premenopausal women during menstrual cycle

Qiuju Fan ${ }^{1}$, Hui Tan ${ }^{1}$, Nan Yu ${ }^{1}$, Qi Yang ${ }^{1}$, Shaoyu Wang ${ }^{2}$, and Yong Yu ${ }^{1}$

${ }^{1}$ Affiliated Hospital of Shaanxi University of Chinese Medicine, Xianyang, China, ${ }^{2}$ Siemens Healthcare, Scientific marketing, Shanghai, China

DTI and IVIM can provide valuable information on tissue microstructure, microcirculation and pathophysiology that has been extensively used on the breast cancer ${ }^{[1,2]}$. However, the breast is a hormonally responsive organ and undergoes periodic variations according to the menstrual cycle. Thus, the periodic variations of DTI and IVIM-derived measurements need to be considered.

Kurtosis as a potential tool to differentiate breast hematological malignancies from breast cancer

Mizue Suzuki ${ }^{1}$, Masako Kataoka ${ }^{1}$, Mami lima $^{1}$, Shotaro Kanao ${ }^{1}$, Kanae Kawai Miyake ${ }^{1}$, Rena Sakaguchi ${ }^{1}$, Ayami Ohno Kishimoto $^{1}$, Maya Honda $^{1}$, Tadakazu Kondo $^{2}$, Tatsuki Kataoka ${ }^{3}$, Takaki Sakurai ${ }^{3}$, Masakazu Toi ${ }^{4}$, and Kaori Togashi ${ }^{1}$

${ }^{1}$ Department of Diagnostic Imaging and Nuclear Medicine, Graduate School of Medicine, Kyoto University, Kyoto, Japan, ${ }^{2}$ Department of Hematology and Oncology, Graduate School of Medicine, Kyoto University, Kyoto, Japan, ${ }^{3}$ Department of Diagnostic Pathology, Graduate School of Medicine, Kyoto University, Kyoto, Japan, ${ }^{4}$ Department of Breast Surgery, Graduate School of Medicine, Kyoto University, Kyoto, Japan

Since breast hematological malignancies show various image findings, it is not easy to differentiate them from breast cancer using conventional MRI. Non-Gaussian diffusion MRI is a relatively new method using multi b values from low to high, reflecting the interaction of water molecules with tissue features. We compared non-Gaussian parameters of breast hematological malignancies and breast cancer to investigate the advantage of non-Gaussian diffusion imaging. Our preliminary results suggest potential advantage of kurtosis as a marker of cellular structure and usefulness in differential diagnosis between breast hematological malignancies and breast cancer. 
Rosa Elena Ochoa Albiztegui ${ }^{1}$, Joao Vicente Machado Horvat ${ }^{1}$, Sunitha Thakur ${ }^{1}$, Blanca Bernard-Davila ${ }^{1}$, Siegfried Trattnig ${ }^{2}$, Thomas Helbich ${ }^{2}$, Elizabeth Morris ${ }^{1}$, and Katja PinkerDomenig $^{1}$

${ }^{1}$ Radiology, Memorial Sloan Kettering Cancer Center, New York, NY, United States, ${ }^{2}$ Biomedical Imaging and Image-guided Therapy, Medical University Vienna, Vienna, Austria

To investigate ultra-high field DCE-MRI of the breast at 7T with pharmacokinetic modeling for differentiation of benign and malignant breast tumors and molecular breast cance subtypes. 37 patients with 43 breast lesion were included and underwent a 7T DCE-MRI of the breast. Quantitative pharmacokinetic imaging biomarkers ktrans and kep aid in the differentiation of benign and malignant breast tumors. Selection of ROI- using a whole tumor and a $10 \mathrm{~mm}^{2}$ ROI- does not influence diagnostic accuracy. Quantitative pharmacokinetic differentiation of benign and malignant breast tumors. Selection of ROI- using a whole tumor and

New frontiers: the role of Arterial Spin Labeling (ASL) and Diffusion Tensor Imaging (DTI) to differentiate between malignant and benign breast lesions.

Akshaykumar Nana Kamble ${ }^{1}$ and Manju Popli ${ }^{1}$

${ }^{1}$ Radio-diagnosis, Institute of Nuclear Medicine and Allied Sciences (INMAS), Delhi, India

There was the time when contrast enhancement was critical to identify and differentiate malignant from benign tumor, but as the field of MR has made strides towards advanced imaging, we now can use the methods which doesn't require contrast. It is especially helpful in end stage renal patients. As the world demographic is slowly tilting towards geriatric population it will soon become essential to come up with alternative ways to detect the malignant pathologies independent of exogenous contrast. In our study we have demonstrated by plotting the ROC curve that ASL and DTI are promising methods to detect breast cancer.

Breast phyllodes tumor: histogram analysis of the apparent diffusion coefficient for assessment of tumor grading

Wenrui Tang ${ }^{1}$, Yan Zhang ${ }^{1}$, Dandan Zheng ${ }^{2}$, and Jingliang Cheng ${ }^{1}$

${ }^{1}$ Department of MRI, The First Affiliated Hospital of Zhengzhou University, Zhengzhou, China, ${ }^{2}$ GE Healthcare, China, Beijing, China

Phyllodes tumors are uncommon, biphasic, fibroepithelial lesions of the breast, characterized by leafy stromal fronds capped by benign bilayered epithelium. Grading of breast phyllodes tumors is critical for diagnosis, treatment options and preoperative evaluation. This study is to assess the feasibility of diffusion weighted image (DWI) for determining phyllodes tumors grades in the femoral breast. Our results reveal that histogram analysis of apparent diffusion coefficient (ADC) parameters derived from DWI can be used to classify the benign and malignant breast phyllodes tumors patients. This can be applied for clinical diagnose and treatment.

Correlation of MR Imaging Features with PIK3CA Mutation Status in Patients with Invasive Breast Cancer: A Preliminary Study

Min Sun Bae ${ }^{1}$, Mary C. Hughes ${ }^{1}$, Maxine Jochelson ${ }^{1}$, Elizabeth A. Morris ${ }^{1}$, and Katja Pinker-Domenig ${ }^{1}$

${ }^{1}$ Department of Radiology, Memorial Sloan Kettering Cancer Center, New York, NY, United States

PIK3CA mutation frequency ranges from $8 \%$ to $40 \%$ in breast cancer. PIK3CA mutations have been shown to be associated with favorable clinicopathologic features including estrogen receptor positive status. In this study, we investigated whether MRI features are correlated with PIK3CA mutation status in patients with invasive breast cancer. Of the 54 patients, 20 $(37 \%)$ had a PIK3CA mutation. PIK3CA mutated tumors were significantly less likely to show intratumoral T2 high signal intensity compared to wild type $(P=.004)$. In conclusion, intratumoral signal intensity on T2-weighted MR images is significantly associated with PIK3CA mutation status.

Preoperative diagnostic value of DKI combined with quantitative dynamic contrast - enhanced MRI in breast lesions

Ting $\mathrm{Li}^{1}$, Siying Wang ${ }^{2}$, Yun Xiong ${ }^{3}$, and Kangan $\mathrm{Li}^{1}$

${ }^{1}$ Shanghai General Hospital, Shanghai, China, ${ }^{2}$ PhilipsHealthcare, Shanghai, China, ${ }^{3}$ Fudan University, Shanghai, China

The aim of this study is to evaluate the diagnostic efficacy of 3.0T MRI diffusion kurtosis imaging and quantitative dynamic contrast enhancement in benign and malignant breast lesions, and to explore the differential diagnosis ability of different pathological types and molecular subtype lesions. 
${ }^{1}$ Radiology, Memorial Sloan Kettering Cancer Center, New York, NY, United States, ${ }^{2}$ Medical Physics, Memorial Sloan Kettering Cancer Center, New York, NY, United States, ${ }^{3}$ Medical University of Vienna, Vienna, Austria

Molecular subtype classification of breast tumor is of paramount importance in determining aggressiveness and prognosis. The ability to use diffusion weighted imaging (DWI) for the prediction of molecular subtypes may improve management in breast cancer. In this study, two radiologists retrospectively evaluated different metrics on apparent diffusion coefficient maps of 107 patients with invasive breast cancer. ER and PR positive lesions had lower ADC values while HER2 positive and high-proliferating had higher values. Luminal cancers had lower $A D C$ values than other subtypes, thus DWI may be used to predict tumor subtype in breast cancer.

Traditional Poster

\section{Xe \& 3He Imaging}

Regional Lung Function Quantification by Combining Gas-Phase Saturation with Hyperpolarized Xenon-129 Dissolved-Phase MRI

Kai Ruppert ${ }^{1}$, Hooman Hamedani ${ }^{1}$, Faraz Amzajerdian ${ }^{1}$, Luis Loza ${ }^{1}$, Yi Xin $^{1}$, Ian F. Duncan ${ }^{1}$, Harilla Profka ${ }^{1}$, Sarmad Siddiqui ${ }^{1}$, Mehrdad Pourfathi ${ }^{1}$, Stephen Kadlecek ${ }^{1}$, and Rahim R. Rizi $^{1}$

Hyperpolarized xenon-129 MRI has previously been used to assess pulmonary gas exchange between the alveolar volume and lung tissue. In this work, we quantified changes in the downstream xenon dissolved-phase signal in the left ventricle in response to a regional saturation of the pulmonary gas-phase signal. This approach permitted us to extract the relative gas-exchange efficiency of the lung volume unaffected by the GP signal saturation, demonstrating increased gas exchange efficiency in the posterior regions of the lung in supine rabbits. The proposed technique might be especially valuable in lung transplantation, during pharmaceutical interventions, or for lung-volume reduction surgeries.

Observing Pulmonary Gas-Transport Dynamics Using Rapid 1D Hyperpolarized Xenon-129 Dissolved-Phase Measurements

Kai Ruppert ${ }^{1}$, Hooman Hamedani ${ }^{1}$, Faraz Amzajerdian ${ }^{1}$, Luis Loza ${ }^{1}$, Yi Xin ${ }^{1}$, Ian F. Duncan ${ }^{1}$, Harilla Profka ${ }^{1}$, Sarmad Siddiqui ${ }^{1}$, Mehrdad Pourfathi ${ }^{1}$, Stephen Kadlecek ${ }^{1}$, and Rahim R. Rizi $^{1}$

${ }^{1}$ Radiology, University of Pennsylvania, Philadelphia, PA, United States

Monitoring the dissolved xenon-129 signal in a central downstream location such as the left ventricle of the heart provides a convenient measure of the lung's gas transport dynamics, and thereby of total lung function. To demonstrate the feasibility of this approach, we combined a rapid simultaneous gas-phase / dissolved-phase 1D-projection acquisition with regional gas-phase saturation to monitor the gas-transport dynamics of the lung as signal variations in the heart of a rat model of radiation-induced lung injury. Our measurements indicate that this method can identify the reductions in regional lung function associated with partial lung irradiation.

Measuring the Impact of PEEP on Pulmonary Gas Transport Using Hyperpolarized Xenon-129 Dissolved-Phase MRI

Kai Ruppert ${ }^{1}$, Hooman Hamedani ${ }^{1}$, Faraz Amzajerdian ${ }^{1}$, Luis Loza ${ }^{1}$, Yi Xin ${ }^{1}$, Ian F. Duncan ${ }^{1}$, Harilla Profka ${ }^{1}$, Sarmad Siddiqui ${ }^{1}$, Mehrdad Pourfathi ${ }^{1}$, Maurizio F. Cereda ${ }^{1}$, Stephen Kadlecek ${ }^{1}$, and Rahim R. Rizi ${ }^{1}$

${ }^{1}$ Radiology, University of Pennsylvania, Philadelphia, PA, United States

Higher positive end-expiratory pressure (PEEP) during mechanical ventilation can result in improved oxygenation, but it can also give rise to ventilator-induced lung injury. In this work, we used a rabbit model to evaluate the sensitivity of a hyperpolarized xenon-129 MRI technique that allows a comprehensive assessment of the pulmonary gas-transport by the entire lung for monitoring the impact of PEEP on lung function. We observed that increased PEEP resulted in a large decrease in pulmonary gas transport that is most likely linked to a lengthened pulmonary transit time.

Hyperpolarized 129Xe MR functional imaging to monitor the response of the human lungs after segmental lipopolysaccharide challenge

Agilo Luitger Kern ${ }^{1,2}$, Heike Biller ${ }^{2,3}$, Filip Klimes ${ }^{1,2}$, Andreas Voskrebenzev ${ }^{1,2}$, Marcel Gutberlet ${ }^{1,2}$, Alexander Rotärmel ${ }^{1,2}$, Christian Schönfeld ${ }^{1,2}$, Julius Renne $^{1,2}$, Olaf Holz $^{2,3}$, Kun $^{2}$ Qing $^{4}$, Kai Ruppert ${ }^{5}$, Frank Wacker ${ }^{1,2}$, Jens Hohlfeld ${ }^{2,3}$, and Jens Vogel-Claussen ${ }^{1,2}$

${ }^{1}$ Institute of Diagnostic and Interventional Radiology, Hannover Medical School, Hannover, Germany, ${ }^{2}$ Biomedical Research in Endstage and Obstructive Lung Disease Hannover (BREATH), German Center for Lung Research (DZL), Hannover, Germany, ${ }^{3}$ Department of Clinical Airway Research, Fraunhofer Institute for Toxicology and Experimental Medicine, Hannover, Germany, ${ }^{4}$ Department of Radiology and Medical Imaging, University of Virginia, Charlottesville, VA, United States, ${ }^{5}$ Department of Radiology, University of Pennsylvania, Philadelphia, PA, United States 
Hyperpolarized ${ }^{129} \mathrm{Xe}$ MRI has been shown to be sensitive to inflammatory changes after lung provocation by lipopolysaccharide (LPS) in an animal model. The purpose of this work was to investigate feasibility of monitoring the response of the human lungs after segmental LPS challenge using ${ }^{129}$ Xe MRI. Dissolved-phase imaging and chemical shift saturation recovery were employed to assess inflammatory changes and to compare MRI results with inflammatory cell counts from bronchoalveolar lavage. Both MRI methods show a significant reduction of the ${ }^{129} \mathrm{Xe}$ in red blood cells and lung tissue ratio in the affected region but no significant correlations with inflammatory cell counts.

Revealing Pulmonary Gas Transport Dynamics using a 3D Radial Hyperpolarized Xenon MRI Acquisition with Variable Flip Angles

Faraz Amzajerdian ${ }^{1}$, Kai Ruppert ${ }^{1}$, Hooman Hamedani ${ }^{1}$, Yi Xin ${ }^{1}$, Ian F. Duncan ${ }^{1}$, Harrilla Profka ${ }^{1}$, Mehrdad Pourfathi ${ }^{1}$, Sarmad Siddiqui ${ }^{1}$, Luis Loza ${ }^{1}$, Stephen Kadlecek ${ }^{1}$, and Rahim R. Rizi $^{1}$

${ }^{1}$ Radiology, University of Pennsylvania, Philadelphia, PA, United States

We demonstrated that reducing the flip angle drives the distribution of acquired dissolved-phase xenon downstream towards the heart. By exploiting this principle, the dynamics of pulmonary gas transport were captured through a single 3D double golden means radial acquisition with linearly decreasing flip angles. Reconstruction with a sliding window generated a series of consecutive images with declining average flip angles, depicting the gradual uptake and accumulation of xenon by the heart and lungs.

${ }^{129}$ Xe signal dynamics and chemical shift in the cardio-pulmonary circuit using cardiac-gated hyperpolarized ${ }^{129}$ Xe NMR

Graham Norquay ${ }^{1}$ and Jim M Wild ${ }^{1}$

${ }^{1}$ University of Sheffield, Sheffield, United Kingdom

The sensitivity of the ${ }^{129} \mathrm{Xe}$ chemical shift to red blood cell oxygenation makes hyperpolarized ${ }^{129} \mathrm{Xe}$ MR spectroscopy a promising technique for measurement of blood oxygenation in vivo. In addition, dissolved phase ${ }^{129} \mathrm{Xe}$ MRS is of interest as a biomarker of gas exchange and interstitial lung disease. Both the signal dynamics and chemical shift of ${ }^{129}$ Xe have been shown to be modulated by the cardiac cycle, potentially adding confounding effects to interpretation of the ${ }^{129}$ Xe MRS chemical shift. In this study, we demonstrate that cardiac-gating in ${ }^{129} \mathrm{Xe}$ MRS reduces the variability in the measured dissolved ${ }^{129} \mathrm{Xe}$ signal and chemical shift in the cardio-pulmonary circuit.

Using a hybrid multibreath hyperpolarized (HP) 129Xe imaging technique for simultaneous assessment of lung function and structure in a two-hit radiation induced lung injury (RILI) model.

Sarmad Siddiqui ${ }^{1}$, Hooman Hamedani ${ }^{1}$, Yi Xin ${ }^{1}$, Luis Loza ${ }^{1}$, Faraz Amzajerdian ${ }^{1}$, Mehrdad Pourfathi ${ }^{1}$, Stephen Kadlecek ${ }^{1}$, Kai Ruppert ${ }^{1}$, Harrilla Profka ${ }^{1}$, Rahim R. Rizi ${ }^{1}$, Shampa Chatterjee $^{2}$, and lan Duncan ${ }^{1}$

${ }^{1}$ Radiology, University of Pennsylvania, Philadelphia, PA, United States, ${ }^{2}$ Physiology, University of Pennsylvania, Philadelphia, PA, United States

In this study we developed a two-hit hemi-thorax radiation-induced lung injury (RILI) model that better simulates the etiology of the disease in humans, and characterized it via a multibreath hyperpolarized (HP) 129Xe imaging technique to assess lung function and structure one month post-radiation. We observed an increased PAO2 of $145 \pm 41$ Torr in the radiated lung compared to $124 \pm 40$ Torr in the contralateral lung. We also observed a corresponding decrease in oxygen uptake in the radiated lung. The preliminary findings suggest that HP 129Xe-derived functional parameters, particularly changes in the alveolar oxygen tension and oxygen uptake can serve as biomarkers during the early fibrotic stage of RILI.

Fast Imaging of Hyperpolarized Xe-129 in the Airspace, Barrier and Red Blood Cells in the Human Lung

Junshuai Xie ${ }^{1,2}$, Haidong $\mathrm{Li}^{1}$, Huiting Zhang ${ }^{1}$, Xiuchao Zhao ${ }^{1}$, Xianping Sun ${ }^{1,2}$, Chaohui Ye ${ }^{1,2}$, and Xin Zhou ${ }^{1,2}$

${ }^{1}$ State Key Laboratory of Magnetic Resonance and Atomic and Molecular Physics, National Center for Magnetic Resonance in Wuhan, Wuhan Institute of Physics and Mathematics, Chinese Academy of Sciences, Wuhan, China, ${ }^{2}$ University of Chinese Academy of Sciences, Beijing, China

Xe-129 in the barrier and red blood cells could be separated by the dissolved-phase (DP) Xe-129 MRI with radial sampling strategy. However, the number of the RF pulse was usually large and thus resulted in long acquisition time. An MRI strategy in the Cartesian coordinate has been used to for high-resolution rodent lung imaging of He- 3 in the airspace. The concept was introduced into fast acquisition of the DP Xe-129 in the human lung with the multi-point Dixon method. The number of the RF pulse reduced and the results of TP/Gas and $\mathrm{RBC} / \mathrm{Gas}$ agreed with the previous study.

Panayiotis Nikolaou ${ }^{1}$, Aaron M Coffey², Bryce Kidd ${ }^{3}$, Megan Murphy ${ }^{3}$, Boyd M Goodson ${ }^{3}$, Michael J Barlow ${ }^{4}$, and Eduard Y Chekmenev ${ }^{5}$

${ }^{1}$ VUIIS, Vanderbilt University Medical Center, Nashville, TN, United States, ${ }^{2}$ Vanderbilt University Medical Center, Nashville, TN, United States, ${ }^{3}$ Southern Illinois University Carbondale, Carbondale, IL, United States, ${ }^{4}$ University of Nottingham, Nottingham, United Kingdom, ${ }^{5}$ Radiology, Vanderbilt University Medical Center, Nashville, TN, United States 
Over the last two decades there have been many advances in the field of hyperpolarized (HP) noble gas production and imaging, largely enabled by the development of low-cost, highpower frequency-narrowed laser diode arrays (LDAs) and the improvement of ${ }^{129}$ Xe polarizer technology in general. Here we present the development and features of the new $3{ }^{\text {rd }}$ generation Batch-Mode ${ }^{129}$ Xe hyperpolarizer. As with most previous ${ }^{129}$ Xe polarizers, the new device utilizes Spin Exchange Optical Pumping (SEOP), a process in which resonant, circularly polarized photons optically pump Rb electrons, which in turn hyperpolarize the ${ }^{129}$ Xe nuclear spins via hyperfine interactions (the "spin-exchange" process).

A paired approach to the segmentation of proton and hyperpolarized gas MR images of the lungs

Alberto M Biancardi ${ }^{1,2}$, Laure Acunzo ${ }^{1}$, Helen Marshall ${ }^{1}$, Bilal A Tahir ${ }^{1,3}$, Paul JC Hughes ${ }^{1}$, Laurie Smith ${ }^{1,4}$, Nicholas D Weatherley ${ }^{1}$, Guilhelm J Collier ${ }^{1}$, and Jim M Wild ${ }^{1,2}$

${ }^{1}$ Polaris, The University of Sheffield, Sheffield, United Kingdom, ${ }^{2}$ INSIGNEO, The University of Sheffield, Sheffield, United Kingdom, ${ }^{3}$ Academic Unit of Clinical Oncology, The University of Sheffield, Sheffield, United Kingdom, ${ }^{4}$ Sheffield Children's Hospital, Sheffield Children's NHS Foundation Trust and Sheffield Teaching Hospitals NHS Foundation Trust, Sheffield, United Kingdom

Quantitative analyses of hyperpolarized gas and ${ }^{1} \mathrm{H}$ lung MRI together provide quantitative information on lung obstruction. Quantification requires segmentation of the ventilated and non-ventilated regions of the hyperpolarized gas MRI and definition of the lung cavity from the paired ${ }^{1} \mathrm{H}$ MRI. Spatial fuzzy c-means segmentation was developed to segment these image pairs simultaneously. Error measures with respect to manual reference segmentations and qualitative grading showed significant improvements when compared to an established method. This work may help towards standardisation and automation of lung ventilation image analysis, and help improve accuracy and reproducibility

A Study of Lung Function Variability in Chronic Obstructive Pulmonary Disease Using Hybrid Hyperpolarized 3He Imaging

Hooman Hamedani ${ }^{1}$, Ryan Baron ${ }^{1}$, Sarmad Siddiqui ${ }^{1}$, Yi Xin ${ }^{1}$, Mary Spencer ${ }^{1}$, Faraz Amzajerdian ${ }^{1}$, Stephen Kadlecek ${ }^{1}$, Kai Ruppert ${ }^{1}$, Mehrdad Pourfathi $^{1}$, Luis Loza ${ }^{1}$, Ian Duncan ${ }^{1}$, Tahmina Achekzai ${ }^{1}$, Maurizio Cereda ${ }^{2}$, and Rahim R. Rizi ${ }^{1}$

2443

${ }^{1}$ Radiology, University of Pennsylvania, Philadelphia, PA, United States, ${ }^{2}$ Anesthesiology and Critical Care, University of Pennsylvania, Philadelphia, PA, United States

To better understand variable lung function in COPD, we imaged a subset of COPDGene subjects at baseline, one week post-baseline and one month post-baseline using a multifaceted hyperpolarized (HP) ${ }^{3} \mathrm{He}$ scheme to measure apparent diffusion coefficient (ADC), fractional ventilation $(\mathrm{FV})$, alveolar oxygen tension $\left(P_{\mathrm{A}} \mathrm{O}_{2}\right)$ and oxygen uptake $(\mathrm{R})$ variability.

Traditional Poster

\section{Body Imaging: Fetal/Placenta \& Pelvis}

MT contrast in the Post-mortem Neonate: A pilot study

Amy R McDowell ${ }^{1}$, Susan Shelmerdine ${ }^{2}$, Sara Lorio ${ }^{1}$, Owen Arthurs ${ }^{2}$, and David Carmichael

${ }^{1}$ UCL GOS Institute of Child Health, London, United Kingdom, ${ }^{2}$ Department of Radiology, Great Ormond Street Hospital for Children NHS Trust, London, United Kingdom

Post-mortem MRI imaging (PMMR) is rapidly becoming a useful tool in the minimally invasive autopsy of fetal and perinatal death allowing clinical diagnosis and assessment of major congenital abnormalities. A recent study suggested that magnetisation transfer values may be a more specific measure of post-mortem heart abnormalities, but there has little application of MT imaging in this area. We performed a preliminary exploration of MT contrast and MT pulse optimisation in whole body PMMR in neonates as part of a multi-parameter mapping protocol.

High Resolution Rapid Neonatal Whole Body Composition Using 3.0 Tesla Chemical Shift Magnetic Resonance Imaging

Jonathan P Dyke ${ }^{1}$, Amanda C Garfinkel ${ }^{2}$, Alan M Groves ${ }^{2}$, and Arzu Kovanlikaya ${ }^{1}$

To evaluate a whole body rapid imaging technique to calculate neonatal lean body mass and percentage adiposity using 3.0 Tesla chemical shift Magnetic Resonance Imaging (MRI). A rapid 2-Point Dixon MRI technique was used to calculate whole body fat and water images at 3.0 Tesla in term ( $n=10)$ and preterm ( $n=15)$ infants in 42 seconds/scan. MRI calculated whole body mass correlated closely with measured body weight (R2=0.87; $<<0.001)$. Scan-rescan analysis demonstrated a $95 \%$ limit of agreement of $1.3 \%$ adiposity. At term corrected age, former preterm infants had significantly reduced lean body mass compared to term born controls $1935 \mathrm{~g}$ versus $2416 \mathrm{~g}(\mathrm{p}=0.002)$. 
Qiaoyan Chen ${ }^{1,2}$, Guoxi Xie ${ }^{3}$, Chao Luo ${ }^{1,2}$, Xing Yang ${ }^{4}$, Jin Zhu ${ }^{5}$, Jo Lee ${ }^{1,2}$, Xiaoliang Zhang ${ }^{6,7}$, Xin Liu ${ }^{1,2}$, and Ye Li $i^{1,2}$

${ }^{1}$ Lauterbur Imaging Research Center, Shenzhen Institutes of Advanced Technology, Chinese Academy of Sciences, Shenzhen, China, ${ }^{2}$ Shenzhen Key Laboratory for MRI, Shenzhen, China, ${ }^{3}$ School of Basic Science, Guangzhou Medical University, Guangzhou, China, ${ }^{4}$ High-Field Magnetic Resonance Brain Imaging Key Laboratory of Sichuan Province, School of Life Science and Technology, University of Electronic Science and Technology of China, Chengdu, China, ${ }^{5}$ Shenzhen People's Hospital, Shenzhen, China, ${ }^{6}$ Department of Radiology and Biomedical Imaging, University of California San Francisco, San Francisco, CA, United States, ${ }^{7}$ UCSF/UC Berkeley Joint Graduate Group in Bioengineering, San Francisco, CA, United States

Due to lack of dedicated fetal imaging coils, the standard commercial abdominal coil is often used for fetal imaging, of which the performance is limited by its insufficient coverage and element number. In this work, a dedicated 36 -channel coil array for fetal imaging was designed, capable of covering a range of pregnancy from 20 to $37+$ weeks. Compared to a commercial abdominal coil array, the proposed 36-channel fetal coil provides improved performance in SNR, parallel imaging capability, and image quality.

Fetal non-contrast MR angiography in second and early third trimester

Uday Krishnamurthy ${ }^{1}$, Swati Mody ${ }^{1}$, Brijesh Yadav $^{1}$, Pavan Kumar Jella ${ }^{1}$, Edgar Edgar Hernandez-Andrade ${ }^{2,3}$, Anabela Trifan ${ }^{1}$, Ewart Haacke ${ }^{1}$, Roberto Romero ${ }^{3}$, and Jaladhar Neelavalli ${ }^{1}$

${ }^{1}$ Radiology, Wayne State University, Detroit, MI, United States, ${ }^{2}$ Obstetrics and Gynecology, Wayne State University, Detroit, MI, United States, ${ }^{3}$ Perinatology Research Branch, NICHD/NIH/DHHS, Detroit, MI, United States

To evaluate the robustness and utility of non-contrast MRA as a means to visualize fetal vasculature, particularly in fetuses younger than 30 weeks gestation.

Non-rigid motion correction for arterial spin labeled (ASL) perfusion imaging of the placenta using ANTs

Zhengjun Li ${ }^{1}$, Eileen Hwuang ${ }^{1}$, Jeffrey Duda ${ }^{2}$, Marta Vidorretta ${ }^{1}$, Nadav Schwartz ${ }^{3}$, John Detre ${ }^{1,2}$, Walter Witschey ${ }^{2}$, and Dylan Tisdall ${ }^{2}$

${ }^{1}$ Dept. of Neurology, University of Pennsylvania, Philadelphia, PA, United States, ${ }^{2}$ Dept. of Radiology, University of Pennsylvania, Philadelphia, PA, United States, ${ }^{3}$ Dept. of Obstetrics and Gynecology, University of Pennsylvania, Philadelphia, PA, United States

Non-rigid motion of the placenta due to maternal breathing and fetal movement is one of the main challenges in placental MRI. In this study, we evaluated non-rigid motion correction of the placenta during arterial spin labeled (ASL) perfusion imaging, using Advanced Normalization Tools (ANTs). The results showed that non-rigid motion correction with ANTs improved the resulting perfusion images as evidenced by reduced the residual power of control-label regression, increased the tSNR, and reduced the power of respiration in the signal.

Diffusion Tensor Imaging for Differentiating Borderline From Malignant Epithelial Ovarian Tumors

XU HAN ${ }^{1}$, MEI-YU SUN ${ }^{1}$, MENG-YAO WANG ${ }^{1}$, LI-ZHI XIE $^{2}$, and RUI FAN ${ }^{1}$

${ }^{1}$ The First Affiliated Hospital of Dalian Medical University, Dalian, China, ${ }^{2}$ GE Healthcare, Beijing, China

To assess the fitted parameters of DTI in ovarian tumors and to investigate their potential in distinguishing borderline from malignant epithelial ovarian tumors, which can provide detailed information for clinical treatment. DC avg, Exat, FA and VRA in DTI were valuable information in distinguishing borderline from malignant epithelial ovarian tumors and can be used as non-enhancement quantitative indexes, which has a good application prospect.

A Subspace Approach to Accelerated HASTE Acquisition for Fetal Brain MRI

Bo Zhao ${ }^{1,2}$, Borjan Gagoski ${ }^{2,3}$, Justin P. Haldar ${ }^{4}$, Elfar Adalsteinsson ${ }^{5}$, Ellen Grant ${ }^{3,6}$, and Lawrence L. Wald ${ }^{1,2}$

${ }^{1}$ Athinoula A. Martinos Center for Biomedical Imaging, Chalestown, MA, United States, ${ }^{2}$ Harvard Medical School, Boston, MA, United States, ${ }^{3}$ Boston Children's Hospital, Boston, MA, United States, ${ }^{4}$ Electrical Engineering, University of Southern California, Los Angeles, CA, United States, ${ }^{5}$ Electrical Engineering and Computer Science, Massachusetts Institute of Technology, Cambridge, MA, United States, ${ }^{6}$ Radiology, Harvard Medical School, Boston, MA, United States

HAlf-fourier Single-shot Turbo spin Echo (HASTE) acquisition is widely used in fetal MR imaging due to its $\mathrm{T}_{2}$ contrast and motion robustness, but speed and $\mathrm{T}_{2}$-blurring remain a problem for fully sampled acquisitions. In the work, we describe a new reconstruction approach based on low-rank and subspace modeling of local k-space neighborhood to accelerate HASTE acquisition. The proposed approach decreases the echo-train length with improved image quality and noise robustness compared to conventional reconstruction. It is compatible with the vendor-provided acquisition. The effectiveness and utility of the proposed approach is evaluated with both retrospectively and prospectively undersampled fetal imaging data. 


\section{Thoracic MRI}

Usefulness of morphological characteristics for the differentiation of benign from malignant peripheral solitary pulmonary lesions using MR T1-weighted 3D Star VIBE Shan Dang ${ }^{1}$, Haifeng Duan ${ }^{1}$, Dong Han ${ }^{1}$, QI Yang ${ }^{1}$, Xin Tian ${ }^{1}$, Nan Yu ${ }^{1}$, Yuxin Lei ${ }^{1}$, Shaoyu Wang ${ }^{2}$, Sujue Lu ${ }^{3}$, and Guangming Ma

${ }^{1}$ Department of Radiology, Affiliated Hospital of Shaanxi University of traditional Chinese Medicine, XianYang, China, ${ }^{2}$ Siemens Healthcare, Scientific marketing, China, Shanhai, China, ${ }^{3}$ Shaanxi University of traditional Chinese Medicine, Xian Yang, China

Can MR T1-weighted 3D Star VIBE alternate the MSCT in morphological features of the peripheral solid pulmonary lesions?

Free-breathing T1-weighted 3D STAR VIBE: versus Thin-Section Computed Tomography for the Assessment of Pulmonary Parenchyma Diseases

Zhanli Ren ${ }^{1}$, Shan Dang ${ }^{2}$, Yuxin $\mathrm{Lei}^{2}$, Nan $\mathrm{Yu}^{2}$, Yong $\mathrm{Yu}^{2}$, and Taiping $\mathrm{He}^{2}$

${ }^{1}$ Shaanxi University of Chinese Medicine, Xianyang, China, ${ }^{2}$ Affiliated Hospital of Shaanxi University of Chinese Medicine, Xianyang, China

Free-breathing T1-weighted 3D star vibe is useful for lung and mediastinum assessment and evaluation of radiological findings for patients with various pulmonary parenchyma diseases.

MRI Ventilation Texture Features Discriminate Severe Asthmatics with and without Eosinophilic Airway Inflammation

Sarah Svenningsen ${ }^{1,2,3}$, Nanxi Zha ${ }^{1}$, Rachel Eddy ${ }^{2}$, Dante Capaldi ${ }^{2}$, Melanie Kjarsgaard $^{3}$, Katherine Radford $^{3}$, Parameswaran Nair ${ }^{1,3}$, and Grace Parraga $^{2}$

${ }^{1}$ McMaster University, Hamilton, ON, Canada, ${ }^{2}$ Robarts Research Institute, Western University, London, ON, Canada, ${ }^{3}$ Firestone Institute for Respiratory Health, St Joseph's Healthcare Hamilton, ON, Canada

Previous work suggests that inhaled gas MRI conceals minable features that are distinctly different between severe asthma inflammatory endotypes and these may be used to predict inflammatory endotype. We evaluated the performance of inhaled gas MRI ventilation defect percent, ventilation coefficient of variation and texture features to discriminate severe asthmatics with and without the eosinophilic inflammatory endotype. MRI measurements of ventilation significantly discriminated asthmatics with eosinophilic inflammation from those without eosinophilic inflammation. Non-invasive MRI-based biomarkers and signatures of asthma inflammatory endotype may serve to guide treatment selection in individual asthmatics or evaluate the effectiveness of anti-inflammatory treatments in clinical trials.

Extraction of fractional ventilation from dynamic oxygen enhanced MRI experiments: preliminary results

Marta Tibiletti ${ }^{1}$, Jose Ulloa ${ }^{1,2}$, and Geoff JM Parker ${ }^{1,2}$

$2454 \quad{ }^{1}$ Bioxydyn Ltd, Manchester, United Kingdom, ${ }^{2}$ Centre for Imaging Sciences, University of Manchester, Manchester, United Kingdom

Fractional ventilation (FV) weighted maps were extracted from free-breathing dynamic $\mathrm{O}_{2}$ enhanced (dynOE) experiment in cystic fibrosis patients. $\mathrm{FV}$ is related to the local expansion of the tissue due to gas arrival in inspiration, while dynOE maps the local rate of the arrival of $\mathrm{O}_{2}$ and the maximum enhancement obtained. These parameters can be extracted from the same acquisition, providing complementary information regarding local lung function.

Comparative study of 3D inversion recovery centric ordered fast field echo in lung dynamic oxygen enhanced MRI at $1.5 \mathrm{~T}$ and $3 \mathrm{~T}$

Marta Tibiletti ${ }^{1}$, Jose Ulloa ${ }^{1,2}$, Alexandra R Morgan ${ }^{1}$, and Geoff JM Parker ${ }^{1,2}$

${ }^{1}$ Bioxydyn Ltd, Manchester, United Kingdom, ${ }^{2}$ Centre for Imaging Sciences, University of Manchester, Manchester, United Kingdom

Dynamic oxygen-enhanced MRI (dOE-MRI) techniques have previously been apply to study the rate and level of $\mathrm{O}_{2}$ enhancement in the lung. Lung MRI investigations are mostly conducted at 1.5T, because signal loss due to stronger susceptibility artefacts in lung tissue is expected at higher field strength. In this work, we demonstrate the feasibility of dOE-MRI at $3 \mathrm{~T}$ on healthy volunteers. The observed signal enhancement is comparable between $1.5 \mathrm{~T}$ and $3 \mathrm{~T}$, but translates in a lower relative T1 change due to higher baseline T1 at 3T. Fitting performance of $\mathrm{O}_{2}$ wash-in curve may be reduced by the lower SNR at 3T. 
${ }^{1}$ Medical Physics, University of Wisconsin - Madison, Madison, WI, United States, ${ }^{2}$ Center for Pulmonary Imaging Research, Division of Pulmonary Medicine and Department of Radiology, Cincinnati Children's Hospital Medical Center, Cincinnati, OH, United States, ${ }^{3}$ Physics, Washington University in St. Louis, St. Louis, MO, United States, ${ }^{4}$ Imaging Research Center, Cincinnati Children's Hospital Medical Center, Cincinnati, OH, United States, ${ }^{5}$ Radiology, University of Missouri, Columbia, MO, United States, ${ }^{6}$ Physics, University of Cincinnati, Cincinnati, $\mathrm{OH}$, United States, ${ }^{7}$ Perinatal Institute, Division of Neonatology and Pulmonary Biology, Cincinnati Children's Hospital Medical Center, Cincinnati, $\mathrm{OH}$, United States

We estimate pulmonary tissue densities (TD) and $\mathrm{R}_{2}{ }^{*}$ in neonatal intensive care unit patients with and without diagnoses of lung disease as well as in healthy adults using multi-echo $3 \mathrm{D}$ ultrashort echo time MRI. As anticipated, a clear negative relationship between TD and $R_{2}{ }^{*}$ is evident. However, after correcting for TD variation, we find significant differences in $R_{2}{ }^{*}$ between diseased and non-diseased neonates, suggesting that MRI can probe differences in susceptibility and/or sub-voxel tissue geometry which may increase understanding of neonatal lung tissue pathologies.

Implementation of the FLORET Ultrashort Echo-Time Sequence for Lung Imaging

Matthew M. Willmering ${ }^{1}$, Ryan K. Robison ${ }^{2}$, Hui Wang ${ }^{3}$, James G. Pipe ${ }^{4}$, and Jason C. Woods ${ }^{1,5}$

${ }^{1}$ Center for Pulmonary Imaging Research, Cincinnati Children's Hospital Medical Center, Cincinnati, OH, United States, ${ }^{2}$ Phoenix Children's Hospital, Phoenix, AZ, United States, ${ }^{3}$ Philips Healthcare, Gainesville, FL, United States, ${ }^{4}$ Barrow Neurological Institute, Phoenix, AZ, United States, ${ }^{5}$ Department of Radiology, Cincinnati Children's Hospital Medical Center, Cincinnati, $\mathrm{OH}$, United States

MRI of lungs is inherently challenging due to the short $\mathrm{T}_{2}{ }^{*}$ and intrinsic motion from the respiratory and cardiac cycles. Ultrashort echo-time (UTE) sequences are often implemented for their shorter echo times and relative insensitivity to motion. Spiral UTE sequences have been touted recently as having greater k-space sampling efficiencies than radial UTE, but few are designed well for the shorter $\mathrm{T}_{2}{ }^{*}$ of lung. In this study, FLORET (Fermat looped, orthogonally encoded trajectories), a recently-developed spiral 3D UTE sequence, was implemented in human lungs for the first time and outperformed traditional radial UTE for imaging of lung tissue.

The Impact of Inspiration Levels on the Repeatability of Quantitative Pulmonary Perfusion DCE-MRI in Patients with Chronic Obstructive Pulmonary Disease and Cystic Fibrosis

Marilisa Schiwek ${ }^{1,2}$, Frank Risse ${ }^{1}$, Simon M. F. Triphan ${ }^{2,3}$, Monika Eichinger ${ }^{2,3,4}$, Sabine Wege $^{5}$, Mirjam Stahl ${ }^{3,6}$, Olaf Sommerburg ${ }^{3,6}$, Marcus A. Mall ${ }^{3,6,7}$, Hans-Ulrich Kauczor $^{2,3,4}$, Michael U. Puderbach ${ }^{2,3,4,8}$, Ralf Eberhardt ${ }^{5}$, Claus P. Heussel ${ }^{2,3,4}$, Gudula Heussel ${ }^{2,3,4}$, and Mark O. Wielpütz ${ }^{2,3,4}$

${ }^{1}$ Transl. Medicine + Clin. Pharmacology, Boehringer Ingelheim Pharma GmbH \& Co. KG, Biberach an der Riss, Germany, ${ }^{2}$ Diagnostic and Interventional Radiology, University Hospital of Heidelberg, Heidelberg, Germany, ${ }^{3}$ Translational Lung Research Center Heidelberg (TLRC), German Lung Research Center (DZL), Heidelberg, Germany, ${ }^{4}$ Diagnostic and Interventional Radiology with Nuclear Medicine, Thoraxklinik at the University Hospital of Heidelberg, Heidelberg, Germany, ${ }^{5}$ Pulmonology and Respiratory Medicine, Thoraxklinik at the University Hospital of Heidelberg, Heidelberg, Germany, ${ }^{6}$ Pediatric Pulmonology \& Allergy and Cystic Fibrosis Center, Pediatrics, University of Heidelberg, Heidelberg, Germany, ${ }^{7}$ Translational Pulmonology, University Hospital Heidelberg, Heidelberg, Germany, ${ }^{8}$ Diagnostic and Interventional Radiology, Hufeland Hospital, Bad Langensalza, Germany

The objective of this study was to investigate the 4-week repeatability of contrast-agent based pulmonary perfusion quantification in clinically stable patients with COPD and CF. Software including fully automated lung segmentation was used to determine pulmonary blood flow (PBF). While a good agreement of PBF was found in the majority of patients, high variabilities were found. Several influence factors were considered as explanations. Differences in SNR due to different inspiratory levels are likely to influence whether quantification in each voxel succeeds. Thus, it may be necessary to modify voxel-based quantification to compensate for differences in inspiratory levels and low SNR.

Magnetic Resonance Imaging of Pulmonary Nodules

Chi Wan Koo ${ }^{1}$, Aiming Lu ${ }^{1}$, Edwin A Takahashi ${ }^{1}$, Jessica Magnuson ${ }^{1}$, Peter D Kollasch ${ }^{2}$, Jennifer R Geske ${ }^{3}$, Julie An ${ }^{4}$, Dennis Wigle ${ }^{5}$, and Tobias Peikert ${ }^{6}$

${ }^{1}$ Radiology, Mayo Clinic, Rochester, MN, United States, ${ }^{2}$ Siemens Medical Solution USA, Inc, Minneapolis, MN, United States, ${ }^{3}$ Biomedical Statistics and Informatics, Mayo Clinic, Rochester, MN, United States, ${ }^{4}$ Northeast Ohio Medical University, Rootstown, OH, United States, ${ }^{5}$ Thoracic Surgery, Mayo Clinic, Rochester, MN, United States, ${ }^{6}$ Pulmonary and Critical Care Medicine, Mayo Clinic, Rochester, MN, United States

Magnetic resonance imaging had been explored as a potential alternative to computed tomography but the majority of prior MRI nodule studies was performed with 1.5-T scanners and not with the most up to date sequences. Our study demonstrated that biomarkers derived from state of the art $3 \mathrm{~T}$ MRI sequences can distinguish benign from malignant pulmonary nodules and correlate with morphologic and physiologic values derived from commonly used noninvasive imaging modalities.

Pulmonary Perfusion MR Imaging with Ultra-Short TE: Comparison of Capability for Regional Perfusion Assessment and Postoperative Lung Function Prediction with Perfusion SPECT and/ or Conventional CT Methods

Yoshiharu Ohno ${ }^{1,2}$, Masao Yui ${ }^{3}$, Yu Chen ${ }^{4}$, Yuji Kishida ${ }^{5}$, Shinichiro Seki ${ }^{1,2}$, Katsusuke Kyotani ${ }^{6}$, and Takeshi Yoshikawa ${ }^{1,2}$ 
${ }^{1}$ Division of Functional and Diagnostic Imaging Research, Department of Radiology, Kobe University Graduate School of Medicine, Kobe, Japan, ${ }^{2}$ Advanced Biomedical Imaging Research Center, Kobe University Graduate School of Medicine, Kobe, Japan, ${ }^{3}$ Toshiba Medical Systems Corporation, Otawara, Japan, ${ }^{4}$ Toshiba Medical Systems (China) Co., Ltd., Beijin, China, ${ }^{5}$ Division of Radiology, Department of Radiology, Kobe University Graduate School of Medicine, Kobe, Japan, ${ }^{6}$ Center for Radiology and Radiation Oncology, Kobe University Hospital, Kobe, Japan

Gadolinium-based blood volume (Gd-based BV) map generated between unenhanced and contrast-enhanced UTE-MRIs may have a potential for regional perfusion assessment like lung perfused BV map on dual-energy CT in patients with pulmonary diseases. We hypothesized that Gd-based BV map has a potential to regional perfusion assessment and postoperative lung function prediction as well as perfusion SPECT and/ or conventional CT methods in NSCLC patients. The purpose of this study was to directly compare the capability of Gd-based BV map for regional perfusion assessment and/ or postoperative lung function prediction in NSCLC patients with perfusion SPECT and conventional CT methods.

Differentiation of Malignant and Benign Pulmonary Lesions with DCE-MR imaging

Xin Sui ${ }^{1}$, Xiaoli Xu ${ }^{1}$, Lan Song ${ }^{1}$, Tianyi Qian ${ }^{2}$, Yi Sun ${ }^{3}$, Wei Song ${ }^{1}$, and Zhengyu Jin ${ }^{1}$

${ }^{1}$ Radiology, Peking Union Medical College Hospital, Beijing, China, ${ }^{2}$ Siemens Healthcare, MR Collaborations NE Asia, Beijing, China, ${ }^{3}$ Siemens Healthcare, MR Collaborations NE Asia, Shanghai, China

The aim of this study was to estimate the diagnostic accuracy of DCE-MR in the differential diagnosis between malignant and benign pulmonary lesions. Thirty patients with suspected lung cancer were recruited. 13 malignancies were proved by pathology. The DCE-MR data was acquired with the TWIST-VIBE technique, and quantitative parameters (Ktrans, Kep, and Ve) were calculated by the Tofts model. Our results demonstrated that malignant lesions had significant higher Ktrans and kep values than benign lesions. The Ktrans and Kep derived from DCE-MR are promising quantification parameters for differentiating lung lesions.

Pre-treatment DCE MRI predicts overall survival in patients with primary lung cancer

Wei Wu ${ }^{1}$, Daniel S Hippe ${ }^{2}$, Nina A Mayr ${ }^{3}$, William Yuh ${ }^{2}$, Liming Xia ${ }^{1}$, and Stephen R Bowen ${ }^{3}$

${ }^{1}$ Radiology, Tongji Medical college affiliated to Huazhong University of Science and Technology, Wuhan, China, ${ }^{2}$ Radiology, University of Washington, Seattle, WA, United States,

${ }^{3}$ Radiation Oncology, University of Washington, Seattle, WA, United States

We tested whether pre-treatment standard DCE MRI imaging and clinical features can predict overall survival (OS) of 37 patients with primary lung cancer. Primary tumor volume (hazard ratio $[\mathrm{HR}]=3.19$ per $1-\mathrm{SD}$ increase, $\mathrm{P}=0.001$ ) and minimum intensity of the peak enhancement phase on $\mathrm{DCE} M R I(H R=0.45, \mathrm{P}=0.012)$ were significant predictors of $\mathrm{OS}$ on univariate Cox regression analysis. Univariate primary tumor volume model (c-index $=0.76, P=0.002)$ and multivariate LASSO Cox models based on DCE MRI features ( $C$-index $=0.69$, $\mathrm{P}=0.046)$ were positive predictors for OS with no statistically significant difference in performance $(\mathrm{P}=0.36)$.

Machine learning of DCE MRI intensity histogram radiomic features for pulmonary lesion classification

Wei Wu ${ }^{1}$, Chunyan Duan ${ }^{2}$, Nina A Mayr ${ }^{2}$, William T Yuh ${ }^{3}$, Liming $\mathrm{Xia}^{1}$, Daniel S Hippe ${ }^{3}$, and Stephen R Bowen ${ }^{2}$

${ }^{1}$ Radiology, Tongji Medical college affiliated to Huazhong University of Science and Technology, Wuhan, China, ${ }^{2}$ Radiation Oncology, University of Washington, Seattle, WA, United States, ${ }^{3}$ Radiology, University of Washington, Seattle, WA, United States

To classify malignant/benign lesions can be challenging and non-invasive means to further improve the diagnostic accuracy would have major impact on management in patients with pulmonary lesions. 62 patients with histologically confirmed pulmonary lesions were retrospectively reviewed. Intensity voxel histogram (IH) features were extracted from DCE-MRI. The efficacy of IH features to classify pulmonary lesions were assessed by correlation with pathology. Under cross-validation, a support vector machine algorithm achieved a diagnostic accuracy, sensitivity and specificity of $95 \%, 99$ and $86 \%$. Our results demonstrate that machine learning of DCE-MRI IH features has potential for accurately classifying pulmonary lesions for clinical translation.

Temporal and spatial evaluation of pulmonary blood flow using multiple delay PCASL at 1.5 Tesla

Ferdinand Seith ${ }^{1}$, Rolf Pohmann ${ }^{2}$, Martin Schwartz ${ }^{3,4}$, Thomas Küstner ${ }^{3,4}$, Klaus Scheffler $^{2,5}$, Konstantin Nikolaou $^{1}$, Fritz Schick ${ }^{3}$, and Petros Martirosian ${ }^{3}$

${ }^{1}$ Department of Diagnostic and Interventional Radiology, University of Tübingen, Tübingen, Germany, ${ }^{2}$ Max Planck Institute for Biological Cybernetics, Tübingen, Germany, ${ }^{3}$ Section on Experimental Radiology, University of Tübingen, Tübingen, Germany, ${ }^{4}$ Institute of Signal Processing and System Theory, University of Stuttgart, Stuttgart, Germany, ${ }^{5}$ Department of Biomedical Magnetic Resonance, University of Tübingen, Tübingen, Germany

Pseudo-continuous-arterial-spin-labeling (PCASL) has been successfully applied in abdominal organs to image organ perfusion. The aim of this work was to evaluate the pulmonary blood flow in dependence on the cardiac cycle using PCASL at 1.5T. Labeling of pulmonary blood flow was achieved by ECG triggering and an labeling plane perpendicular to the pulmonary trunk (tagging duration $300 \mathrm{~ms}$ ). In five volunteers, eight measurements were acquired with fast True-FISP imaging (in-plane-resolution, $2.5 \times 2.5 \mathrm{~mm}{ }^{2}$, coronal view) with postlabeling delays between 100 and $1500 \mathrm{~ms}$. The PCASL-True-FISP technique was able to precisely assess blood flow of pulmonary arteries, as well as perfusion of the lung parenchyma. 
GRE bSSFP vs. FLASH based Fourier Decomposition lung MRI at 1.5T: evaluation of image quality, fractional ventilation and lung perfusion in healthy volunteers

Alexander Rotärmel ${ }^{1,2}$, Andreas Voskrebenzev ${ }^{1,2}$, Filip Klimes ${ }^{1,2}$, Marcel Gutberlet ${ }^{1,2}$, Frank Wacker ${ }^{1,2}$, and Jens Vogel-Claussen ${ }^{1,2}$

${ }^{1}$ Institute of Diagnostic and Interventional Radiology, Medical School Hannover, Hannover, Germany, ${ }^{2}$ German Center for Lung Research, Hannover, Germany

The comparison between different MRI sequences for assessment of lung ventilation and perfusion using phase-resolved functional lung MRI post-processing (PREFUL) needs further evaluation to support clinical translation. Our study compares two gradient echo (GRE) balanced steady state free precession (bSSFP) sequences (one commercially available and one modified by Bauman et al.) and one GRE Fast Low Angle Shot (FLASH) sequence regarding signal-to-noise ratio, fractional ventilation and lung perfusion. In summary, the bSSFP sequence modified by Bauman provides significantly higher SNR values and better perfusion values in the lung parenchyma compared to the commercially available bSSFP and FLASH sequences using PREFUL.

UTE-SENCEFUL: high resolution 3D ventilation weighted maps

Lenon Mendes Pereira ${ }^{1}$, Andreas M. Weng ${ }^{1}$, Tobias Wech ${ }^{1}$, Manuel Stich ${ }^{1}$, Christian Kestler ${ }^{1}$, Simon Veldhoen ${ }^{1}$, Andreas S. Kunz $^{1}$, Thorsten A. Bley ${ }^{1}$, and Herbert Köstler ${ }^{1}$

${ }^{1}$ Department of Diagnostic and Interventional Radiology, University Hospital Wurzburg, Wurzburg, Germany

In this work we present a method to assess lung ventilation in 3D by combining Self-gated Non-Contrast-enhanced Functional Lung MRI (SENCEFUL) with an ultra-short echo time (UTE) acquisition and a 3D image registration technique. Ventilation weighted maps were generated and the quantitative ventilation value for a healthy volunteer was assessed. Lung ventilation and image quality were compared between the new UTE-SENCEFUL and the standard 2D-SENCEFUL methods. UTE-SENCEFUL was able to present a 3D reconstruction of the breathing cycle, 3D ventilation weighted maps with high resolution and quantitative ventilation values in agreement with the literature.

Contributions of Large Versus Small Airways to MRI Ventilation Heterogeneity in Asthmatics

Rachel L Eddy ${ }^{1,2}$, Heather M Young ${ }^{1,2}$, Andrea Kassay ${ }^{1,2}$, Dante PI Capaldi ${ }^{1,2}$, Sarah Svenningsen ${ }^{1,3}$, David G McCormack ${ }^{4}$, and Grace Parraga ${ }^{1,2}$

${ }^{1}$ Robarts Research Institute, London, ON, Canada, ${ }^{2}$ Medical Biophysics, Western University, London, ON, Canada, ${ }^{3}$ Medicine, McMaster University, Hamilton, ON, Canada, ${ }^{4}$ Medicine, Division of Respirology, Western University, London, ON, Canada

Pulmonary functional MRI identifies the exact location of functional abnormalities within the asthmatic lung, however the relative contributions of large and small airways to ventilation heterogeneity in a given patient are unknown. Here, we differentiated hyperpolarized noble gas MRI ventilation into regions corresponding to the large and small airways using patientspecific airway trees and calculated the ventilation defect percent (VDP) related to large and small airways independently. The classification of small and large airway VDP may help with clinical treatment decisions for individualized therapies.

Assessment of the diaphragm morphology in upright seated and supine position

Christoph Arthofer ${ }^{1}$, Charlotte E Bolton ${ }^{1,2}$, Zhenghao Wang ${ }^{1,2}$, Andrew Cooper ${ }^{3}$, Andrew Peters ${ }^{3}$, Michael Barlow ${ }^{1,4}$, Dorothee Auer ${ }^{1,4}$, Richard Bowtell $^{1,3}$, Ian Hall $^{1,2}$, and Penny Gowland ${ }^{1,3}$

${ }^{1}$ National Institute for Health Research (NIHR) Nottingham Biomedical Research Centre, Nottingham, United Kingdom, ${ }^{2}$ Respiratory Medicine, University of Nottingham, Nottingham, United Kingdom, ${ }^{3}$ Sir Peter Mansfield Imaging Centre, University of Nottingham, Nottingham, United Kingdom, ${ }^{4}$ Clinical Neuroscience, University of Nottingham, Nottingham, United Kingdom

The morphology of the diaphragm is an important factor in the consideration of dyspnoea and treatment of respiratory diseases. The acquisition of images with commonly used methods is limited by the patient position or duration of the procedure. We present the first images of the diaphragm acquired in an upright MR scanner, and estimate repeatability and differences in morphology depending on posture.

Dynamic contrast-enhanced MRI in the lung - evaluation of measures of pulmonary oedema and pulmonary endothelial permeability in healthy subjects and patients with chronic heart failure

Alexandra R. Morgan ${ }^{1}$, Joseph Cheriyan²,3,4, Caleb Roberts ${ }^{5}$, Martin J. Graves ${ }^{4}$, Ilse Patterson ${ }^{4}$, Rhys A. Slough ${ }^{4}$, Rosemary Schroyer ${ }^{6}$, Disala Fernando ${ }^{2}$, Linda Henderson ${ }^{7}$, Subramanya Kumar ${ }^{2}$, Geoffrey J.M. Parker ${ }^{5,8}$, Dennis Sprecher $^{7}$, and Robert L. Janiczek ${ }^{1}$

${ }^{1}$ GSK, Stevenage, United Kingdom, ${ }^{2}$ GSK Clinical Unit, Cambridge, United Kingdom, ${ }^{3}$ Experimental Medicine \& Immunotherapeutics, University of Cambridge, Cambridge, United Kingdom, ${ }^{4}$ Cambridge University Hospitals, Cambridge, United Kingdom, ${ }^{5}$ Bioxydyn Ltd, Manchester, United Kingdom, ${ }^{6} \mathrm{GSK}$, Collegeville, PA, United States, ${ }^{7} \mathrm{GSK}$, King of Prussia, PA, United States, ${ }^{8}$ Centre for Imaging Sciences, The University of Manchester, Manchester, United Kingdom 
MRI has previously demonstrated increased lung water content in patients with heart failure (HF), but has not yet been used to distinguish between intravascular and extravascular water in these patients. This study evaluated dynamic contrast-enhanced MRI (DCE-MRI) for measuring pulmonary oedema and endothelial permeability in healthy volunteers (HV) and chronic HF patients at rest and post-exercise. DCE-MRI showed a redistribution of lung water towards the interstitial space in chronic HF, as compared to HV, suggesting this method may have value as a novel endpoint for dose-ranging and proof-of-mechanism studies in chronic HF. No exercise-induced change was seen in either group.

Optimization of Steady-state Free Precession with ${ }^{19} \mathrm{~F}$ Perfluoropropane for Increased Signal-to-Noise for Human Lung Ventilation Imaging at $3 \mathrm{~T}$

Adam Maunder ${ }^{1}$, Madhwesha Rao ${ }^{1}$, and Jim Wild ${ }^{1}$

${ }^{1}$ POLARIS, Academic Radiology, University of Sheffield, Sheffield, United Kingdom

Fluorinated gas MRI is an alternative modality to hyperpolarized gas MR for imaging lung ventilation, but is constrained by lower SNR. Improvement of the signal-to-noise ratio of human lung ventilation images with ${ }^{19} \mathrm{~F}$ the steady-state free precession (SSFP) sequence was previously explored at 1.5T. Here, we present optimization of SSFP for imaging lung ventilation at 3T. The achievable improvement of in-vivo imaging quality with realistic relaxation parameters is demonstrated with comparison against the spoiled gradient echo sequence. Limits in applying the SSFP sequence due to specific absorption ratio at $3 \mathrm{~T}$ and the dependence on $\mathrm{T}_{2}{ }^{*}$ within the lungs are detailed.

Probing changes in lung physiology in COPD using CT, perfusion MRI and hyperpolarized xenon-129 MRI

Kun Qing ${ }^{1}$, Nicholas J. Tustison ${ }^{1}$, John P. Mugler, III ${ }^{1}$, Jaime F. Mata ${ }^{1}$, Zixuan Lin ${ }^{1}$, Li Zhao ${ }^{2}$, Da Wang ${ }^{3}$, Xue Feng ${ }^{1}$, Kai Ruppert ${ }^{4}$, Talissa A. Altes ${ }^{5}$, Joanne M. Cassani ${ }^{5}$, and Y. Michael Shim 1

${ }^{1}$ University of Virginia, Charlottesville, VA, United States, ${ }^{2}$ Beth Israel Deaconess Medical Center, Boston, MA, United States, ${ }^{3}$ University of Washington, Seattle, WA, United States, ${ }^{4}$ University of Pennsylvania, Philadelphia, VA, United States, ${ }^{5}$ University of Missouri School of Medicine, Columbia, MO, United States

In this study, by using chest CT, Gadolinium-enhanced perfusion MRI, and hyperpolarized xenon-129 ventilation and gas uptake MRI, we assessed the quantitative changes in tissue density, pulmonary perfusion and gas uptake in patients with COPD compared to normal subjects. We found evidence for compensatory pulmonary vasoconstriction to match impairment of ventilation, and also pulmonary shunt and dead space. By incorporating a new lobar segmentation method for proton MRI, we performed statistical analysis to evaluate the regional interrelationships among different measures. We demonstrated that xenon-129 MRI has high potential to identify changes of multiple aspects of lung physiology in one acquisition.

Combination of Perfluoropropane and oxygen-enhanced MRI-derived washout kinetics for detection of ischemic injury to lungs in a porcine ex-vivo perfusion system

Julius Renne ${ }^{1,2}$, Marcel Gutberlet ${ }^{1,2}$, Andreas Voskrebenzev ${ }^{1,2}$, Agilo Kern ${ }^{1,2}$, Till Kaireit ${ }^{1,2}$, Jan Bernd Hinrichs ${ }^{1,2}$, Peter Braubach ${ }^{3}$, Christiane S Falk ${ }^{2,4}$, Klaus Höffler ${ }^{5}$, Gregor Warnecke $^{2,5}$, Axel Haverich ${ }^{5}$, Frank Wacker ${ }^{1}$, Jens Vogel-Claussen ${ }^{1,2}$, and Norman Zinne ${ }^{2,5}$

${ }^{1}$ Diagnostic and Interventional Radiology, Hannover Medical School, Hannover, Germany, ${ }^{2}$ Integrated Research and Treatment Center Transplantation (IfB-Tx), Hannover, Germany, ${ }^{3}$ Institute for Pathology, Hannover Medical School, Hannover, Germany, ${ }^{4}$ Institute for Transplant Immunology, Hannover Medical School, Hannover, Germany, ${ }^{5}$ Clinic for Cardiothoracic and Transplantation Surgery, Hannover Medical School, Hannover, Germany

Ex-vivo lung perfusion and ventilation systems are a promising new tool for conditioning marginal lung allografts. However, reliable biomarkers for evaluating graft function are missing. In this study MRI-derived fluorine and oxygen washout times are to be evaluated as lung function parameters in a porcine model of ischemia. Washout time for oxygen is prolonged while fluorine washout is not in lungs after warm ischemia compared to normal controls, which might reflect pulmonary edema limiting oxygen diffusion. Determination of fluorine and oxygen washout is feasible in an ex-vivo lung perfusion system and seems to be promising tools for evaluating graft function.

Mapping of Ventilation/Perfusion Ratios in the human lung using 19F MRI of Perfluoropropane

Arnd Obert ${ }^{1,2}$, Marcel Gutberlet ${ }^{1,2}$, Alexander Rotärmel $^{1,2}$, Frank Wacker $^{1,2}$, and Jens Vogel-Claussen ${ }^{1,2}$

${ }^{1}$ Institute for Diagnostic and Interventional Radiology, Hannover Medical School, Hannover, Germany, ${ }^{2}$ Biomedical Research in Endstage and Obstructive Lung Disease Hannover (BREATH), German Center for Lung Research (DZL), Hannover, Germany

In this work, the correlation between longitudinal relaxation time $\left(\mathrm{T}_{1}\right)$, alveolar partial pressure and ventilation-perfusion ratio (V/Q) of an inhaled fluorinated gas is used to compute quantitative V/Q maps of the human lung. The trapping of inert Perfluoropropane $\left(\mathrm{C}_{3} \mathrm{~F}_{8}\right)$ in poorly ventilated regions of the lung (low V/Q) leads to an increase of its alveolar partial pressure which is detectable as an increase of $\mathrm{T}_{1}$ in ${ }^{19} \mathrm{~F}$ MR Imaging. Here, V/Q maps of three patients with Chronic Obstructed Pulmonary Disease (COPD) were calculated and compared to a V/Q map of a healthy volunteer. 
MRI of inhaled perfluoropropane offers a safely repeatable modality for mapping pulmonary ventilation. However, as a thermally polarised gas, signal is scarce and acquisitions are limited to breath hold durations or require respiratory gating. Improving the temporal resolution would present the opportunity to implement dynamic imaging or improve image quality in breath hold acquisitions. In this study, the acquisition time was reduced by partially sampling k-space using a compressed sensing technique. A 3-fold decrease in acquisition time was achieved whilst maintaining visually similar image quality. An average SNR of $25: 1$ was measured in a $6 s$ D acquisition in healthy volunteers.

Microporous Lung Phantoms for 19F-MRI of Inhaled Imaging Agents with Physiologically Representative Relaxation Times

Mary Neal ${ }^{1}$, Helena Sexton ${ }^{1}$, Eric Hughes ${ }^{1}$, and Pete Thelwall ${ }^{1}$

${ }^{1}$ Newcastle Magnetic Resonance Centre, Newcastle University, Newcastle upon Tyne, United Kingdom

A primary characteristic of ${ }^{19} \mathrm{~F}-\mathrm{MRI}$ of pulmonary ventilation is the short in vivo $\mathrm{T}_{2}{ }^{*}$ of the inhaled imaging agent caused by the inhomogeneous magnetic environment proximal to the alveolar walls. This study describes two novel methods for fabrication of phantoms that mimic the physical and magnetic properties of alveolar tissue. In both cases the perfluorinated gas phase imaging agent is suspended in a stable microporous foam medium. The fabrication techniques permitted precise control of either bubble size or gas/liquid ratio. Highly monodisperse stable foams were formed with a perfluoropropane $\mathrm{T}_{2}{ }^{*}$ of $2 \mathrm{~ms}$, comparable to that measured in the human lung.

Assessment of ventilation heterogeneity using hyperpolarized gas MRI histogram analysis

Paul J.C. Hughes ${ }^{1}$, Laurie Smith ${ }^{1,2}$, Felix Horn ${ }^{1}$, Alberto M. Biancardi ${ }^{1}$, Neil Stewart ${ }^{1}$, Graham Norquay ${ }^{1}$, Madhwesha Rao ${ }^{1}$, Ina Aldag ${ }^{2}$, Chris Taylor ${ }^{2}$, Helen Marshall ${ }^{1}$, Guilhem Collier ${ }^{1}$, and Jim M. Wild ${ }^{1}$

${ }^{1}$ POLARIS, Academic Unit of Radiology, University of Sheffield, Sheffield, United Kingdom, ${ }^{2}$ Sheffield Children's Hospital, Sheffield Children's NHS Foundation Trust; and Sheffield Teaching Hospitals NHS Foundation Trust, Sheffield, United Kingdom

Development of sensitive imaging biomarkers to differentiate health from disease is an important research topic in pulmonary MRI. This work aimed to make use of the rich spatial and signal intensity information in hyperpolarized gas MR ventilation images to determine metrics of ventilation heterogeneity. Retrospective analysis was performed on ${ }^{3} \mathrm{He}$ ventilation images acquired from healthy volunteers and patients with cystic fibrosis, asthma and chronic obstructive pulmonary disease.

SNR and Dose Requirements for Quantitative 6-Zone Analysis of Hyperpolarized (129)Xe Ventilation MRI

Fei $\operatorname{Tan}^{1}$, Mu He${ }^{2}$, Leith Rankine ${ }^{3}$, Rohan S. Virgincar ${ }^{1}$, John C. Nouls ${ }^{4}$, Steven Shipes ${ }^{4}$, and Bastiaan Driehuys $s^{1,3,4}$

${ }^{1}$ Department of Biomedical Engineering, Duke University, Durham, NC, United States, ${ }^{2}$ Department of Electrical and Computer Engineering, Duke University, Durham, NC, United States, ${ }^{3}$ Department of Medical Physics, Duke University, Durham, NC, United States, ${ }^{4}$ Department of Radiology, Duke University, Durham, NC, United States

Hyperpolarized (HP) ${ }^{129}$ Xe ventilation MRI can be used for non-invasive assessment of lung obstruction. However, the minimum ${ }^{129}$ Xe dose to obtain HP ${ }^{129}$ Xe ventilation MRI with sufficient signal-to-noise ratio (SNR) for reliable quantitative analysis has not yet been established. In this work, we introduced the reader-based six-zone analysis, which is used with ${ }^{133} \mathrm{Xe}$ and ${ }^{99 \mathrm{~m}} \mathrm{Tc}$ ventilation and perfusion scintigraphy, and applied to Rician noise degraded ${ }^{129} \mathrm{Xe}$ ventilation MRI of COPD patients. We found that the minimum required SNR for 6 zone quantification of ventilation is $4.4 \pm 5.8$ (mean $\pm S D$ ), which suggests a minimum required ${ }^{129}$ Xe dose equivalent of $89.2 \mathrm{ml}$ for this resolution.

Hyperpolarized 129Xe gas and ultra-short echo MRI for evaluation of structure-function correlates in cystic fibrosis lung disease: a comparison of analysis methods

Robert Thomen ${ }^{1}$, Laura Walkup ${ }^{2}$, David Roach ${ }^{2}$, Nara Higano ${ }^{2}$, Zackary Cleveland ${ }^{2}$, Andrew Schapiro ${ }^{3}$, Alan Brody ${ }^{3}$, John P Clancy ${ }^{4}$, and Jason Woods ${ }^{2}$

${ }^{1}$ Radiology and BioEngineering, University of Missouri, Columbia, MO, United States, ${ }^{2}$ Center for Pulmonary Imaging Research, Cincinnati Children's Hospital, Cincinnati, OH, United States, ${ }^{3}$ Radiology, Cincinnati Children's Hospital, Cincinnati, $\mathrm{OH}$, United States, ${ }^{4}$ Pulmonary Medicine, Cincinnati Children's Hospital, Cincinnati, OH, United States

A number of techniques for analysis of hyperpolarized gas (HPG) images have emerged and demonstrated sensitivity to lung disease severity. However, the precise extent of lung function decline due to specific pathologies associated with obstructive lung disease has not been established. Here we have performed HPG ${ }^{129}$ Xe analysis using 3 common methods from the literature (mean-anchored, percentile-anchored, and k-means methods) in order to evaluate correlations with structural pathologies identified in ultra-short echo-time (UTE) images. The presence of bronchiectasis and mucus plugging correlated best with whole-lung ventilation defect percentage (VDP). Consolidation and air-trapping demonstrated weaker (though still significant) correlation with VDP. 
${ }^{1}$ Physics and Astronomy, University of North Carolina at Chapel Hill, Chapel Hill, NC, United States, ${ }^{2}$ Biomedical Research Imaging Center, University of North Carolina at Chapel Hill, Chapel Hill, NC, United States, ${ }^{3}$ Applied Physical Sciences, University of North Carolina at Chapel Hill, Chapel Hill, NC, United States

Dissolved-phase ${ }^{129} \mathrm{Xe}$ (DPXe) chemical shift (CS) measurements could benefit from a robust reference system that can provide consistent CS values independently of gas partial pressures, lung inflation, subject position, and shimming conditions. We demonstrate that, by referencing the DPXe frequency to that of nearby protons, consistent CS values can be obtained, both in vitro and in vivo, enabling correct assignment of some of the spectral lines observed in vivo.

Quantifying Regional Lung Function in Interstitial Lung Disease with Hyperpolarized Xenon-129 3D SB-CSI

Mackenzie Carlson ${ }^{1}$, Borna Mehrad ${ }^{2}$, Yun Shim ${ }^{1}$, Nicholas Tustison ${ }^{1}$, John Mugler ${ }^{1}$, Talissa Altes ${ }^{1,3}$, Lucia Flors ${ }^{3}$, Grady Miller ${ }^{1}$, and Jaime Mata ${ }^{1}$

${ }^{1}$ University of Virginia, Charlottesville, VA, United States, ${ }^{2}$ University of Florida, Gainesville, FL, United States, ${ }^{3}$ University of Missouri, Columbia, MO, United States

In this study, lung ventilation and gas uptake/exchange was assessed in healthy and interstitial lung disease (ILD) subject populations using 3D Single-Breath Chemical Shift Imaging, a combination of MR spectroscopic imaging and hyperpolarized xenon-129 gas imaging. By probing metrics such as Tissue/RBC, Tissue/Gas, RBC/Gas, T2* and chemical shifts in lung parenchyma and red blood cells, we find statistically significant distinctions in the lung physiology between healthy and ILD subjects.

Traditional Poster

\section{Pancreas/GI}

Tumor necrosis factor (TNF) antagonist therapy in small bowel Crohn's disease (CD): association of the apparent diffusion coefficient (ADC) with treatment response.

Bradley Spieler ${ }^{1}$, Hector De Jesus ${ }^{1}$, Christopher Rouse ${ }^{1}$, Catherine Hudson ${ }^{2}$, Scott Kleinpeter ${ }^{3}$, Catherine Batte ${ }^{4}$, Raman Danrad ${ }^{1}$, and Kara De Felice ${ }^{5}$

${ }^{1}$ Radiology, LSUHSC New Orleans, New Orleans, LA, United States, ${ }^{2}$ Internal Medicine, LSUHSC New Orleans, New Orleans, LA, United States, ${ }^{3}$ School of Medicine, LSUHSC New Orleans, New Orleans, LA, United States, ${ }^{4}$ LSU, Baton Rouge, LA, United States, ${ }^{5}$ Gastroenterology, LSUHSC New Orleans, New Orleans, LA, United States

Diffusion weighted imaging (DWI) has proven beneficial in the assessment of disease activity and therapeutic response in a myriad of pathology. Studies have shown an inversely proportional correlation between bowel inflammation in Crohn's disease (CD) and apparent diffusion coefficient (ADC) values of involved bowel wall. This beckons an intriguing opportunity for gauging treatment response, particularly with respect to some of the most commonly used agents, tumor necrosis factor (TNF) antagonists. This study retrospectively measured the ADC value of affected small bowel segments before and after anti-TNF infusion therapy and compares it to the clinical response in patients with active CD.

Semi-automatic method for generating multiplanar reformatting views of MR post-contrast T1-weighted images for visualizing and assessing pediatric Crohn's disease

Yechiel Lamash ${ }^{1}$, Sila Kurugol ${ }^{1}$, Moti Freiman ${ }^{1}$, and Simon K Warfield ${ }^{1}$

${ }^{1}$ Radiology, Boston Childrens Hospital and Harvard Medical School, Boston, MA, United States

In this proposed study, we aim to develop a semi-automated method for generating multiplanar reformatting images (MPR) of pediatric Crohn's disease (pCD) segments from T1weighted post-contrast MR image data. We demonstrate that this method can efficiently visualize and assess this disease. Importantly, the centerline length can be used as a reliable measure of the extent of disease. Moreover, the MPR image can be used as a platform for intestinal wall segmentation and for more accurate depiction of luminal narrowing. We also expect such MPR views to be used as a unified parametric platform for evaluating disease progression in follow-up scans.

MRI assessed small bowel dysmotility and its relationship with patient reported symptoms: An exploration of automated vs subjective assessment techniques

Ruaridh Malcolm Gollifer ${ }^{1}$, Alex Menys ${ }^{1}$, Andrew Plumb ${ }^{1}$, Frans Vos $^{2,3}$, Jaap Stoker ${ }^{2}$, Stuart A Taylor ${ }^{1}$, and David Atkinson ${ }^{1}$

${ }^{1}$ Centre for Medical Imaging, University College London (UCL), London, United Kingdom, ${ }^{2}$ Radiology and Nuclear Medicine, Academic Medical Center (AMC), Amsterdam, Netherlands,

${ }^{3}$ Quantitative Imaging Group, Delft University of Technology, Delft, Netherlands

The pathophysiology of chronic abdominal symptoms in Crohn's disease (CD) is complex. Recent pilot data using automated quantification of motility MRI suggests reduced variation in apparently normal bowel may underpin symptoms, including pain and diarrhoea. This two-centre validation study tests this association and compares automated measurements with subjective radiologist bowel motility assessment. We confirmed that reduced spatial variation of motility is significantly associated with the severity of abdominal symptoms, although the correlation was not strong. Automated measurement had superior inter-reader variability than subjective radiologist assessment, and showed a stronger association with patient symptoms. 
The workflow for the validation of USPIO-enhanced MRI for the detection of lymph node metastases in rectal cancer

Rutger C.H. Stijns ${ }^{1}$, Bart W.J. Philips ${ }^{1}$, Chella van der Post ${ }^{2}$, Iris D. Nagtegaal ${ }^{2}$, Carla Wauters ${ }^{3}$, Luc J.A. Strobbe ${ }^{4}$, Fatih Polat ${ }^{4}$, Johannes H.W. de Wilt ${ }^{5}$, Stefan H.G. Rietsch ${ }^{6,7}$, Sascha Brunheim $^{6,7}$, Stephan Orzada ${ }^{6}$, Harald H. Quick ${ }^{6,7}$, Jurgen F. Fütterer ${ }^{1}$, and Tom W.J. Scheenen ${ }^{1,6}$

${ }^{1}$ Radiology and Nuclear medicine, Radboudumc, Nijmegen, Netherlands, ${ }^{2}$ Pathology, Radboudumc, Nijmegen, Netherlands, ${ }^{3}$ Pathology, Cansius Wilhelmina hospital, Nijmegen, Netherlands, ${ }^{4}$ Surgery, Cansius Wilhelmina hospital, Nijmegen, Netherlands, ${ }^{5}$ Surgery, Radboudumc, Nijmegen, Netherlands, ${ }^{6}$ Erwin L. Hahn Institute for MR Imaging, University of Duisburg-Essen, Essen, Germany, ${ }^{7}$ High Field and Hybrid MR Imaging, University Hospital Essen, Essen, Germany

For patients with rectal cancer, the presence of lymph node metastases is an important risk factor for determining prognosis and stratifying for treatment. Clinically, lymph node staging is very challenging, especially when lymph nodes are small $(<5 \mathrm{~mm})$. By using ultrasmall superparamagnetic iron oxide (USPIO) particles combined with (ultra) high magnetic field imaging (combidex-enhanced MRI), the detection rate of these metastatic lymph nodes may improve significantly. In this abstract we present the workflow for validating combidex-enhanced MRI by performing a node to node comparison of in vivo combidex-enhanced MRI findings with histopathological examination.

Quantitative assessment of pancreatic proton density fat fraction (PDFF) and R2* with preoperative T2* corrected multi-echo chemical-shift-encoded MRI in patients undergoing pancreatic resection: comparison with single-voxel $1 \mathrm{H}-\mathrm{MRS}$

Yali Qu ${ }^{1}$, Mou Li ${ }^{1}$, Zhen Zhang ${ }^{1}$, Zixing Huang ${ }^{1}$, Chunchao Xia ${ }^{1}$, and Bin Song ${ }^{1}$

$2485 \quad{ }^{1}$ Radiology, West China Hospital, Sichuan University, Chengdu, China

Many studies have shown multi-echo chemical-shift-encoded magnetic resonance imaging (CSE-MRI) has good performance for the evaluation of fat and iron in liver. However, the relevant studies in pancreas are fewer. We found that pancreatic PDFF and $\mathrm{R}^{*}{ }^{*}$ estimated by $\mathrm{T}^{*}$ corrected multi-echo CSE-MRI showed a moderate correlation with ${ }^{1} \mathrm{H}-\mathrm{MRS}$ results in patients undergoing pancreatic resection. In addition, our study showed that pancreatic PDFF was not to be significantly associated with clinically relevant postoperative pancreatic fistula.

The value of IDEAL-IQ in evaluating pancreatic fat quantification in patients with non-alcoholic fatty liver disease(NAFLD)

Qinhe Zhang ${ }^{1}$, Ailian Liu ${ }^{1}$, and Lizhi Xie ${ }^{2}$

${ }^{1}$ Department of Radiology, the First Affiliated Hospital of Dalian Medical University, Dalian, China, ${ }^{2}$ Department of Radiology, the First Affiliated Hospital of Dalian Medical University, Beijing, China

The study aims to assess the pancreatic fatty quantitation in NAFLD by use of IDEAL-IQ. It was concluded that IDEAL-IQ is a new way to evaluate the pancreatic fat quantification in patients with NAFLD. The fat fraction of the pancreas in patients with NAFLD is significantly higher than that in normal subjects, and the distribution of pancreatic fat in various regions of the pancreas in the NAFLD patients is well.

Quantitation of metabolites in human tumour (paraganglioma and GIST) tissues with mitochondrial mutations (SDH and IDH1) by HRMAS 1H NMR spectroscopy

Basetti Madhu ${ }^{1}$, Ruth T Casey ${ }^{2,3}$, Benjamin G Challis ${ }^{3}$, Graeme R Clark ${ }^{2}$, Alison Marker ${ }^{4}$, Olivier Giger ${ }^{4}$, Venkata R Bulusu ${ }^{5}$, Mary A McLean ${ }^{1}$, Ferdia A Gallagher ${ }^{6}$, and Eamonn R Maher $^{2}$

${ }^{1}$ Imaging Core, Cancer Research UK Cambridge Institute, Cambridge, United Kingdom, ${ }^{2}$ Department of Medical Genetics, University of Cambridge, Cambridge, United Kingdom, ${ }^{3}$ Department of Endocrinology, Cambridge University Hospital NHS Foundation Trust, Cambridge, United Kingdom, ${ }^{4}$ Department of Histopathology, Cambridge University Hospital NHS Foundation Trust, Cambridge, United Kingdom, ${ }^{5}$ Department of Medical Oncology, Cambridge University Hospital NHS Foundation Trust, Cambridge, United Kingdom, ${ }^{6}$ Department of Radiology, Cambridge University NHS Foundation Trust, Cambridge, United Kingdom

In this study we report, for the first time, the detection of $2 \mathrm{HG}$ in IDH1 mutated human GIST tumour tissues by HRMAS ${ }^{1} \mathrm{H}$ NMR spectroscopy. We quantified the levels of Succinate and $2 \mathrm{HG}$ in human paraganglioma and GIST tissues. The lactate, glutamate and glycero-phosphocholine (GPC) concentrations were significantly lower in SDHx mutated tumours compared to wild type (WT) tumour tissues, Detection of higher levels of Succinate in SDH mutated tumour tissue and 2HG in IDH1 mutated tissue and their quantitation will be helpful in the stratification of patient treatment in the clinics.

Assessment of Colonic Motility Using Magnetic Resonance Imaging: Reproducibility of a Macrogol Challenge

Victoria Wilkinson-Smith ${ }^{1,2}$, Alex Menys ${ }^{3}$, Christopher Bradley ${ }^{2,4}$, Maura Corsetti ${ }^{1,2}$, Luca Marciani $^{1}{ }^{12}$, David Atkinson ${ }^{5}$, Carol Coupland ${ }^{6}$, Stuart Taylor ${ }^{5}$, Penny Gowland ${ }^{4}$, Robin Spiller ${ }^{1,2}$, and Caroline Hoad ${ }^{2,4}$ 
${ }^{1}$ Nottingham Digestive Diseases Centre, University of Nottingham, Nottingham, United Kingdom, ${ }^{2}$ National Institute for Health Research (NIHR) Nottingham Biomedical Research Centre, Nottingham University Hospitals NHS Trust and University of Nottingham, Nottingham, United Kingdom, ${ }^{3}$ Motilent Ltd, London, United Kingdom, ${ }^{4}$ Sir Peter Mansfield Imaging Centre, University of Nottingham, Nottingham, United Kingdom, ${ }^{5}$ Centre for Medical Imaging, University College London, London, United Kingdom, ${ }^{6}$ Division of Primary Care, University of Nottingham, Nottingham, United Kingdom

This study assessed the reproducibility of a previously developed diagnostic test using a macrogol stimulus and MRI measures to assess colonic motility. This test was performed twice on healthy volunteers and the results were compared. The data showed some variability across visits representing both variability in baseline data and the physiological response of the colon to the stimulus. Correlation data suggested that although intra-subject variability existed the maximum measured MRI parameters all increased post stimulus. This colonic stimulus test allows us greater insight into potential pathologies behind $\mathrm{Gl}$ disorders and as such may be of value here.

Case report: Three-dimensional visualization of the normal human perirectal muscle with diffusion tensor imaging (DTI)

Koji Tokunaga ${ }^{1}$, Shigeki Arizono ${ }^{1}$, Koji Fujimoto² ${ }^{2}$ Tomoaki Okada $^{3}$, Katsutoshi Murata ${ }^{4}$, Hiroyoshi Isoda ${ }^{1}$, and Kaori Togashi ${ }^{1}$

${ }^{1}$ Department of Diagnostic Imaging and Nuclear Medicine, Kyoto University Graduate School of Medicine, Kyoto, Japan, ${ }^{2}$ Human Brain Research Center, Kyoto University Graduate School of Medicine, Kyoto, Japan, ${ }^{3}$ Division of Gastrointestinal Surgery, Department of Surgery, Kyoto University Graduate School of Medicine, Kyoto, Japan, ${ }^{4}$ Siemens Healthcare K. K., Tokyo, Japan

Diffusion tensor imaging (DTI) can provide the directionality of water diffusion in tissues, informing on its underlying microstructures and microdynamics. There has been no previous report on the visualization of anterior portion of the longitudinal anal muscle (aLAM). In this case study, we present the 3D visualization of the aLAM in normal male subjects with DTI. By adjusted parameters for DTI sequence, we could successfully visualize thin smooth muscle layer of the rectum. This technique could be useful when planning operation for rectal and anal diseases.

Traditional Poster

\section{Body Imaging: Renal}

Characterization of Renal Solid Masses Using Multiparametric Diffusion-Weighted Imaging

Jianjian Zhang ${ }^{1}$, Guangyu $\mathrm{Wu}^{1}$, and Yongming $\mathrm{Dai}^{2}$

${ }^{1}$ Renji Hospital, School of Medicine, Shanghai Jiao Tong University, Shanghai, China, ${ }^{2}$ United Imaging Healthcare, Shanghai, China

Preoperative characterization of the renal lesions has clinical significance in determining the appropriate treatment strategy and evaluating prognosis. The current study aims to investigate the potential of multiparametric DWI models, including monoexponential, biexponential, stretched-exponential, and kurtosis models in distinguishing between benign and malignant renal lesions, different tumor types as well as different grading of RCC. Compared with monoexpontial model, these highly parameterized non-Gaussian diffusion models may provide more information in the characterization of renal lesions, which would be helpful in improving therapy strategies and prognoses in the future, and further evaluation are required.

Intravoxel incoherent motion-diffusion weighted imaging (IVIM-DWI) parameters distinguish kidney allografts with delayed graft function

Eyesha Hashim ${ }^{1}$, Darren Yuen ${ }^{2,3}$, General Leung ${ }^{1,4}$, and Anish Kirpalani ${ }^{1,4}$

${ }^{1}$ Medical Imaging, St. Michael's Hospital, Toronto, ON, Canada, ${ }^{2}$ Nephrology, St. Michael's Hospital, Toronto, ON, Canada, ${ }^{3}$ Keenan Research Centre for Biomedical Science, St. Michael's Hospital, Toronto, ON, Canada, ${ }^{4}$ Li Ka Shing Knowledge Institute, St. Michael's Hospital, Toronto, ON, Canada

Delayed graft function (DGF) complicates $21-36 \%$ of all deceased donor kidney transplants, and leads to early inpatient post-transplant dialysis, higher risk of graft failure and death. In this abstract, we show that IVIM-derived flow (f)-fraction, is significantly different in kidney allografts exhibiting DGF compared to those that do not develop DGF. Furthermore, f fraction shows a significant negative correlation with time to recovery and a positive trend with renal function at 3 months post-transplant as measured with eGFR.

Compensating for Bulk Motion in Feed and Wrap Renal Dynamic Radial VIBE DCE-MRI using Bulk Motion Removal and Non-Rigid Registration

Sila Kurugol ${ }^{1}$, Onur Afacan ${ }^{1}$, Catherine Seager ${ }^{1}$, Richard S Lee ${ }^{1}$, Jeanne S Chow ${ }^{1}$, and Simon K. Warfield ${ }^{1}$ 
Dynamic Radial VIBE DCE-MRI enables motion-robust imaging with high spatiotemporal resolution for accurate estimation of kidney function. However, in feed and wrap DCE-MRI, bulk motion during infant's sleep reduces the quality of images affected by motion and limits clinical utility of this method for imaging without sedation. This work evaluated the ability of detecting bulk motion using the center-of-k-space line, removing corrupted volumes, and compensating for motion using non-rigid registration for improved parameter estimation accuracy. Results showed that volumes affected by motion were successfully detected and removed in all patients, and the goodness-of-fit to the tracer kinetic model was improved.

A Preliminary Study of the Longitudinal Changes in a Reversible Unilateral Ureteral Obstruction Rat Model using Intravoxel Incoherent Motion and Arterial Spin Labeling Imaging Genwen $\mathrm{Hu}^{1}$, Xianyue Quan ${ }^{2}$, Jianmin $\mathrm{Xu}^{1}$, Liangping Luo ${ }^{3}$, Yingjie $\mathrm{Mei}^{4}$, and Siying Wang ${ }^{5}$

${ }^{1}$ Department of Radiology, The Second Clinical Medical College of Jinan University, Shenzhen People's Hospital, Shenzhen, China, ${ }^{2}$ Zhujiang Hospital, Southern Medical University, Guangzhou, China, ${ }^{3}$ The First Affiliated Hospital of Jinan University, Guangzhou, China, ${ }^{4}$ MR Clinical Science, Philips Healthcare, Guangzhou, China, ${ }^{5}$ MR Clinical Science, Philips Healthcare, Shanghai, China

The longitudinal changes of intravoxel incoherent motion (IVIM) and arterial spin labeling (ASL) imaging in a RUUO model

Application of T1rho and T1 mapping MRI in Tracking Renal Ischemia Reperfusion Injury Process in Rats

Yangguang Yuan ${ }^{1,2}$, Jingjing Huang ${ }^{1}$, Yingjie Mei ${ }^{3}$, Siying Wang ${ }^{4}$, and Wen Liang ${ }^{1}$

${ }^{1}$ Medical Image Center, Zhujiang Hospital, Southern Medical University, Guangzhou, China, ${ }^{2}$ Radiology Department, Shenzhen Luohu People's Hospital, Shenzhen, China, ${ }^{3}$ Philips Healthcare, Guangzhou, China, ${ }^{4}$ Philips Healthcare, Shanghai, China

Previous studies using T1rho and T1 mapping in the liver and heart demonstrated that T1rho value and T1 relaxation time can be used to assess acute injury and long-term tissue fibrosis1. However, to the best of our knowledge, these techniques have not been explored to evaluate acute kidney ischemia damage. In our study, we found that T1rho value and T1 relaxation time showed high specificity and sensitivity in a rat renal ischemia reperfusion injury (IRI) model.

R1rho dispersion in human kidney

Ping Wang ${ }^{1}$ and John C. Gore ${ }^{1}$

$R_{1 \rho}\left(=1 / T_{1 \rho}\right)$ imaging has been applied in many human organs to characterize tissue biochemical changes. However, $R_{1 \rho}$ imaging in human kidney has been rarely reported partly due to the challenges associated with field inhomogeneities and respiratory motion. We developed an $\mathrm{R}_{1}$ imaging protocol for human kidney which used adiabatic half passage pulse and volume shimming to overcome field inhomogeneities. In addition, $\mathrm{R}_{1 \rho}$ dispersion was evaluated via a simple method with a fixed locking time but different locking frequencies. The volunteer scans exhibited characterized $\mathrm{R}_{1 \rho}$ maps in kidney, also there was greater $\mathrm{R}_{1 \mathrm{\rho}}$ dispersion between locking frequencies of $100 \mathrm{~Hz}$ and $300 \mathrm{~Hz}$.

\section{Body: Fat Imaging}

A Dedicated Protocol for Fat Fraction Mapping in Obese Patients: Preliminary Findings in Skeletal Muscle

Naomi S Sakai ${ }^{1}$, Timothy Bray ${ }^{1}$, Alan Bainbridge ${ }^{2}$, Rachel Batterham ${ }^{3}$, Stuart Taylor ${ }^{1}$, and Margaret Hall-Craggs ${ }^{1}$

${ }^{1}$ Centre for Medical Imaging, University College London, London, United Kingdom, ${ }^{2}$ Department of Medical Physics, University College London Hospital, London, United Kingdom,

${ }^{3}$ Centre for Obesity Research, University College London, London, United Kingdom

Obesity is associated with ectopic fat deposition and chronic inflammation in skeletal muscle (SM), which contributes to insulin resistance. Novel treatments for obesity such as bariatric surgery can reduce insulin resistance by reducing ectopic fat deposition, but this effect is inconsistent and poorly understood. Therefore, we need a fast, non-invasive method that can help to study the link between ectopic fat deposition and insulin resistance. Here, we describe a protocol for scanning obese patients, which is fast, tolerable and accurate, and reveals significant changes in SM proton density fat fraction (PDFF) in obese patients. 
Kyu-Ho Song ${ }^{1}$, Min-Young Lee ${ }^{1}$, Chi-Hyeon Yoo ${ }^{1}$, Song-I Lim ${ }^{1}$, and Bo-Young Choe ${ }^{1}$

${ }^{1}$ Department of Biomedical Engineering and Research Institute of Biomedical Engineering, College of Medicine, The Catholic University of Korea, Seoul, Republic of Korea

Magnetic resonance spectroscopy (MRS) with optimized relaxation time provides an effective means for quantifying lipid content and characterizing hepatic steatosis. The aim of this study was to quantify the difference in hepatic lipid content with metabolic changes and determine effect of diet on high-fat diet (HFD)-fed mice by measuring the main localized MRS sequence with relaxation times.

Differentiating supraclavicular from gluteal adipose tissue based on simultaneous PDFF and T2* mapping using a twenty-echo gradient echo acquisition

Daniela Franz ${ }^{1}$, Maximilian N. Diefenbach ${ }^{1}$, Jan Syväri ${ }^{1}$, Dominik Weidlich ${ }^{1}$, Ernst J. Rummeny ${ }^{1}$, Hans Hauner ${ }^{2}$, Stefan Ruschke ${ }^{1}$, and Dimitrios C. Karampinos ${ }^{1}$

${ }^{1}$ Ismaninger Str. 22, Department of Diagnostic and Interventional Radiology, Technical University of Munich, Munich, Germany, ${ }^{2}$ Else Kröner Fresenius Center for Nutritional Medicine, Technical University of Munich, Munich, Germany

PDFF and T2* have been previously proposed as two important parameters in quantitative MRI of adipose tissue. This study investigates the difference between gluteal and supraclavicular adipose tissue $T 2^{*}$ and the relationship between adipose tissue T2* and PDFF using a twenty-echo multi-echo gradient echo acquisition. A highly significant difference between the PDFF in different fat regions was detected in water-fat separation results when using either the first 6 echoes or the full 20 echoes. However, T2* values were only significantly different between fat regions, when using the full 20 echoes and not when using the first 6 echoes. PDFF also correlated with T2* when using the full 20 echoes.

Magnetic Resonance Imaging and Spectroscopic Investigation of interscapular BAT and Skeletal Muscle IMCL in High Intensity Exercise Trained Rats

Venkatesh Gopalan ${ }^{1}$, Rengaraj Anantharaj ${ }^{1}$, Le Thi Thu Giang ${ }^{1}$, Sanjay Kumar Verma ${ }^{1}$, Jadegoud Yaligar ${ }^{1}$, Anna Ulyanova ${ }^{1}$, Karthik Mallilankaraman $^{2}$, and S Sendhil Velan ${ }^{1}$

${ }^{1}$ Laboratory of Molecular Imaging, Singapore Bioimaging Consortium, Agency for Science, Technology and Research (A*STAR), Singapore, Singapore, ${ }^{2}$ Mitochondrial Physiology and Metabolism Lab, Department of Physiology, National University of Singapore, Singapore, Singapore

There is a large interest in developing non-pharmacological approaches such as exercise and nutritional compounds for activating BAT to improve metabolic health. In this study, we have investigated the effect of high intensity exercise on interscapular BAT and Intramyocellular lipids (IMCL) from skeletal muscle of rats. Exercise-induced adrenergic receptor stimulation improves quality of iBAT by remodeling of WAT into beige fat and improved mitochondrial fatty acid oxidation. Skeletal muscle IMCL also reduced with exercise along with increased PGC-1 $\alpha$ expression due to energy expenditure.

Abdominal and organ fat content quantification in PROFAST trial (Probiotics and intermittent fasting to improve pre-diabetes)

Dech Dokpuang ${ }^{1}$, Rinki Murphy² ${ }^{2}$ Lindsay Plank $^{2}$, Reza Nemati ${ }^{1}$, and Jun Lu ${ }^{3,4}$

${ }^{1}$ Auckland University of Technology, Auckland, New Zealand, ${ }^{2}$ University of Auckland, Auckland, New Zealand, ${ }^{3}$ School of Science, Auckland University of Technology, Auckland, New Zealand, ${ }^{4}$ College of Life Sciences, Shenzhen University, Shenzhen, China

The primary objective of this study was to test quantification protocols on human abdominal and organ fat data acquired using magnetic resonance (MR) imaging or spectroscopy. Liver, pancreatic, visceral and subcutaneous fat in 10 obese patients with prediabetes were measured before and after a 12-week intermittent fasting programme with daily probiotic or placebo supplementation. All participants were scanned by a Siemens 3.0T MR scanner. The quantification of fat contents was performed using ImageJ (for MRI data) and SIVIC software (for MRS data). Two methods of quantifying pancreas fat were compared.

Metabolic Imaging and Characterization of Browning Adipose Tissue by DCE-MRI and Dixon Imaging

Jadegoud Yaligar ${ }^{1}$, Sanjay Kumar Verma ${ }^{1}$, Venkatesh Gopalan ${ }^{1}$, Rengaraj Anantharaj ${ }^{1}$, Giang Le Thi Thu ${ }^{1}$, and S. Sendhil Velan ${ }^{1}$

${ }^{1}$ Laboratory of Molecular Imaging, Singapore Bioimaging Consortium, Singapore, Singapore

Browning of white adipose tissues is emerging as a promising strategy to increase whole body energy expenditure and to reduce obesity. At a whole body level, increasing the beige or BAT volume and enhancing its functional activity is a promising strategy for management of obesity. There is a lack of non-invasive methods for imaging the browning process. For the first time we demonstrated the feasibility of non-invasive imaging of browning adipose tissue by fat fraction imaging and DCE-MRI. The browning adipose tissues show significant reduction in fat fraction and increase in tissue perfusion parameters including $\mathrm{K}^{\text {trans }}$ and $\mathrm{v}_{\mathrm{e}}$ 
Jadegoud Yaligar ${ }^{1}$, Rengaraj Anantharaj ${ }^{1}$, Le Thi Thu Giang ${ }^{1}$, Sanjay Kumar Verma ${ }^{1}$, Venkatesh Gopalan ${ }^{1}$, Bhanu Prakash K N Karthik Mallilankaraman $^{2}$, and S. Sendhil Velan ${ }^{1}$

${ }^{1}$ Laboratory of Molecular Imaging, Singapore Bioimaging Consortium, Singapore, Singapore, ${ }^{2}$ Department of Physiology, National University of Singapore, Singapore, Singapore

High-GI diet has been linked with insulin resistance, type 2 diabetes and cardiovascular risk factors. Brown fat activity positively correlates with increased energy expenditure during $\beta 3$ agonist/cold induced BAT activation, suggesting regulatory link between BAT and energy metabolism. In this study we evaluated long term metabolic effects of high and low-GI diets on brown adipose tissue metabolism and ectopic fat accumulation in liver and abdomen by MRI and MRS. Low-GI diet fed animals were responsive to prolonged BAT activation for metabolizing the fat. Weight and volumes of iBAT increased with $\beta 3$-agonist treatment, implying potential remodeling of WAT into Beige.

Metabolic Imaging of brown adipose tissue in leucine deficient diet fed mice.

Anna Ulyanova ${ }^{1}$, Jadegoud Yaligar ${ }^{1}$, Anantharaj Rengaraj ${ }^{1}$, Giang Le Thi Thu ${ }^{1}$, Sanjay K Verma ${ }^{1}$, Venkatesh Gopalan ${ }^{1}$, and S Sendhil Velan ${ }^{1}$

${ }^{1}$ Laboratory of Molecular Imaging, Singapore Bioimaging Consortium, Singapore, Singapore

Brown adipose tissue plays an important role in energy expenditure. The deficiency of the essential amino acid leucine has been linked with CREB/TRH pathway and regulation of energy expenditure and food intake. Here we investigated the effect of leucine deficient diet on interscapular brown adipose tissue (iBAT) in mice. Dixon imaging was performed to assess fat fraction changes within iBAT followed by RNA analysis. There was a decrease in fat fraction for leucine deficient diet fed mice together with increased UCP1 expression indicating that short term leucine deprivation leads to iBAT activation.

Identification and Characterization of Brown and White Adipose Tissue Depots in Rats by 3D Whole Body Imaging

Rengaraj Anantharaj ${ }^{1}$, Sanjay Kumar Verma ${ }^{1}$, Jadegoud Yaligar ${ }^{1}$, Julian Gan ${ }^{2}$, Giang Le Thi Thu ${ }^{1}$, Kavita Kaur ${ }^{1}$, Venkatesh Gopalan ${ }^{1}$, Kuan Jin Lee ${ }^{1}$, and S. Sendhil Velan ${ }^{1}$

${ }^{1}$ Laboratory of Molecular Imaging, Singapore Bioimaging Consortium, Singapore, Singapore, ${ }^{2}$ Siemens Healthcare Pte. Ltd, SINGAPORE, Singapore

Excess body adiposity results in obesity and metabolic dysfunction. Identification and characterization of various white, brown and browning adipose tissues and the possibility of reversing pre-diabetic pathology is of current clinical interest for combating obesity and diabetes. In this study, we have identified and characterized various brown and white fat depots by whole body imaging in rats using a Siemens $3 T$ Skyra system.

Evaluation of Simultaneous MRI/PET of Supraclavicular BAT for Detecting Adaptive Thermogenesis after Sympathetic Nervous System Activation

Sanjay K Verma ${ }^{1}$, Lijuan Sun ${ }^{2}$, Suresh Anand Sadananthan ${ }^{3}$, Navin Michael ${ }^{3}$, Hui Jen Goh $^{2}$, Priya Govindharajulu², John Totman ${ }^{4}$, David Townsend ${ }^{4}$, Houchun H Hu ${ }^{5}$, Melvin KheeShing Leow ${ }^{2,6}$, and S Sendhil Velan ${ }^{1,3}$

${ }^{1}$ Laboratory of Molecular Imaging, Singapore Bioimaging Consortium, Agency for Science Technology and Research (A*STAR), Singapore, Singapore, ${ }^{2}$ Clinical Nutrition Research Centre, Agency for Science, Technology and Research (A*STAR), Singapore, Singapore, ${ }^{3}$ Singapore Institute of Clinical Sciences, Agency for Science, Technology and Research (A*STAR), Singapore, Singapore, ${ }^{4}$ Clinical Imaging Research Centre, Agency for Science, Technology and Research (A*STAR), Singapore, Singapore, ${ }^{5}$ Department of Radiology, Nationwide Children's Hospital Columbus, Columbus, $\mathrm{OH}$, United States, ${ }^{6}$ Department of Endocrinology, Tan Tock Seng Hospital, Singapore, Singapore

There is a large interest in detecting and quantifying brown adipose tissue (BAT) in humans for evaluating its potential to design therapeutic strategies to combat obesity-related metabolic dysfunction. In the current study, we evaluated the use of simultaneous PET/MRI of supraclavicular BAT (sBAT) for distinguishing subjects with high or low adaptive thermogenesis after sympathetic nervous system activation by cold exposure and capsinoids ingestion. As a sub-study, We also evaluated the duration of cold-exposure for changes in 18F-FDG uptake and Dixon-based fat-fraction. We found that adaptive thermogenesis after capsinoids ingestion was too low to be detected by either modality, while PET was successful in identifying high responders to cold stimulation.

In Vivo Diffusion Magnetic Resonance Spectroscopy of Brown and White Adipose tissues

Sanjay K Verma ${ }^{1}$, Kavita Kaur ${ }^{1}$, Jadegoud Yaligar $^{1}$, Navin Michael ${ }^{2}$, Anantharaj Rengaraj ${ }^{1}$, Le Thi Thu Giang ${ }^{1}$, Venkatesh Gopalan ${ }^{1}$, Suresh Anand Sadananthan ${ }^{2}$, Melvin Khee-Shing Leow $^{3,4}$, and S Sendhil Velan ${ }^{1,2}$

${ }^{1}$ Laboratory of Molecular Imaging, Singapore Bioimaging Consortium, Agency for Science Technology and Research (A*STAR), Singapore, Singapore, ${ }^{2}$ Singapore Institute of Clinical Sciences, Agency for Science, Technology and Research (A*STAR), Singapore, Singapore, ${ }^{3}$ Clinical Nutrition Research Centre, Agency for Science, Technology and Research (A*STAR), Singapore, Singapore, ${ }^{4}$ Department of Endocrinology, Tan Tock Seng Hospital, Singapore, Singapore

There is a large interest in understanding the biophysical properties of BAT, WAT, and beige adipose tissues for evaluating its potential to improve whole body metabolism. Diffusion properties of tissues provide information on microstructure, anisotropy, and pathology. In the presence of cellular and sub-cellular barriers, and heterogeneity the lipid diffusion is restricted. Water diffusion has been well characterized in several organs. Fat diffusion has not been studied due to the hardware limitations. In this study, we have implemented diffusion-weighted spectroscopy for investigating in-vivo diffusion properties of BAT and WAT. 


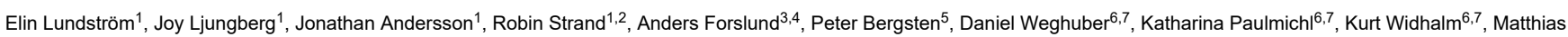
Meissnitzer ${ }^{8}$, Håkan Ahlström ${ }^{1,9}$, and Joel Kullberg ${ }^{1,9}$

${ }^{1}$ Department of Radiology, Uppsala University, Uppsala, Sweden, ${ }^{2}$ Department of Information Technology, Uppsala University, Uppsala, Sweden, ${ }^{3}$ Department of Women's and Children's Health, Uppsala University, Uppsala, Sweden, ${ }^{4}$ Children Obesity Clinic, Uppsala University Hospital, Uppsala, Sweden, ${ }^{5}$ Department of Medical Cell Biology, Uppsala University, Uppsala, Sweden, ${ }^{6}$ Department of Paediatrics, Paracelsus Medical University, Salzburg, Austria, ${ }^{7}$ Obesity Research Unit, Paracelsus Medical University, Salzburg, Austria, ${ }^{8}$ Department of Radiology, Paracelsus Medical University, Salzburg, Austria, ${ }^{9}$ Antaros Medical, BioVenture Hub, Mölndal, Sweden

Investigating the role of brown fat (BAT) in child/adolescent metabolism and obesity is important for elucidating its potential as an antiobesity/antidiabetes therapeutic target. This study presents associations between MRI estimates of BAT (by cervical-supraclavicular adipose tissue fat fraction and $\mathrm{T}_{2}{ }^{*}$ ) and abdominal adiposity and glucose metabolism parameters in children/adolescents. Associations between the BAT estimates and adiposity were observed, supporting previous indications of decreasing BAT amounts with increasing adiposity. Additional associations between the BAT estimates and important glucose metabolism parameters may reflect a role for BAT in glucose and energy metabolism and potentially a link to development of type 2 diabetes.

2-phase Dixon technique to assay dermal white adipose tissue loss as potential early diagnostic biomarker of scleroderma using genetic Fra-2 mice

Nicola Bertolino ${ }^{1}$, Roberta Goncalves Marangoni ${ }^{2}$, Daniele Procissi ${ }^{1}$, Cynthia Yang ${ }^{1}$, Sol Misener ${ }^{1}$, Warren G Tourtellotte ${ }^{3}$, and John Varga ${ }^{2}$

${ }^{1}$ Radiology, Feinberg School of Medicine, Northwestern University, Chicago, IL, United States, ${ }^{2}$ Division of Rheumatology, Feinberg School of Medicine, Northwestern University, Chicago, IL, United States, ${ }^{3}$ Pathology, Neurology, Neurosurgery and Regenerative Medicine, Cedars Sinai Medical Center, Los Angeles, CA, United States

Scleroderma is an autoimmune disease leading to fibrosis resulting in stiff skin, formation of ulcers, joint contractures and ultimately functional incapacity. Loss of dermal white adipose tissue (dWAT) was observed ex-vivo prior to the development of fibrosis. In this study we demostrated the feasibility of employing CSE MRI Dixon technique to detect and quantify invivo dWAT thickness using a genetic Fra-2 fibrosis mice model. The proposed non-invasive diagnostic method to evaluate or predict skin fibrosis would greatly improve clinicians' ability to track progression and response to treatment and also provide a tool to investigate pathogenesis in animal models.

Using water-fat MRI to detect remodeling of adipose tissue

Amanda DV MacCannell ${ }^{1}$, Kevin J Sinclair ${ }^{2}$, James F Staples ${ }^{1}$, and Charles A McKenzie ${ }^{2}$

${ }^{1}$ Biology, Western University, London, ON, Canada, ${ }^{2}$ Medical Biophysics, Western University, London, ON, Canada

Hibernating mammals use brown adipose tissue (BAT) as a primary source of heat production for arousal from torpor. In hibernators, both white adipose tissue (WAT) and BAT volumes increase in autumn even when temperatures are warm, unlike non-hibernators which require cold exposure for BAT growth. Differentiation of WAT from BAT between depots in close proximity can be achieved using IDEAL water-fat MRI. Hibernating mammals exposed to constant warm environments showed drastic molecular changes to their BAT depots that could ultimately be detected my MRI, proving IDEAL's versatility and specificity.

Novel model of alcoholic hepatitis and alcoholic steatohepatitis using C57BL/6N mice and magnetic resonance imaging/spectroscopy

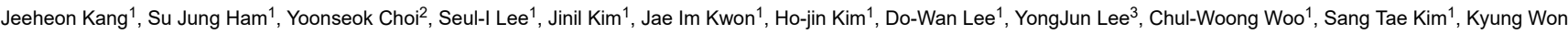
$\mathrm{Kim}^{1}$, and Dong-Cheol Woo ${ }^{1}$

Alcoholic liver disease is classified into two subgroups: alcoholic hepatitis (AH) and alcoholic steatohepatitis (ASH). These differ in most characteristics, including clinicopathologic features and treatment. However, animal models of $\mathrm{AH}$ and $\mathrm{ASH}$ are not well established. Noninvasive monitoring is essential for evaluating chronic diseases such as $\mathrm{AH}$ and $\mathrm{ASH}$. Magnetic resonance imaging and spectroscopy (MRI/S) have recently gained considerable attention as noninvasive monitoring tools for chronic liver disease. The aim of this study, therefore, was to develop a comprehensive animal model of $\mathrm{AH}$ and $\mathrm{ASH}$ that can be monitored noninvasively using MRI/S.

ADC quantification of lipids with high b-value stimulated echo-prepared diffusion-weighted 2D single shot TSE

Dominik Weidlich ${ }^{1}$, Stefan Ruschke ${ }^{1}$, Barbara Cervantes ${ }^{1}$, Andreas Hock ${ }^{2}$, and Dimitrios C. Karampinos ${ }^{1}$

${ }^{1}$ Department of Diagnostic and Interventional Radiology, Technical University of Munich, Munich, Germany, ${ }^{2}$ Philips Healthcare, Hamburg, Germany 
The prevalence of the metabolic syndrome is rapidly growing over the past decade. Fat plays a central role in the incidence and the progression of the metabolic syndrome and despite the successful clinical translation of quantitative fat MRI biomarkers into applications, current MRI biomarkers cannot answer questions about fat cell microstructure in different fat depots. This work proposes an acquisition imaging method that probes the diffusion properties of lipids, compares the proposed method to single-voxel diffusion-weighted MRS in vivo in the tibia bone marrow and investigates in vivo the dependency of $A D C$ quantification on voxel size in gluteal fat.

Traditional Poster

\section{Body: Animal Models}

Quantitative evaluation of gadolinium deposition after various gadolinium-based contrast agent injection in the rat abdominal organs .

Hyewon $\mathrm{Oh}^{1}$, PanKi Kim ${ }^{2}$, ChanGyu Joo ${ }^{3}$, and YongEun Chung ${ }^{1}$

${ }^{1}$ Radiology, BK21 PLUS Project for Medical Science, Yonsei University College of Medicine, Seoul, Republic of Korea, ${ }^{2}$ Research Institute of Radiological Science, Severance Hospital, Yonsei University Health System, Seoul, Republic of Korea, ${ }^{3}$ Yonsei Biomedical Science Institute, Yonsei University College of Medicine, Seoul, Republic of Korea

Gadolinium-based Contrast agent (GBCA) is likely to deposit in the rat abdominal organs.

Individual Time Series Analysis of p53 Knockout Medaka by in vivo Magnetic Resonance Microscopy

Hajime Morizumi ${ }^{1}$, Takahiro Nishigaki ${ }^{2}$, Naozo Sugimoto ${ }^{2}$, and Tomohiro Ueno ${ }^{2}$

Tumor suppressor gene p53 knockout medaka has been generated. The tumor spectrum of this medaka model, however, remains unknown. In this study, we performed individual time series analysis of p53 knockout medaka using a 14.1T MR microscopy. Extracting size change of kidney of the medaka model, we found early indications of disease and difference in phenotype due to location difference of point mutation in the p53 gene. Since p53 knockout medaka showed rather large variations in kidney slice, importance of individual time series analysis was confirmed.

$\mathrm{Mn}^{2+}$-free chow reduces gastrointestinal signal for $\mathrm{T}_{1}$-weighted $\mathrm{MRI}$ of the mouse abdomen

Veerle Kersemans ${ }^{1}$, Stuart Gilchrist ${ }^{1}$, Paul Kinchesh ${ }^{1}$, and Sean Smart ${ }^{1}$

${ }^{1}$ University of Oxford, CRUK/MRC Oxford Institute for Radiation Oncology, Oxford, United Kingdom

Standard commercial chow given to laboratory animals may contain high levels of paramagnetic $\mathrm{Mn}^{2+}$-ions which act as a $\mathrm{T}_{1}$-reducing contrast agent. Signal intensities where $\mathrm{Mn}^{2+}$ is present are increased when using short-TR, $T_{1}$ W-MRI imaging and the GI-tract appears brighter than the rest of the body. As peristalsis is an inherently unstable motional process, high intensity and temporally unstable signals are formed in the Gl-tract, creating image-ghosting and decreasing resolution from that prescribed. We present images acquired before and after transition from $\mathrm{Mn}^{2+}$-bearing to $\mathrm{Mn}^{2+}$-free food to show that these deleterious image effects can be reduced through simple dietary formulation change.

Whole-Body Cardio-Respiratory Synchronised DCE-MRI in the Mouse

Veerle Kersemans ${ }^{1}$, Philip Danny Allen ${ }^{1}$, Stuart Gilchrist ${ }^{1}$, Ana L Gomes ${ }^{1}$, Paul Kinchesh ${ }^{1}$, and Sean Smart ${ }^{1}$

${ }^{1}$ University of Oxford, CRUK/MRC Oxford Institute for Radiation Oncology, Oxford, United Kingdom

Prospective gating of constant, short TR scans enables rapid imaging to be performed in conjunction with cardiac and respiratory synchronisation. We show that prospectively-gated, dynamic contrast enhanced MRI (DCE-MRI) can be performed over the whole mouse body with a time resolution of ca. $15 \mathrm{~s} /$ frame such that multiple organs can be examined simultaneously. 
In this study we try to develop a renal IRI model which leads to fibrosis. Fibrosis markers indicate the best effect after 7 days of reperfusion. We also investigate the possibility of using $23 \mathrm{Na} \mathrm{T} 2^{*}$ to evaluate fibrosis, this gave rise to a correlation with fibrosis markers only when normalizing to water transport from cortex to medulla.

eNOS-/- mice fed with HFD develop progressive non-alcoholic fatty liver disease (NAFLD) which is partially reversible with antihypertensive and hypoglycemic therapy

Begoña Lavin Plaza ${ }^{1}$, Marcelo E Andia ${ }^{2}$, Thomas Eykyn ${ }^{1}$, Alkystis Phinikaridou ${ }^{1}$, Aline Xavier ${ }^{2}$, and Rene M Botnar ${ }^{1}$

${ }^{1}$ Imaging Sciences and Biomedical Engineering, King's College London, London, United Kingdom, ${ }^{2}$ Radiology department, School of Medicine, Pontificia Universidad Catolica de Chile, Santiago, Chile

Liver steatosis or non-alcoholic fatty liver disease (NAFLD) is the most common liver disease in Western countries. However, the cause and treatments are still controversial. Nitric oxide (NO) and its derivatives play important roles in the physiology and pathophysiology of the vascular system and liver metabolism. We quantified intraperitoneal fat and liver fat-fraction using 3T MRI in eNOS-/- mice fed with HFD and investigated (1) whether pharmacological treatments for type 2 diabetes and hypertension reduced fat deposition and (2) if the phenotype could be recapitulated by administration of an inhibitor of endothelial NO synthesis (L-NAME) in wild type mice.

Traditional Poster

\section{Body: Technical Advances}

Exhibition Hall 2518-2535

Stretched-Exponential Diffusion-Weighted Imaging Model for Abdominal MR

Takeshi Yoshikawa ${ }^{1}$, Katsusuke Kyotani ${ }^{2}$, Yoshiharu Ohno ${ }^{1}$, Yoshimori Kassai ${ }^{3}$, Seiya Kai ${ }^{3}$, Eiji Takeda ${ }^{2}$, Shinichiro Seki ${ }^{1}$, and Yuji Kishida $^{4}$

${ }^{1}$ Advanced Biomedical Imaging Research Center, Kobe University Graduate School of Medicine, Kobe, Japan, ${ }^{2}$ Center of Radiology and Radiation Oncology, Kobe University Hospital, Kobe, Japan, ${ }^{3}$ Toshiba Medical Systems Co., Otawara, Japan, ${ }^{4}$ Radiology, Kobe University Graduate School of Medicine, Kobe, Japan

Stretched-exponential model can be used as an excellent alternative to mono-exponential model in evaluation of abdominal organs and diseases.

Measuring Abdominal Wall Muscle Deformation using MR Tissue Tagging

Lawrence Dougherty ${ }^{1}$, Pilla J. James ${ }^{1}$, and Anil Chauhan ${ }^{1}$

${ }^{1}$ Radiology, University of Pennsylvania, Philadelphia, PA, United States

The current state of hernia repair relies heavily on clinical evaluation of patients, which is ultimately a poor predictor of outcomes for patients going into surgery. There are currently no reliable data, standard imaging modalities, or guidelines available to predict successful fascial closure in hernia repair. A method using MR tissue tagging with synchronous displacement of the abdominal wall was developed. This will allow analysis of the mechanical properties of muscle for noninvasive, diagnostic tool for pre-operatively predicting successful fascial closure in hernia repair.

Whole body Quantitative Susceptibility Mapping using Automated Preconditioning

Zhe Liu ${ }^{1,2}$, Yan Wen ${ }^{1,2}$, Pascal Spincemaille ${ }^{1}$, Thanh Nguyen ${ }^{1}$, and Yi Wang ${ }^{1,2}$

${ }^{1}$ Radiology, Weill Cornell Medical College, New York, NY, United States, ${ }^{2}$ Biomedical Engineering, Cornell University, Ithaca, NY, United States

An automated method is proposed for generating an optimal preconditioner for a given field input for performing preconditioned total field inversion quantitative susceptibility mapping. In gradient echo data acquired in healthy subjects and patients in various anatomic regions, the obtained preconditioner leads to the same optimal susceptibility map quality as a manually selected preconditioner. 
${ }^{1}$ Imaging Physics, MD Anderson Cancer Center, Houston, TX, United States, ${ }^{2}$ Radiation Oncology, MD Anderson Cancer Center, Houston, TX, United States, ${ }^{3}$ Diagnostic Radiology, MD Anderson Cancer Center, Houston, TX, United States

A Fully Convolutional Network (FCN) was developed and applied to the task of contouring esophageal tumors on diffusion weighted images. After proper training, tumor classification by the FCN demonstrated excellent agreement with tumor contours from an inter-reader agreement study in the validation images. The FCN was able to achieve correct tumor classification in most cases with respect to different tumor position and shapes, and in the presence of intratumoral esophageal lumen.

Detection of liver fibrosis from MRlusing histogram of strains

Yasmine A. Safwat ${ }^{1}$, Rasha S. Hussein ${ }^{2}$, Ayman Khalifa ${ }^{3}$, Ahmed S. Ibrahim ${ }^{4}$, Ahmed Samir ${ }^{5}$, Heba Abdallah ${ }^{6}$, and Ahmed S Fahmy ${ }^{7}$

${ }^{1}$ Center for Informatics Science, Nile University, Cairo, Egypt, ${ }^{2}$ Radiodiagnosis, Ain Shams University, Cairo, Egypt, ${ }^{3}$ Biomedical Engineering Department, Helwan University, Cairo, Egypt, ${ }^{4}$ Radiodiagnosi, Ain Shams University, Cairo, Egypt, ${ }^{5}$ Tropical Department, Ain Shams University, Cairo, Egypt, ${ }^{6}$ Tropical Medicine Department, Ain Shams University, Cairo, Egypt, ${ }^{7}$ Biomedical Engineering Department, Cairo University, Cairo, Egypt

In this work, we present the results of a novel method for detecting liver fibrosis from tagged MRI images. The method is based on extracting a set of features representing the liver deformations induced by the heart motion. First, the tagged MRI images are analyzed to calculate the liver tissue strain induced by the heart motion. The histogram of the peak strain values at each point within the liver are used as feature vectors to classify normal from patients with liver fibrosis. Classification using support-vector-machines using data of 34 subject (15 normal, 19 patients) showed sensitivity and specificity of $89 \%$, and $80 \%$ respectively.

Changes of T2 signal intensities of abdominal organs between pre- and post-enhanced HASTE using ferumoxytol

Woo Kyoung Jeong ${ }^{1,2}$, Kim-Lien Nguyen ${ }^{3}$, Puja Shahrouki ${ }^{1}$, and J. Paul Finn ${ }^{1}$

${ }^{1}$ Radiology, David Geffen School of Medicine at UCLA, Los Angeles, CA, United States, ${ }^{2}$ Radiology, Samsung Medical Center, Seoul, Republic of Korea, ${ }^{3}$ Cardiology, David Geffen School of Medicine at UCLA, Los Angeles, CA, United States

This study was designed to investigate the signal intensities (SI) of abdominal organs on pre- and post-enhanced HASTE images using ferumoxytol at a dose of $4 \mathrm{mg} / \mathrm{kg}$, and to compare the differences of enhancement effect among the organs. We found that the SI of liver, spleen, and pancreas were significantly decreased on HASTE after ferumoxytol administration. The greatest effects on SI were observed in liver and spleen. Little change in SI of muscle and fat was noted. The findings suggest that normal liver and spleen undergo profound decrease in signal intensity following ferumoxytol injection, likely reflecting their high blood volume. This observation suggests a potential role for ferumoxytol in detection and characterization of focal lesions of the liver and spleen.

Gadoxetic acid-enhanced dynamic MR imaging using optimized integrated combination with parallel imaging and compressed sensing technique

Nobuyuki Kawai ${ }^{1}$, Satoshi Goshima ${ }^{1}$, Kimihiro Kajita ${ }^{2}$, Tomoyuki Okuaki ${ }^{3}$, Masatoshi Honda ${ }^{3}$, Hiroshi Kawada ${ }^{1}$, Yoshifumi Noda ${ }^{1}$, Yukichi Tanahashi ${ }^{1}$, Shoma Nagata $^{1}$, and Masayuki Matsuo ${ }^{1}$

Gadoxetic acid-enhanced MRI represents an essential part in the assessment of hepatic diseases, however, dynamic imaging especially in hepatic arterial phase is still challenging for patients with limited breath-hold capabilities. We assessed prototype sequence using optimized integrated combination with parallel imaging and compressed sensing technique (Compressed-SENSE) for liver imaging, which enabled significant reduction of acquisition time resulting in excellent image quality with less motion artifact, especially in hepatic arterial phase, compared with conventional method. Our results demonstrated the significance and usefulness of Compressed-SENSE in clinical use for gadoxetic acid-enhanced dynamic MR imaging.

Hepatobiliary phase imaging using optimized integrated combination with parallel imaging and compressed sensing technique.

Nobuyuki Kawai ${ }^{1}$, Satoshi Goshima ${ }^{1}$, Kimihiro Kajita ${ }^{2}$, Tomoyuki Okuaki ${ }^{3}$, Masatoshi Honda ${ }^{3}$, Hiroshi Kawada ${ }^{1}$, Yoshifumi Noda ${ }^{1}$, Yukichi Tanahashi ${ }^{1}$, Shoma Nagata $^{1}$, and Masayuki Matsuo ${ }^{1}$

Gadoxetic acid-enhanced MRI plays an important role in the assessment of hepatic diseases. Hepatobiliary phase image has an amazing tissue contrast for the lesions with or without functional hepatocytes, however, which is still challenging for patients with limited breath-hold capabilities. We assessed prototype sequence using optimized integrated combination with parallel imaging and compressed sensing technique (Compressed-SENSE) for liver imaging. Our results demonstrated that Compressed-SENSE technique enabled significant reduction of acquisition time without image quality degradation resulting in higher spatial resolution and excellent image quality compared with conventional method. 
Development of a fast 4D-MRI with sub-second volumetric frame rate for respiratory motion tracking in abdominal radiotherapy

Jing Yuan ${ }^{1}$, Yihang Zhou ${ }^{1}$, Oilei Wong ${ }^{1}$, KinYin Cheung ${ }^{1}$, and Siu Ki Yu ${ }^{1}$

${ }^{1}$ Medical physics and research department, Hong Kong Sanatorium \& Hospital, Happy Valley, Hong Kong

Time-resolved volumetric MRI (4D-MRI) is gaining more interests for better tumor motion characterization than 4D-CT in abdominal radiotherapy, while 3D sequence has limited use for 4D-MRI acquisition due to its slow volume-frame-rate (VFR) and various motion artifacts. We developed a fast 4D-MRI technique based on CAIPIRINHA accelerated 3D spoiled gradient echo sequence and a 1.63 frames-per-second $(615 \mathrm{~ms} /$ frame, $\sim 1 / 7$ of normal respiratory cycle of $4-5 \mathrm{~s})$ VFR was achieved. This 4D-MRI was demonstrated for whole abdomen respiratory motion tracking in healthy volunteers, indicating its great potentials for internal-target-volume definition in radiotherapy treatment planning and image guidance of MR-guidedradiotherapy.

A study of correlation of the SUVmax and ADC in malignant breast tumors using simultaneous PET-MRI

Jing Yuan ${ }^{1}$, Gladys Goh Lo ${ }^{2}$, Garrett CL Ho ${ }^{3}$, Sirong Chen ${ }^{1}$, Helen HL Chan ${ }^{2}$, Victor HG Ai ${ }^{2}$, William SK Cheung ${ }^{3}$, Catherine YH Wong ${ }^{3}$, Suk Yee Polly Cheung ${ }^{4}$, and Ting Ting Wong ${ }^{4}$

${ }^{1}$ Medical physics and research department, Hong Kong Sanatorium \& Hospital, Happy Valley, Hong Kong, ${ }^{2}$ Department of diagnostic \& interventional radiology, Hong Kong Sanatorium \& Hospital, Happy Valley, Hong Kong, ${ }^{3}$ Department of nuclear medicine \&positron emission tomography, Hong Kong Sanatorium \& Hospital, Happy Valley, Hong Kong, ${ }^{4}$ Breast Care Center, Hong Kong Sanatorium \& Hospital, Happy Valley, Hong Kong

We studied the correlation of simultaneous DWI-ADC and 18F-FDG SUVmax in invasive ductal carcinoma (IDC) tumors ( $n=41)$, and their association with different diagnostic factors using integrated PET-MRI. An insignificant inverse correlation was found $(r=-0.214, p=0.179)$ between SUVmax and ADCmean. SUVmax was significantly associated with tumor T-stage $(p=0.024)$. ADCmean of the index IDC was significantly smaller in the patients with pathologically confirmed regional lymph node metastasis $(p=0.0488)$ and estrogen receptor status $(p=0.0254)$. An insignificantly larger SUVmax $(p=0.1352)$ was found in triple negative IDCs. Our results showed that SUVmax and ADCmean might potentially have complementary roles in breast cancer characterization.

Comparison of liver motion measured by dynamic MRI and respiration signals obtained by an optical sensor

Julien Sénégas ${ }^{1}$, Sascha Krueger ${ }^{1}$, Daniel Wirtz ${ }^{1}$, Ger Kersten², Mukul Rocque ${ }^{3}$, Ivan E. Dimitrov ${ }^{4}$, Andrea J. Wiethoff ${ }^{5}$, Keith Hulsey ${ }^{6}$, Ivan Pedrosa ${ }^{6}$, and Ananth J. Madhuranthakam ${ }^{6}$

${ }^{1}$ Philips Research Laboratories, Hamburg, Germany, ${ }^{2}$ Philips Innovation Services, Eindhoven, Netherlands, ${ }^{3}$ Philips Research Laboratories, Eindhoven, Netherlands, ${ }^{4}$ Philips Healthcare, Gainesville, FL, United States, ${ }^{5}$ Philips Research North America, Cambridge, MA, United States, ${ }^{6}$ Department of Radiology and Advanced Imaging Research Center, UT Southwestern Medical Center, Dallas, TX, United States

To monitor the breathing status of a subject and to synchronize data acquisition with respiration, external sensors, such as respiratory bellows, are routinely used. These sensors probe only the local breathing motion, and, hence, their signal quality can vary significantly depending on the individual subject's physiology and morphology as well as the experience of the MR operator. Recently, optical sensors were proposed as an alternative. The purpose of this work was to compare the signals obtained by an optical sensor and by a pressure-based sensor with respect to their ability to represent the true liver motion during breathing.

Optical unobtrusive physiology sensor for respiratory-triggered MRI acquisitions

Sascha Krueger ${ }^{1}$, Julien Sénégas ${ }^{1}$, Daniel Wirtz ${ }^{1}$, Marek Bartula² ${ }^{2}$ Vincent Jeanne ${ }^{3}$, Thiru Kanagasabapathi ${ }^{2}$, and Ger Kersten ${ }^{4}$

${ }^{1}$ Philips Research, Hamburg, Germany, ${ }^{2}$ Philips Research, Eindhoven, Netherlands, ${ }^{3}$ Philips Healthcare, Bothell, WA, United States, ${ }^{4}$ Philips Innovation Services, Eindhoven, Netherlands

A prototype in-bore camera-based physiology sensor was developed and applied for respiratory-triggered MR acquisitions on volunteers. The camera-based physiology sensor allows unobtrusive measurement of breathing activity by derivation of respiratory signals from video stream in real-time. The camera-based breathing sensor provided high quality breathing signal reliably under all tested circumstances of a volunteer study. The breathing signal quality was rated to be superior compared to the bellows in terms of SNR and signal characteristics. Potential false triggers where significantly reduced by the camera. The resulting image quality was on average superior when triggering off the camera compared to when triggering off the bellows.

Towards efficient free breathing dynamic liver MRI using Cartesian k-space sampling with compressed sensing

Caizhong Chen ${ }^{1}$, Shengxiang Rao ${ }^{1}$, Guobin $\mathrm{Li}^{2}$, Zhaopeng $\mathrm{Li}^{2}$, Jiayu Zhu², Jinguang Zong ${ }^{2}$, Xixi Wen², and Mengsu Zeng ${ }^{1}$

${ }^{1}$ Department of Radiology, Zhongshan Hospital, Fudan University, Shanghai, China, ${ }^{2}$ United Imaging Healthcare, Shanghai, China

An efficient imaging method using Cartesian k-space undersampling with compressed sensing, and automatic detection of respiration was proposed to enable free-breathing dynamic liver imaging with a temporal resolution up to $1.0 \mathrm{sec} / \mathrm{phase}$ 
MR Imaging Perfusion and Diffusion analysis to assess preoperative Short Course Radiotherapy response in locally advanced rectal cancer: Standardized Index of Shape by DCE-MRI and Intravoxel Incoherent Motion derived parameters by DW-MRI

Roberta Fusco ${ }^{1}$ and Antonella Petrillo ${ }^{1}$

Aim of this study is to determine the diagnostic performance of MR imaging for the assessment of tumor response after Short Course Radiotherapy (SCR) in patients with LARC using Standardized Index of Shape (SIS) obtained by DCE-MRI and using ADC, DKI and IVIM derived parameters obtained by DW-MRI.

We demostrated that SIS is a hopeful DCE-MRI angiogenic biomarker to assess preoperative treatment response after SCR with delayed surgery and it permits to discriminate pCR allowing to direct surgery for tailored and conservative treatment.

Validation of Reproducibility of Both Zoom Diffusion Imaging And Conventional Full Field of View Method in The Kidney Study

Hsuan Wen $\mathrm{Yu}^{1,2}$, Feng Mao Chiu ${ }^{3}$, Cheng Ping Chien ${ }^{2}$, and You Yin Chen ${ }^{1}$

${ }^{1}$ National Yang-Ming University, Taipei, Taiwan, ${ }^{2}$ Taipei Beitou Health Management Hospital, Taipei, Taiwan, ${ }^{3}$ Philips Healthcare, Taipei, Taiwan

Diffusion Tensor Imaging (DTI) is a reliable tool for investigating renal microstructure and renal function, the imaging stability remains challenging. Recently, the image-quality improvement by zoom DTI technique (reduced Field-Of-View diffusion) is reported ${ }^{1}$. We scanned 10 healthy volunteers by this technique via the respiration-triggered acquisition, and we assessed different ROIs within the medulla and the cortex of the kidney. In this study, the reproducibility between different subjects in zoom DTI was more promising when compared to full-FOV DTI. More DTI scalars were compared between zoom and full-FOV DTI in cortex and medulla and these may be potential parameters to detect pathological changes in kidney.

Improvement of ADC Precision in Left Liver Lobe by Weighted Averaging

Takashi Nishihara $^{1}$, Masahiro Takizawa ${ }^{1}$, Ryuji Shirase $^{1}$, Takenori Murase ${ }^{1}$, and Masayuki Isobe ${ }^{1}$

${ }^{1}$ Hitachi, Ltd. Healthcare Business Unit, Tokyo, Japan

The signal intensity in liver DWI was induced by the cardiac motion. A new post-processing method using weighted image averaging is evaluated to mitigate these signal loss of pixels. The proposed method suppressed the signal loss and the precision of ADC was improved.

Comparison of quiet diffusion-weighted imaging with standard DWI in the abdomen: preliminary evaluation in the assessment of abdominal organs

Xianyun Cai ${ }^{1}$, Guangbin Wang ${ }^{2}$, Tianyi Qian ${ }^{3}$, David Grodzki ${ }^{4}$, Sai Shao ${ }^{2}$, Cong Sun ${ }^{1}$, and Huihua $\mathrm{Li}^{2}$

${ }^{1}$ Shandong Medical Imaging Research Institute, Shandong University, Jinan, China, ${ }^{2}$ Shandong Medical Imaging Research Institute, Jinan, China, ${ }^{3}$ Siemens Healthcare, MR Collaborations NE Asia, Beijing, China, ${ }^{4}$ Siemens Healthcare, Application Development, Erlangen, Germany

This study aimed to evaluate the diagnostic value of a quiet DWI (q-DWI) sequence in abdominal organs. Twenty-four patients underwent MR scans, including standard DWI and q-DWI. Quantitative and qualitative assessments regarding the signal-to-noise ratio (SNR), contrast-to-noise ratio (CNR), lesion conspicuity, the level of artifacts, and overall image quality, were measured. The qualitative rating by two radiologists shows that there were differences in lesion conspicuity, but these were not significant. The CNR and SNR of q-DWI were significantly higher than those of regular-DWI(r-DWI). For those patients who were intolerant to noise, the q-DWI technique could be more suitable.

Computer aided cancer detection based on volumetric DCE-MRI analysis

Barbara Ilse Bennani-Baiti ${ }^{1}$ and Pascal Andreas Baltzer ${ }^{1}$

2535 'Department of Biomedical Imaging and Image-guided Therapy, Medical University of Vienna, Vienna, Austria

While CAD is already routinely employed in conventional mammography, the data available on CAD cancer detection at MRI so far are limited and mostly include evaluation of lesion size, vascularization kinetics and tumor extent. Our data from two different approaches based on the percentage of voxel volume enhancement of either the ipsilateral breast alone or accounting for background parenchymal enhancement measured in the contralateral breast suggest both to be viable approaches for breast cancer detection with excellent reproducibility, that should be further developed. 


\section{Body: Liver}

Non-Invasive Assessment of Mesenteric Hemodynamics with 4D flow MRI

Grant S Roberts ${ }^{1}$, Alejandro Roldan-Alzate ${ }^{2}$, Christopher J Francois ${ }^{2}$, and Oliver Wieben ${ }^{1,2}$

${ }^{1}$ Medical Physics, University of Wisconsin - Madison, Madison, WI, United States, ${ }^{2}$ Radiology, University of Wisconsin - Madison, Madison, WI, United States

Chronic mesenteric ischemia ( $\mathrm{CMI}$ ) is caused by inadequate blood flow to the intestines. This study investigates the use of 4D flow MRI to non-invasively assess the hemodynamics of the mesenteric circulation in patients with CMI and controls. Flow was measured in 9 vessels before and after meal challenges for 19 subjects suspected of CMI and 6 controls. Postprandial flow increased significantly in the supraceliac aorta, superior mesenteric artery, superior mesenteric vein, and portal vein. The flow increase was drastically stunted in patients with CMI. This demonstrates the potential for 4D flow MRI in assisting the challenging diagnosis of CMI.

Hemodynamic Evaluations of Hepatic Vasculatures using 4D-PCA and MRFD for Liver Disease Assessment

Takeshi Yoshikawa ${ }^{1}$, Katsusuke Kyotani ${ }^{2}$, Yoshiharu Ohno ${ }^{1}$, Shinichiro Seki ${ }^{1}$, Yuji Kishida ${ }^{3}$, and Eiji Takeda ${ }^{2}$

${ }^{1}$ Advanced Biomedical Imaging Research Center, Kobe University Graduate School of Medicine, Kobe, Japan, ${ }^{2}$ Center of Radiology and Radiation Oncology, Kobe University Hospital, Kobe, Japan, ${ }^{3}$ Radiology, Kobe University Graduate School of Medicine, Kobe, Japan

4D-PCA and MRFD can characterize liver vessels and measured WSSs provide additional information in liver disease assessments.

Combination of compressed sensing and two-dimensional parallel imaging can reduce the scan time for arterial phase image of gadoxetic acid enhance liver MR without degradation of image quality compared to parallel imaging alone

Dong Ho Lee ${ }^{1}$, Hyo-jin Kang ${ }^{1}$, Eun Ju Kim² ${ }^{2}$ Jeong Min Lee ${ }^{1}$, and Hwaseong Ryu ${ }^{1}$

${ }^{1}$ Radiology, Seoul National University Hospital, Seoul, Republic of Korea, ${ }^{2}$ Philips Healthcare, Seoul, Republic of Korea

Using combination of compressed sensing and parallel imaging for arterial phase image acquisition in gadoxetic acid enhanced liver MR

Ancillary imaging features for differentiation of hypervascular hepatic tumors on Gadoxetic acid-enhanced MR imaging

Hyun Jeong Park ${ }^{1}$ and Young Kon $\mathrm{Kim}^{2}$

${ }^{1}$ radiology, Chung-ang university hospital, Seoul, Republic of Korea, ${ }^{2}$ radiology, Samsung medical center, Seoul, Republic of Korea

There are many types of hypervascular tumors that need to be differentiated from hepatocellular carcinoma (HCC) including focal nodular hyperplasia (FNH), hepatocellular adenoma (HCA), neuroendocrine tumor (NET), and intrahepatic cholangiocarcinoma (ICC). Since each tumor requires different treatment strategies, awareness and recognition of reliable imaging features that help precisely distinguish among these hypervascular tumors. Since these hypervascular tumors occasionally manifest overlapping imaging features, the accurate diagnosis of these tumors can still be challenging on MRI. Therefore, we conducted this study to determine ancillary imaging features that help differentiation of hypervascular hepatic tumors on gadoxetic acid-enhanced MRI.

Analysis of the Value of Texture Feature Calculated From Contrast-Enhanced MR Images in Differentiating FNH and HCC

Zhuo Shi ${ }^{1}$, Lizhi Xie ${ }^{2}$, XinMing Zhao ${ }^{1}$, and Han Ou-Yang ${ }^{1}$

${ }^{1}$ National Cancer Center/Cancer Hospital, Chinese Academy of Medical Sciences and Peking Union Medical College, Beijing, China, ${ }^{2}$ GE Healthcare, China, Beijing, China

For atypical FNH and HCC, conventional MR still has some limitations in differential diagnosis. Texture features can reflect the internal heterogeneity of the lesions. The purpose of this study was to find the texture features' differences between $\mathrm{FNH}$ and $\mathrm{HCC}$, in order to provide auxiliary diagnosis for the lesions which have difficulties in identification. 
${ }^{1}$ Department of Radiology, The First Affiliated Hospital of Dalian Medical University, Dalian, China, ${ }^{2}$ Huiying Medical Technology Co., Ltd., Beijing, China, ${ }^{3}$ GE Healthcare, MR Research, Beijing, China

Recently, radiomics has drawn attention in radiological research. Many scholars believe that radiomics may provide effective information for cancer diagnosis. In present study, we aim to distinguish hepatocellular carcinoma $(\mathrm{HCC})$ and hepatic cavernous hemangioma $(\mathrm{HCH})$ by precontrast MRI based radiomics and conclude that T2WI based radiomics using logistic regression classifier showed optimal diagnostic performance.

Liver Imaging Reporting and Data System Category 5: 3.0 T MR Predictors of Microvascular Invasion and Early Recurrence after Hepatectomy for Hepatocellular Carcinoma

Jingbiao Chen ${ }^{1}$, Qungang Shan ${ }^{1}$, Yao Zhang ${ }^{1}$, Hao Yang ${ }^{1}$, Ying Deng ${ }^{1}$, Jun Wu ${ }^{1}$, Bingjun $\mathrm{He}^{1}$, Sichi Kuang ${ }^{1}$, Claude B Sirlin ${ }^{2}$, and Jin Wang ${ }^{1}$

${ }^{1}$ Department of Radiology, the Third Affiliated Hospital of Sun Yat-sen University(SYSU), Guangzhou, China, ${ }^{2}$ Department of Radiology, University of California San Diego, San Diego, CA, Armenia

Hepatocellular carcinoma ( $\mathrm{HCC}$ ) is the fifth most common malignancy worldwide. Tumor microvascular invasion (MVI) predicts early posthepatectomy HCC recurrence, but usually cannot be determined until the tumor is surgically removed and analyzed histologically. The capability preoperatively to predict MVI and early postsurgical recurrence would represent an advance by informing optimal selection of surgical candidates. Here we show that in combination with a AFP (a tumor biomarker), two Liver Imaging Reporting and Data System (LIRADS) imaging features (mosaic architecture, corona enhancement) can predict MVI and three features (tumor number, mosaic architecture, absence of intralesional fat) can predict early recurrence.

Validation of a radiomics nomogram for preoperative prediction of early recurrence in hepatocellular carcinoma less than $5 \mathrm{~cm}$

Xiaohong Ma ${ }^{1}$, Jianyong Zhu ${ }^{1}$, Shuang Wang ${ }^{1}$, Meng Liang ${ }^{1}$, Bing Feng ${ }^{1}$, Jiangfen $\mathrm{Wu}^{2}$, Chunwu Zhou ${ }^{1}$, and Xinming Zhao

${ }^{1}$ Diagnostic Radiology, National Cancer Center/ Cancer Hospital, Chinese Academy of Medical Sciences and Peking Union Medical College, Beijing, China, ${ }^{2}$ GE Healthcare, China, Beijing, China

A high early recurrence $(E R)(\leq 1$ year) rate of hepatocellular carcinoma $(\mathrm{HCC})$ remains a significant concern. It is an important problem to find a powerful preoperative tool for predicting ER. This study aimed to development and validation of a Radiomics Nomogram for Preoperative Prediction ER in hepatocellular carcinoma less than $5 \mathrm{~cm}$. We found that the textural signature was a significant predictor for ER in HCC, and Radiomics nomogram performed better for preoperative prediction of ER in HCC.

The Effects of Helical Flow Patterns, Confluence Angle, and Flow Distribution in the Portal Vein

David Richard Rutkowski ${ }^{1,2}$, Scott B Reeder ${ }^{2,3,4,5,6}$, and Alejandro Roldán-Alzate ${ }^{1,2,4}$

${ }^{1}$ Mechanical Engineering, University of Wisconsin-Madison, Madison, WI, United States, ${ }^{2}$ Radiology, University of Wisconsin-Madison, Madison, WI, United States, ${ }^{3}$ Medical Physics, University of Wisconsin-Madison, Madison, WI, United States, ${ }^{4}$ Biomedical Engineering, University of Wisconsin-Madison, Madison, WI, United States, ${ }^{5}$ Medicine, University of Wisconsin-Madison, Madison, WI, United States, ${ }^{6}$ Emergency Medicine, University of Wisconsin-Madison, Madison, WI, United States

The hemodynamics of the liver in normal and diseased conditions are not fully understood. In this study, 4D flow MRI and computational modeling were used to analyze the effects of portal venous flow patterns at the spleno-mesenteric confluence. Specifically, the geometric configuration of the confluence on intra-hepatic portal circulation in healthy subjects and cirrhotic patients before and after a meal challenge was analyzed. Significant correlations between flow distribution, helicity, geometry, and flow patterns were observed, and differences between normal and pathological flow were also characterized.

MRI in the evaluation of liver involvement in pediatric patients with cystic fibrosis

Katherine J Carey ${ }^{1,2}$, Scott B Reeder ${ }^{1,2}$, Mark Kliewer $^{2}$, R. Paul Guillerman ${ }^{3}$, Diego Hernando ${ }^{1,2}$, and Scott K Nagle ${ }^{2}$

${ }^{1}$ Medical Physics, University of Wisconsin, Madison, WI, United States, ${ }^{2}$ Radiology, University of Wisconsin, Madison, WI, United States, ${ }^{3}$ Radiology, Texas Children's Hospital, Houston, TX, United States

In this prospective study of 15 pediatric cystic fibrosis subjects, we show that non-sedated comprehensive quantitative liver MRI is feasible. Furthermore, free-breathing $2 \mathrm{D}$ IDEAL IQ outperformed breath-held 3D IDEAL IQ in both image quality and repeatability of proton density fat fraction. Short term 1-2 week repeatability of MR elastography stiffness measurements were comparable with ultrasound elastography. Quantitative liver MRI in the pediatric cystic fibrosis population offers the ability to visualize structure and quantify hepatic steatosis and liver stiffness in a single exam. 
Paul de Heer ${ }^{1}$, Anne-Sophie van Schelt ${ }^{1}$, Jasper Schoormans ${ }^{2}$, Gustav J. Strijkers ${ }^{2}$, Bram F. Coolen ${ }^{2}$, Jurgen H. Runge ${ }^{1}$, Jaap Stoker ${ }^{1}$, and Aart J. Nederveen ${ }^{1}$

${ }^{1}$ Radiology and Nuclear Medicine, Academic Medical Center Amsterdam, Amsterdam, Netherlands, ${ }^{2}$ Biomedical Engineering \& Physics, Academic Medical Center Amsterdam, Amsterdam, Netherlands

In body MR many acquisitions respiratory motion correction is of great importance. In this study we compared self-gated motion-state binning to binning by both the pencil-beam navigator and a respiration belt by looking at the resulting image quality for each method. A 3D T1-weighted radial stack-of-stars turbo field echo (TFE) was acquired in three volunteers. The self-gated respiratory motion binning outperformed the other two methods in image quality and smoothness between the respiratory states. More subjects should be included in the study but for now it can be concluded that self-gating would be the preferred method of respiratory binning.

Evaluating of segmental liver function by using Gd-EOB-DTPA-enhanced MRI

Jiyun ZHANG $^{1}$ and Jian $\mathrm{LU}^{1}$

${ }^{1}$ Department of Radiology, the Third People's Hospital of Nantong, Nantong, China

The aim of this study is to investigate the value of Gd-EOB-DTPA-enhanced MRI in evaluating segmental liver function. Statistical analysis was used to evaluate the relationship between the $\triangle \mathrm{LMR}$ of each liver segment and liver function, as well as the $\triangle \mathrm{LMR}$ of different liver segments. Our quantitative study demonstrated that Gd-EOB-DTPA intake into hepatocytes was strongly affected by liver function. The segmental liver function can be evaluated via Gd-EOB-DTPA-enhanced MRI and calculation of the $\triangle \mathrm{LMR}$ may be a novel optional.

Amide Proton Transfer (APT) MR imaging and Magnetization Transfer (MT) MR imaging of liver cirrhosis: a clinical feasibility study

Xin Chen ${ }^{1,2}$, Guangbin Wang ${ }^{1}$, Jinyuan Zhou ${ }^{2}$, Yi Zhang ${ }^{2,3}$, Weibo Chen ${ }^{4}$, and Huihua $\mathrm{Li}^{1}$

${ }^{1}$ Shandong Medical Imaging Research Institute, Jinan, China, ${ }^{2}$ Department of Radiology, Johns Hopkins University School of Medicine, Baltimore, MD, United States, ${ }^{3}$ Center for Brain Imaging Science and Technology, Department of Biomedical Engineering, Zhejiang University, Hangzhou, China, ${ }^{4}$ Philips Healthcare, Shanghai, China

This study aimed to demonstrate the feasibility of the APT and MT MR imaging in depicting the liver cirrhosis at 3.0T. We compared MTR and APTw values in 11 healthy livers and 8 liver cirrhosis. The patients with liver cirrhosis showed a lower APTw values than healthy volunteers indicating APT imaging detectable mobile protein levels in the liver tissues. The patients with liver cirrhosis exhibited a higher MTR values than the healthy volunteers. Liver cirrhosis exhibited a significantly higher MTR, indicating indicating a higher concentration of biochemistry components in liver cirrhosis. We have shown that it is clinically feasible to perform APT and MT MR imaging of liver cirrhosis.

Vessel Size Imaging for Liver Fibrosis Staging Based on Dynamic Susceptibility Contrast Using SE/GRE-EPI Sequence: Comparison with US Elastography and Histopathological Correlation

Ruo-kun Li ${ }^{1}$, Fu-hua Yan ${ }^{1}$, Wei-bo Chen ${ }^{2}$, and He Wang ${ }^{3}$

${ }^{1}$ Radiology, Ruijin Hospital, Shanghai Jiaotong University School of Medcine, Shanghai, China, ${ }^{2}$ Philips Healthcare, Shanghai, China, ${ }^{3}$ Institute of Science and Technology for BrainInspired Intelligence, Shanghai, China

The study investigated the value of vessel size imaging (VSI) based on dynamic susceptibility contrast using SE/GRE-EPI sequence for liver fibrosis staging, compared with US elastography and correlated with histopathological results. We found that VVF and Nu value based on VSI were independent predicative factors of liver fibrosis (R2 $=0.566$, $P=0.002$ ). They had correlation with hepatic sinusoidal structures including parenchymal area (PA), sinusoidal area (SA), hepatocyte area (HA), sinusoidal perimeter (SP), SA/SP ratio, SA/SP index, and HA/SP index. Microvessel density (MVDdensity) and area (MVDarea). VSI has potential for liver fibrosis staging with good diagnostic capability similar to US elastography.

Model-based volumetric T2 mapping of the liver

Jeong Hee Yoon ${ }^{1}$, Yohan Son ${ }^{2}$, Berthold Kiefer $^{3}$, and Jeong Min Lee ${ }^{1}$

${ }^{1}$ Seoul National University Hospital, Seoul, Republic of Korea, ${ }^{2}$ Siemens Healthcare Korea, Seoul, Republic of Korea, ${ }^{3}$ Siemens Healthcare, Erlangen, Germany

T2 relaxation time estimation is able to aid liver tissue characterization by providing quantitative information of the tissue. 
${ }^{1}$ Sir Peter Mansfield Imaging Centre, University of Nottingham, Nottingham, United Kingdom, ${ }^{2}$ National Institute for Health Research (NIHR) Nottingham Biomedical Research Centre, Nottingham, United Kingdom

This study explores the feasibility of measuring magnetisation transfer in both the liver and kidney through acquisition of a full z-spectrum. In this study we developed a protocol to reduce artefacts from respiration and blood flow pulsatility and measured relative amounts of MT in both the liver and kidney medulla by modelling MT with a super-Lorentzian lineshape and fitting to the acquired spectrum. This will be relevant in monitoring fibrosis in abdominal organs.

Expanding the limits of cardiovascular MR: amyloid detection in the liver and spleen

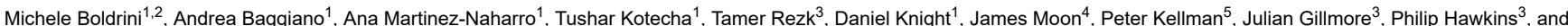
Marianna Fontana ${ }^{1}$

${ }^{1}$ CMR department, National Amyloidosis Center, UCL Royal Free Hospital, London, United Kingdom, ${ }^{2}$ Internal Medicine, Università degli Studi di Pavia, Pavia, Italy, ${ }^{3}$ National Amyloidosis Center, UCL Royal Free Hospital, London, United Kingdom, ${ }^{4}$ Barts Health - Barts Heart Centre, London, United Kingdom, ${ }^{5}$ National Institute of Health, Washington, MD, United States

In this study we evaluated the utility of bolus-only ECV maps in extra-cardiac AL amyloidosis by comparing it with SAP scintigraphy findings in liver and spleen. These two techniques where performed in a large prospective cohort of patients with suspected systemic AL amyloidosis and where compared in terms of (1) diagnostic accuracy in liver and spleen amiloidosis; (2) quantification of the liver and spleen amyloid deposits. This was done using a standard acquisition for cardiac studies, with no extra image acquisition or optimization for hypochondriac regions.

Traditional Poster

\section{Prostate}

Optimization of the Contrast-to-noise Ratio between Malignant and Non-malignant Prostate Tissue in T2-weighted MRI.

Shirin Sabouri ${ }^{1}$, Silvia D. Chang ${ }^{2}$, Edward C. Jones ${ }^{3}$, S. Larry Goldenberg ${ }^{4}$, Peter C. Black ${ }^{4}$, and Piotr Kozlowski ${ }^{2}$

${ }^{1}$ Physics and Astronomy, University of British Columbia, Vancouver, BC, Canada, ${ }^{2}$ Radiology, University of British Columbia, Vancouver, BC, Canada, ${ }^{3}$ Pathology and Laboratory Medicine, University of British Columbia, Vancouver, BC, Canada, ${ }^{4}$ Urologic Sciences, University of British Columbia, Vancouver, BC, Canada

T2W imaging is an important sequence in the PIRADSv2 guideline for scoring prostatic lesions. The apparent contrast between malignant and non-malignant tissues on T2W images depends on the time of echo (TE). In this study we have investigated the effect of TE on the contrast-to-noise ratio (CNR) between malignant and non-malignant tissues. We have acquired and analyzed T2W data from 12 patients. Our results show that CNR increases abruptly for TEs between 25 and $175 \mathrm{~ms}$. After CNR reaches its maximum at $175 \mathrm{~ms}$ it gradually decreases. Our findings may be used toward improvement of $\mathrm{T} 2 \mathrm{~W}$ protocols for diagnosis of prostatic carcinoma.

The Influence of Temporal Resolution on the Diagnostic Accuracy of DCE-MRI in Evaluation of Prostate Cancer.

Shirin Sabouri ${ }^{1}$, Silvia D. Chang ${ }^{2}$, Edward C. Jones ${ }^{3}$, S. Larry Goldenberg ${ }^{4}$, Peter C. Black ${ }^{4}$, and Piotr Kozlowski ${ }^{2}$

${ }^{1}$ Physics and Astronomy, University of British Columbia, Vancouver, BC, Canada, ${ }^{2}$ Radiology, University of British Columbia, Vancouver, BC, Canada, ${ }^{3}$ Pathology and Laboratory Medicine, University of British Columbia, Vancouver, BC, Canada, ${ }^{4}$ Urologic Sciences, University of British Columbia, Vancouver, BC, Canada

DCE-MRI is widely used for cancer detection, and is a part of PIRADS v2 guideline for scoring prostatic lesions. Diagnostic accuracy of DCE-MRI may depend on the rate of temporal sampling. In this study we have investigated the relationship between the rate of temporal sampling of DCE-MRI and the accuracy of detection of prostatic carcinoma. We have acquired and analyzed DCE-MRI data from 15 patients. Our results show that the accuracy of DCE-MRI in detection of prostatic carcinoma is not affected by sampling rates between 3.4 to 13.6 seconds.

Magnetic resonance spectroscopy of localized prostate cancer: assessment of antitumor effects of intra-prostatic hormone deprivation therapy

Jan Weis ${ }^{1}$, Michael Häggman² ${ }^{2}$ Sam Ladjevardi², Niklas Axen ${ }^{3}$, and Carl-Gustaf Gölander ${ }^{3}$

${ }^{1}$ Department of Medical Physics, Uppsala University Hospital, Uppsala, Sweden, ${ }^{2}$ Department of Urology, Uppsala University Hospital, Uppsala, Sweden, ${ }^{3}$ LIDDS AB, Uppsala, Sweden 
A novel controlled release formulation based on calcium sulphate as drug carrier loaded with the antiandrogen 2-hydroxiflutamide as the active pharmaceutical agent was injected locally into the prostate in patients with prostate cancer. Single-voxel and 2D MRSI using a surface coil were used to investigate the treatment efficiency. The results demonstrate usefulness of both MRS techniques to detect metabolic atrophy caused by long-term local hormone-deprivation therapy. The presence of metabolic atrophy reflects the antitumor effects of the study drug formulation 6 weeks after the intraprostatic injections.

Effect of Rectal Gas on Susceptibility Artifact in Prostate DWI

Eun Bin Lee ${ }^{1}$, Ely Felker ${ }^{2}$, David Lu², Kari Sorge ${ }^{2}$, and Kyunghyun Sung

${ }^{1}$ Bioengineering, Radiological Sciences, University of California, Los Angeles, Los Angeles, CA, United States, ${ }^{2}$ Radiological Sciences, University of California, Los Angeles, Los Angeles, CA, United States

Diffusion-weighted imaging (DWI) is a key component of multi-parametric prostate MRI; however, DWI is prone to susceptibility artifact occurring in the peripheral zone, where $70 \%$ of tumors are found. The purpose of this study was to qualitatively and quantitatively assess the effect of rectal gas on the presence of this artifact. The study found that in cases with no rectal gas $\left(<2 \mathrm{~cm}^{3}\right)$, image quality is excellent as a rule. When more than $2 \mathrm{~cm}^{3}$ of gas is present, a range of image quality is seen that is not correlated to the amount of gas present.

Whole-body MRI for prostate cancer at primary staging: interobserver concordance, diagnostic accuracy and protocol optimisation

Edward William Johnston ${ }^{1}$, Arash Latifoltojar ${ }^{1}$, Harbir Sidhu ${ }^{1}$, Elisenda Bonet-Carne ${ }^{1}$, Magdalena Sokolska ${ }^{1}$, Alan Bainbridge ${ }^{1}$, and Shonit Punwani

${ }^{1}$ Centre for Medical Imaging, University College London, London, United Kingdom

Whole body (WB) MRI is developing as a cancer staging platform in primary prostate cancer, although has not yet been adopted into clinical practice. In this study, we show that WB-MRI provides high levels of interobserver concordance, intermodality concordance and diagnostic accuracy for both nodal and metastatic bone disease, with higher levels of sensitivity than BS for metastatic disease, and similar performance to PET/CT. We also show that T2W and post contrast mDixon have no additive diagnostic value above T1W and DWI alone.

Image quality of WB-MRI in staging recurrent prostate cancer: a multicentre, multinational, multivendor, multiscanner study.

Edward William Johnston ${ }^{1}$, Alan Bainbridge ${ }^{1}$, Glenn Bauman ${ }^{2}$, Sue Chua ${ }^{3}$, lan Davis ${ }^{4}$, Rod Hicks ${ }^{5}$, Ur Metser ${ }^{6}$, Frederic Pouliot ${ }^{7}$, Andrew Scott $^{8}$, Jonathan Thiessen $^{2}$, Nina Tunariu $^{3}$, Andrew Weickhardt ${ }^{8}$, Louise Emmett ${ }^{9}$, and Shonit Punwani ${ }^{1}$

${ }^{1}$ Centre for Medical Imaging, University College London, London, United Kingdom, ${ }^{2}$ London Health Sciences Centre, London, ON, Canada, ${ }^{3}$ Royal Marsden Hospital, Sutton, United Kingdom, ${ }^{4}$ Monash, Melbourne, Australia, ${ }^{5}$ Peter McCallum Cancer Centre, Melbourne, Australia, ${ }^{6}$ University of Toronto, Toronto, ON, Canada, ${ }^{7}$ Laval Quebec, Quebec, QC, Canada, ${ }^{8}$ Olivia Newton-John Cancer Centre, Melbourne, Australia, ${ }^{9}$ St Vincents Cancer Center, Sidney, Australia

Whilst whole body (WB) MRI offers substantial promise in cancer staging, considerations regarding image quality are lacking in the literature, yet are essential for the effective delivery of the technique. Here we report the image quality of WB-MRI in 86 patients with suspected biochemical recurrence in prostate cancer in a trial carried out over 3 continents (Australia, America and Europe). We show that the image quality of WB-MRI varies substantially between anatomical sites and centres, particularly for diffusion-weighted sequences, which emphasizes the need to optimise sequences carefully prior to establishing a WB-MRI practice.

Comparison of Prostate Volume Measured by Transrectal Ultrasonography and Magnetic Resonance Imaging with the Actual Prostate Volume Measured after Radical Prostatectomy Sung Bin Park ${ }^{1}$ and Haesun $\mathrm{Choi}^{2}$

${ }^{1}$ Radiology, Chung-Ang University Hospital, Seoul, Republic of Korea, ${ }^{2}$ Diagnostic Radiology, MD Anderson Cancer Center, Houston, TX, United States

A determination of prostate gland volume facilitates an assessment of prostate disorders and, for prostate cancer, in conjunction with other parameters, can help predict the pathologic stage of disease, offer insights into the prognosis, and help predict treatment response Prostate volume can also be used for calculating prostate-specific antigen density (PSAD) when selecting active surveillance candidates.

The measuring the volume of prostate removed by radical prostatectomy as performed in the present study may be an appropriate way to assess the actual prostate volume even though it may be cancerous. The present study aimed to compare the prostate volume, as measured by TRUS and by MRI, with that of the actual prostate volume measured after a radical prostatectomy. 
${ }^{1}$ Department of Radiodiagnosis, All India Institute of Medical Sciences, New Delhi, India, ${ }^{2}$ Department of Urology, All India Institute of Medical Sciences, New Delhi, India, ${ }^{3}$ Department of NMR, All India Institute of Medical Sciences, New Delhi, India

We report a prospective evaluation of prostate imaging reporting, archiving and data system version 2 (PIRADSv2) for multiparametric MRI (mpMRI) taking histopathology of radical prostatectomy specimens as reference standard. PIRADSv2 for mpMRI is easy to apply for detection of cancer of prostate (CaP) at optimal cut-offs of $\geq 3$ for cancer as a whole and $\geq 4$ for intermediate and high grade cancers. It is an accurate system to diagnose clinically significant disease. 26 patients having a biopsy-proven CaP, were investigated at $3.0 \mathrm{~T}$ using mpMRI, followed by radical prostatectomy within 1 month. Gleason grade group from radical prostatectomy specimens and ROC curve analysis was used to determine the accuracy for cut-offs for scores of PIRADSv2

Multi-parametric MRI evaluation of prostate cancer volume: correlation with whole mount pathology

Chongpeng Sun ${ }^{1,2}$, Aritrick Chatterjee ${ }^{1}$, Ambereen Yousuf $^{1}$, Tatiana Antic ${ }^{3}$, Scott Eggener ${ }^{4}$, Gregory Karczmar ${ }^{1}$, and Aytekin Oto ${ }^{1}$

${ }^{1}$ Department of Radiology, University of Chicago, Chicago, IL, United States, ${ }^{2}$ Department of Radiology, Guangzhou Medical University, Guangzhou, China, ${ }^{3}$ Department of Pathology, University of Chicago, Chicago, IL, United States, ${ }^{4}$ Department of Urology, University of Chicago, Chicago, IL, United States

This study compared prostate cancer volume determined on different multi-parametric MRI sequences: T2W imaging, ADC map and DCE-MRI with whole mount pathology in 17 patients. Tumor volumes were measured on T2W images, ADC maps and DCE-MRI by 2 radiologists and compared with reference standard volume measured from pathology. While lesion volume estimated using mpMRI sequences showed good correlation with pathology, T2W and ADC significantly underestimated, whereas DCE-MRI showed no significant difference. Therefore, DCE-MRI is the most effective sequence for estimating PCa volume with the highest accuracy compared to T2W-imaging and ADC maps and has similar good correlation and precision.

A comparison of biexponetial fitting and spectral modelling methods for T2 mapping of prostate cancer

Dominic Carlin ${ }^{1,2}$, Matthew R Orton ${ }^{1,2}$, Veronica A Morgan ${ }^{1,2}$, David J Collins ${ }^{1,2}$, and Nandita M deSouza ${ }^{1,2}$

${ }^{1}$ CRUK Imaging Centre, Institute of Cancer Research, London, United Kingdom, ${ }^{2}$ MRI Unit, The Royal Marsden NHS Foundation Trust, Sutton, United Kingdom

Spectral modelling and model fitting were compared for quantitative T2 mapping of the prostate. 32-echo data were acquired from 11 patients with biopsy-proven prostate cancer at 3T. There was excellent correlation between the two approaches for estimates of T2-short, T2-long and luminal water fraction ( $r=0.96,0.71,0.94$ respectively). Luminal water fractions were significantly higher in normal peripheral and transition zones using the model fitting approach $(P=0.04$ and $<0.01$ respectively), but were comparable in tumor. The larger quantitative difference between tumour and normal tissue could mean model fitting is superior for qualitative assessment in prostate cancer.

Characterising prostate tumour growth patterns in men on active surveillance: linking ADC features to growth kinetics

Dominic Carlin ${ }^{1,2}$, Veronica A Morgan ${ }^{1,2}$, Chris Parker ${ }^{1,3}$, and Nandita M deSouza ${ }^{1,2}$

${ }^{1}$ CRUK Imaging Centre, Institute of Cancer Research, London, United Kingdom, ${ }^{2}$ MRI Unit, The Royal Marsden NHS Foundation Trust, Sutton, United Kingdom, ${ }^{3}$ Urology Department, The Royal Marsden NHS Foundation Trust, Sutton, United Kingdom, Sutton, United Kingdom

Tumor growth kinetics of low-risk prostate cancer in 15 men managed by active surveillance with size increase on repeat MRI were correlated with ADC histogram metrics. Measurements were made over 3 time-points at least 1 year apart (mean $3.6 \pm 0.95$ years). Median growth was $23.1 \%$ in the first interval and $49.8 \%$ in the second. ADC reduced over time. Accelerated growth during the second time interval correlated with the increase in interquartile range $(r=0.6, p=0.02)$ and shift to more positive skew $(r=-0.56, p=0.03)$ seen during the first time interval, suggesting that increasing heterogeneity and reducing ADC may signal accelerated growth.

Comparison of multiparametric MRI and MRI-ultrasound fusion guided biopsy for prostate cancer diagnosis

Renee F. Cattell ${ }^{1,2}$, James J. Kang ${ }^{2}$, Sarah Dacosta ${ }^{2}$, Matthew A. Barish² ${ }^{2}$ Howard L. Adler ${ }^{3}$, Massimiliano Spaliviero ${ }^{3}$, Marlene Zawin² ${ }^{2}$ Haifang Li², and Tim Q. Duong ${ }^{2}$

${ }^{1}$ Department of Biomedical Engineering, Stony Brook University, Stony Brook, NY, United States, ${ }^{2}$ Department of Radiology, Stony Brook University, Stony Brook, NY, United States,

${ }^{3}$ Department of Urology, Stony Brook University, Stony Brook, NY, United States

High false-positive rates of prostate cancer diagnosis techniques have resulted in unnecessary biopsies and increased costs of care. This study compared the prostate cancer diagnosis by multiparametric MRI and MRI-ultrasound fusion guided biopsy at our institution, with the ultimate goal of improving MRI diagnosis of prostate cancer. At our institution, multiparametric MRI PI-RADS scores and MRI-ultrasound fusion guided biopsy Gleason scores agreed $46-57 \%$ of the time which falls within the ranges in literature. 
Multi parametric magnetic resonance imaging for the detection of prostate cancer: combination of T2-weighted, diffusion tensor imaging and magnetic resonance spectroscopic imaging

Neda Gholizadeh ${ }^{1}$, Peter B Greer ${ }^{2,3}$, John Simpson $^{2,3}$, Jim Denham $^{4}$, Peter Lau ${ }^{5,6}$, and Saadallah Ramadan 1,6

${ }^{1}$ Health Science, The University of Newcastle, Newcastle, Australia, ${ }^{2}$ Radiation oncology, Calvary Mater Newcastle, Newcastle, Australia, ${ }^{3}$ Medical Physics, The University of Newcastle, Newcastle, Australia, ${ }^{4}$ Radiation oncology, calvary mater newcastle, Newcastle, Australia, ${ }^{5}$ Radiology, Calvary Mater Newcastle, Newcastle, Australia, ${ }^{6}$ Imaging Centre, Hunter Medical Research Institute (HMRI), Newcastle, Australia

The aim of this study was to determine the diagnostic performance of mp-MRI using T2WI, DWI, DTI and MRSI for prostate cancer patients with various Gleason scores. mp-MRI using T2WI, DWI, DTI and MRSI on 12 prostate cancer patients. The area under receiver operating characteristic (ROC) curve of T2WI+DWI and T2WI+DWI+DTI+MRSI images were generated and used to evaluate the performance of $\mathrm{mp}-\mathrm{MRI}$ for discriminating cancer and healthy regions. Our results suggest that mp-MRI using DWI, DTI and MRSI in combination with structural T2WI improve performance for discrimination of cancer and healthy prostate tissues.

A framework for intensity-based affine registration of multiparametric prostate MRI via mutual information and genetic algorithms

Ethan Leng ${ }^{1}$, David Porter ${ }^{2}$, Andrew Larson ${ }^{1}$, Xiaoxuan $\mathrm{He}^{1}$, Benjamin Spilseth ${ }^{3}$, and Gregory J. Metzger ${ }^{1}$

${ }^{1}$ Center for Magnetic Resonance Research, University of Minnesota, Minneapolis, MN, United States, ${ }^{2}$ Minnesota Supercomputing Institute, University of Minnesota, Minneapolis, MN, United States, ${ }^{3}$ Department of Radiology, University of Minnesota, Minneapolis, MN, United States

An image registration framework was developed to perform 3D, affine, intensity-based co registration of multiparametric MRI series using mutual information as the similarity metric. The proposed methods include corrections to compensate for the effects of an endorectal coil, which is commonly used in prostate MRI. Experiments to characterize the registration method demonstrate that it is theoretically accurate to within $1.0 \mathrm{~mm}$ (when estimating the translation component). Qualitatively, significant improvements are seen in the co-localization of parametric maps with the anatomic images. The proposed framework may readily be integrated into a CAD system for prostate cancer detection.

Radiomics assessment of prostate cancer grade using texture features from DWI,T1WI and T2WI

ZHANG LI ${ }^{1}$, ZHANG XIAOLING ${ }^{1}$, and ZHUO ZHIZHENG ${ }^{2}$

${ }^{1}$ Shaanxi Provincial People's Hospital,Xi'an, Xi'an, China, ${ }^{2}$ Philips Healthcare,Beijing, China, Beijing, China

The purpose of this study was to investigate the value and diagnostic efficiency of DWI,T1WI and T2WI using texture analysis for discriminating the gleason scores of prostate cancer. The results of this study indicate that texture analysis may provide a new method for Gleason classification of prostate cancer. A radiomics model of textural features from T2WI and $A D C$ maps have a good diagnostic accuracy in patients of a prostate cancer. Quantitative textural analysis may help distinguish low cancers form high- or intermediate-grade cancer with high sensitivity and moderate specificity.

Prostate imaging at 7T using multi-acquisition SSFP with parallel transmission and low SAR RF pulses.

Benjamin R Knowles ${ }^{1}$, Arthur W Magill ${ }^{1}$, and Mark E Ladd ${ }^{1}$

${ }^{1}$ Medical Physics in Radiology, German Cancer Research Center (DKFZ), Heidelberg, Germany

Prostate imaging at Ultra High Field suffers from SAR limitations and B1 inhomogeneity. This is especially effects Turbo Spin Echo for T2 weighted imaging, although this contrast holds significant clinical value. Steady-State Free Precession offers a T2/T1 contrast with lower flip angles and potentially lower SAR. In this study, multi-contrast CISS imaging was investigated for prostate imaging. VERSE pulses were implemented to reduce SAR. Results show good contrast in the prostate between the peripheral and transitional zones, comparable to that observed in TSE images. The use of VERSE pulses greatly reduces SAR and maintains contrast.

Evaluating the Role of PIRADS V2 and TRUSgBX for Improving Detection of Clinically Significant Prostate Cancer at Radical Prostatectomy

Alireza Ziaei ${ }^{1}$, Francesco Alessandrino ${ }^{1,2}$, Mark Vangel $^{3}$, Tina Kapur ${ }^{1}$, Clare Mary Tempany $^{1}$, and Fiona Mary Fennessy ${ }^{1,2}$

${ }^{1}$ Dept. of Radiology, Harvard Medical School, Brigham and Women's Hospital, Boston, MA, United States, 2Dept. of Imaging, Dana-Farber Cancer Institute, Boston, MA, United States, ${ }^{3}$ Dept. of Radiology, Harvard Medical School, Massachusetts General Hospital, Charlestown, MA, United States

The aim of this retrospective study was to determine a role for PIRADS V2 in conjunction with TRUSgBX to predict the presence of clinically significant prostate cancer (csPCa) in treatment naïve men with pathology-proven prostate cancer who underwent TRUSgBX, followed by $3 \mathrm{~T}$ mp-MRI prostate, and subsequently underwent RP. Our findings suggest that adding PIRADS V2 assessment to TRUSgBX improves the prediction of final pathology for presence of indolent disease and csPCa, and may help alleviate the rate of upgrading at RP. 
mpMRI-based Machine-Learning Classifier Comparison for Gleason 4 Pattern Detection in Transition Zone and Peripheral Zone Prostate Lesions

Michela Antonelli^1 , Edward W Johnston ${ }^{\wedge 2}$, Sebastien Ourselin*1,3, and Shonit Punwani²

${ }^{1}$ Translational Imaging Group, CMIC, University College London, London, United Kingdom, ${ }^{2}$ Centre for Medical Imaging, University College London, London, United Kingdom,

${ }^{3}$ Dementia Research Centre, Department of Neurodegenerative Disease, UCL Institute of Neurology, London, United Kingdom

Multi-parametric MRI (mpMRI) can be used to non-invasively predict the presence of a Gleason 4 pattern in transition zone (TZ) and peripheral zone (PZ) prostate cancers. Here the performance of five machine-learning classifiers, which use mpMRI and clinical features, were compared. Analysis included a five-fold cross validation and a temporally separated validation to prove the generalisability of the classifiers. The results showed that PZ models can predict the presence of a Gleason 4 pattern better than TZ models. The statistically better PZ classifier is a linear regression model while for TZ the best classifier is Naïve Bayes model.

Ex vivo ultra-high-field 9.4-Tesla magnetic resonance elastography (MRE) in comparison to whole-mount pathology for improved prostate cancer diagnostics.

Rolf Otto Reiter ${ }^{1,2}$, Shreyan Majumdar ${ }^{1}$, Steven Kearney ${ }^{1}$, Thomas Royston ${ }^{1}$, Brandon Caldwell ${ }^{3}$, Rong-Wen Tain ${ }^{4}$, Kejia Cai $^{4}$, Cristian Luciano $^{1}$, Andre Kajdacsy-Balla ${ }^{5}$, Winnie Mar ${ }^{4}$, Michael Abern ${ }^{3}$, and Dieter Klatt ${ }^{1}$

${ }^{1}$ Richard and Loan Hill Department of Bioengineering, University of Illinois at Chicago, Chicago, IL, United States, ${ }^{2}$ Department of Radiology, Charité - Universitätsmedizin Berlin, corporate member of Freie Universität Berlin, Humboldt-Universität zu Berlin, and Berlin Institute of Health, Berlin, Germany, ${ }^{3}$ Department of Urology, University of Illinois at Chicago, Chicago, IL, United States, ${ }^{4}$ Department of Radiology, University of Illinois at Chicago, Chicago, IL, United States, ${ }^{5}$ Department of Pathology, University of Illinois at Chicago, Chicago, IL, United States

Despite the success of multiparametric magnetic resonance imaging (mpMRI) for the assessment of prostate cancer, it suffers from limitations such as a moderate inter-reader reliability and sub-optimal diagnostic accuracy. This is the first study for the assessment of 6 human prostate specimens without pathology fixation or prior radiation therapy using ex vivo $9.4-$-Tesla magnetic resonance elastography (MRE). Using whole-mount pathology as a reference, preliminary results show a sensitivity and specificity of $86 \%$ and $52 \%$, respectively. MRE has the potential to improve the differentiation of benign prostatic hyperplasia nodules from malignant lesions, which is a known limitation of mpMRI.

Motion-tolerant super-resolution reconstruction from multi-stack MR data

Sachin Jambawalikar ${ }^{1,2}$, Daniel Litwiller ${ }^{3}$, Michael Liu ${ }^{1}$, Rami Vanguri ${ }^{4}$, Simukayi Mutasa ${ }^{5,6}$, Zhengchao Dong $^{7,8}$, and Hiram Shaish ${ }^{1}$

${ }^{1}$ Radiology, Columbia University Medical Center, New York, NY, United States, ${ }^{2}$ Radiology, New York Presbyterian Hospital, New York, NY, United States, ${ }^{3}$ Global MR Applications and Workflow, GE Healthcare, New York, NY, United States, ${ }^{4}$ Biomedical Informatics, Columbia University, New York, NY, United States, ${ }^{5}$ Radiology, Columbia University Medical Center, NY, NY, United States, ${ }^{6}$ Radiology, New York Presbyterian Hospital, NY, NY, United States, ${ }^{7}$ New York State Psychiatric Institute, New York, NY, United States, ${ }^{8}$ Psychiatry, Columbia University, 10032, NY, United States

Image super resolution reconstruction (ISRR) is a technique that may be useful for generating fast, motion tolerant 3D reconstructed images from multi stack data. We provide initial results of a multi-step ISRR approach using patch-to-volume reconstruction(PVR) followed by a slice-by-slice convolutional neural network to further improve spatial resolution. Our methods provide improved measures of peak-SNR, and could be used to rapidly generate 3D volumes from multiple 2D stacks in fetal and abdominal imaging where constant motion requires short scan times as well as in pelvic imaging where high SNR requirements lead to long scan times and motion artifact. Motion artifact is a significant obstacle in these MRI applications resulting in image quality degradation and potentially limited diagnostic ability.

Correlation of Perfusion Parameters Between Intravoxel Incoherent Motion Diffusion-Weighted Imaging and Texture Analysis of Dynamic Contrast Enhancement Imaging for Diagnosis of Prostate Cancer in Central Zone and Hyperplasia

Dan Guo ${ }^{1}$, Ailian Liu ${ }^{1}$ Lihua Chen ${ }^{1}$, and Lizhi Xie ${ }^{2}$

${ }^{1}$ Radiology Department of The First Affiliated Hospital of Dalian Medical University, Dalian, China, ${ }^{2}$ GE Healthcare, MR Research China, Beijing, Beijing, China

This work assessed the diagnostic value of intravoxel incoherent motion diffusion-weighted (IVIM-DWI) and texture analysis of dynamic contrast enhancement (DCE) in prostate cancer in central zone $(\mathrm{CZ})$ and hyperplasia and the correlation of perfusion parameters of them.

Comparison of Radiomics and Quantitative ADC Measurements of Prostate PI-RADS v2 Lesions to Prospective Radiologist Performance

David Bonekamp ${ }^{1}$, Simon Kohl ${ }^{1}$, Manuel Wiesenfarth ${ }^{1}$, Patrick Schelb ${ }^{1}$, Jan-Philipp Radtke ${ }^{2}$, Michael Götz ${ }^{1}$, Philipp Kickingereder ${ }^{2}$, Kaneschka Yaqubi ${ }^{1}$, Bertram Hitthaler ${ }^{2}$, Nils Gählert ${ }^{1}$, Tristan Anselm Kuder ${ }^{1}$, Fenja Deister ${ }^{1}$, Martin Freitag ${ }^{1}$, Markus Hohenfellner ${ }^{2}$, Boris Hadaschik ${ }^{3}$, Heinz-Peter Schlemmer ${ }^{1}$, and Klaus Maier-Hein ${ }^{1}$

${ }^{1}$ German Cancer Research Center, Heidelberg, Germany, ${ }^{2}$ University Hospital Heidelberg, Heidelberg, Germany, ${ }^{3}$ University Hospital Essen, Essen, Germany 
Multiparametric MRI (mpMRI) has recently seen further standardization by introduction of the PI-RADS version 2 system. mpMRI/transrectal ultrasound (TRUS)-guided fusion biopsies have demonstrated ability to closely match the histopathology seen after radical prostatectomy. Radiomics is a novel approach to extract a large number of quantitative features from medical imaging and combination with machine learning has demonstrated potential in the classification of mpMRI of the prostate. Here, we aim to compare state of the art radiomics and machine learning with ADC measurements, and prospective radiologist assessment using PI-RADS version 2 (PIRADSv2) in the evaluation of cancer suspicious lesions of the prostate.

Voxel Level Radiologic-Pathologic Validation of DCE-MRI with ISUP Grade in Prostate Cancer

Qing Zhang ${ }^{1}$, Xiaoyu Lv ${ }^{1}$, Chengwei Zhang ${ }^{1}$, Qinglei Zhang ${ }^{2}$, Ming Li ${ }^{2}$, Yao Fu ${ }^{3}$, Jun $\mathrm{Xie}^{4}$, Jiangfen Wu ${ }^{5}$, Bing Zhang ${ }^{2}$, and Hongqian Guo ${ }^{1}$

${ }^{1}$ Department of Urology, Drum Tower Hospital, Medical School of Nanjing University, Institute of Urology, Nanjing University, Nanjing, China, ${ }^{2}$ Department of Radiology, Drum Tower Hospital, Medical School of Nanjing University, Nanjing, China, ${ }^{3}$ Department of Pathology, Drum Tower Hospital, Medical School of Nanjing University, Nanjing, China, ${ }^{4}$ United Imaging Healthcare Co., Ltd, Shanghai, China, ${ }^{5}$ GE Healthcare, Nanjing, China

The biggest challenge in patients with newly diagnosed PCa is shifting from cancer detection or staging alone to identifying them with aggressive disease. The PI-RADS $v 2$ recognizes the role of DCE-MRI is limited but is essential. This work presented a radiology pathology correlation framework that enabled identification of promising in vivo DCE MRI markers of PCa risk at voxel level. The relationship between ISUP grade and DCE-MRI (Ktrans and Kep) suggests that it may be used as a component of active surveillance to noninvasively detect high-grade PCa and affect staging and treatment.

In-bore MR guided prostate biopsy using multiparametric MRI to avoid unnecessary biopsies

Sujeet K Mewar ${ }^{1}$, Sanajy Sharma ${ }^{2}$, Ekta Dhamijia ${ }^{3}$, Rupsa Bhattacharjee ${ }^{4}$, Sanjay Thulkar $^{3}$, Pradeep Kumar ${ }^{1}$, Virendra Kumar ${ }^{1}$, Senthil S Kumaran ${ }^{1}$, Siddhartha D Gupta ${ }^{5}$, Rajeev Kumar $^{6}$, and Naranamangalam R Jagannathan ${ }^{1}$

${ }^{1}$ Department of NMR and MRI Facility, All India Institute of Medical Sciences, New Delhi, India, ${ }^{2}$ Department of Radiology, All India Institute of Medical Sciences, New Delhi, India, ${ }^{3}$ Department of Radiology, IRCH, All India Institute of Medical Sciences, New Delhi, India, ${ }^{4}$ Philips Health Systems, Philips India Ltd., Gurgaon, India, ${ }^{5}$ Department of Pathology, All India Institute of Medical Sciences, New Delhi, India, ${ }^{6}$ Department of Urology, All India Institute of Medical Sciences, New Delhi, India

We report the results of the pilot study carried out using multiparametric $(\mathrm{mp}) \mathrm{MRI}$ and in-bore MRI-guided prostate biopsy for detection of PCa to reduce the number of unnecessary biopsies. 11 patients were recruited based on prostate specific antigen $>4 \mathrm{ng} / \mathrm{ml}$ and abnormal digital rectal examination. In-bore MRI targeted lesions with high PIRADS scores ( 3 to 5 ) were correlated with the histopathological findings. The average ADC in PCa patients was significantly lower than the prostatitis and BPH patients. Out of 11 patients, 3 showed adenocarcinoma, 5 prostatitis and $3 \mathrm{BPH}$

Multiparametric MRI methods development for clinical prostate imaging at $7 \mathrm{~T}$

Gregory J. Metzger ${ }^{1}$, Ryan Kalmoe ${ }^{1}$, Arcan Erturk ${ }^{1}$, Xiaoxuan $\mathrm{He}^{1}$, Sudhir Ramanna ${ }^{1}$, Ethan Leng ${ }^{1}$, Christopher Warlick ${ }^{1}$, and Benjamin Spilseth ${ }^{2}$

The advantages of increased SNR drive the spread of applications to 7T. While methods and hardware continue to improve, the potential to perform a full multiparametric exam exploiting the advantages of ultrahigh magnetic fields becomes possible but has yet to be investigated. We explore a full MRI exam including anatomic, diffusion and dynamic contrast enhanced MRI (DCEMRI) methods at $7 \mathrm{~T}$ and compare them against $3 \mathrm{~T}$ acquisitions in a patient population with various coil configurations: surface coils and surface combined endorectal coils.

Traditional Poster

\section{Body: Liver Fat \& NASH}

Exhibition Hall 2578-2587

Wednesday 16:15 - 18:15

In Vivo MRI Monitoring of the Induction and Reversal of Non-Alcoholic Steatohepatitis in a Rat Model

Amy H Herlihy ${ }^{1}$, Antigoni Ekonomou ${ }^{2}$, Camilla Simmons ${ }^{2}$, Matteo Milanesi ${ }^{1}$, Catherine Kelly ${ }^{1}$, and Po-Wah So ${ }^{2}$

${ }^{1}$ Perspectum Diagnostics Ltd, Oxford, United Kingdom, ${ }^{2}$ Neuroimaging, King's College London, London, United Kingdom

Steatosis and steatohepatitis (NASH) may be attenuated by calorie restriction and/or exercise if intervention is early enough. However, steatosis/NASH is generally asymptomatic, and when clinical signs are observed, simple lifestyle interventions are no longer effective. Thus, there is a real clinical need to detect steatosis/NASH early but also to monitor putative therapies. A methionine-choline-deficient diet leads to NASH in rats, that is readily reversible when rats are placed back on a methionine-choline replete diet. This model will be used to assess the ability of MRI to detect the induction of, and reversal of steatosis, in vivo. 
Non-alcoholic Fatty Liver Disease Assessment in Obese and Non-obese Pregnant Women with Water-Fat MRI

Stephanie A Giza ${ }^{1}$, Simran Sethi ${ }^{1}$, Takashi Hashimoto ${ }^{2,3}$, Barbra de Vrijer $^{2,4}$, and Charles A McKenzie ${ }^{1,2}$

${ }^{1}$ Medical Biophysics, Western University, London, ON, Canada, ${ }^{2}$ Division of Maternal, Fetal and Newborn Health, Children's Health Research Institute, London, ON, Canada, ${ }^{3}$ Obstetrics and Gynecology, Kagoshima City Hospital, Kagoshima, Japan, ${ }^{4}$ Obstetrics and Gynaecology, Western University, London, ON, Canada

Proton density fat fraction (PDFF) was used to assess fatty liver of pregnant women with normal and obese body mass indexes (BMI). No significant difference was found in the mean hepatic PDFF between the two groups $(\mathrm{p}=0.28)$. One normal BMI woman and one obese woman had elevated hepatic PDFF measurements.

Validation of magnetic resonance imaging-proton density fat fraction for hepatic fat content in healthy Asian population

Sonal Krishan ${ }^{1}$

${ }^{1}$ Radiology, Medanta Hospital, Gurgaon, India

The primary purpose of this work was to determine the precision of clinical MR imaging-PDFF hepatic fat quantification, to look at spatial heterogeneity in all the Couinad segments, to establish normative data and least significant change in Indian population. Our study has shown that there is no systematic or significant difference in the right versus left lobe, or any of the liver segments in patients with grade 0 steatosis. The least significant change of liver fat that can be measured reliably using MR imaging-PDFF is $2.1 \%$. Mean hepatic fat content calculated by MR imaging-PDFF is $2.89 \%(95 \% \mathrm{Cl}, 1 \%-6.8 \%)$ in normal Indian population. The current study is the first study determining normative data of hepatic fat content in histologically proven grade 0 steatosis population from India.

Validation of goose liver fat measurement by CSE-MRI with biochemical extraction as reference

$\mathrm{Li} \mathrm{Xu}^{1}$, Yangyang Duanmu ${ }^{1}$, Xiaoqi Wang ${ }^{2}$, Glen M Blake ${ }^{3}$, Peng Wang ${ }^{4}$, Manling Zhang ${ }^{5}$, Chao Wang ${ }^{6}$, and Xiaoguang Cheng

${ }^{1}$ Department of Radiology, Beijing Jishuitan Hospital, Beijing, China, ${ }^{2}$ Philips Healthcare, Beijing, China, ${ }^{3}$ Biomedical Engineering Department, King's College London, London, United Kingdom, ${ }^{4}$ Department of Pathology, Capital Medical University Affiliated Beijing Ditan Hospital, Beijing, China, ${ }^{5}$ China national food \& safety supervision and inspection center, Beijing, China, ${ }^{6}$ Department of Statistics, Beijing Jishuitan Hospital, Beijing, China

This study aimed to validate chemical shift encoded magnetic resonance imaging (CSE-MRI) to assess hepatic steatosis. Twenty-two geese with a wide range of hepatic steatosis were collected, and proton density fat fraction by MRI (MRI-PDFF), biochemical triglyceride content, and histology were performed within the left lobe, upper and lower half of the right lobe of the geese livers. MRI correlated highly with chemical extraction $(r=0.949(p<0.001))$. Chemically extracted triglyceride was accurately predicted by MRI-PDFF $(Y=-1.8+0.773 * X)$. In conclusion, CSE-MRI measurement of goose liver fat was accurate and reliable compared with biochemical measurement.

Relationship between Proton Density Fat Fraction and Liver Triglyceride Composition estimated by 1H MR Spectroscopy

Gavin Hamilton ${ }^{1}$, Alexandra N Schlein ${ }^{1}$, Adrija Mamidipalli ${ }^{1}$, Yesenia Covarrubias ${ }^{1}$, Jonathan C Hooker ${ }^{1}$, Walter C Henderson ${ }^{1}$, Ethan Z Sy ${ }^{1}$, Jennifer Y Cui ${ }^{1}$, Rohit Loomba ${ }^{2}$, and Claude B Sirlin ${ }^{1}$

${ }^{1}$ Liver Imaging Group, Department of Radiology, University of California, San Diego, La Jolla, CA, United States, ${ }^{2}$ NAFLD Research Center, Division of Gastroenterology, Department of Medicine, University of California, San Diego, La Jolla, CA, United States

Liver triglyceride composition was estimated using ${ }^{1} \mathrm{H}$ MRS and compared to MRS estimated Proton Density Fat fraction (PDFF) to see if liver fat composition changes with PDFF. STEAM liver spectra were acquired in 263 adult subjects at 3 Tesla using breath-held, long-TR, multi-TE MRS to estimate PDFF and respiratory gated water-sated single TE MRS to estimate triglyceride composition. There is a significant change in the triglyceride composition of liver with changing PDFF, with the liver fat becoming more saturated as PDFF increases.

Diurnal Variation of Liver Fat Concentration

Timothy J Colgan ${ }^{1,2}$, Andrew J Van Pay ${ }^{1}$, Samir D Sharma ${ }^{1}$, and Scott B Reeder ${ }^{1,2,3,4,5}$

${ }^{1}$ Radiology, University of Wisconsin - Madison, Madison, WI, United States, ${ }^{2}$ Medical Physics, University of Wisconsin - Madison, Madison, WI, United States, ${ }^{3}$ Medicine, University of Wisconsin - Madison, Madison, WI, United States, ${ }^{4}$ Biomedical Engineering, University of Wisconsin - Madison, Madison, WI, United States, ${ }^{5}$ Emergency Medicine, University of Wisconsin - Madison, Madison, WI, United States 
Abnormal accumulation of intracellular triglycerides (hepatic steatosis) is the earliest and hallmark feature of nonalcoholic fatty liver disease (NAFLD). Confounder-corrected quantitative chemical-shift encoded MRI (CSE-MRI) is an accurate, precise and reproducible biomarker of hepatic steatosis as quantified by the proton density fat fraction (PDFF). However, the effect of meals and diurnal variability has not been established. In this study, we examined the variability of PDFF measurements resulting from meals, diurnal variation and between visits on different days. This study demonstrates that CSE-MRI liver fat estimation is not significantly affected by diurnal changes.

Effect of Signal to Noise Ratio and Estimator Type on Bias of Hepatic Proton Density Fat Fraction Measurement

Edward M Lawrence ${ }^{1}$, Nathan T Roberts ${ }^{1,2}$, Diego Hernando ${ }^{1,3}$, and Scott B Reeder ${ }^{1,3,4,5,6}$

${ }^{1}$ Radiology, University of Wisconsin-Madison, Madison, WI, United States, ${ }^{2}$ Electrical \& Computer Engineering, University of Wisconsin-Madison, Madison, WI, United States, ${ }^{3}$ Medical Physics, University of Wisconsin-Madison, Madison, WI, United States, ${ }^{4}$ Medicine, University of Wisconsin-Madison, Madison, WI, United States, ${ }^{5}$ Emergency Medicine, University of Wisconsin-Madison, Madison, WI, United States, ${ }^{6}$ Biomedical Engineering, University of Wisconsin-Madison, Madison, WI, United States

Proton-density fat-fraction (PDFF) is typically measured by calculating the mean PDFF value within a region of interest (ROI). However, the mean estimator has been shown to result in bias when signal-to-noise ratio (SNR) is low. This work characterizes the accuracy of median and maximum likelihood estimator (MLE) as alternative estimators for the measurement of liver PDFF. Our results demonstrate that at low-SNR, the mean estimator has a larger error than either the median or MLE values obtained from the same ROIs, when compared to the PDFF value obtained from spectroscopy, and had a bias of approximately $-1 \%$.

Measurement of Hepatic Lipid During Free Breathing with T2-Corrected Multiecho ${ }^{1} \mathrm{H}$ MR Spectroscopy

Jack Knight-Scott ${ }^{1}$, Adina Alazraki ${ }^{1,2}$, Miriam Vos ${ }^{2}$, Xiaodong Zhong ${ }^{3}$, and Brian Dale ${ }^{4}$

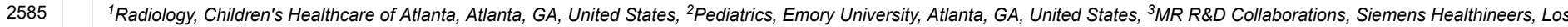
Angeles, CA, United States, ${ }^{4} M R R \& D$ Collaborations, Siemens Healthineers, Cary, NC, United States

Current MR techniques for quantifying hepatic fat through measurement of the proton density fat fraction (PDFF) require a breath hold that many patients find challenging. In this work, we show that when employing single voxel multiecho spectroscopy for measurement of the liver PDFF, breath holding and free breathing acquisitions yield similar results.

Long-term and short-term repeatability of hepatic proton density fat fraction measurement across MR field strengths in nonalcoholic fatty liver disease subjects and a phantom

Bohyun Kim ${ }^{1}$, Hye Jin Kim¹, Jei Hee Lee ${ }^{1}$, Hyo Jung Cho², and Jai Keun Kim ${ }^{1}$

${ }^{1}$ Radiology, Ajou University Hospital, Suwon, Republic of Korea, ${ }^{2}$ Internal Medicine, Ajou University Hospital, Suwon, Republic of Korea

Long-term and short-term repeatability of hepatic proton density fat fraction measurements was assessed across MR field strengths in nonalcoholic fatty liver disease subjects and a phantom. Our results showed that PDFF measurement have high short-term and long-term repeatability across the fields strengths, and patients undergoing a longitudinal PDFF measurement may be scanned regardless of MR field strength.

A study on the weighting factor investigating of liver parenchyma for 6-point interference Dixon fat percentage imaging accuracy in non-alcoholic fatty liver disease Seung-Man $\mathrm{Yu}^{1}$

The aim of this study was to determine the most accurate weighting factor for precise quantification of fatty liver when the 6-point interference Dixon fat percentage imaging technique is used by analyzing changes in WFs of fatty acid metabolites in liver. The importance of accurate WFs in the calculation of 6-pt-DIXON-based FP was confirmed in the phantom experiment. This study proposes average WF values that can be effectively used to acquire accurate 6-pt-DIXON FP images for non-alcoholic fatty liver. In addition, if the WFs of liver parenchyma FMs are applied, the accuracy of 6-pt-DIXON FP imaging can further increase.

Traditional Poster

\section{Body: MRE}


Jun Chen ${ }^{1}$, Robert Laird ${ }^{2}$, Qingyun Liu ${ }^{2}$, Brad Jr. Bolster ${ }^{3}$, Kevin Glaser ${ }^{1}$, Marianna Baum², and Richard Ehman ${ }^{1}$

${ }^{1} 2001$ st St Sw, Mayo Clinic, Rochester, MN, United States, ${ }^{2}$ Florida International University, Miami, FL, United States, ${ }^{3}$ Siemens Healthineers, Salt Lake City, UT, United States

As a non-invasive imaging technique for detecting and staging liver fibrosis, MR Elastography (MRE) is highly sensitive and specific. Conventional $2 \mathrm{D}$ liver GREMRE is very effective, and only takes about 1-2 minutes with multiple breath-holds (11-16 seconds, each). However, shorter acquisition times and fewer breath-holds are always desired for these examinations, especially when patients have difficulty holding their breath. In this study, we developed an 11-second hepatic MRE protocol based on SE-EPIMRE sequence, which was performed in a single breath-hold comfortably; the repeatability of repeated MRE scans was also assessed.

MR Elastography in Primary Sclerosing Cholangitis: Interobserver Agreement for Liver Stiffness Measurement

Safa Hoodeshenas ${ }^{1}$, Bogdan Dzyubak ${ }^{1}$, John E Eaton ${ }^{2}$, Richard L Ehman ${ }^{1}$, and Sudhakar K Venkatesh ${ }^{1}$

${ }^{1}$ Radiology, Mayo Clinic, Rochester, MN, United States, ${ }^{2}$ Gastroenterology and Hepatology, Mayo Clinic, Rochester, MN, United States

Primary sclerosing cholangitis (PSC) is a chronic liver disease characterized by heterogeneous distribution of increased stiffness in periphery, segmental or lobar pattern. The heterogeneity of liver stiffness has raised concerns for reproducibility of liver stiffness measurement (LSM). We performed interobserver agreement analysis for LSM with two readers drawing manual regions of interest (ROI) and with an automated algorithm. Our study results show that large geographical ROls including the focal regions of increased liver stiffnesses have excellent agreement between readers and automated method. Therefore large geographical ROIs using either manual or automated methods should be used for LSM in PSC patients.

The role of MRE in predicting the degree of esophageal varices in patients with hepatitis $B$ cirrhosis

Da-wei Yang ${ }^{1}$, zheng-han Yang ${ }^{1}$, zhen-chang Wang ${ }^{1}$, and Hon You ${ }^{2}$

${ }^{1}$ Captial medical university, Beijing friendship hospital, Beijing, China, ${ }^{2}$ Hepatology, Captial medical university, Beijing friendship hospital, Beijing, China

This abstract showed that liver and spleen stiffness value based on MRE was correlated well with the degree of esophageal varices, and they can be used to predict the degree of esophageal varices on hepatitis $b$ cirrhosis patients.

Inter reader agreement for liver Magnetic Resonance Elastography region-of-interest (ROI)-size, -overlap, -placement, and stiffness estimation in adults in a clinical trial

Adrija Mamidipalli ${ }^{1}$, Walter C. Henderson ${ }^{1}$, Jonathan C. Hooker ${ }^{1}$, Tanya Wolfson ${ }^{2}$, Yesenia Covarrubias ${ }^{1}$, Anthony Gamst ${ }^{2}$, Nikolaus Szeverenyi ${ }^{1}$, Gavin Hamilton ${ }^{1}$, Rohit Loomba ${ }^{3}$, and Claude B. Sirlin ${ }^{1}$

${ }^{1}$ Liver Imaging Group, Radiology, UCSD, San Diego, CA, United States, ${ }^{2}$ Computational and Applied Statistics Laboratory, UCSD, San Diego, CA, United States, ${ }^{3}$ NAFLD Research Center, Division of Gastroenterology, Department of Medicine, UCSD, San Diego, CA, United States

MR elastography (MRE) is an established technique for the non-invasive assessment of hepatic stiffness and fibrosis, and is commonly performed using a gradient-echo-acquisition of four slices through the widest portion of the liver. The mean liver-stiffness is calculated as the average of the ROI pixel values over all four stiffness map slices. Identification (drawing) of these ROIs is subjective, relying on reader judgment to assess the wave-quality. This study examines the inter-reader agreement of MRE-ROI-size, overlap and placement, and how they affect the MRE shear-stiffness values in adults with known or suspected nonalcoholic fatty liver disease.

Comparison of Breath-Hold (BH) and Respiratory-Triggered (RT) Fast Field Echo (FFE) Hepatic MR Elastography (MRE)

Hui Wang ${ }^{1}$, Tom Cull ${ }^{2}$, Jean Tkach ${ }^{3}$, Suraj D. Serai ${ }^{3}$, Andrew Trout $^{3}$, Charles Dumoulin $^{3}$, and Jonathan R. Dillman ${ }^{3}$

${ }^{1}$ Philips, Cincinnati, OH, United States, ${ }^{2}$ Philips, Wickliffe, $\mathrm{OH}$, United States, ${ }^{3}$ Radiology, Cincinnati Children's Hospital Medical Center, Cincinnati, OH, United States

We compared breath-hold $(\mathrm{BH})$ and respiratory-triggered $(\mathrm{RT})$ two-dimensional (2D) fast field echo (FFE) MR elastography (MRE) liver stiffness measurements in adult volunteers showing comparable results between $t$ techniques.

Can MR elastography be used to measure liver stiffness in patients with iron overload?

Suraj D Serai ${ }^{1}$ and Andrew T Trout ${ }^{2}$

${ }^{1}$ Radiology, CHOP, Philadelphia, PA, United States, ${ }^{2}$ Radiology, CCHMC, Cincinnati, OH, United States 
Untreated, iron overload causes hepatic fibrosis and cirrhosis, diabetes mellitus, hypogonadism, cardiomyopathy, dysrhythmias, and sudden death. In patients with liver iron overload, GRE based MRE techniques most likely fail due to very low signal from the liver. 2D Spin echo echo planar imaging (SE-EPI) based sequences have higher wave SNR compared with 2D GRE based MR elastography because of a higher number of wave cycles encoded per trigger (60 wave cycles per trigger vs three wave cycles per trigger in the typical 2D GRE acquisition sequence), which enables higher signal-intensity sampling of the phase waveform used to calculate the shear stiffness. In this study, our goal was to assess and demonstrate the applicability of a modified short TE, SE-EPI based MRE for staging liver fibrosis in select patients with liver iron overload conditions.

Assessment of Treatment Outcome in Chronic Hepatitis C Virus Infected Patients with Liver Stiffness Measured by Magnetic Resonance Elastography

Stephan Rodrigo Marticorena Garcia ${ }^{1}$, Heiko Tzschätzsch ${ }^{1}$, Christian Althoff ${ }^{1}$, Christian Burkhardt ${ }^{1}$, Michael Dürr ${ }^{2}$, Fabian Halleck ${ }^{2}$, Klemens Budde ${ }^{2}$, Korinna Jöhrens $^{3}$, Bernd Hamm ${ }^{1}$, Jürgen Braun ${ }^{4}$, Thomas Fischer ${ }^{1}$, Ingolf Sack ${ }^{1}$, and Jing Guo ${ }^{1}$

${ }^{1}$ Radiology, Charité - Universitätsmedizin Berlin, Berlin, Germany, ${ }^{2}$ Nephrology, Charité - Universitätsmedizin Berlin, Berlin, Germany, ${ }^{3}$ Pathology, Charité - Universitätsmedizin Berlin, Berlin, Germany, ${ }^{4}$ Medical Informatics, Charité - Universitätsmedizin Berlin, Berlin, Germany

High-resolution stiffness maps of the liver and kidney transplant (KTx) were generated after direct-acting antiviral therapy using multifrequency magnetic resonance elastography (MRE) and tomoelastography data processing in $\mathrm{KTx}$ recipients with chronic hepatitis $\mathrm{C}$ infection. Changes in liver stiffness after viral clearance were related to the immediate reduction in the inflammatory response in the early period and were stable until one year after end of treatment. MRE promises to be an early predictor for therapeutic success in HCV treatment.

Impact of Motion-Encoding Gradient (MEG) Direction, Slice Position and Slice Orientation on the estimation of Liver Stiffness using Magnetic Resonance Elastography (MRE) in clinical patients

Jiming Zhang ${ }^{1}$, Claudio Arena ${ }^{1}$, Debra Dees ${ }^{1}$, Melissa Andrews ${ }^{1}$, Afis Ajala ${ }^{2}$, and Raja Muthupillai ${ }^{1}$

${ }^{1}$ Diagnostic and Interventional Radiology, Baylor St Luke's Medical Center, Houston, TX, United States, ${ }^{2}$ Physics and Texas Center for Superconductivity, University of Houston, Houston, TX, United States

As an extension of our previous work done in healthy subjects, we evaluate the impact of the direction of motion-encoding gradient (MEG), slice orientation, and coverage on the estimation of LS in 99 clinical patients referred for MRE. The results from the study show that: (a) liver stiffness (LS) measured with MEG superimposed over RL and AP directions was higher than that of LS measured with MEG in the FH direction; (b) Slight variations in the angulation of the transverse slice has negligible impact on LS estimates; and (c) The percentage area of the liver in which LS can be confidently measured (confidence map area) can have substantial variations (independent of direction of MEG) between slices and therefore, it may be beneficial to acquire more than one slice in a clinical setting.

Comparison of the QIBA MRE ROI-drawing method to a method standardized by tracing liver parenchyma boundaries

Walter C Henderson ${ }^{1}$, Alexandra N Schlein ${ }^{1}$, Jonathan C Hooker ${ }^{1}$, Yesenia Covarrubias ${ }^{1}$, Tanya Wolfson ${ }^{2}$, Adrija Mamidipalli ${ }^{1}$, Jennifer Y Cui ${ }^{1}$, Yingzhen Zhang ${ }^{1}$, Ethan Z Sy ${ }^{1}$, Nikolaus M Szeverenyi ${ }^{1}$, Rohit Loomba ${ }^{3}$, and Claude B Sirlin ${ }^{1}$

${ }^{1}$ Liver Imaging Group, Department of Radiology, UC San Diego, La Jolla, CA, United States, ${ }^{2}$ Computational and Applied Statistics Laboratory, UC San Diego, La Jolla, CA, United States, ${ }^{3}$ NAFLD Research Clinic, Division of Gastroenterology, Department of Medicine, UC San Diego, La Jolla, CA, United States

While the Quantitative Imaging Biomarker Alliance (QIBA) draft recommendations on ROI placement in 2D MRE image analysis prescribe that only linear waves and parenchyma at least $1 \mathrm{~cm}$ from the liver edge be included, another abstract submitted to this meeting (Mamidipalli et al.) has found that analysts with equivalent experience and skill level draw significantly different ROIs when using these guidelines. This study compares the QIBA method of ROI placement to a method that is more standardized and inclusive, and compares the agreement and bias between each method on 2D MRE liver stiffness measurements.

Traditional Poster

\section{Body: Liver Iron}


Non-invasive estimation of liver iron concentration (LIC) by R2-MRI is often used for detection, grading and treatment monitoring in patients with suspected or known iron overload. The only current R2-MRI LIC estimation method with regulatory clearance is FerriScan $₫$, a proprietary analysis for biexponential R2-relaxometry. We implemented a nonproprietary biexponential R2-relaxometry using a "dictionary-search" algorithm, to reproduce the FerriScan $®$ results. In 38 patients with known or suspected iron overload, we demonstrated excellent reproducibility (by linearity and absolute agreement) in R2 and LIC between FerriScan $®$ and dictionary-search analyses, suggesting generalizability of the R2-MRI approach for LIC estimation.

R2*-Relaxometry Can Replace Histology for Detecting Slight Iron Overload in Patients with Early Stage Chronic Liver Disease: A Comparison of R2*, Histology, and Mass-Spectrometry

Markus Karlsson ${ }^{1}$, Mattias Ekstedt ${ }^{2}$, Mikael F Forsgren ${ }^{3}$, Nils Dahlström ${ }^{4}$, Bengt Norén ${ }^{3}$, Olof Dahlqvist-Leinhard ${ }^{4}$, Stergios Kechagias ${ }^{2}$, and Peter Lundberg ${ }^{1}$

${ }^{1}$ Department of Radiation Physics, and Department of Medical and Health Sciences and Center for Medical Image Science and Visualization (CMIV), Linköping University, Linköping, Sweden, ${ }^{2}$ Department of Medical and Health Sciences and Department of Gastroenterology and Hepatology, Linköping University, Linköping, Sweden, ${ }^{3}$ Center for Medical Image Science and Visualization (CMIV), Linköping University, Linköping, Sweden, ${ }^{4}$ Department of Medical and Health Sciences and Center for Medical Image Science and Visualization (CMIV), Linköping University, Linköping, Sweden

R2*-relaxometry can be used to non-invasively detect hepatic iron overload. However, most previous studies included patients with very high iron content. We sought to investigate if relaxometry reliably can detect lower levels of hepatic siderosis. R2* was therefore measured in patients with suspected chronic liver diseases of varying etiologies. We compared the relaxation rates to histological semiquantitative assessment as well as total liver iron content using mass spectrometry. There was good correlation between $\mathrm{R} 2^{*}$ and liver iron content. We also showed that R2*-relaxometry is better than histology when detecting slight iron overload.

Dynamic Monitoring of Liver Iron Overload and Chelation Therapy using Magnetic Resonance Imaging

Gregory Simchick ${ }^{1,2}$, Zhi Liu ${ }^{3}$, May Xiong ${ }^{3}$, and Qun Zhao ${ }^{1,2}$

${ }^{1}$ Physics and Astronomy, University of Georgia, Athens, GA, United States, ${ }^{2}$ Bio-Imaging Research Center, University of Georgia, Athens, GA, United States, ${ }^{3}$ Pharmaceutical \& Biomedical Sciences, University of Georgia, Athens, GA, United States

Many diseases have been associated with excessive iron in the liver. Therefore, the non-invasive detection of liver iron overload and the monitoring of iron chelation therapy is highly desirable. Presented here is a method to demonstrate the feasibility of this using MR-based $\$ \$ \$ R^{\wedge}\left\{{ }^{*}\right\}\{2\} \$ \$ \$$ and magnetic susceptibility quantification. Significant increases in $\$ \$ \$ R^{\wedge}\left\{{ }^{*}\right\}\{2\} \$ \$ \$$ and susceptibility (Glass' $\Delta$ values in the ranges of $[-4.29-3.23]$ and $[-2.55-2.23]$, respectively) are observed in iron overloaded livers in comparison to baseline measurements. After six doses of Polyrotaxane conjugated with Deferoxamine (rPR-DFO) iron chelation therapy administered over twelve days, $\Delta$ values of 0.13 and -0.09 are observed for $\$ \$ \$ R^{\wedge}\left\{{ }^{*}\right\}\{2\} \$ \$ \$$ and susceptibility, respectively, indicating that the differences are no longer significant and the treatment is effective.

Noise-corrected R2* estimation using 3D multi-gradient-echo Dixon for hepatic iron overload: Comparisons with 2D multi-gradient-echo sequences

Huimin $\mathrm{Lin}^{1}$, Stephan Kannengiesser ${ }^{2}$, Caixia Fư ${ }^{3}$, Jun Shen ${ }^{1}$, and Fuhua Yan ${ }^{1}$

${ }^{1}$ Ruijin Hospital, Shanghai Jiaotong University School of Medicine, Shanghai, China, ${ }^{2}$ MR Application Predevelopment, Siemens Healthcare, Erlangen, Germany, ${ }^{3}$ Application Development, Siemens Shenzhen Magnetic Resonance Ltd., Shanghai, China

Different combinations of acquisition and postprocessing for R2* estimation were compared: 3D multi-gradient-echo Dixon vs. 2D multi-gradient-echo, with/without fat saturation (FS); noise-corrected (NC) vs. fat-and-noise-corrected (FNC) fitting. Twenty patients suspected of hepatic iron overload, but not having steatosis, were included. 3D_NC_R2* showed excellent agreement with 2D NC_R2*. Up to medium R2*, this held also for 3D FNC R2* vs. 2D NC_R2*; at high R2*, fat modeling reduced R2*. 2DFS NC_R2* was also reduced. R2* standard deviation was lowest in 3D_FNC, and highest in 2DFS_NC. 3D multi-echo Dixon with noise correction is a promising technique for whole-liver iron quantification, but further analyses are necessary.

Gradient-Echo MRI for Liver Iron Content Determination employing $\mathrm{R}_{2}{ }^{*}$ Relaxometry: Influence of Gender and Disease

Arthur Peter Wunderlich ${ }^{1}, 2$, Sabrina Schweyer ${ }^{1}$, Daniel Frisch ${ }^{1}$, Justin Brosig $^{1},{\text { Holger } \text { Cario }^{3}, \text { Meinrad Beer }^{1} \text {, and Stefan Andreas Schmidt }}^{1}$

${ }^{1}$ Diagnostic and Interventional Radiology, University Ulm, Medical Center, Ulm, Germany, ${ }^{2}$ Section for Experimental Radiology, University Ulm, Medical Center, Ulm, Germany, ${ }^{3}$ Department of Pediatrics and Adolescent Medicine, University Ulm, Medical Center, Ulm, Germany

To investigate the relation between $\mathrm{R}_{2}{ }^{*}$ gained from gradient echo (GRE) MRI and liver iron concentration (LIC), we studied the influence of patient characteristics. 205 patients (92 $\mathrm{f}$, $113 \mathrm{~m}$; 98 with Thalassemia major, 31 with Sickle Cell Anemia, 15 with Diamond-Blackfan-Anemia) suspected for liver iron overload were scanned according to Ferriscan ${ }^{\circledR}$ with spin echo MRI to obtain reference LIC values, and GRE protocols suitable for LIC determination. GRE analysis based on manually drawn liver ROIs and relaxometry yielded $R_{2}{ }^{*}$ values. Correlation analysis of $R_{2}{ }^{*}$ to reference LIC revealed different correlation parameters between patient subgroups concerning disease and gender 
Ramin Jafari ${ }^{1}$, Anne Koehne de Gonzalez ${ }^{2}$, Yi Wang ${ }^{1,3}$, Thanh Nguyen ${ }^{3}$, Alexey Dimov ${ }^{1}$, Kofi Mawuli Deh ${ }^{3}$, Zhe Liu $^{1}$, Gary Brittenham $^{2}$, Martin Prince $^{3}$, and Pascal Spincemaille ${ }^{3}$

${ }^{1}$ Cornell University, Ithaca, NY, United States, ${ }^{2}$ Columbia University Medical Center, New York, NY, United States, ${ }^{3}$ Weill Cornell Medicine, New York, NY, United States

Precise measurement of liver iron content (LIC) in patients with transfusional iron overload is important in iron-chelation therapy. MRI can be used as a non-invasive method to measure iron levels in the liver. Typically, R2 and R2* based methods are used for this purpose. In this work, we use human liver explants to demonstrate the degree to which steatosis and fibrosis are confounding factors for $\mathrm{R}^{*}$ and quantitative susceptibility mapping in LIC measurement.

Ultrashort Echo Time Imaging for Quantification of Hepatic Iron Overload: Comparison of Current Acquisition and Fitting Methods via Simulations and Phantom Data

Aaryani Tipirneni-Sajja ${ }^{1}$, Ralf B. Loeffler ${ }^{1}$, Andrea N. Sajewski ${ }^{1}$, Jane S. Hankins ${ }^{2}$, and Claudia M. Hillenbrand ${ }^{1}$

$2603 \quad{ }^{1}$ Diagnostic Imaging, St. Jude Children's Research Hospital, Memphis, TN, United States, ${ }^{2}$ Hematology, St. Jude Children's Research Hospital, Memphis, TN, United States

Assessment of hepatic iron content by R2*-MRI is a non-invasive alternative to liver biopsy. R2* is typically measured by a multiecho gradient-echo (GRE) sequence, however, GRE fails in high iron cases when T2* decay is rapid. In recent years, ultrashort echo time (UTE) imaging has been proposed to increase the accuracy in R2* measurements in high and massive iron overload. Still, the accuracy of R2* measurements depends on acquisition parameters and curve fitting algorithms, which vary between institutions. The purpose of this study is to compare current $\mathrm{R} 2^{*}$ acquisition and fitting methods, and identify the optimal acquisition and fitting methods for clinical use.

Demonstration of linear correlation between R2* and liver iron concentration across multiple MR acquisition parameters at $1.5 \mathrm{~T}$ and $3 \mathrm{~T}$.

Richard Hayden Jones ${ }^{1}$, Jason Bentley ${ }^{2}$, Valentina Taviani ${ }^{3}$, Diego Hernando ${ }^{4}$, Scott Reeder ${ }^{5}$, and Shreyas Vasanawala ${ }^{1}$

${ }^{1}$ Radiology, Lucile Packard Children's Hospital, Palo Alto, CA, United States, ${ }^{2}$ Stanford University of Medicine Quantitative Sciences Unit, Stanford University, Palo Alto, CA, United States, ${ }^{3}$ GE Healthcare, Sunnyvale, CA, United States, ${ }^{4}$ Radiology, Medical Physics, University of Wisconsin, Madison, WI, United States, ${ }^{5}$ Radiology, Biomedical Engineering, Medical Physics, University of Wisconsin, Madison, WI, United States

We demonstrate a robust linear relationship between the concentration of liver iron and R2* measurements taken in any liver segment, with various planes of acquisition, slice thickness, flip angle, and echo spacing at 1.5T or 3T. As compared with Ferriscan, R2* imaging is faster, lower-cost, and requires no post-processing, and has better geographic availability compared to the gold standard of superconducting quantum interference devices.

Quantification of multiple organ iron deposition in transfusion dependent diseases using mDIXON-Quant technique

Qiaoling Wu ${ }^{1}$, Zhizheng Zhuo ${ }^{2}$, and Hongyan $\mathrm{Ni}^{3}$

${ }^{1}$ Tianjin University of Traditional Chinese Medicine, Tianjin, China, ${ }^{2}$ Clincial Science, Philips Healthcare, Beijing, China, ${ }^{3}$ Tianjin First Center Hospital, Tianjin, China

Iron overload is a common complication of transfusion dependent patients. Magnetic resonance imaging can be used for quantitative detection of iron deposition in transfusion dependent patients. A total intake of iron for transfusion was evaluated based on the mDIXON-Quant and 3D-FFE sequence respectively. Because the mdixon-quant can avoid the effect of fat on the iron overload evaluation, the mDIXON-Quant sequence can more accurately quantify iron deposition in liver and pancreas than 3D-FFE sequence. The quantitative application of mDIXON-Quant in detection of iron deposition in patients can provide reliable basis for iron chelation therapy in clinic.

Hepatic Iron overload estimation by proton density mDIXON Quant technique

Ane Ugarte ${ }^{1}$, Javier Sánchez-González ${ }^{2}$, Coloma Álvarez-de-Eulate ${ }^{1}$, José María Alustiza ${ }^{3}$, and Jose Ignacio Emparanza ${ }^{1,4}$

This work evaluates the utility of R2* obtained from multi-point multi-peak proton density fat fraction to assess iron overload and the accuracy of provided relaxation maps compared with more established multi-echo gradient echo sequence. 
Purpose: To develop a method to estimate total iron load of the whole liver. Methods: Multi gradient-echo pulse sequence was applied to 17 patients with varying degrees of liver iron content (LIC). LIC was measured via T2* mapping on a voxel-by-voxel basis. Liver was segmented with a semi-automated dual-clustering method. Total iron load was estimated by numerically integrating the LIC histogram. Results: This assessment of iron load presents a noninvasive whole liver alternative to liver biopsies. Conclusion: T2* relaxometry and segmentation provide a novel method for iron content quantification at the organ level that can easily be adapted in clinics.

Test-retest Repeatability of R2* Mapping and Quantitative Susceptibility Mapping for Liver Iron Quantification

Ante Zhu' ${ }^{1,2}$, Timothy J. Colgan², Scott B. Reeder ${ }^{1,2,3,4,5}$, and Diego Hernando ${ }^{2,3}$

${ }^{1}$ Biomedical Engineering, University of Wisconsin-Madison, Madison, WI, United States, ${ }^{2}$ Radiology, University of Wisconsin-Madison, Madison, WI, United States, ${ }^{3}$ Medical Physics, University of Wisconsin-Madison, Madison, WI, United States, ${ }^{4}$ Medicine, University of Wisconsin-Madison, Madison, WI, United States, ${ }^{5}$ Emergency Medicine, University of WisconsinMadison, Madison, WI, United States

Liver iron concentration is widely recognized as the best overall metric of total body iron content. Accurate and precise (repeatable) non-invasive measurements of liver iron concentration are needed. In this work, we assessed the test-retest repeatability of R2* mapping and quantitative susceptibility mapping (QSM) in patients with liver iron overload at $1.5 \mathrm{~T}$. Our test-retest measurements demonstrate good agreement in different protocols for R2* quantification but large limits of agreements for QSM susceptibility estimates. Further optimization of QSM techniques is needed to improve test-retest repeatability.

Robust multi-parametric mapping for abdomen imaging

Young-Joong Yang ${ }^{1}$, Jong-Hyun Yoon ${ }^{1}$, Jin-Soo Kim ${ }^{1}$, and Chang-Beom Ahn ${ }^{1}$

A robust abdominal multi-parametric mapping using multi-echo data is proposed. Reconstructed maps are water, fat images, quantitative susceptibility map (QSM), and R2* map. Fat fraction and iron deposition in the liver may be important parameters for diagnosis. Challenges to the abdominal mapping include large field inhomogeneity, phase wrapping, phase variations from water and fat signal, chemical shift, and physiological motions. We applied simultaneous unwrapping phase and error recovery from inhomogeneity (SUPER) technique to correct field inhomogeneity and phase wrapping. The technique is stably applicable to objects containing water and fat signal, and is also useful as a preprocessing for QSM.

Traditional Poster

\section{Body: Liver Imaging Using Perfusion, Diffusion, T1, \& T1rho}

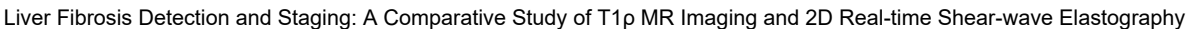

Ruo-kun Li' ${ }^{1}$ Fu-hua Yan ${ }^{1}$, Xin-pin Ren², and Wei-bo Chen ${ }^{3}$

${ }^{1}$ Radiology, Ruijin Hospital, Shanghai Jiaotong University School of Medcine, Shanghai, China, ${ }^{2}$ Ultrasound, Ruijin Hospital, Shanghai Jiaotong University of Medcine, Shanghai, China,

There was moderate positive correlation between fibrosis stage and T1 $\rho$ values ( $r=0.566 ; 95 \% \mathrm{Cl} 0.291-0.754 ; \mathrm{P}<0.0001)$, and LS value $(r=0.726 ; 95 \% \mathrm{Cl} 0.521-0.851 ; \mathrm{P}=0.003)$. T1 $\rho$ values showed moderate positive correlations with LS values ( $r=0.693$; $95 \%$ confidence interval $[\mathrm{Cl}]: 0.472-0.832 ; \mathrm{P}<0.0001)$. Areas Under ROC (AUROCs) were 0.861 (95\% Cl: $0.705-$

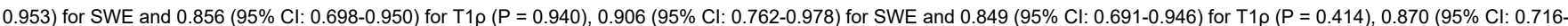

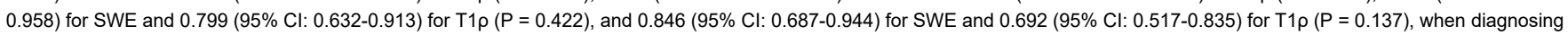
liver fibrosis with $\geq F 1, \geq F 2, \geq F 3$ and $F 4$, respectively. There was moderate positive correlation between inflammatory activity and $T 1 \rho$ values $(r=0.520 ; 95 \% \mathrm{Cl} 0.158-0.807 ; \mathrm{P}=0.013$ ).

Markus Karlsson ${ }^{1}$, Thobias Romu ${ }^{2,3}$, Amir Razavi ${ }^{4}$, Nils Dahlström ${ }^{5}$, Mikael F Forsgren ${ }^{6}$, Olof Dahlqvist-Leinhard ${ }^{3,7}$, Bengt Norén ${ }^{6}$, Mattias Ekstedt ${ }^{8}$, Stergios Kechagias ${ }^{8}$, and Peter Lundberg ${ }^{1}$

${ }^{1}$ Department of Radiation Physics, and Department of Medical and Health Sciences and Center for Medical Image Science and Visualization (CMIV), Linköping Univeristy, Linköping, Sweden, ${ }^{2}$ Department of Biomedical Engineering and Center For Medical Image Science and Visualization (CMIV), Linköping University, Linköping, Sweden, ${ }^{3}$ Amra AB, Linköping, Sweden, ${ }^{4}$ Department of Radiology, and Department of Medical and Health Sciences, Linköping University, Norrköping, Sweden, ${ }^{5}$ Department of Medical and Health Sciences and Center for Medical Image Science and Visualization (CMIV), Linköping University, Linköping, Sweden, ${ }^{6}$ Center for Medical Image Science and Visualization (CMIV), Linköping University, Linköping, Sweden, ${ }^{7}$ Department of Medical and Health Sciences and Center for Medical Image Science and Visualization (CMIV), Linköping Univeristy, Linköping, Sweden,

${ }^{8}$ Department of Medical and Health Sciences and Department of Gasteroenterology and Hepatology, Linköping University, Linköping, Sweden 
We measured T1 relaxation times in a prospectively recruited cohort of patients with suspected chronic liver disease. Our aim was to investigate the predictive value of T1 for staging hepatic fibrosis. Furthermore, we sought to test if $\mathrm{T} 1$ values are confounded by inflammation or presence of iron. We found that $\mathrm{T} 1 \mathrm{was}$ confounded by iron and that $\mathrm{T} 1 \mathrm{alone}$ had a poor ability to stage hepatic fibrosis.

Bi-exponential T1rho Relaxation of In Vivo Human Liver

Weitian Chen ${ }^{1}$, Vincent Wong ${ }^{2}$, Queenie Chan ${ }^{3}$, Yi-Xiang Wang ${ }^{1}$, and Winnie Chu ${ }^{1}$

${ }^{1}$ Department of Imaging and Interventional Radiology, The Chinese University of Hong Kong, Shatin, Hong Kong, ${ }^{2}$ Department of Medicine \& Therapeutics, The Chinese University of Hong Kong, Shatin, Hong Kong, ${ }^{3}$ Philips Healthcare, Hong Kong, China, Shatin, Hong Kong

Quantitative T1rho imaging is reported a promising non-invasive diagnostic tool for detection of liver fibrosis at its early stage. T1 rho relaxation is often estimated by a mono-exponential relaxation model. However, bi-exponential relaxation may occur due to compartmentation of the liver tissue. Bi-exponential T1rho relaxation has been reported in rat muscle and human knee cartilage. In this work, we provided our observation and analysis of bi-exponential T1rho relaxation of in vivo human liver.

The influence of glycogen on shortened modified Look-Locker inversion recovery (shMOLLI) T1 maps of the liver

Ferenc Emil Mozes ${ }^{1}$, Elizabeth Mary Tunnicliffe ${ }^{1}$, Michael Pavlides ${ }^{1,2}$, and Matthew David Robson ${ }^{1}$

${ }^{1}$ University of Oxford Centre for Magnetic Resonance Research, University of Oxford, Oxford, United Kingdom, ${ }^{2}$ Translational Gastroenterology Unit, University of Oxford, Oxford, United Kingdom

Dynamic physiological changes in the liver may influence the increased variability of shMOLLI $T_{1}$ of healthy livers relative to normal myocardial shMOLLI $T_{1}$ variability. Since glycogen concentration varies over relatively short time periods, this may contribute to the variability. This study explores two possible pathways by which glycogen might influence shMOLLI measurements: chemical exchange saturation transfer (CEST) effects and direct change of liver water relaxation. Simulations, phantom and human experiments suggest that the CEST effect is negligible in vivo and a $7 \%$ shortening of $\mathrm{T}_{1}$ at high glycogen concentration is driven by direct relaxation effects.

More than Hepatobiliary Relative Enhancement Ratio by Gd-EOB-DTPA for Liver Fibrosis Estimation, Hepatocyte Fraction method by T1 mapping measurement Shen Pan ${ }^{1}$, Xiaoqi Wang ${ }^{2}$, and Qiyong Guo ${ }^{1}$

${ }^{1}$ Sheng Jing Hospital, Shenyang, China, ${ }^{2}$ Philips Healthcare, Beijing, China

We calculated the hepatocytefraction (Hep) and reduction rate of T1 relaxation time (RE) based on T1changes in the hepatocyte due to pharmacokinetics of gadoliniumethoxybenzyldiethylenetriamine pentaacetic acid (Gd-EOB-DTPA) uptake in liver.Both Hep and RE were compared with liver fibrosis stage according to theMETAVIR scoring system. And we found that Hep significantly correlated withfibrosis stage, and indicate it a good quantitative biomarker for liver fibrosis estimation.

Comparison between MR T1p imaging and acoustic radiation force impulse for noninvasive assessment of liver fibrosis: repeatability, reproducibility, and diagnostic performance in rat models

Jinning $\mathrm{Li}^{1}$, Huanhuan Liu ${ }^{1}$, Caiyuan Zhang ${ }^{1}$, Shuyan Yang ${ }^{1}$, Yanshu Wang ${ }^{1}$, Weibo Chen ${ }^{2}$, and Dengbin Wang ${ }^{1}$

${ }^{1}$ Radiology, Xinhua Hospital, Shanghai Jiao Tong University School of Medicine, Shanghai, China, ${ }^{2}$ Philips Healthcare, Shanghai, China

The purpose of this study was to validate the repeatability, reproducibility, and diagnostic performance of MR T1 $\rho$ imaging for staging liver fibrosis, compared with ultrasound-based acoustic radiation force impulse (ARFI). The cross-sectional study was performed in rat models with carbon tetrachloride $\left(\mathrm{CCl}_{4}\right)$. The results of histopathological analysis were used as reference standard. T1 $\rho$ imaging showed comparable repeatability and reproducibility with ARFI, however, manifested more accurate diagnostic performance for staging liver fibrosis, especially for detecting early stage of fibrosis.

Assessment of Liver fibrosis using Exchange dual-input dual-compartment pharmacokinetic model of Dynamic Contrast-enhanced MRI

Lan Zhang ${ }^{1}$ and Zhi Zheng Zhuo ${ }^{2}$

${ }^{1}$ MRI, The 1 st affiliated hospital of Henan University of TCM, Zhengzhou, China, ${ }^{2}$ PHILIPS Healthcare, Beijing, China 
The clinical need in the development of non-invasive methods for liver fibrosis assessment has emerged. At 3.0T, human in-vivo studies have demonstrated DCE-MRI using Exchange dual-input and dual-compartment pharmacokinetic model has potential to detect and assess the vascular permeability modification of liver fibrosis. DCE-MRI pharmacokinetic quantitative parameters including Ktrans, Ve and Vp can be used for diagnosing and staging liver fibrosis. Ktrans is the best index and predictor for discriminating normal livers from fibrotic livers.

Diffusion MRI alteration following the induction of mild liver fibrosis in a rabbit model

Matteo Figini ${ }^{1}$, Liang Pan ${ }^{1,2}$, Chong Sun ${ }^{1,3}$, Bin Wang ${ }^{1,4}$, Junjie Shangguan ${ }^{1}$, Kang Zhou ${ }^{1,5}$, Na Shang $^{1}$, Quanhong Ma ${ }^{1}$, and Zhuoli Zhang ${ }^{1}$

${ }^{1}$ Radiology, Northwestern University, Chicago, IL, United States, ${ }^{2}$ Radiology, The Third Affiliated Hospital of Soochow University, Changzhou, China, ${ }^{3}$ Orthopedics, Qilu Hospital, Shandong University, Jinan, China, ${ }^{4}$ General Surgery, Nanfang Hospital, Southern Medical University, Guangzhou, China, ${ }^{5}$ Radiology, Peking Union Medical College Hospital, Chinese Academy of Medical Science and Peking Union Medical College, Beijing, China

Nine rabbits were injected with carbon tetrachloride for 6 weeks to induce liver fibrosis. At the end of this period, these rabbits and 15 controls injected with saline underwent an imaging protocol including diffusion MRI. Histology showed mild fibrosis throughout the liver of the CCl4-injected animals. The Apparent Diffusion Coefficient in the liver of the fibrotic rabbits was significantly higher than in the controls. This counterintuitive result can be explained by the presence of many conflicting mechanisms during the early stage of fibrosis. If confirmed, the $\mathrm{ADC}$ could become a valuable tool for the early detection of liver fibrosis

Comprehensive analysis of advanced liver fibrosis in rats using multi-parameter MRI

Qing $\mathrm{Li}^{1}$, Shuangshuang $\mathrm{Xie}^{2}$, Hanxiong $\mathrm{Qi}^{2}$, Zhizheng Zhuo ${ }^{3}$, Yue Cheng ${ }^{2}$, and Wen Shen ${ }^{2}$

${ }^{1}$ Tianjin First Center Hospital, Tianjin, China, ${ }^{2}$ Radiology department, Tianjin First Center Hospital, Tianjin, China, ${ }^{3}$ Philips healthcare, Beijing China, Beijing, China

This study investigated the value of multi-parametric analysis using IVIM, DKI and MR T1 $\rho$ for the diagnosis of advanced liver fibrosis. Sixteen healthy rats and fifteen rats with advanced liver fibrosis (F4 confirmed by liver pathological examination) were scanned with IVIM, DKI and MR T1 $\rho$. IVIM derived D*, D, f, DKI derived MD, K value and MR T1 $\rho$ derived T1 $\rho$ value were compared between the above two groups. Our results showed $D^{*}, D, f$ and $M D$ decreased while $K$ value and T1 $\rho$ value increased in rats with advanced liver fibrosis. And $D^{*}, D, f$, $K$ value and $T 1 \rho$ value demonstrated significant difference $(P<0.05)$. We therefore conclude that decreased $D^{*}$, $D$ and $f$ and increased $K$ value and $T 1 \rho$ value could be useful in the diagnosis of liver fibrosis.

Diagnostic value of intravoxel incoherent motion (IVIM) diffusion-weighted imaging in hepatic sinusoidal obstruction syndrome: an experimental study in a rat model - preliminary results Eun Kyoung Hong ${ }^{1}$, ljin Joo ${ }^{1}$, and Kyoungbun Lee ${ }^{2}$

${ }^{1}$ Department of Radiology, Seoul National University Hospital, Seoul, Republic of Korea, ${ }^{2}$ Department of Pathology, Seoul National University Hospital, Seoul, Republic of Korea

Hepatic sinusoidal obstruction syndrome (SOS), a toxic liver injury, needs an accurate diagnosis and serial monitoring for an effective management. Intravoxel incoherent motion (IVIM) DWI, which allows separate estimation of molecular diffusion and microcirculation, potentially provides information regarding hepatic parenchymal abnormalities. This study investigated the diagnostic value of IVIM-DWI in the assessment of hepatic SOS using a monocrotaline-induced rat SOS model. Our study results showed that ADC, true diffusion coefficient, and perfusion fraction showed significant correlation with the severity of SOS, which would suggest that IVIM-DWI may serve as a noninvasive method in the quantitative assessment of hepatic SOS.

Assessment of the Hepatocyte Fraction Combined with Liver Volume for the estimation of liver function

Ke Wang ${ }^{1}$, Xiaochao Guo ${ }^{1}$, He Wang ${ }^{1}$, Zhizheng Zhuo ${ }^{2}$, and Xiaoying Wang ${ }^{1}$

${ }^{1}$ Radiology, Peking University First Hospital, Beijing, China, ${ }^{2}$ Philips Healthcare, Beijing, China

Hepatocyte fraction (HeF) and uptake function based on $\mathrm{k}$ map have becoming new biomarkers based on EOB-MR in estimation of hepatic function. Liver volume is another factor that influence the liver function. The purpose of our study was to determine whether liver function can be estimated quantitatively from EOB-MR combined with liver volume.

Comparison of the diagnostic performances of three methods of ROI placement for the measurements of Intravoxel incoherent motion diffusion-weighted MR imaging parameters in hepatocellular carcinoma

Yi Wei $^{1}$, Bin Song ${ }^{2}$, and Dandan Zheng ${ }^{3}$

${ }^{1}$ Radiology, West China Hospital, Sichuan University, Chengdu, China, ${ }^{2}$ Radiology, West Chian Hospital, Sichuan University, Chengdu, China, ${ }^{3}$ GE Healthcare, China, Beijing, China 
The pathological differentiated grade is heavily associated with the hepatocellular carcinoma prognosis. Through a prospectively research, we sought to determine the diagnostic performances of three methods of ROI placement for measurements of IVIM parameters in the grading of hepatocellular carcinoma. According to the results, we found that different ROI positioning methods used significantly affects the IVIM and ADC parameters measurements. Measurements of ADCslow value derived from whole tumor volume method entailed the highest diagnostic performance in grading hepatocellular carcinoma. These results suggested that ADCslow value derived from whole tumor volume method might be useful in assessing the differentiated grade of carcinoma, and which might be helpful in predicting the patients' prognosis.

In primary sclerosing cholangitis, diffusion weighted magnetic resonance imaging correlates better with liver stiffness than Gadoxetate disodium enhanced MR imaging

Jin Yamamura ${ }^{1}$, Jan Sedlacik ${ }^{2}$, Tillmann Schuler ${ }^{1}$, Ralph Buchert ${ }^{3}$, Dr. Maxim Avanesov ${ }^{1}$, Hendrik Kooijman-Kurfuerst ${ }^{4}$, Christoph Schramm ${ }^{5}$, Gerhard Adam ${ }^{1}$, and Sarah Keller ${ }^{1}$

${ }^{1}$ Diagnostic and Interventional Radiology, University Medical Center Hamburg-Eppendorf, Hamburg, Germany, ${ }^{2}$ Neuroradiology, University Medical Center Hamburg-Eppendorf, Hamburg, Germany, ${ }^{3}$ Nuclear Medicine, University Medical Center Hamburg-Eppendorf, Hamburg, Germany, ${ }^{4}$ MRI, Philips Medical Systems, Hamburg, Germany, ${ }^{5}$ Internal Medicine, University Medical Center Hamburg-Eppendorf, Hamburg, Germany

Several disadvantages of DCE-MRI, such as long examination time, application of intravenous contrast agents and elaborative postprocessing and the higher sensitivity of the ADC to differentiate several stages of fibrosis, favorites DWI over DCE-MRI for diagnosis and staging of fibrosis in routine clinical MRI of PSC patients.

Dynamic Contrast-Enhanced MRI to Assess Hepatocellular Carcinoma Response to Transarterial Chemoembolization: a Pilot Study.

Alana Thibodeau-Antonacci ${ }^{1,2}$, Léonie Petitclerc ${ }^{1,2}$, Guillaume Gilbert ${ }^{3}$, Laurent Bilodeau ${ }^{2}$, Hélène Castel ${ }^{4}$, Simon Turcotte ${ }^{5}$, Damien Olivié ${ }^{2}$, Catherine Huet $^{2}$, Pierre Perreault ${ }^{2}$, Gilles Soulez ${ }^{2}$, An Tang ${ }^{1,2}$, and Samuel Kadoury ${ }^{1,2,6}$

${ }^{1}$ Centre de Recherche du Centre Hospitalier de I'Université de Montréal, Montreal, QC, Canada, ${ }^{2}$ Radiology, Centre Hospitalier de I'Université de Montréal, Montreal, QC, Canada, ${ }^{3}$ Philips Healthcare Canada, Markham, ON, Canada, ${ }^{4}$ Gastroentology and Hepatology, Centre Hospitalier de I'Université de Montréal, Montreal, QC, Canada, ${ }^{5}$ Surgery, Hepatopancreatobiliary and Liver Transplantation Division, Centre Hospitalier de I'Université de Montréal, Montreal, QC, Canada, ${ }^{6}$ Polytechnique Montréal, Montreal, QC, Canada

Hepatocellular carcinoma response to transarterial chemo-embolization is traditionally assessed by qualitative interpretation of imaging features and enhancement dynamics. However, quantitative parameters derived by fitting a dual-input single-compartment model on dynamic contrast-enhanced-MRI data show promise, as they may help discriminate non-viable from viable tumors after treatment. Peak enhancement ratio significantly decreased after transarterial chemo-embolization in tumors with complete response (i.e. non-viable tumor group). This pilot study suggests that quantitative dynamic contrast-enhanced-MRI parameters may be used to assess treatment response.

Dynamic contrast-enhanced MRI and intravoxel incoherent motion diffusion-weighted imaging for the evaluation of HCC response to $90 \mathrm{Yttrium}$ radioembolization

Stefanie Hectors ${ }^{1}$, Paul Kennedy ${ }^{1}$, Octavia Bane ${ }^{1}$, Maxwell Segall ${ }^{1}$, Sara Lewis ${ }^{1,2}$, Myron Schwartz ${ }^{3}$, Edward Kim ${ }^{2}$, and Bachir Taouli ${ }^{1,2}$

${ }^{1}$ Translational and Molecular Imaging Institute, Icahn School of Medicine at Mount Sinai, New York, NY, United States, ${ }^{2}$ Department of Radiology, Icahn School of Medicine at Mount Sinai, New York, NY, United States, ${ }^{3}$ Recanati/Miller Transplantation Institute, Icahn School of Medicine at Mount Sinai, New York, NY, United States

The goal of this study is to assess whether DCE-MRI and IVIM-DWI can be used to predict response of hepatocellular carcinoma (HCC) to $90 \mathrm{Yttrium}$ radioembolization (RE). In a preliminary cohort, significant changes were observed in both DCE-MRI and IVIM-DWI parameters at 6 weeks after treatment, which suggest that both techniques are sensitive to treatment effects of RE to HCC tissue. The exact utility of the DCE-MRI and IVIM-DWI parameters will be tested in a larger cohort.

Estimating Liver Function by Gadoxetate Enhanced MRI: Comparison of Pharmacokinetic Models in a Clinical Setting

Markus Karlsson ${ }^{1}$, Gunnar Cedersund ${ }^{2}$, and Peter Lundberg ${ }^{1}$

${ }^{1}$ Department of Radiation Physics, and Department of Medical and Health Sciences and Center for Medical Image Science and Visualization (CMIV), Linköping Univeristy, Linköping, Sweden, ${ }^{2}$ Department of Biomedical Engineering, Linköping University, Linköping, Sweden

The hepatic uptake rate of Gadoxetate is a possible biomarker for liver function and several different pharmacokinetic models have been developed. However, no one has ever compared these models using the same data. We compared three different models using imaging data with low temporal, but high spatial resolution. We showed that two of the models estimates almost the same values of the hepatic uptake rate. The fact that two different pharmacokinetic models can produce the same parameter values validates the entire pharmacokinetic modelling approach, indicating that it is not just a model-specific parameter being estimated, but the actual transport rate. 
${ }^{1}$ Medical Imaging, University of Arizona, Tucson, AZ, United States, ${ }^{2}$ Electrical and Computer Engineering, University of Arizona, Tucson, AZ, United States, ${ }^{3}$ Biomedical Engineering, University of Arizona, Tucson, AZ, United States, ${ }^{4}$ Siemens Medical Solutions USA, Inc, Tucson, AZ, United States

Chronic liver disease (CLD) is known to affect 3.9 million of Americans. Collagen deposition in CLD affects the perfusion of the liver parenchyma and dynamic contrast enhanced MRI (DCE-MRI) can be used for the non-invasive diagnosis of CLD. Here we present a liver perfusion technique based on a free-breathing 3D radial golden-angle stack-of-stars acquisition along with a compressed sensing reconstruction to generate DCE data with 4-sec temporal resolution. Perfusion parameters are estimated by fitting the DCE data to a dual-input two compartment pharmacokinetic model and used to evaluate hepatic fibrosis in CLD.

Traditional Poster

\section{Value of MRI}

The value of MRI in radiation therapy

Olga L Green ${ }^{1}$, Hiram A Gay ${ }^{1}$, Paragh Parikh ${ }^{1}$, Stacie L Mackey² , Sasa Mutic ${ }^{1}$, Thomas G Dvergsten ${ }^{1}$, Mo Kadbi ${ }^{3}$, and H Michael Gach ${ }^{1}$

${ }^{1}$ Radiation Oncology, Washington University in St Louis, St Louis, MO, United States, ${ }^{2}$ Radiation Oncology, Barnes-Jewish Hospital, St Louis, MO, United States, ${ }^{3}$ MRI, Philips Healthcare, Cleveland, $\mathrm{OH}$, United States

During the last decade, the role of MRI in radiation therapy (RT) grew dramatically. The soft-tissue benefits from MRI simulations complement the geometric accuracy and photon attenuation maps from computed tomography in RT treatment planning. MR for calculating attenuation (MRCAT) is being used for MRI-only treatment planning. The clinical utilization of hybrid MRI-guided radiation therapy (MR-IGRT) systems began in January 2014. MR-IGRT enables real-time tracking of tumors and highly conformal treatments that enable improved patient outcomes. Hence, the value of MRI in RT is rapidly rising. Examples of MRI's role in, and value to, RT are presented.

Comparison of MRI and CT Characterizations of Lung Lesions from Pulmonary Tuberculosis

Liya Wang ${ }^{1,2}$, Zhou Liư ${ }^{3}$ Lijian Liu³ ${ }^{3}$ Zhiqing Wu ${ }^{1}$, Yuzhong Zhang ${ }^{1}$, and Hui Mao ${ }^{2}$

${ }^{1}$ Radiology, The People's Hospital of Longhua, Shenzhen, China, ${ }^{2}$ Radiology and Imaging Sciences, Emory University School of Medicine, Atlanta, GA, United States, ${ }^{3}$ Radiology, Cancer Hospital Chinese Academy of Medical Science, Shenzhen Center, Shenzhen, China

Lung MRI can be applied to imaging and characterize the abnormalities and lesions in patients with history of pulmonary tuberculosis (TB). By comparing with the images obtained from the routine clinical CT from the same patients, this work shows that MRI is comparable to CT as a non-radiation alternative for lung imaging with good diagnostic image quality. In addition, MRI can provide additional information on lung soft tissue properties not available from CT.

An optimised, MRI-PET based clinical protocol for improving the differential diagnosis of Late-life Depression and Alzheimer's Disease

Louise Emsell ${ }^{1,2,3,4}$, Kristof Vansteelandt ${ }^{2}$, François-Laurent De Winter ${ }^{2,4}$, Filip Bouckaert ${ }^{2,5}$, Lene Claes ${ }^{4}$, Danny Christiaens ${ }^{4}$, Lies Van Assche ${ }^{2,4}$, Jan Van den Stock ${ }^{2,4}$, Rik Vandenberghe $^{6}$, Stefan Sunaert ${ }^{1,3}$, and Mathieu Vandenbulcke ${ }^{2,4}$

${ }^{1}$ Translational MRI, Department of Imaging \& Pathology, KU Leuven, Leuven, Belgium, ${ }^{2}$ Old Age Psychiatry, UPC KU Leuven, Leuven, Belgium, ${ }^{3}$ Radiology, UZ Leuven, Leuven, Belgium, ${ }^{4}$ Laboratory for Translational Neuropsychiatry, Dept Neurosciences, KU Leuven, Leuven, Belgium, ${ }^{5}$ Academisch Centrum voor ECT en Neuromodulatie (AcCENT), UPC KU Leuven, Kortenberg, Belgium, ${ }^{6}$ Laboratory for Cognitive Neurology, KU Leuven, Leuven, Belgium

Owing to overlapping symptomatology, differentiating between late-life depression (LLD) and Alzheimer's Disease (AD), is clinically challenging. Amyloid PET may be used to improve AD diagnosis, however it is expensive and not widely available. Here we apply a two-step MRI driven approach exploiting the different degree of hippocampal volume loss that is present in both disorders to derive hippocampal volume thresholds for identifying patients who could be diagnosed without a PET exam. Using the more cost-effective hippocampal volumetry approach, we could correctly classify half of the patient sample. This increased to $90 \%$ when adding $18 \mathrm{~F}$-flutametamol PET for the remaining patients.

Do MRI structured reportsispis for diabetic foot contain concise information for clinical application?

Li Guo ${ }^{1}$, Xiaoying Wang ${ }^{1}$, Xin $\mathrm{Qi}^{2}$, Yufeng $\mathrm{Xu}^{1}$, Yong Huang ${ }^{1}$, and Xueying $\mathrm{Li}^{3}$

${ }^{1}$ Department of Radiology, Peking University First Hospital, Beijing, China, ${ }^{2}$ Department of Plastic Surgery \& Burn, Peking University First Hospital, Beijing, China, ${ }^{3}$ Department of Biostatistics, Peking University First Hospital, Beijing, China 
The aim of this study is to evaluate if structured reporting of MRI in diabetic foot(DF) contain concise information for clinical application compared with nonstructured reporting. Thirty nonstructured foot MRI reports of patients with DF were included, and another structured report was written for each patient. Three readers (A, B\&C) evaluated the nonstructured and structured reports. Statistical analysis included Wilcoxon signed ranks tests and chi-square tests. All readers needed shorter time to understand the structured reports. For the 8 features for DF, two readers could understand bone edema significantly more often when reading structured versus nonstructured reports. All readers needed to evaluate images when reading nonstructured reports, 2 radiologists (reader $\mathrm{A} \& \mathrm{C}$ ) needed to evaluate images when reading structured reports, and reader $\mathrm{B}$ (doctor of burn \& plastic surgery) only needed $4(13.3 \%$ ) to evaluate images when reading the structured reports. All readers missed Charcot joint and fracture when reading nonstructured reports, but only reader $\mathrm{A}$ missed fracture and reader $\mathrm{C}$ missed Charcot joint when reading structured reports. All readers found another abscess when reading structured reports. In conclusion, structured reports of MRI in patients with DF provided more concise information for clinical application than nonstructured reports.

Scanner Status Tool (STATS): towards increasing the value of MR

Ed Mojahed ${ }^{1}$

${ }^{1}$ Clinical Science, Philips, Nashville, TN, United States

Scanner Status tool (STATS) is a light Perl-based script that runs on the MR scanner's Host computer and picks up critical information about the status of the background processes and informs users when everything is OK or when there is an error detected so they could subsequently take an informed action. This will result in a smoother workflow, reduction in wasted time, promotion of First Time Right imaging, and increased value of MR.

Quantitative validation of the image contrast generated by MAgnetic Resonance image Compilation (MAGiC) technique

Chia-Wei Li ${ }^{1}$, Chien-Yuan Lin ${ }^{2}$, Ai-Ling Hsu ${ }^{3}$, and Wing P. Chan ${ }^{1,4}$

${ }^{1}$ Department of Radiology, Wan Fang Hospital, Taipei Medical University, Taipei, Taiwan, ${ }^{2}$ GE Healthcare, Taipei, Taiwan, ${ }^{3}$ Graduate Institude of Biomedical Electronics and Bioinformatics, National Taiwan University, Taipei, Taiwan, ${ }^{4}$ Department of Radiology, School of Medicine, College of Medicine, Taipei, Taiwan

MAGiC scan could provide several different clinical relevant weighted images and quantitative tissue relaxation time with use of a multi-slice, multi-echo, and multi-delay acquisition in the single scan. In this study, a homemade phantom containing 7 tubes with various concentrations of aqueous $\mathrm{CuSO}_{4}$ was used to quantitatively validate the image quality of a MAGiC scan. Results show that overall diagnostic image quality using MAGiC is comparable to that using conventional scanning, but a slight contrast difference is seen where $T_{1}$ values are low (outside the range of brain $\mathrm{T}_{1}$ values, less than $500 \mathrm{~ms}$ )

Feasibility of high throughput scanning at $7 \mathrm{~T}: 13$ subjects per hour

Tijl van der Velden ${ }^{1}$, Erwin Krikken ${ }^{1}$, Catalina Arteaga ${ }^{1}$, Fredy Visser ${ }^{2}$, and Dennis Klomp ${ }^{1}$

${ }^{1}$ Radiology, University Medical Center Utrecht, Utrecht, Netherlands, ${ }^{2}$ Philips Healthcare, Best, Netherlands

While substantial acceleration in MRI acquisitions have been demonstrated in the last decades (particularly at high fields where SNR is not limited), substantial patient and scan preparation time have been reported that seem to prohibit high patient throughput for clinical MRI. In this study we demonstrate that robust head MRI can be obtained at a throughput of more than 13 subjects per hour, including patient management and scan preparations (even faster than typical X-ray exams). Increased throughput may be an alternative way to "killer applications" in making high field MRI economically viable.

Added value of a management software tool for optimization of clinical MRI workflow

Timo De Bondt ${ }^{1,2}$, Mahdi Kalai ${ }^{2}$, Floris Vanhevel ${ }^{1}$, Olivier Morhedec ${ }^{2}$, Florian Sarrazin ${ }^{2}$, Donat Thery ${ }^{2}$, Federica Zanca ${ }^{2}$, and Paul M Parizel ${ }^{1}$

${ }^{1}$ Radiology, Antwerp University Hospital, Antwerp, Belgium, ${ }^{2}$ GE Healthcare, Buc, France

MRI has important drawbacks like slow speed and high cost, which makes it a challenge to maintain cost-effectiveness in context of the changing healthcare economic environment. We show that a management software tool, giving easy access to operational and clinical data, can provide insights into everyday clinical workflow. Additionally, it has the potential to facilitate optimization of protocols, and improve patient safety.

Jonas Denck ${ }^{1}$, Wilfried Landschütz ${ }^{2}$, Knud Nairz ${ }^{3}$, Johannes T. Heverhagen ${ }^{3}$, Andreas Maier ${ }^{1}$, and Eva Rothgang ${ }^{4}$

${ }^{1}$ University of Erlangen-Nuremberg, Erlangen, Germany, ${ }^{2}$ Siemens Healthineers, Erlangen, Germany, ${ }^{3}$ Inselspital, University of Bern, Bern, Switzerland, ${ }^{4}$ Technical University of Applied Sciences Amberg-Weiden, Amberg, Germany 
We developed an algorithm that is capable of retrieving MRI billing codes from MRI log data. This proof-of-concept work is applied to Tarmed, the Swiss fee-for-service tariff system for outpatient services, and is tested on two MRI scanners, a MAGNETOM Aera and a MAGNETOM Skyra (Siemens Healthcare, Erlangen, Germany), of a single radiology site. A machine learning approach for automated MRI billing code retrieval from MRI log data is implemented. The proposed algorithm reliably predicts medical billing codes for MRI exams (F1-score: $97.1 \%)$. Integrated in the clinical environment, this work has the potential to reduce the workload for technologists, prevent coding errors and enable scanner-specific expense and turnover analysis.

Patient Acceptance on a Compact 3T is Generally Superior to a Whole-Body Scanner

Erin Gray ${ }^{1}$, John Huston $\mathrm{III}^{1}$, Yunhong Shu ${ }^{1}$, Myung-Ho $\ln ^{1}$, Shengzhen $\mathrm{Tao}^{1}$, Joshua Trzasko ${ }^{1}$, Eric Fiveland ${ }^{2}$, Thomas K.F. Foo ${ }^{2}$, and Matt A Bernstein ${ }^{1}$

${ }^{1}$ Radiology, Mayo Clinic, Rochester, MN, United States, ${ }^{2}$ GE Global Reserach, Niskayuna, NY, United States

A compact 3T scanner was developed under a Bioengineering Research Partnership as a technology demonstrator. To assess patient acceptance on the compact 3T compared with a whole-body $3 \mathrm{~T}$ MR, 33 consecutive patients completed a series of survey questions to report their subjective experience. The survey results demonstrate that the Compact $3 \mathrm{~T}$ is equal or superior to a whole-body scanner for patient acceptance.

Synthesis and analysis of low b value Diffusion Weighted Images at $10 \mathrm{mT}$

Seema S Bhat ${ }^{1}$, Pavan Poojar ${ }^{1,2}$, Marta da Silva Ferreira ${ }^{3}$, Hanumantharaju M C ${ }^{4}$, Rita G Nunes ${ }^{3}$, and Sairam Geethanath ${ }^{5,6}$

${ }^{1}$ Dayananda Sagar Institutes, Bangalore, Bangalore, India, ${ }^{2}$ Wipro-GE Healthcare, BANGALORE, India, ${ }^{3}$ Institute for Systems and Robotics /Department of Bioengineering, Instituto Superior Técnico, Universidade de Lisboa, Lisbon, Portugal, Lisbon, Portugal, ${ }^{4}$ BMS Institute of Technology and Management, BANGALORE, India, ${ }^{5}$ MIRC, Dayananda Sagar Institutes,Bangalore, BANGALORE, India, ${ }^{6}$ Dept. of Radiology, Columbia University Medical Center, New York, NY, New York, NY, United States

DWI MRI is a well established method for stroke imaging, within the critical operating window of approximately four to six hours. This requires an accessible, portable and cost effective MR solution typically achieved at very low magnetic fields. In this work, simulation of low b value DWI images at $10 \mathrm{mT}$ has been performed in comparison with $1.5 \mathrm{~T}$. Also, the affect of pulse sequence design parameters has been explored to arrive at a potentially useful DWI acquisition scheme. Future work includes prospective implementations of the sequence on a $10 \mathrm{mT}$ scanner and; denoising and reconstruction of low field images using deep learning

Mapping metabolic activation as FDG-PET/Amyloid-PET using Contrast-free MRI and Deep Learning

Enhao Gong ${ }^{1}$, Kevin Chen², Jia Guo ${ }^{2}$, Audrey Fan², John Pauly ${ }^{1}$, and Greg Zaharchuk ${ }^{2}$

${ }^{1}$ Electrical Engineering, Stanford University, Stanford, CA, United States, ${ }^{2}$ Radiology, Stanford University, Stanford, CA, United States

MRI has great clinical values to distinguish soft-tissues without contrast or radiation. By using the hybrid-modality information from MRI and PET, here we developed deep learning method to synthesize metabolic activity mapping from contrast-free multi-contrast MRI images. Trained on clinical datasets, we demonstrated the feasibility to estimate metabolic biomarker from contrast-free MRI and validated on both FDG-PET/MRI and Amyloid-PET/MRI in-vivo datasets. This technique can be used for more efficient, low-cost, multi-tracer biomarker from contrast-free MRI and validated on both FDG-PET/MRI and Amyloid-PET/MRI in-vivo datasets. This technique can be used for mor
functional imaging, exploring anatomy-function relationship, visualizing new bio-markers and improving the workflow for both MRI and PET/MRI.

Free-Breathing Motion Insensitive T1-Weighted Spine MRI in Children Using a Radial Acquisition at 3 Tesla

Houchun Harry Hu${ }^{1}$, Thomas Benkert ${ }^{2}$, Mark Smith ${ }^{1}$, Jerome Rusin ${ }^{1}$, Aaron McAllister ${ }^{1}$, Jeremy Jones ${ }^{1}$, Ramkumar Krishnamurthy ${ }^{1}$, and Kai Tobias Block ${ }^{1}$

MRI methods that are insensitive to physiological motion are attractive in pediatric applications. In this work, we compare a 3D T1-weighted radial acquisition with conventional multislice TSE in post-contrast spine imaging at 3T in seven patients. Images were rated by three neuroradiologists. Radial data were perceived as more diagnostic than TSE and Cartesian TSE data were significantly more impacted by motion and pulsation. Qualitatively, radial images yielded improved spinal cord to CSF signal contrast and better conspicuity of nerve roots than TSE data. In evaluating secondary CSF tumor spread, radial spine MRI provides a confident "first-time-right" protocol than TSE scans. 
Detection of early stage Chronic Kidney Disease (CKD) is essential to improve patient outcome but remains a challenge. In this study we generated T1 and T2 maps of renal cortex at 3.0 T in CKD patients and healthy volunteers $(n=16)$. Modified Look-Locker sequence with simulated ECG and a multi-echo Gradient and Spin-Echo sequence were used to generate T1 and T2 maps respectively. T1 of CKD kidney $(1752 \pm 45 \mathrm{~ms})$ was significantly higher $(\mathrm{P}<0.001)$ than that of healthy kidney $(1538 \pm 37 \mathrm{~ms})$. There was no significant difference between the groups in T2, FWHM and skewness of T2.

MAGNETIC RESONANCE IMAGING TEXTURE ANALYSIS (MRTA) ON T1WI, T2WI AND T1WI CONTRAST: DIAGNOSTIC ACCURACY OF CEREBRAL GLIOMA.

Mame Fatou KEITA ${ }^{1}$, Liang Fatou Han², YANWEI MIAO², and Mahammed MOHAMUD²

${ }^{1}$ Radiology, The First Affiliated Hospital of Dalian Medical University, Dalian, China, ${ }^{2}$ Radiology, The first affiliated Hospital of Dalian Medical University, Dalian, China

Cerebral gliomas are the most common primary malignant brain tumor in adults and include Astrocytoma, Oligodendroglioma and Oligoastrocytoma. Due to its multi-parametric approach, MRI was used to quantify tumor heterogeneity with Texture Analysis (TA). To avoid unnecessary surgeries and set-up good treatment's plan, the analysis of conventional MRI sequences was performed and showed a strong level of discrimination between the three gliomas on each sequence. TA has shown promise in the discrimination between lesions on MR images and provided satisfactory results.

MRI TEXTURE ANALYSIS: DIFFERENTIAL DIAGNOSIS OF CEREBRAL GLIOMAS FOLLOWING WHO 2016 CLASSIFICATION OF CNS TUMOURS

Mame Fatou KEITA 1 , Liang Fatou $\mathrm{Han}^{2}$, and YANWEI MIAO²

${ }^{1}$ Radiology, The First Affiliated Hospital of Dalian Medical University, Dalian, China, ${ }^{2}$ Radiology, The first affiliated Hospital of Dalian Medical University, Dalian, China

For the first time in 2016, the World Health Organisation (WHO) Classification of Tumours of the Central Nervous System used molecular parameters in addition to histology to define many tumour entities, thus formulating a concept for how CNS tumour diagnose should be structured in the molecular era and in that way is both a conceptual and practical advance over its 2007 predecessor. The strength of non-invasive diagnosis using textural analysis of conventional MRI sequences was evaluated and gave satisfactory results comparing grade II, III and IV including their genetic status.

Dynamic Contrast-enhanced MR imaging of rabbit VX2 bone tumor: Model Selection, repeatability and Validation

Wei Gong ${ }^{1}$ and Yunfei Zha ${ }^{1}$

${ }^{1}$ Renmin Hospital of Wuhan University, Wu han, China

To compare the repeatability and availability of the quantitative parameters for dynamic contrast-enhanced MR imaging that is based on the Reference-Region model and Tofts model with the microcirculation perfusion and permeability characteristics in rabbit $\mathrm{VX} 2$ bone tumor.

Baseline mrEMVI as an independent prognostic factor for locally advanced rectal cancer with neoadjuvant chemoradiotherapy: recommendations for risk stratification

XIAO-YAN ZHANG ${ }^{1}$, SHUAI WANG ${ }^{1}$, XIAO-TING LI ${ }^{1}$, YING-PING WANG ${ }^{2}$, YAN-JIE SHI ${ }^{1}$, LIN WANG ${ }^{3}$, AI-WEN WU $^{2}$, and YING-SHI SUN ${ }^{1}$

${ }^{1}$ Radiology, Key laboratory of Carcinogenesis and Translational Research (Ministry of Education), Department of Radiology, Peking University Cancer Hospital \& Institute, BEIJING, China, ${ }^{2}$ Key laboratory of Carcinogenesis and Translational Research (Ministry of Education), Department of Radiology, Peking University Cancer Hospital \& Institute, BEIJING, China, ${ }^{3}$ Radiology, Key laboratory of Carcinogenesis and Translational Research (Ministry of Education), Department of Radiology, Peking University Cancer Hospital \& Institute, BEIJING, Christmas Island

Extramural venous invasion status is a potential prognostic factor for identifying rectal cancer patients with a high risk of distant metastasis or local recurrence. It is currently unclear what impact extramural venous invasion status as defined by magnetic resonance imaging before neoadjuvant chemoradiotherapy (pre-NCRT mrEMVI) has on survival outcomes in patients with locally advanced rectal cancer. Moreover, the incorporation of baseline mrEMVI into risk stratification is poorly understood.This study has demonstrated that pre-NCRT mrEMVI status can be reliably evaluated and can serve as an independent prognostic factor for distant and local recurrence and overall survival in patients with locally advanced rectal cancer. We have provided important evidence that pre-NCRT mrEMVI status should be considered for managing risk stratification in baseline locally advanced rectal cancer. Finally, we recommend that mrEMVI evaluation be included in routine pre-NCRT MR reports to support an individualized treatment strategy, considering positive pre-NCRT mrEMVI may serve as an indicator for neoadjuvant chemotherapy. 
${ }^{1}$ Imaging, NingXia People's Hospital, Yinchuan, China, ${ }^{2}$ Imaging, NingXia People's Hospital, YinChuan, China, ${ }^{3}$ Imaging Systemt Clinical Science Philips Healthcare, Philips(China) Investment co.Ltd., Beijing, China

This study aims to explore the efficacy of NAC assessed by quantitative multi-parameter utilized bi-exponential diffusion weighted imaging in breast cancer. In this study, there is significant difference in $\Delta \mathrm{F}_{\text {fast }}$ value between groups, mainly results from the rise of $\mathrm{F}_{\text {fast }}$ value of tumor because of chemotherapy. Nonetheless, the diagnosis efficacy is mild for $N A C$ assessment using $\Delta \mathrm{F}_{\text {fast }}$ value.

Evaluation of spiral trajectories for very low field MR imaging of the brain

pavan poojar ${ }^{1}$, Imam Ahmed Shaik ${ }^{1}$, Girish Koulagi ${ }^{1}$, Seema S Bhat ${ }^{1}$, and Sairam Geethanath ${ }^{1,2}$

${ }^{1}$ Medical Imaging Research Centre, Dayananda Sagar Institutions, Bangalore, India, ${ }^{2}$ Dept. of Radiology, Columbia University Medical Center, New York, NY, United States

Very low field (VLF) MRI systems provide cost effective, accessible solutions for brain imaging. However, VLF MRI typically suffers from significantly lower signal-to-noise ratio and hence longer acquisition times. This work explores the utilization of spiral acquisitions at VLF as it provides efficient sampling of kspace and accelerated acquisitions compared to Cartesian trajectories. Spiral trajectories were designed for $10 \mathrm{mT}$ without violating the hardware constraints resulting in potential accelerated acquisitions. Retrospective reconstruction of brain images was performed using Non uniform Fast Fourier Transform and Graphical Programming Interface. Future work involves prospective implementation on a home built scanner being currently pursued.

Crossed cerebellar diaschisis: diagnostic \& prognostic value of BOLD fMRI cerebrovascular reactivity

Marco Piccirelli ${ }^{1}$, Martina Sebök ${ }^{2}$, Christiaan van Niftrik ${ }^{2}$, Oliver Bozinov ${ }^{2}$, Susanne Wegener ${ }^{3}$, Giuseppe Esposito ${ }^{2}$, Athina Pangalu ${ }^{1}$, Antonios Valavanis $^{1}$, Alfred Buck $^{4}$, Andreas Luft $^{3}$, Luca Regli², and Jorn Fierstra ${ }^{2}$

${ }^{1}$ Neuroradiology, University Hospital Zurich, Zurich, Switzerland, ${ }^{2}$ Neurosurgery, University Hospital Zurich, Zurich, Switzerland, ${ }^{3}$ Neurology, University Hospital Zurich, Zurich, Switzerland, ${ }^{4}$ Nuclear Medicine, University Hospital Zurich, Zurich, Switzerland

Crossed cerebellar diaschisis (CCD) is associated with poorer stroke outcome and is traditionally measured with [15O]-H2O-PET.

BOLD-CVR can detect CCD with high specificity and sensitivity. Furthermore, CCD subjects identified with BOLD-CVR also had a poorer clinical status at baseline and at three months follow-up. These encouraging results suggest that BOLD-CVR might be considered as a diagnostic and prognostic test for CCD subjects, comparable to the gold standard [15O]-H2O$\mathrm{PET}$ - but without the $\sim 1 \mathrm{mSv}$ radiation dose.

Traditional Poster

\section{Motion Correction: Cleaning up in the Brain}

High resolution imaging at $7 \mathrm{~T}$ using interleaved prospective motion correction (iMOCO)

Vincent Boer ${ }^{1}$, Mads Andersen ${ }^{2}$, Anouk Marsman ${ }^{1}$, and Esben Thade Petersen ${ }^{1,3}$

${ }^{1}$ Danish Research Centre for Magnetic Resonance, Centre for Functional and Diagnostic Imaging and Research, Copenhagen University Hospital Hvidovre, Hvidovre, Denmark, ${ }^{2}$ Philips Healthcare, Copenhagen, Denmark, ${ }^{3}$ Center for Magnetic Resonance, Department of Electrical Engineering, Technical University of Denmark, Lyngby, Denmark

Subject motion is a major problem in MRI, leading to less diagnostic information in the clinic and lowering data quality in research. Especially at high field, the relatively long scan times applied for high resolution imaging makes motion one of the major challenges. A promising solution is to update the field-of-view in real time based on tracking with MRI-based navigators. Here we show an implementation for prospective motion correction using MRI navigators at $7 \mathrm{~T}$. The framework was very flexible, as the navigator and target sequence are simply defined as two different scans, which can be interleaved at any sequence level.

Prospectively Motion Corrected DWI by Projection Fat Navigators

Johan Berglund ${ }^{1}$, Henric Rydén ${ }^{1,2}$, Enrico Avventi ${ }^{1,2}$, Tim Sprenger $^{1,3}$, Ola Norbeck ${ }^{1,2}$, and Stefan Skare ${ }^{1,2}$

${ }^{1}$ Department of Clinical Neuroscience, Karolinska Institutet, Stockholm, Sweden, ${ }^{2}$ Neuroradiology, Karolinska University Hospital, Stockholm, Sweden, ${ }^{3}$ GE Healthcare, Stockholm, Sweden 
A projected fat navigator module was added to a diffusion weighted EPI sequence to allow prospective rigid body motion correction without additional hardware. Improved image quality was demonstrated by imaging the brain of a volunteer subject who performed prescribed patterns of large motion with and without prospective correction. Improvements were most evident for through-plane motion. For in-plane motion only, the image quality was comparable to images acquired without motion. Ghosting due to gradient delays following FOV updates was avoided by acquiring phase reference lines directly after the excitation pulse.

Comparing TAMER (TArgeted Motion Estimation and Reduction) reduced modeling to alternating minimization for data consistency based motion mitigation

Melissa W. Haskell ${ }^{1,2}$, Stephen F. Cauley ${ }^{1,3}$, and Lawrence L. Wald ${ }^{1,3,4}$

${ }^{1}$ A. A. Martinos Center for Biomedical Imaging, Department of Radiology, MGH, Charlestown, MA, United States, ${ }^{2}$ Graduate Program in Biophysics, Harvard University, Cambridge, MA, United States, ${ }^{3}$ Harvard Medical School, Boston, MA, United States, ${ }^{4}$ Harvard-MIT Division of Health Sciences and Technology, MIT, Cambridge, MA, United States

Retrospective motion correction techniques offer minimal disruptions to sequences and clinical workflows. The computational burden of retrospective techniques can be eased either with alternating minimizations, or true joint estimation but on a reduced model. We provide computational experiments demonstrating the tightly coupled nature of the optimization variable types (motion and voxel values) which hinders the alternating based approaches. The alternating techniques can have an average search direction error of $75 \%$, vs. $22 \%$ with reduced modeling. We demonstrate a computational speedup of 17x using our reduced model approach, and present in vivo imaging results comparing TAMER to a state-of-the-art alternating minimization.

Optical prospective motion correction for brain imaging at $7 \mathrm{~T}$ without a mouthpiece

Phillip DiGiacomo ${ }^{1}$, Julian Maclaren ${ }^{1}$, Murat Aksoy ${ }^{1}$, Brian Burns ${ }^{2}$, Roland Bammer ${ }^{1}$, Brian Rutt ${ }^{1}$, and Michael Zeineh

$2651 \quad{ }^{1}$ Department of Radiology, Stanford University, Stanford, CA, United States, ${ }^{2}$ MR Applied Science Lab, GE Healthcare, Menlo Park, CA, United States

The advancements in signal to noise ratio, contrast, and resolution enabled by high-field MR systems provide great potential for visualizing more nuanced brain anatomy. However, in order to translate these advancements to the discovery and clinical implementation of novel neuroimaging biomarkers, motion artifact resulting from long scan times must be addressed. Here, we demonstrate proof-of-concept of a novel prospective optical motion tracking and correction system using a coil-mounted camera without a mouthpiece, visualizing an optical marker placed on the cheek of human subjects in a 7T MR system.

Pediatric Head Motion Detection using Free Induction Decay Navigators

Tess E Wallace ${ }^{1}$, Kristina Pelkola ${ }^{2}$, Monet Dugan ${ }^{2}$, Simon K Warfield ${ }^{1}$, and Onur Afacan ${ }^{1}$

${ }^{1}$ Radiology, Boston Children's Hospital, Harvard Medical School, Boston, MA, United States, ${ }^{2}$ Radiology, Boston Children's Hospital, Boston, MA, United States

Free induction decay navigators (FIDnavs) are sensitive to head motion and can be rapidly acquired using standard scanner hardware, making them an attractive approach for motion detection in pediatric MRI. In this study, we perform a head-to-head comparison of various FIDnav motion detection algorithms in controlled volunteer experiments and in pediatric patients scanned under typical conditions using a modified MPRAGE sequence. We demonstrate that computing the change in cross-correlation coefficient between FIDnav signal vectors results in excellent detection accuracy in both volunteers and patients, based on concurrent ground-truth RMS displacements measured using an electromagnetic tracking system.

A Novel Framework for Head Motion Measurement using Free Induction Decay Navigators from Multi-Channel Coil Arrays

Tess E Wallace ${ }^{1}$, Onur Afacan ${ }^{1}$, and Simon K Warfield ${ }^{1}$

FID navigators (FIDnavs) encode substantial quantitative rigid-body motion information; however, current implementations require subjects to cooperate for a choreographed training session, which is impractical in many clinical scenarios. We present a new approach that uses simulation of the acquisition physics and effect of motion on the measured FIDnav from each coil. This method is tested in three volunteers scanned at 3T with a 32-channel head coil using a 3D FLASH sequence, each performing a series of repeating motion patterns. Submillimeter and sub-degree tracking accuracy was achieved across all volunteers, demonstrating the efficacy of this approach for real-time head motion measurement.

Patricia Johnson ${ }^{1,2}$, Reggie Taylor ${ }^{3,4}$, Tim Whelan $^{1}$, and Maria Drangova ${ }^{1,2}$

${ }^{1}$ Robarts Research Institute, London, ON, Canada, ${ }^{2}$ Medical Biophysics, Western University, London, ON, Canada, ${ }^{3}$ Lawson Health Research Institute, London, ON, Canada, ${ }^{4}$ Siemens Canada, Oakville, ON, Canada 
Head motion during brain imaging with hybrid PET-MR degrades the quality of both the PET and MR images. Simultaneous acquisition with the two modalities provides the opportunity for MR motion measurement techniques to be used for correction of PET data. In this study, spherical navigator echoes (SNAV) were used for retrospective motion correction of PET images. A phantom was repositioned several times during a list mode acquisition. The list mode data was binned into motion states based on the SNAV measured motion, and a motion-corrected PET reconstruction was performed. SNAV motion correction successfully removed blurring in the PET images.

Artifact Detection Using Correlation Analyses Applied to MEGA-PRESS Data Containing Subject Head Movements

Sofie Tapper ${ }^{1,2}$, Anders Tisell ${ }^{1,2}$, Gunther Helms ${ }^{3}$, and Peter Lundberg ${ }^{1,2}$

${ }^{1}$ Department of Radiation Physics, and Department of Medical and Health Sciences, Linköping University, Linköping, Sweden, ${ }^{2}$ Center for Medical Image Science and Visualization, Linköping University, Linköping, Sweden, ${ }^{3}$ Department of Medical Radiation Physics, Lund University, Lund, Sweden

Subject movements and other disturbances might contaminate the Magnetic Resonance Spectroscopy data, and these artifacts can be misinterpreted as actual metabolite signals by the quantification program. Thus, an automatic method could be very helpful for finding artifacts and eliminating them. In this work, an approach of using correlation analyses was tested in order to evaluate if motion contaminated data could be identified. A total of $296 / 320$ spectra were correctly categorized according to the movement-paradigm. This procedure could be suitable for identifying data that are affected by subject motion or other artifacts that would reduce the quality of the result.

Motion correction of $\mathrm{T}^{*}$-weighted MRI with consideration of $\mathrm{B} 0$ and $\mathrm{B} 1$ effect

Jiaen Liu ${ }^{1}$, Peter van Gelderen ${ }^{1}$, Jacco A. de Zwart ${ }^{1}$, and Jeff H. Duyn ${ }^{1}$

${ }^{1}$ National Institute of Neurological Disorders and Stroke, National Institutes of Health, Bethesda, MD, United States

$\mathrm{T}_{2}{ }^{*}$-weighted MRI has broad applications in the brain and can provide both functional and (micro) anatomical information. Unfortunately, it has proven rather sensitive to subtle head motion, and the associated changes in $B_{0}$ and to a lesser extent $B_{1}$. In this study, the collective impact of pose-dependent $B_{0}$ and $B_{1}$ on $T_{2}{ }^{*}$-weighted gradient echo MRI was investigated. A conjugate-gradient method was utilized for reconstructing MR images collected during variation of head poses.

Retrospective motion correction of head motion using electromagnetic sensors

Onur Afacan ${ }^{1}$, Tess E. Wallace ${ }^{1}$, and Simon K. Warfield ${ }^{1}$

${ }^{1}$ Radiology, Boston Children's Hospital and Harvard Medical School, Boston, MA, United States

Motion artifacts pose significant problems for the acquisition of MR images, especially in pediatric populations. In this work we developed a retrospective motion correction framework that uses motion information from two electromagnetic sensors attached to the forehead of subjects. We evaluated our retrospective motion correction strategy on 12 different cases and show that that motion traces from the EM tracker can be used to retrospectively improve image quality.

Blurring and Ghosting Effects Under Beats Formation in Magnetic Resonance Imaging Under Source Vibration

Dhiraj Sinha ${ }^{1}$, Pranay Prateek ${ }^{2}$, Simon Lui ${ }^{2}$, and Shaoying Huang ${ }^{3}$

${ }^{1}$ Electrical Engineering and Computer Science, Massachusetts Institute of Technology, Cambridge, MA, United States, ${ }^{2}$ Information Systems Technology and Design, Singapore University of Technology and Design, Singapore, Singapore, ${ }^{3}$ Engineering Product Development, Singapore University of Technology and Design, Singapore, Singapore

A key challenge of MRI is development of an accurate model of noise generation which are integral to generation of high-resolution images. Currently, motion induced noise is addressed at algorithmic level. Here, we present a novel physical model which incorporates the role of mechanical vibration of body parts in generation of additional frequency components in the emitted radio frequency spectrum around the precession frequency. The mathematical model was validated through a computational simulation which led to the discovery that beats generated as a result of mechanical vibrations of the source lead to ghosting and blurring effects.

Pseudo-3D PROPELLER

Ola Norbeck ${ }^{1,2}$, Enrico Avventi ${ }^{1,2}$, Henric Ryden ${ }^{1,2}$, Johan Berglund $^{2}$, Tim Sprenger $^{3}$, and Stefan Skare ${ }^{1,2}$

${ }^{1}$ Neuroradiology, Karolinska University Hospital, Stockholm, Sweden, ${ }^{2}$ Clinical Neuroscience, Karolinska Instituet, Stockholm, Sweden, ${ }^{3}$ MR Applied Science Laboratory Europe, GE Healthcare, Stockholm, Sweden 
A thin-sliced (pseudo-3D) SMS accelerated PROPELLER with retrospective motion correction is demonstrated and compared to prospectively motion corrected 3D RARE using spiral navigators. The results show that our pseudo 3D PROPELLER sequence can produce higher image quality than 3D RARE, even in reformatted views, with and without the presence of head motion.

Reduction of respiratory motion artifact in c-spine imaging using deep learning: Is substitution of navigator possible?

Hongpyo Lee ${ }^{1}$, Kanghyun Ryu ${ }^{1}$, Yoonho Nam² ${ }^{2}$ Jaeho Lee ${ }^{1}$, and Dong-Hyun Kim ${ }^{1}$

Deep learning methods are starting to be widely used in medical images. Here, we propose a deep learning approach to compensate respiratory induced artifacts. A deep convolutional neural network was designed to train the ghosting artifact caused by respiratory motion in c-spine imaging. Using deep learning, compensation can be applied without additional data such as navigator echo.

\section{Pulses, Sequences, Motion \& Artefacts}

Can scans with different TR be combined to improve UTE T2* measurements?

Dirk H.J. Poot ${ }^{1,2}$, Paul Baron ${ }^{1}$, and Juan A. Hernandez-Tamames ${ }^{1}$

We investigated combining UTE sequences with different TR without requiring knowledge of T1, to enable increasing the number of short TE scans for T2* quantification. Many short T2* tissues have multiple compartments with ultra-short and somewhat longer T2 values. To quantify both a substantial number of images with ultra-short TE and images with a substantial maximal TE are required. The large maximal TE requires relatively large TR and hence long scan times, while the ultra-short TE scans have to be acquired separately. Hence, being able to combine images with different TR would be beneficial for such studies.

Image reconstruction in low field MRI: a super-resolution approach

Merel de Leeuw den Bouter ${ }^{1}$, Martin van Gijzen ${ }^{1}$, Andrew Webb² ${ }^{2}$ and Rob Remis ${ }^{3}$

${ }^{1}$ Delft Institute of Applied Mathematics, Delft University of Technology, Delft, Netherlands, ${ }^{2}$ Gorter Centre, Leiden University Medical Centre, Leiden, Netherlands, ${ }^{3}$ Circuits and Systems, Delft University of Technology, Delft, Netherlands

Inexpensive MRI scanners based on permanent magnets present a promising diagnostic tool for developing countries. For very inhomogeneous fields an ill-posed system of equations has to be solved in order to obtain an image. Due to the low signal-to-noise ratio, direct attempts at generating high resolution images yield poor results. In this research, superresolution reconstruction is considered as an alternative. By first obtaining low resolution images and then applying super-resolution, high resolution images of better quality can be obtained.

Properties optimization of pads configurations on CST to minimize B1+ field inhomogeneities at $7 \mathrm{~T}$ in the temporal lobes and cerebellum

Zo Raolison ${ }^{1}$, Marc Dubois ${ }^{2}$, Luisa Neves² ${ }^{2}$ Stefan Enoch², Nicolas Malléjac ${ }^{3}$, Pierre Sabouroux ${ }^{2}$, Anne-Lise Adenot-Engelvin ${ }^{3}$, Alexandre Vignaud ${ }^{1}$, and Redha Abdeddaïm²

$2663 \quad{ }^{1}$ CEA-Neurospin, Paris, France, ${ }^{2}$ Institut Fresnel, Marseille, France, ${ }^{3}$ CEA-Le Ripault, Monts, France

A simple and efficient way to enhance the $\mathrm{B}_{1}{ }^{+}$field dark areas appearing in the temporal lobes and cerebellum at $7 \mathrm{~T}$ in MRI is to use pads with relative High-Dielectric Constant materials. We present here simulations of different pads configurations aiming to reduce those dark areas. It has been found that the educated guess consisting in using a three pads configuration localized in front of each area is less efficient than two pads above the ears for the temporal lobes or a single pad on the neck for the cerebellum. 
${ }^{1}$ CEA-Neurospin, Paris, France, ${ }^{2}$ Institut Fresnel, Marseille, France, ${ }^{3}$ Siemens Healthineers, Saint Denis, France, ${ }^{4}$ CEA-Le Ripault, Monts, France

A simple and efficient way to enhance the $\mathrm{B}_{1}{ }^{+}$field dark areas appearing in the temporal lobes at $7 \mathrm{~T}$ in MRI is to use pads with relative High-Dielectric Constant materials which most promising ones are perovskites mixed with water. As their performance drops over time, those materials are still not currently used in clinical routine. A novel high lifespan material made of 4-Fluoro 1.3-dioxalan-2-one and Polyethylene glycol mixed with silicon carbide particles is presented here. It is shown that their performances are on pair with $\mathrm{BaTiO}_{3}$ water mixture through permittivity measurements and MRI scans a $7 \mathrm{~T}$.

Enabling long excitation pulses in algebraic ZTE imaging by dead-time reduction via dual acquisition with alternative RF modulations

Romain Froidevaux ${ }^{1}$, Markus Weiger ${ }^{1}$, and Klaas Paul Pruessmann ${ }^{1}$

${ }^{1}$ ETH Zurich and University of Zurich, Zurich, Switzerland

MRI of tissues with short transverse relaxation times raises both scientific and clinical interest and can be performed with zero echo time MRI. However, as RF excitation is done under the radial encoding gradient, flip angle amplitudes and uniformity are limited. This issue can be circumvented by using longer modulated pulses. However, pulse length is limited by dead-time-induced central k-space gaps getting too large for robust image reconstruction. In this work, we propose a new approach that enables the use of long RF pulses in algebraic ZTE by utilizing their intrinsic encoding properties to fill part of the dead-time gap.

Distribution-controlled and optimally spread non-Cartesian sampling curves for accelerated in vivo brain imaging at 7 Tesla

Carole Lazarus ${ }^{1}$, Pierre Weiss ${ }^{2}$, Loubna El Gueddari ${ }^{1}$, Franck Mauconduit ${ }^{3}$, Alexandre Vignaud ${ }^{4}$, and Philippe Ciuciu

${ }^{1}$ CEA/NeuroSpin - INRIA/Parietal, Gif-sur-Yvette, France, ${ }^{2}$ CNRS - ITAV, Toulouse, France, ${ }^{3}$ Siemens Healthineers, Saint-Denis, France, ${ }^{4}$ CEA/NeuroSpin/UNIRS/METRIC, Gif-surYvette, France

This work reports the use of new non-Cartesian k-space trajectories whose improved efficiency allows to significantly reduce MR scan time with minimum deterioration of image quality. Instead of using simple geometrical patterns, we introduce an approach inspired from stippling techniques, which automatically designs optimized sampling patterns along any distribution by taking full advantage of the hardware capabilities. Our strategy leads to drastically accelerated acquisitions, as demonstrated by our experimental results at $7 \mathrm{~T}$ on in vivo human brains. We compare our method to widely-used non-Cartesian trajectories (spiral,radial) and demonstrate its superiority regarding image quality and robustness to system imperfections.

Accelerated SMS-FSE with Long Hard Pulse Trains and Spatially Invariant FID Suppression

Eun Ji Lim ${ }^{1}$ and Jaeseok Park ${ }^{1}$

${ }^{1}$ Department of Biomedical Engineering, Sungkyunkwan University, Suwon, Republic of Korea

Simultaneous multi-slice (SMS) FSE in [1] was shown to be efficient for slice acceleration without much loss of signals. Despite its gains, conventional SMS-FSE, which employs highflip-angle, spatially selective multi-band RF pulses in both excitation and refocusing, remains challenging particularly on high magnetic field due to high energy deposition and limited echo train length (ETL), eventually leading to low imaging efficiency. To alleviate this problem, we recently introduced a variable-flip-angle (VFA) SMS-FSE imaging with long hard pulse trains in which spatially selective multi-band RF pulses are used only for excitation while all refocusing RF pulses are short and non-selective2. Nevertheless, this approach still remains sub-optimal due to the $180^{\circ}$ phase cycling in the refocusing pulse trains over two averages for FID suppression. Thus, the purpose of this work is to develop a novel, accelerated SMSFSE with long hard pulse trains and spatially invariant FID suppression in which sharable FID artifacts are directly constructed using only 2-TR calibration scan instead of 2-average phase cycling scan and then subtracted. It is demonstrated that the proposed SMS-FSE with an SMS factor of 7 makes it possible to complete whole brain imaging only in 15 sec without apparent artifacts and noise.

Rapid dynamic contrast-enhanced MRI for small animals at 7T using 3D UTE-GRASP

Jin Zhang ${ }^{1}$, Li Feng ${ }^{1}$, Ricardo Otazo ${ }^{1}$, and Sungheon Gene Kim ${ }^{1}$

${ }^{1}$ Center for Biomedical Imaging (CBI), Center for Advanced Imaging Innovation and Research (CAI2R), New York University School of Medicine, New York, NY, United States

It remains challenging to achieve simultaneous high spatial isotropic resolution and high temporal resolution in dynamic contrast enhanced (DCE) MRI of small animals, due to the relatively low signal to noise ratio (SNR) from small voxels. The purpose of this study is to develop a highly accelerated, high-spatial and high-temporal resolution DCE-MRI method for small animal imaging at 7T using 3D ultrashort echo time (UTE) golden-angle radial sampling with a combined compressed sensing and parallel imaging approach based on the GRASP technique. Our preliminary results demonstrate that the proposed UTE-GRASP method has the potential to improve both spatial and temporal resolution. 
José P. Marques ${ }^{1}$, Daniel Gomez ${ }^{1}$, and David G. Norris ${ }^{1}$

${ }^{1}$ Donders Centre for Cognitive Neuroimaging, Radboud University, Nijmegen, Netherlands

In this work we explore the added incoherence introduced when shifting the undersampling pattern in the phase enconding direction in successive slices, both when doing standard inplane acceleration in 2D imaging or Simultaneous Multi-Slice (SMS) imaging with CAIPI trajectories. To be able to explore this incoherence, we treat both the 2D imaging and SMS imaging as one volumetric problem where the physically successive slices are forced to be coherent.

Concomitant B1 Field in Low-Field MRI: Potential Contributions to TRASE Image Artefacts

Christopher P Bidinosti ${ }^{1}$, Pierre-Jean Nacher ${ }^{2}$, and Geneviève Tastevin ${ }^{2}$

${ }^{1}$ Department of Physics, University of Winnipeg, Winnipeg, MB, Canada, ${ }^{2}$ Laboratoire Kastler Brossel, ENS-PSL Research University, CNRS, UPMC-Sorbonne Université, Collège de France, Paris, France

TRansmit Array Spatial Encoding (TRASE) MRI uses trains of rf pulses produced by transmit coils which generate transverse fields of uniform magnitude and spatially varying directions. These coils also unavoidably generate concomitant rf fields, which in turn affect magnetisation dynamics during rf flips in low-field NMR. Bloch's equation are numerically solved to show that $\pi$-pulses imperfectly reverse transverse magnetisation and that the resulting error in azimuthal angle linearly increases with $B_{1} / B_{0}$, with the number of pulses in the TRASE pulse train, and with distance from the coil axis in the sample. This may induce significant image distortions or artefacts. Supporting experiments performed at $2 \mathrm{mT}$ will be reported.

Exploring the Limits of Super-Resolution MRI with Phaseless Encoding

Rui Tian ${ }^{1}$, Franciszek Hennel ${ }^{1}$, and Klaas P Pruessmann ${ }^{1}$

${ }^{1}$ Institute for Biomedical Engineering, University of Zurich and ETH Zurich, Zurich, Switzerland

The recently proposed method of Super-resolution (SR) MRI with phaseless subpixel encoding simultaneously samples three neighboring k-space bands and provides resolution enhancement factor up to 3.0. We now demonstrate an almost five-fold resolution enhancement by applying additional encoding steps of higher modulation frequency, which allows five bands to be acquired without compromising the methods' immunity to phase fluctuations. Since the signal-to-noise ratio at high resolution becomes critical, we derived and experimentally verified the optimum flip angle of the encoding (tagging) sequence. A possibility to correct artefacts caused by flip angle inhomogeneity is also shown based on simulation.

Banding-Free Balanced SSFP Cardiac Cine using Frequency Modulation and Phase-Cycle Redundancy

Anjali Datta ${ }^{1}$, Dwight G Nishimura ${ }^{1}$, and Corey A Baron ${ }^{1}$

${ }^{1}$ Electrical Engineering, Stanford University, Stanford, CA, United States

For banding-artifact reduction in cardiac cine bSSFP imaging, we present a highly accelerated frequency-modulated sequence that can be used to acquire three phase-cycles within a short breath-hold. A reconstruction that exploits redundancies between the phase-cycles enables the high acceleration. Acquiring more phase-cycles facilitates a flatter spectral profile short breath-hold. A reconstruction that exploits redundancies between the phase-cycles enables the high acceleration. Acquiring more phase-cycles facilitates a flatter spectral profile
after phase-cycle combination. We formulate a regularization term for the reconstruction that is general to any number of phase-cycles to consistently achieve good image quality in multiple subjects.

T1-weighted bipolar fat/water separated spin-echo PROPELLER acquired with dual bandwidths

Henric Rydén ${ }^{1,2}$, Johan Berglund ${ }^{1}$, Enrico Avventi ${ }^{1,2}$, Tim Sprenger $^{1,3}$, Ola Norbeck $^{1,2}$, and Stefan Skare ${ }^{1,2}$

${ }^{1}$ Department of Clinical Neuroscience, Karolinska Institutet, Stockholm, Sweden, ${ }^{2}$ Neuroradiology, Karolinska University Hospital, Stockholm, Sweden, ${ }^{3}$ GE Healthcare, Stockholm, Sweden

A bipolar fat/water separated T1-weighted dual-bandwidth spin-echo PROPELLER sequence is proposed which achieves strong and homogenous fat suppression without any dead time. Dual bandwidth sequences are compared against a corresponding fat saturated sequence in terms of SNR and CNR efficiency.

Development of a spiral spin- and gradient-echo (spiral-SAGE) approach for improved dynamic contrast neuroimaging

Ashley M. Stokes ${ }^{1}$, Ryan K. Robison ${ }^{2,3}$, Ashley G. Anderson III², James G. Pipe ${ }^{2}$, and C. Chad Quarles 
${ }^{1}$ Translational Bioimaging Group, Barrow Neurological Institute, Phoenix, AZ, United States, ${ }^{2}$ Magnetic Resonance Technology Design Group, Barrow Neurological Institute, Phoenix, AZ, United States, ${ }^{3}$ Phoenix Children's Hospital, Phoenix, AZ, United States

The purpose of this study is to develop a spiral-based combined spin- and gradient-echo (spiral-SAGE) pulse sequence for simultaneous dynamic contrast-enhanced (DCE-MRI) and dynamic susceptibility contrast MRI (DSC-MRI). Using this sequence, we obtained gradient-echo TEs of 1.69 and $26 \mathrm{ms,} \mathrm{a} \mathrm{SE} \mathrm{TE} \mathrm{of} 87.72 \mathrm{~ms}$, with a TR of $1663 \mathrm{~ms}$. Using an iterative SENSE reconstruction followed by deblurring, spiral-induced image artifacts were minimized. Comparison of spiral-SAGE images with conventional EPI-SAGE images illustrates substantial improvements in image distortion and image intensity variations. Spiral-SAGE provides a significant improvement for the assessment of perfusion and permeability in various neuropathologies.

A Two-Dimensional Spiral Multi-Echo Turbo-Spin-Echo Technique

Zhiqiang Li ${ }^{1}$, Ashley G Anderson III ${ }^{1}$, Melvyn B Ooi ${ }^{1,2}$, and James G Pipe ${ }^{1}$

TSE is widely used for T2 weighted imaging in routine clinical neuro exams. However, the concerns with TSE include its high specific absorption rate (SAR), and difference in contrast compared to conventional SE. In this work we propose a 2D spiral multi-echo TSE technique, which is insensitive to the T2-decay induced signal variation that affects other spiral TSE techniques. This technique provides improved contrast, high signal to noise ratio, and substantially reduced SAR, compared to Cartesian TSE.

T2 Mapping Using ZTE Combined with Burst Encoding (BURZTE)

Rolf F Schulte ${ }^{1}$ and Ana Beatriz Solana ${ }^{1}$

${ }^{1}$ GE Healthcare, Munich, Germany

ZTE acquisition is combined with spin-echo burst encoding for quiet T2 mapping. An initial ZTE excitation train encodes multiple 3D radial spokes, which get refocused by reversing the gradients. A double spin-echo leads to T2 decay, from which T2 maps are extracted by exponential fitting. Accuracy is validated in the Eurospin TO5 relaxation phantom, while in vivo feasibility is demonstrated by $\mathrm{T} 2$ mapping in healthy brains.

A Data Driven Nyquist Ghost and Gradient Delay Correction for Navigator-Free 3D Planes on a Paddlewheel (POP) EPI

Daniel Stäb ${ }^{1}$, Tobias Wech ${ }^{2,3}$, and Markus Barth ${ }^{1}$

${ }^{1}$ The Centre for Advanced Imaging, The University of Queensland, Brisbane, Australia, ${ }^{2}$ Department of Diagnostic and Interventional Radiology, University Hospital Würzburg, Würzburg, Germany, ${ }^{3}$ Comprehensive Heart Failure Centre, University Hospital Würzburg, Würzburg, Germany

3D planes-on-a-paddlewheel (POP) echo-planar imaging (EPI) is an effective non-Cartesian readout scheme realized by rotating conventional EPI readout planes about the phase encoding axis. Navigator based phase correction schemes are typically employed to account for gradient timing errors, associated trajectory errors and artifacts. In this work, we propose to use "Self Consistency for an Iterative Trajectory Adjustment" SCITA for an improved and purely data-driven removal of trajectory misalignment artifacts. As the actual k-space trajectory is derived from the imaging data, navigator acquisitions can be omitted and echo, repetition and acquisition times may be considerably shortened.

Tailored SEMs for wave modulations in SMS imaging

Sebastian Littin ${ }^{1}$, Stefan Kroboth ${ }^{1}$, Huijun $\mathrm{Yu}^{1}$, Feng Jia ${ }^{1}$, Ying-Hua Chu ${ }^{1}$, Yi-Cheng Hsu ${ }^{1}$, and Maxim Zaitsev ${ }^{1}$

${ }^{1}$ Department of Radiology, Medical Physics, Medical Center, University of Freiburg, Faculty of Medicine, Freiburg, Germany

The use of a matrix gradient coil enables to tailor spatial encoding magnetic Fields (SEMs) for slice specific frequency shifts. Applying such shifts in oscillatory manner allows for novel methods of signal separation in SMS imaging.

Phase corrected Hadamard acquisition compared with three-dimensional (3D) Fourier encoding for functional MRI

Seul Lee ${ }^{1}$ and Gary Glover ${ }^{2}$

${ }^{1}$ Electrical Engineering, Stanford University, Stanford, CA, United States, ${ }^{2}$ Radiology, Stanford University, Stanford, CA, United States 
Three-dimensional (3D) functional MRI (fMRI) can be superior in localization of activation signals compared to two-dimensional (2D) fMRI because higher spatial resolution can be acquired due to potentially higher signal-to-noise ratio (SNR) and thinner slices. However in 3D, physiological noise reduces SNR due to higher signal at the k-space center; thus the number of slices should be decreased to reduce physiological noise. With Fourier encoding, acquiring a small number of slices results in excessive Gibbs ringing. In this study, we propose Hadamard reconstruction for 3D fMRI acquisition to avoid the artifact caused from Fourier encoding and return higher SNR.

Improved Automatic Deblurring Using a Novel Objective Function Paired with a Retraced Spiral Acquisition Trajectory

Steven P Allen ${ }^{1}$, Xue Feng ${ }^{1}$, Samuel Fielden ${ }^{2}$, and Craig H Meyer ${ }^{1}$

${ }^{1}$ Biomedical Engineering, University of Virginia, Charlottesville, VA, United States, ${ }^{2}$ Autism and Developmental Medicine Institute, Geisinger Health System, Lewisburg, PA, United States

We introduce a novel objective function for automatic deblurring of images acquired with non-2DFT trajectories. When paired with the recently introduced retraced, spiral-in-out trajectory, this objective function provides two advantages over previously established functions: it is invariant with incidental phase and is less susceptible to spurious extrema. These advantages lead to effective deblurring over a larger range of off resonance conditions and readout durations. Here, using simulations and phantom studies, we compare the sensitivity of this objective function to spurious extrema to a previously proposed function.

Influence of Parameter Optimization and Segmentation on the Accuracy of Various Registration Approaches for Multi-parametric 3D Breast MRI Data

Subhajit Chatterjee ${ }^{1,2,3}$, Snekha Thakran ${ }^{1}$, Rakesh Kumar Gupta ${ }^{4}$, and Anup Singh ${ }^{1,5}$

${ }^{1}$ Centre for Biomedical Engineering, Indian Institute of Technology Delhi, New Delhi, India, ${ }^{2}$ C-DOT India, New Delhi, India, ${ }^{3}$ Computer Science and Engineering, Indian Institute of Technology Delhi, New Delhi, India, ${ }^{4}$ Department of Radiology, Fortis Memorial Research Institute, Gurgaon, India, New Delh, India, ${ }^{5}$ Department of Biomedical Engineering, All India Institute of Medical Sciences Delhi, New Delhi, India

Registration of human Breast MRI images is challenging due to its elastic deformable nature. In this study, various existing rigid and non-rigid registration methods were evaluated and compared in terms of accuracy and computation time. This work investigated influence of different registration parameters and showed possible ways to achieve better registration results. Experiential result revealed that the combination of Affine and B-spline method provided more time efficiency and accuracy than other methods.

Radius Segmented Multi-shot Spiral for Diffusion Imaging

Yukari Yamamoto ${ }^{1}$, Shinji Kurokawa² ${ }^{2}$ Yoshitaka Sato², and Hisaaki Ochi ${ }^{1}$

${ }^{1}$ Research \& Development Group, Hitachi, Ltd., Tokyo, Japan, ${ }^{2}$ Healthcare Business Unit, Hitachi, Ltd., Chiba, Japan

Single-shot echo-planar imaging (EPI) is usually used in diffusion-weighted imaging (DWI); however, it is difficult to apply to examining the entire brain because of image distortion due to susceptibility inhomogeneity. In addition, multi-shot imaging, in which image distortion is relatively small, is affected by pulsation artifacts and aliasing. We propose a multi-shot spiral method in which a spiral trajectory is divided in the radial direction. DWI studies were performed on the brain of a healthy volunteer. The proposed method could sample k-space data for each shot without aliasing, and sufficient correction for pulsation artifacts could be obtained.

PET/MR dynamic imaging of an inflatable phantom with self-gated UTE-MRI

Fatiha Andoh ${ }^{1}$, Tanguy Boucneau ${ }^{1}$, Marina Filipovic Pierucci ${ }^{2}$, Simon Stute ${ }^{2}$, Brice Fernandez ${ }^{3}$, Peder Larson $^{4}$, and Xavier Maître ${ }^{1}$

${ }^{1}$ Imagerie par Résonance Magnétique Médicale et Multi-Modalités, IR4M, CNRS, Univ. Paris Sud, Orsay, France, ${ }^{2}$ Imagerie Moleculaire In Vivo, IMIV Laboratory, CEA, Orsay, France,

${ }^{3}$ Applications \& Workflow, GE Healthcare, Orsay, France, ${ }^{4}$ Department of Radiology and Biomedical Imaging, University of California - San Francisco, San Francisco, CA, United States

MRI offers many advantages for chest imaging such as the absence of irradiation and the opportunity to obtain images with various contrasts in soft tissues. Developing MRI lung imaging would provide solutions to a real public health problem related to lung disease. Besides, PET is relevant for the study of metabolic changes caused by parenchymatous affections. Hence PET-MRI is a promising route for the characterization of lung diseases. One of the immediate issues lung imaging raises is motion. Physiological motion needs to be taken into account during the imaging process to avoid blurring or ghosting artifacts in both imaging modalities. 
${ }^{1}$ Radiology, University of California, San Diego, San Diego, CA, United States, ${ }^{2}$ GE Healthcare, San Diego, CA, United States, ${ }^{3}$ VA San Diego Healthcare System, San Diego, CA, United States

Conventional T2 and T1 $\rho$ have limited values in evaluating short T2 tissues, and are affected by the magic angle effect. Ultrashort echo time (UTE) sequences can detect short T2 tissues. Magnetization transfer (MT) modeling and adiabatic T1 $\rho$ (AdiabT1 $\rho$ ) seem to be insensitive to the magic angle effect. The combination of 3D UTE-Cones sequence with MT (3D UTE-Cones-MT) and AdiabT1 $\rho$ (3D UTE-Cones-AdiabT1 $\rho$ ) may resolve those limitations. However, patient motion may occur during the relatively long scan time. This study aims to develop 3D UTE-Cones-MT and UTE-Cones-AdiabT1 $\rho$ with an elastix registration technique to compensate for motion during the scans.

Rotating Outer Volume Suppression for Reduced Field of View PROPELLER Imaging

Daniel V Litwiller ${ }^{1}$, Valentina Taviani² ${ }^{2}$ Suchandrima Banerjee ${ }^{2}$, Lloyd Estkowski ${ }^{2}$, Yuval Zur ${ }^{3}$, Ali Ersoz $^{4}$, and Ersin Bayram

${ }^{1}$ Global MR Applications \& Workflow, GE Healthcare, New York, NY, United States, ${ }^{2}$ Global MR Applications \& Workflow, GE Healthcare, Menlo Park, CA, United States, ${ }^{3}$ GE Healthcare, Haifa, Israel, ${ }^{4}$ GE Healthcare, Waukesha, WI, United States, ${ }^{5}$ Global MR Applications \& Workflow, GE Healthcare, Houston, TX, United States

We present a modified PROPELLER pulse sequence that incorporates rotating outer volume suppression for reduced field of view imaging. In vivo results are presented, demonstrating comparable imaging performance with conventional PROPELLER imaging.

Reformattable MAVRIC-SL Using Robust Principal Component Analysis and Variable Density Complementary Poisson Disc Sampling

Philip K. Lee ${ }^{1,2}$, Daehyun Yoon ${ }^{2}$, Xinwei Shi ${ }^{1,2}$, Evan G. Levine ${ }^{1,2}$, Yuxin $\mathrm{Hu}^{1,2}$, and Brian A. Hargreaves ${ }^{1,2}$

${ }^{1}$ Electrical Engineering, Stanford University, Stanford, CA, United States, ${ }^{2}$ Radiology, Stanford University, Stanford, CA, United States

MAVRIC-SL resolves metal-induced artifacts at the cost of additional scan time. A reconstruction using Robust Principal Component Analysis (RPCA) has been shown to considerably reduce scan times with minimal loss in image quality. We apply this scan time reduction to acquire isotropic MAVRIC-SL data that can be reformatted to all three planes, combining multiple high-resolution scans into a single, short, isotropic scan. We show retrospectively undersampled isotropic MAVRIC-SL RPCA reconstructions reformatted to three planes for the case of a hip phantom, and a volunteer with a titanium hip replacement. The RPCA reconstruction offers good image quality in multiple planes at clinically feasible scan times, with shorter scan times than separate high-resolution acquisitions.

Accurate localization of individual DBS contacts by MRI using zero-TE phase images

Sathish Ramani ${ }^{1}$, Rolf Schulte ${ }^{2}$, Graeme Mckinnon ${ }^{3}$, Jeffrey Ashe ${ }^{1}$, Julie Pilitsis ${ }^{4}$, and Ileana Hancu ${ }^{1}$

${ }^{1}$ GE Global Research, Niskayuna, NY, United States, ${ }^{2}$ GE Healthcare, Munich, Germany, ${ }^{3}$ GE Healthcare, Waukesha, WI, United States, ${ }^{4}$ Albany Medical Center, Albany, NY, United States

The goal of our work was to demonstrate improved DBS contact visualization and localization by using a zero-TE (ZTE) acquisition. Signal dephasing during sequence readout, proportional to the electrode-induced field inhomogeneity, enables high-contrast visualization of individual electrode contacts. Matching measured ZTE-phase maps to simulations of orientation dependent, susceptibility induced field inhomogeneity created by the electrode is shown to result in significantly more accurate and precise contact localization than by using standard SPGR acquisitions. Electrode center differences of $0.69 \pm 0.45 \mathrm{~mm} / 0.32 \pm 0.09 \mathrm{~mm}$ were seen between SPGR/ZTE[phase] and CT.

Measured k-space based RF Compensation Effect Analysis within Various 2D Excitation Volume in 7T pTx system

Sanghoon $\mathrm{Kim}^{1}$ and Mark Lowe

${ }^{1}$ Radiology, Cleveland Clinic, CLEVELAND, OH, United States

This work presents simple method for RF compensation effect analysis. For the RF compensation, we used previously presented measured k-space based method. We analyzed three different $2 \mathrm{D}$ excitation volume data using simple histogram based method and found that not only for small volume excitation region, larger volume excitation region shows significant and more dominant compensation effect. This finding will help inform the design of RF profiles in In-vivo 2D excitation applications in pTx system.

$\mathrm{K}_{\mathrm{T}}$-Points Pulses Reduce $\mathrm{B}_{1}$ Shading at $3 \mathrm{~T}$ : Demonstration in Routine Abdominal DCE-MRI and Evaluation of Reliability

Raphaël Tomi-Tricot ${ }^{1}$, Vincent Gras ${ }^{1}$, Franck Mauconduit ${ }^{2}$, François Legou ${ }^{3}$, Nicolas Boulant ${ }^{1}$, Matthias Gebhardt ${ }^{4}$, Dieter Ritter ${ }^{4}$, Berthold Kiefer ${ }^{4}$, Pierre Zerbib $^{3}$, Alain Rahmouni ${ }^{3,5}$, Alexandre Vignaud ${ }^{1}$, Alain Luciani ${ }^{3,5,6}$, and Alexis Amadon ${ }^{1}$ 
${ }^{1}$ CEA/DRF/Joliot/NeuroSpin/UNIRS, Gif-sur-Yvette, France, ${ }^{2}$ Siemens Healthcare SAS, Saint-Denis, France, ${ }^{3}$ Department of Radiology, AP-HP, CHU Henri Mondor, Créteil, France,

${ }^{4}$ Siemens Healthcare $\mathrm{GmbH}$, Erlangen, Germany, ${ }^{5}$ Université Paris-Est Créteil Val-de-Marne, Créteil, France, ${ }^{6}$ INSERM Unité U955, Equipe 18, Créteil, France

At high field, MRI systems offer a higher signal-to-noise ratio, but $\mathrm{B}_{1}{ }^{+}$-inhomogeneity-induced artefacts in large organs can lead to shading and erroneous contrast. In this work, subjecttailored $\mathrm{k}_{\mathrm{T}}$-points pulse design performance was evaluated in clinical routine on liver DCE-MRI at $3 \mathrm{~T}$, against that of patient-specific RF shimming. Both excitation homogeneity simulation and image quality assessment were performed on a variety of patients. The interest of $\mathrm{k}_{\mathrm{T}}$-points is clearly demonstrated, as well as the reliability of the approach.

$\mathrm{k}_{\mathrm{T}}$-spokes: combining $\mathrm{k}_{\mathrm{T}}$-points with spokes to ease ramp pulse design for TOF slab selection with parallel transmission at 7T

Gaël Saïb ${ }^{1}$, Vincent Gras ${ }^{1}$, Franck Mauconduit ${ }^{2}$, Alexandre Vignaud ${ }^{1}$, Denis Le Bihan ${ }^{1}$, Laurent Le Brusquet ${ }^{3}$, Nicolas Boulant ${ }^{1}$, and Alexis Amadon ${ }^{1}$

${ }^{1}$ CEA/DRF/Joliot/NeuroSpin/UNIRS, Gif-sur-Yvette, France, ${ }^{2}$ Siemens Healthineers France, Saint-Denis, France, ${ }^{3}$ Laboratoire des Signaux et Systèmes, Université ParisSaclay/CentraleSupélec/CNRS, Gif-sur-Yvette, France

TONE pulses counteract blood saturation through the imaged slab in TOF sequences, but their ramp profile is hampered by RF inhomogeneities at Ultra High Field. On the other hand, $\mathrm{k}_{\mathrm{z}}$-spokes are known to compensate for in-plane $\mathrm{B}_{1}{ }^{+}$heterogeneities in slice or slab selection. However, their design doesn't address thru-slab heterogeneities. To address them, a new pulse type called " $\mathrm{k}_{\mathrm{T}}$-spokes" is introduced. As TONE pulses, $\mathrm{k}_{\mathrm{T}}$-spokes efficacy is demonstrated with $\mathrm{pTx}$ at $7 \mathrm{~T}$ in comparison with mere equivalent $\mathrm{k}_{\mathrm{z}}$-spokes.

K-Space Trajectory Correction for UTE Sequence with Multi-Echo Radial Acquisition

Liao Ying ${ }^{1}$, Paul Kyu Han ${ }^{2}$, Shuang $\mathrm{Hu}^{2,3}$, Kui Ying ${ }^{4,5}$, Chao $\mathrm{Ma}^{2}$, and Georges El Fakhri ${ }^{2}$

${ }^{1}$ Biomedical Engineering, Tsinghua University, Beijing, China, ${ }^{2}$ Gordon Center for Medical Imaging, Radiology, Massachusetts General Hospital, Harvard Medical School, Boston, MA, United States, ${ }^{3}$ Nuclear Medicine, West China Hospital, Sichuan University, Sichuan, China, ${ }^{4}$ Engineering Physics, Tsinghua University, Beijing, China, ${ }^{5}$ Key Laboratory of Particle and Radiation Imaging, Ministry of Education, Medical Physics and Engineering Institute, Tsinghua University, Beijing, China

UTE allows imaging of rapidly decaying short- $\mathrm{T}_{2}$ components and are often combined with multi-echo radial acquisition for PET attenuation correction applications. However, UTE is inherently susceptibility to gradient errors due to the usage of radial acquisition and simple time delay corrections render impractical to correct deviations from the ideal trajectory when UTE is combined with multi-echo radial acquisition scheme. In this work, we describe a simple, one-time calibration method that allows k-space trajectory correction for UTE sequence combined with multi-echo radial acquisition. The performance of the proposed method is shown via a phantom and an in vivo experiment, using a calibration scan previously acquired from a water phantom

Spin Lock Adiabatic Correction (SLAC) Excitation

Edward M Green ${ }^{1}$, James C Korte ${ }^{1}$, Bahman Tahayori ${ }^{2,3}$, Peter M Farrell ${ }^{4}$, and Leigh A Johnston ${ }^{1}$

${ }^{1}$ Dept. of Biomedical Engineering, University of Melbourne, Melbourne, Australia, ${ }^{2}$ Dept. Electrical and Computer Systems Engineering, Monash University, Melbourne, Australia, ${ }^{3}$ Dept. of Medical Physics and Biomedical Engineering, Shiraz University of Medical Sciences, Shiraz, Iran (Islamic Republic of), ${ }^{4}$ Dept. of Electrical and Electronic Engineering, University of Melbourne, Melbourne, Australia

A new form of B1-insensitive excitation is introduced, termed Spin-Lock Adiabatic Correction (SLAC) excitation, that combines a Spin-Locking excitation with an orthogonal Adiabatic Correction to more uniformly flip the magnetisation across a range of B1 strengths. SLAC pulses achieve adiabatic-like excitation, in terms of B1-insensitivity, in faster excitation time while not increasing the delivered power. We demonstrate the advantages of SLAC pulses in both simulation and phantom experiments. Decreasing the pulse duration causes performance breakdown of the adiabatic pulse due to violation of the adiabatic condition, while the SLAC pulse maintains control of magnetisation across the range of B1 strengths.

Comparison of Efficacy of Multiple EPI Distortion Correction Techniques on Toddler Data

Vinai Roopchansingh ${ }^{1}$, Jerry Judson French $\mathrm{Jr}^{2}$, Daniel Glen ${ }^{3}$, Richard Reynolds ${ }^{3}$, Dylan Miles Nielson ${ }^{4}$, Robert William Cox ${ }^{3}$, Audrey Thurm ${ }^{2}$, and Susan Elizabeth Swedo ${ }^{2}$

${ }^{1}$ Functional MRI Facility / NIMH, National Institutes of Health, Bethesda, MD, United States, ${ }^{2}$ Section on Behavioral Pediatrics / NIMH, National Institutes of Health, Bethesda, MD, United States, ${ }^{3}$ Scientific and Statistical Computing Core / NIMH, National Institutes of Health, Bethesda, MD, United States, ${ }^{4}$ Data Science and Sharing Team / NIMH, National Institutes of Health, Bethesda, MD, United States

Echo-planar data acquired from a group of toddlers was distortion corrected using combinations of different data, algorithms, and software packages. Performance was evaluated by comparing mutual information scores of how well corrected versus uncorrected EPI data aligned with structural $\mathrm{T}_{1}$-weighted data. 
Evaluating $\mathrm{T} 2^{*}$ bias impact and correction strategies in quantitative proton density mapping

Evelyne Balteau ${ }^{1}$, Tobias Leutritz ${ }^{2}$, Nikolaus Weiskopf ${ }^{2}$, Enrico Reimer ${ }^{2}$, Antoine Lutti ${ }^{3}$, Martina F Callaghan ${ }^{4}$, Siawoosh Mohammadi ${ }^{5}$, and Karsten Tabelow $^{6}$

${ }^{1}$ Cyclotron Research Centre - GIGA-CRC in vivo imaging, University of Liege, Liege, Belgium, ${ }^{2}$ Department of Neurophysics, Max Planck Institute for Human Cognitive and Brain Sciences, Leipzig, Germany, ${ }^{3}$ Laboratoire de Recherche en Neuroimagerie, CHUV, University of Lausanne, Lausanne, Switzerland, ${ }^{4}$ Wellcome Trust Centre for Neuroimaging, University College London, London, United Kingdom, ${ }^{5}$ Institut für Systemische Neurowissenschaften, Universitätsklinikum Hamburg-Eppendorf, Hamburg, Germany, ${ }^{6}$ Weierstrass Institute for Applied Analysis and Stochastics, Berlin, Germany

Bias correction is an important step for achieving accurate and precise parameter quantification in MRI. Residual $\mathrm{T}_{2}{ }^{*}$-weighting in quantitative proton density maps estimated from short echo time FLASH images is often considered negligible, despite the potential bias. Using the hMRI toolbox, we analyse simulated FLASH-based multiparameter mapping datasets with variable noise levels. Using the quantitative maps on which the simulations are based as a gold standard, we quantified the bias caused by residual $T_{2}{ }^{*}$-weighting. Furthermore, we evaluated a number of estimation methods in terms of their sensitivity and/or effectiveness at correcting this $\mathrm{T}_{2}{ }^{*}$-weighting bias, and in terms of their robustness to background noise.

A Simple Method for Improved Correction of EPI Odd-Even Line Inconsistency

Yuan Zheng ${ }^{1}$, Yu Ding ${ }^{1}$, Qing Wei ${ }^{2}$, and Weiguo Zhang ${ }^{1}$

${ }^{1}$ UIH America, Inc., Houston, TX, United States, ${ }^{2}$ United Imaging Healthcare, Shanghai, China

We have developed a simple method for EPI Nyquist ghosting artifacts removal. Our technique borrows the idea of GRAPPA, and extracts a non-biased kernel from imperfect multichannel EPI data to correct the odd-even line inconsistency. We have demonstrated both in-vivo and in-vitro that this strategy can significantly reduce Nyquist ghosts. The proposed method is quite simple and can be conveniently used with many current EPI correction techniques to generate ghosting-free images.

Real-time cardiac MR imaging based on a radial bSSFP sequence with trajectory auto-correction

Guoxi Xie ${ }^{1}$, Xiaoyong Zhang ${ }^{2,3}$, Wenlong $\mathrm{Lv}^{2}$, Caiyun $\mathrm{Shi}^{2}$, Shi Su${ }^{2}$, Bensheng Qiu ${ }^{4}$, and Xin $\mathrm{Liu}^{2}$

${ }^{1}$ Department of Biomedical Engineering, Guangzhou Medical University, Guangzhou, China, ${ }^{2}$ Shenzhen Institutes of Advanced Technology, Chinese Academy of Sciences, Shenzhen, China, ${ }^{3}$ MR Collaborations NE Asia, Siemens Healthcare, Shenzhen, China, ${ }^{4}$ Centers for Biomedical Engineering, University of Science and Technology of China, Hefei, China

Conventional cardiac cine imaging is based on ECG-triggering, which is difficult to be used in arrhythmic patients. Real-time cardiac cine technique based on radial sampling scheme is an alternative approach for imaging the arrhythmic patients. However, the technique is often hampered in trajectory error due to system gradient delay. To address this issue, a novel real-time cardiac cine technique was developed based on a radial bSSFP sequence with trajectory error auto-correction. Preliminary results demonstrated that the proposed technique can improve the image quality and has potential to be clinically useful for the arrhythmic patients.

A novel method for video-based cardiac gating in 7T MR angiography using a video of the foot

Nicolai Spicher ${ }^{1}$, Stephan Orzada ${ }^{2}$, Stefan Maderwald², Mark E. Ladd ${ }^{2,3}$, and Markus Kukuk ${ }^{1}$

${ }^{1}$ Department of Computer Science, University of Applied Sciences and Arts Dortmund, Dortmund, Germany, ${ }^{2}$ Erwin L. Hahn Institute for Magnetic Resonance Imaging, University Duisburg-Essen, Essen, Germany, ${ }^{3}$ Division of Medical Physics in Radiology, German Cancer Research Center, Heidelberg, Germany

In ultra-high-field MRI, cardiac gating is problematic because electrocardiography is prone to magnetohydrodynamic artifacts and pulse oximetry suffers from signal loss during long examinations. The goal of this work is to investigate practical feasibility of cardiac gating based on a video from the sole of the foot that is leaned to a glass plate. We combined this novel setup with an open-source software for video-based cardiac gating (https://github.com/nspi/vbcg) and performed ultra-high-field non-enhanced angiography in one volunteer. As reference, we performed pulse oximetry gating and comparison of maximum intensity projection images shows a similar image quality. Future work will evaluate the feasibility of this novel cardiac gating method in a larger cohort.

Anna Scherman Rydhög ${ }^{1}$, Ofer Pasternak ${ }^{2}$, Freddy Ståhlberg ${ }^{1,3,4}$, Ronnie Wirestam ${ }^{1}$, Linda Knutsson ${ }^{1,5}$, and André Ahlgren ${ }^{1}$

${ }^{1}$ Department of Medical Radiation Physics, Lund University, Lund, Sweden, ${ }^{2}$ Departments of Psychiatry and Radiology, Harvard Medical School, Boston, MA, United States, ${ }^{3}$ Department of Diagnostic Radiology, Lund University, Lund, Sweden, ${ }^{4}$ Lund University Bioimaging Center, Lund University, Lund, Sweden, ${ }^{5}$ The Russell H. Morgan Department of Radiology and Radiological Science, The Johns Hopkins University School of Medicine, Baltimore, MD, United States 
In conventional intravoxel incoherent motion (IVIM) imaging, the blood fraction is estimated using a two-compartment model (blood and tissue). However, blood fraction estimation is hampered by cerebrospinal fluid (CSF) contamination and tissue-dependent relaxation times. We propose a three-compartment model (blood, tissue, CSF), which accounts for compartment-specific diffusion and relaxation properties. Estimation of gray and white matter blood fractions using this model is demonstrated with in-vivo human data of variable diffusion weightings, echo times and inversion times. In comparison with two-compartment models (with and without relaxation), the proposed three-compartment model yielded lower estimates of the blood fraction, suggesting a better separation from CSF.

Data driven sampling of k-space using GO-Active technique

Pavan poojar ${ }^{1,2}$, Ashok kumar reddy ${ }^{2}$, Amaresha Shridhar Konar ${ }^{1}$, Ramesh Venkatesan $^{2}$, and Sairam Geethanath ${ }^{1,3}$

${ }^{1}$ Medical Imaging Research Centre, Dayananda Sagar Institutions, Bangalore, India, ${ }^{2}$ GE, Healthcare, Bangalore, India, ${ }^{3}$ Dept. of Radiology, Columbia University Medical Center, New York, NY, United States

The extensive coverage of k-space data on a standard MRI scanner requires long acquisition times. In dynamic MRI methods such as DCE-MRI, cardiac MRI, DWI, etc., the shape of the significant values in k-space depends on the structure of the organ and temporal events. The proposed method generates the arbitrary k-space trajectory and optimizes the gradient waveforms by utilizing GO-Active. Design constraints of gradient system are slew rate and gradient amplitude are accounted for by using convex optimization. All images were acquired on a GE 1.5T scanner. Image reconstruction was performed in graphical programming interface.

Optimal Choice of Echo Times for Gradient Echo B0 Field Mapping

Yasmin Geiger ${ }^{1}$ and Assaf Assaf Tal

${ }^{1}$ Chemical and Biological Physics, Weizmann Institute of Science, Rehovot, Israel

Field maps are essential in spectroscopy, shimming, MR thermometry and geometric distortion correction. Minimizing the noise in acquired field maps is therefore potentially important to all of these applications. When using a multi-gradient echo, the choice of echo times has a marked effect on the noise on the acquired field maps. Here, we derive the optimal echo times which minimize the amount of noise in the resulting field maps.

Unconventional trajectories on the Bloch Sphere: A closer look at the effects and consequences of the breakdown of the rotating wave approximation

Christopher Bidinosti ${ }^{1}$, Pierre-Jean Nacher ${ }^{2}$, and Geneviève Tastevin ${ }^{2}$

${ }^{1}$ Physics, University of Winnipeg, Winnipeg, MB, Canada, ${ }^{2}$ Laboratoire Kastler Brossel, ENS-PSL Research University, Paris, France

TRASE MRI uses rapid m-pulses of phase gradient fields, and in general requires as many as two distinct phase-gradient coils per encoding direction. This tends to restrict one to large amplitude, linear $\mathrm{B}_{1}$ fields, which in low $\mathrm{B}_{0}$ field leads to a breakdown of the rotating wave approximation. We have studied this regime both numerically and experimentally. Our results show a rich behavior involving a complex interplay of the Bloch-Siegert shift, the $B_{1}$ start and stop phase, and $B_{1}$ amplitude transients.

Respiratory-Gated $B_{0}$ Field Stabilisation for High Resolution Mouse Brain Imaging

Paul Kinchesh ${ }^{1}$, Stuart Gilchrist ${ }^{1}$, Niloufar Zarghami ${ }^{1}$, Alexandre A Khrapitchev ${ }^{1}$, Nicola R Sibson ${ }^{1}$, and Sean Smart ${ }^{1}$

${ }^{1}$ CRUK/MRC Oxford Institute for Radiation Oncology, University of Oxford, Oxford, United Kingdom

The echoes of a 3D multi gradient echo (MGE) scan are typically combined for detection of USPIO and MPIO. The echo combination requires $B_{0}$ to be constant throughout the scan to achieve good image fidelity at high resolution. A navigator acquisition embedded in the MGE scan maintains the MR steady state and enables a real-time adaptive $B_{0}$ correction. It is demonstrated that a respiratory-gated correction scheme outperforms ungated correction in mouse brain for the detection of micron sized iron-oxide particles coupled with anti-vascular cell adhesion molecule antibody (VCAM-MPIO) to identify inflammation in vessels.

Flexible spatial encoding strategy using receive coil aggregates for Halbach magnet array based magnetic resonance imaging

Dong Wei Lu ${ }^{1}$, Zhi Hua Ren ${ }^{1}$, and Shao Ying Huang

${ }^{1} E P D$ Pillar, Singapore University of Technology and Design, Singapore, Singapore 
To make a MRI system portable, a practical approach is applying Halbach magnet array and nonlinear spatial encoding strategy. Here, the rotation of a magnet array for imaging is replaced by electrically forming RF receive coil aggregates with phase delay. For the resultant system with a new encoding matrix, Truncated-Singular-Value-Decomposition with an optimal regularization parameter is proposed which reconstructs images with good quality. An accelerated L-curve method is proposed to obtain the optimal regularization parameter. Results show that the proposed strategy provides considerable improvement of the image quality compared to existing method, e.g. Kaczmarz iteration, without rotating the magnet array.

Simultaneous Multi-Contrast Imaging in Combination with in-plane Parallel Imaging

Nora-Josefin Breutigam ${ }^{1}$, Matthias Günther ${ }^{1,2,3}$, and David Andrew Porter ${ }^{4}$

${ }^{1}$ MR Physics, Fraunhofer MEVIS, Bremen, Germany, ${ }^{2}$ University Bremen, Bremen, Germany, ${ }^{3}$ mediri GmbH, Heidelberg, Germany, ${ }^{4}$ MRI Physics, Imaging Centre of Excellence, College of Medical, Veterinary \& Life Sciences, University of Glasgow, Glasgow, Scotland

Simultaneous Multi-Contrast (SMC) Imaging enables a synchronous acquisition of multiple image contrasts within one measurement. The technique reduces patient examination times and facilitates accurate image registration between contrasts. Previous work used readout-segmented EPI (rs-EPI) to perform high-resolution, navigator-corrected, diffusion-weighted imaging simultaneously with a T2*-weighted acquisition. This combination of contrasts has clinical significance in acute stroke. These previous studies did not use in-plane acceleration to reduce spatial distortion caused by the EPI readout. This study introduces an updated version of the SMC technique that incorporates in-plane acceleration with GRAPPA to allow an improved image quality for future clinical studies.

3D Cones acquisition for human extremities using a $1.5 \mathrm{~T}$ compact superconducting magnet and unshielded gradient coil

Ayana Setoi ${ }^{1}$ and Katsumi Kose ${ }^{1}$

${ }^{1}$ University of Tsukuba, Tsukuba, Japan

We developed 3D Cones sequences for human extremities on a $1.5 \mathrm{~T}$ MRI system using a compact superconducting magnet ( $280 \mathrm{~mm}$ bore) equipped with an unshielded gradient coil. Linear eddy fields were measured using a spherical phantom and eddy current effects on the 3D Cones sequences were evaluated using a 3D water phantom. As a result, effects of higher-order eddy fields proportional to $z^{2} x$ and $z^{2} y$ spatial distributions were clearly observed. The 3D Cones sequences were applied to UTE imaging of a porcine hoof sample and a human forearm, which demonstrated their promise in UTE imaging.

GPU-optimized fast 3D MRI simulator for arbitrary trajectory sampling

Ryoichi Kose ${ }^{1}$, Ayana Setoi ${ }^{2}$, and Katsumi Kose ${ }^{2}$

${ }^{1}$ MRTechnology, Inc., Tsukuba, Japan, ${ }^{2}$ University of Tsukuba, Tsukuba, Japan

We developed a GPU-optimized fast 3D MRI simulator for arbitrary trajectory sampling. The performance of the simulator was evaluated using stack of 2D spiral and 3D Cones sequences. The result demonstrated that our simulator is a powerful tool for studies of non-Cartesian sampling as well as Cartesian sampling imaging sequences.

DIXON-type pulse sequence for MRI-only external beam radiotherapy of prostate cancer

Souha Aouadi ${ }^{1}$, Satheesh Paloor ${ }^{1}$, Ana Vasic ${ }^{1}$, Tarraf Torfeh ${ }^{1}$, Maeve McGarry ${ }^{1}$, Primoz Petric ${ }^{1}$, Hadi Fayad ${ }^{1}$, Rabih Hammoud ${ }^{1}$, and Noora Al-Hammadi ${ }^{1}$

${ }^{1}$ Department of Radiation Oncology, National Center for Cancer Care and Research, Hamad Medical Corporation, Doha, Qatar

Water-fat separated images provided by the DIXON-type pulse sequence were combined with the multi-scale and dual-contrast patch-based method to generate synthetic-CT (sCT) for MR-only external beam radiotherapy treatment planning of prostate cancer. The benefit of such sequence was demonstrated by retrospective geometric and dosimetric evaluation of SCT on five patients. Compared to reference CT, the mean absolute error was $89.07 \pm 14.2 \mathrm{HU}$, the dice coefficient in soft tissues was $0.93 \pm 0.01$. Good agreement with conventional planning techniques was obtained; the highest percentages of dose metrics deviations were below $0.7 \%$ for PTV, $0.05 \%$ for the rectum, and $0.01 \%$ for the bladder.

Hsu-Lei Lee ${ }^{1,2}$, Zengmin Li' ${ }^{1}$, and Kai-Hsiang Chuang ${ }^{1,2}$ 
Acceleration of rodent brain functional MRI using parallel imaging techniques is not widely used due to the limited availability of high-density phased-array coil on pre-clinical scanners. In this study we demonstrated a POMP-EPI method to enable simultaneous multi-slice acquisition for fast mouse brain imaging without a phased array coil. A four-fold multiband acceleration was achieved without using coil sensitivity information. This method can be used to increase the spatial or temporal resolution of mouse fMRI acquisition, which will benefit the study of dynamics of neural activity and connectivity.

Analysis of diffusion effects in SSFP sequences with extended phase graphs

Yangzi Qiao ${ }^{1}$, Chao Zou ${ }^{1}$, Chuanli Cheng ${ }^{1,2}$, Qian Wan ${ }^{1}$, Changjun Tie ${ }^{1}$, Xin Liu ${ }^{1}$, and Hairong Zheng

${ }^{1}$ Shenzhen Institutes of Advanced Technology, Chinese Academy of Sciences, Shenzhen, China, ${ }^{2}$ University of Chinese academy of Sciences, Beijing, China

EPG simulation was applied to analysis the diffusion effect of two SSFP-FID signals, FISP and ES. The influence of T1, T2, and unbalanced gradient on signal intensity with consideration of diffusion effect was studied. The EPG simulation have a good consistency with the experimental data, indicating it can efficiently and precisely calculate the diffusion effect of SSFP signals. Both the simulation and phantom study reveals that for some specific tissues and imaging parameters, positive diffusion contrast can be obtained in FISP and ES sequence. For quantitative method based on SSFP signals, such as TESS relaxometry, the diffusion effect should be considered while large unbalanced gradients and small flip angle were employed for high resolution imaging in high field system.

Simple algorithm for the correction of MRI image artefacts due to random phase fluctuations

P. James Ross ${ }^{1}$, Lionel M. Broche ${ }^{1}$, and David J. Lurie ${ }^{1}$

${ }^{1}$ Aberdeen Biomedical Imaging Centre, University of Aberdeen, Aberdeen, United Kingdom

Here we present a simple post-processing algorithm that is able to correct ghosting caused by a slow off-resonance drift caused by the use of a resistive magnet. The algorithm is described and validated in simulations, phantoms and in vivo.

Traditional Poster

\section{Machine Learning for Cancer Applications}

Radiomics analysis for preoperative prediction of synchronous distant metastasis in patients with rectal cancer

Huanhuan Liu ${ }^{1}$, Caiyuan Zhang ${ }^{1}$, Jinning $\mathrm{Li}^{1}$, Weibo Chen ${ }^{2}$, and Dengbin Wang ${ }^{1}$

${ }^{1}$ Radiology, Xinhua hospital, Shanghai Jiaotong University School of Medicine, Shanghai, China, ${ }^{2}$ Philips Healthcare, Shanghai, China

Rectal cancer is one of the most common malignant tumors in gastrointestinal tract. Tumor metastasis is still a major cause of death in patients with rectal cancer. The distant metastasis rate for rectal cancer remains constant at 20-50\%1. Prediction of synchronous distant metastasis is important for the choice of personalized treatment strategies. Radiomics can extract quantitative features from digital images, which are related to the underlying pathophysiology2. We developed a radiomics model based on the MR radiomics features in combination with independent clinico-radiologic risk factors, which help to predict the synchronous distant metastasis in patients with rectal cancer

Computer-aided diagnosis of hepatocellular carcinoma and hepatic cavernous hemangioma using non-enhanced MRI with a random forest classifier

Jingjun Wu${ }^{1}$, Ailian Liu ${ }^{1}$, Jingjing $\mathrm{Cui}^{2}$, and Lizhi $\mathrm{Xie}^{3}$

${ }^{1}$ Department of Radiology, The First Affiliated Hospital of Dalian Medical University, Dalian, China, ${ }^{2}$ Huiying Medical Technology Co., Ltd., Beijing, China, ${ }^{3}$ GE Healthcare, MR Research, Beijing, China

The current study aims to develop a computer-aided diagnosis (CAD) system and assess its ability in identification of hepatocellular carcinoma (HCC) and hepatic cavernous hemangioma $(\mathrm{HCH})$ using non-enhanced MRI with a random forest classifier. Good performance was observed in this $\mathrm{CAD}$ system based on out-phase images. 
Deep learning has become the method of choice for tumor segmentation. Most deep learning algorithms incorporate a multi-modal approach, as different MR modalities are optimized to detect different aspects of tumor. However, modalities are often missing or unusable due to artifacts. In such cases, it is difficult to perform robust automatic tumor segmentation. We demonstrate that a convolutional neural network can be used to synthesize FLAIR MR images that have high similarity with real FLAIR images. Furthermore, we show that the use of these synthetic images can improve segmentation performance.

Development and Validation of a Classifier for Prediction of Distant Metastasis in Nasopharyngeal Carcinoma at Initial Staging

Bin Zhang ${ }^{1}$

${ }^{1}$ The first affiliated hospital of Jinan university, Guangzhou, China

we sought to improve the prediction of DM in NPC patients by developing a novel combined classifier to stratified patients into high-risk and low-risk groups with significant differences in 5-year survival. To our best of knowledge, our study is the first to integrate intratumor heterogeneity with EBV DNA for predicting DM in NPC patients, and found the combined classifier achieved superior prognostic performance than either the radiomic signatures or the clinical variables alone, which with a higher AUC, sensitivity, and specificity improvement.

Motion Detection and Quality Assessment of MR images with Deep Convolutional DenseNets

Sandro Braun ${ }^{1}$, Xiao Chen ${ }^{1}$, Benjamin Odry ${ }^{1}$, Boris Mailhe ${ }^{1}$, and Mariappan Nadar ${ }^{1}$

${ }^{1}$ Medical Imaging Technologies, Siemens Healthineers, Princeton, NJ, United States

We use simulated motion-corrupted images to compute associated image quality metrics and quantify the corresponding severity of motion. We train models with four different inputs (full image, Foreground only, Background only or both Foreground and Background in two channels) to regress to those metrics. To obtain a ground-truth as acceptable or not acceptable image quality, we choose acceptance thresholds within a reasonable range, depending on the level of tolerable motion. The network shows high accuracy within this range. For both metrics used (MSSIM and NRMSE), BG-models perform better than FGBG-models.

A multi-channel convolutional neural network for segmentation of breast lesions in DCE-MRI

Karl Spuhler ${ }^{1}$, Mario Serrano Sosa ${ }^{1}$, Jie Ding ${ }^{1}$, Tim Duong $^{2}$, and Chuan Huang ${ }^{1,2,3}$

${ }^{1}$ Biomedical Engineering, Stony Brook University, Stony Brook, NY, United States, ${ }^{2}$ Radiology, Stony Brook Medicine, Stony Brook, NY, United States, ${ }^{3}$ Psychiatry, Stony Brook Medicine, Stony Brook, NY, United States

Radiomics offers a highly quantitative and high-dimensional view of the tumor microenvironment which no conventional imaging technique allows. It is the ideal strategy for personalizing care in heterogeneous cancers such as in the breast. Most approaches require time consuming, manual region of interest segmentation. Here, we present a fast and accurate neural network approach for breast lesion segmentation which can be adapted to accept any number of imaging modalities and shows reliability across many types of lesion.

Segmentation of Bone Tumor with MR imaging using Machine Learning

Amit Mehndiratta ${ }^{1}$, Akshay Kumar Gupta ${ }^{2}$, Esha Baidya Kayal ${ }^{1}$, Devasenathipathy Kandasamy ${ }^{3}$, Sameer Bakhshi ${ }^{4}$, and Raju Sharma

${ }^{1}$ Centre for Biomedical Engineering, Indian Institute of Technology Delhi, New Delhi, India, ${ }^{2}$ Department of Computer Science and Engineering, Indian Institute of Technology Delhi, New Delhi, India, ${ }^{3}$ Department of Radiology, All India Institute of Medical Sciences, New Delhi, India, ${ }^{4}$ Department of Medical Oncology, IRCH, All India Institute of Medical Sciences, New Delhi, India

There has been a lot of work in segmentation of tumors in organs like the brain. Segmentation of bone tumor with MRI is not widely studied. Manual segmentation can be costly and time consuming. We study three automatic 3D segmentation techniques: Energy-based graph cuts, deep feed forward neural networks and mean shift clustering. Results show that, these methods can perform good quality segmentation (dice coefficient $>70 \%$ ) even with no human intervention. Tumor ADC values computed using these methods are comparable with those obtained from manual segmentation, showing that these methods can be used as a screening tool. 
Noninvasive identification of IDH-mutational status in glioma patients using ${ }^{1} \mathrm{H}-\mathrm{MRS}$ is diagnostically and prognostically valuable. However, the most widely used short TE method is reported to be more subject to false diagnosis due to the severe spectral overlap of $2 \mathrm{HG}$. We explored the potential applicability of deep learning in addressing this issue. A deep neural network that was trained on a large number of simulated spectra substantially improved the overall diagnostic accuracy on the patient spectra, compared to the LCModel analysis. As no spectral fitting is involved, our results are not subject to ambiguity arising from the CRLB-based data interpretation.

Evaluation of 2D and 3D convolutional neural network methods for generating pelvic synthetic CT from T1-weighted MRI

Jie Fu ${ }^{1}$, Yingli Yang ${ }^{2}$, Kamal Singhrao ${ }^{1}$, Dan Ruan², Daniel A. Low ${ }^{2}$, Anand P. Santhanam², and John H. Lewis ${ }^{2}$

${ }^{1}$ David Geffen School of Medicine, UCLA, Los Angeles, CA, United States, ${ }^{2}$ Department of Radiation Oncology, UCLA, Los Angeles, CA, United States

Synthetic CT ( $\mathrm{SCT}$ ) must be generated directly from MRI scans to achieve MRI-only radiotherapy. We propose $2 \mathrm{D}$ and $3 \mathrm{D}$ convolution neural network models for generating pelvic $\mathrm{SCT}$ and evaluate their performance. Five-fold cross-validation is performed using paired T1-weighted MRI and CT scans from 20 patients. Our results show the $2 \mathrm{D}$ model generates accurate $\mathrm{SCT}$ for all patients in this study. The average mean absolute error (MAE) between CT and SCT across all patients is $38.0 \pm 3.9 \mathrm{HU}$ in the $2 \mathrm{D}$ model. The average MAE is $55.9 \pm 28.4 \mathrm{HU}$ in the $3 \mathrm{D}$ model. This large variation is possibly due to the limited number of $3 \mathrm{D}$ training volumes.

The Weakest Link in the Chain: How MR Data Quality influences Convolutional Neural Network Performance

Lars Bielak ${ }^{1}$, Hatice Bunea ${ }^{2}$, Nicole Wiedenmann², Anca-Ligia Grosu² ${ }^{2}$ and Michael Bock ${ }^{1}$

${ }^{1}$ Dept. of Radiology, Medical Physics, Medical Center - University of Freiburg, Faculty of Medicine, University of Freiburg, Freiburg, Germany, ${ }^{2}$ Department of Radiation Oncology, Medical Center - University of Freiburg, Faculty of Medicine, University of Freiburg, German Cancer Consortium (DKTK), Partner Site Freiburg, Freiburg, Germany

In this work, tumor segmentation performance of a convolutional neural network is tested with respect to input data quality. 19 patients suffering from head and neck tumors underwent multi-parametric MRI including diffusion weighted imaging. The network was trained on multiparametric MR images with and without geometrically corrected diffusion data. With distortion correction, the Dice coefficient could be increased by $22 \%$ over uncorrected data showing the necessity for geometric image pre-processing in neural network analysis.

Computer aided quantification of prostate cancer diffusion-weighted imaging: repeatability analysis of radiomics as biomarkers for Gleason score prediction Ileana Montoya Perez ${ }^{1,2}$, Jussi Toivonen ${ }^{1,2}$, Parisa Movahedi ${ }^{1,2}$, Harri Merisaari ${ }^{2,3}$, Janne Verho ${ }^{2}$, Pekka Taimen ${ }^{4}$, Peter J. Boström ${ }^{5}$ Tapio Pahikkala ${ }^{1}$, Hannu J. Aronen $^{2,6}$, and Ivan Jambor $^{2,6}$

${ }^{1}$ Department of Future Technologies, University of Turku, Turku, Finland, ${ }^{2}$ Department of Diagnostic Radiology, University of Turku, Turku, Finland, ${ }^{3}$ Turku PET Centre, University of Turku, Turku, Finland, ${ }^{4}$ Department of Pathology, University of Turku and Turku University Hospital, Turku, Finland, ${ }^{5}$ Department of Urology, University of Turku and Turku University Hospital, Turku, Finland, ${ }^{6}$ Medical Imaging Centre of Southwest Finland, Turku University Hospital, Turku, Finland

We evaluated the repeatability of apparent diffusion coefficient, derived using monoexponential function (ADCm) from prostate cancer DWI ( $12 \mathrm{~b}$ values, $0-2000 \mathrm{~s} / \mathrm{mm}^{2}$ ), radiomics of prostate cancer and their potential to predict prostate cancer Gleason score (histological grading system of prostate cancer aggressiveness). Statistical features (mean, median, $10^{\text {th }}$, $25^{\text {th }}$ percentile) and Gabor texture feature of DWI ADCm parametric maps showed high repeatability and correlated significantly with Gleason score. In contrast, homogeneity gray-level co-occurrence matrix showed low repeatability despite having significant correlation with Gleason score.

Locating hypoxia-related tumour regions in NSCLC: utility and repeatability of data-driven segmentation of combined OE/DCE-MRI data

Adam K Featherstone ${ }^{1}$, Ahmed Salem ${ }^{1,2,3}$, Ross A Little ${ }^{1}$, Yvonne Watson ${ }^{1}$, Susan Cheung ${ }^{1}$, Corrine Faivre-Finn ${ }^{2,3}$, James PB O'Connor ${ }^{2,4}$, Julian C Matthews ${ }^{1}$, and Geoff JM Parker ${ }^{1,5}$

${ }^{1}$ Division of Informatics, Imaging \& Data Sciences, The University of Manchester, Manchester, United Kingdom, ${ }^{2}$ Division of Cancer Sciences, The University of Manchester, Manchester, United Kingdom, ${ }^{3}$ Department of Clinical Oncology, Christie NHS Foundation Trust, Manchester, United Kingdom, ${ }^{4}$ Department of Radiology, Christie NHS Foundation Trust, Mancester United Kingdom, ${ }^{5}$ Bioxydyn Ltd., Manchester, United Kingdom

There is a need to develop tumour hypoxia biomarkers for patient stratification and for tracking tumour response to therapy. We apply our preclinically-optimised, data-driven segmentation of combined OE-MRI/DCE-MRI data to a cohort of non small-cell lung cancer (NSCLC) patients, aiming to map tumour hypoxia non-invasively. Tissue classes with different oxygenation and perfusion characteristics are located, and we discuss challenges specific to use in the clinical setting. Further optimisation of the technique is needed to improve its repeatability and its ability to enable the identification of definitively hypoxic regions in these types of data. 
Daiki Tamada ${ }^{1}$, Utaroh Motosugi ${ }^{1}$, and Hiroshi Onishi ${ }^{1}$

${ }^{1}$ Department of Radiology, University of Yamanashi, Chuo, Japan

Diffusion-weighted imaging (DWI) of the liver using a single-shot EPI sequence suffer from motion artifact caused by cardiac motion. The reconstruction of DWI with multiple numbers of excitation including the corrupted echoes due to systolic cardiac motion results in a severe signal loss in the left lobes, even if other echoes in diastolic phase had no artifact. In this study, we propose a selection algorithm to reject the corrupted echoes using convolutional neural network was proposed. The volunteer studies demonstrated that the proposed method improves the image quality of liver DWI.

Repeatability of Selected Multiparametric Prostate MRI Radiomics Features

Michael Schwier ${ }^{1,2}$, Joost van Griethuysen ${ }^{3}$, Mark G Vangel ${ }^{2,4}$, Steve Pieper ${ }^{5}$, Sharon Peled ${ }^{1,2}$, Clare M Tempany ${ }^{1,2}$, Hugo Aerts ${ }^{2,6}$, Ron Kikinis ${ }^{1,2}$, Fiona M Fennessy ${ }^{1,2,6}$, and Andrey Fedorov ${ }^{1,2}$

${ }^{1}$ Brigham and Women's Hospital, Boston, MA, United States, ${ }^{2}$ Harvard Medical School, Boston, MA, United States, ${ }^{3}$ Netherlands Cancer Institute / Maastricht University, Amsterdam, Netherlands, ${ }^{4}$ Massachusetts General Hospital, Charlestown, MA, United States, ${ }^{5}$ Isomics, Inc., Cambridge, MA, United States, ${ }^{6}$ Dana-Farber Cancer Institute, Boston, MA, United States

In this study we assess the repeatability of selected radiomics features for small prostate tumors in ADC and T2-weighted images. We used a prostate mpMRI test-retest dataset for our evaluation. Different configurations of preprocessing were compared. The intraclass correlation coefficient was employed as a measure of repeatability. Our results show that several of the selected features have good repeatability, however, only when specific preprocessing was applied. Based on our data, texture computation should be done in 2D. Normalization improves repeatability for ADC features, but not in T2-weighted images.

Quantitative texture analysis of apparent diffusion coefficient (ADC) for evaluating histologic differentiated grade of head and neck squamous cell carcinoma

Yu Chen ${ }^{1}$, Yanan Zhao ${ }^{1}$, Huadan Xue ${ }^{1}$, Zhuhua Zhang ${ }^{1}$, and Zhengyu Jin ${ }^{1}$

${ }^{1}$ Peking Union Medical College Hospital, Beijing, China

To investigate the feasibility of using texture analysis (TA) of apparent diffusion coefficient (ADC) to distinguish between well- and moderate- differentiated head and neck squamous cell carcinoma (HNSCC). A total of 22 patients were retrospectively analyzed, including: well-differentiated degree SCC (WSCC, $\mathrm{n}=11)$ and moderate-differentiated degree SCC (MSCC, $n=11)$. A Mean $>101.38$ at coarse texture scale $(S S F=6 m m)$ identified $W S C C$ and $M S C C$ with the highest $A U C$ of $0.843 \pm 0.083(S e=72.7 \%, S p=81.8 \%, P P V=80 \%, P V=75 \%$, and accuracy $=77.3 \%$ ). Texture analysis of ADC proved to be a feasible tool for differentiating WSCC from MSCC, and had better diagnostic performance than ADC value.

Traditional Poster

\section{Machine Learning for Tissue Segmentation \& Classification}

Deep learning-based whole head segmentation for simultaneous PET/MR attenuation correction

Jakub Baran ${ }^{1,2}$, Kamlesh Pawar ${ }^{1,3}$, Nicholas Ferris ${ }^{1,4}$, Sharna Jamadar ${ }^{1,3,5}$, Marian Cholewa ${ }^{2}$, Zhaolin Chen ${ }^{1,6}$, and Gary Egan ${ }^{1,3,5}$

${ }^{1}$ Monash Biomedical Imaging, Monash University, Clayton, Australia, ${ }^{2}$ Department of Biophysics, Faculty of Mathematics and Natural Sciences, University of Rzeszow, Rzeszow, Poland, ${ }^{3}$ Monash Institute of Cognitive and Clinical Neurosciences and School of Psychological Sciences, Monash University, Clayton, Australia, ${ }^{4}$ Monash Imaging, Monash Health, Clayton, Australia, ${ }^{5}$ Australian Research Council Centre of Excellence for Integrative Brain Function, Monash University, Clayton, Australia, ${ }^{6}$ Department of Electrical and Computer Systems Engineering, Monash University, Clayton, Australia

Estimation of an accurate PET attenuation correction factor is crucial for quantitative PET imaging, and is an active area of research in simultaneous PET/MR. In this work, we propose a deep learning-based image segmentation method to improve the accuracy of PET attenuation correction for simultaneous PET/MR imaging of the human head. We compare segmentation methods for accurate tissue segmentation and attenuation map generation. We demonstrate improved PET image reconstruction accuracy using the proposed deep learning-based method. 
${ }^{1}$ Department of Computer Science, Johns Hopkins University, Baltimore, MD, United States, ${ }^{2}$ The Russell H. Morgan Department of Radiology and Radiological Science, Johns Hopkins University School of Medicine, Baltimore, MD, United States, ${ }^{3}$ Sidney Kimmel Cancer Center, Johns Hopkins University School of Medicine, Baltimore, MD, United States

Artificial intelligence(AI) and deep learning techniques are increasingly being used in radiological applications. The true potential of deep learning in MRI applications can only be achieved by developing an Al that can learn the underlying MRI physics rather than a task that is specific to an organ or a particular tissue pathology. To that end, we developed and tested a multiparametric deep learning model capable of tissue segmentation and characterization in both breast cancer and stroke.

Brain Segmentation in Rodent MR-Images Using Convolutional Neural Networks

Björn Sigurðsson ${ }^{1}$, Sune Darkner ${ }^{2}$, Stefan Sommer ${ }^{2}$, Kristian Nygaard Mortensen ${ }^{1}$, Simon Sanggaard ${ }^{3}$, Serhii Kostrikov ${ }^{4}$, and Maiken Nedergaard ${ }^{1,5}$

${ }^{1}$ Center for translational neuromedicine, University of Copenhagen, Copenhagen, Denmark, ${ }^{2}$ Department of Computer Science, University of Copenhagen, Copenhagen, Denmark, ${ }^{3}$ Department of Anesthesiology, Yale School of Medicine, New Haven, CT, United States, ${ }^{4}$ Institut for Mikro- og Nanoteknologi, Technical University of Denmark, Kgs. Lyngby, Denmark, ${ }^{5}$ Department of Neurosurgery, University of Rochester, Rochester, NY, United States

This study compares two different methods for the task of brain segmentation in rodent MR-images, a convolutional neural network (CNN) and majority voting of a registration based atlas (RBA), and how limited training data affect their performance. The CNN was implemented in Tensorflow.

The RBA performs better on average when using a training set with fewer than 20 images but the CNN achieves a higher median dice-score with a training set of 19 images.

A Comparison of Deep Learning Convolutional Neural Networks for Liver Segmentation in Radial Turbo Spin Echo Images

Lavanya Umapathy ${ }^{1}$, Mahesh Bharath Keerthivasan ${ }^{1}$, Jean-Philippe Galons ${ }^{2}$, Wyatt Unger ${ }^{2}$, Diego Martin ${ }^{2}$, Maria Altbach², and Ali Bilgin ${ }^{1,3}$

${ }^{1}$ Electrical and Computer Engineering, University of Arizona, Tucson, AZ, United States, ${ }^{2}$ Medical Imaging, University of Arizona, Tucson, AZ, United States, ${ }^{3}$ Biomedical Engineering, University of Arizona, Tucson, AZ, United States

Motion-robust 2D-RADTSE can provide a high-resolution composite, T2-weighted images at multiple echo times (TEs), and a quantitative T2 map, all from a single k-space acquisition. We use deep-learning CNN for segmentation of liver in abdominal RADTSE images. An enhanced UNET architecture with generalized dice loss based objective function was implemented. Three nets were trained, one for each image type obtained from the sequence. On evaluating net performances on the validation set, we found that nets trained on TE images or T2 maps had higher average dice scores than the one trained on composites, implying information regarding T2 variation aids in segmentation.

Deep learning Based Liver Segmentation from MR Images Using 3D Mutli-Resolution Convolutional Neural Networks

Mootaz Eldib ${ }^{1}$ and Jonathan Riek ${ }^{1}$

${ }^{1}$ BioTelemetry Research, Rochester, NY, United States

A deep learning based image segmentation algorithm is presented for the liver in volumetric MRI data. The fully automated state-of-the-art algorithm was trained with a large dataset resulting in excellent segmentation accuracy as compared to the trained radiologist performance.

2D Single Plane Big Data Convolutional Neural Network for Skull-Stripping

Oeslle Lucena ${ }^{1}$, Roberto Souza ${ }^{2}$, Richard Frayne ${ }^{2}$, Letícia Rittner ${ }^{1}$, and Roberto Lotufo ${ }^{1}$

${ }^{1}$ University of Campinas, Campinas, Brazil, ${ }^{2}$ University of Calgary, Calgary, AB, Canada

Convolutional neural networks for MR image segmentation require a large amount of labelled data. Nevertheless, medical image datasets with expert manual segmentation, which is usually the gold standard for that task, are scarce as this step is both time-consuming and labor intensive. We propose a deep-learning-based skull-stripping (SS) method trained using data provided by consensus-based data augmentation through silver standard masks. Silver standard masks are generated using Simultaneous Truth and Performance Level Estimation (STAPLE) consensus algorithm. Our results indicate comparable performance to state-of-the-art-methods, but computationally effcient even under CPU-based processing

Accurate Cerebellum segmentation using a 3D Convolutional Neural Network and fully connected CRF

Nina Jacobsen ${ }^{1}$, Andreas Deistung ${ }^{1,2,3}$, Dagmar Timmann ${ }^{2,3}$, Jürgen R. Reichenbach ${ }^{1}$, and Daniel Güllmar ${ }^{1}$ 
${ }^{1}$ Medical Physics Group, Institute for Diagnostic and Interventional Radiology, Jena University Hospital, Jena, Germany, ${ }^{2}$ Section of Experimental Neurology, Department of Neurology, Essen University Hospital, Essen, Germany, ${ }^{3}$ Erwin L. Hahn Institute for Magnetic Resonance Imaging, University Duisburg-Essen, Essen, Germany

Subject-specific information about the cerebellum serves as an important biomarker in the clinical setting, however segmentation of the cerebellum is a challenging task. We demonstrate the feasibility of automatic cerebellum segmentation using a 3D convolutional neural network followed by a fully connected conditional random fields algorithm. The network was trained using 12 preprocessed T1-weighted images and corresponding manually refined ground truth segmentations. The new approach revealed robustness and similar DICE coefficients with respect to the conventional FreeSurfer approach.

Sciatic Nerve Segmentation in MRI Volumes of the Upper Leg via 3D Convolutional Neural Networks

Matthew Hancock ${ }^{1}$, Shashank Manjunath ${ }^{1}$, Jun $\mathrm{Li}^{2}$, and Richard Dortch ${ }^{3,4}$

${ }^{1}$ Vanderbilt University, Nashville, TN, United States, ${ }^{2}$ Neurology, Vanderbilt University Medical Center, Nashville, TN, United States, ${ }^{3}$ Radiology, Vanderbilt University Medical Center, Nashville, TN, United States, ${ }^{4}$ Biomedical Engineering, Vanderbilt University, Nashville, TN, United States

In Charcot-Marie-Tooth disease (CMT) diseases, sciatic nerve (SN) hypertrophy may be a viable biomarker of patient impairment. Estimating nerve diameters currently requires laborintensive manual segmentations. Our goal was to use 3D convolutional neural networks (CNN), which have been applied successfully in other biomedical imaging applications, to segment the SN. Using a 3D U-Net architecture developed in Keras 2.0 and Python 2.7, we trained CNNs on data partitioned from 38 control and 34 CMT patients with manually defined region-of-interests $(\mathrm{ROI})$. We found that batch-normalizing 3D CNNs achieved the highest performance, demonstrating CNN's ability to automatically produce high-quality segmentations of the SN.

Automatic Myocardium Segmentation using Fully Conventional Network (FCN)

Yan Wang ${ }^{1}$, Peng $\mathrm{Cao}^{1}$, Karen Ordovas ${ }^{1}$, and Jing Liu ${ }^{1}$

${ }^{1}$ University of California, San Francisco, San Francisco, CA, United States

We introduce a new methodology that combines deep learning and level set for the automated segmentation of the myocardium from cardiac cine magnetic resonance (MR) data. The method employs deep learning algorithm to learn the segmentation task from the ground truth data. The inferred shape is incorporated into level set model to improve the accuracy and robustness of the segmentation.

U-net: Convolutional Networks for Carotid Artery Wall Segmentation in Simultaneous Non-Contrast Angiography and intra-Plaque hemorrhage (SNAP) imaging

Mingquan LIN ${ }^{1}$, Bernard Chiu ${ }^{1}$, Qiang Zhang ${ }^{2}$, Huiyu Qiao ${ }^{2}$, Jiaqi Dou ${ }^{3}$, Binbin Sui ${ }^{4}$, Shuo Chen ${ }^{2}$, Xihai Zhao ${ }^{2}$, Zhensen Chen ${ }^{2}$, and Huijun Chen ${ }^{2}$

${ }^{1}$ Department of Electronic Engineering, City University of Hong Kong, Hong Kong, Hong Kong, ${ }^{2}$ Center of Biomedical Imaging Research, Tsinghua University, Beijing, China, ${ }^{3}$ Beijing Jiaotong University, Beijing, China, ${ }^{4}$ Beijing Tian Tan Hospital, Beijing, China

The purpose of this study is to develop a U-net deep learning model to segment the carotid artery wall using a single 3D Simultaneous Non-Contrast Angiography and intra-Plaque hemorrhage (SNAP) acquisition. Using U-net convolutional Networks can achieve acceptable dice similarity coefficient. In addition, by adding more SNAP imaging such as phasecorrected images $(C R)$, the magnitude of REF and the real part of IR as well as excluded the slice that cannot register and has low image quality may further improve the result.

Breast MRI Tissue Classification and Partial Volume Estimation using Different Methods: Evaluation on T1, T2 and PD-weighted TSE Images

Subhajit Chatterjee ${ }^{1,2,3}$, Snekha Thakran ${ }^{1}$, Rakesh Kumar Gupta ${ }^{4}$, and Anup Singh ${ }^{1,5}$

${ }^{1}$ Centre for Biomedical Engineering, Indian Institute of Technology Delhi, New Delhi, India, ${ }^{2} \mathrm{C}-\mathrm{DO}$ I India, New Delhi, India, ${ }^{3}$ Computer Science and Engineering, Indian Institute of Technology Delhi, New Delhi, India, ${ }^{4}$ Department of Radiology and Imaging, Fortis Memorial Research Institute, Gurgaon, India, New Delhi, India, ${ }^{5}$ Department of Biomedical Engineering, All India Institute of Medical Sciences Delhi, New Delhi, India

Partial volume effect(PVE) is caused by the insufficient spatial resolution of MRI images. Boundaries of different tissue-types are considered as partial volume(PV) prone area where each voxel can be mixture more than one tissue-type. PVE can introduce errors in inner segmentation and Breast density estimation. In this study we have identified PV voxels and estimated the proportion of each tissue-type within a PV voxel using fat and nonfat saturated MRI data. Experimental results revealed that difference method (difference between nonfat and fat saturated images) can provide similar tissue classification and estimation accuracy as compared to existing methods. 
MR-only simulation is increasingly more popular because of superior soft-tissue contrast and radiation dose-free for conventional and adaptive radiotherapy, as compared to CT simulation. Identifying bones is crucial towards successful MR-only simulation, particularly in cranial and head-and-neck regions where radio-sensitive soft-tissues densely present. This abstract proposed a framework exhibiting self-learning compatibility to capture case-specific information to perform skull segmentation. Without manual input and training information, the proposed framework utilized a clustering technique to collectively analyze images from multiple MR sequences. Evaluated in eight volunteer cases, it was shown that the proposed unsupervised-learning framework well-suited MR-based skull segmentation.

Reconstruction of MR images by combining k-spaces of multi-contrast MR data through deep learning

Won-Joon Do ${ }^{1}$, Yo Seob Han ${ }^{1}$, Seung Hong $\mathrm{Choi}^{2}$, Jong Chul Ye ${ }^{1}$, and Sung-Hong Park ${ }^{1}$

${ }^{1}$ Department of Bio and Brain Engineering, KAIST, Daejeon, Republic of Korea, ${ }^{2}$ Department of Radiology, Seoul National University Hospital, Seoul, Republic of Korea

We propose a new deep neural network (Y-net) that can utilize images acquired with a different MR contrast for reconstruction of down-sampled images. K-space center of downsampled T2-weighted images and k-space edge of full-sampled T1-weighted images were combined through one Y-net, and desired high-resolution T2-weighted images were generated by another $\mathrm{Y}$-net. The proposed network not only improved spatial resolution but also suppressed ringing artifacts caused by the down-sampling at the k-space center. The developed technique potentially enables to accelerate the multi-contrast MR imaging in routine clinical studies.

Traditional Poster

\section{Classification \& Prediction for Function \& Disease}

Application of machine learning for MRI case studies

Nagesh Adluru ${ }^{1}$, Cole Korponay ${ }^{1}$, Robin I Goldman ${ }^{1}$, Andrew L Alexander ${ }^{1}$, and Richard J Davidson ${ }^{1}$

${ }^{1}$ University of Wisconsin Madison, Madison, WI, United States

Machine learning can be used to train a model that maps MRI features to clinical phenotype covariates. We present the application of such a framework in the context of MRI case studies. While the presented framework is general in its applicability for individual level analysis, it has particular appeal in the context of case studies where the data can be extraordinarily rare or precious. Specifically, the framework was applied to study the case of an extraordinary long term meditator whose MRI data was acquired over four different time points over a period of fifteen years. Thanks to standardization of image processing and sparsity enhancing regularization methods in machine learning, the case study was performed by including the existing prior data in training the model.

Deep Recurrent Neural Network Based Learning for Determining Structural Changes in Brain MRE: Towards Early Detection of Alzheimer's

Raghuprasad $\mathrm{M} \mathrm{S}^{1}$

${ }^{1} M R I$, GE Healthcare, Bangalore, India

Alzheimer's Disease (AD) is a type of dementia which is now known to be the leading cause of death in the United States. Hence, early detection of AD is crucial for treatment planning and preventive measures before patient develops irreversible brain trauma. Deep learning (DL) is a robust machine learning technique used for classification to extract low-to high-leve features. Previous studies have used DL to classify functional MRI data of Alzheimers subjects. However, none have employed DL to classify the ealsticity changes in brain MRE data. As a first step towards early diagnosis of $A D$ we have developed a deep recurrent neural learning scheme to classify structural and elasticity changes in brain MRE.

Is it possible to estimate recanalization effect for acute ischemic stroke patients using a single deep learning model?

Anne Nielsen ${ }^{1,2}$, Mikkel Bo Hansen ${ }^{1}$, and Kim Mouridsen ${ }^{1}$

$2741 \quad{ }^{1}$ Center of Functionally Integrative Neuroscience and MINDLab, Department of Clinical Medicine, Aarhus University, Aarhus, Denmark, ${ }^{2}$ Cercare Medical, Aarhus, Denmark

Every year, 13 million people suffer acute ischemic stroke. Brain tissue infarcts permanently within hours after stroke onset and rapid recanalization is therefore of utmost importance. In this project, we aim to estimate recanalization effect by a single convolutional neural network customized to include magnetic resonance imaging biomarkers as well as individual recanalization information. This is in contrast to the traditional approach which is splitting the data set according to the recanalization information and training several models. We find a significant recanalization effect and believe this to be an important step towards an automated decision support system. 
Prognostic-value of imaging markers for the prediction of the clinical evolution in Alzheimer's disease

Cécilia Damon ${ }^{1}$, Guillaume Magnien ${ }^{2}$, Urielle Thoprakarn ${ }^{1}$, Bruno Vegreville ${ }^{1}$, Jinpeng Li $^{1}$, Jean-Baptiste Martini ${ }^{1}$, and Clarisse Longo dos Santos ${ }^{1}$

${ }^{1}$ Qynapse, Paris, France, ${ }^{2}$ École polytechnique fédérale de Lausanne, Paris, France

Predicting the individual clinical course remains a major issue in biomarker research in Alzheimer's disease to adapt the therapeutic care of patients. Imaging data may contain valuable early markers of the clinical evolution of $A D$. In this study, we investigated the prognostic value of some imaging markers for the prediction of the clinical evolution of mild cognitive impairment $(\mathrm{MCl})$ and $A D$ patients over 24 months through both the conversion and the cognitive decline problems. With a rigorous validation scheme, for each clinical outcome, we built competitive predictive models on the ADNI cohort which are highly generalizable to other independent cohorts (OASIS and AddNeuroMed).

Optimization of Asymmetric Spin Echo MRI for Oxygen Extraction Fraction Mapping in the Brain and Initial Experience with Moya-Moya Patients

Dharmesh Tailor ${ }^{1}$, John J Lee ${ }^{2}$, Hongyu $\mathrm{An}^{3}$, and Colin Derdeyn ${ }^{4}$

${ }^{1}$ Radiology, Florida Hospital \& University of Central Florida School of Medicine, Orlando, FL, United States, ${ }^{2}$ Mallinckrodt Institute of Radiology, Washington University School of Medicine, St. Louis, MO, United States, ${ }^{3}$ Washington University School of Medicine, St. Louis, MO, United States, ${ }^{4}$ University of lowa School of Medicine, Iowa City, IA, United States

Asymmetric Spin Echo (ASE) MRI has been previously applied for quantitative cerebral oxygen extraction mapping. In this study we optimize this technique using O-15 PET as a gold standard for oxygen extraction fraction (OEF) quantitation and apply the optimized ASE approach for studying brain lesions in Moya-Moya patients. Results suggest that optimized OEF maps from ASE MRI have the potential to detect brain lesions unseen with conventional MRI sequences. These lesions detected by ASE appear to provide information that is statistically independent from the information provided by conventional MRI approaches.

Early Prediction of Total Knee Replacement using Structural MRI and 3D Deep Convolutional Neural Networks

Kevin Leung ${ }^{1}$, Gregory Chang ${ }^{2}$, Kyunghyun $\mathrm{Cho}^{3}$, and Cem Deniz ${ }^{4,5}$

${ }^{1}$ Courant Institute of Mathematical Sciences and Leonard N. Stern School of Business, New York University, New York, NY, United States, ${ }^{2}$ Department of Radiology, Center for Musculoskeletal Care, New York University Langone Medical Center, New York, NY, United States, ${ }^{3}$ Courant Institute of Mathematical Science and Center for Data Science, New York University, New York, NY, United States, ${ }^{4}$ Department of Radiology, Center for Advanced Imaging Innovation and Research (CAI2R) and Bernard and Irene Schwartz Center for Biomedical Imaging, New York University Langone Medical Center, New York, NY, United States, ${ }^{5}$ Sackler Institute of Graduate Biomedical Sciences, New University School of Medicine, New York, NY, United States

The early prediction of individuals who will eventually require total knee replacement (TKR) remains a challenging problem. In this project, we propose to use $3 \mathrm{D}$ deep convolutional neural networks (CNN) to predict the likelihood of a patient receiving a TKR within nine years using 718 subjects from the Osteoarthritis Initiative ${ }^{1}$ (OAI) dataset. We found that our model results in better performance compared to a logistic regression model using clinical risk factors ${ }^{2}$ (AUC: $0.8480 \pm .0173$ vs $0.7716 \pm .0229$ and accuracy: $77.15 \pm 1.88 \%$ vs. $71.16 \pm 2.70 \%$ ).

Classification of Different Episodic Memory Tasks by Time Points using a Deep Neural Network

Zhengshi Yang ${ }^{1}$, Xiaowei Zhuang ${ }^{1}$, Karthik Sreenivasan ${ }^{1}$, Virendra Mishra ${ }^{1}$, Christopher Bird ${ }^{1}$, Tim Curran $^{2}$, Sarah J Banks ${ }^{1}$, and Dietmar Cordes ${ }^{1,2}$

Classification of different episodic memory tasks by time points is challenging because the signal-to-noise ratio in affected brain regions of the medial temporal lobes is low and similar brain regions (such as the hippocampus) contribute to memory activation. No studies have implemented a deep neural network (DNN) to classify memory tasks at each fMRI time point using whole-brain data. We have implemented a region-of-interest based DNN framework and applied it to classify three different episodic memory tasks. Results indicate that this DNN classifier can accurately discriminate between all these tasks.

Resting-state Brain Networks using Spectral Clustering Analysis

Jason Barrett ${ }^{1}$, Haomiao Meng ${ }^{2}$, Song Chen ${ }^{1}$, Li Zhao ${ }^{3}$, David Alsop ${ }^{3}$, Xingye Qiao ${ }^{2}$, and Weiying Dai ${ }^{1}$

${ }^{1}$ Computer Science, State University of New York at Binghamton, Vestal, NY, United States, ${ }^{2}$ Mathematical Sciences, State University of New York at Binghamton, Vestal, NY, United States, ${ }^{3}$ Department of Radiology, Beth Israel Deaconess Medical Center and Harvard Medical School, Boston, MA, United States

Seed-based correlation method and independent component analysis (ICA)-based method have been used to extract the resting-state brain networks from fMRI data. Both methods require either prior knowledge of brain anatomy or selection of unordered spatial sources. Here, we investigate a data-driven spectral clustering algorithm to study brain networks for resting-state arterial spin labeling (ASL) and blood-oxygen-level dependent (BOLD) fMRI data. The spectral clustering algorithm successfully separates the brain resting-state networks and rank the non-neural noises at last. It is of great benefit to use ASL to study brain resting-state networks because of the largely reduced non-neural noise sources. 
Deep learning based MR image diagnostic quality deduction to reduce patient recall

Arathi Sreekumari ${ }^{1}$, Ileana Hancu ${ }^{2}$, Dirk Beque ${ }^{3}$, Keith Park ${ }^{2}$, Uday Patil ${ }^{1}$, Desmond Teck Beng Yeo ${ }^{2}$, Thomas K Foo ${ }^{2}$, and Dattesh Shanbhag ${ }^{1}$

${ }^{1}$ GE Global Research, Bangalore, India, ${ }^{2}$ GE Global Research, Niskayuna, NY, United States, ${ }^{3}$ GE Global Research, Garching bei München, Germany

In this abstract, we describe a fast and robust methodology to highlight on-console, the diagnostic quality of acquired MRI imaging data. Specifically, using convolutional neural networks we flag the MRI volumes affected by motion and consequently hinder the diagnosis by clinician at the time of reading the exam. By prospectively flagging such exams at acquisition console itself and re-acquiring them with improved protocol will obviate the need for costly patient recall and re-scan in clinical setting.

MRI-based radiomics signature for head and neck squamous cell carcinoma patients

Ling Dong ${ }^{1}$, Ying Yuan ${ }^{2}$, Xiaofeng Tao ${ }^{2}$, Di Dong ${ }^{3}$, Zhenyu Liu ${ }^{3}$, Yali Zang ${ }^{3}$, and Jie Tian ${ }^{4}$

${ }^{1}$ University of Electronic Science and Technology of China, Beijing, China, ${ }^{2}$ Shanghai Ninth People's Hospital, Shanghai, China, ${ }^{3}$ CAS Key Laboratory of Molecular Imaging, Institute of Automation, Chinese Academy of Sciences, Beijing P.R. China; University of Chinese Academy of Sciences, Beijing P.R. China., Beijing, China, ${ }^{4}$ CAS Key Laboratory of Molecular Imaging, Institute of Automation, Chinese Academy of Sciences, Beijing, China

To assess overall survival (OS) of head and neck squamous cell carcinoma (HNSCC) patients and the radiomics features, a large number of quantitative radiomics features were extracted from MRI and selected by machine learning methods. Based on these features, a multivariate Cox proportional hazards model was built as a independent predictor to identify patients. Seven features was found to have association with OS (training cohort, $\mathrm{P}<0.0001$; testing cohort, $\mathrm{P}=0.0013$ ). In the training cohort, the radiomics signature yielded a C-index of $0.73(95 \% \mathrm{Cl}, 0.63-0.84)$, which was $0.71(95 \% \mathrm{Cl}: 0.59-0.82)$ in the testing cohort. The potential association between MRI-based radiomics signature and OS was explored.

Radiomics based strategy for identifying poorly differentiated HCC by using precontrast MRI

Jingjun $\mathrm{Wu}^{1}$, Ailian $\mathrm{Liu}^{1}$, Jingjing $\mathrm{Cui}^{2}$, and Lizhi $\mathrm{Xie}^{3}$

${ }^{1}$ Department of Radiology, The First Affiliated Hospital of Dalian Medical University, Dalian, China, ${ }^{2}$ Huiying Medical Technology Co., Ltd., Beijing, China, ${ }^{3}$ GE Healthcare, MR Research, Beijing, China

This work aimed for a radiomics based strategy to identify poorly differentiated hepatocellular carcinoma (HCC) which may own a high risk of recurrence or metastasis. By comparing the performance of four classifiers (decision tree, DT; random forest, RF; k-nearest neighbors, KNN; logistic regression, LR) on dual-echo T1WI (in-phase and out-phase), T2WI and DWI images, we found that LR achieved the best result (AUC: 0.95 ; sensitivity: 0.75 ; specificity: 0.85 ) on DWI images, forming a valuable strategy for clinical practice.

STAGE Imaging at 1.5T: A Rapid Brain Protocol Providing More Images As Well As Quantitative Data

Yu Wang ${ }^{1,2}$, Feng Huang ${ }^{1}$, Wei Xu1 ${ }^{1}$, Tiecheng $\mathrm{Li}^{1}$, Hongyu Guo ${ }^{1}$, Yongsheng Chen ${ }^{3,4,5}$, and Ewart Mark Haccke ${ }^{2,3,5}$

${ }^{1}$ Neusoft Medical System, Shanghai, China, ${ }^{2}$ Shanghai Key Laboratory of Magnetic Resonance, East China Normal University, Shanghai, China, ${ }^{3}$ The MRI Institute for Biomedical Research, Detroit, MI, United States, ${ }^{4}$ Sino-Dutch Biomedical and Information Engineering School, Northeastern University, Shenyang, China, ${ }^{5}$ Department of Radiology, School of Medicine, Wayne State University, Detroit, MI, United States

Many image contrasts are necessary in clinical magnetic resonance imaging (MRI) including qualitative and quantitative images, which traditionally take a long acquisition time. STrategically Acquired Gradient Echo (STAGE) $)^{1,2,3}$ is a rapid imaging method which can acquire multiple qualitative and quantitative images with good resolution and SNR in just 5 minutes at 3 T. In this work, the STAGE concept is optimized, and further extended to $1.5 T$. A total of 11 high quality clinically meaningful images, and 2 field maps, were produced with $0.67 \times 1.33 \times 2.7 \mathrm{~mm}^{3}$ resolution in a single 9-min scan on a NMS $1.5 \mathrm{~T}$ system covering the whole brain.

Radiomics using multi parametric MRI for pre-treatment prediction of complete response to neo-adjuvant treatment in locally advanced rectal cancer

Stefano Trebeschi ${ }^{1}$, Joost J. M. van Griethuysen ${ }^{1}$, Doenja M. J. Lambregts ${ }^{1}$, Max J Lahaye ${ }^{1}$, Frans C. H. Bakers ${ }^{2}$, Roy F.A. Vliegen ${ }^{3}$, Emile Voest ${ }^{4}$, Regina G.H. Beets-Tan ${ }^{1}$, and Hugo J.W.L. Aerts 5

${ }^{1}$ Radiology, Netherlands Cancer Institute, Amsterdam, Netherlands, ${ }^{2}$ Radiology, Maastricht University Medical Center, Maastricht, Netherlands, ${ }^{3}$ Radiology, Zuyderland Medical Center Heerlen, Heerlen, Netherlands, ${ }^{4}$ Medical Oncology, Netherlands Cancer Institute, Amsterdam, Netherlands, ${ }^{5}$ Radiation Oncology and Radiology, Dana Farber Cancer Institute, Boston, MA, United States

Aim of this investigation was to assess the predictive value of MR Radiomics as predictive biomarker for locally advanced rectal carcinoma. Through univariate analysis and unsupervised biclustering we found significant associations between diffusion radiomic textures and complete response in a multi-center cohort. The results suggest the viability of Radiomics as biomarker and puts emphasis on image quality. 
Traditional Poster

\section{Quantitative MRI}

A unified signal readout improves denoising of multi-modal spinal cord MRI

Francesco Grussu ${ }^{1,2}$, Marco Battiston ${ }^{1}$, Jelle Veraart ${ }^{3}$, Torben Schneider ${ }^{4}$, Julien Cohen-Adad ${ }^{5,6}$, Manuel Jorge Cardoso ${ }^{7,8}$, Daniel C. Alexander ${ }^{2}$, Dmitry S. Novikov ${ }^{3}$, Els Fieremans ${ }^{3}$, and Claudia Angela Gandini Wheeler-Kingshott ${ }^{1,9,10}$

${ }^{1}$ Queen Square MS Centre, UCL Institute of Neurology, Faculty of Brain Sciences, University College London, London, United Kingdom, ${ }^{2}$ Centre for Medical Image Computing, Department of Computer Science, University College London, London, United Kingdom, ${ }^{3}$ Center for Biomedical Imaging, Department of Radiology, New York University School of Medicine, New York, NY, United States, ${ }^{4}$ Philips UK, Guildford, Surrey, United Kingdom, ${ }^{5}$ NeuroPoly Lab, Institute of Biomedical Engineering, Polytechnique Montréal, Montréal, QC, Canada, ${ }^{6}$ Functional Neuroimaging Unit, CRIUGM, Université de Montréal, Montréal, QC, Canada, ${ }^{7}$ Centre for Medical Image Computing, Department of Medical Physics and Biomedical Engineering, University College London, London, United Kingdom, ${ }^{8}$ Dementia Research Centre, UCL Institute of Neurology, Faculty of Brain Sciences, University College London, London, United Kingdom, ${ }^{9}$ Brain MRI $3 T$ Research Centre, C. Mondino National Neurological Institute, Pavia, Italy, ${ }^{10}$ Department of Brain and Behavioural Sciences, University of Pavia, Pavia, Italy

Denoising based on Marčenko-Pastur principal component analysis (MP-PCA) is a versatile model-free method proposed for brain imaging. Here, we assess the potential of the technique for multi-modal quantitative spinal cord MRI. We analyse a unique data set consisting of multi-modal cervical scans obtained with a unified signal readout, and corroborate in vivo findings with simulations. We show that MP-PCA denoising is a valid tool for pre-processing a variety of signal contrasts in the spinal cord. In particular, the overall performance of denoising can be enhanced further on multi-modal acquisitions with matched signal readout, due to increased data redundancy.

SNR-Efficient 3D GRE T1 $\rho$ Mapping of the Brain using Tailored Variable Flip Angle Scheduling

Casey P. Johnson ${ }^{1}$, Daniel R. Thedens ${ }^{2}$, and Vincent A. Magnotta ${ }^{2}$

${ }^{1}$ Center for Magnetic Resonance Research, University of Minnesota, Minneapolis, MN, United States, ${ }^{2}$ Radiology, University of lowa, lowa City, IA, United States

We introduce a new 3D GRE acquisition strategy to greatly improve the SNR efficiency of quantitative 3D T1 $\rho$ mapping. Unlike the state-of-the-art 3D MAPSS method, the proposed approach assigns a unique variable flip angle schedule for each spin-lock preparation pulse duration. This enables the use of larger flip angles and greater flexibility in selection of imaging parameters to improve SNR efficiency. In this work, we evaluate this technique for T1 $\rho$ mapping of the brain, but this method can also be applied to other regions of the body and used with a variety of magnetization preparation pulses.

Rapid whole brain qMT imaging with inter-slice MT effects and database-driven fitting approach

Jae-Woong Kim ${ }^{1}$, Sul-Li Lee ${ }^{1}$, Seung Hong Choi ${ }^{2}$, and Sung-Hong Park ${ }^{1}$

${ }^{1}$ Department of Bio and Brain Engineering, Korea Advanced Institute of Science and Technology, Daejeon, Republic of Korea, ${ }^{2}$ Department of Radiology, Seoul National University Hospital, Seoul, Republic of Korea

Quantitative magnetization transfer (qMT) imaging provides unique tissue contrast, but suffers from prolonged scan time and processing time. The current study suggests inter-slice MT acquisition and database-driven qMT parameter fitting in order to mitigate the problems. Inter-slice scanning takes advantage of incidental MT effects, and thus does not require separate MT preparation. It enabled us to complete the whole brain data acquisition within a clinically reasonable scan time of $\sim 10$ min. The employment of pre-defined database also greatly reduced the qMT processing time, while revealing consistent qMT maps compared to those from the conventional method. The proposed database-driven inter-slice qMT method can be a promising alternative of qMT imaging.

Predicting Histological Stainings of Brain Tissue from MRI Data using Artificial Neural Networks

Riccardo Metere ${ }^{1}$, Henrik Marschner ${ }^{1}$, Katja Reimann $^{2,3}$, André Pampel $^{1}$, and Harald E. Möller ${ }^{1}$

${ }^{1}$ NMR Unit, Max Planck Institute for Human Cognitive and Brain Sciences, Leipzig, Germany, ${ }^{2}$ Paul Flechsig Institute for Brain Research, University of Leipzig, Leipzig, Germany, ${ }^{3}$ Max Planck Institute for Human Cognitive and Brain Sciences, Leipzig, Germany

The generation of contrast in MRI relies on a variety of physical processes (e.g. relaxation, magnetization transfer, etc.) that produces a relatively rich amount of information for biological samples. However, given the complex microstructure of tissues, some histological information of relevance in biology and medicine are obtained more easily using optical acquisition techniques on specifically stained specimens. Here, we propose a machine-learning-based method of replicating the contrast information from optical microscopy by exploiting the richness of MRI acquisitions (which will limit the final resolution). The approach exploits the properties of multi-layer feed-forward neural networks as universal function approximators. 
Multiple dynamics gradient-echo EPI acquisitions for quantitative susceptibility mapping

Vanessa Wiggermann ${ }^{1,2,3}$, Enedino Hernández-Torres ${ }^{2,3}$, Christian Kames ${ }^{1,3}$, and Alexander Rauscher ${ }^{1,2,3,4}$

${ }^{1}$ Physics and Astronomy, University of British Columbia, Vancouver, BC, Canada, ${ }^{2}$ UBC MRI Research Centre, University of British Columbia, Vancouver, BC, Canada, ${ }^{3}$ Pediatrics, University of British Columbia, Vancouver, BC, Canada, ${ }^{4} B C$ Children's Research Centre, University of British Columbia, Vancouver, BC, Canada

In this work we demonstrate the feasibility to utilize EPI read-out schemes in combination with multiple dynamics to acquire multi-echo like data sets with the freedom of variable echo times, allowing to acquire fast, high-resolution quantitative susceptibility maps (QSM) images. Assessing the quality of the QSM scans in a region-of-interest based analysis as well as via structural and feature similarities we observed high qualitative and quantitative agreement between QSM images from multi-dynamic EPI acquisitions and multi-echo gradient echo scans.

Evaluation of Marchenko-Pastur PCA denoising on Multi-Exponential Relaxometry

Mark D. Does ${ }^{1}$, Jonas Lynge Olesen ${ }^{2}$, Kevin D Harkins ${ }^{3}$, Teresa Serradas-Duarte ${ }^{4}$, Sune N Jespersen ${ }^{2}$, and Noam Shemesh ${ }^{4}$

${ }^{1}$ Biomedical Engineering, Vanderbilt University, Nashville, TN, United States, ${ }^{2}$ Aarhus University, Aarhus, Denmark, ${ }^{3}$ Vanderbilt University, Nashville, TN, United States, ${ }^{4}$ Champalimaud Centre for the Unknown, Lisbon, Portugal

MRI relaxometry is a powerful tool for characterizing tissue at the sub-voxel level, such as for myelin water imaging. However, a major impediment to its use is the high signal-to-noise ratio requirement. Here, we propose Marchenko-Pastur principal component analysis—previously proposed for diffusion MRI-to denoise relaxometry data. Experimental studies and simulations exemplify the utility of this denoising, and its potential to accelerate data acquisition by $6-8 \mathrm{X}$ or more without bias in fitted relaxometry measures or degradation of image resolution. This simple yet important denoising step thus paves the way for broader applicability of relaxometry.

A novel strategy for rapid multiparameter mapping based on SPGR with continuous steady state longitudinal magnetization

Jinhyeok Choi ${ }^{1}$ and Hyeonjin $\mathrm{Kim}^{1,2}$

${ }^{1}$ Department of Biomedical Sciences, Seoul National University, Seoul, Republic of Korea, ${ }^{2}$ Department of Radiology, Seoul National University, Seoul, Republic of Korea

A method is proposed for simultaneous $\mathrm{T} 1, \mathrm{~T}^{*}$ and M0 mapping on a single scan by removal of inter-scan time delays based on the analytically found arrays of flip angles and TRs that maintain longitudinal magnetization in a steady state throughout the scan. Our preliminary results are in support of potential application of the proposed method in rapid multiparametric MRI in combination with a suitable undersamping strategy.

\section{MAGNETIC SUSCEPTIBILITY OF HUMAN KNEE AT 7T USING ULTRASHORT ECHO MR DATA}

Shaeez Usman Abdulla ${ }^{1}$, David C Reutens ${ }^{1}$, and Viktor Vegh ${ }^{1}$

${ }^{1}$ Centre for Advanced Imaging, University of Queensland, Australia, Brisbane, Australia

Ultra-short echo time quantitative susceptibility mapping (QSM) is a promising tool for the study of tissues with short relaxation times. At ultra-high field, the reconstruction of quality phase images is challenging because of the absence of a reference coil. We propose the use of selective channel combination of phase-offset-corrected signal phase data for ultra-short echo time QSM. We compared our findings against an established channel combination method. Qualitative and quantitative analyses of combined phase and QSM images were performed at three echo times. Selective combination of individual channel phase images results in improved ultra-short echo time susceptibility maps.

Multi-Parameter Mapping with $500 \mu \mathrm{m}$ Resolution Using a Flexible 23-Channel RF Coil

Kerrin J Pine ${ }^{1}$, Lenka Vaculciakova ${ }^{1}$, Evgeniya Kirilina ${ }^{1}$, Nico Scherf ${ }^{1}$, and Nikolaus Weiskopf ${ }^{1}$

$2760 \quad{ }^{1}$ Department of Neurophysics, Max Planck Institute for Human Cognitive and Brain Sciences, Leipzig, Germany

To better understand the human brain's microstructure, there is a need for in-vivo myelin and iron mapping methods which have sufficient resolution to map mesoscopic intra-cortical structures (e.g. lamina). However, resolution is critically SNR-limited. We show that by using a mechanically flexible RF coil array which conforms to the subject's own individual skull shape, sufficient SNR is gained to map the main MR contrast parameters and the line of Gennari within the superficial primary visual cortex. The work demonstrates the feasibility of laminar analysis of myelination at widely available modest field strengths. 
Mohammad-Reza Nazem-Zadeh ${ }^{1}$, Kost V. Elisevich ${ }^{2}$, and Hamid Soltanian-Zadeh ${ }^{3,4}$

${ }^{1}$ Research Center for Molecular and Cellular Imaging, Tehran University of Medical Sciences, Tehran, Iran (Islamic Republic of), ${ }^{2}$ Clinical Neurosciences, Spectrum Health Medical Group, Grand Rapids, MI, United States, ${ }^{3}$ Radiology and Research Administration, Henry Ford Health System, Detroit, MI, United States, ${ }^{4}$ Control and Intelligent Processing Center of Excellence (CIPCE), School of Electrical and Computer, University of Tehran, Tehran, Tehran, Iran (Islamic Republic of)

In this work, multivariate response-driven lateralization models were developed using MRI, DTI, and SPECT attributes and logistic regression, to determine the side of epileptogenicity in TLE patients. The proposed response models were capable of handling missing data points using imputation of missing attributes by their mean values measured on a control cohort. Additionally, the proposed response model can be further generalized by integrating attributes of additional modalities (such as PET- positron emission tomography) into the process. Increased reliability in lateralizing TLE cases using the proposed response model reinforces the notion that ECoG in a number of cases may be circumvented.

Body Phantom with Prostate Mimic for Evaluation of Quantitative MRI

Ryan M Kalmoe ${ }^{1}$, Elizabeth Mirowski², and Gregory J Metzger ${ }^{1}$

${ }^{1}$ Center for Magnetic Resonance Research and Department of Radiology, University of Minnesota, Minneapolis, MN, United States, ${ }^{2}$ Imaging Standards Division, High Precision Devices, Inc., Boulder, CO, United States

A body phantom, containing a prostate mimic with traceable T1/T2/ADC standards, was designed and manufactured to assess acquisition-, system-, and RF coil- dependent variances of quantitative MRI parameters. In order to explore the potential of the phantom as a quality assurance tool, two phantoms were constructed and evaluated with two receive coil configurations across two scanners over a period of three weeks. It is demonstrated that this phantom is a useful prostate specific quality assurance tool and provide the information needed to harmonize results thus minimizing the impact of multiple dependencies on quantitative results.

Improved muscle T2 estimation by maximum-likelihood parameter estimation using an extended-phase-graph signal model with locally estimated Rician noise levels

Nick Zafeiropoulos ${ }^{1}$, Stephen Wastling ${ }^{1}$, Christopher Sinclair ${ }^{1}$, Tarek Yousry ${ }^{1}$, Enrico De Vita ${ }^{1}$, Robert Janiczek ${ }^{2}$, and John Thornton ${ }^{1}$

${ }^{1}$ Institute of Neurology, London, United Kingdom, ${ }^{2}$ Glaxo Smith Kline, London, United Kingdom

Maximum likelihood model parameter estimation accounting for the Rician noise distribution in MRI acquisitions, combined with the extended graph formalism and incorporating slice profile considerations, offers higher precision and less bias with regards to the predicted parameters in T2 relaxometry. In this work this was tested by simulations and validated in phantom and in vivo data from healthy volunteers.

Improved ADC Estimation Technique Using Regularized Nonlinear Least Squares Fitting

Eric A. Borisch ${ }^{1}$, Adam T. Froemming ${ }^{1}$, Roger C. Grimm ${ }^{1}$, Yunhong Shu ${ }^{1}$, Ashley T. Tao ${ }^{1}$, Stephen J. Riederer ${ }^{1}$, and Joshua D. Trzasko ${ }^{1}$

${ }^{1}$ Radiology, Mayo Clinic, Rochester, MN, United States

A high-performance model-based regularized non-linear-least-squares ADC fitting technique has been designed and implemented. Phantom testing shows a reduction in noise with significant retention of detail, while providing $<10 \mathrm{sec}$ computation for 3D acquisitions with $4 \mathrm{~b}$-values.

Analysis of magnetization transfer (MT) effect on Bloch-simulation based T2 mapping accuracy, demonstrated on in vitro urea phantom

Dvir Radunsky ${ }^{1}$ and Noam Ben-Eliezer ${ }^{1,2,3}$

${ }^{1}$ Department of Biomedical Engineering, Tel Aviv University, Tel Aviv, Israel, ${ }^{2}$ Center for Advanced Imaging Innovation and Research, New York University, New York, NY, United States,

${ }^{3}$ Sagol School of Neuroscience, Tel Aviv University, Tel Aviv, Israel

Accurate quantification of $\mathrm{T}_{2}$ values hold high value for a variety of clinical and research applications, yet is highly challenged by the inherent bias of rapid multi-SE (MSE) protocols due to stimulated and indirect echoes. Recently, we introduced the echo modulation curve (EMC) algorithm, which successfully overcomes this problem to produce accurate quantification of $\mathrm{T}_{2}$ values that are stable across scanners and scan settings. In this work, we investigate the effect of magnetization transfer on MSE signal, and specifically on EMC-derived $T_{2}$ values for different $T_{2}$ baselines, number of slices, and slice gaps, using an in vitro urea model. 
The parameters estimated in DCE-MRI studies vary greatly depending on the arterial input function. Moreover, a more complex model than the extended Tofts' model (ETM) is needed in glioma patients. In this work, a method to generate a voxel-specific input function (VIF) is introduced and used with a two-tissue compartment model (2TCM) that separates the fast and slow kinetics of the Gd contrast agent. The VIF provided more accurate results in the superior sagittal sinus (SSS) than the internal carotid artery and combined with the $2 \mathrm{TCM}$, significantly improved the fits to the tumour over the ETM using the SSS.

Joint T1/T2 mapping with frequency-modulated SSFP, radial sampling, and subspace reconstruction

Volkert Roeloffs ${ }^{1}$, Jost M. Kollmeier ${ }^{1}$, Nick Scholand ${ }^{2,3}$, Dirk Voit ${ }^{1}$, Sebastian Rosenzweig ${ }^{2,3}$, H. Christian M. Holme ${ }^{2,3}$, Martin Uecker $^{2,3}$, and Jens Frahm ${ }^{1,3}$

${ }^{1}$ Biomed NMR, Max Planck Institute for Biophysical Chemistry, Goettingen, Germany, ${ }^{2}$ Institute for Diagnostic and Interventional Radiology, University Medical Center, Goettingen, Germany, ${ }^{3}$ Partner site Goettingen, German Centre for Cardiovascular Research (DZHK), Goettingen, Germany

In this work, we propose frequency-modulated SSFP imaging with 3D stack-of-stars encoding to perform joint T1/T2 mapping. In contrast to phase-cycled SSFP, inefficient preparation phases are avoided and a subspace-constrained reconstruction allows efficient handling of large data sets. Quantitative mapping is realized by projecting the reconstructed subspace coefficients onto a precomputed piece-wise linear approximation of the Bloch-response manifold. General feasibility is proven by comparison to Gold Standard measurements on a home-brew $T_{1} / T_{2}$ phantom. The investigated approach is a promising candidate for multi-parametric mapping in vivo.

Accurate and rapid dictionary-based T2 mapping using multi-echo turbo spin echo sequences with reduced refocusing angle

Julian Emmerich ${ }^{1}$ and Sina Straub ${ }^{1}$

${ }^{1}$ Medical Physics in Radiology, German Cancer Research Center (DKFZ), Heidelberg, Germany

In this work, we present a fast and accurate T2 mapping method based on standard multi-echo turbo spin echo sequences (ME-TSE) that are widely available on clinical scanners. Estimation of T2 values is done by a Bloch simulation-based algorithm. As this method can account for stimulated echoes that occur during the echo train within a TSE sequence, its use for sequences with reduced refocusing flip angle is feasible to avoid SAR problems at higher field strength.

Towards measurement of normal Blood-Brain Barrier leakage in individual subjects using DCE-MRI

Nicholas G Dowell ${ }^{1}$, Samira N Bouyagoub ${ }^{1}$, Naji Tabet ${ }^{1}$, Neil A Harrison ${ }^{1}$, Mara Cercignani ${ }^{1}$, and Paul S Tofts ${ }^{1}$

${ }^{1}$ Department of Neuroscience, Brighton and Sussex Medical School, Brighton, United Kingdom

The ability to measure normal BBB leakage in individual subjects would provide a technique to quantify extremely subtle BBB abnormalities in neurological disease. The technique is extremely demanding of scanner stability and vulnerable to low-level (invisible) artefacts. In phantom and healthy control scans without Gd machine stability is good when using Ernstangle scanning. Image artefact currently limits precision in measuring BBB permeability, and is probably caused by pulsatile motion of the Superior Sagittal Sinus (SSS). Image noise is insignificant when optimised imaging parameters (e.g. $F A=30^{\circ}, \mathrm{TR}=30 \mathrm{~ms}$ ) are used. Blood signal is significant and can probably be modelled using SSS signal.

Analytical Characterization of Statistical Bias in Multi-Point Apparent Diffusion Coefficient (ADC) Measurements: Application to Prostate Cancer Imaging

Joshua D. Trzasko ${ }^{1}$, Brent A. Warndahl ${ }^{1}$, Stephen J. Riederer ${ }^{1}$, and Adam T. Froemming ${ }^{1}$

${ }^{1}$ Mayo Clinic, Rochester, MN, United States

In most diffusion studies, two or more DW images are acquired and an apparent diffusion coefficient (ADC) map is generated, with the goal of providing quantitative diffusion information that is independent of acquisition settings or secondary tissues properties. However, ADC values can vary significantly following protocol changes. In this work, we analytically determine the statistical bias in ADC maps generated from multi-point DWI acquisitions, and show how the derived model rationalizes noise-based error propagation as the source of ADC inconsistencies observed in our own clinical practice. 
Proton density fat fraction (PDFF) measurements can be confounded by small effects that are not properly accounted for in modeling. This abstract seeks to understand the empirically observed correlation between R2* and PDFF in terms of the susceptibility difference between water and fat. Numerical fitted values were found to be close to literature values for triglyceride unsaturation and magnetic susceptibility in liver.

Quantitative Synthetic T1 Mapping of the Brain from Structural Imaging using Deep Learning

Samuel Anthony Hurley ${ }^{1,2}$, Jacob M Johnson ${ }^{1}$, Barbara B Bendlin ${ }^{3}$, and Alan B McMillan ${ }^{1}$

${ }^{1}$ Radiology, University of Wisconsin, Madison, WI, United States, ${ }^{2}$ Neuroscience, University of Wisconsin, Madison, WI, United States, ${ }^{3}$ Medicine, University of Wisconsin, Madison, WI, United States

We propose a method to generate synthetic T1 maps directly from conventional T1-weighted imaging. Rather than rely on fitting an explicit signal model or precomputing a dictionary from a closed form equation (e.g. Bloch equations or extended phase graph), we employ deep learning combined with training data from variable flip angle (VFA) T1 mapping experiments to generate an implicit machine learning model of T1 signal. The use of deep learning to enable quantitative imaging directly from an acquired T1-weighted image is a provocative approach with promising capability, as demonstrated herein with less than $3 \%$ error compared to a VFA approach.

Fat Content and Fatty Acid Composition Quantification Using a 3D Stack-of-Radial Trajectory With Adaptive Gradient Calibration

Manuel Schneider ${ }^{1}$, Felix Lugauer ${ }^{1}$, Elisabeth Hoppe ${ }^{1}$, Dominik Nickel ${ }^{2}$, Brian M Dale ${ }^{3}$, Berthold Kiefer ${ }^{2}$, Andreas Maier ${ }^{1}$, and Mustafa R Bashir ${ }^{4,5}$

${ }^{1}$ Pattern Recognition Lab, Department of Computer Science, Friedrich-Alexander-Universität Erlangen-Nürnberg, Erlangen, Germany, ${ }^{2}$ MR Application Predevelopment, Siemens Healthcare GmbH, Erlangen, Germany, ${ }^{3}$ MR R\&D Collaborations, Siemens Healthineers, Cary, NC, United States, ${ }^{4}$ Radiology, Duke University Medical Center, Durham, NC, United States, ${ }^{5}$ Center for Advanced Magnetic Resonance Development, Duke University Medical Center, Durham, NC, United States

The purpose of this study was to evaluate the effect of an adaptive gradient calibration technique for a 3D stack-of-radial sequence with regard to magnitude- and complex-based fat content quantification and triglyceride saturation estimation. In-vivo measurements in two healthy volunteers showed that gradient calibration improved the accuracy of complex fitted fat fraction and fatty acid maps. Gradient calibration only had a minor impact on magnitude-based fat fraction results.

T2-based MR oximetry with background-suppressed T2-bSSFP to reduce partial volume errors

Michael C Langham ${ }^{1}$, Ana E Rodríguez-Soto ${ }^{2}$, Nadav Schwartz ${ }^{2}$, and Felix W Wehrli ${ }^{2}$

${ }^{1} 3400$ Spruce St, University of Pennsylvania, Philadelphia, PA, United States, ${ }^{2}$ University of Pennsylvania, Philadelphia, PA, United States

In small tortuous vessels in the presence of motion it is not possible to prescribe the imaging slice perpendicular to minimize the partial volume effect, which is a significant source of error in $\mathrm{T}_{2}$-based oximetry. We propose background suppression (BS) commonly used in ASL prior to $\mathrm{T}_{2}$-preparation. BS reduces SNR but can be compensated with increased slice thickness and reduced inplane resolution. We tested the method in a controlled experiment via quantification of femoral vein blood oxygenation, which has been measured extensively in our laboratory. The utility of the method is further demonstrated in human umbilical vessels in vivo.

In vivo feasibility of T1-corrected Dual-TR Chemical Shift Encoded Fat Quantification Method

Xiaoke Wang ${ }^{1}$, Diego Hernando ${ }^{2,3}$, and Scott Reeder ${ }^{1,2,3,4,5}$

${ }^{1}$ Biomedical Engineering, University of Wisconsin-Madison, Madison, WI, United States, ${ }^{2}$ Radiology, University of Wisconsin-Madison, Madison, WI, United States, ${ }^{3}$ Medical Physics, University of Wisconsin-Madison, Madison, WI, United States, ${ }^{4}$ Medicine, University of Wisconsin-Madison, Madison, WI, United States, ${ }^{5}$ Emergency Medicine, University of WisconsinMadison, Madison, WI, United States

In chemical shift encoded (CSE) fat quantification techniques, a low flip angle is most commonly used to avoid T1 bias at the expense of SNR. Alternatively, dual flip angle (DFA) acquisitions can be used for T1-corrected fat quantification, however DFA doubles the scan time. A dual TR (DTR) method is proposed where a small percentage increase of scan time allows the independent estimation of T1 of water and fat, and T1-correced fat quantification. This work demonstrates the feasibility of DTR in phantoms and liver imaging.

Simultaneous acquisition of MR angiography and 3D quantitative MR parameter maps

Tomoki Amemiya ${ }^{1}$, Suguru Yokosawa ${ }^{1}$, Yo Taniguchi ${ }^{1}$, Toru Shirai ${ }^{1}$, Ryota Sato ${ }^{1}$, Yoshihisa Soutome ${ }^{1}$, and Hisaaki Ochi ${ }^{1}$ 
We proposed a method to obtain MRA simultaneously with 3D quantitative MR parameter maps. The method calculates MRA by combining images and maps obtained using MR parameter mapping with weights that change in the head-to-neck direction in order to correct for the effect of blood flow. The method was evaluated with five healthy volunteers. It visualized the visibility of blood vessels and correlation of intensity with time-of-flight MRA more effectively than conventional calculation method. This suggests that the proposed method is effective for simultaneously obtaining computational MRA and MR parameter maps.

Reproducibility of Native Renal T1 mapping for Renal Tissue Characterization

Ilona Alexandra Dekkers ${ }^{1}$, Elisabeth Paiman ${ }^{1}$, Aiko de Vries ${ }^{2}$, and Hildo Lamb ${ }^{1}$

${ }^{1}$ Radiology, Leiden University Medical Center, Leiden, Netherlands, ${ }^{2}$ Nephrology, Leiden University Medical Center, Leiden, Netherlands

Advanced renal disease is characterized by adverse changes in renal structure, however non-invasive diagnostic imaging techniques are currently lacking. Here we describe the assessment and reproducibility of native T1 mapping for renal tissue characterization. Renal native T1 mapping was performed in 15 healthy human volunteers using the Modified LookLocker Imaging (MOLLI) $5 \mathrm{~s}(3 \mathrm{~s}) 3 \mathrm{~s}$ sequence on a clinical 3.0 T MR system. Found intra- and inter-examination ICCs for renal cortex $(0.77,0.65)$ and medulla $(0.65,0.99)$ indicate good intra- and inter-examination reproducibility, combined with the Bland-Altman analysis showing good agreement. Renal native T1-mapping is a promising reproducible technique for renal tissue characterization.

Physical parameterization of relaxation curves in GRE sequences

Alexey Vladimirovich Protopopov ${ }^{1}$ and Michael Bock ${ }^{1}$

${ }^{1}$ Dept.of Radiology, Medical Physics, Medical Center University of Freiburg, Faculty of Medicine, University of Freiburg, Freiburg, Germany

The parameter $\mathrm{T}_{2}{ }^{*}$ is often used to describe the apparent rate of spin-spin relaxation in the presence of local magnetic field gradients, which is commonly assumed to be monoexponential. However, the behavior of the transverse relaxation is more complex, since structural characteristics of biological tissues are encoded in the shape of relaxation curve which cannot be described by a single parameter. Several attempts have been made to introduce more accurate relaxation models. In this work we present a concept for the quantitative analysis of the relaxation curve shape in gradient-recalled echo (GRE) imaging based on physical parameters of the signal.

Synthetic MRI of the Knee: ISMRM/NIST Phantom Validation and In-Vivo Qualitative, Quantitative and Diagnostic Comparison with Conventional MRI of the Diagnosis of Internal Derangement

Neil Kumar ${ }^{1}$, Benjamin Fritz ${ }^{2}$, Steven Stern ${ }^{3}$, Marcel Warntjes ${ }^{4}$, Yen Mei Lisa Chuah ${ }^{5}$, and Jan Fritz ${ }^{1}$

${ }^{1}$ Radiology, Johns Hopkins Hospital, Baltimore, MD, United States, ${ }^{2}$ Balgrist University Hospital, Zurich, Switzerland, ${ }^{3}$ Bond Business School, Gold Coast, Australia, ${ }^{4}$ Center for Medical Imaging Science and Visualization (CMIV), Linköping University, Linköping, Sweden, ${ }^{5}$ Siemens Healthcare GmbH, Erlangen, Germany

Knee MRI protocols containing morphologic and quantitative pulse sequences allow comprehensive evaluation of multiple tissues. However, separate quantitative and qualitative image acquisitions are time consuming. We demonstrated excellent native and error-calibrated accuracy of synthetic MRI of the knee for T1, T2 and proton density quantification with use of an ISMRM/NIST phantom, and show excellent intra-day and inter-day repeatability in living human subjects. Synthetic MRI improves contrast-to-noise ratios of cartilage and menisci and yields improvements in artifact reduction and fat suppression. We demonstrate equivalent subjective ratings and diagnostic performance for internal derangement between conventional and synthetic MRI.

The statisitical error in FISP-MRF experiments

Danielle Kara ${ }^{1}$, Jesse Hamilton ${ }^{2}$, Mingdong Fan ${ }^{1}$, Nicole Seiberlich ${ }^{2,3}$, and Robert Brown ${ }^{1}$

${ }^{1}$ Physics, Case Western Reserve University, Cleveland, $\mathrm{OH}$, United States, ${ }^{2}$ Biomedical Engineering, Case Western Reserve University, Cleveland, OH, United States, ${ }^{3}$ Radiology, Case Western Reserve University, Cleveland, $\mathrm{OH}$, United States

The MRF framework has significant freedom in sequence design, increasing its utility and scope, but also the difficulty of determining an optimally efficient experiment. To address this challenge, a statistical analysis of MRF is used to develop a model relating the error in relaxation time quantification and the resulting experimental efficiencies to the number of repetitions in a FISP-MRF experiment. In general, $T_{1}$ and $T_{2}$ efficiencies peak prior to 1000 time steps, then decrease to constant values for larger time step totals. Therefore, the derived model can be used to design efficient MRF experiments. 
Complex-valued residual network learning for parallel MR imaging

Shanshan Wang ${ }^{1}$, Huitao Cheng ${ }^{1}$, Ziwen Ke ${ }^{1}$, Leslie Ying ${ }^{2}$, Xin Liu ${ }^{1}$, Hairong Zheng ${ }^{1}$, and Dong Liang ${ }^{1}$

${ }^{1}$ Paul C. Lauterbur Research Center for Biomedical Imaging, SIAT, Chinese Academy of Sciences, Shenzhen, China, ${ }^{2}$ Department of Biomedical Engineering and Department of Electrical Engineering, The State University of New York, Buffalo, NY, United States

Applying deep learning to fast MR imaging has been new and highly evolved. This direction utilizes networks to draw valuable prior information from available big datasets and then assists fast online imaging. Nevertheless, most existing works adopt real-valued network structures while MR images are complex-valued. This paper proposes a complex-valued residual network learning framework for parallel MR imaging. Specifically, complex-valued convolution and initialization strategy are provided. Residual connections are also adopted to learn a more accurate prior. Experimental results show that the proposed method could achieve improved complex-valued image reconstruction with much less time compared to GRAPPA and SPIRiT.

A Neural Network for Referenceless Reconstruction in Simultaneous Multi-Slice Imaging

Klaus Eickel ${ }^{1,2}$ and Matthias Günther $r^{1,2,3}$

${ }^{1}$ Fraunhofer MEVIS, Bremen, Germany, ${ }^{2}$ mediri GmbH, Heidelberg, Germany, ${ }^{3}$ University Bremen, Bremen, Germany

The unwrapping of simultaneous multi-slice images without extra reference data is presented. A trained deep neural network disentangles overlapping image content and creates the final magnitude images. The results are compared to established techniques (split slice-GRAPPA), especially where correct reference data are missing.

Deep Generative Adversarial Networks for High Resolution fMRI using Variable Density Spiral Sampling

Tianle $\mathrm{CaO}^{1}$, Xuesong $\mathrm{Li}^{1}$, Yan $\mathrm{Tong}^{2}$, and Hua Guo ${ }^{1}$

${ }^{1}$ Center for Biomedical Imaging Research, Department of Biomedical Engineering, School of Medicine, Tsinghua University, Beijing, China, ${ }^{2}$ University of Oxford, London, United Kingdom

An approach to fMRI image reconstruction for variable density radial trajectories is proposed in this abstract. We have employed Generative Adversarial Networks (GAN), which is made up of a generator and a discriminator, to map input aliasing images to gold standard images. Different from the large computation requirements of CS-based methods, the proposed method is able to both boost reconstruction efficiency and achieve a good image quality in the meantime.

Auto-calibrated Parallel Imaging Reconstruction using Fully Connected Recurrent Neural Networks

Tianle Cao ${ }^{1}$, Jiahao Lin $^{2}$, and Kyunghyun Sung ${ }^{2}$

${ }^{1}$ Center for Biomedical Imaging Research, Department of Biomedical Engineering, School of Medicine, Tsinghua University, Beijing, China, ${ }^{2}$ Radiological Sciences, University of California, Los Angeles, Los Angeles, CA, United States

A new approach to auto-calibrating, coil-by-coil parallel imaging reconstruction is presented. It is a generalized reconstruction framework based on deep learning. A neural network consisting of three Dense layer (Fully connected layer) units, an RNN layer and an output Dense unit is designed and trained to identify the mapping relationship between the zero-filled and fully-sampled k-space data. The training process could be separated into two steps: pre-training and fine-tuning. Results show our proposed model could be robust to arbitrary undersampling patterns in k-space and shows a higher structural similarity index compared with traiditional k-space based methods.

FLAIR MR Image Synthesis By Using 3D Fully Convolutional Networks for Multiple Sclerosis

Wen Wei ${ }^{1,2,3}$, Emilie Poirion ${ }^{2}$, Benedetta Bodini² ${ }^{2}$ Stanley Durrleman ${ }^{2,3}$, Olivier Colliot ${ }^{2,3}$, Bruno Stankoff $^{2}$, and Nicholas Ayache

${ }^{1}$ Asclepios project-team, Inria, Sophia Antipolis, France, ${ }^{2}$ Sorbonne Universités, UPMC Univ Paris 06, Inserm, CNRS, Institut du cerveau et la moelle (ICM), AP-HP-Hôpital PitiéSalpêtrière, Paris, France, ${ }^{3}$ Aramis project-team, Inria, Paris, France

Fluid-attenuated inversion recovery (FLAIR) MRI pulse sequence is used clinically and in research for the detection of WM lesions. However, in a clinical setting, some MRI pulse sequences can be missing because of patient or time constraints. We propose 3D fully convolutional neural networks to predict a FLAIR MRI pulse sequence from other MRI pulse sequences. We evaluate our approach on a real multiple sclerosis disease dataset by assessing the lesion contrast and by comparing our approach to other methods. Both the qualitative and quantitative results show that our method is competitive for FLAIR prediction. 
Investigation of convolutional neural network based deep learning for cardiac imaging

Shanshan Wang ${ }^{1}$, Ziwen $\mathrm{Ke}^{1}$, Huitao Cheng ${ }^{1}$, Leslie Ying ${ }^{2}$, Xin Liu ${ }^{1}$, Hairong Zheng ${ }^{1}$, and Dong Liang ${ }^{1}$

${ }^{1}$ Paul C. Lauterbur Research Center for Biomedical Imaging, SIAT, Chinese Academy of Sciences, Shenzhen, China, ${ }^{2}$ Department of Biomedical Engineering and Department of Electrical Engineering, The State University of New York, Buffalo, New York, Armenia

Deep learning based fast MR imaging has been very popular lately. Nevertheless, the empirical nature of existing approaches still leave quite a few questions open. To address this, this paper designs different convolutional neural networks to investigate various factors, such as direct CNN mapping, noise stimulation, data consistency and data sharing, for deep learning based cardiac imaging. We find out that if K-space manipulation strategy is not adopted, CNN still needs dedicated sampling patterns or more complicated structures to remove global corruptions. Furthermore, K-space updating strategy are encouraged to be incorporated with deep learning for better final performances.

Synthetic CT Generation using MRI with Deep Learning: How does the selection of input images affect the resulting synthetic CT?

Andrew Palmera Leynes ${ }^{1,2}$ and Peder Eric Zufall Larson ${ }^{1,2}$

${ }^{1}$ Department of Radiology and Biomedical Imaging, University of California San Francisco, San Francisco, CA, United States, ${ }^{2}$ UC Berkeley - UC San Francisco Joint Graduate Program in Bioengineering, Berkeley and San Francisco, CA, United States

Most recently, synthetic CT generation methods have been utilizing deep learning. One major open question with this approach is that it is not clear what MRI images would produce the best synthetic CT images. We investigated how the selection of MRI inputs affect the resulting output using a fixed network. We found that Dixon MRI may be sufficient for quantitatively accurate synthetic CT images and ZTE MRI may provide additional information to capture bowel air distributions.

Learning multichannel coil combination with Automated Transform by Manifold Approximation (AUTOMAP) using complex-valued neural networks

Bo Zhu ${ }^{1,2}$, Stephen Cauley ${ }^{1}$, Bruce R. Rosen ${ }^{1}$, and Matthew S Rosen ${ }^{1,2}$

${ }^{1}$ A.A. Martinos Center for Biomedical Imaging, Massachusetts General Hospital, Harvard Medical School, Boston, MA, United States, ${ }^{2}$ Department of Physics, Harvard University, Cambridge, MA, United States

End-to-end learning of the image reconstruction domain transform with AUTOMAP (Automated Transform by Manifold Approximation) has been demonstrated on a variety of spatial encoding strategies previously limited to single-channel data. We extend this framework to learning reconstruction of highly undersampled multichannel k-space data solely from pairs of multichannel k-space and image training data without employing conventional parallel imaging formulations such as SENSE or GRAPPA, and show improved RMSE and artifact reduction with the trained AUTOMAP reconstruction network.

Accelerated EPI DWI using a Deep-learning-based Reconstruction.

Yuhsuan $\mathrm{Wu}^{1}$, Erpeng Dai ${ }^{1}$, Chun Yuan ${ }^{1,2}$, and Hua Guo ${ }^{1}$

${ }^{1}$ Center for Biomedical Imaging Research, Department of Biomedical Engineering, School of Medicine, Tsinghua University, Beijing, China, ${ }^{2}$ Vascular Imaging Laboratory, Department of Radiology, University of Washington, Washington, WA, United States

In this work, we preliminarily demonstrate the deep-learning-based reconstruction can be used for under-sampled diffusion imaging. By integrating the sharable information from multiple diffusion directions, the under-sampled data can be nicely recovered.

Deep Learning Reconstruction for Tailored Magnetic Resonance Fingerprinting

Amaresha Shridhar Konar ${ }^{1}$, Vineet Vinay Bhombore ${ }^{1}$, Imam Ahmed Shaik ${ }^{1}$, Seema Bhat ${ }^{1}$, Rajagopalan Sundaresan², Sachin Jambawalikar ${ }^{3}$, Ramesh Venkatesan ${ }^{2}$, and Sairam Geethanath ${ }^{1,3}$

${ }^{1}$ MIRC, Dayananda Sagar Institutions, Bangalore, India, ${ }^{2}$ MRI, GE Healthcare, Bangalore, India, ${ }^{3}$ Radiology, Columbia University, New York, NY, United States

Magnetic Resonance Fingerprinting (MRF) is an accelerated acquisition and reconstruction method employed to generate multiple parametric maps. Tailored MRF (TMRF) coupled with deep learning based reconstruction has been proposed to overcome the shortcoming of $T_{2}$ under estimation and the need for dictionaries respectively. A generalized approach with training of natural images and a specific approach with training of brain data are detailed in this work. Both approaches are demonstrated, compared and quantified. 
${ }^{1}$ Pattern Recognition Lab, Department of Computer Science, Friedrich-Alexander-Universität Erlangen-Nürnberg, Erlangen, Germany, ${ }^{2}$ Siemens Healthcare, Application Development, Erlangen, Germany, ${ }^{3}$ Friedrich-Alexander-Universität Erlangen-Nürnberg, Erlangen, Germany

This work demonstrates the successful application of Deep Learning with phantom and human measurements for the reconstruction in Magnetic Resonance Fingerprinting (MRF). State-of-the-art MRF reconstruction yields quantitative maps of e.g. $T_{1}$ and $T_{2}$ by acquiring multiple undersampled images with various acquisition parameters, commonly referred to as fingerprints. Every measured fingerprint (per voxel) is compared with a dictionary of simulated fingerprints for possible parameter combinations. This time-consuming step can be replaced with a neural network, which directly predicts the parameters from a fingerprint. This was previously shown with simulated data. Here, we extend this approach to real measurements.

Tailored Magnetic Resonance Fingerprinting: optimizing acquisition schedule and intelligent reconstruction using a block approach

Imam Shaik ${ }^{1}$, Amaresha Shridhar Konar ${ }^{1}$, Vineet Vinay Bhombore ${ }^{1}$, Rajagopalan Sundaresan ${ }^{2}$, Shivaprasad Ashok Chikop ${ }^{1,2}$, Gul Moonis $^{3}$, Prachi Dubey $^{3}$, Sachin Jambawalikar $^{3}$, Ramesh Venkatesan², and Sairam Geethanath ${ }^{1,3}$

${ }^{1}$ Medical Imaging Research Center, Dayananda Sagar Institutions, Bangalore, India, ${ }^{2}$ Wipro GE Healthcare, Bangalore, India, ${ }^{3}$ Dept.of Radiology, Columbia University Medical Center NewYork, NY, United States

Magnetic Resonance Fingerprinting technique concurrently generates multiple parametric maps providing for accelerated quantitative imaging. However, quantification of tissues with long T2 such as Cerebrospinal Fluid (CSF) remains a challenge. The main aim of this study is to design acquisition parameters to quantify tissues with long T2 values employing a block based, contrast Tailored MRF (TMRF) approach. In addition, this work emphases on a Neural Network (NN) approach that does not demand noise simulation and/or dictionaries.

Data-Driven Image Contrast Synthesis from Efficient Mixed-Contrast Sequences

Jonathan I Tamir ${ }^{1}$, Valentina Taviani², Shreyas S Vasanawala ${ }^{3}$, and Michael Lustig ${ }^{1}$

${ }^{1}$ Electrical Engineering and Computer Sciences, University of California, Berkeley, Berkeley, CA, United States, ${ }^{2}$ MR Applications and Workflow, GE Healthcare, Menlo Park, CA, United States, ${ }^{3}$ Radiology, Stanford University, Stanford, CA, United States

Synthetic MR is an attractive paradigm for generating diagnostic MR images with retrospectively chosen scan parameters. Typically, synthetic MR images are produced by collecting measurements at multiple measurement times and fitting to a physical model. Here we propose a two-step approach to contrast synthesis. First, we solve a regularized linear inverse problem to reconstruct images at multiple measurement times. Second, we classify spatio-temporal signals and apply different linear combinations based on the classification. We demonstrate the approach on retrospectively under-sampled T1 Shuffling data, in which 3D FSE is collected at relatively short repetition times (TR), and combined to synthesize image contrast with a long TR. The data-driven approach may be useful for synthesizing MR contrasts from acquisitors with varying measurement parameters.

Synthetic FLAIR image from multi-echo GRE using U-Net

Jiyong Park ${ }^{1}$, Kanghyun Ryu ${ }^{1}$, Yoonho Nam² ${ }^{\text {, Jaewook Shin }}{ }^{1}$, Jaeho Lee ${ }^{1}$, and Dong-Hyun Kim ${ }^{1}$

${ }^{1}$ School of Electrical and \& Electronic Engineering, Yonsei University, Seoul, Republic of Korea, ${ }^{2}$ Seoul St. Mary's Hospital, College of Medicine, The Catholic University of Korea, Seoul, Republic of Korea

The fluid-attenuated inversion recovery(FLAIR) image is one of the most frequently scanned images useful for detecting and diagnosing various lesions. The FLAIR technique suppresses cerebrospinal fluid(CSF) signal by using specific TR and long TE. The WM-GM contrast is similar to the T2-weighted image, except that CSF signal is suppressed. Multiecho GRE(mGRE) has increasingly been used for medical diagnosis. Here, we used the mGRE images to create a synthetic FLAIR image using deep learning

Improved Synthetic MRI from Multi-echo MRI Using Deep Learning

Enhao Gong ${ }^{1}$, Suchandrima Banerjee ${ }^{2}$, John Pauly $^{1}$, and Greg Zaharchuk ${ }^{3}$

${ }^{1}$ Electrical Engineering, Stanford University, Stanford, CA, United States, ${ }^{2}$ GE Healthcare, Menlo Park, CA, United States, ${ }^{3}$ Radiology, Stanford University, Stanford, CA, United States

Synthetic MRI enables reconstruction of multiple MRI contrasts from a single (multi-echo) scan which significantly improves scanning efficiency. However, the existing state-of-the-art voxel-wise model-fitting method is not optimal. The model-fitting method often results in inaccurate parameter estimation and undesired artifacts, especially for T2-FLAIR synthesis as shown in clinical studies. Here a deep learning method is proposed to improve the contrast synthesis from multi-delay multi-echo MR imaging. With T2-FLAIR synthesis as an example, the proposed method outperforms existing model-fitting based method to overcome artifacts and improve synthesis accuracy. The proposed method is an essential component for delivering reliable and accurate synthetic MRI, further accelerating scanning and improving quantitative parameter mapping. 
Integrating Spatial and Temporal Correlations into a Deep Neural Network for Low-delay Reconstruction of Highly Undersampled Radial Dynamic Images

Hidenori Takeshima ${ }^{1,2}$

${ }^{1}$ Clinical Application Research Department, Research and Development Center, Toshiba Medical Systems Corporation, Kanagawa, Japan, ${ }^{2}$ Analytics Al Laboratory, Corporate Research \& Development Center, Toshiba Corporation, Kanagawa, Japan

This paper proposes a novel method for the reconstruction of dynamic images from highly undersampled radial k-space data. In order to take advantage of spatial and temporal correlations and reducing the reconstruction time delay, a deep neural network (DNN) was trained with additional input images displaying the aforementioned correlations. It is shown that the image quality from the proposed method is superior to that of the method based on the conventional DNN reconstruction scheme from a single input to a single output.

Noise Level Adaptive Deep Convolutional Neural Network for Image Denoising

Kenzo Isogawa ${ }^{1}$, Takashi Ida ${ }^{1}$, Taichiro Shiodera ${ }^{1}$, Tomoyuki Takeguchi ${ }^{1}$, Yuichi Yamashita ${ }^{2}$, and Hiroshi Takai ${ }^{3}$

${ }^{1}$ Corporate research and development center, Toshiba corporation, Kawasaki, Japan, ${ }^{2}$ MRI system division, Toshiba Medical Systems Corporation, Otawara, Japan, ${ }^{3}$ MRI Systems Development Department, Toshiba Medical Systems Corporation, Otawara, Japan

For integrated diagnosis, MRI provides various types of images related to different acquisition parameters. The change of the acquisition parameters affects noise levels of the provided image in meaningful ways. To adapt the change of the noise level, it is desirable for denoising methods to be adaptive to the noise level, but deep neural network methods are not adaptive, despite their high performance. We propose a deep convolutional neural network (CNN) adjustable to noise levels. The activation functions of the CNN use soft shrinkage whose threshold is proportional to noise level of the input image.

Iterative Cross-Domain Deep-Learning Approach for Reconstructing Undersampled Radial MRI

Doohyun Park ${ }^{1}$, Taejoon Eo ${ }^{1}$, Taeseong Kim¹ ${ }^{1}$ Jinseong Jang ${ }^{1}$, and Dosik Hwang ${ }^{1}$

${ }^{1}$ Yonsei University, Seoul, Republic of Korea

The purpose of this study is to eliminate the aliasing artifacts in accerelated radial MRI. We designed a Cross-Domain deep-learning network, called SISI-Net(Sinogram-ImageSinogram-Image Network). This is an architecture to gradually solves data sparsity problems by iteratively learning the radial sampling data in the sinogram domain and the reconstructed data in the image domain. As a result, proposed network could remove aliasing artifacts effectively while maintaining structural information.

Deep Sinogram Learning for Radial MRI: Comparison with k-space and Image Learning

Taeseong Kim ${ }^{1}$, Taejoon Eo ${ }^{1}$, Doohyun Park ${ }^{1}$, Yohan Jun ${ }^{1,2}$, and Dosik Hwang ${ }^{1}$

${ }^{1}$ Yonsei University, Seoul, Republic of Korea, ${ }^{2}$ Philips Korea, Seoul, Republic of Korea

Deep Sinogram Learning for Radial MRI: Comparison with k-space- and image learning. We demonstrated that singoram learning was more effective than k-space- or image learning in terms of restoring tissue structures and removal of streaking artifacts while not making those as real structures.

Convolutional neural network segmentation of skeletal muscle NMR images

Eduard Snezhko ${ }^{1}$, Noura Azzabou ${ }^{2,3}$, Pierre-Yves Baudin $^{4}$, and Pierre G. Carlier ${ }^{2,3}$

${ }^{1}$ Mathematical Cybernetics, United Institute of Informatics Problems, Minsk, Belarus, ${ }^{2}$ NMR Laboratory, Institute of Myology, Paris, France, ${ }^{3}$ NMR Laboratory, CEA,DRF,IBFJ,MIRCen, Paris, France, ${ }^{4}$ Consultants for Research in Imaging and Spectroscopy, Tournai, Belgium

The purpose of this work was to investigate the ability of deep convolutional neural networks (CNN) to segment muscle groups in NMR images. To this end, we used lower limb scans of patients with different neuromuscular diseases and various levels of fatty infiltration. Thigh and leg muscle groups were first segmented manually and then used in the training and validation processes of the CNN. The mean Dice coefficient of the obtained segmentations was 0.9 , demonstrating the effectiveness of the technique in automatically segmenting both healthy and pathological muscle groups. 
${ }^{1}$ Radiology, Athinoula A. Martinos Center for Biomedical Imaging, Massachusetts General Hospital, Charlestown, MA, United States, ${ }^{2}$ Harvard Medical School, Boston, MA, United States, ${ }^{3}$ Physics, Harvard University, Cambridge, MA, United States

Due to very low Boltzmann polarization, MR images acquired at ultra-low field (ULF), MR images are mostly corrupted with noise, thus resulting in low signal-to-noise. In the aim of improving image quality at ULF, we apply the deep neural network image reconstruction technique, AUTOMAP, to low SNR datasets acquired at $6.5 \mathrm{mT}$. The performance of AUTOMAP (Automated Transform by Manifold Approximation) versus the conventional Inverse Fast Fourier Transform (IFFT) on this data was evaluated. The results for AUTOMAP reconstruction show a significant noise reduction, leading to more than $30 \%$ gain in signal to noise ratio as compared to standard IFFT.

Machine Learning Using the BART Toolbox - Implementation of a Deep Convolutional Neural Network for Denoising

Martin Uecker ${ }^{1,2}$

${ }^{1}$ University Medical Center Göttingen, Göttingen, Germany, ${ }^{2}$ Partner-site Göttingen, DZHK (German Centre for Cardiovascular Research), Göttingen, Germany

Deep convolutional neural networks (DCNNs) tend to outperfom conventional image processing algorithms in recent benchmarks for classifcation, segmentation, denoising, and many other image processing tasks. Here, we show how DCNNs can be implemented using existing building blocks already provided by the BART image reconstruction toolbox. As proof-ofprinciple we discuss the implementation of an image denoising tool based on a pre-trained DCNN.

MR Image Super-resolution Reconstruction Via Enhanced Recursive Residual Network

Ye Fuze ${ }^{1}$ and Lijun Bao ${ }^{1}$

${ }^{1}$ Department of Electronic Science, Xiamen University, Xiamen, China

Magnetic resonance image (MRI) super-resolution (SR) algorithms have been applied to increase the spatial resolution of scans after acquisition, thus facilitating the clinical diagnosis. Motivated by the great success of deep convolutional neural network in computer vision, we introduced an Enhanced Recursive Residual Network (ERRN) for MRI SR. We show that the performance of our method exceeds conventional learning based methods (sparse coding-based ScSR, CNN-based SRCNN and VDSR) in terms of reconstruction error, peak-signal-tonoise-ratio (PSNR) and structure similarity index (SSIM) value.

Reconstruction in deep learning of highly under-sampled T2-weighted image with T1- weighted image

Lei Xiang ${ }^{1}$, Weitang Chang ${ }^{2}$, Yong Chen ${ }^{2}$, Weili Lin ${ }^{2}$, Qian Wang ${ }^{1}$, and Dinggang Shen ${ }^{2}$

${ }^{1}$ School of Biomedical Engineering, Shanghai Jiao Tong University, shanghai, China, ${ }^{2}$ Department of Radiology and BRIC, University of North Carolina at Chapel Hill, chapel hill, NC, United States

T1-weighted image (T1WI) and T2-weighted image (T2WI) are routinely acquired in MRI protocols, which can provide complementary information to each other. However, the acquisition time for each sequence is non-trivial, making clinical MRI a slow and expensive procedure. With the purpose to shorten MRI acquisition time, we present a deep learning approach to reconstruct $\mathrm{T} 2 \mathrm{WI}$ from $\mathrm{T} 1 \mathrm{WI}$ and highly under-sampled T2WI. Our results demonstrate that the proposed method could achieve 8 or higher acceleration rate while keeping high image quality of the reconstructed T2WI.

Real-time cardiac cine using supervised machine learning and compressed sensing with radial trajectory

Jingyuan Lyu ${ }^{1}$, Yu Ding ${ }^{1}$, Qi Liu ${ }^{1}$, and Jian $\mathrm{Xu}^{1}$

${ }^{1}$ UIH America., Houston, TX, United States

2D Real-time cardiac cine imaging is valuable for myocardiac function studies. Compared with Cartesian trajectory, Golden-angle (GA) radial acquisition is promising in patients with impaired breath-hold capacity [1]. The GA radial acquisition is an easy-to-implement and promising technique that features improved spatial-temporal resolution, and overcuts Cartesian sampling trajectories in reducing motion artifacts.

Deep-learned STIR imaging via Deep Learning with multi-contrast MRI

Hanbyol Jang ${ }^{1,2}$, Jinseong Jang ${ }^{1}$, Kihun Bang ${ }^{1,2}$, and Dosik Hwang ${ }^{1}$

${ }^{1}$ Yonsei University, Seoul, Republic of Korea, ${ }^{2}$ Philips Korea, Seoul, Republic of Korea 
The goal of this study is to make STIR MRI using deep learning with multi-contrast MRI. First, we simulated the phantom image created by the bloch equation, which is the basic formula for making MRI, and confirmed that the convolution neural network learns the bloch equation. We also showed the feasibility of making STIR image with in-vivo T1- and T2-weighted, and GRE images in the knee.

\section{Multivariate pattern analysis of multi-band MRI k-space}

Scott J Peltier ${ }^{1,2}$, Krisanne Litinas ${ }^{1}$, Anne $u^{2}$, Jonathan Lisinski ${ }^{3}$, and Stephen LaConte ${ }^{3}$

${ }^{1}$ Functional MRI Laboratory, University of Michigan, Ann Arbor, MI, United States, ${ }^{2}$ Biomedical Engineering, University of Michigan, Ann Arbor, MI, United States, ${ }^{3}$ Carillon Research Institute, Virginia Tech, Roanoke, VA, United States

Multi-band MRI allows for accelerated MR acquisition. However, the reconstruction algorithms, being more complex, require increased reconstruction time without advanced hardware. In this work, we extend classification of MR k-space data to multi-band imaging, enabling rapid prediction of brain state without the need for image reconstruction. We also demonstrate that high prediction accuracy can be achieved even with reduced k-space coverage.

Traditional Poster

\section{Acquisition, Reconstruction \& Analysis: Sparse \& Low-Rank Models}

Parameter-free Parallel Imaging and Compressed Sensing

Jonathan I Tamir ${ }^{1}$, Frank Ong ${ }^{1}$, Shreyas S Vasanawala ${ }^{2}$, and Michael Lustig ${ }^{1}$

We demonstrate an end-to-end parallel imaging and compressed sensing reconstruction that does not rely on parameter tuning. We combine noise pre-whitening, auto-tuned coil sensitivity estimation, and a noise-constrained compressed sensing reconstruction to eliminate the need to select parameters such as soft threshold regularization. The method is validated across a large corpus of phantom and in vivo data at different levels of SNR and with different types of coils in 2D and in 3D. An end-to-end reconstruction is shown for 2D variable density single-shot fast spin-echo with reconstruction times of less than one minute.

Accelerating Non-Cartesian, Sparsity-Promoting Image Reconstruction Via Line Search FISTA

Matthew J. Muckley ${ }^{1}$, Jeffrey A. Fessler ${ }^{2}$, and Marcelo V. W. Zibetti ${ }^{1}$

${ }^{1}$ Center for Biomedical Imaging, New York University School of Medicine, New York, NY, United States, ${ }^{2}$ Electrical Engineering and Computer Science, University of Michigan, Ann Arbor, MI, United States

Iterative reconstruction algorithms for non-Cartesian MRI can have slow convergence due to the nonuniform density of k-space samples. Convergence speed can be improved by including the density compensation function into the algorithm, but current techniques for doing so can lead to SNR penalties or algorithm divergence. Here, we combine the use of density compensation with a line search under the MFISTA framework. The method has the convergence guarantees of MFISTA while gaining the speed improvements of using the density compensition function. The algorithm generalizes further to any FISTA algorithm.

Rapid acquisition for MSK applications using compressed sensing with small coils

Laura Bernadette Lane ${ }^{1}$, Nicolás Schlotterbeck ${ }^{1}$, Gabriel della Maggiora ${ }^{1,2}$, Carlos Castillo-Passi ${ }^{1,2}$, Pablo Besa ${ }^{3}$, Sebastián Irarrazaval ${ }^{3}$, Alvaro Burdiles ${ }^{4}$, Cristián Montalba ${ }^{1}$, and Pablo Irarrazaval $1,2,5$

${ }^{1}$ Biomedical Imaging Center, Pontificia Universidad Católica de Chile, Santiago, Chile, ${ }^{2}$ Department of Electrical Engineering, Pontificia Universidad Católica de Chile, Santiago, Chile, ${ }^{3}$ Department of Orthopedics and Traumatology, School of Medicine, Pontificia Universidad Católica de Chile, Santiago, Chile, ${ }^{4}$ Department of Radiology, School of Medicine, Pontificia Universidad Católica de Chile, Santiago, Chile, ${ }^{5}$ Institute for Biological and Medical Engineering, Pontificia Universidad Católica de Chile, Santiago, Chile

There is a need for faster acquisitions of the MSK system. Particularly, for assessing the ACL at different degrees of flexion, and even better, for dynamic studies. Our work proposes how to obtain quasi-static images of the MSK, in particular for the study of the Anterior Cruciate Ligament (ACL), using smaller and less rigid coils by undersampling and compressed sensing reconstruction. 
A Matrix Completion-Based Reconstruction of In Vivo Eye Images from Undersampled Cartesian 7T MRF Data

Kirsten Koolstra ${ }^{1}$, Andrew Webb ${ }^{1}$, Jan-Willem Beenakker ${ }^{1,2}$, Peter Koken ${ }^{3}$, Mariya Doneva ${ }^{3}$, and Peter Börnert ${ }^{1,3}$

${ }^{1}$ Radiology, Leiden University Medical Center, Leiden, Netherlands, ${ }^{2}$ Ophthalmology, Leiden University Medical Center, Leiden, Netherlands, ${ }^{3}$ Philips Research Hamburg, Hamburg, Germany

Eye motion is the main challenge in ocular MRF scans. To achieve good MRF image quality on one side and to improve patient comfort on the other side, scan times need to be reduced. In this single-channel coil approach with Cartesian sampling, high undersampling can be supported by using the appropriate reconstruction approach. In this work, a matrix completion-based reconstruction was adopted. Resulting parameter maps are compared to maps obtained after a compressed sensing reconstruction, showing that for matrix completion even much greater undersampling factors result in more accurate parameter maps.

MRI denoising using image patch prior based on Gasussian mixture model

Yuhan Zhang ${ }^{1,2}$, Shurong Zou ${ }^{1}$, Ying $\mathrm{Fu}^{1,2}$, and Jia $\mathrm{He}^{1}$

${ }^{1}$ School of Computer Science, Chengdu University of Information and Technology, Chengdu, China, ${ }^{2}$ Collaborative Innovation Center for Image and Geospatial Information, Chengdu University of Information and Technology, Chengdu University of Information and Technology, Chengdu, China

MRI is prone to noise pollution in imaging process.MRI with noise seriously affects the doctor's diagnosis of disease.In order to remove noise in MRI,this abstract considers a patchbased method that integrates Gaussian mixture models(GMMs) learning its parameters from external MRI patches with the clustering of desired patches guided by learned GMMs. The last step is to estimate the clear image by low-rank approximation process.Experimental results show the effectiveness of our method.Compared with the classical MRI denoising algorithm-NLM(Non Local Mean) and ADF(Anisotropic Diffusion Filter), our method achieves better results both visually and numerically.

L1, Lp, L2, and Elastic Net Penalties for Regularization of Two-Gaussian Component Distributions in One-dimensional Magnetic Resonance Relaxometry

Christiana Sabett ${ }^{1}$, Ariel Hafftka ${ }^{1}$, Kyle Sexton ${ }^{2}$, and Richard Spencer ${ }^{2}$

${ }^{1}$ Applied Mathematics \& Statistics, and Scientific Computation (AMSC), University of Maryland, College Park, College Park, MD, United States, ${ }^{2}$ National Institute on Aging (NIA), Baltimore, MD, United States

Magnetic resonance (MR) relaxometry time distributions are recovered via the inverse Laplace transform (ILT), an ill-posed problem that is generally stabilized using Tikhonov regularization. Recent work has considered other penalties, such as the $L_{1}$ penalty for locally narrow distributions. $L_{p}$ penalties, $1<p<2$, may be appropriate for distributions consisting of both narrow and broad components; a linear combination of $L_{1}$ and $L_{2}$ penalties, the elastic net (EN), may similarly be useful. However, there is little guidance regarding the choice of regularization penalty for the recovery of transverse relaxation distributions. We compare the effectiveness of each penalty for representative relaxation data.

Compressed Sensing 3D Double Inversion Recovery (DIR) in the Brain

Tom Hilbert ${ }^{1,2,3}$, Esther Raitel ${ }^{4}$, Jean-Philippe Thiran ${ }^{2,3}$, Reto Meuli ${ }^{2}$, Christoph Forman ${ }^{4}$, and Tobias Kober ${ }^{1,2,3}$

${ }^{1}$ Advanced Clinical Imaging Technology, Siemens Healthcare AG, Lausanne, Switzerland, ${ }^{2}$ Department of Radiology, University Hospital (CHUV), Lausanne, Switzerland, ${ }^{3}$ LTS5, École Polytechnique Fédérale de Lausanne, Lausanne, Switzerland, ${ }^{4}$ Siemens Healthcare GmbH, Erlangen, Germany

Double Inversion Recovery (DIR) provides a clinical valuable contrast, especially to study gray matter tissue alterations. However, long acquisition times hinder its use in clinical routine. Assuming that the inherent sparsity of the contrast is well suited for compressed sensing, we tested an incoherently undersampled 3D variable-flip-angle fast spin echo sequence with subsequent compressed sensing reconstruction. The reconstructed fourfold accelerated images exhibit image quality similar to both the clinical standard (twofold GRAPPA-accelerated) and to the fully sampled acquisition. The proposed sequence with $\sim 4$ min acquisition time may allow a more frequent use of DIR in clinical routine.

Effect of compressed sensing acceleration on high spectral and spatial resolution (HiSS) breast MRI image quality

Milica Medved ${ }^{1}$, Marco Vicari ${ }^{2}$, and Gregory S Karczmar ${ }^{1}$

${ }^{1}$ Radiology, University of Chicago, Chicago, IL, United States, ${ }^{2}$ Fraunhofer MEVIS, Bremen, Germany

Strong T2* weighting has allowed high sensitivity of HiSS breast MRI to cancer, but in whole-breast imaging, contrast is compromised due to necessarily shorter echo trains. k-space under-sampling techniques such as compressed sensing (CS) yield time savings that can be traded for longer echo trains and stronger T2* weighting, potentially increasing breast HiSS $\mathrm{MRI}$ performance in screening and diagnostic applications. Our CS simulation resulted in minimal reduction in spatial resolution for acceleration factor $\mathrm{R}=2$, showing $\mathrm{CS}$ to be a promising acceleration strategy for HiSS MRI, allowing longer echo trains and stronger $\mathrm{T}^{*}$ weighting. 
Automatic Selection of Optimal Regularization Parameters in Compressed Sensing using No Reference Magnetic Resonance Image Quality Assessment.

Kihun Bang ${ }^{1,2}$, Jinseong Jang ${ }^{1}$, Yohan Jun ${ }^{1,2}$, Hanbyol Jang ${ }^{1,2}$, Hojoon Lee ${ }^{3}$, and Dosik Hwang ${ }^{1}$

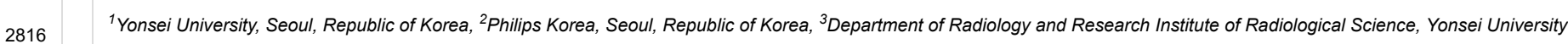
College of Medicine, Seoul, Republic of Korea

Compressed Sensing can reconstruct image without artifacts from the undersampled data, however setting the regularization parameters in CS optimization problem is difficult. Empirically selected parameters or extracted from L-curve method have less reliability. This abstract proposes CS reconstructed MR image quality assessment without ground truth and it can select proper regularization parameters automatically much faster and much reliable.

Real-time 4D Flow MRI with Arbitrary Acquisition Duration

Yichen Zheng ${ }^{1}$, Aiqi Sun ${ }^{2}$, Shuo Chen ${ }^{1}$, Xiaole Wang ${ }^{1}$, Chun Yuan ${ }^{1,3}$, and Rui $\mathrm{Li}^{1}$

${ }^{1}$ Center for Biomedical Imaging Research, Department of Biomedical Engineering, School of Medicine, Tsinghua University, Beijing, China, ${ }^{2}$ Neusoft Medical System, Shanghai, China,

${ }^{3}$ Vascular Imaging Laboratory, Department of Radiology, University of Washington, Seattle, WA, United States

Real-time 4D flow MRI, without ECG gating and respiration control, has been developed as an effective tool to evaluate hemodynamics. With the benefits of low rank and partial separable model, it could be reconstructed with arbitrary acquisition duration. In this study, we investigated the relationship between acquisition duration and image quality of real-time 4D Flow MRI, and proposed optimized acquisition duration considering both image quality and acquisition efficiency.

Combination of Narrow-band KWIC and GROWL for Multiple T1-weighted Images Reconstruction Based on 3D Golden Angle Radial MR Sequence

Yajie Wang ${ }^{1}$, Haikun $\mathrm{Qi}^{1}$, Yishi Wang ${ }^{1}$, Feng Huang ${ }^{2}$, and Huijun Chen ${ }^{1}$

${ }^{1}$ Center for Biomedical Imaging Research, School of Medicine, Tsinghua University, Beijing, China, ${ }^{2}$ Neusoft Medical System, Shanghai, China

Radial sampling has been an increased application due to its insensitivity to motion. A reconstruction method combined the 3D GRAPPA operator for wider radial bands (GROWL) and narrow-band k-space weighted image contrast (KWIC) was proposed and used in GOAL-SNAP sequence. The proposed reconstruction method showed lower image RMSE, accurate T1 map estimation in simulation and higher image quality in in-vivo experiments with shorter computation time.

Phase sensitive receiver combination using prescan singular value decomposition derived receiver sensitivities

Olivia W Stanley ${ }^{1,2}$, Ravi S Menon ${ }^{1,2}$, and L Martyn Klassen ${ }^{1,2}$

${ }^{1}$ Medical Biophysics, The University of Western Ontario, London, ON, Canada, ${ }^{2}$ Centre for Functional and Metabolic Mapping, The University of Western Ontario, London, ON, Canada

Phase sensitive imaging with multi-channel radio-frequency arrays requires sophisticated channel combination. Combining signal from multiple channels without considering the spatial sensitivity profile of those channels can lead to destructive interference and poor quality phase images. This work outlines a phase combination method which interpolates SVD derived relative sensitivity estimates from a prescan using a solid harmonic basis to allow for phase alignment that is extensible to the remainder of the imaging session. Furthermore, this phase alignment method is computationally efficient and applicable to any coil configuration.

Impact of ICA-based denoising of ASL data in clinical settings

Davide Carone ${ }^{1,2}$, George Harston ${ }^{1}$, Thomas Okell ${ }^{3}$, Michael Chappell ${ }^{3,4}$, and James Kennedy ${ }^{1}$

${ }^{1}$ Acute Vascular Imaging Centre, Radcliffe Department of Medicine, University of Oxford, Oxford, United Kingdom, ${ }^{2}$ Laboratory of Experimental Stroke Research, Department of Surgery and Translational Medicine, Milan Center of Neuroscience, University of Milano Bicocca, Monza, Italy, ${ }^{3}$ Wellcome Centre for Integrative Neuroimaging, Oxford Centre for Functional MRI of the Brain, Nuffield Department of Clinical Neurosciences, University of Oxford, Oxford, United Kingdom, ${ }^{4}$ Institute of Biomedical Engineering, Department of Engineering Science, University of Oxford, Oxford, United Kingdom

ASL data has a low signal to noise ratio (SNR) and is sensitive to motion. Independent component analysis (ICA) has been successfully applied to denoise similar quality data in fMRI We explored the effects of using an ICA approach on ASL data acquired in two different clinical settings. Mean cerebral blood flow (CBF) values were identical pre- and post- ICA indicating good signal preservation. However, the variance of $C B F$ and bolus arrival time measures was significantly reduced suggesting a reduction in noise. These results suggest that ICA based denoising represents a promising strategy to improve ASL data quality. 
A Fast and General Non-Cartesian GRAPPA Reconstruction Method

Tianrui Luo ${ }^{1}$, Douglas C. Noll ${ }^{1}$, Jeffrey A. Fessler ${ }^{1}$, and Jon-Fredrik Nielsen ${ }^{1}$

${ }^{1}$ University of Michigan, Ann Arbor, MI, United States

Iterative parallel imaging reconstruction can be very time-consuming for dynamic imaging applications such as functional MRI. GRAPPA is non-iterative but is generally not well-suited for non-Cartesian acquisitions. In this work, we propose a generalization of GRAPPA applicable to arbitrary non-Cartesian readouts. Our non-Cartesian GRAPPA method works by associating a unique kernel with each unsampled (missing) k-space location, and synthesizing non-Cartesian autocalibration (ACS) data by phase-shifts. This approach requires calibrating a very large number of distinct patterns, for which we propose an efficient NUFFT-like algorithm. With this approach we demonstrate fast reconstruction of 3D stack-of-spirals and stack-of-stars images.

A Fourier Spectrum Features Based Patch Clustering Method for Inverse Problems In MRI Processing

Lijun Bao ${ }^{1}$

${ }^{1}$ Department of Electronic Science, Xiamen University, Xiamen, China

Patch clustering is involved into a number of inverse problems in MRI processing, such as image denoising, cross modality synthesis, parallel imaging reconstruction, super-resolution, under-sampled reconstruction, image registration and even segmentation. Considering that the MR signals are acquired in the k-space and then are Fourier transformed into the spatial domain, in this work we propose a new clustering method based on the features extracted from the frequency spectrum, which can be either applied alone for patch or image clustering, or combined with feature descriptors in the spatial domain to facilitate inverse problems processing in MRI.

Blind Simultaneous MultiSlice (SMS) Reconstruction with Application to Phase Contrast Flow Imaging

Suhyung Park ${ }^{1}$, Liyong Chen ${ }^{2}$, and David A Feinberg ${ }^{1,2}$

${ }^{1}$ Helen Wills Neuroscience Institute, University of California, Berkeley, CA, United States, ${ }^{2}$ Advanced MRI Technologies, Sebastopol, CA, United States

Phase contrast MRI (PC-MRI) has been evolved into a practical and widely used technique for quantification of blood flow velocity and volume, which provides useful insights into pathophysiology. However, PC-MRI requires a long acquisition time to build up phase contrast, requiring flow-reference and flow-encoded datasets over multiple heartbeats, and limiting its general use of flow imaging as a clinical routine. To enable higher acceleration rates, in this work we proposed a generalized coil-by-coil approach to simultaneous multislice (SMS) reconstruction in conjunction with inplane acceleration called as bline SMS (b-SMS) by incorporating slice separation and inplane reconstruction into a single optimization framework that is formulated as an inverse problem with data fidelity.

Zero-padding reconstruction for wave-CAIPI images with improved accuracy, and its application in ViSTa myelin water images

Zhe $\mathrm{Wu}^{1}$, Berkin Bilgic ${ }^{2,3}$, Hongjian $\mathrm{He}^{1}$, Yi Sun ${ }^{4}$, Yiping $\mathrm{Du}^{5}$, Kawin Setsompop ${ }^{2,3}$, and Jianhui Zhong ${ }^{1,6}$

${ }^{1}$ Center for Brain Imaging Science and Technology, Department of Biomedical Engineering, Zhejiang University, Hangzhou, China, ${ }^{2}$ Athinoula A. Martinos Center for Biomedical Imaging, Department of Radiology, Massachusetts General Hospital, Charlestown, MA, United States, ${ }^{3}$ Department of Radiology, Harvard Medical School, Boston, MA, United States, ${ }^{4}$ MR Collaboration NE Asia, Siemens Healthcare, Shanghai, China, ${ }^{5}$ School of Biomedical Engineering, Shanghai Jiao Tong University, Shanghai, China, ${ }^{6}$ Department of Imaging Sciences, University of Rochester, Rochester, NY, United States

This study presents an intuitive zero-padding (ZP) reconstruction method for wave-encoded images with an improved accuracy. It was shown to be effective in reducing the residual point spread function (PSF) for all wave-encoded images. ZP reduced the errors between the wave-encoded and Cartesian GRE for all wave gradient configurations in simulation and reduced the side-main lobe intensity ratio from $34 \%$ to $16 \%$ in the thin-slab in vivo Visualization of Short Transverse relaxation time component (ViSTa) images. ZP is applicable for the reconstruction of wave-CAIPI, a recent proposed parallel imaging method using wave-encoding with negligible g-factor penalty under high acceleration factor.

Enhanced ADMM-Net for Compressed Sensing MRI

Guanyu Li', Jiaojiao Xiong ${ }^{1}$, and Qiegen Liu ${ }^{1}$

${ }^{1}$ Department of Electronic Information Engineering, Nanchang University, Nanchang, China

Compressed sensing is an effective approach for fast magnetic resonance imaging (CSMRI) that employs sparsity to reconstruct MR images from undersampled k-space data. Synthesis and analysis sparse models are two representative directions. This work aims to develop an enhanced ADMM-Net on the basis of SADN model, which unifies synthesis and analysis prior by means of the convolutional operator. The present SADN-Net not only promotes the generative sparse feature maps to be sparse, but also enforces the convolution between the filter and trained images to be sparse. Besides, it uses optimized parameters learned from the training data. Experiments show that the proposed algorithm achieves higher reconstruction accuracies. 
DCE-MRI Perfusion Analysis with L1-Norm Spatial Regularization

Michal Bartoš ${ }^{1}$, Michal Šorel ${ }^{1}$, Marie Mangová ${ }^{2}$, Pavel Rajmic ${ }^{2}$, Michal Standara ${ }^{3}$, and Radovan Jiřík

${ }^{1}$ The Czech Academy of Sciences, Institute of Information Theory and Automation, Prague, Czech Republic, ${ }^{2}$ Department of Telecommunications, Brno University of Technology, Brno, Czech Republic, ${ }^{3}$ Masaryk Memorial Cancer Institute, Brno, Czech Republic, ${ }^{4}$ The Czech Academy of Sciences, Institute of Scientific Instruments, Brno, Czech Republic

DCE-MRI perfusion analysis suffers from low reliability, especially when $2^{\text {nd }}$-generation pharmacokinetic models are used to estimate perfusion parameter maps (voxel-by-voxel estimation) in low SNR conditions. These models provide estimates of plasma flow and capillary permeability in addition to the commonly used parameters $\mathrm{K}^{\text {trans }}, \mathrm{k}_{\mathrm{ep}}$. This contribution presents a method for estimation of perfusion maps using the tissue homogeneity model with incorporated spatial regularization in the form of total variation. The algorithm is based on the proximal minimization methods well established in image reconstruction problems. The use of state-of-the-art minimization and image regularization techniques stabilizes the estimates of perfusion parameter maps and keeps the computational demands low.

Laplacian pyramid based data fusion for high resolution dynamic MRI

Liad Pollak Zuckerman ${ }^{1}$, Lior Weizman², Yonina C. Eldar ${ }^{2}$, Dafna Ben Bashat ${ }^{3}$, Moran Arzi ${ }^{3}$, and Michal Irani ${ }^{1}$

${ }^{1}$ Faculty of Mathematics and Computer Science, Weizmann Institute of Science, Rehovot, Israel, ${ }^{2}$ Department of Electrical Engineering, Technion - Israel Institute of Technology, Haifa,

Dynamic contrast-enhanced (DCE) MRI is useful for tumor diagnosis and treatment. In DCE, there is a tradeoff between the spatial and temporal resolutions. Improving the spatial resolution while preserving the temporal dynamics is essential for better diagnosis/treatment. We present a method (LAPFUD) for enhancing the spatial frequency without compromising on temporal resolution. LAPFUD combines information from a static high-resolution image acquired at baseline, with each low-resolution frame. By making local decisions it preserves details from both inputs without changing the temporal behavior. Experiments show that LAPFUD provides superior performance (spatially and temporally) compared to the commonly used keyhole method.

Traditional Poster

\section{Image Analysis \& Post-Acquisition Computing}

Quantification of liver function by linearization of a 2-compartment model of gadoxetic-acid uptake using dynamic contrast enhanced magnetic resonance imaging

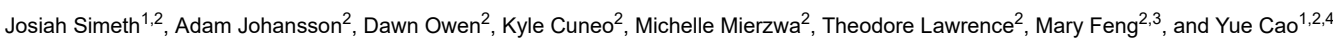

${ }^{1}$ Biomedical Engineering, University of Michigan, Ann Arbor, MI, United States, ${ }^{2}$ Radiation Oncology, University of Michigan, Ann Arbor, MI, United States, ${ }^{3}$ Radiation Oncology, University of California San Francisco, San Francisco, CA, United States, ${ }^{4}$ Radiology, University of Michigan, Ann Arbor, MI, United States

This study used the uptake of gadoxetic acid contrast into the hepatocytes as a means of quantifying liver function. A linearized form of the dual-input two-compartment model was developed to estimate the uptake robustly and efficiently. Validation was obtained relative to the predictions of the accepted dual-input two-compartment model, and independent measurements of whole liver function. The linearized approach allows the creation of a spatially resolved quantitative image of liver function, using standard clinical acquisitions, and removes the requirement for impractical, high temporal resolution scans.

Pseudo-CT generation from 3D multi-echo gradient-echo MRI

Véronique Fortier ${ }^{1,2}$ and Ives R. Levesque $e^{1,2,3}$

${ }^{1}$ Medical Physics Unit, McGill University, Montreal, QC, Canada, ${ }^{2}$ Biomedical Engineering, McGill University, Montreal, QC, Canada, ${ }^{3}$ Research Institute of the McGill University Health Centre, Montreal, QC, Canada

MRI-based treatment planning in radiotherapy is limited by the lack of electron density information and by the difficulty to differentiate air from bone regions. A completely automatic method to produce a pseudo-CT from a 3D gradient-echo dataset through voxel-wise assignment of computed tomography (CT) numbers (Hounsfield unit (HU)) was developed. The HU assignment is based on a combination of relative fat and water content and magnetic susceptibility estimates. The proposed method avoids registration errors and allows for HU variability in each tissue class. An improved quantitative susceptibility mapping algorithm for regions with large susceptibility and negligible signal is also presented. 
We propose a new tool in AFNI-FATCAT to estimate the above RSFC parameters even when time series are censored, using the Lomb-Scargle (L-S) periodogram. The L-S approach for estimating RSFC parameters is useful and generalizable for FMRI data, where censoring is nearly always performed during processing. The method shows minimal bias of parameter estimation, and also allows for the estimation of confidence intervals for the parameters.

The fractal dimension of the tendon-microstructure and its relevance for the detection of permanent changes in micromorphology due to strong mechanical load: a T2* MR-microscopy study using very short detection time

Andreas Georg Berg ${ }^{1}$ and Martin Stoiber ${ }^{1}$

${ }^{1}$ Center for Medical Physics and Biomedical Engineering, Medical University of Vienna, Vienna, Austria

The tendon-structure is hierarchically organized: the endotenon soft tissue separates the collagen fibre bundles in sub-segments with decreasing diameter. MR-microscopy at pixel size below $80 \mu \mathrm{m}$ is capable to differentiate microstructure up to the second hierarchical level and demonstrate self-similarity of the sub-segmentations. Can this self-similarity of the tendon be characterized by a fractal dimension? Is the fractal dimension sensitive to microstructural permanent changes as a consequence of strong mechanical load? We present our investigations obtained within a pilot study using short-TE Multislice-T2*-microscopy with a pixel-size of 39x35 $\mu \mathrm{m} 2$ indicating the importance of the crimp filament structure.

Noise mitigation of high-resolution 7T MRI images

Tales Santini ${ }^{1}$, Fabrício Brito ${ }^{2}$, Sossena Wood ${ }^{1}$, Tiago Martins ${ }^{1}$, Joseph Mettenburg ${ }^{1}$, Howard Aizenstein ${ }^{1}$, Marcelo Vieira ${ }^{2}$, and Tamer S. Ibrahim ${ }^{1}$

${ }^{1}$ University of Pittsburgh, Pittsburgh, PA, United States, ${ }^{2}$ University of Sao Paulo, Sao Carlos, Brazil

High-resolution images typically present lower signal-to-noise ratio (SNR) due to the reduced voxel size. In this work, the BM4D filter was applied to high-resolution MPRAGE images acquired at 7T MRI. Original and denoised images were compared using two different acquisition resolutions: $0.7 \mathrm{~mm}$ isotropic and $0.54 \mathrm{~mm}$ isotropic. The method shows good results for higher-resolution images, greatly improving the SNR while keeping the useful clinical information and the small details which are not discerned using the lower-resolution acquisitions.

\section{A Comparison of Brain Subnetwork Extraction Methods}

Elizabeth Ceiridwen Anne Powell ${ }^{1,2}$, Ferran Prados ${ }^{2,3}$, Baris Kanber ${ }^{2,3}$, Wallace Brownlee ${ }^{2}$, Sara Collorone ${ }^{2}$, Sebastien Ourselin ${ }^{3}$, Olga Ciccarelli $^{2}$, Jonathan D Clayden $^{4}$, Ahmed Toosy $^{2}$, and Claudia Angela Gandini Wheeler-Kingshott ${ }^{2}$

${ }^{1}$ Medical Physics and Biomedical Engineering, University College London, London, United Kingdom, ${ }^{2}$ Queen Square MS Centre, UCL Institute of Neurology, Faculty of Brain Sciences, University College London, London, United Kingdom, ${ }^{3}$ Translational Imaging Group, Centre for Medical Image Computing, Medical Physics and Biomedical Engineering, University College London, London, United Kingdom, ${ }^{4}$ Developmental Imaging and Biophysics Section, Great Ormond Street Institute of Child Health, University College London, London, United Kingdom

In the complex network model of the brain it is often noted that a subset of nodes, or subnetwork, plays a central role in network architecture, whose damage could have a disproportionate effect on network resilience to injury. The identification of "important" nodes in a network is non-trivial though, and several fundamentally different methods exist; it is currently unclear to what extent these methods agree. In this work we demonstrate that subnetworks extracted using rich club and principal network analysis share $60 \%$ of nodes, suggesting a core subset of nodes are important to network architecture independently of analysis model.

Beyond high resolution: Pitfalls in quantification of cortical thickness based on higher and ultra-high resolution data

Falk Lüsebrink ${ }^{1}$ and Oliver Speck ${ }^{1,2,3,4}$

${ }^{1}$ Biomedical Magnetic Resonance, Otto-von-Guericke University, Magdeburg, Germany, ${ }^{2}$ Center for Behavioral Brain Sciences, Magdeburg, Germany, ${ }^{3}$ Leibnitz Institute for Neurobiology, Magdeburg, Germany, ${ }^{4}$ German Center for Neurodegenerative Disease (DZNE), Magdeburg, Germany

It was shown that higher resolution data increases the accuracy of the brain segmentation resulting in a decrease of cortical thickness estimates. However, data is still mostly acquired at a spatial resolution of $1 \mathrm{~mm}$ for quantifying cortical thickness. Several software packages allow processing of higher resolution data. However, FreeSurfer constitutes the de facto standard due to its prevalence. Therefore, we investigate the effects of resolution and SNR at two important stages of its standard processing pipeline: the skull stripping and white matter segmentation. 
A major challenge to facial de-identification in $3 \mathrm{D}$ brain MR images is to find a trade-off between patient privacy protection and retaining the usefulness of the image data. An efficient facial de-identification method is proposed. The method can efficiently conceal identifiable facial details in the 3D brain MR images while maintaining the usefulness of the data. The experimental results indicated the proposed method can achieve the state-of-the-art performance and retain more image data in comparison with the currently available tools.

Segmentation of Gray Matter, White Matter and Cerebrospinal Fluid with MP2RAGE

Yishi Wang ${ }^{1}$, Yajie Wang ${ }^{1}$, Zhe Zhang ${ }^{1}$, Yuhui Xiong ${ }^{1}$, Qiang Zhang ${ }^{1}$, Chun Yuan ${ }^{1,2}$, and Hua Guo ${ }^{1}$

${ }^{1}$ Center for Biomedical Imaging Research, Department of Biomedical Engineering, School of Medicine, Tsinghua University, Beijing, China, ${ }^{2}$ Vascular Imaging Laboratory, Department of Radiology, University of Washington, Seattle, WA, United States

Segmentation of gray matter (GM), white matter (WM) and cerebrospinal fluid (CSF) is an important tool for brain MRI research. A few challenges remain for the segmentation such as the image intensity non-uniformity induced by $B_{1}$ field inhomogeneity, suboptimal data acquisition protocols and long processing time. We propose a fast automatic method which combines the data acquisition with segmentation and is insensitive to $B_{1}$ field inhomogeneity using MP2RAGE. The proposed method has high accuracy and superior performance for the segmentation of subcortical gray matter and is applicable for a wide age range.

A software package designed to integrate advanced fMRI methods for presurgical mapping and clinical studies (IClinfMRI)

Ai-Ling Hsu ${ }^{1,2}$, Ping Hou ${ }^{1}$, Jason M Johnson ${ }^{3}$, Changwei W Wu ${ }^{4}$, Kyle R Noll ${ }^{5}$, Sujit S Prabhu ${ }^{6}$, Sherise D Ferguson ${ }^{6}$, Vinodh A Kumar ${ }^{3}$, Donald F Schomer ${ }^{3}$, John D Hazle ${ }^{1}$, Jyh-Horng Chen ${ }^{2}$, and Ho-Ling Liu ${ }^{1}$

${ }^{1}$ Department of Imaging Physics, The University of Texas MD Anderson Cancer Center, Houston, TX, United States, ${ }^{2}$ Graduate Institute of Biomedical Electronics and Bioinformatics, National Taiwan University, Taipei, Taiwan, ${ }^{3}$ Department of Diagnostic Radiology, The University of Texas MD Anderson Cancer Center, Houston, TX, United States, ${ }^{4}$ Graduate Institute of Humanities in Medicine, Taipei Medical University, Taipei, Taiwan, ${ }^{5}$ Section of Neuropsychology, Department of Neuro-Oncology, The University of Texas MD Anderson Cancer Center, Houston, TX, United States, ${ }^{6}$ Department of Neurosurgery, The University of Texas MD Anderson Cancer Center, Houston, TX, United States

Task-evoked and resting-state (rs) fMRI techniques have been applied to clinical management of neurological diseases, exemplified by pre-surgical functional mapping. Moreover, recent studies recommended incorporating cerebrovascular reactivity imaging into clinical fMRI to evaluate the risk of lesion-induced neurovascular uncoupling. However, a specialized clinical software that integrates the three complementary fMRI techniques and promptly outputs results to clinical PACS and surgical navigation system remains lacking. Here, we developed the Integrated fMRI for Clinical Research (IClinfMRI) software package to incorporate these advanced fMRI methods with streamlined processing and shortened the processing time for presurgical mapping and other clinical applications.

The Change of Adipose Tissues and Organ Fat-fraction in Patients with Morbid Obesity Before and After Bariatric Surgery

Steve Cheuk Ngai Hui ${ }^{1}$, Simon Kin Hung Wong ${ }^{2}$, Qiyong Ai ${ }^{1}$, David Ka Wai Yeung ${ }^{3}$, and Winnie Chiu Wing Chu ${ }^{1}$

${ }^{1}$ Department of Imaging and Interventional Radiology, The Chinese University of Hong Kong, Hong Kong, Hong Kong, ${ }^{2}$ Department of Surgery, The Chinese University of Hong Kong, Hong Kong, Hong Kong, ${ }^{3}$ Department of Clinical Oncology, The Chinese University of Hong Kong, Hong Kong, Hong Kong

The purpose of this study was to investigate the change of brown and white adipose tissue, as well as fat content in liver and pancreas, in patients with morbid obesity before and after bariatric surgery. mDixon sequence and proton MRS were used to measure fat content. Results indicated that weight, BMI, waist circumference, pancreatic fat, liver fat, subcutaneous and visceral adipose tissues were significantly reduced $6-12$ months after surgery. The present study suggested that bariatric surgery effectively reduced the weight in patients with morbid obesity.

Infant brain extraction in T2 weighted MR images using k-means clustering and spatial information

Inyoung Bae ${ }^{1}$, JungHyun Song ${ }^{1}$, Seonyeong Shin ${ }^{1}$, Jun-Young Chung ${ }^{1}$, Sung-Ho Woo ${ }^{2}$, Dongchan Kim ${ }^{3}$, and Yeji Han ${ }^{1}$

${ }^{1}$ Gachon Advanced Institute for Health Science and Technology (GAIST), Gachon University, Incheon, Republic of Korea, ${ }^{2}$ Neuroscience Research Institute, Incheon, Republic of Korea, ${ }^{3}$ College of Health Science, Gachon University, Incheon, Republic of Korea

Brain extraction is an essential pre-processing step for brain image analysis. In this work, a new brain extraction technique for T2 weighted image of an infant brain with pathological characteristics is proposed to reduce the error of conventional techniques caused by variations in contrast and brain size of infant brain from that of the adult brain. We used k-means clustering, spatial information, and morphological approaches to improve brain extraction technique. Quantitative analysis was conducted using the dice ratio compared with the results of manual segmentation. 
Random Forest based Calf Muscle Segmentation from MR data incorporating Prior Information

Marc Fischer ${ }^{1,2}$, Martin Schwartz ${ }^{1,2}$, Bin Yang $^{2}$, and Fritz Schick ${ }^{1}$

${ }^{1}$ Section on Experimental Radiology, Department of Diagnostic and Interventional Radiology, University Hospital of Tübingen, Tübingen, Germany, ${ }^{2}$ Institute of Signal Processing and System Theory, University of Stuttgart, Stuttgart, Germany

Delineation of muscle structures from MR images is an intricate but essential step for quantitative morphological assessment in many areas. In this work segmentation of muscles in the right calf from 2D MR data has been performed. Since challenging conditions prevail, prior information was incorporated in a Machine Learning driven approach. Versatile Random Forests were employed making use of annotated atlases as well as defined landmarks. It was demonstrated that incorporation of this prior information results in a feasible and fully automatic muscle segmentation.

Volumetric Mesh-based Mapping of the Placenta to a Canonical Template for Visualization of Regional Anatomy and Function

S. Mazdak Abulnaga ${ }^{1,2}$, Esra Abaci Turk ${ }^{3}$, Jie Luo ${ }^{4}$, Justin Solomon ${ }^{1,2}$, Lawrence L. Wald ${ }^{5,6,7}$, Elfar Adalsteinsson ${ }^{1,7}$, Carolina Bibbo ${ }^{8}$, Julian N. Robinson ${ }^{8}$, William H. Barth, Jr. ${ }^{9}$, Drucilla J. Roberts ${ }^{10}$, P. Ellen Grant ${ }^{3}$, and Polina Golland ${ }^{1,2}$

${ }^{1}$ Electrical Engineering and Computer Science, Massachusetts Institute of Technology, Cambridge, MA, United States, ${ }^{2}$ Computer Science and Artificial Intelligence Laboratory, Massachusetts Institute of Technology, Cambridge, MA, United States, ${ }^{3}$ Fetal-Neonatal Neuroimaging and Developmental Science Center, Boston Children's Hospital, Harvard Medical School, Boston, MA, United States, ${ }^{4}$ School of Biomedical Engineering, Shanghai Jiao Tong University, Shanghai, China, ${ }^{5}$ Radiology, Harvard Medical School, Boston, MA, United States, ${ }^{6}$ Athinoula A. Martinos Center for Biomedical Imaging, Massachusetts General Hospital, Charlestown, MA, United States, ${ }^{7}$ Institute for Medical Engineering and Science, Massachusetts Institute of Technology, Cambridge, MA, United States, ${ }^{8}$ Maternal and Fetal Medicine, Brigham and Women's Hospital, Harvard Medical School, Boston, MA, United States, ${ }^{9}$ Maternal and Fetal Medicine, Obstetrics \& Gynecology, Massachusetts General Hospital, Harvard Medical School, Boston, MA, United States, ${ }^{10}$ Obstetrics and Perinatal Pathology, Massachusetts General Hospital, Harvard Medical School, Boston, MA, United States

We demonstrate a volumetric mesh-based mapping of the placenta to a canonical template that resembles the better-known ex vivo shape. Placental shape presents significant challenges for visualization of the associated signals. No standard framework exists for visualizing the organ in vivo. Our approach is to flatten a volumetric mesh that captures subjectspecific placental shape while penalizing local distortion to maintain anatomical fidelity. The resulting algorithm produces an invertible transformation to the canonical template. To demonstrate the promise of the proposed approach, we present visualization of BOLD MRI intensity and oxygenation measures after mapping them to a flattened placenta template.

Interactive and flexible quality control in $\mathrm{FMRI}$ sequence evaluation: the uniQC toolbox

Saskia Bollmann ${ }^{1}$, Lars Kasper ${ }^{2,3}$, Klaas Pruessmann ${ }^{2}$, Markus Barth ${ }^{1}$, and Klaas Enno Stephan ${ }^{3}$

${ }^{1}$ Centre for Advanced Imaging, The University of Queensland, Brisbane, Australia, ${ }^{2}$ Institute for Biomedical Engineering, ETH Zurich and University of Zurich, Zurich, Switzerland,

${ }^{3}$ Translational Neuromodeling Unit (TNU), Institute for Biomedical Engineering, University of Zurich and ETH Zurich, Zurich, Switzerland

We present a unified neuroimaging quality control (uniQC) toolbox that enables flexible, interactive assessment of various quality measures on n-dimensional imaging data in Matlab. Key features are its seamless integration in the interactive Matlab command window and the intuitive concatenation of imaging and plot operations using operator overloading that enables fast prototyping of artefact detection and data analysis pipelines. The object-oriented design provides a general framework for $n$-dimensional data handling that can be utilized for fMRI sequence development and quality control.

Interactive Tool to Create Adjustable Anatomical Atlases for Mouse Brain Imaging

Markus Sack ${ }^{1,2}$, Lei Zheng ${ }^{2,3}$, Natalia Gass ${ }^{1,2}$, Alexander Sartorius ${ }^{2,4}$, Gabriele Ende $^{1}$, and Wolfgang Weber-Fahr ${ }^{1,2}$

${ }^{1}$ Neuroimaging, Central Institute of Mental Health, Medical Faculty Mannheim, Heidelberg University, Mannheim, Germany, ${ }^{2}$ RG Translational Imaging, Central Institute of Mental Health Medical Faculty Mannheim, Heidelberg University, Mannheim, Germany, ${ }^{3}$ Experimental Radiation Oncology, Department of Radiation Oncology, University Medical Center Mannheim, Heidelberg University, Mannheim, Germany, ${ }^{4}$ Psychiatry and Psychotherapy, Central Institute of Mental Health, Medical Faculty Mannheim, Heidelberg University, Mannheim, Germany

Brain atlases enable researchers to focus their investigations on specific anatomically defined brain regions and are used in many MRI applications like fMRI, morphometry, whole brain spectroscopy, et cetera. Despite their great use and numerous variants they usually consist of rigid predefined brain regions with a given level of detail often degrading them a non-ideal tool in special research topics. We present a GUI application which allows researchers to easily create mouse brain atlases with an adjustable level of detail and coverage to match specific research questions.

A High Performance Computing Cluster Implementation Of Compressed Sensing Reconstruction For MR Histology

Robert James Anderson ${ }^{1}$, Nian Wang ${ }^{1}$, James J Cook $^{1}$, Gary P Cofer ${ }^{1}$, Russell Dibb ${ }^{1,2}$, G. Allan Johnson ${ }^{1}$, and Alexandra Badea ${ }^{1}$ 
We report the generation of a software pipeline for accelerated MR image reconstruction in a high-performance computing environment, motivated by the shift in time demands from the acquisition to the computational burden of reconstruction in compressed sensing.

Cerebral white matter lesions in multiple sclerosis: optimized automated segmentation and longitudinal follow-up

Philippe $\operatorname{Tran}^{1,2}$, Domitille Dempuré ${ }^{1}$, Ludovic Fillon ${ }^{2,3}$, Marie Chupin ${ }^{2,3}$, Urielle Thoprakarn ${ }^{1}$, and Jean-Baptiste Martini ${ }^{1}$

${ }^{1}$ Qynapse, Paris, France, ${ }^{2}$ Sorbonne Universités, UPMC Univ Paris 06, Inserm, CNRS, Institut du cerveau et de la moelle épinière (ICM) - Hôpital Pitié-Salpêtrière, Boulevard de I'hôpital, F-75013, Inria Paris, Aramis project-team, Paris, France, ${ }^{3} \mathrm{CATI}$, Paris, France

In Multiple Sclerosis (MS), detection of T2-hyperintense white matter lesions on MRI has become a crucial criterion for early diagnosis and monitoring. In this study, we propose an accurate and reliable automated method for lesion segmentation and longitudinal follow-up, using color-scaled maps of lesion evolution depicting increasing and decreasing patterns. Validation of the cross-sectional segmentation has been performed on large samples of MS patients and shows good agreement with manual tracing. Through its reliability and robustness, the measures provided by our automated method of lesion quantification could be a valuable tool for clinical routine and clinical trials.

Reconstruction of quantitative proton density maps from routine clinical data

Antonio Ricciardi ${ }^{1,2,3}$, Francesco Grussu ${ }^{1,3}$, Rebecca Samson ${ }^{1}$, Daniel C Alexander ${ }^{3}$, and Claudia Angela Gandini Wheeler-Kingshott ${ }^{1,4,5}$

${ }^{1}$ Queen Square MS Centre, Department of Neuroinflammation, UCL Institute of Neurology, Faculty of Brain Sciences, University College London, London, United Kingdom, ${ }^{2}$ Department of Medical Physics and Biomedical Engineering, University College London, London, United Kingdom, ${ }^{3}$ Centre for Medical Image Computing, Department of Computer Science, University College London, London, United Kingdom, ${ }^{4}$ Department of Brain and Behavioral Sciences, University of Pavia, Pavia, Italy, ${ }^{5}$ Brain MRI $3 T$ Research Centre, C. Mondino National Neurological Institute, Pavia, Italy

Quantitative proton density (qPD) mapping can be used to measure tissue water content, whose alteration are often linked to pathological conditions. Quantitative MRI methods have been developed in order to make results numerically coherent, but require specific sequences often missing in standard clinical protocols. In this study, an existing approach for the reconstruction of qPD maps from clinical data was corrected to take into account excitation B1 field inhomogeneities, and compared to qPD maps obtained via multi parametric mapping (MPM). The applied correction made clinical-derived qPD maps more similar to the MPM reference than the uncorrected method, without the need of additional specific sequences.

Morphometric Thresholded Fractional Anisotropy for robust quantitative assessment and enhanced visualization of whole-brain white matter alterations in rodent models of Lupus Daniele Procissi ${ }^{1}$, Hadijat-Kubura Moradeke Makinde ${ }^{2}$, Nicola Bertolino ${ }^{1}$, Bradley Allen ${ }^{1}$, Cynthia Yang ${ }^{1}$, and Carla M Cuda ${ }^{2}$

${ }^{1}$ Radiology, Feinberg School of Medicine, Northwestern University, Chicago, IL, United States, ${ }^{2}$ Rheumatology, Feinberg School of Medicine, Northwestern University, Chicago, IL, United States

The study describes a novel voxel-wise brain fractional anisotropy (FA) analysis approach based on morphometrics evaluation of 3D surfaces. These surfaces were generated using thresholded segmentation of two dimensional FA maps and used for both quantitative analysis and enhanced visualization of differences between a control group and two rodent Lupus models with different degrees of white matter alterations. The methods described, if appropriately translated, could enable integration of DTI-MRI in the diagnostic pipeline in a clinical setting.

A Simplified Framework for MR Image Processing \& 3D Printing in Healthcare Applications

Amit Mehndiratta ${ }^{1}$, Manish Kumar Arya ${ }^{1,2}$, Ravi Singh $^{3}$, Shankhabrata $\mathrm{Nag}^{4}$, and Sonal Krishan ${ }^{5}$

${ }^{1}$ Centre for Biomedical Engineering, Indian Institute of Technology Delhi, New Delhi, India, ${ }^{2}$ Department of Electronics \& Communication Engineering, G L Bajaj Institute of Technology and Management, Greater Noida, India, ${ }^{3}$ Department of Electrical Engineering, Indian Institute of Technology, New Delhi, India, ${ }^{4}$ Department of Chemical Engineering, Indian Institute of Technology, New Delhi, India, ${ }^{5}$ Department of Radiology, Medanta: The Medicity Hospital, Gurugram, India

This work summarized a simplified framework that can be used to generate 3D printed prototype from 3D MRI images, with the help of widely available processing tools. The process is conceptually divided into three steps: image acquisition, image post-processing and 3D printing. The utility of the streamlined framework is demonstrated by building 3D prototype of Liver, Spleen and Kidneys using Selective Laser Sintering (SLS) and Fused Deposition Modeling (FDM) technology based 3D printers. The simplified approach has been suggested to assist users in creating 3D anatomical model from medical imaging data using relevant open source tools. 
${ }^{1}$ Academy for Advanced Interdisciplinary Studies, Peking University, Beijing, China, ${ }^{2}$ Department of Radiology, Peking University First Hospital, Beijing, China, ${ }^{3}$ College of Engineering, Peking University, Beijing, China

Automatic segmentation both in the whole prostate gland and the peripheral zone is a meaningful work, because there are different evaluation criteria for different regions according to prostate imaging reporting and data system's advice. Here we show a new method base on deep learning which can get the prostate outer contour and the peripheral zone contour fast and accurately without any manual intervention. The mean segmentation accuracies for 262 images are $94.87 \%$ ( the whole prostate gland) and $85.66 \%$ (the peripheral zone). Even in some extreme cases, such as hyperplasia and cancer, our method shows relatively good performance.

\section{A STATISTICAL FRAMEWORK FOR EVALUATING THE RELIABILITY OF MYELIN IMAGING}

Agah Karakuzu ${ }^{1,2}$, Cyril Pernet ${ }^{3}$, Tanguy Duval ${ }^{1}$, Julien Cohen-Adad ${ }^{1,4}$, and Nikola Stikov ${ }^{1,2}$

${ }^{1}$ Polytechnique Montreal, Montreal, QC, Canada, ${ }^{2}$ Montréal Heart Institute, Montréal, QC, Canada, ${ }^{3}$ Brain Research Center, Division of Clinical Neurosciences, University of Edinburgh, Edinburgh, Scotland, ${ }^{4}$ Functional Neuroimaging Unit, CRIUGM, Universite De Montreal, Montreal, QC, Canada

Given the importance of myelin in brain structure and function, the advancement of MR-based myelin imaging techniques has drawn a great deal of attention. In this abstract we propose a statistical framework for analyzing myelin imaging, taking us one step closer to standardizing and industrializing MR-based myelin biomarkers. In a nutshell, we are computing Pearson correlation coefficients for scan-rescan reliability and taking their differences to determine if some myelin techniques are more reliable than others. We tested this framework in ex vivo dog spinal cord and found the differences between myelin metrics to be subtle, indicating that one metric can often serve as a surrogate for another.

QuantiCEST: Bayesian Model-based Analysis of CEST MR

Paula L. Croal ${ }^{1}$, Yunus Msayib ${ }^{1}$, Kevin J. Ray ${ }^{2,3}$, Martin Craig $^{1}$, and Michael Chappell ${ }^{1}$

${ }^{1}$ Institute of Biomedical Engineering, University of Oxford, Oxford, United Kingdom, ${ }^{2}$ CRUK \& MRC Oxford Institute for Radiation Oncology, University of Oxford, Oxford, United Kingdom, ${ }^{3}$ Wellcome Centre for Integrative Neuroimaging, FMRIB, Nuffield Department of Clinical Neurosciences, University of Oxford, Oxford, United Kingdom

QuantiPhyse, a python-based software tool for quantitative image processing, has recently been released, to increase the accessibility of physiological modelling and quantification. Here, we present QuantiCEST, a QuantiPhyse plug-in offering Bayesian model-based analysis for quantification of CEST MRI. Using either the graphical user interface or command line, users can easily specify a multipool model of the Bloch-McConnell equations to quantify CEST data acquired with any combination of offset frequencies, saturation power and field strength. Additional information, such as relaxation times, can also be incorporated in the model, allowing flexibility to suit individual research needs. A typical analysis pipeline is presented.

A fully automatic territory segmentation method for prostate MR images by multi-atlas matching

Lian Ding ${ }^{1}$, Ge Gao ${ }^{2}$, Yi Zhu ${ }^{1}$, Xiaodong Zhang ${ }^{2}$, Jue Zhang ${ }^{3}$, Jing Fang ${ }^{3}$, and Xiaoying Wang ${ }^{2}$

${ }^{1}$ Biomechanics and Medical Engineering, Academy for Advanced Interdisciplinary Studies, Peking University, Beijing, China, ${ }^{2}$ Department of Radiology, Peking University Frist Hospital, Beijing, China, ${ }^{3}$ Biomechanics and Medical Engineering, Academy for Advanced Interdisciplinary Studies/ College of Engineering, Peking University, Beijing, China

Approximately $70 \%-75 \%$ of prostate cancers originate in the peripheral zone (PZ) and $20 \%-30 \%$ in the transition zone (TZ). According to the Prostate Imaging Reporting and Data System (PIRADS), the diagnostic criteria for PZ and TZ is different. To accomplish fully automatic segmentation for the PZ and TZ, we proposed a territory segmentation method for prostate MR images by multi-atlas matching. This novel segmentation method could not only segment the whole prostate (WP) region, but also the PZ and TZ. The proposed method is fully automatic and could achieve high segmentation accuracy.

Making Quantitative Susceptibility Mapping (QSM) a clinical reality: a one minute Morphology Enabled Dipole Inversion using GPU computing

Mengyuan Wan ${ }^{1}$, Zhe Liu ${ }^{2,3}$, Pascal Spincemaille ${ }^{2}$, and Yi Wang ${ }^{2,3}$

${ }^{1}$ Software Engineering, Wuhan University, Wuhan, China, ${ }^{2}$ Radiology, Weill Cornell Medical College, New York, NY, United States, ${ }^{3}$ Biomedical Engineering, Cornell University, Ithaca, NY, United States

In this work, we demonstrate the feasibility of using GPU computing to achieve a 15 fold acceleration of the most time consuming parts of the Morphology Enabled Dipole Inversion (MEDI) method for Quantitative Susceptiblity Mapping (QSM) leading to an overall 5 fold reduction in total processing time, allowing a one minute susceptibility map reconstruction. 
${ }^{1}$ Laboratory of Neuro Imaging, USC Stevens Neuroimaging and Informatics Institute, Keck School of Medicine of USC, Los Angeles, CA, United States, ${ }^{2}$ Department of Computer Science, Brown University, Providence, RI, United States

Computational tools are increasingly important to MR imaging research, as they can make experiments more reproducible, improve our ability to share our findings and methods, and facilitate hypothesis generation. We aim to contribute a software package to the research community named the Quantitative Imaging Toolkit (QIT). QIT was developed to provide tools for 3D visualization, data exploration, and computational analysis of neuroimaging datasets. While meant to be generally useful for neuroimaging, the tools have extensively developed features for analyzing diffusion MRI data, running large population imaging analyses, and developing new algorithms.

\section{ISMRM Raw Data Viewer}

Benjamin E. Dietrich ${ }^{1}$, Bertram J. Wilm ${ }^{1}$, and Klaas P. Prüssmann ${ }^{1}$

${ }^{1}$ Institute for Biomedical Engineering, ETH Zurich, Zurich, Switzerland

The ISMRM raw data format enables vendor agnostic, reproducible image reconstruction research. So far, the ISMRM raw data format ecosystem did not have a format specific, fast data browser, which is capable of handling large datasets and displaying the data in a convenient form. In this work, we present such a software tool, open-source and platform independent.

Single Image Super-Resolution using the Similarity of Sub-Images in FREBAS Transformed Space

satoshi ITO'

${ }^{1}$ Research Division of Intelligence and Infromation Science, Graduate School of Engineering, Utsunomiya University, Utsunomiya, Japan

In this paper, we propose a new fast image interpolation method involving super-resolution effects. We use FREBAS transform to obtain multi-directional multi-resolution sub-images. By using the similarity of sub-images between different size images, sub-images beyond the Nyquist frequency is estimated using the FREBAS transformed images corresponding scaling parameter. Experiments showed that obtained images have much more sharpened structure than super resolution method based on dictionary learning. PSNR and SSIM are improved and calculation cost is very small compared to learning based method.

FPGA based real-time sensitivity maps estimation using pre-scan method.

Tooba Khan ${ }^{1}$, Muhammad Faisal Siddiqui ${ }^{1}$, and Hammad Omer $^{1}$

${ }^{1}$ Electrical Engineering, COMSATS Institute of Information Technology, Islamabad, Islamabad, Pakistan

Accurate estimation of the receiver coil sensitivities is critical for an error-free image reconstruction from under-sampled data in SENSE. This work proposes an FPGA (Field Programmable Gate Array) based application specific hardware, for real-time sensitivity maps estimation using pre-scan method. In the proposed work, SENSE reconstructions are performed using the sensitivity maps (computed from the proposed design) and the under-sampled data. The results show that the proposed architecture computes receiver coil sensitivity maps in only $1.466 \mathrm{~ms}$ for 8 receiver coils. Also, SENSE reconstructed images show a good mean SNR $(30+\mathrm{dB})$ and low artefact-power $\left(<6 \times 10^{-4}\right)$.

Active learning for automated reference-free MR image quality assessment: decreasing the number of required training samples by reduction of intra-batch redundancy.

Annika Liebgott ${ }^{1,2}$, Damian Boborzi ${ }^{2}$, Sergios Gatidis ${ }^{1}$, Fritz Schick ${ }^{3}$, Konstantin Nikolaou ${ }^{1}$, Bin Yang ${ }^{2}$, and Thomas Küstner $^{2,3}$

${ }^{1}$ Department of Diagnostic and Interventional Radiology, University Hospital of Tuebingen, Tuebingen, Germany, ${ }^{2}$ Institute of Signal Processing and System Theory, University of Stuttgart, Stuttgart, Germany, ${ }^{3}$ Section on Experimental Radiology, University Hospital of Tuebingen, Tuebingen, Germany

Active learning aims to reduce the amount of labeled data required to adequately train a classifier by iteratively selecting samples carrying the most valuable information for the training process. In this study, we investigate the influence of redundancy within the batch of selected samples per iteration, aiming to further reduce the amount of labeled data for automated assessment of MR image quality. An SVM and a DNN are trained with images labeled by radiologists according to the perceived image quality. Approaches to reduce redundancy are compared. Results indicate that reducing the intra-batch correlation for SVM needs fewest labeled samples.

Generic feature extraction accompanied by support vector classification: an efficient and effective way for MR image quality determination

Dirk Bequé ${ }^{1}$, Arathi Sreekumari ${ }^{2}$, Dattesh Shanbhag ${ }^{2}$, Keith Park ${ }^{3}$, Desmond Teck Beng Yeo ${ }^{3}$, Thomas K.F. Foo ${ }^{3}$, and Ileana Hancu ${ }^{3}$ 
${ }^{1}$ GE Global Research, Garching bei München, Germany, ${ }^{2}$ GE Global Research, Bangalore, India, ${ }^{3}$ GE Global Research, Niskayuna, NY, United States

Support vector machine image classification is performed on MR brain images to determine the need to repeat the MR acquisition. However, the image feature extraction is completely brain image agnostic. It is performed either directly on image slices or simple transformations thereof, like e.g. by fore/background thresholding or 1 -level wavelet decomposition. 120 image features and meta-data entries are used to classify images as sufficient to diagnose or not. $84 \%$ accuracy is demonstrated, even after reducing the feature space to only 20 features. Such feature computation is fast enough to perform image quality assessment in real time, immediately after scan completion.

Automatic Brain MR Sequence Classification for Quality Control using Support Vector Machines and Convolutional Neural Networks

Luis A. Souto Maior Neto ${ }^{1,2}$, Heather Charette ${ }^{3,4}$, Marina Salluzzi ${ }^{4,5}$, Mariana Bento ${ }^{4,5}$, and Richard Frayne ${ }^{1,2,4,5}$

${ }^{1}$ Biomedical Engineering Graduate Program, University of Calgary, Calgary, AB, Canada, ${ }^{2}$ Seaman Family MR Research Centre, University of Calgary, Calgary, AB, Canada, ${ }^{3}$ Schulich School of Engineering, University of Calgary, Calgary, AB, Canada, ${ }^{4}$ Calgary Image Processing and Analysis Centre, Calgary, AB, Canada, ${ }^{5}$ Radiology, and Clinical Neurosciences, Hotchkiss Brain Institute, Calgary, AB, Canada

Medical imaging core lab centres face increasing quality control (QC) challenges as studies/trials become larger and more complex. Many $\mathrm{QC}$ processes are performed manually by experts, a time-consuming process. Most of the work on automated medical image $Q C$ in the literature focuses on text-based metadata correction, thus automated QC algorithms that are able to detect inconsistencies with image data only are needed. We propose two different methods for classification of anonymized MR images by acquisition method (T1-w, T2-w, T1 post contrast, or FLAIR). The classifiers were trained on the MICCAI-BRATS 2016 dataset and achieved accuracies of $85.7 \%$ and $93.8 \%$.

Integration of the BART Toolbox into Gadgetron Streaming Framework for Inline Cloud-Based Reconstruction

Mahamadou Diakite ${ }^{1}$, Adrienne E. Campbell-Washburn ${ }^{1}$, and Hui Xue ${ }^{1}$

${ }^{1} \mathrm{NHLBI}$, National Institutes of Health, Bethesda, MD, United States

BART toolbox is a free open-source framework that consists of a rich set of libraries for common operations in medical image reconstruction. Although the libraries provide highly efficient image reconstruction algorithms and toolbox of command-line programs, it does not, by itself, provide seamless integration with commercial MRI systems. Therefore, the goal of the present work is to enable the deployment of BART in clinical research environment for real-time image reconstruction using Gadgetron streaming framework.

MR-only Radiation Therapy Planning workflow optimization for Head and Neck: Zero TE based pseudo CT conversion with body coil.

Cristina Cozzini ${ }^{1}$, Sandeep Kaushik ${ }^{2}$, and Florian Wiesinger ${ }^{1}$

${ }^{1}$ GE Healthcare, Munich, Germany, ${ }^{2}$ GE Global Research, Bangalore, India

Proton Density (PD) weighted Zero Echo Time (ZTE) imaging has been recently developed to provide bone, soft-tissue and air classification suitable for PET/MR Attenuation Correction and Radiation Therapy Planning (RTP). Here we demonstrate ZTE based derivation of pseudo CT using an optimized body coil protocol, which enables patient positioning in the MRI with the RT fixation devices, while preserving the image quality and reproducibility needed for pseudo $\mathrm{CT}$ conversion. The method was tested for the head and neck application on $\mathrm{N}=5$ volunteers for different resolutions. The results were compared versus a high SNR surface coil, previously demonstrated suitable for pseudo CT conversion and dose calculation.

Data processing methods for the extraction of novel FFC-MRI biomarkers

Lionel Broche ${ }^{1}$, Vasileios Zampetoulas ${ }^{1}$, and David Lurie

${ }^{1}$ University of Aberdeen, Aberdeen, United Kingdom

Fast Field-Cycling (FFC) MRI generates images with $T_{1}$-dispersion contrast that provide new insights for medical applications. No model of such dispersion data exists for biological tissues therefore a phenomenological approach is chosen here that minimises data loss while isolating meaningful information by curve fitting. This approach provided promising biomarkers in several pilot studies spanning a range of applications: osteoarthritis, liver fibrosis, breast cancer, glioma and fatty tissues. This shows that a dispersion-based approach of FFC-MRI data is an interesting and novel approach for the discovery of novel biomarkers.

Traditional Poster

\section{Acquisition, Reconstruction, Analysis}


A Principled Approach to Combining Inversion Recovery Images

Antal Horvath ${ }^{1}$, Christoph Jud ${ }^{1}$, Simon Pezold ${ }^{1}$, Matthias Weigel ${ }^{1,2}$, Charidimos Tsagkas ${ }^{3}$, Katrin Parmar $^{3}$, Oliver Bieri ${ }^{1,2}$, and Philippe Cattin ${ }^{1}$

${ }^{1}$ Department of Biomedical Engineering, University of Basel, Allschwil, Switzerland, ${ }^{2}$ Division of Radiological Physics, Department of Radiology, University Hospital Basel, Basel, Switzerland, ${ }^{3}$ Department of Neurology, University Hospital Basel, Basel, Switzerland

The averaged magnetization inversion recovery acquisitions (AMIRA) spinal cord imaging sequence acquires images of different inversion contrasts. Despite the different contrasts the images can be combined to even enhance tissue contrast. We give a principled justification for such averaging. Using energy optimization, we describe how to automatically optimize the contrast-to-noise ratio between different tissues using a compressed sensing inspired approach. We show that the uniform weights in the recently proposed AMIRA sequence are close to the optimum but can nevertheless still be improved. As an example we optimize the contrast-to-noise ratio between different compartments in the spinal cord.

High Resolution Restoration of Neonatal Images: Matlab Based Framework

Nurten Ceren Askin ${ }^{1}$, Peter Lichard ${ }^{2}$, Sebastien Courvoisier ${ }^{1}$, Petra Huppi ${ }^{3}$, Michel Kocher ${ }^{4}$, and Francois Lazeyras ${ }^{1}$

${ }^{1}$ Department of Radiology and Medical Informatics, University of Geneva, Genève, Switzerland, ${ }^{2}$ School of Engineering, École Polytechnique Fédérale de Lausanne, Lausanne, Switzerland, ${ }^{3}$ Department of Pediatrics, University Hospitals of Geneva, Genève, Switzerland, ${ }^{4}$ Biomedical Imaging Group, École Polytechnique Fédérale de Lausanne, Lausanne, Switzerland

A MATLAB based graphical user interface (GUI) was created to apply super resolution (SR) technique on low resolution neonate MR images for obtaining high resolution volume. The user has options to compute HR volume with registration and different reconstruction and regularization methods. In quantitative analysis section, root mean square error, signal-to-noise ratio values could be computed.

Correcting for contrast differences across 3D T1 acquisitions

Sean N Hatton ${ }^{1,2}$, Donald J Hagler ${ }^{1,3,4}$, Joshua Kuperman $2,3,4$, William S Kremen ${ }^{1,2}$, and Anders M Dale ${ }^{1,2,4,5}$

${ }^{1}$ Department of Psychiatry, University of California, San Diego, La Jolla, CA, United States, ${ }^{2}$ Center for Behavior Genetics of Aging, University of California, San Diego, La Jolla, CA, United States, ${ }^{3}$ Department of Radiology, University of California, San Diego, La Jolla, CA, United States, ${ }^{4}$ Center for Multimodal Imaging and Genetics, University of California, San Diego, La Jolla, CA, United States, ${ }^{5}$ Department of Neurosciences, University of California, San Diego, La Jolla, CA, United States

The aim of this study was to correct volumetric differences between images acquired with different MRI parameters. We scanned six subjects on the same $3.0 \mathrm{~T}$ MRI scanner using different T1-weighted imaging sequences. Images were corrected for gradient warping and intensity inhomogeneity, then we applied a novel white matter intensity scaling and a voxelwise image intensity normalization process. The correction improved the goodness of fit, precision and accuracy of the volumetric segmentation of the target image to each test sequence (typically $<1 \%$ difference). This procedure is particularly effective for voxel-wise segmentation techniques over surface-based approaches.

A Tailored Functional Form for Increased Accuracy in CEST B1 Calibration Curves

Abigail T.J. Cember ${ }^{1,2}$, Hari Hariharan ${ }^{2,3}$, and Ravinder Reddy 2,3

${ }^{1}$ Graduate Group in Biochemistry and Biophysics, Perelman School of Medicine, University of Pennsylvania, Philadelphia, PA, United States, ${ }^{2}$ Center for Magnetic Resonance and Optical Imaging, Perelman School of Medicine, University of Pennsylvania, Philadelphia, PA, United States, ${ }^{3}$ Department of Radiology, Perelman School of Medicine, University of Pennsylvania, Philadelphia, PA, United States

Correction for the amplitude of $\mathrm{B}_{1}$ (RF pulse) in CEST experiments is currently done using calibration curves fitted ad hoc with polynomial functions. We have previously found that these polynomial-based correction curves sometimes produce unreasonable results, especially in measurements with large $B_{1}$ variation. Here, we use Bloch-McConnell simulations of CEST as a function of $B_{1}$ strength to demonstrate a new, Lorentzian-type functional form and fitting strategy, expected to lead to an increase in both accuracy and precision in processing of CEST data.

Spatially regularized multi-exponential transverse relaxation times estimation from magnitude MRI images under Rician noise.

Christian EL HAJJ' ${ }^{1,2}$, Guylaine COLLEWET ${ }^{2}$, Maja MUSSE ${ }^{2}$, and Saïd MOUSSAOUI ${ }^{1}$

${ }^{1}$ ECN, LS2N UMR CNRS 6004, Nantes, France, ${ }^{2}$ IRSTEA, UR OPAALE, Rennes, France

This work aims at improving the estimation of multi-exponential transverse relaxation times from noisy magnitude MRI images. A spatially regularized Maximum-Likelihood estimato accounting for the Rician distribution of the noise was introduced. This approach is compared to a Rician corrected least-square criterion with the introduction of spatial regularization. To deal with the large-scale optimization problem, a majoration-minimization approach was used, allowing the implementation of both the maximum-likelihood estimator and the spatial regularization. The importance of the regularization alongside the rician noise incorporation is shown both visually and numerically on magnitude MRI images acquired on fruit samples. 
Multi-Contrast 3D MR Image Reconstruction from Incomplete Measurements with Spatially Adaptive Priors

Hyunkyung Maeng ${ }^{1}$, Sugil Kim ${ }^{1,2}$, Suhyung Park ${ }^{1}$, Eun ji Lim ${ }^{1}$, and Jaeseok Park ${ }^{1}$

${ }^{1}$ Department of Biomedical Engineering, Sungkyunkwan University, Suwon, Republic of Korea, ${ }^{2}$ Department of Brain and Cognitive Engineering, Korea University, Seoul, Republic of Korea

Magnetic resonance imaging (MRI) is well established as a clinical routine in which multiple sets of data are typically acquired to produce various image contrasts such as T1, T2, FLAIR, etc. Despite the versatile nature of MRI, multi-contrast data acquisition is highly time consuming particularly when $3 \mathrm{D}$ encoding is needed. To address this issue, in this work we propose a novel, multi-contrast 3D MR image reconstruction with spatially adaptive priors by exploiting sharable information across the contrast dimension: edge and coil sensitivity maps. The proposed method consists of the following three steps: 1) estimation of edge maps common over all contrasts, 2) estimation of contrast-specific edge maps, and 3) multi-contrast image reconstruction with spatially adaptive, contrast-specific edge priors. In vivo experimental studies show that the proposed method enables T1, T2, and FLAIR $3 \mathrm{D}$ isotropic (1 $\mathrm{mm}^{3}$ ) imaging roughly in 5-6 minutes.

Banding-Free Reconstruction in Frequency-Modulated bSSFP using Virtual Coils with Regularized Non-Linear Inversion

H. Christian M. Holme ${ }^{1,2}$, Nick Scholand ${ }^{1,2}$, Sebastian Rosenzweig ${ }^{1,2}$, Robin N. Wilke ${ }^{1,2}$, and Martin Uecker ${ }^{1,2}$

$2870 \quad{ }^{1}$ Department of Diagnostic and Interventional Radiology, University Medical Center Göttingen, Göttingen, Germany, ${ }^{2}$ Partner Site Göttingen, German Center for Cardiovascular Research (DZHK), Göttingen, Germany

We propose a method for banding-free reconstruction of bSSFP images using Regularized Non-Linear Inversion (NLINV). Instead of using only a few different phase cycles, we dynamically change the phase cycle in each frame, which only slightly changes the steady state. By stacking all frames together as virtual coils and using NLINV, images free of banding artifacts can be reconstructed. Since no new steady states need to be prepared, dead time is eliminated from the acquisition.

Matrix analysis of Hybrid Multidimensional MRI for the diagnosis of prostate cancer

Aritrick Chatterjee ${ }^{1}$, Xiaobing Fan ${ }^{1}$, Shiyang Wang ${ }^{1}$, Tatjana Antic ${ }^{2}$, Scott Eggener ${ }^{3}$, Aytekin Oto ${ }^{1}$, and Gregory S Karczmar ${ }^{1}$

${ }^{1}$ Department of Radiology, University of Chicago, Chicago, IL, United States, ${ }^{2}$ Department of Pathology, University of Chicago, Chicago, IL, United States, ${ }^{3}$ Department of Urology, University of Chicago, Chicago, IL, United States

This study investigates the feasibility of diagnosing prostate cancer through matrix analysis of Hybrid Multidimensional MRI (HM-MRI) data. Data was acquired with all combinations of TE $(47,75,100 \mathrm{~ms})$ and $b$-values $\left(0,750,1500 \mathrm{~s} / \mathrm{mm}^{2}\right)$, resulting in a $3 \times 3$ matrix associated with each voxel. Matrix analysis parameters: trace, eigenvalues and eigenvectors were calculated for benign tissue and prostate cancer. Prostate cancer showed significantly increased trace, eigenvalue 1 , eigenvector components $v_{12}$ and $v_{13}$ and reduced $v_{11}$ compared to normal tissue. PCa diagnosis is feasible using matrix analysis of HM-MRI data with parameters showing good differentiation between PCa and benign prostatic tissue (AUC $0.80-0.96$ on ROC analysis).

Cerebral vasoreactivity latency correction: a clinical case study

Olivier Rossel ${ }^{1}$, Jérémy Deverdun ${ }^{1}$, Amel Benali ${ }^{1}$, Victor Vagné ${ }^{1}$, Nicolas Menjot de Champfleur ${ }^{1}$, and Emmanuelle Le Bars ${ }^{1}$

In MR cerebral vasoreactivity (CVR) experiments, responses to the gas stimuli are expected to be with no major time lag. However, it has been shown that different brain regions could respond with different timing during a vasoreactivity. The presence of potential latencies could lead to a misinterpretation of the resulting CVR maps. We attempt to correct CVR maps for physiological latency differences, and propose an alternative way to display both corrected CVR and CVR latency. The data from a Moya Moya patient highlight that even without a strong perfusion alteration, the vasoreactivity is strongly delayed or even completely disrupted. 
The dual-plane co-RASOR sequence is able to differentiate between a LDR brachytherapy seeds and gold fiducial markes used in prostatic imaging, by exploiting signal pileups and rewinding them radially inwards using different off-resonant frequency offsets and has the potential to avoid a radiological CT scan that's clinically used to differentiate the two in boost therapy.

SNR analysis of retrospectively gated DENSE at $7 \mathrm{~T}$ for the measurement of brain tissue pulsatility

Ayodeji L. Adams ${ }^{1}$, Jacob-Jan Sloots ${ }^{1}$, Peter R. Luijten¹, and Jaco J. M. Zwanenburg ${ }^{1}$

${ }^{1}$ Radiology, University Medical Center Utrecht, Utrecht, Netherlands

Measurements of brain tissue pulsatility can provide information about viscoelastic tissue properties and assess microvasculature blood volume pulsations as a biomarker. VCGtriggered DENSE is capable of acquiring micrometer-level tissue displacements and volumetric strain. However, it is slow and suffers from triggering issues, especially at 7T. In this work, retrospectively-gated DENSE using a pulse oximeter was implemented at 7T. Assessment of its performance showed maintained SNR within half the scan time of triggered DENSE. The high SNR and reduced scan time simplifies its application in future studies assessing the potential of DENSE-derived brain tissue displacements as a biomarker for neurological diseases.

Quantification and management of MR image spatial accuracy for applications in radiation therapy

Teo Stanescu ${ }^{1}$, Joanne Moseley ${ }^{2}$, Callum Moseley $^{2}$, Mostafa Shahabi $^{2}$, and David Jaffray ${ }^{1}$

${ }^{1}$ Medical Physics, Radiation Medicine Program, Princess Margaret Cancer Centre, University Health Network \& University of Toronto, Toronto, ON, Canada, ${ }^{2}$ Medical Physics, Radiation Medicine Program, Princess Margaret Cancer Centre, University Health Network, Toronto, ON, Canada

An automated imaging pipeline was developed and validated to handle the management of MR image spatial accuracy with a focus on applications for radiation therapy (RT). Protoco enforcement was implemented to accept/reject datasets based on expected clinical sequence parameters. System and patient related image spatial distortions were quantified using numerical simulations and measurements. Vector field maps were rendered and stored for automatic filtering and correction of patient MR images. Data and process monitoring was enabled via a web application. The imaging pipeline was deployed clinically to automatically validate patient image data required for RT planning and in-room MR-guided treatment delivery.

Quality Assurance of physiologic signal measures in HCP resting state fMRI data

Wanyong Shin ${ }^{1}$ and Mark J Lowe

${ }^{1}$ Radilogy, Cleveland Clinic, Cleveland, $\mathrm{OH}$, United States

Recently, a physiological log file timing error with $\mathrm{FMRI}$ acquisition was fixed and physiologic data with corrected timing was uploaded to the WU-MINN human connectome project (HCP) cloud. While HCP preprocessing pipeline for resting state ( $r s-)$ and $\mathrm{fMRI}$ has been proposed, the physiologic noise correction sub-pipeline has not been established yet and the physiologic noise data has had less attention in HCP community. In this study, we investigate the quality of HCP physiologic data and propose a standard physiological noise quality assurance.

Sulcal ridge alignment for laminar fMRI at $7 \mathrm{~T}$

Pierre-Louis Bazin ${ }^{1,2,3}$, Wietske van der Zwaag ${ }^{1}$, Ritu Bhandari ${ }^{2}$, Christian Keysers ${ }^{2,4}$, and Valeria Gazzola ${ }^{2,4}$

${ }^{1}$ Spinoza Centre for Neuroimaging, Amsterdam, Netherlands, ${ }^{2}$ Netherlands Institute for Neuroscience, Amsterdam, Netherlands, ${ }^{3}$ Max Planck Institute for Human Cognitive and Brain Sciences, Leipzig, Germany, ${ }^{4}$ Psychology, University of Amsterdam, Amsterdam, Netherlands

Laminar fMRI at 7T typically involves imaging small slabs of cortex, and requires precise alignment of the anatomical and functional data to transfer intra-cortical depth information to the fMRI data. We present a method taking advantage of the high resolution of the fMRI data and extracted sulcal patterns.

Short-term Fourier Transform Analysis of Respiratory- and Cardiac-driven Pulsation of Cerebrospinal Fluid under Free Breathing

Tetsuya Tokushima ${ }^{1}$, Satoshi Yatsushiro ${ }^{2}$, Saeko Sunohara ${ }^{1}$, Mitsunori Matsumae ${ }^{3}$, Hideki Atsumi ${ }^{3}$, and Kagayaki Kuroda ${ }^{1,2}$

${ }^{1}$ Cource of Electrical and Electric Engineering, Guraduate School of Engineering, Tokai University, Hiratsuka, Kanagawa, Japan, ${ }^{2}$ Guraduate School of Information Science and Technology, Tokai University, Hiratsuka, Kanagawa, Japan, ${ }^{3}$ Department of Neurosurgery, Tokai University, Isehara, Kanagawa, Japan 
To separate the respiratory- and cardiac-driven motions of cerebrospinal fluid (CSF) under free breathing, CSF velocity in 7 healthy volunteers and 3 hydrocephalus patients were observed by asynchronous phase contrast $(\mathrm{PC})$ technique with monitoring respiratory and ECG signals. Spectrograms of CSF velocity and respiratory signal obtained by short-term Fourier transform (STFT) with 8-sec length Hamming window revealed that the peak respiratory motion appeared in 0-0.5 Hz band, while the cardiac motion appeared around 1-1.5 $\mathrm{Hz}$. These results suggest that the separation of the two motion components is possible by sliding the frequency bands temporarily according to the spectrogram.

Improved MR Neurography of Brachial Plexus at 3.0 T with iMSDE and DIXON methods

Xiaoqi Wang ${ }^{1}$ and $\mathrm{Li} \mathrm{X \textrm {X } ^ { 2 }}$

${ }^{1}$ Philips Healthcare, Beijing, China, ${ }^{2}$ Beijing Jishuitan Hospital, Beijing, China

In this study we developed a sequence for neurography with robust fat and blood suppression for increased conspicuity of nerves. Improved Motion-Sensitized Driven-Equilibrium Preparation (iMSDE) was applied to null blood and lymphatic motions. DIXON TSE readout with long TE was used to generate water images representing nerves, in brachial plexus where other fat suppression pulses were challenged by B0 and B1 complexities. Preliminary test in brachial plexus showed its advantages and stability.

Towards a Routine Clinical Application of Chemical Exchange Sensitive MRI

Patrick Schuenke ${ }^{1}$, Ralf Omar Floca ${ }^{2,3}$, Caspar Jonas Goch ${ }^{2}$, Marco Nolden², Moritz Zaiss ${ }^{4}$, Johannes Windschuh ${ }^{5}$, Hoai Nam Dang ${ }^{1}$, Christian David ${ }^{6}$, Volkert Roeloffs ${ }^{7}$, Peter Bachert ${ }^{1}$, and Mark E. Ladd ${ }^{1}$

${ }^{1}$ Division of Medical Physics in Radiology, German Cancer Research Center (DKFZ), Heidelberg, Germany, ${ }^{2}$ Division of Medical Image Computing, German Cancer Research Center (DKFZ), Heidelberg, Germany, ${ }^{3}$ Heidelberg Institute of Radiation Oncology (HIRO), National Center for Radiation Research in Oncology (NCRO), Heidelberg, Germany, ${ }^{4}$ Max Planck Institute for Biological Cybernetics, Tuebingen, Germany, ${ }^{5}$ Center for Biomedical Imaging, NYU Langone Medical Center, New York, NY, United States, ${ }^{6}$ Division of X-Ray Imaging and Computed Tomography, German Cancer Research Center (DKFZ), Heidelberg, Germany, ${ }^{7}$ Max Planck Institute for Biophysical Chemistry, Goettingen, Germany

Chemical exchange saturation transfer (CEST) and chemical exchange sensitive spinlock (CESL) were shown to have potential to provide molecular information for diagnosing a wide range of diseases. However, the lack of standardized acquisition protocols and freely available post-processing software prohibited the widespread application of these promising techniques until now. In this work, we present a modularly designed CEST/CESL preparation block that is easy to operate and can be used with arbitrary MRI readouts. Further, we developed and provide a $\mathrm{C}++$ based open-source software that offers many CEST/CESL specific functionalities for the post-processing of the acquired data.

Observation of the kinetics of antioxidant action in blood serum as measured by NMR relaxation

Magdalena Witek ${ }^{1}$, Dorota Wierzuchowska², and Barbara Blicharska ${ }^{3}$

${ }^{1}$ Food Technology, University of Agriculture in Krakow, Krakow, Poland, ${ }^{2}$ Pedagogical University, Krakow, Poland, ${ }^{3}$ Institute of Physics, Jagiellonian University, Krakow, Poland

The exposure of blood serum to reactive oxygen species creates free radicals and damages the structure of biomolecules. It causes proton NMR relaxation times to change over time. In aqueous protein solutions, $T_{1}$ and $T_{2}$ decay curves were fitted by single exponential components. Following addition of hydrogen peroxide to blood serum which contained endogenous antioxidants, relaxation times initially decreased then increased towards initial values. The character of these curves and their fitting parameters depend on kinetics of oxidation processes. We hope that MR imaging may help to investigate these important processes also in vivo.

Visualizing the Spatial Propagation of Ventilation and Perfusion Signal with Fourier-Decomposition MR

David Bondesson ${ }^{1,2}$, Thomas Gaaß ${ }^{1,2}$, Moritz Schneider ${ }^{1,2}$, Bernd Kühn ${ }^{3}$, Olaf Dietrich ${ }^{1}$, and Julien Dinkel ${ }^{1,2}$

${ }^{1}$ Department of Radiology, University Hospital Munich, LMU, Munich, Germany, ${ }^{2}$ Comprehensive Pneumology Center Munich (CPC-M), Helmholtz Zentrum München, Munich, Germany, ${ }^{3}$ Siemens healthcare $\mathrm{GmbH}$, Erlangen, Germany

Fourier decomposition (FD) MRI offers functional imaging without exposing patient to contrast agents during free breathing measurements, facilitating the examination of patients with impaired respiration. The presented method visualizes signal progression in the lung by using the phase information of the FD-method and addresses recently raised concern that variable-frequency signals can lead to errors in ventilation and perfusion phase estimates. With the signal progression maps it is further shown how localized signal delays caused by pathologies can be identified.

Dynamic field map correction based on reversed-gradient design for non-Cartesian single-shot fast fMRI

Fei Wang ${ }^{1}$, Bruno Riemenschneider ${ }^{1}$, Juergen Hennig ${ }^{1}$, and Pierre LeVan ${ }^{1}$

${ }^{1}$ Dept. of Radiology, Medical Center - University of Freiburg, Freiburg, Germany 
A dynamic field map correction technique based on reversed-gradient design is introduced to non-Cartesian single-shot fast fMRI to correct the off-resonance artifacts. The field map estimated from dual-TE GRE scan could not capture that from field drift and eddy currents, so the off-resonance artifacts could not be corrected completely. This technique acquires two images with reversed slow-encoding directions in each time frame, which is generally used in EPI, and updates field map iteratively based on conjugate gradient reconstruction. After correction, the off-resonance artifacts are significantly reduced.

Improving radial cardiac cine with higher-order total-variation regularizations

Renjie He${ }^{1}$, Qi Liu², Yao Ding ${ }^{1}$, and Ruobing $\mathrm{He}^{3}$

${ }^{1}$ MDACC, Houston, TX, United States, ${ }^{2}$ UIHA, Houston, TX, United States, ${ }^{3}$ IUFW, Fort Wayne, IN, United States

In cardiac cine MRI, radial data acquisition will make the motion effects being more noise-like in image domain, and to achieve high temporal resolution, sparse sampling will inevitably lead to streaking artifacts using conventional image reconstruction methods. Golden angle radial reordering which provides continuously change in angle direction will eliminate the coherence of (streaking) artifacts in the temporal dimension. While GRASP-like reconstruction method applies 1D total-variation (TV) regularization on the reconstructed temporal signal, the spatial consistence of the reconstructed images are not ensured. Here we propose a reconstruction strategy using a higher-order TV to promote the spatial imaging quality.

Correction of Ferromagnetic Object Artifacts Using Simulated Off-Resonance Map

Sina Amirrajab ${ }^{1}$, Vahid Ghodrati ${ }^{2,3}$, and Abbas Nasiraei Moghaddam ${ }^{1}$

${ }^{1}$ Biomedical Engineering, Amirkabir University of Technology (Tehran Polytechnic), Tehran, Iran (Islamic Republic of), ${ }^{2}$ Department of Radiological Sciences, University of California, Los Angeles, Los Angeles, CA, United States, ${ }^{3}$ Biomedical Physics Interdepartmental Program, University of California, Los Angeles, Los Angeles, CA, United States

The aim of this study is to quantitatively investigate the potential of field-mapping method in areas around a ferromagnetic object in the post processing approach. As a worst-case scenario, for regions farther than around 10 times the radius of a ferromagnetic foreign object, the post processing approach (based on the simulated off-resonance map) can be useful, even at 3 Tesla, to correct image distortions. At this distance, the exact shape of the object is not important and results obtained for the sphere, remain valid for most objects with the same volume.

Real-time Personalized Acquisition Optimization: 30\%-50\% reconstruction improvements from a 10-second undersampling optimization

Ke Wang ${ }^{1,2}$, Enhao Gong ${ }^{2}$, Suchandrima Banerjee ${ }^{3}$, John M Pauly ${ }^{2}$, and Greg Zaharchunk ${ }^{4}$

${ }^{1}$ Department of Biomedical Engineering, Tsinghua University, Beijing, China, ${ }^{2}$ Department of Electrical Engineering, Stanford University, Stanford, CA, United States, ${ }^{3}$ GE Healthcare, Menlo Park, CA, United States, ${ }^{4}$ Department of Radiology, Stanford University, Stanford, CA, United States

Improved undersampling designs can effectively improve the acquisition and resulting reconstruction accuracy. However, existing undersampling optimization methods are timeconsuming and the limited performance prevent their clinical applications. Here we proposed an improved undersampling trajectory optimization scheme to generate an optimized trajectory within seconds and apply for subsequent multi-contrast MRI datasets on a per-subject basis. By using a data-driven method combined with improved algorithm design, GPU acceleration and more efficient computation, the proposed method can optimize a trajectory within $5-10$ seconds and achieve $30 \%-50 \%$ reconstruction improvement with the same acquisition cost, which makes real-time under-sampling optimization possible for clinical applications.

Speedup of iterative k-t SENSE reconstruction using the multidimensional fast Fourier transform for arbitrary periodic grids

Adam Johansson ${ }^{1}$, James M Balter ${ }^{1}$, and Yue Cao ${ }^{1,2,3}$

${ }^{1}$ Radiation Oncology, University of Michigan, Ann Arbor, MI, United States, ${ }^{2}$ Radiology, University of Michigan, Ann Arbor, MI, United States, ${ }^{3}$ Biomedical Engineering, University of Michigan, Ann Arbor, MI, United States

The multidimensional fast Fourier transform (MFFT) for arbitrary n-dimensional periodic grids allows images with non-cuboid voxels and fields of view (FOV) to be reconstructed efficiently. In this study, we apply the MFFT with a modified Smith normal form to iterative k-t SENSE reconstruction of data from a golden-angle stack-of-stars DCE-MRI sequence to produce a 4-D image with a non-cuboid FOV tailored to fit tight around the imaged subject. Compared to FFT-based reconstruction on a tight-fitting cuboid FOV, the non-cuboid MFFT reduced reconstruction time by $18 \%$ and memory usage by $11 \%$ while producing voxel values identical to those found in the reconstruction domain of the cuboid FOV.

MR Fingerprinting Reconstruction using Convolutional Neural Network (MRF-CNN)

Qiang Zhang ${ }^{1}$, Rui Guo ${ }^{1}$, Huikun $\mathrm{Qi}^{1}$, Di Cui ${ }^{2}$, Edward S Hui ${ }^{2}$, Shuo Chen ${ }^{1}$, Hua Guo $^{1}$, and Huijun Chen ${ }^{1}$

${ }^{1}$ Department of Biomedical Engineering, Tsinghua University, Beijing, China, ${ }^{2}$ Department of Diagnostic Radiology, The University of Hong Kong, Hong Kong, China 
The purpose of this study is to develop a MR fingerprinting (MRF) reconstruction algorithm using convolutional neural network (MRF-CNN). Better MRF reconstruction fidelity was achieved using our MRF-CNN compared with that of the conventional approach ( $R^{2}$ of $T_{1}: 0.98$ vs $0.97, R^{2}$ of $T_{2}: 0.97$ vs 0.59 ). This study further demonstrated the performance of our MRF-CNN, which was retrained using MR signal evolutions in the continuous parameter space with various levels of Gaussian noise, amidst noise contamination, suggesting that it may likely be a better alternative than the conventional MRF dictionary matching approach.

Interior-Point and Particle-Swarm Optimization of an Inversion-Recovery Prepared Spoiled Gradient Echo Magnetic Resonance Fingerprinting Sequence

Jingwen $\mathrm{Yao}^{1,2}$, Zhaohuan Zhang ${ }^{1,2}$, Kyunghyun Sung ${ }^{1}$, and Holden $\mathrm{H} . \mathrm{Wu}^{1}$

${ }^{1}$ Department of Radiological Sciences, David Geffen School of Medicine, University of California, Los Angeles, Los Angeles, CA, United States, ${ }^{2}$ Department of Bioengineering, University of California, Los Angeles, Los Angeles, CA, United States

Magnetic Resonance Fingerprinting (MRF) permits simultaneous quantitative mapping of multiple MR parameters. We propose a framework for optimizing the sequence parameters of an inversion recovery prepared spoiled gradient echo (IR spoiled-GRE) based MRF sequence, with interior-point (IP) method and particle-swarm optimization (PSO). By using an designed exponential cost function to maximize the discrimination between tissue types, with combined sinusoidal wave functions as input to generate sequence parameters, substantial improvement of accuracy for $\mathrm{T}_{1}$ and $\mathrm{T}_{2}{ }^{*}$ quantification can be achieved. Simultaneous high accuracy of $\mathrm{T}_{1}$ and $\mathrm{T}_{2}{ }^{*}$ estimations can be achieved within $0.7 \mathrm{~s}$ for $\mathrm{SNR} \geq 5$; within $0.5 \mathrm{~s}$ for SNR $\geq 10$.

CoverBLIP: scalable iterative matched-filtering for MR Fingerprint recovery

Mohammad Golbabaee ${ }^{1}$, Zhouye Chen², Yves Wiaux², and Michael Davies ${ }^{1}$

${ }^{1}$ School of Engineering, University of Edinburgh, Edinburgh, United Kingdom, ${ }^{2}$ School of Engineering and Physical Sciences, Heriot-Watt University, Edinburgh, United Kingdom

Current popular methods for Magnetic Resonance Fingerprint (MRF) recovery are bottlenecked by the heavy computations of a matched-filtering step due to the size and complexity of the fingerprints dictionary. In this abstract we investigate and evaluate the advantages of incorporating an accelerated and scalable Approximate Nearest Neighbour Search (ANNS) scheme based on the Cover trees structure to shortcut the computations of this step within an iterative recovery algorithm and to obtain a good compromise between the computational cost and reconstruction accuracy of the MRF problem.

Matching Error Evaluation in Magnetic Resonance Fingerprinting with a Fast Imaging with Steady Precession sequence using Bloch Equation Simulations with a Diffusion Propagator

Shota Hodono ${ }^{1}$, Yun Jiang ${ }^{2}$, Gregor Körzdörfer ${ }^{3,4}$, Naren Nallapareddy ${ }^{5}$, Vikas Gulani² ${ }^{2}$ and Mark Griswold²

${ }^{1}$ Physics and Astronomy, Ohio Northern University, Ada, OH, United States, ${ }^{2}$ Radiology, Case Western Reserve University, Cleveland, OH, United States, ${ }^{3}$ Siemens Healthcare, Erlangen, Germany, ${ }^{4}$ Friedrich-Alexander Universität Erlangen-Nürnberg, Erlangen, Germany, ${ }^{5}$ Biomedical Engineering, Case Western Reserve University, Cleveland, OH, United States

The robustness of $T_{1}, T_{2}$ values derived from Magnetic Resonance Fingerprinting (MRF) is limited in certain situations because MRF dictionaries have in general not included apparent diffusion coefficients (ADC). In this study, the potential estimated $T_{1}, T_{2}$ errors due to the omission of diffusion were evaluated for the MRF-fast imaging with steady precession sequence. Dictionaries with $A D C$ values were generated by using Bloch equations with a diffusion propagator. The generated signal evolutions with $A D C$ were matched to those generated by Bloch equation simulations without ADC by employing a template-matching algorithm.

In vivo parametric mapping using piecewise constant flip angle and multi shot EPI MR Fingerprinting

Zaid Bin Mahbub ${ }^{1}$, Mohammad Golbabaae ${ }^{2}$, Arnold Julian Vinoj Benjamin ${ }^{1,2}$, Mike Davies $^{2}$, and lan Marshall ${ }^{1}$

${ }^{1}$ Centre for Clinical Brain Sciences, University of Edinburgh, Edinburgh, United Kingdom, ${ }^{2}$ School of Engineering, Institute for Digital Communications, University of Edinburgh Edinburgh, United Kingdom

Previous MR fingerprinting studies have used smooth variations in TR and flip angles. In this study we introduce a piecewise constant flip angle train into a standard gradient echo multi shot EPI sequence. The resulting T1, T2 and proton density maps were obtained from a phantom and healthy volunteers using only 3 distinct flip angle values (obtained by optimization over 8 different flip angles) and using iterative reconstruction. The method generates steady states covering full k-space, producing alias-free maps.

Tianyu Han ${ }^{1}$, Teresa Nolte ${ }^{1,2}$, Nicolas Gross-Weege ${ }^{1}$, and Volkmar Schulz ${ }^{1,3}$

${ }^{1}$ Department for Physics of Molecular Imaging Systems, RWTH Aachen University, Aachen, Germany, ${ }^{2}$ Multiphysics and Optics, Philips Research Europe, Eindhoven, Netherlands, ${ }^{3}$ Oncology Solutions, Philips Research Europe, Eindhoven, Netherlands 
MR fingerprinting offers a rapid way to accurately map multiple tissue parameters. The dictionary based reconstruction under the influence of Gaussian noise is identified as a convex optimization problem and solved by a Nelder-Mead simplex algorithm. Instead of a lengthy and uniform sampling proposed by dictionay matching, the new approach using a heuristic and incoherent sampling in the $\$ \$ \$ T$ T $1 \$ \$ \$-\$ \$ T$ T $2 \$ \$ \$$ space. More robust $\$ \$ \$$ T_ $1 \$ \$ \$$ estimations are obtained even under severe noise environments. Thus, a robust and fast MR fingerprinting reconstruction can be made without any dictionary.

Towards Unified Colormaps for Quantitative MRF Data

Mark Griswold ${ }^{1}$, Jeffrey Sunshine ${ }^{1}$, Nicole Seiberlich ${ }^{1}$, and Vikas Gulani ${ }^{1}$

${ }^{1}$ Case Western Reserve University, 44106, $\mathrm{OH}$, United States

The goal of quantitative methods such as MRF is to provide a quantitative characterization of tissue physiology and pathology. These data are displayed as images to convey both geographic/anatomical information and quantitative physical property measurements. But this also means that the manner in which the information is displayed is critical. Here we propose several color map alternatives that have been optimized for use in MRF. It is hoped that the use and further optimization of these maps by the community will further improve our ability to visualize and understand this kind of quantitative data.

Simultaneous Quantification of T1, T2, and Off-resonance Using FISP-MRF with a Rosette Trajectory and Readout Segmentation

Yuchi Liu ${ }^{1}$, Jesse Hamilton ${ }^{1}$, Mark Griswold ${ }^{1,2}$, and Nicole Seiberlich ${ }^{1}$

${ }^{1}$ Biomedical Engineering, Case Western Reserve University, Cleveland, $\mathrm{OH}$, United States, ${ }^{2}$ Radiology, University Hospitals, Cleveland, OH, United States

Artifacts due to off-resonance effects are a significant challenge for non-Cartesian MRI. In FISP-based MRF sequences, if the entire spiral readout is employed to generate a highly undersampled image, any off-resonance during the readout will lead to blurring. However, short portions of the readout will be mostly free of dephasing due to off-resonance effects. By gridding only segments of the readout, it may be possible to quantify the resonance frequency along with $\mathrm{T}_{1}$ and $\mathrm{T}_{2}$. This work shows a proof-of-principle application of this idea using the cardiac MRF sequence with the rosette trajectory in simulations.

Traditional Poster

\section{Tissue Characterization}

Simultaneous high-resolution cardiac T1 mapping and cine imaging using model-based iterative image reconstruction

Kirsten Miriam Becker ${ }^{1}$, Jeanette Schulz-Menger ${ }^{2,3,4}$, Tobias Schaeffter ${ }^{1,5}$, and Christoph Kolbitsch ${ }^{1,5}$

${ }^{1}$ Physikalisch-Technische Bundesanstalt (PTB), Braunschweig and Berlin, Germany, ${ }^{2}$ Working Group on Cardiovascular Magnetic Resonance, Experimental and Clinical Research Center (ECRC), DZHK partner site Berlin, Berlin, Germany, ${ }^{3}$ Charité Medical Faculty University Medicine, Berlin, Germany, ${ }^{4}$ Department of Cardiology and Nephrology, HELIOS Klinikum Berlin Buch, Berlin, Germany, ${ }^{5}$ Division of Imaging Sciences and Biomedical Engineerin, King's College London, London, United Kingdom

Native myocardial T1 mapping provides information for the detection of diffuse fibrosis in different cardiac diseases. Here we present simultaneous T1 mapping and cine imaging with a resolution of $1.3 \times 1.3 \mathrm{~mm}^{2}$ using model-based iterative reconstruction. T1 times in the septum were $1285 \pm 46 \mathrm{~ms}$ compared to $1240 \pm 28 \mathrm{~ms}$ obtained with MOLLI. In contrast to MOLLI, the proposed approach did not show any heart rate dependence of T1. In addition, the approach allows T1 mapping of challenging structures, such as the right ventricle and the apex. Functional assessment of reconstructed cine images did not show any significant differences compared to a standard Cartesian cine scan.

Prospective correction of patient-specific respiratory motion in T1 and T2 mapping

Michael Bush ${ }^{1}$, Rizwan Ahmad ${ }^{1}$, Yingmin Liu ${ }^{1}$, Ning Jin ${ }^{2}$, Juliet Varghese ${ }^{1}$, and Orlando Simonetti ${ }^{1}$

${ }^{1}$ The Ohio State University, Columbus, $\mathrm{OH}$, United States, ${ }^{2}$ Cardiovascular MR R\&D, Siemens Medical Solutions USA, Inc., Columbus, OH, United States

Respiratory motion in cardiovascular MRI presents a challenging problem with many solutions. Current approaches require breath-holds, neglect through-plane motion or significantly increase scan time. Our patient-specific prospective motion correction strategy addresses these issues and corrects for respiratory motion in real time. Numerous cardiac imaging applications stand to benefit from our approach, including perfusion imaging, parameter mapping, and late gadolinium enhancement. By modeling on a patient-specific basis, and prospectively correcting for respiratory motion, we expect to significantly improve the reliability and efficiency of CMR. For demonstration, the proposed strategy was applied to improve the accuracy of free-breathing T1 and T2 mapping. 
Dynamic Nitroxide-Enhanced MRI Detects Oxidative Stress in Myocardial Infarction

Sophia Xinyuan Cui ${ }^{1}$, Soham A. Shah ${ }^{1}$, Christopher D. Waters ${ }^{1}$, Lanlin Chen ${ }^{1}$, Rene J. Roy ${ }^{2}$, Brent A. French ${ }^{1,2}$, and Frederick H. Epstein ${ }^{1,2}$

Oxidative stress plays an important role in the pathogenesis of myocardial repair and remodeling after myocardial infarction (MI). Nitroxide free radicals have been used as redoxsensitive MRI contrasts agents in preclinical studies to assess tumor redox status. We tested the hypothesis that dynamic nitroxide-enhanced MRI can detect oxidative stress in MI. Imaging was performed in healthy control mice and in mice one day post-MI. The ratio of the MRI signal decay between the infarcted anterolateral wall and the noninfarcted septum was significantly higher in mice after MI, indicating that nitroxide-enhanced MRI can detect increased oxidative stress in infarcted myocardium.

Isotropic 3D Late Gadolinium Enhancement Imaging using 3D Patch-Based Super-Resolution

Aurelien Bustin ${ }^{1,2,3}$, Damien Voilliot ${ }^{3,4}$, Jacques Felblinger ${ }^{3,5,6}$, Laurent Bonnemains ${ }^{3,7}$, and Freddy Odille $e^{3,5,6}$

${ }^{1}$ School of Biomedical Engineering and Imaging Sciences, King's College London, London, United Kingdom, ${ }^{2}$ Department of Computer Science, Technical University of Munich, Munich, Germany, ${ }^{3}$ IADI, INSERM U947 and Universite de Lorraine, Nancy, France, ${ }^{4}$ Department of cardiology, University Hospital of Brabois, Nancy, France, ${ }^{5}$ CIC-IT 1433, INSERM, Nancy, France, ${ }^{6}$ Pole Imagerie, CHRU de Nancy, Nancy, France, ${ }^{7}$ Department of Cardiac Surgery, CHU Strasbourg, Strasbourg, France

Cardiac late gadolinium enhancement (LGE) imaging has become a reference clinical tool for assessing myocardial scar and viability. Despite superior signal-to-noise-ratio of 3D LGE techniques, current 3D breath-hold acquisitions are still limited by scan time and low-resolution, especially in the through-plane direction. Consequently, most clinical protocols include three anisotropic LGE acquisitions in different views to better visualize myocardial fibrosis in different orientations. Nevertheless, assessing myocardial viability in different views remains tedious and time-consuming. In this study, we sought to achieve isotropic 3D LGE by combining low-resolution anisotropic acquisitions using a 3D patch-based super-resolution reconstruction.

Cardiac relaxometry in childhood acute lymphoblastic leukemia survivors.

Delphine Perie-Curnier ${ }^{1}$, Mohamed Aissiou ${ }^{1}$, Louise Leleu ${ }^{1}$, Farida Cheriet ${ }^{2}$, Tarik Hafyane ${ }^{3}$, Maja Krajinovic ${ }^{4}$, Caroline Laverdiere ${ }^{4}$, Daniel Sinnett ${ }^{4}$, Gregor Andelfinger ${ }^{4}$, and Daniel Curnier ${ }^{5}$

${ }^{1}$ Mechanical Engineering, Polytechnique Montreal, Montreal, QC, Canada, ${ }^{2}$ Computing and software engineering, Polytechnique Montreal, Montreal, QC, Canada, ${ }^{3}$ Research Center, Montreal Heart Institute, Montreal, QC, Canada, ${ }^{4}$ Research Center, CHU Sainte-Justine, Montreal, QC, Canada, ${ }^{5}$ Kinesiology, University of Montreal, Montreal, QC, Canada

The aim of this study was to evaluate T1 pre- and post-gadolinium enhancement and T2 relaxation times sensitivity to detect myocardial changes induced by doxorubicin-based chemotherapy in childhood acute lymphoblastic leukemia survivors. Myocardial changes such as increased fibrosis index and injury due to associated changes in myocardial free water content were found between risk groups of cancer survivors, suggesting T2, post-gadolinium T1 and particularly the partition coefficient as early indices for myocardial tissue damages in the onset of doxorubicin-induced cardiotoxicity. These computing tools will be pivotal in patient follow-up to anticipate pathology evolution.

Age, gender and heart rate dependency of spin echo based diffusion tensor imaging measurements in healthy hearts

Alexander Gotschy ${ }^{1,2}$, Constantin von Deuster ${ }^{1}$, Robbert J.H. van Gorkum ${ }^{1}$, Ella Vintschger ${ }^{1}$, Robert Manka ${ }^{2,3}$, Christian T. Stoeck ${ }^{1}$, and Sebastian Kozerke $^{1}$

${ }^{1}$ Institute for Biomedical Engineering, University and ETH Zurich, Zurich, Switzerland, ${ }^{2}$ Department of Cardiology, University Hospital Zurich, Zurich, Switzerland, ${ }^{3}$ Institute of Diagnostic and Interventional Radiology, University Hospital Zurich, Zurich, Switzerland

Cardiac diffusion tensor imaging (cDTI) is a novel non-invasive method that allows assessing changes in myocardial microstructure in various cardiomyopathies. To identify pathologies, however, the distribution of CDTI parameters and their subject specific dependencies in normal hearts need to be known. Therefore, we investigated age, gender and heart rate dependencies of quantitative parameters derived from spin-echo based cDTI in healthy subjects. Our results display the variation of cDTI parameters in normal hearts and thereby allow gauging at which level of expected pathological changes sex and age matched reference values will be needed in future clinical practice.

Comparison of GRE, SSFP, and CINE CMR Acquisitions for Measuring Magnetization Transfer at 3T

Matthew Jacob Van Houten ${ }^{1}$, Yang Yang ${ }^{1}$, and Michael Salerno ${ }^{1}$

${ }^{1}$ Biomedical Engineering, University of Virginia, Charlottesville, VA, United States

We developed and compared multiple magnetization transfer (MT) pulse sequence strategies to characterize myocardial fibrosis without the need for gadolinium contrast at 3T. We demonstrated in an initial study of 4 healthy volunteers that a free-breathing, single-shot GRE is the most effective technique for producing high quality myocardial MT ratio maps. We will continue refining and investigating this sequence as a method for quantifying both focal and diffuse fibrosis in patients with heart failure. 
Fully Automatic SegmenTal analysis of myocardial Relaxometry (FASTR) - Initial results using T1 mapping

Venkat Ramanan ${ }^{1,2}$, Nitishkumar Bhatt ${ }^{1}$, LaBonny Biswas ${ }^{1,2}$, Idan Roifman ${ }^{2}$, Graham Wright $^{1,2,3}$, and Nilesh Ghugre ${ }^{1,2,3}$

${ }^{1}$ Physical Sciences Platform, Sunnybrook Research Institute, Toronto, ON, Canada, ${ }^{2}$ Schulich Heart Program, Sunnybrook Research Institute, Toronto, ON, Canada, ${ }^{3}$ Department of Medical Biophysics, University of Toronto, Toronto, ON, Canada

Relaxometric techniques, particularly T1 mapping, have gained clinical importance recently. T1 and ECV are usually calculated by manually drawing contours on the maps. This is laborious particularly for large volume studies. Here we present a fully automated framework (FASTR) for segmental analysis of T1 maps (both native and post-contrast) and partitioncoefficient values. Since CINE images are usually always acquired in the studies, we use CINE derived epi/endocardial contours and make further adjustments on T1 maps. This results in more accurate and robust segmentation of the myocardial wall, which works even in the presence of edema, infarct and minor artifacts.

High-Resolution T1 Mapping using Parameter-Free Low Rank Denoising

Sebastian Weingärtner ${ }^{1,2,3}$, Steen Moeller ${ }^{2}$, Chetan Shenoy ${ }^{4}$, and Mehmet Akcakaya ${ }^{1,2}$

${ }^{1}$ Electrical and Computer Engineering, University of Minnesota, Minneapolis, MN, United States, ${ }^{2}$ Center for Magnetic Resonance Research, University of Minnesota, Minneapolis, MN, United States, ${ }^{3}$ Computer Assisted Clincial Medicine, Heidelberg University, Mannheim, MA, Germany, ${ }^{4}$ Department of Cardiology, University of Minnesota, Minneapolis, MN, United States

Myocardial T1 mapping has become increasingly established for tissue characterization in numerous cardiomyopathies. However, the commonly used end-diastolic single-shot imaging imposes restrictions on the spatio-temporal resolution. In this work, we explored increased parallel imaging accelerations and higher resolutions, in conjunction with an image denoising technique that exploits inter-dependencies between the multiple images using random matrix theory. Following parallel imaging reconstruction, common noise characteristics across the images are extracted from the singular value decomposition of a Gaussian random matrix and denoised using locally low-rank regularization. Application of this technique to SAPPHIRE T1 mapping shows no corruption of the T1 time and enables parallel imaging acceleration up to 4 with an in-plane resolution of $1.1 \times 1.1 \mathrm{~mm}^{2}$ at clinical image quality.

Cardiac MR multi-modal imaging: role in diagnosis and differential diagnosis of fulminant myocarditis in children

Cuiyan Wang ${ }^{1}$, Haipeng Wang ${ }^{2}$, Guangbin Wang ${ }^{1}$, Bin Zhao ${ }^{1}$, and Bin Zhao

2905 'Shandong Medical Imaging Research institute, Jinan, China, ${ }^{2}$ Shandong Provincial Hospital, Jinan, China

Clinical characteristics, cardiac morphology, function parameters and myocardial tissue characterization on MRI of three groups (FM, AM and CM) were retrospectively compared to find that higher myocardial thickness and T2 ratio were seen in FM than in AM and CM with statistical significance; the LVEF and incidence of LGE in FM were higher than that in CM with statistical significance. So that CMR has values in the diagnosis and differential diagnosis of FM.

Myocardial T1 Measurement and Relationship with Myocardial T2 and Black Blood T2* at 3.0T MRI for Thalassemia Major Patients

Aamish Zahir Kazi ${ }^{1}$ and Bhavin Govindji Jankharia ${ }^{2}$

${ }^{1}$ Radiodiagnosis, Picture - This by Jankharia Imaging, Mumbai, India, ${ }^{2}$ Picture - This by Jankharia Imaging, Mumbai, India

Black blood T2* mapping on $1.5 \mathrm{~T}$ is currently the gold standard for iron load assessment in patients with iron overload and plays a crucial role in patient management. Inaccuracies in $\mathrm{T}^{*}$ quantification at $3 \mathrm{~T}$ due to greater artifact levels and higher B0 and B1 inhomogeneities have resulted in a lack of multi-center and multi-vendor validation studies to standardize T2* for iron overload assessment on $3 \mathrm{~T}$. In-vivo and In-vitro studies on $1.5 \mathrm{~T}$ have suggested that $\mathrm{T} 1$ and $\mathrm{T} 2$ could be potential alternatives to T2* In this study, we have demonstrated linear relationships for T1 Vs T2*, T2 Vs T2* and T1 Vs T2 at 3T suggesting that T1 or T2 could be potential alternatives to T2*.

Iron-Ceroid Complex From Apoptotic Siderophage-Derived Foam Cells Promotes Perpetual Macrophage Ingress and Localized Edema Formation in Hemorrhagic Myocardial Infarctions: Histopathology and Immunohistochemistry Findings to MRI Correlates

Ivan Cokic ${ }^{1}$, Guan Wang ${ }^{1}$, Kolja Wawrowsky ${ }^{1}$, Hsin-Jung Yang ${ }^{1}$, Richard LQ Tang ${ }^{1}$, and Rohan Dharmakumar ${ }^{1}$

The capacity of macrophages (MФ) to oxidize LDL, produce ceroid (CR), and transform into foam cells $(F C)$ is enhanced following erythrophagocytosis. During the process of FC formation, part of hemoglobin-derived iron forms a complex with CR. CR is cytotoxic; and over time, it can lead to FC apoptosis. Release of CR from apoptotic FC into the surrounding tissue may cause dysfunction and apoptosis of newly invading MФ. Given that lipid and iron deposits within hemorrhagic $\mathrm{MI}$ (hMI) typically colocalize in the infarct periphery, we hypothesized that CR from apoptotic FC promotes perpetual MФ ingress and localized edema formation in hMI. 


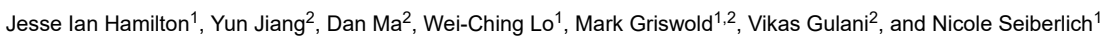

Because different CMRF pulse sequences may have different sensitivities to confounding factors, the generation of accurate and precise T1 and T2 maps may require detailed modeling of spin dynamics. This work studies the importance of modeling slice profile, B1, and relaxation during adiabatic inversion and T2 preparation pulses in cMRF. The ISMRM/NIST system phantom was scanned using cMRF sequences with different patterns of flip angles, TRs, and preparation pulses. Modeling these additional effects leads to higher correlation (using linear regression and concordance correlation coefficients) between NIST and cMRF measurements and better consistency between different cMRF sequences.

Assessing myocardial infarct in lymphatic insufficient mice by rotating frame relaxation times

Elias Yla-Herttuala ${ }^{1}$, Taina Vuorio ${ }^{1}$, Johanna Laakkonen ${ }^{1}$, Svetlana Laidinen ${ }^{1}$, Seppo Yla-Herttuala ${ }^{1}$, and Timo Liimatainen ${ }^{1,2}$

Relaxation times $T_{2}, T_{1 \rho}$ and RAFFn were applied to study alterations in myocardial infarct (MI) in control and in mice with insufficient lymphatic system (VEGFR3). The findings are supported by cardiac functional parameters and histology. We found significant difference between VEGFR3 and control in $\mathrm{T}_{\text {RAFF4 }}(\mathrm{p}<0.05) 8$ days after MI and between pre-MI and post-MI time points in $T_{2}(p<0.01)$. Relaxation times increased significantly $(p<0.05)$ after $M I$ in all measurements. We conclude that $T_{R A F F}$ gain information of alterations of fibrosis in lymphatic insufficiency after MI.

Simultaneous Multi-Slice Gradient Echo Spin Echo EPI (SMS-GESE-EPI) enables simultaneous cardiac T2 and T2* imaging and mapping across six slices within a single heartbeat

Maaike van den Boomen ${ }^{1,2}$, Mary Kate Manhard ${ }^{2}$, Christopher Nguyen ${ }^{3,4}$, SoHyun Han² ${ }^{2}$ Kyre E. Emblem ${ }^{5}$, Riemer H.J.A. Slart ${ }^{6,7}$, Ciprian Catana ${ }^{2}$, Niek H.J. Prakken ${ }^{1}$, Bruce Rosen ${ }^{2}$, Ronald J.H. Borra ${ }^{6,8}$, and Kawin Setsompop ${ }^{2,9,10}$

${ }^{1}$ Department of Radiology, University of Groningen, University Medical Center Groningen, Groningen, Netherlands, ${ }^{2}$ A.A. Martinos Center for Biomedical Imaging, Massachusetts General Hospital, Charlestown, MA, United States, ${ }^{3}$ Cardiovascular Research Center, Massachusetts General Hospital and Harvard Medical School, Boston, MA, United States,

${ }^{4}$ Biomedical Imaging Research Institute, Cedars-Sinai Medical Center, Los Angeles, CA, United States, ${ }^{5}$ Oslo University Hospital, Oslo, Norway, ${ }^{6}$ Department of Nuclear Medicine and Molecular Imaging, University of Groningen, University Medical Center Groningen, Groningen, Netherlands, ${ }^{7}$ Department of Photonic Imaging, University of Twente, Enschede, Netherlands, ${ }^{8}$ Medical Imaging Centre of Southwest Finland, Turku University Hospital, Turku, Finland, ${ }^{9}$ Department of Radiology, Harvard Medical School, Boston, MA, United States, ${ }^{10}$ Division of Health Sciences and Technology, Harvard-MIT, Cambridge, MA, United States

Cardiac $\mathrm{T}_{2}{ }^{*}$ and $\mathrm{T}_{2}$-based techniques suffer from variabilities introduced by acquisition over multiple heartbeats and breath holds. We demonstrate the use of a dual-echo SMS-GESEEPI sequence that can simultaneously provide $\mathrm{T}_{2}{ }^{*}$ - and $\mathrm{T}_{2}$-weighted images from six slice locations within a single heartbeat and breath-hold. Introduction of 5 -echos also enabled dynamic $T_{2}{ }^{*}$ - and $T_{2}$-mapping per heartbeat within a breath-hold. These dynamically acquired $T_{2}{ }^{*}-$ and $T_{2}$-maps remained stable over ten heartbeats. Several applications might benefit from these modified GESE sequences, such as BOLD measurements and vessel architecture imaging of the myocardium.

Multi-Parametric Cardiac MRI is Needed for Accurate Staging of Reperfused Hemorrhagic Myocardial Infarctions

Guan Wang ${ }^{1,2}$, Hsin-Jung Yang ${ }^{1}$, Ivan Cokic ${ }^{1}$, Avinash Kali ${ }^{1}$, Richard Tang ${ }^{1}$, Joseph Francis ${ }^{3}$, Songbai Li ${ }^{2}$, and Rohan Dharmakumar ${ }^{1,4}$

${ }^{1}$ Pacific Theatres Suite 400. 8700 Bevely Boulevard, Cedars-Sinai Medical Center BIRI, Los angeles, CA, United States, ${ }^{2}$ Dept of Radiology, The First Affiliated Hospital of China Medical University, Shenyang, China, ${ }^{3}$ Dept of Veterinary Medicine, Louisiana State University, Baton Rouge, LA, Baton Rouge, LA, United States, ${ }^{4}$ David Geffen School of Medicine, University of California, Los angeles, CA, United States

Cardiac MRI (CMR) based staging of myocardial infarction (MI) with or without contrast agents relies on the resolution of edema in the chronic phase, which is typically determined on the basis of T2-based MRI. However, whether T2 CMR is sufficient for staging all MI types has not been studied. We investigated this using animal models with and without hemorrhagic Mls. Our results show that non-hemorrhagic Mls can be staged based on T2 changes in the MI territory. However, the incomplete resolution of T2 elevations in the peripheral layers of hemorrhagic Ml territories can confound staging of hemorrhagic Mls.

Fast and precise myocardial T1 mapping using a segmented golden angle radial MOLLI sequence with bSSFP readout

Jiaxin Shao ${ }^{1}$, Ziwu Zhou ${ }^{1}$, Fei Han ${ }^{1}$, and Peng Hu${ }^{2}$

${ }^{1}$ Radiology, UCLA, Los Angeles, CA, United States, ${ }^{2}$ UCLA, Los Angeles, CA, United States 
Among the various myocardial T1 mapping sequences developed, the radial variants of the MOLLI acquisitions (raMOLLIs) are promising. As raMOLLIs can decrease the acquisition time down to a few heartbeats while keeping good T1 estimation precision due to a large number of images reconstructed along the T1 relaxation recovery curve. The previous raMOLLIs use FLASH readout due to the sensitivity of bSSFP readout to image artifacts. As bSSFP readout has high SNR, a variant of radial MOLLI with bSSFP readout was developed to ensure accurate and precise myocardial T1 mapping by using segmented golden angle radial acquisition.

Investigating extra-cellular volume fraction in patients with Becker Muscular Dystrophy and Limb Girdle Muscular Dystrophy 21.

Alex Murphy ${ }^{1}$, David M Higgins ${ }^{2}$, Volker Straub ${ }^{1}$, and Kieren Grant Hollingsworth ${ }^{3}$

${ }^{1}$ Institute of Genetic Medicine, Newcastle University, Newcastle upon Tyne, United Kingdom, ${ }^{2}$ Philips Healthcare, Guildford, United Kingdom, ${ }^{3}$ Newcastle Magnetic Resonance Centre, Newcastle University, Newcastle upon Tyne, United Kingdom

The development of gradual and diffuse myocardial fibrosis is a key pathology in muscular dystrophy and it is possible that extracellular volume (ECV) measurement may be a useful biomarker. Thirteen participants with muscular dystrophy and ten healthy controls were recruited to undergo cardiac MRI, including cardiac tagging, LGE and ECV measurement to determine whether significant global or local differences in ECV could be detected, and their relationship to cardiac dysfunction as indicated by cine imaging and cardiac tagging. Global ECV was not different but there were significant segmental differences between muscular dystrophy and controls.

Accelerated 3D saturation-recovery based myocardial T1 mapping using fewer saturation time points and denoising

Giovanna Nordio ${ }^{1}$, Aurelien Bustin ${ }^{1}$, Torben Schneider ${ }^{2}$, Markus Henningsson ${ }^{1}$, Claudia Prieto ${ }^{1}$, and René Botnar

${ }^{1}$ King's College London, London, United Kingdom, ${ }^{2}$ Philips Healthcare, London, United Kingdom

In this study we propose to accelerate the 3D saturation-recovery (3D SASHA) T1-mapping technique by using a reduced number of saturation time points while maintaining accuracy and precision using a 3D denoising method. No statistical difference was found in terms of accuracy and precision (respectively $p=0.14$ and $p=0.99$ ) between the $T 1$-maps reconstructed after denoising using different number of T1-weighted images (between three and nine). After application of 3D denoising, the precision was independent of the number of T1-weighted images used for the fitting, which may permit to considerably accelerate the 3D SASHA acquisition.

3D SASHA myocardial T1 mapping with high accuracy and improved precision

Giovanna Nordio ${ }^{1}$, Aurelien Bustin ${ }^{1}$, Markus Henningsson ${ }^{1}$, Freddy Odille $^{2,3}$, Claudia Prieto $^{1}$, and René Botnar ${ }^{1}$

${ }^{1}$ School of Biomedical Engineering and Imaging Sciences, King's College London, London, United Kingdom, ${ }^{2}$ Imagerie Adaptative Diagnostique et Intervenionelle, INSERM U947 et Université de Lorraine, Nancy, France, ${ }^{3}$ CIC-IT 1433, INSERM, Université de Lorraine, Nancy, France

In this study we propose to further improve the precision of free-breathing 3D saturation-recovery based T1 mapping (3D SASHA), while keeping its high accuracy, by employing a nove 3D denoising method which exploits spatio-temporal correlations in the T1-weighted images. The proposed approach has been tested on ten healthy subjects and four patients with cardiovascular disease. For all subjects, no statistical difference was observed between the precision measured on 3D denoised SASHA and 2D MOLLI T1 maps ( $p=0.62$ ), while preserving the T1 accuracy. There was an improvement in the precision after denoising on the 3D SASHA T1 maps acquired in healthy subjects and patients.

Single breath-hold MR T1-mapping in the heart: comparison of hybrid MOLLI and MOLLI53

Yu Chun-Yang ${ }^{1}$, Huang Teng-Yi ${ }^{2}$, and Chung Hsiao-Wen

${ }^{1}$ National Taiwan University, Taipei, Taiwan, ${ }^{2}$ National Taiwan University of Science and Technology, Taipei, Taiwan

A hybrid MOLLI method that integrated saturation recovery with the classical inversion recovery sequence was proposed for quantitative T1 mapping in the myocardium within one single breath-hold. By replacing the second inversion pulse of the original MOLLI53 technique with a saturation pulse, the long recovery time could be alleviated in hybrid MOLLI, thereby allowing more images to be sampled from the T1 relaxation curve. Phantom and healthy subject experiments conducted in comparison with the classical MOLLI53 demonstrated that the proposed method was able to provide comparable image quality as well as precise T1 quantification in the myocardium.

Pan ki Kim ${ }^{1}$, Chul Hwan Park², Yoo Jin Hong ${ }^{1}$, and Byoung Wook Choi ${ }^{1}$

${ }^{1}$ Department of Radiology and Research Institute of Radiological Science, Severance Hospital, Yonsei University Medical Center, Seoul, Republic of Korea, ${ }^{2}$ Department of Radiology and Research Institute of Radiological Science, Gangnam Severance Hospital, Yonsei University Medical Center, Seoul, Republic of Korea 
Chemical Exchange Saturation Transfer (CEST) has been attracting attention as a molecular imaging method to investigate myocardial muscle energetics according to creatine changes. In this study, we proposed a robust CEST imaging technique from cardiac and respiratory motion using golden angle radial readout to achieve CEST imaging at the heart of the rat. We investigated the feasibility of the proposed method for the creatine phantom and a normal rat.

Simulation-Aided Contrast Agent Washout Analysis in Patients with Acute Myocarditis

Leili Riazy ${ }^{1,2}$, Tobias Schaeffter ${ }^{3}$, Marc Olbrich ${ }^{3,4}$, Johannes Schueler ${ }^{5,6}$, Florian von Knobelsdorff-Brenkenhoff ${ }^{5,7}$, Thoralf Niendorf ${ }^{1,2}$, and Jeanette Schulz-Menger ${ }^{5,6}$

${ }^{1}$ Berlin Ultrahigh Field Facility, Max-Delbrueck-Centrum Berlin-Buch, Berlin, Germany, ${ }^{2}$ DZHK (German Centre for Cardiovascular Research), Berlin, Germany, ${ }^{3}$ Medical Physics and Metrological Information Technology, Physikalisch-Technische Bundesanstalt, Berlin, Germany, ${ }^{4}$ Technical University Berlin, Berlin, Germany, ${ }^{5}$ Working Group on Cardiovascular Magnetic Resonance, Experimental and Clinical Research Center (ECRC), Berlin, Germany, ${ }^{6}$ Department of Cardiology and Nephrology, HELIOS Klinikum Berlin Buch, Berlin, Germany, ${ }^{7}$ Clinic Agatharied, Dept. of Cardiology, University of Munich, Hausham, Germany

Contrast-enhancement techniques allow the visualization of small myocardial injuries in acute myocarditis, which cannot be detected by any other noninvasive technique. Late Gadolinium Enhancement (LGE) has been shown predictive for the development of heart failure. Early Gadolinium Enhancement (EGE) was identified as parameter for detection of disease activity. We analyze the contrast agent washout during 10 minutes after tracer administration. Our aim is to characterize parameter values of patients with myocarditis in a 3D spatially distributed contrast agent flow model.

Evaluation of MOLLI fitting algorithms robustness to partial volume effects due to fat

Andreia S Gaspar ${ }^{1}$ and Rita G Nunes

$2919{ }^{1}$ Institute for Systems and Robotics/Department of Bioengineering, Instituto Superior Técnico, Universidade de Lisboa, Lisbon, Portugal

The MOLLI sequence for myocardium T1 quantification is widely applied in the clinical setting. The standard 3-parameter fitting algorithm allows a high precision in the T1 estimates but it comes at the cost of a low accuracy. The accuracy can be improved using the instantaneous signal loss (InSiL) approximation method for signal fitting. In this work we evaluated the robustness of the InSiL algorithm when fat also contributes to the signal. The results show that InSiL enables to increase the accuracy of the MOLLI sequence even in the presence of partial volume effects due to fat.

Myocardial T1 mapping with second-based MOLLI scheme for reduced heartrate variation: A phantom validation study at $1.5 \mathrm{~T}$ and $3.0 \mathrm{~T}$

Shuo Zhang ${ }^{1,2}$, Jennifer Ann Bryant ${ }^{2}$, Evelyn Shi Shi Quah ${ }^{3}$, Calvin Chin ${ }^{2,4}$, Derek Hausenloy ${ }^{2,4}$, Jouke Smink ${ }^{5}$, Ru San Tan², ${ }^{2,}$ Yeong Shyan Lee ${ }^{3}$, and Stuart A Cook ${ }^{2,4}$

${ }^{1}$ Philips, Singapore, Singapore, ${ }^{2}$ National Heart Centre Singapore, Singapore, Singapore, ${ }^{3}$ Tan Tock Seng Hospital, Singapore, Singapore, ${ }^{4}$ Duke-NUS Medical School, Singapore, Singapore, ${ }^{5}$ Philips, Best, Netherlands

Current standard myocardial T1 mapping is based on the modified Look-Locker inversion recovery (MOLLI) technique and single-shot readout per image acquisition. A second-based scheme has recently been proposed to mitigate the dependence of imaging times on heartrate and also to increase robustness in T1 estimation. Here we report a phantom-based study with ECG simulation comparing it with the original beat-based MOLLI acquisition scheme at both 1.5 and 3.0T. We demonstrate the advantage of this approach with reduced heartrate dependence and variation for an improved T1 quantification reliability.

Understanding the material behaviour of ex-vivo porcine hearts using MR-Elastography and Rheology

Myrianthi Hadjicharalambous ${ }^{1}$, Adela Capilnasiu ${ }^{1}$, Ayse Sila Dokumaci ${ }^{1}$, Daniel Fovargue ${ }^{1}$, Gerhard Sommer $^{2}$, Gerhard Holzapfel ${ }^{2}$, Ralph Sinkus ${ }^{1}$, and David Nordsletten

${ }^{1}$ Biomedical Engineering, King's College London, London, United Kingdom, ${ }^{2}$ Institute of Biomechanics, Graz University of Technology, Graz, Austria

Myocardial stiffness has been shown to correlate with heart disease, nevertheless, reliable stiffness estimates are hindered by the remarkably complex behaviour and function of the heart muscle. In this work, we use MR-Elastography and rheological experiments to obtain a better understanding of the myocardial material behaviour. MR-Elastography and cyclic shear tests are performed on ex-vivo porcine hearts, under varying frequencies. Our results demonstrate important tissue properties and highlight the viscoelastic properties of the myocardium which are often neglected. Improving our understanding of the interlinked material properties of the heart is a critical step towards the accurate prediction of myocardial stiffness. 
${ }^{1}$ Yonsei University College of Medicine, Seoul, Republic of Korea, ${ }^{2}$ Biomedical Imaging Research Institute, Cedars-Sinai Medical Center, Los Angeles, CA, United States,

${ }^{3}$ Cardiovascular Research Center, Massachusetts General Hospital and Harvard Medical School, Charlestown, MA, United States, ${ }^{4}$ Department of Bioengineering, University of California, Los Angeles, CA, United States

In hypertrophic cardiomyopathy, myocardial fiber disarray, and interstitial fibrosis interfere with regional systolic myocardial function despite clinically hyperdynamic systolic function. We quantitatively assessed the difference in myocardial fiber orientation between diseased and normal cardiac segments using diffusion tensor imaging. Further, these fiber microstructure were compared to the regional global longitudinal strain to evaluate whether the structure-function relationship changes according to the disease involvement.

Single-shot Radial Fast Spin-Echo T2 Mapping Pulse Sequence

KyungPyo Hong ${ }^{1}$, Hassan Haji-valizadeh ${ }^{2}$, Nivedita Kikkeri Naresh ${ }^{1}$, and Daniel Kim ${ }^{1}$

${ }^{1}$ Radiology, Northwestern University, Chicago, IL, United States, ${ }^{2}$ Northwestern University, Chicago, IL, United States

Cardiac T2 mapping is a proven imaging test for myocardial tissue characterization. Standard cardiac T2 mapping involved in cardiac MRI protocol requires a breath-hold duration of 10 sec and usually samples three short-axis planes of the heart. This limited spatial coverage may miss the focal lesion. In this study, we developed a single-shot cardiac T2 mapping pulse sequence and reconstructed multiple T2-weighted images from a single T2-decay data, using k-Space weighted image contrast and compressed sensing technique. We tested its performance in patients with suspected infiltrative cardiomyopathy, and it yielded $7.9 \%$ difference in myocardial T2 values compared to standard T2 mapping

Traditional Poster

\section{Velocity \& Flow}

A combined 4D Flow MRI-modelling approach for assessing the subject-specific effects of dobutamine on left ventricular function

Belen Casas ${ }^{1,2}$, Federica Viola ${ }^{1}$, Gunnar Cedersund ${ }^{3}$, Ann F Bolger $^{4}$, Matts Karlsson ${ }^{2,5}$, Carl-Johan Carlhäll ${ }^{1,2}$, and Tino Ebbers ${ }^{1,2}$

${ }^{1}$ Department of Medical and Health Sciences, Linköping University, Linköping, Sweden, ${ }^{2}$ Center for Medical Image Science and Visualization (CMIV), Linköping University, Linköping, Sweden, ${ }^{3}$ Department of Biomedical Engineering, Linköping University, Linköping, Sweden, ${ }^{4}$ Department of Medicine, University of California San Francisco, San Francisco, CA, United States, ${ }^{5}$ Department of Management and Engineering, Linköping University, Linköping, Sweden

This study applies a previously developed imaging-modelling approach to investigate the subject-specific effects of dobutamine on left ventricular contraction and relaxation patterns in healthy subjects. We created personalized models for nine subjects at rest and under dobutamine stress. The personalized parameter values were in agreement with the effects of inotropy and lusitropy reported in previous studies, and demostrated the anticipated variability in individual responses to dobutamine. With further validation, the given approach has the potential to generate advanced metrics of cardiovascular physiology and pathophysiology that could extend beyond conventional techniques for both diagnosis and optimization of a personalized medical regimen.

Fast Self-Navigated Wall Shear Stress Measurements in the Murine Aortic Arch Using Radial 4D-PC-MRI at 17.6T

Kristina Andelovic ${ }^{1,2}$, Patrick Winter ${ }^{2}$, Thomas Kampf ${ }^{2,3}$, Julius Heidenreich ${ }^{3}$, Anton $\mathrm{Xu}^{2}$, Peter M. Jakob ${ }^{2}$, Wolfgang R. Bauer ${ }^{1}$, and Volker Herold ${ }^{2}$

${ }^{1}$ Medicine I, Cardiology, University Hospital Wuerzburg, Wuerzburg, Germany, ${ }^{2}$ Experimental Physics V, University of Wuerzburg, Wuerzburg, Germany, ${ }^{3}$ Interventional and Diagnostic Radiology, University Hospital Wuerzburg, Wuerzburg, Germany

4D phase contrast (PC)-MRI is a non-invasive tool for the assessment of cardiovascular hemodynamics or the Wall Shear Stress (WSS) to study atherosclerotic risks in vivo. Major limitations of conventional triggered methods are the long measurement times needed for high-resolution data sets and the requirement of stable ECG triggering, which is diffcult at high magnetic field strengths. In this work, an ECG-free, retrospectively synchronized method is presented that enables fast high-resolution measurements of 4D flow and wall shear stress in the murine aortic arch.

The impact of left ventricular ejection fraction on cardiovascular blood flow

Merih Cibis ${ }^{1}$, Carl-Johan Carlhäll ${ }^{1}$, Jan Engvall ${ }^{1}$, and Tino Ebbers ${ }^{1}$ 
The impact of left ventricular (LV) ejection fraction (LVEF) on cardiovascular blood flow is not completely understood. We used a method, called "Atlas heart generation", to investigate cardiovascular flow of patients with ischemic heart disease $(n=62)$. The patients underwent 4D-Flow MRI and were stratified according to LVEF. We found that the lower LVEF group had lower velocities throughout the aorta, in a portion of LV and left atrium, at peak-systole. At early-diastole, differences were observed in the aortic arch, and in the apical-septal segments of LV. The suggested method can detect changes in cardiovascular flow and add to pathophysiological understanding.

4D flow MRI Investigation of link between Aortic Stiffness and Embolic Pathway of Aortic Flow Reversal in Patients with Cryptogenic Stroke

Kelly Jarvis ${ }^{1}$, Alireza Vali ${ }^{1}$, Shyam Prabhakaran ${ }^{2}$, Jeremy D. Collins ${ }^{1}$, and Michael Markl ${ }^{1}$

${ }^{1}$ Radiology, Northwestern University, Chicago, IL, United States, ${ }^{2}$ Neurology, Northwestern University, Chicago, IL, United States

Reverse aortic flow causing plaque embolization from the descending aorta (DAo) has been identified as a new source of stroke but the underlying cause of flow reversal is unclear. There is evidence that aortic stiffness can cause flow reversal but no study has investigated this relationship in detail. This study used high-temporal resolution 4D flow MRI to evaluate aortic stiffness and regional aortic flow reversal in patients with cryptogenic stroke. Elevated PWV was associated with reverse flow in areas of the aortic arch and DAo providing evidence for aortic stiffness and flow reversal as a potential embolic mechanism.

4D Flow MRI-Based Aortic Pulse Wave Velocity: Systematic Analysis of the Impact of Temporal Resolution on Estimation in Patients with Aortic Atherosclerosis and Age-matched Controls

Kelly Jarvis ${ }^{1}$, Alireza Vali ${ }^{1}$, Shyam Prabhakaran ${ }^{2}$, Jeremy D. Collins ${ }^{1}$, and Michael Markl ${ }^{1}$

${ }^{1}$ Radiology, Northwestern University, Chicago, IL, United States, ${ }^{2}$ Neurology, Northwestern University, Chicago, IL, United States

Elevated pulse wave velocity (PWV) is a measure of aortic stiffness and an indicator of cardiovascular disease. Pulse waves propagate quickly along the aorta and high-temporal resolution measurement of velocity data with full spatial coverage is needed to improve the precision of PWV estimation. This study used high-temporal resolution 4D flow MRI to assess PWV and investigate the impact of temporal resolution on PWV estimation methods (i.e. time-to-foot and cross-correlation) in patients with known atherosclerosis. The findings suggest that using cross-correlation to estimate the time-delay between flow waveforms is optimal, particularly at inferior temporal resolutions.

4D Flow Imaging with Reduced Field-of-Excitation

Clarissa Wink ${ }^{1}$, Giulio Ferrazzi ${ }^{1}$, Jean Pierre Bassenge ${ }^{1}$, Sebastian Flassbeck $^{2}$, Simon Schmidt ${ }^{2}$, Tobias Schaeffter ${ }^{1}$, and Sebastian Schmitter ${ }^{1}$

${ }^{1}$ Physikalisch-Technische Bundesanstalt (PTB), Braunschweig and Berlin, Germany, ${ }^{2}$ Medical Physics in Radiology, German Cancer Research Center (DKFZ), Heidelberg, Germany

4D flow MRI suffers from long scan times which limit maximum spatial resolutions. A promising approach is to restrict the field-of-excitation (FOX) to the region of interest and therefore reduce the field-of-view (FOV) not only in partition/slab direction, but also in phase encoding direction. In this work, we replace the slab-selective excitation of a 4D flow sequence by a $2 \mathrm{D}$ spatially-selective excitation with reduced FOX to enable reduced FOV imaging. We investigate the impact of the excitation on velocity encoding and demonstrate correct velocity quantification with $\$ \$ \$ 101 \% \$ \$$ reduced scan times in phantoms and in-vivo.

Estimating highly-accurate velocity maps from FVE MRI data using a PDE-constrained optimization

Vinicius Carvalho Rispoli ${ }^{1,2}$, Joao Luiz Azevedo Carvalho ${ }^{3}$, Cristiano Jacques Miosso ${ }^{2}$, Fabiano Araujo Soares ${ }^{2}$, Giordanno Bruno Borges ${ }^{1}$, and Ivan Rosa Siqueira ${ }^{4}$

${ }^{1}$ Department of Mathematics, University of Brasilia, Brasilia, Brazil, ${ }^{2}$ Engineering Faculty at Gama, University of Brasilia, Brasilia, Brazil, ${ }^{3}$ Department of Electrical Engineering, University of Brasilia, Brasilia, Brazil, ${ }^{4}$ Department of Chemical and Biomolecular Engineering, Rice University, Houston, TX, United States

Fourier velocity encoding (FVE) is a technique capable of delivering clinically treatable data at short acquisition times. FVE resolves the velocity distribution in each voxel of the image with high signal-to-noise ratio. This makes it suitable for the calculation of relevant biomarkers (e.g. wall shear rate and oscillatory shear index). However, it does not provide the blood flow velocity field directly. Techniques to estimate the actual blood flow from FVE velocity distributions have been previously presented. In this work, we present a novel method for velocity map estimation based on a PDE-constrained optimization that provides better results than previous methods.

Flow-encoding Arterial Structure Acquired using Silent-MRA: A Preliminary Study

Chia-Wei Li ${ }^{1}$, Chien-Yuan Eddy Lin ${ }^{2}$, Charng-Chyi Shieh ${ }^{2}$, Chen-Syuanms Lin ${ }^{1}$, Chia-Yuen Chen ${ }^{1}$, and Wing P. Chan ${ }^{1,3}$

${ }^{1}$ Department of Radiology, Wan Fang Hospital, Taipei Medical University, Taipei, Taiwan, ${ }^{2}$ GE Healthcare, Taipei, Taiwan, ${ }^{3}$ Department of Radiology, School of Medicine, College of Medicine, Taipei Medical University, Taipei, Taiwan 
Silent magnetic resonance angiography (Silent-MRA), which combines the Silent Scan algorithm to achieve a zero echo time with an arterial spin-labelling method, has recently been introduced as a novel MRA technique. Many studies of Silent-MRA focused on evaluating vascular structure; however, reports of further investigations into the flow information generated by Silent-MRA cannot be found. To this end, we compared the flow-encoding Silent-MRA signal with phase contrast flow imaging and found a linear correlation between the two. This preliminary study demonstrates the potential power of using flow-encoding Silent-MRA in assessing complicated vascular disease.

Wall shear stress analysis after anatomically pre-shaped $90^{\circ}$ - and straight ascending aortic grafts: A comparison between prostheses and age-matched volunteers using 4D Flow MRI

Malte Maria Sieren ${ }^{1}$, Jennifer Schlüter ${ }^{1}$, Thekla Helene Oechtering ${ }^{1}$, Michael Scharfschwerdt ${ }^{2}$, Christian Auer $^{2}$, Markus Hüllebrand ${ }^{3}$, Hans-Hinrich Sievers ${ }^{2}$, Jörg Barkhausen ${ }^{1}$, and Alex Frydrychowicz ${ }^{1}$

${ }^{1}$ Department of Radiology and Nuclear Medicine, University Hospital Schleswig-Holstein, Campus Lübeck, Lübeck, Germany, ${ }^{2}$ Department of Cardiac and Cardiothoracic Vascular Surgery, University Hospital Schleswig-Holstein, Campus Lübeck, Lübeck, Germany, ${ }^{3}$ Fraunhofer MEVIS, Bremen, Germany

Patients with aortic prostheses following aneurysm/dissection repair demonstrate an increased number of secondary aortic flow patterns. These may result in elevated forces acting on the vessel wall and thus preterm degenerative changes. Anatomically pre-shaped $90^{\circ}$-prostheses promise more physiological flow patterns and wall shear stress (WSS). The aim of this study was to compare WSS of patients with straight prostheses $(n=8), 90^{\circ}$-prostheses $(n=9)$ and healthy volunteers $(n=12)$ based on $4 D$ Flow MRI. Results revealed a tendency towards decreased WSS in regions distal to the $90^{\circ}$-prostheses, whereas in comparison to healthy volunteers, WSS values in patients with both prostheses were significantly increased.

Measuring cardiac output and leg blood flow with phase-contrast MRI during supine cycling exercise.

Thijs Schoots ${ }^{1}$, Berit Wassenaar ${ }^{1}$, Anita Kuiper ${ }^{2}$, Hareld Kemps ${ }^{1}$, Jeroen Jeneson ${ }^{2}$, and Remco Renken ${ }^{2}$

${ }^{1}$ Flow, Maxima Medical Center, Eindhoven, Netherlands, ${ }^{2}$ Medical University Center Groningen, Groningen, Netherlands

Patients with chronic heart failure suffer from diminished leg blood flow (LBF). Question remains to what extent the distribution or the cardiac output (CO) is responsible. This study investigates whether $\mathrm{CO}$ and LBF could be measured reliably using phase contrast MRI during supine exercise. 10 healthy subjects performed a supine exercise test in the MRI at two days at different exercise intensities. Comparison between both days showed promising reproducibility of measuring CO and LBF during supine cycling in the MRI although LBF measurements proved more challenging

A Comparison of PC-MRI Eddy Current Correction Methods in the Presence of Noise

Avinash Pramod Chinchali ${ }^{1}$, Michael Loecher ${ }^{2}$, and Daniel B Ennis ${ }^{1,2}$

${ }^{1}$ Bioengineering, UCLA, Los Angeles, CA, United States, ${ }^{2}$ Radiological Sciences, UCLA, Los Angeles, CA, United States

Eddy current induced phase errors lead to PC-MRI velocity errors that must be corrected. Static tissue fitting is commonly implemented to correct these phase errors. The aim of this work was to quantitatively compare corrections made using local and global static tissue fitting techniques over a wide range of SNR. Average correction differences between local and global strategies in static tissue were on the order of $0.9 \mathrm{~cm} / \mathrm{s}$ for low SNR protocols and $0.1 \mathrm{~cm} / \mathrm{s}$ for high SNR protocols. Local correction introduced phase error in $\sim 5 \%$ ROIs (always when SNR<30). Local correction is therefore suitable for higher SNR PC-MRI acquisitions.

Local Pulse Wave Velocity from 4D-Flow MR applied in Familial Hypercholesterolemia patients.

Joaquin Mura ${ }^{1}$, Julio Sotelo ${ }^{1,2}$, Animesh Tandon ${ }^{3,4}$, Tarique Hussain ${ }^{3,4},{\text { Andrew } \operatorname{Tran}^{3,4} \text {, Cristian Tejos }}^{1,2,5}$, and Sergio Uribe ${ }^{1,5,6}$

${ }^{1}$ Biomedical Imaging Center, Pontificia Universidad Catolica de Chile, Santiago, Chile, ${ }^{2}$ Electrical Engineering, Pontificia Universidad Catolica de Chile, Santiago, Chile, ${ }^{3}$ Radiology, University of Texas Southwestern, Dallas, TX, United States, ${ }^{4}$ Biomedical Engineering, University of Texas Southwestern, Dallas, TX, United States, ${ }^{5}$ Institute for Biological and Medical Engineering, Pontificia Universidad Catolica de Chile, Santiago, Chile, ${ }^{6}$ Radiology, Pontificia Universidad Catolica de Chile, Santiago, Chile

We propose an improved methodology ${ }^{1}$ to automatically estimate local $3 \mathrm{D}$ Pulse Wave Velocity (PWV) measurements for quantifying local alterations due to aortic distensibility using $4 \mathrm{D}$ flow data. 18 volunteers and 25 patients with Familial Hypercholesterolemia (FH) were evaluated using the proposed method. The results show the prevalence of higher values of PWV in $\mathrm{FH}$ patients than volunteers, particularly in the ascending aorta (AAo) and proximal descending aorta (pDAo). This semi-automatic 3D method is less user dependent and uses multiple correlations to improve accuracy. We demonstrate an excellent agreement with expect values. 
Here, a Y-shaped pulsatile flow phantom is used to evaluate the flow quantification error, as measured by 2D CINE PC, caused by magnetic susceptibility in the presence of clinically used MR-conditional ferromagnetic stents, even in ROIs where the artifact is not visualized in magnitude image. Our results indicate that flow measurements should be performed more than $12 \mathrm{~mm}$ away from the proximal or distal part of the stent to achieve accurate flow measurements.

Can cardiac 2D Phase-Contrast MRI velocity measurements be used to characterize left ventricle hemodynamics?

Stephanie Marchesseau ${ }^{1}$, Teresa Yeung ${ }^{2,3}$, and John J Totman ${ }^{1}$

${ }^{1}$ Clinical Imaging Research Centre, A*STAR, Singapore, Singapore, ${ }^{2}$ Cardiovascular Research Institute, National University Heart Centre, Singapore, Singapore, ${ }^{3}$ Department of Surgery, NUS, Singapore, Singapore

Phase-contrast MRI were proposed to estimate intracardiac pressure gradients, but it is still unclear if this acquisition can be reliably used in the assessment of hemodynamics of the left ventricle. In this study, we performed reproducibility and test-retest experiments to evaluate the clinical use of PC-MRI and concluded that pressure and velocity parameters measured from the inflow (from atrium to LV) seem to be reliably measured using PC-MRI but it was not the case for the outflow parameters. Moreover, test-retest experiment showed that individual parameters were not constant over time, which therefore questions the diagnostic value of PC-MRI pressure measurement.

Blood flow measurement using 3D cine PC MRI within the abdominal aortic aneurysm and visceral arteries in pre- and post-EVAR condition; blood flow in the SMA might be improved after EVAR.

Masataka Sugiyama ${ }^{1}$, Yasuo Takehara ${ }^{2}$, Tetsuya Wakayama ${ }^{3}$, Atsushi Nozaki ${ }^{3}$, Marcus Alley ${ }^{4}$, Takasuke Ushio ${ }^{1}$, Shinji Naganawa ${ }^{5}$, and Harumi Sakahara ${ }^{1}$

${ }^{1}$ Radiology, Hamamatsu University School of Medicine, Hamamatsu, Japan, ${ }^{2}$ Department of Fundamental Development for Advanced Low Invasive Diagnostic Imaging, Nagoya University, Graduate School of Medicine, Nagoya, Japan, ${ }^{3}$ Applied Science Laboratory Asia Pacific, GE Healthcare Japan, Hino, Japan, ${ }^{4}$ Radiology, Stanford University School of Medicine, Stanford, CA, United States, ${ }^{5}$ Radiology, Nagoya University School of Medicine, Nagoya, Japan

The blood flow volume within the visceral arteries were measured and compared between pre-and post-EVAR conditions using 4DFlow MRI. The maximum systolic flow volume ratio in the SMA to that of the aorta showed significant increase after EVAR. 4DFlow might be useful for evaluation of the blood flow dynamics of the aorta and visceral arteries in pre- and postEVAR condition.

Usefulness of 4D flow in the Diagnosis of Atrial Septal Defects in Adults

Mamoru Takahashi ${ }^{1}$, Yasuo Takehara ${ }^{2}$, Norihiro Tooyama ${ }^{1}$, Katsutoshi Ichijo ${ }^{1}$, Tomoyasu Amano ${ }^{1}$, Yoshikazu Nagura ${ }^{1}$, Kouichi Mizuno ${ }^{1}$, Takuya Matsumoto ${ }^{1}$, Tomoyuki Okuaki ${ }^{3}$, and Harumi Sakahara ${ }^{4}$

${ }^{1}$ Seirei Mikatahara General Hospital, Hamamatsu, Japan, ${ }^{2}$ Nagoya University, Graduate School of Medicine, Nagoya, Japan, ${ }^{3}$ Philips Healthcare AsiaPacific, Tokyo, Japan, ${ }^{4}$ Hamamatsu University School of Medicine, Hamamatsu, Japan

We tested whether 4D flow can offer useful hemodynamic information in the diagnosis of atrial septal defects in Adults. 4D PCA was clearly able to visualize abnormal shunts from the left antrum to the right antrum of ASD patients.

Background phase correction in the presence wrap-around artifact: Application in 4D flow imaging

Aaron A. Pruitt ${ }^{1}$, Ning $\mathrm{Jin}^{2}$, Yingmin $\mathrm{Liu}^{3}$, Orlando Simonetti ${ }^{4}$, and Rizwan Ahmad

${ }^{1}$ Biomedical Engineering, The Ohio State University, Columbus, OH, United States, ${ }^{2}$ Cardiovascular MR R\&D, Siemens Medical Solutions USA, Inc., Columbus, OH, United States, ${ }^{3}$ Davis Heart and Lung Research Institute, The Ohio State University, Columbus, $\mathrm{OH}$, United States, ${ }^{4}$ Internal Medicine, The Ohio State University, Columbus, OH, United States

Residual background phase offsets due to eddy-currents limit the accuracy of flow quantification in 4D flow imaging. Commonly utilized polynomial regression of stationary voxels to correct background phase, however, is unreliable in the presence of wrap-around artifact. Here, we present an automated approach to identify and exclude regions of wrap-around from the fit, and validate its effectiveness in phantom and in vivo. 
4D flow MRI offers the ability to measure and visualize the temporal evolution of complex blood flow patterns within an 3D volume. Some studies have been performed to validate 4D PC flow measurements, such as the comparison of $4 \mathrm{D}$ PC flow measurements to two-dimensional (2D) flow and to phantoms measurements as a reference standard. We hypothesized that 4D flow could be used to evaluate the hemodynamic parameters in patients with tetralogy of Fallot compare with 2D flow.

2D phase-contrast MRI as an integrative method for the evaluation of patients with chronic thromboembolic pulmonary hypertension before and after pulmonary endarterectomy Christoph P. Czerner ${ }^{1}$, Christian Schoenfeld ${ }^{1}$, Serghei Cebotari ${ }^{2}$, Julius Renne ${ }^{1}$, Till F. Kaireit ${ }^{1}$, Hinrich B. Winther ${ }^{1}$, Gesa H. Hauck ${ }^{1}$, Marius Hoeper ${ }^{3}$, Frank Wacker ${ }^{1}$, and Jens VogelClaussen ${ }^{1}$

${ }^{1}$ Institute for Diagnostic and Interventional Radiology, Hannover Medical School, Hannover, Germany, ${ }^{2}$ Department of Cardiothoracic, Transplantation and Vascular Surgery, Hannover Medical School, Hannover, Germany, ${ }^{3}$ Clinic for Pneumology, Hannover Medical School, Hannover, Germany

Pulmonary endarterectomy (PEA) is an established method for treatment of chronic thromboembolic pulmonary hypertension (CTEPH). MRI is currently proposed as novel tool for treatment monitoring. We evaluated $2 \mathrm{D}$ phase-contrast (PC) MRI of the main pulmonary artery as perioperative monitoring method in relation to cardiac and parenchymal perfusion MRI as well as to clinical parameters. $32 \mathrm{CTEPH}$ patients who underwent MRI before and after PEA were analyzed. Results show improved postoperative pulmonary hemodynamics. 2D PC MRI data correlate well with cardiac as well as perfusion changes and clinical parameters which makes this method a simple tool for treatment monitoring after PEA.

Cardiovascular Magnetic Resonance Imaging : a Tool for Non-invasive Absolute Aortic Blood Pressure Estimation

Khalil Rachid ${ }^{1}$ and Dima Rodriguez ${ }^{2}$

${ }^{1}$ IR4M, Université Paris-Sud, Paris, France, ${ }^{2} I R 4 M$, Université Paris-Sud, Orsay, France

Cardiovascular Magnetic Resonance Imaging (CMRI) is a well-established modality that allows not only non-invasive accurate blood flow quantification but also provides anatomical and biomechanical information about large vessel properties (e.g. aortic wall elasticity and distension) and central hemodynamics. The aim of our study is to use an MR-compatible aortic flow setup including two different elastic phantoms and validate MR-based pressure waveforms predicted by 1D blood flow model against invasive pressure measurements.

Quantitative phase-contrast CMR of blood flow in fetal vessels gated by Doppler ultrasound: comparison with metric optimized gating

Erik Hedström ${ }^{1,2}$, Katarina Steding-Ehrenborg ${ }^{1,3}$, Sebastian Bidhult ${ }^{1,4}$, Christian Ruprecht ${ }^{5,6}$, Fabian Kording ${ }^{5,6}$, and Anthony $\mathrm{H} \mathrm{Aletras}^{1,7}$

${ }^{1}$ Lund University, Skåne University Hospital, Department of Clinical Sciences Lund, Clinical Physiology, Lund, Sweden, ${ }^{2}$ Lund University, Skåne University Hospital, Department of Clinical Sciences Lund, Diagnostic Radiology, Lund, Sweden, ${ }^{3}$ Lund University, Department of Health Sciences, Physiotherapy, Lund, Sweden, ${ }^{4}$ Lund University, Department of Biomedical Engineering, Faculty of Engineering, Lund, Sweden, ${ }^{5}$ Department of Diagnostic and Interventional Radiology, University Medical Center Hamburg-Eppendorf, Hamburg, Germany, ${ }^{6}$ northh medical GmbH, Martinistraße 52, 20246, Hamburg, Germany, ${ }^{7}$ Laboratory of Computing, Medical Informatics and Biomedical - Imaging Technologies, Aristotle University of Thessaloniki, School of Medicine, Thessaloniki, Greece

The recent Doppler UltraSound (DUS) triggering method, which utilizes an MR-compatible ultrasound device to assess fetal heart contractions to provide a triggering/gating signal may improve fetal quantitative flow assessment by phase-contrast CMR. We evaluated the DUS method for blood flow measurements in the fetal descending aorta and umbilical vein, in comparison with the metric optimized gating method. Fetal quantitative blood flow by phase-contrast CMR is feasible using the DUS method. This further increases usability of fetal $\mathrm{CMR}$, as post-processing is not needed.

A Validation of MR Flow Velocity Mapping with Automated Phase Offset Correction Using a Gel Flow Phantom Controlled by a Motorized Piston in MR Phase Contrast Cine Flow Measurement

Kwan-Jin Jung ${ }^{1}$, Youssef Jaber ${ }^{2}$, and Frank C Sup IV ${ }^{2}$

${ }^{1}$ Human Magnetic Resonance Center, Institute of Applied Life Sciences, University of Massachusetts Amherst, Amherst, MA, United States, ${ }^{2}$ Department of Mechanical and Industrial Engineering, University of Massachusetts Amherst, Amherst, MA, United States

The accuracy of MR flow velocity measurement has been compromised due to a phase offset induced from the eddy current of gradient pulses. An automated correction method of the phase offset had been developed using an image-based algorithm. In order to validate the correction method and the measured velocity accuracy, we developed a flow phantom with a constant flow cross-section and used a servo motor controlled actuator to move the flow phantom accurately. The controlled movement of the new flow phantom allowed us to validate the phase offset correction method and the accuracy of the MR velocity measurement. 
${ }^{1}$ Radiology, Medical Physics, Medical Center - University of Freiburg, Faculty of Medicine, University of Freiburg, Freiburg, Germany, ${ }^{2}$ Institute for Fluid Mechanics and Aerodynamics, Department of Mechanical Engineering, Technische Universitaet Darmstadt, Darmstadt, Germany, ${ }^{3}$ Institute for Numerical Analysis and Scientific Computing, Department of Mathematics, Technische Universitaet Darmstadt, Darmstadt, Germany

Wall shear stress (WSS) quantifies the frictional force that flowing blood exerts on a vessel wall and can be estimated from MR-based flow measurements via numerical differentiation. Correct assessment of WSS remains difficult because of the limited spatial resolution, partial volume effects and the per-se unknown position of the wall. It has been shown that such WSS evaluations tend to underestimate. We investigate an alternative method to evaluate WSS using the Clauser-plot method - a graphical way to estimate the WSS in fully developed turbulent stationary flow. We briefly describe the Clauser-plot method and present experimental validation in a straight tube.

Correlation of Aortic Flow and Cardiac Function in Patients With Fabry Disease

Yi-Xian Li ${ }^{1}$, Bo-Yan Chuang ${ }^{1}$, Ming-Ting $\mathrm{Wu}^{2}$, and Hsu-Hsia Peng ${ }^{1}$

${ }^{1}$ Department of Biomedical Engineering and Environmental Sciences, National Tsing Hua University, Hsinchu, Taiwan, ${ }^{2}$ Department of Radiology, Kaohsiung Veterans General Hospital, Kaohsiung, Taiwan

We aim to explore the potential correlation of aortic flow and cardiac function in patients with Fabry disease (FD). The decreased total flow and increased maximum acceleration illustrated altered aortic hemodynamics. The left ventricular peak ejection rate (LVPER) negatively associated with the aortic total flow might be a mechanism to compensate the decreased aortic total flow in FD group. Besides, the positive correlation between LVPER and the systolic maximum acceleration described the interaction between cardiac function and aortic flow. In conclusion, the quantitative aortic flow-related parameters could help to elucidate altered aortic characteristics and the possible correlation with cardiac function.

Accelerating Dual Venc 4D Flow Using Compressed Sensing with Locally Low Rank along Velocity Encoding

Peng Lai ${ }^{1}$, Fatih Suleyman Hafalir ${ }^{2}$, Joseph Y Cheng ${ }^{3}$, Jonathan I Tamir ${ }^{4}$, Shreyas S Vasanawala ${ }^{3}$, Anja C.S Brau ${ }^{1}$, and Martin A Janich ${ }^{5}$

${ }^{1}$ Global MR Applications and Workflow, GE Healthcare, Menlo Park, CA, United States, ${ }^{2}$ Technical University of Munich, Munich, Germany, ${ }^{3}$ Radiology, Stanford University, Stanford, CA, United States, ${ }^{4}$ Electrical Engineering and Computer Science, University of California, Berkeley, CA, United States, ${ }^{5}$ Global MR Applications and Workflow, GE Healthcare, Munich, Germany

Dual Venc has been developed to improve the accuracy of conventional 4D flow in high dynamic range of velocity. However, dual Venc acquisition doubles scan time. This work explored a high dimensional compressed sensing method to accelerate dual Venc 4D flow by utilizing additional data redundancy in the velocity encoding dimension.

Traditional Poster

\section{Cardiac Function \& Myocardial Perfusion}

Right-ventricular Longitudinal Strain Reference Values of Healthy Volunteers by Age and Gender as Measured with CMR Tissue Tracking

Yangyang $\mathrm{Qu}^{1,2}$, Jan Paul ${ }^{1}$, Dominik Buckert ${ }^{1}$, Genshan $\mathrm{Ma}^{3}$, and Volker Rasche ${ }^{1}$

${ }^{1}$ Internal Medicine II, University Hospital of Ulm, Ulm, Germany, ${ }^{2}$ Medical School of Southeast University, Nanjing, China, ${ }^{3}$ Cardiology Department, Zhongda Hospital Southeast University, Nanjing, China

Our study measured RV longitudinal strain (RVGLS) by CMR 2D tissue tracking and investigated its diagnostic role in patients with RV heart failure.

150 healthy volunteers in three age groups ( $\mathrm{G}_{20-40}$ years, $\mathrm{G}_{41-60}$ years, and $\mathrm{G}_{61-80 \text { years }}$ ) and 30 patients diagnosed as $\mathrm{DCM}$ were recruited.

Normal RVGLS was $-23.9 \% \pm 5.2 \%$ with significant higher values in females in $\mathrm{G}_{41-60}$ and $\mathrm{G}_{61-80}$. The cut-off value identified as $-13.71 \%$ showed good sensitivity, specificity, positive and negative predictive value in diagnosing RV contractile dysfunction among DCM patients.

In summary, RVGLS were increased in females, and it benefited the evaluation of RV contractile function. 
We report that a bias in the assessment of left ventricular function (LVF) is due to the compressed sensing (CS) CINE. In cardiac CINE MRI EDV (or ESV) is assessed when blood volume is a maximum (or a minimum). Practically a time window (given by VPS) is used to reduce scan time. For CS-CINE the time window is expanded by adopting data at nearby cardiac frames. The expanded acquisition window reduces EDV and increases ESV due to time average effect. Note that the changes of the quantities are not random, thus they should be removed for a better diagnosis.

The right ventricular deformation in type 2 diabetes mellitus patients: insights from cardiac magnetic resonance feature-tracking

Bi-yue Hu${ }^{1}$, Zhi-gang Yang ${ }^{1}, \mathrm{Xi} \mathrm{Liu}^{1}, \mathrm{Ke} \mathrm{Shi}^{1}$, Hua-yan $\mathrm{Xu}^{1}$, and Ying-kun Guo ${ }^{2}$

${ }^{1}$ Department of Radiology, West China Hospital, Sichuan University, 37\# Guo Xue Xiang, Chengdu, Sichuan 610041, China., Chengdu, China, ${ }^{2}$ Department of Radiology, West China Second University Hospital, Sichuan University, 20\# Section 3 South Renmin Road, Chengdu, Sichuan 610041, China., Chengdu, China

Aim of this study was to clarify the feasibility of cardiovascular magnetic resonance (CMR)-derived feature-tracking for assessing right ventricle (RV) myocardial deformation in patients with type 2 diabetes mellitus (T2DM). Seventy T2DM patients and 22 healthy controls were enrolled. Cardiac volumes and function, and RV tissue-tracking parameters were determined by CMR. Compared with healthy subjects, significantly lower values of some global and regional strain parameters in T2DM (all p<0.05). Our results concluded that abnormal RV myocardial deformation could be monitored using CMR feature-tracking in T2DM; and the systolic and diastolic dysfunction was associated with RV volumes, HDL, and HbA1c.

Patterns of myocardial strain are unique in $\mathrm{HIV}+$ patients with heart failure with preserved ejection fraction

Bradley D Allen ${ }^{1}$, Amer Ahmed Syed ${ }^{1}$, James C Carr ${ }^{1}$, Matthew J Feinstein ${ }^{2}$, and Jeremy D Collins ${ }^{1}$

${ }^{1}$ Radiology, Northwestern University, Chicago, IL, United States, ${ }^{2}$ Medicine - Cardiology, Northwestern University, Chicago, IL, United States

Human immunodeficiency virus (HIV) infection is associated with impaired cardiac function beyond what is expected from coronary artery disease alone. Our aim in the current study was to compare myocardial strain in a cohort HIV+ patients and uninfected controls with adjudicated heart failure (HF) using cardiovascular MRI feature tracking. Our results demonstrate unique Ecc and Err strain patterns in HIV+ patients, with relative apical sparing in HIV+ patients with EF>50\%, but relative mid-LV and global strain reduction in HIV+ patients with $\mathrm{EF}<50 \%$. This constellation of findings suggests that patterns of myocardial functional impairment may be unique in HIV+ HF patients.

Cardiac Balanced SSFP 2D Cine DENSE for Myocardial Strain with comparison to Spiral 2D Cine DENSE

Ronald J. Beyers ${ }^{1}$, Nouha Salibi ${ }^{1,2}$, and Thomas S. Denney ${ }^{1}$

${ }^{1}$ MRI Research Center, Auburn University, Auburn, AL, United States, ${ }^{2}$ Siemens Healthineers, Malvern, PA, United States

Quantification of myocardial strain has been previously demonstrated with echo-planar and spiral sequence versions of Displacement Encoding with Stimulated Echoes (DENSE). However, the non-conventional k-space acquisition of these previous efforts has hindered their integration into mainstream cardiac MRI application. Here we present a more conventional balanced SSFP (bSSFP) version of 2D Cardiac Cine DENSE and compare its performance to 2D Spiral Cine DENSE in normal human subjects. In vivo human scans at 3T demonstrated good agreement of myocardial radial (Err) and circumferential (Ecc) strain values between bSSFP Cine DENSE and Spiral Cine DENSE that also agree with previous literature.

Left Ventricle 2D and 3D Strain Phantoms Generation Using a Python Finite Element-based Library

Hernán Mella ${ }^{1,2}$, Joaquin Mura ${ }^{1}$, Julio Sotelo ${ }^{1,2}$, and Sergio Uribe ${ }^{1,3,4}$

${ }^{1}$ Biomedical Imaging Center, Pontificia Universidad Católica de Chile, Santiago, Chile, ${ }^{2}$ Electrical Engineering Department, Pontificia Universidad Católica de Chile, Santiago, Chile, ${ }^{3}$ Radiology Department, Pontificia Universidad Católica de Chile, Santiago, Chile, ${ }^{4}$ Institute for Biological and Medical Engineering, School of Engineering, Medicine and Biological Sciences, Pontificia Universidad Católica de Chile, Santiago, Chile

The strain in the left ventricle is a well-known biomarker for cardiac diseases. Nowadays, several acquisition techniques have been developed to improve the diagnose of this kind of conditions. Usually, strain biomarkers are obtained by mean of image post-processing techniques using different deformation metrics. In this work we present a numerical framework for the generation of left-ventricle strain phantoms using three different acquisition sequences in order to provide a broad database of patients and volunteers with different types of diseases. Our library provides a robust image generation tool to compare and develop new post-processing methods for quantifying strain phantoms 
${ }^{1}$ Berlin Ultrahigh Field Facility (B.U.F.F.), Max Delbrück Center for Molecular Medicine in the Helmholtz Association (MDC), Berlin, Germany, ${ }^{2}$ DZHK (German Centre for Cardiovascular Research), partner site Berlin, Berlin, Germany, ${ }^{3}$ Department of Experimental Pharmacology and Toxicology, University Medical Center Hamburg-Eppendorf, Hamburg, Germany, ${ }^{4}$ DZHK (German Centre for Cardiovascular Research), partner site Hamburg/Kiel/Lübeck, Hamburg, Germany, ${ }^{5}$ Experimental and Clinical Research Center, Charite Medical Faculty and the Max Delbrueck Center for Molecular Medicine in the Helmholtz Association, Berlin, Germany

Mutations in gene MYBPC3, encoding cardiac myosin-binding protein $\mathrm{C}$, cause hypertrophic cardiomyopathy (HCM), which is characterized by left ventricular hypertrophy (LVH), diastolic dysfunction, increased interstitial fibrosis, and may lead to sudden cardiac death and heart failure. In spite of the advances in translational medicine, we know very little about $\mathrm{HCM}$. The HCM progression is complex and shows heterogeneous phenotypes. The missing linkage of in vivo imaging and pathology has hindered the investigation of detail mechanisms of HCM. We therefore investigated Mybpc3-targeted mouse models using CMR markers for understanding HCM pathophysiology and to get closer to complete pictures of HCM progression.

Protective effect of Resveratrol against cardiac dysfunction and impaired energy metabolism of type 2 diabetic female GK rat heart submitted to Ischemia-Reperfusion injury

Natacha Fourny ${ }^{1}$, Carole Lan ${ }^{1}$, Eric Sérée ${ }^{2}$, Laurent Pechere $^{3}$, Monique Bernard ${ }^{1}$, and Martine Desrois ${ }^{1}$

${ }^{1}$ Aix Marseille Univ, CNRS, CRMBM, Marseille, France, ${ }^{2}$ Aix-Marseille Univ, UMR Inserm 1062/ INRA 1260, NORT, Marseille, France, ${ }^{3}$ YVERY sarl, Marseille, France

Type 2 diabetes doubles the risk of myocardial infarction in women. New treatments need to be found to reduce cardiovascular mortality. Consequently, we investigated the effect of Resveratrol (RSV) on the tolerance to ischemia-reperfusion (IR) injury of type 2 diabetic female Goto-Kakizaki (GK) rat heart. We used a multiparametric approach allowing simultaneous measurement of cardiac function, energy metabolism by ${ }^{31} \mathrm{P}$ MRS and endothelial function. Oral RSV treatment improved myocardial performance, coronary flow and energy metabolism during reperfusion in GK rats. Consequently, RSV might be an interesting therapeutic approach to improve survival to myocardial IR injury of type 2 diabetic women.

Longitudinal follow-up of endothelial function after ischemia reperfusion injury treated with a novel regenerative therapy by albumin-based DCE MRI

Maaike van den Boomen ${ }^{1}$, Patricia Y.W. Dankers ${ }^{2,3,4}$, Leonie B.P. Niesen ${ }^{1}$, Carlijn V.C. Bouten ${ }^{2}$, and Katrien Vandoorne ${ }^{1}$

${ }^{1}$ Biomedical Engineering, Biomedical NMR, Eindhoven University of Technology, Eindhoven, Netherlands, ${ }^{2}$ Biomedical Engineering, Soft Tissue Biomechanics \& Tissue Engineering, Eindhoven University of Technology, Eindhoven, Netherlands, ${ }^{3}$ Institute for Complex Molecular Systems, Eindhoven, Netherlands, ${ }^{4}$ Biomedical Engineering, Organic Chemistry, Eindhoven University of Technology, Eindhoven, Netherlands

Dynamic contrast enhanced (DCE) MRI in combination with gadolinium-labeled albumin enabled longitudinal follow up of a novel hydrogel based regenerative therapy to treat myocardial infarction (MI). The local fractional blood volumes (fBVa measure for microvascular density) and permeability surface areas in the myocardium were increased at day 3 after MI due to the growth factors released from the hydrogel. This increase might indicate angiogenesis, which improves the inflammatory response. At day 7 the vascular density and permeability went back to normal again, which possibly avoid excessive extension of the MI.

Global and segmental cardiac magnetic resonance tissue tracking of hypertrophic cardiomyopathy: How does hypertrophy and fibrosis contribute to myocardial deformation?

Ruo-yang Shi ${ }^{1}$, Bing-hua Chen ${ }^{1}$, Dong-aolei $\mathrm{An}^{1}$, Rui Wu ${ }^{1}$, Liang $\mathrm{Du}^{2}$, Jiani Hu${ }^{3}$, Meng Jiang ${ }^{4}$, Wei-bo Chen ${ }^{5}$, Lian-ming Wu ${ }^{1}$, and Jian-rong Xu ${ }^{1}$

${ }^{1}$ Department of Radiology, Ren Ji Hospital, School of Medicine, Shanghai Jiao Tong University, Shanghai, China, ${ }^{2}$ Robotics Institute, Shanghai Jiao Tong University, Shanghai, China, ${ }^{3}$ Department of Radiology, Wayne State University, Detroit, MI, United States, ${ }^{4}$ Department of Cardiology, Ren Ji Hospital, School of Medicine, Shanghai Jiao Tong University, Shanghai, China, ${ }^{5}$ Philips Healthcare, Shanghai, China

In the patients with $\mathrm{HCM}$, subtle LV deformation can be observed and measured clinically before the onset of general LV functional changes. Both hypertrophy and fibrosis influenced the extent of LV deformation. Our study demonstrated the 2D CS as a stable global parameter to assess LV functional and ECV changes. At the segmental level, hypertrophy and LGE (+) antagonistic affected 2D RS and diastolic RSR. Despite the excitement surrounding these pertinent clinical findings, further research is warranted as the mechanism of the phenomenon still needs to be explored. 
According to our study findings, BOLD MRI detected greater deoxygenated hemoglobin in HTN LVMH(measured by T2* BOLD MRI)compared with HTN non-LVMH and control groups. Lower T2* BOLD MRI values were associated with higher ECV values and correlated with reductions in circumferential and longitudinal strain, strain rate and displacement. Higher LVMI was associated with an increase in ECV and nativeT1, and a decrease inT2* BOLD MRI values. To our knowledge, this is the first study to assess the influence of myocardial oxygenation on cardiac function in hypertensive patients by applying combined T2* BOLD MRI, T1mapping and strain analysis. Assessing myocardial capillary oxygenation by BOLD MRI relies on the measurement of BOLD MRI relaxation time through endogenous contrast of deoxygenated hemoglobin. Myocardial microvascular oxygenation could reflect a balance or imbalance between oxygen supply and demand.

Pilot Tone Navigation Enables Contactless Prospective Cardiac Triggering: Initial Volunteer Results for Prospective Cine

Mario Bacher ${ }^{1}$, Peter Speier ${ }^{1}$, Jan Bollenbeck ${ }^{1}$, Matthias Fenchel $^{2}$, and Matthias Stuber ${ }^{3}$

${ }^{1}$ Siemens Healthcare, Erlangen, Germany, ${ }^{2}$ Siemens Medical Solutions USA, New York, NY, United States, ${ }^{3}$ CHUV, Département de Radiologie Médicale, Lausanne, Switzerland

Pilot Tones are a contactless, electromagnetic navigator that offers monitoring of cardiac and respiratory motion independently of the acquisition. Here we present initial volunteer results in utilizing the cardiac Pilot Tone signal to prospectively trigger a segmented cardiac Cine acquisition without the need for ECG.

Simultaneous Multi Slice (SMS) SSFP first-pass myocardial perfusion imaging with iterative reconstruction at 1.5 Tesla.

Muhummad Sohaib Nazir ${ }^{1}$, Radhouene Neji ${ }^{1}, 2$, Peter Speier ${ }^{3}$, Daniel Staeb ${ }^{4}$, Michaela Schmidt ${ }^{3}$, Christoph Forman ${ }^{3}$, Reza Razavi ${ }^{1}$, Sven Plein $^{1}$, Tevfik Ismail ${ }^{1}$, Amedeo Chiribiri $^{1}$, and Sebastien Roujol ${ }^{1}$

${ }^{1}$ Biomedical Engineering and Imaging Sciences, King's College London, London, United Kingdom, ${ }^{2}$ MR Research Collaborations, Siemens Healthcare Limited, Frimley, United Kingdom, ${ }^{3}$ Siemens Healthcare, Erlangen, Germany, ${ }^{4}$ The Centre for Advanced Imaging, The University of Queensland, Brisbane, Australia

Myocardial perfusion imaging is recommended for ischaemia testing in patients although spatial coverage is limited to 3 slices in clinical practice. Simultaneous Multi Slice (SMS) imaging combined with iterative reconstruction was evaluated to provide greater heart coverage with minimal signal-to-noise penalty. 8 patients underwent two contrast enhanced dynamic perfusion scans at rest to compare the standard 3 slice with the SMS 6 slice protocol. Subjective image quality was found to be comparable to a standard 3 slice approach. This technique may have clinical utility in patients with suspected coronary artery disease through detection of ischaemia with greater heart coverage.

Rest Perfusion within Chronic Infarctions Depends on Type of Acute Myocardial Infarction: Insights from a Serial MRI Study in Patients

Eric Johnson ${ }^{1,2}$, Andreas Kumar ${ }^{3}$, and Rohan Dharmakumar ${ }^{1,2}$

${ }^{1}$ Biomedical Imaging Research Institute, Cedars Sinai Medical Center, Los Angeles, CA, United States, ${ }^{2}$ Dept of Bioengineering, University of California, Los Angeles, Los Angeles, CA, United States, ${ }^{3}$ Northern Ontario School of Medicine, Sudbury, ON, Canada

Excessive iron in tissue can impair endothelial function and reduce microcirculatory blood flow. We hypothesized that resting blood flow in chronic hemorrhagic myocardial infarction (hMI) territories, where iron concentration is known to be significantly elevated, would be lower than in non-hMI territories. We studied this in patients with reperfused myocardial infarction using cardiac MRI over a 6-month period following infarction. Mean relative perfusion index of hMls were significantly lower than non-hMls. This finding supports the notion that hypoperfusion within hMl territories may be an important pathological contributor to adverse cardiac remodeling commonly observed in patients with hMls.

Multiple sets of simultaneous multi-slice (SMS) for improved short and long axis coverage of myocardial DCE perfusion

Edward DiBella ${ }^{1}$, Jason Mendes ${ }^{1}$, Mark Ibrahim² ${ }^{2}$, Ye Tian ${ }^{3}$, Brent Wilson ${ }^{2}$, and Ganesh Adluru

${ }^{1}$ Radiology and Imaging Sciences, University of Utah, Salt Lake City, UT, United States, ${ }^{2}$ Cardiology, University of Utah, Salt Lake City, UT, United States, ${ }^{3}$ Physics, University of Utah, Salt Lake City, UT, United States

We propose a unique perfusion acquisition that offers improved coverage and confidence of detecting true ischemia and artifacts in cardiac perfusion dynamic acquisitions. Three slices are acquired simultaneously after each saturation pulse, and there is time to acquire 3 sets of such slices even at high heartrates. The ability to simultaneously acquire multiple slices opens up many new possibilities. The approach proposed here can acquire for example 6 short axis slices and 3 long axis slices each heartbeat, which allows detection of small areas of ischemia and can provide additional volume coverage and confidence. Preliminary results show the promise of this multi-plane SMS approach.

Evaluation of extended GROG and Toeplitz pre-reconstruction interpolation methods on radial simultaneous multi slice MRI

Ye $\operatorname{Tian}^{1,2}$, Ganesh Adluru${ }^{1}$, Jason Mendes ${ }^{1}$, and Edward DiBella ${ }^{1}$

${ }^{1}$ UCAIR, University of Utah, Salt Lake City, UT, United States, ${ }^{2}$ Physics and Astronomy, University of Utah, Salt Lake City, UT, United States 
The purpose of this study is to develop and extend GRAPPA operator gridding (GROG) for fast iterative reconstruction of radial SMS data, and to compare extended GROG (EGROG) with GROG, Toeplitz and NUFFT methods. Simulation and in-vivo tests were done to compare these methods. Our results show that EGROG improves reconstruction by providing better Cartesian k-space estimation, it outperforms Toeplitz and GROG at oversampling factor 2, and a speed up factor of $\sim 2$ was achieved compared to NUFFT.

Hybrid Estimation of the Arterial Input Function Using Blind Deconvolution and the Measured Blood Pool Signal

Radovan Jirik ${ }^{1,2}$, Jason Mendes², Ye Tian², Ganesh Adluru², and Edward DiBella ${ }^{2}$

${ }^{1}$ Institute of Scientific Instruments of the ASCR, Brno, Czech Republic, ${ }^{2}$ Utah Center for Advanced Imaging and Research, University of Utah, Salt Lake City, UT, United States

In Dynamic Contrast-Enhanced (DCE) MRI, inaccurate estimation of the arterial input function (AIF) is still a major cause of the low reliability of kinetic parameter estimates. We propose a new method of AIF estimation. It combines AIF measured from the blood-pool signal and multichannel blind deconvolution. The weights of the measured AIF are based on its analytically derived uncertainty and a model relating signal intensity and gadolinium concentration.

The method has been evaluated on simulated myocardial perfusion data, mimicking real noise and kinetic parameter distributions. The hybrid method gave better results compared to the blood-pool or blind-deconvolution approaches alone.

Fully-automated motion correction and probability-based segmentation of myocardial perfusion MRI data

Cian Michael Scannell ${ }^{1}$, Adriana Villa ${ }^{1}$, Jack Lee $^{1}$, Marcel Breeuwer $^{2,3}$, and Amedeo Chiribiri ${ }^{1}$

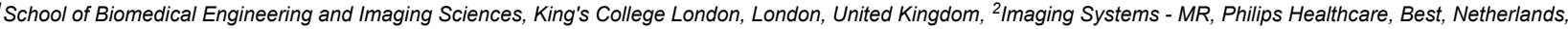

${ }^{3}$ Department of Biomedical Engineering, Eindhoven University of Technology, Eindhoven, Netherlands

This work presents a fully-automated framework for the pre-processing of free-breathing myocardial perfusion MRI data. Image series are first split into low-rank and sparse components using RPCA. This allows estimation of the deformation fields required to motion correct the image series, in the absence of dynamic contrast enhancement. Once motion corrected, pixels are clustered into anatomically relevant clusters using perfusion-superpixels which groups nearby pixels that have similar time dynamics. A LDA classifier is trained which allows the generation of myocardial probability maps and active contours are fit to the high probability regions to give a delineation of the myocardium.

Validation of MR multitasking myocardial perfusion reserve measurements against simultaneous $13 \mathrm{~N}$-ammonia PET

Anthony G Christodoulou ${ }^{1}$, Damini Dey ${ }^{1,2}$, Behzad Sharif ${ }^{1}$, Richard Tang ${ }^{1}$, Wafa Tawackoli ${ }^{1,3,4}$, Rohan Dharmakumar ${ }^{1,2,5}$, Piotr J Slomka ${ }^{1,5}$, Daniel S Berman ${ }^{1,5}$, and Debiao Li ${ }^{1,2}$

${ }^{1}$ Biomedical Imaging Research Institute, Cedars-Sinai Medical Center, Los Angeles, CA, United States, ${ }^{2}$ Department of Bioengineering, University of California, Los Angeles, Los Angeles, CA, United States, ${ }^{3}$ Department of Surgery, Cedars-Sinai Medical Center, Los Angeles, CA, United States, ${ }^{4}$ Board of Governors Regenerative Medicine Institute, Cedars-Sinai Medical Center, Los Angeles, CA, United States, ${ }^{5}$ Department of Medicine, David Geffen School of Medicine, University of California, Los Angeles, Los Angeles, CA, United States

Measurements from myocardial perfusion MRI have previously been compared against separate PET measurements. However, MR quantification is complicated by signal nonlinearity (leading to a dual-bolus paradigm) and ECG misfires; furthermore, physiological variation in between separate PET and MR assessments are a confounding factor in validation. This work leverages the recent advent of multimodal PET-MR systems to perform a preliminary validation of quantitative MPR measurements from MR multitasking-a new framework allowing single-bolus, non-ECG perfusion quantification-against simultaneous $13 \mathrm{~N}$-ammonia PET-MR measurements in pigs. Excellent agreement was found between modalities (no bias, $p=0.66$; intraclass correlation coefficient $=0.95$ )

Left Atrial Surface Strain from Cine MRI Data in Patients with Mitral Regurgitation

Xiaoxia Zhang ${ }^{1,2}$, Himanshu Gupta ${ }^{3}$, James Davis ${ }^{3}$, Steven G. Lloyd ${ }^{3}$, Louis Dell'Italia ${ }^{3}$, and Thomas S. Denney Jr. ${ }^{1,2}$

${ }^{1}$ Auburn University MRI Research Center, Auburn University, Auburn, AL, United States, ${ }^{2}$ Department of Electrical and Computer Engineering, Auburn University, Auburn, AL, United States, ${ }^{3}$ Division of Cardiovascular Disease, University of Alabama at Birmingham, Birmingham, AL, United States

Mitral regurgitation (MR) is a common form of valvular disease where degeneration of the mitral valve causes blood from the left ventricle to be regurgitated into the left atrium (LA). For some MR patients, surgery to repair or replace the mitral valve is an option, but it can be difficult to determine when to do the surgery. Volumetric remodeling in the left atrium in MR patients has been reported, and could precede remodeling of the left ventricle (LV) and damage to the LV wall. Here, we investigate changes in endocardial surface strain in the LA in patients with MR compared to normal. Changes in LA volume and deformation may be useful in determining the severity and chronicity of valvular regurgitation and have clinical potentials in optimizing surgery timing and patient management.

Right and left ventricular myocardial strain in healthy adolescents: Establishing normal reference values

Joseph Lang ${ }^{1}$, Greg Barton ${ }^{1}$, Arij Beshish ${ }^{1}$, Kara Goss ${ }^{1}$, Marlowe Eldridge ${ }^{1}$, and Christopher J Francois 
Tissue-tracking, a post-processing technique using routinely-acquired cine images, can assess strain, a multidimensional measure of myocardial contraction. In this prospective study, we measured left ventricular and right ventricular peak global radial, circumferential and longitudinal strain in 28 healthy adolescents ages $12-14$ years. The data from this study provide normative global strain values to be used for future clinical and translational CMR studies.

Traditional Poster

\section{Cardiovascular Image Processing}

Comparison of cardiac MRI myocardial strain quantification techniques demonstrates systematic differences between feature tracking and heart deformation analysis

Amer Ahmed Syed ${ }^{1}$, Bradley D Allen ${ }^{1}$, Eric J Keller ${ }^{1}$, James C Carr ${ }^{1}$, Matthew J Feinstein ${ }^{2}$, Susanne Schnell ${ }^{1}$, Michael Markl ${ }^{1,3}$, and Jeremy D Collins ${ }^{1}$

${ }^{1}$ Department of Radiology, Northwestern University, Chicago, IL, United States, ${ }^{2}$ Medicine - Cardiology, Northwestern University, Chicago, IL, United States, ${ }^{3}$ Biomedical Engineering,

Myocardial strain is commonly performed at transthoracic echocardiography and is a sensitive technique for detecting subclinical disease. Feature tracking (CMR-FT) and heart deformation analysis (HDA) are two techniques that can be applied to balanced steady state free precession cinegraphic images, enabling the assessment of Lagrangian strains at cardiac MRI. We compared myocardial strains derived using these techniques in a cohort of $30 \mathrm{HIV}+$ patients. CMR-FT and HDA derived myocardial strains were significantly different, with CMR-FT consistently yielding higher strain values than HDA. Our results highlight technique dependence of CMR strain and underscore the need for technique specific normative reference values.

Automated Segmentation of the Carotid Bifurcation using Region Growing and Support Vector Machines

Magnus Ziegler ${ }^{1,2}$, Max Gefvert ${ }^{1,2}$, Jan Engvall ${ }^{1,2,3}$, Ebo de Muinck ${ }^{1,2,3}$, and Petter Dyverfeldt ${ }^{1,2}$

${ }^{1}$ Linköping University, Linköping, Sweden, ${ }^{2}$ Center for Medical Image Science and Visualization (CMIV), Linköping, Sweden, ${ }^{3}$ University Hospital Linköping, Linköping, Sweden

Roughly 1 in 40 deaths worldwide are caused by strokes resulting from emboli that reach the brain from ruptured atherosclerotic plaques in the carotid artery. Segmentation of the carotid artery bifurcation in MR is necessary enables further analysis. Unfortunately, this is a slow and difficult task that is often performed manually. Two segmentation methods, one based on Region Growing (RG), and one using Support Vector Machines (SVM), were implemented for segmenting the carotid bifurcation in contrast-enhanced MR Angiograms (CE-MRA). Both methods were tested quantitatively, against ground truth segmentations using the DICE and true-positive ratio (TPR) and were also scored qualitatively using visual inspection. Both methods scored highly (RG $0.890 \pm 0.022$, SVM $0.890 \pm 0.022$ ) using the DICE score and true-positive ratio (RG $0.938 \pm 0.026$, SVM $0.931 \pm 0.285$ ). During qualitative assessments, RG and SVM both scored highly with median score $4 / 5$.

Intracranial vessel wall segmentation on 3D black-blood MRI using convolutional neural network

Hao Liu ${ }^{1}$, Dongye $\mathrm{Li}^{1,2}$, Xuesong Li ${ }^{3}$, Qiang Zhang ${ }^{1}$, Guanhua Wang ${ }^{1}$, Yishi Wang ${ }^{1}$, Xihai Zhao ${ }^{1}$, and Huijun Chen ${ }^{1}$

Intracranial artery atherosclerosis is a major cause of stroke. manually segmenting intracranial artery vessel wall is laborious and time-consuming. we proposed an automatic intracranial artery vessel wall segmentation framework to find the centerline of the intracranial artery from SNAP images to segment the final lumen and outer-wall contours on the cross-sectional 2D slices perpendicular to the centerline.

Jacob A Macdonald ${ }^{1}$, Grant S Roberts ${ }^{1}$, and Oliver Wieben ${ }^{1,2}$ 
MRI during exercise stress can be a powerful tool in discerning abnormal cardiac behavior not apparent at rest. As a result of increased cardiac and respiratory motion, robust gating is essential for high-quality acquisitions during exercise. Due to increased patient motion, however, missed ECG triggers are more likely during exercise than at rest. For reconstructions with retrospective gating, such missed triggers can result in data attributed to the wrong portion of the cardiac cycle. In this work, we present an algorithm to identify and correct missed ECG triggers, allowing for exercise scans otherwise compromised by poor gating to be salvaged.

Phase Unwrapping of 4D Flow Data with Graph Cuts

Andrew Justice ${ }^{1}$, Sean Callahan², Jung won $\mathrm{Cha}^{2}$, and Amir Amini ${ }^{2}$

${ }^{1}$ Electrical and Computer Engineering, University of Louisville, Louisville, $K Y$, United States, ${ }^{2}$ University of Louisville, Louisville, KY, United States

A common problem with 4D flow magnetic resonance imaging is aliasing that occurs as a result of a low velocity encoding parameter. Consequently, an efficient and robust algorithm is needed to unwrap this data. We propose an iterative graph cuts algorithm to perform the necessary phase unwrapping and attain correct velocity values. The graph cuts algorithm utilizes a global energy minimization framework. This method is shown to accurately unwrap the aliased data more accurately than existing techniques for $4 \mathrm{D}$ Flow data. This included unwrapping synthetic data with Vencs down to $20 \%$ of the max velocity and SNRs down to 2 .

Automatic lumen size measurement in carotid atherosclerosis with phase sensitive magnetic resonance angiography(MRA) using self-trained radial basis function kernel support vector machine

Daniel S Hippe ${ }^{1}$, Jie Sun ${ }^{1}$, Chun Yuan ${ }^{1}$, and Haining Liu ${ }^{1}$

${ }^{1}$ Radiology, University of Washington, SEATTLE, WA, United States

A self-trained algorithm based on Ostu's method and a radial basis function (RBF) kernel support vector machine (SVM) model was developed for automatic lumen detection and quantification for the negative polarity map of SNAP magnetic resonance angiography(MRA). Based on an analysis of 15 arteries with carotid stenosis, the proposed automatic lumen segmentation algorithm demonstrated good agreement with manual lumen segmentation of SNAP MRA (intraclass correlation coefficient (ICC $=0.95$ ). The automated method also had good agreement with manual segmentation of CE-MRA $(I C C=0.90)$, which was comparable to the agreement between manually segmented SNAP MRA and CE-MRA $(I C C=0.93)$.

Evaluation of e-prime with cardiac magnetic resonance cine imaging—preliminary feasibility study with comparison to echocardiography

Felicia Seemann ${ }^{1,2,3}$, Ricardo Gonzales ${ }^{3}$, Chenxi Hu${ }^{3}$, Michael Quail ${ }^{4}$, Karl Grunseich ${ }^{3}$, Lauren Baldassarre ${ }^{4}$, Albert Sinusas ${ }^{4}$, Judith Meadows ${ }^{4}$, Hamid Mojibian ${ }^{3}$, and Dana C. Peters ${ }^{3}$

${ }^{1}$ Department of Clinical Physiology, Skåne University Hospital, Lund University, Lund, Sweden, ${ }^{2}$ Department of Biomedical Engineering, Faculty of Engineering, Lund University, Lund, Sweden, ${ }^{3}$ Department of Radiology and Biomedical Imaging, Yale School of Medicine, Yale University, New Haven, CT, United States, ${ }^{4}$ Section of Cardiovascular medicine, Department of Internal Medicine, Yale School of Medicine, Yale University, New Haven, CT, United States

Diastolic dysfunction is commonly assessed by echocardiography, but not by cardiovascular magnetic resonance (CMR). To evaluate diastolic function, the mitral annular flow (E) and velocity (e') at the early rapid filling phase are measured. While E can be accurately measured by CMR, methods for measuring e' need to be established. In this study a feature tracking based method for measuring $e^{\prime}$ is applied to CMR images, and validated against echocardiography. There was an agreement between the methods, but sources of disparities between CMR and echocardiographic e' measurements need to be further studied in order to improve the accuracy of e' measurement by CMR.

High-resolution Imaging with a priori Knowledge Incorporating the Navier-Stokes equations and the discontinuous Galerkin method (4D flow HIKING): towards flow reconstruction constrained by computational fluid dynamics

Johannes Töger ${ }^{1}$, Matthew J Zahr² ${ }^{2}$ Karin Markenroth Bloch ${ }^{3}$, Marcus Carlsson ${ }^{4}$, and Per-Olof Persson ${ }^{2}$

${ }^{1}$ Diagnostic Radiology, Department of Clinical Sciences, Skane University Hospital, Lund University, Lund, Sweden, ${ }^{2}$ Department of Mathematics, Lawrence Berkeley Laboratory and University of California, Berkeley, CA, United States, ${ }^{3}$ Lund University Bioimaging Center, Lund University, Lund, Sweden, ${ }^{4}$ Clinical Physiology, Department of Clinical Sciences, Skane University Hospital, Lund University, Lund, Sweden

Magnetic resonance 4D flow imaging is a promising technique for diagnosis and follow-up of disease. However, 4D flow is limited by long scan times and low resolution. This work presents phantom validation of a new method for 4D flow scan acceleration, called 4D flow high-resolution imaging with a priori knowledge incorporating the Navier-Stokes equations and the discontinuous Galerkin method (4D flow HIKING). Excellent agreement with laser particle image velocimetry (PIV) was found, demonstrating the potential of the framework for scan time reduction and enhanced data quality in $4 \mathrm{D}$ flow. 
Hyungkyu Huh ${ }^{1}$, Menhel Kinno ${ }^{2}$, James D Thomas ${ }^{2}$, Michael Markl ${ }^{1,3}$, and Alex J Barker ${ }^{1}$

${ }^{1}$ Department of Radiology, Northwestern University, Chicago, IL, United States, ${ }^{2}$ Department of Cardiology, Feinberg Medical School, Chicago, IL, United States, ${ }^{3}$ Department of Biomedical Engineering, Northwestern University, Chicago, IL, United States

The purpose of this study was to compare the aortic valve effective orifice area (EOA) estimated between Doppler echocardiography and 4D flow MRI using a consecutive same-day study design to minimize inter-modality variability. Peak velocity and left ventricular outflow tract area were higher for MRI but velocity time integral was higher for echo. These differences were compensatory when computing EOA, which resulted in good agreement despite discrepancies in echo vs MRI. Volumetric 3D velocity information has the potential to better estimate EOA in the presence of eccentric jets. This potential strength will be studied in aortic stenosis patients.

Pixel-wise quantitative myocardial perfusion mapping with cloud based non-linear iterative reconstruction using Gadgetron framework

Hui Xue ${ }^{1}$, Sven Plein ${ }^{2}$, Amedeo Chiribiri $^{3}$, and Peter Kellman ${ }^{1}$

${ }^{1} \mathrm{NHLBI}$, NIH, Bethesda, MD, United States, ${ }^{2}$ University of Leeds, Leeds, United Kingdom, ${ }^{3}$ King's College London, London, United Kingdom

In this abstract, we present a solution to speed up the non-linear reconstruction for myocardial perfusion imaging and demonstrate its clinical usage through the Gadgetron cloud deployed at Microsoft Azure infrastructure. We also achieved pixel-wise myocardial blood flow mapping on the non-linearly reconstructed images, given the computing power on the cloud. All these processing steps were inline integrated on the clinical MR scanners. As a result, the proposed solution allows us to deploy non-linear perfusion imaging with quantitative flow mapping as a clinical application.

Quantitative Classification of Atherosclerotic Plaque Compositions in Carotid Arteries: An in vivo T1 Mapping Study

Huiyu Qiao ${ }^{1}$, Haikun $\mathrm{Qi}^{1}$, Dongye $\mathrm{Li}^{1}{ }^{1,2}$, Dongxiang $\mathrm{Xu}^{3}$, Huijun Chen ${ }^{1}$, Chun Yuan ${ }^{1,3}$, and Xihai Zhao ${ }^{1}$

${ }^{1}$ Center for Biomedical Imaging Research, Department of Biomedical Engineering, Tsinghua University, Beijing, China, ${ }^{2}$ Center for Brain Disorders Research, Capital Medical University, Beijing, China, ${ }^{3}$ Department of Radiology, University of Washington, Seattle, WA, United States

This study sought to investigate the usefulness of in vivo T1 mapping in quantitative classification of compositions and vulnerability of carotid artery atherosclerotic plaques. We found that it is feasible to quantify the T1 values of atherosclerotic plaque compositions in carotid artery with in vivo T1 mapping. Significant differences in T1 values between fibrous tissue and other plaque compositions indicate that it is possible to classify plaque compositional features using T1 mapping. In addition, our findings of IPH and LRNC with significant different T1 values from other plaque compositions suggest the potential of T1 mapping in classification of plaque vulnerability.

Automatic bullseye analysis of myocardial T1 values: a segmentation approach based on deep learning

Yu-Nian Ou ${ }^{1}$, Tsai-Ling Yang ${ }^{1}$, Teng-Yi Huang ${ }^{1}$, and Ming-Ting Wu ${ }^{2}$

${ }^{1}$ Department of Electrical Engineering, National Taiwan University of Science and Technology, Taipei, Taiwan, ${ }^{2}$ Department of Radiology, Kao-Hsiung Veterans General Hospital, KaoHsiung, Taiwan

The study presents an automatic segmentation method for short-axis MOLLI data sets. We used a deep learning method based on convolutional neural network to accurately extract walls and blood pool regions of left and right ventricle. We compared the results with a layer-growing method presented in ISMRM 2017 and found that the accuracy of segmentation was significantly improved when using the deep learning method.

Traditional Poster

\section{Vascular}

Dariusch Reza Hadizadeh ${ }^{1}$, Gregor Jost ${ }^{2}$, Julian Lütckens ${ }^{1}$, Vera Catharina Keil ${ }^{1}$, Christoph Endler ${ }^{1}$, Hubertus Pietsch ${ }^{2}$, Hans Heinz Schild ${ }^{1}$, and Winfried Albert Willinek ${ }^{3}$

${ }^{1}$ Radiology, University of Bonn, Bonn, Germany, ${ }^{2} \mathrm{MR}$ and CT Contrast Media Research, Bayer AG, Berlin, Germany, ${ }^{3}$ Radiology, Neuroradiology, Sonography and Nuclear Brüderkrankenhaus, Trier, Germany 
This study systematically evaluated the impact of contrast agent $(\mathrm{CA})$ doses both quantitatively and regarding image quality on time-resolved contrast enhanced MR-angiography (4DMRA). The intra-individual study-design under highly standardized conditions was realized using an animal model. 5 anesthetized Göttingen minipigs received thoracic-abdominal $4 \mathrm{D}$ MRA at $1.5 \mathrm{~T}$ at five CA doses from $0.02-0.10 \mathrm{mmol} / \mathrm{kgBW}$. We observed that the further the CA traveled along the circulation, the more a dose reduction resulted in weaker peak signal enhancement and low image quality. We conclude that CA dose reduction has varying effects on image quality in 4D-MRA with respect to vessel types and sizes.

Triple Accelerated NCE-MRA with optimised sampling patterns

Hao Li ${ }^{1}$, Andrew Nicholas Priest ${ }^{1}$, Martin John Graves ${ }^{1}$, and David John Lomas

${ }^{1}$ Department of Radiology, University of Cambridge, Cambridge, United Kingdom

In this study, we developed an acceleration technique combining compressed sensing (CS), parallel imaging (PI) and partial Fourier (PF) for the fresh blood imaging (FBI) sequence. Then, we evaluated the influence of the pattern design parameters and explored the optimal values for these parameters. By using the optimised sampling patterns, the FBI acquisition can be accelerated up to 10 times while the image quality is maintained.

Whole-heart coronary MRA at 3.0T: Comparison between conventional method and new acceleration technique by compressed SENSE.

Shinichi Takase ${ }^{1}$, Masaki Ishida ${ }^{1}$, Yoshitaka Goto ${ }^{1}$, Shiho Isoshima ${ }^{1}$, Wakana Makino ${ }^{1}$, Haruno Sakuma ${ }^{1}$, Makoto Obara ${ }^{2}$, Tsunehiro Yamahata ${ }^{1}$, Katsuhiro Inoue $^{1}$, Kakuya Kitagawa $^{1}$, and Hajime Sakuma ${ }^{1}$

${ }^{1}$ Department of Radiology, Mie University Hospital, Tsu, Mie, Japan, ${ }^{2}$ MR Clinical Science, Philips Japan, Ltd., Tokyo, Japan

Compressed SENSE (CSENSE) is a novel method to combine Sensitivity Encoding (SENSE) and compressed sensing for rapid MR imaging. CSENSE can achieve a reduction factor higher than those achieved by SENSE while preserving the image quality by the denoising effect with iterations. In this work, CSENSE was applied to 3.0T free-breathing whole-heart coronary MRA to reduce the acquisition time. As compared to the conventional acquisition method using SENSE, CSENSE allows for up to 2.5 -fold reduction of acquisition time without significant degradation of image quality of whole-heart coronary MRA at 3.0T, especially after the contrast injection.

Central thoracic vein imaging without Gadolinium: diagnostic confidence of DANTE-based 3D subtractive NCE-MRA and comparison with 2D bSSFP

Andrew Nicholas Priest ${ }^{1}$, Ilse Patterson ${ }^{1}$, Nadeem Shaida ${ }^{1}$, Nicholas J Hilliard ${ }^{1}$, Sarah Hilborne ${ }^{1}$, and David John Lomas ${ }^{1}$

${ }^{1}$ Radiology, Addenbrooke's Hospital and University of Cambridge, Cambridge, United Kingdom

Imaging of the central thoracic veins is often challenging due to renal failure and/or difficult venous access, which render contrast agent administration problematic. This work assesses a non-contrast-enhanced free-breathing 3D subtractive MR angiography method for thoracic central vein imaging in a group of 18 patients. Evaluation by experienced radiologists demonstrated that angiograms obtained with this new method give high diagnostic confidence, which is significantly better than our standard 2D breath-hold approach in the absence of contrast medium. There is also better agreement between readers for the new sequence.

Accelerated Acquisition of Vessel-Encoded Arterial Spin Labelling Angiograms with Compressed Sensing

S Sophie Schauman ${ }^{1}$, Mark Chiew ${ }^{1}$, and Thomas W Okell

${ }^{1}$ Wellcome Centre for Integrative Neuroimaging, FMRIB, Nuffield Department of Clinical Neurosciences, University of Oxford, Oxford, United Kingdom

Vessel-selective ASL angiography provides information about cerebral blood supply not achievable by other non-invasive techniques. It is, however, limited by long acquisition times. Here we demonstrate the benefit of using compressed sensing to reconstruct undersampled vessel-selective angiograms and furthermore, consider how the vessel-encoding process affects the choice of sampling strategy compared to non-selective imaging. We show that vessel-selective angiograms arising from three brain-feeding arteries can be reconstructed with excellent fidelity in the same scan time normally required for non-selective ASL angiography.

Large Field-of-View Nonenhanced Neurovascular MR Angiography Using Ungated Radial Quiescent-Interval Slice-Selective (QISS)

loannis Koktzoglou ${ }^{1,2}$, Ali Serhal ${ }^{3}$, Jianing Pang ${ }^{4}$, and Robert R Edelman ${ }^{1,3}$

${ }^{1}$ Radiology, NorthShore University HealthSystem, Evanston, IL, United States, ${ }^{2}$ Radiology, University of Chicago Pritzker School of Medicine, Chicago, IL, United States, ${ }^{3}$ Radiology, Northwestern University Feinberg School of Medicine, Chicago, IL, United States, ${ }^{4}$ Siemens Healthineers, Chicago, IL, United States 
We report a prototype ungated radial quiescent-interval slice-selective technique for nonenhanced magnetic resonance angiography of the extracranial carotid, vertebrobasilar and proximal intracranial circulations. The proposed method efficiently covers a large field-of-view, provides improved image quality with respect to Cartesian sampling, and provides flexibility to shorten the acquisition time via radial undersampling.

Breath-hold Three-dimensional Quiescent-Interval Slice-Selective (QISS) MR Angiography using a Fast-Interrupted Steady-State (FISS) Readout: Application to the Coronary and Renal Arteries

Robert R Edelman ${ }^{1,2}$, Jianing Pang ${ }^{3}$, and loannis Koktzoglou ${ }^{1,4}$

${ }^{1}$ Radiology, NorthShore University HealthSystem, Evanston, IL, United States, ${ }^{2}$ Radiology, Feinberg School of Medicine, Northwestern University, Chicago, IL, United States, ${ }^{3}$ Siemens Medical Solutions, Chicago, IL, United States, ${ }^{4}$ Radiology, Prtizker School of Medicine, University of Chicago, Chicago, IL, United States

Quiescent-interval slice-selective (QISS) is a robust nonenhanced 2D MRA technique, but has potential limitations regarding minimum slice thickness, slice profile, and fat suppression We therefore implemented a breath-hold prototype 3D version of QISS which uses a thin-slab RF excitation, stack-of-stars k-space trajectory, and fast interrupted steady-state (FISS) readout instead of bSSFP. 2D and 3D QISS were compared for imaging of the coronary and renal arteries. Benefits of 3D QISS included better depiction of small branch vessels and improved quality for multi-planar reconstructions.

Artifact Reduction in 3D Radial Whole-Heart Imaging Using Slab-Selective RF Excitation

Jianing Pang ${ }^{1}$, Davide Piccini ${ }^{2}$, Christoph Forman ${ }^{3}$, and Michaela Schmidt ${ }^{3}$

${ }^{1}$ Siemens Medical Solutions USA Inc, Chicago, IL, United States, ${ }^{2}$ Advanced Clinical Imaging Technology, Siemens Healthcare AG, Lausanne, Switzerland, ${ }^{3}$ Siemens Healthcare, Erlangen, Germany

To date, most 3D radial kooshball imaging implementations had used non-selective (NS) radiofrequency pulses for volumetric excitation. However, given the undersampled nature of radial imaging, signal from excited regions in the periphery increases the streaking level in the central area of the field-of-view. In this work, we implemented slab-selective (SS) excitation for 3D radial whole-heart imaging. Results on 10 volunteers showed that SS excitation improved mean apparent signal- and contrast-to-noise ratio by $24 \%$ and $40 \%$, respectively, with a mean scan time increase of $26 \%$ due to longer TR.

Retrospective Multi-Phase Non-Contrast-Enhanced Magnetic Resonance Angiography (ROMANCE MRA) for Robust Angiogram Separation in the Presence of Cardiac Arrhythmia

Hahnsung Kim ${ }^{1,2}$, Suhyung Park ${ }^{1,3}$, Eung Yeop Kim ${ }^{4}$, Chul-Ho Sohn ${ }^{5}$, and Jaeseok Park

${ }^{1}$ Department of Biomedical Engineering, Sungkyunkwan University, Suwon, Republic of Korea, ${ }^{2}$ Biomedical Imaging Research Institute, Cedars-Sinai Medical Center, Los Angeles, CA, United States, ${ }^{3} \mathrm{Helen}$ Wills Neuroscience Institute, University of California, Berkeley, CA, United States, ${ }^{4}$ Department of Radiology, Gachon University Gil Medical Center, Incheon, Republic of Korea, ${ }^{5}$ Department of Radiology, Seoul National University Hospital, Seoul, Republic of Korea

In the proposed ROMANCE MRA, data were continuously acquired over all cardiac phases using retrospective, multi-phase flow-sensitive single-slab 3D fast spin echo (FSE) with variable refocusing flip angles, while an external pulse oximeter was in sync with pulse repetitions in FSE to record real-time information on cardiac cycles. Data were then sorted into $\mathrm{k}$ bin space using the real-time cardiac information. Angiograms were reconstructed directly from k-bin space by solving a constrained optimization problem with both subtraction-induced sparsity and low rank priors. Peripheral MRA was performed in a normal volunteer and a volunteer with cardiac arrhythmia using conventional fresh blood imaging (FBI) and the proposed ROMANCE MRA for comparison.

Breath Hold Non-contrast Enhanced Angiography of Renal Arteries at 3T using Compressed SENSE Acceleration

Brian Johnson ${ }^{1,2}$, Ivan E. Dimitrov ${ }^{1,3}$, Sandeep Ganji ${ }^{1}$, Yasutomo Katsumata ${ }^{4}$, Mariya Doneva ${ }^{5}$, Ali Pirasteh ${ }^{2}$, Johannes Peeters ${ }^{6,7}$, and Ivan Pedrosa ${ }^{2}$

${ }^{1}$ Philips Healthcare, Gainesville, FL, United States, ${ }^{2}$ Radiology, University of Texas Southwestern Medical Center, Dallas, TX, United States, ${ }^{3}$ Advanced Imaging Research Center, University of Texas Southwestern Medical Center, Dallas, TX, United States, ${ }^{4}$ Philips Healthcare, Tokyo, Japan, ${ }^{5}$ MR Resarch \& Development, Philips Healthcare, Hamburg, Germany, ${ }^{6}$ MR Clinical Science, Philips Healthcare, Best, Netherlands, ${ }^{7}$ MR Resarch \& Development, Philips Healthcare, Best, Netherlands

Non-Contrast Enhanced angiography of the renal arteries is an important technology for patients with chronic kidney disease. Existing techniques, like b-TRANCE, have long acquisition times, which makes them sensitive to motion artifacts. Respiratory triggering or navigation can be used to improve motion robustness. This however results in even longer scan times. Compressed SENSE is an effective way for accelerating 3D acquisitions and can be used to substantially reduce scan times. In this study, we report preliminary results of a breath hold and free breathing approaches for contrast-free renal angiography by combining b-TRANCE with compressed SENSE. 
${ }^{1}$ Siemens Shenzhen Magnetic Resonance Ltd, Shen Zhen, China, ${ }^{2}$ Siemens Healthcare China Ltd, Shang Hai, China

In this work, we present a Keyhole method for fast Time Of Flight (TOF) imaging. We compare it with a recently published segmented k-space filling scheme. Moreover, we demonstrate the feasibility of combing the Keyhole and segmented methods for further acceleration. Such a hybrid TOF can be potentially suited for high-resolution angiograms at ultra-high field.

Improved Non-Contrast Renal Angiography Using Respiratory and Cardiac Gating with Dynamically Determined Inversion Times: A Simulation Study

Xiaoxuan $\mathrm{He}^{1}$, Naoharu Kobayashi ${ }^{1}$, Xiufeng $\mathrm{Li}^{1}$, and Gregory J. Metzger ${ }^{1}$

${ }^{1}$ Center for Magnetic Resonance Research, University of Minnesota, Minneapolis, MN, United States

In this simulation study, we aim to demonstrate the feasibility of improving non-contrast enhanced renal MRA by adding a cardiac gate with dynamically determined inversion times. The benefits of the proposal include higher contrast due to better background suppression and improved inflow enhancement, which may be clinically significant for delineating occluded vessels.

Comparison of Whole-Heart Noncontrast-Enhanced 3T MR Angiography and CT Angiography in Detection of Coronary Artery Disease

Jingwen Dai ${ }^{1}$, Jian Cao ${ }^{1}$, Jing $A n^{2}$, Lu Lin ${ }^{1}$, Yining Wang ${ }^{1}$, and Zhengyu Jin ${ }^{1}$

${ }^{1}$ Peking Union Medical College Hospital, Peking Union Medical College, Chinese Academy of Medical Sciences, Beijing, China, ${ }^{2}$ Siemens Healthcare, MR Collaborations NE Asia, Beijing, China

The aim of this study was to investigate the diagnostic performance of noncontrast-enhanced coronary MR angiography in the detection of clinical significant coronary artery stenosis by using CTA as a reference. The preliminary results indicate that the noncontrast-enhanced coronary MR angiography has an excellent consistency in evaluating coronary artery disease in comparison to CTA. Noncontrast-enhanced coronary MR angiography may be suitable as a screening tool for coronary artery disease.

An accelerated peripheral MRA based on velocity-selective RF pulse using radial-MAGGULLI

Dongchan Kim ${ }^{1}$, Yeji Han ${ }^{1}$, Jun-Young Chung ${ }^{1}$, and HyunWook Park ${ }^{2}$

${ }^{1}$ Gachon University, Incheon, Republic of Korea, ${ }^{2}$ KAIST, Daejeon, Republic of Korea

We recently proposed a new peripheral MRA technique using velocity-selective gradient-echo (VS-GRE) sequence. Despite the high CNR and background suppression of the VS-GRE technique, this technique suffered from the reduced CNR efficiency, which was caused by the reduced sampling efficiency of radial trajectory in the peripheral region with anisotropic FOV. In this work, we propose a combination of the proposed peripheral MRA and the simultaneous multi-slice (SMS) imaging technique in the radial trajectory. In-vivo experiment results show that the proposed method could produce peripheral MRA with the reduced imaging time by radial-MAGGULLI.

Free Breathing Multiple Delays Renal Perfusion MRI using Hadamard encoded pCASL

Naoyuki Takei ${ }^{1}$, Shota Ishida ${ }^{2}$, Nobuyuki Kosaka ${ }^{3}$, R Marc Lebel $^{4}$, Yuki Matta ${ }^{2}$, Hirohiko Kimura ${ }^{3}$, and Hiroyuki Kabasawa ${ }^{1}$

${ }^{1}$ Global MR Applications \& Workflow, GE Healthcare, Tokyo, Japan, ${ }^{2}$ Radiological Center, University of Fukui Hospital, Fukui, Japan, ${ }^{3}$ Department of Radiology, University of Fukui, Fukui, Japan, ${ }^{4}$ Global MR Application \& Workflow GE Healthcare, Calgary, AB, Canada

Current pCASL renal perfusion imaging is typically restricted to a single post label delay (PLD) time. While multiple PLD (mPLD) times can be achieved with sequential scans with different PLD times, this procedure is time consuming. A rapid acquisition was developed using Hadamard encoding for mPLD pCASL imaging combined with a motion robust timing and readout strategy to permit free breathing renal ASL. The feasibility study explores the application of Hadamard encoding to renal perfusion imaging where spin labeling is affected by pulsatile flow and demonstrated that a cardiac triggered scan provided stable perfusion images achieving ATT corrected renal blood flow with seven PLD acquisition

Free-breathing zoomed whole heart coronary MRA without respiratory gating using small-FOV 3D stack-of-stars radial sequence with pseudo-golden angle sampling

Takashige Yoshdia ${ }^{1,2}$, Masami Yoneyama ${ }^{3}$, Kohei Yuda ${ }^{1}$, Takumi Koyano ${ }^{1}$, Yuki Furukawa ${ }^{1}$, Mariko Okura ${ }^{4}$, Nobuo Kawauchi ${ }^{4}$, and Haruo Saito ${ }^{2}$

${ }^{1}$ Radiology, Tokyo Metropolitan Police Hospital, Tokyo, Japan, ${ }^{2}$ Graduate school of Medicine, Division of Diagnostic Image Analysis, Tohoku University, Miyagi, Japan, ${ }^{3}$ MR Clinical

Science, Philips Japan, Tokyo, Japan, ${ }^{4}$ Diagnosis of radiology, Tokyo Metropolitan Police Hospital, Tokyo, Japan 
One of the problem of whole heart coronary MRA is the prolongation of acquisition time. It is caused for degrade image quality. However, the radial sampling technique is able to obtain image of inconspicuous artifact such as aliasing and motion; furthermore, the sequence is possible to reduce scan time by understate data sampling. Hence the zoomed whole heart coronary MRA with pseudo golden angle radial sampling was improved image quality without extend scan time.

The feasibility of a homemade dielectric pad using commercially available ultrasound gel with Gadolinium contrast material to improve B1 homogeneity for non-enhanced peripheral MR angiography

Akiyoshi Yamamoto ${ }^{1}$, Akikazu Harada ${ }^{1}$, Yuji Shintani ${ }^{1}$, Daiji Uchiyama ${ }^{1}$, Seigo Yoshida ${ }^{1}$, Katsumi Nakamura ${ }^{2}$, and Mitsue Miyazaki ${ }^{3}$

${ }^{1}$ Tobata Kyouritsu Hospital, Kitakyusyu,Fukuoka, Japan, ${ }^{2}$ Tobata General Hospital, Kitakyusyu,Fukuoka, Japan, ${ }^{3}$ Radiology, UC, San Diego, La Jolla, CA, United States

We investigated an effect of homemade dielectric pads with using commercially available ultrasound (US) gel for improvement of B1 inhomogeneity in the peripheral artery examination using non-contrast fresh blood imaging (FBI) at 3T. We designed the two-bottle phantom mimics the iliac-femoral region, where often observed signal loss in peripheral non-contrast MRA due to B1 inhomogeneity. The result of the phantom study using US gel indicated uniform RF penetration in the B1 map. The US-gel pad improved the RF power penetration under the condition of B1 inhomogeneity and superior visualization of the left superficial femoral artery.

Real-time low-field cardiac MRI using an integrated MRI-guided radiotherapy system

H Michael Gach ${ }^{1}$, Sayantan Bhadra ${ }^{2}$, Austen N Curcuru ${ }^{1}$, Roger Nana ${ }^{3}$, Clifford G Robinson ${ }^{1}$, Phillip S Cuculich ${ }^{4}$, Sasa Mutic ${ }^{1}$, and Mark A Anastasio

${ }^{1}$ Radiation Oncology, Washington University in St Louis, St Louis, MO, United States, ${ }^{2}$ Computer Science and Engineering, Washington University in St Louis, St Louis, MO, United States, ${ }^{3}$ ViewRay, Oakwood Village, OH, United States, ${ }^{4}$ Electrophysiology, Washington University in St Louis, St Louis, MO, United States, ${ }^{5}$ Biomedical Engineering, Washington University in St Louis, St Louis, MO, United States

The efficacy of stereotactic body radiation therapy (SBRT) cardiac radiosurgery in resolving cardiac arrhythmias was recently reported from a small clinical trial (NCT02919618). However, real-time tracking of the cardiac lesion is challenging using conventional cone-beam CT guided radiotherapy. MRI-guided radiotherapy (MRIgRT) systems integrate real-time MRI for lesion tracking with radiation therapy and can provide excellent cardiac tissue image quality at high frame rates. Real-time cardiac MRI using sparsely-sampled radial acquisitions is demonstrated with iterative reconstruction methods at low-field $(0.35 \mathrm{~T})$. The performance goal is to image the heart and track the lesion at $30 \mathrm{~Hz}$ with $2.5 \mathrm{~mm}$ in-plane resolution.

Wideband Inversion Recovery Late Gadolinium Enhancement Sequence improves Image Quality in Patients with Cardiac Implanted Electronic Devices

Jadranka Stojanovska ${ }^{1}$, Mason Runge ${ }^{2}$, El-Sayed Ibrahim ${ }^{3}$, Anil K. Attili ${ }^{1}$, Thomas Chenevert ${ }^{1}$, Maryam Ghadimi-Mahani ${ }^{1}$, and Frank Bogun ${ }^{4}$

${ }^{1}$ Radiology, Michigan Medicine, Ann Arbor, MI, United States, ${ }^{2}$ University of Michigan Medical School, Ann Arbor, MI, United States, ${ }^{3}$ Radiology, University of Michigan, Ann Arbor, MI, United States, ${ }^{4}$ Cardiovascular Medicine, Michigan Medicine, Ann Arbor, MI, United States

Late gadolinium enhancement is a gold standard for myocardial scar assessment in patients with ventricular tachycardia before their ablation. The presence of cardiac implantable electronic devices degrade the image quality by producing the hyper signal intensity and make the image non-diagnostic. The modified wideband inversion recovery sequence alleviates these hyper signal intensity artifacts and render diagnostic images.

The Prevalence of Pulmonary Vein Stenosis Post Radio-Frequency Catheter Ablation in Atrial Fibrillation Patients

Hana Sheitt ${ }^{1}$, Julio Garcia ${ }^{1,2}$, Andrew Howarth ${ }^{1,3}$, Stephen Wilton ${ }^{1}$, Carmen P. Lydell ${ }^{1,4}$, and James A. White ${ }^{1,3}$

${ }^{1}$ Stephenson Cardiac Imaging Center, Libin Cardiovascular Institute of Alberta, Calgary, AB, Canada, ${ }^{2}$ Department of Cardiac Science, University of Calgary, Calgary, AB, Canada, ${ }^{3}$ Department of Medicine, University of Calgary, Calgary, AB, Canada, ${ }^{4}$ Diagnostic Imaging, University of Calgary, Calgary, AB, Canada

This study is demonstrating the rule of cardiac MRI in evaluating pulmonary veins (PV) stenosis in atrial fibrillation patients before and after radio-frequency catheter ablation (RFCA). 
Intracranial and extracranial atherosclerotic disease are major causes of ischemic stroke. Recently, an improved DANTE-prepared 3D variable-flip-angle turbo spin echo (SPACE) imaging method was developed for high resolution simultaneously imaging of intracranial and extracranial arterial vessel wall with enhanced cerebrospinal fluid suppression. The purpose of this study was to evaluate the scan-rescan, intra-and inter-observer reproducibility when using the method for comprehensive assessment of intracranial and extracranial vessel wall morphology. In conclusion, the improved 3D simultaneous vessel wall imaging technique provided good to excellent reproducibility for intracranial and extracranial arterial vessel wall measurements.

Assessment of carotid atherosclerosis: a comparison between 2D and 3D multi-contrast vessel wall magnetic resonance imaging

Yunduo Li ${ }^{1}$, Hanyu Wei ${ }^{1}$, Xihai Zhao ${ }^{1}$, Gador Canton ${ }^{2}$, Jie Sun ${ }^{2}$, Zechen Zhou ${ }^{3}$, Shuo Chen ${ }^{1}$, Rui Li ${ }^{1}$, and Chun Yuan ${ }^{1,2}$

${ }^{1}$ Center for Biomedical Imaging Research, Department of Biomedical Engineering, Tsinghua University, Beijing, China, ${ }^{2}$ Department of Radiology, University of Washington, Seattle, WA, United States, ${ }^{3}$ Philips Research North America, Cambridge, MA, United States

In this study, we compared morphological measurements and identification of plaque components in carotid artery between 2D and 3D multi-contrast vessel wall MRI techniques. 3D multi-contrast vessel wall imaging, with $0.8 \mathrm{~mm}$ isotropic resolution and $15 \mathrm{~min}$ total scan time, showed good inter-reader reproducibility and provided comparable morphological information as $2 \mathrm{D}$ multi-contrast imaging, and more importantly, has its potential to improve visualization of plaque components.

High prevalence of intraplaque hemorrhage in peripheral artery disease is indicated by large coverage femoral vessel wall MRI

Niranjan Balu ${ }^{1}$, Jie Sun ${ }^{1}$, Thomas Hatsukami², Daniel Isquith ${ }^{3}$, Susan McKeeth ${ }^{3}$, Chun Yuan ${ }^{1}$, and Xue-Qiao Zhao ${ }^{3}$

${ }^{1}$ Radiology, University of Washington, Seattle, WA, United States, ${ }^{2}$ Vascular Surgery, University of Washington, Seattle, WA, United States, ${ }^{3}$ Cardiology, University of Washington, Seattle, WA, United States

Intraplaque hemorrhage (IPH) is known to be a high-risk atherosclerotic plaque feature based on carotid imaging but its prevalence is unknown in peripheral artery disease (PAD). Since PAD is a diffuse disease that can occur along a long stretch of the femoral artery, large coverage 3D vessel wall MRI is required to identify IPH prevalence in PAD. This study reports the high prevalence of IPH in patients with an abnormal ankle-brachial index (ABI) using IPH specific large coverage 3D vessel wall MRI.

Preliminary Investigation of Extravascular Fluid Transport along Arterial Adventitia of Human Lower Extremity

Hongyi Li ${ }^{1}$, Wentao $\mathrm{Liu}^{2}$, Yang Fan ${ }^{3}$, Liang $\mathrm{Xu}^{4}$, Yupeng $\mathrm{Cao}^{2,5}$, Fang Wang ${ }^{1}$, Dong Han ${ }^{2,5}$, and Min Chen ${ }^{4}$

${ }^{1}$ Cardiology Division, Beijing Hospital, Beijing, China, ${ }^{2}$ CAS Center for Excellence in Nanoscience, National Center for Nanoscience and Technology, Chinese Academy of Sciences, Beijing, China, ${ }^{3}$ MR Research China, GE Healthcare, Beijing, China, ${ }^{4}$ Radiological department, Beijing Hospital, Beijing, China, ${ }^{5}$ School of Future Technology, University of Chinese Academy of Sciences, Beijing, China

Extravascular fluid transport have been reported both in human and animal studies during recent decades. Our previous work demonstrated a long-distance extravascular fluid transport which is consisted of oriented fibrous connective tissues in venous adventitia, arterial adventitia and dermis of amputated lower extremities. To further explore the pattern of fluid transport along lower extremity arteries, we implemented contrast enhanced MRI in volunteers and tracked the longitudinal contrast agent transportation. The periarterial regions near tibia showed high signal intensity after contrast agent administration suggest an unexplored extravascular fluid transport. This study may provide a novel diagnosis method of PAD.

The Characteristics of Chronic Internal Carotid Artery Occlusion for Successful Endovascular Intervention by 3D MR Vessel Wall Imaging

Jin Zhang ${ }^{1}$, Huilin Zhao ${ }^{1}$, Beibei Sun ${ }^{1}$, Xiaosheng Liu ${ }^{1}$, Jieqing Wan ${ }^{2}$, Weibo Chen $^{3}$, Xihai Zhao ${ }^{4}$, Chun Yuan ${ }^{5}$, and Jianrong Xu1

${ }^{1}$ Radiology, Renji hospital, Shanghai Jiaotong University, Shanghai, China, ${ }^{2}$ Neurosutgery, Renji hospital, Shanghai Jiaotong University, Shanghai, China, ${ }^{3}$ Philips Healthcare, Shanghai, China, ${ }^{4}$ Biomedical Engineering \& Center for Biomedical Imaging Research, Tsinghua University, Beijing, China, ${ }^{5}$ Radiology, University of Washington, Seattle, WA, United States

Visualization of the extent and components of internal carotid artery chronic total occlusion (CTO) may play an important role in deciding whether patients can undergo the endovascular intervention successfully or not. This study sought to investigate the characteristics of internal carotid artery CTO for successful endovascular intervention by $3 \mathrm{D}$ MR Vessel Wall Imaging. We found that patients with lower extent of occlusion and IPH at the proximal occlusion site had a higher success rate of recanalization. The results suggest 3D MR vessel wall imaging might be useful of patient selection for more possibly successful endovascular intervention. 
${ }^{1}$ Berlin Ultrahigh Field Facility (B.U.F.F.), Max Delbrück Center for Molecular Medicine (MDC) in the Helmholtz Association, Berlin, Germany, ${ }^{2}$ Technische Universität, Berlin, Germany, ${ }^{3}$ Experimental and Clinical Research Center (ECRC), a joint cooperation between the Charité Medical Faculty and the Max Delbrück Center for Molecular Medicine in the Helmholtz Association, Berlin, Germany

Inversion recovery prepared cardiac black blood RARE techniques (IR-RARE) are routinely applied at clinical field strengths while still facing numerous challenges at 7.0 T. Realizing the clinical importance of IR-RARE and the benefits of UHF, this study aims at the design of a double inversion recovery prepared imaging technique at $7.0 \mathrm{~T}$. The inversion efficiency and signal suppression efficiency of hyperbolic secant (HS4 and HS8) inversion pulses were analyzed in phantom experiments. First preliminary in-vivo applications using the implemented HSn pulses showed promising results.

Traditional Poster

\section{Novel Concepts, Techniques \& Methods}

Non-invasive quantitative estimation of blood oxygen saturation with MRI: feasibility of machine learning

Juliet Varghese ${ }^{1}$, Rizwan Ahmad ${ }^{1,2}$, Subha Raman ${ }^{1,3,4}$, Lee C Potter ${ }^{5}$, and Orlando P Simonetti ${ }^{1,3,4}$

${ }^{1}$ Dorothy M. Davis Heart and Lung Research Institute, The Ohio State University, Columbus, OH, United States, ${ }^{2}$ Department of Biomedical Engineering, The Ohio State University, Columbus, $\mathrm{OH}$, United States, ${ }^{3}$ Division of Cardiovascular Medicine, Department of Internal Medicine. The Ohio State University, Columbus, OH, United States, ${ }^{4}$ Department of Radiology, The Ohio State University, Columbus, $\mathrm{OH}$, United States, ${ }^{5}$ Department of Electrical and Computer Engineering, The Ohio State University, Columbus, OH, United States

Non-invasive estimation of intra-cardiac blood oxygen (O2) saturation by magnetic resonance (MR) imaging would be useful in evaluating shunt severity in congenital heart disease, and oxygen delivery and consumption energetics in heart failure and pulmonary hypertension. Accurate estimation of blood $\mathrm{O} 2$ saturation from MR data may be limited, however, by the lack of an accurate model to characterize the dependence on $\mathrm{T} 2$ relaxation of blood on its $\mathrm{O} 2$ saturation level. The present study explores the feasibility of machine learning to accurately predict blood $\mathrm{O} 2$ saturation; the performance is evaluated in a preliminary cohort of patients against the Luz-Meiboom model.

Differentiation of blood clot hematocrit and age in vitro using R2* and quantitative susceptibility mapping at 3T

Spencer D Christiansen ${ }^{1,2}$, Junmin Liu ${ }^{1}$, Joy Dunmore-Buyze ${ }^{1}$, Michael B Boffa ${ }^{3}$, and Maria Drangova ${ }^{1,2}$

${ }^{1}$ Imaging Research Laboratories, Robarts Research Institute, Western University, London, ON, Canada, London, ON, Canada, ${ }^{2}$ Dept. of Medical Biophysics, Schulich School of Medicine \& Dentistry, Western University, London, ON, Canada, London, ON, Canada, ${ }^{3}$ Dept. of Biochemistry, Schulich School of Medicine \& Dentistry, Western University, London, ON Canada, London, ON, Canada

Thrombus composition and age in ischemic occlusion can significantly influence treatment efficacy, yet current MR characterization methods are qualitative and cannot distinguish between the effects of red blood cell age and concentration (hematocrit). We examined the ability of $R_{2}{ }^{*}$ and quantitative susceptibility (QS) maps derived simultaneously from multiecho GRE acquisition to discriminate between blood clots of varied hematocrit formed in vitro and monitored over a six-day ageing period. Fresh clots (age $<6$ hours) of different hematocrit were distinguishable using either $\mathrm{R}_{2}{ }^{*}$ or $\mathrm{QS}$ values, while aged clots were distinguishable only when both values were considered.

Impact of empagliflozin on cardiac energy status and function in diabetic $\mathrm{db} / \mathrm{db}$ mice

Desiree Abdurrachim ${ }^{1}$, Emmy Manders $^{1}$, Klaas Nicolay ${ }^{1}$, Eric Mayoux ${ }^{2}$, and Jeanine J Prompers ${ }^{1,3}$

${ }^{1}$ Biomedical Engineering, Eindhoven University of Technology, Eindhoven, Netherlands, ${ }^{2}$ Cardiometabolic Diseases Research, Boehringer Ingelheim, Biberach, Germany, ${ }^{3}$ Radiology, University Medical Center Utrecht, Utrecht, Netherlands

Diabetes is associated with impaired cardiac energetics and diastolic dysfunction. A substrate shift toward ketones has been proposed to explain the benefits of empagliflozin on cardiovascular outcome in diabetes patients. We investigated the effects of empagliflozin on cardiac energetics and function in diabetic db/db mice using ${ }^{31} \mathrm{P}-\mathrm{MRS}$ and MRI. After a single dose of empagliflozin, cardiac PCr/ATP ratio was higher compared with placebo-treated controls, which was associated with increased plasma ketone levels and lower cardiac load. After 6 weeks of treatment, cardiac diastolic function tended to be improved, while plasma ketones and cardiac PCr/ATP ratio were not different from placebo. 
The circle of Willis (COW) is an important intracranocervical collateral circulation system. We hypothesized that the integrity of COW may affect the characteristics of carotid plaques by influencing carotid hemodynamics. This study investigated the relationship between incompleteness of COW and the compositional features of atherosclerotic plaques in carotid arteries. We found that the incompleteness of circle of Willis is associated with vulnerability of carotid artery atherosclerotic plaques. Our findings suggest that integrity of circle of Willis may play a role in occurence of high risk plaque features, particularly intraplaque hemorrhage and fibrous cap rupture.

Evaluation myocardial fibrosis in diabetes with cardiac magnetic resonance T1-mapping: correlation with the metabolic and diabetic duration

Yue Gao ${ }^{1}$, Zhigang Yang ${ }^{1}, \mathrm{Xi} \mathrm{Liu}^{1}$, Linjun Xie ${ }^{2}$, Li Jiang ${ }^{1}$, Biyue Hü ${ }^{1}$, and Yingkun Guo

${ }^{1}$ west china hospital, sichuan university, chengdu, China, ${ }^{2}$ West China Second University Hospital, chengdu, China

In order to clarify the relationship among diffuse myocardial fibrosis and abnormal metabolic and duration and diabetes, we compared the T1 mapping parameters on cardiac magnetic resonance (CMR). type 2 diabetes (T2DM) patients and normal controls were enrolled and performed CMR both. Our results showed ECV were higher in T2DM than controls, and positively associated with high level glycosylated hemoglobin and longer duration diabetes. Diabetes myocardial fibrosis could be effectively detected by CMR T1 mapping. The trend of myocardial fibrosis in patients with hyperglycemia and long-term duration is more obvious

In Vivo Hyperpolarized MRI Reveals Metabolic Changes Following Treatment with Mildronate in the Control and Diabetic Heart.

Dragana Savic ${ }^{1}$, Lorenz Holzner ${ }^{1}$, Vicky Ball ${ }^{1}$, M. Kate Curtis ${ }^{1}$, Lisa C. Heather ${ }^{1}$, and Damian J. Tyler ${ }^{1}$

${ }^{1}$ University of Oxford, Oxford, United Kingdom

L-carnitine acts as a buffer of acetyl-CoA units in the mitochondria, as well as facilitating transport of fatty acids. Mildronate can block the biosynthesis of L-carnitine and its uptake by inhibiting CPT-1. The purpose of this study was to investigate the effect of Mildronate treatment on cardiac function and metabolism in the healthy and the diabetic rat heart. We show that daily injections of Mildronate can alter cardiac metabolism in the in-vivo diabetic and healthy rat heart, without any functional changes, and surprisingly Mildronate can increase flux through pyruvate dehydrogenase. Such studies will allow a better understanding of the interactions between metabolism and function in the diabetic heart and may provide new insight into novel therapeutics.

Cardiac MRI with the Siemens Terra 7T System: Initial Experience and Optimization of Default Protocols

David Lohr ${ }^{1}$, Maxim Terekhov ${ }^{1}$, Aleksander Kosmala ${ }^{1}$, Maria Roxana Stefanescu ${ }^{1}$, Michael Hock ${ }^{1}$, and Laura Maria Schreiber ${ }^{1}$

${ }^{1}$ Chair of Cellular and Molecular Imaging, Comprehensive Heart Failure Center (CHFC), University Hospital, Wuerzburg, Germany

The demand for the application of Ultra-High Field (B0 $\geq 7 \mathrm{~T})$ MR-scanners in cardiovascular MRI grows permanently despite of technical challenges increasing significantly with the static magnetic field strength. We report initial experience with the new 7T system Siemens Magnetom ${ }^{\mathrm{TM}}$ Terra for acquiring MR-images of the human heart. A standard workflow for cardiac assessment has been developed and tested in $\mathrm{N}=18$ healthy volunteers in single transmit mode. Currently CINE scans with $14-17$ slices covering up to 35 heart phases are well suited for clinical volumetric heart function characterization. Diagnostic image quality can be provided for subsequent volunteers.

Cardiac MRI assessment of the effects of dietary Eicosapentaenoic acid (EPA) on the adverse cardiac consequences of sepsis in rat

Amidou Sissou Traore ${ }^{1}$, Thibault Leger ${ }^{2}$, Guilhem Pagès ${ }^{1}$, Lucie Cassagnes ${ }^{3,4}$, Azarnoush Kasra ${ }^{5}$, Jean-Marie Bonny ${ }^{1}$, and Luc Demaison ${ }^{2,6}$

${ }^{1}$ UR270 QuaPA, INRA, Saint-Genès Champanelle, France, ${ }^{2}$ UNH, INRA, Clermont-Ferrand, France, ${ }^{3}$ Department of diagnostic and interventional radiology, Clermont Ferrand University Hospital, Clermont-Ferrand, France, ${ }^{4}$ IGT, Institut Pascal, UMR 6602, CNRS, Clermont-Ferrand, France, ${ }^{5}$ Heart Surgery Department, Clermont Ferrand University Hospital, ClermontFerrand, France, ${ }^{6} \mathrm{CRNH}$, Clermont Auvergne University, Clermont-Ferrand, France

Severe sepsis is one of the leading cause of death in the intensive care units (ICU) or in short time after discharge from ICU. Developing a rat model of early sepsis involving caecal ligation and puncture, we undertaken this cardiac MRI study to quantitatively assess myocardial function and the protective effect of dietary EPA. Our results showed that, in the exception of the rate of contraction, cardiac functions are less impacted in the early hyperdynamic phase of sepsis with no/or milder modulation of dietary EPA.

Frequency Dependence of Anisotropic Material Properties Estimated form Cardiac Magnetic Resonance Elastography: An In Silico Study

Renee Miller ${ }^{1,2}$, Arunark Kolipaka ${ }^{3}$, Martyn P Nash ${ }^{2,4}$, and Alistair A Young ${ }^{1,2}$

${ }^{1}$ Anatomy and Medical Imaging, University of Auckland, Auckland, New Zealand, ${ }^{2}$ Auckland Bioengineering Institute, University of Auckland, Auckland, New Zealand, ${ }^{3}$ The Ohio State University Wexner Medical Center, Ohio State University, Columbus, OH, United States, ${ }^{4}$ Department of Engineering Science, University of Auckland, Auckland, New Zealand 
Despite the anisotropy of myocardium, previous cardiac MR elastography studies have investigated isotropic stiffness of heart tissue. Anisotropic material properties could provide a better understanding of structural changes that occur in the heart due to pathologies such as diastolic heart failure. However, optimal imaging parameters to measure anisotropic properties are yet unknown. This study investigates the optimal loading frequency that accurately recovers anisotropic stiffness measurements using simulations of cardiac MR elastography experiments in the presence of Gaussian noise and known fibre orientations. The optimised virtual fields method is used as an inversion method to translate harmonic displacements to stiffness parameters.

Integrated, 3D Printed Cost Effective Phantom solution for MR Imaging of Cardiac Structure and Function

Shivaprasad Ashok Chikop ${ }^{1}$, Amaresha Shridhar Konar ${ }^{1}$, Nithin Vajuvalli $^{1}$, Ramesh Venkatesan ${ }^{2}$, and Sairam Geethanath ${ }^{1,3}$

${ }^{1}$ Medical Imaging Research Centre, Dayananda Sagar Institutions, Bangalore, India, ${ }^{2}$ Healthcare, Wipro-GE, bangalore, India, ${ }^{3}$ Department of Radiology, Columbia University Medical Centre, New YOrk, NY, United States

An integrated cardiac phantom solution was developed to correlate with clinically relevant parameters entered through a user interface (UI). Mimicking of human heart was achieved through integration of a flexible 3D printed heart model and peristaltic pump. Results depict the correlation between the input parameters to output parameters obtained through image processing of the phantom MR images. The work illustrates the structural features and motion measures of the cardiac phantom. The phantom can therefore be employed to assess novel acquisition and reconstruction methods. The utilization of 3D printing enables the use of subject specific phantom to study diverse cardiovascular scenarios.

18F-FDG PET/MRI Allows Early Detection of Foam Cell Formation and Fat Deposition in Hemorrhagic Myocardial Infarctions

Ivan Cokic ${ }^{1}$, Jane Sykes ${ }^{2}$, John Butler ${ }^{2}$, Michael S Kovacs ${ }^{2}$, Hsin-Jung Yang ${ }^{2}$, Damini Dey ${ }^{1}$, Frank S Prato ${ }^{2}$, and Rohan Dharmakumar ${ }^{1}$

$3018 \quad{ }^{1}$ Cedars-Sinai Medical Center, Los Angeles, CA, United States, ${ }^{2}$ Lawson Health Research Institute, London, ON, Canada

Inability of macrophages (MФ) to switch from pro-inflammatory (M1, glycolytic) to anti-inflammatory (M2, oxidative) phenotype can lead to increased glucose transporter 1 (GLUT1)mediated glucose metabolism, decreased fatty acid (FA) beta oxidation, increased intracellular lipid accumulation, and M $\Phi$-to-foam cell transformation. Recent studies in the field of chronic venous leg ulcers have shown that iron-overloaded $M \Phi$ fail to switch from M1 to M2 phenotype. In this study we hypothesized that inability of iron-overloaded MФ to switch from M1 to M2 phenotype underlies fatty degeneration of hemorrhagic myocardial infarction via MФ lipid accumulation and their transformation into foam cells

A Least Squares Approach for Relative Pressure Measurement from 4D flow PC-MRI

Sina Hooshyar ${ }^{1,2}$, Sean Callahan ${ }^{2}$, MJ Negahdar ${ }^{2}$, Saeed Kermani ${ }^{1}$, and Amir Amini ${ }^{2}$

3019 'Biomedical Engineering, School of Advanced Technologies in Medicine, Isfahan University of Medical Science, Isfahan, Iran (Islamic Republic of), ${ }^{2}$ Electrical and Computer Engineering, University of Louisville, Louisville, KY, United States

Noninvasive determination of relative transstenotic pressure drop from 4D flow MRI has been investigated by a fast 3D matrix method based on the Least-Squares strategy. The method was tested with CFD velocity data as input as well as MRI phantom data. While results are comparable to the conventional pressure-poisson equation approach, the method is computationally more efficient.

Traditional Poster

\section{MR/PET}

Radiotracer dose reduction in 18F-FDG whole-body PET/MR: Effects on image quality and quantification

Maike E. Lindemann ${ }^{1}$, Vanessa Stebner ${ }^{2}$, Alexander Tschischka ${ }^{3}$, Julian Kirchner ${ }^{3}$, Lale Umutlu ${ }^{4}$, and Harald H. Quick ${ }^{1,5}$

${ }^{1}$ Highfield- and Hybrid MR Imaging, University Hospital Essen, Essen, Germany, ${ }^{2}$ Department of Nuclear Medicine, University Hospital Essen, Essen, Germany, ${ }^{3}$ Department of Diagnostic and Interventional Radiology, University Hospital Düsseldorf, Düsseldorf, Germany, ${ }^{4}$ Department of Diagnostic and Interventional Radiology and Neuroradiology, University Hospital Essen, Essen, Germany, ${ }^{5}$ Erwin L. Hahn Institute for Magnetic Resonance Imaging, Essen, Germany

The study goal is to investigate how the simulated reduction of injected radiotracer affects PET image quality and quantification in whole-body PET/MR in patients with oncologic findings. PET data of fifty-one patients was reconstructed with 4, 3, 2 and 1 minute/bed time interval. Image quality parameters were analyzed. As expected, the image quality decreases with shorter PET image acquisition times. Besides the two key factors acquisition time and injected activity, the image quality is influenced by the BMI. A lower BMI results in better image quality parameters. 2 minutes acquisition time per bed is sufficient to provide accurate lesion detection. 
Investigating the relationship between perfusion and glucose metabolism by simultaneous PET/MRI in frontotemporal dementia.

Rebecca M.E. Steketee ${ }^{1}$, Mariachiara Longarzo ${ }^{2}$, Vincenzo Alfano ${ }^{2}$, Carlo Cavaliere ${ }^{2}$, Dario Grossi ${ }^{3}$, Marion Smits ${ }^{1}$, and Marco Aiello ${ }^{2}$

${ }^{1}$ Radiology \& Nuclear Medicine, Erasmus MC - University Medical Center Rotterdam, Rotterdam, Netherlands, ${ }^{2}$ NAPLAB - SDN NeuroAnatomy and image Processing LABoratory, IRCCS SDN, Naples, Italy, ${ }^{3}$ Department of Psychology, University of Campania Luigi Vanvitelli, Caserta, Italy

Arterial spin labeling (ASL)-magnetic resonance imaging (MRI) and fluorodeoxyglucose (FDG)-positron emission tomography (PET) both have diagnostic value for dementia, particularly frontotemporal dementia (FTD). By using simultaneous FDG-PET/ASL-MRI, we investigated the relationship between brain metabolism and perfusion in FTD, to evaluate their suitability and complementarity. Exploratory analysis of simultaneous FDG-PET/ASL-MRI in 15 dementia patients showed that metabolism and CBF correlate well on a global level, both visually and quantitatively. On a regional level, one-on-one correlations are limited, supposedly to disease-specific regions such as frontotemporal, subcortical and parietal regions. These results will be substantiated in a larger and better differentiated dementia cohort.

An Evaluation of Radial GRE Attenuation Correction Maps for Cardiac and Coronary PET-MRI Studies

Gillian Macnaught ${ }^{1,2}$, Jack Andrews ${ }^{2}$, David Brian ${ }^{1}$, Kenneth Dolan $^{1}$, Philip M. Robson ${ }^{3}$, Zahi A. Fayad ${ }^{3,4}$, Tim P Clark $^{1,5}$, Alison Fletcher ${ }^{1,5}$, Matthias Fenchel $^{6}$, Scott Semple ${ }^{1,2}$, Edwin J.R. van Beek ${ }^{1}$, David E. Newby ${ }^{1,2}$, and Marc R. Dweck ${ }^{2}$

${ }^{1}$ Edinburgh Imaging facility QMRI, The University of Edinburgh, Edinburgh, United Kingdom, ${ }^{2}$ The British Heart Foundation/University of Edinburgh Centre for Cardiovascular Sciences, The University of Edinburgh, Edinburgh, United Kingdom, ${ }^{3}$ Translational and Molecular Imaging Institute, Icahn School of Medicine at Mount Sinai, New York, NY, United States, ${ }^{4}$ Cardiovascular Institute, Icahn School of Medicine at Mount Sinai, New York, NY, United States, ${ }^{5}$ Department of Nuclear Medicine, NHS Lothian, Edinburgh, United Kingdom, ${ }^{6}$ Siemens Healthcare $\mathrm{GmbH}$, Erlangen, Germany

MR-based attenuation correction of PET images is essential for PET-MRI studies. An intensity threshold method for creating attenuation correction maps ( $\mu$ maps) from 3D golden-angle radial spoiled gradient echo (radial GRE) images is presented. PET reconstructions using the Threshold $\mu$ maps, an existing radial GRE method for creating $\mu$ maps and the manufacture Dixon VIBE $\mu$ maps are compared for quantification of ${ }^{18} \mathrm{~F}$ Sodium Fluoride $\left({ }^{18} \mathrm{~F} \mathrm{NaF}\right)$ uptake in the aorta. Radial GRE $\mu$ maps better delineate the trachea and heart-lung boundaries. Dixon $\mu$ maps produced PET images with significantly lower aorta wall SUVmax values than radial GRE $\mu$ maps. $\mu$ maps must be characterised prior to implementation.

Multi-contrast MRI Enhance Ultra-low-dose PET Reconstruction

Junshen $\mathrm{Xu}^{1,2}$, Enhao Gong ${ }^{2,3}$, Mehdi Khalighi ${ }^{4}$, John Pauly ${ }^{2}$, and Greg Zaharchuk ${ }^{3}$

${ }^{1}$ Engineering Physics, Tsinghua University, Beijing, China, ${ }^{2}$ Electrical Engineering, Stanford University, Stanford, CA, United States, ${ }^{3}$ Radiology, Stanford University, Stanford, CA, United States, ${ }^{4}$ GE Healthcare, Menlo Park, CA, United States

Simultaneous PET/MRI is a powerful multimodality imaging technique for both anatomical and functional imaging. Here we propose a novel method for high-quality PET image reconstruction from ultra-low-dose (more than $99 \%$ reduction compared to current practice) PET scanning by using multi-contrast-MRI. A multi-scale fully convolutional network was developed for solving the reconstruction. The proposed method is compared with other methods on a Glioblastoma(GBM) clinical dataset. Results show that our method achieves superior image quality compared with state-of-the-art methods in low-dose PET reconstruction. Besides, quantitative and qualitative evaluations indicate that multi-contrast MRI significantly improves the reconstruction quality with better structural details.

A Voxelwise Analysis of PET/MR DATA towards Characterization of Prostate Cancer

Yachao Liu ${ }^{1}, \mathrm{Mu} \mathrm{Lin}^{2}, \mathrm{Xu} \mathrm{Yan^{2 }}$, and Baixuan Xu${ }^{1}$

${ }^{1}$ PLA 301 General Hospital, Nuclear Medicine Department, Beijing, China, ${ }^{2}$ Siemens Healthcare, MR Collaborations NE Asia, Shanghai, China

The combined use of diffusion-weighted and 11C-Choline PET images can provide complementary information on prostate cancer. However, it is still unknown how to combine these multiple parameters to give a simple indication for malignant lesions. Based on a scatterplot analysis of standardized uptake values (SUVs) and apparent diffusion coefficient (ADC) values, we clustered voxels into groups corresponding to different tissue types. The proposed method shows promising results in differentiating the lesion of tumor from normal tissue.

ADC-corrected SUV derived from voxel-based SUV-ADC scatter plots for the evaluation of soft-tissue tumor treatment response in FDG-PET/MR hybrid imaging

Sungtak Hong ${ }^{1}$, Yuji Watanabe ${ }^{2}$, Daiki Shinyama ${ }^{1}$, Keisuke Ishimatsu $^{3}$, Koji Sagiyama ${ }^{3}$, and Hiroshi Honda ${ }^{3}$

${ }^{1}$ Philips Japan, Tokyo, Japan, ${ }^{2}$ Department of Molecular Imaging and Diagnosis, Kyushu University, Fukuoka, Japan, ${ }^{3}$ Department of Clinical Radiology, Kyushu University, Fukuoka, Japan 
It is often difficult to quantify tumor treatment response with SUVmax because a single voxel measurement does not always represent a whole tumor. In this study, we developed a new parameter called cellular SUV (cSUV) from the SUV-ADC scatter plots. Cluster analysis also applied to the cSUV-measurement of a tumor consisting of multiple components such as liposarcoma, necrotic tumor after treatment, etc. The percent change in cSUV between pre- and post-treatment correlated better with the RECIST1.0 assessment than that of SUVmax. The cSUV combined with cluster analysis could be a promising bio-imaging marker for monitoring treatment response of soft-tissue tumors.

Hybrid Liver Multiparametric MRI and F18-FDG PET/MR in Diagnosing and Staging of Intrahepatic Cholangiocarcinoma: An initial Experience

Ming Yang ${ }^{1}$, Alvin C. Silva ${ }^{1}$, Mitesh J. Borad ${ }^{2}$, Andrew E. Liguori ${ }^{1}$, Anshuman Panda ${ }^{1}$, Ba D. Nguyen ${ }^{1}$, Thomas DeLeon ${ }^{2}$, Michael C. Roarke ${ }^{1}$, and Yuxiang Zhou

${ }^{1}$ Radiology, Mayo Clinic in Arizona, Scottsdale, AZ, United States, ${ }^{2}$ Hematology/Oncology, Mayo Clinic in Arizona, Scottsdale, AZ, United States

Intrahepatic cholangiocarcinoma (ICC) is an uncommon biliary tract malignancy with an unfavorable prognosis given its complicated clinical and imaging manifestations. In this preliminary study, we investigated the role of hybrid liver mpMRI and F18-FDG PET/MR in diagnosing and staging of ICC. Our preliminary data show promising value of this "one-stop" imaging modality in providing complementary morphological and functional information in detecting viable tumor burden, defining nodal and distant metastasis utilizing both MRI and PET molecular imaging biomarkers.

A Motion Correction Method Based on Navigator for Simultaneous PET/MR abdominal Imaging

Ke Meng ${ }^{1,2,3}$, Lingzhi $\mathrm{Hu}^{4,5}$, and Qun $\mathrm{Chen}^{5}$

${ }^{1}$ Shanghai Advanced Research Institute, Shanghai, China, ${ }^{2}$ University of Chinese Academy of Sciences, Beijing, China, ${ }^{3}$ Shanghai Tech University, Shanghai, China, ${ }^{4}$ UIH America Inc, Houston, TX, United States, ${ }^{5}$ United Imaging Healthcare, Shanghai, China

The integrated PET/MR combines the advantages of functional imaging device PET and high resolution high contrast MRI, simultaneously acquiring PET and MR images at the same position, improving image fusion accuracy. However, respiratory motion during abdominal imaging causes notorious motion artifact in the MRI images and blurring the PET images. A PET/MR motion correction method based on real-time 2D excitation navigator has been implemented and evaluated. Phantom and human imaging result implies that this technique can precisely acquire object motion and effectively eliminate motion blurring. Without additional operation and device, it offers a simple and cost-down way for clinical use.

Truly simultaneous preclinical PET-MRI in a $20 \mathrm{~cm} 9.4$ Tesla magnet with a retrofitted miniature detector: Initial results in the twitcher mouse model of Krabbe disease Ferdinand Schweser ${ }^{1,2}$, Akshay V Dhamankar ${ }^{1}$, Poonam Choudhary ${ }^{1,3}$, Nadav Weinstock ${ }^{4,5}$, Cheryl Knapp ${ }^{2}$, Marilena Preda ${ }^{1,2}$, Daesung Shin ${ }^{4,5}$, Robert Zivadinov $^{1,2}$, and Lawrence Wrabetz ${ }^{4,5,6}$

${ }^{1}$ Buffalo Neuroimaging Analysis Center, Department of Neurology, Jacobs School of Medicine and Biomedical Sciences, University at Buffalo, The State University of New York, Buffalo, NY, United States, ${ }^{2}$ Center for Biomedical Imaging, Clinical and Translational Science Institute, University at Buffalo, The State University of New York, Buffalo, NY, United States, ${ }^{3}$ Department of Medical Physics, University at Buffalo, The State University of New York, Buffalo, NY, United States, ${ }^{4}$ Hunter James Kelly Research Institute, Jacobs School of Medicine and Biomedical Sciences, University at Buffalo, The State University of New York, Buffalo, NY, United States, ${ }^{5}$ Department of Biochemistry, Jacobs School of Medicine and Biomedical Sciences, University at Buffalo, The State University of New York, Buffalo, NY, United States, ${ }^{6}$ Department of Neurology, Jacobs School of Medicine and Biomedical Sciences, University at Buffalo, The State University of New York, Buffalo, NY, United States

While the potential of PET-MRI is increasingly being explored in the clinical setting, preclinical PET-MRI is only slowly leaving the proof-of-concept stage, which may be explained by technical difficulties due to the size-constraints and strong magnetic fields used in preclinical MRI. In the current work, we present results from a first in vivo application of ${ }^{18} \mathrm{~F}$-FDG PETMRI using a retrofitted micro-PET detector in a commercial $9.4 \mathrm{~T}$ magnet. We studied the twitcher mouse model of Krabbe disease, in which an altered glucose metabolism had been suggested.

Assessment of Metastatic Lymph Node in Head and Neck Cancer Using Simultaneous 18F-FDG-PET and DCE-MRI

Akshay Wadera ${ }^{1,2,3}$, Mari Hagiwara ${ }^{4}$, Roy Raad ${ }^{4}$, Kent Friedman ${ }^{4}$, Brian Schmidt ${ }^{5}$, Babak Givi ${ }^{5}$, Adam Jacobson ${ }^{5}$, Theresa Tran ${ }^{5}$, Mark DeLacure ${ }^{5}$, Cheng Z. Liu ${ }^{6}$, Elcin Zan ${ }^{4}$, and S. Gene $\mathrm{Kim}^{1,2}$

${ }^{1}$ Center for Advanced Imaging Innovation and Research (CAI2R), Radiology, NYU School of Medicine, New York, NY, United States, ${ }^{2}$ Center for Biomedical Imaging, Radiology, NYU, NYU School of Medicine, New York, NY, United States, ${ }^{3}$ New York Medical College, Valhalla, NY, United States, ${ }^{4}$ Department of Radiology, NYU School of Medicine, New York, NY, United States, ${ }^{5}$ Otolaryngology-Head and Neck Surgery, NYU School of Medicine, New York, NY, United States, ${ }^{6}$ Pathology, NYU School of Medicine, New York, NY, United States

Regional lymph node metastasis is one of the important predictors of poor prognosis in head and neck cancer. Detecting small nodes with micro-metastases remains challenging for currently available diagnostic imaging methods, including positron emission tomography with 18F-fluorodeoxyglucose (18F-FDG PET) and dynamic contrast enhanced magnetic resonance imaging (DCE-MRI). The purpose of this study is to demonstrate the synergistic role of FDG PET and DCE-MRI in detecting lymph nodes with metastatic potential. Our preliminary results demonstrate that the combined modeling of MR and FDG PET kinetic parameters has the potential to detect lymph node microenvironment changes and assess potentially metastatic lymph nodes. 
Rasmus Birn ${ }^{1}$, Samuel Anthony Hurley ${ }^{2,3}$, Abigail Z Rajala² ${ }^{2}$ Caitlynn N Filla², Austin M Patrick ${ }^{4}$, Dillon J Gwozdz ${ }^{2}$, Walter F Block ${ }^{4,5}$, Andrew L Alexander $1,4,6$, Alexander K Converse ${ }^{6}$, Rick L Jenison ${ }^{7}$, Bradley T Christian ${ }^{4}$, Alan B McMillan ${ }^{3}$, and Luis C Populin²

${ }^{1}$ Psychiatry, University of Wisconsin, Madison, WI, United States, ${ }^{2}$ Neuroscience, University of Wisconsin, Madison, WI, United States, ${ }^{3}$ Radiology, University of Wisconsin, Madison, WI, United States, ${ }^{4}$ Medical Physics, University of Wisconsin, Madison, WI, United States, ${ }^{5}$ Biomedical Engineering, University of Wisconsin, Madison, WI, United States, ${ }^{6}$ Waisman Center, University of Wisconsin, Madison, WI, United States, ${ }^{7}$ Psychology, University of Wisconsin, Madison, WI, United States

Higher-order cognitive functions result from dynamic interactions of distributed networks comprised of anatomically, physiologically, and pharmacologically separate components of the nervous system. To further our understanding the basic mechanisms and functions of such networks, as well as how they are affected by the administration of therapeutic drugs, we have developed a PET-fMRI platform to take simultaneous measurements of neural activity (fMRI), and concentration of dopamine (PET) during the same physiological state, and without the confounding effects of anesthetics. With this platform, we have measured for the first time in a primate brain the effects of administering different doses of methylphenidate on extracellular levels of dopamine and functional connectivity.

Neurovascular coupling to D2/D3 partial agonist antipsychotic drug occupancy using simultaneous PET/fMR

Christin Y Sander ${ }^{1,2}$, Bruce R Rosen ${ }^{1,2}$, and Joseph B Mandeville ${ }^{1,2}$

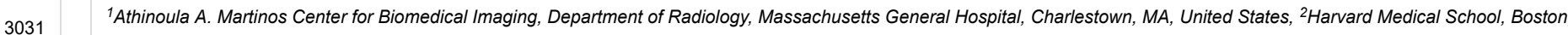
MA, United States

Drug-receptor interactions are the basis of signal modulation in the brain, yet, in vivo mechanisms of the action of many drugs are not well understood. In this study, we characterize the in vivo profile of a current third generation antipsychotic drug at the D2/D3 dopamine receptor using simultaneous PET and fMRI. The results are compared to full D2/D3 antagonists and agonists profiles and show that functional differences can be distinguished with occupancy-matched fMRI responses.

Feasibility study of multinuclear MR at 9.4T and PET in a rat brain tumour mode

Chang-Hoon Choi ${ }^{1}$, Carina Stegmayr ${ }^{1}$, Aliaksandra Shymanskaya ${ }^{1}$, Wieland A. Worthoff ${ }^{1}$, Nuno A da Silva ${ }^{1}$, Jörg Felder ${ }^{1}$, Karl-Josef Langen ${ }^{1,2}$, and N. Jon Shah ${ }^{1,3}$

${ }^{1}$ Forschungszentrum Juelich, Juelich, Germany, ${ }^{2}$ Department of Nuclear Medicine and Neurology, RWTH Aachen University Hospital, Aachen, Germany, ${ }^{3}$ Faculty of Medicine, Department of Neurology, RWTH Aachen University, JARA, Aachen, Germany

Multinuclear MR provides important information concerning cell integrity or energy metabolism. PET uses radioactive tracers to gain valuable insights into physiological and metabolic processes with both a high level of sensitivity and specificity. Here, we explored the combination of sequential multinuclear MR and PET in a rat brain tumour model. This allows in vivo multinuclear MR-PET experiments to be carried out without compromising the performance of either multinuclear MR or PET. In vivo multinuclear MR and PET images and spectra from rats with/without brain tumours confirmed the potential use of the different X-nuclei derived metabolic information.

Traditional Poster

\section{Molecular Imaging}

Smart thermosensitive liposomes for effective solid tumor therapy with MRI tracking at 21.1 T

Jens T. Rosenberg ${ }^{1}$, Kevin Affram² ${ }^{2}$, Ofonime Udofot ${ }^{2}$, Mandip Singh ${ }^{2}$, Sunil Krishnan ${ }^{3}$, Renee Reams ${ }^{2}$, and Edward Agyare ${ }^{2}$

${ }^{1}$ The National High Magnetic Field Laboratory, Florida State University, Tallahassee, FL, United States, ${ }^{2}$ College of Pharmacy and Pharmaceutical Sciences, Florida A\&M University, Tallahassee, FL, United States, ${ }^{3}$ University of Texas MD Anderson Cancer Center, Houston, TX, United States

Here we show the ability of using Gadolinium (Gd) labeled thermosensitive liposomal nanoparticle (TSLnp) as a delivery system for anticancer drug, gemcitabine (Gem) to human pancreatic tumors. Pancreatic cancer ( $\mathrm{PCa}$ ) due to its high malignancy, poor prognosis and resistance to chemotherapy is one of the leading cancer-associated death in the United States. The proposed agent showed significant Gem accumulation in heated tumor relative to free Gem. Gd labeled TSLnp (Gd-TSLnp) show contrast in ex vivo tumor tissue. The GdTSLnp show increased $\mathrm{T}_{1}$ contrast in vivo with an implanted tumor compared to $\mathrm{Gd}$ and targets the tumor tissue. 
${ }^{1}$ Chemistry, Lakehead University, Thunder Bay, ON, Canada, ${ }^{2}$ Chemistry, University of Rhode Island, Kingston, RI, United States, ${ }^{3}$ Thunder Bay Regional Health Research Institute, Thunder Bay, ON, Canada, ${ }^{4}$ Biology, Northern Ontario School of Medicine, Thunder Bay, ON, Canada

We have recently advanced the field of hyperpolarized (HP) ${ }^{129}$ Xe magnetic resonance imaging (MRI) with the in vivo detection of cucurbit[6]uril (CB6), a highly sensitive MR contrast agent. CB6 is biochemically inactive, which makes its natural bio-distribution non-specific; thus, it cannot be precisely localized within a living mammalian body using HP ${ }^{129}$ Xe MRI. We have previously identified cyclodextrin-based pseudorotaxanes as conjugatable scaffolds for xenon biosensors; in this work, we introduce a second class of conjugatable scaffolds, with the hyperCEST detection of benzene-appended CB6, a potential precursor to a wide variety of targeted molecular imaging probes.

Prospects of ${ }^{31} \mathrm{P}$ Contrast Media for ${ }^{31} \mathrm{P}-\mathrm{MRS}$

Louise R. Tear ${ }^{1,2}$, Mahon L. Maguire ${ }^{2}$, Gogulan Karunanithy ${ }^{3}$, Deborah Sneddon ${ }^{4}$, Nicola J. Farrer ${ }^{1}$, Andrew Baldwin ${ }^{3}$, Stephen Faulkner ${ }^{1}$, and Jurgen E. Schneider ${ }^{2,5}$

${ }^{1}$ Chemistry Research Laboratory, University of Oxford, Oxford, United Kingdom, ${ }^{2}$ BHF Experimental MR Unit (BMRU), University of Oxford, Oxford, United Kingdom, ${ }^{3}$ Physical and Theoretical Chemistry Laboratory, University of Oxford, Oxford, United Kingdom, ${ }^{4}$ Radiobiology Research Institute, University of Oxford, Oxford, United Kingdom, ${ }^{5}$ Leeds Institute of Cardiovascular and Metabolic Medicine, University of Leeds, Leeds, United Kingdom

${ }^{31} \mathrm{P}$-MRS can be used to determine the relative ratios of phosphate species in vivo to aid clinical diagnosis, but is limited by poor SNR and long acquisition times associated with the ${ }^{31} \mathrm{P}$ nucleus. This work investigates the potential of ${ }^{31} \mathrm{P} \mathrm{T}_{1}$ contrast agents based on Gd.DO3A derivatives by using ${ }^{31} \mathrm{P}$-MRS. These compounds demonstrate significant relaxation enhancement of ${ }^{31} \mathrm{P} \mathrm{R}_{1}$ for ATP, PCr and $\mathrm{P}_{\mathrm{i}}$, therefore showing excellent potential as ${ }^{31} \mathrm{P}$ contrast agents. Cell studies indicate the Gd.DO3A derivatives investigated do not come into contact with intracellular phosphate metabolites, which limits these initial complexes to use as extracellular contrast agents.

Dysprosium based liver-specific ultra-high field MRI T2 contrast agent

Ah Rum Baek ${ }^{1}$, Heekyung Kim²,3, Soyeon Kim ${ }^{1}$, Garam Choi ${ }^{1}$, Bokyung Sung ${ }^{1}$, MD. Kamrul Islam ${ }^{1}$, Taekwan Lee ${ }^{4}$, DongKyu Kim ${ }^{4}$, Hoesu jung ${ }^{4}$, and Yongmin Chang ${ }^{1,2,5}$

${ }^{1}$ Department of Medical \&Biological Engineering, Kyungpook National Univesity, Daegu, Korea, Democratic People's Republic of, ${ }^{2}$ Department of Molecular Medicine \& BK21 Plus KNU Biomedical Convergence Program, Kyungpook National Univesity, Daegu, Korea, Democratic People's Republic of, Institute of Biomedical Engineering Research, Kyungpook National University, Daegu, Korea, Democratic People's Republic of, ${ }^{4}$ Laboratory Animal Center, Daegu-Gyeongbuk Medical Innovation Foundation, Daegu, Korea, Democratic People's Republic of, ${ }^{5}$ Department of Radiology, Kyungpook National Univesity, Daegu, Korea, Democratic People's Republic of

[Dy(EOB-DO3A)] is prepared according to the general synthetic methods, and characterized by spectroscopic analysis. Therelaxivities that measured at $9.4 \mathrm{~T}$ animal MRI are $r_{l}=1.01, r_{2}=2.80 \mathrm{mM}^{-1} \mathrm{~s}^{-1}$. We observe acceptablenegative-enhancement with liver $\mathrm{T}_{2}$-weighted image, alsoconfirm about $30 \%$ liver accumulation within $1 \mathrm{~h}$ post-injection at inductively coupled plasma (ICP) spectrometer data.

A Hyperpolarized 129Xe "OFF-ON" MRI Biosensor Triggered by Diamine Oxidase

Bin Zhang ${ }^{1}$, Qing Luo ${ }^{2}$, Qianni Guo ${ }^{2}$, Xiaoxiao Zhang ${ }^{1}$, Qingbin Zeng ${ }^{2}$, Longhui Zhao ${ }^{2}$, Yaping Yuan ${ }^{2}$, Weiping Jiang ${ }^{2}$, Chaohui Ye ${ }^{2}$, and Xin Zhou ${ }^{2}$

${ }^{1}$ State Key Laboratory of Magnetic Resonance and Atomic and Molecular Physics, National Center for Magnetic Resonance in Wuhan, Wuhan Institute of Physics and Mathematics, Chinese Academy of Sciences, 430071, Wuhan China-Wuhan National Laboratory for Optoelectronics, Huazhong University of Science and Technology (HUST), 430074, Wuhan, China, ${ }^{2}$ State Key Laboratory of Magnetic Resonance and Atomic and Molecular Physics, National Center for Magnetic Resonance in Wuhan, Wuhan Institute of Physics and Mathematics, Chinese Academy of Sciences, 430071, Wuhan, China

Benefiting from ultra-high sensitivity of Hyper-CEST method, ${ }^{129}$ Xe biosensors possess an obvious advantage in sensitivity over other MRI sensors. However, due to its indirect detection mode the Hyper-CEST spectra resolution is relatively low limiting chemical shift to be an effective indicator in traditional NMR. In order to solve this problem, a ${ }^{129}$ Xe biosensor based on a new "turn-on" strategy is designed, which exhibits high detection specificity for an enzyme diamine oxidase (DAO). This ${ }^{129}$ Xe biosensor possesses very high detection sensitivity, and can be tested in Small intestinal villus epithelial cells. Using this strategy, lots of disease-related enzyme can be detected.

Fluorine-19 MRI hot-spot imaging of lung metastasis in rodents

Deanne Lister ${ }^{1}$, Hongyan $\mathrm{Xu}^{1}$, and Eric T Ahrens ${ }^{1}$

${ }^{1}$ Radiology, University of California, San Diego, CA, United States

Lung cancer is the leading cause of cancer deaths. Safe and specific MRI probes are needed to enable early detection of lesion presence and therapeutic response. Injected PFC nanoemulsion, taken up by tumor associated macrophages (TAMs), can be used as a biomarker to detect metastases using ${ }^{19} \mathrm{~F}$ MRI. In a metastatic lung cancer mouse model, we show that PFC is effectively taken up by TAMs and vividly displays lung metastasis using ${ }^{19} \mathrm{~F}$ MRI. Validation assays using in vivo bioluminescence and histology support the MRI findings. Overall, ${ }^{19} \mathrm{~F}$ hot-spot imaging offers a highly-specific marker of tumor burden in lung parenchyma. 
A Small Molecule NIR-19F MR Contrast Agent of Aza-BODIPY for Bimodal In Vivo Imaging

Lianhua Liu ${ }^{1}$, Yaping Yuan ${ }^{1}$, Yuqi Yang ${ }^{1}$, McMahon T. Michael ${ }^{2,3}$, Shizhen Chen ${ }^{1}$, and Xin Zhou ${ }^{1}$

${ }^{1}$ State Key Laboratory of Magnetic Resonance and Atomic and Molecular Physics, Wuhan Institute of Physics and Mathematics, Chinese Academy of Sciences-Wuhan National Laboratory for Optoelectronics, Wuhan, China, ${ }^{2}$ Russell H. Morgan Department of Radiology and Radiological Science, The Johns Hopkins University School of Medicine, Baltimore, MD, United States, ${ }^{3}$ F.M. Kirby Research Center for Functional Brain Imaging, Kennedy Krieger Institute, Baltimore, MD, United States

Accurate and early diagnosis of diseases is most import for medical imaging. MRI is one of the most promising techniques for the non-invasive visualization. Compared to ${ }^{1} \mathrm{H}$ MRI, ${ }^{19} \mathrm{~F}$ MRI provides high-contrast images without endogenous background signals, but low sensitivity. To address the limitation, our strategy is to combination of ${ }^{19} \mathrm{~F}$ MRI and a more sensitive NIR fluorescence imaging technique to develop a bimodal contrast agent BDPF. Both ex vivo and in vivo experimental results indicated BDPF had excellent optical and ${ }^{19} \mathrm{~F}$ MRI properties. Thus, the NIR- ${ }^{19} \mathrm{~F}$ MR bimodal imaging may provide a new way to detect tumor.

Manganese enhanced MRI in organotypic rat hippocampus slices: A correlative study with synchrotron X-ray nanoprobe analysis and electron microscopy.

Alexia Daoust ${ }^{1}$, Natalia B Pivovarova ${ }^{1}$, Emily Petrus ${ }^{1}$, S Brian Andrews ${ }^{1}$, Barry Lai $^{2}$, Si Chen $^{2}$, Maria A Aronova ${ }^{3}$, Richard D Leapman $^{3}$, and Alan P Koretsky ${ }^{1}$

${ }^{1}$ National Institute of Neurological Disorders and Stroke, National Institutes of Health, Bethesda, MD, United States, ${ }^{2}$ Argonne National Laboratory, Advanced Photon Source, Argonne, IL, United States, ${ }^{3}$ National Institute of Biomedical Imaging and Bioengineering, National Institutes of Health, Bethesda, MD, United States

Manganese $(\mathrm{Mn2}+)$ Enhanced Magnetic Resonance Imaging (MEMRI) can be used for different applications such as tracing neuronal connections or functional imaging. However, Mn ${ }^{2+}$ uptake and transport mechanisms are still unclear. These mechanisms were studied by imaging sub-cellular Mn2+ in an organotypic hippocampal slice culture by coupling MEMRI, TEM and $\mathrm{X}$-ray methodologies. The data indicates that $\mathrm{Mn}^{2+}$ is located at synapses but not in mitochondria.

Camelid single-domain antibodies bioconjugate for the magnetic resonance imaging of Alzheimer's disease.

Clémence Dudeffant ${ }^{1}$, Matthias Vandesquille ${ }^{2}$, Tengfei Li ${ }^{3}$, Christelle Ganneau ${ }^{2}$, Ihsen Youssef ${ }^{3}$, Benoît Delatour ${ }^{3}$, Pierre Lafaye ${ }^{4}$, Sylvie Bay $^{2}$, and Marc Dhenain ${ }^{1}$

${ }^{1}$ Molecular Imaging Research Center (MIRCen), Commissariat à l'énergie atomique et aux énergies alternatives (CEA), Fontenay aux Roses, France, ${ }^{2}$ Unité de Chimie des Biomolécules, Institut Pasteur, Paris, France, ${ }^{3}$ Institut du cerveau et de la moelle épinière (ICM), Paris, France, ${ }^{4}$ Plateforme d'Ingénierie des Anticorps, Institut Pasteur, Paris, France

Detection of intracerebral targets with imaging probes is challenging due to the non-permissive nature of blood-brain barrier (BBB). Camelid single-domain antibody-fragments (VHH) are small and stable antibodies able to potentially cross the BBB. Here, we selected VHH specifically targeting amyloid-beta deposits, one of the main lesions of Alzheimer's disease and labeled them with the contrastophore gadolinium. These innovative contrast agents allowed MRI detection of amyloid deposits in postmortem brain tissues of a mouse model of amyloidosis. The ability to produce $\mathrm{VHH}$ conjugates that cross the BBB opens the way for future development of tailored imaging probes targeting intracerebral antigens.

Investigating Off-Resonance Fat Modulations in the TurboSPI Signal to Improve R2* Mapping for Quantitative Cell Tracking

Zoe O'Brien-Moran ${ }^{1,2}$, Chris V Bowen ${ }^{1,2}$, James A Rioux ${ }^{* 1,2}$, and Kimberly D Brewer ${ }^{* 1,2}$

${ }^{1}$ Biomedical Translational Imaging Centre (BIOTIC), Halifax, NS, Canada, ${ }^{2}$ Physics, Dalhousie University, Halifax, NS, Canada

TurboSPI has the potential to offer quantitative cell tracking with high fidelity $\mathrm{R}_{2^{*}}$ mapping. However, early in vivo studies demonstrated that accuracy of the $\mathrm{R}_{2^{*}}$ fitting deteriorates in the presence of off-resonance fat signal. In this work, we investigate these findings further with an in vitro study. We used in silico and in vitro data to develop and test a more comprehensive decay model that accounts for fat oscillations in the TurboSPI signal. The proposed model results in improved $R_{2^{\star}}$ estimates in the presence of fat.

Improved Sensitivity of Cellular MRI Using Phase-cycled Balanced SSFP of Ferumoxytol Nanocomplex Labeled Macrophages at Ultra-high Field

Yelong Shen ${ }^{1,2}$, Lirong Yan ${ }^{1}$, Xingfeng Shao ${ }^{1}$, Bin Zhao ${ }^{2}$, Jinlun Bai ${ }^{3}$, Wange $\mathrm{Lu}^{3}$, and Danny JJ Wang ${ }^{1}$

${ }^{1}$ Laboratory of FMRI Technology (LOFT), Mark \& Mary Stevens Neuroimaging and Informatics Institute, Keck School of Medicine, University of Southern California (USC), Los Angeles, CA, United States, ${ }^{2}$ Shandong Medical Imaging Research Institute, School of Medicine, Shandong University, Jinan, China, ${ }^{3}$ Broad Stem Cell Institute, Keck School of Medicine, University of Southern California (USC), Los Angeles, CA, United States

This study aimed to investigate the feasibility and sensitivity of cellular MRI with ferumoxytol nanocomplex labeled macrophages at ultrahigh magnetic field of 7T. Different labeling strategies, labeling times, magnetic field strengths, imaging sequences and post processing methods were evaluated to achieve the optimal protocol. Combining ferumoxytol, heparin and protamine (HFP nanocomplex) labeled macrophages with balanced steady-state free precession (bSSFP) sequence on a 7T MRI scanner and post processed by root mean square (RMS) combination of multiple phases showed the best contrast in phantom and ex vivo experiments, reaching a sensitivity for detecting a few tens of cells. 
Unexpected accumulation of iron in liver of immune compromised mice: Implications for cell tracking experiments

Christiane Mallett ${ }^{1,2}$, Matti Kiupel ${ }^{3}$, and Erik Shapiro ${ }^{1,2}$

${ }^{1}$ Radiology, Michigan State University, East Lansing, MI, United States, ${ }^{2}$ Institute for Quantitative Health Science and Engineering, Michigan State University, East Lansing, MI, United States, ${ }^{3}$ Veterinary Diagnostic Laboratory, Michigan State University, East Lansing, MI, United States

An increased iron load was observed in immune-deficient mice which may mean that they are not suitable for iron oxide based cell tracking experiments. This was not seen in healthy controls fed a similar diet. It was resolved by feeding a low-iron diet.

Stem Cell Tracking Using Effective Self-Assembled Peptide-Modified Superparamagnetic Nanoparticles

Lei $\mathrm{Gu}^{1}$ and $\mathrm{Min} \mathrm{Wu}^{2}$

${ }^{1}$ Huaxi MR Research Center (HMRRC), Sichuan University, ChengDu, China, ${ }^{2}$ Department of Radiology, Molecular Imaging Program at Stanford (MIPS), Stanford University, Stanford, $C A$, United States

In cell therapies and regeneration medicine, superparamagnetic iron oxide nanoparticles (SPIONs) have been developed as excellent magnetic resonance imaging (MRI) contrast agents for stem cell labeling and tracking due to their biocompatibility. Here, we designed a self-assembled peptide amphiphile (PA) replace the transfection agents. This PA was conjugated to the surfaces of SPIONs to label rat mesenchymal stem cells (MSCs), which enhanced the contrast and labeling effects. The labeled cells showed that peptide-SPIONs had improved internalization, efficiency and $T_{2}$-weight relaxivity and were nontoxic to the MSCs. The results demonstrated that these self-assembled peptide-modified SPIONs are potential candidates to label MSCs for tracking stem cells using MRI in vivo.

A proof-of-concept study on the quantification of gene expression levels with doxycycline-inducible MR reporter gene

Seul-I Lee ${ }^{1}$, Jeeheon Kang ${ }^{1}$, Yoonseok Choi ${ }^{2}$, Jinil Kim ${ }^{1}$, Jae-Im Kwon ${ }^{1}$, Ho-jin Kim ${ }^{1}$, Su Jung Ham ${ }^{3}$, Sang-Tae Kim ${ }^{1}$, Chul Woong Woo ${ }^{1}$, Do-Wan Lee ${ }^{1}$, Dong-Cheol Woo ${ }^{1}$, and Kyung Won $\mathrm{Kim}^{3}$

${ }^{1}$ MR Core, Asan medical Center, Seoul, Republic of Korea, ${ }^{2}$ GangNeung Asan Medical Center, GangNeung, Republic of Korea, ${ }^{3}$ Radiology, Asan medical Center, Seoul, Republic of Korea

Recent research on MR reporter genes has demonstrated their potential for use in transgene expression monitoring. We have conducted a preliminary study on the development of a new MRI reporter system [organic anion transporting polypeptide (OATP) 1B1] that can analyze gene expression level using MR reporter genes. By establishing doxycycline-inducible cell line, we observed T1 shortening on MRI, which indicated increased expression level of the OATP1B1 gene. A strong correlation was observed between conventional methods for measurement of gene expression and rrT1 of MR imaging. In this study, we provide preliminary evidence of the potential application of MRI to determine gene expression.

GMP-grade nanoparticle imaging agent for 19F MR, photoacoustic, and fluorescence imaging

Edyta Swider ${ }^{1}$, Khalid Daoudi ${ }^{2}$, Eric van Dinther ${ }^{1}$, Alexander H. J. Staal ${ }^{1}$, N. Koen van Riessen ${ }^{1}$, Olga Koshkina ${ }^{1}$, I. Jolanda M. de Vries ${ }^{1,3}$, Chris L. de Korte ${ }^{2}$, and Mangala Srinivas ${ }^{1}$

${ }^{1}$ Tumor Immunology, Radboud Institute for Molecular Life Sciences, Nijmegen, Netherlands, ${ }^{2}$ Radiology and Nuclear Medicine, Radboud University Medical Center, Nijmegen, Netherlands, ${ }^{3}$ Medical Oncology, Radboud University Medical Center, Nijmegen, Netherlands

Cellular therapies hold great promise for the treatment of various diseases. Its success strongly depends on the imaging modality and cell tracking, which can be achieved by the addition of an imaging label to cells, for example in the form of nanoparticles. Here, we report on polymeric nanoparticles encapsulating perfluorocarbon and dye, which can be used for cell loading and can be detected with several imaging modalities. This will further give information about cell numbers and localization in vivo.

An Improved CEST MRI Reporter Gene for Molecular Imaging of Cell and Viral Based Therapeutics

Christian T. Farrar ${ }^{1}$, Hirotaka Ito ${ }^{2}$, Hiroshi Nakashima ${ }^{2}$, E. Antonio Chiocca ${ }^{2}$, and Assaf A. Gilad ${ }^{3,4,5}$

${ }^{1}$ Martinos Center for Biomedcial Imaging, Department of Radiology, Massachusetts General Hospital and Harvard Medical School, Charlestown, MA, United States, ${ }^{2}$ Department of Neurosurgery, Brigham \& Women's Hospital and Harvard Medical School, Boston, MA, United States, ${ }^{3}$ Department of Biomedical Engineering, Michigan State University, East Lansing, MI, United States, ${ }^{4}$ The Institute of Quantitative Health Science and Engineering, Michigan State University, East Lansing, MI, United States, ${ }^{5}$ Department of Radiology, Michigan State University, East Lansing, MI, United States

The ability to image cell- or viral-based therapeutics is critical for optimizing therapeutic strategies and assessing efficacy. A lysine rich protein (LRP) chemical exchange saturation transfer (CEST) MRI reporter gene has previously been developed and successfully used to image oncolytic viruses and tumor cells. However, the highly repetitive nature of the LRP reporter gene sequence lead to DNA recombination events and the expression of a range of truncated LRP protein fragments, thereby greatly limiting the CEST sensitivity. Here we report the use of a redesigned LRP reporter (rdLRP), which demonstrated excellent stability and CEST sensitivity. 
Magnetic Resonance Tracking of Iron-Labeled Stem Cells After Osteochondral Defect in Ovine Model

Joshua Kaggie ${ }^{1,2}$, Martin J Graves ${ }^{1,2}$, James MacKay ${ }^{1,2}$, Scott Reid ${ }^{3}$, Hareklea Markides ${ }^{4}$, Alicia El Haj ${ }^{4}$, Stephen McDonnell ${ }^{2,5}$, Fiona J Gilbert ${ }^{1,2}$, Andrew McCaskie ${ }^{2,5}$, and Frances Henson $^{6}$

${ }^{1}$ Radiology, University of Cambridge, Cambridge, United Kingdom, ${ }^{2}$ Addenbrooke's Hospital, Cambridge University Hospitals NHS Foundation Trust, Cambridge, United Kingdom, ${ }^{3}$ GE Healthcare, Amersham, United Kingdom, ${ }^{4}$ Institute of Science and Technology in Medicine, Keele University, Newcastle, United Kingdom, ${ }^{5}$ Division of Trauma and Orthopaedic Surgery, University of Cambridge, Cambridge, United Kingdom, ${ }^{6}$ Veterinary Medicine, University of Cambridge, Cambridge, United Kingdom

Multipotent mesenchymal stem cells (MSCs) can be labeled with superparamagnetic iron-oxide nanoparticles (SPION) particles to track single cells with MRI, and thereby follow MSC infiltration. However, a limitation with conventional MR sequences is that their long echo times are unable to measure fast signal decays, which occur in dense bone tissue and with high SPION infiltration. Ultra-short echo time (UTE) MRI can capture these rapidly decaying signals. In this work, we use 3D cones to track tissue development after injection of SPION labeled MSCs in an ovine model.

Mapping of spatial distribution of the olfactory bulb new neurons at single cell level using iron oxide assisted-MRI

Nikorn Pothayee ${ }^{1}$, Claire Perez ${ }^{2}$, Stephen Dodd ${ }^{1}$, and Alan P. Koretsky ${ }^{1}$

$3050 \quad{ }^{1}$ National Institutes of Health, Bethesda, MD, United States, ${ }^{2}$ University of Guam, Mangiloa, Guam

In this study, we aimed to develop a method that could quantitatively track new neurons in the olfactory bulb (OB). We first established that MRI signals detected in the OB were those of single labeled new neurons that migrate from the neurogenic niche into the OB. Further, we combined the anatomical MRI enhancing properties of $\mathrm{Mn}^{2+}$ to evaluate the preference of new neurons for specific layers within the $\mathrm{OB}$ and to determine whether sensory enrichment affects distribution of adult-born neurons within the $\mathrm{OB}$ layers.

A Carbon-Fibre Sheet Resistor for MR, CT, SPECT and PET-compatible Temperature Maintenance in Small Animals

Veerle Kersemans ${ }^{1}$, Stuart Gilchrist ${ }^{1}$, Philip Danny Allen ${ }^{1}$, Paul Kinchesh ${ }^{1}$, and Sean Smart ${ }^{1}$

${ }^{1}$ University of Oxford, CRUK/MRC Oxford Institute for Radiation Oncology, Oxford, United Kingdom

A resistive heater that is compatible with MR, CT, SPECT and PET imaging has been produced from a commercially available carbon-fibre sheet. Adequacy of temperature maintenance and insensitivity of MR and CT imaging to the presence and use of the heater is shown. Multimodal MR-CT-PET-SPECT imaging of the lower abdomen is demonstrated in vivo in the physiologically maintained and viable anaesthetised mouse.

Traditional Poster

\section{Hyperpolarised MR}

Application of a novel $13 \mathrm{C}$ hyperpolarized metabolic tracer for $\mathrm{y}$-Glutamyl transferase activity in vivo tumor xenograft

Tomohiro Seki ${ }^{1}$, Marino Itoda $^{2}$, Shun Kishimoto ${ }^{1}$, Kazu Yamamoto ${ }^{1}$, Yoichi Takakusagi ${ }^{3}$, Jeffery Brender ${ }^{1}$, Ronja M. Malinowski ${ }^{4}$, Tatsuya Nishihara ${ }^{2}$, Hikari A. I. Yoshihara ${ }^{5}$, Hiroshi Nonaka $^{2}$, Keita Saito ${ }^{1}$, Nobu Oshima ${ }^{1}$, Jan H. Ardenkjaer-Larsen ${ }^{4}$, James B. Mitchell ${ }^{1}$, Murali C. Krishna ${ }^{1}$, and Shinsuke Sando ${ }^{2}$

${ }^{1}$ Radiation Biology Branch, CCR, NCl, NIH, Bethesda, MD, United States, ${ }^{2}$ Department of Chemistry and Biotechnology, Graduate School of Engineering, UT, Bunkyo-ku, Tokyo, Japan, ${ }^{3}$ Department of Molecular Imaging \& Theranostics, QST, Chiba-shi, Japan, ${ }^{4}$ Electrical Engineering, Department of Electrical Engineering, DTU, Lyngby, Denmark, ${ }^{5}$ Institute of Physics of Biological Systems, EPFL, Lausanne, Swaziland

This research aimed to develop the non-invasive in vivo detection of $\mathrm{y}$-glutamyl transferase (GGT) activity by a novel GGT molecular probe, $\mathrm{y}$-Glu-[1- $\left.{ }^{13} \mathrm{C}\right] \mathrm{Gly}$, in combination with hyperpolarized (HP) ${ }^{13} \mathrm{C}$ Magnetic Resonance (MR) spectroscopy. We succeed in detecting the strong HP ${ }^{13} \mathrm{C}$ MR signal of $\mathrm{Y}$-Glu- $\left[1-{ }^{13} \mathrm{C}\right] \mathrm{Gly}$ from tumor xenograft in vivo. Detecting HP ${ }^{13} \mathrm{C}$ MR signal from the metabolite of this novel probe in tumor xenograft is our next challenge.

Variable Resolution Echo-Planar Imaging for Improved Quantification of Hyperpolarized 13C Metabolism

Jeremy W Gordon ${ }^{1}$, Eugene Milshteyn ${ }^{1}$, Daniel B Vigneron ${ }^{1}$, and Peder EZ Larson ${ }^{1}$ 
Unlike ionizing imaging modalities, the SNR in MRI is proportional to voxel volume, but downsampling or voxel averaging after acquisition only improves SNR by the square root of the voxel volume. To take advantage of this distinction, we use a frequency selective imaging approach to independently excite the hyperpolarized ${ }^{13} \mathrm{C}$ substrate (pyruvate) and downstream metabolites (lactate, alanine, and bicarbonate). This allows us to tailor the spatial resolution for each metabolic product, yielding high-resolution images for pyruvate as well as quantification at a coarser resolution for the lower SNR metabolites, such as bicarbonate, which would be undetectable at the higher resolution.

Dynamic Metabolic Imaging of Co-Polarized [2-13C]Pyruvate and [1,4-13C2]Fumarate Using 3D-Spiral CSI with Alternate Spectral Band Excitation

Maninder Singh ${ }^{1}$, Sonal Josan ${ }^{2}$, and Dirk Mayer ${ }^{1}$

${ }^{1}$ Diagnostic Radiology and Nuclear Medicine, University of Maryland, Baltimore, MD, United States, ${ }^{2}$ Siemens Healthcare, Erlangen, Germany

Metabolic imaging of biologically-relevant hyperpolarized agents allows measurement of metabolic processes in real time in-vivo. We demonstrate dynamic metabolic imaging of a single bolus of co-polarized $\left[2-{ }^{13} \mathrm{C}\right]$ pyruvate and $\left[1,4-{ }^{13} \mathrm{C}\right.$ ] fumarate in control as well as in rats with liver necrosis. Chemical shift imaging (CSI) of such a mixture is challenging due to the large spectral dispersion of resulting resonances, which could lead to severe chemical shift displacement artifacts if acquired by conventional slice-selective excitation pulses. Here we obtain CSI information by a volumetric method using alternate 3D spectrally-selective excitation of sub-bands containing fewer resonances.

Simple and fast hyperpolarization of a biomolecule: Theory and Experiment

Stephan Berner ${ }^{1,2,3}$, Stephan Knecht ${ }^{1,4}$, Andreas Benjamin Schmidt ${ }^{1,4}$, Mirko Zimmermann ${ }^{1}$, Jürgen Hennig ${ }^{1}$, Dominik von Elverfeldt ${ }^{1}$, and Jan-Bernd Hövener ${ }^{4}$

${ }^{1}$ Department of Radiology, Medical Physics, Medical Center-University of Freiburg, Faculty of Medicine, University of Freiburg, Freiburg, Germany, ${ }^{2}$ DKTK, Freiburg, Germany, ${ }^{3}$ DKFZ, Heidelberg, Germany, ${ }^{4}$ Department of Radiology and Neuroradiology, Section Biomedical Imaging, MOIN CC University Medical Center Schleswig-Holstein, University of Kiel, Kiel, Germany

Hyperpolarization overcomes the biggest limitation of MRI: its low sensitivity, and enables metabolite mapping. Hyperpolarized ${ }^{13} \mathrm{C}$ magnetization can be produced by transferring the spin order of parahydrogen into ${ }^{13} \mathrm{C}$ by hydrogenation followed by a sequence of ${ }^{1} \mathrm{H}$ and ${ }^{13} \mathrm{C}$ pulses. However, it is possible to hyperpolarize $\mathrm{AA}$ ' $\mathrm{X}$ spin systems by two pulses on ${ }^{13} \mathrm{C}$. Theoretical models were developed to describe the polarization transfer and significant signal increase was observed for the biomolecule succinate after spin order transfer directly in the magnet of a commercial MRI system. The experimental data is well described by theoretical calculations except for an overall scaling factor.

Ex-vivo real-time measurement of ethanol induced changes in brain metabolism of hyperpolarized [1-13C]-pyruvate

Benjamin Grieb ${ }^{1}$, Assad Azar ${ }^{1}$, Talia Harris ${ }^{1}$, Gal Sapir ${ }^{1}$, Atara Nardi-Schreiber ${ }^{1}$, Ayelet Gamliel ${ }^{1}$, Sivaranjan Uppala ${ }^{1}$, Jacob Sosna ${ }^{1}$, J. Moshe Gomori ${ }^{1}$, and Rachel Katz-Brull ${ }^{1}$

${ }^{1}$ Department of Radiology, Hadassah-Hebrew University Medical Center, Jerusalem, Israel

Changes in brain metabolism during acute alcohol intoxication either reflect global inhibition or changes in the utilized energy substrates. NMR spectroscopy of hyperpolarized metabolites offer the opportunity to investigate the metabolic changes due to alcohol intoxication in the brain in real time. Here we present preliminary data showing decreased [113C]lactate formation from hyperpolarized [1-13C]pyruvate in perfused and ethanol incubated rat brain slices. This approach may offer a future innovative tool to non-invasively image brain metabolism in real-time during alcohol intoxication.

Impurities of $\left[1-{ }^{13} \mathrm{C}\right]$ pyruvic acid and their potential effects on the interpretation of hyperpolarized pyruvate metabolism studies

Talia Harris ${ }^{1}$, Ayelet Gamliel ${ }^{1}$, Jacob Sosna ${ }^{1}$, J. Moshe Gomori ${ }^{1}$, and Rachel Katz-Brull ${ }^{1}$

${ }^{1}$ Hadassah-Hebrew University Medical Center, Jerusalem, Israel

Commercially available $\left[1-{ }^{13} \mathrm{C}\right]$ pyruvic acid contains impurities that have chemical shifts similar to pyruvate's metabolic products. We show that these observed impurity peaks possess long $\mathrm{T}_{1} \mathrm{~s}$ and for several peaks the chemical shift is very sensitive to the $\mathrm{pH}$ in the narrow physiological range measured. We concluded that in order to reliably identify low concentration metabolic products of hyperpolarized pyruvate it is crucial to characterize in situ the $\mathrm{pH}$ dependent impurity spectrum of the batch of $\left[1-{ }^{13} \mathrm{C}\right] \mathrm{pyruvic}$ acid used. 
Translating the hyperpolarized signal observed in the brain in vivo to cerebral metabolic rates is not straightforward, as the observed signals reflect also the influx of metabolites produced in the body, the cerebral blood volume and flow, and the rate of transport across the blood brain barrier. We introduce a robust method to study rapid metabolism of hyperpolarized substrates ex vivo in viable rat brain slices and demonstrate its ability to characterize rates of LDH and PDH activities. Despite variations in these measured rates, we saw that the Lactate to Bicarbonate ratio is highly reproducible across all samples.

In vivo hyperpolarization transfer in a clinical MRI scanner

Cornelius von Morze ${ }^{1}$, Galen D. Reed ${ }^{2}$, Peder E. Larson ${ }^{1}$, Daniele Mammoli ${ }^{1}$, Albert P. Chen ${ }^{2}$, James Tropp ${ }^{3}$, Mark Van Criekinge ${ }^{1}$, Michael A. Ohliger ${ }^{1}$, John Kurhanewicz ${ }^{1}$, Daniel B. Vigneron ${ }^{1}$, and Matthew E. Merritt ${ }^{4}$

${ }^{1}$ Department of Radiology \& Biomedical Imaging, UCSF, San Francisco, CA, United States, ${ }^{2}$ GE Healthcare, San Francisco, CA, United States, ${ }^{3}$ Berkshire Magnetics, Berkeley, CA, United States, ${ }^{4}$ Department of Biochemistry, University of Florida, Gainesville, Gainesville, FL, United States

The purpose of this study was to investigate the feasibility of in vivo ${ }^{13} \mathrm{C}->{ }^{1} \mathrm{H}$ hyperpolarization transfer, which has significant potential advantages for detecting the distribution and metabolism of hyperpolarized ${ }^{13} \mathrm{C}$ probes, in a clinical MRI scanner. A standalone pulsed ${ }^{13} \mathrm{C} \mathrm{RF}$ transmit channel was developed for operation in conjunction with the standard ${ }^{1} \mathrm{H}$ channel of a clinical 3T MRI scanner. Operation of the custom pulsed ${ }^{13} \mathrm{C}$ RF channel resulted in effective ${ }^{13} \mathrm{C}$ - ${ }^{1} \mathrm{H}$ hyperpolarization transfer, as confirmed by the characteristic antiphase appearance of ${ }^{1} \mathrm{H}$-detected, ${ }^{1} \mathrm{~J} \mathrm{CH}$-coupled doublets. ${ }^{1} \mathrm{H}$ detection of $\mathrm{HP}\left[2-{ }^{13} \mathrm{C}\right]$ lactate generated in vivo was achieved in a rat liver slice.

Novel Metabolic Markers for Therapeutic Approaches Targeting Serine Synthesis Pathway in Leukemia

Sangmoo Jeong ${ }^{1,2}$, Madeleine A. Gao ${ }^{3}$, Alexandra Schurer ${ }^{2}$, Nathaniel T. Kim ${ }^{1,2}$, Yuanming Cheng ${ }^{2}$, Roozbeh Eskandari ${ }^{1,2}$, Michael G. Kharas ${ }^{2,4}$, and Kayvan R. Keshari ${ }^{1,2,4}$

${ }^{1}$ Department of Radiology, Memorial Sloan Kettering Cancer Center, New York, NY, United States, ${ }^{2}$ Molecular Pharmacology Program, Memorial Sloan Kettering Cancer Center, New

The serine synthesis pathway (SSP), which provides precursors for redox homeostasis and nucleotide synthesis, has emerged as a critical metabolic pathway in cancer. However, the assessment of therapeutic approaches targeting the SSP has been challenging due to a lack of distinct biomarkers. We have identified that the SSP inhibition increases reactive oxygen species (ROS) levels and, intriguingly, glycolytic rate in leukemia cells. Using hyperpolarized dehydroascorbate and pyruvate magnetic resonance, we assessed therapeutic responses earlier than any significant changes in cell viability. This approach has broad implications as an effective methodology for monitoring therapeutic responses with SSP inhibition in multiple cancers.

In vitro and in vivo $13 \mathrm{C}$ metabolic imaging of pyruvate to lactate conversion with high spatial and temporal resolution using a me-bSSFP sequence

Christoph Alexander Müller ${ }^{1,2}$, Christian Hundshammer ${ }^{3}$, Miriam Braeuer $^{3}$, Jason Graham Skinner ${ }^{1}$, Adam Espe Hansen ${ }^{4}$, Sven Mansson ${ }^{5}$, Franz Schilling ${ }^{3}$, Jochen Leupold ${ }^{1}$, Dominik von Elverfeldt ${ }^{1}$, Jan Henrik Ardenkjaer-Larsen ${ }^{6}$, Markus Schwaiger ${ }^{3}$, Jürgen Hennig ${ }^{1}$, and Jan-Bernd Hövener ${ }^{7}$

${ }^{1}$ Department of Radiology, Medical Physics, Medical Center - University of Freiburg, Faculty of Medicine, University of Freiburg, Freiburg, Germany, ${ }^{2}$ German Consortium for Translational Cancer Research (DKTK), Partnersite Freiburg, German Center for Cancer Research (DKFZ), Heidelberg, Germany, ${ }^{3}$ Department of Nuclear Medicine, Klinikum rechts der Isar, Technical University of Munich, München, Germany, ${ }^{4}$ Department of Clinical Physiology, Nuclear Medicine \& PET, Rigshospitalet, University of Copenhagen, Copenhagen, Denmark, ${ }^{5}$ Medical Radiation Physics, Malmö University Hospital, Lund University, Malmö, Sweden, ${ }^{6}$ Department of Electrical Engineering, Technical University of Denmark, Copenhagen, Denmark, ${ }^{7}$ Section for Biomedical Imaging, Department of Radiology and Neuroradiology, University Medical Center Schleswig-Holstein, University of Kiel, Kiel, Germany

In order to use the transient signal of hyperpolarized tracers and their metabolites efficiently, dedicated imaging sequences are required. Here, we present a multi-echo $b S S F P$ sequence with Dixon-based iterative reconstruction to obtain metabolite maps of hyperpolarized $\left[1-{ }^{13} \mathrm{C}\right]$ pyruvate and the product of an enzymatic conversion $\left[1-{ }^{13} \mathrm{C}\right]$ lactate on a human $3 \mathrm{~T}$ PETMRI system in vitro and in vivo. When comparing to other methods (i.e. CSI and non-localized NMR spectra) we found that me-bSSFP provides good metabolite separation and reliable quantitative kinetic data more than 16 times faster than CSI (350 ms vs. $5.8 \mathrm{~s}$ ), while consuming a similar amount of hyperpolarized magnetization.

In Vivo Spectroscopic Detection of Arginase Enzyme Activity with Hyperpolarized [6-13C,15N3]-Arginine

Andrew $\mathrm{Cho}^{1}$, Roozbeh Eskandari ${ }^{2}$, and Kayvan Keshari ${ }^{2}$

${ }^{1}$ Weill Cornell Graduate School, New York, NY, United States, ${ }^{2}$ Memorial Sloan Kettering Cancer Center, New York, NY, United States

Aberrations in arginase enzyme expression are associated with a variety of pathologies, and an in vivo probe to quantify flux through this pathway may hold utility towards patient stratification. We propose the use our custom synthesized compound, $\left[6-{ }^{13} \mathrm{C},{ }^{15} \mathrm{~N}_{3}\right]$-arginine, as a hyperpolarized MRI probe for arginase activity. ${ }^{15} \mathrm{~N}$ enrichment reduces quadrupolar relaxation and extends $T_{2}$, facilitating in vivo imaging. We were able to acquire ${ }^{13} \mathrm{C}$ spectroscopic data on a healthy mouse and detected in vivo conversion of hyperpolarized arginine to urea, which warrants further exploration of this imaging probe in the future. 
Thomas Theis ${ }^{1}$, Johannes Colell ${ }^{1}$, Zijian Zhou ${ }^{1}$, Shannon Eriksson ${ }^{2}$, Jacob Lindale ${ }^{1}$, Yi-Fen Yen ${ }^{3}$, Matthew Rosen ${ }^{3,4}$, Eduard Chekmenev ${ }^{5}$, and Warren Warren ${ }^{6}$

${ }^{1}$ Chemistry, Duke University, Durham, NC, United States, ${ }^{2}$ Medicine, Duke University, Durham, NC, United States, ${ }^{3}$ A. A. Martinos Center for Biomedical Imaging, Massachsetts General Hospital, Charlestown, MA, United States, ${ }^{4}$ Physics, Harvard University, Cambridge, MA, United States, ${ }^{5}$ Vanderbilt Univesity Institute for Imaging Science, Nashville, TN, United States, ${ }^{6}$ Physics, Chemistry, BME, Radiology, Duke University, Durham, NC, United States

NMR and MRI are inherently low sensitivity techniques. Hyperpolarization technology overcomes this problem by enhancing MR signals by 10,000 -fold or more. However, most hyperpolarization techniques are complex, expensive and slow. We describe hyperpolarization chemistry that is simple, low-cost, and fast or even continuous. Specifically, we describe recent advances in parahydrogen-induced polarization, combined with various MR detection schemes to establish 1) miniaturized NMR spectrometers, 2) NMR structural elucidation with reduced limits of detection, and 3 ) low-cost biomolecular imaging.

Boosting SABRE-SHEATH hyperpolarization with Coherent Control of Spin Dynamics

Thomas Theis ${ }^{1}$, Shannon Eriksson ${ }^{1}$, Johannes Colell ${ }^{1}$, Zijian $^{2}$ hou ${ }^{1}$, Jacob Lindale ${ }^{1}$, and Warren Warren ${ }^{2}$

${ }^{1}$ Chemistry, Duke University, Durham, NC, United States, ${ }^{2}$ Physics, Chemistry, BME, Radiology, Duke University, Durham, NC, United States

Signal Amplification By Reversible Exchange (SABRE) is a parahydrogen based hyperpolarization modality that is particularly simple, low-cost, and fast or even continuous. A more recent variant, SABRE-SHEATH (SABRE in SHield Enables Alignment Transfer to Heteronuclei) enables targeting ${ }^{15} \mathrm{~N}$ and ${ }^{13} \mathrm{C}$ nuclei in a wide range of substrates, where hyperpolarization lifetimes can be particularly long. However, both SABRE and SABRE-SHEATH are limited by the incoherent nature of the hyperpolarization transfer process. Here we describe a pulsed variant of SABRE-SHEATH that takes coherent control over the spin dynamics and more than doubles achievable hyperpolarization levels. In addition, the pulsed SABRE-SHEATH experiments provide a new way of probing the hyperpolarization transfer, shedding new light on the limiting factors of this emerging technology

Super-resolution Hyperpolarized C13 Imaging with 2D-Linear Prediction and Trigonometric Curves

Jack J J J Miller ${ }^{1,2,3}$, Sofia Dimoudi ${ }^{1}$, Aaron Hess ${ }^{1}$, and Damian J Tyler ${ }^{1,3}$

${ }^{1}$ Oxford Centre for Clinical Magnetic Resonance Research, University of Oxford, Oxford, United Kingdom, ${ }^{2}$ Department of Physics, University of Oxford, Oxford, United Kingdom,

${ }^{3}$ Department of Physiology, Anatomy and Genetics, University of Oxford, Oxford, United Kingdom

Hyperpolarized $\$ \$ \$ \wedge\{13\}$ text $\{C\} \$ \$ \$$-imaging techniques a powerful and clinically translatable method to image metabolism. However, owing to the finite and non-renewable magnetisation available to the technique, all proposed imaging sequences necessarily have a comparatively small matrix size compared to conventional anatomical imaging. Typically hyperpolarized images are therefore reconstructed with a large degree of zero-filling. We show here that a modified form of $2 \mathrm{D}$ least-squares linear prediction that uses the known analytic properties of trigonometric curves can extrapolate unmeasured Fourier coefficients and hence improve the apparent reconstructed resolution of hyperpolarized images.

Assessing Y-glutamyl transpeptidase activity in kidney using hyperpolarized $\mathrm{Y}$-Glu-[1-13C]Gly

Steffen F. Frank ${ }^{1}$, Hikari A. I. Yoshihara ${ }^{1}$, Marino Itoda $^{2}$, Shinsuke Sando ${ }^{2}$, and Rolf Gruetter $3,4,5$

${ }^{1}$ Laboratory for Functional and Metabolic Imaging, EPFL, Lausanne, Switzerland, ${ }^{2}$ Department of Chemistry and Biotechnology, The University of Tokyo, Tokyo, Japan, ${ }^{3}$ Department of Radiology, University of Geneva, Geneva, Switzerland, ${ }^{4}$ Department of Radiology, University of Lausanne, Lausanne, Switzerland, ${ }^{5}$ Centre for Biomedical Imaging, EPFL, Lausanne, Switzerland

Hyperpolarized $\mathrm{y}$-Glu-[1-13 $\mathrm{C}]$ Gly provides a non-invasive means to detect $\mathrm{y}$-glutamyl transpeptidase (GGT) enzyme activity in vivo and indicates its potential for application in functional imaging. Since GGT is most abundant in the proximal tubules of the kidney, and since the properties of $\mathrm{Y}-\mathrm{Glu}-\left[1-{ }^{13} \mathrm{C}\right] \mathrm{Gly}$ are suitable for in vivo hyperpolarized ${ }^{13} \mathrm{C}$ metabolic analysis, it was proposed as a molecular probe to study kidney function. The aim of the present study is to identify the dose of $\mathrm{Y}$-Glu-[1- $\left.{ }^{13} \mathrm{C}\right] \mathrm{Gly}$ that gives high NMR sensitivity in the unsaturated state of the GGT enzyme.

Dynamic Hyperpolarized 13C MRSI using the SPICE technique: A feasibility study

JaeEun Song ${ }^{1}$, Jaewook Shin ${ }^{1}$, Hansol Lee ${ }^{1}$, Young-suk Choi ${ }^{2}$, Chan Gyu Joo ${ }^{3}$, Ho-Taek Song ${ }^{2}$, and Dong-Hyun Kim ${ }^{1}$

${ }^{1}$ Department of Electrical and Electronic Engineering, Yonsei University, Seoul, Republic of Korea, ${ }^{2}$ Yonsei University College of Medicine, Seoul, Republic of Korea, ${ }^{3}$ Yonsei Biomedical Science Institute, College of Medicine, Yonsei University, Seoul, Republic of Korea

In this study, we investigated the use of SPICE (SPectroscopic Imaging by exploiting spatiospectral CorrElation) technique for dynamic hyperpolarized ${ }^{13} \mathrm{C}$ MRSI by in vitro phantom experiment and in vivo mouse experiment. In vitro phantom experiment, the dynamic images from SPICE were compared to the dynamic data from FIDCSI. In vivo experiment, the dynamic images were acquired in normal and high fat diet (HFD) mouse kidney. 
Non-invasive redox molecular imaging of atopic dermatitis using in vivo dynamic nuclear polarization MRI

Fuminori Hyodo ${ }^{1,2}$, Hinako Eto ${ }^{2}$, Gaku Tsuji ${ }^{2}$, Masutaka Furue ${ }^{2}$, and Masayuki Matsuo ${ }^{1}$

Atopic dermatitis $(A D)$ is a chronic inflammatory condition with complex etiology. Redox imbalance caused by excessive oxidative stress has been shown to mediate disease activity of $A D$. We have established such a technique that can detect and visualize the redox status of the skin using in vivo dynamic nuclear polarization(DNP) MRI. We utilized an AD mouse model that was generated by repeated topical application of mite antigen in NC/Nga mice. We revealed that AD skin lesions demonstrated more rapid reduction rates of image intensity than normal skin, indicating that our technique can monitor oxidative stress in $A D$ skin.

Monitoring effect of rapamycin on pyruvate metabolism in SCC tumor using hyperpolarized 13C-MRI

Keita Saito ${ }^{1}$, Shingo Matsumoto ${ }^{1,2}$, Yoichi Takakusagi ${ }^{1,3}$, Masayuki Matsuo ${ }^{1,4}$, Hellmut Merkle ${ }^{5}$, James B Mitchell ${ }^{1}$, and Murali C Krishna ${ }^{1}$

${ }^{1}$ National Cancer Institute, Bethesda, MD, United States, ${ }^{2}$ Hokkaido University School of Engineering, Sapporo, Japan, ${ }^{3}$ National Institutes for Quantum and Radiological Science and Technology, Chiba, Japan, ${ }^{4}$ Gifu University School of Medicine, Gifu, Japan, ${ }^{5}$ National Institute of Neurological Disorder and Stroke, Bethesda, MD, United States

Effect of an mTOR inhibitor rapamycin on pyruvate metabolism in squamous cell carcinoma (SCC) xenografts was investigated using hyperpolarized ${ }^{13} \mathrm{C}-\mathrm{MRI}$. $\left[1-{ }^{13} \mathrm{C}\right]$ lactate to $\left[1-{ }^{13} \mathrm{C}\right]$ pyruvate ratio $(\mathrm{Lac} / \mathrm{Pyr})$ in the SCC tumors increased as tumor grew in non-treated control mice, whereas it significantly dropped after 2 days of the rapamycin treatment. Inhibition of mTOR caused a drop of LDH protein level and the activity in the SCC tumor, and perfusion in the tumor was improved by the rapamycin treatment. Lac/Pyr monitored using hyperpolarized ${ }^{13} \mathrm{C}-\mathrm{MRI}$ would become a useful marker for tumor response to mTOR inhibitors.

Multiscale Imaging of Breast Cancer Metabolism using Fluorescence Lifetime Imaging Microscopy and Hyperpolarized Magnetic Resonance Spectroscopy

Sarah J. Erickson-Bhatt ${ }^{1}$, Ben Cox $^{2}$, Erin Adamson ${ }^{2}$, Suzanne Ponik² ${ }^{2}$ Matthew Conklin², Brett Morris², David Inman², Joseph Szulczewski², Patricia Keely², M. Elizabeth Meyerand², Caroline Alexander ${ }^{2}$, Kevin Eliceiri' ${ }^{2}$, and Sean Fain ${ }^{2}$

${ }^{1} 1111$ Highland Ave., University of Wisconsin, Madison, WI, United States, ${ }^{2}$ University of Wisconsin, Madison, WI, United States

Every day in the U.S. 100 women die of metastatic breast cancer. Current clinical methods cannot determine from the primary site which tumors will metastasize and spread to other areas of the body. Herein, multiple imaging scales are used to assess the metabolic signatures of metastatic and dormant tumor cell lines. Fluorescence lifetime imaging microscopy (FLIM) and hyperpolarized magnetic resonance spectroscopy (hMRS) imaging studies are performed in 3D cell culture using an MRI compatible bioreactor and in vivo mouse models to evaluate metabolic signatures at the individual cellular and tumor mass scales to predict metastasis versus dormancy.

Comparison of Asymmetric and Symmetric K-space Sampling in EPI for 3D Time-Resolved Hyperpolarized 13C MRI with [1-13C]Pyruvate

Benjamin J. Geraghty ${ }^{1,2}$, Casey Y. Lee ${ }^{1,2}$, Albert P. Chen ${ }^{3}$, and Charles H. Cunningham ${ }^{1,2}$

${ }^{1}$ Department of Medical Biophysics, University of Toronto, Toronto, ON, Canada, ${ }^{2}$ Physical Sciences, Sunnybrook Research Institute, Toronto, ON, Canada, ${ }^{3}$ GE Healthcare, Toronto, ON, Canada

The minimum echo-time for hyperpolarized ${ }^{13} \mathrm{C}$ echo-planar imaging can be reduced with partial sampling along the blipped direction in k-space. To investigate the extent to which echotime shortening can improve signal-to-noise ratio, we've employed an experimental design that toggles between two different spatial encoding strategies during a time-resolved hyperpolarized $\left[1{ }^{13} \mathrm{C}\right]$ pyruvate acquisition. Using clinically approved hardware with a pre-clinical animal model, we compared symmetric with asymmetric echo-planar imaging. Considerable signal-to-noise ratio gains for asymmetric vs symmetric sampling were observed without artifacts. On the basis of this study, our group will employ asymmetric sampling in our forthcoming human trials.

Hyperpolarized $13 \mathrm{C}$ chemical shift imaging of transient focal ischemia reperfusion injury in developing rat brain

Shu-Juan Fan ${ }^{1}$, Amara Larpthaveesarp ${ }^{2}$, Yiran Chen $^{1,3}$, Sukumar Subramaniam ${ }^{1}$, Robert Bok ${ }^{1}$, Fernando Gonzalez ${ }^{2}$, and Duan Xu ${ }^{1}$

${ }^{1}$ Dept. of Radiology and Biomedical Imaging, University of California San Francisco, San Francisco, CA, United States, ${ }^{2}$ Dept. of Pediatrics, University of California San Francisco, San Francisco, CA, United States, ${ }^{3}$ Joint UCSF/UC Berkeley Graduate Group in Bioengineering, San Francisco, CA, United States

We investigated the use of hyperpolarized $\left[1-{ }^{13} \mathrm{C}\right]$ pyruvate magnetic resonance chemical shift imaging in monitoring energy metabolism in developing rat brain following transient focal ischemia-reperfusion injury, which is the most common form of stroke in neonates. We show that the conversion from $\left[1-{ }^{13} \mathrm{C}\right]$ pyruvate to $\left[1-{ }^{13} \mathrm{C}\right]$ lactate was higher in the injured cerebral hemisphere as compared with that in the contralateral hemisphere, which lasted for up to 7 days after the ischemia-reperfusion injury. This phenomenon can be potentially used as a biomarker to facilitate long-term prognosis, characterize therapeutic responses and study the mechanisms of injury repair in neonates with transient focal ischemic stroke. 
Quantitative Data Analysis of in vivo Hyperpolarized 13C NMR Data: 1D vs 2D

Frits H.A. van Heijster ${ }^{1}$, Ron de Beer ${ }^{2}$, Arend Heerschap ${ }^{1}$, and Dirk van Ormondt ${ }^{2}$

3073

${ }^{1}$ Radiology and Nuclear Medicine, Radboud University Medical Center, Nijmegen, Netherlands, ${ }^{2}$ Applied Physics, TU Delft, Delft, Netherlands

${ }^{13} \mathrm{C}$-DNP hyperpolarization (HP) in MR allows for single shot detection of ${ }^{13} \mathrm{C}$-labeled metabolites in vivo. The dynamic acquisition of ${ }^{13} \mathrm{C}$ MR signals after injection of a HP ${ }^{13} \mathrm{C}$-substrate results in a two-dimensional time-domain dataset. Often the 1D NMR time domain is fitted first and the results are fed into a kinetic model. We present a 2D method, in which all data points in both NMR and kinetic time dimensions are fitted simultaneously. This results in an improved accuracy for all determined kinetic parameters compared to the 1D method, in particular for low-SNR metabolites. CRBs are significantly smaller using $2 \mathrm{D}$ analysis.

HyperSIFT: Temoporal Denoising of Hyperpolarized Data Improves SNR while Perserving Dynamic Information

Kristin L Granlund ${ }^{1,2}$ and Kayvan R Keshari²,3

${ }^{1}$ Radiology, Memorial Sloan Kettering Cancer Center, New York, NY, United States, ${ }^{2}$ Molecular Pharmacology, Memorial Sloan Kettering Cancer Center, New York, NY, United States, ${ }^{3}$ Weill Cornell Medical College, New York, NY, United States

We apply a temporal denoising algorithm to hyperpolarized MRI. The SIFT method filters out temporal frequencies with low amplitudes, reducing noise while preserving dynamic information. We demonstrate this in a bioreactor setting, introducing hyperpolarized [1-13C] pyruvate to cells and human subjects and observing the conversion of pyruvate to lactate. 